\title{
8th World Congress on Developmental Origins of Health and Disease
}

\section{DOHaD13-1362}

Identification of micrornas of the mammary gland associated with milk production of dairy cows

Kuljeet Singh ${ }^{1, *}$, Paul H Maclean ${ }^{1}$, Regan Murney ${ }^{1}$, Kim Oden ${ }^{1}$, Kerst Stelwagen ${ }^{2}$

${ }^{1}$ Ruakura Research Centre, AgResearch Ltd; ${ }^{2}$ SciLactis Ltd, Waikato Innovation Park, Hamilton, New Zealand

Background: A potential role of epigenetic mechanisms in manipulation of mammary function in the dairy cow is emerging. Short-term changes of milking frequency in early lactation can have an immediate and a long term effect on milk yield. The effect is controlled locally within mammary glands and is thought to be a function of secretory mammary epithelial cell number and/or activity, but the definitive mechanism remains unknown. The aim of this study was to identify microRNAs (miRNAs) which may be involved in the control of milk production within the bovine mammary gland.

Method: Ten multiparous cows $(5 \pm 2$ postpartum (pp)) were unilaterally milked for 14 days; udder halves were either milked once a day (1x) or four times a day (4x). On day 14 , mammary tissue was collected from both udder halves and total RNA extracted. The cows were then milked twice daily (2x) for the remainder of lactation ( $\sim 200$ days pp). By the end of the treatment period the $4 \mathrm{x}$-milked udder halves were producing 2 -fold $(\mathrm{P}<0.001)$ more milk than the $1 \mathrm{x}$-milked udder halves and continued to produce $15 \%(\mathrm{P}<0.01)$ more milk than $1 \mathrm{x}$-udder halves once returned to $2 \mathrm{x}$ milking. Next Generation Sequencing was used to enumerate miRNAs present in both $4 \mathrm{x}$ and $1 \mathrm{x}$ udder halves from 4 cows.

Results: A number of miRNAs were found to be higher in the $4 \mathrm{x}$ samples, including miR-125a, miR-125b, miR-99b, miR-100.

Conclusion: These miRNAs have been shown to regulate genes important to mammary gland function including mTOR and TNF-alpha.

Disclosure of Interest: None Declared.

Key words: None.

\section{DOHaD13-1319}

Milk leptin and IGF1 in early lactating sows according to body composition and mammary gland position

Latifa Abdennebi-Najar ${ }^{1, *}$, Marie Daupley ${ }^{2}$, Samir DOU ${ }^{1}$, Jean Yves Madec ${ }^{2}$, Claude-Narcisse NIAMBA ${ }^{2}$, Duane H Keisler ${ }^{3}$
${ }^{1}$ Institut Polytechnique LaSalle, UPSP EGEAL; ${ }^{2}$ Institut Polytechnique LaSalle, Beauvais, France; ${ }^{3}$ Division of Animal Sciences, University of Missouri, Missouri, United States

Background: The presence of leptin and IGF1 in colostrum and milk can influence physiological development of suckling IUGR neonates and affect their predisposition to obesity and metabolic syndrome. The purpose of this study was to investigate the relationship between leptin and IGF1 in colostrum and milk secreted from sow mammary gland in relation to sow body composition and mammary gland (MG) position and determine whether this relationship influenced subsequent growth of the IUGR piglet.

Method: Based on backfat depth at parturition, nine healthy sows were classified as FAT $(>23 \mathrm{~mm}, n=5)$, or THIN $(<16 \mathrm{~mm}, n=4)$. Colostrum and milk were collected from day 1 to day 6 after parturition and leptin and IGF1 levels were determined by RIA assays ${ }^{1,2}$.

Results: Both leptin and IGF1 concentrations varied with sow body composition $(\mathrm{p}<0.001)$. During early lactation, fat sows produced milk in the middle $\mathrm{MG}$ region with a leptin content $52 \%$ higher $(\mathrm{p}<0.001)$, and IGF1 content $69 \%$ higher $(\mathrm{p}<0.001)$, than that of lean sows. In fat sows concentrations of leptin from the middle MG were greater $(\mathrm{p}<0.05)$ than milk from posterior MG while no difference was observed in leptin and IGF content in lean sows.

Conclusion: Collectively, these data provide evidence that sow body fat composition during early lactation and MG position affects leptin and IGF1 levels in both colostrum and milk and may affect offspring growth performance.

\section{References}

1. Lamberson WR, Safranski TJ, Bates RO, Keisler DH, Matteri RL J Anim Sci. 1995; 73. 3241-3245.

2. Berg EP, McFadin EL, Maddock RR, Goodwin N, Baas TJ, Keisler DHJ Anim Sci. 2003 Jan;81(1):167-71.

Disclosure of Interest: None Declared.

\section{DOHaD13-1252}

A macaque model of IUGR shows investment in bone and brain only on a high fat diet

Ai Ling Teh ${ }^{1, *}$, Louiza Chan ${ }^{1}$, Yong Chee Tan ${ }^{1}$, Pei Fang $\operatorname{Tan}^{1}$, Christopher Yeung ${ }^{1}$, Melvir Singh ${ }^{1}$, Johnny Wong ${ }^{1}$, Peter Gluckman ${ }^{1}$, Joanna Holbrook ${ }^{2}$, Michael Meaney ${ }^{1,3}$, Keefe Chng ${ }^{1}$ 
${ }^{1}$ Singapore Institute of Clinical Sciences (SICS), $A^{*} S T A R$;

${ }^{2}$ Singapore Institute of Clincial Sciences (SICS), A*STAR, Singapore, Singapore; ${ }^{3}$ Douglas University Mental Health Institute, McGill University, Quebec, Canada

Background: Evidence from observational and experimental studies links adverse exposures in early life to disease susceptibility in adulthood. In particular human infants with intra-uterine growth retardation (IUGR) have a higher disease risk for metabolic, cardiovascular and neurodevelopmental disorders. We developed a cynomolgous macaque model of IUGR in which animals born small were exposed to high fat or control diets, and responses compared to that of control animals. Method: We induce mild IUGR by reducing maternal food intake by $35 \%$ between days $32-70$ of gestation and $30 \%$ from day 71 onwards to term (gestation length: 155-170 days). At term, the IUGR infant macaques have a lower mean weight compared to controls. All infants were followed and intensively phenotyped by morphometric and metabolic measurements. At 15 months the subjects were stratified by diet, resulting in four test groups: control - high fat diet, control - control diet, IUGR - high fat diet, IUGR - control diet.

Results: There was no difference in weight gain for control animals fed on a high fat compared to a control diet. In contrast, IUGR animals exposed to a high-caloric diet from 15 to 24 months of age show accelerated weight gain compared to animals on a maintenance diet and morphometric growth through an increase in height and head circumference compared to animals on a maintenance diet. Interestingly the IUGR animals on the high-fat diet had similar growth to controls, whilst IUGR animals on the control diet were smaller at all timepoints. In addition IUGR animals demonstrated rapid glucose clearance in intravenous glucose tolerance tests (IVGTTs) at 15 months.

Conclusion: A differential response to diet was present only in the IUGR background. This recapitulates the phenotype of IUGR human infants as does the insulin sensitivity seen in IUGR cases. That the IUGR animals on a high-fat diet invest in bone at a similar level to the control animals has implications to high-calorie intervention for IUGR and SGA human infants.

Disclosure of Interest: None Declared.

\section{DOHaD13-1394}

A murine model for maternal smoking to study the role of micrornas in transgenerational asthma transmission

Stefan Dehmel ${ }^{1}$, Petra Nathan ${ }^{1}$, Katrin Milger ${ }^{1}$, Raphael Prungnaud ${ }^{1}$, Rabea Imker ${ }^{1}$, Martin Irmler ${ }^{2}$, Johannes Beckers $^{2,3}$, Oliver Eickelberg ${ }^{1}$, Susanne Krauss-Etschmann ${ }^{1, *}$

${ }^{1}$ Comprehensive Pneumology Center CPC; ${ }^{2}$ Institute of Experimental Genetics, Helmholtz Zentrum Muenchen, Muenchen; ${ }^{3}$ Chair of Experimental Genetics, Technische Universitaet Muenchen, Freising, Germany
Background: Epidemiologic studies showed that maternal smoking during pregnancy is a main risk factor for the offspring to develop asthma later in life ${ }^{1-4}$. One explanation for this is that environmental exposures active during early critical developmental stages lead to a mal-programming of disease risk later in life ${ }^{5}$. MicroRNAs (miRs) are short, non-coding RNAs that regulate gene expression. While being subject to epigenetic regulation and imprinting ${ }^{6}$, miRs also function as regulators of epigenetic control elements ${ }^{7,8}$ and respond to environmental factors (e.g. LPS, tobacco smoke ${ }^{9,10}$ ). Importantly, miRs act as master regulators during fetal development of bronchoalveolar tissues $^{11}$ and the immune system ${ }^{12}$. We therefore asked how maternal smoking during pregnancy affects growth and pulmonary miR/target mRNA networks in offspring mice.

Method: Pregnant BALB/c mice were exposed to filtered air (FA) or mainstream cigarette smoke (MCS) daily starting on embryonic day (E) 2.5 (=treatment day (TD) 0 ). Weights of pregnant and non-pregnant mice were recorded daily and ANOVA with Bonferroni posttests was used for statistical analysis. On E18.5 (=TD16) offspring mice were removed by caesarean section and body and lung weights were recorded. For mRNA/ miR profiling (Affymetrix GeneChips ${ }^{\circledR}$ ) we used pulmonary RNAs of E18.5 pups ( $n=6 /$ group). Inverse correlation of MCSregulated mRNAs and miRs (Ingenuity ${ }^{\circledR}$ IPA $^{\circledR}$ ) is currently conducted to identify miR regulated pathways.

Results: Pregnant MCS mice showed reduced body weights on TD11-16 (each TD: $\mathrm{p}<0.05$, vs FA mice). While body weights decreased significantly in non-pregnant MCS mice on TD8-14 compared to FA mice (each TD: $\mathrm{p}<0.001$ ), normalization of maternal body weights to litter sizes showed that maternal weight was not affected by MCS-exposure in pregnant mice. Furthermore, MCS dams had slightly reduced numbers of viable pups per litter $(6.75 \pm 1.49)$ when compared to FA dams $(7.60 \pm 1.17)$ but the difference did not reach statistical significance. The number of resorptions was unaffected by MCS-exposure. Fetal body weights were significantly reduced by in utero MCS exposure in male (MCS (mean $\pm \mathrm{SD}$ ): $0.95 \pm 0.12$ g, FA: $1.05 \pm 0.15 \mathrm{~g}, \mathrm{p}<0.05, \mathrm{n}>20$ ) and female pups (MCS: $0.89 \pm 0.12 \mathrm{~g}$, FA: $1.04 \pm 0.17 \mathrm{~g}, \mathrm{p}<0.001$, $\mathrm{n}>30$ ). Fetal lung weights were significantly reduced by in utero MCS exposure in male (MCS: $31.55 \pm 5.84 \mathrm{mg}$, FA: $34.95 \pm 5.53 \mathrm{mg}, \mathrm{p}<0.05, \mathrm{n}>20$ ) and female pups (MCS: $27.16 \pm 6.61 \mathrm{mg}$, FA: $34.78 \pm 7.15 \mathrm{mg}, \mathrm{p}<0.0001, \mathrm{n}>30$ ).

Conclusion: Our model of prenatal smoke exposure is in agreement with findings in humans where maternal smoking during pregnancy is associated with small for the gestational age in offspring ${ }^{4}$. We anticipate identifying effects of maternal smoking on miR-regulated pathways with a critical function in the early pulmonary development that may affect disease development later in life.

\section{References}

1. Burke, H., Pediatrics, 2012.

2. Li, Y.-F., Chest, 2005.

3. Neuman, Å., AJRCCM, 2012. 
4. Bjerg, A., Pediatrics, 2011.

5. Barker, D.J., Mol Med Today, 1995.

6. Williams, A.E., Developmental Dynamics, 2007.

7. Pan, W., J Immunol, 2010.

8. Grandjean, V., Development, 2009.

9. Moschos, S.A., BMC genomics, 2007.

10. Pottelberge, G.R. Van, AJRCCM, 2011.

11. Bhaskaran, M., Physiol Genomics, 2009.

12. Rodriguez, A., Science, 2007.

Disclosure of Interest: None Declared.

\section{DOHaD13-1384}

A novel model for retinopathy of prematurity: effects in adult mice after neonatal exposure to hyperoxic gas

Sheena Bouch ${ }^{1, *}$, Xiangting Chen ${ }^{1}$, Foula Sozo ${ }^{1}$, Richard Harding $^{1}$, Paul McMenamin ${ }^{1}$

\section{${ }^{1}$ Department of Anatomy and Developmental Biology, Monash} University, Melbourne, Australia

Background: Retinopathy of prematurity (ROP) is a significant cause of visual morbidity in very preterm infants (born $<32$ weeks of gestation) and usually follows prolonged neonatal exposure to hyperoxic gas. The incidence of ROP is $5-8 \%$ in developed countries and can reach up to $30 \%$ in developing countries. Mouse models are commonly utilised to investigate the disease processes underlying ROP. Previous murine studies of ROP have largely focused on short-term effects of neonatal exposure to hyperoxic gas on the developing eye. In this study our aim was to determine the long-term effects of neonatal exposure to clinically relevant levels of $\mathrm{O}_{2}$ exposure on the development of retinopathy in mice using live in vivo imaging and histological analysis.

Method: Neonatal mice (C57BL/6J) were raised in either $40 \%$ or $65 \% \mathrm{O}_{2}$ (hyperoxia groups) from birth until postnatal day $7(\mathrm{P} 7 \mathrm{~d})$ and then raised in room air until early adulthood (P56d) or middle-age (10 months; P10 m). Controls $(\mathrm{CON})$ breathed room air for the duration of the experiment. At P56d (CON n $=11,40 \% \mathrm{O}_{2} \mathrm{n}=20,65 \%$ $\left.\mathrm{O}_{2} \mathrm{n}=10\right)$ and $\mathrm{P} 10 \mathrm{~m}\left(\mathrm{CON} \mathrm{n}=15,65 \% \mathrm{O}_{2} \mathrm{n}=15\right)$, in vivo brightfield and fluorescent angiographic imaging of the retinal fundus was performed with a Micron III camera under anaesthesia with subsequent recovery. At autopsy (P56d and P10 m), eyes were collected for histological examination and whole-mount retinal immunohistochemistry.

Results: In vivo imaging of the fundus indicated that at P56d the $40 \%$ and $65 \% \mathrm{O}_{2}$ groups and at $\mathrm{P} 10 \mathrm{~m}$ the $65 \% \mathrm{O}_{2}$ group had a persistent hyaloid vasculature with an increased number of vascular branches, multiple large retinal lesions and abnormal retinal vasculature compared to the $\mathrm{CON}$ groups. Histological examination of the $65 \% \mathrm{O}_{2}$ group at P56d revealed thinning of the peripheral retina, multiple retinal folds, presence of pseudorosettes in the retina, and presence of hyaloid vessels in the vitreous extending to the posterior lens surface. The degree of pathology in the $40 \% \mathrm{O}_{2}$ group was apparently less severe than in the $65 \% \mathrm{O}_{2}$ group. Conclusion: These preliminary data demonstrate for the first time that neonatal exposure to hyperoxic gas from birth results in $\mathrm{O}_{2}$ concentration dependent pathology and longterm retinal injury, resembling the pathology observed in human ROP following very preterm birth and exposure to hyperoxia. In addition, the use of in vivo fundal imaging enabled us to monitor and evaluate the condition of the eye without having to sacrifice the animal; this feature will likely be beneficial in evaluating the effectiveness of possible treatments for ROP. Further studies are required to understand the underlying mechanisms contributing to the abnormal retinal vasculature that we observed following neonatal hyperoxia exposure, as well as the persistence of hyaloid vessels, which would normally regress around P7dP13d.

Disclosure of Interest: None Declared.

\section{DOHaD13-1420}

An animal model of low grade chronic inflammation and prenatal programming of offspring disease

Anete Dudele ${ }^{1, *}$, Tobias Wang ${ }^{1}$, Sten Lund ${ }^{2}$

${ }^{1}$ Bioscience, AARHUS UNIVERSITY; ${ }^{2}$ Clinical Medicine-The Department of Endocrinology and Diabetes, AARHUS

UNIVERSITY HOSPITAL, Aarhus, Denmark

Background: Maternal obesity during pregnancy significantly increases the risk for the offspring to develop obesity, type 2 diabetes and cardiovascular disease later in life, but the underlying causal mechanisms responsible for this programming remain to be understood. It is well known that a state of chronic low grade inflammation causally links obesity and the related lifestyle diseases and that experimental activation of immune response during pregnancy induces behavioral changes in the offspring. Thus, we propose that obesityrelated chronic inflammation during pregnancy represents a potential mechanism for prenatal programming of offspring lifestyle diseases. To study these effects, we set out to create a functional animal model where chronic inflammation is induced in female mice without affecting their adiposity or glucose metabolism.

Method: To induce low grade chronic inflammation, a slow release pellet (Innovative Research of America, USA) delivering Lipopolysaccharide (LPS - a potent, naturally occurring endotoxin) at a constant rate for 60 days was implanted intraperitoneally in C57BL/6JBomTac female mice, while a control group of mice was implanted with a mock pellet. During the following 60 days body mass and food intake were measured weekly and oral glucose tolerance was assessed 6 weeks after pellet implantation. Mice were 
euthanized 60 days after pellet implantation for measurements of body composition and organ size.

Results: Two days after implantation females receiving LPS showed a significant decrease in body mass, compared to sham-treated mice $(10.8 \%$ vs. $5.4 \%$ decrease, respectively) indicative of immune response to LPS. These differences disappeared one week after operation and mice in both groups had similar body masses throughout the remaining experimental period. LPS did not affect glucose tolerance, but lead to elevated spleen mass, consistent with the presence of an inflammatory state. Overall adiposity was not affected by LPS, but females receiving LPS had significantly larger mesenteric fat depots $(0.44 \mathrm{~g}$ in LPS treated animals vs. $0.36 \mathrm{~g}$ in control group, $\mathrm{P}=0.004)$.

Conclusion: In conclusion, chronic LPS administration induced inflammation in female mice, but did not affect their adiposity and glucose metabolism. As in the present animal model LPS can be continuously administered for 60 days, it provides a possibility to study effects of maternal inflammation during the periods of pregnancy and lactation on offspring physiology, in absence of such confounding factors as maternal hyperglycemia and hyperlipidemia.

Disclosure of Interest: None Declared.

\section{DOHaD13-1659}

An animal model of paternal obesity programs metabolic disturbances in two generations of mice and alters the transcriptional profile of founder testis and sperm microrna content

Tod Fullston ${ }^{1, *}$, Nicole O. McPherson ${ }^{1}$, Julie A. Owens ${ }^{1}$, Michelle Lane ${ }^{1}$

${ }^{1}$ Discipline of Obstetrics \& Gynaecology, School of Paediatrics and Reproductive Health, Robinson Institute, The University of Adelaide, Adelaide, Australia

Background: Obesity and comorbid pathologies such as type 2 diabetes and sub-fertility are becoming increasingly prevalent worldwide. Although the paternal genomic contribution to the next generation is clear, less is known about how epigenetic alterations might impact offspring health. We have previously documented that a paternal high fat diet (HFD) increased adiposity without overt diabetes and transmitted diminished reproductive viability through two subsequent generations.

Method: Here we recapitulated a HFD fed founder C57Bl6 male mouse phenotype of paternal obesity, hyperlipidaemia and hyperletinaemia without alterations to glucose/insulin homeostasis (blood glucose/insulin concentrations and glucose/insulin tolerance). Control diet/HFD fed founder mice (CD/HFD- $\mathrm{F}_{0}$; $n=10$ each) were mated to normal weight $\mathrm{CD}$ females.

Results: HFD- $\mathrm{F}_{0}$ sired first generation $\left(\mathrm{F}_{1}\right)$ animals exhibited muted responses to glucose and insulin, acutest in $F_{1}$ females.
At 8 weeks old $F_{1}$ females were already glucose intolerant $\left(\mathrm{CD}-\mathrm{F}_{0} \quad 267 \pm 33 ; \quad\right.$ HFD-F $\left.\mathrm{F}_{0} 295 \pm 16 ; \quad P<0.05\right)$ with reduced insulin sensitivity by 16 weeks $\left(\mathrm{CD}-\mathrm{F}_{0} 158 \pm 28\right.$; HFD- $\left.\mathrm{F}_{0} 90 \pm 18 ; P<0.05\right)$ that persisted throughout life (8-39 weeks), concomitant with increased adiposity $(+65.6 \% ; P<0.0001)$. Although $\mathrm{F}_{1}$ males also displayed glucose intolerance at 8 weeks old $\left(\mathrm{CD}-\mathrm{F}_{0} 378 \pm 59\right.$; HFD$\left.\mathrm{F}_{0} 476 \pm 87 ; P<0.05\right)$, reduced insulin sensitivity presented later at 26 weeks $\left(\mathrm{CD}-\mathrm{F}_{0} 220 \pm 29\right.$; HFD $-\mathrm{F}_{0} 129 \pm 33$; $P<0.05)$ without increased adiposity.

Strikingly, metabolic derangements were further transmitted to the second generation $\left(\mathrm{F}_{2}\right)$, albeit with incomplete penetrance. $3 / 4$ $\mathrm{F}_{2}$ animal groups from a HFD- $\mathrm{F}_{0}$ lineage (except of $\mathrm{F}_{2}$ males from $F_{1}$ males) exhibited muted insulin sensitivity, with glucose intolerance and increased adiposity $(+24.1 \% ; P<0.05)$ limited to $F_{2}$ males from $F_{1}$ females. Milder metabolic disturbances observed in grand-paternal HFD lineage $\mathrm{F}_{2}$ offspring imply that $\mathrm{F}_{3}$ metabolic health might not be compromised.

Founder male obesity reduced global DNA methylation in testes $(-27.0 \% ; P<0.01)$ and spermatids $(-24.9 \%$; $P<0.0001)$, and altered microRNA content in testes and sperm.

Conclusion: This study associates paternal obesity with epigenetic alterations to testis and sperm, which might partly explain intergenerational transmission of impaired metabolism and obesity observed through two generations.

Disclosure of Interest: None Declared.

\section{DOHaD13-1667}

\section{Animal models and fetal programming: an integrative literature approach}

Narges Bahi-Jaber ${ }^{1 * *}$, Andreï Mogoutov ${ }^{2}$, Ghada Elmhiri ${ }^{1}$, Latifa Abdennebi-Najar ${ }^{1}$

${ }^{1}$ UPSP EGEAL, INSTITUT LASALLE BEAUVAIS, BEAUVAIS; ${ }^{2} I F R I S$ - institut Francilien Recherche Innovation Sociéte', MARNE-LA-VALLE'E, France

Background: Despite the substantial epidemiological evidence for fetal origins of adult disease, there are intrinsic limitations in long-term retrospective studies. Some aspects can, however, be focused on by using the controlled conditions afforded by animal models, a number of which have been developed to study this in utero programming phenomenon. Animal models allow study of the pathophysiology of disease, and afford a means to study the underlying biochemical and molecular biological mechanisms, whilst they cannot be used entirely as a substitute for the study of human diseases. Analyzing the contribution of animal models in our understanding of fetal programming as well as their limits requires a systematic review of the existing literature.

Method: We used a novel methodology, i.e. the automated extraction of information from electronically published 
sources (PubMed and Web of Science), based on the online platform CorText (manager.cortext.net) for text-mining and heterogeneous data analysis. This integrative approach allow us to collect and visualize the available data to determine the contribution of animal models on programming and determine the interaction among animal models, pathology and gene expression in a systemic manner.

Results: The analysis was done on a database of more than 7000 papers published during last twenty years. This approach allows us to show the dynamics of the research field and related sub-fields (obstetrics, metabolic diseases, neuroscience etc). We provide an exhaustive mapping of the species used as model, their relations with pathologies, thematic sub-fields and their time evolution.

Conclusion: This innovative research tool for meta-analysis of scientific literature allows us to understand the trends, detect the emerging sub-fields and to target the new innovative research directions in fetal programming for preventing the development of adult diseases.

Disclosure of Interest: None Declared.

\section{DOHaD13-1042}

\section{Antigen capture therapy (ACT) for the prevention of intrauterine inflammation, fetal injury and preterm birth}

Matthew Kemp ${ }^{1, *}$, Masatoshi Saito ${ }^{2}$, Matthew Payne ${ }^{3}$, Demelza Ireland ${ }^{3}$, Suhas Kallapur ${ }^{4}$, Alan Jobe ${ }^{4}$, Boris Kramer $^{5}$, Yuichro Miura ${ }^{3}$, John Newnham ${ }^{3}$, Jeffrey Keelan ${ }^{3}$

${ }^{1}$ School of Women's and Infants' Health, University of Western Australia, Perth, Australia; ${ }^{2}$ Division of Perinatal Medicine, Tohoku University Hospital, Sendai, Japan; ${ }^{3}$ School of Women's and Infants' Health, The University of Western Australia, Perth, Australia; ${ }^{4}$ Division of Pulmonary Biology, Cincinnati Children's Hospital Medical Centre, Cincinnati, United States; ${ }^{5}$ Department of Pediatrics, Maastricht University Medical Centre, Maastricht, Netherlands

Background: Uterine infection is a leading cause of respiratory disease, neurological injury, cognitive and learning disabilities and cerebral palsy in preterm infants ${ }^{1}$. Strong experimental evidence suggests that the inflammation caused by uterine infection is responsible for both preterm labour and injury to the developing fetus ${ }^{2}$. Antibiotic therapy alone is unlikely to resolve fetal injury or prevent preterm delivery due to continued exposure of gestational tissue to proinflammatory microbial agonist. The use of agents to inhibit ubiquitously expressed inflammatory signalling pathways (e.g. NF- $\kappa \mathrm{B}$ signalling) that play an important role in normal fetal development/homeostasis may adversely affect fetal development ${ }^{3}$. We hypothesised that intraamniotic delivery of polymyxin-b (PMXB; a cyclic, cationic peptide antibiotic with a high binding affinity for the lipid A moiety of LPS) as an Antigen Capture Therapy (ACT) to bind and inactivate
E.coli lipopolysaccharide (LPS) would inhibit uterine inflammation in our pregnant sheep model.

Method: Pregnant sheep carrying single fetuses at $122 \mathrm{~d}$ gestational age were randomised to receive a single intraamniotic injection of either: i) $10 \mathrm{mg}$ LPS (O55:B5, Sigma Aldrich, St. Louis, MO) $(\mathrm{n}=8)$; ii) $10 \mathrm{mg}$ LPS $+10 \mathrm{mg}$ PMXB (Sigma Aldrich) $(\mathrm{n}=8)$; or iii) $10 \mathrm{mg}$ PMXB $(\mathrm{n}=8)$. PMXB dose was informed by in vitro dose-response studies. Animals were euthanised after $48 \mathrm{~h}$. Amniotic fluid, fetal lung, skin and spleen were dissected and snap frozen in liquid nitrogen for inflammatory protein/mRNA analyses. Data were normality tested and grouped differences assessed using parametric or nonparametric one-way ANOVA as appropriate.

Results: qPCR analysis demonstrated a 98\% reduction in IL$1 \beta, 90 \%$ reduction in TNF- $\alpha, 90 \%$ reduction in IL- 6 , $97.5 \%$ reduction in IL-8, and a 93\% reduction in MCP-2 mRNA expression in lung tissue of fetuses exposed to LPS + PMXB relative to those treated with LPS alone. Small, non-significant increases in IL- $1 \beta$ and MCP-2 mRNA expression were identified in the spleen of fetuses exposed to LPS alone. LPS-induced increases in fetal skin IL-8 and MCP-2 mRNA were not altered by PMXB delivery. Interestingly, PMXB + LPS treatment resulted in a $83 \%$ reduction in the concentration of amniotic fluid IL- 6 and a $50 \%$ reduction in the concentration of amniotic fluid IL-8, relative to fetuses exposed to LPS alone.

Conclusion: In the present study, intraamniotic injection of PMXB significantly reduced inflammation in the lung of fetuses exposed to LPS but did not inhibit inflammatory cytokine expression in the fetal skin. Interestingly, treatment with PMXB did yield marked reductions in the concentration of pro-inflammatory mediators IL-6 and IL-8 in the amniotic fluid. These data suggest that the development of an ACT targeting preterm birth-associated organisms (i.e. Ureaplasma spp.), delivered in conjunction with an appropriate antibiotic may provide a means of controlling intrauterine inflammation, preventing preterm birth and injurious changes in fetal development.

\section{References}

1. Goldenberg RL, et al. Lancet 2008; 371(9606): 75.

2. Bastek JA, et al. Clin. Perinatol. 2011; 38(3): 385.

3. Rinaldi SF, et al. Exp. Rev.Clin. Immunol. 2011; 7(5): 675.

Disclosure of Interest: None Declared.

\section{DOHaD13-1173}

Early weaning in rats modifies glucagon-like peptide 1 (GLP 1) profile contributing to the obesity development in adult life

Elaine Oliveira $^{1, *}$, Patricia C. Lisboa ${ }^{1}$, Fernanda T. Quitete ${ }^{1}$, Jessica L. Nobre ${ }^{1}$, Egberto G. Moura ${ }^{1}$

${ }^{1}$ Physiology, State University of Rio de Janeiro, Rio de Janeiro, Brazil 
Background: Early weaning causes undernutrition and programs to central obesity, hyperleptinemia and leptin resistance at adulthood $^{1,2}$. GLP 1 is a gut-derived peptide that has been reported to improve insulin resistance ${ }^{3}$, and recently that also promotes preadipocyte differentiation in 3T3-L1 cells that presented an increase numbers of small adipocytes4. In the present study, we evaluated the GLP 1 in serum, small gut and visceral adipose tissue and its receptor in rats that were early weaned as well as the repercussions at adulthood. Method: Wistar lactating rats were separated into: EW group (early weaning) - dams were involved with a bandage to interrupt the lactation in the last 3 days of lactation, and C group (control) - dams whose pups had free access to milk during all lactation (21 days). Rats were killed at 21 and 180 days; blood, gut and visceral adipose tissues were collected for analysis. Serum GLP 1 was determined by ELISA kit, GLP 1 and GLP $1 \mathrm{R}$ content in small gut and GLP $1 \mathrm{R}$ in adipose tissue were determined by Western blotting. All significant data were $\mathrm{p}<0.05$.

Results: As expected, at 21 days-old, EW pups showed lower body weight, visceral adipose content and lower visceral adipocytes area; at 180 days-old, offspring presented hyperphagia, overweight, higher visceral adipose tissue and higher visceral adipocytes area. At weaning (21 days-old), EW pups presented higher GLP 1 in serum but lower levels in small gut. Concerning GLP 1R, these rats showed lower content in small gut but higher content in visceral adipose tissue. On the other hand, at adulthood, EW offspring only displayed lower GLP 1 content in small gut.

Conclusion: At weaning, the lower GLP 1 content in the small gut together with higher serum GLP 1 may be due to an increase of GLP 1 secretion. Possible, the higher GLP 1R content in adipose tissue resulted in a lower adipocyte area and hyperplasia. This imprinting on the adipocyte may result in a programming effect that resulted in adipocytes hypertrophy with consequent alterations in glucose homeostasis and insulin resistance with normoinsulinemia, which suggest impairment in insulin secretion. Thus, the lower gut GLP1 may suggest a lower production that could contribute for the lower insulin secretion. Thus, exclusive breastfeeding, in addition to strengthening the ties between mother and child, it is essential to establishment of future metabolic profile.

\section{References}

1. Lima NS et al., Developmental plasticity of endocrine disorders in obesity model primed by early weaning in dams. Horm Metab Res. 45:22-30, 2013.

2. Nobre JL et al., Calcium supplementation prevents obesity, hyperleptinaemia and hyperglycaemia in adult rats programmed byearly weaning. Br J Nutr. 107:979-88, 2012.

3. Burmeister MA et al., Acute activation of central GLP-1 receptors enhances hepatic insulin action and insulin secretion in high-fat-fed, insulin resistant mice. Am J Physiol Endocrinol Metab. 302:E334-43, 2012.
4. Yang J et al., Glucagon-like peptide 1 regulates adipogenesis in 3T3-L1 preadipocytes. Int $\mathrm{J}$ Mol Med. 31: 1429-35, 2013.

Disclosure of Interest: None Declared.

\section{DOHaD13-1330}

Effect of low dose intra-amniotic endotoxin on kidney development in rats

Keiji Suzuki ${ }^{1, *}$, Hidehiro D. Takahashi ${ }^{2}$

${ }^{I}$ Department of Pediatrics, Tokai University School of Medicine, Isehara, ${ }^{2}$ Division of Neonatology, Perinatal Center, Saitama Medical Center, Kawagoe, Japan

Background: Chorioamnionitis (CAM) is one of the major causes of preterm delivery and known to be associated with impairment of various developing organs. However, it is not clear how CAM affects development of the kidney. The aim was to study effects of low dose intra-amniotic lipopolysaccharide (LPS), on development of the kidney into adulthood. Method: At 20 days of gestation, pregnant SD rats (term $22.5 \mathrm{~d})$ were anesthetized and the uterus exposed under general anesthesia. The uterine wall was punctured and $0.1 \mu \mathrm{g}$ LPS dissolved in $0.1 \mathrm{ml}$ saline injected into each amniotic cavity. In the control group, $0.1 \mathrm{ml}$ saline was injected. At $22 \mathrm{~d}$ (term), the fetuses were delivered spontaneously and vaginally. The pups were nursed by their own mother until 3 weeks and weaned thereafter. At postnatal day 56 (adolescence), rats were euthanized and the left kidneys were removed. The kidneys were weighed, fixed with formaldehyde and embedded in paraffin. The kidney was cut into sections and stained with periodic acidSchiff. We measured the glomerular number $(\mathrm{Ng})$ and the mean glomerular volume $(\mathrm{Vg})$, and calculated the index of glomerular filtration capacity $\left(\mathrm{Ng}^{*} \mathrm{Vg}^{2 / 3}\right)$.

Results: Males had heavier kidney weight than females. After adjustment for body weight however, it was not different between males and females $(4.47 \pm 0.12$ vs $4.16 \pm 0.09$ $\mathrm{g} / \mathrm{kgBW})$. There were no differences in body weight or kidney weight between LPS and control rats. In females compared to males, $\mathrm{Ng}$ was significantly higher and $\mathrm{Vg}$ tended to be lower. $\mathrm{Ng}^{*} \mathrm{Vg}^{2 / 3}$ was higher in females. In males, there were no differences in $\mathrm{Ng}, \mathrm{Vg}$ or $\mathrm{Ng}^{*} \mathrm{Vg}^{2 / 3}$ between LPS rats and controls. In females however, $\mathrm{Ng}$ and $\mathrm{Ng}^{*} \mathrm{Vg}^{2 / 3}$ were increased in LPS-exposed rats (Ng: $271 \pm 22$ vs $368 \pm 37 /$ gBW, $\mathrm{p}<0.05)\left(\mathrm{Ng}^{*} \mathrm{Vg}^{2 / 3}: 14.9 \pm 0.3\right.$ vs $18.4 \pm 1.0 \times 10^{4} \mu \mathrm{m}^{2} /$ kgBW; $\mathrm{p}<0.05)$.

Conclusion: Antenatal intra-amniotic exposure to LPS resulted in an increased nephron number only in females. Low dose LPS may have a favorable effect on kidney development (increase in nephron number and glomerular filtration capacity) which is influenced by gender.

Disclosure of Interest: None Declared. 


\section{DOHaD13-1704}

\section{Effect of vitamin D deficiency on skeletal development across the early lifecourse}

Tsiloon $\mathrm{Li}^{1,2, *}$, Stuart A. Lanham ${ }^{2}$, Philipp J. Thurner ${ }^{1}$, Richard O. Oreffo ${ }^{2}$

${ }^{1}$ Bioengineering Research Group; ${ }^{2}$ Bone and Joint Research Group, University of Southampton, Southampton,

United Kingdom

Background: Bone health and maintenance is modulated by osteogenic cell activity and changes in cellular function will likely cause bone material and structural rearrangements, altering the mechanical properties of the bone tissue. Vitamin $\mathrm{D}$ is known to be important for bone health and has key roles in inducing differentiation towards pathways favourable for osteogenesis and calcium and phosphorus uptake. However, the role of vitamin D during early development are less well established and with current estimations of around one billion people worldwide recorded as having insufficient levels of vitamin D [1], we hypothesised that vitamin D deficiency in early life causes changes at molecular level, inhibiting bone cell behaviour that persists throughout life and reflected, critically, through detrimental changes in material and structural properties of bone.

Method: A murine model of Sprague-Dawley rats with complete vitamin $\mathrm{D}$ depletion during in-utero life was used and evaluated at 140 days of age. Femora were excised from each sample, with osteoblast gene expression measured from femur epiphyses and whole femora used for reference point micro-indentation [2] analysis and micro-CT imaging to investigate associated micromechanical and microarchitectural changes respectively. Significance of results between Vitamin D deplete and control groups were measured using student t-tests for each gender.

Results: Investigation into mRNA expression showed that for both male and female levels of RUNX2, osteocalcin, osteopontin and collagen I were raised in the deplete group (except for RUNX2 and osteocalcin in the female cohort). Ossteopontin expression in males was found to be statistically significant $(p=0.02)$. Computer tomography confimred thicker cortical bone at the distal femur region with increased bone volume for control animals whilst the trabecular microarchitecture of the same bone region was found to be improved in the male control group; however, the reverse was true for females. Microindentation data exhibited a reduced capability of control femurs to resist applied indentation forces. However, this did not reach statistical significance.

Conclusion: While a more distinct effect was expected as a result of the complete deficiency of vitamin D during early life, it is likely the lack of molecular and physical difference between the vitamin $\mathrm{D}$ deplete group and control group is a prospective consequence of the early time point analysed, which is comparable to an early adult in humans. It is likely that changes will not manifest until much later in life.
Critically, these studies highlight unknown compensatory mechanisms, which have arisen as a consequence of early nutritional challenge, maintaining skeletal development. These results indicate that early vitamin $\mathrm{D}$ deficiency has no immediate effect on skeletal development but emphasise the need for additional longitudinal studies to delineate the compensatory mechanisms active and the effects of vitamin D deficiency at further age points.

\section{References}

1. Hewinson et al, JBMR, 25(1):11-13, 2010.

2. Diez-Perez et al, JBMR, 25(8): 1877-1885, 2010.

Disclosure of Interest: None Declared.

\section{DOHaD13-1726}

Effects of experimental hyperleptinaemia in neonatal rats on subsequent sucrose preference and reward-related receptor density

Tim South ${ }^{1, *}$, Sung Eun Bae ${ }^{1}$, Jennifer C. Roberts ${ }^{1}$, Amandine Mullier ${ }^{1}$, Lucilla Poston ${ }^{1}$, Paul D. Taylor ${ }^{1}$, Clive W. Coen ${ }^{1}$

${ }^{1}$ Division of Women's Health, King's College London, London, United Kingdom

Background: Our previous work has shown that the offspring of obese rat dams display a heightened and prolonged post-natal leptin surge. We hypothesise that this may predispose them to hyperphagia and obesity in adulthood. The present study investigated whether neonatal hyperleptinaemia leads to alterations in food preference and in the density of reward-related receptors in the central nervous system (CNS) prior to the onset of obesity.

Method: Male Sprague Dawley rats were treated intraperitoneally with leptin $(0.3 \mathrm{mg} / \mathrm{kg}, \mathrm{n}=6)$ or vehicle (saline, $\mathrm{n}=6$ ) twice daily from Day 9 for seven days. At Day 30, the animals were euthanised and brains were removed to determine receptor binding levels by autoradiography. An overnight two-bottle sucrose preference test was undertaken on Day 30 on a separate cohort of leptin- or vehicle-treated animals ( $\mathrm{n}=10$ /group).

Results: A sugar preference test established that the leptintreated rats had a greater preference for sucrose (\% intake of $2 \%$ sucrose solution Versus water) than the saline-treated control animals (94.6\% and $84.7 \%$ respectively). D1 binding in the leptin-treated rats was reduced in the nucleus accumbens (NAcc) core and in the lateral caudate putamen $(\mathrm{CPu})$ compared to the receptor density observed in the saline-treated rats. D2 binding was also reduced in the NAcc and $\mathrm{CPu}$ of the leptin-treated animals. In contrast, $\mathrm{CB} 1$ receptor binding in the leptin-treated rats was elevated in the NAcc.

Conclusion: Prolonged neonatal hyperleptinaemia results in alterations in feeding preference and in CNS reward-related 
receptor binding density. These may predispose the individuals to behaviour that leads to obesity.

Disclosure of Interest: None Declared.

\section{DOHaD13-1068}

\section{Establishing an animal model of passive exposure to tobacco smoke during pregnancy}

Isabel C. R. Werlang ${ }^{1,2, *}$, Mariana D. Schiffner ${ }^{2}$, Carmem Pilla ${ }^{3}$, Patricia P. Silveira ${ }^{1}$, Carla Dalmaz ${ }^{4}$, Marcelo Z. Goldani $^{1,2}$, Fernanda U. Fontella ${ }^{2}$

${ }^{1}$ Departamento de Pediatria e Puericultura, Universidade Federal do Rio Grande do Sul; ${ }^{2}$ Laboratório de Pediatria Translacional; ${ }^{3}$ Laboratório de Análises Clinicas, Hospital de Clinicas de Porto Alegre; ${ }^{4}$ Departamento de Bioquímica, Universidade Federal do Rio Grande do Sul, Porto Alegre, Brazil

Background: Many studies have linked maternal smoking during pregnancy with increased risk for adverse outcomes in newborns, such as intrauterine growth restriction and low birth weight. In this work, we proposed an animal model of tobacco passive exposure during gestation to investigate its effects on fetal growth and biochemical parameters, using cotinine and carboxyhemoglobin as exposure markers.

Method: Exposure to tobacco smoke during pregnancy: pregnant rats were randomly assigned to one of the three groups: control, manipulated control and tobacco (exposed to a one cigarette twice a day for 21 days of gestation). The manipulated control group went through the same intervention of tobacco group, without suffering smoke exposure; control group remained intact in the home cage. On $22^{\text {nd }}$ day of pregnancy pups were surgically delivered. Immediately after, maternal trunk blood was collected for carboxyhemoglobin, cotinine, and biochemical measurements. Trunk blood of the pups was also collected and fetal biometry measured.

Results: The proposed experimental model of tobacco exposure during pregnancy was successful in inducing high levels of carboxyhemoglobin, showing an approximate increase of $1.5 \%$ in the tobacco group when compared with the manipulated control group $(P<0.0001)$. We found a mean cotinine value of $42.5 \mathrm{ng} / \mathrm{mL}$ in the exposed animals, whereas the control and manipulated control groups had undetectable values. In addition, tobacco exposure during the intrauterine period significantly decreased the birth weight $(P=0.005)$, serum glucose $(P<0.0001)$ and insulin $(P<0.009)$ of the pups.

Conclusion: The model was able to mimic alterations observed in human newborns whose mothers were exposed to tobacco during gestation.

\section{References}

1. Bassi JA, Rosso P, Moessinger AC, Blanc AB, James LS. 1984. Fetal growth retardation due to maternal tobacco smoke exposure in the rat. Pediatr Res 18: 127-130.
2. Carmines EL, Rajendran N. 2008. Evidence for Carbon Monoxide as the Major Factor Contributing to Lower Fetal Weights in Rats Exposed to Cigarette Smoke. J Toxicol Sci 102: 383-391.

3. Esposito ER, Horn KH, Greene RM, Pisano MM. 2008. An animal model of cigarette smoke-induced in utero growth retardation. Toxicology 246: 193-202.

4. Martin-Gronert MS, Ozanne SE. 2007. Experimental IUGR and later diabetes. J Intern Med 261: 437-452.

5. Mello PRB, Okay TS, Dores EFGC, Botelho C. 2005a. Marcadores de exposição tabágica em ratas lactantes utilizando um modelo de exposição passiva desde o início da gestação. Pulmão RJ 14: 289-293.

Disclosure of Interest: None Declared.

\section{DOHaD13-1251}

Experimental induction of anaemia in preterm lambs: feasibility, survival, growth and haematological outcomes in adulthood

Alexandra H. Wallace ${ }^{1, *}$, Stuart Dalziel ${ }^{1,2}$, Anita Wylie ${ }^{1}$, Amanda van $\mathrm{Zyl}^{1}$, Jane Harding ${ }^{1}$, Anne Jaquiery ${ }^{1}$

${ }^{1}$ Liggins Institute, University of Auckland; ${ }^{2}$ Children's Emergency Department, Starship Hospital, Auckland, New Zealand

Background: In sheep, fetal anaemia alters coronary conductance and increases susceptibility to ischaemia-reperfusion injury in adulthood. We have shown that human fetal anaemia secondary to rhesus disease may also have cardiovascular consequences in adulthood. As most preterm infants become anaemic after birth at a similar postmenstrual age to that of fetuses suffering rhesus disease, it is possible that anaemia of prematurity may also alter cardiovascular outcome in later life. The aim of this study was to develop a reliable protocol for the induction of anaemia in preterm lambs and seek preliminary evidence that this may have long-term cardiovascular consequences.

Method: Ewes were randomly allocated to delivery preterm (138 days gestation) or at term ( 147 days, term controls, TC). Lambs born preterm were randomly allocated to one of 3 groups: preterm controls (PC); or experimental groups in which anaemia was induced by daily venesection until reaching a target haematocrit (Hct) of 50\% (PT50\%) or $30 \%$ (PT30\%) of baseline. Haematological and growth parameters of all lambs were monitored regularly to 12 months of age. Groups were compared using ANOVA with Tukey-Kramer post-hoc corrections or Wilcoxon tests as appropriate. Data are mean \pm SD.

Results: 11 PT50\%, 10 PT30\%, 10 PC and 12 TC lambs were included. All PT50\% and PC lambs survived to term equivalent age (TEA) but 3 PT30\% lambs died before TEA from complications of anaemia, with Hct less than $10 \%$ on the day of death. However, survival at 12 months was similar 
in all groups (PT50\% 91\%; PT30\% 70\%; PC 100\%; TC $83 \%, p=0.23)$. Target $\mathrm{Hct}$ was reached at a median gestational age of 144 days in PT $50 \%$ lambs and 145 days in PT30\% lambs. As intended, Hct decreased 52\%, to $22 \pm 4 \%$ in PT $50 \%$ lambs and $70 \%$, to $12 \pm 3 \%$ in PT $30 \%$ lambs. In PC lambs Hct decreased 39\%, to reach a nadir of $28 \pm 1 \%$ at 14 days post TEA. By 28 days post TEA Hct was similar in all groups (PT50\% $34 \pm 3 \%$; PT30\% $33 \pm 2$; PC $34 \pm 3 \%$; TC $35 \pm 4 \%, \mathrm{p}=0.67$ ). At 12 months, haemoglobin concentration in PT50\% and PT30\% lambs was higher than in PC lambs, but similar in TC lambs (PT50\% $116 \pm 7$; PT30\% 115 \pm 6 ; PC $106 \pm 8$; TC $112 \pm 7 \mathrm{~g} / \mathrm{l}$, $\mathrm{p}=0.01)$. Size at birth was similar in all preterm lambs (PT50\% 4.4 $40.5 ; \quad$ PT30\% $4.2 \pm 0.6 ; \quad$ PC $4.1 \pm 0.1$, $\mathrm{p}=0.31)$. At TEA, PT $50 \%$ and PC lambs were heavier than PT30\% lambs and all preterm lambs were heavier than TC lambs (PT50\% 7.0 \pm 0.8; PT30\% 5.7 \pm 0.9 ; PC 6.9 \pm 0.4 ; TC $4.8 \pm 0.7 \mathrm{~kg}, \mathrm{p}<0.001)$. At 12 months PT30\% lambs were

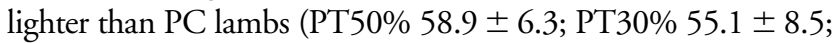
PC $63.2 \pm 3.6$; TC $57.0 \pm 5.7 \mathrm{~kg}, \mathrm{p}=0.05)$ but growth parameters were otherwise similar in all groups.

Conclusion: Anaemia can be successfully induced in preterm lambs, although survival of lambs was compromised below a haematocrit of $10 \%$. Severe but not moderate preterm anaemia impaired growth into adulthood. Increased haemoglobin at 12 months in both anaemic lamb groups suggests that preterm anaemia may influence the programming of haematological parameters in later life. This experimental approach will allow investigation of long-term outcomes of preterm anaemia and may help determine the implications of different transfusion thresholds in the preterm infant.

Disclosure of Interest: None Declared.

\section{DOHaD13-1734}

Experimental neonatal hyperglycaemia and adult glucose homeostasis

Errol Richardson ${ }^{1, *}$, Ben Ellis ${ }^{2}$, Emmanuel A. Domfey ${ }^{2}$, Christina Georgallou ${ }^{2}$, Paul D. Taylor ${ }^{3}$, Lucilla Poston ${ }^{2}$

${ }^{1}$ Division of Women's Health, King's College London; ${ }^{2}$ Division of Women's Health, King's College London; ${ }^{3}$ Division of Women's Health, King's College London, London, United Kingdom

Background: The mechanisms by which maternal obesity programmes offspring metabolic dysfunction remain poorly understood. In rodents, an exaggerated postnatal leptin surge has repeatedly been shown to play an important role. We have demonstrated that milk produced by obese rat dams contains higher concentrations of glucose, free fatty acid, triglycerides and cholesterol compared to milk produced by control dams. This in turn has been observed to cause hyperglycemia and hyperinsulinemia in neonatal offspring.
We hypothesised that neonatal hyperglycemia may precipitate the exaggerated neonatal leptin surge and in so doing contribute to the observed phenotype of offspring of obese dams (1).

Method: Glucose $(2 \mathrm{~g} / \mathrm{kg}$ ) (G-Tx) or saline (S-Tx) was administered twice daily via subcutaneous injection to naïve neonatal rats at postnatal day 2-7. Plasma insulin and blood glucose were analysed 0,30 and $60 \mathrm{~min}$ after the second injection on postnatal day 7. Plasma leptin was assayed at postnatal days 2, 7,9 and 13. At postnatal day 20 rats were weaned onto standard laboratory chow. Food intake was measured at 1, 2, 4, 12 and $24 \mathrm{hr}$ following a leptin challenge. Insulin sensitivity was estimated using the homeostasis model assessment of insulin resistance (HOMA-IR).

Results: Neonatal glucose administration resulted in marked hyperglycemia at $30 \mathrm{~min}$ post injection $(P=<0.001$ vs. Sx). Plasma insulin was elevated in G-Tx animals at 30 and $60 \mathrm{~min}$ post injection ( $P=<0.01$ vs. $\mathrm{Sx}$ at both time points). Contrary to our hypothesis, neonatal glucose administration did not influence the neonatal leptin surge. Despite this, neonatal hyperglycemia and resultant hyperinsulinemia elicited a mild, sex-specific, adult phenotype. Male G-Tx rats were resistant to leptin at 1 and $2 \mathrm{hr}$ post administration. Insulin sensitivity, measured by HOMA-IR, was found to be significantly improved in female $\mathrm{Gx}$ rats $(1.22 \pm 0.21$ vs. $1.76 \pm 0.11 \mathrm{p}<0.05)$ at postnatal day 60, but did not differ from S-Tx animals at PND120. There was no apparent effect of experimental hyperglycaemia on glucose homeostatsis in male Gx.

Conclusion: Results suggest that hyperglycemia may be involved in neonatal programming of metabolism but is not sufficient to recapitulate the effects of maternal obesity.

\section{Reference}

1. Kirk, S.L., et al., Maternal obesity induced by diet in rats permanently influences central processes regulating food intake in offspring. PLoS One, 2009. 4(6): p. e5870.

Disclosure of Interest: None Declared.

\section{DOHaD13-1529}

Exposure to a cafeteria diet during the suckling period increases fat mass at weaning independent of prenatal dietary exposure

Mini A. Vithayathil ${ }^{1, *}$, Zhi Ong ${ }^{1}$, Robert Gibson ${ }^{1}$, Beverly Muhlhausler ${ }^{1}$

${ }^{1}$ FOODplus Research Centre, UNIVERSITY OF ADELAIDE, Adelaide, Australia

Background: Excess maternal intake of palatable diets high in fat and sugar during pregnancy and lactation is associated with an increased risk of obesity in the offspring. The adipose tissue is a key target of developmental programming and 
exposure to an excess nutrient supply before birth has been associated with an increased capacity for fat storage in adipose depots in postnatal life. However, the relative contribution of prenatal and early postnatal nutritional exposures to the early programming of the adipocyte and obesity, and the extent to which the impact of prenatal exposure to an increased nutrient supply can be mitigated by restoring appropriate nutrition in the early postnatal period, are yet to be defined. Method: Nulliparous Albino Wistar rats were fed either standard rat chow (Control, $\mathrm{n}=14$ ) or high-fat, high-sugar cafeteria diet $(\mathrm{HF}, \mathrm{n}=12)$ during pregnancy and lactation. After birth, all pups were cross-fostered onto another dam from either the same or different feeding group. Milk samples were collected from dams in the $2^{\text {nd }}$ week of lactation. Total protein content of the milk samples was determined by Bradford assay and fatty acid composition assessed by gas chromatography. Offspring were euthanized at 3 weeks of age (weaning) for the determination of body fat mass. qRT-PCR was used to determine mRNA expression of key adipogenic and lipogenic transcription factors, Sterol Regulatory Element Binding Protein 1c (SREBP1c) and Peroxisome Proliferator Activated Receptor gamma (PPAR $\gamma$ ) in subcutaneous and visceral fat depots.

Results: At 3 weeks of age, \% body fat mass was higher in both male and female offspring suckled by a HF dam, independent of whether they had been born to a Control or HF dam $(P<0.001)$. Milk from HF dams had a higher total fat $\%$ and higher content of saturated fat $(P<0.01)$ and omega-6 polyunsaturated fatty acids $(P<0.05)$ compared to milk from Control dams. There was no difference in the protein content of the milk between the Control and HF groups. In females, but not in males, PPAR $\gamma$ mRNA expression in subcutaneous adipose tissue was higher in offspring suckled by HF dams compared to those suckled by Control dams, independent of prenatal nutritional exposure $(P<0.05)$. The expression of SREBP1-c, however, was lower in offspring reared by HF mothers compared to offspring reared by Control mothers in both subcutaneous and retroperitoneal tissues in both males and females $(P<0.01)$. Conclusion: Our findings support the hypothesis that exposure to a high-fat, high-sugar diet during the suckling period in rodents is a more important determinant of fat mass at weaning than exposure before birth, and suggest that this may be driven by the increased fat content of the maternal milk. In females, the increase in fat mass may be mediated by the upregulation of the key adipogenic/lipogenic transcription factor PPAR $\gamma$, however this does not appear to be the case in males. In conclusion, these data suggest that restoring appropriate nutrition in the early postnatal period has the potential to mitigate the negative impact of prenatal dietary exposures on fat mass and further studies to investigate whether these effects are maintained longer term are clearly warranted.

Disclosure of Interest: None Declared.

\section{DOHaD13-1335}

Genetic and experiential influences on the development of stress vulnerability and resilience in rhesus macaques

Dario Maestripieri ${ }^{1, *}$, Stephen Lindell ${ }^{2}$, Christina Barr ${ }^{2}$, Karen Parker ${ }^{3}$

${ }^{1}$ University of Chicago, Chicago; ${ }^{2}$ NIAA/NIH, Rockville;

${ }^{3}$ Stanford University, Palo Alto, United States

Background: Our research conducted with the free-ranging population of rhesus macaques on Cayo Santiago, Puerto Rico, tests the core hypothesis that variation in the quality of early maternal behavior affects the development of stress vulnerability and resilience in the offspring. This research also seeks to identify some of the physiological mechanisms underlying these maternal influences and to investigate the possible role of other risk or protective factors such as polymorphisms in the SERT and OPRM1 genes and the availability of social support.

Method: Two cohorts of 45 infants and their mothers are recruited in the first and in the third year of the study, respectively, and then followed longitudinally from birth through their first 3 years of age. Within each cohort, infants are assigned to one of three groups with low, moderate, and high rates of maternal rejection on the basis of observations conducted in the first 3 months of life.

Results: Preliminary results obtained during the infants' first year of life indicate that plasma cortisol responses to a 30-min separation from the mother are predicted in part by genotype, and in part by early experience. Infants with 1 or 2 copies of the short SERT allele and infants whose mothers had 1 or 2 copies of the short SERT allele had higher cortisol responses to separation than infants with 2 copies of the long SERT allele, or infants whose mothers had 2 copies of the long SERT allele. Moreover, infants exposed to moderate levels of maternal rejection in the first 2 months of life had lower cortisol response to separation than infants exposed to low or high maternal rejection rates. Infants exposed to moderate maternal rejection also had the highest levels of both CSF and plasma oxytocin.

Conclusion: Maternal programming of infant oxytocin levels therefore may be one of the mechanisms through which exposure to early moderate stress promotes greater resilience later in life.

Disclosure of Interest: None Declared.

Key words: None.

\section{DOHaD13-1300}

Increased hippocampal gene expression is associated with low birth weight in female non-human primate neonates Jan P. Buschdorf ${ }^{1, *}$, Shirlene X. Ong ${ }^{1}$, Michael Meaney ${ }^{1,2}$, Joanna Holbrook ${ }^{1}$, Mei Lyn Ong ${ }^{1}$, Keefe Chng ${ }^{1}$

${ }^{1}$ Singapore Institute for Clinical Sciences, Singapore, Singapore;

${ }^{2}$ Douglas Mental Health University Institute, Montreal, Canada 
Background: Low birth weight predicts reduced hippocampal volume as well as impaired cognitive abilities. Increased in utero exposure to glucocorticoids correlates with low birth weight and compromised neural development. However, little is known about the differences in gene expression in late fetal or early postnatal development which might mediate these effects. The aim of this study was to identify changes in hippocampal gene expression early in development which are associated with low birth weight.

Method: We compared the relative mRNA expression of genes involved in glucocorticoid signalling in the hippocampus of non-human primate neonates (Macaca fascicularis) of naturally occurring normal and low birth weights by qPCR and Western-blotting. We then used microarray expression profiling to conduct an open search for genes regulated by birth weight.

Results: We detected a decreased mineralocorticoid receptor/ glucocorticoid receptor (MR/GR) ratio in the hippocampus of low birth weight animals on the transcriptional as well as on protein level. No significant differences were detected for the glucocorticoid and mineralocorticoid receptors individually (Ong et al. 2013 J Mol Endocrinol; doi: 10.1530/JME-120218). Analysis of our microarray data revealed an almost complete separation of hippocampal samples according to birth weight based on individual geneexpression signatures derived from 24,154 probe sets. Gene ontology and pathway analysis suggests increased transcriptional activity as well as enhanced cellular outgrowth and synapse formation in the hippocampus of low-birth weight animals. Disease-related GO terms indicate an up-regulation of numerous genes associated with mental disorders (e.g., ANK3, SAP97, TCF4).

Conclusion: Our data suggest that impaired fetal growth associates with altered hippocampal transcription of genes linked to multiple forms of psychopathology.

Disclosure of Interest: None Declared.

\section{DOHaD13-1364}

\section{Inheritance of stress-dependent epigenetic change in drosophila melanogaster}

Ki-Hyeon Seong ${ }^{1, *}$, Dong $\mathrm{Li}^{1}$, Shunsuke Ishii ${ }^{1}$

${ }^{1}$ RIKEN institute, Tsukuba, Japan

Background: There are numerous epidemiological data of the effect of various stresses on several disease incidences in their adulthood and offspring. At present, the experimental evidences of epigenetic inheritance caused by stresses are also beginning to accumulate in some animal models. However, molecular mechanism and biological meaning underlying these phenomena has not been clearly understood yet. Previously, I showed that Drosophila transcription factor-2 (dATF-2) is involved in heterochromatin formation and that phosphorylation of dATF-2 by various stresses, such as heat shock and osmotic stresses, induces heterochromatin disruption. I also revealed these stress dependent epigenome changes mediated by dATF- 2 can be inherited to next generations in a non-Mendelian manner (Seong et al., 2011). dATF-2 colocalized with HP1 not only on heterochromatin but also at specific euchromatic regions, suggesting that dATF-2 may regulate expression of euchromatic genes in the same manner of heterochromatin by stress.

Method: We mainly used Drosophila $w^{1118}$ and dATF-2 mutant flies for analysis. To measure heterochromatin state, we have used $w^{m 4}$ strain. We have performed expression microarray analysis of male progenies derived from male flies reared in rich and poor nutritional media. For lifespan test, 30 flies were transferred to flesh poor media every 3 days and the number of dead flies were counted.

Results: We have tried to identify the specific stresses that could induce dATF-2 dependent epigenetic change. To induce metabolic stress, I used nutritional stress because I found heterochromatin state of Drosophila dramatically decreased at the high nutritional condition and the nutritional effect did not observed in the dATF-2 mutant background. I have performed gene expression array analysis of $\mathrm{F} 1$ progenies which derived from male parent reared on two different nutritional conditions, poor and rich medium. Surprisingly, the array data showed that almost gene expressional change by nutritional stress might be mediated by dATF- 2 in F1 generation. Moreover, I found the progenies from the male parent reared on rich medium showed shorter lifespan than the progeny from males on poor medium.

Conclusion: These results suggest that the nutritional stress on parents could affect gene expression and lifespan of their offspring in dATF-2 dependent manner.

\section{Reference}

1. Seong KH, Li D, Shimizu H, Nakamura R \& Ishii S. Inheritance of Stress-induced, ATF-2-Dependent Epigenetic Change. Cell. 145(7), 1049-1061, 2011.

Disclosure of Interest: None Declared.

\section{DOHaD13-1610}

Intrauterine growth restriction induces early onset of age induced alterations in the estrous cycle in female growth restricted rats

Suttira Intapad ${ }^{1, *}$, Barbara T. Alexander ${ }^{1}$

${ }^{1}$ Physiology \& Biophysics, UNIVERSITY OF MISSISSIPPI

MEDICAL CENTER, Jackson, United States

Background: The natural transition to reproductive senescence is an important physiological process that occurs with aging and results in menopause in woman and diminished or lost fertility in most mammalian species. In the Sprague Dawley (SD) rat the regular estrous cycle pattern has 4-5 day 
cycle followed by an increase in cycle length prior to a transition into persistent estrous and eventual persistent diestrus by 18 months of age indicative of a peri-menopausal state. Intrauterine growth restriction (IUGR) induced by placental insufficiency at day 14 of gestration in the SD rat programs hypertension in IUGR offspring prior to puberty. However, after puberty female IUGR offspring are normotensive until one year of age when blood pressure is significantly elevated relative to age-matched female control. Hypertension at one year of age in female IUGR is associated with a significant increase in total body fat and visceral fat not observed in age-matched female control offspring. Recent human studies indicate that onset of menopause may occur at an earlier age in low birth weight women. Thus, this study tested the hypothesis that estrous cycling is altered at an earlier age in the female IUGR relative to age-matched female control.

Method: The stage of estrous cycle was determined by vaginal smear in the early morning for a 30 day period starting at 5 and 11 months of age. At 6 and 12 months of age uterine weight was collected and serum estradiol levels were determined.

Results: Estradiol levels (12 month: $42.43 \pm 4.1 \mathrm{pg} / \mathrm{ml}$ and $62.18 \pm 14.2 \mathrm{pg} / \mathrm{ml})$ and uterine weight (12 month: $2.05 \pm 0.18 \mathrm{~g} / \mathrm{kgBW}$ and 12 months; $2.34 \pm 0.22 \mathrm{~g} / \mathrm{kgBW})$ were not statistically different in control relative to IUGR at 6 and 12 months of age, respectively (data for 6 months not shown). Both control and IUGR rats had a regular pattern of cycling that consisted of all stages and exhibited a normal length of 4-5 days at 6 months of age. However, by 11 months of age control rats exhibited an extension of cycle length by 15 days with all phases present (diestrus, midestrus, proestrus and estrous) whereas IUGR rats by 11 months of age exhibited persistent estrous with only the estrous phase present.

Conclusion: Thus, these findings suggest that the female IUGR rat is already in the peri-menopausal state by 11 months of age indicating that an insult during fetal life in the female IUGR rat programs early aging of reproductive system.

Disclosure of Interest: S. Intapad Grant/Research support from: 12POST11980021, B. Alexander Grant/Research support from: HL074927, HL51971.

\section{DOHaD13-1194}

Long term hypoxia during gestation alters perirenal adipose tissue in the lamb: a trigger for adiposity?

Charles Ducsay ${ }^{1, *}$, Elizabeth Newby ${ }^{1}$, Caleb Cato ${ }^{1}$, Krista Singleton $^{2}$, Dean Myers ${ }^{2}$

${ }^{1}$ Center for Perinatal Biology, Loma Linda University, Loma Linda; ${ }^{2} \mathrm{Ob} / \mathrm{Gyn}$, University of Oklahoma Health Sciences Center, Oklahoma CIty, United States

Background: There is a significant deposition of adipose tissue during the final third of gestation in both sheep and human fetuses, particularly in perirenal/abdominal fat.
Further, the fetal adipose depot has characteristics of both brown and white fat. We previously reported increased expression of genes governing white fat adipogenesis or white adipose function (PPAR $\gamma, 11 \beta$-hydroxysteroid dehydrogenase-1 [HSD11B1]) in perirenal fat of the late gestation long-term hypoxic ovine fetus (1). In addition, perirenal adipose expression and fetal plasma leptin was elevated (2), a hallmark of activated adipose tissue. Perirenal adipose from LTH fetuses also exhibited enhanced expression of brown-fat specific genes (PRDM16, uncoupling protein-1 [UCP-1], PGC1 $\alpha$, Deiodinase-II [DIO2]). The present study was designed to test the hypothesis that this brown fat phenotype is maintained post-birth.

Method: Pregnant ewes were maintained at high altitude $(3,820 \mathrm{~m}, \mathrm{LTH})$ from $\sim$ day 40 of gestation through $12-14$ days post-delivery. Perirenal adipose tissue was collected from the LTH lambs $(n=7)$ and age-matched normoxic controls $(\mathrm{n}=5)$ for analysis of UCP-1, PGC1a, PPAR $\gamma$, PRDM16 and DIO2 mRNA by qRT-PCR. Cyclophilin was used as a housekeeping mRNA. Data are expressed in fg mRNA/50 ng RNA, mean \pm SEM.

Results: All values listed are control compared to LTH $\left({ }^{*} \mathrm{p}<0.05\right)$ :

UCP1 $\left(317.0 \pm 195\right.$ vs. $\left.31.7 \pm 11.0^{*}\right)$

PGC1 $\alpha\left(3.0 \pm 0.6\right.$ vs. $\left.1.2 \pm 0.2^{*}\right)$

PRDM16 $\left(0.20 \pm 0.04\right.$ vs. $\left.0.08 \pm 0.03^{*}\right)$

DIO2 $\left(0.67 \pm 0.35\right.$ vs. $\left.0.20 \pm 0.09^{*}\right)$

PPAR $\gamma(17.1 \pm 2.8$ vs. $22.6 \pm 1.5)$

Expression of all genes studied, except PPAR $\gamma$, was significantly reduced in the LTH lambs.

Conclusion: In marked contrast to our previous data in the LTH fetus, lambs exposed to gestational LTH exhibited a pronounced loss of brown fat specific gene expression compared to control lambs. However, PPAR $\gamma$, a major transcription factor governing white adipose tissue was maintained. We speculate that the loss of the brown fat phenotype after birth in the LTH offspring will promote obesity due to decreased energy expenditure, while favoring white adipose deposition. This would profoundly increase the propensity for adipogenesis in these offspring. Supported by NIH Grant HD31226.

\section{References}

1. Am J Physiol Regul Integr Comp Physiol. 2008 294(4): R1312-8. PMID: 18287225.

2. Am J Physiol Regul Integr Comp Physiol. 2006 291(5): R1406-13. PMID: 16825421.

Disclosure of Interest: None Declared.

\section{DOHaD13-1116}

Low insulin-like growth factor 1 in breast milk is associated with lower birth weight and a lower growth curve in a cynomolgus macaque model

Yong Chee $\operatorname{Tan}^{1, *}$, Carine $\operatorname{Lim}^{1}$, Louiza Chan ${ }^{1}$, Yap Seng Chong $^{2}$, Peter D. Gluckman ${ }^{1}$, Keefe Chng ${ }^{1}$ 
${ }^{1}$ Singapore Institute for Clinical Sciences, Agency for Science, Technology and Research, ${ }^{2}$ Department of Obstetrics \& Gynaecology, Yong Loo Lin School of Medicine, National University of Singapore, Singapore, Singapore

Background: Research has shown the association between intrauterine growth restricted (IUGR), low birth weight (LBW) and early onset of metabolic diseases in later life. Insulin-like growth factor 1 (IGF1) is one of the parameters which has been found to differ between normal and low birth weight infants in many animal models. Many studies are focused on IGF1 related genes, free circulating IGF1 in infants and how they impact on LBW infant growth. However, little attention has been paid to the IGF1 concentration in mothers' breast milk on which infants are fed. This study investigates the amount of IGF1 in milk produced by dams of normal and LBW offsprings using a cynomolgus macaque model. The study also explores the effect of the growth trajectory after being fed on maternal milk with high or low levels of IGF1.

Method: After pregnancy detection, control dams were given $100 \%$ of their required caloric intake throughout gestation, while IUGR dams were given $65-70 \%$ of the caloric intake of control dams from gestational day 32 to the end of pregnancy. All neonates were delivered naturally and their birth weights and morphometrics were measured at birth. Follow up measurements were done every 3 months until 15 months of age. Milk collection was done 1 month after delivery. The IGF1 concentration in milk was determined using an IGF1 ELISA kit. All statistical analysis was carried out using SPSS 19.0. Independent t-tests and Pearson correlation coefficient were used for IGF1 analysis. Mixed model analysis was used for growth analysis. p-value of less than 0.05 was considered statistically significant for all tests.

Results: In general, dams with LBW infants produced breast milk with lower concentration of IGF1 compared to control dams. Significant moderate positive correlation was observed between the IGF1 concentration in breast milk and the offsprings' birth weight $(\mathrm{r}=0.489, \mathrm{p}=0.015)$. Offspring from dams with low level of IGF1 in maternal milk had a lower growth curve at 15 months compared to offspring from dams with high level of IGF1 in maternal milk.

Conclusion: Low birth weight infants are associated with lower IGF-1 levels in breast milk produced by dams, and a lower growth trajectory over 15 months.

Disclosure of Interest: None Declared.

\section{DOHaD13-1147}

\section{Maternal administration of glucocorticoid in early pregnancy alters postnatal pituitary-adrenal responses to challenge in young sheep}

Shaofu Li ${ }^{1, *}$, John P. Newnham ${ }^{1}$, Ilias Nitsos ${ }^{2}$, Graeme R. Polglase ${ }^{2}$, Timothy J. Moss ${ }^{2}$, Thorsten Braun ${ }^{3}$, John R. G. Challis ${ }^{1,4}$
${ }^{1}$ School of Women's and Infants' Health, The University of Western Australia, Perth; ${ }^{2}$ The Ritchie Centre, Monash Institute of Medical Research \& Department of Obstetrics and Gynaecology, Monash University, Melbourne, Australia; ${ }^{3}$ Department of Obstetrics, Charite' University Berlin, Berlin, Germany; ${ }^{4}$ Departments of Physiology and Obstetrics and Gynecology, University of Toronto, Toronto, Canada

Background: Synthetic corticosteroid treatment is used in early human pregnancy to prevent virilisation of female fetuses in cases of suspected congenital adrenal hyperplasia. However, the long-term consequences of this treatment on development of the fetal hypothalamic-pituitary-adrenal (HPA) axis are not well understood. We hypothesized that a 2-day exposure to glucocorticoids early in pregnancy would alter HPA axis responsiveness in offspring at 7 months of age. We employed metyrapone to challenge the HPA axis of young lambs through its action to block cortisol synthesis by inhibiting steroid $11 \beta$-hydroxylase activity.

Method: Pregnant ewes carrying singleton fetuses were randomized to control ( $2 \mathrm{ml}$ saline/ewe) or dexamethasone (dex) treatment $(0.14 \mathrm{mg} / \mathrm{kg}$ ewe weight) consisting of four intramuscular injections at 12-hourly intervals over 48 hours on days 40-42 (term 150 days). At 7 months postnatal age, catheters were implanted into a jugular artery and vein. Five days later, we gave a bolus intravenous injection of metyrapone $(30 \mathrm{mg} / \mathrm{kg})$ and arterial blood samples were taken at $-30,-15,0$, 5, 10, 20, 30 60, 90, 120 and 180 minutes. Plasma ACTH and cortisol levels were measured by ${ }^{125}$ I radioimmunoassay.

Results: Metyrapone produced a decrease in plasma cortisol levels at 5 minutes although the difference was only significant in the treated males $(\operatorname{dex}-\mathrm{M})(\mathrm{p}<0.001)$. The fall in cortisol at 5 minutes was associated with a rise in ACTH in all groups, but this ACTH response was lower in treatment females (dex-F) than in control females (C-F) from 5 to 90 minutes $(\mathrm{p} \leq 0.03)$ and was lower in dex-M than in control (C-M) from 90 to 180 minutes $(\mathrm{p} \leq 0.04)$. ACTH levels in dex-F were lower than dex-M at 20 and 30 minutes ( $\mathrm{p} \leq 0.03$ ). In females, the ACTH area under the curve (AUC) response to metyrapone challenge was significantly lower in dextreated animals than in controls $(p=0.03)$. Cortisol levels in response to the change in $\mathrm{ACTH}$ in dex-F were lower than in $\mathrm{C}$ F from 20 to 90 minutes $(\mathrm{P} \leq 0.03)$ but cortisol levels in dex-M were higher than in C-M from 30 to 90 minutes $(p \leq 0.04)$. Cortisol AUC was lower in dex-M than in C-M ( $p=0.007)$, was lower in dex-F than C-F $(\mathrm{p}<0.001)$ and was lower in dex$\mathrm{M}$ compare to dex-F ( $\mathrm{p}=0.002)$.

Conclusion: Exposure of fetal sheep to dexamethasone in early pregnancy resulted in blunted ACTH levels in response to challenge in both sexes. Responses of ACTH and cortisol levels were different in dexamethasone-treated male and female sheep, providing evidence of sex differences in HPA responses to corticosteroid exposure in early gestation.

Disclosure of Interest: None Declared. 


\section{DOHaD13-1621}

\section{Maternal obesity results in lower energy expenditure in adult offspring when challenged with an obesogenic diet}

Xanthi Maragkoudaki ${ }^{1, *}$, Matthew Naylor ${ }^{1}$, Timothy South ${ }^{1}$, Joaquim M. Pombo ${ }^{1}$, Lucilla Poston ${ }^{1}$, Paul D. Taylor ${ }^{1}$

${ }^{1}$ Division of Women's Health, King's College, London, United Kingdom

Background: Maternal obesity is an independent risk-factor for childhood obesity and metabolic syndrome. We have developed a murine model of maternal diet-induced obesity, which gives rise to offspring hyperphagia and obesity [1]. We investigated whether maternal obesity alters offspring energy expenditure and the effect of an obesogenic diet in adulthood. Method: Female mice were fed standard chow or an obesogenic diet 6-weeks before mating, throughout pregnancy and lactation. Offspring were weaned onto standard chow. At 3-months of age energy intake, energy expenditure (EE) and respiratory exchange ratio (RER) were measured continuously for $24 \mathrm{hrs}$ by indirect calorimetry, (Labmaster, TSE) and intraperitoneal glucose tolerance tests (GTTs) performed. Following a 3-week challenge with an obesogenic diet EE, RER and food intake were again measured. All data was expressed as the average \pm SEM. Statistical significance was considered when $\mathrm{P}$ value was $\leq 0.05$. Student's t-test was used to compare averages between groups. Correlation coefficients were calculated for EE and energy intake for each group.

Results: Male and female offspring of obese mothers (OffOb, $\mathrm{n}=11$ ) had lower RER $(\mathrm{P}<0.05)$ than controls (OffCon, $\mathrm{n}=10$ ) at all times. Glucose tolerance was significantly lower in OffOb females compared to OffCon (OffCon AUC: $59.87 \pm 0.75$ vs OffOb AUC: $80.23 \pm 7.8, \quad \mathrm{P}=0.01)$. Female OffCon showed a positive correlation between energy intake and EE; a measure of diet-induced thermogenesis $(\mathrm{r}=0.52, \mathrm{P}=0.01)$, which was absent in OffOb females. Following exposure to the obesogenic diet, female OffOb showed significantly reduced night-time EE compared to OffCon (OffCon: $12.12 \pm 0.75 \mathrm{kcal} / \mathrm{h} / \mathrm{kg}, \mathrm{n}=11$; vs OffOb:10.76 $\pm 0.31 \mathrm{kcal} / \mathrm{h} / \mathrm{kg}, \mathrm{n}=10 \mathrm{P}<0.01$ ).

Conclusion: Maternal obesity was associated with lower RER in young adults and with gender specific glucose intolerance, prior to development of obesity. Maternal obesity showed an interaction with the adult obesogenic diet, significantly influencing EE. This could, in part, be explained by underlying impairment of diet-induced thermogenesis.

\section{Reference}

1. Samuelsson, A.M., et al., Diet-induced obesity in female mice leads to offspring hyperphagia, adiposity, hypertension, and insulin resistance: a novel murine model of developmental programming. Hypertension, 2008. 51(2): p. 383-92.

Disclosure of Interest: None Declared.

\section{DOHaD13-1185}

Maternal pre-implantation or gestational diet alters fetal bone development

Stuart Lanham ${ }^{1}$, Stephanie Marfy-Smith ${ }^{1}$, Franky Lock ${ }^{1}$, Judith Eckert ${ }^{1}$, Adam Watkins ${ }^{1}$, Emma Lucas ${ }^{1}$, Richard O. Oreffo $^{1}$, Tom Fleming ${ }^{1}$, Stephanie Meakins ${ }^{1, *}$

${ }^{1}$ UNIVERSITY OF SOUTHAMPTON, Southampton, United Kingdom

Background: Maternal protein deficiency during pregnancy induces bone structural and strength alterations in female offspring in old age, and a maternal high fat diet can also influence bone structure in older offspring. However, little is known about the effect of maternal diet manipulation on the development of bone in the growing fetus. We have investigated, in a mouse model, how a low protein (LP) or high fat (HF) diet during pregnancy can alter bone development in the growing fetus.

Method: Female MF1 mice (7-8.5 weeks) were naturally mated and fed either normal protein diet (NP, 18\% casein), LP (9\% casein), Emb-LP (LP until gestational day 3.5 then NP), normal fat diet (NF, 10\% fat), HF (22.5\% fat), Emb$\mathrm{HF}$ (HF until gestational day 3.5 then NF). Fetuses were collected at gestational day 17.5. As the developing skeleton was almost complete by this time, a method of assessing peripheral skeletal development was devised. This consisted of adding the number of visible vertebrae below the pelvis to the development of the right forepaw (value of 1 if only metacarpal were visible, 2 if proximal phalanges were visible, and value of 3 if intermediate phalanges were visible).

Results: For the low protein diet, the most developed skeleton was seen in the Emb-LP group $(p<0.001 \mathrm{v}$ controls). Mean fetal mass was increased in Emb-LP, but not to a significant level $(\mathrm{p}=0.07)$. Compared to controls, Emb-LP males were less affected $(p=0.08)$ than females $(p=0.02)$. No differences in mass were found when analysed by gender.

For the high fat diet, the most developed skeleton was seen in the HF group $(p<0.001 \mathrm{v}$ controls, $p=0.02 \mathrm{v}$ Emb-HF), although the Emb-HF group also showed increased skeletal development $(p=0.04 \mathrm{v}$ controls). Mean fetal mass was increased in the Emb-HF group, but not to a significant level. When sexes were compared, both showed increased development in the HF group (both male and female $\mathrm{p}=0.02 \mathrm{v}$ controls), but not the Emb-HF group. Mean fetal mass was increased only in males in the Emb-HF group $(p=0.02 \mathrm{v}$ controls).

Conclusion: Timing and nature of the maternal dietary insult can alter bone development in the growing fetus. In addition, there were sex differences in the fetal response, whereby only bones in female fetuses were affected by low protein diet, but both male and female fetuses showed alteration of bone development by maternal high fat diet. The clear implications of this study are that the high level of 
worldwide dietary fat may lead to increased levels of bone disorders in both males and females in later life.

Disclosure of Interest: None Declared.

\section{DOHaD13-1592}

\section{Maternal undernutrition alters DNA methylation profiles in rat embryonic kidney}

Mariko Hida ${ }^{1}{ }^{*}$, Midori Awazu ${ }^{1}$

${ }^{1}$ Pediatrics, Keio University School of Medicine, Tokyo, Japan

Background: Maternal undernutrition leads to low nephron number. We found that ureteric bud branching, a crucial factor in determining nephron number, is reduced by maternal undernutrition in rats. Reduced nephron number by a nutritional insult is transmitted to the second generation in rats. Furthermore, maternal nutrient restriction alters global methylation in the baboon kidney. We therefore investigated the effect of maternal undernutrition on genomewide DNA methylation in the rat embryonic kidney.

Method: The embryonic day 18 kidneys of dams given food ad libitum $(\mathrm{CON})$ and those subjected to $50 \%$ food restriction throughout pregnancy (NR) were examined. The DNA methylation landscape around promoter $\mathrm{CpG}$ islands was analyzed using methylated DNA immunopreciitation (MeDIP) coupled with microarray (NimbleGen Rat ChIP-chip 385 K Promoter array) comparing methylated fractions of CON and NR. MeDIP probe significances were generated using the Kolmogorov-Smirnov test as implemented by NimbleScan. These values were transformed $(-\log 10)$ to give peak scores, which reflect the probability of methylation at a p-value of less than 0.01. Glomerular number was determined by acid maceration at 3 weeks.

Results: Glomerular number of NR was significantly reduced by $20 \%$. Of 15911 promoter regions included in the array, 7330 regions were hypomethylated and 6310 regions were hypermethylated in NR compared with CON. In NR, nearest genes to methylated regions were categorized as, in descending order of freuency, G-protein coupled receptor signaling pathway, detection of chemical stimulus involved in sensory perception of smell, transcription, transport, apoptosis, development, and others. Hypermethylated genes in NR important in kidney development are, in descending order of peak score, $\beta$ catenin, heparin-binding EGF-like growth factor,activin A receptor, integrin $\beta 4$, HGF, BMP $4 / S \operatorname{mad} 5$, TGF- $\beta 1 /$ Smad4, MMP9, cAMP dependent kinase, integrin linked kinase, PKC $\delta$, and kinases in MAP kinase cascades. Most of these are critical for ureteric branching.

Conclusion: Maternal nutrient restricition changes DNA methylation of genes involved in ureteric branching, which may contribute to reduced nephron number and transgenerational transmission.

Disclosure of Interest: None Declared.

\section{DOHaD13-1470}

\section{Melatonin prevents prenatal dexamethasone-induced programmed hypertension in a rat model}

You-Lin Tain ${ }^{1, *}$, Chien-Ning $\mathrm{Hsu}^{2}$, Ting-Hsin $\mathrm{Wu}^{1}$, Li-Tung Huang ${ }^{1}$

${ }^{1}$ Pediatrics, ${ }^{2}$ Pharmacy, Kaohsiung Chang Gung Memorial Hospital and Chang Gung University, College of Medicine, Taiwan, Kaohsiung, Taiwan

Background: Antenatal corticosteroid is recommended to accelerate fetal lung maturation. In experimental model, maternal dexamethasone (DEX) exposure results in reduced nephron number, contributing to programmed offspring hypertension. We recently fount that early melatonin treatment restores nitric oxide $(\mathrm{NO}) /$ reactive oxygen species (ROS) balance and ameliorates the development of hypertension in young spontaneously hypertensive rats. Nephrogenesis genes can be epigenetically controlled via class I histone deacetylases (HDACs). Glucocorticoids, oxidative stress, and epigenetic changes are proposed mechanisms involved in the developmental programming. Thus we intended to elucidate whether melatonin can modulate oxidative stress or epigenetic regulation to prevent prenatal DEX-induced programmed hypertension.

Method: Pregnancy SD rats received intraperitoneal dexamethasone $(0.1 \mathrm{mg} / \mathrm{kg} \mathrm{BW})$ or vesicle daily from gestational day 16 to 22 to conduct a prenatal DEX exposure model. Male offspring were assigned to four groups ( $\mathrm{n}=5-7 /$ group): control, DEX, control + melatonin, and DEX + melatonin. Melatonin-treated pregnancy rats received $0.01 \%$ melatonin in drinking water during the whole period of pregnancy and lactation. All rats were sacrificed at 16 weeks of age. Renal superoxide and $\mathrm{NO}$ levels were determined by electron paramagnetic resonance. Renal mRNA expression of nephrogenesis-related genes, HDAC, and RAS components were analyzed by real-time PCR.

Results: The DEX group developed hypertension at 16 weeks of age, which was prevented by melatonin therapy. DEX exposure had no effect on nephron number and related genes. However, maternal melatonin treatment increased nephron numbers in control and DEX-treated offspring. In addition, the gene expression of fibroblast growth factor-2 (FGF2) and bone paired box gene 2 (PAX2), responsible for nephrogenesis were increased in 16-week-old DEX offspring kidney. Next, DEX significantly upregulated renin, angiotensin II type 1 receptor (AT1R), and Mas expression in the kidney. Maternal melatonin treatment increased renal expressions of renin, prorenin receptor, AT1R, AT2R, and Mas in control rats. Nevertheless, melatonin treatment increased renal renin, prorenin receptor, ACE2, and AT1R while decreased AT2R and Mas in DEX exposure rats. Renal levels of superoxide and NO were not different among the four groups. Next, we found DEX upregulated HDAC-1 expression in the kidney. However, maternal melatonin therapy increased renal 
expression of HDAC-1, $-2,-3$, and -8 in both control and DEX offspring.

Conclusion: We concluded that prenatal DEX exposureinduced programming hypertension, related to dysregulation of RAS in the kidney. Nephron numbers and restoration of RAS balance contributed to the protective mechanism of melatonin on DEX-induced programming hypertension. Given that class I HDAC isoforms were differentially regulated by DEX and melatonin, the possible epigenetic regulation for the regulation of hypertension in later life awaits further evaluation.

Disclosure of Interest: None Declared.

\section{DOHaD13-1533}

Metabolic imprinting effect during early growth on extra cellular matrix construction in Japanese Black steers

Aoi Nomura ${ }^{1}{ }^{1}$, Ryosuke Fujimura ${ }^{1}$, Sithyphone Khounsakunalath $^{1}$, Kunihiko Saito ${ }^{2}$, Kaori Sakuma ${ }^{2}$, Tuyoshi Abe ${ }^{2}$, Shuichi Kaneda ${ }^{2}$, Tetsuji Etoh $^{1}$, Yuuji Shiotsuka ${ }^{1}$, Hideyuki Takahashi ${ }^{1}$, Takafumi Gotoh ${ }^{1}$

${ }^{1}$ Animal Production and ecology, Kuju Agricultural Research Center, Kyushu University, Taketa; ${ }^{2}$ National Livestock Breeding Center, Nishishirakawa-gun, Japan

Background: In muscle, the extra cellular matrix (ECM) supports the contraction of myofibers and is an important factor affecting the tenderness of meat. A previous study reported that supplementation with grain increased the quantity of heat-soluble collagen and improved meat tenderness in beef. This phenomenon is referred to as "metabolic imprinting or metabolic programming" based on medical research regarding "the developmental origins of health and disease $(\mathrm{DOHaD})$ ". We examined how metabolic imprinting during early nutrition affects the expression of genes related to the ECM in muscles of Japanese Black cattle (JB) fattened on grass.

Method: Twenty-three steers were divided into two feeding groups. One group was intensively nursed with milk replacer and was fed concentrate and roughage after weaning (HE: $n=12$ ). The other group was normally nursed with milk replacer and was fed roughage alone after weaning ( $R$ : $\mathrm{n}=11$ ). The longissimus thoracis muscle (LT) was sampled at $3,10,14,20$ and $30 \mathrm{mo}$.

Results: In this study, we focused on Type I, III, IV and VI collagen, Fibronectin and Laminin. Expression levels of some of these genes were significantly different between the HE and $\mathrm{R}$ groups.

Conclusion: In conclusion, our data suggest that early nutrition may affect the formation of the ECM in the muscle of JB.

Disclosure of Interest: None Declared.

\section{DOHaD13-1753}

Metabolic imprinting effect in beef production: influence of nutrition manipulation during an early growth stage on PPARg2 and PMRT5 a expressions in the longissimus muscle in wagyu (Japanese Black)

Sithyphone Khounsaknalath ${ }^{1, *}$ K Saito ${ }^{2}$, Stefan $\mathrm{Maak}^{3}$, A Saito ${ }^{4}$ and R Fujimura ${ }^{1}, \mathrm{~K} \mathrm{Etoh}^{1}, \mathrm{~K}$ Sakuma ${ }^{2}, \mathrm{~K}$ Kaneda ${ }^{2}$, $\mathrm{T} \mathrm{Abe}^{2}$, T Etoh ${ }^{1}, \mathrm{Y}$ Shiotsuka ${ }^{1}, \mathrm{H}$ Hasebe ${ }^{2}, \mathrm{H}$ Hasebe $^{2}$, A Elke $^{3}, \mathrm{H}$ Takahashi ${ }^{1}$, T Gotoh $^{* 1}$

${ }^{1}$ Kuju Agricultural Research Center, Kyushu University, Takeda City, Oita Prefecture; ${ }^{2}$ National Livestock Breeding Center, Nishigo, Fukushima, Japan; ${ }^{3}$ Farm Animal Biology, Leibniz Institute, Dummerstorf, Germany; ${ }^{4}$ Zenrakuren, Tokyo, Japan

Background: Japanese Black cattle, or "Wagyu", are known to accumulate high levels of intramuscular fat. This experiment was conducted to clarify how early nutrition affected expressions of PPARg2 (Peroxisome ProliferatorActivated Receptor $\gamma 2$ ) and PMRT5 (Protein Arginine Methyltransferase 5) in Japanese Black steers fattened on roughage.

Method: Japanese Black steers were randomly allocated into two groups. The high-energy group (Imp: $\mathrm{n}=12$ ) received intensified nursing (maximum intake of $1.8 \mathrm{~kg}$ per day) until 3 months of age and was then fed a high-concentrate diet from 4 to 10 months of age. The roughage group (Cont: $\mathrm{n}=11$ ) received normal nursing (maximum intake of $0.6 \mathrm{~kg}$ per day) until 3 months of age and was then fed only roughage (orchard grass hay) ad libitum from 4 to 10 months of age. From 10 months of age, both groups were fed only roughage (orchard grass hay) ad libitum until 14 months of age. All animals were then put onto the same pasture and grazed until 20 months of age, fed only roughage (orchard grass hay) ad libitum from 21 to 31 months of age, and then slaughtered at 31 months of age. Fresh tissue samples were biopsied from the longissimus muscle (LM) at 3, 10, 14, 20 and 30 months of age. The expressions of PPARg2 and PMRT5 were investigated in each LM sample by qPCR analysis (Applied Biosystems StepOneSystem, Life Technologies Co., Ltd., USA). Intramuscular fat content was measured by Soxhlet methods in LM in both groups.

Results: The intramuscular fat content in LM was significantly larger in group Imp $(13.2 \pm 4.7 \%)$ than in group Cont $(9.4 \pm 3.6 \%)(\mathrm{p}<0.05)$. PPARg2 is as one of isoforms of PPARg which is a master gene of adipogenesis. The expression of PPARg2 was significantly higher in group Imp than in group Cont at 3 and 30 months of age $(\mathrm{P}<0.05, \mathrm{P}<0.01$, respectively). PMRT5 is an epigenetic signaling gene in association with adipogenesis process of CCATT/enhancer binding protein (C/EBP)b, PPARg, and CCATT/enhancer binding protein (C/EBP)a.The expression of PMRT5 was also significantly higher in group Imp than in group Cont at 3 and 30 months of age $(\mathrm{P}<0.05, \mathrm{P}<0.01$, respectively). 
Conclusion: These findings indicated that PMRT5 would acts as co-activator for the gene expression in adipogenesis which promotes adipogenic differentiation. Our study clearly showed that metabolic imprinting by early nutrition had a strong effect on gene expressions of adipogenesis at ending period in the Japanese Black Cattle.

Disclosure of Interest: None Declared.

\section{DOHaD13-1377}

Metabolic phenotype of adult offspring rats exposed to intrauterine mild hyperglycemia and high fat diet in early life

Kai Zhang ${ }^{1, *}$, Shuang Wang ${ }^{1, *}$, Jingmei $\mathrm{Ma}^{1,1}$, Huixia Yang $^{1}$, Huixia Yang ${ }^{1}$

\section{${ }^{1}$ OBGYN, Peking University 1st Hospital, Beijing, China}

Background: Hyperglycemia during pregnancy, as one of common pregnancy complications, has great influences on series of long-term issues, including high risk of noncommunicable diseases (NCDs), such as metabolism syndrome in their offspring' adult period, which has been proved by a lot of evidence from epidemic and animal models. With new criteria for the diagnosis of GDM gradually promoted in China, greater number of pregnancies is in need of careful glucose control with a high proportion of mild hyperglycemia and macrosomia newborn. Animal model provide a window to investigate the mechanism of maternal-fetal complications in GDM. So this study is to explore the long-term effects of intrauterine mild hyperglycemia and postnatal high fat diet on metabolic phenotype of adult offspring.

Method: Twenty pregnant Wistar rats were randomly divided into intrauterine hyperglycemia group and control group. Streptozotocin (STZ, $25 \mathrm{mg} / \mathrm{kg}$ ) was given to hyperglycemia group with a single intraperitoneal injection to induce intrauterine mild hyperglycemia model (blood glucose between $10-20 \mathrm{mmol} / \mathrm{L}$ ). Their offsprings were divided into 4 groups according to intrauterine blood glucose level and dietary patterns after weaning: (1) hyperglycemia and fed with normal diet after weaning (group DN); (2) hyperglycemia and fed with high-fat diet after weaning (group DF); (3) control and fed with normal diet after weaning (group $\mathrm{CN}$ ); (4) control and fed with high-fat diet after weaning (group CF). Each group had 8 randomly selected male and female rats. The blood glucose levels, blood pressure and birth weights of pregnant rats were recorded weekly after weaning. At the age of 28 weeks which is the adult period, visceral fat content (including mesenteric fat content, epididymal fat content, perirenal fat) and metabolic markers: FPG, FINS, TG and HDL-C were measured in all four groups.

Results: 1. The average blood glucose level of intrauterine hyperglycemia group was significantly higher than control group ( $\mathrm{P}<0.01) ; 2$. On birth day, 3 weeks and 4 weeks, the body weights of DN and DF were remarkably increased (DN vs. $\mathrm{CN}$ and $\mathrm{DF}$ vs. $\mathrm{CF})(\mathrm{P}<\mathrm{O} .05) ; 3$. At the age of 28 weeks, compared with group $\mathrm{CN}$, the perirenal fat contents of $\mathrm{DN}, \mathrm{CF}$ and $\mathrm{DF}$ were remarkably increased $(\mathrm{P}<\mathrm{O} .05) ; 4$. At the age of 28 weeks, FPG, FINS and HOMA-IR in four groups had no statistical difference ( $\mathrm{P}>\mathrm{O} .05)$; Compared to group $\mathrm{CN}$, the $\mathrm{TG}$ levels of $\mathrm{DN}, \mathrm{CF}$ and $\mathrm{DF}$ were significantly increased $(\mathrm{P}<\mathrm{O} .05)$. Arterial pressure of male offspring was increased from 12 weeks (DN vs. CN).

Conclusion: Exposed to intrauterine mild hyperglycemia, the offsprings, especially male offspring showed some evident metabolic changes, including body weight increasing on birth day and early postnatal period, visceral fat content increasing and lipid metabolism disorders, which would be aggravated by postnatal high fat diet.

Disclosure of Interest: None Declared.

\section{DOHaD13-1713}

Modelling developmental origins of adult based diseases (obesity) using mesenchymal stem - cellular, molecular and functional approaches

Vijayalakshmi Venkatesan $^{1, *}$, Soundarya Madhira ${ }^{2}$, Sowmya $\mathrm{K}^{2}$, Sireesha Ganneru ${ }^{2}$, Rajanna $\mathrm{A}^{3}$, Venkata $\mathrm{M}^{4}$, Raghunath $\mathrm{M}^{5}$

${ }^{1}$ Stem Cell Research Lab, National Institute of Nutritrion;

${ }^{2}$ Stem Cell Research Lab, National Institute of Nutrition;

${ }^{3}$ Stem Cell Research Laboratory, National Institute of Nutritrion; ${ }^{4}$ Stem Cell Research Laboratory; ${ }^{5}$ Endocriniology and Metabolism, National Institute of Nutrition, Hyderabad, India

Background: Given the high prevalence of Micronutrient deficiencies (MNs) globally vis -a -vis chronic diseases Obesity T2D, CVD, IR, inflammation, hypertension etc, necessitates understanding of nutrient - gene interactions and programming closer to the in vivo scenario. Development of model systems (in vitro and in vivo) have helped to a large extent, in bridging gap to understand the mechanism(s) of disease. Mesenchymal stem cells (MSCs), form dynamic model system with capacity for differentiation into adipogenic, osteogenic and chondrogenic lineages as well as their transdifferentiation ability.

Aims and Objectives: The present study was carried out to explore the potential (s) of BM-MSCs as an in vitro model to decipher the mechanisms (including inflammation) underlying the deficiency of B Vitamins such as folic acid and B12 (70\%), which forms an important risk factor for the adult onset degenerative diseases including diabesity - of major global concern.

Method: We have used Primary cultures of BM-MSCs (third passage) maintained either in Normal medium/Control or B12 and Folate restricted medium (MN Restricted) were 
characterized for: proliferation markers, cellular stress (ROS), inflammation (TNF $\alpha$ ) and adipogenesis (Triglyceride) and for Leptin as adipogenic lineage marker as per our published protocols.

Results: The data showed that BM-MSCs from MNR group demonstrated significant increase in proliferation rates $(\mathrm{CD} 90+/ \mathrm{CD} 29+$, 'S' phase of cell cycle by FACS, TNF $\alpha(\leftarrow 1.68)$, ROS $(\leftarrow 1.23)$ by fluroscence and functional assessment by accelerated adipogenesis $((\leftarrow 2.5)$ with a quantitative increase in adipocytes/ generation and TG content $(\leftarrow \mathbf{1 . 1 0 5})$. In addition an increase in transcript levels for adipogenic lineage marker - Leptin was upregulated $(\leftarrow 1.8)$ as compared to the Control. Further, these observations were in compliance with the anthropometric data.

Conclusion: Given the ease of accessibility and availability of MSCs, the present study form the basis to report for the first time, application of BM-MSCs as an in vitro model towards development of suitable dietary interventional strategies for the better management of Non communicable disease - of major global concern.

\section{Reference}

1. SL Madhira, SS Challa, M Chalasani, G Nappanveethl, R Ajumeera, RR Bhonde, V Venkatesan. Promise(s) of Mesenchymal Stem Cells as an In Vitro Model System to Depict Pre-Diabetic/ Diabetic Milieu in WNIN/GR-Ob Mutant Rats. PLoS One 2012 7:e48061 IF 4.092.

2. Mitnala Sasikala, GuduruVenkat Rao, V. Vijaya lakshmi, Rebala Pradeep, Suresh Pothani, Pondugala Pavan Kumar, Radhika Gaddipati, Ganneru Sirisha, Ramji Cheemalakonda, Manu Tandan, Chivukula Subramanyam, Seshadri Vasudevan, D. Nageshwar Reddy. Long-term functions of encapsulated islets grafted in non-human primates without immunosuppresssion. 2013, Transplantation (Press). IF 4.17.

Disclosure of Interest: None Declared.

\section{DOHaD13-1241}

\section{Mouse models of FGR show an altered metabolic profile which is reversible following treatment with sildenafil citrate}

Karolina Sulek ${ }^{1, *}$, Joanna L. Stanley ${ }^{1,2}$, David I. Broadhurst ${ }^{2}$, Irene J. Andersson ${ }^{2}$, Wen Hong ${ }^{3}$, Sandra T. Davidge ${ }^{4}$, Louise C. Kenny ${ }^{5}$, Colin P. Sibley ${ }^{6}$, Rupasri Mandal ${ }^{7}$, David Wishart ${ }^{7}$, Philip N. Baker ${ }^{1,2,4}$

${ }^{1}$ Liggins Institute, University of Auckland, Auckland, New Zealand; ${ }^{2}$ Department of Medicine, University of Alberta, Edmonton, Canada; ${ }^{3}$ Department of Obstetrics and Gynecology, Zhejiang University, Hangzhou, China; ${ }^{4}$ Departments of Obstetrics and Gynecology/Physiology, University of Alberta, Edmonton, Canada; ${ }^{5}$ The Anu Research Centre, Departemtn of Obstetrics and Gynecology,
Cork University Maternity Hospital, Cork, Ireland;

${ }^{6}$ Maternal and Fetal Health Research Centre, University of Manchester, Manchester, United Kingdom; ${ }^{7}$ Metabolomics Innovation Centre, University of Alberta, Edmonton, Canada

Background: Fetal Growth Restriction (FGR) is defined as a fetus that fails to reach its genetic growth potential. Smaller size at birth is strongly associated with longer-term health consequences, such as an increased risk of developing cardiovascular/metabolic diseases. Animal studies and limited in vivo human studies suggest that the phosphodiesterase-5 inhibitor Sildenafil citrate (Viagra) might be a possible therapeutic option for FGR. In order to further investigate this potential, we looked for pathological mechanisms of action responsible for FGR, as well as Sildenafil-induced rescue of fetal growth, via aberration of the global metabolic profile in mouse models.

Method: We have previously reported that Catechol-Omethyltransferase $\left(\mathrm{COMT}^{-/-}\right)$and endothelial nitric oxide synthase $\left(\mathrm{eNOS}^{-1-}\right)$ knockout mice show features of FGR. Metabolomic profiles of $\mathrm{COMT}^{-1-}(\mathrm{n}=12)$ and $\mathrm{eNOS}^{-1-}$ mice $(n=8)$ were compared with those of wild type C57BL6/J (WT) mice $(\mathrm{n}=12)$. On day 18.5 of gestation dams were sacrificed and blood samples were collected by cardiac puncture (set on ice to clot; centrifuged at $4^{\circ} \mathrm{C}, 5 \mathrm{~min}$, $5000 \mathrm{rpm}$ ). Serum was snap-frozen and stored at $-80^{\circ} \mathrm{C}$ until further analysis. We applied a targeted quantitative metabolomics approach using a combination of DI-MS (Absolute$I D Q^{\mathrm{TM}}$ Kit) with a reverse-phase LC-MS/MS Kit (BIOCRATES Life Sciences AG, Austria) on ABI 4000 QTrap (Applied Biosystems/MDS Sciex, CA) MS. 180 metabolites were identified and quantified. The effects of Sildenafil citrate on the metabolomic profiles of $\mathrm{COMT}^{-/-}$ and WT mice were then determined. $\mathrm{COMT}^{-1-}$ and WT mice were randomly assigned to either Sildenafil $(0.2 \mathrm{mg} / \mathrm{ml})$ or placebo group from gestational day 12.5-18.5.

Results: In untreated animals, the FGR phenotype was reflected in a markedly different metabolomic profile in $\mathrm{COMT}^{-1-}$ mice, as compared to WT mice; 9 metabolites with $\mathrm{P}<0.01$ and 9 metabolites with $\mathrm{P}<0.05$. There were fewer identified differences in the eNOS ${ }^{-1-}$ vs. WT mice (4 metabolites). Primarily, glycerophospholipids, sphingolipids, acylcarnitines and biogenic amines were the metabolites altered. Sildenafil treatment had little effect on the WT metabolomic profile but significantly altered the $\mathrm{COMT}^{-1-}$ profile such that differences $(\mathrm{P}<0.05)$ in only $5 / 18$ metabolites remained.

Conclusion: Metabolomic profiling can provide insights into pathogenesis of FGR as well as mechanisms of therapeutic effects. Sildenafil treatment normalised concentrations of metabolites, such as kynurenine, which have previously been linked to pregnancy disorders.

Disclosure of Interest: None Declared. 


\section{DOHaD13-1678}

\section{Offspring of high fat fed rats do not exhibit evidence of augmented sympathetic function}

Sarah Henry ${ }^{1, *}$, Ryan Wood-Bradley ${ }^{1}$, Roger Evans ${ }^{2}$, Luise Cullen-McEwen ${ }^{1}$, John Bertram ${ }^{1}$, James Armitage ${ }^{3}$

${ }^{1}$ Anatomy \& Developmental Biology; ${ }^{2}$ Physiology, Monash University, Melbourne; ${ }^{3}$ Optometry, Deakin University, Waurn, Australia

Background: We have previously reported that excessive maternal exposure to fat during pregnancy results in both renal and cardiovascular dysfunction in 1 year-old male and female offspring of fat fed rats (OHF). The exact mechanisms contributing to this dysfunction are, however, unclear. We investigated whether maternal high fat feeding results in increased sympathetic nerve activity in OHF.

Method: Female Sprague-Dawley rats $(\mathrm{n}=5-8)$ were fed either a control (C) (7\% canola oil) or high fat (HF) (3\% canola oil and $20 \%$ lard) diet for 3 weeks prior to mating, and during pregnancy and lactation. From weaning, offspring were chow fed ad libitum. At 1 year of age whole body noradrenaline spillover was estimated in anaesthetized rats. ${ }^{3 H}$ Noradrenaline was infused and whole body noradrenaline spillover was calculated. Sodium nitroprusside (SNP) was then infused to determine changes in sympathetic arousal in response to acute hypotension. Mean arterial pressure (MAP) and heart rate were simultaneously monitored in unconscious animals. In addition, renal noradrenaline content was determined during development (embryonic day 20) and in postnatal animals at 21 days and lyear as a proxy measure for long-term sympathetic nerve activity. Data were analysed using a mixed linear model, weighted for litter with maternal diet and offspring sex as main factors.

Results: There was no impact of maternal diet on whole noradrenaline spillover in male and female $\mathrm{OHF}$ at 1 year $\left(\mathrm{P}_{\text {Diet }}=0.17, \mathrm{P}_{\text {Sex }}=0.97, \mathrm{P}_{\text {Diet }}\right.$ Sex $\left._{\text {S }}=0.40\right)$. In addition, there was no difference in acute sympathetic arousal in male and female

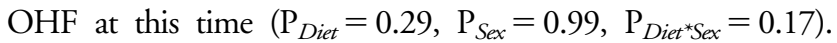
Finally, analysis of regional noradrenaline content demonstrated that there was no effect of maternal diet on sympathetic activity to the kidneys during development $\left(\mathrm{P}_{\text {Diet }}=0.75\right)$ or during postnatal life at 21 days $\left(\mathrm{P}_{\text {Diet }}=0.64, \mathrm{P}_{\text {Sex }}=0.45, \mathrm{P}_{\text {Diet } \text { t }_{\text {Sex }}}=\right.$ $0.79)$ and 12 months $\left(\mathrm{P}_{\text {Diet }}=0.22, \mathrm{P}_{\text {Sex }}<0.01, \mathrm{P}_{\text {Diet }}\right.$ Sex $\left._{\text {S }}=0.65\right)$. Conclusion: In contrast to our hypothesis, we found that exposure to a high fat diet during fetal and early postnatal life does not result in programmed activation of the sympathetic nervous system in male and female rats at 1 year of age. This suggests that a high fat diet during pregnancy does not alter sympathetic nervous system function, and may be ruled out as a major contributing mechanism behind the renal and cardiovascular dysfunction observed in this model. Phenotypically, both control and OHF body weight and adiposity were similar and may be one explanation as to why sympathetic activity was comparable between groups.

Disclosure of Interest: None Declared.

\section{DOHaD13-1105}

Repeated ischemia reperfusion in a maternal protein restriction model increases the risk of fetal brain hemorrhage via P53-signal activation

Takuya Ito $^{1, *}$, Kenichi Funamoto ${ }^{2}$, Kiyoe Funamoto ${ }^{1}$, Clarissa L. Velayo ${ }^{3}$, Yupeng Dong ${ }^{1}$, Miyuki Endo ${ }^{1}$, Rika Sugibayashi ${ }^{4}$, Keita Iida ${ }^{1}$, Toshiyuki Hayase ${ }^{2}$, Nobuo Yaegashi ${ }^{3}$, Yoshitaka Kimura ${ }^{1}$

${ }^{1}$ Advanced Interdisciplinary Biomedical Engineering; ${ }^{2}$ Institute of Fluid Science, Tohoku University; ${ }^{3}$ Department of Gynecology and Obstetrics, Tohoku University Hospital; ${ }^{4}$ Graduate School of Medicine, Tohoku University, Sendai, Japan

Background: Brain hemorrhage is a major cause of neonatal morbidity. More than $90 \%$ of term infants with encephalopathy, including brain hemorrhage, had acute perinatal brain damage. Hypoxic-ischemic events induced by intrapartal asphyxia are thought to be a cause of brain hemorrhage,however, the mechanism by which these events occur have not been fully elucidated. In this study, fetal brain hemorrhage induction through ischemia/reperfusion was investigated using a murine model of maternal protein restriction. Both the presence and timing of brain hemorrhage were observed by means of microscopy and ultrasonography. Sequential fetal brain blood flow measurements by Doppler ultrasound combined with microarray analysis of gene networks provided possible explanations for the pathogenic mechanism of observed brain hemorrhage.

Method: Female C57BL/6N mice were given low protein or regular food for 2 weeks before mating and during pregnancy. In addition, for the protein restriction group alone at gestational days 15.5 and 16.5, a number of maternal mice comprising a third group were given subcutaneous injections of a p53-inhibitor. At day 17.5 of gestation, maternal uterine horns in all groups were exposed. From each pair, one uterine horn was chosen for ischemic/reperfusion cycles which consisted of a 5 minute occlusion by clamping both ovarian and uterine arteries and subsequent release for 5 minutes. This cycle was repeated three times (total of $30 \mathrm{~min}$ ). During these ischemia reperfusion challenges, fetal heart rate and B-mode images of the fetal brain were monitored by electrocardiography and ultrasound-based micro-imaging. After the challenge, the fetal brains were collected for histological evidence of brain hemorrhage. Furthermore, the gene expression of $\mathrm{p} 53$, a marker for severe hypoxia, was quantified using microarray analysis in both low protein and regular food groups of fetuses.

Results: Fetuses of pregnant mice given the low protein diet were highly susceptible to perinatal hypoxic-ischemia as compared to controls. In the low protein diet group with ischemia/reperfusion, the gray scale changes in B-mode ultrasound images were monitored in areas where hemorrhage was detected histologically. Changes in intensity in the low protein diet group were monitored from the $2^{\text {nd }}$ occluding period. 
In the regular diet group with ischemia/reperfusion, no gray scale changes were detected.

Microarray data showed expression levels of p53 and its downstream genes were regulated by a low protein diet and subsequent ischemia/reperfusion. In addition, fetal brain hemorrhage was inhibited by maternal p53-inhibitor treatment indicating a correlation between susceptibility to hemorrhage and activation of p53-signals.

Conclusion: This murine experimental system is a useful tool for understanding the mechanism of fetal brain hemorrhage and p53-signalling is an important target for its prevention.

Disclosure of Interest: None Declared.

\section{DOHaD13-1700}

The long term consequences of prenatal stress on steroid production and functional zonation of the adrenal gland in the spiny mouse

Tracey Quinn ${ }^{1, *}$, Karen Moritz ${ }^{2}$, Hayley Dickinson ${ }^{1}$, David Walker ${ }^{1,3}$

${ }^{1}$ The Ritchie Centre, MONASH INSTITUTE OF MEDICAL RESEARCH, Melbourne; ${ }^{2}$ School of Biomedical Sciences, University of Queensland, Brisbane; ${ }^{3}$ Department of Obstetrics and Gynecology, Monash Universtiy, Melbourne, Australia

Background: Antenatal stress results in elevation of maternal plasma glucocorticoids, disturbs the development of the fetal hypothalamic-pituitary-adrenal axis and steroidogenic activity of the adrenal cortex. However, the long-term effects on adrenal steroidogenesis have not been elucidated. We recently reported fetal adrenal production of dehydroepiandrosterone (DHEA) in the precocial spiny mouse (Acomys cabirinus) ${ }^{1}$. We therefore determined the effect of brief maternal exposure to high glucocorticoids (dexamethasone, DEX) at mid and late pregnancy on adrenal structure and the production of the C19 steroids DHEA and testosterone, as well as the glucocorticoid cortisol in adult offspring.

Method: Pregnant spiny mice were treated for $60 \mathrm{~h}$ with either $125 \mu \mathrm{g} / \mathrm{kg}$ DEX or saline s.c by osmotic minipump at day $20(0.5$ term $)$ or $30(0.75$ term $)$ of gestation. Protein expression of tyrosine hydroxylase $(\mathrm{TOH})$, steroidogenic acute regulatory protein (StAR), 3 $\beta$-hydroxysteroid dehydrogenase (3ßHSD), 17-hydroxylase and 17-20 lyase (p450c17), and cytochrome b5 (cytb5) were determined by immunohistochemistry in adrenal glands at 20-25 weeks of age. DHEA, Testosterone, and cortisol were measured by radioimmunoassay.

Results: Maternal DEX at 20 days gestation did not affect adrenal structure, but expression of StAR, p450c17, and cytb5 were significantly reduced in the adult adrenal zona reticularis (ZR), with significantly greater change in male vs female offspring $(p<0.05)$. Plasma DHEA was significantly decreased in male offspring from DEX-treated $(3.8 \pm 0.6 \mathrm{ng} / \mathrm{ml})$ vs saline-treated $(8.6 \pm 0.8 \mathrm{ng} / \mathrm{ml} ; \mathrm{p}=0.001) \mathrm{dams}$; plasma cortisol was unaffected in either sex, so that the DHEA:cortisol ratio was significantly lower in males only $(p<0.05)$. In contrast, Testosterone levels were significantly increased in male offspring from DEX-treated $(266.03 \pm 50.75 \mathrm{pg} / \mathrm{ml})$ vs saline $(83.47 \pm 32.3 \mathrm{pg} / \mathrm{ml}, p<0.05)$ treated dams. DEX treatment at 30 days of gestation had no effect on adrenal steroid enzyme expression or plasma cortisol, DHEA or testosterone in either sex at 20-25 weeks of age.

Conclusion: Brief exposure to excess glucocorticoid has a long-term impact on the $\mathrm{ZR}$ and adrenal steroidogenesis, affecting secretion of DHEA and Testosterone in male offspring, an effect produced at 0.5 but not 0.75 gestation. DHEA is important for brain development, and its suppression in adult life might contribute to the neurobehavioral pathologies that can arise after illness and stress during pregnancy.

\section{Reference}

1. Quinn et al, Endocrinology 154:1190-201.

Disclosure of Interest: None Declared.

\section{DOHaD13-1386}

The male disadvantage following preterm birth: role of lung maturation

Noreen Ishak ${ }^{1, *}$, Takushi Hanita ${ }^{1}$, Foula Sozo ${ }^{1}$, Robert De Matteo $^{1}$, Richard Harding ${ }^{1}$

${ }^{1}$ School of Biomedical Sciences, Monash University, Melbourne, Australia

Background: Male preterm infants have a greater risk of respiratory insufficiency than females, which may have longterm consequences for neurological development ${ }^{1}$. However, the mechanisms responsible for this 'male disadvantage' in respiratory function are unknown. Our aim was to identify the physiological basis for the 'male disadvantage' in respiratory function following preterm birth, using an established ovine model of preterm birth and respiratory insufficiency ${ }^{2}$. In particular, we examined the composition of pulmonary surfactant.

Method: We delivered unanaesthetised lambs at 0.9 of term ( 8 female, 6 male) and studied them for 8 hours after delivery. At $8 \mathrm{~h}$, the lambs were euthanized for measurement of static lung compliance and collection of bronchoalveolar lavage fluid (BALF) and lung tissue. We later measured the surfactant phospholipid composition and protein concentration of BALF and the gene expression of surfactant proteins $(S P)-A,-B,-C$ and $-D$ and the protein expression of SP-A and pro-SP-C in lung tissue. Data were analysed by either oneway repeated measures ANOVA or the unpaired Student's t-test. Values are presented as mean \pm SEM and statistical significance is denoted as $\mathrm{P}<0.05$. 
Results: At $4-8 \mathrm{~h}$ after birth, male lambs had significantly lower arterial $\mathrm{pH}(7.229 \pm 0.020$ vs $7.319 \pm 0.018)$ and lactate concentration $(1.87 \pm 0.18$ vs $2.62 \pm 0.17 \mathrm{mmol} / \mathrm{l})$, and higher $\mathrm{PaCO}_{2} \quad(61.7 \pm 4.0$ vs $46.2 \pm 1.4 \mathrm{mmHg})$ compared to females; blood glucose concentration was similar in males and females. Inspiratory effort assessed from intrapleural pressure was greater in males than in females (7.9 \pm 0.4 vs $5.6 \pm 0.3 \mathrm{mmHg}$; $\mathrm{P}<0.05$ ), while respiratory rate was not different. Mean arterial pressure (Males (M): $52.5 \pm 1.7$ vs Females (F): $50.6 \pm 1.8 \mathrm{mmHg}$ ) and heart rate (M: $181 \pm 3$ vs F: $179 \pm 6$ beats $/ \mathrm{min}$ ) were not significantly different between sexes. Static lung compliance at $8 \mathrm{~h}$ was $24 \%$ lower in males $(\mathrm{P}=0.007)$. Total protein concentration of BALF (M: $2.94 \pm 0.33$ vs F: $3.28 \pm 0.43 \mathrm{mg} / \mathrm{ml}$ ) and the expression of $S P-A,-B,-C$ and $-D$ and SP-A protein were not different between sexes. Pro-SP-C, expressed as a fold-change relative to females, was significantly reduced by $24 \%$ in males $(0.8 \pm 0.1)$ compared to females $(1.0 \pm 0.1 ; \mathrm{P}<0.05)$. Phospholipid analysis of BALF revealed significantly lower proportions of PC 32:1 (M: $20.8 \pm 0.7$ vs F: $22.6 \pm 0.5 \%$ ) and PE 36:2 (M: $36.5 \pm 1.5$ vs F: $39.9 \pm 0.8 \%)$ in males compared to females $(\mathrm{P}<0.05)$.

Conclusion: Males have poorer respiratory adaptation after preterm birth than females. At $8 \mathrm{~h}$ after preterm birth, male preterm lambs have stiffer lungs than females, resulting in $\mathrm{CO}_{2}$ retention, acidemia and greater inspiratory effort. These differences are likely due to sex differences in the composition of surfactant and pro-SP-C protein expression which together could result in lower lung compliance in males. Poorer lung function and oxygenation in male preterm infants could increase the risk of neurological impairment.

\section{References}

1. Peacock et al. Pediatric Research, 71:305-10, 2012.

2. De Matteo et al. Reproductive Sciences, 17:724-33, 2010.

Disclosure of Interest: None Declared.

\section{DOHaD13-1349}

Transgenerational inheritance of a response to nutritional challenge, and the genetic mechanisms underlying predictive adaptive responses

Amy J. Osborne ${ }^{1, *}$, Sarah M. Morgan ${ }^{1}$, Praneeta Ramsey ${ }^{1}$, Peter K. Dearden ${ }^{1}$

${ }^{1}$ Biochemistry, University of Otago, Dunedin, New Zealand

Background: Developmental pathways are established in early life and differences in these pathways can influence response to environmental exposure during adulthood. For example, a fetus exposed to signals of nutritional deficiency will set its metabolic trajectory to expect such an environment; the fetus has predicted its future environment in response to maternal cues, known as a predictive adaptive response (PAR). If this prediction is incorrect (and the offspring is biologically mismatched to its environment), the individual's response to later environmental exposures can be affected, either positively or negatively. This has been demonstrated in many species, and advantages gained from the predictive adaptive response are able to persist across generations; if the environment remains constant, the predictive trait may become fixed (genetic assimilation).

Method: Here we use the fruitfly Drosophila melanogaster to investigate PARs and the specific mechanisms that lead to transgenerational inheritance of the resulting traits. We are manipulating the diet of $D$. melanogaster by altering the carbohydrate and protein ratios of their food whilst maintaining constant caloric content. We are using quantitative PCR to investigate differential gene expression resulting from this manipulation in the F1 and F2 generations, on both matched and mismatched diets, to enable assessment of the nature of the PAR and consequences for correct and incorrect prediction. This work also extends to the F3 generation and beyond. Our research will provide us with a phenotype through which to investigate the exact genetic nature of the PAR mechanism via whole transcriptome sequencing.

Results: Results from these experiments will be integral in understanding how transduction of environmental cues from mother to offspring can elicit a genetic response and how this can be maintained through the germ line to the F2 generation, F3 and beyond.

Conclusion: Crucially, these data will provide an insight into how development can influence future health outcomes, aiding the understanding of the developmental origins of health and disease.

Disclosure of Interest: None Declared.

\section{DOHaD13-1385}

Up-Regulation of prolyl-hydroxylase 3 and surfactant proteins in the fetal lung after maternal exposure to chronic hypoxia

Erin V. McGillick ${ }^{1}$, Sandra Orgeig ${ }^{1}$, Beth J. Allison ${ }^{2}$, Kirsty L. Brain ${ }^{2}$, Youguo $\mathrm{Niu}^{2}$, Nozomi Itani ${ }^{2}$,

Katie L. Skeffington ${ }^{2}$, Andrew D. Kane ${ }^{2}$, Emilio A. Herrera ${ }^{2}$, Dino A. Giussani ${ }^{2}$, Janna L. Morrison ${ }^{1, *}$

${ }^{1}$ School of Pharmacy \& Medical Sciences, Sansom Institute for Health Research, University of South Australia, Adelaide, Australia; ${ }^{2}$ Department of Physiology, Development and Neuroscience, University of Cambridge, Cambridge, United Kingdom

Background: There is controversy surrounding the risk of respiratory distress syndrome in the intrauterine growth restricted (IUGR) fetus. This is reflected in sheep models of IUGR where some demonstrate an increase and others a 
decrease in the gene expression of surfactant proteins, which are important for the transition to air breathing at birth. IUGR has many aetiologies and the range of sheep models reflects the different mechanisms that may impact on lung development. In this study, we focus on the effect of maternal chronic hypoxia during late gestation on surfactant protein gene expression in the fetal lung.

Method: Chronically catheterised pregnant Welsh Mountain sheep carrying male singletons were exposed to normoxia $(\mathrm{N}$; $\mathrm{n}=8)$ or hypoxia $\left(\mathrm{H} ; \mathrm{n}=7 ; 10 \% \mathrm{O}_{2}\right)$ for the last third of gestation (105-138 days (d); term, $\sim 145 \mathrm{~d})$. At $138 \mathrm{~d}$, fetuses underwent post mortem, weights were recorded and tissues collected. qRT-PCR was used to quantify lung mRNA expression of surfactant proteins (SP-A, -B, -C \& -D), glucocorticoid (GC) regulatory genes (11- $\beta$ hydroxysteroid dehydrogenase (11ßHSD) -1 and -2 isoforms \& glucocorticoid receptor (GR)), hypoxia inducible factor (HIF) action (HIF-1 $\alpha,-1 \beta$ and $-2 \alpha \&$ prolyl hydroxylase (PHD) enzymes 1, 2 and 3) and HIF target genes (vascular endothelial growth factor (VEGF) and adrenomedullin $(\mathrm{ADM}))$. Gene data is presented as mean normalised expression \pm SEM. Data were analysed using the Student's unpaired $t$-test. $P<0.05$ was considered statistically significant.

Results: Maternal $\mathrm{PaO}_{2}$ decreased, while haemoglobin $[\mathrm{Hb}]$ increased during $\mathrm{H}$ relative to $\mathrm{N}\left(\mathrm{PaO}_{2}: 106 \pm 2\right.$ vs. $47 \pm 1 \mathrm{mmHg} ;[\mathrm{Hb}]: 10.0 \pm 0.2$ vs. $11.7 \pm 0.1 \mathrm{~g} / \mathrm{dl}$, both $P<0.05) . \mathrm{H}$ induced asymmetric fetal growth restriction (Fetal body weight: $4.0 \pm 0.1$ vs. $3.2 \pm 0.3 \mathrm{~kg}$; Ratio of bi-parietal diameter to hind limb lower length: $3.7 \pm 0.1 \mathrm{vs}$. $6.5 \pm 0.6$, both $P<0.05)$ but did not alter relative lung weight $(26.1 \pm 1.4$ vs. $23.0 \pm 1.2 \mathrm{~g} / \mathrm{kg}, \quad P=0.16) . \quad \mathrm{H}$ increased the mRNA expression of fetal lung SP-B, -C and -D (SP-B: $0.85 \pm 0.07$ vs. $1.30 \pm 0.15$; SP-C: $3.44 \pm 0.37$ vs. $5.33 \pm 0.74 ; \quad S P-D: 0.03 \pm 0.003$ vs. $0.04 \pm 0.004$, all $P<0.05)$. There was, however, no significant difference in expression of GC regulatory genes (11ßHSD-1, $11 \beta \mathrm{HSD}-2$ \& GR). Interestingly, mRNA expression of PHD3 increased significantly following $\mathrm{H}(0.01 \pm 0.001$ vs. $0.03 \pm 0.002$, $P<0.05)$, but there was no change in the expression of the HIFs (HIF- $1 \alpha$, HIF-1 $\beta \&$ HIF-2 $\alpha$ ) or in the HIF responsive genes, VEGF and ADM.

Conclusion: These findings demonstrate that prenatal exposure to maternal hypoxia may play a protective role in the developing lung by promoting maturation of the surfactant system. The molecular mechanisms underlying this effect remain unclear, however are unlikely to be GC or HIF dependent. These results suggest that exposure to maternal hypoxia during late gestation may enhance fetal lung maturation and aid in preparing a fetus for the successful transition to air breathing at birth.

Supported by the British Heart Foundation and the NHMRC of Australia.

Disclosure of Interest: None Declared.

\section{DOHaD13-1282}

Uteroplacental insufficiency acutely affects the expression of adrenal genes encoding rate limiting enzymes of the catecholamine- and steroid-biosynthesis in the fetus impact on kidney function in later life?

Eva Nüsken ${ }^{1}$, Maria Wohlfarth ${ }^{1}$, Gregor Lippach ${ }^{1}$, Jörg Dötsch ${ }^{1}$, Kai D. Nüsken ${ }^{1, *}$

${ }^{1}$ Department of Pediatrics and Adolescent Medicine, University of Cologne, Cologne, Germany

Background: The adrenal glands of fetal rats develop during the $2^{\text {nd }}$ half of gestation, beginning on gestational day E13 (delivery on day E23). Concurrently, fetal kidney development takes place, partially controlled by the renin-angiotensin-aldosterone system (RAAS) [1]. Ligation (LIG) of the uterine arteries and veins in rats on day E19 serves as a model of placental insufficiency with subsequent developmental programming of kidney function $[2,3]$. We hypothesized that LIG affects kidney function of the offspring in later life because of acute alteration of fetal catecholamine and steroidbiosynthesis and subsequent activation of the RAAS during a critical window of kidney development.

Method: We studied fetuses of rat dams that either underwent bilateral uterine artery and vein LIG or sham operation (SOP) on day E19 of their first pregnancy. Offspring of dams without treatment served as controls (C). Fetuses were removed from the dams by cesarean section on day E19 (4h after surgery; LIG, $\mathrm{n}=20$; SOP, $\mathrm{n}=24$, C, $\mathrm{n}=12$ ), day E20 (24h after surgery; LIG, $\mathrm{n}=27$; SOP, $\mathrm{n}=19 ; \mathrm{C}, \mathrm{n}=12)$ and day E22 (72h after surgery; LIG, $\mathrm{n}=10 ;$ SOP, $\mathrm{n}=20 ; \mathrm{C}, \mathrm{n}=24$ ). Fetal kidneys and adrenal glands were extracted and immediately shock frozen. Gene expressions of tyrosine hydroxylase $(\mathrm{TH})$, dopamine-Phydroxylase $(\mathrm{DpH}), 11 \beta$-hydroxylase (CYP11B1) and aldosterone synthase (CYP11B2) in fetal adrenal glands and of renin in fetal kidneys were measured by qRT-PCR.

Results: Expressions of all investigated adrenal genes were altered in LIG fetuses $24 \mathrm{~h}$ after surgery (day E20), compared to fetuses of group C. Expressions of genes encoding rate limiting enzymes of catecholamine synthesis $(\mathrm{TH}, \mathrm{DpH})$ were elevated $(p<0.001)$, expressions of genes encoding rate limiting enzymes of corticosterone- (CYP11B1) and aldosterone-biosynthesis (CYP11B2) were reduced $(\mathrm{p}<0.001)$. 4h and $72 \mathrm{~h}$ after surgery, expressions of fetal adrenal genes were similar in all groups. In kidneys of LIG fetuses, expression of renin was elevated $24 \mathrm{~h}$ and $72 \mathrm{~h}$ after surgery $(\mathrm{p}<0.001)$.

Conclusion: In our model, LIG offspring shows significant modification of adrenal factors affecting the RAAS in a critical window of kidney development. Increased expression of $\mathrm{TH}$ and $\mathrm{DpH}$ may indicate increased fetal exposition to catecholamines. Subsequently, renin expression may be induced via renal $\beta$-adrenergic receptors. Aldosterone synthesis may concomitantly be reduced. As LIG offspring shows reduced fractional sodium reabsorption $(\mathrm{FeNa})$ and increased 
urinary aldosterone excretion at 7 weeks of age, programming of tubular kidney function by adrenal-renal interaction is conceivable.

\section{References}

1. Dötsch J, et al. Fetal programming of renal function. Pediatr Nephrol. 2012;27:513-20.

2. Nüsken KD, et al. Uteroplacental insufficiency after bilateral uterine artery ligation in the rat: impact on postnatal glucose and lipid metabolism and evidence for metabolic programming of the offspring by sham operation. Endocrinology. 2008;149:1056-63.

3. Plank C, Nüsken KD, et al. Intrauterine growth restriction following ligation of the uterine arteries leads to more severe glomerulosclerosis after mesangioproliferative glomerulonephritis in the offspring. Am J Nephrol. 2010;32:287-95.

Disclosure of Interest: None Declared.

\section{DOHaD13-1600}

Functional capacity and physical activity levels in children and adolescents with congenital heart disease

Lucia C. Pellanda ${ }^{1,2, *}$, Camila Schaan ${ }^{3}$, Beatriz D. Schaan ${ }^{4,5}$

${ }^{1}$ Post graduation Program, Fundação Universitária de Cardiologia do Rio Grande do Sul (FUCRS); ${ }^{2}$ Public Health

Department, Universidade Federal de Ciencias da Saúde (UFCSPA); ${ }^{3}$ Fundaçáo Universitária de Cardiologia do Rio Grande do Sul (FUCRS); ${ }^{4}$ Endocrine Division, Hospital de Clinicas de Porto Alegre; ${ }^{5}$ Department of Internal Medicine, Universidade Federal do Rio Grande do Sul, Porto Alegre, Porto Alegre, Brazil

Background: With advances in surgical techniques and postoperative support, there was a significant increase in survival of children with congenital heart disease (CHD), with consequent increase in long-term morbidity. Children and adolescents with CHD tend to be sedentary, with low levels of physical activity when compared to healthy individuals, and more often become sedentary adults with risk factors for coronary heart disease. Thus, the objective of the present study was to evaluate the physical activity level and functional capacity of children and adolescents with CHD. We also investigated the existence of possible correlations with surgical and echocardiographic findings, metabolic and inflammatory profile and differences between patients with acyanotic vs. cyanotic CHD.

Method: Children with cyanotic and acyanotic CHD followed in a tertiary Pediatric Cardiology Clinic reference ceter were evaluated with the six-minute walk test (6MWT). to assess functional capacity, of the International Physical Activity Questionnaire (IPAQ -short form) questionnaire to evaluate physical activity levels. Laboratory examinations included blood glucose, triglycerides, cholesterol, complete blood count, C-reactive protein and insulin levels. All patients also underwent a comprehensive transthoracic echocardiographic study.

Results: Twenty-five individuals were evaluated, the mean age was $12.0 \pm 3.7$ years, and $20(80 \%)$ of them were male. The IPAQ showed that six individuals (24\%) were very active, eight (32\%) were active, nine (36\%) had irregular physical activity and two (8\%) were sedentary. The mean distance walked in the 6MWT, considering all individuals studied, was $464.7 \pm 100.4$ meters, which was $181.4 \pm 42.0$ meters less than the predicted $(\mathrm{p}=0.005)$.

Conclusion: Children and adolescents with CHD have low functional capacity and low physical activity levels. It is important to assess physical activity level and functional capacity can in CHD children and adolescents, to allow for the application of preventive health measures early during the lifecourse.

Disclosure of Interest: None Declared.

\section{DOHaD13-1440}

Intergenerational associations between parental height and cardiovascular disease risk in the offspring-findings from the Andhra Pradesh Children and Parents Study (APCAPS)

Poornima Prabhakaran ${ }^{1, *}$, Dimple Kondal ${ }^{1}$, Radhakrishna $\mathrm{KV}^{2}$, Srinath Reddy ${ }^{1}$, Yoav Ben-Shlomo ${ }^{3}$, George Davey $\mathrm{Smith}^{3}$

${ }^{1}$ Public Health Foundation of India, New Delhi; ${ }^{2}$ National Institute of Nutrition, Hyderabad, India; ${ }^{3}$ University of Bristol, Bristol, United Kingdom

Background: Parental influences on cardiovascular disease (CVD) risk in their children can be linked to their own earlier circumstances and adult height is a sensitive proxy marker for this. The association of parental height with offspring CVD risk was examined in the Andhra Pradesh Children and Parents Study (APCAPS).

Method: APCAPS, a retrospective family-based study builds on a birth cohort (1987-1990) born within a nutrition supplementation trial of pregnant mothers in 29 villages in Andhra Pradesh, India. Parents and siblings of nearly 1800 index children were retraced to build this family cohort. Interviewer administered questionnaires were used to collect data on demographic, nutrition, physical activity and health status. Anthropometric, body composition, biochemical and vascular measures were collected using standardised techniques.

We present data from subjects in 1129 households $(\mathrm{N}=4369)$ in 20 villages. The association of parental height (Mothers $\mathrm{N}=987$, Fathers $\mathrm{N}=798$ ) with CVD risk factors in children $(\mathrm{N}=2581$, mean age $=22.3 \mathrm{yrs}$, Males $=58.9 \%)$ was assessed using multiple regression analyses, adjusting for 
potential confounders and familial clustering. CVD risk factors included height, weight, body mass index, waist and hip circumference, skin folds, total body fat and fat-free mass, fasting glucose, insulin, HOMA-Insulin Resistance, lipids, blood pressure, pulse wave velocity and augmentation index. Results: Maternal and paternal height were positively associated with offspring height $[\beta=0.302(0.274,0.331)$ and $0.271(0.239$, $0.302)]$, weight $[\beta=0.170(0.137,0.203)$ and $0.167(0.128$, $0.205)]$, body mass index $[\beta=0.034(-0.003,0.070)$ and $0.042(-0.001,0.0084)]$, waist $[\beta=0.066(0.033,0.099)$ and $0.068(0.033,0.105)]$ and hip $[\beta=0.140(0.105,0.176)$ and $0.152(0.109,0.194)]$ circumferences, total body fat $[\beta=0.102(0.062,0.142)$ and $0.082(0.043,0.122)]$ and fatfree mass $[\beta=0.152(0.122,0.181)$ and $0.173(0.140,0.206)]$. Most of these effects were however attenuated or lost on adjusting for child's current socio-economic status and height. Maternal height showed a positive association, robust to adjustment, with HOMA and diastolic blood pressure only among female offspring ( $\mathrm{p}$ for interaction $=0.02$ and $<0.001$ respectively). Paternal height was positively associated with LDL$\mathrm{C}$ and inversely with systolic and diastolic blood pressure in both genders, after accounting for confounders. There was no association with other outcomes studied. Comparison of maternal versus paternal height influences showed true differences only for fasting insulin, HOMA and diastolic blood pressure.

Conclusion: Taller maternal height (reflective of better early nutritional and environmental circumstances) leading to improved birth outcomes (developmental origins hypothesis) and consequent better cardiovascular risk profile in the next generation has been shown in Western populations. In our study, increasing parental height was associated with increases, rather than decreases in most offspring CVD risk factors, after accounting for potential confounders. This suggests that the role of post-natal lifestyle factors such as poor diet and physical activity, associated with increased urbanization, may be the causative pathway rather than in-utero influences.

Disclosure of Interest: None Declared.

\section{DOHaD13-1535}

\section{Is socio-economic transition in a rural area of India associated with cardiovascular risk in children?}

Kalyanaraman Kumaran ${ }^{1,2 *}$, Smita Kulkarni ${ }^{1}$, Anjali $\mathrm{Radkar}^{3}$, Sanjeevanee Mulay ${ }^{3}$, Chittaranjan Yajnik ${ }^{1}$

${ }^{1}$ Diabetes Unit, KEM Hospital Research Centre, Pune, India; ${ }^{2} M R C$ Lifecourse Epidemiology Unit, University of Southampton, Southampton, United Kingdom; ${ }^{3}$ Population Studies, Gokhale Institute of Politics and Economics, Pune, India

Background: The rise in cardiovascular disease (CVD) and type 2 diabetes mellitus (T2DM) in India has partly been attributed to the rapid socio-economic transition and urbanisation resulting in adoption of increasingly energy dense diets and reduced physical activity. The concept of socio-economic transition is multi-disciplinary and is difficult to measure. The change in socio-economic status (SES) and its indicators may be used to understand and partly quantify the transition. The Pune Maternal Nutrition Study (PMNS) was set up in 1993 in a rural area around Pune, India to prospectively study the influence of maternal nutrition during pregnancy on fetal growth and future disease risk in their offspring. We had an opportunity to examine the change in SES in this rural cohort and compare their status against the national and state indicators. We also examined the association between their SES and body size and diet in childhood.

Method: Data on the mothers was collected between 1993-96 and subsequently data on their offspring was collected at 6 years $(\sim 2001)$ and $12(\sim 2007)$ years of age. Information on SES was collected using standardised questionnaires (Parikh and Trivedi at 1993-96 and 2001; Standard of Living Index at 2001 and 2007) enabling us to compare changes in SES within the cohort. Comparisons with rural Maharashtra and India were made using data from the National Family Health Survey 1999 and 2006 (NFHS II and III). Information on diet was collected at 12 years. We followed up 700 live births of whom information on SES was available at all three time points in 570 families. We restrict this analysis to change between 6 and 12 years in offspring. Results: There was an increase in those classified as high SES from 64 to $77 \%$; in comparison the increase in rural Maharashtra was from 10 to $30 \%$. Overall $47 \%$ were classified as high SES in India in 2006. There was an increase in the percentage of nuclear families from $36 \%$ in 2001 to $46 \%$ in 2007 (57 and 61\% in rural Maharashtra and India respectively). The percentage of those receiving piped drinking water increased from 17 to $42 \%$ (23 to $37 \%$ for rural Maharashtra). The percentage of those using wood for cooking declined from 64 to $47 \%$. There was an increase in those living in 'pucca' (bricks and stone) houses and decline in those living in 'kaccha' (mud and thatch) houses. There was also an increase in material possessions. High SES at 6 and 12 years was associated with greater weight and height at both ages and with greater body fat percent at 12 years. Those in high SES had greater total cholesterol but lower total WBC levels at both ages. At 12 years, they also had a greater total calorie, fat and protein intakes but similar carbohydrate intake.

Conclusion: Our data suggests that our cohort is more affluent than rural Maharashtra and that there has been an improvement in living standards in this rural community. The rise in living standards is associated with increases in height and weight in the children along with an increase in risk factors for CVD. The effects of transition at the beginning will be felt by the upper SES and it is possible that generations of undernutrition followed by excessive catch up growth accounts for their greater risk. Population measures to reduce CVD in India may therefore need to be tailored for targeting specific groups.

Disclosure of Interest: None Declared. 


\section{DOHaD13-1137}

The unborn siblings of preterm infants, role of maternal age - a follow up register-linkage study of the 1987-1990 Finnish birth cohort

Suvi Turkka ${ }^{1, *}$, Eero Kajantie ${ }^{1,2}$, Marja Vääräsmäki ${ }^{3,4}$, Mika Gissler ${ }^{1}$, Anneli Pouta ${ }^{3}$, Petteri Hovi ${ }^{1,2}$

${ }^{1}$ National Institute for Health and Welfare; ${ }^{2}$ Children's Hospital, Helsinki University Central Hospital and University of Helsinki, Helsinki; ${ }^{3}$ National Institute for Health and Welfare; ${ }^{4}$ Oulu University Hospital, Oulu, Finland

Background: Families of newborn preterm infants may encounter crisis or at least suffer from decreased quality of life which may lead to longer interbirth intervals, i.e. time between two subsequent births. Maternal age could modify this association. The purpose of this comprehensive Finnish nationwide register-linkage study was to describe interbirth interval after a preterm singleton birth.

Method: We obtained data from Finnish Medical Birth Register (MBR) that encompasses detailed pregnancy and newborn data (available from 1987 onwards) for each infant born in Finland. We included all the children born between Jan 1, 1987 and Sep 30, $1990(n=235$ 624). Sibling data (from Jan 1, 1987 to May 31, 2012) came from the National Population Register Centre (PRC). We linked the data by unique personal identification numbers allocated for every resident of Finland.

The number of singleton births was 230 378, 9983 (4.3\%) were preterm. Interbirth interval was defined as the time between an index child's birth during 1987-1990 and the subsequent birth to a same mother. The effect of gestational age was analyzed by Cox-regression for proportional hazards. In addition, index child sex, maternal age and the number of mother's previous children were taken account as covariates. We excluded births of 1178 infants (555 preterm, 5.5\%) who died before $1^{\text {st }}$ birthday and 2210 because of stillbirth. Data on gestational age was missing for 2916 (1.3\%).

Results: There was $56.8 \%$ probability that a subsequent sibling was born to an index child, the average interbirth interval being 3.7 years.

The number of subjects in five subgroups according to gestation weeks - breakpoints at 28, 32, 37 and 42 weeks - were 225, 769, 8211,205 218 and 9648. At age 2, percentages of index children with a sibling in these subgroups were $10.7 \%, 12.5 \%$, $14.9 \%, 15,9 \%, 18.8 \%$ and $16.0 \%$. Having a teenage mother changed these percentages into $0.0 \%, 34.1 \%, 21.1 \%, 25.2 \%$, and $26.1 \%$. The mothers-age*gestation -interaction p-values were $>0.068$. At age 3 percentages of index children with a sibling were $20.9 \%, 23.5 \%, 29.3 \%, 31.0 \%$, and $35.9 \%$. Percentages for teenage mothers were $33.3 \%, 43.9 \%, 35.9 \%$, $43.8 \%$, and $45.6 \%$, with interaction p-values $>0.01$.

At the end of follow-up, number of those with a sibling, in the preterm groups pooled, was 39 per thousand less than expected.
We compared interbirth intervals to those born at term (37-42 weeks of gestation) first with an unadjusted Cox model. In the three respective preterm-child subgroups breakpoints at 28and 32 weeks, the hazard ratios for having a sibling were 0.64 ( 0.52 to 0.78$), 0.71$ (0.62 to 0.80$)$ and 0.92 (0.89 to 0.95$)$. For nulliparous $(\mathrm{N}=91004)$ these hazard ratios were 0.48 ( 0.37 to 0.63$), 0.57(0.50$ to 0.65$)$ and 0.82 $(0.79$ to 0.85$)$. In models adjusted for maternal age, index child sex and number of previous deliveries (if appropriate), the hazard ratios were approximately the same.

Conclusion: The likelihood of having younger siblings was lower for preterm children as compared to those born at term, and the role of maternal age was not prominent. Preterm birth has a double legacy on the population: of each thousand children born alive preterm, 55 die during infancy; for the remaining, 39 siblings remain unborn.

Disclosure of Interest: None Declared.

\section{DOHaD13-1539}

The placental origins of later cancer risk: results from the Dutch famine birth cohort study

Tessa Rosebom ${ }^{1, *}$, Rebecca Painter ${ }^{1}$, Susanne De Rooij ${ }^{1}$, Karin Michels ${ }^{2}$, David Barker ${ }^{3}$

${ }^{1}$ Academic Medical Centre Amsterdam, Amsterdam, Netherlands; ${ }^{2}$ Harvard Medical School, Boston, United States;

${ }^{3}$ University of Southampton, Southampton, United Kingdom

Background: There are indications that certain types of cancer originate in utero. Breast cancer, ovarian cancer and prostate cancer are more prevalent among individuals who were larger at birth. Recently, a study in Finland found the size of the placental surface was associated with lung cancer, independently from size at birth. Here, we examined the associations between the size of the mother's pelvis, the size of the baby and placenta at birth and later cancer mortality in other populations, and to which degree maternal nutrition confounds this association.

Method: The Dutch Famine Birth Cohort consists of 2414 term singletons born alive in the Wilhelmina Gasthuis in Amsterdam between 1943 and 1947. Ration distribution records were used to determine maternal famine exposure. Medical birth records provided information about the mother, the size of the baby and placenta at birth. Causes of death until December 31st 2007 were retrieved for 2254 cohort members. In total, 98 cohort members died of cancer. Results: Placental length, breadth, area and volume were all positively associated with cancer mortality. For every $40 \mathrm{~cm}^{2}$ increase in placental area the hazard ratio (HR) for cancer mortality was 1.19 (95\%CI: 1.06-1.33). This association was independent of birth weight, it was similar for men and women, and it was not affected by prenatal famine exposure. Large pelvic size of the mother was associated with increased 
cancer mortality in men $(\mathrm{HR}=1.19[1.02-1.39])$. This association was independent of size at birth and famine exposure. Although birth weights tended to be higher among those who died of cancer, the association did not reach statistical significance.

Conclusion: Large placental size is associated with increased cancer mortality. Our findings add to the evidence that cancer may originate in utero.

Disclosure of Interest: None Declared.

\section{DOHaD13-1258}

Tobacco use in rural South India: patterns, smoking dependence, quit attempts and response to a group counseling intervention for smoking cessation

Jayakrishnan Radhakrishnan ${ }^{1, *}$, Aleyamma Mathew ${ }^{2}$, Paul Sebastian ${ }^{3}$

${ }^{1}$ Division of Community Oncology; ${ }^{2}$ Division of Epidemiology and Biostatistics; ${ }^{3}$ Director, Regional Cancer Centre,

Trivandrum, India

Background: The association between tobacco and various types of cancers is well established. More than $80 \%$ of lung cancers occurring among men in the world are related to tobacco smoking. In India, more than one third of adults use tobacco in one form or the other. Every year 63,000 lung cancer cases are reported in the country. In this background this study was conducted to understand the tobacco use patterns, smoking dependence, quit attempts in one year and to explore the feasibilityof conducting a group counseling session for smoking cessation intervention.

The objective of the study was to assess the patterns of tobacco use among men in the age group 18-60 years, nicotine dependence, smoking quit attempts in one year and participation rates for group counseling in smoking cessation intervention programmes conducted after 2-3 weeks of completion of the baseline survey.

Method: 11 clusters were identified from 4 Community Development Blocks (lower level of administrative system) in a rural district of Kerala state in South India using multistage sampling technique for an ongoing community based smoking cessation intervention trial. Information on tobacco use, nicotine dependency using the 6 item Fagerstrom Test for Nicotine Dependence (FTND) and smoking quit attempts by study subjects in one year were assessed using semi structured pretested questionnaire. Data collection was done using trained volunteers. Group counseling cum medical camps were conducted in 5 clusters of the intervention area within 2-3 weeks of completion of the baseline survey after sensitizing the community.

Results: 3304 subjects were interviewed on the tobacco use pattern through a house to house survey. 928 subjects were identified as current daily smokers which constituted a prevalence of $28 \%$ (mean age 44.4, SD 9.2). Prevalence of smokeless tobacco and alcohol were reported as $9.8 \%$ and $18.1 \%$ respectively. Among smokers more than 50\% smoked cigarette, $14 \%$ smoked bidi and $28 \%$ used both forms. A significant association was also found between age and tobacco use. $(p<0.0001)$. The overall FTND score among current daily smokers was 5.04 (SD: 5.05). The FTND scores increased with age and decreased with higher literacy and socioeconomic status. As far as smoking is concerned, 19.2\% reported quitting smoking habit at least for 24 hours in a span of one year, while $28.4 \%$ reported reduction in the frequency of smoking though they were unable to quit the habit totally. 474 participants were requested to attend the group counseling cum general medical camp in the intervention area. Only $16 \%$ attended the medical camp and received group counselling.

Conclusion: Tobacco use continues to be a public health problem in the rural areas of the country. Tobacco control requires a multipronged strategy. The study points to the fact that instead of adopting a single strategy for tobacco cessation, the feasibility of utilizing multiple cost effective approaches has to be explored to increase response to tobacco cessation programmes in the rural community.

Disclosure of Interest: None Declared.

\section{DOHaD13-1246}

A longitudinal analysis of blood pressure in a sample of term small for gesational age and appropriate for gestational age infants

John M. D. Thompson ${ }^{1, *}$, Rinki Murphy ${ }^{2}$, Karen E. Waldie $^{3}$, Clare R. Wall ${ }^{4}$, Edwin A. Mitchell ${ }^{1}$

${ }^{1}$ Paediatrics: Child and Youth Health; ${ }^{2}$ Medicine; ${ }^{3}$ Psychology;

${ }^{4}$ Discipline of Nutrition, University of Auckland, Auckland, New Zealand

Background: Increased blood pressure in childhood and adoescence continues to track through to adulthood, hence increased blood pressure in early life is likely to lead to higher blood pressure in adulthood and an increaed risk of metabolic syndrome. It is thus of potential importance to determine what factors are potentially modifiable in early life to attempt to decrease the chance of high blood pressure in later lide. Thus we aimed to determine risk factors associated with blood pressure (BP) throughout childhood, particularly those relating to prenatal events, parental factors, early childhood growth; and to assess their impact over time.

Method: In a longitudinal study of 871 Caucasian infants sampled disproportionately for small for gestational age (SGA): approximately half had birthweight $<10^{\text {th }}$ percentile when delivered at term in Auckland, New Zealand; BP and body mass index (BMI) was measured at visits at $3 \frac{1}{2}$, 7 and 11 years of age and analysed using a repeated measures model. 
Prenatal events assessed were SGA status, exposure to maternal smoking or hypertension in-utero. Parental factors included socioeconomic status, maternal and paternal prepregnancy BMI, maternal age at delivery and parity. Early childhood factors assessed were age, gender, breastfeeding and weight SDS change during three time windows (birth to nine months, 9months to 3.5 years, 3.5 years to 7 years). Each risk factor was examined for an interaction with time.

Results: Increased systolic and diastolic BP was most strongly associated with the child's current BMI z-score, maternal hypertension in pregnancy, and their age. Increased systolic $\mathrm{BP}$ was also associated with male gender and maternal BMI. Increased diastolic BP was also associated with maternal age at delivery (under 25 or over 30). Only maternal hypertension in pregnancy history showed an amplification in association with offspring BP with time.

Conclusion: The strongest determinants of childhood BP is their age and current BMI z-score. Maternal hypertension during pregnancy predicts a small proportion of childhood $\mathrm{BP}$, however this effect increases during the childhood period of 3.5 to 11 years.

Disclosure of Interest: None Declared.

\section{DOHaD13-1455}

Accelerated infant weight gain and risk for non-alcoholic fatty liver disease in early adulthood

Laura Breij $^{1, *}$, Gerthe Kerkhof ${ }^{1}$, Anita Hokken-Koelega ${ }^{1}$

${ }^{1}$ Department of Pediatrics, Subdivision of Endocrinology, Erasmus University Medical Center/Sophia Children's Hospital, Rotterdam, Netherlands

Background: Non-alcoholic fatty liver disease (NAFLD) is considered the hepatic metabolic syndrome. Some studies demonstrated an association between small size at birth and NAFLD. Rapid catch-up often follows small birth size and has been associated with metabolic syndrome, but its association with NAFLD remained unknown.

Method: In 268 adults aged $18-24 \mathrm{yrs}$, BMI, waist circumference, triglyceride, gamma-glutamyltransferase $(\gamma$ GT), alkaline phosphatase (ALP), alanine aminotranserase (ALT), and aspartate aminotransferase (AS) levels were determined. Fatty liver index (FLI, 0-100) was calculated. Associations of birth weight SDS and first year gain in weight and length SDS were determined with FLI and other liver markers. Comparisons were performed between subjects with and without rapid catch-up in weight in the first year of life. Furthermore, an FLI-score (low, intermediate, high risk for NAFLD) was assigned to each participant to determine clinical relevance, and ordinal regression analyses were performed.

Results: Gain in weight in the first three months of life was associated with FLI as a continuous variable, whereas low birth weight was not. There were no significant associations with $\gamma$-GT, ALT, or AST. Subjects with rapid catch-up had significantly higher FLI in early adulthood than subjects with slow catch-up. Higher gain in weight for length in the first three months of life, and rapid catch-up in weight, were both associated with a higher FLI-score at 21 yrs.

Conclusion: Accelerated gain in weight for length in the first three months of life is associated with a higher risk for NAFLD in early adulthood, whereas small size at birth is not.

Disclosure of Interest: None Declared.

\section{DOHaD13-1115}

Adult survivors of severe fetal anaemia have smaller, thicker walled left ventricles, reduced coronary perfusion and increased cardiovascular risk

Alexandra Wallace ${ }^{1, *}$, Stuart Dalziel ${ }^{2}$, Kent Thornburg ${ }^{3}$, Craig Broberg ${ }^{3}$, Alistair Young ${ }^{4}$, Brett Cowan ${ }^{4}$, Jane Harding ${ }^{1}$

${ }^{1}$ Liggins Institute, University of Auckland; ${ }^{2}$ Children's Emergency Department, Starship Children's Hospital, Auckland, New Zealand; ${ }^{3}$ Heart Research Center, Oregon Health and Sciences University, Portland, United States; ${ }^{4}$ Centre for Advanced MRI, University of Auckland, Auckland, New Zealand

Background: Adult sheep exposed to fetal anaemia have increased infarction area following ischaemic insult, but the cardiovascular effects of fetal anaemia in adult humans are unknown. The purpose of this study was to compare cardiovascular function, risk factors and myocardial blood flow (MBF) in adults who received intrauterine transfusion for fetal anaemia due to rhesus disease with that of their unaffected siblings.

Method: Participants were adults who received intrauterine transfusion at National Women's Hospital in Auckland from 1963-92 and their unaffected sibling(s). Assessments included anthropometry, blood pressure, lipids, heart rate variability and cardiac MRI, including analysis of $\mathrm{MBF}$ at rest and with hyperaemia induced by adenosine infusion. Statistical analysis was by multiple regression adjusted for age, sex, and birth weight z-score, with participants nested in their sibling groups.

Results: Affected participants $(n=95)$ were younger than unaffected $(\mathrm{n}=92$, mean \pm SEM: $33.7 \pm 1.0$ vs. $40.1 \pm 1.1$ years, $\mathrm{p}<0.001)$ and born at lower gestation $(34.3 \pm 0.2$ vs. $39.5 \pm 0.2$ weeks, $\mathrm{p}<0.001)$ but had higher birth weight z-scores $(0.82 \pm 0.1$ vs. $-0.14 \pm 0.1, \mathrm{p}<0.001)$. Affected participants had lower end diastolic volume $(154.6 \pm 2.6$ vs. $166.2 \pm 2.6 \mathrm{ml}, \mathrm{p}=0.003)$, end systolic volume $(58.1 \pm 1.4$ vs. $64.1 \pm 1.5 \mathrm{ml}, \mathrm{p}=0.007)$, and stroke volume $(96.3 \pm 1.6$ vs. $102.1 \pm 1.7 \mathrm{ml}, \mathrm{p}=0.02)$, increased relative left ventricular free wall thickness $(0.62 \pm 0.01$ vs. $0.58 \pm 0.01$, $\mathrm{p}=0.04)$ and a trend towards reduced left ventricular mass index $\left(64.7 \pm 0.9\right.$ vs. $\left.67.2 \pm 0.9 \mathrm{~g} / \mathrm{m}^{2}, \mathrm{p}=0.06\right)$. MBF was reduced in affected participants at rest (global $\mathrm{MBF}$ 
$0.24 \pm 0.01$ vs. $0.29 \pm 0.01 \mathrm{ml} / \mathrm{min} / \mathrm{g}, \quad \mathrm{p}=0.009)$, with significant reduction in resting $\mathrm{MBF}$ in the left ventricular free wall $(0.28 \pm 0.01$ vs. $0.37 \pm 0.01 \mathrm{ml} / \mathrm{min} / \mathrm{g}, \mathrm{p}=0.0001)$ but not the septum $(0.37 \pm 0.02$ vs. $0.42 \pm 0.02 \mathrm{ml} / \mathrm{min} / \mathrm{g}$, $\mathrm{p}=0.08)$. During adenosine infusion, there was a trend towards reduced $\mathrm{MBF}$ in affected participants $(1.21 \pm 0.18$ vs. $1.73 \pm 0.18 \mathrm{ml} / \mathrm{min} / \mathrm{g}, \mathrm{p}=0.06)$. Affected participants also had reduced high density lipoprotein concentration $(1.44 \pm 0.04$ vs. $1.56 \pm 0.04 \mathrm{mmol} / \mathrm{L}, \mathrm{p}=0.04)$ and augmented sympathovagal tone indicated by increased low frequency to high frequency ratio on assessment of heart rate variability $(2.3 \pm 0.3$ vs. $1.5 \pm 0.3, \mathrm{p}=0.04)$.

Conclusion: Adult survivors of fetal anaemia have smaller volume, relatively thicker walled hearts than unaffected siblings, with reduced MBF at rest, potentially indicating increased risk of ischaemic injury. Together with reduced HDL and augmented sympathetic tone, these findings provide the first evidence in humans that fetal anaemia is associated with an increased cardiovascular risk profile in adulthood.

Disclosure of Interest: None Declared.

\section{DOHaD13-1088}

An ultrasound assessment of the heart, kidneys and common carotid arteries in three month old lambs born moderately preterm

Paul Lombardo ${ }^{1, *}$, Michal Schneider ${ }^{1}$, Robert DeMatteo ${ }^{2}$, Richard Harding ${ }^{2}$, Vivian Nguyen ${ }^{2}$, Graeme Polglase ${ }^{3}$, M Jane Black ${ }^{2}$

${ }^{1}$ Medical Imaging and Radiations Science; ${ }^{2}$ Anatomy \& Developmental Biology, Monash University; ${ }^{3}$ The Ritchie Centre, Monash Institute of Medical Research, Clayton, Australia

Background: Structural changes to the heart, vasculature and kidneys in response to preterm birth may lead to an increased risk of cardiovascular disease in later life. We conducted an in vivo ultrasound assessment of preterm and term lambs three months after birth to examine growth of the heart, blood vessels and kidneys.

Method: Moderately preterm birth (0.9 of term) was induced (Epostane) in seventeen Border Leicester x Merino ewes after antenatal corticosteroids (Celestone Chronodose: $11.4 \mathrm{mg}$ i.m.; 2 doses, 24 hrs apart). Eight female and nine male lambs were born vaginally at $132 \pm 1$ days gestational age (dGA). A control group of lambs was induced to be born at term ( $147 \pm 1$ days dGA) and comprised six female and eleven males. Ultrasound imaging was conducted on all lambs at approximately three months ( $13 \pm 1$ weeks) after birth to measure the structure and function of the left ventricle, kidney dimensions and renal artery blood flow velocities, as well as the diameter and blood flow velocities within the aortic root, pulmonary arteries and the right and left common carotid arteries. Three measurements of each parameter were obtained and averaged, then corrected for body weight and compared using a two-way ANOVA and Tukey post-hoc analysis of preterm/term and male/female factors and their interaction.

Results: At three months of age, preterm lambs weighed less than term lambs $(15.8 \%, p=0.002)$. Left ventricular hypertrophy was evident in the hearts of preterm lambs, with an increase in the anterior $(16.5 \%, \mathrm{p}<0.05)$ and posterior $(14.2 \%, p=0.003)$ wall systolic thickness of the left ventricle and in the systolic $(15.7 \%, \mathrm{p}=0.011)$ and diastolic $(12.2 \%$, $\mathrm{p}=0.034)$ thickness of the interventricular septum. The percentage fractional shortening of the cardiac muscle was also increased $(12.7 \%, \mathrm{p}=0.011)$ and the aortic root diameter was enlarged $(11.1 \%, \mathrm{p}=0.043)$ in the preterm lambs. Systolic blood flow velocity in the aortic root was increased $(17.8 \%, \mathrm{p}=0.037)$ and the diameter of the right $(16.1 \%, p=0.009)$ and left $(13.9 \%, p=0.044)$ common carotid arteries were increased in the preterm lambs compared to term lambs. The right kidney length $(10.7 \%, \mathrm{p}<0.05)$ and width $(9.7 \%, \mathrm{p}=0.012)$ dimensions were also increased compared to term lambs. The left kidney could not be demonstrated adequately using ultrasound. There were no significant sex differences found in any of the comparisons.

Conclusion: Lambs born moderately preterm exhibit a thicker left ventricular wall, increased left ventricular contraction and larger common carotid vessel diameter than term lambs when adjusted for body weight at three months of age. These findings are likely to be an adaptive response to preterm birth and/or glucocorticoid administration. The relationship between these findings and subsequent cardiovascular vulnerability into adulthood will be examined.

Disclosure of Interest: None Declared.

\section{DOHaD13-1567}

Associations of cardiovascular risk factors among parents and their offspring of the New Delhi birth cohort

Dorothy Lall ${ }^{1, *}$, Shika Sinha ${ }^{2}$, Poornima Prabhakaran ${ }^{3}$, Nikhil Tandon ${ }^{4}$, Ramakrishnan Lakshmy ${ }^{5}$, Santosh Bhargava $^{6}$, HPS Sachdev ${ }^{7}$, D Prabhakaran ${ }^{1}$

${ }^{1}$ Cardiovascular research, Centre for Chronic Disease Control;

${ }^{2}$ Research, Sitaram Bhartia Institute of Science and Research;

${ }^{3}$ Research, Public Health Foundation of India; ${ }^{4}$ Endocrinology, All India Institute of Medical Sciences; ${ }^{5}$ Biochemistry, All India Institute of Medical Sciences; ${ }^{6}$ Paediatrics, Sunderlal Jain Hospital; ${ }^{7}$ Paediatrics, Sitaram Bhartia Institute of Science and Research, New Delhi, India

Background: The intergenerational association of cardiovascular risk factors has been reported from several different populations ${ }^{1}$. However, there is considerable heterogeneity in the strength of this association between generations. In this study we aim to explore the correlaion between parent and 
offspring cardiovascular risk factors namely, sum of skin fold thickness, Systolic blood pressure (SBP), Diastolic blood pressure (DBP), Body mass index (BMI), Total cholesterol (TC), High density cholesterol (HDL), Triglyceride (TG), and Fasting plasma glucose (FPG) in an urban Indian birth cohort and also determine the strength of these associations. Method: The study population consisted of 398 parents (F1), $40 \%$ male, average age $37.3( \pm 1.6)$ years and their 548 adolescent offspring (F2), 58\% male, average age $13.0( \pm 2.4)$ years of the New Delhi Birth Cohort. The strength of association is reported as the standardised $\beta$ coefficients of multiple regression analyses. The TC, TG, HDL, FPG and sum of skin fold thickness were log transformed for analyses. The model adjusted for age and gender of both the cohorts, clustering between siblings and for socioeconomic status, summarised as a score derived as the first principal component from a correlation matrix of 17 binary variables indicating material possessions ${ }^{2}$. Child and parent BMI were also adjusted for, except for BMI and skinfold thickness associations.

Results: Risk factors in the offspring were significantly associated with their parent's risk factors except for FPG. The $\beta$ coefficients (SE), 95\% CI were 0.19(0.04), 0.07-0.24 for SBP, 0.15(0.56), (0.04-0.22) for DBP, 0.32(0.67), $0.34-0.61$ for BMI and $0.22(0.01), 0.01-0.05$ for sum of skin fold thickness. The lipid profile (logarithmic) associations were as follows, $0.37(0.7), 0.28-0.56$ for TC, $0.37(0.06), 0.23-0.46$ for HDL and $0.38(.06), 0.23-0.44$ for TG between offspring and their parents.

Conclusion: Most risk factors are significantly associated among parents and their offspring after adjustments for potential confounders and clustering between siblings. This has important implications in its scope to be used for communication and screening in offspring of parents with cardiovascular risk factors to prevent cardiovascular disease in the next generation.

\section{References}

1. Vik KL, Romundstad P, Nilsen TI. Tracking of cardiovascular risk factors across generations: family linkage within the population-based HUNT study, Norway. J Epidemiol Community Health 2013;0:1-7.

2. Huffman MD, Prabhakaran D, Osmond C, et al. Incidence of cardiovascular risk factors in an Indian urban cohort results from the New Delhi birth cohort. J Am Coll Cardiol 2011;57:1765-74.

Disclosure of Interest: None Declared.

\section{DOHaD13-1464}

\section{Developmental changes in cardiac expression of micrornas and their targets involved in cardiomyocyte proliferation in fetal and newborn sheep}

Janna Morrison ${ }^{1, *}$, Doug Brooks ${ }^{2}$, Caroline McMillen ${ }^{1}$, Enzo Porrello ${ }^{3}$, Song Zhang ${ }^{1}$, Kimberley Botting ${ }^{1}$
${ }^{1}$ Early Origins of Adult Health Research Group, Sansom Institute for Health Research; ${ }^{2}$ Mechanisms in Cell Biology and Diseases Research Group, Sansom Institute for Health Research, University of South Australia, Adelaide; ${ }^{3}$ Laboratory for Cardiac Regeneration, School of Biomedical Sciences, University of

Queensland, Brisbane, Australia

Background: Recent studies in zebrafish and mice implicate microRNAs (miRs) in the regulation of genes responsible for cell cycle progression in the heart, in particular the miR-15 family (miR-15a, -15b, -16, -195 and -497), miR-133, miR199a and miR-590. miRs are small non-coding RNAs that regulate the abundance of protein by targeting mRNA for degradation or inhibiting translation. In mice, members of the miR-15 family are upregulated in early postnatal life, corresponding to a time when cardiomyocytes lose the ability to proliferate and regenerate a heart after injury. The timing of cardiomyocyte maturation in mice differs from humans, such that the ability of murine cardiomyocytes to proliferate is lost between 4-10d postnatal life, but the transition to terminally differentiated cardiomyocytes begins in late gestation in humans and in sheep. We aim to test the hypothesis that miRs responsible for the regulation of proliferation will increase with increasing age and the mRNA expression of their target genes will decrease in the fetal and postnatal sheep heart.

Method: We have measured the cardiac expression of miRs involved in cell cycle entry in the sheep fetus at $90 \mathrm{~d}$ gestation (when all cardiomyocytes are mononucleated and capable of proliferation; term $=150 \mathrm{~d}$ ), $121 \mathrm{~d}$ gestation (when $\sim 75 \%$ of cardiomyocytes are capable of proliferation), 141d gestation (when $\sim 25 \%$ of cardiomyocytes are capable of proliferating), $5 \mathrm{~d}$ and $21 \mathrm{~d}$ after birth (when almost all cardiomyocytes are terminally differentiated).

Results: Fold change analysis to $90 \mathrm{~d}$ gestation indicates that miR-497 increases with increasing age and that miR-15a is increased at $21 \mathrm{~d}$. The miR-15 family target genes (Chek1, Cdc2a, Birc5 and Spag5) decrease with increasing age. The expression of miR-199a increases with increasing gestation, but returns to the level of expression observed at $90 \mathrm{~d}$ gestation at $21 \mathrm{~d}$ postnatal life. Interestingly, miR-590 does not change with increasing age. miR-199a and miR-590 target genes, $H_{o p} X$ and Crim1, do not change with increasing age, however, Clic5 unexpectedly increases into postnatal life. miR-133 increases with increasing age, but unexpectedly, so too does the mRNA expression of its target genes Pgam1 and Connexin43.

Conclusion: The overall changes in expression of the miR-15 family and their target genes are consistent with the changes observed in mice across the period of proliferative to terminally differentiated cardiomyocytes in early postnatal life. Interestingly, however, miR-133, -199a and -590 and their target genes are not consistent with the timing of changes in their expression in mice. This suggests that proliferation of sheep cardiomyocytes is subtly different to 
that of mice, but suggests that the sheep heart may be capable of regeneration during fetal life.

Disclosure of Interest: None Declared.

\section{DOHaD13-1657}

Diet in early life and the development of cardiometabolic disease risk factors in young adulthood in Cebu, Philippines

Nanette Lee Mayol ${ }^{1, *}$, Isabelita Bas ${ }^{1}$, Judith Borja ${ }^{1}$, Paulita Duazo $^{1}$, Linda Adair ${ }^{2}$

${ }^{1}$ USC Office of Population Studies Foundation, Inc., Cebu City, Philippines; ${ }^{2}$ Nutrition, University of North Carolina at Chapel Hill, Chapel Hill, United States

Background: Studies have explored the potential programming role of early diet on the development of cardiometabolic disease (CMD) risk factors later in life but few, if any, come from developing countries like the Philippines. We examined how protein and fat intakes from complementary foods at 6 , 12,18 and 24 months (mos) relate to young adult body mass index (BMI), waist circumference (WC), body fat (\%BF), blood pressure $(\mathrm{BP})$, fasting glucose $(\mathrm{FG})$ and lipid levels (total, low and high density lipoprotein cholesterol and triglycerides (TC, LDL, HDL, TG)).

Method: We used data from 1613 participants (54\% male) of the Cebu Longitudinal Health and Nutrition Survey who were born between 1983 and 1984. Diet data in the first 2 yrs of life were collected prospectively from the mother using 24-hour recall. Energy adjusted protein and fat intakes from complementary foods at 6, 12, 18 and 24 mos were standardized to allow comparability across time points. Infants who were exclusively breastfed at these time points were excluded. Outcome measures were collected in 2005 when participants were between 20 to 22 yrs old. To estimate the association between early diet measures and the outcomes, we used regression models adjusted for total energy intake and breastfeeding status at each time point from 6 mos to 2 yrs, birthweight, gestational age, sex, baseline maternal age and education, household income, and urbanicity. Alpha was set at 0.05 .

Results: Mean protein intake from 6 to 24 mos ranged from 10 to $12 \%$ while mean fat intake ranged from 12 to $13 \%$ of total energy intake. Formula milk was a major source of protein across all time points, but its contribution decreased as the child got older. Infant cereal was a top protein source at 6 mos while seafoods and bakery products became prominent sources from 12 mos onwards. For fat intake, formula milk and cooking oil were the main sources at 6 mos. Cooking oil's contribution to fat intake increased as the child got older. In 2005, 11\% had high TC $(>=200 \mathrm{mg} / \mathrm{dL}), 10 \%$ had high LDL $(>=200 \mathrm{mg} / \mathrm{dL}), 48 \%$ had low HDL $(<40 \mathrm{mg} / \mathrm{dL})$ and $17 \%$ had high TG $(>=150 \mathrm{mg} / \mathrm{dL})$ levels. About $6 \%$ had high BP $(>=140 / 90 \mathrm{mmHg}$ ) and $9 \%$ were overweight $(\mathrm{OW}=\mathrm{BMI}>=25 \mathrm{~kg} / \mathrm{sqm})$. Fat and protein intakes were associated with lipid levels. Having high TC and LDL levels were predicted by fat intake at 18 mos [odds ratio $(95 \% \mathrm{CI})$ : $1.27(1.04,1.54)$ and $1.28(1.04,1.56)$, respectively] while having low HDL levels were associated with protein intake at $12 \operatorname{mos}[1.14(1.01,1.29)]$. Fat and protein intake in early childhood but not in infancy also influenced obesity measures. Specifically, fat intake at 18 and 24 mos and protein intake at 18 mos were associated with being OW [0.78 (0.62, 0.98), $1.28(1.06,1.56)$, and $1.24(1.02,1.50)$, respectively]. Moreover, fat intake at 24 mos was significantly associated with adult WC and \%BF [linear regression coeff (95\% CI): $0.57(0.11,1.04)$, and 0.41 (0.09, 0.72), respectively]. No significant early diet effects on TG, BP and FG were observed.

Conclusion: Results suggest that early life diet composition may influence chronic disease risk in the Philippines. The latter part of the first 2 yrs of life provides opportunities for dietary intervention to prevent adult CMD. These information are particularly valuable in a transitioning country with increasing levels of CMD morbidity and mortality.

Disclosure of Interest: None Declared.

\section{DOHaD13-1055}

Early detection of cardiac dysfunction after preterm birth by speckle-tracking echocardiography

Ulf Schubert $^{1, *}$

${ }^{1}$ Neonatology, Karolinska Sweden, Stockholm, Sweden

Background: Preterm birth is associated with adverse cardiovascular events later in life. The underlying mechanisms are unknown. We undertook a sequential analysis of cardiac function after preterm birth by speckle-tracking echocardiography (STE) and compared the results to a healthy control group. Method: Evaluation of cardiac function of 25 very preterm infants (GA 26-30 weeks) at birth, at term-equivalent age and at 3 months of corrected age and comparison to the findings in 30 healthy term children ( $1^{\text {st }}$ investigation intrauterine). We measured longitudinal strain (\%), strain rate $(1 / \mathrm{sec})$ and tissue velocities $(\mathrm{cm} / \mathrm{s})$ in both ventricles in systole and diastole to characterize myocardial performance and compared the results to conventional echocardiography. Results: At 3 months of corrected age, very preterm infants exhibit significantly lower left ventricular (LV) strain values (19.9 vs $22.0 \%, \mathrm{p}<0.001)$, systolic (5.8 vs $6.4 \mathrm{~cm} / \mathrm{s}, \mathrm{p}=0.01)$ and diastolic ( 7.8 vs $10.6 \mathrm{~cm} / \mathrm{s}, \mathrm{p}<0.001)$ tissue velocities and early diastolic strain-rate values $(3.9 / \mathrm{s}$ vs $4.7 / \mathrm{s}, \mathrm{p}<0.001)$ compared to healthy control infants born at term. There was a trend to lower values even in the right ventricle- though not statistically significant. Conclusion: Left ventricular systolic and diastolic dysfunction emerges already 6 months after very preterm birth and 
can be identified by STE while conventional echocardiography is not able to detect abnormal myocardial performance at this age. LV dysfunction might occur because of premature adaption towards higher systemic afterload and incomplete re-modelling of the LV early in life.

Disclosure of Interest: None Declared.

\section{DOHaD13-1631}

Early life stress dysregulates growth and angiogenic factors and increases microvascular density in the kidney

Analia Loria ${ }^{1, *}$

${ }^{1}$ Experimental Medicine, Georgia Regents University, Augusta, United States

Background: Previously, we have shown that maternal separation (Msep), a model of chronic behavioral stress during early life, does not change blood pressure at baseline but exacerbates the cardiovascular response to a chronic hypertensive stimulus. Therefore, we focused in the kidney since it is the organ involved in long-term blood pressure regulation. We found that Msep rats show reduced glomerular filtration rate and signs of proteinuria. Thus, we hypothesized that Msep primes the renal and cardiovascular system to chronic disease. The aim of this study was to determine the renal microvascular density (MV) of Msep weanlings and adult rats. In addition, to test the mRNA expression of growth and angiogenic factors in the kidney of Msep early in postnatal life.

Method: Msep was performed in WKY by separating approx half of the male pups from the dam $3 \mathrm{hr}$ a day from days 2-14 of life. Renal tissue from 3 and 12 week old rats were perfused with intravascular contrast agent $(2 \mathrm{~mL} / \mathrm{min})$, which was a freshly mixed radio-opaque silicone polymer containing lead chromate (Microfil MV122) for micro-computed tomography (CT) analysis. In addition, renal tissue from 1-week-old rats was harvested and snap frozen for PCR studies. The $\mathrm{RT}^{2}$ Profiler ${ }^{\mathrm{TM}} \mathrm{PCR}$ Array profiles the expression of 84 key genes encoding enzymes known or predicted to modify rat growth and angiogenic factors and therefore gene expression in kidney.

Results: In 3 week old rats, MV 0-200 $\mu \mathrm{m}$ diameter was greater in cortex and medulla of Msep rats (428 \pm 51 and $\left.313 \pm 46 \mathrm{MV} / \mathrm{mm}^{2}\right)$ compared to control $(279 \pm 33$ and $\left.209 \pm 30 \mathrm{MV} / \mathrm{mm}^{2}, \mathrm{p}<0.05\right)$. In 12 week old rats $\mathrm{MV}$ density $0-200 \mu \mathrm{m}$ was greater in cortex of Msep rats $\left(316 \pm 48\right.$ and $\left.241 \pm 34 \mathrm{MV} / \mathrm{mm}^{2}\right)$ compared to control $\left(193 \pm 18\right.$ and $\left.149 \pm 23 \mathrm{MV} / \mathrm{mm}^{2}, \mathrm{p}<0.05\right)$ as well. MV density in cortex and medulla $(0-500 \mu \mathrm{m}$ diameter $)$ at 3 and 12 weeks was also increased $(\mathrm{p}<0.05)$. In addition, in oneweek-old pups we found that ANGPTL1 (blood vessel maturation and stability, 2 folds), ADAMSTS1 (normal growth, and organ morphology and function, 3 folds) and
AMOT (vessel endothelial cell migration and morphogenesis of new blood vessels, 5 folds) were significantly increased $(\mathrm{p}<0.05)$ in Msep rats.

Conclusion: As vasculogenesis occurs in response to local cues (such as growth factors) to form new vessels during development, we propose that Msep (or adverse environment in early stages of development) induces an abnormal vascular development in the kidney leading to a permanent change of the renal vascular structure and function and therefore, impairing chronic blood pressure control in the adulthood.

\section{References}

1. Loria AS, Pollock DM, Pollock JS. Early life stress sensitizes rats to angiotensin II-induced hypertension and vascular inflammation in adult life. Hypertension. Feb;55(2): 494-9, 2010.

2. Loria AS, Kang KT, Pollock DM, Pollock JS. Early life stress enhances Angiotensin II-mediated vasoconstriction by reduced endothelial nitric oxide buffering capacity. Hypertension. 58(4):619-26., 2011.

3. Loria AS, Yamamoto T, Pollock DM, Pollock JS. "Early Life Stress Induces Renal dysFunction in Adult Male But Not Female Rats". Am J. Physiol Regul Integr Comp Physiol, 15;304(2):R121, 2012.

4. Loria AS, Brands MW, Pollock DM, Pollock JS. "Early life stress sensitizes the renal and systemic sympathetic system in rats". Am J. Physiol Renal. Epub ahead of print.

Disclosure of Interest: None Declared.

\section{DOHaD13-1422}

Effects of reactive oxygen species on vascular function in the offspring of protein-restricted stroke-prone spontaneously hypertensive rats

Kumiko Takemori $^{1, *}$, Hiroyuki Ito $^{2}$

${ }^{1}$ Department of Food and Nutrition, Faculty of Agriculture, Kinki University, Nara: ${ }^{2}$ Department Biomedical Engineering, Faculty of Biology-Oriented Science and Technology, Kinki University, Wakayama, Japan

Background: It has been already revealed that reactive oxygen species (ROS) has an important roles in the potential mechanism underlying the programming of hypertension in utero. As ROS are known to induce epigenetic changes, cardiovascular dysfunction in the offspring of protein-restricted dams may be due to epigenetic mechanisms triggered by ROS. However, it is still unclear how ROS may induce hypertension or vascular dysfunction in the offspring of protein-restricted dams. In this study, we investigated the effects of ROS on vascular function in the stroke-prone spontaneously hypertensive rats (SHRSP) offspring of protein-restricted dams.

Method: Male and female SHRSPs were used. After confirming successful mating by the appearance of a vaginal 
plug, female rats were fed either a control diet containing $20 \%$ casein or a protein-restricted diet containing $9 \%$ casein, with pair feeding until gestation. At 12 weeks of age, an osmotic mini pump filled with phorbol 12-myristate 13acetate (PMA, NADPH oxidase activator with a protein kinase $\mathrm{C}$ activation) was inserted subcutaneously and the connected cannula was inserted into a femoral vein to infuse PMA at a dose of $10 \mathrm{nmol} / \mathrm{h}$ for 4 weeks. Anti-oxidant enzyme activities in red blood cells fraction and plasma diacron-reactive oxygen metabolites (dROMs, mainly due to hydroperoxids) contents were assessed. We further investigated the vascular reactivity and the expression of endothelial nitric oxide synthase (eNOS) and soluble guanylic acid cyclase (sGC) in thoracic aorta.

Results: There were no significant differences in body weight of dams fed the protein-restricted or control diet. However, the birth weight of male and female offspring of proteinrestricted dams was lower than that of offspring of control dams. No significant differences found in blood pressure of offspring of protein-restricted dams or control dams. To determine the effects of oxidative stress on vascular function in the offspring of protein-restricted SHRSP, oxidative stress were induced in 12-week-old offspring of protein-restricted and control SHRSP for 4 weeks. Plasma d-ROMs level was significantly higher in offspring of protein-restricted dams than in control dams. However, anti-oxidant enzyme activities (glutathione peroxidase and superoxide dismutase) were similar in both groups. In the thoracic aorta, although no significant difference was found in contractile response to phenylephrine, acetylcholine-induced relaxation was significantly reduced in offspring of protein-restricted dams. Expression of endothelial eNOS was lower and expression of $s \mathrm{GC}$ was higher in offspring of protein-restricted dams.

Conclusion: Our present study revealed that the SHRSP offspring of maternal protein-restricted dams were hyperresponsive to ROS and displayed evidence of vascular dysfunction. We conclude protein restriction during pregnancy increases ROS sensitivity in SHRSP offspring. Thus, appropriate treatments, such as administration of an antioxidant, may be useful to prevent or treat hypertensive vascular injury in mature offspring exposed to a poor intrauterine environment.

Disclosure of Interest: None Declared.

\section{DOHaD13-1132}

Epinephrine, norepinephrine and heart rate response after psychosocial stress in young adults born preterm at very low birth weight - the Helsinki study of very low birth weight adults

Nina Kaseva ${ }^{1, *}$, Karoliina Wehkalampi ${ }^{1}$, Riikka Pyhälä ${ }^{2}$, Kimmo Feldt ${ }^{2}$, Anu-Katriina Pesonen ${ }^{2}$, Kati Heinonen ${ }^{2}$, Petteri Hovi ${ }^{1}$, Anna-Liisa Järvenpää ${ }^{3}$, Sture Andersson ${ }^{3}$, Johan G. Eriksson ${ }^{1}$, Katri Räikkönen ${ }^{2}$, Eero Kajantie ${ }^{1}$
${ }^{1}$ Department of Chronic Disease and Diabetes Prevention, National Institute for Health and Welfare; ${ }^{2}$ Institute of Behavioral Sciences, University of Helsinki; ${ }^{3}$ Children's Hospital, Helsinki University Central Hospital and University of Helsinki, Helsinki, Finland

Background: Preterm birth is associated with increased risk factors for cardiovascular disease in later life. Sympathetic nervous system response to psychosocial stress could in part mediate this link. The aim of our study was to investigate the role of preterm birth at very low birth weight (VLBW, $\leq 1500 \mathrm{~g}$ ) on epinephrine (E), norepinephrine (NE) and HR response after psychosocial stress in adult life.

Method: We studied 50 young adults, aged 19-27 years, born preterm at VLBW and 39 term-born controls, groupmatched for age, sex and birth hospital. The participants underwent a standardized psychosocial stress test, the Trier Social Stress Test (TSST). E, NE and HR were repeatedly measured during the test. Data were analyzed with mixed effects and linear regression models, adjusted for age, body mass index, hormonal contraception, time of day, menstrual cycle phase and highest parental education.

Results: $\mathrm{E}$ and NE concentrations and HR increased significantly after stress. Baseline concentrations, peak after stress, increments and area-under-the-curve for $\mathrm{E}$ and $\mathrm{NE}$ were similar in VLBW and control groups in both men and women ( $\mathrm{p}$ for difference $>.2$ ). Analysis of sex interaction suggested that the difference between VLBW and term participants was greater in women ( $\mathrm{p}$-values for interaction time*sex*VLBW status 0.01 for $\mathrm{E}$ and 0.06 for NE). For example, during TSST NE concentrations were $27.7 \%$ lower $(95 \% \mathrm{CI} ; 3.1$ to 52.2$)$ in VLBW-women, while in men no statistically significant difference between groups was found. Also E concentrations were similar for VLBW and control groups, in both men and women. Mean HR at baseline, during stress task and HR reactivity was similar in VLBW and controls $(\mathrm{p}>.1)$.

Conclusion: We found no evidence of increased E, NE or HR response to psychosocial stress in young adults born preterm at VLBW. VLBW women may have a lower NE response than their peers born at term. If replicated, this could be a protective characteristic.

Disclosure of Interest: None Declared.

\section{DOHaD13-1230}

Exercise does not confer cardioprotection or improve vascular function in adult male offspring born growth restricted

Laura M. Reyes ${ }^{1,2,3, *}$, Jude S. Morton ${ }^{2,3}$, Raven Kirschenman ${ }^{2,3}$, Darren DeLorey ${ }^{4}$, Sandra T. Davidge ${ }^{1,2,3}$

${ }^{1}$ Physiology; ${ }^{2}$ Obstetrics and Gynecology; ${ }^{3}$ Women and Children's Health Research Institute; ${ }^{4}$ Physical Education \& Recreation, UNIVERSITY OF ALBERTA, Edmonton, Canada 
Background: Intrauterine growth restriction (IUGR) has been associated with increased susceptibility to myocardial ischemia/reperfusion (I/R) injury and endothelial dysfunction later in life. Exercise is an effective preventive intervention for cardiovascular diseases. However, it is unknown whether exercise can be an effective intervention for improving cardiovascular function in adult offspring born growth restricted. We hypothesized that exercise will decrease myocardial susceptibility to ischemia insult and improve vascular endothelial function in adult offspring born following hypoxia-induced IUGR.

Method: Since hypoxia is a critical insult causing IUGR in many pregnancy complications, we used a model of hypoxiainduced IUGR. Pregnant Sprague Dawley rats were exposed to control (room air) or hypoxia ( $11 \%$ oxygen) conditions from gestational day 15 to 21 . At 10 weeks of age, control and IUGR, male and female offspring were randomized to either an exercise-training (6 weeks; 5 days/week, $30 \mathrm{~min} /$ day at $20 \mathrm{~m} / \mathrm{min}$ ) or sedentary group. Twenty-four hours after cessation of exercise, offspring were euthanized and their hearts perfused for $10 \mathrm{~min}$ in retrograde Langendorff mode. Hearts were then switched to working heart mode and global, normothermic, no flow ischemia was induced for $10 \mathrm{~min}$.Following ischemia, hearts were reperfused for $40 \mathrm{~min}$. Second order mesenteric arteries were isolated and mounted on a wire myograph. Cumulative concentration response curves to phenylephrine (PE) and methacholine $(\mathrm{MCh})$ were performed in the presence of the nitric oxide synthase inhibitor (L-NAME, $100 \mu \mathrm{M} / \mathrm{L}$ ).

Results: Exercise improved baseline cardiac performance by $60 \pm 4.4 \%(p<0.001)$ in control but not IUGR male offspring. Surprisingly, exercise decreased cardiac recovery from $\mathrm{I} / \mathrm{R}$ in IUGR male offspring $(1.2 \pm 0.2 \%$ vs. $51.5 \pm 10.5 \%$ recovery from baseline; $p=0.002$ ) but did not affect cardiac recovery from $\mathrm{I} / \mathrm{R}$ in control male offspring $(60.8 \pm 8.5 \%$ vs. $67.4 \pm 15.5 \%$ recovery from baseline; $p=0.73)$. In females, exercise did not affect cardiac recovery from $\mathrm{I} / \mathrm{R}$ in either control or IUGR offspring. Exercise enhanced nitric oxide-mediated vasodilation in only control female offspring $(p=0.02)$ with a trend for this enhancement in control male offspring $(p=0.06)$. Exercise did not have an effect on vascular function in IUGR offspring.

Conclusion: Contrary to our hypothesis, exercise decreased the ability of the heart to recover from ischemia in male offspring following hypoxia-induced IUGR, and had no effect in female offspring. Our vascular function data suggest that exercise enhanced nitric oxide-mediated vasodilation in control offspring but not IUGR offspring. Results from the present study highlight that understanding mechanisms by which exercise impacts the cardiovascular system in a susceptible population is essential. The impact of exercise may be an additional insult not well tolerated for those born IUGR.

Disclosure of Interest: None Declared.

\section{DOHaD13-1307}

Exposure to early life vitamin D insufficiency has lifelong implications for vascular and renal function

Marianne Tare ${ }^{1, *}$, Kristen Bubb ${ }^{1}$, Michelle Kett ${ }^{1}$, Harold Coleman ${ }^{1}$, Kate Denton ${ }^{1}$, Helena Parkington ${ }^{1}$

${ }^{1}$ Physiology, Monash University, Melbourne, Australia

Background: Worldwide, many women of reproductive age are vitamin $\mathrm{D}(\mathrm{Vit} \mathrm{D})$ deficient. In adults VitD deficiency is linked with increased risk of cardiovascular disease. In contrast, the effects of early life exposure to VitD deficiency on lifelong cardiovascular health are poorly understood. In this study we examined the effects of in utero and early life VitD insufficiency in rats on cardiovascular and renal function in the offspring.

Method: Female Sprague-Dawley rats were fed either a VitDdeplete or -replete diet for 6 weeks before, and throughout pregnancy and lactation. Offspring were maintained on the same diet as their mothers until 3 months of age, after which time, all rats were fed a VitD replete diet. Arterial pressure was measured at 2 and 12 months of age in conscious rats. Vascular function was assessed in arteries from a variety of vascular beds using wire and pressure myography. Kidney function was measured in anaesthetised rats by clearance methods at 8 months of age.

Results: While VitD deficient, young male and female offspring have elevated mean arterial pressure (MAP) and heart rate (HR) and endothelial vasodilator dysfunction, as previously reported. We now show that following long term restoration of $\mathrm{VitD}$ status, MAP, HR and endothelial function had normalised in both male and female offspring. Strikingly, in animals that experienced VitD deficiency only in early life, there were marked alterations in the function of the kidney and its vasculature. Small renal lobar arteries had enhanced responsiveness to angiotensin II, with the maximum constriction increased by $4-9$ fold $(\mathrm{P}<0.05)$ in arteries from males and females, respectively. These arteries also exhibited enhanced vasoconstriction to perivascular nerve stimulation, and renal glomerular filtration rate was almost halved $(\mathrm{P}<0.002)$.

Conclusion: Early life VitD insufficiency causes early disturbances in cardiovascular function. Most of the adverse cardiovascular effects are resolved with subsequent $\mathrm{VitD}$ supplementation, except for effects on the kidney and its vasculature which are permanent. Thus, ensuring VitD sufficiency in women of reproductive age is important for the renal health of their offspring.

Disclosure of Interest: None Declared.

\section{DOHaD13-1100}

Fetal growth, early postnatal growth and arterial adventitial thickness in later childhood

Tim Sullivan ${ }^{1}$, Jason Harmer ${ }^{1}$, Brett Toelle ${ }^{2}$, Guy Marks ${ }^{2}$, David Celermajer ${ }^{3}$, Michael Skilton ${ }^{4, *}$ 
${ }^{1}$ Royal Prince Alfred Hospital; ${ }^{2}$ Woolcock Institute of Medical Research; ${ }^{3}$ University of Sydney, Sydney, Australia; ${ }^{4}$ Boden Institute, University of Sydney, Sydney, Australia

Background: The arterial adventitia appears to be an important player in the atherosclerotic disease process, being involved in paracrine signaling with the arterial intima and media, and undergoing structural modification, including thickening, in response to cardiovascular risk factors. The relationship of early life risk factors, specifically fetal growth and early postnatal weight gain, with arterial adventitial thickness, has not been described.

Method: We analyzed data from 379 non-diabetic children recruited antenatally, who had carotid extra-medial thickness, a noninvasive measure of arterial adventitial thickness (1), assessed by high resolution ultrasound at age 8-years. Fetal growth was assessed as birth weight adjusting for gestational age. Early weight gain was assessed as change in weight from 0-18 months, adjusted for concurrent height change. Analyses were stratified by sex.

Results: Fetal growth was not associated with carotid extramedial thickness in either boys $(8.3 \mu \mathrm{m}$ [SE 12.6] per $\mathrm{kg}$ birth weight, $P=0.51)$ or girls $(0.4 \mu \mathrm{m}$ [SE 14.3] per kg birth weight, $P=0.98$ ). Early weight gain, however, was associated with carotid extra-medial thickness in boys $(18.5 \mu \mathrm{m}$ [SE 5.3] per $\mathrm{kg}$ height-adjusted weight gain, $P<0.001)$, although not in girls $(-0.6 \mu \mathrm{m}$ [SE 6.0] per $\mathrm{kg}$ height-adjusted weight gain, $P=0.92)$. The association in boys was independent of size at birth, carotid intima-media thickness, height and weight at age 8-years.

Conclusion: Early weight gain between 0-18 months, beyond that predicted from change in stature, is a significant predictor of carotid extra-medial thickness in 8 year old boys, consistent with excessive early postnatal growth altering arterial adventitial structure.

\section{Reference}

1. Skilton MR, et al. JACC Cardiovasc Imaging 2009; 2:176-182.

Disclosure of Interest: None Declared.

\section{DOHaD13-1513}

Fetal origins of autonomic control in Peruvian children

Monica L. Mispireta ${ }^{1, *}$, Laura E. Caulfield ${ }^{2}$, Nelly Zavaleta ${ }^{3}$, Mario Merialdi ${ }^{4}$, Diane L. Putnick ${ }^{5}$, Marc H. Bornstein ${ }^{5}$, Janet A. DiPietro ${ }^{6}$

${ }^{1}$ Division of Health Sciences, Idaho State University, Pocatello; ${ }^{2}$ Department of International Health, Johns Hopkins Bloomberg School of Public Health, Baltimore, United States; ${ }^{3}$ Instituto de Investigacion Nutricional, Lima, Peru; ${ }^{4}$ Department of Reproductive Health and Research, World Health Organization, Geneva, Switzerland; ${ }^{5}$ Eunice Kennedy Shriver National
Institute of Child Health and Human Development, National Institutes of Health, Bethesda; ${ }^{6}$ Department of Population, Family and Reproductive Health, Johns Hopkins Bloomberg School of Public Health, Baltimore, United States

Background: Imbalances in autonomic nervous activity are associated with negative health outcomes in adults. Differences in cardiac measures of autonomic control are observed in the fetal period and remain stable into the postnatal period. This study examines associations between several cardiac measures of autonomic control from mid-gestation into childhood.

Method: Electronic fetal monitoring was conducted at 20, $24,28,32,36$, and 38 wk gestation for 50 minutes. Fetal heart rate (FHR) was quantified, and the following measures of FHR variability were computed: 1) standard deviation of FHR (FHR-SD), 2) range of FHR, and 3) number of FHR accelerations. When children were $4.5 \mathrm{y}$ of age, 5 minutes of heart rate data were collected using electrocardiography with children at rest. Heart period (HP), the interval between R-R waves, was quantified, and the following measures of FHR variability were computed: 1) standard deviation of HP (HP$\mathrm{SD}), 2$ ) range of $\mathrm{HP}, 3)$ mean square of successive differences (MSSD), a time-dependent method of analyzing variation in successive $\mathrm{HP}$; and 4 ) vagal tone ( $V$ ), a measure of respiratory sinus arrhythmia.

Multiple linear regression models were fitted using the available cardiac measures at $4.5 \mathrm{y}$ as outcomes (HP, HP-SD, range of $\mathrm{HP}, \mathrm{MSSD}$, and $V$ ), and the available fetal cardiac measures (mean FHR, mean FHR-SD, range of FHR at the end of pregnancy, and number of FHR accelerations at the end of pregnancy) as covariates. For each outcome, nested models were developed using a stepwise approach, introducing one FHR measure at the time and comparing each model with the previous one using the Likelihood Ratio Test (LRT) to determine the best fitting models.

Results: Fetal heart rate (FHR) was inversely associated with child heart period (HP) $(\beta=-2.91,95 \% \mathrm{CI}:-4.66,-1.17$; $\mathrm{p}=0.001)$ and four measures of cardiac variability including standard deviation of HP (HP-SD) $(\beta=-0.04,95 \% \mathrm{CI}$ : $-0.06,-0.01 ; p=0.003)$, range of $\mathrm{HP}(\beta=-2.40$, $95 \% \mathrm{CI}:-4.15, \quad-0.66 ; \mathrm{p}=0.007)$, mean square of successive differences (MSSD) $(\beta=-0.56,95 \% \mathrm{CI}$ : -0.95 , $-0.17 ; \mathrm{p}=0.005)$, and a measure of vagal tone $(V)$ $(\beta=-0.04,95 \% \mathrm{CI}:-0.08,-0.01 ; p=0.025)$ at $4.5 \mathrm{y}$. Two measures of cardiac variability or patterning in the fetal period, HR range and the number of episodic accelerations of heart rate, were also significantly associated with childhood measures of HP, HR variability (HP-SD, MSSD, range of $\mathrm{HP}$ ), and vagal tone, although in opposite directions.

Conclusion: Results from this study confirm persistent associations between multiple cardiac measures of autonomic control during the fetal period and comparable cardiac measures of autonomic control in childhood. Our results are consistent with previous findings showing stability of heart rate and variability in children from the United States from 
the prenatal to the postnatal period, and further expand these findings to a sample of Peruvian children from different sociodemographic background. Together, these results provide strong evidence that individual differences in autonomic control of heart rate patterns have prenatal origins.

Disclosure of Interest: None Declared.

\section{DOHaD13-1221}

Higher maternal vitamin D status in late pregnancy is associated with reduced aortic stiffness in 9-year old children assessed using magnetic resonance imaging

Jennifer Bryant ${ }^{1,2,3, *}$, Charles Peebles ${ }^{2}$, Mark Hanson ${ }^{3,4}$, Lucy Davies ${ }^{1}$, Hazel Inskip ${ }^{1}$, Nick Harvey ${ }^{1}$, Cyrus Cooper ${ }^{1}$, Keith M. Godfrey ${ }^{1,3,4}$

${ }^{1}$ MRC Lifecourse Epidemiology Unit; ${ }^{2}$ Cardiothoracic Radiology; ${ }^{3}$ NIHR Southampton Biomedical Research Centre, University of Southampton and University Hospital Southampton NHS Foundation Trust; ${ }^{4}$ Institute of Developmental Sciences, University of Southampton, Southampton, United Kingdom

Background: Recent studies have shown an association between lower vitamin D status and greater arterial stiffness. Greater arterial stiffness, a recognised risk factor for cardiovascular disease, can be assessed by measurement of aortic distensibility and compliance. Lower aortic distensibility indicates increased stiffness of the aortic wall. Poor early development has been linked to increased risk of cardiovascular disease in later life, and we examined the association between maternal $25(\mathrm{OH})$-vitamin $\mathrm{D}$ status in late pregnancy and vascular function in the child at the age of 9 years.

Method: Non-invasive assessment of local aortic root stiffness (arterial distensibility and compliance) was performed in 196 children aged 9 years using high-resolution steady state free precession cine magnetic resonance imaging (MRI). Subjects were participants in the Southampton Women's Survey (SWS), in which maternal $25(\mathrm{OH})$-vitamin D concentrations had been measured in late pregnancy.

Results: Higher late pregnancy maternal 25(OH)-vitamin D concentration was associated with greater aortic distensibility ( $\beta=0.002 \mathrm{mmHg}^{-1} / \mathrm{nmol} / \mathrm{l}, \quad[95 \% \mathrm{CI} \quad 0.0004$ to 0.004$]$, $\mathrm{p}=0.015, \mathrm{n}=196)$ and compliance $(\beta=0.002 \mathrm{AU} / \mathrm{nmol} / \mathrm{l}$, [95\%CI 0.0004 to 0.004 ], $\mathrm{p}=0.014, \mathrm{n}=196$ ). Adjustment for the child's sex and height had little effect on the findings.

Conclusion: Our data suggest an effect of maternal vitamin $\mathrm{D}$ status during pregnancy on vascular development in utero, leading to changes in arterial structure in the offspring. Although the effect is modest, even small favourable changes in childhood aortic structure may have substantial beneficial consequences, setting the child on a lower trajectory for cardiovascular risk later in the lifecourse.

This work was supported by funding from the Medical Research Council, Arthritis Research UK, the British Heart
Foundation and the National Institute for Health Research (NIHR) Southampton Biomedical Research Centre.

Disclosure of Interest: J. Bryant: None Declared., C. Peebles: None Declared., M. Hanson: None Declared., L. Davies: None Declared., H. Inskip: None Declared., N. Harvey: None Declared., C. Cooper: None Declared., K. Godfrey Other: I have acted as a consultant to Abbott Nutrition and Nestle Nutrition, and received reimbursement for speaking at Abbott Nutrition, International Life Sciences Institute (ILSI Europe) and Nestle Nutrition Institute workshops. I am part of an academic consortium that has received research funding from Abbott Nutrition, Nestec and Danone.

\section{DOHaD13-1677}

In vitro culture and embryo transfer alters the regulation of blood pressure in early postnatal life

Monalisa Padhee ${ }^{1, *}$, I. Caroline McMillen ${ }^{1}$, Severence M. MacLaughlin ${ }^{1}$, Song Zhang ${ }^{1}$, David O. Kleemann ${ }^{2}$, Simon K. Walker ${ }^{2}$, Jen Kelly ${ }^{2}$, Skye Rudiger ${ }^{2}$, Janna L. Morrison ${ }^{1}$

${ }^{1}$ Sansom Institute for Health Research, School of Pharmacy and Medical Sciences, University of South Australia; ${ }^{2}$ Turretfield Research Centre, South Australian Research and Development Institute, Adelaide, Australia

Background: Previous studies have shown that nutrient restriction during the early stages of the embryo development results in altered blood pressure in the offspring. In this study, we have investigated whether in vitro culture and transfer of the sheep embryo alters the blood pressure and heart rate in response to $\alpha$ adrenergic agonist (phenylephrine) in the postnatal lamb.

Method: Embryos were either transferred to an intermediate ewe (ET) or cultured in vitro in the absence (IVC) or presence of human serum (IVCHS) and methyl donor (IVCHS $+\mathrm{M}$ ) for 6d. Naturally mated (NM) ewes were controls. At 22-23 wks of age, phenylephrine was administered intravenously (4, 8,16 and $20 \mu \mathrm{g} / \mathrm{kg}$ ). Baroreflex sensitivity (the relationship between mean arterial pressure (MAP) and heart rate (HR)) was determined using linear regression analysis. The impact of embryo transfer and in vitro culture treatments on systolic blood pressure (SBP), diastolic blood pressure (DBP), MAP and $\mathrm{HR}$ were analysed using multifactorial ANOVA with repeated measures.

Results: There was a significant inverse relationship between MAP and HR in the NM, ET and IVCHS groups in response to phenylephrine. This relationship was blunted, however, in the IVC and IVCHS + M groups. There were no differences in delta SBP, DBP, MAP but an increase in delta HR in the ET, IVC and IVCHS + M group compared to IVCHS group in response to the low dose of phenylephrine. There were no differences in delta DBP and MAP but an increase in delta $\mathrm{SBP}$ in the males of IVCHS group with no change in females 
in response to the high dose of phenylephrine. There was also an increase in delta HR in IVC and IVCHS + M groups in response to the high dose of the phenylephrine.

Conclusion: This study demonstrates that there is a blunting of baroreflex sensitivity and an increase in delta HR in response to both low and high dose of phenylephrine in the offspring exposed to IVC and IVCHS + M. This may suggest that there is an increased dependence on sympathetic tone for the maintenance of MAP and HR in these groups.

Disclosure of Interest: None Declared.

\section{DOHaD13-1046}

Leukocyte telomere length is associated with elevated plasma glucose and HBAlc in young healthy men independently of birth weight

Louise Groth Grunnet $^{1, *}$, Kasper Pilgaard ${ }^{2}$, Christine B. Jensen $^{3}$, Charlotte Brøns ${ }^{1}$, Amra Alibegovic ${ }^{4}$, Susan Ozanne ${ }^{5}$, Martin Bennett ${ }^{6}$, Allan Vaag ${ }^{1}$

${ }^{1}$ Diabetes \& Metabolism, Rigshospitalet, Copenhagen N; ${ }^{2}$ Hvidovre Hospital, Hvidovre; ${ }^{3}$ Novo Nordisk, Soborg; ${ }^{4}$ Steno Diabetes Center, Gentofte, Denmark; ${ }^{5}$ Department of Clinical Biochemistry, University of Cambridge; ${ }^{6}$ Division of Cardiovascular Medicine, Addenbrooke's Hospital, Cambridge, United Kingdom

Background: Telomeres are nucleoprotein caps flanking DNA and telomere length is supposed to be a biomarker of aging. Shorter blood cell telomere lengths are associated with premature coronary artery disease. Low birth weight is associated with type 2 diabetes and cardiovascular disease. We therefore investigated a possible early relationship between low birth weight and telomere length.

Method: A cohort of low birth weight $(\mathrm{n}=55)$ and normal birth weight $(\mathrm{n}=61)$ men, average age 22.4 years, were phenotyped using multiple anthropometric measurements, blood pressure, fasting blood samples and a hyperinsulinemic euglycemic clamp in order to determine insulin sensitivity. Telomere length was determined using a validated qPCR method. Results: Low birth weight was not associated with short blood telomere length. Telomere length was negatively associated with elevated plasma glucose and HbA1c. Low birth weight men had significantly shorter adult height, increased abdominal obesity (waist-to-hip-ratios), elevated fasting plasma glucose and insulin levels, and evidence of insulin resistance as measured by HOMA-IR, predominantly reflecting insulin action in the liver, but not as measured by the insulin clamp technique predominantly reflecting insulin action in muscle.

Conclusion: The association between low birth weight and cardiovascular disease can in young healthy men not be explained by shorter telomere length. Shorter telomere length was negatively associated with elevated plasma glucose and
HbA1c early in life, before the development of overt cardiovascular disease.

Disclosure of Interest: None Declared.

\section{DOHaD13-1437}

Maternal B12 insufficiency independently predicts the metabolic risk factors of the offspring at birth

Antonysunil Adaikalakoteswari ${ }^{1}$, Alexander Lawson ${ }^{2}$, Craig Webster ${ }^{2}$, Catherine Wood ${ }^{3}$, Neil Anderson ${ }^{3}$, Philip G. McTernan ${ }^{1}$, Gyanendra Tripathi ${ }^{1}$, Manu Vatish ${ }^{1}$, Ponnusamy Saravanan ${ }^{1,3, *}$

${ }^{1}$ Division of Metabolic and Vascular Health, University of Warwick, Warwick; ${ }^{2}$ Department of Pathology, Heartlands Hospital, Birmingham; ${ }^{3}$ Academic department of Diabetes, George Eliot Hospital, Nuneaton, United Kingdom

Background: Studies in population with high vegetarianism show that vitamin B12 insufficiency is common in pregnancy and independently predict adiposity and insulin resistance in the offspring. As B12 is crucial for DNA methylation along with folic acid, epigenetic programming is postulated as the cause of such higher metabolic risk. A recent multi-ethnic study in the UK showed that both South Asian and White Caucasian women with anaemia due to vitamin B12 deficiency is associated with high cardiovascular risk profile. However, it is not known whether B12 insufficiency is common in the women of childbearing age or during pregnancy in the UK. The aims of our study were to (1) investigate the level of B12 in childbearing women in a population representative of the UK and (2) investigate whether maternal B12 levels in White Caucasian women predict metabolic risk of the offspring.

Method: The prevalence of B12 insufficiency in the UK representative population $(\mathrm{n}=3704)$ was studied using the publicly available dataset of British National Diet and National Survey (NDNS) between July 2000 and June 2001. 1256 subjects had full biochemical analysis which included lipid profiles, B12, folate and homocysteine. We analysed women of childbearing age:19-39 ( $\mathrm{n}=315)$. To assess the effect of B12 during pregnancy, paired maternal and cord blood samples $(n=91)$ were collected in a cohort of women undergoing elective caesarean section at University Hospital of Coventry and Warwickshire (UHCW cohort). Serum vitamin-B12 and folate were determined by electrochemiluminescent immunoassay and serum homocysteine by liquid-chromatography with tandem-mass spectrometric detection (LC-MS/MS).

Results: NDNS: The prevalence of serum vitamin B12 insufficiency $(<150 \mathrm{pmol} / \mathrm{L})$ among child bearing women was $12 \%$. There was a non-significant trend of higher BMI and waist-hip ratio in women with low B12 levels. Women with low B12 levels had higher total cholesterol, LDL cholesterol, cholesterol to HDL ratio and homocysteine 
(all $\mathrm{p}<0.05$ ). UHCW cohort: None of these mothers had overt anemia and $45 \%$ were smokers. B12 and folate insufficiency $(<7 \mathrm{nmol} / \mathrm{L})$ were $40 \%$ and $11 \%$ respectively. Low B12 mothers had higher BMI, triglycerides, cholesterol, LDL-cholesterol and homocysteine (all $\mathrm{p}<0.05$ ). The neonates of low B12 mothers had lower HDL-cholesterol, higher triglycerides, leptin and homocysteine (all p <0.05). In regression analysis, adjusted for age, BMI, glucose, smoking, parity and folate supplement use, maternal B12 independently predicted neonatal HDL-cholesterol $(\beta=0.169$; $\left.p=0.044 ; \quad R^{2}=8.6 \%\right)$, leptin $(\beta=-0.662 ; p=0.002$; $\left.\mathrm{R}^{2}=12.7 \%\right), \quad$ homocysteine $\quad(\beta=-0.302 ; \quad \mathrm{p}=0.001 ;$ $\mathrm{R}^{2}=14.7 \%$ ) but not triglycerides or HOMA-IR.

Conclusion: Our study showed that B12 insufficiency was common in women of childbearing age in a nationwide cohort, representative of the UK population and in a small cohort of White Caucasian pregnant women at the time of delivery. Maternal B12 insufficiency independently predicted metabolic risk of the offspring. These findings need to be replicated in a larger study ideally in early pregnancy or before pregnancy. Such findings will have a huge potential to reduce the burden of metabolic disorders in the offspring, if the causality is proven.

Disclosure of Interest: None Declared.

\section{DOHaD13-1279}

\section{Maternal diet-induced obesity programmes cardiovascular dysfunction in the offspring}

Heather L. Blackmore ${ }^{1, *}$, Denise Fernandez-Twinn ${ }^{1}$, Yougou Niu ${ }^{2}$, Dino A. Giussani ${ }^{2}$, Susan E. Ozanne ${ }^{1}$

${ }^{1}$ Insitute of Metabolic Sciences; ${ }^{2}$ Department of Physiology, Development and Neuroscience, University of Cambridge, Cambridge, United Kingdom

Background: Evidence derived from human and animal studies suggests that exposure to suboptimal conditions in early life increases the risk of adult-onset diseases, such as cardiovascular disease. Studies initially focused on the impact of early under-nutrition. However, with rising levels of obesity including women of childbearing age, there is accumulating interest in the long-term impact of early exposure to overnutrition and obesity. This study determined the impact on the adult offspring cardiovascular phenotype following exposure to maternal diet-induced obesity in mice.

Method: Obesity was induced in female mice by feeding a highly palatable diet rich in simple sugars and saturated fat for 6 weeks prior to pregnancy and throughout pregnancy and lactation. Control females were fed standard chow throughout life. Offspring were weaned onto standard chow. Male offspring were studied at 3, 5, 8 and 12 weeks for structural, functional and molecular analyses of the cardiovascular phenotype. Frozen cardiac sections were stained with wheat germ agglutinin for cardiomyocyte area quantification. Stereology was used to quantify cardiac wall and lumen widths, areas and volumes. Fetal gene expression was also determined by RTPCR. At 12 weeks of age, isolated Langendorff heart preparations investigated cardiac function. Assessment of basal cardiac function included left ventricular developed pressure (LVDP), left ventricular end diastolic pressure (LVEDP), minimum and maximum velocity of cardiac contraction. Chronotropic and ionotropic effects to a muscarinic agonist (Carbachol) and a beta-adrenergic agonist (Isoprenaline) were also determined.

Results: Offspring from obese dams had significantly larger hearts [absolute $(\mathrm{p}<0.05)$ and relative $(\mathrm{p}<0.01)$ to bodyweight] at 3, 5 and 8 weeks of age. Cardiomyocyte cell area was significantly elevated in offspring of obese dams at all four time points $(\mathrm{p}<0.0001)$. At 8 weeks, offspring exposed to maternal diet-induced obesity had significantly greater left ventricular free wall width, left ventricular area and volume $(\mathrm{p}<0.05)$. Re-expression of fetal genes occurred as early as 3 weeks in offspring of obese dams showing increased mRNA expression of NPPB $(\mathrm{p}<0.01)$ and ACTA1 $(\mathrm{p}<0.05)$ and attenuated mRNA expression of $M Y H 6$ and ATP2A2 (both $\mathrm{p}<0.05)$. From 5 to 12 weeks of age, a switch in myosin to a fetal profile was evident with an increase in the MYH7:MYH6 ratio $(\mathrm{p}<0.05)$. Cardiac functional studies at 12 weeks of age revealed strong evidence of systolic and diastolic dysfunction, impaired myocardial contractility and relaxability and a switch in the cardiac phenotype to one of sympathetic dominance with enhanced ionotropic responses to isoprenaline while diminished ionotropic responses to Carbachol in offspring exposed to maternal obesity (all $\mathrm{p}<0.05$ ).

Conclusion: Maternal diet induced obesity programmes cardiovascular dysfunction in the adult offspring even when they are fed a healthy postnatal diet. Specifically, functional and molecular data suggest an increased risk of heart failure. These results have implications for future generations of offspring born to obese women in relation to their increased risk of cardiovascular disease.

Disclosure of Interest: None Declared.

\section{DOHaD13-1596}

Maternal parameters influencing the developmental programming of offspring metabolic disease following maternal diet-induced obesity

Maria Alfaradhi ${ }^{1, *}$, Heather Blackmore ${ }^{1}$, Denise FernandezTwinn $^{1}$, Susan Ozanne ${ }^{1}$

${ }^{1}$ University of Cambridge Metabolic Research Laboratories, Wellcome Trust-MRC Institute of Metabolic Science, Cambridge, United Kingdom

Background: Investigation into the rapid rise of metabolic and cardiovascular disorders throughout the western world in 
recent decades has highlighted the importance of early nutrition in the programming of these diseases. Epidemiological studies have established a link between maternal obesity and increased risk of metabolic disease in the offspring. Models of diet-induced maternal obesity have been widely used to dissect the mechanisms driving offspring metabolic disease. However, relatively few studies have explored the maternal programming factors that may mediate these effects. Here we used a mouse model of maternal diet-induced obesity to examine the effects of an obesogenic diet on maternal metabolic parameters and offspring physical characteristics.

Method: Female C57BL/6 mice were fed either a standard chow diet or an obesogenic high fat, high sugar diet before mating and during gestation and lactation. Offspring from both groups (Control and Mat-Ob) were weaned onto a standard diet on postnatal day 21 and maintained on this for the duration of the study. Dam and offspring food intake and body weight was measured weekly. Maternal serum was analysed at the end of lactation. Male offspring were studied at 8 weeks of age.

Results: Dams fed an obesogenic diet were significantly heavier than controls at the time of mating and throughout gestation and lactation. At the end of lactation obese dams were hyperinsulinaemic, hypercholesterolaemic and hyperleptinaemic. There were no differences in body weight and adiposity in offspring of obese dams (Mat-Ob) compared to controls at 8 weeks of age. However, at this age Mat-Ob offspring had significantly greater absolute and relative heart weights compared to control offspring. Offspring heart weight was significantly correlated with maternal cholesterol (Pearson $\mathrm{r} 0.61, p<0.01$ ) and HDL (Pearson r 0.70, $p<0.001$ ) levels. Together, these account for $43.7 \%$ of the variance in offspring heart weight $(p<0.01)$. Maternal triglycerides and glucose levels, however, did not correlate with offspring heart weight.

Conclusion: Maternal cholesterol levels were a strong predictor of offspring heart weight. These findings provide insight into the relative contribution of different maternal factors in mediating programming effects in the offspring, could serve as a maternal biomarker predictive for offspring heart disease and will help in the development of potential intervention strategies.

Disclosure of Interest: None Declared.

\section{DOHaD13-1442}

\section{Maternal smoking behavior in early pregnancy is associated with parasympathetic nervous system activation in the offspring at age 5-6 years: the ABCD-study}

Tanja Vrijkotte ${ }^{1, *}$, Maaike Gademan ${ }^{1}$, Susanne D. Rooij ${ }^{2}$, Tessa Roseboom ${ }^{3}$, Marcel Twickler ${ }^{4}$

${ }^{1}$ Public Health; ${ }^{2}$ Clinical Epidemiology and Biostatistics; ${ }^{3}$ Obstetrics and Gynaecology, Academic Medical Center, Amsterdam, Netherlands; ${ }^{4}$ Endocrinology, Diabetology en Metabolic Diseases, Antwerp University Hospital, Antwerp, Belgium
Background: Maternal smoking behavior during pregnancy might have long lasting effects on the cardiovascular system in the offspring with sex specific effects, but evidence is inconclusive. We studied the association between maternal smoking behavior during early pregnancy and cardiac autonomic nervous system (ANS) activity in the offspring at the age of 5-6 years. Mediation by fetal growth restriction or shorter gestational age, as well as possible sex differences in the associations were studied.

Method: Data were collected within the ABCD-study, a multi-ethnic prospective birth cohort in Amsterdam. Smoking was self-reported by questionnaire at median gestation 16 weeks (IQR 12-20 weeks). Indicators of cardiac ANS activity were: heart rate (HR), pre-ejection-period (PEP; sympathetic activity) and respiratory sinus arrhythmia (RSA; parasympathetic activity), measured with electrocardiography and impedance cardiography in resting supine and sitting position. Results were adjusted for maternal (parity, prepregnancy BMI, age, socioeconomic status, hypertension) and child characteristics (time of the day at measurement, domestic smoke exposure, ethnicity, amount of screen time per day, height, BMI and age). Standardized birth weight was used as an indicator of fetal growth. Linear mixed models were applied.

Results: 3102 mother-child pairs were included. $74.1 \%$ of the mothers were non-smokers, $16.9 \%$ stopped smoking, $6.2 \%$ smoked $1-5$ cigarettes/day and $2.8 \%$ smoked $>5$ cigarettes/day during early pregnancy. Maternal smoking was associated with decreased HR and increased RSA in the offspring during supine as well as sitting position. HR results for supine position were (compared to non-smokers): stopped smoking: $-.30 \mathrm{bpm}, 1-5$ cigarettes/day: $-1.05 \mathrm{bpm},>5$ cigarettes/day: $-2.53 \mathrm{bpm}$ (p-value 0.006) and for RSA (compared to non-smokers): stopped smoking: .17 msec, 1-5 cigarettes/day: $5.52 \mathrm{msec},>5$ cigarettes/day: $22.36 \mathrm{msec}$ (pvalue 0.008 ). HR and RSA results for sitting position were comparable. Interactions between supine and sitting position (indicator of altered ANS reactivity to posture changes) were not significant ( $\mathrm{p}$-value all $>0.25)$. The results were not altered when standardized birth weight and gestational age were added to the models, indicating no mediation by fetal growth restriction or shorter gestational age. The results were similar in boys and girls ( $\mathrm{p}$-value for interaction all $>0.359$ ). Smoking was not associated with alterations in PEP.

Conclusion: Smoking during early pregnancy is associated with increased cardiac parasympathetic activity (dominant vagotonus), in according with a decreased heart rate in the offspring at young age. This association is not mediated by fetal growth restriction or shorter gestational age. This observation is puzzling as it suggests that intensity of mothers' smoking behavior during pregnancy is not negatively affecting the offspring's vagotonus; hitherto, an acknowledged risk factor in premature cardiovascular disease and diabetes later in life. Subsequently, future research regarding effects of high nicotine exposure in early pregnancy on 
development of brain structures determining the vagal balance after infancy is of interest.

Disclosure of Interest: None Declared.

\section{DOHaD13-1487}

\section{Moderate preterm birth in a sheep model: effects on postnatal body growth and arterial pressure}

\author{
Vivian Nguyen $^{1, *}$, Robert De Matteo ${ }^{1}$, Richard Harding ${ }^{1}$, \\ Graeme Polglase ${ }^{2}$, Mary Jane Black ${ }^{1}$ \\ ${ }^{1}$ Anatomy and Developmental Biology, Monash University; \\ ${ }^{2}$ The Ritchie Centre, Monash Institute of Medical Research, \\ Clayton, Australia
}

Background: Preterm birth affects $12 \%$ of all live births, with the majority being moderately preterm (i.e. birth at $32-<37$ weeks gestation). Epidemiological studies show that individuals born preterm have an increased risk of developing hypertension in adulthood, which is concerning, as hypertension is a major risk factor for cardiovascular disease. The greater arterial pressure in subjects born preterm may relate to altered body growth after preterm birth. In this study, using a sheep model, we compared postnatal growth, arterial pressure and heart rate in sheep born moderately preterm with those born at term.

Method: Border-Leicester-Merino crossbred ewes carrying singleton fetuses were induced to deliver vaginally (Epostane; $50 \mathrm{mg}$ in $2 \mathrm{ml} \mathrm{EtOH}$ i.v.) at 0.9 of term (131 \pm 1 days of gestation, $\mathrm{n}=16$ ); controls were born at term (147 \pm 1 days, $\mathrm{n}=17$ ). To facilitate survival of preterm lambs, betamethasone $(11.4 \mathrm{mg}$, i.m.) was administered to the ewe 24 and 48 hours before expected preterm birth. After birth, body weight, heart rate, arterial pressure, crown-rump length, thoracic girth and limb lengths were measured weekly for 12 weeks then monthly afterwards. Data were analysed using a 3 way ANOVA (factors: sex, age of delivery and postnatal age).

Results: At birth, preterm lambs were significantly lighter than term controls $(4.19 \pm 0.56 \mathrm{~kg}$ vs $6.44 \pm 1.16 \mathrm{~kg}$, $\mathrm{p}<0.0001)$. After birth, preterm lambs remained significantly lighter over the study period $(\mathrm{p}<0.0001)$ gaining an average of $1.56 \pm 0.03 \mathrm{~kg} /$ week whilst term lambs grew at $1.85 \pm 0.04 \mathrm{~kg} /$ week, which indicates an $18 \%$ greater growth rate in term lambs. By 5 months of age, body weight plateaued in both groups. In accordance with body weights, all morphometric measurements of postnatal growth were significantly lower in preterm lambs compared to term lambs. Over the study period, sex differences were also observed, with males exhibiting a greater front limb length $(p=0.014)$ and ponderal index $(\mathrm{p}<0.0001)$, but smaller thoracic girth $(p=0.012)$ compared to females. There was a significant overall reduction in systolic blood pressure $(\mathrm{p}<0.0001)$, diastolic blood pressure $(p=0.013)$ and mean arterial pressure $(\mathrm{p}<0.0001)$ in preterm lambs; however no difference in heart rate was observed.
Conclusion: Unexpectedly, during the first 7 months of life, there was a reduction in systolic, diastolic and mean arterial pressure in lambs born moderately preterm compared to those born at term; this may relate to their attenuated postnatal growth. Our continued monitoring of these lambs will establish whether the reduction in blood pressure in preterm lambs persists into adulthood.

Disclosure of Interest: None Declared.

\section{DOHaD13-1297}

Pre-weaning growth hormone treatment reverses maternal undernutrition-induced endothelial dysfunction, hypertension and alters heart development in adult offspring

Clint Gray ${ }^{1,2, *}$, Minglan $\mathrm{Li}^{1}$, Clare Reynolds ${ }^{1}$, Mark Vickers ${ }^{1}$

${ }^{1}$ Liggins Institute, University of Auckland, Auckland, New Zealand; ${ }^{2}$ Gravida: National Centre for Growth \& Development, University of Auckland, Auckland

Background: Maternal undernutrition is known to cause cardiac hypertrophy, elevated systolic blood pressure (SBP) and endothelial dysfunction in adult offspring. Few studies have investigated interventions during the early life period of developmental plasticity to ameliorate the programmed cardiovascular phenotypes. Using a model of moderate maternal undernutrition we examined the effects of neonatal growth hormone $(\mathrm{GH})$ treatment on adult offspring SBP, vascular function and cardiac remodelling.

Method: Female Sprague-Dawley rats were fed either a standard control diet (CON) or $50 \%$ of CON intake throughout pregnancy (UN). From neonatal day 3 until weaning (day 21), $\mathrm{CON}$ and $\mathrm{UN}$ pups received either saline (CON-S, UN-S) or GH $(2.5 \mathrm{ug} / \mathrm{g} /$ day) (CON-GH, UN-GH). All dams were fed ad libitum throughout lactation. Male offspring were fed a standard diet until the end of the study. Systolic BP (SBP) was measured at day 150 by tail cuff plethysmography. At day 160, vascular function of mesenteric vessels was assessed by pressure myography. Histological analysis and siRNA arrays were used to investigate cardiac remodelling and siRNA expression in adult offspring hearts. Results: Despite no change in heart rate, SBP was increased in UN-S groups and normalised in UN-GH groups (CON-S $121 \pm 2 \mathrm{mmHg}$, CON-GH $115 \pm 3$, UN-S $146 \pm 3$, UN$\mathrm{GH} 127 \pm 2)$. Pressure mediated dilation was reduced in UN-S offspring and normalised in UN-GH groups. Vessels from UN-S offspring demonstrated a reduced constrictor response to phenylephrine and reduced vasodilator response to acetylcholine. Preliminary histological analysis of heart development and structure revealed larger hearts, left ventricular hypertrophy in the UN-S offspring, with a beneficial remodelling of heart structure in UN-GH offspring hearts. PCR siRNA array data is currently being analysed in these hearts. 
Conclusion: Pre-weaning GH treatment reverses the negative effects of maternal UN on SBP and vascular function of adult offspring from mothers undernourished during pregnancy. These changes were paralleled by an altered cardiac structure indicative of $\mathrm{GH}$-induced cardiac remodelling. These data clearly suggest that developmental cardiovascular programming is potentially reversible by early life $\mathrm{GH}$ treatment and that $\mathrm{GH}$ can reverse the cardiac and vascular adaptations resulting from early life undernutrition.

Disclosure of Interest: None Declared.

\section{DOHaD13-1181}

Pregnancy as a critical period for the development of cardiovascular risks for women

Abdullah Mamun ${ }^{1, *}$

${ }^{1}$ School of Population Health, The University of Queensland, Brisbane, Australia

Background: Pregnancy as a critical period for the development of cardiovascular risks for mothers is under investigated. We aim to examine whether pregnancy factors including gestational obesity, gestational weight gain (GWG), hypertensive disorder in pregnancy (HDP) and smoking in pregnancy predict long-term cardiovascular risks of mothers including hypertension, postpartum weight retention (PPWR), obesity and diabetes.

Method: We followed a subsample of 2000 women from an original cohort of 7223 women who gave birth in Brisbane, Australia, between 1981 and 1983. GWG in kg per gestational week and Institute of Medicine (IOM) categories (inadequate, adequate and excess weight gain) of combined prepregnancy BMI and GWG were considered. HDP was measured and mothers prospectively self-reported their smoking status during and after pregnancy. Maternal PPWR, overweight, obesity and diabetes were measured at $21 \mathrm{y}$ postdelivery. Potential confounding factors are considered in the multivariate analyses.

Results: Analyses with IOM categories showed a greater postnatal increase in BMI for women defined as having excessive GWG (3.7, on average; 95\% CI: 3.1, 4.3) than for women with adequate GWG. The women who gained excess weight during pregnancy had increased odds of being overweight [OR: 2.2; 95\% CI: 1.6, 2.8] or obese (OR:4.5; $95 \%$ CI; 3.4, 5.9) $21 \mathrm{y}$ after the index pregnancy. Similarly, the women who gained excess weight during pregnancy were at greater odds of experiencing diabetes (OR 1.5, 95\% CI: $1.1,1.9)$ at $21 \mathrm{y}$. Mothers who experienced HDP were at greater risk to experience hypertension at 21y post-partum. All these associations were independent of potential confounding factors.

Conclusion: Findings of this study suggest that pregnancy is a critical period for the development of cardiovascular risks including obesity, hypertension and diabetes for mothers in long-term.

Disclosure of Interest: None Declared.

\section{DOHaD13-1565}

The effect of early life nutrition on baroreceptor control mechanisms of blood pressure in adult sheep

Kirsten R. Poore ${ }^{1}$, Francesca Paltenghi ${ }^{1}$, Jane K. Cleal ${ }^{1}$, Mark A. Hanson ${ }^{1}$, Lucy R. Green ${ }^{1, *}$

${ }^{I}$ Institute of Developmental Sciences, University of Southampton, Southampton, United Kingdom

Background: Epidemiological and animal studies link early-life nutrition and cardiovascular disease in adult life. In sheep, early gestation undernutrition alters fetal cardiovascular control via a resetting of the baroreflex ${ }^{(1)}$. Adult sheep cardiovascular dysfunction induced by early life undernutrition is reduced when the mismatch between preand postnatal environments is minimised ${ }^{(2)}$ and in the current study of these animals we examined baroreflex function. Since the action of angiotensin II in the nucleus tractus soliatarii (NTS) is implicated in modulating the baroreflex in adults ${ }^{(3)}$, baroreceptor function was also examined during concurrent infusion of the angiotensin converting enzyme inhibitor, captopril.

Method: Welsh Mountain ewes received 100\% (C, $\mathrm{n}=34)$ or $50 \%$ of nutrient requirements $(\mathrm{U}, \mathrm{n}=35)$ from $1-31$ days of gestation, and $100 \%$ thereafter. Offspring were fed ad libitum (CC, $\mathrm{n}=19$; UC, $\mathrm{n}=19$ ) or fed at a level that reduced body weight to $85 \%$ of individual target weight from 12 to 25 weeks postnatal age and ad libitum thereafter (CU, $\mathrm{n}=17 ; \mathrm{UU}, \mathrm{n}=20$ ). In 2.5 year-old offspring (males and females, singletons and twins), measurements of baseline blood pressure (BP) and baroreceptor function in response to phenylephrine $(35 \mu \mathrm{g} / \mathrm{kg}$ i.v. $)$ were made with and without the concurrent infusion of the angiotensin converting enzyme inhibitor, captopril $(500 \mu \mathrm{g} / \mathrm{kg} / \mathrm{h}, 5 \mathrm{ml} / \mathrm{h})$. In the minutes after PE administration, plots of beat-to-beat R-R interval (taken from an ECG recording) vs. systolic BP were used to assess baroreceptor function in terms of operating point (BP at $50 \%$ of maximal R-R response), sensitivity (slope of the linear portion of the plot) and $\Delta$ maximum systolic $\mathrm{BP}$ relative to baseline. Data were tested using repeated measures ANOVA.

Results: In all animals, regardless of previous nutritional exposure, captopril infusion reduced $(P<0.001)$ basal systolic, diastolic and mean arterial $\mathrm{BP}$, baroreflex operating point and $\Delta$ maximum systolic BP achieved following PE administration. Baroreflex sensitivity (slope) was not affected by captopril infusion. There was no influence per se of nutritional group, offspring sex or twinning on the observations.

Conclusion: Blockade of angiotensin II production decreased the baroreflex operating point, without any change in 
sensitivity, suggesting a resetting of the baroreflex to the reduced basal blood pressure during captopril infusion. Pre- or postnatal undernutrition did not affect baroreflex function, even when a layer of cardiovascular control has been removed by captopril infusion ${ }^{(4)}$. This suggests that our previous observations of an altered baroreflex in fetal life following prenatal undernutrition ${ }^{(1)}$ may not have longer term implications for adult baroreflex function.

\section{References}

1. Hawkins, P et al. (2000) Am J Physiol Reg Integ Comp Physiol 279: R340.

2. Cleal, JK et al. (2007) PNAS 104: 9529.

3. Reid, IA (1992) Am J Physiol Endocrinol Metab 262: E763.

4. Green, LR et al. (1998) J Physiol 507.3: 857.

Supported by British Heart Foundation

Disclosure of Interest: None Declared.

\section{DOHaD13-1520}

The role of programming, growth trajectories and infant feeding in explaining childhood blood pressure: the ABCD study

Marieke De Beer ${ }^{1, *}$, Manon van Eijsden ${ }^{2}$, Tanja Vrijkotte ${ }^{3}$, Caroline Fall ${ }^{4}$, Clive Osmond ${ }^{4}$, Reinoud Gemke

${ }^{1}$ Pediatrics, VU University Medical Center; ${ }^{2}$ Department of Epidemiology, Documentation and Health Promotion, Municipal Health Service; ${ }^{3}$ Public Health, Academic Medical Center, University of Amsterdam, Amsterdam, Netherlands; ${ }^{4} M R C$ Lifecourse Epidemiology Unit, Southampton General Hospital, University of Southampton; Southampton, United Kingdom;

${ }^{5}$ VU University Medical Center Amsterdam, Amsterdam, Netherlands

Background: Fetal growth retardation and postnatal catch up growth are identified as risk factors for the development of cardiovascular disease in adulthood. Accordingly, lower birth weight and increased infant and childhood growth have been associated with higher blood pressure (BP) later in life. Duration of breastfeeding and timing of introduction of complementary feeding have been associated with infant growth and may therefore also affect childhood BP. We hypothesized that 1) slower prenatal growth and faster infant and childhood growth are risk factors for higher BP in childhood; 2) shorter duration of breastfeeding and/or earlier introduction of complementary feeding are risk factors for higher BP in childhood.

Method: This study was part of a prospective pregnancy cohort study (Amsterdam Born Children and their Development). We assessed how BP in 2338 children aged 5-6 years relates to birth weight, infant feeding (duration of full breastfeeding and timing of introduction of complementary feeding), and to statistically independent measures representing linear growth (height gain) and relative weight gain (weight gain accounting for height gain) in 5 periods: $0-1,1-3,3-6$, 6-12 months (infancy) and 12 months-5 years (childhood). We used 2 different approaches: (1) a prospective approach in which we included linear growth and relative weight gain in both infancy and childhood; (2) a retrospective approach in which we included linear growth and relative weight gain in infancy and further adjusted for subsequent height and BMI at age 5-6 years. By using a prospective approach we aimed to examine the relative importance of growth in different time periods. The retrospective approach intended to explore the background of the associations, i.e. to disentangle programming effects from effects of faster growth trajectories.

Results: We found that lower birth weight, slower linear growth and slower relative weight gain in the first month were associated with higher childhood BP. Faster linear growth and faster relative weight gain after 1 month of age were associated with higher childhood BP. The effect sizes for the associations of $+1 \mathrm{SD}$ of relative weight gain with systolic $\mathrm{BP}$ and diastolic BP ranged from 0.71 to $1.35 \mathrm{~mm} \mathrm{Hg}$ and 0.26 to $0.92 \mathrm{~mm} \mathrm{Hg}$ respectively. Childhood growth had strongest associations with childhood BP. Full breastfeeding longer than 3 months and introduction of complementary feeding after 6 months were associated with lower childhood BP.

Conclusion: We infer that the inverse associations of prenatal growth and growth in the first month with childhood BP may reflect a programming effect. The positive association between growth after 1 month and childhood BP probably reflects an effect of faster growth trajectories. A longer duration of breastfeeding and appropriate weaning aiming at introduction of complementary feeding after 6 months may have benefits for later BP. Strongest associations with childhood BP were found for linear growth and relative weight gain in the childhood period, this period may therefore also be very eligible for public health interventions.

Disclosure of Interest: None Declared.

\section{DOHaD13-1355}

Ultrasound evaluation of the structure and blood flow in the heart, kidneys and major arteries of moderately preterm lambs at term equivalent age.

Paul Lombardo ${ }^{1, *}$, Michal Schneider ${ }^{1}$, Robert De Matteo ${ }^{2}$, Richard Harding ${ }^{2}$, Vivian Nguyen ${ }^{2}$, Graeme Polglase ${ }^{3}$, M Jane Black ${ }^{2}$

${ }^{1}$ Medical Imaging and Radiations Science; ${ }^{2}$ Anatomy o Developmental Biology; ${ }^{3}$ The Ritchie Centre, Monash Institute of Medical Research, Monash University, Clayton, Australia

Background: The adaptations of the cardiovascular system in response to preterm birth are not well understood and may 
contribute to the onset of cardiovascular disease in adulthood. We compared the structure and blood flow in the heart, major arteries and kidneys of moderately preterm lambs at term equivalent age, to lambs born at term using ultrasound. Method: Moderately preterm birth (0.9 of term) was induced (Epostane) in twenty-one Border Leicester x Merino ewes after antenatal corticosteroids (Celestone Chronodose: $11.4 \mathrm{mg}$ i.m.; 2 doses, $24 \mathrm{hrs}$ apart). Nine female and twelve male lambs were born vaginally at $132 \pm 1$ days gestational age (dGA). A control group of six female and eleven male lambs were induced to be born at term (147 \pm 1 dGA). Ultrasound imaging was conducted on all lambs at $148 \pm 1$ days post-conceptional age, defined as the sum of dGA and postnatal age. The structure and function of the left ventricle, the size of both kidneys and renal artery blood flow velocities, in addition to the diameter and blood flow velocities within the aortic root, pulmonary arteries and both common carotid arteries were recorded. Several measurements of each parameter were obtained and averaged, then corrected for bodyweight and compared using a two-way ANOVA with preterm/term and male/female as factors.

Results: Preterm lambs at term equivalent age had a greater bodyweight than term lambs $(20.9 \%, \mathrm{p}=0.0002)$. When adjusted to bodyweight, the anterior wall of the left ventricle was thinner in systole $(18.3 \%, \mathrm{p}=0.0011)$ and diastole $(27.5 \%, \mathrm{p}<0.0001)$ in preterm lambs compared to term lambs, as was the thickness of the interventricular septum in systole $(21.6 \%, \mathrm{p}<0.0001)$ and diastole $(24.1 \%$, $\mathrm{p}<0.0001)$. The aortic root diameter was smaller $(11.8 \%$, $\mathrm{p}=0.0159)$ and the maximum systolic blood flow velocity was lower in the main $(29.3 \%, \mathrm{p}<0.0001)$, right $(31.2 \%$, $\mathrm{p}<0.0013)$ and left $(39.3 \%, \mathrm{p}<0.0001)$ pulmonary arteries in preterm lambs. The right kidney length $(17.5 \%$, $\mathrm{p}=0.0002)$ and thickness $(12.9 \%, \mathrm{p}=0.032)$ and the left kidney length $(14.3 \%, \mathrm{p}=0.0013)$ and thickness $(12.8 \%$, $\mathrm{p}=0.003)$ were lower in preterm lambs than in controls. The right common carotid artery diameter $(19.7 \%, \mathrm{p}<0.0001)$, Resistive Index, RI, $(9.8 \%, \mathrm{p}=0.0181)$ and left common carotid artery diameter $(20.9 \%, \mathrm{p}=0.0002)$ and $\mathrm{RI}(10.6 \%$, $\mathrm{p}=0.0125)$ were lower in preterm lambs compared to term lambs. There were no significant sex differences found in any of the comparisons.

Conclusion: This study demonstrates bodyweight related differences in heart structure, kidney size and the diameter and blood flow velocities in major arteries in moderately preterm lambs compared to term lambs at term equivalent age. It is likely that these differences have arisen in response to preterm birth, owing to cardiovascular immaturity at the time of the haemodynamic transition at birth. The relationship between these findings and subsequent susceptibility to cardiovascular disease in later life will be investigated with serial ultrasound follow up as these lambs develop into adulthood.

Disclosure of Interest: None Declared.

\section{DOHaD13-1372}

Vascular-related pregnancy complications are associated with morphological alterations in umbilical cord vasculature

Emilie Herzog ${ }^{1, *}$, Anniek Reijnierse ${ }^{1}$, Ronald de Krijger ${ }^{2}$, Irwin Reiss ${ }^{3}$, Eric Steegers ${ }^{1}$, Régine Steegers-Theunissen ${ }^{1,4}$

${ }^{1}$ Obstetrics and Gynaecology; ${ }^{2}$ Pathology; ${ }^{3}$ Neonatology;

${ }^{4}$ Clinical Genetics, Erasmus MC, University Medical Centre Rotterdam, The Netherlands, Rotterdam, Netherlands

Background: Children born after pregnancies complicated by preeclampsia (PE), foetal growth restriction (FGR) or preterm birth (PTB) are associated with an increased risk of cardiovascular disease in later life. Disturbed structural development of embryonic and extra-embryonic vasculature during pregnancy may be involved.

Hypothesis: we hypothesize that the easily accessible umbilical cord vasculature is a good model for studying foetal vascular development in these pregnancies.

Therefore, we examined the morphology of the umbilical cord vasculature in complicated (PE, FGR, spontaneous PTB) and uncomplicated control pregnancies.

Method: In the Rotterdam Predict study, a hospital-based cohort study, we conducted a case-control study of 105 patients consisting of PE $(\mathrm{n}=31)$, FGR $(\mathrm{n}=26)$, spontaneous PTB $(\mathrm{n}=24)$ and controls $(\mathrm{n}=24)$. Macroscopic umbilical cord measurements were performed and umbilical cord samples were formalin-fixed within 1 hour after birth for histomorphometric analysis of digitised paraffin sections. Haematoxylin/eosin staining was used for area and wallthickness measurements and Elastic Van Gieson staining for the examination of elastic fibre quantity. ANOVA testing was applied for statistical analysis.

Results: The macroscopic measurements showed shorter umbilical cord lengths in the PE group (mean $43.0 \mathrm{~cm}, \mathrm{SD}$ 13.8, $\mathrm{p}=0.010$ ) compared to controls (mean $53.8 \mathrm{~cm}$, SD 13.7). After adjustment for cord length and gestational age, the number of coils per $\mathrm{cm}$ umbilical cord was comparable in every study group. Microscopic measurements revealed a smaller total umbilical cord area in PE (mean $73.7 \mathrm{~mm}^{2}$, SD 23.9, $\mathrm{p}=0.003$ ) and FGR (mean $65.8 \mathrm{~mm}^{2}$, SD 23.3, $\mathrm{p}<0.001$ ) compared to controls (mean $99.8 \mathrm{~mm}^{2}$, SD 35.1). In the PE-group only, both the artery and vein wall thicknesses were smaller (artery wall thickness mean $0.72 \mathrm{~mm}$, SD 0.08, $\mathrm{p}=0.019$; vein wall thickness mean $0.55 \mathrm{~mm}, \mathrm{SD} 0.14, \mathrm{p}=0.001$ ) compared to controls (artery wall thickness mean $0.85 \mathrm{~mm}, \mathrm{SD} 0.11$ and vein wall thickness mean $0.81 \mathrm{~mm}, \mathrm{SD} 0.21$ ). Finally, the percentage of elastic fibres in the vein was lower in PE (mean 7.2\%, SD 2.0, $\mathrm{p}=0.012)$ and FGR (mean 7.3\%, SD 2.0, $\mathrm{p}=0.030)$ than in controls (mean $9.0 \%$, SD 2.5).

Conclusion: We demonstrated alterations of the umbilical cord vasculature in children born after PE and FGR. Future studies should elucidate whether similar vascular changes exist 
in newborns, and focus on the association with cardiovascular disease-risk in adulthood. To understand the impact of vascular changes of the umbilical cord, long term follow up of mothers with PE, FGR and their offspring is necessary.

Disclosure of Interest: None Declared.

\section{DOHaD13-1476}

Woman in delivered with low birth weight have a risk of pregnancy-induced hypertension especially gestational hypertension in pregnancy of herself

Hiroaki Aoki ${ }^{1,2, *}$, Naoko Arata ${ }^{3}$, Naoko Sakamoto $^{4}$, Aikou Okamoto $^{1}$, Haruhiko Sago ${ }^{2}$, Atsuko Murashima ${ }^{3}$

${ }^{1}$ Obstetrics and Gynecology, The Jikei University School of Medicine; ${ }^{2}$ Center for Maternal-Fetal and Neonatal Medicine; ${ }^{3}$ Obstetric Medicine; ${ }^{4}$ Epidemiology, National Center for Child Health and Development, Tokyo, Japan

Background: It was known that low birth weight is linked to subsequent hypertension in adulthood, and that pregnancyinduced hypertension (PIH) is risk of her subsequent chronic hypertension. However, the relation between maternal low birth weight and PIH, especially separation into gestational hypertension $(\mathrm{GH})$ and preeclampsia (PE) subgroups, has not been well studied. We performed hospital based case-control study to assess association between PIH (GH and PE) and maternal low birth weight.

Method: This case-control study included 139 singleton cases with PIH, 90 were GH and 49 were PE, and 837 singleton controls. Maternal own birth weight and known risk factors were extracted from the maternity health record and electronic chart. We computed using logistic regression analysis adjusted for maternal age, parity, pre-pregnancy BMI, and gestational diabetes mellitus. Unadjusted and adjusted odds ratios (OR) and 95\% confidence intervals (CI) were determined.

Results: Maternal low birth weight $(<2500 \mathrm{~g})$ were at risk of $\mathrm{PIH}$ in her pregnancy (unadjusted OR 2.56, 95\% CI 1.27-5.13, adjusted OR 2.36, 95\% CI 1.11-4.71) and especially of GH (unadjusted OR 4.23, 95\%CI 2.07-8.63, adjusted OR 3.99, 95\% CI 1.86-8.13), as compared with maternal moderate birth weight $(2500-3999 \mathrm{~g})$. On the other hand, there were no PE cases in maternal low birth weight. Maternal over weight $(<=4000 \mathrm{~g})$ were not at risk of PIH (unadjusted OR 1.42, 95\% CI 0.57-3.51, adjusted OR 1.27, 95\% CI 0.46-3.04), nor GH (unadjusted OR 1.96, 95\% CI $0.73-5.25$, adjusted OR $1.81,95 \%$ CI $0.58-4.62$ ), and PE (unadjusted OR 0.60, 95\% CI 0.08-4.48, adjusted OR 0.54, 95\% CI 0.03-2.69).

Conclusion: Maternal low birth weight is risk factor of PIH, especially for GH.

Disclosure of Interest: None Declared.

\section{DOHaD13-1694}

Experiences of pediatric health care professionals while dealing with intersex children and their parents: a qualitative study in a pediatric setting of Karachi, Pakistan

Shela A. A. Hirani ${ }^{1, *}$

${ }^{1}$ School of Nursing and Midwifery, Aga Khan University, Karachi, Pakistan

Background: Clinical presentation of ambiguous genitalia is one of the rare but challenging situations that emerge various bioethical issues at the Pediatric settings. In Pakistan very few initiatives have been undertaken to explore the challenges encountered by Pediatric health care professionals while dealing with intersex children and their parents. Therefore, this study aims at exploring the experiences of Pakistani pediatric healthcare professionals while caring for intersex children and parents.

Method: While utilizing descriptive exploratory design, this study was conducted at a private tertiary care hospital of Karachi, Pakistan. The study received approval from the Ethical Review Committee of the indentified setting. A semi-structure interview guide was utilized to conduct in-depth interviews with the pediatric health care professionals, including two Pediatric nurses and two Pediatric physicians, each from outpatient and inpatient unit of the selected Pediatric setting.

Results: All the study participants indicated that caring for intersex children and their families is challenging as the condition is considered as taboo and as one of the social emergencies in the Pakistani pediatric setting. The commonly faced challenges of Pediatric health care professionals were highlighted as: the dilemma of declaring the child's exact gender, parental preference for male sex, limited expertise of health care professionals, non-compliance of treatment, time constrains, and long term management of the condition.

Conclusion: In Pakistan, care of Intersex children and their families presents several challenges for the pediatric health care professionals. To effectively care for intersex children and their parents at Pakistani pediatric setting, need was viewed to enhance collaborative approach among pediatric health team and assure proper training of pediatric health care professionals during their undergraduate studies.

\section{References}

1. Blizzard, R. M. (2002). Intersex Issues : A Series of Continuing Conundrums. Pediatrics, 110 (3), 616-621.

2. Frader, J., Alderson, P., Asch, A., Aspinall, C., Davis, D., Derger, A., Edwards, J., Feder, E.K., Frank, A., Hedley, L.A., Kittay, E., Marsh, J., Miller, P.S., Mourdian, W., Nelson, H., \& Parens, E. (2004). Health Care Professionals and Intersex conditions. Archives of Pediatrics \& Adolescent medicine, 158 (5), 426-428.

3. Lee, P.A., Houk, C. P., \& Ahmed, F. (2006). Consensus statement on management of intersex disorders. Pediatrics, 118 (2), 488-500. 
4. Stein, M. T. (2004). A newborn infant with a disorder of sexual differentiation. Pediatrics, 114 (5), 1473-1477.

Disclosure of Interest: None Declared.

\section{DOHaD13-1071}

Is offspring health influenced by maternal vitamin $D$ status in pregnancy? A systematic review and meta-analysis

Nicholas C. Harvey ${ }^{1}$, Chris Holroyd ${ }^{1}$, Georgia Ntani ${ }^{1}$, Kassim Javaid ${ }^{2}$, Philip Cooper ${ }^{1}$, Rebecca Moon ${ }^{1}$, Zoe Cole ${ }^{1}$, Tannaze Tinati ${ }^{1}$, Nicholas Bishop ${ }^{3}$ on behalf of UK Vitamin D in Pregnancy Working Group, Keith Godfrey ${ }^{1,4}$, Elaine Dennison $^{1, *}$, Janis Baird ${ }^{1}$, Cyrus Cooper ${ }^{1,2,4}$ and UK Vitamin D in Pregnancy Working Group

${ }^{1}$ MRC Lifecourse Epidemiology Unit, University of Southampton, Southampton; ${ }^{2}$ NIHR Musculoskeletal Biomedical Research Unit, University of Oxford, Oxford; ${ }^{3}$ Academic Unit of Child Health, Department of Human Metabolism, University of Sheffield, Sheffield; ${ }^{4}$ NIHR Southampton Biomedical Research Centre, University of Southampton and University Hospital Southampton NHS Foundation Trust, Southampton, United Kingdom

Background: We performed a systematic review to explore whether 1) low maternal circulating $25(\mathrm{OH})$-vitamin D $[25(\mathrm{OH}) \mathrm{D}]$ during pregnancy is associated with impairment of offspring health; and 2) maternal supplementation with vitamin $\mathrm{D}$ in pregnancy might ameliorate these effects.

Method: Major electronic databases were searched up to June 2012 covering both published and grey literature. Bibliographies of selected papers were hand-searched for additional references. Relevant authors were contacted for any unpublished findings and additional data if necessary. All reviews, data extraction and quality assessments were performed by two reviewers according to CRD guidelines. Eligible studies included pregnant women and their offspring, and one or more relevant exposures (either assessment of vitamin $\mathrm{D}$ status [dietary intake, sunlight exposure, circulating $25(\mathrm{OH}) \mathrm{D}$ ] or supplementation of participants with vitamin $\mathrm{D}$ or vitamin $\mathrm{D}$ containing food e.g. oily fish) and outcomes (offspring birth weight, birth length, head circumference, bone mass, anthropometry and body composition, risk of asthma and atopy, small for gestational dates, preterm birth, type 1 diabetes, low birth weight, serum calcium concentration, blood pressure and rickets). Maternal health outcomes were also addressed.

Results: 76 studies were included. There was considerable heterogeneity between the studies and for most outcomes there was conflicting evidence. Indeed, no convincing evidence was found for any association between maternal vitamin $\mathrm{D}$ status and offspring asthma, atopy, type 1 diabetes or blood pressure. However, modest positive relationships were identified between maternal $25(\mathrm{OH}) \mathrm{D}$ and 1$)$ offspring cord blood or postnatal calcium concentrations (meta-analysis of 6 intervention studies, mean difference $0.05 \mathrm{mmol} / \mathrm{l}(95 \%$ CI 0.02, 0.05); studies all had high risk of bias); 2) offspring birth weight (meta-analysis of 3 observational studies using log-transformed $25(\mathrm{OH}) \mathrm{D}$ concentrations, pooled regression coefficient adjusting for potential confounding factors $5.63 \mathrm{~g} /$ $10 \%$ change in maternal $25(\mathrm{OH}) \mathrm{D}(95 \%$ CI $1.11,10.16)$, but no association in 4 studies using natural units, or across intervention studies); and 3) offspring bone mass (in observational studies judged to be of good quality, but which did not permit meta-analysis).

Conclusion: There was modest evidence to support associations between maternal 25(OH)-vitamin D status and offspring serum calcium concentrations, birth weight and bone mass. However, these findings were limited by their observational nature or risk of bias. High-quality intervention studies to investigate these outcomes are now required, as the current evidence base cannot adequately inform clinical practice.

Acknowledgements: The UK Vitamin D in Pregnancy Working Group.

Disclosure of Interest: None Declared.

\section{DOHaD13-1357}

Risk factors for retinopathy of prematurity in preterm low birth weight neonates during peripartum

Yan Chen ${ }^{1, *}$, Jun Zhang ${ }^{1}$

${ }^{1}$ Shanghai Key Laboratory of Children's Environmental Health, Xinhua Hospital, Shanghai Jiao Tong University School of

Medicine, Shanghai, China, shanghai, China

Background: Retinopathy of prematurity (ROP) formerly known as retrolental fibroplasia (RLF) is an enigmatic disease of the developing retinal vasculature of the prematurely born infants, which potentially leading to blindness and severe visual impairment. ROP is manifestly not an exclusively environmental disease. The difference between intrauterine and extra-uterine may result in abnormal developing of immature retinal vasculature. The incidence increases with decreasing GA and BW. With improved survival of very low birth weight infants, ROP is emerging as a significant problem. It is a disease of modern times. As many as $27 \% \sim 35 \%$ infants less than $1500 \mathrm{~g} \mathrm{BW}$ and 16\% 48\% infants less than $1000 \mathrm{~g} \mathrm{BW}$ develop some degree of ROP. Apart from prematurity, there are many of other risk factors result in ROP.

Method: We retrospectively analyzed records of preterm infants who were born in neonatal intensive care unit (NICU) of Xinhua Hospital and Shanghai Children's Medical Center from January 2011 to June 2012 and were screened for ROP in ophthalmic center of Xinhua Hospital. A total of 55 preterm births with birth weight $<1500 \mathrm{~g}$ were eligible and screened. All mothers had regular antenatal care. The mother and baby medical records were complete.

Results: 55 neonates were screened during the study period, of whom $21(38 \%)$ and $5(9.1 \%)$ infants developed ROP 
of stage $1 \sim 2$ and stage 3 5 , respectively. The mean birth weight (BW) of infants without ROP, with ROP of stage $1 \sim 2$ and with ROP of stage 3 4 were $1362.8 \pm 101.2 \mathrm{~kg}$, $1283.8 \pm 174.8 \mathrm{~g}$ and $1110.0 \pm 164.9 \mathrm{~g}$. The mean gestational age (GA) of infants without ROP, with ROP of stage $1 \sim 2$ and with ROP of stage 3 4 were $31.74 \pm 1.8$ wks, $30.4 \pm 1.3 \mathrm{wks}, 28.8 \pm 1.6 \mathrm{wks}$. The following risk factors were found to be significant for ROP on univariate analysis with covariates of gestation and birth weight: duration from birth to the day which energy reaching $70 \mathrm{kal} / \mathrm{kg} /$ day by parenteral nutrition (PN) and enteral nutrition (EN), sum of protein receiving during 2 wks after birth, the highest endotoxin level in 2 wks after birth, the minimum hemoglobin $(\mathrm{Hb})$ concentration of mother during pregnancy. The risk factors found to be significant on rank sum test were sepsis, antenatal steroids, and pathological changes of placenta. A multivariate step-wise logistic regression showed that gestational age (GA), duration from birth to the day which energy reaching $70 \mathrm{kal} / \mathrm{kg} /$ day by $\mathrm{PN}$ and $\mathrm{EN}$, the highest endotoxin level during 2 wks after birth, antenatal steroids, pathological changes of placent were statistically significant.

Conclusion: Although ROP is a multifactorial disease, low GA and BW are the most important. Other factors such as energy and protein supplied during early period after birth, endotoxin level in $2 \mathrm{wks}$ after birth, the maternal minimum $\mathrm{Hb}$ in pregnancy, antenatal steroids and pathological changes of the placenta are closely associated with ROP.

Disclosure of Interest: None Declared.

\section{DOHaD13-1052}

Lessons learned on teaching that early life lasts a lifetime since Portland DOHaD 7

Stephen Bezruchka ${ }^{1, *}$

${ }^{1}$ Global Health, University of Washington, Seattle, United States

Background: Efforts to present $\mathrm{DOHaD}$ concepts were presented in a poster at $\mathrm{DOHaD} 7$. In the ensuring two years more has been learned to build on those materials.

Method: University level courses have been taught face-toface and online to undergraduate and graduate students in the health sciences. Students have been mentored in their research and capstone activities related to DOHaD. Public speaking to various audiences has been critically examined.

Results: Continually innovating ways of presenting the importance of early life concepts is necessary to discover effective means of communication and to instill critical thinking skills among health care workers, public health audiences and the general public.

Online courses with discussion forums may allow for more reflective disclosure regarding $\mathrm{DOHaD}$ concepts than in face-to-face courses.
One-liners, short pithy phrases that capture attention are needed to spur interest in the importance of early life. Telling stories works better than presenting data. Creating strong graphical statements enhances communication.

Attention focussed on the increased prevalence of chronic diseases in the world represents a compelling opportunity to engage people in their developmental origins. There is a huge industry devoted to treating chronic diseases that hampers presenting concepts of primordial prevention.

Relating $\mathrm{DOHaD}$ concepts to clinical cases is more effective in creating awareness of early life's importance than the mere presentation of research.

Success depends on the receptivity of a nation to scientific concepts. Deliberate attempts to create ignorance by vested economic interests have often hampered public understanding. There are as yet no comprehensive, easily accessible sources of materials to allow interested ordinary people to capture the strength and intensity of the developmental origins paradigm. Conclusion: Much attention needs to be paid to communicating research findings and their applicability to ordinary life.

Creating forums for sharing what works may help enhance public understanding of the importance of the early years for adult health.

\section{References}

1. Gould, L., E. Mogford, et al. (2010). "Successes and Challenges of Teaching the Social Determinants of Health in Secondary Schools: Case Examples in Seattle, Washington." Health Promot Pract 11(3_suppl): 26S-33S.

2. Mogford, E., L. Gould, et al. (2011). "Teaching critical health literacy in the US as a means to action on the social determinants of health." Health Promotion International 26(1): 4-13.

Disclosure of Interest: None Declared.

\section{DOHaD13-1686}

Association between breast feeding and childhood obesity among Pakistani school-going children

Saima A. A. Sachwani ${ }^{1, *}$, Rozina Karmaliani ${ }^{1}$, Shela Hirani ${ }^{1}$, Asif Raza Khowaja ${ }^{2}$

${ }^{1}$ School of Nursing and Midwifery; ${ }^{2}$ Dept of pediatrics, Aga Khan University, Karachi, Pakistan

Background: A substantial body of evidences has suggested breast feeding, as being protective against childhood obesity. This study aimed to investigate the association between breast feeding and childhood obesity in Pakistani school going children.

Method: 132 cases and 396 controls, school going children (aged 5-14 years of age) matched on age and gender, were recruited from public and private schools. Anthropometric data was collected, and a pre-tested structured questionnaire 
on socio-demographic profile, and lifestyle domains (dietary patterns, physical activity, sedentarism, and breast feeding) was administered to each from children and their mothers of the study. Conditional logistic regression was done using the (SPSS version 19.0), and (SAS version 9.2).

Results: Association between breast feeding and childhood obesity were linked in a dose-response relationship manner. History of no exclusively breast feeding [mORadj $=5.5 ; 95 \%$ CI (2.3-12.9)], exclusively breast feeding $<3$ months $[\operatorname{mORadj}=4,95 \% \mathrm{CI}(2.0-7.8)]$, and exclusively breast feeding for 4-5 months [mORadj $=2.6$; 95\% CI (1.5-4.7)] was associated with a higher obesity risk. However, moderately active $[\mathrm{mORadj}=0.5 ; 95 \%$ CI $(0.3-0.8)]$, as well as lower extent of participation [mORadj $=0.3 ; 95 \% \mathrm{CI}$ $(0.15-0.7)]$ in moderate to vigorous physical activity was protective against childhood obesity.

Conclusion: Longer duration of exclusive breast feeding have a protective effect against childhood obesity in a doseresponse manner. In Pakistan, future longitudinal studies are urged to confirm this association because of its implications for public health.

\section{References}

1. Arenz, S., Rückerl, R., Koletzko, B., \& Von Kries, R. (2004). Breast-feeding and childhood obesity:a systematic review. International Journal of Obesity, 28(10),1247-1256.

2. Balaban, G., \& Silva, G. A. P. (2004).Protective effect of breastfeeding against childhood obesity.Jornal de pediatria, 80 (1), 7-16.

3. Fall, C. H. D., Borja, J. B., Osmond, C., Richter, L., Bhargava,S. K., Martorell, R., et al. (2010). Infant-feeding patterns and cardiovascular risk factors in young adulthood: data from five cohorts in low- and middle-income countries. International Journal of Epidemiology, 40(1), 47-62.

4. Jafar, T. H., Qadri, Z., Islam, M., Hatcher, J., Bhutta, Z. A., \& Chaturvedi, N. (2008a). Rise in childhood obesity with persistently high rates of undernutrition among urban schoolaged Indo-Asian children. Archives of Disease in Childhood, 93(5), 373-378.

Disclosure of Interest: None Declared.

\section{DOHaD13-1499}

Changes in levels of milk long chain polyunsaturated fatty acids and neurotrophins across lactation in preeclampsia

Sadhana Joshi ${ }^{1, *}$ on behalf of Nutritional Medicine, Kamini Dangat ${ }^{1}$, Anitha Kilari ${ }^{1}$, Sanjay Lalwani ${ }^{2}$, Savita Mehendale ${ }^{3}$

${ }^{1}$ Nutritional Medicine, Interactive Research School for Health Affairs; ${ }^{2}$ Pediatrics; ${ }^{3}$ Obstetrics and Gynecology, Bharati Medical College and Hospital, Pune, India

Background: Our earlier studies have shown 1) reduced maternal plasma omega 3 fatty acids especially docosahex- aenoic acid (DHA) and higher milk DHA levels at postnatal day 3 in women with preeclampsia than normotensive women. 2) Higher milk brain derived neurotrophic factor (BDNF) levels but similar milk nerve growth factor (NGF) levels were observed at day 3 of lactation in preeclampsia group as compared to normotensive women. These components may change over the course of lactation. Therefore, the present study examines simultaneously the levels of milk fatty acids and neurotrophins in mothers with preeclampsia and compares them with controls at various time points during lactation.

Method: Women with preeclampsia $(\mathrm{n}=101)$ and normotensive women $(n=144)$ with singleton pregnancies were recruited. Mothers and their babies were followed-up at day $3,1.5,2.5,3.5$ and 6 months post delivery. Milk samples were collected and anthropometry was recorded at every time point. Long chain polyunsaturated fatty acid (LCPUFA) composition of milk samples was analyzed using gas chromatography, and neurotrophins were analyzed by Emax Immuno Assay System using Promega kits.

Results: Milk DHA levels were higher $(\mathrm{p}<0.05)$ at day 3, 1.5 and 3.5 months in mothers with preeclampsia than controls. Milk NGF levels were lower $(345.56 \pm 168.46 \mathrm{pg} /$ $\mathrm{ml}$ Vs $447.17 \pm 221.08 \mathrm{pg} / \mathrm{ml}$ ) at 1.5 months; $(599.28 \pm$ $455.12 \mathrm{pg} / \mathrm{ml}$ Vs $812.21 \pm 522.19 \mathrm{pg} / \mathrm{ml}$ ) at 3.5 months in the preeclampsia group. Milk BDNF levels was lower only at 1.5 months in the preeclampsia group $(303.2 \pm 52.61 \mathrm{pg} / \mathrm{ml})$ as compared to control group $(338.95 \pm 52.63 \mathrm{pg} / \mathrm{ml})$.

Conclusion: The present study for the first time examines reports the levels of human milk fatty acids and neurotrophins in mothers with preeclampsia and compares them with controls at various time points during lactation and also examines their association with growth of the infant. This study suggests that there is differential regulation of DHA and neurotrophins in breast milk in preeclampsia over the course of lactation. Lower levels of neurotrophins like BDNF and NGF could plausibly influence brain development and function in children born to mothers with preeclampsia. Future studies should explore the associations between milk LCPUFA, neurotrophins with neurodevelopment in children.

Disclosure of Interest: None Declared.

\section{DOHaD13-1543}

\section{Does breast milk adiponectin affect infant adiposity?}

Hamid Jan Jan Mohamed ${ }^{1 *}$, See Ling Loy ${ }^{1}$, Marhazlina Mohamed $^{1}$, Yu Wang ${ }^{2}$

${ }^{1}$ Nutrition Program, Universiti Sains Malaysia, Kubang Kerian, Malaysia; ${ }^{2}$ Pharmacology Department, The University of Hong Kong, Hong Kong, China

Background: Breastfeeding has been suggested to prevent childhood obesity, but with inconclusive biochemical evidence. 
Specific constituents of human milk such as the adiponectin may play a role in this relationship by protecting against insulin resistance and adiposity. The present study was therefore aimed to examine the role of breast milk adiponectin on infant adiposity.

Method: Between April 2011 and December 2012, a total of 138 pairs healthy mother-infant were enrolled in the Universiti Sains Malaysia Birth Cohort Study in Kelantan, Malaysia. Breast milk sample was collected within 1-5 days after delivery and analyzed for adiponectin concentration by enzyme-linked immunosorbent assay. Infant growth was assessed by using the World Health Organization Growth Reference 2007. Infant weight and body mass index-for-age $\mathrm{Z}$ score (BAZ) at aged 12 months were used as the adiposity indicator. Statistic analysis was performed by using analysis of covariance and simple linear regression.

Results: Overall, $13.9 \%$ infants were overweight at 12 months of age according to BAZ. Mean adiponectin in milk was $16.78 \mathrm{ng} / \mathrm{ml}$. In comparison to infants who were breastfed for at least 12 months, infants with stopped breastfeeding at aged 12 months had a higher body weight after adjusting for maternal age, pre-pregnancy body mass index, hair nicotine level, infant sex, gestational age and birth weight $(p=0.003)$. No significant association was observed between breast milk adiponectin at birth and infant weight $(\beta=0.01, p=0.787)$ and $\mathrm{BAZ}(\beta=0.01, p=0.440)$ at 12 months.

Conclusion: Breastfeeding is protective against infant adiposity development until the aged of 12 months. However, adiponectin in breast milk did not seem to affect infant adiposity. The protective effect of breastfeeding on childhood overweight and obesity is suggested to be contributed by other bioactive compounds of breast milk.

Disclosure of Interest: None Declared.

\section{DOHaD13-1658}

\section{Exclusive breastfeeding reduces adolescent metabolic disease in individuals at high genetic risk}

Scott White ${ }^{1, *}$, Leon Adams ${ }^{2}$, John Olynyk ${ }^{2}$, Koya Ayonrinde $^{2}$, Stephen Lye ${ }^{3}$, John Newnham ${ }^{1}$, Craig Pennell ${ }^{1}$

${ }^{1}$ School of Women's and Infants' Health; ${ }^{2}$ School of Medicine and Pharmacology, The University of Western Australia, Perth, Australia; ${ }^{3}$ Samuel Lunenfeld Research Institute, Toronto, Canada

Background: Metabolic disease imposes a substantial burden upon global health. A large body of evidence supports the role of early life environmental events in the pathogenesis of adult metabolic disease. Gene-environment interactions underlie some of this association. Early life nutrition is a potentially modifiable contributor to adult metabolic risk.
Method: The Western Australian Pregnancy Cohort (Raine) Study is an ongoing pregnancy cohort study with participants followed from 18 weeks gestation into adulthood. Infants were assessed for growth at birth. Nutritional and genetic data was obtained at cohort assessments from age 1 to 21 years. At age 17 years 993 participants underwent ultrasound assessment of hepatic steatosis and assessment of body mass index (BMI). Risk scores for non-alcoholic fatty liver disease (NAFLD) and obesity were calculated reflecting the summed additive genotype of 52 and 29 single nucleotide polymorphisms, respectively, previously demonstrated to be associated with NAFLD or obesity and with significant associations in the Raine cohort. Generalised linear modelling and multivariate linear regression were used to identify associations.

Results: The risk scores were significantly associated with NAFLD and BMI. NAFLD prevalence was $9 \%, 17 \%$ and $24 \%$ in those at low (lower quartile), medium (middle quartiles) and high (upper quartile) genetic risk (adiposityadjusted odds ratio (OR) 1.13 per allele, $\mathrm{p}=0.0002$ ). Increasing ponderal index was associated with a reduction in NAFLD (OR 0.82 per $\left.\mathrm{kgm}^{-3}, \mathrm{p}=0.0001\right)$. BMI increased with genetic risk $\left(+0.02 \mathrm{kgm}^{-2}\right.$ per allele, $\left.\mathrm{p}=1 \times 10^{-13}\right)$, with $9 \%, 12 \%$ and $25 \%$ of individuals at low, medium and high risk of obesity having BMI $>25 \mathrm{~kg}$ $\mathrm{m}^{-2}$. Increasing birth weight and greater postnatal weight gain were associated with increased BMI in adolescence. Exclusive breastfeeding (EBF) was associated with decreased adolescent BMI and NAFLD risk with EBF of three and six months, respectively. The benefit of EBF was greatest in those at highest genetic risk, with obesity in $22 \%$ of $\mathrm{EBF}$ individuals versus $28 \%$ of non-EBF individuals with obesity risk score in the upper quartile, and NAFLD in $18 \%$ of EBF individuals versus $34 \%$ of non-EBF individuals with NAFLD risk score in the upper quartile. These associations were independent of an effect of EBF upon postnatal weight gain. Conclusion: These data demonstrate an influence of early life factors upon adult metabolic risk. There was a substantial reduction in NAFLD and obesity in those EBF in infancy. The greatest reduction was in those at greatest genetic risk, in whom one case of NAFLD could be prevented for every six individuals who are EBF. This has important implications for the promotion of EBF in the prevention of adult metabolic disease. The lack of an association with postnatal weight gain suggests that the influence of EBF may be related to the programming effect of breastfeeding itself, perhaps related to the increased frequency of feeding in EBF. Alternatively, the benefit of EBF may relate to differing hormonal and nutrient compositions of breastmilk and formula, which may warrant investigation for potential pharmacologic interventions. The identification of individuals at greatest risk and with the greatest benefit could allow a targeted approach to optimise the efficiency of intervention strategies.

Disclosure of Interest: None Declared.. 


\section{DOHaD13-1177}

\section{Impact of cesarean section on autism in offspring: a systematic review and meta-analysis}

Hong-Tian $\mathrm{Li}^{1}{ }^{1, *}$, Qingjun Huang ${ }^{2}$, Zhi-long Wang ${ }^{1}$, Yu-bo Zhou ${ }^{1}$, Jian-meng Liu ${ }^{1}$

${ }^{1}$ Institute of Reproductive and Child Health/ Ministry of Health Key Laboratory of Reproductive Health, School of Public Health, Peking University Health Science Center, Beijing; ${ }^{2}$ Mental Health Center, Shantou University, Shantou, China

Background: Indirect evidence suggests an association of caesarean section with autism spectrum disorders, but results from epidemiological studies are inconsistent. We aimed to assess the risk of autism spectrum disorders associated with cesarean births by performing a review and meta-analysis.

Method: Pubmed, Web of Science, ScienceDirect, Wiley-Blackwell, and Ovid were searched for relevant articles published up to September 2012 using two groups of search terms: "cesarean" and "autism". Case-control or cohort studies investigating the association of cesarean section with autism spectrum disorders were considered to be eligible for the review and meta-analysis. Both crude and multivariable-adjusted relative risk estimates were extracted and pooled separately for total, elective, and emergency cesarean section using random-effects models.

Results: The search yielded 2250 publications, from which 17 were eligible for the meta-analysis. A pooled analysis of crude risk estimates showed that cesarean versus vaginal delivery was associated with a $47 \%$ increased risk of autism spectrum disorders (relative risk $=1.47,95 \% \mathrm{CI}=1.29-1.69$ ); the risk magnitude was attenuated to $27 \%$ (relative risk $=1.27,95 \%$ $\mathrm{CI}=0.97-1.66)$ when four multivariable-adjusted risk estimates were pooled. Subgroup meta-analyses further showed that elective rather than emergency cesarean section was significantly associated with autism spectrum disorders. The pooled relative risk of crude risk estimates for elective cesarean section was 1.53 (95\% CI $=1.25-1.86)$; the risk magnitude was attenuated to 1.34 (95\% CI $=1.10-1.63)$ when four multivariable-adjusted risk estimates were pooled.

Conclusion: The results of this meta-analysis support that cesarean section, especially elective cesarean section, is associated with an increased risk of autism spectrum disorders. However, because only a few studies reported multivariable-adjusted results, further studies conducted with extensive adjustment for confounders should be carried out to strengthen the findings.

Disclosure of Interest: None Declared.

\section{DOHaD13-1133}

\section{Impact of delivery mode on placental transfusion and iron-related hematological indices in term neonates: a systematic review and meta-analysis}

Yu-Bo Zhou ${ }^{1}{ }^{1 *}$, Hong-tian $\mathrm{Li}^{1}$, Li-ping $\mathrm{ZHU}^{2}$, Jian-meng Liu ${ }^{1}$
${ }^{1}$ Department of Epidemiology and Biostatistics, School of Public Health, Peking University Health Science Center, Institute of Reproductive and Child Health/ Ministry of Health Key Laboratory of Reproductive Health, Peking University Health Science Center, Beijing; ${ }^{2}$ Shanghai First Maternity and Infant Hospital Affiliated to Tongji University, Shanghai Women's Health Institute, Shanghai, China

Background: Evidence suggests that cesarean section is likely associated with a reduced placental transfusion and poor hematological status in neonates, but clinical studies have reported somewhat inconsistent results. We conducted a systematic review and meta-analysis to examine whether delivery mode affects placental transfusion and iron-related hematological indices in cord blood and neonates' peripheral blood.

Method: Pubmed, Web of Science, ScienceDirect, and Ovid were searched for relevant studies that reported the association of cesarean versus vaginal delivery with one of the following outcome measures: hematocrit level, hemoglobin concentration, and erythrocyte count in cord/peripheral blood; volume of placental residual blood and placental transfusion. Eligible studies were classified into high, medium, and low quality according to the Newcastle-Ottawa scale. Weighted mean differences (WMD) were calculated based on randomeffects model. Sensitivity analyses were performed to explore the robustness of pooled results by excluding medium/low quality studies or the study with the largest sample size. Subgroup analyses were further performed to test whether the pooled results differed by types of cesarean section (elective/emergency) and types of blood sample (cord/ peripheral blood).

Results: Sixteen studies, involving a total of 11950 participants, were eligible for the meta-analysis. Of these studies, 9 focused on cord blood, one on peripheral blood (6 hours after birth), one on both cord and peripheral blood (3 hours after birth), 4 on placental residual blood volume, and one on placental transfusion volume. Compared with neonates born vaginally, those born by cesarean section had a lower level of hematocrit (WMD, - 2.91\%; 95\% confidence internal [CI], $-4.16 \%$ to $-1.65 \%$ ), hemoglobin (WMD, $-0.51 \mathrm{~g} / \mathrm{dL} ; 95 \% \mathrm{CI},-0.74 \mathrm{~g} / \mathrm{dL}$ to $-0.27 \mathrm{~g} / \mathrm{dL}$ ) and erythrocyte (WMD, $-0.16^{\prime} 10^{12} / \mathrm{L} ; 95 \% \mathrm{CI},-0.30^{\prime} 10^{12} / \mathrm{L}$ to $-0.01^{\prime} 10^{12} / \mathrm{L}$ ), and a higher placental residual blood volume (WMD, $16.39 \mathrm{ml}$; 95\%CI, $14.41 \mathrm{ml}$ to $18.37 \mathrm{ml}$ ). The study in which placental transfusion volume was assessed using two different methods reported inconsistent results (mean difference, $28.6 \mathrm{ml}$ and $-16.2 \mathrm{ml}$, respectively). We conducted sensitivity and subgroup analyses only for hematocrit, but not for other outcome measures due to the limited number of available studies. The significant WMD remained after excluding medium quality studies or the study with the largest sample size; the WMD was substantially lower for peripheral blood $(-6.94 \% ; 95 \% \mathrm{CI},-9.15 \%$ to $-4.73 \%)$ than that for cord blood $(-1.75 \%$; 95\%CI, $-2.82 \%,-0.68 \%$ ) (P for interaction $<0.001)$, but did not materially differ by types of cesarean section $(\mathrm{P}$ for interaction $=0.72$ ). 
Conclusion: Cesarean section is associated with a reduced placental transfusion and a reduced level of iron-related hematologic indices in both cord and peripheral blood, suggesting that offspring delivered by cesarean section, compared with those born vaginally, might be more likely affected by iron-deficiency anemia in later life.

Disclosure of Interest: None Declared.

\section{DOHaD13-1045}

Research on the association between feeding practices and weight status of the infants and young children in Kongjiang community of Shanghai

Jing-Qiu Ma ${ }^{1, *}$, Xiao-Yang Sheng ${ }^{1}$

${ }^{1}$ Department of Child and Adolescent Healthcare, Xinhua

Hospital Affiliated to Shanghai Jiaotong University School of

Medicine, Shanghai, China

Background: The infant feeding patterns, feeding environment, caregiver's feeding beliefs and over-feeding behaviors may have great effect on an infant's growth and development. Our aim is to investigate the associations between infant and child feeding practices and growth outcomes in the first 18 months of life, and further to explore the possible practices may lead to overweight.

Method: The 6-month-old infants and their main caregivers entering the Kongjiang Community Health Center for a routine well-child check were invited to take part in this study and were followed up every 6 months over 12 months. Questionnaire survey was carried out through on-site face-toface interview with the main caregivers of children using the self-designed Infant and Young Child Feeding Questionnaire, which included infant feeding patterns, feeding beliefs/ attitudes, caregivers' feeding behaviors and feeding environment at each visit. Meanwhile, the weight and length of the children were also measured at each visit. The underlying factor structure of the feeding questionnaire was determined by factor analysis. Mean factor scores from questionnaires were compared between overweight and normal weight infants and young children.

Results: Among 262 infants who had completed the investigation at 12 months, $86(32.82 \%)$ infants were overweight [BMI-for-age $\mathrm{z}$ scores $(\mathrm{BAZ})>+1$ ]. The overweight infants had significantly greater increases in BAZ between birth -6 months, 6-12 months and birth- 12 months than normal weight infants (all $P<0.001$ ). In normal weight infants, caregivers worried more about infants being "underweight" $(Z=-5.270, P<0.001)$ and showed more overfeeding behaviors $(Z=-2.757, P=0.006)$, whereas caregivers with overweight infants worried more about infants "eating too much" and being "overweight" $(Z=-2.057$, $P=0.040$ and $Z=-5.779, P<0.001$, respectively). In 86 overweight infants, the scores of "worrying about infants being underweight" were positively correlated with the increase in BAZ between 6-12 months (Bata $=0.240$, $P=0.029)$ by multiple linear regression. Among 197 young children who had finally completed the investigation at 18 months, 64 (32.49\%) children were overweight (BAZ > +1). The overweight children had significantly greater increases in BAZ between birth- 6 months, birth-12 months, $12-18$ months, 6-18 months and birth-18 months than normal weight children ( $P$ all $<0.001)$. The mother's pre-pregnancy BMI of overweight children was higher than that of normal weight children $(t=-2.470, P=0.014)$. In normal weight infants, caregivers worried more about infants being "underweight" and "eating less" $(Z=-3.260, P=0.001)$, whereas caregivers with overweight infants worried more about infants "eating too much" and being "overweight" $(Z=-4.035, P<0.001)$. In 64 overweight infants, the scores of "pushing child to eat up a certain amount of food" were positively correlated with the increase in BAZ between 12-18 months (Bata $=0.293$, $P=0.029)$ by multiple linear regression.

Conclusion: A large increase in BMI z score of the overweight infants and young children is associated with some inappropriate feeding beliefs and behaviors of caregivers.

Disclosure of Interest: None Declared.

\section{DOHaD13-1738}

DOHaD theories and ethical issues: a literature search

Antonio Cunha ${ }^{1, *}$, Marcelo Land ${ }^{1}$

${ }^{1}$ Pediatrics, Federal University of Rio de Janeiro, Rio de Janeiro, Brazil

Background: DOHad proposes new models of causality and mechanisms of diseases. Investigations in this new field may result in impact on disease prevention and on health promotion. However, ethical implications may arise as well. For instance the concepts of fetus as patients, fetal rights, right to life and right to be born healthy. The purpose of this study is to present the results of a literature search on the topic ethical issues in DOHad and discuss potential implications of the findings.

Method: An electronic literature search was conducted in PUBMED and in ScIELO (Scientific Electronic Library Online) using the following MEsH terms: DOHad[All Fields], ethics[All Fields] and DOHad[All Fields] AND ethics[All Fields]. References found for the term DOHad were examined manually searching for the discussion of ethical issues. PubMed is a free database accessing primarily the MEDLINE database of references and abstracts on life sciences and biomedical topics. As of 19 May 2013, PubMed had over 22.7 million records going back to 1966 . SciELO is a bibliographic database and a model for cooperative electronic publishing in developing countries originally from Brazil, in partnership with the Latin American and Caribbean 
Center on Health Sciences Information. The database contains 985 scientific journals from different countries in free and universal access, full-text format.

Results: The search was conducted in the first week of June 2013. In PUBMED we found for the term DOHad 84 references, for Ethics 168,927 and for both terms together no reference. In Scielo we found for the term DOHad one reference, for Ethics 2,399 and for both terms no reference. By searching the titles and the abstracts of the references found for DOHad in both sources we found only one reference mentioning ethical issues (Silveira, P et al. J. Pediatr. (Rio J.) 83(6): 494-504, ILUS. 2007 Dec). However, this was a general review article not specifically on ethics.

Conclusion: We found only one study in our searches that discuss, although not exclusively, the ethical implications of DOHad. Even considering the limitations of searching only two sources and the fact that we did not read all articles that we found for the term DOHad, this is a clear evidence that the literature is very limited in this topic. It is possible that the discussion of ethical implications in the context of DOHad is limited as well. The results suggest that more work should be done in this area given the potential implications, related to the new concepts and knowledge emerging from this new field, in human relations and health.

\section{Reference}

1. Silveira PP, Portella AK, Goldani M, Barbieri MA. Developmental origins of health and disease (DOHaD). J. Pediatr. (Rio J.) 83(6): 494-504, ILUS. 2007 Dec.

Disclosure of Interest: None Declared.

\section{DOHaD13-1331}

Effects of maternal gestational diabetes mellitus on offspring overweight and obesity in childhood in China

Yaling Zhao ${ }^{1, *}$, Runmei $\mathrm{Ma}^{1}$ and Obstetrics and Gynecology, Pediatricsthe First Affiliated Hospital of Kunming Medical University

${ }^{1}$ Obstetrics and Gynecology, the First Affiliated Hospital of Kunming Medical University, Kunming Yunnan, 650032, China, Kunming, China

Background: Little is known about the magnitude of risk for offspring obesity that attributable to maternal diabetes in utero in Chinese Mainland. Based on the above research background, the purpose of the study is to investigate the long-term effects of maternal perinatal factors including GDM, obesity and weight gain during pregnancy on childhood overweight and obesity from 1.1 to 10 years of age in Chinese population.

Method: A study was conducted on 1068 children of mothers with gestational diabetes mellitus and 1756 children of mothers with normal glucose tolerance who were born between from January 1, 2003 to December 31, 2011 in the first affiliated hospital of Kunming medical university. The height and weight of children were obtained from parental reports through telephone interview. Multiple Logistic regressions were used to determine perinatal risk factors correlating with overweight and obesity of offspring in childhood.

Results: There were 178 overweight children (16.6\%) and 97 obesity children (9.1\%) in GDM group. Before 5 years old, there was no significant difference in the prevalence of overweight between GDM and control groups. After 5 years old, AGA and LGA offspring in GDM group had an increased prevalence of overweight and obesity compared with control group. Compared with the offspring of mothers with normal weight prepregnancy, offspring of mothers with prepregnancy overweight had a higher risk of overweight [OR: 1.036(95\% CI: 1.007 1.067)], but after adjusting for 0-3 month feeding mode, this correlation disappeared [OR: 1.034(95\% CI: 0.998 1.071)]. LGA offspring had a higher risk of overweight [OR: 2.267(95\% CI: 1.533 3.351)].

Conclusion: Before 5 years old, the prevalence of overweight and obesity was reduced in offspring of mothers with GDM compared with control group. After 5 years old, offspring of mothers with GDM had a higher prevalence of overweight and obesity than that of control group. The risk of overweight was highest in LGA offspring with concomitant exposure to GDM and maternal prepregnancy overweight. However, adjusting for the 0 to 3 feeding mode after birth attenuated the association.

Disclosure of Interest: None Declared.

\section{DOHaD13-1332}

Growth patterns in appropriate for gestational age infants of gestational diabetic mothers during the first year

Yaling Zhao ${ }^{1, *}$, Runmei $\mathrm{Ma}^{1}$ and Obstetrics and Gynecology, Pediatrics, the First Affiliated Hospital of Kunming Medical University

${ }^{1}$ Obstetrics and Gynecology, the First Affiliated Hospital of Kunming Medical University, Kunming Yunnan, 650032, China, Kunming, China

Background: It is unclear whether the growth pattern in infants of mother with GDM is a continuation of the fetal growth pattern. Whether the special growth pattern in offspring of mother with GDM is the outcome of the influence of intrauterine hyperglycemia environment on offspring? To answer these questions, we performed a prospective cohort study to follow-up AGA infants of mother with GDM to explore the growth pattern in infant of mother with GDM.

Method: The objects of this study were offspring of women who delivered in our hospital from 2011 January to 2011 December. The GDM group included 70 AGA infants 
mother with GDM. The control group included 154 AGA infants of women with normal glucose tolerance. Body weight and body length of infants in two groups were measured at 3, 6 and 12 months age respectively.

Results: Male AGA infants in GDM group had greater body weight and BMI than that of control group. There was no significantly difference in birth weight and body mass index between female AGA infants in two groups. Body weight, body length and BMI of male AGA infants in GDM group were less than that of control group at 3 months and 6 months age, but more than that of control group at 12 months age, there were no significant differences between two group $(P>0.05)$. Body weight, body length and BMI of female AGA infants in GDM group were similar to that of control group at 3 months, 6 months and 12 months age. The weight and height gain during infancy (0-3 months, 3-6 months) of male AGA infants in GDM group were lower than that of control group, but the difference was statistically significant only at 3-6 months. According to the data of general growth patterns of Chinese infants at $0-18$ years, the weight and height gain during infancy (0-3 months, 3-6 months) of male AGA infants in GDM group were lower than that of the third percentile. The weight and height gain during infancy (6-12 months) of male AGA infants of gestational diabetic mothers were higher than that of control group, but the difference was not statistically significant. There were no significant differences in weight and height gain during infancy (0-3 months, 3-6 months and 6-12 months age) of female AGA infants between two groups $(\mathrm{P}>0.05)$.

Conclusion: Male infants of gestational diabetic mothers grew slowly during their infancy of 0-6 months and then their growth becomes increasingly fast, which suggested that the influence of intrauterine hyperglycemia environment of GDM mothers on fetal growth might continue after birth.

Disclosure of Interest: None Declared.

\section{DOHaD13-1564}

\section{Higher glucose, insulin and insulin resistance (HOMA-IR) in childhood predict adverse cardiovascular risk profile in early adulthood: Pune Children Study}

Chittaranjan Yajnik ${ }^{1, *}$, Prachi Katre ${ }^{2}$, Suyog Joshi ${ }^{1}$, Kalyanaraman Kumaran ${ }^{1}$, Anand Pandit ${ }^{3}$, Caroline Fall ${ }^{4}$

${ }^{1}$ Diabetes Unit, KEM Hospital Research Centre; ${ }^{2}$ Life Sciences, Persistent Systems Ltd; ${ }^{3}$ Pediatrics Unit, KEM Hospital Research Centre, Pune, India; ${ }^{4}$ MRC Lifecourse Epidemiology Unit, University of Southampton, Southampton, United Kingdom

Background: Since the demonstration of an association between small birth size and higher risk of type 2 diabetes and cardiovascular disease (CVD) by David Barker and his colleagues, a number of birth cohorts have been established to document the life-course evolution of chronic non-communicable diseases. Very few of these cohorts have been followed into adult age. We set up the Pune Children Study (PCS) in 1991 in children born between 1987-89 in KEM Hospital, Pune, India. At 4 and at 8 years of age we found an inverse association between birth weight and risk factors for type 2 diabetes and CVD. We have now followed these children at 21 years of age to study a range of risk factors for type 2 diabetes and CVD. We report the relationship between childhood glucose, insulin and insulin resistance (HOMA-IR) and CVD risk factors at 21 years of age.

Method: At 21 years, we followed 357 of 477 children who had glucose and insulin measurements at 8 years. We performed a $75 \mathrm{gm}$ oral GTT (fasting, 30 and $120 \mathrm{~min}$ glucose and insulin measurements) and measured anthropometry, lipids and blood pressure. We calculated HOMAIR using online calculator (www.dtu.ox.ac.uk), and used regression and relative risk (RR) analyses for studying associations between 8 and 21 year measurements.

Results: Higher fasting glucose during childhood predicted higher cholesterol, fasting and $120 \mathrm{~min}$ glucose and insulin, and HOMA-IR at 21 years. Higher fasting insulin and HOMA-IR predicted higher body mass index (BMI), waist hip ratio, diastolic blood pressure, triglycerides, fasting glucose, fasting and $120 \mathrm{~min}$ insulin and insulin resistance (HOMA-IR) and lower HDL-cholesterol at 21 years. Residuals of childhood fasting insulin and HOMA-IR (i.e. independent of childhood BMI and sum of skin folds) similarly predicted higher BMI, waist hip ratio, triglyceride and insulin concentrations, HOMA-IR and lower HDL-cholesterol at 21 years. The RR of the individuals being in the highest risk quartile of CVD parameters (BMI, WHR, systolic and diastolic BP, triglycerides, $120 \mathrm{~min}$ insulin and HOMA-IR) at 21 years was increased if they were in the highest quartile of HOMA-IR at 8 years $(\mathrm{RR}=1.48$ to $1.81, \mathrm{p}<0.05$ for all $)$. Higher fasting glucose independent of fasting insulin was related to higher cholesterol, higher fasting glucose and insulin, and to higher HOMA-IR. Higher insulin independent of fasting glucose was related to higher BMI, WHR, blood pressure, triglyceride, fasting glucose and insulin, and HOMA-IR, and to lower HDL-cholesterol. Childhood measurements had very high negative predictive value but relatively lower positive predictive value.

Conclusion: PCS is a unique dataset which allows investigation of life-course evolution of CVD; there are few such comprehensive datasets anywhere in the world. We found that higher glucose, insulin and HOMA-IR in childhood predict higher levels of a range of cardiovascular risk factors at 21 years of age, suggesting that the seeds of CVD are sown in early life. It implies that the current strategies of CVD prevention started in middle life are less than satisfactory.

Disclosure of Interest: None Declared. 


\section{DOHaD13-1663}

\section{Identification of biological pathways influenced by multiple maternal factors in $\mathbf{4}$ different cell types of newborn twins using genome-wide methylation arrays}

Yuk Jing Loke ${ }^{1,2, *}$, Lavinia Gordon ${ }^{3}$, John Galati ${ }^{4}$, Richard Saffery ${ }^{2,5}$, Jeff Craig ${ }^{1,2}$

${ }^{1}$ Early Life Epigenetics, Murdoch Childrens Research Institute (MCRI); ${ }^{2}$ Paediatrics, University of Melbourne; ${ }^{3}$ Clinical Epidemiology and Biostatistics Unit (CEBU); ${ }^{4}$ Clinical Epidemiology ad Biostatistics Unit (CEBU), Murdoch Childrens Research Institute (MCRI), Parkville, Australia; ${ }^{5}$ Cancer, Disease and Developmental Epigenetics, Murdoch Childrens Research Institute (MCRI), Parkville, Austria

Background: Epigenetic events are crucial for early development, and can be influenced by environmental factors, potentially programming the genome for later health outcomes. DNA methylation is the most widely studied mechanism of epigenetic modification. Most environmental studies to date have only focused on associations of one or two maternal factors with gene-specific methylation at birth with only a single cell type. This study aimed to further investigate the influences of multiple maternal factors on DNA methylation at birth in a genome-wide scale.

Method: Genome-wide methylation data of 20 pairs of twins from 4 cell types (buccals, human umbilical vein endothelial cells (HUVECs), cord blood mononuclear cells (CBMCs), and placenta) were obtained from Illumina HumanMethylation $27 \mathrm{k}$ and $450 \mathrm{k}$ BeadArrays. The data were normalized and analysed against multiple maternal factors, specifically periconceptional folate, alcohol, smoking, macronutrient and vitamin B12 intake, and maternal stress. The RUV (Remove Unwanted Variation) method ${ }^{1}$ was used to analyse for any biological variation in the analysis. DAVID bioinformatics and IPA analyses were used to identify biological pathways that were enriched by genes that were identified to have significant biological variation.

Results: We found different significantly-enriched biological pathways corresponding to different maternal factors, specific to each cell type. The most interesting gene ontology finding was the enrichment of negative regulation of cell death in CBMCs in association with periconceptional alcohol intake. Negative regulation of cell death can cause irregularities in cell cycle.

Conclusion: This is the first newborn twins study to have looked at epigenetic influence of multiple maternal factors on multiple cell types on a genome-wide scale. This agrees with our previous studies that epigenetic influences are cell-specific and each cell types are epigenetically programmed in a unique way. This genome-wide data also provides a platform for further studies to identify genes that are more enriched by certain maternal factors, and warrants for further investigation at a gene-specific level.

\section{Reference}

1. Ganon-Bartsch J.A. and Speed T. (2012) Using control genes to correct for unwanted variation in microarray data. Biostatistics, 13, 3, pp.539-552.

Disclosure of Interest: None Declared.

\section{DOHaD13-1715}

Including DOHaD elements in a model of public policy for achieving adequate early nutrition in the obstetric control program: contrólate y progresa, in Caracas, Venezuela

Marianella Herrera $^{1, *}$, Julio Castro ${ }^{2}$, Jorge Díaz-Polanco ${ }^{1}$, Jorge Hernández-Rojas ${ }^{2}$, Carlos Walter ${ }^{1}$

${ }^{1}$ Development and Health; ${ }^{2}$ Universidad Central de Venezuela, Caracas, Venezuela, Bolivarian Republic Of

Background: There is a gap between scientists and policy makers moreover when results are long-term expected. Venezuela is a country in Nutrition Transition with the consequences of the coexistence of obesity and undernutrition in its population, along with this fact, is among the countries with highest rates of pregnancy in adolescents in Latin America. Intergenerational cycle of maternal "double burden" might be present in Venezuelan Pregnant women. "Contrólate y Progresa" is the program offered by Alcaldía de Sucre in Caracas to pregnant women. The aim of this project is to review the program and propose a model of public policies in which Dohad elements are present for prevention of diseases later in life and ameliorating the consequences in health of socio-economic disparities.

Method: Extensive Review of the variables monitored at "Contrólate y Progresa" through digital clinical records (unique in Venezuela), and adherence to this program.

Results: first control starts usually after first trimester, ultrasound is performed at first (when possible), second and third trimesters, along with laboratory checkups, few data is know about pre-pregnancy weight, BMI and body composition.

Conclusion: Due to the increasing prevalence of obesity, being among the first five diagnosis at this municipality, and coexisting with diseases congruent with undernutrition, early prevention of non communicable diseases including obesity/undernutrition is advised through assessment of pre-pregnancy nutritional status, subsequent checkups and educational programs for women in fertile age a model for introducing Dohad elements in public policies at ambulatory primary care is proposed as shown in the graphics.

\section{References}

1. American Dietetic Association. Nutrition and Lifestyle for a Healthy Pregnancy Outcome. J Am Diet Assoc. 2008; 108:553-561. 
2. Seligman HK and Schillinger D. Hunger and Socioeconomic disparities in Chronic Disease. NEJM 363;1 July 12010.

Disclosure of Interest: None Declared.

\section{DOHaD13-1183}

Intra uterine or post natal growth matters for the metabolic profile of Sri Lankan children

Vithanage P. Wickramasinghe ${ }^{1, *}$, Carukshi Arambepola ${ }^{2}$, P Bandar $^{1}$, M. Abeysekera ${ }^{1}$, S. Kuruppu ${ }^{1}$, P. Dilshan ${ }^{1}$, B.S. Dissanayake ${ }^{1}$

\section{${ }^{1}$ Paediatrics; ${ }^{2}$ Community Medicine, University of Colombo, Colombo, Sri Lanka}

Background: Backer hypothesized that poor intrauterine growth is directly responsible for metabolic derangements later in life. However, this was challenged by Singhal and Lucas with "accelerated post natal growth" hypothesis. The objective was to identify the effects of intrauterine and later growth on metabolic derangements among 5-15year old school children in Colombo district.

Method: A cross sectional descriptive study was conducted based on two-stage probability proportionate cluster sampling technique.

Fasting blood glucose and lipid profile was done after a 12 hour overnight fast. 2 hour OGTT was done by giving $1.75 \mathrm{~g} / \mathrm{kg}$ body weight anhydrous glucose (max $75 \mathrm{~g}$ ). Height, weight, Fat mass (FM) and blood pressure (BP) was measured and BMI calculated.

Sample was stratified by age $(5-10$ yrs and $>10-15$ yrs $)$ and each group was categorized by birth weight and current BMI tertile. Therefore $3 \times 3$ tables were constructed for metabolic parameters.

Results: 833 children were studied (boys 494). 3.2\% were obese according to IOTF classification.

In the 5-10 year age category, significant metabolic abnormalities were noted in the lowest birth weight and highest BMI tertile category (\%FM 27.8\%; SBP $95.9 \mathrm{mmHg}$; DBP $59.8 \mathrm{mmHg}$; RBS $92.1 \mathrm{mg} / \mathrm{dl}$; Cholesterol $176.1 \mathrm{mg} / \mathrm{dl}$; TG $83.6 \mathrm{mg} / \mathrm{dl}$; HDL $47.3 \mathrm{mg} / \mathrm{dl}$ LDL $111.6 \mathrm{mg} / \mathrm{dl}$ ) than those who were in lowest birth and lowest current BMI tertile (\%FM 14.0\%; SBP $87.7 \mathrm{mmHg}$; DBP $53.5 \mathrm{mmHg}$; RBS $87.5 \mathrm{mg} / \mathrm{dl}$; Cholesterol $158.7 \mathrm{mg} / \mathrm{dl}$; TG $63.4 \mathrm{mg} / \mathrm{dl}$; HDL $54.9 \mathrm{mg} / \mathrm{dl}$ LDL $91.1 \mathrm{mg} / \mathrm{dl})$.

In the $>10-15$ year old age group also similar abnormalities were noted. Metabolic derangements in the lowest birth and highest BMI tertile (\%FM 32.6\%; SBP $109.5 \mathrm{mmHg}$; DBP $67.2 \mathrm{mmHg}$; RBS $93.1 \mathrm{mg} / \mathrm{dl}$; Cholesterol $169.7 \mathrm{mg} / \mathrm{dl}$; TG $98.5 \mathrm{mg} / \mathrm{dl}$; HDL $43.9 \mathrm{mg} / \mathrm{dl}$ LDL $106.1 \mathrm{mg} / \mathrm{dl}$ ) were significantly impaired than those in lowest birth and lowest current BMI tertile (\%FM 16.1\%; SBP $97.9 \mathrm{mmHg}$; DBP $60.5 \mathrm{mmHg}$; RBS $85.4 \mathrm{mg} / \mathrm{dl}$; Cholesterol $162.8 \mathrm{mg} / \mathrm{dl}$; TG $72.7 \mathrm{mg} / \mathrm{dl}$; HDL $53.3 \mathrm{mg} / \mathrm{dl}$ LDL $94.9 \mathrm{mg} / \mathrm{dl})$.
When compared across the three birth weight tertiles, no significant differences were noted in the metabolic parameters. However, when compared across BMI tertiles, those in the highest BMI tertile had significantly impaired metabolic profile than the other two.

Increase in the weight in children is mainly due to accumulation of fat.

Conclusion: This study shows that rise in BMI leads to deranged metabolic parameters even at younger age. Low birth weight independently is not a risk factor to have abnormal metabolic profile in childhood, but a gain of high BMI later in life, is associated with many metabolic derangements, than those who were born small and remain small. This favours the accelerated post natal growth hypothesis. Most gain weight by disproportionately accumulation fat.

Therefore it is important to correctly feed and monitor growth of low birth weight individuals rather than insisting on "catch up growth".

Disclosure of Interest: None Declared.

\section{DOHaD13-1497}

Low whole grain intake among pregnant mothers in Singapore as indicated by plasma alkylresorcinols and estimated intake from dietary records

Alastair B. Ross ${ }^{1}$, Majorelee Colega ${ }^{2}$, Ai Lin Lim³ ${ }^{3}$, Irma Silva-Zolezzi ${ }^{4}$, Catherine Macé ${ }^{4}$, Seang Mei Saw ${ }^{2}$, Kenneth Kwek ${ }^{5}$, Peter Gluckman ${ }^{6}$, Keith M. Godfrey ${ }^{7}$, Yap Seng Chong ${ }^{2}$, Mary F.-F. Chong ${ }^{3, *}$ and GUSTO

${ }^{1}$ Chalmers University of Technology, Gothenbury, Sweden; ${ }^{2}$ National University of Singapore; ${ }^{3}$ Singapore Institute for Clinical Sciences, $A^{*} S T A R$, Singapore, Singapore; ${ }^{4}$ Nestle' Research Centre, Lausanne, Switzerland; ${ }^{5} K K$ Woman's and Children's Hospital, Singapore, Singapore; ${ }^{6}$ Liggins Institute, Auckland, New Zealand; 7 University of Southampton, Southampton, United Kingdom

Background: Maternal nutrition can induce epigenetic modifications that alter gene expression in the foetus, which in turn leads to phenotypic changes that can impact on the health outcomes of offspring. Compared with refined grains, whole grains are substantially higher in many micronutrients, including folate and betaine (trimethylglycine), which are known to provide methyl groups for DNA methylation. In addition, higher intakes of wholegrain foods have been consistently associated with a lower risk of chronic disorders including cardiovascular disease and diabetes. However, there is limited data on wholegrain intake in the general population, and no data on the wholegrain intakes of pregnant mothers in Singapore or other Asian countries.

Method: To evaluate wholegrain intake, $24 \mathrm{~h}$ recalls $(n=1000)$ were analysed for wholegrain foods and blood samples taken for measurement of plasma alkylresorcinols by 
LC-MS/MS $(\mathrm{n}=100)$ during $26^{\text {th }}-28^{\text {th }}$ week of gestation in pregnant mothers from the GUSTO birth cohort study.

Results: Initial results $(\mathrm{n}=100)$ found very low plasma total alkylresorcinol concentrations [median $(\mathrm{IQR})=9(3,15)$ $\mathrm{nmol} / \mathrm{l}$, suggesting low intake of wholegrain wheat in this group. Plasma alkylresorcinol concentrations were higher in those who consumed whole grains (22\% of mothers) compared with those who did not consume whole grains $(78 \%)[$ median $(\mathrm{IQR})=21(7.5,60.5)$ vs $7.5(2.75,14)]$ $\mathrm{nmol} / \mathrm{l}$, respectively; $\mathrm{p}<0.0001)$. Based on dietary records, those who consumed whole grains consumed $57 \mathrm{~g} /$ day (IQR $30,169 \mathrm{~g}$ per day). Even at these low levels of whole grain intake, plasma alkylresorcinols were correlated with wholegrain wheat intake $\left(r^{2}=0.366 ; \mathrm{p}<0.001\right)$.

Conclusion: Wholegrain intake among pregnant mothers in Singapore is well below the 2-3 (60-95 g) servings of whole grains per day recommended by the Singapore Health Promotion Board. This may have implications for both maternal and infant health outcomes and efforts to increase wholegrain intake should be supported to encourage people to choose whole grains over refined grains in their diet.

Disclosure of Interest: None Declared.

\section{DOHaD13-1638}

\section{Nutritional insufficiency among pre-pregnant and pregnant women in Bogor, West Java, Indonesia}

Siti Madanijah ${ }^{1,2}$, Dodik Briawan ${ }^{1,2}$, Rimbawan Rimbawan ${ }^{1}$, Zulaikhah Zulaikhah ${ }^{1}$, Nuri Andarwulan ${ }^{1,3}$, Lilis Nuraida ${ }^{1,3}$, Tonny Sundjaya ${ }^{4}$, Laksmi Murti ${ }^{5}$, Priyali Shah ${ }^{6}$, Jacques Bindels ${ }^{7}, *$

${ }^{1}$ SEAFAST Centre, Bogor Agricultural University; ${ }^{2}$ Community Nutrition; ${ }^{3}$ Food Science \& Technology, Bogor Agricultural University, Bogor; ${ }^{4}$ Research \& Development, PT Saribusada Generasi Mahardhika; ${ }^{5}$ Research \& Development, PT Nutricia Indonesia Sejahtera, Jakarta, Indonesia; ${ }^{6}$ Danone Research, Centre for Specialised Nutrition; ${ }^{7}$ Danone Research, Centre for Specialised Nutrition, Singapore

Background: Balanced nutrient intake during pre-pregnancy\&pregnancyis one of the key determinants of healthy pregnancy outcome related to later life health. Earlier studies concluded that diet in child-bearing-age Indonesian women is relatively poor, posing increased risk for morbidity and mortality. As limited data are available, a cross-sectional community based study was performed in Bogor, West Java, Indonesia to investigate nutrient insufficiency.

Method: Two hundred women $(20-40 \mathrm{yr})$ in pre-pregnancy (PPW) \& pregnant women group (PW) each, equally distributed over local economic quintiles (Q2,Q3,Q4) were enrolled for the study. PPW were women planning for their pregnancy, PW were women in their $2^{\text {nd }}$ trimester of pregnancy. Women with chronic disease affecting their dietary intake or with any acute morbidity condition on day of survey were excluded. Dietary intake data was collected by $24 \mathrm{hr}$ dietary recall. Nutrient intakes (energy, protein, iron, calcium, zinc) were calculated utilizing Indonesian Food Composition Tables. Adequacy of nutrient intake was determined by comparing with Indonesian (AKG) or South East Asia (SEA) recommendations (protein intake: adjusted for $70 \%$ protein quality; zinc:moderate bioavailability; iron: $7.5 \%$ bioavailability). AKG provides range of energy \& zinc levels for PPW \& PW, lower levels were considered as recommendations. Due to data variability, median nutrient intakes $\&$ inter quartile ranges are shown. Nutrient intakes $<70 \%$ for energy $\&$ protein and $<77 \%$ of recommendations for iron, calcium\&zinc were considered as "insufficient".

Results: Median (IQR) ranges of nutrient intakes were energy $1360 \mathrm{kcal}(1098 ; 1695)$, protein $43.1 \mathrm{~g}(34.6 ; 55.0)$, iron $16.0 \mathrm{mg}$ (12.1;21.2), calcium 503.2 mg (331.8;721.3) \& zinc $10.5 \mathrm{mg}(6.4 ; 16.0)$ for PPW. Similar analysis for PW showed intake of energy $1534 \mathrm{kcal}(1173 ; 1950)$, protein $43.5 \mathrm{~g}$ $(32.0 ; 61.5)$, iron $15.0 \mathrm{mg}(10.0 ; 22.0)$, calcium $684.5 \mathrm{mg}$ (402.0;1046.5), \& zinc $9.8 \mathrm{mg}(7.1 ; 15.1)$. Results indicate $>50 \%$ women were unable to meet sufficiency levels for all nutrients except zinc (\%AKG). Higher calcium intake was observed among PW due to higher milk intake during pregnancy (PW $181.6 \mathrm{~g} / \mathrm{d}$ vs. PPW $64.6 \mathrm{~g} / \mathrm{d}$ ). Median energy intakes were comparable between groups (PPW $75.6 \%$ vs. PW $73.0 \%$ AKG). Further analysis indicated that 1 in 4 women had insufficient intake (AKG) for all nutrients (SEA, all nutrients except zinc). Combined insufficient iron \& calcium intakes (AKG) were 50.5 \& $48.8 \%$ for PPW \& PW, respectively, with a gradient across socio economic classes. A similar trend was observed for combined energy, protein \& calcium insufficiency.

Conclusion: More than half of Indonesian pre-pregnant and pregnant women had intakes below recommendations across socio-economic classes, indicating that consumed food sources did not provide recommended nutrient levels. There is a need to improve food quantity \& quality to increase intake of these specific macro- $\&$ micronutrients, albeit that affordability/poverty may still be an issue in the lowest wealth quintile. Clearly, more nutrition education is needed across all socio economic classes to increase understanding of nutrient requirements during this specific stage in life.

Disclosure of Interest: None Declared.

\section{DOHaD13-1295}

People in the United States of America die younger than those in other rich nations partly because of neglect of DOHaD concepts

Stephen Bezruchka ${ }^{1, *}$

${ }^{1}$ Global Health, University of Washington, Seattle, United States 
Background: In the early 1950s, the United States of America could claim to be one of the world's longest lived nations with some of the best mortality outcomes. Since then, other nations have had more rapid declines in mortality so that now mortality indicators of health place the USA behind the other rich nations and for some indicators on a par with poor countries.

Method: The Institute of Health Metrics and Evaluation, a part of the Department of Global Health at the University of Washington, has produced web-based materials that allow easy access to mortality data trends around the world. Examples of population health data display will be presented. Results: The evolving trend of health outcomes are traced for the last forty years using the IHME web-based data visualizations. These show, for example, that US men are more likely to die in the prime of life (45q15) than are men in Tunisia, a middle-income country, while for women, those aged 15 in Sri Lanka, a poor nation, have better chances of being alive at age 60 than US women.

Maternal mortality trends demonstrate an increase in the United States over the last fifteen years, something not seen in the other rich nations.

Comparisons can be made of US counties that have the life expectancies of entire nations for both men and women in ten year sequences from 1989 to 2009. These demonstrate that for 2009 there are no US counties with life expectancies for women comparable to women in the entire nation of Japan which represents a significant change over the previous two decades. Other data visualizations on the IHME website demonstrate absolute declines in life expectancy for women in close to $30 \%$ of US counties for the period 1987-2007.

The United States of America has the least federal government expenditure directed towards early life issues, including no paid ante-natal or parental leave policies. Welfare support directed towards families is also among the lowest for rich nations.

The United States spends 18\% of its Gross National Product on health care services, almost twice that of any other nation. In total the amounts are close to half of the world's expenditures.

Conclusion: IHME data visualizations can be used to inform the public of the health status of various nations making comparisons on mortality indicators with the United States and its counties.

While the USA spends an enormous amount of money on medical care, health as measured for longevity or not dying in the prime of life eludes these people. Lack of attention focussed on early life issues and neglect of the Developmental Origins of Health and Disease paradigm have enormous health costs.

\section{Reference}

1. http://www.healthmetricsandevaluation.org/tools/datavisualizations.

Disclosure of Interest: None Declared.

\section{DOHaD13-1410}

Skinfold thickness and body fat in urban 6-9 year old children: influence of socioeconomic status

Rita S. Patil ${ }^{1, *}$, Shobha A. Udipi ${ }^{2}$

${ }^{I}$ Foods and Nutrition, Maniben Nanavati Women's College, S.N.D.T Women's University; ${ }^{2}$ Food Science and Nutrition, S.N.D.T Women's University, Mumbai, India

Background: There is increasing prevalence of childhood overweight/ obesity and the consequent risks for developing non communicable diseases at an early age in India. In this context, the present study was undertaken in Mumbai, India on 3600 school children aged 6-9 years.

Method: Height, weight and skinfold at triceps, biceps, subscapular and suprailiac were measured in triplicate. Body Mass Index and body fat were calculated.

Results: The mean skinfold measurements were: triceps $7.1 \pm 2.6 \mathrm{~mm}$, biceps $-4.8 \pm 1.9 \mathrm{~mm}$, subscapular skinfold $6.4 \pm 2.0 \mathrm{~mm}$ and suprailiac $-6.3 \pm 2.2 \mathrm{~mm}$. The mean percent body fat was $12.9 \pm 4.3$ for all children in the study. Overall, $24.4 \%$ children were underweight, $14.7 \%$ were overweight and $7.5 \%$ were obese. Although prevalence of overweight and obesity was significantly lower among the low socioeconomic status (SES) children compared to their high SES peers, $22.8 \%$ of low SES children were overweight + obese (based on Cole's classification) compared to $32.4 \%$ children from upper SES. Low SES children had significantly lower mean skinfold thicknesses and body fat compared to children of the upper SES categories. However, distribution of low SES children among the four quartiles of body fat indicated that $2.5 \%$ of underweight children were in the third (second highest) quartile of body fat, $27.5 \%$ of normal weight children were in the third quartile and $4 \%$ were in the fourth and highest body fat quartile. Among overweight children, $68.4 \%$ were in the highest (fourth) quartile.

Conclusion: The data indicated that considerable percentage of children from low SES are overweight and have more body fat which is of concern.

Disclosure of Interest: None Declared.

\section{DOHaD13-1398}

A higher maternal cardiovascular disease risk score
reduces embryonic growth in IVF/ICSI pregnancies

Kim Wijnands ${ }^{1}$,*, Evelyne van Uitert ${ }^{1}$, Remco Visser ${ }^{1}$, Jeanine Roeters van Lennep ${ }^{2}$, Anton Koning ${ }^{3}$, Niek Exalto ${ }^{1}$, Eric Steegers ${ }^{1}$, Régine Steegers-Theunissen ${ }^{1,4}$

${ }^{1}$ Obstetrics and Gynecology; ${ }^{2}$ Internal Medicine; ${ }^{3}$ Bioinformatics; ${ }^{4}$ Clinical Genetics, Erasmus MC, University Medical Center, Rotterdam, Netherlands

Background: Maternal cardiovascular diseases (CVD) detrimentally affect pregnancy course and outcome. Less is known 
about the effects of CVD risk factors in women without CVD. Our aim was to estimate the influence of a maternal CVD risk score on embryonic growth trajectories in pregnancies with reliable pregnancy dating.

Method: In the Rotterdam Predict study, a prospective periconception cohort study, we analyzed 111 singleton pregnancies with nonmalformed and live birth outcomes of women without CVD. We included spontaneous pregnancies with a reliable first day of the last menstrual period and a regular menstrual cycle of 25 to 31 days only $(n=66)$ and IVF/ICSI pregnancies $(n=45)$. Women underwent weekly three-dimensional ultrasound scans (3D US) from six up to thirteen weeks of gestational age (GA). To estimate embryonic growth trajectories, crown-rump length (CRL) measurements were performed using V-Scope software in the BARCO I-Space. Maternal characteristics and CVD risk factors were obtained using self-administered questionnaires. CVD risk score comprised maternal age, body-mass index, CVD in parents, diet and periconceptional smoking. To assess the association between the CVD risk score and embryonic growth trajectories, we performed linear mixed model analysis with hierarchical backward elimination using square root transformed CRL to achieve linearity with GA.

Results: In 111 pregnancies with 696 3D US we measured 637 (91.5\%) CRLs. In IVF/ICSI pregnancies, CVD risk score was inversely associated with embryonic growth trajectories (CRL $\beta=-0.05 \mathrm{~mm} ; P=0.025$, adjusted for GA and fetal gender including time interaction using GA). In spontaneous pregnancies, CVD risk score was not significantly associated with embryonic growth trajectories.

Conclusion: This study shows that a higher CVD risk score in women without CVD is associated with slightly reduced embryonic growth in IVF/ICSI pregnancies, but not in spontaneous pregnancies. Future research will have to elucidate whether the increased vulnerability due to the IVF/ICSI treatment or differences in reliability of pregnancy dating can explain these results.

Disclosure of Interest: None Declared.

\section{DOHaD13-1480}

Cryopreservation of embryos reduces the risk of small for gestational age

Michael Davies ${ }^{1, *}$, Alice Rumbold ${ }^{1}$, Jennifer Marino ${ }^{2}$, Kristyn Willson ${ }^{3}$, Lynne Giles ${ }^{3}$, Vivienne Moore ${ }^{3}$

${ }^{1}$ Robinson Institute, UNIVERSITY OF ADELAIDE, Adelaide; ${ }^{2}$ Department of Obstetrics and Gynaecology, University of Melbourne, Melbourne; ${ }^{3}$ Public Health, UNIVERSITY OF ADELAIDE, Adelaide, Australia

Background: Interventions to substantially reduce the incidence of fetal growth restriction in humans are limited in number. The study of specialised populations or clinical conditions that modify fetal growth may have important implications for developing future interventions. Reduced fetal growth is a common outcome of infertility treatments, due to technical limitations of to provide optimal conditions for fetal development, and the metabolic impairment associated with infertility. However, birthweight and gestational age vary across treatment modalities. Therefore, the aim of this study was to compare birthweight and gestational age for births after in vitro fertilisation (IVF) and intracyctoplasmic sperm injection (ICSI) with and without the step of cryopreservation of the embryo.

Method: A cohort of all patients in South Australia receiving infertility treatment 1986-2002 were linked to the state-wide perinatal collection (all live births and stillbirths of at least 20 weeks' gestation or $400 \mathrm{~g}$ birth weight, and all terminations). Some 4,260 births after infertility treatment including 1,394 after IVF and 911 after ICSI were compared to 292,141 births from spontaneous conceptions. Odds ratios were calculated for a range of routinely reported perinatal outcomes for birth weight and gestation stratified by mode of conception and multiplicity, and adjusted for maternal age, BMI, pre-existing and emergent diseases in pregnancy.

Results: Singleton births after IVF and ICSI using 'fresh' embryos were $240 \mathrm{gms}$ and $139 \mathrm{gms}$ lighter respectively compared to births to fertile couples, and the corresponding odds ratios for SGA were $1.5(\mathrm{CI}=1.3,1.8)$ and 1.5 $(\mathrm{CI}=1.3,1.9)$.

Frozen embryo transfer eliminated the birthweight deficit for singleton IVF births +3 gms $(\mathrm{CI}=-61,5)$, and significantly reduced the risk of SGA compared to the fertile population $(\mathrm{OR}=0.6, \mathrm{CI}=0.4,0.8)$. Frozen embryo transfer after ICSI increased the mean birth weight compared to the fertile population $+81 \mathrm{gms}(\mathrm{CI}=19,143)$ and eliminated the risk of SGA $(\mathrm{OR}=0.7, \mathrm{CI}=0.4,1.1)$.

However, the risk of large for gestational age was increased after frozen embryo transfer for both IVF $(\mathrm{OR}=1.4$, $\mathrm{CI}=1.0,1.8)$ and ICSI $(\mathrm{OR}=1.5,1.0,2.3)$.

A similar but weaker pattern of outcomes was also observed for twins.

Conclusion: With over 4 million live births worldwide, infertility treatment has created a large scale natural experiment on fetal development. Cryopreservation is an increasingly common strategy to optimise the use of multiple embryos created during infertility treatment. Embryo freezing appears to select against developmentally compromised embryos, but may also induce epigenetic changes in the growth axis, as indicted by the concerning increase in the risk of LGA births in both singletons and twins. The study of this population may identify basic mechanisms that can be developed for use beyond infertility treatment, where embryo vitrification is already being adopted as a strategy to alleviate the growth restricting effects of infertility treatment.

Disclosure of Interest: None Declared. 


\section{DOHaD13-1545}

Disruption of methionine metabolism may cause aberrant protein expression of TEAD4 and CDX2 in bovine preimplantation embryos in vitro

Shuntaro Ikeda ${ }^{1, *}$

${ }^{1}$ Laboratory of Animal Physiology and Functional Anatomy, Graduate School of Agriculture, KYOTO UNIVERSITY, Kyoto, Japan

Background: Animals ingest various kinds of dietary nutrients that affect the physiological functions of preimplantation embryos. Methionine is a dietary essential amino acid that plays multiple biological roles including those as a precursor of the essential methyl donor for methylation reactions (S-adenosylmethionine, SAM) and the methylation of DNA and histones are fundamental means for the epigenetic regulation of gene expression. We previously reported that in vitro treatment with ethionine, an antimetabolite of methionine, of bovine preimplantation embryos increased the mRNA expression of TEA domain family member 4 (Tead4) and impaired the blastocyst development. TEAD4 was reported to play a decisive role at the morula stage in the specification of the TE lineage, involving the activation of TE-regulatory genes such as caudal type homeobox $2(\mathrm{Cdx} 2)$. In the present study, in order to further investigate the implication of methionine metabolism in preimplantation development, we investigated the effects of ethionine treatment on the protein expressions of TEAD4 and CDX2 in bovine preimplantation embryos.

Method: In vitro produced bovine embryos that had developed to the 8-cell stage or more on day3 after the commencement of in vitro fertilization (IVF) were then cultured until day $6(\mathrm{IVF}=$ day 0$)$ in medium supplemented with 0 (control) or $5 \mathrm{mM}$ ethionine. The day 6 embryos in each group were subjected to immunofluorescence analysis of TEAD4 or CDX2 using monoclonal antibodies against these proteins. The nuclei of embryos were counterstained with bisbenzimide to count cell numbers. The expression levels represented by the immunofluorescence were compared between the treatment groups.

Results: Ethionine treatment at $5 \mathrm{mM}$ did not affect the cell number of day 6 embryos. Immunofluorescence analysis of TEAD4 revealed the higher expression in ethionine-treated embryos compared with the control. When compared within embryos that had relatively smaller cell numbers, the nuclear expression level of CDX2 was higher in the ethionine-treated group than in the control.

Conclusion: The ethionine treatment of bovine preimplantation embryos caused the aberrant higher expression of TEAD4 protein and premature nuclear localization of CDX2. These results suggest that the disruption of methionine metabolism affects the expressions of developmentally important proteins during mammalian preimplantation development.

Disclosure of Interest: None Declared.

\section{DOHaD13-1479}

Exogenous hormones for the treatment of infertility induces sex-specific variations in birthweight and length of gestation

Michael Davies ${ }^{1, *}$, Alice Rumbold ${ }^{1}$, Jennifer Marino ${ }^{2}$, Kristyn Willson ${ }^{3}$, Lynne Giles ${ }^{3}$, Vivienne Moore ${ }^{3}$

${ }^{1}$ Robinson Institute, UNIVERSITY OF ADELAIDE, Adelaide;

${ }^{2}$ Department of Obstetrics and Gynaecology, University of Melbourne, Melbourne; ${ }^{3}$ Public Health, UNIVERSITY OF ADELAIDE, Adelaide, Australia

Background: Endocrine disruptors have emerged as candidates for a wide range of developmental disorders. There is seldom the opportunity to examine controlled, timed, exposures in humans during the periconception period. Infertility treatment commonly involves exposure of women to drugs, commonly a selective estrogen receptor modulator (SERM) or follicle stimulating hormone (FSH) to induce ovulation. Perinatal outcomes after this treatment indicate a pattern of compromised fetal growth and reduced gestation. While perinatal outcomes after exposure to these drugs have been reported, effects of exposure by fetal sex have not been considered.

The aim of this study was to compare associations of ovulation induction for infertility with perinatal outcomes for males and females in a defined population.

Method: A cohort of all patients in South Australia receiving infertility treatment 1986-2002 were linked to the state-wide perinatal collection (all live births and stillbirths of at least 20 weeks' gestation or $400 \mathrm{~g}$ birth weight, and all terminations). A total of 4,260 infertility treatment births, including 340 singleton births after ovulation induction alone, were compared to 292,141 births from spontaneous conceptions. Odds ratios were calculated for a range of routinely reported perinatal outcomes for birth weight, gestation, and fetal death stratified by mode of conception and fetal sex and compared to the fertile general population with adjustment for a range of confounders including maternal age, parity, BMI, social position, pre-existing diseases and diseases in pregnancy.

Results: As a group, babies conceived by ovulation induction treatment were at increased risk of being born with low birth weight $(\mathrm{OR}=1.8, \mathrm{CI}=1.2,2.6)$, and born preterm $(\mathrm{OR}=$ $1.7, \mathrm{CI}=1.2,2.6)$ when compared to spontaneously conceived births. Mean birthweight was not significantly reduced $(-43$ gms, CI $=-109,2)$.

Sex differences were noted. Ovulation induction was associated with the risk of very low $(\mathrm{OR}=4.6 ; 1.9-11.4)$ low birth weight $(\mathrm{OR}=3.2 ; 1.9-5.4)$, preterm birth $(\mathrm{OR}=$ 1.9;1.1-3.3), very small size for gestational age $(\mathrm{OR}=$ $2.9 ; 1.6-5.5)$, and small size for gestational age $(\mathrm{OR}=2.1$; 1.4-3.2) among male singletons. There were no significant differences for females.

Conclusion: Exogenous hormones used for ovulation induction differentially influence male and female fetal growth. 
As assisted conception is generally associated with restricted fetal growth, the observed result may be due to the estrogenic maternal environment differentially increasing the growth trajectory of the female fetus, as opposed to restricting the growth of the male. The sexually dimorphic result suggests this is a treatment effect, rather than due to patient characteristics. OI is a potentially important exposure of interest as an endocrine disrupter, given its widespread and longstanding status as firstline therapy for anovulatory infertility.

Disclosure of Interest: None Declared.

\section{DOHaD13-1438}

In vitro fertilization and intracytoplasmic sperm injection are associated with increased glucose levels at the age of five to six years: the Amsterdam born children and their development study

Martina Pontesilli ${ }^{1,2, *}$, Rebecca C. Painter ${ }^{2}$, Iris J. Grooten ${ }^{1}$, Joris A. M. van der Post ${ }^{2}$, Ben Willem $\mathrm{Mol}^{2}$, Tanja Vrijkotte ${ }^{3}$, Sjoerd Repping ${ }^{4}$, Tessa J. Roseboom ${ }^{5}$

${ }^{1}$ Dept. of Obstetrics \& Gynaecology, Medical Centre Alkmaar, Alkmaar; ${ }^{2}$ Dept. of Obstetrics \& Gynaecology; ${ }^{3}$ Dept. of Social Medicine; ${ }^{4}$ Centre for Reproductive Medicine; ${ }^{5}$ Dept. of Clinical Epidemiology, Biostatistics and Bioinformatics, Academic Medical Centre Amsterdam, Amsterdam, Netherlands

Background: Conditions around the time of conception affect the offspring's growth and cardiovascular physiology. There is growing evidence that assisted reproduction techniques (ART) including In Vitro Fertilization (IVF) and Intracytoplasmic Sperm Injection (ICSI) may have lasting consequences for the health of individuals conceived through these techniques. We here investigate effects of ART on cardiovascular and metabolic profiles of 5-6 year old children through analysis of prospectively collected data.

Method: The Amsterdam Born Children and their Development study (ABCD-study) is a large multi-ethnic cohort study that was set up in Amsterdam to include pregnant women who registered for antenatal care between January 2003 and March 2004. In this cohort, we prospectively collected information on conception mode, lifestyle, nutrition and socio-demographic background, and the women as well as their children were followed.

At the age of 5-6 years, 3260 singletons were examined at which blood pressure, height and weight as well as lipid profile and fasting glucose were measured. Blood samples were taken in a subgroup $(n=2023)$. We compared cardiovascular and metabolic profiles of children conceived through intrauterine insemination (IUI), ovulation induction (OI) and IVF and ICSI to those of spontaneously conceived children.

Results: Of the 3260 children, 51 were conceived through IUI, 33 through OI and 29 through IVF or ICSI. Birth weight and gestational age did not differ statistically significant between children conceived through IUI, OI, IVF/ICSI and children conceived spontaneously. At the age of 5-6 years, there were no significant differences in height, weight and BMI between the groups. Fasting glucose levels were higher among children who were conceived through OI, IVF and ICSI compared to those who were conceived spontaneously. Adjustments for maternal characteristics (BMI, maternal age and parity) and for child characteristics (gender and BMI at the age of 5-6) did not attenuate these difference (adjusted difference for OI: $0.37 \mathrm{mmol} / 1,95 \% \mathrm{CI}$ 0.16 to $0.59, \mathrm{p}<0.05$; adjusted difference for IVF/ICSI: $0.26 \mathrm{mmol} / \mathrm{l}, 95 \%$ CI 0.02 to $0.5, \mathrm{p}<0.05$ ).

No differences were found in cardiovascular and metabolic profiles between spontaneously conceived children from subfertile women (defined as more than 12 months to conception) and children from non-subfertile women.

Conclusion: Children conceived through ART (except for IUI) had higher glucose levels at the age of 5-6 years compared to spontaneously conceived children, a finding that is in agreement with findings from other studies. This may suggest that these forms of assisted reproduction may have lasting consequences for infant's health. Optimising the early environment of individuals conceived through these techniques (through optimising culture media and hormone stimulation), as well as a renewed consideration of the indication of these treatments, may provide a new challenge in the prevention of disease in future generations.

Disclosure of Interest: None Declared.

\section{DOHaD13-1503}

Association between maternal adherence to Ramadan fasting during pregnancy and birth weight of the newborn Ary I. Savitri ${ }^{1, *}$, Nasim Yadegari ${ }^{2}$, Julia Bakker ${ }^{2}$, Reyn J. van Ewijk $^{3}$, Diederick E. Grobbee ${ }^{1}$, Rebecca C. Painter ${ }^{2}$, Cuno S. Uiterwaal $^{1}$, Tessa J. Roseboom ${ }^{2,4}$

${ }^{1}$ Department of Epidemiology, Julius Center for Health Sciences and Primary Care, University Medical Center, Utrecht;

${ }^{2}$ Department of Obstetrics and Gynecology, Academic Medical Center, Amsterdam, Netherlands; ${ }^{3}$ Institute of Medical Biostatistics, Epidemiology, and Informatics, University Medical

Center, Mainz, Germany; ${ }^{4}$ Department of Clinical Epidemiology, Biostatistics and Bioinformatics, Academic Medical Center, Amsterdam, Netherlands

Background: Many Muslim women worldwide are pregnant during Ramadan and adhere to Ramadan fasting during pregnancy. We determined whether maternal adherence to Ramadan fasting during pregnancy has an impact on the birth weight of the newborn, and whether the effects differed according to trimester in which Ramadan fasting took place. Method: A prospective cohort study was conducted in 130 pregnant Muslim women who attended antenatal care in 
Amsterdam and Zaanstad, The Netherlands. Data on Ramadan fasting adherence during pregnancy and demographics were selfreported by pregnant women, and the outcome of the newborn was retrieved from medical records after delivery

Results: Half of all the women adhered to Ramadan fasting. With strict adherence to Ramadan fasting in pregnancy, newborns' birth weight tended to be lower than with nonfasting, although this was not statistically significant $(-198 \mathrm{~g}$, 95\% CI: -447 to $51, \mathrm{p}=0.12)$. Children of mothers who fasted in the first trimester of pregnancy were lighter at birth than those whose mothers had not fasted $(-272 \mathrm{~g}, 95 \% \mathrm{CI}$ : -547 to $3, p=0.05)$. There were no differences in birth weight between children whose mothers had or had not fasted if Ramadan fasting had taken place later in pregnancy.

Conclusion: Ramadan fasting during pregnancy may lead to lower birth weight of newborns. These findings call for further confirmation in larger numbers of women to investigate potential implications for perinatal and long-term morbidity and mortality.

Disclosure of Interest: None Declared.

\section{DOHaD13-1108}

\section{Associations of third trimester fetal blood flow distribution and kidney size with childhood kidney function}

Marjolein Kooijman ${ }^{1,2, *}$, Hanneke Bakker ${ }^{1,2,3}$, Albert Hofman ${ }^{1}$, Vincent Jaddoe ${ }^{1,2,3}$

${ }^{1}$ Department of Epidemiology; ${ }^{2}$ The Generation R Study Group; ${ }^{3}$ Department of Pediatrics, Erasmus MC, Rotterdam, Netherlands

Background: An adverse fetal environment may lead to smaller kidneys with a reduced number of nephrons, and subsequently to impaired kidney function in later life. An early marker of an adverse intra-uterine environment is a change in fetal blood flow distribution with preferential blood flow to the brains at expense of the trunk. Our aim was to evaluate the associations of third trimester fetal blood flow distribution and kidney size with childhood kidney function. Method: In a population-based prospective cohort study among 923 pregnant women, we measured third trimester (median gestational age 30.3 weeks (95\% range $28.5-32.7$ )) fetal growth characteristics, blood flow distribution by measurements of resistance of the umbilical and cerebral artery and kidney biometrics. We performed follow up measurements of kidney volume, creatinine and cystatin $\mathrm{C}$ blood levels, albuminuria and blood pressure in childhood (median 5.9 years (95\% range 5.7-6.7)). The glomerular filtration rate was estimated using the Schwartz formula.

Results: Higher third trimester fetal umbilical/cerebral artery pulsality index, which reflects a preferential blood flow to the upper body parts, was associated with a smaller childhood combined kidney volume $\left(-1.73 \mathrm{~cm}^{3}\right.$ per SDS increase in pulsality index, 95\% CI $-3.09,-0.38)$ and higher creatinine levels $(0.71 \mu \mathrm{mol} / 1$ per SDS increase in pulsatlity index, 95\% CI 0.24, 1.18). Larger third trimester combined fetal kidney volume was associated with lower creatinine levels $(-0.77 \mu \mathrm{mol} / \mathrm{l}$ per SDS increase in kidney volume, 95\% CI $-1.28,-0.26)$, lower cystatin C levels $(-14 \mu \mathrm{g} / \mathrm{l}$ per SDS increase in kidney volume, 95\% CI $-22,-6)$ and higher glomerular filtration rate $\left(2.86 \mathrm{ml} / \mathrm{min}\right.$ per $1.73 \mathrm{~m}^{2}$ per SDS increase in kidney volume, $95 \%$ CI 1.37, 4.36), but not with micro albuminuria and blood pressure in childhood.

Conclusion: Redistribution of fetal blood flow to the upper body parts in third trimester of pregnancy and smaller fetal kidney volume affect kidney function in childhood. Whether these adaptations lead to greater risks of renal disease in adult life should be further studied.

Disclosure of Interest: None Declared.

\section{DOHaD13-1511}

Diurnal changes in glucose during pregnancy are associated with macrosomia in the newborn

Graham R. Law ${ }^{1, *}$

${ }^{1}$ Division of Biostatistics, University of Leeds, Leeds, United Kingdom

Background: Large birth weight, macrosomia, is the commonest complication of diabetic pregnancy. Although macrosomia is thought to be attributed to high blood glucose, the actual continuous glucose profile across the day and night for macrosomia has not been described. This study aimed to measure glucose over the 24 hour period continuously and this will allow more accurate diagnostic and prognostic assessments, and optimize new therapeutic technologies in insulin administration.

Method: A prospective observational study (between 2003-2006) involving longitudinal continuous glucose measurements in two secondary care antenatal clinics for diabetes in the UK was analysed. Pregnant women with Type 1 or Type 2 diabetes who were free of severe medical and psychological comorbidities and were willing to wear a continuous glucose monitor, were recruited. Macrosomia, defined as a birth weight on or above the $90^{\text {th }}$ centile for sex and gestational age, was the outcome. Functional data analysis and functional regression were used to examine the association with the levels of glucose and macrosomia.

Results: There were 49 pregnant women recruited with 256640 glucose measurements over 171 separate sessions of measurements. The functional regression model showed that Type 2 diabetes, compared to Type 1 diabetes, have significantly lower levels of glucose during the night time, from $10 \mathrm{pm}$, to noon the following day. There was little difference between trimester 2 and trimester 1 . A lowering of glucose from around $9 \mathrm{pm}$ until 4 am is seen in trimester 2 . Trimester 3 has a greater difference with a similar reduction 
in glucose from around $8 \mathrm{pm}$ until 10am the following morning. There is a slight increase in trimester 2 and trimester 3 (compared to trimester 1) during the morning from 9am to noon. Mothers with babies who have macrosomia have higher levels of glucose from around 9am until approximately $1 \mathrm{am}$. Then there is a reduction in glucose from 1 am until 9am.

Conclusion: This study is the first to demonstrate the diurnal variation in glucose that is associated with the development of macrosomia in diabetic pregnancy. It confirms established evidence that postprandial hyperglycemia during the day is associated with macrosomia, but gives novel information about the contribution of nocturnal glucose control and suggests that relative hypoglycemia has an important role to play. This information has the potential to aid diagnostic, prognostic and therapeutic decision making in everyday clinical practice. Additional evidence is now required to assess whether normalising the diurnal profile of glucose translates into a reduced risk of macrosomia.

Disclosure of Interest: None Declared.

\section{DOHaD13-1229}

Early and late childbearing as risk factors for fetal growth and adverse birth outcomes: evidence from Sao Paulo, Brazil

Alexandra Brentani ${ }^{1, *}$, Ana Maria de Ulhôa Escobar ${ }^{1}$, Filumena M. D. S. Gomes ${ }^{1}$, Maria H. Valente ${ }^{1}$, Sandra J. Grisi ${ }^{1}$, Günther Fink ${ }^{2}$

${ }^{1}$ Pediatrics, Faculdade de Medicina Universidade de São Paulo, São Paulo, Brazil; ${ }^{2}$ Global Health and Population, Harvard School of Public Health, Cambridge, United States

Background: Beyond the potentially large long-term health consequences of intrauterine growth restrictions predicted by the Barker hypothesis, gestational complications constitute a primary risk factor for neonatal and infant mortality. In an effort to achieve Millennium Development Goals (MDGs) 4 and 5, Brazil has made substantial progress in child health, but continues to struggle with continued high rates of teenage pregnancies as well as with a simultaneous increase in pregnancies from mothers age 40 and older.

Objectives: To analyze the relationship between maternal age and fetal development as well as pregnancy outcomes in the urban setting of São Paulo, Brazil.

Method: Complete digital medical records were extracted for 34,977 births delivered at the University Hospital- HU in Sáo Paulo between 2003 and 2013. Four outcomes were analyzed using multivariate logistic models: child survival at birth, low birth weight adjusted for gestational age, preterm deliveries, and child health in the first 10 minutes after birth (APGAR scores 1, 5 and 10).

Results: No significant correlation between teenage pregnancy and stillbirth were found, while maternal age above
40 increased the odds of stillbirth by a factor of 3 (OR 4.14, $95 \%$ CI [2.321, 7.386], p-value, <0.001). Adolescent pregnancies increased the odds of intra-uterine growth restrictions (IUGR) by 41\% (OR 1.41, 95\% CI [1.260, 1.581 ], p-value $<0.001)$, while maternal age 40 and older increased the odds of IUGR by 75 percent (OR 1.75, 95\% CI [1.356, 2.258], p-value <0.001). Similar patterns were observed for pre-term deliveries. Adolescent pregnancies increased the odds of preterm deliveries by 60\% (OR 1.60, 95\% CI [1.427, 1.787], p-value <0.001), and maternal age of 40 and older increased the odds by 33\% (OR 1.31, 95\% CI $[0.993,1.785]$, p-value $=0.056)$. No significant association was found between teenage pregnancy and low Apgar scores. Maternal age of 40 and older increased the odds of low Apgar scores by a factor of 2 (OR 2.66, 95\% CI [1.6949, 4.1809], p-value $<0.001$ ).

Conclusion: The findings suggest that maternal age constitutes a primary risk factor for fetal development and child health in Sao Paulo. Increased medical attention during the pre- and postnatal periods are needed to attenuate the negative health consequences of pregnancies by younger and older mothers.

Disclosure of Interest: None Declared.

\section{DOHaD13-1721}

Estimating the adverse effect of low maternal BMI, smoking, on preterm birth and decreased fetal growth, in Japanese women

Naho Morisaki ${ }^{1,2, *}$, Kenji Takeda ${ }^{1}$

${ }^{1}$ Department of Health Policy, National Center for Child Health and Development; ${ }^{2}$ Department of Pediatrics, University of Tokyo, Tokyo, Japan

Background: Increase in preterm birth and low birth weight (birthweight $<2500 \mathrm{~g}$ ) is a serious issue in Japan. Acknowledging the most effective interventions is important policy wise.

Method: Using a preexisting cross-sectional data set of a multi-level sample of all children born in Japan during 1994-2010 and alive at time of study, we investigated the association between maternal BMI, smoking, and age with birth weight and risk of preterm birth in 16238 singletons.

Results: $5.2 \%$ of the children were born preterm, and average birth weight was 3031 grams. Average maternal BMI was 20.6, with $18.9 \%$ underweight (BMI < 18.5) and $8.0 \%$ overweight $(\mathrm{BMI}>25)$. $5.1 \%$ were smokers, $11.7 \%$ were over 35 years old, 1.3\% were below 20 years old at delivery. In multivariate logistic regression adjusting for maternal characteristics, maternal smoking [odds ratio (OR) 1.41(1.10-1.81)], BMI $<18.5$ [OR 1.40 (1.10; 1.76)], $\mathrm{BMI}>25$ [OR $1.37(1.17 ; 1.85)]$, age $<20[\mathrm{OR} 2.40$ $(1.38 ; 4.18)]$, age $>35$ [OR $1.62(1.27 ; 2.09)$ ], were all risk 
factors for preterm birth. In multivariate linear regression adjusting for maternal characteristics and gestational length, maternal smoking [102 g $(92,112)]$, lower BMI [20.3 g (18.5, 22.1)/unit) and higher age $[20.2 \mathrm{~g}(8.3,32.1) / 10$ years], were all risk factors for smaller fetal growth.

Furthermore, through standardization using these models we estimated that eliminating maternal smoking would decrease preterm birth by $3 \%$ and increase average birth weight by $14 \mathrm{~g}$. Similarly if all underweight mothers increased weight by $2 \mathrm{~kg}$, preterm birth would decrease by $3 \%$ and average birth weight would increase by $26 \mathrm{~g}$. Combination of both interventions would decrease preterm birth by $6 \%$, and increase average birth weight by 35 grams.

Conclusion: For the individual, the adverse effect of being underweight is similar to that of smoking. On a population basis, in our current situation with a large proportion of underweight mothers, advocating to increase pre-pregnancy weight may be an intervention nearly as effective as eliminating smoking, to reduce preterm and increasing average birth weight in Japan.

Disclosure of Interest: None Declared.

DOHaD13-1717

Experience of major life events during childhood and development of obesity in adulthood: a co-twin control study.

Marianne E. Vámosi ${ }^{1, *}$, Berit L. Heitmann ${ }^{2,3}$, Kyle Raymond ${ }^{3}$, Jindong Ding ${ }^{4}$

${ }^{1}$ Institute of Public Health, Aahus University, Aarhus;

${ }^{2}$ National Institute of Public Health, University of Southern

Denmark, Odense; ${ }^{3}$ Institute of Preventive Medicine, Research

Unit for Dietary Studies, Bispebjerg and Frederiksberg Hospitals - a part of Copenhagen University Hospital, The Capital Region, Copenhagen; ${ }^{4}$ Institute of Public Health, University of Southern Denmark, Odense, Denmark

Background: The etiology of adult obesity is still poorly understood, even if often simply attributed to too much food and too little exercise. A few studies have suggested that adverse psychological factors also be determinants. We examined if separation from parents before the age of 17 , parental loss, and living in a "children's home" during childhood were associated with development of adult obesity, independent of the early environment and genetic factors.

Method: The study used the co-twin control study discordant for a trait to determine influences of environmental factors. DZ twins were also included.

Same sex adult pairs, aged $<50$ years in 2002, and discordant for BMI, i.e. one twin having a BMI $>30 \mathrm{~kg} / \mathrm{m}^{2}$ and the co- twin a BMI between 20 and $25 \mathrm{~kg} / \mathrm{m}^{2}$, were selected. The subjects were invited to participate in a structured interview regarding adverse childhood experiences, including whether the child had been living in a children's home and if one of the parents died, got separated or got divorced during childhood. We used the The Childhood Family Relationship Questionnaire. All were interviewed without any relatives, including the co-twin, being present and by the same interviewer.

Results: A total of 288 twins were contacted and, 236 (82\%) interviews were completed. In total $20.8 \%$ had been separated from mother, and $26.1 \%$ had been separated from father. Furthermore $9.3 \%$ of the children had been living in children's home and $6.8 \%$ had lost their father. Only twins who were normal weight as children were included in the intra - pair analysis, which showed no association between ever living in a children's home and adult obesity $\mathrm{OR}=1.95$ (0.19-5.37), or being separated from father or mother OR = $1.22(0.46-3.34)$ and $\mathrm{OR}=0.9(0.32-2.46)$, respectively.

Conclusion: The results of this study indicate that early major life events such as separation from parents before age of 17 , or having stayed at a children's home are not related to obesity in adulthood.

\section{References}

Disclosure of Interest: None Declared.

\section{DOHaD13-1050}

\section{Gestational diabetes mellitus (GDM) and changes in retinal microvasculature 6 months after delivery}

Lingjun $\mathrm{Li}^{1, *}$, Yap Seng Chong ${ }^{1}$, Kenneth Kwek ${ }^{2}$, Peter Gluckman ${ }^{3}$, Seang Mei Saw ${ }^{1}$, Tien-Yin Wong ${ }^{1}$

${ }^{1}$ National University of Singapore; ${ }^{2} K K$ Women and Children's Hospital, Singapore; ${ }^{3} A^{*} S T A R$, National University of Singapore, singapore, Singapore

Background: Gestational diabetes mellitus (GDM) is associated with long-term risk of diabetes in pregnant women. Although the exact mechanisms are unknown, a role for the microcirculation has been implicated. We examined mid-pregnancy GDM and changes in the retinal microvasculature in a cohort of Singapore pregnant women 6 months after delivery.

Method: Pregnant women aged 18-46 years were recruited during their early pregnancy from the Growing Up in Singapore Towards Healthy Outcomes (GUSTO) study and followed up 6 months after delivery. Blood pressure, anthropometric measurements, fasting plasma glucose level, 2-hr plasma glucose level were measured at the 26 weeks gestation week following a standardized protocol. Questionnaires on socio-economic status and life style were ascertained by trained clinic staff. GDM were diagnosed using OGTT data according to WHO guideline. Retinal photography was examined at 26 weeks gestation and 6 months after delivery. Quantitative retinal vascular parameters were assessed by a 
semi-automated computer program (Singapore I Vessel Assessment [SIVA], version 3.0). Changes of retinal vascular parameters are defined as percentage changes in retinal vascular parameters between 6 months after delivery and 26 weeks gestation.

Results: Among the 280 pregnant women who included in this study, $47(16.8 \%)$ were diagnosed as GDM at 26 weeks gestation. In multivariate analysis, GDM patients tended to have more changes in retinal arteriolar widening than nonGDM subjects ( $35.16 \%$ vs. $31.48 \%$, p trend $<00.01)$. Even though GDM was associated with 26 weeks smaller retinal arteriolar fractal dimension (1.206 vs. $1.228 \mathrm{Df}, \mathrm{p}$ trend $<0.01)$ and smaller retinal venular fractal dimension $(1.210$ vs. $1.227 \mathrm{Df}, \mathrm{p}$ trend $=0.02$ ) than non-GDM subjects, these associations were attenuated after delivery.

Conclusion: GDM was associated with postnatal early retinal vascular changes consistent with risk of type 2 diabetes. Our finding suggested a possible impact of GDM on the microcirculation during pregnancy, which may be a pathophysiologic pathway for evidence on the development of future metabolic diseases in women.

Disclosure of Interest: None Declared.

\section{DOHaD13-1669}

Impact of fetal smoke exposure on respiratory outcomes in school- aged children. The Generation R study

Martijn Den Dekker ${ }^{1, *}$, Agnes M. Sonnenschein - van der Voort $^{1}$, Johan C. De Jongste ${ }^{2}$, Albert Hofman ${ }^{3}$, Vincent V. Jaddoe $^{4}$, Liesbeth Duijts ${ }^{5}$

${ }^{1}$ Pediatric pulmonology/Epidemiology; ${ }^{2}$ Pediatric pulmonology, Erasmus MC - Sophia Children's Hospital; ${ }^{3}$ Epidemiology,

Erasmus MC; ${ }^{4}$ Epidemiology/pediatrics; ${ }^{5}$ Pediatric pulmonology \& division of neonatology, Erasmus $M C$ - Sophia Children's

Hospital, Rotterdam, Netherlands

Background: Results of previous studies on the adverse effects of fetal smoke exposure with respiratory outcomes in school aged children are conflicting. We examined whether maternal smoking during pregnancy is associated with longitudinal wheezing patterns, doctor- diagnosed asthma and airway resistance (Rint) at the age of 6 years.

Method: This study was embedded in Generation R, a population-based cohort study from fetal life onwards. Parental smoking during pregnancy was assessed by questionnaires. Wheezing was reported from age 1 to 6 years. Wheezing patterns were defined as never wheezing, early wheezing $(<=3$ years only), late wheezing ( $>3-6$ years only) and persistent wheezing (both $<=3$ years and $>3-6$ years). Parental report of doctor diagnosed asthma and measurements of airway resistance (Rint) were obtained at 6 years. Multivariate analyses were based on 5,755 children. Results: Of all children, $\mathrm{n}=510(8.9 \%)$ were exposed to first trimester maternal smoking, and $\mathrm{n}=921 \quad(16.0 \%)$ were exposed to continued maternal smoking during pregnancy. Compared with no smoke exposure during pregnancy, we observed no associations of first-trimester-only smoking with wheezing patterns, asthma, or Rint. Maternal continued smoking during pregnancy was associated with higher risks of persistent wheezing; odds ratio ( $95 \%$ confidence interval): $1.42(1.05,1.91)$. No associations of continued smoking during pregnancy with asthma or Rint were observed.

Conclusion: Continued smoking during pregnancy is associated with persistent wheezing in school aged children. Further studies are needed to explore the underlying mechanisms.

Disclosure of Interest: None Declared.

\section{DOHaD13-1182}

Maternal caffeine intake and risk of low birth weight: a meta-analysis

Ling-Wei Chen ${ }^{1, *}$, Yi Wu ${ }^{1}$, Nithya Neelakantan ${ }^{1}$, Mary F.-F. Chong', Rob M. van Dam ${ }^{3}$

${ }^{1}$ Saw Swee Hock School of Public Health, National University of Singapore; ${ }^{2}$ Singapore Institute for Clinical Sciences, $A^{*} S T A R$; ${ }^{3}$ Saw Swee Hock School of Public Health and Yong Loo Lin School of Medicine, National University of Singapore,

Singapore, Singapore

Background: Emerging epidemiological evidence suggests harmful effect of maternal caffeine intake during pregnancy on birth weight, but results have been inconsistent. We aim to elucidate the relationship between maternal caffeine intake and birth weight in a meta-analysis.

Method: We searched MEDLINE and SCOPUS through October 2011 and identified 10 eligible prospective studies of caffeine and birth weight. Of these, 5 studies reported data on low birth weight (LBW) or intrauterine growth restriction (IUGR) and 3 studies reported data on differences in birth weight (2 studies reported data on both types of outcomes). Caffeine categories reported in the original studies were categorized into "low" (51-150 mg/day), "moderate" (151-350 mg/day), "high" (>350 mg/day) and a reference category (lowest consumption group in respective studies). Summary relative risks (RR) and differences in birth weight with $95 \%$ confidence intervals (CI) were calculated using random-effects models and the I-squared statistic $\left(\mathrm{I}^{2}\right)$ and $\mathrm{p}$-value for heterogeneity ( $\mathrm{p}$-het) were calculated as measures of between-study heterogeneity.

Results: The summary RR of LBW or IUGR was 1.66 (95\% CI: $1.15,2.41 ; \mathrm{I}^{2}=68.7 \%$, p-het $\left.=0.004\right)$ for high caffeine intake, 1.37 for moderate caffeine intake $(95 \% \mathrm{CI}: 1.08$, $1.74 ; \mathrm{I}^{2}=37.7 \%$, p-het $\left.=0.16\right)$, and $1.25(95 \% \mathrm{CI}: 1.03$, $1.52 ; \mathrm{I}^{2}=0.0 \%, \mathrm{p}$-het $=0.91$ ) for low caffeine intake as compared with reference caffeine intake group of the respective studies. Similar trends were observed when analysis 
was done separately for LBW and IUGR. The birth weight difference was $-71.7 \mathrm{~g}\left(95 \% \mathrm{CI}:-106.0,-37.5 ; \mathrm{I}^{2}=0.0 \%\right.$, $\mathrm{p}$-het $=0.59)$ for high caffeine intake, $-31.7 \mathrm{~g}(95 \% \mathrm{CI}$ : $-69.5,6.1 ; \mathrm{I}^{2}=27.4 \%$, p-het $\left.=0.27\right)$ for moderate caffeine intake, and $-9.5 \mathrm{~g}\left(95 \% \mathrm{CI}:-35.3,16.4 ; \mathrm{I}^{2}=0.0 \%\right.$, $\mathrm{p}$-het $=0.63)$ for low caffeine intake.

Conclusion: In this meta-analysis, we observed significant increased risk of LBW or IUGR for high, moderate, and low caffeine intake following a dose-response relationship. These results suggest that maternal caffeine intake during pregnancy can increase risk of LBW or IUGR.

Disclosure of Interest: None Declared.

\section{DOHaD13-1066}

\section{Maternal obesity and offspring premature mortality: a follow-up of 1,323,275 person-years}

Rebecca M. Reynolds ${ }^{1, *}$, Keith M. Allan ${ }^{2}$, Edwin A. Raja ${ }^{2}$, Sohinee Bhattacharya ${ }^{2}$, Geraldine McNeill ${ }^{2}$, Phil Hannaford ${ }^{2}$, Amanda J. Lee ${ }^{2}$, Siladitya Bhattacharya ${ }^{2}$, Jane E. Norman ${ }^{1}$

${ }^{1}$ University of Edinburgh, Edinburgh; ${ }^{2}$ University of Aberdeen, Aberdeen, United Kingdom

Background: Obesity in pregnancy is associated with cardiometabolic risk factors in the offspring. We hypothesised that maternal obesity is also associated with increased risk of offspring death from cardiovascular events. We aimed to test this hypothesis using a large database of pregnancy data, the Aberdeen Maternity and Neonatal Databank (AMND), linked to offspring national death and morbidity records to generate a total of 1,323,275 person-years follow-up.

Method: Birth records of 37,709 children from 1950-current from the Aberdeen Maternity and Neonatal databank were linked to the General Register of Deaths, Scotland and the Scottish Morbidity Record systems to identify outcomes of death and hospital admissions for cardiovascular events up to 01/01/2012. Maternal BMI was calculated from height and weight measured at the first antenatal visit. The effect of maternal obesity on offspring outcomes was tested using timeto-event analysis using Cox-proportional hazard regression to compare outcomes in offspring of mothers in underweight, overweight or obese categories of BMI, compared to offspring of women with normal BMI. Ethical approval was obtained from the North of Scotland Research Ethics Service (REC reference: 10/S11034/15). Approvals were also obtained from the steering committee of the AMND and the Privacy Advisory Committee of ISD, Scotland.

Results: All-cause mortality was increased in offspring of obese mothers $\left(\mathrm{BMI}>30 \mathrm{~kg} / \mathrm{m}^{2}\right.$ ) compared to normal BMI mothers after adjustment for maternal age at delivery, socioeconomic status, offspring gender, birthweight, gestation at delivery and gestational age of BMI measurement (Hazard ratio $[\mathrm{HR}](95 \%$ confidence interval) $1.35(1 \cdot 17,1 \cdot 55)$.
In adjusted models, offspring of obese mothers also had increased risk of hospital admission for a cardiovascular event (HR 1.29 $(1.06,1.57)$ compared to normal BMI mothers. The offspring of overweight mothers also had a higher risk of adverse outcomes. Findings remained significant after adjustment for offspring current socio-economic status.

Conclusion: Maternal obesity increases risk of premature death in her offspring. As one in five women in the UK is currently obese at antenatal booking, strategies to optimise weight prior to pregnancy are urgently required.

Disclosure of Interest: None Declared.

\section{DOHaD13-1684}

Maternal prenatal distress predicts adolescent reported internalising behaviour problems in a prospective birth cohort

Kim S. Betts ${ }^{1, *}$, Gail M. Williams ${ }^{1}$, Jacob M. Najman ${ }^{2}$, Rosa Alati ${ }^{1}$

${ }^{1}$ School of Population Health; ${ }^{2}$ School of Social Science and Population Health, University of Queensland, Brisbane, Australia

Background: Studies have shown a link between maternal prenatal mental health or stress symptoms and early child behaviour problems. Further research is needed to investigate outcomes among adolescent offspring and better account for residual environmental confounding.

Method: Participants included 3,925 mother-offspring pairs from the Mater University Study of Pregnancy (MUSP), an Australian prospective, pre-birth cohort study. Latent Class Growth Analysis with parallel processes was used to empirically derive trajectories of maternal depression, anxiety and stress over four measurement periods between the mothers' first clinic visit and 5 years post-pregnancy. We used Achenbach's Youth Self Report to predict adolescent report internalising and externalising problem behaviour by maternal distress trajectories. In multivariate logistic regression analyses we adjusted for confounders, including birth weight, maternal alcohol and smoking in pregnancy, mother's education and father's history of mental health, in addition to factors which may mediate the relationship between early postnatal distress and offspring behaviour such as maternal life events and relationship quality and contact with the new born.

Results: Seven trajectories of maternal distress were identified, including one which isolated high levels of anxiety, depression and stress during pregnancy. After adjustment for confounders this was the only group of mothers which predicted internalising problems, while no group predicted externalising problems.

Conclusion: We found evidence for a prenatal distress effect, whereby maternal distress in early pregnancy uniquely increased the risk of internalising behaviour problems in adolescence.

Disclosure of Interest: None Declared. 


\section{DOHaD13-1339}

\section{Maternal smoking during pregnancy and adiposity among contemporary Portuguese children}

\author{
Leah $\mathrm{Li}^{1, *}$, Helen Peters ${ }^{1}$, Cristina Padez ${ }^{2}$ \\ ${ }^{1}$ Centre for Paediatric Epidemiology, UCL Institute \\ of Child Health, London, United Kingdom; ${ }^{2}$ Department \\ of Life Sciences, and Research Centre for Anthropology \\ and Health, University of Coimbra, Coimbra, \\ Portugal
}

Background: There is increasing evidence suggesting that maternal smoking during pregnancy is associated with an increase in risk of childhood overweight and obesity defined by BMI. Less is known about its association with other adiposity measures. There is little evidence on its association with CVD risk factors in childhood.

Method: We used data from a study of 17,286 school children aged 3-10 y across all mainland Portuguese districts, from March 2009 to January 2010. Anthropometric measurements, blood pressure, and resting pulse rate were measured by trained technicians using a standardized procedure. The sum of three (triceps, subscapular, and suprailiac) skinfolds was derived. We applied Quantile regression models, with a fractional polynomial function of age, to examine the association of maternal smoking during pregnancy (retrospectively reported by the mother) with offspring adiposity, blood pressure, and resting pulse rate. The models were further adjusted for parental BMI, maternal age and education, parity, birth weight, gestational age, infant feeding, hours spent watching TV, and consumption of sweet drinks.

Results: Overall $13.7 \%$ of children were born to mothers who smoked during pregnancy. Maternal smoking was associated with higher median BMI (by $0.4 \mathrm{~kg} / \mathrm{m}^{2}$ ), waist circumference (by $0.6-0.8 \mathrm{~cm}$ ), and the sum of skinfolds (by $0.9-1.4 \mathrm{~mm}$ ) after adjustment for pre-natal and early life factors, compared to children of nonsmokers. The differences were also seen for the $90^{\text {th }}$ centile of the adiposity measures. While the associations were evident among 3-year-olds, there was indication that the effect of maternal smoking on adiposity measures was greater among older than younger children, although the interaction between maternal smoking and offspring age was non-significant. There was little evidence that maternal smoking is associated with childhood BP and resting pulse rate.

Conclusion: Our results show that the association between maternal smoking during pregnancy and increased adiposity was apparent in children from age 3 years. The association with a range of adiposity measures was present across all age groups up to 10 years, with some suggestion of a greater association in older children.

Disclosure of Interest: None Declared.

\section{DOHaD13-1259}

$\mathrm{N}-3$ long-chain polyunsaturated fatty acids in breast milk and cognitive function in childhood

Carolien G. Herraets $^{1,2}$, Lenie Van Rossem ${ }^{1, *}$, Johan C. de Jongste $^{3}$, Gerard H. Koppelman ${ }^{4}$, Alet H. Wijga ${ }^{5}$, Henriette A. Smit ${ }^{1}$

${ }^{1}$ Julius Center for Health Sciences and Primary Care, University Medical Center Utrecht, Utrecht; ${ }^{2} V U$ Medical Center, Amsterdam; ${ }^{3}$ Department of Paediatrics, Erasmus University Medical Center, Sophia Children's Hospital, Rotterdam; ${ }^{4}$ Department of Pediatric Pulmonology and Pediatric Allergology, Beatrix Children's Hospital, University Medical Center Groningen, University of Groningen, Groningen; ${ }^{5}$ Center for Prevention and Health Services Research, National Institute of Public Health, Bilthoven, Netherlands

Background: N-3 long-chain poly-unsaturated fatty acids (LC PUFAs) play an important role in neurodevelopment during pregnancy and early life. We studied the association between n-3 LC PUFA composition, specifically DHA and EPA, of breast milk and child's cognition at age 12 .

Method: Fatty acids in breast milk samples were analyzed for 157 mothers in the Prevention and Incidence of Asthma and Mite Allergy (PIAMA) study, a population-based birth cohort study that started in 1996/1997 in the Netherlands. EPA and DHA content of breast milk (in weight percentage of total fatty acids, wt $\%$ ) were dichotomized into 'low' (below median) and 'high' (above median). Child's cognition was measured with a standardized national academic achievement test at the age of 12 (possible range: 501-550). We used multiple linear regression to assess the difference in cognitive scores between 3 groups of children:

1) children who received a relatively high amount of n-3 LC PUFAs in breast milk (above median wt $\%$ )

2) children who received a relatively low amount of n-3 LC PUFAs in breast milk (below median wt\%)

3) the reference group of 277 children who were not breastfed and received formula without n-3 LC PUFAs.

Confounders including parental educational level, maternal smoking during pregnancy, birth weight, gestational age, gender, attendance of nursery school, child's mental wellbeing, and child's current fish intake were taken into account. Results: Mean cognitive score was 538.9 (SD: 7.9). In fully adjusted models, children who received breast milk with a relatively high content of n-3 LC PUFAs had higher cognitive scores compared to formula-fed children $\left(b_{\text {DHA }}=1.73,95 \%\right.$ CI: $\left.-0.16,3.63 ; b_{\mathrm{EPA}}=1.28,95 \% \mathrm{CI}:-0.66,3.22\right)$. This association was present in girls $\left(b_{\mathrm{DHA}}=3.36,95 \% \mathrm{CI}: 0.73\right.$, 5.99; $\mathrm{b}_{\mathrm{EPA}}=2.79$, 95\% CI: $0.15,5.42$ ), but not in boys $\left(b_{\mathrm{DHA}}=0.14,95 \% \mathrm{CI}:-2.65,2.94 ; \mathrm{b}_{\mathrm{EPA}}=-0.40,95 \% \mathrm{CI}\right.$ : $-3.34,2.55)$. The cognitive scores did not differ between children who received breast milk with a relatively low content of n-3 LC PUFAs and formula-fed children $\left(b_{\mathrm{DHA}}=0.57,95 \%\right.$ 
CI: $\left.-1.42,2.45 ; b_{\mathrm{EPA}}=1.02,95 \% \mathrm{CI}:-0.87,2.91\right)$. This was also the case when stratifying by gender.

Conclusion: A high DHA and EPA content in breast milk is associated with higher cognitive scores in girls at the age of 12 .

Disclosure of Interest: None Declared.

\section{DOHaD13-1112}

Prenatal antibiotic and antifungal medication use are associated with atopic dermatitis and allergic sensitization in 2 year-old children

Ganesa Wegienka ${ }^{1, *}$, Suzanne Havstad ${ }^{1}$, Edward Zoratti ${ }^{2}$, Dennis Ownby ${ }^{3}$, Christine Johnson ${ }^{1}$

${ }^{1}$ Department of Public Health Sciences; ${ }^{2}$ Internal Medicine, HENRY FORD HEALTH SYSTEM, Detroit; ${ }^{3}$ Pediatrics, Georgia Regents University, Augusta, United States

Background: Recent studies of allergic diseases have begun to focus on the early life microbial exposure of a child as it relates to their allergic disease risk. Atopic dermatitis (AD) and allergic sensitization (AS) have increased in prevalence at the same time that antibiotic use increased in healthcare. Factors that affect the vaginal microbiome such as antibiotics and antifungal medications could potentially be associated with allergic disease outcomes. For example, there have been studies of a potential role for the usage of antibiotics in pregnancy in the subsequent development of allergies and asthma in the children and these studies have yielded mixed results. Meanwhile, cesarian delivery is now perceived to increase risk of allergic disease due to lack of exposure to the diversity of the vaginal microbiome. The work presented here investigates the associations between maternal prenatal use of prescription antibiotics and antifungals (any types) and the incidence of $\mathrm{AD}$ and $\mathrm{AS}$ in children 2 years of age stratified by mode of delivery.

Method: Data are from a birth cohort study called WHEALS based in the Detroit, Michigan, USA area. Women were recruited during their last trimester from the obstetrics clinics of Henry Ford Health System. Prenatal medical charts were reviewed for all prescription medications. The child underwent a study clinic visit at age 2 years to have a physician assess whether the child ever had AD. Blood was also collected to measure specific $\operatorname{IgE}(\mathrm{sIgE})$ to 10 allergens: dust mite, dog, cat, ragweed, Alternaria, egg, milk, peanut and cockroach. A child with at least $1 \mathrm{sIgE}>0.35 \mathrm{IU} / \mathrm{mL}$ was considered to have AS. Mantel-Haenszel chi-square tests were used to assess associations.

Results: The results from 331 children are included (128/ 331, 38.7\% were c-sections). Among the mothers, $16.9 \%$ used an antifungal and $57.1 \%$ used some type of antibiotic during pregnancy. Among children born vaginally, 15.6\% of children whose mothers took no antifungal medications in pregnancy and $26.7 \%$ of children whose mothers did take them had $\mathrm{AD}$. These rates were less than that of children born via c-section $(27.3 \%, \mathrm{MH} \mathrm{p}=0.04)$. The rates of AS were $48.2 \%$ among children born vaginally to mothers who took no antifungal medications in pregnancy, 53.6\% among children whose mothers did, and $59.0 \%$ among children born via-section $(\mathrm{MH} \mathrm{p}=0.10)$. Children born vaginally to mothers who did not use antibiotics prenatally had the lowest rates of $\mathrm{AD}$ (13.5\%) versus those born either vaginally (used prenatal antibiotics) or via c-section $(20.2 \%$ and $27.3 \%$, respectively, $\mathrm{MH} \mathrm{p}=0.01)$. Children born via c-section also had the highest rates of atopy $(59.0 \%)$ compared to children born vaginally to mothers who did not take prenatal antibiotics (44.7\%) and mothers who did take them (52.9\%) $(\mathrm{MH} \mathrm{p}=0.06)$.

Conclusion: Study of antibiotic and antifungal use during pregnancy with consideration of delivery type is worthy of continued investigation in investigations of allergic disease etiology. The results of this work further support examination of the prenatal mircobiome in the study of the child's subsequent health.

Disclosure of Interest: None Declared.

\section{DOHaD13-1317}

\section{Prenatal exposure to air pollution and newborn blood pressure}

Lenie Van Rossem ${ }^{1,2, *}$, Sheryl L. Rifas-Shiman ${ }^{3}$, Steven J. Melly ${ }^{2}$, Itai Kloog ${ }^{2}$, Heike Luttmann-Gibson ${ }^{2}$, Antonella Zanobetti ${ }^{2}$, Brent Coull ${ }^{4}$, Joel Schwartz ${ }^{2}$, Murray Mittleman ${ }^{2,5}$, Emily Oken ${ }^{3}$, Matthew W. Gillman ${ }^{3,6}$, Petros Koutrakis ${ }^{2}$, Diane R. Gold ${ }^{2,7}$

${ }^{1}$ Julius Center for Health Sciences and Primary Care, University Medical Center Utrecht, Utrecht, Netherlands; ${ }^{2}$ Department of Environmental Health, Harvard School of Public Health; ${ }^{3}$ Obesity Prevention Program, Department of Population Medicine, Harvard Medical School and Harvard Pilgrim Health Care Institute; ${ }^{4}$ Department of Biostatistics, Harvard School of Public Health; ${ }^{5}$ Cardiovascular Epidemiology Research Unit, Department of Medicine, Beth Israel Deaconess Hospital and Harvard Medical School; ${ }^{6}$ Department of Nutrition, Harvard School of Public Health; ${ }^{7}$ Channing Laboratory, Department of Medicine, Brigham and Women's Hospital; and Harvard Medical School, Boston, United States

Background: To examine associations of antenatal exposure to ambient air pollution with newborn systolic blood pressure (BP).

Method: We studied 1,131 mother-infant pairs in Project Viva, a Boston-area pre-birth cohort. We calculated average exposures by trimester and during the 2 to 7, 14, 30, 60 and 90 days before birth for temporally-resolved particulate matter $(\mathrm{PM})_{2.5}$, black carbon $(\mathrm{BC}), \mathrm{NO}_{\mathrm{X}}, \mathrm{NO}_{2}$, and $\mathrm{O}_{3}$ measured at stationary monitoring sites, and for spatiotemporally-resolved estimates of $\mathrm{PM}_{2.5}$ and $\mathrm{BC}$ at the residence level. 
We measured newborn BP up to 5 times with an automated device. We used mixed effects models to examine associations between air pollutant exposures and BP, adjusting for measurement sequence; child's birth weight, ethnicity, and state during BP measurement; mother's age, socio-economic position, and $3^{\text {rd }}$ trimester $\mathrm{BP}$, and long-term time trend. Estimates represent differences in BP for the $75^{\text {th }}$ vs. $25^{\text {th }}$ percentile (IQR) of pollution.

Results: Mean (SD) systolic BP in newborns was 72.5 (9.0) mmHg. Higher mean $\mathrm{PM}_{2.5}, \mathrm{BC}, \mathrm{NO}_{\mathrm{X}}$ or $\mathrm{NO}_{2}$ exposures before birth were associated with higher newborn BP [e.g., $1.1 \mathrm{mmHg}$, (95\% CI: 0.2 to 2.0) per IQR increase in $\mathrm{PM}_{2.5}$ in the 90 days before birth and $7.9 \mathrm{mmHg}(95 \% \mathrm{CI}: 4.2$ to 11.5) per IQR of $\left.\mathrm{NO}_{\mathrm{X}}\right]$. In contrast, higher $\mathrm{O}_{3}$ in all trimesters was associated with lower newborn BP [e.g. $-6.8 \mathrm{mmHg}(95 \%$ CI: -10.4 to -3.2 ) for the 90 day period before birth]. In a multi-pollutant model including $\mathrm{O}_{3}$ and $\mathrm{NO}_{\mathrm{X}}$, higher $\mathrm{O}_{3}$ was still associated with lower newborn BP $(-2.5 \mathrm{mmHg}, 95 \% \mathrm{CI}$ : $-7.9,2.9)$ in the 90 days before birth and higher $\mathrm{NO}_{\mathrm{X}}$ was associated with higher newborn BP $(6.0 \mathrm{mmHg}, 95 \% \mathrm{CI}$ : 0.4, 11.6) in the 90 days before birth.

Conclusion: Higher exposures to $\mathrm{PM}_{2.5}, \mathrm{BC}, \mathrm{NO}_{2}$, and $\mathrm{NO}_{\mathrm{X}}$ in late pregnancy were associated with higher newborn $\mathrm{BP}$, but $\mathrm{O}_{3}$ exposure was associated with lower BP. The implications of these findings for BP in later childhood and adulthood are unknown.

Disclosure of Interest: None Declared.

\section{DOHaD13-1656}

Relationship between maternal clinic/home blood pressure during pregnancy and birth weight of newborns in normotensive women: the Boshi Study

Noriyuki Iwama ${ }^{1, *}$, Hirohito Metoki ${ }^{1}$, Takayoshi Ohkubo ${ }^{2}$, Mikiko Katagiri $^{3}$, Mami Ishikuro ${ }^{3}$, Taku Obara ${ }^{3}$, Katuyo Yagihashi ${ }^{4}$, Ryusuke Inoue ${ }^{3}$, Hidekazu Nishigori ${ }^{5}$, Takashi Sugiyama ${ }^{5}$, Junichi Sugawara ${ }^{3}$, Nobuo Yaegashi ${ }^{5}$, Kazuhiko Hoshi ${ }^{6}$, Masakuni Suzuki ${ }^{6}$, Shinichi Kuriyama ${ }^{3}$, Yutaka Imai ${ }^{3}$

${ }^{I}$ Tohoku University Graduate School of Medicine, Sendai;

${ }^{2}$ Teikyo University School of Medicine, Tokyo; ${ }^{3}$ Tohoku University,

Sendai; ${ }^{4}$ Suzuki Memorial Hospital, Iwanuma; ${ }^{5}$ Obstetrics

and Gynecology, Tohoku University, Sendai; ${ }^{6}$ Obstetrics and

Gynecology, Suzuki Memorial Hospital, Iwanuma, Japan

Background: Previous studies showed newborns with small for gestational age (SGA) or large for gestational age (LGA) were associated with future several diseases. Birth weight (BW) is influenced by maternal complication such as pregnancy-induced hypertension (PIH). Although several studies revealed that PIH was associated with SGA or LGA, some women without PIH deliver SGA or LGA newborns. No studies investigated the relationship between maternal clinic BP (CBP) during pregnancy and the risk of SGA or LGA in normotensive women. Home BP (HBP) is measured optionally in clinical field in addition to CBP. But the difference of the above relationship among CBP and HBP is unknown. Therefore, the aim of this study is to clarify them. Method: This study is a part of prospective cohort study which was conducted near Sendai, Miyagi prefecture in Japan. 579 pregnant women who didn't develop PIH were included. We evaluated $\mathrm{CBP}$ and $\mathrm{HBP}$ between 12 and 37 weeks gestation. CBP data were collected from medical record and women were asked to measure their HBP during pregnancy. Mean $\mathrm{CBP}$ and HBP during pregnancy were divided into tertiles. BWs of newborns were categorized into SGA, appropriate for gestational age (AGA) and LGA based on distribution of gestational age specific BW in Japanese. SGA and LGA were defined below 10\%tile and no less than $90 \%$ tile, respectively. AGA was defined between them.

Multivariate multinomial logistic regression analyses were applied to analyze the association between tertiles of CBP/ $\mathrm{HBP}$ and above categories with possible confounding factors. Two models were also analyzed by logistic regression analyses. (Model 1; Missing CBP/HBP were not compensated. Model 2; Missing $\mathrm{CBP} / \mathrm{HBP}$ were compensated using each regression equations which were calculated by marginal model.)

Results: The numbers of women were 34, 491 and 54 in SGA, AGA and LGA, respectively. There were no significant associations between SGA and CBP in model 1 and 2 with using multivariate multinomial logistic regression model. ORs for SGA tended to be higher as home SBP increased in both model, however, it was not significant statistically. ORs for SGA in the subjects with the highest home DBP was statistically significant (Model 1; OR 3.6, 95\%CI 1.4-9.4, Model 2; OR 3.9, 95\%CI 1.5-10.1). ORs of CBP and HBP were not statistically significant in both models about tertiles of CBP/HBP and LGA.

Conclusion: Although CBP was not associated with SGA and LGA significantly, high home DBP was related to SGA. "PIH-like" pathological status which can be detected by HBP might be present in women who delivered newborns with $S G A$, even if their CBP values were within normal range.

\section{References}

1. Ishikuro $\mathrm{M}$ et al. Blood pressure measured in the clinic and at home during pregnancy among nulliparous and multiparous women: the BOSHI study. Am J Hypertens. 2013 Jan;26(1):141-8

Disclosure of Interest: None Declared.

\section{DOHaD13-1544}

Relevance of precision in newborn weight in neonatal research Harshad Sane ${ }^{1, *}$, Harsha Chopra ${ }^{1}$, Monika Dayama ${ }^{1}$, Patsy Coakley ${ }^{2}$, Vanessa Cox ${ }^{2}$, Meera Gandhi ${ }^{1}$, Sirazul Sahariah ${ }^{1}$, Caroline Fall ${ }^{2}$, Ramesh Potdar ${ }^{1}$ 
${ }^{1}$ Centre for the Study of Social Change, Mumbai, India;

${ }^{2}$ Lifecourse Epidemiology Unit, Medical Research Council, University of Southampton, Southampton, United Kingdom

Background: Newborn weight is a proxy for intrauterine growth. The relevance of birth weight alters with the perspective of the user and the location. We have carried out a large randomised controlled trial of food-based micronutrient supplementation among women living in Mumbai slums (Mumbai Maternal Nutrition Project). The supplementary foods were provided for at least three months before, and throughout, pregnancy. A primary outcome was birth weight. We have compared birth weights recorded by our research team with routine birth weights recorded by hospital staff.

Hypothesis: Hospital-recorded birth weights are too imprecise to use for research.

Aim: To compare birth weights taken with a research objective and by a trained team (group 1) with weights of the same babies recorded by routine hospital staff (group 2).

Method: Out of 1976 live singleton newborns, we obtained both birth weights for 942 babies. In group 1, the weights were taken on a single weighing scale (SECA, to the nearest $10 \mathrm{~g}$ ) by specially trained research nurses over a period of 6 years. Group 2 babies were weighed by the hospital staff of a total of 35 hospitals (7 public hospitals and 28 private maternity homes). We collected group 2 birth weights from hospital registers or discharge papers.

Results: Of the 942 newborns, 499 were boys. The mean birth weight in group 1 was $2606 \mathrm{~g}$ and that in group 2 was $2746 \mathrm{~g}$. Although birth weights in the two groups were highly correlated $(\rho=0.840)$, the mean (SD) difference between the two was $140(229) \mathrm{g}(\mathrm{p}<0.001)$, a difference of approximately $6 \%$. The prevalence of low birth weight in group 1 was $37 \%$, compared with $21 \%$ in group 2; 145 babies were misclassified as normal weight according to the hospital birth weight. Histograms of birth weights in each group showed that in group 1, the bars between 2000-2400 g included from $40 \%-70 \%$ of babies, compared to $30 \%-40 \%$ for group 2. In group 2 there was extreme clumping of the data at 2500, 3000 and $3500 \mathrm{~g}$, while in group 1 the histogram showed a smooth bell-shaped distribution.

Conclusion: These results show that hospital-recorded birth weights lack precision, which leads to frequent misclassification of babies as low or normal birthweight. This could lead to inappropriate management and follow-up and render data unsuitable for research. The likely reasons for imprecision are that: 1) the approach taken to measurement of routine birth weights is too casual; 2) there is insufficient staff training or monitoring of the accuracy of measurements; 3 ) equipment is old and/or poorly maintained; and 4) clinical objectives do not require greater precision unless the baby is sick. The significance of accurate birth weights should be highlighted through education campaigns aimed at all stakeholders (mothers, midwives, obstetricians, paediatricians and public health authorities).
Acknowledgements: The project was funded by the Wellcome Trust and Medical Research Council, UK, ICICI Social Initiatives Group, Mumbai and Parthenon Trust, Switzerland.

Disclosure of Interest: None Declared.

\section{DOHaD13-1601}

\section{Use of a neural model for prediction of preterm birth}

Sâmara P. Costa ${ }^{1}$, Antonio Augusto M. da Silva ${ }^{1}$, Rosangela F. L. Batista ${ }^{1}$, Viviane C. Cardoso ${ }^{2}$, Marco A. Barbieri $^{2}$, Marcelo Z. Goldani ${ }^{3} *$, José Eduardo R. Rabelo ${ }^{1}$, Heloisa Bettiol ${ }^{2}$, Alcione M. dos Santos ${ }^{1}$

${ }^{1}$ Public Health, Federal University of Maranhão, Sao Luis; ${ }^{2}$ Pediatrics, Faculty of Medicine of Ribeirao Preto, University of Sao Paulo, Ribeirao Preto; ${ }^{3}$ Pediatrics, Faculty of Medicine, Federal University of Rio Grande do Sul, Porto Alegre, Brazil

Background: Preterm birth, defined as the one that occurs between 20 and 37 gestational weeks, is the most concerning perinatal issue due to its association with significant early life morbidity and mortality rates. Only one third of all preterm births are related to classical risk factors. Furthermore, the associations between socioeconomic and educational factors and other unidentified causes with preterm birth make its prevention a true challenge. Clinical assessment is often difficult due to the complexity of the phenomenon, the disorganization of data and information overload, as well as the lack of reliable and valid tools to measure and predict the risk of preterm birth. The objective of this study was to determine a neural model to identify preterm births in a sample of pregnant women from the city of Sao Luis, Maranhao, Brazil.

Method: We considered 68 cases (preterm delivery at $<37$ weeks of gestation) and 126 term controls. The data was obtained from a sample of pregnant women belonging to a birth cohort study named BRISA, recruited in public maternity hospitals of Sao Luis, and had their first obstetric ultrasound performed at less than 20 weeks of gestation for gestational age definition. Several artificial neural networks were trained to predict preterm birth, using explicative variables concerning socioeconomic conditions, lifestyle, reproductive characteristics and blood cytokine levels. Data set was divided into training and test set. The neural networks were developed using MATLAB 12.0. Neural network performance was evaluated by measuring the accuracy, sensitivity, specificity, predictive values and geometric mean in the test set.

Results: The neural network presented accuracy between $43 \%$ and $55 \%$, specificity between $32 \%$ and $54 \%$, and sensitivity between $46 \%$ and $64 \%$. The predictive negative value was $88 \%$ and the geometric mean showed consistency between the measurements; thus the proposed neural model was considered useful in detecting negative cases of preterm births. 
Conclusion: The neural network demonstrated difficulty in predicting preterm births. However, the high negative predictive value and the geometric mean showing balance between measures, suggest that the proposed neural model can be an useful tool in detecting negative cases. It can be used in combination with other methods, to prevent preterm births and rank the risks associated with this birth outcome.

Disclosure of Interest: None Declared.

\section{DOHaD13-1439}

Validity of recalled birthweight versus a gold standard measurement: systematic review and meta-analysis

Mark Zhang ${ }^{1}$, Susan Shenkin ${ }^{1}$, Supriya Mathur ${ }^{1}$, Rebecca M. Reynolds ${ }^{1, *}$

${ }^{1}$ University of Edinburgh, Edinburgh, United Kingdom

Background: Numerous studies have demonstrated an association between low birth weight and adverse health outcomes in child and adulthood. many of these studies have used self- or maternal recall of birth weight due to lack of availabiligy of official birth weight records but the validity of this method is not known. We aimed to carry out a systematic review and meta-analysis of studies testing the validity of recalled birth weight when compared with a 'gold standard' measurement.

Method: Studies were identified by a comprehensive search of online databases and reference lists of relevant papers. Inclusion criteria were studies exploring the question: Does individual recall of birth weight (either self or parental) correlate with the recorded birth weight? Publications not in English language or with unrepresentative populations eg specific mental or physical illnesses were excluded. Data were extracted by two independent reviewers and were pooled in a meta-analysis using a fixed effect model. Quality of each manuscript was scored.

Results: 777 records were reviewed from which 42 eligible studies with 21895 participants were identified. 29 studies (69\%) were from USA or Europe. 30 studies (71\%) reported maternal recall of birth weight. Time from birth date to recall ranged from 5 days to 80 years. Correlation coefficients between recalled and verified birth weight ranged from 0.64 to 1 . The mean differences (MD) of the recalled birth weight and verified birth weight were small, ranging from -1 to $+129 \mathrm{~g}$. The combined $\mathrm{MD}$ was $-1.75 \mathrm{~g}(95 \%$ CI: $-12.41-8.91 \mathrm{~g}$ ) and $79 \%$ of studies reported a MD within $50 \mathrm{~g}$ indicating very similar agreement between recalled and verified birth weight. There was no strong evidence for an effect of time elapsed since birth event or mode of recall (maternal vs. self) on the validity of recalled birth weight. Quality of manuscripts was generally good though 28 studies had small sample sizes $(<500$ subjects).
Conclusion: The excellent agreement between recalled and actual birth weight suggests recalled birth weight is suitable for use in similar epidemiological studies.

Disclosure of Interest: None Declared.

\section{DOHaD13-1417}

A genetic study of steroid resistance nephrotic syndrome: relationship between $-173 \mathrm{G}$ To $\mathrm{C}$ polymorphism of macrophage inhibitory factor gene and level of serum macrophage inhibitory factor in children

Oke Rina Ramayani ${ }^{1, *}$, Partini Pudjiastuti Trihono ${ }^{2}$, Nanan Sekarwana ${ }^{3}$, Ahmad Hamim Sadewa ${ }^{4}$, Aznan Lelo ${ }^{5}$

${ }^{1}$ Pediatric, Pediatric Department Sumatera Utara University, Medan; ${ }^{2}$ Pediatrics, Pediatrics Department Indonesia University, Jakarta; ${ }^{3}$ Pediatrics, Pediatrics Department Padjajaran University, Bandung; ${ }^{4}$ Biochemistry, Biochemistry Department Gadjah Mada University, Jogjakarta; ${ }^{5}$ Pharmacologic and Therapeutic, Pharmacologic and therapeutic department Sumatera Utara University, Medan, Indonesia

Background: There is no satisfactory explanation why some nephrotic syndrome (NS) patients respond to glucocorticoid and others do not. Polymorphism of the macrophage migration inhibitory factor (MIF) gene is one of the various genes that have been proved as risk factor to steroid resistance in many diseases. It has been demonstrated that MIF have counter-regulator activities of glucocorticoid. To examine whether frequency of $\mathrm{C}$ allele of polymorphism of MIF gene and level of serum MIF are higher in SRNS pediatric patients compared with those of SSNS and healthy children. In addition, to define whether there is any correlation among those variables.

Method: During a period of November 2011 to September 2012, one hundred twenty consecutive children consist of three groups (healthy children, steroid resistant and steroid sensitive nephrotic syndrome) were examined. Healthy children was defined by normally estimated glomerular filtration rate and spot urinary albumin creatinine ratio $<150 \mathrm{ug} / \mathrm{mg}$ creatinine. The SRNS was diagnosed in children had not responded to the usual doses of steroids within 4 weeks of initiating treatment. The SSNS patient was defined as remission after usual doses steroid. Patients and controls were excluded if they i). had transient or orthostatic proteinuria, ii).were in an unstable clinical condition. Genotype of $-173 \mathrm{G}$ to $\mathrm{C}$ polymorphism of MIF gene was determined with PCR RFLP methods. Serum MIF concentration were measured with sandwich ELISA. Data was analyzed using chi square test, Kruskall Wallis test and multivariate analysis. A p value less than 0.05 was considered as significantly different.

Results: The genotype frequencies of $-173 \mathrm{G}$ to $\mathrm{C}$ polymorhism of MIF gene were as follow: GG 35\%, 
GC 55\%, CC 10\% among SRNS, GG 70\%, GC 27.5\%, CC $2.5 \%$ among SSNS and GG 60\%, GC 32.5\%, CC $7.5 \%$ among healthy children ( $p=0.005$ after combined GC and $\mathrm{CC}$ into one cell). The frequency of $\mathrm{C}$ allele of this polymorphism was three times higher in SRNS compared with that of SSNS patients (OR 2.74; 95\% CI 1.13-6.84). The level of serum MIF was higher in SRNS (31.9; range $14.3-117.2 \mathrm{ng} / \mathrm{mL})$ compared with SSNS (25.7; range $10.4-64.8 \mathrm{ng} / \mathrm{mL}$ ) and healthy children $(27.4 ; 11.4-96 \mathrm{ng} / \mathrm{mL}$ ) (Kruskall Wallis $\mathrm{p}=0.04$ ). In manova multivariate analysis, polymorphism of MIF gene and serum MIF were interaction together among SRNS, SSNS and healthy children ( $\mathrm{p}$ interaction $=0.008)$.

Conclusion: The $\mathrm{C}$ allele of $-173 \mathrm{G}$ to $\mathrm{C}$ polymorphism of MIF gene is a risk factor for SRNS in this study. There is a correlation between polymorhism and level of MIF in SRNS, SSNS and healthy children

Key word: macrophage inhibitory factor; steroid resistance nephrotic syndrome; steroid sensitive nephrotic syndrome; polymorphism

Part of these study have been presented in Congress APFCB 2013 in Denpasar

Disclosure of Interest: None Declared.

\section{DOHaD13-1201}

\section{Association of FTO and MC4R with child adiposity in SGA, and gene environment interactions with activity and dietary pattern}

John M. D. Thompson ${ }^{1, *}$, Rinki Murphy², Angharad Morgan ${ }^{3,4}$, Rod Lea ${ }^{5}$, Clare Wall ${ }^{3}$, Karen Waldie ${ }^{6}$, Lynnette Ferguson ${ }^{3,4}$, Edwin Mitchell ${ }^{1}$

${ }^{1}$ Paediatrics: Child and Youth Health; ${ }^{2}$ Medicine; ${ }^{3}$ Discipline of Nutrition, University of Auckland; ${ }^{4}$ Nutirgenomics, Auckland, New Zealand; ${ }^{5}$ Genomics Research Centre, Griffith University, Gold coast, Australia; ' ${ }^{6}$ sychology, University of Auckland, Auckland, New Zealand

Background: Small for gestational age (SGA) is associated with increased risk of obesity and metabolic disorders later in life. We hypothesised that if those born SGA have underlying metabolic programming favouring energy conservation, then the inheritance of common obesity risk alleles would magnify their susceptibility for increased body fat as part of geneenvironment interactions

Method: A longitudinal study of 546 children, approximately half of whom had been born SGA. We tested 2 obesity related SNPs in FTO and MC4R in relation to PBF at 7 years of age, including interactions with SGA, dietary pattern scores and physical activity.

Results: The FTO SNP rs6499640 was found to be significantly related to PBF. The MC4R SNP rs17700633 was found to show a significant interaction with SGA status. Increased healthy dietary pattern scores were found to ameliorate the effects of FTO and MC4R risk genotypes, as well as vigorous physical activity on the impact of FTO.

Conclusion: FTO was confirmed to be related to PBF in this population. The impact of MC4R on increasing PBF was confined to those born AGA. The interactions between MC4R and FTO risk genotypes and dietary pattern score, as well as physical activity, need confirmation in other cohorts.

Disclosure of Interest: None Declared.

\section{DOHaD13-1637}

Cellular energy substrate balance in intrauterine life and developmental programming of birth phenotypes: role of mitochondrial DNA variation and haplogroups

Pathik Wadhwa $^{1, *}$, Dimitra Chalkia ${ }^{2}$, Olga Derbeneva ${ }^{2}$, Masha Lvova ${ }^{2}$, Lawrence Shimmin ${ }^{3}$, James Hixson ${ }^{3}$, Sonja Entringer ${ }^{4}$, Claudia Buss ${ }^{4}$, Hyagriv Simhan ${ }^{5}$, Douglas Wallace $^{2}$

${ }^{1}$ Psychiatry, University of California, Irvine, Irvine; ${ }^{2}$ Center for Mitochondrial and Epigenomic Medicine, The Children's Hospital of Philadelphia Research Institute, Philadelphia; ${ }^{3}$ Human Genetics Center, University of Texas Health Science Center, Huston; ${ }^{4}$ Pediatrics, University of California, Irvine, Orange; ${ }^{5}$ Obstetrics \& Gynecology, University of Pittsburgh, Pittsburgh, United States

Background: Energetic state-related cues represent among the most salient signals that the developing embryo and fetus relies upon to shape phenotypic specificity. It is well established that newborn birth phenotypes represent markers of intrauterine developmental conditions and predictors of subsequent health and disease risk related outcomes. Across adverse birth phenotypes preterm birth (PTB) represents the single most important global problem in maternal and child health. The fundamental role of mitochondria in cellular energetics and of mitochondrial genetic variation in key aspects of mitochondrial function, coupled with evidence that supports maternal and non-Mendelian inheritance of PTB and its association with race/ethnicity, led to the hypothesis examined in the current study that mtDNA haplogroups and variants or haplogroups contribute to the etiology of PTB.

Method: We examined the association of mtDNA haplogroups and variants with PTB in a representative, population-based cohort of 645 mother-child dyads from Southern California and Central Pennsylvania. Child mtDNA sequences were determined by automated Sanger Sequence, with interpretation and rectification by MITOMASTER. Mitochondrial haplogroups were organized by continental clustering. mtDNA haplogroups were correlated with key pregnancy and birth outcomes.

Results: Two major findings have emerged to date: First, among women harboring European mtDNAs, those that 
belong to $\mathrm{H}-\mathrm{HV}-\mathrm{V}$ lineage are at increased risk for PTB $(\mathrm{OR}=2.8, p=0.038)$, while those that harbor lineage $\mathrm{J}-\mathrm{T}$ $\mathrm{mtDNAs}$ are at decreased risk $(\mathrm{OR}=0.24, p=0.026)$. Second, women with African mtDNAs (macrohaplogroup L lineage) are at a markedly increased risk for reduced gestational length and fetal growth (birth weight) compared to women with European or Asian mtDNAs. This association does not appear to simply be a product of African "ethnicity" since a preliminary analysis suggests that women who harbor African mtDNAs but self-reported as "non-Black" still show an effect of the mtDNA lineage.

Conclusion: Mitochondrial haplogroups represent human population migration patterns over time that, in turn, reflect ancestral adaptations in terms of the balance between cellular energy generation and heat production to the context of geography (energy substrate, temperature). These data represent novel and, to the best of our knowledge, the first findings linking mitochondrial markers of cellular energy dynamics during intrauterine life with key birth phenotypes. The incorporation of mitochondrial genetics to the $\mathrm{DOHaD}$ paradigm offers a unique window for achieving a clearer understanding of energetic-state related adaptations with critical developmental and health implications.

Disclosure of Interest: None Declared.

\section{DOHaD13-1306}

Epigenetic memory of gestational diabetes in the infant

Hong Pan ${ }^{1}$, , Maggie Lim ${ }^{1}$, Mei Lyn Ong ${ }^{1}$, Clara Cheong ${ }^{1}$, Jun Hao Tan ${ }^{1}$, Kok Hian Tan ${ }^{1}$, Yap-Seng Chong ${ }^{2}$, Michael J. Meaney, ${ }^{3,4}$, Peter Gluckman ${ }^{4}$, Walter Stunkel ${ }^{4}$, Joanna Holbrook ${ }^{4}$

${ }^{1}$ Institute of Clinical Sciences (SICS), A*STAR, Singapore;

${ }^{2}$ Yong Loo Lin School of Medicine, National University of Singapore, Singapore; ${ }^{3}$ Douglas University Mental Health Institute, McGill University, Quebec, Canada; ${ }^{4}$ Singapore Institute of Clinical Sciences (SICS), $A^{*}$ STAR, Singapore, Singapore

Background: Environment in utero has been shown to modify the risk of adult-onset disease. Gestational diabetes mellitus (GDM) has a high incidence in Singapore and higher glucose levels in pregnant mothers, even in the absence of GDM, affects infant adiposity and later metabolic phenotype. We hypothesise that high glucose in the in utero environment modifies the fetal epigenome and that epigenetic mark is transmitted to later life to affect metabolism. Using umbilical cords collected in the GUSTO (Growing Up in Singapore Toward Healthy Outcomes) birth cohort, we measured genome-wide DNA methylation of infants born to gestationally diabetic mothers and controls.

Method: Pregnant women enrolled in the GUSTO birth cohort were subjected to oral glucose tolerance (OGTT) tests at 26 weeks pregnant. Infant umbilical cord methylomes were interrogated using the Infinium $450 \mathrm{~K}$ array which measured DNA methylation at $>450,000 \mathrm{CpG}$ sites. Candidate markers were validated in an expanded sample set using Sequenom Epityper.

Results: We surveyed the DNA methylomes of the umbilical cords of 24 Chinese neonates, born to mothers that had high two hour OGTT scores $(>7.8 \mathrm{mg} / \mathrm{dL})$ and 24 Chinese neonates born to mothers with low two hour OGTT scores $(\leq 6.0 \mathrm{mg} / \mathrm{dL})$. Cases and controls were matched for maternal BMI and age; and infant gender and gestational age. 1073 CpG probes had significant differences between the two groups (Wilcoxon test $\mathrm{p}<0.05$ ) and absolution methylation difference of more than $5 \%$. We were also able to identify 61 significantly differentially methylated regions (DMRs) which contained contiguous $\mathrm{CpGs}$ with differential methylation between groups. Gene category and pathway enrichment analysis showed enrichment for genes implicated in this screen to metabolic disorders. Candidate marks were validated across an expanded sample set and investigated for interaction of subject genotype.

Conclusion: Epigenetic differences can be discerned between babies born to hyperglycaemic compared to normative mothers. We suggest that these form part of the molecular memory of gestational diabetes that persists to infancy and beyond.

Disclosure of Interest: None Declared.

\section{DOHaD13-1450}

\section{Fetal genetic polymorphisms associated with birth weight and body mass index and intrauterine fetal growth restriction}

Fumihiro Sata ${ }^{1, *}$, Akiko Eto ${ }^{1}$, Yasuhiro Kanatani ${ }^{1}$, Atsuko Araki $^{2}$, Takahiko Mitsui ${ }^{2}$, Reiko Kishi ${ }^{2}$, Katsuya Nonomura ${ }^{2}$

${ }^{1}$ NATIONAL INSTITUTE OF PUBLIC HEALTH, Wako;

${ }^{2}$ Hokkaido University, Sapporo, Japan

Background: Intrauterine fetal growth restriction (IUGR) is a multifactorial disorder, and its etiology includes both environmental and genetic components. Recent life-course epidemiological studies have shown numbers of evidences of anthropometric parameters in newborns and infants linked to adult chronic diseases such as type 2 diabetes, hypertension, cardiovascular and kidney diseases. We aimed to investigate whether fetal genetic polymorphisms associated with birth weight and body mass index (BMI) affected fetal growth.

Method: We conducted a prospective cohort study of 213 mother-newborn pairs delivered singleton live births in Sapporo, Japan ${ }^{1}$. Forty-seven single nucleotide polymorphisms (SNPs), which were reported to be associated with birth weight and BMI in recent genome-wide association studies $(\mathrm{GWAS})^{2,3}$, were detected in Japanese newborns. Multiple logistic regression and linear regression models were used to 
evaluate the associations between fetal genotypes and IUGR, defined as birth weight $<10$ th percentile, and birth weight with adjustments for maternal age, parity, smoking status during pregnancy and infant gender.

Results: The odds ratio (OR) for the risk of IUGR, in fetal CT + CC genotype groups in rs6548238 near the TMEM18 gene in 2 p25.3 region were 5.9 (95\% confidence interval [CI], 1.4-23.8) compared to the TT genotype group. The estimated reductions in birth weight were $257 \mathrm{~g}$ (standard error $[\mathrm{SE}], 130 \mathrm{~g}$ ) for the AA genotype group in rs2331841 near the $M C 4 R$ gene in $18 \mathrm{q} 21.31$ region compared to the AG + GG genotype groups.

Conclusion: Our findings suggest that rs6548238 near the TMEM18 gene may be a susceptible marker to IUGR in a Japanese population.

\section{References}

1. Kishi et al. Cohort profile: the Hokkaido study on environment and children's health in Japan. Int J Epidemiol 2011;40:611-618.

2. Willer et al. Six new loci associated with body mass index highlight a neuronal influence on body weight regulation. Nat Genet 2009;41:25-34.

3. Okada et al. Common variants at CDKAL1 and KLF9 are associated with body mass index in east Asian populations. Nat Genet 2012;44:302-306.

Disclosure of Interest: None Declared.

\section{DOHaD13-1536}

\section{Genome-Wide association analysis suggests influence of glycan metabolic pathway on vitamin $B_{12}$ levels in Indian population}

Suraj S. Nongmaithem ${ }^{1, *}$, Charu V. Joglekar ${ }^{2}$, Smita Kulkarni ${ }^{2}$, Chittaranjan S. Yajnik ${ }^{2}$, Giriraj R. Chandak ${ }^{1}$

${ }^{1}$ CSIR-Centre for Cellular and Molecular Biology, Hyderabad;

${ }^{2}$ Diabetes Unit, KEM Hospital and Research Centre, Pune, India

Background: Vitamin $\mathrm{B}_{12}$ is an important cofactor of onecarbon metabolic (OCM) cycle which controls the key processes of life including DNA synthesis and epigenetic regulation. Observations from Pune Maternal Nutrition Study have established low $\mathrm{B}_{12}$ levels as the major determinant of hyperhomocysteinemia, which is important risk factor for fetal growth restriction, developmental defects, childhood insulin resistance, and cardiovascular disorders. Earlier candidate gene studies using common single nucleotide polymorphisms (SNPs) in OCM pathway failed to identify strong genetic determinants of $\mathrm{B}_{12}$ and homocysteine levels in Indians.

Method: We have performed genome-wide association study (GWAS) for circulating vitamin $\mathrm{B}_{12}$ levels in 1000 normal individuals with directly genotyped SNPs from Affymetrix
SNP 6.0 and imputation SNPs based on 1000 Genome data. Furthermore, pathway based analysis was used to identify possible biologically significant pathways that are enriched with genes associated with vitamin $B_{12}$ levels.

Results: We have identified several novel genetic loci that predict vitamin $\mathrm{B}_{12}$ levels in Indians. Although many of them do not reach GWAS significance $\left(\mathrm{p}<10^{-8}\right)$, they show strong biological relevance. We detected association of other SNPs that are in strong linkage disequilibrium with variants reported to be associated with vitamin $\mathrm{B}_{12}$ levels, suggesting some commonality in genetic contribution to $\mathrm{B}_{12}$ levels. Pathway-based analysis of genes or nearby genes of SNPs $\left(\mathrm{p}<10^{-3}\right)$ shows enrichment of glycan biosynthesis and metabolism and many gastrointestinal infections such as Vibrio cholerae, Helicobacter pylori.

Conclusion: Glycan metabolism plays a key role in hostmicrobiome interaction. Enrichment of genes participating in glycan metabolism and gastrointestinal infection pathway suggests a possible role of host microbiome interaction in regulation of $\mathrm{B}_{12}$ levels in Indians. Our observations provide a unique opportunity to understand the role of vitamin $B_{12}$ in developmental programming and future risk of health and metabolic diseases.

Disclosure of Interest: None Declared.

\section{DOHaD13-1170}

Ghrelin and obestatin levels with polymorphisms of the ghrelin/obestatin gene in small for gestational age infants Shulian Zhang ${ }^{1, *}$, Chao Chen ${ }^{1}$

\section{${ }^{1}$ Department of Neonatology, Children's Hospital of Fudan} University, Shanghai, China

Background: The preproghrelin (ghrelin/obestatin) gene encodes two peptides, ghrelin and obestatin, which have opposite effects on development and energy balance. We investigated the role of ghrelin and obestatin with polymorphisms of preproghrelin in small for gestational infants (SGA).

Method: Plasma ghrelin, obestatin and leptin were measured after birth in 150 newborn babies, including 30 term appropriate for gestational age (AGA), 30 term SGA, 30 term large for gestational age (LGA), 30 preterm AGA and 30 preterm SGA infants. The same factors were measured by ELISA in different AGA infants $(\leq 30,31-33,34-36$, 37-39, 40-42 weeks) and term AGA neonates at different days (D1, D7, D14, D21, D28). Three single nucleotide polymorphisms (SNPs) (Arg51Gln, Leu72Met, Gln90Leu) were measured by TaqMan ${ }^{\text {TM }}$ technology in 496 neonates admitted in 2010 including 150 neonates above.

Results: The levels of ghrelin, obestatin and leptin were low during neonatal period. There was no difference in ghrelin between any groups. Plasma obestatin was higher in term 
SGA compared with term LGA $(213.46 \pm 15.62$ vs $166.33 \pm 10.02, \mathrm{P}=0.014)$. The level of obestatin was lower in preterm SGA infants at D1 than at D7. Howerer, the level of leptin was higher in preterm SGA infants at D1 than D7 as well as between preterm AGA infants at D1 and D7. There were no significant changes of ghrelin, obestatin and leptin between different gestational age infants. The level of obestatin increased gradually during the neonatal period, in contrast the ghrelin/obestatin ratio and leptin levels decreased during the same period. The allele frequency of Leu72Met was significantly different between preterm SGA and preterm AGA. Otherwise, there was no significant difference in Arg51Gln, Leu72Met, Gln90Leu genotypes and allele frequency between term SGA and term AGA neonates as well as preterm SGA and preterm AGA neonates.

Conclusion: The level of ghrelin, obestatin and leptin were low after birth. There were opposite changes of ghrelin and leptin in neonates at different days, which suggested they may be involved in growth after birth. There were no ghrelin/ obestatin polymorphisms which might contribute to SGA infants. The role of all these factors on development need to be further investigated.

Disclosure of Interest: None Declared.

\section{DOHaD13-1609}

Increased SLC6A4 DNA methylation at birth is associated with an increased fat mass at $\mathbf{6}$ years

Karen Lillycrop ${ }^{1,1, *}$, AiLing Teh $^{2}$, Emma Garratt ${ }^{3}$, Rebecca Clarke-Harris ${ }^{3}$, Robert Murray ${ }^{3}$, Paula Costello ${ }^{3}$, Shelia Barton ${ }^{4}$, Graham Burdge ${ }^{3}$, Hazel Inskip ${ }^{4}$, Cyrus Cooper ${ }^{4}$, Joanna Holbrook ${ }^{2}$, Peter Gluckman ${ }^{5}$, Nicholas C. Harvey ${ }^{4}$, Mark Hanson ${ }^{3}$, Keith Godfrey ${ }^{4}$ and The Epigen Consortium

${ }^{1}$ Centre for Biological Sciences, University of Southampton, Southampton, United Kingdom; ${ }^{2}$ Singapore Institute for Clinical Sciences, $A^{*}$ Star, Singapore, Singapore; ${ }^{3}$ Human Development and Health Academic Unit; ${ }^{4} M R C$ Life Course Epidemiology Unit, University of Southampton, Southampton, United Kingdom; ${ }^{5}$ Liggins Institute, University of Auckland, Auckland, New Zealand

Background: Early life environment has been linked to longterm changes in neuropsychological development. The mechanism by which environmental cues in early life are transmitted to the fetus and the process by which different, stable phenotypes are induced may involve the altered epigenetic regulation of genes. However to date there is no direct evidence in humans that early life environment may affect later neuropsychological function through an epigenetic mechanism. To investigate the contribution of epigenetic processes to later neuropsychological function, we used a genome wide methylation assay (MBD-array) to identify methylation differences at birth associated with neuropsychological function at age 4 years.

Method: Genomic DNA from umbilical cord samples was obtained from 24 children from the Southampton Women's Survey (SWS) and analysed using a MBD array. Their Wechsler Preschool and Primary Scale of Intelligence (WPPSI) scores at age 4 years were grouped into 4 categories with group 1 having the lowest WPPSI scores and group 4 having the highest. Absolute methylation levels were estimated using the Bayesian algorithm BATMAN. Pathway analysis was performed to search for known interactions between the genes containing differentially methylated regions of interest (DMROIs). Sodium bisulfite pyrosequencing was used to validate the DMROIs on a further 176 samples from the SWS cohort.

Results: The bioinformatics analysis identified 110 DMRs and 38 DMROIs. Pathway enrichment analyses found that the top pathway enriched amongst the DMROIs in the GO process category were diencephalon development (4/59 genes, $\mathrm{p}=0.000033$ ). Differential methylated CpGs which were contained in the diencephalon development pathway were selected for further validation. The sodium bisulfite pyrosequencing validation confirmed that umbilical cord methylation of specific CpGs at birth was associated with later cognitive function. Functional validation of the $\mathrm{CpG}$ loci associated with later cognitive performance is ongoing.

Conclusion: This study shows that methylation of specific genes at birth are associated with later cognitive outcomes providing novel evidence regarding the importance of the developmental contribution to later cognitive function. Moreover such epigenetic marks at birth may have prognostic value and could be used for monitoring programmes to optimize maternal health and nutrition for long-term benefits to the offspring.

This work was supported by funding from the Medical Research Council, British Heart Foundation, NIHR Southampton Biomedical Research Centre, University of Southampton and University Hospital Southampton NHS Foundation Trust, UK Foods Standards Agency and Abbott Nutrition.

Disclosure of Interest: None Declared.

\section{DOHaD13-1308}

Inter-individual differences in the methylome of neonates: what proportion is driven by in utero environment?

Ai Ling Teh ${ }^{1}$, Hong Pan ${ }^{1}$, Li Chen ${ }^{1}$, Mei Lyn Ong ${ }^{1}$, Johnny Wong ${ }^{1}$, Sarah $\mathrm{Mah}^{2}$, Yap-Seng Chong ${ }^{4}$, Kenneth Kwek ${ }^{3}$, Clara Cheong ${ }^{1}$, Walter Stunkel ${ }^{1}$, Michael Kobor ${ }^{5}$, Michael J. Meaney ${ }^{1,6}$, Peter Gluckman ${ }^{1}$, Joanna Holbrook ${ }^{1, *}$

${ }^{1}$ Singapore Institute of Clinical Sciences (SICS), $A^{*} S T A R$, Singapore, Singapore; ${ }^{2}$ Centre for Molecular Medicine and Therapeutics, University of British Columbia, British Columbia, 
Canada; ${ }^{3} K K$ Women's and Children's Hospital, Singapore; ${ }^{4}$ Yong Loo Lin School of Medicine, National University of Singapore, Singapore; ${ }^{5}$ Centre for Molecular Medicine and Therapeutics, University of British Columbia; ${ }^{6}$ Douglas University Mental Health Institute, McGill University, Quebec, Canada

Background: Environment in utero modifies the risk of adult-onset disease, a phenomenon dubbed 'fetal programming'. Since epigenetic marks can persist and thus affect disease risk in later life, in utero environmental effect on the epigenome is a putative molecular mechanism for fetal programming. DNA methylation at particular site, associates with measures of in utero environment and later phenotype. However, DNA methylation marks can be specified by sequence context. In humans, at least, some variation in DNA methylation is a consequence of nucleotide polymorphism, precluding an influence of environment. These polymorphisms are defined as methylation quantitative trait loci (methQTLs). However it is not clear if methQTLs account for all inter-individual variation or a minority. We surveyed the extent of inter-individual differences in genome-wide DNA methylation profiles at birth and determined the proportion that can be explained by genotype.

Method: We surveyed the DNA methylomes of the umbilical cords of 100 neonates using the Infinium $450 \mathrm{~K}$ array, which quantitatively measures DNA methylation at $>450,000$ CpG sites. We determined regions that contain more than two CpGs that are variable between individuals with a median absolute deviation score above the $95^{\text {th }}$ percentile. We also surveyed the DNA genotypes of the same 100 neonates using the Illumina omniexpress + exome array. Methylation values at each $\mathrm{CpG}$ were compared to every genotype assayed using logistic regression controlling for batch, gender and gestational age.

Results: 1741 variably methylated regions (VMRs) were determined across the 100 neonates. The most variable $\mathrm{CpG}$ in each VMR was chosen to represent the VMR. 15 VMRs are disrupted by a polymorphism. 59 (3\%) were correlated with $\mathrm{R}^{2}>0.9$ to a particular SNP genotype (not within the $\mathrm{CpG}$ ) and so designated genotype dependent. 58 of the genotype-dependent VMRs were in cis and one was in trans. $1076(62 \%)$ VMRs had no association with any of the genotypes assayed. 606 (35\%) VMRs showed some association with a genotype (usually in cis 561 vs 31), but were not fully explained by a single genotype, suggesting either a multifactorial effect of multiple SNPs or modification by environment.

Conclusion: Our results evidence that a proportion interindividual differences in methylation are due to the effect of genotype. As we only measure the one million SNPs present on the arrays, we probably under-estimate the proportion of genotype-dependence. $62 \%$ of inter-individual differences are not driven by any of the genotypes assayed and so are likely to be a result of differential in utero environment. In addition, we found $35 \%$ of inter-individual differences are partially influenced by genotype, suggesting that environment mediated by genotype may determine the epigenetic state of the majority of neonate methylomes.

Disclosure of Interest: None Declared.

\section{DOHaD13-1603}

\section{Methylation of a $\mathrm{CpG}$ in the promoter region of the NOS3 gene is associated with pre-school wheeze in SWS children}

Shelia Barton ${ }^{1, *}$, Allan Shepherd ${ }^{2}$, Sherry Ngo ${ }^{2}$, ChandraKanth Bhoothpur ${ }^{2}$, Hazel Inskip ${ }^{1}$, Katherine Pike ${ }^{1}$, Emma Garratt $^{3}$, Tejal Bhatt ${ }^{3}$, Mark Hanson ${ }^{3}$, Peter Gluckman ${ }^{2}$, Cyrus Cooper ${ }^{1}$, Karen Lillycrop ${ }^{4}$, Keith Godfrey ${ }^{1}$ and The Epigen Consortium

${ }^{1}$ MRC Life Course Epidemiology Unit, University of Southampton, Southampton, United Kingdom; ${ }^{2}$ Liggins Institute, University of Auckland, Auckland, New Zealand; ${ }^{3}$ Human Development and Health Academic Unit; ${ }^{4}$ Centre for Biological Sciences, University of Southampton, Southampton, United Kingdom

Background: Asthma is a chronic inflammatory disease of the airways characterized by airway obstruction, epithelial damage and airway hyperresponsiveness. There is now increasing evidence from both human and animal studies that asthma and atopy may originate at least in part in utero, through the influence of early life nutrition on the epigenetic regulation of genes. Thus measurement of the methylation of CpGs within the promoter of NOS3, a key regulator of airway smooth muscle tone in human fetal tissues at birth, might be able to predict later asthma and allergy phenotypes. Method: The methylation of candidate genes selected on the basis of potential involvement in airway disease was determined using Sequenom Massarray mass-spectrometry in a study of $\sim 550$ Southampton Women's Survey (SWS) umbilical cord samples. Methylation of $5 \mathrm{CpGs}$ in the promoter region of the NOS3 gene was measured. Distributions of methylation values were investigated for consistency between different Sequenom runs using boxplots. CpGs were excluded if the difference between the $5^{\text {th }}$ and $95^{\text {th }}$ percentiles was less than $5 \%$ methylation. Pre-school wheeze in the SWS cohort was defined as wheezing at any of the following time points 6 months, 12 months, 2 or 3 years old. Associations between preschool wheeze and methylation percentages were investigated

Results: 2 CpGs were excluded from analysis as the difference between the $5^{\text {th }}$ and $95^{\text {th }}$ percentiles was less than $5 \%$ methylation. As the methylation measurements were not Normally distributed, non parametric (Mann-Whitney U) tests were used to test association with phenotypes. $1 \mathrm{CpG}$ in the promoter region of NOS3 showed statistically significant association with pre-school wheeze $(\mathrm{n}=551, \mathrm{p}=0.045)$. The median methylation in the pre-school wheeze group was $65 \%$, and $59.5 \%$. in the control group. 
Conclusion: These results suggest that higher methylation of the promoter region of NOS3 could contribute to the development of pre-school wheeze. Confirmation of this will require replication in a different cohort and validation in vitro. This work was supported by funding from the Medical Research Council, British Heart Foundation, Danone, NIHR Southampton Biomedical Research Centre, University of Southampton and University Hospital Southampton NHS Foundation Trust, and the National Research Centre for Growth and Development New Zealand

Disclosure of Interest: None Declared.

\section{DOHaD13-1655}

The RS13266634 type 2 diabetes-risk variant in SLC30A8 is associated with increased fetal growth: the fetal glucose hypothesis

Scott White ${ }^{1, *}$, Lawrie Beilin ${ }^{2}$, Trevor Mori ${ }^{2}$, Stephen Lye ${ }^{3}$, John Newnham ${ }^{1}$, Craig Pennell ${ }^{4}$

${ }^{1}$ School of Women's and Infants' Health; ${ }^{2}$ School of Medicine and Pharmacology, The University of Western Australia, Perth, Australia; ${ }^{3}$ Samuel Lunenfeld Research Institute, Toronto, Canada; ${ }^{4}$ School of Women's and Infant's Health, The

University of Western Australia, Perth, Australia

Background: Type 2 diabetes mellitus (T2DM) is associated with size at birth, with those born both small and large for gestational age at increased risk. Genetic variation in the insulin pathway is likely to contribute to this association by virtue of the dual roles of insulin as a fetal growth factor and in glucose homeostasis. The fetal insulin hypothesis suggests that genetically determined insulin resistance leads to reduced insulin-driven growth in the fetus and hyperglycaemia in the adult. The rs13266634 single nucleotide polymorphism (SNP) in SLC30A8 is associated with a $50 \%$ increased risk of T2DM. We aimed to investigate the associations of maternal and fetal rs13266634 genotype with fetal growth and adult glucose homeostasis.

Method: 1203 mother-fetus pairs of the Western Australian Pregnancy Cohort (Raine) Study were followed from 18 weeks gestation to early adulthood. Serial ultrasound assessments of fetal growth were performed. Fetal abdominal circumference (AC) was assessed as the best descriptor of fetal growth and adiposity. Offspring fasting glucose and insulin were measured at age 17 years. Maternal and fetal DNA was extracted at either the 14- or 17-year follow up assessment and genotype for the rs13266634 SNP was obtained. Genetic associations were sought using multivariate linear regression for adult fasting glucose, insulin, and homeostasis model assessment of insulin resistance (HOMA-IR), and using linear mixed effects models for fetal AC trajectory. Institutional ethics approval and participant informed consent were obtained.

Results: Adult glucose homeostasis parameters were associated with rs13266634 genotype, with risk allele carriers having increased fasting glucose $(p=0.032)$, decreased fasting insulin $(p<0.001)$, and decreased HOMA-IR $(p=0.004)$. These associations are in keeping with the known functional implications of this SNP. Fetal abdominal growth was associated with maternal and fetal rs13266634 genotype, with both maternal and fetal genotype contributing to accelerated fetal growth in risk-allele carriers. Compared to riskallele non-carrier pairs (AC at 38 weeks $333 \mathrm{~mm}, 45^{\text {th }}$ percentile), risk-allele carrier pairs had increased AC $(343 \mathrm{~mm}$, $73^{\text {rd }}$ percentile, $\left.\mathrm{p}=0.008\right)$, as did risk-allele non-carrier fetuses of carrier mothers $\left(346 \mathrm{~mm}, 79^{\text {th }}\right.$ percentile, $\left.p=0.005\right)$. There was a trend to increased AC in risk-allele carrier fetuses of noncarrier mothers $\left(340 \mathrm{~mm}, 66^{\text {th }}\right.$ percentile, $\left.\mathrm{p}=0.090\right)$.

Conclusion: The rs13266634 T2DM risk-allele is associated with accelerated fetal abdominal growth. This is in contrast to the fetal insulin hypothesis. We propose the fetal glucose hypothesis to describe the pathophysiology underlying this association, in which accelerated fetal growth occurs in response to insulin hypersensitivity (as described by decreased HOMA-IR in adulthood) in a fetus who has an inadequate insulin response to a transplacental glucose load (decreased insulin) and therefore becomes hyperglycaemic (increased glucose). Where the fetal insulin hypothesis may explain part of the association between fetal growth restriction and T2DM at one end of the birth weight spectrum, the fetal glucose hypothesis may explain part of the association between fetal macrosomia and T2DM at the other.

Disclosure of Interest: None Declared.

\section{DOHaD13-1253}

Two thirds of the genetic variants associated with fetal growth trajectories have established and validated associations with obesity and the metabolic syndrome

Craig E. Pennell ${ }^{1, *}$, Qi W. Ang ${ }^{1}$, Julie A. Marsh ${ }^{2}$, Scott W. White $^{1}$, Laurent Briollais ${ }^{3}$, Melanie K. White ${ }^{1}$, Lawrence Beilin $^{4}$, John P. Newnham ${ }^{1}$, Stephen J. Lye

${ }^{1}$ School of Women's and Infants' Health; ${ }^{2}$ School of Population Health, The University of Western Australia, Perth, Australia;

${ }^{3}$ Samuel Lunenfeld Research Institute, Mount Sinai Hospital, Toronto, Canada; ${ }^{4}$ School of Medicine and Pharmacology, The University of Western Australia, Perth, Australia; ${ }^{5}$ Samuel Lunenfeld Research Institute, Mount Sinai Hospital, University of Toronto, Toronto, Canada

Background: The regulation of fetal growth is complex, and influenced by genetics (maternal and fetal genome) and the environment. To date, no genome-wide association study (GWAS) has been performed on longitudinal fetal biometry. Aims: The aims of this study were to (i) perform the first GWAS investigating the relationships between single nucleotide polymorphisms (SNPs) and antenatal ultrasound biometry [abdominal circumference (AC), head circumference $(\mathrm{HC})$ 
and femur length (FL) utilising longitudinal analyses] and (ii) evaluate the longterm associations of these SNPs.

Method: A GWAS was performed on AC, HC and FL measures recorded at multiple time points from 18-38 weeks gestation, in the Western Australian Pregnancy (Raine) Cohort $(\mathrm{n}=2900)$. Nonlinear mixed-effects models were utilized to model the longitudinal data with suitable correlation structure. Covariates included maternal smoking, fetal sex, gestational age and the $1^{\text {st }}$ two principal components (fixed effects); gestational age was included in the random effects. Summary statistics were calculated at 20 and 30 weeks gestation; p-values were based on log-likelihood ratio tests. SNPs with a p-value of $<9.9 \times 10^{-6}$ will be taken to replication in Generation $\mathrm{R}$, the Infancia y Medio Ambiente [INMA] and Southampton Women's Study [SWS] cohorts. Meta-analysis will be based on statistics extracted at 20 and 30 weeks gestation from the participating populations.

Results: Multiple SNPs $(n=139)$ in 48 genetic regions spread over 17 chromosomes were associated with HC, AC and FL growth trajectories.

The 139 identified SNPs were distributed across 48 genetic regions in 17 chromosomes. Of these 48 regions, there were 3 that overlapped: 2 between FL and HC; and 1 between AC and FL. Only one specific SNP (rs10762562) located in PRKG1 was associated with multiple biometric markers.

Of the 48 genetic regions identified from the antenatal growth GWAS, 30 (63\%) have established associations with metabolic diseases (i.e. obesity, blood pressure, ischaemic heart disease, diabetes, fasting insulin, HOMA, lipoproteins etc.) in large population studies validated in multiple independent populations. This included $11 / 18$ regions associated with $\mathrm{AC}, 8 / 16$ with $\mathrm{FL}$ and $11 / 14$ with $\mathrm{HC}$.

\begin{tabular}{lccccc}
\hline Gestational \\
Week & Biometry & $\begin{array}{c}\text { Number } \\
\text { SNPs (n) } \\
(\mathrm{p}<9.9 \times \\
10-6)\end{array}$ & $\begin{array}{c}\text { Number } \\
\text { Independent } \\
\text { Regions (n) }\end{array}$ & $\begin{array}{c}\text { Beta } \\
\text { Range }\end{array}$ & p-Value Range \\
\hline 20 & HC & 13 & 5 & $-7.55-2.43$ & $3.33 \times 10-6-9.24 \times 10-6$ \\
30 & HC & 22 & 6 & $-3.59-12.65$ & $3.61 \times 10-6-9.76 \times 10-6$ \\
20 & AC & 9 & 6 & $-3.29-12.34$ & $2.12 \times 10-6-9.87 \times 10-6$ \\
30 & AC & 62 & 9 & $-3.66-13.91$ & $1.55 \times 10-6-9.68 \times 10-6$ \\
20 & FL & 14 & 6 & $-0.46-0.53$ & $5.00 \times 10-7-9.38 \times 10-6$ \\
30 & FL & 19 & 11 & $-2.49-1.26$ & $2.05 \times 10-7-8.35 \times 10-6$ \\
\hline
\end{tabular}

Conclusion: These data suggest that genetic influences on fetal growth may be different for each biometric measure. The regions identified are novel and have not previously been associated with fetal growth, but have been associated with adult morphology and non-communicable diseases. These data support a significant role of genetics in the relationship between fetal growth and adult disease. Validated signals will be further explored in maternal DNA.

Disclosure of Interest: None Declared.

\section{DOHaD13-1672}

Adherence to the maternal one-carbon-rich dietary pattern is associated with birth weight and DNA methylation of leptin in very young children

Jennifer J.F.M. Verheijden-Paulissen ${ }^{1, *}$, Willemijn M. Meijer ${ }^{1}$, Paul H.C. Eilers ${ }^{2}$, Jeanne H.M. de Vries ${ }^{3}$, P. Eline Slagboom ${ }^{4,5}$, Bastiaan T. Heijmans ${ }^{4,5}$, Régine P.M. Steegers-Theunissen ${ }^{1,6}$

${ }^{1}$ Obstetrics and Gynaecology; ${ }^{2}$ Biostatistics, Erasmus MC, Rotterdam; ${ }^{3}$ Human Nutrition, Wageningen University and Research Centre, Wageningen; ${ }^{4}$ Molecular Epidemiology, Leiden University Medical Centre; ${ }^{5}$ Netherlands Consortium for Healthy Ageing, Leiden;

${ }^{6}$ Clinical Genetics, Erasmus MC, Rotterdam, Netherlands

Background: Maternal dietary intake and folic acid use affect embryonic and fetal development and birth weight. Periconceptional folic acid use increases the methylation of the insulin-like growth factor 2 gene differentially methylated region (IGF2 $D M R$ ), which is also inversely associated with birth weight. Dietary patterns are rather constant throughout life. We investigated whether adherence to a specific dietary pattern by the mother affects birth weight through modification of DNA methylation of the fetal growth genes IGF2 DMR and leptin (LEP).

Method: In 120 mothers we assessed the habitual dietary patterns from food frequency data using principal component analysis at the standardized study moment of 17 months after birth (SD 3 months) of the index-child. At the same moment also blood samples were obtained from the child for the determination of methylation of the IGF2 DMR (4 CpGs) and $L E P$ (7 CPGs) loci. The average methylation and the individual $\mathrm{CpG}$ sites per locus were measured using a mass spectrometry-based method.

Results: First we identified a maternal One Carbon-rich dietary pattern of which the adherence was strongly associated with a higher birth weight $(\beta=0.230 ; \mathrm{P}=0.002)$ and a lower overall methylation of $L E P(\beta=-0.8 ; \mathrm{P}=0.026)$, in particular due to three out of $7 \mathrm{CpG}$ sites. A $4.4 \%$ increase of the methylation percentage of $L E P$ was associated with a 586 grams (1 SD) decrease in birth weight. No significant association was shown between this maternal dietary pattern and the overall methylation of IGF2 DMR.

Conclusion: This data suggests that the maternal One Carbon-rich dietary pattern is positively associated with birth weight. This association may be explained in part by epigenetic programming of $L E P$ thereby contributing to fetal and postnatal growth of very young children.

Disclosure of Interest: None Declared.

\section{DOHaD13-1557}

Association between hyponatremia in preterm infants during neonatal period and later elevation of blood pressure in childhood

Takayuki Kaneko ${ }^{1 *}$, Masaki Wada ${ }^{1}$, Akihiko Saitoh ${ }^{1}$, Satoshi Kusuda ${ }^{2}$ 
${ }^{1}$ Pediatrics, Niigata University School of Medicine, Niigata;

${ }^{2}$ Maternal and Perinatal Center, Tokyo Women's Medical

University, Tokyo, Japan

Background: Preterm infants are known to have a risk of developing metabolic syndrome such as hypertension, obesity, and diabetes in later life. Nutritional and metabolic conditions before and after birth seem to be strongly related to the development of the diseases. In contrast, preterm infants are also known to have a high incidence of severe hyponatremia during neonatal period, which is mainly caused by sodium loss from renal tubules and deficit in sodium supplement. Sodium balance during neonatal period might have a prolonged effect on circulation and water balance. Thus, we hypothesized that the sodium imbalance in preterm infants during neonatal period has been continuously affecting the electrolyte homeostasis, and may cause hypertension in later life.

Method: Subjects were 51 very low birth weight (VLBW) infants admitted to the NICU at Niigata University Medical \& Dental Hospital. They have been followed at the outpatient clinic and currently aged from 3 to 6 years. Extensive perinatal and neonatal data, including gestational age, birth weight, gender, and a clinical course of neonatal period were collected from the medical records. A lowest sodium concentration in serum during neonatal period (low $\mathrm{Na}$ ) or, a maximum daily sodium supplement $(\max \mathrm{Na})$ to maintain sodium balance within normal range was used as an indicator for the sodium deprivation. We defined the sodium deprivation (SD) group as low $\mathrm{Na}<130 \mathrm{mEq} / \mathrm{l}$ or $\max$ $\mathrm{Na}>4.0 \mathrm{mEq} / \mathrm{kg} /$ day. Blood pressure (BP) of the infants was measured at the outpatient clinic. The relationship between sodium deprivation in neonatal period and BP in childhood was evaluated.

Results: Among the 51 VLBW infants (51\%female), the median birth weight was 974 (IQR: 812-1120) $\mathrm{g}$ and the median gestational age was 29 (IQR: 26-30) weeks. The median low $\mathrm{Na}$ was 131 (IQR: 128-133) $\mathrm{mEq} / \mathrm{l}$, and the median $\max \mathrm{Na}$ was 4.2 (IQR: $2.2-5.5$ ) $\mathrm{mEq} / \mathrm{kg} /$ day. The SD group showed higher BP than non-SD group at 3 to 5 year-old-age. At 3 years of age, the averages of systolic BP (sBP) between SD and non-SD group were 97.9 and $91.8 \mathrm{mmHg}$, respectively $(\mathrm{p}=0.214)$, and the averages of diastolic BP (dBP) between SD and non-SD group were 60.7 and $52.0 \mathrm{mmHg}$, respectively $(\mathrm{p}=0.06)$. At 4 years of age, the averages of $s B P$ between $S D$ and non-SD group were 104.8 and $93.0 \mathrm{mmHg}$, respectively $(\mathrm{p}=0.09)$, and the averages of dBP between $\mathrm{SD}$ and non-SD group were 60.5 and $56.0 \mathrm{mmHg}$, respectively $(\mathrm{p}=0.383)$. At 5 years of age, the averages of $\mathrm{sBP}$ between $\mathrm{SD}$ and non-SD group were 92.5 and $81.4 \mathrm{mmHg}$, respectively $(\mathrm{p}=0.009)$, and the averages of $\mathrm{dBP}$ between $\mathrm{SD}$ and non-SD group were 51.5 and $48.6 \mathrm{mmHg}$, respectively $(\mathrm{p}=0.612$ ).

Conclusion: In this study, we found that the VLBW infants with sodium deprivation during neonatal period have a tendency towards having higher BP in childhood. These data suggest that the electrolyte regulation during neonatal period among VLBW infants continuously influenced the circulatory condition in childhood. This disturbance of sodium metabolism during neonatal period may be risk factors for hypertension in later life of childhood.

Disclosure of Interest: None Declared.

\section{DOHaD13-1708}

Association of imprinting status of IGF2 with the pathophysiology of intrauterine growth retardation rats

Meihui Zhang ${ }^{1}$, Xiuyun $Z^{1}{ }^{1}{ }^{1}$, Lihong Liao ${ }^{1}$, Xiaoping Luo ${ }^{1, *}$

${ }^{1}$ Pediatrics, Tongji Hospital, Tongji Medical College, Huazhong University of Science and Technology, Wuhan, China

Background: To explore the correlation of imprinting status of IGF2 and IUGR in rats. And to investigate the changes of IGF2 imprinting status during the catch-up growth.

Method: We established the IUGR and CG-IUGR rats models with maternal nutritional restriction during the whole pregnant period and reducing the litter size of IUGR offspring after birth. After the establishment of rat model of IUGR, placenta and liver tissues of rats in the control group and the IUGR group were collected. The mRNA levels of IGF2 in placenta and liver tissues were detected via Real-time PCR respectively.Then we extracted the genomic DNA from the tissues of placenta and liver of newborn, 4 weeks and 8 weeks rats. With bisulfite sequencing, pyrosequencing was used to test the DNA methylation level of the CTCF binding regions in imprinting control region IGF2/H19DMR.

Results: IGF2 mRNA expression of placenta, newborn liver and liver of 4 weeks rats dramatically declined in IUGR group compared with control group.The IGF2 mRNA expression in liver tissues of 8 weeks rats could not be detected.Our DNA methylation sequencing data showed that three of four complements of CTCF sites exhibited distinct alteration in the placenta,liver of 4 weeks and 8 weeks. The methylation levels of the CpG dinucleotides in CTCF1 and CTCF2 binding regions were declined in placenta of IUGR rats.And methylation levels of the $\mathrm{CpG}$ dinucleotides in CTCF1 and CTCF3 binding regions were declined significantly in fetal liver,4weeks liver and 8 weeks liver of IUGR rats. But the pyrosequencing showed no significant changes in methylation levels of the $\mathrm{CpG}$ dinucleotides in H19DMR CTCF4 binding region of IUGR groups.

Conclusion: The mRNA level of IGF2 was down-regulated in the IUGR model, which could play an important role in the pathogenesis of IUGR. And the down regulation is the result of the aberrant imprinting status of the imprinting 
control regionH19DMR. We conclude the expression level of IGF2 could be down regulated through aberrant imprinting status to mediate the development of IUGR.

Disclosure of Interest: None Declared.

\section{DOHaD13-1269}

Association of maternal and nutrient supply line factors with DNA methylation at the imprinted IGF2/H19 locus in multiple tissues of newborn twins.

Jeffrey M. Craig ${ }^{1,2, *}$, Yuk Jing Loke ${ }^{1,2}$, John Galati ${ }^{3}$, Richard Saffery ${ }^{1,2}$

${ }^{1}$ Murdoch Childrens Research Institute; ${ }^{2}$ Paediatrics, University of Melbourne; ${ }^{3}$ Cebu, Murdoch Childrens Research Institute,

Melbourne, Australia

Background: Epigenetic events are crucial for early development, and can be influenced by environmental factors, potentially programming the genome for later altered health outcomes. The insulin-like growth factor $2(I G F 2) / H 19$ locus is crucial for prenatal growth and the epigenetic state at this locus is environmentally labile. Recent studies have implicated maternal factors in the regulation of DNA methylation at this locus, although data are often conflicting in the direction and magnitude of effect. Most studies have focused on single or two tissues, and on single or two differentially-methylated regions (DMRs) regulating IGF2/ H19 expression.

Method: We investigated the relationship between multiple shared and non-shared gestational/maternal factors and DNA methylation at four IGF2/H19 DMRs in 5 newborn cell types from 67 pairs of monozygotic and 49 pairs of dizygotic twins. Data on maternal and non-shared supply line factors were collected during the second and third trimesters of pregnancy and DNA methylation was measured via mass spectrometry using Sequenom MassArray EpiTyper analysis.

Results: Our findings showed that the site of umbilical cord insertion into the placenta in monochorionic twins has the strongest positive association with methylation in all IGF2/ H19 DMRs and tissues $(\mathrm{p}<0.05)$. However, we also observed that the other maternal factors examined affect DMRs and tissues differently.

Conclusion: The consistently strong positive association between cord insertion and methylation in all DMRs and tissue examined suggest the importance of the quality of cord insertion on methylation. Also, evidence for tissue- and locusspecific effects were observed for the other maternal factors, emphasizing that responsiveness to environmental exposures in utero cannot be generalized across genes and tissues, and has implications for all epigenetic studies investigating the developmental origins of health and disease.

Disclosure of Interest: None Declared.

\section{DOHaD13-1390}

Characterization of epigenetic changes in muscle precursor cells during the early onset of insulin resistance

Wenqing Jean Lee ${ }^{1, *}$, Yonghui $\mathrm{Wu}^{1}$, Radiance Lim ${ }^{1}$, Hilary Chua ${ }^{1}$, Shaillay Dogra ${ }^{1}$, Ai Ling Teh ${ }^{1}$, Mei Lyn Ong ${ }^{2}$, Peter Gluckman ${ }^{1}$, E Shyong Tai ${ }^{3}$, Melvin K. Leow ${ }^{1,3}$, Yung Seng Lee ${ }^{1,3}$, Craig McFarlane ${ }^{1}$, Joanna Holbrook ${ }^{1}$, Ravi Kambudar ${ }^{1}$

${ }^{1}$ Singapore Institute of Clinical Sciences (SICS), A*STAR;

${ }^{2}$ Singapore Institute of Clinical Sceinces (SICS), A*STAR;

${ }^{3}$ Yong Loo Lin School of Medicine, National University of

Singapore, Singapore, Singapore

Background: Characterization of epigenetic changes in muscle precursor cells during the early onset of insulin resistance

Lee Wenqing Jean ${ }^{1, *}$, Wu Yonghui ${ }^{1, *}$, Radiance $\operatorname{Lim}^{1}$, Hilary Chua ${ }^{1}$, Shaillay Dogra ${ }^{1}$, Ai Ling Teh ${ }^{1}$, Mei-Lyn Ong ${ }^{1}$, Peter D Gluckman ${ }^{1}$, Tai E Shyong ${ }^{2}$, Melvin K S Leow ${ }^{1,2}$, Lee Yung Seng ${ }^{1,2}$, Craig McFarlane ${ }^{1}$, Joanna D Holbrook ${ }^{1}$, Ravi Kambadur ${ }^{1}$

*These authors contributed equally

${ }^{1}$ Singapore Institute of Clinical Sciences (SICS), $A^{*}$ STAR, Brenner Centre for Molecular Medicine, 30 Medical Drive, Singapore; ${ }^{2}$ Yong Loo Lin School of Medicine, National University of Singapore, National University Health System, Singapore

Background: Type 2 diabetes is one of the most prevalent diseases in the world. Evidence from observational and experimental studies links adverse exposures in early life to diabetes disease susceptibility in adulthood. We hypothesise that the molecular mechanism whereby early environment cues are transmitted to disease risk is epigenetic and therefore, there would be epigenetic marks present at the very early stages of the disease. Currently little is known about these early mechanisms, particularly in metabolically active tissues such as skeletal muscle. Skeletal muscle is a critical insulin target tissue that is highly involved in glucose disposal and homeostasis and hence influences the progression of diabetes. The Singapore Adult Metabolism Study (SAMS) has collected muscle biopsies from overweight insulin resistant men and matched lean controls. Human primary myoblast lines from these biopsies were established and were further shown to recapitulate the insulin sensitivity of their source individual. Therefore these primary cultures were used to identify epigenetic marks specifying insulin resistance. In this study we surveyed the transcriptome and DNA methylomes of the SAMS myocyte cultures to find marks associating with insulin resistance.

Method: RNA from the myoblast cultures of eight insulin sensitive (IS) and eight insulin resistant (IR) SAMS subjects were analyzed using Agilent ( 8 × $60 \mathrm{~K})$ human expression arrays. The DNA methylome from a subset of these 16 samples was further interrogated by both highly quantitative genome-wide 
Infinium $450 \mathrm{~K}$ analysis (6 IR vs 6 IS) and quantitative whole-genome MeDIP-seq analysis (5 IR vs 5 IS).

Results: Methylome profiles from both the Infinium and MeDIP-seq data showed high concordance. Marks correlated with both Homeostasis Model Assessment (HOMA) and Insulin sensitivity index (ISI) were detected in both the transcriptomics and methylome datasets. Genes perturbed at both the transcriptomics and epigenetic levels were prioritised. Pathway analysis revealed enrichment in pathways associated with metabolic disease and cell fate specification.

Conclusion: Epigenetic marks which have a consequence in the muscle transcriptome are present at the very early stages of diabetes disease progression. The marks may be a consequence of early life environment or later adiposity, predisposing individuals to type 2 diabetes.

Disclosure of Interest: None Declared.

\section{DOHaD13-1573}

Comparative methylome analysis of $B_{12}$ intervention shows enrichment of genes and pathways involved in obesity and type 2 diabetes

Dilip Kumar Yadav ${ }^{1, *}$, Charu V. Joglekar ${ }^{2}$, Hong Pan $^{3}$, Caroline H. Fall ${ }^{4}$, Karen A. Lillycrop ${ }^{5}$, Joanna D. Holbrook ${ }^{3}$, Chittaranjan S. Yajnik ${ }^{2}$, Giriraj R. Chandak ${ }^{1}$

${ }^{1}$ CSIR-Center For Cellular amd Molecular Biology, Hyderabad; ${ }^{2}$ Diabetes Unit, King Edward Memorial Hospital \& Research Centre, Pune, India; ${ }^{3}$ Singapore Institute of Clinical Sciences, Singapore, Singapore; ${ }^{4}$ MRC Life course Epidemiology Unit;

${ }^{5}$ Institute of Developmental Science, University of Southampton, Southampton, United Kingdom

Background: Pune Maternal Nutrition Study (PMNS) has provided strong evidence that maternal hyperhomocysteinemia is risk factor for low birth weight, higher adiposity and insulin resistance in their children; phenotypes that are intermediate traits for future risk of type 2 diabetes and cardiovascular disorders. We have also demonstrated that $\mathrm{B}_{12}$ intervention in PMNS children significantly increases $B_{12}$ levels with consequent reduction in homocysteine levels. Since $B_{12}$ is central to conversion of homocysteine to S-adenosyl methionine, which provides the methyl group for DNA methylation reactions, we investigated the effect of $\mathrm{B}_{12}$ and/or folate intervention on gene methylation.

Method: A double-blind randomised trial was conducted in PMNS children (mean age $=9$ years) to test three levels of $\mathrm{B}_{12}$ supplementation, (B0 $\mu \mathrm{g}, \mathrm{B} 2 \mu \mathrm{g}$ and $\mathrm{B} 10 \mu \mathrm{g}$ ) along with two levels of folic acid, (F0 $\mu \mathrm{g}$ and F200 $\mu \mathrm{g}$ ) thus forming six groups (B0F200, B0F0, B2F200, B2F0, B10F200 and $\mathrm{B} 10 \mathrm{~F} 0)$. We randomly selected 6 males and 6 females each (Pre- and post- intervention) from four groups (B0F200, B0F0, B10F200 and B10F0) and compared their methylome by Infinium HumanMethylation $450 \mathrm{~K}$ bead array. Differentially methylated loci were validated by pyrosequencing.

Results: We did not observe any significant differences in methylation levels in placebo (B0F0) or only folate intervened group (B0F200). However, over 1400 differentially methylated CpGs (DMCpGs, $\mathrm{p} \leq 4.8 \times 10^{-4}$, FDR $\leq$ 0.049 ) and over 50 differentially methylated regions (DMRs, $\mathrm{p} \leq 0.001, \mathrm{FDR} \leq 0.05)$ were identified in the group with only $\mathrm{B}_{12}$ intervention (B10F0). Gene ontology analysis revealed that the DMROIs were enriched for pathways associated with obesity, insulin resistance and type 2 diabetes. Differential methylation of $\mathrm{CpG}$ loci within FTO and TCF7L2, identified from the array was confirmed by pyrosequencing analysis. The analysis of other loci is in progress.

Conclusion: Preliminary findings of this study suggest a possible epigenetic role of one carbon metabolism in obesity and insulin resistance. These studies also give some understanding of the epigenetic mechanism of $\mathrm{B}_{12}$ action.

Disclosure of Interest: None Declared.

\section{DOHaD13-1710}

Differentially methylated genes associated with immune function in pregnant women colonized with group B streptococcus

Michelle L. Wright ${ }^{1, *}$, Cindy M. Anderson ${ }^{2}$, Jody L. Ralph ${ }^{1}$, Joyce E. Ohm ${ }^{3}$

${ }^{1}$ Nursing, University of North Dakota, Grand Forks; ${ }^{2}$ Nursing, The Ohio State University, Columbus; ${ }^{3}$ Biochemistry and Molecular Biology, University of North Dakota, Grand Forks, United States

Background: Group B streptococcus (GBS) is the leading cause of neonatal morbidity and mortality due to infection in the United States. Maternal GBS colonization is the primary risk factor associated with the development of neonatal GBS sepsis. Between 10-30\% of pregnant women are colonized with GBS and risk factors for maternal colonization are ambiguous and inconclusive. The significance of the problem surrounding maternal GBS colonization is compounded by the fact that current guidelines to prevent neonatal GBS do not prevent poor pregnancy outcomes associated with the bacteria prior to delivery and infants continue to be infected despite intrapartum antibiotic treatment. The objective of this study was to investigate the functional relevance of differential DNA methylation in maternal peripheral blood during early pregnancy that has may contribute to susceptibility for colonization with GBS.

Method: DNA methylation was quantified at approximately $450 \mathrm{~K}$ individual $\mathrm{CpG}$ dinucleotides in white blood cells from biobanked samples of 40 women collected early in pregnancy. A delta-beta criteria of $>0.2$ or $<-.02$ was used 
to determine a significant gain or loss in methylation, representing a $20 \%$ change respectively. Significance in mean methylation changes was determined at $\mathrm{p}<0.05$. To identify potential for clinically relevant findings, functional roles of differentially methylated genes were determined using Database for Annotation, Visualization and Integrated Discovery v6.7 (DAVID, http://david.abcc.ncifcrf.gov). Using a classification stringency of "high" to determine functional annotation clusters, enrichment scores were used to rank the importance $(\geq 1.3)$ of genes into functional groups.

Results: Among genes with $\mathrm{CpG}$ methylation loss, cluster 1 was associated with immune function and had an enrichment score of 3.96, containing the GO Terms of major histocompatibility protein complex, immunoglobulin/major histocompatibility and immune response. Methylation loss associated with immune function was also identified in examination of gene ontology and associated diseases. Of particular significance was the identification of methylation loss in the AGPAT1 gene, associated with regulation of cytokine production. Similarly, functional clustering of genes with CpG methylation gain was also associated with immune function (cluster 2, enrichment score 2.31), containing GO Terms of major histocompatibility II, intestinal immune network for IgA production and immune response, confirmed by gene ontology and associated disease conditions.

Conclusion: These data suggests that DNA methylation changes in genes associated with immune function and inflammation could be related to maternal serum levels of associated protein products contribute to GBS colonization susceptibility. Identification of these products allows for investigation into alternative targeted prevention methods. Addressing predisposition to GBS colonization early in pregnancy may prevent miscarriages, stillbirths, and neonatal sepsis associated with GBS.

Disclosure of Interest: None Declared.

\section{DOHaD13-1054}

DNA methylation, gene expression and plasma levels of leptin in young low birth weight men and controls, before and after a 36 hours fasting intervention.

Line Hjort $^{1, *}$, Sine W. Jørgsensen ${ }^{1}$, Charlotte Brøns ${ }^{1}$, Linn Gillberg ${ }^{1}$, Elin Hall ${ }^{2}$, Charlotte Ling ${ }^{2}$, Allan Vaag ${ }^{1}$

${ }^{1}$ Department of Endocrinology, Rigshospitalet, Copenhagen, Denmark; ${ }^{2}$ Department of Clinical Sciences Malmö, Lund University, Lund, Sweden

Background: Low birth weight (LBW) is associated with increased risk of Type 2 Diabetes (T2D), increased fat mass and differential expression of adipokines. Operating in the window between genetic and environmental factors, epigenetic mechanisms may play a role in regulating expression of adipokine genes involved in T2D, including leptin $(L E P)$. Knowing that fasting causes dramatic decrease in plasma leptin levels (p-leptin), we aimed to investigate if this is associated with a change in LEP transcription in the adipose tissue caused by changes in DNA methylation degree. Furthermore, we wanted to investigate if leptin expression was regulated differently in LBW and normal birth weight (NBW) individuals before and after a fasting challenge.

Method: 20 LBW men and 17 matched NBW controls were studied during a 36 hour fasting intervention. After 8-12 weeks, a subset of both groups $(n=6 \mathrm{NBW}, 7 \mathrm{LBW})$ participated in an overnight fasting control study with identical study settings. Body composition was studied with Dual-energy X-ray Absorptiometry (DXA) scanning. After 36 hours fasting or after the overnight control fast, subcutaneous adipose tissue biopsies were excised immediately before initiating an intra venous glucose tolerance test. P-leptin levels were measured after 12, 36, 39 and 42 hours. DNA/RNA was extracted from the adipose tissue biopsies and DNA methylation degree was studied at $39 \mathrm{CpG}$ sites in the proximal $L E P$ promoter region using the EpiTYPER MassArray approach. LEP mRNA expression was measured by real-time PCR.

Results: Average DNA methylation degree of the LEP promoter was after the overnight fasting control study found to be higher $(\sim 3 \%)$ in LBW compared to NBW subjects $(p<0.05)$. After 36 hours of fasting the average methylation degree of the $L E P$ promoter was increased ( 5\%) compared to the control study in NBW subjects only. No differences were found in adipose tissue LEP mRNA expression between the two birth weight groups in either the control or fasting study, neither did LEP mRNA expression change with fasting. When studying p-leptin levels during the fasting study, we found a higher overnight-fasting (after 12 hours) p-leptin level $(p=0.04)$ in LBW subjects compared to NBW subjects. After 36 hours of fasting p-leptin was decreased more than 3 -fold in both groups $(p<0.0001)$, but remained higher among the LBW subjects. When comparing p-leptin levels between the control study and fasting study at all four time points of p-leptin measurement, the changes in p-leptin were found to be larger among the NBW subjects, compared to the LBW subjects.

Conclusion: LBW subjects are found to have a higher baseline DNA methylation degree at proximal regions of the LEP promoter in adipose tissue. A short-time metabolic fasting challenge induces small but significant changes in $L E P$ promoter DNA methylation in NBW subjects only. LBW individuals are characterized with higher baseline p-leptin levels and NBW individuals are found more capable of regulating p-leptin during a fasting challenge than LBW individuals. No evidence was found to support the hypothesis that DNA methylation changes of the $L E P$ promoter regulate the drastic decrease in p-leptin during fasting.

Disclosure of Interest: None Declared. 


\section{DOHaD13-1396}

DNA methylation of SIRT1, the longevity gene, in blood from children at 5-7 years exhibits temporal stability and predicts adiposity in adolescence

Rebecca Clarke-Harris ${ }^{1}$, Joanne Hosking ${ }^{2}$, Terence J. Wilkin $^{3}$, Jonathan Pinkney ${ }^{2}$, Alison Jeffery ${ }^{2}$, Brad Metcalf ${ }^{2}$, Keith M. Godfrey ${ }^{4}$, Linda D. Voss ${ }^{2}$, Graham C. Burdge ${ }^{1, *}$, Karen A. Lillycrop ${ }^{1}$

${ }^{1}$ University of Southampton, Southampton; ${ }^{2}$ University of Plymouth, Plymouth; ${ }^{3}$ University of Exeter, Exeter; ${ }^{4} M R C$ Lifecourse Epidemiology Unit, University of Southampton, Southampton, United Kingdom

Background: It has been proposed that epigenetic processes may be an important mechanism linking early life environmental exposures with patterns of risk of non-communicable diseases in later life (1). Since epigenetic processes contribute to the aetiology of metabolic disease (1), epigenetic marks in proxy tissues collected in early life may have utility in predicting disease in later childhood and adolescence. Our preliminary findings show that the methylation status of specific $\mathrm{CpG}$ loci in the peroxisomal proliferator- $\gamma$-coactivator-1alpha (PCG1a) promoter in blood at age 5-7 years predicted later adiposity in the children age 14 years. PGC1a is a downstream effector of Sirtuin 1 (SIRT1), a gene which has been shown to control lifespan in a range of species and which is known to act as a major systemic metabolic sensor that directly links nutrient availability to both glucose and lipid metabolic homeostasis (2). We, therefore, analysed the DNA methylation status of the SIRT1 promoter in blood from 40 children (20 boys) collected annually between 5 and 14 years. Method: We used sodium bisulphite pyrosequencing to analyse a region of the SIRT1 promoter between -668 and -880 bp $5^{\prime}$ to the transcription start site that contains $7 \mathrm{CpG}$ loci and encompasses regulatory transcription factor response elements. Methylation values were averaged between 5-7 years to produce complete data sets. There were no significant associations between SIRT1 methylation and leukocyte populations (all $\mathrm{P}>0.1$ ). Body composition was measured annually by dual X-ray absorptiometry and physical activity by accelerometry. Mixed effects modelling was used to assess the association between methylation at 5-7 years and adiposity (\% body fat) between 9-14 years, taking into account age, sex and physical activity.

Results: Methylation of individual CpG dinucleotides in the SIRT1 promoter differed between loci from $13.2 \pm 3.5 \%$ to $64.2 \pm 6.8 \%$. The methylation status of these loci was stable between 5-7 and 14 years (year on year associations, $r=0.58$ to $0.96, \mathrm{P}<0.001)$. Methylation of two $\mathrm{CpG}$ loci at 5-7 years was associated inversely with future adiposity. For each $10 \%$ difference in methylation at 5-7 years, adiposity differed by $3.0 \%$ (95\% CI: 6.0 to 1.0 ) at CpG -880 and $4.8 \%$ (8. 2 to 1.4 ) at $\mathrm{CpG}-865$ such that methylation of these loci at 5-7 years predicted up to $8 \%$ and $12 \%$ of the variation in adiposity at 14 years, respectively.
Conclusion: Together these findings suggest that, at least for some loci, methylation patterns that are established by early childhood and which are not subsequently influenced by later environmental factors are consistent with the paradigm that epigenetics is a causal process that links the early life environment to the future phenotype. These data also suggest that developmentally-induced epigenetic modulation of SIRT1 and of its downstream effectors influences adiposity in childhood and adolescence.

\section{References}

1. Gluckman PD et al. N Engl J Med 2008;359:61.

2. Leibiger IB \& Berggren PO Nature Medicine - 12, 34-36 (2006)

Disclosure of Interest: None Declared.

\section{DOHaD13-1618}

Early environmental toxicant exposure leads to mesenchymal stem cell epigenomic remodeling

Joyce Ohm $^{1, *}$, Derek Hovick ${ }^{1}$, Anastasia Lindahl ${ }^{2}$, Dedrick $\operatorname{Dan}^{2}$

${ }^{1}$ School of Medicine; ${ }^{2}$ University of North Dakota, Grand Forks, United States

Background: Epidemiological studies have long suggested a critical, but poorly understood, link between toxic environmental exposures early in development and the occurence of human disease later in life. Environmental toxins may disrupt epigenetic regulation of gene expression in cells via the induction of alterations in both DNA methylation patterns and chromatin structure. Our ongoing research objective is to determine whether is exposure to common environmental contaminants during development or during key windows of susceptibility (WOS) may induce abnormal DNA methylation changes in differentiating stem cells and to identify the molecular mechanisms responsible.

Method: Gene expression analysis and global methylation quantification of individual $\mathrm{CpG}$ dinucleotides in mesenchymal stem cell (MSC) before and after exposure to the environmental toxicant paraquat was performed using the Illumina Infinium $450 \mathrm{~K}$ methylation arrays and compared to gene expression data for key regulatory proteins.

Results: We show that exposure of MSC to paraquat alters the expression of key stem cell and epigenetic regulatory proteins including the DNA methyltransferases, several members of the jumonji family of histone/DNA demethylases, and bromodomain proteins such as BRD4 and induces abnormal DNA methylation patterns during cellular differentation in vitro.

Conclusion: Our data implicate environmental toxin exposure in the deregulation of stem cell signaling pathways and epigenomic remodeling. This potential link between environmental stress and epigenetic changes in a cell has 
direct implications for a variety of human diseases including cancer, infertility, and neurodegenerative disorders.

Disclosure of Interest: None Declared.

\section{DOHaD13-1434}

Epigenetic changes of CDKN2A/2B in peripheral blood mononuclear cells for type 2 diabetes

Ju Luan ${ }^{1,2, *}$, Ying Wang ${ }^{1}$, Heung Man Lee ${ }^{1,2}$, Zhibo Gao ${ }^{3}$, Xin Liu ${ }^{3}$, Vincent, K.L Lam ${ }^{1}$, Kevin Yip ${ }^{4}$, Ting Fung Chan ${ }^{5}$, Wing Hung Tam ${ }^{6}$, Wing Yee So ${ }^{1,2}$, Keith M. Godfrey ${ }^{7}$, Mark A. Hanson ${ }^{8}$, Juliana C.N. Chan ${ }^{1,2,9}$, Ronald C.W. Ma ${ }^{1,2,9}$

${ }^{1}$ Department of Medicine and Therapeutics; ${ }^{2} \mathrm{Li} \mathrm{Ka}$ Shing Institute of Health Sciences, Chinese University of Hong Kong, Hong Kong; ${ }^{3}$ BGI-Shenzhen, Shen Zhen; ${ }^{4}$ Department of Computer Science and Engineering; ${ }^{5}$ School of Life Sciences; ${ }^{6}$ Department of Obstetrics and Gynaecology, Chinese University of Hong Kong, Hong Kong, China; ${ }^{7}$ MRC Lifecourse Epidemiology Unit and NIHR Southampton Biomedical Research Centre, University of Southampton and University Hospital Southampton NHS Foundation Trust; ${ }^{8}$ Institute of Developmental Sciences, University of Southampton, Southampton, United Kingdom; ${ }^{9}$ Hong Kong Institute of Diabetes and Obesity, Chinese University of Hong Kong, Hong Kong, China

Background: In order to discover genetic and epigenetic changes associated with maternal diabetes, we have applied a multi-omic approach in a family trio with maternal diabetes utilizing next generation sequencing technology. CDKN2A/2B, two T2D susceptibility loci, were confirmed and CDKN2A expression levels were decreased in T2D subjects compared to controls. In the present study, our aims were to 1) evaluate the use of peripheral blood mononuclear cells (PBMCs) for validating epigenetic biomarkers; and 2) set up a highthroughput platform to perform methylation analysis for differential methylation regions (DMRs) identified in this multi-omic study.

Method: We recruited 65 subjects with T2D and 65 gender and age-matched controls for the validation study. PBMCs were isolated from whole blood of participants at their first visit and genomic DNA was extracted. Methylation of DMRs identified in CDKN2A intron 1(chr9: 21964356-21963957, hg18) and in CDKN2B promoter (chr9: 22000961-22001288, hg18) were measured with EpiTYPER platform (Sequenom), respectively. Mann-Whitney $U$ test was performed to assess the significance of methylation ratios between the cases and control groups. Multivariate statistics were used to calculate differences in individual and total DNA methylation between the case and control subjects adjusted for sex and age. Statistical calculations were carrying out using SPSS 18 (SPSS, USA).

Results: Using DNA obtained from the PBMCs, twelve of fifteen CpGs (80\%) in CDKN2A intron 1 and eight of ten CpGs $(80 \%)$ in CKDN2B promoter were detected by
MALDI-TOF mass spectrometry, respectively. The total methylation level and most individual CpGs in the CDKN2B promoter showed significant hypermethylation $(p<0.01)$ adjusted for sex and age in T2D relative to controls. For example, \% methylation of CpG (Chr9:22001207+) was $23.2 \% \pm 5 \%$ in health control and $25.6 \% \pm 4 \%$ in T2D $(p=0.004)$. The methylation levels of CDKN2A DMRs were not increased of T2D compared to control.

Conclusion: We have successfully developed a platform to perform methylation analysis for the DMRs of CDKN2A/2B. Our results show that methylation changes of $\mathrm{CpG}$ in the DMRs of CDKN2B were replicated in PBMCs in an independent validation cohort. Further work is currently underway to evaluate relationships between the signature and clinical phenotype. The results suggest EpiTYPER as an efficient method for methylation validation analysis and highlight the potential utility of PBMCs in the discovery and replication of epigenetic biomarkers associated with T2D.

Acknowledgement: Supported by the Focused Investment Scheme of the Chinese University of Hong Kong, and the EFSD/CDS/Lilly Collaborative Research Programme.

Disclosure of Interest: None Declared.

\section{DOHaD13-1693}

Epigenetic changes in survivors of preterm birth: effect of gestational age and evidence for a long term legacy

Mark Cruickshank ${ }^{1}$, Christiane Theda ${ }^{2,3, *}$, Alicia Oshlack ${ }^{4}$, David Martino $^{5}$, Penenope Sheehan ${ }^{2,3}$, Richard Saffery ${ }^{5,6}$, Peter Davis ${ }^{2,3}$, Lex Doyle ${ }^{2,3}$, Jeffrey M. Craig ${ }^{5,6}$

${ }^{1}$ Telethon Institute for Child Health Research, University of Western Australia, Perth; ${ }^{2}$ Department of Obstetrics and Gynaecology, University of Melbourne; ${ }^{3}$ Royal Women's Hospital; ${ }^{4}$ Bioinformatics; ${ }^{5}$ MURDOCH CHILDRENS RESEARCH INSTITUTE; ' Paediatrics, University of Melbourne, Melbourne, Australia

Background: Preterm birth confers a high risk of adverse long-term health outcomes for survivors, yet the underlying molecular mechanisms are unclear. We hypothesized that effects of preterm birth can be mediated through measurable epigenetic changes throughout development. We therefore used a longitudinal birth cohort to measure the epigenetic mark of DNA methylation at birth and 18 years comparing survivors of extremely preterm birth with infants born at term.

Method: Using 12 extreme preterm birth cases and 12 matched, term controls, we extracted DNA from archived neonatal blood spots and blood collected in a similar way at 18 years of age. DNA methylation was measured at 347,789 autosomal locations throughout the genome using Infinium HM450 arrays. Representative methylation differences were confirmed by Sequenom MassArray EpiTyping. 
Results: At birth we found 1,555 sites with significant differences in methylation between term and preterm babies. At 18 years of age, these differences had largely resolved, suggesting that DNA methylation differences at birth are mainly driven by factors relating to gestational age, such as cell maturity and to some extent, cell composition. Using matched longitudinal samples, we found suggestive evidence for an epigenetic legacy of preterm birth, identifying persistent methylation differences at several genomic loci. Longitudinal comparisons uncovered a significant overlap between sites that were differentially-methylated at birth and those that changed with age. We found an unexpectedly high number of birth- and age-differentially-methylated probes (314) showing a higher level of DNA methylation in term infant's blood compared to both preterm infants' and adult blood, suggesting that developmentally-associated epigenetic trajectories may not always be linear.

Conclusion: We present evidence for widespread methylation differences between extreme preterm and term infants at birth that are largely resolved by 18 years of age. These results are consistent with methylation changes associated with blood cell development, immune induction, cellular composition and age at these time points. Finally, using this small sample size we identified a number of sites that were persistently altered in the preterm group and may be associated with a long term epigenetic legacy of preterm birth.

Disclosure of Interest: None Declared.

\section{DOHaD13-1303}

\section{Epigenetic memory of maternal depression in the infant}

Li Chen ${ }^{1 * *}$, Sarah $\mathrm{Mah}^{2}$, Yap-Seng Chong ${ }^{3}$, Helen Chen ${ }^{4}$, Jan Paul Buschdorf $f^{5}$, Walter Stunkel ${ }^{1}$, Peter Gluckman ${ }^{1}$, Anne Rifkin-Graboi ${ }^{5}$, Michael Kobor ${ }^{6}$, Michael J. Meaney ${ }^{1,7}$, Joanna Holbrook ${ }^{1,1}$

${ }^{1}$ Singapore Institute of Clinical Sciences (SICS), $A^{*}$ STAR, Singapore, Singapore; ${ }^{2}$ Centre for Molecular Medicine and Therapeutics, University of British Columbia, British Columbia, Canada; ${ }^{3}$ Yong Loo Lin School of Medicine, National University of Singapore; ${ }^{4} K K$ Women's and Children's Hospital; ${ }^{5}$ Singapore Institute of Clinical Sciences (SICS), Singapore, Singapore;

${ }^{6}$ Centre for Molecular Medicine and Therapeutics, University of British Columbia; ${ }^{7}$ Douglas University Mental Health Institute, McGill University, Quebec, Canada

Background: Environment in utero has been shown to modify the risk of adult-onset disease. Prenatal maternal depression and anxiety has been shown to adversely affect infant neurocognitive development. We hypothesise that signals associated with maternal emotional well being in the in utero environment modify the fetal epigenome and that epigenetic mark is transmitted to later life to affect neurodevelopment. Using umbilical cords collected in the
GUSTO (Growing Up in Singapore Toward Healthy Outcomes) birth cohort, we measured genome-wide DNA methylation of infants born to mothers across the normal range of mood.

Methods: Pregnant women enrolled in the GUSTO birth cohort were assessed for depression using the Edinburgh postnatal depression scale (EPDS) and State-Trait anxiety inventory (STAI) at 26 weeks pregnant. Infant umbilical cord methylomes were interrogated using the Infinium $450 \mathrm{~K}$ array which measured DNA methylation at $>450,000 \mathrm{CpG}$ sites.

Results: We surveyed the DNA methylomes of the umbilical cords of 100 neonates, born to mothers across a range of EPDS and STAI scores. 1,828 CpG probes had significant associations with EPDS and absolution methylation difference across the range of more than $5 \%$. We also identified significantly differentially methylated regions (DMRs) which contained contiguous CpGs with differential methylation between groups. Gene category and pathway enrichment analysis showed enrichment for genes implicated in this screen to neuronal functioning.

Conclusions: Epigenetic differences can be discerned between babies born to depressed mothers, compared to normative controls. We suggest that these form part of the molecular memory of maternal depression that persists to infancy and beyond.

Disclosure of Interest: None Declared.

\section{DOHaD13-1620}

Functional relevance of early pregnancy DNA methylation hypomethylation in preeclampsia

Cindy M. Anderson ${ }^{1, *}$, Jody L. Ralph ${ }^{2}$, Michelle L. Wright ${ }^{2}$, Joyce E. $\mathrm{Ohm}^{3}$

${ }^{1}$ Nursing, The Ohio State University, Columbus; ${ }^{2}$ Nursing;

${ }^{3}$ Biochemistry and Molecular Biology, University of North

Dakota, Grand Forks, United States

Background: Preeclampsia (PE) affects $1-8 \%$ of women in the US, representing a major cause of acute maternal morbidity with potential to influence future cardiovascular risk in mothers and their offspring. Children of women with preeclampsia have an increased risk of preeclampsia during their own pregnancies or those of their spouses, suggesting a complex, non-Mendelian transgenerational inheritance pattern. The availability of early maternal screening to determine PE risk is currently unavailable, limiting early recognition and potential intervention. We previously identified methylation loss in key maternal genes with potential utility in screening for preeclampsia during early pregnancy (Anderson et al., 2013). The objective of this study was to investigate the functional relevance of DNA hypomethylation in maternal peripheral blood during early pregnancy that has may underlie the pathogenesis of PE. Method: In this prospective study, blood was collected from nulliparous women in the first trimester of pregnancy. 
DNA methylation was quantified at approximately $450 \mathrm{~K}$ individual $\mathrm{CpG}$ dinucleotides in white blood cells from women who subsequently developed PE and from matched normotensive controls $(\mathrm{n}=6$ /group) across the genome (Illumina Infinium). Average beta scores for each individual CpG dinucleotide were compiled based on percent methylation, ranging from 0 ( $0 \%$ methylation) to 1 (100\% methylation), with significant methylation change established at a delta.beta of $\leq-0.2$ ( $\geq 20 \%$ loss of methylation) or $\geq 0.2$ ( $\geq 20 \%$ gain in methylation) between groups. Samples were also independently analyzed using the NIMBL (Numerical Identification of Methylation Biomarker Lists) Infinium analysis package, specifically designed to identify biomarkers in clinical samples. Functional interpretation of genes was completed using the Database for Annotation, Visualization and Integrated Discovery (DAVID, http://david.abcc.ncifcrf.gov) using a classification stringency of high to determine functional annotation clusters.

Results: Twenty differentially methylated sites were identified as potential biomarkers based on the greatest absolute distance between samples from women with $\mathrm{PE}$ and those with normotensive pregnancy. Of the top 20 differentially methylated sites, 13 were associated with known genes of which $85 \%$ had significant loss of methylation. Functional relevance of genes associated with the 11 hypomethylated sites was associated with inflammation, hemostasis, inherited connective tissue disease, oocyte and embryonic/stem cell expression, counteraction of mitogenic function and maintenance of endothelial junction integrity.

Conclusion: Hypomethylated biomarker sites identified in early pregnancy among women who subsequently developed PE are linked to cellular functions relevant to the pathogenesis of preeclampsia. Further investigation may lead to mechanistic targets and potential therapies that reduce maternal risk for PE and mitigate intrauterine influences that contribute to alteration of the fetal epigenome, predisposing to future risk for preeclampsia.

\section{References}

1. Anderson CM, Ralph JL, Wright ML, Linggi B, Ohm JE. (2013). DNA methylation as biomarker for heritable preeclampsia. Biological Research for Nursing. (In press)

Disclosure of Interest: None Declared.

\section{DOHaD13-1407}

Gene expression and DNA methylation of PGC-1alpha in liver and muscle of IUGR rats with catch-up growth

Xuemei Xie ${ }^{1}$, Lihong Liao ${ }^{1}$, Meihui Zhang ${ }^{1}$, Xiaoping Luo ${ }^{1, *}$

${ }^{1}$ Pediatrics, Tongji Hospital, Tongji Medical College, Huazhong University of Science and Technology, Wuhan, China

Background: Epigenetic modification via DNA methylation is associated with metabolic disease. Intrauterine growth retardation (IUGR) individuals with catch-up growth are prone to insulin resistance. We hypothesized that the change in gene expression and DNA methylation of peroxisome proliferator-activated receptor gamma (PPARgamma) coactivator-1 alpha (PGC-1alpha) might contribute to insulin resistance in catch-up growth IUGR rats (CG-IUGR).

Method: A CG-IUGR rat model was established through maternal nutritional restriction and reduction of litter size from postnatal day 1 . The 8 -week rats were sacrificed, and the RNA, protein and DNA of hind-limb skeletal muscle and liver tissue were extracted. Real-time PCR and western blot were used to determine the expression level of PGC- $1 \alpha$. After PCR amplification of the bisulfite treated DNA, Pyrosequencing was used to analyse the methylation level of specific $\mathrm{CpG}$ sites in PGC-1alpha promoter. The mitochondrial content in liver and muscle was assessed by real-time PCR as well.

Results: CG-IUGR showed increase in the methylation level of specific sites -787 and -803 in PGC-1alpha promoter, and decrease in the PGC-1alpha expression and mitochondrial content compare with control. The methylation level of specific sites in PGC-1alpha promoter was positively correlated with plasma fasting insulin level.

Conclusion: Our findings suggest that the DNA methylation of PGC-1alpha promoter probably plays a potential role in metabolic programming in CG-IUGR rats.

Disclosure of Interest: None Declared.

\section{DOHaD13-1195}

Genome wide analysis of comparative transcriptional networks in adipocytes differentiated from umbilical cord derived mesenchymal stem cells, isolated from growth restricted and normal babies

Roy Joseph ${ }^{1, *}$, Jeremie Poschmann ${ }^{2}$, Yhee Cheng Chng ${ }^{1}$, Rami Sukarieh ${ }^{1}$, Jun Hao Tan ${ }^{1}$, Ai Ling Teh ${ }^{1}$, Joanna D. Holbrook ${ }^{1}$, Kai Lyn $\mathrm{Ng}^{3}$, Yap Seng Chong ${ }^{3}$, Peter D. Gluckman $^{1}$, Shyam Prabhakar ${ }^{2}$, Walter Stünkel ${ }^{1}$

${ }^{1}$ Growth, Development \& Metabolism Programme, Singapore Institute for Clinical Sciences, Agency for Science Technology and Research (A*STAR); ${ }^{2}$ Integrated Genomics, Genome Institute of Singapore, Agency for Science Technology and Research (A*STAR); ${ }^{3}$ Obstetrics \& Gynaecology, NUHS, Singapore, Singapore

Background: Individuals, born small for gestational age (SGA) face an increased risk of metabolic syndrome including adiposity later in life. Mesenchymal stem cells (MSCs) from Wharton's jelly of umbilical cord (UC) tissue are multipotent and therefore serve as an ideal model system for studying genes at various levels of adipocyte differentiation. Our hypothesis is that SGA as a proxy for an impaired intrauterine environment is associated with specific epigenetic marks and altered biochemical pathways which become physiologically apparent only in the fully differentiated adipocyte state. 
Method: We established an adipocyte differentiation protocol using UC-MSCs isolated from the umbilical cords obtained from SGA and normal neonates born at the National University Hospital (NUH) in Singapore. Using next generation ChIP-sequencing, we generated chromatin state maps for histone marks H3K27ac and H3K27me3 and their enrichment at various cycles of differentiation. We identified genomic regions of interest such as enhancers and promoters, as well as their regulated genes. In particular, we tested whether functional enhancer and promoter specific histone $\mathrm{H} 3 \mathrm{~K} 27 \mathrm{ac}$ or polycomb-related $\mathrm{H} 3 \mathrm{~K} 27 \mathrm{me} 3$ marks were associated with up or down-regulated genes assayed on a global scale via gene expression microarray studies. We selected more than 30 genes with possible involvement in the adipocyte differentiation process, as well as putative roles in metabolism, and extended RT-PCR validation experiments to additional differentiated UC-MSC primary lines from SGA and control groups using the Fluidigm qPCR platform. Results: We found that the gene expression pattern for the large majority of these genes was highly consistent with the ChIP-seq data sets. We identified candidate genes of interest such as TFAP $2 \alpha$, ACSL1, and SOX6, whose expression was found to be statistically different in the SGA compared to the control group.

Conclusion: Using adipocytes differentiated from UC-MSC lines as model systems and global profiling of histone marks along with gene expression data, we have identified candidate genes in growth restricted babies which are associated with a putative poor metabolic developmental trajectory.

Disclosure of Interest: None Declared.

\section{DOHaD13-1594}

Glucocorticoids alter DNA methylation in the adult male germline resulting in altered steroid receptor expression and DNA methylation in offspring

Sophie Petropoulos ${ }^{1, *}$, Stephen G. Matthews ${ }^{2}$, Moshe Szyf ${ }^{1}$

${ }^{1}$ Pharmacology and Therapeutics, McGill University, Montreal; ${ }^{2}$ Physiology, University of Toronto, Toronto, Canada

Background: Synthetic glucocorticoids (sGCs) are commonly prescribed for the management of inflammatory and endocrine disorders. However, nothing is known regarding the effects of GCs on germline methylome and whether these effects can be transmitted to the next generation. We hypothesized that administration of $s G C$ to adult males alters global DNA methylation in mature sperm and modifies the transcription and methylation of steroid receptors in the hippocampus and kidney $\mathrm{F} 1$ offspring.

Method: Adult C57BL/6 males ( $\mathrm{n}=10$ /group) were injected (s.c.) with dexamethasone (sGC; $1 \mathrm{mg} / \mathrm{kg}$ ) or vehicle (saline) for 5 consecutive days. Mice were euthanized 60 days after treatment or bred with control females. Global DNA methylation of mature sperm was determined. Receptor expression (qPCR) and DNA methylation (MeDIP) were assessed in $\mathrm{F} 1$ offspring hippocampus and kidney at postnatal day (PND) 50 and PND240.

Results: A significant increase in global non-CpG methylation was observed in sperm $(\mathrm{P}<0.05)$. In the hippocampus of male offspring (PND50) a significant increase in mineralocorticoid receptor (MR) and decrease in estrogen receptor alpha (ERa) were observed at $(\mathrm{P}<0.01, \mathrm{P}<0.05)$; no change in the glucocorticoid receptor $(\mathrm{GR})$ was observed compared to control. In the kidney, MR and GR expression significantly decreased $(\mathrm{P}<0.05)$ compared to control, while ERa showed a trend toward decreased expression $(P=0.06)$. No differences in expression were observed in the female tissues. By PND240, male hippocampal MR and GR expression significantly declined $(\mathrm{P}<0.05)$, while ERa rebounded to levels matching control. In the kidney, MR expression significantly decreased $(\mathrm{P}<0.05)$. Significant demethylation of MR, GR and ER $(P<0.05)$ were observed. Conclusion: This is the first demonstration that pharmacological exposure to GC can reprogram the adult male germline methylome and the expression and methylation of key steroid nuclear receptors in male offspring. These findings are of crucial importance given that changes to the germline methylome may be transmitted to the embryo and program offspring for disease later in life, in a sex specific manner.

Disclosure of Interest: None Declared.

\section{DOHaD13-1096}

Glucocorticoid effects on germline epigenetic reprogramming in the rat $-\mathbf{a}$ mechanism for the transmission of programming effects across generations?

Catherine M. Rose ${ }^{1, *}$, Sander van den Driesche ${ }^{2}$, Ashley K. Boyle ${ }^{2}$, Richard M. Sharpe ${ }^{2}$, Richard R. Meehan ${ }^{3}$, Amanda J. Drake ${ }^{1}$

${ }^{1}$ Endocrinology Unit, University/BHF Centre for Cardiovascular Science, University of Edinburgh; ${ }^{2} M R C$ Centre for Reproductive Health, University of Edinburgh; ${ }^{3}$ Medical Research Council Human Genetics Unit, Edinburgh, United Kingdom

Background: Exposure of the fetus to excess glucocorticoid is associated with low birth weight and increased cardiovascular disease risk in the first generation offspring and such 'programmed effects' can be transmitted across generations through both male and female lines. Although the mechanisms are unclear, it is hypothesised that disruption of germline epigenetic reprogramming in fetal germ cells may account for the transmission of programmed effects across generations. Epigenetic reprogramming of germ cells is characterised by genome-wide erasure and subsequent re-establishment of DNA methylation. This process has not been described for the rat which is commonly used in models of intergenerational 
cardiovascular disease transmission. Furthermore, the potential involvement of the more recently identified cytosine modifications; 5-hydroxymethylcytosine $(5 \mathrm{hmC})$, 5-formylcytosine (5fC) and 5-carboxylcytosine $(5 \mathrm{caC})$, has not been characterised during germ cell ontogeny. We have therefore characterised the global dynamics of $5 \mathrm{mC}, 5 \mathrm{hmC}, 5 \mathrm{fC}$ and $5 \mathrm{caC}$, the de-novo methyltransferases (DNMT3a and $3 \mathrm{~b}$ ) and the effects of fetal Dexamethasone (Dex) exposure in the developing rat germ line during the re-establishment phase of epigenetic reprogramming.

Method: Pregnant female Wistar rats were treated with Dex $(100 \mu \mathrm{g} / \mathrm{kg} / \mathrm{day})$ or vehicle from E15.5 onwards. Testes were collected between E14.5 and E21.5, and the localisation of $5 \mathrm{mC}, 5 \mathrm{hmC}, 5 \mathrm{fC}, 5 \mathrm{caC}$, DNMT3a and DNMT3b identified by immunofluorescence; some co-localisation studies with a germ cell-specific marker (Deleted in Azoospermia-Like (DAZL)) were also conducted.

Results: $5 \mathrm{hmC}, 5 \mathrm{fC}$ and $5 \mathrm{caC}$ were present between E14.5 and $\mathrm{E} 16.5$ but absent thereafter. In contrast, $5 \mathrm{mC}$ was absent during this time but was present in a subset of germ cells at E19.5, and in all germ cells by e20.5. Dex administration was associated with a greater number of $5 \mathrm{mC}$-positive germ cells in the fetus at E19.5, compared to controls, suggesting premature re-methylation. DNMT3a and $3 \mathrm{~b}$ were detected in germ cells throughout the re-methylation phase in both treatment and control groups.

Conclusion: Our observations indicate that dynamic changes in $5 \mathrm{hmC}, 5 \mathrm{fC}$ and $5 \mathrm{caC}$ occur in germ cells throughout gestation, suggesting that these modifications may have a role in DNA reprogramming events during germ cell development. This study also indicates that the re-methylation phase of epigenetic reprogramming is conserved between rat and mouse and begins prenatally in the male germline. This remethylation phase appears to occur asynchronously in the final stages of rat gestation. Glucocorticoid exposure may affect epigenetic reprogramming within the developing germ cell, and this may be one mechanism for the transmission of cardiovascular disease risk across generations.

Disclosure of Interest: None Declared.

\section{DOHaD13-1604}

\section{Human fetal growth associates with altered DNA hydroxymethylation of $\mathrm{H19}$ and with expression and DNA methylation of placental IGF2}

Chinthika Piyasena ${ }^{1,2, *}$, Khulan Batbayar ${ }^{1}$, Gopi Menon ${ }^{2}$, Jonathan R. Seckl ${ }^{1}$, Rebecca M. Reynolds ${ }^{1}$, Amanda J. Drake ${ }^{1}$

${ }^{1}$ Endocrinology, Queen's Medical Research Institute, University of Edinburgh; ${ }^{2}$ Simpson Centre for Reproductive Health, Royal Infirmary of Edinburgh, Edinburgh, United Kingdom

Background: Low and high birthweight associate with adverse health outcomes throughout life. Altered placental expression of imprinted genes including insulin like growth factor 2 (IGF2) may be one mechanism linking the environment and later disease risk. IGF2 expression is controlled by DNA methylation at differentially methylated regions (DMR0 and DMR2) proximal to the IGF2 gene and at the imprinting control region (ICR) upstream of the neighbouring $\mathrm{H} 19$ gene. Alterations in cytosine methylation $(5 \mathrm{mC})$ at these DMRs have been reported in syndromic and non-syndromic fetal growth abnormalities. The newly described DNA modification, 5-hydroxymethylcytosine $(5 \mathrm{hmC})$, is an intermediate step in DNA demethylation, however the positive relationship between $5 \mathrm{hmC}$ profiles and transcription suggests that $5 \mathrm{hmC}$ may be a useful signature of transcriptional state. Previous studies have not been able to technically discriminate $5 \mathrm{mC}$ from $5 \mathrm{hmC}$ and therefore the role of the latter and less well-studied modification may have been underestimated. We studied $5 \mathrm{mC}$ and $5 \mathrm{hmC}$ within the IGF2/H19 locus in term human placenta and related this to birthweight.

Method: Placental samples from 72 women with a singleton pregnancy at term ( $>37$ weeks) representing a wide variation in birthweight (range $2.16-4.61 \mathrm{~kg}$, median $3.39 \mathrm{~kg}$ ) were obtained from the Edinburgh Reproductive Tissue BioBank. Samples had been snap frozen/stored in RNAlater as appropriate. Pregnancies complicated by congenital abnormalities or diabetes were excluded. Standard deviation scores ( $\mathrm{z}$ scores) for birthweight were derived from "British 1990 reference data, re-analysed 2009"1. Gene expression was analysed using real-time PCR and geNorm and Normfinder, both recommended software, were used to determine a stable housekeeping gene. Enrichment of $5 \mathrm{mC}$ was performed using the Active Motif MethylCollector Ultra and enrichment of $5 \mathrm{hmC}$ using a hydroxymethyl DNA immunoprecipitation technique. Percentage enrichment at DMR0, DMR2, the H19 ICR and the H19 gene body was analysed using real-time PCR. Data were analysed using SPSS version 19.

Results: Placental IGF2 mRNA levels correlated positively with birthweight $\mathrm{z}$ score when adjusted for maternal body mass index and parity using linear regression $(r=0.301$ $p=0.021$, adjusted $p=0.03$ ) but not when pre-eclampsia and maternal smoking were added into the model (adjusted $p=0.106) .5 \mathrm{mC}$ levels at DMR0 correlated with birthweight $\mathrm{z}$ score in adjusted analyses $(\mathrm{r}=0.538, p=0.0001$, adjusted $p=0.0001$ ) but $5 \mathrm{mC}$ levels at DMR2 and the H19 ICR were not related to size at birth. $5 \mathrm{hmC}$ levels within the $\mathrm{H} 19$ gene body correlated with birthweight $\mathrm{z}$ score in adjusted analyses $(\mathrm{r}=0.337, p=0.004$, adjusted $p=0.002)$.

Conclusion: We present further evidence that placental DNA methylation at IGF2 is involved in the regulation of birthweight and additionally, show that size at birth may be associates with differences in 5-hydroxymethylation. This may bring more insight into the biologic mechanisms determining birthweight and the origins of adult chronic disease. 


\section{References}

1. LMS growth, Version 2.71, 2011. http://www.healthfor allchildren.co.uk/

Disclosure of Interest: None Declared.

\section{DOHaD13-1623}

\section{Human placenta drives developmental plasticity through} imprinting control

Benedetta Izzi ${ }^{1,2}$, Alexandra Binder ${ }^{3}$, Timothy Barrow ${ }^{1,2}$, Karin Michels ${ }^{1,2,3, *}$

${ }^{1}$ Institute for Prevention and Tumorepidemiology, University of Freiburg Medical Center, Freiburg, Germany; ${ }^{2}$ Obstetrics and Gyncecology Epidemiology Center, Harvard Medical School; ${ }^{3}$ Department of Epidemiology, Harvard School of Public Health, Boston, United States

Background: Developmental plasticity permits fetal adaptation to varied intrauterine environments. Little is known about the mechanistic underpinnings of fetal plasticity. The placenta is a likely participant due to its role as interface between the fetal and maternal circulations: it regulates the transfer of nutrients and waste, and controls fetal growth, development and metabolism. Genomic imprinting is particularly prevalent in placenta and imprinted genes are strong regulators of growth and development. Differentially methylated regions (DMRs) at specific imprinted gene loci are thought to be remodeled and set prior to germ layer specification and its pattern preserved throughout life in all tissues.

Method: We compared DNA methylation levels of 7 imprinted genes described to be important in several human disorders in 4 perinatal tissues (placenta, cord blood, cord tissue, foreskin) and maternal blood from 6 mother-child dyads selected from the Epigenetic Birth Cohort. Using pyrosequencing methylation for 11 DMRs including IGF2 (DMR0 and DMR2), H19, GRB10, MEST1, SNRPN, KCNQ1, GNAS (NESP, XL and Exon A/B DMRs) was assessed. For each DMR, the average methylation level was modeled as a function of tissue origin using a linear mixed model, accounting for within-person correlation. A likelihood ratio test was used to identify significant disparities in methylation level between tissues; post-hoc pairwise comparisons between tissue types were corrected for multiple testing. IGF2, H19, GNAS-Xlalhpas and GRB10 expression was evaluated within the same individuals by qPCR. A Wilcoxon rank-sum test was used to identify significant differential expression between tissues.

Results: No significant differences in methylation levels were detected between tissues for H19, GRB10, SNRPN, KCNQ1 or MEST1. Placenta GNAS methylation was significantly higher compared to all other tissues while both IGF2 DMR0 and DMR2 were significantly hypomethylated $(\mathrm{p}<0.001$ for each DMR). Significant but smaller differences, were observed for IGF2 DMR0 in cord tissue and foreskin compared to cord blood and maternal blood. Consistent with the observed methylation changes, expression of IGF2 and GNAS-XLalphas displayed significantly higher expression in the placenta than in the cord blood, while no differences were detected for GRB10. H19 expression was also significantly higher in placenta compared to cord blood despite their similar methylation pattern.

Conclusion: This is the first study focusing on the comparison of DNA methylation and gene expression for imprinted genes between human perinatal tissues. For both IGF2 DMRs and the three GNAS DMRs, tissue origin was a significant predictor of methylation level. The correlation between methylation level and gene expression was modest reflecting the complexity of gene regulation, which depends also on chromatin structure and nucleosome positioning. As GNAS and IGF2 control growth and development, the specific methylation pattern observed for these genes in placenta coupled with their higher expression levels compared to cord blood, suggest that plasticity of human placenta in response to the intrauterine environment might be driven by epigenetic mechanisms.

Disclosure of Interest: None Declared.

\section{DOHaD13-1509}

\section{Identification of CDKN2A as a perinatal epigenetic marker of later adiposity}

Karen Lillycrop ${ }^{1}$, Robert Murray ${ }^{2, *}$, Ai Ling Teh ${ }^{3}$, Clara Cheong ${ }^{3}$, Shaillay Dogra ${ }^{3}$, Rebecca Clarke-Harris ${ }^{2}$, Sheila Barton ${ }^{4}$, Paula Costello ${ }^{2}$, Emma Garratt ${ }^{2}$, Graham Burdge ${ }^{2}$, Cyrus Cooper ${ }^{4}$, Hazel Inskip ${ }^{4}$, Fabian Yap ${ }^{5}$, Lee Yung Seng ${ }^{5}$, Chong Yap Seng ${ }^{5}$, Joanna D. Holbrook ${ }^{3}$, Peter Gluckman ${ }^{6}$, Nick Harvey ${ }^{4}$, Mark Hanson ${ }^{2,7}$, Keith Godfrey ${ }^{2,4,7}$ and EpiGen Consortium

${ }^{1}$ Centre for Biological Sciences; ${ }^{2}$ Human Development and Health Academic Unit, University of Southampton, southampton, United Kingdom; ${ }^{3}$ Singapore Institute for Clinical Sciences, Singapore, Singapore; ${ }^{4}$ MRC Lifecourse Epidemiology Unit, University of Southampton, southampton, United Kingdom; ${ }^{5}$ Yong Loo Lin School of Medicine, National University of Singapore, Singapore, Singapore; ${ }^{6}$ Liggins Institute, University of Auckland, Auckland, New Zealand; ${ }^{7}$ NIHR Southampton Biomedical Research Centre, University Hospital Southampton NHS Foundation Trust and University of Southampton, southampton, United Kingdom

Background: Experimental studies have provided strong evidence that developmental influences acting through epigenetic processes induce long term metabolic effects. This has led to the hypothesis that developmentally induced changes in epigenetic gene regulation, might affect susceptibility to human obesity in later life. Identifying such epigenetic changes may provide insights into the mechanisms underlying the development of adiposity and allow the identification of individuals at 
risk of metabolic disease. To this end, we used a Methyl binding domain (MBD) array to screen for methylation differences in umbilical cord (UC) at birth which were associated with adiposity of the children aged 6 years.

Method: UC DNA was extracted from 21 infants in the Southampton Women's Survey (SWS), a population based mother-offspring cohort and analysed using a MBD array. Subjects were selected as they represented a range of $\%$ fat values at 6 years (assessed by DXA scan) between the $5^{\text {th }}$ and the $95^{\text {th }}$ percentiles. Absolute methylation levels were estimated using the Bayesian algorithm BATMAN. The resulting differentially methylated regions of interest (DMROIs) were validated by pyrosequencing in a larger subset of samples from the SWS cohort, with replication carried out on $\mathrm{n}=127$ subjects from the GUSTO (Growing Up in Singapore Towards healthy Outcomes) cohort.

Results: Bioinformatic analysis identified 185 DMRs and 61 DMROIs associated with \% fat mass at 6 years. Sodium bisulfite pyrosequencing of the nine CpG's within the DMROI of CDKN2A in the SWS cohort confirmed the association between umbilical cord CDKN2A methylation (CpGs 1-4, 6, 7) and \% fat mass $(\mathrm{P}<0.05)$ in the children age 6 years. These findings were replicated in the second independent GUSTO cohort where an association was seen between CDKN2A methylation and ponderal index at 6 months of age (CpGs 4, 5,7, and 8, p <0.05).

Conclusion: Umbilical cord methylation of specific $\mathrm{CpG}$ loci within the CDKN2A gene locus was associated with the child's later adiposity in two independent birth cohort studies. GWAS studies have previously identified CDKN2A as a significant contributor to a number of ageing associated diseases. Together, our findings raise the possibility that epigenetic deregulation of the CDKN2A gene locus may also contribute to the development of adiposity.

This work was supported by grants from the MRC, BHF, Arthritis Research UK, National Osteoporosis Society, International Osteoporosis Foundation, Cohen Trust, Nestec, NIHR Musculoskeletal Biomedical Research Unit, University of Oxford and NIHR Southampton Biomedical Research Centre, University of Southampton and University Hospital Southampton NHS Foundation Trust.

Disclosure of Interest: None Declared.

\section{DOHaD13-1416}

\section{Major epigenetic development distinguishing neuronal and non-neuronal cells occurs postnatally in the mouse hypothalamus}

Ge Li ${ }^{1}$, Wenjuang Zhang ${ }^{1}$, Maria S. Baker ${ }^{1}$, Eleonora Laritsky $^{1}$, Richard B. Simerly ${ }^{2}$, Rui Chen ${ }^{3,4}$, Lanlan Shen ${ }^{1}$, Robert A. Waterland ${ }^{1,4, *}$

${ }^{1}$ Department of Pediatrics, Baylor College of Medicine, USDA/ARS Children's Nutrition Research Center, Houston;
${ }^{2}$ Neuroscience Program, The Saban Research Institute, Children's Hospital Los Angeles, University of Southern California, Keck School of Medicine, Los Angeles; ${ }^{3}$ Human Genome Sequencing Center; ${ }^{4}$ Department of Molecular and Human Genetics, Baylor College of Medicine, Houston, United States

Background: An improved understanding of epigenetic development in a heterogeneous tissue like the hypothalamus will require techniques to independently examine specific cellular subpopulations. The two predominant cell types in the central nervous system are neurons and glia. Given that neuronal and non-neuronal cells have distinct epigenetic regulation (1-3), we set out to determine when this is established in the murine hypothalamus.

Method: Hypothalami were dissected from inbred male 'non-agouti' (4) mice at postnatal day 0 (P0) and P21. Nonneuronal and neuronal nuclei were separated via fluorescenceactivated sorting based on staining for the neuron-specific nuclear surface marker $\mathrm{NeuN}$; each sample for sorting was comprised of 2 age-matched hypothalami. Genome-scale DNA methylation profiling was performed by methylation specific amplification (5) coupled with next generation sequencing (MSA-seq) (5 independent samples per age). MSA-seq results were validated by bisulfite pyrosequencing. Gene expression of selected hits was determined by qPCR with RNA extracted from sorted nuclei.

Results: Of the 50,782 genomic regions interrogated by MSA-seq, 327 and 2211 showed significant $(P<0.0001)$ non-neuron vs. neuron methylation differences at $\mathrm{P0}$ and P21, respectively, indicating that the majority of epigenetic differentiation that distinguishes these two classes of hypothalamic cells occurs postnatally. Whereas most (7436) methylation changes from P0 to P21 occurred in both nonneuronal and neuronal cells, 287 were specific to nonneurons, and 1681 were specific to neurons. Pyrosequencing of 16 hits yielded a $100 \%$ validation rate, confirming the reliability of the MSA-seq data. Gene ontology analysis found almost no enriched ontologies among the large number of genes undergoing P0 to P21 methylation changes in both cell types. Numerous neurodevelopmental processes were, however, significantly associated with genes undergoing changes only in specific cell types, suggesting that postnatal DNA methylation plays a key role in regulating cellular neurodevelopmental fate. Developmental changes in gene expression of Bmp4,Shh, and En1 were significantly correlated with changes in DNA methylation, indicating a regulatory role for these cell-type specific epigenetic changes.

Conclusion: Our data indicate that the major epigenetic development that distinguishes gene expression potential of hypothalamic neurons and glia does not occur until after birth. Hence, the postnatal period in the mouse is a critical period for hypothalamic developmental epigenetics. Studies of postnatal epigenetic 'programming' of body weight regulation will clearly benefit from studying separately hypothalamic neuronal and non-neuronal cells. 


\section{References}

1. I. Cheung et al., Proc Natl Acad Sci US A 107, 8824

(May 11, 2010).

2. K. Iwamoto et al., Genome Res 21, 688 (May, 2011).

3. P. J. Skene et al., Mol Cell 37, 457 (Feb 26, 2010).

4. R. A. Waterland, R. L. Jirtle, Mol Cell Biol 23, 5293

(Aug, 2003).

5. L. Shen et al., PLoS Genet 3, 2023 (Oct, 2007).

Disclosure of Interest: None Declared.

\section{DOHaD13-1750}

Mechanism of programmed obesity in intrauterine growth restricted newborns: epigenetic mediated early induction of adipocyte differentiation contributes to enhanced adipogenesis

Mina Desai Ph.D ${ }^{1, *}$, Juanita Jellyman Ph.D ${ }^{1}$, Guang Han M.D. ${ }^{1}$, Marie H. Beall M.D. ${ }^{2}$, Michael G. Ross M.D., M.P.H. ${ }^{1}$

${ }^{1}$ Department of Obstetrics and Gynecology, Los Angeles Biomedical Research Institute at Harbor - UCLA Medical Center, Torrance; ${ }^{2}$ Los Angeles Perinatal Association, Los Angeles, United States

Background: The key feature of gestationally programmed obesity in intrauterine growth restricted (IUGR) newborns is enhanced adipogenesis. Adipogenesis is driven by adipocyte differentiation, a process whereby previously silent adipogenic genes are activated. It is likely that this well-regulated transcriptional occurrence is mediated via epigenetic mechanisms. DNA methyltransferase (DNMT3a) and histone deactetylase (HDAC1) both suppress gene expression. We have previously shown that maternal food restriction results in IUGR newborns that develop adult obesity. Notably at 1 day of age, IUGR newborns have upregulated expression of adipogenic transcription factors (PPAR $\gamma, \mathrm{C} / \mathrm{EBP} \alpha$. We hypothesized that IUGR adipocytes exhibit enhanced adipocyte differentiation as a result of epigenetic mediated premature induction of adipogenic genes. Using primary adipocyte cultures, we determined the time-course of induction of epigenetic modulators, adipogenic transcription factors and their downstream lipogenic target genes (SREBP1, fatty acid synthase, acetyl-CoA carboxylase).

Method: Control dams received ad libitum food, whereas study dams were $50 \%$ food-restricted from pregnancy day 10 to term, resulting in IUGR newborns. Adipose tissue was obtained from 1 day old IUGR and Control newborns and cultured for $48 \mathrm{~h}$ (time 0). Cells were induced to differentiate at day 0 with hormone inducer mixture. Protein was extracted at day 2, 4 and 6 of induction and expression of DNMT3a, $\mathrm{HDAC} 1, \operatorname{PPAR} \gamma, \mathrm{C} / \mathrm{EBP} \alpha$, fatty acid synthase and acetylCoA carboxylase were determined. Values were normalized to GAPDH and presented as fold change.
Results: In IUGR adipocytes, prior to induction at day 0, DNMT3a and HDAC1 expression were upregulated as compared to Controls. With induction, both epigenetic modulators showed progressively reduction in expression, beginning as early as day 2. Concomitantly, there was increased expression of adipogenic and lipogenic genes, peaking at day 4 . In contrast, Control adipocytes showed a significantly delayed induction evident at day 4 .

Conclusion: Early induction of adipogenic genes as a result of reduced DNMT3a and HDAC1 likely contributes to enhanced adipogenesis and obesity in IUGR offspring. These results suggest an intrinsic potential for programmed adipogenesis in IUGR offspring, independent of the body hormonal milieu or offspring diet.

Disclosure of Interest: None Declared.

\section{DOHaD13-1706}

Methylation levels of the placental facilitated transporter TAT1 are related to maternal body composition prior to pregnancy

Claire L. Simner ${ }^{1, *}$, Rohan M. Lewis ${ }^{1}$, Hazel M. Inskip ${ }^{2}$, Cyrus Cooper ${ }^{2}$, Mark A. Hanson ${ }^{1}$, Keith M. Godfrey ${ }^{1,2,3}$, Nicholas C. Harvey ${ }^{2}$, Karen A. Lillycrop ${ }^{4}$, Jane K. Cleal ${ }^{1}$ and The Southampton Woman's Survey Study Group

${ }^{1}$ Human Development and Health Academic Unit; ${ }^{2} M R C$ Lifecourse Epidemiology Unit, University of Southampton;

${ }^{3}$ NIHR Southampton Biomedical Research Centre, University of Southampton and University Hospital Southampton NHS

Foundation Trust; ${ }^{4}$ Centre for Biological Sciences, University of Southampton, Southampton, United Kingdom

Background: The facilitated transporter TAT1 (slc16a10) mediates efflux of amino acids from the placenta to the fetus. TAT1 mRNA expression positively relates to several measures of fetal growth in Southampton Women's Survey (SWS) placentas. The mRNA level may be regulated by modification of the DNA methylation status of specific cytosines in $\mathrm{CpG}$ dinucleotides within the promoter region of the TAT1 gene. Epigenetic regulation is known to be influenced by the maternal environment and this study aimed to establish whether methylation levels within the TAT1 promoter region in human placenta relate to its mRNA expression, or are related to maternal body composition.

Method: We used data and samples from the SWS, a cohort study of 3,159 pregnancies with information collected from the mothers before conception. With informed consent and ethical approval placental tissue samples were collected within $30 \mathrm{~min}$ of delivery. Tissue samples used for this analysis were selected from 300 collected in total, based on availability of neonatal data. DNA was extracted from placental samples $(\mathrm{n}=100)$ and bisulphite converted. Regions of the TAT1 promoter containing predicted transcription factor binding 
sites were amplified by PCR and methylation of 2 individual CpG dinucleotides -666 and -687 base pairs from transcription initiation were analysed by pyrosequencing. Quantitative real-time RT-PCR was used to measure TAT1 mRNA expression. Pearson's correlation $\left(\mathrm{r}_{\mathrm{p}}\right)$ was used to explore the relationship between CpG-666 and CpG-687 methylation levels, TAT1 mRNA levels and pre-pregnancy maternal body composition measures.

Results: CpG-687 had higher levels of methylation in male placentas compared to female placentas $(p=0.04)$. The methylation levels of CpG-687, but not CpG-666, were negatively related to maternal $B M I\left(r_{p}=-0.24, p=0.02\right)$, sum of skinfold thickness $\left(r_{p}=-0.20, p=0.05\right)$, estimated fat mass $(\mathrm{kg})\left(\mathrm{r}_{\mathrm{p}}=-0.23, \mathrm{p}=0.02\right)$, mid-upper arm circumference $\left(r_{p}=0.22, \quad p=0.03\right)$ and calf circumference $\left(r_{p}=0.20\right.$, $\mathrm{p}=0.05)$. The methylation levels of the $2 \mathrm{CpG}$ sites did not correlate with TAT1 mRNA expression in term placenta.

Conclusion: This study has shown that the maternal body composition prior to pregnancy is associated with the methylation level of a $\mathrm{CpG}$ site within the placental TAT1 gene promoter. $\mathrm{CpG}$ methylation was not, however, correlated with TAT1 mRNA expression in term placenta although whether the methylation of these sites may have effects at other points of gestation or in other tissues remains to be determined and needs further investigation.

This work is supported by the Gerald Kerkut Charitable Trust

Disclosure of Interest: None Declared.

\section{DOHaD13-1617}

\section{Micro-RNA let7e in plasma as marker of early metabolic dysfunction in pre-pubertal subjects}

Bernardo J. Krause ${ }^{1, *}$, Ivo Carrasco-Wong ${ }^{1}$, Angelica Dominguez ${ }^{2}$, Pilar Arnaiz ${ }^{3}$, Marcelo Farias ${ }^{1}$, Francisco Mardones ${ }^{2}$, Paola Casanello ${ }^{1}$

${ }^{1}$ Division of Obstetrics and Gynecology; ${ }^{2}$ Public Health;

${ }^{3}$ Division of Pediatric, School of Medicine, Pontificia

Universidad Católica de Chile, Santiago, Chile

Background: Metabolic syndrome (MetS) in adults is commonly defined as the concomitance of cardiometabolic alterations including obesity, insulin resistance, glucose intolerance, dyslipidaemia and hypertension. Growing evidence shows that MetS risk can be detected in children however there is no a clear set of markers to diagnose this condition in paediatrics subjects. Recent studies show in adults that altered levels of specific micro-RNAs are related with diseases associated with MetS. Here we determined the plasma levels of four MetS-associated micro-RNAs (i.e. miR126, diabetes; miR132, obesity; mir145, diabetes; miRlet7e, hypertension) in children with or without trait-risk for cardiometabolic alterations.

Method: Trait-risk for MetS was defined in 156 children (10-12 years old) by the presence of altered lipids, glucose and insulin plasma levels, BMI, waist/hip ratio and systemic blood pressure, considering a trait-risk those levels over the $90^{\text {th }}$ centile regarding a reference population. Children were clustered by the presence of none (control), 1, 2 or $3 \mathrm{Met} S$ traits. Neonatal information (birth weight and size, and gestational age) were included in the analysis. Micro-RNAs were isolated from plasma, and levels of miR126, miR132, miR145 and miRlet7e were determined by Taqman qPCR.

Results: Thirty children presented no MetS traits, whilst 49 showed one trait, 42 two traits and 35 three (or more) traits. None of these children presented altered blood pressure. The presence of 1 or more MetS trait was associated to higher weight, BMI and waist/hip ratio. Two or more MetS traits were further associated by higher triglycerides and VLDL plasma levels, and low HDL. Plasma levels of miR126, miR132 and miR145 showed no differences. Levels of miRlet7e ( $\sim 3.4$ fold) were increased in subjects with 3 or more MetS traits. Increased levels of miRlet7e were mainly associated to higher BMI, waist/hip ratio and triglycerides, along with lower HDL. There were no associations between perinatal information and plasma levels of these miRNA. Furthermore, plasma levels of miR126 positively correlated with BMI $(r=0.185, p=0.020)$, waist $/$ hip ratio $(r=0.220$, $\mathrm{p}=0.006)$, triglycerides $(\mathrm{r}=0.175, \mathrm{p}=0.028)$ and VLDL $(\mathrm{r}=0.173, \mathrm{p}=0.030)$, whilst miRlet7e levels showed a negative correlation with HDL $(\mathrm{r}=-0.168, \mathrm{p}=0.035)$.

Conclusion: Increased risk for MetS in children is associated with altered levels of micro-RNAs previously described in adults, but these changes occur in a different sense (miR-let7e increases and miR-126 decreases in adults). This divergence suggest that early metabolic alterations could trigger a micro-RNA-mediated compensatory response. Altogether these results show that changes in miR-let7e and miR-126 levels represent early markers of metabolic dysfunction in children with MetS traits.

\section{References}

1. Li et al., Circulation. 2011;124:175-84

2. Zampetaki et al., Circ Res. 2010;107:810-7

Disclosure of Interest: None Declared.

\section{DOHaD13-1250}

Molecular harbingers of diabetes in the non-human primate

Mei Lyn Ong ${ }^{1, *}$, Peck Yean $\operatorname{Tan}^{2}$, Jan Paul Buschdorf ${ }^{2}$, Walter Stunkel ${ }^{2}$, Paul Robson ${ }^{3}$, Jun-Tao $\mathrm{Li}^{3}$, Niranjan Nagarajan $^{3}$, Louiza Chan ${ }^{2}$, Peter Gluckman ${ }^{2}$, Keefe Chng ${ }^{2}$, Michael Meaney ${ }^{2,4}$, Joanna Holbrook ${ }^{2}$

${ }^{1}$ Singapore Institute of Clincial Sciences (SICS), $A^{*} S T A R$;

${ }^{2}$ Singapore Institute of Clinical Sciences (SICS), $A^{*} S T A R$;

${ }^{3}$ Genome Institute of Singapore (GIS), $A^{*} S T A R$, Singapore, Singapore, ${ }^{4}$ Douglas University Mental Health Institute, McGill University, Quebec, Canada 
Background: Molecular harbingers of diabetes in the non-human primate

Mei-Lyn Ong ${ }^{1 *}$, Tan Peck Yean ${ }^{1 *}$, Jan-Paul Buschdorf ${ }^{1}$, Walter Stunkel ${ }^{1}$, Paul Robson ${ }^{2}$, Jun-Tao $\mathrm{Li}^{2}$, Niranjan Nagarajan $^{2}$, Louiza Chan ${ }^{1}$, Peter Gluckman ${ }^{1}$, Chng Keefe ${ }^{1}$, Michael J Meaney ${ }^{1,3}$, Joanna D Holbrook ${ }^{1}$

*These authors contributed equally

${ }^{1}$ Singapore Institute of Clinical Sciences (SICS), A*STAR, Brenner Centre for Molecular Medicine, 30 Medical Drive, Singapore; ${ }^{2}$ Genome Institute of Singapore (GIS), $A^{*} S T A R$, Genome, 60 Biopolis Street, Singapore; ${ }^{3}$ Douglas University Mental Health Institute, McGill University, Montreal Canada

Background: Evidence from observational and experimental studies links adverse exposures in early life to susceptibility for diabetes in adulthood. We hypothesise that the molecular mechanism whereby early environmental influences are translated into disease risk is epigenetic and that there would be epigenetic marks present at the very early stages of the disease. We developed a cynomologous macaque model of the obese and pre-diabetic state. We surveyed the transcriptome and DNA methylomes of muscle biopsies from pre-diabetic and control animals to find marks associating with the development of insulin resistance. The genome of the cynomologous macaque was recently elucidated enabling discovery of molecular marks via next generation sequencing approaches.

Method: We induce obesity and insulin resistance by exposure to high fat diets. Seven adult macaques in control group were given the standard lab diet (Laboratory FiberPlus Monkey Diet 5049, 14\% fat content, Lab Diet), while seven macaques in pre-diabetic group were given high fat diet (Obesity induced primate diet, 35\% fat content, Altromin). The animals were biopsied six months after diet treatment and muscle tissues were processed for RNA and DNA extraction. The transcriptomes were assayed by RNAseq and the DNA methylomes by reduced representation bisulfite sequencing (RRBS). Tophat and Cufflinks were used to perform genome mapping and transcriptome quantification and Bsmap was used for genome alignment and methylation calling.

Results: Both the RNA-seq and RRBS datasets yielded high quality alignments. Differential marks were detected in both the transcriptomics and methylome datasets. Genes perturbed at both the transcriptomics and epigenetic levels were prioritised. Pathway analysis revealed enrichment in pathways associated with metabolic disease. Data was compared to a novel analogous human dataset from a Singaporean cohort.

Conclusions: Epigenetic marks that have a consequence for the muscle transcriptome are present at the very early stages of diabetes disease progression. The marks may be a consequence of early life environment or later adiposity.

Disclosure of Interest: None Declared.

\section{DOHaD13-1691}

Novel paternal genome effects on placental and fetal phenotype at midgestation

Consuelo Estrella ${ }^{1,2, *}$, Ruidong Xiang ${ }^{1,2}$, Carolyn Fitzsimmons ${ }^{1}$, Zbignew Kruk ${ }^{1}$, Brian Burns ${ }^{3}$, Claire Roberts ${ }^{2,4}$, Stefan Hiendleder ${ }^{1,2}$

${ }^{1}$ School of Animal and Veterinary Sciences, The University of Adelaide, Roseworthy, South Australia; ${ }^{2}$ Robinson Institute, The University of Adelaide, Adelaide, South Australia; ${ }^{3}$ Centre for Animal Science, Queensland Alliance for Agriculture and Food Innovation, The University of Queensland, Rockhampton; ${ }^{4}$ School of Paediatrics and Reproductive Health, The University of Adelaide, Adelaide, South Australia, Australia

Background: Inequality of maternal and paternal genomes due to genomic imprinting and mitochondrial DNA is well established. However, the magnitude of maternal and paternal genome effects on variation in placental and fetal traits remains to be investigated.

Method: We used Bos taurus and Bos indicus dams and sires to generate both purebred and reciprocal cross fetuses $(n=73)$ that were collected at day 153 of gestation. Weights and measurements of placenta, umbilical cord, fetal fluids and fetal organs were analysed by general linear models with main effects of maternal and paternal genome and fetal sex.

Results: Our results show that parental genomes explained significant amounts of variation $(58-100 \%, \quad \mathrm{P}<0.05-$ $0.0001)$ in twenty-two placental and fetal traits. Specifically, while maternal genome largely explains genetic variation in placental traits (90\%) and fetal organ weights (60-98\%), the paternal genome predominantly contributes to variation in umbilical cord weight and length (73\%), placenta (70\%) and umbilical cord (83\%) efficiencies, and total fetal fluids weight (73\%). Moreover, there are significant paternal genomedetermined correlations between a) fetus and umbilical cord weight $(\mathrm{P}<0.0001)$, b) fetal heart and umbilical cord weight $(\mathrm{P}<0.001)$, c) fetal heart weight and umbilical artery $(\mathrm{P}<0.01)$ and vein diameter $(\mathrm{P}<0.05), \mathrm{d})$ fetal lung and total fetal fluids weight $(\mathrm{P}<0.05)$. The umbilical cord is a channel for maternal resource transfer to the fetus. Another reservoir of bioactive compounds the fetus has immediate access to are the fetal fluids. Both characteristics are influenced by the paternal genome, apparently directed to enhancing fetal growth. On the other hand, the maternal genome, possibly via maternally expressed genes, regulates resource allocation to the fetus through changes in placental morphology and weight.

Conclusion: In conclusion, our study shows for the first time, the extent of maternal and paternal genome contributions to placental and fetal traits. Our data support co-existence of parental genetic conflict and maternal co-adaptation as explanations for parent-of-origin effects at the phenotypic level. 


\section{References}

1. Haig D (2004) GENOMIC IMPRINTING AND KINSHIP: How Good is the Evidence? Annual Review of Genetics 38: 553-85.

2. Wolf JB, Hager R (2006) A Maternal-Offspring Coadaptation Theory for the Evolution of Genomic Imprinting. PLoS Biology 4: e380.

Disclosure of Interest: None Declared.

\section{DOHaD13-1341}

Peroxisomal proliferator activated receptor-gammaco-activator-1alpha promoter methylation in blood from children at 5-7 years predicts their adiposity from 9 to 14 years

Rebecca Clarke-Harris $^{1, *}$, Joanne Hosking ${ }^{2}$, Terence J. Wilkin $^{3}$, Jonathan Pinkney ${ }^{2}$, Alison Jeffery ${ }^{2}$, Brad S. Metcalf ${ }^{2}$, Keith M. Godfrey ${ }^{4}$, Linda Voss ${ }^{2}$, Karen A. Lillycrop $^{1}$, Graham Burdge ${ }^{1}$

${ }^{1}$ University of Southampton, Southampton; ${ }^{2}$ University of Plymouth, Plymouth; ${ }^{3}$ University of Exeter, Exeter; ${ }^{4} M R C$ Lifecourse Epidemiology Unit, University of Southampton, Southampton, United Kingdom

Background: The early environment, acting via epigenetic processes, is associated with differential risk of cardio-metabolic disease (CMD) which can be predicted by epigenetic marks in proxy tissues $(1,2)$. Measurements of epigenetic marks in proxy tissues at time points distant from the health outcome or the environmental exposure may be confounded by intervening stochastic and environmental variation. To address this, we used pyrosequencing to measure DNA methylation in the peroxisomal proliferator-gamma-co-activator-1alpha (PCG1a) promoter in blood from 40 children (20 boys) collected annually between 5 and 14 years(3).

Method: We used pyrosequencing to measure the methylation status of individual $\mathrm{CpG}$ loci in a region located between 500 and $900 \mathrm{bp}$ upstream from the transcription start site that has been shown previously to regulate transcription (4). Body composition was measured annually by dual X-ray absorptiometry, physical activity by accelerometery and pubertal timing by age at peak high velocity (APHV). Methylation values were averaged between 5-7 years to produce complete data sets. We used generalised estimating equations to model the association between methylation at 5-7 years and adiposity. We used electrophoretic mobility shift assays to exemplify the effect of differential methylation at one of the $\mathrm{CpG}$ loci that predicted fat mass on transcription factor binding.

Results: Methylation of individual CpG loci in the PGC1a promoter varied from $20.4 \pm 3.5 \%$ to $64.9 \pm 2.8 \%$. There were no significant differences between sexes. The methylation status of these loci was stable between 5 and 14 years (longitudinal tracking coefficients 0.83 to 0.99 ; all $\mathrm{P}<0.001$ ) and there was no significant relationship with neutrophil or lymphocyte populations (all $\mathrm{P}>0.1$ ). Methylation of $4 / 7 \mathrm{CpG}$ loci identified in this region of the PCG1a promoter at 5-7 years was significantly associated with future adiposity up to 14 years. For each $10 \%$ difference in methylation at 5-7 years, per cent body fat differed by $6.3 \%$ to $12.5 \%$ (all $\mathrm{P}<0.03$ ). There were no significant interactions between methylation at any of these four loci and sex $(\mathrm{P} \geq 0.52)$, APHV ( $\mathrm{P} \geq 0.06)$, physical activity $(\mathrm{P} \geq 0.23$ ) or age (all $\mathrm{P} \geq 0.21$. We found that methylation at this locus led to a swapping of PBX-1 partners in a heterodimeric complex resulting in increased binding of the pro-adipogenic HOXB9/PBX-1 heterodimer. This is consistent with a positive relationship between methylation and adiposity in the children. Conclusion: Together these findings support the role of epigenetic changes in early life in the aetiology of CMD and suggest that temporally stable CpG loci measured in childhood may have utility in predicting CMD risk. Furthermore, differential methylation of specific $\mathrm{CpG}$ loci may modify transcription by altering the nature of regulatory transcription factor complexes.

\section{References}

1. Gluckman PD et al. N Engl J Med 2008;359:61.

2. Godfrey KM et al. Diabetes 2011;60:1528.

3. Voss LD et al. J Pediatr Endocrinol Metab 2003;16:1211.

4. Ling C et al. Diabetologia 2008;51:615.

Disclosure of Interest: None Declared.

\section{DOHaD13-1374}

Pregnancy complications and duration of pregnancy affect umbilical cord blood cell populations: implications for future epigenetic studies

Emilie Herzog ${ }^{1, *}$, Marten van der Zee ${ }^{1}$, Alex Eggink ${ }^{1}$, Jacqueline Lagendijk ${ }^{1}$, Jingzhen Hou ${ }^{1}$, Robert de Jonge ${ }^{2}$, Eric Steegers ${ }^{1}$, Régine Steegers-Theunissen ${ }^{1}$

${ }^{1}$ Obstetrics and Gynaecology; ${ }^{2}$ Clinical Chemistry, Erasmus $M C$, University Medical Centre Rotterdam, The Netherlands, Rotterdam, Netherlands

Background: Alterations in the number of umbilical cord blood (UCB) cells (monocytes, neutrophils, erythrocytes) have been reported in immune-mediated pregnancy complications, such as preeclampsia (PE) and foetal growth restriction (FGR). Haematopoiesis occurs in concurrent stages as pregnancy advances. However, PE and FGR complicated pregnancies often end preterm. Therefore, our objective was to investigate whether the number and distribution of UCB cells are related to PE, FGR, spontaneous preterm birth (PTB) and or the duration of pregnancy.

Method: In the Rotterdam Predict study, a hospital-based cohort study, we conducted a case-control study of 163 pregnancies consisting of pregnancy complications $(n=48)$ comprising of $\mathrm{PE}$ 
$(n=15)$, FGR $(n=19)$, and preterm birth (PTB) $(n=14)$ and uncomplicated pregnancies $(\mathrm{n}=115)$. UCB samples were subjected to flow cytometric analysis within 48 hours after delivery (Sysmex XE-5000, Etten-Leur, The Netherlands) to quantify erythrocytes, thrombocytes and leucocyte differential. ANOVA testing was applied for statistical analysis.

Results: The UCB monocyte count was lower in PE (mean $0.96 \times 10^{9} / \mathrm{L}$, standard deviation 0.39$)(\mathrm{p}=0.022)$ and PTB $\left(0.93 \times 10^{9} / \mathrm{L}, 0.41\right)(\mathrm{p}=0.025)$ than in uncomplicated pregnancies $\left(1.38 \times 10^{9} / \mathrm{L}, 0.53\right)$. This also applied to the neutrophil count in PE $\left(5.17 \times 10^{9} / \mathrm{L}, 3.28\right)(\mathrm{p}=0.046)$ and $\mathrm{PTB}$ $\left(3.90 \times 10^{9} / \mathrm{L}, 3.24\right)(\mathrm{p}=0.001)$ compared to uncomplicated pregnancies $\left(7.00 \times 10^{9} / \mathrm{L}, 2.32\right)$. Nucleated red blood cells (NRBC) per 100 white blood cells were only higher in PE $\left(11.29 \times 10^{9} / \mathrm{L} / 100 \mathrm{WBC}, 7.01\right)(\mathrm{p}=0.013)$ than in uncomplicated pregnancies $\left(6.29 \times 10^{9} / \mathrm{L} / 100 \mathrm{WBC}, 5.22\right)$. The number of UCB thrombocytes was nearly significantly decreased in PE $\left(212.25 \times 10^{9} / \mathrm{L}, 86.81\right)(\mathrm{p}=0.051)$ compared to uncomplicated pregnancies ( $\left.266.37 \times 10^{9} / \mathrm{L}, 70.28\right)$.

Stratified analysis for term PE deliveries only, showed no statistical differences with the uncomplicated pregnancies.

Conclusion: In this study, we demonstrate that PE and PTB are associated with lower numbers of UCB leucocytes (monocytes, neutrophils). The duration of pregnancy, however, showed the strongest effect on the number of these cells. The higher number of NRBC in PE patients suggests an enhanced red blood cell production, which may be due to PE-associated inflammation. Since the duration of pregnancy has a critical influence on the quantity of UCB cell populations, the number and distribution of these cells should be taken into account in future studies on epigenetic programming and pregnancy complications.

Disclosure of Interest: None Declared.

\section{DOHaD13-1090}

\section{Prenatal glucocorticoid overexposure may accelerate maturation of the heme pathway through effects on transcription and DNA methylation in rat liver}

Khulan Batbayar ${ }^{1, *}$, Lincoln Liu ${ }^{1}$, Jonathan R. Manning ${ }^{2}$, Ashley K. Boyle ${ }^{1}$, Catherine M. Rose ${ }^{1}$, Donald R. Dunbar ${ }^{2}$, Jonathan R. Seckl ${ }^{1}$, Amanda J. Drake ${ }^{1}$

${ }^{1}$ Endocrinology Unit; ${ }^{2}$ BHF Centre of Research Excellence Bioinformatics Facility, University/BHF Centre for Cardiovascular Science, University of Edinburgh, Queen's Medical Research Institute, Edinburgh, United Kingdom

Background: Human epidemiological and animal studies have shown an association between early life events and an increased risk of cardiovascular, metabolic, and neuroendocrine disorders in adulthood; termed "developmental programming". Although the underlying mechanisms of programming are not completely elucidated, epigenetic dysregulation is increasingly recognized as an important mediator of heritably maintained gene expression changes. There are two major hypotheses to explain the association of low birthweight with later cardiometabolic disease risk: fetal malnutrition and overexposure to glucocorticoids. We have developed an animal model of glucocorticoid programming in rats in which administration of the synthetic glucocorticoid Dexamethasone (Dex) results in low birthweight and the development of hypertension and insulin resistance in adulthood. In this study we performed systematic transcriptome profiling in this model and explored associations between gene expression and DNA methylation changes at candidate genes.

Method: Pregnant female Wistar rats were injected subcutaneously with either $100 \mathrm{mcg} / \mathrm{kg}$ Dex or Saline (Sal) daily during the last third of gestation. Fetal liver samples were collected at embryonic day 20 from Dex and Sal offspring for total RNA and DNA extraction. Transcriptome analysis was performed using Illumina RatRef-12 Expression Beadchip Arrays (3 biological replicates per group) and locus specific quantitative RT-PCR assays (8 biological replicates per group). DNA methylation was analyzed using Pyrosequencing.

Results: 134 genes were differentially expressed between Dex and Sal offspring among which many changes were relevant to early maturation processes. Pathway analysis revealed marked changes in heme biosynthesis, utilization and degradation pathways. Quantitative RT-PCR validation confirmed that the hepatic expression of 3 genes involved in heme biosynthesis, Alad, Urod and Cpox, and one gene involved in heme degradation, Blvrb, were decreased at least 1.7 fold $(p<0.01)$, whereas the expression of Cyp2c23, involved in heme utilization, was increased 1.9 fold $(\mathrm{p}<0.001)$ following prenatal dex. There was a trend for a decrease in the expression of $\mathrm{Hmbs}$ which is involved in biosynthesis ( 1.5 fold; $p=0.08$ ). The timing and the direction of these changes, which coincides with a switch of hematopoiesis from liver to bone marrow, suggests accelerated maturation. This observation is supported by increased DNA methylation at multiple CpGs within the erythroid-specific alternative promoters of Alad and Hmbs and with decreased methylation at the Cyp2c23 promoter in Dex exposed animals.

Conclusion: Prenatal glucocorticoid programming is associated with changes in liver gene expression in the late-gestation fetus predicting accelerated maturation. These results support the concept that programmed effects on gene expression associate with altered promoter methylation.

Disclosure of Interest: None Declared.

\section{DOHaD13-1074}

Presence of NF-Y as an epigenetic element on regulatory regions of ID genes in human embryonic carcinoma cells Farideh Moeinvaziri ${ }^{1,2, *}$, Raha Favaedi ${ }^{1}$, Maryam Shahhoseini ${ }^{1}$

${ }^{1}$ Department of Genetics at Reproductive Biomedicine Research Center, Royan Institute for Reproductive Biomedicine, ACECR; 


\section{${ }^{2}$ Department of Developmental Biology, University of Science and Culture, ACECR, Tehran, Iran, Islamic Republic}

Background: Epigenetic mechanisms, such as incorporation of histone variants and substitutes, have been shown to play key roles in regulation of gene transcription. Among them, NF-Y, a histone substitute protein, which specifically binds to the CCAAT box, has a significant role in chromatin remodelling of their target genes such as $I D$ (ID1-ID4), a basic helix-loop-helix family genes. ID patterns are important part of signalling pathways involved in development by inhibiting differentiation and stimulating proliferation. Embryonal carcinoma (EC) cells derived from testicular tumors are valuable tools for investigating embryogenesis and developmental biology processes. These cells do provide an amenable and more simplistic experimental model to understand the epigenetic changes and maybe therapeutic purposes in embryology and developmental process.

Method: In this study, the chromatin immunoprecipitation coupled with real-time PCR was performed using anti-NF-Y antibody on chromatin extract from a humanembryonal carcinoma cell line, named NT2/NTERA2, to evaluate incorporation levels of NF-Y on the regulatory region of ID genes.

Results: The results of ChIP real-time PCR analysis clearly showed a significant incorporation of NF-Yon the regulatory region of ID1, ID2 and ID3 in NT2 cells. Due to loss of CCAAT box in promoter regulatory region of ID4 gene, NF-Y protein did not show any incorporation to the regulatory region of this gene.

Conclusion: The current finding implies the dynamic epigenetic role of NF-Y in regulation of IDs which are involved in embryonic development.

\section{References}

1. Matuoka K., Yu Chen K., (1999), Nuclear Factor Y (NF-Y) and Cellular Senescence, Exp Cell Res. 253: 365-371. 2. Dolfini D., Gatta R., Mantovani R., (2012), NF-Y and the transcriptional activation of CCAAT promoters, Crit Rev Biochem Mol Biol., 47: 29-49.

3. Norton J.D., (2000), ID helix-loop-helix proteins in cell growth, differentiation and tumorigenesis, J Cell Sci. 113, 3897-3905.

4. Iavarone A., Lasorella A., (2006), ID proteins as targets in cancer and tools in neurobiology, Trends Mol Med. 12:588-94.

5. Romero-Lanman E.E., Pavlovic S., Amlani B., Chin Y., Benezra R., (2012), Id1 maintains rmbryonic stem cell selfrenewal by up-regulation of nanog and repression of brachyury expression, Stem Cells Dev. 21:384-93.

6. Przyborski S.A., Christie V.B., Hayman M.W., Stewart R., Horrocks G.M., (2004), Human embryonal carcinoma stem cells: models of embryonic development in humans. Stem Cells Dev. 13: 400-8.
7. Shahoseini M., Taei A., Mehrjardi N.Z., Salekdeh G.H., Baharvand H., (2010), Epigenetic analysis of human embryonic cells during RA-induced neural differentiation. Biochem Cell Biol. 88: 527-38.

Disclosure of Interest: None Declared.

\section{DOHaD13-1274}

The impact of parental environment on embryonic development: methylome and transcriptome analysis on the same embryos

Marc A. Sirard ${ }^{1, *}$, Claude Robert ${ }^{1}$, Beatrice deMontera ${ }^{1}$, Habib Shojaei Saadi ${ }^{1}$, Dominic Gagne ${ }^{1}$

${ }^{1}$ Sciences Animales, Université Laval, Quebec, Canada

Background: Early embryonic development represents a very sensitive developmental window during which cells are dynamically remodeled. This remodeling involves erasure and reprogramming of the gametes, which will determine cell differentiation into various lineages. Stresses applied during this period are thus expected to create considerable impacts on embryo development. Incidentally, most reproductive technologies, such as in vitro production, take place during this period and are thus expected to impact embryo development and possibly carry to them to the resulting offspring. Evidences of the impact of the in vitro nutritional environment on developmental rates and the transcriptome have indeed been presented but the mechanisms involved remain unknown. Epigenetics factors are starting to attract attention as possible effectors in these events for their involvement with environmental stress-induced impacts. DNA methylation marks could be carried through cellular divisions and thus provide a mean for long term impacts to be translated.

Method: We set out to develop a unique platform for the study of bovine early embryos methylome. This microarray based platform is based on a subtractive approach comparing Day-7 and Day-12 methylomes to ensure a relevant genomic representation in the oligo set $(400,000$ targets). The platform was then tested in a study measuring the impacts of the addition of methyl donor (S-adenosyl methionin) in the embryonic microenvironment at the transcriptomic and epigenetic (DNA methylation) levels.

Results: Global hypermethylation was observed in treated embryos. A list of differentially expressed genes and long noncoding RNAs was also identified. Targeted cellular functions and molecular networks also showed similarities between datasets. This protocol allows a genome wide direct comparison between gene expression and methylation status. Conclusion: Considering the challenges of tissue limitation, and in an effort to lower cost per sample, The EmbryoGENE DNA Methylation Array (EDMA) was developed. With the help of its streamlined data analysis pipeline, it allows for a rapid high-resolution mapping of global DNA methylation 
using the genetic material of only 10 expanded blastocysts (7-8 ng of gDNA). These platforms will now serve to highlight the embryo response to environmental insults from both the application of assisted reproductive technologies and from maternal nutritional conditions.

Disclosure of Interest: None Declared.

\section{DOHaD13-1098}

Customized versus population based birth weight curves: does it make a difference for ethnic groups?

Tanja Vrijkotte ${ }^{1, *}$, Jason Gardosi ${ }^{2}$, Alike de Roon-Immerzeel ${ }^{3}$, Karien Stronks ${ }^{1}$, Wessel Ganzevoort ${ }^{4}$

${ }^{1}$ Public Health, Academic Medical Center, Amsterdam, Netherlands; ${ }^{2}$ Perinatal Institute, Birmingham, United Kingdom;

${ }^{3}$ Scientific Department/Guideline Development, Dutch Organisation of Midwifes, Utrecht; ${ }^{4}$ Obstetrics and Gynaecology,

Academic Medical Center, Amsterdam, Netherlands

Background: Fetal growth restriction as well as fetal overgrowth are both associated with adverse perinatal outcome. To identify children at both ends of the growth spectrum, it is necessary to determine the optimal fetal growth. In current obstetric practice, population based (parity and sex specific) as well as customized (additionally adjusted for maternal height, pre-pregnancy weight and ethnic origin) birth weight reference curves are used to define the fetal growth potential. With increasing number of pregnant women with a migration background with distinct differences in maternal height and weight, it is important to know whether there are ethnic differences in the prevalence of small-for-gestational age (SGA; $<10^{\text {th }}$ percentile) and largefor-gestational-age (LGA; $>90^{\text {th }}$ percentile) babies, based on the two types of reference curves.

Method: We applied the customised (CUST; based on Gardosi curves ${ }^{1}$ ) and the population (POP; based on PRN curves) reference curves on the pregnancy outcome data from the multi-ethnic ABCD cohort (inclusion 2003-2004 in Amsterdam, pregnancy duration $<25$ weeks excluded, $\mathrm{n}=7826)^{2}$ Ethnic groups were: Dutch $(\mathrm{n}=3850)$, Surinamese-Hindustani $(n=131)$, Surinamese-Creole $(n=197)$, Surinamese-other $(\mathrm{n}=354)$, Turkish $(\mathrm{n}=410)$, Moroccan $(\mathrm{n}=720)$, Ghanaian $(\mathrm{n}=177)$ and other $(\mathrm{n}=1987)$.

Results: Based on CUST-curves, the SGA prevalence in the Dutch group was $8.8 \%$ and varied between $7.4 \%$ (Turkish) and $15.3 \%$ (Ghanaian). Based on POP-curves, this number was the lowest in the Dutch groups $(7.4 \%)$ and the highest in the Surinam-Hindustani group (29.8\%). LGA prevalence was lowest in the Dutch group (10.8\%) and highest in all Surinamese groups (around 17\%) when based on CUSTcurves. Based on POP-curves, LGA prevalence was the highest in the Dutch group (10.5\%) and lowest in the Surinamese-Creole group (4.1\%).
Conclusion: There are large differences in ethnic specific prevalences in SGA and LGA, depending on the applied reference curve. When customised curves are applied, ethnic differences in SGA largely disappear as LGA differences become more prominent. The higher prevalence of LGA in especially Surinamese women is in line with their known more adverse metabolic profile.

\section{References}

1. Gardosi J., Figueras F., Clausson B. and Francis A. The customised growth potential: an international research tool to study the epidemiology of fetal growth. Paediat and Perinatal Epidem. 2010;25:2-10.

2. Eijsden van M., Vrijkotte T.G.M., RJBJ Gemke and Wal, van der M.F. Cohort Profile: The Amsterdam Born Children and their Development (ABCD) Study. Int J Epidemiol 2010.

Disclosure of Interest: None Declared.

\section{DOHaD13-1683}

Diet patterns among low income women of reproductive age in Mumbai

Sarah Kehoe ${ }^{1, *}$, Devi Shivshankaran ${ }^{2}$, Harsha Chopra ${ }^{2}$, Sirazul Sahariah ${ }^{2}$, Meera Gandhi ${ }^{2}$, Harshad Sane ${ }^{2}$, Vanessa Cox ${ }^{1}$, Patsy Coakley ${ }^{1}$, Barrie Margetts ${ }^{1}$, Ramesh Potdar ${ }^{2}$, Caroline Fall ${ }^{1}$

${ }^{1}$ University of Southampton, Southampton, United Kingdom; ${ }^{2}$ Centre for Study of Social Change, Mumbai, India

Background: Poor nutritional status of young women before and during pregnancy can result in sub-optimal fetal development. National survey data indicate that in 2005-6 approximately a quarter of women living in Mumbai slums were chronically undernourished (body mass index $(\mathrm{BMI})<18.5 \mathrm{~kg} / \mathrm{m}^{2}$ ) and $46 \%$ had iron deficiency anaemia $(\mathrm{Hb}<12 \mathrm{~g} / \mathrm{dL})$. There is evidence of low intakes of micronutrient-rich foods such as fruit among women of reproductive age living in Mumbai slums, yet little is known about dietary patterns in this population. Our objective was to identify diet patterns adhered to by low income slum-dwelling women who were planning to become pregnant. We also aimed to study socio-demographic factors that may be associated with diet patterns as well as look at the effect of diet patterns on BMI.

Method: Participants were 6513 women aged $25 \pm 4$ years registered in the Mumbai Maternal Nutrition Project (MMNP). We collected demographic and anthropometric data from the women at registration. A trained project assistant administered a 212-item food frequency questionnaire (FFQ) with a reference period of the past week. The FFQ foods were condensed to 40 food groups and a principal component analysis of the food group variables was conducted. We used univariate and multivariate regression 
models to study associations between pattern scores, BMI and demographic variables (age, religion, education, occupation and standard of living).

Results: The women's median (IQR) BMI was 20.0 (17.9, 22.9) $\mathrm{kg} / \mathrm{m}^{2}$. The diet pattern that accounted for most variance $(8.7 \%)$ was characterised by frequent intakes of snacks, fruit and desserts ('snack and fruit' pattern). The second pattern explained $5.3 \%$ of the variance and was characterised by frequent intakes of biriyani (a rice and meat dish) and mutton and low intakes of legumes and coconut ('non-vegetarian' pattern). The third pattern was named 'fish and coconut' as it was characterised by frequent intakes of these foods and low intakes of milk. Adherence to the 'snack and fruit' pattern was positively associated with skill level of occupation $(p=0.001)$ and standard of living $(p=0.003)$. Adherence to the non-vegetarian pattern was associated with younger age, being Muslim, lower education status and lower standard of living. The 'fish and coconut' pattern was positively associated with education and SLI score $(\mathrm{p}<0.001)$. BMI was positively associated with snack and fruit and non-vegetarian scores $(p<0.01)$. There was a negative association between fish and coconut scores and BMI but this was not statistically significant $(\mathrm{p}=0.118)$.

Conclusion: This study provides new information on diet patterns among low income Indian women. These patterns are different to those frequently observed in high income countries as there is no obvious 'healthy' pattern. This indicates that factors that affect dietary intakes are likely to differ by setting. Adherence to the patterns was related to BMI, age, religion, education and socio-economic status. This information can be used to target dietary interventions to at-risk-groups more effectively and thus improve the nutritional status of young women in low income settings leading to more favourable birth outcomes and reduced risk of chronic disease in the next generation.

Disclosure of Interest: None Declared.

\section{DOHaD13-1653}

Does fructosamine in early pregnancy explain the association between maternal pre-pregnancy bmi and birth weight in a multi-ethnic population?

Adriëtte Oostvogels ${ }^{1, *}$, Nicole Van Melsen ${ }^{2}$, Joke Koelewijn ${ }^{2}$, Manon Van Eijsden ${ }^{3}$, Tanja Vrijkotte ${ }^{1}$

${ }^{1}$ Public Health; ${ }^{2}$ Obstetrics and Gynaecology, AMC; ${ }^{3}$ Epidemiology, Documentation and Health Promotion, Public Health Service, Amsterdam, Netherlands

Background: It is known that maternal pre-pregnancy body mass index (pBMI) is positively associated with birth weight. However we do not know if this association is ethnic specific as a consequence of differences in glycemic control. Fructosamine is the level of glycosylated protein and consists mainly of glycosylated albumin and is considered a measure of short glycemic control (2-3 weeks), both inside and outside pregnancy. We assessed the association between maternal pBMI and birth weight for different ethnic groups and determined if differences in fructosamine levels in early pregnancy, could explain possible differences in these associations.

Method: Data from the prospective multi-ethnic ABCDstudy was used. Ethnicity was based on the country of birth of the pregnant woman's mother: Dutch (reference; $\mathrm{n}=4068$; blood $n=2322)$, Turkish $(n=386$; blood $n=160)$, Moroccan $(n=664$; blood $n=228)$, Black Caribbean $(n=218$; blood $n=108)$, Ghanaian $(n=156$; blood $n=48)$, Hindu $(\mathrm{n}=105$; blood $\mathrm{n}=45)$. pBMI was self-reported in the pregnancy questionnaire (median: 16 weeks of gestation; IQR:14-18 weeks), birth weight was obtained from the national obstetric registry and the Child Health Care Registration. Non-fasting fructosamine levels (subsample) were assessed in blood (median: 13 weeks of gestation; IQR 12-14 weeks). Only singleton pregnancies, not complicated by hypertensive disorders were included. We adjusted for gestational age at blood sampling, maternal age, education and height, smoking, alcohol and physical activity during pregnancy, parity and sex of the child. We used multivariate linear models. Ethnic specific associations were tested with interaction terms, followed by stratified analyses.

Results: In adjusted models, we found an ethnic specific association between $\mathrm{pBMI}$ and birth weight ( $\mathrm{p}$ for interaction $<0.05)$. Every unit increase in pBMI was associated with an increase in birth weight of 17.8 grams in Dutch women $(p<0.001), 10.8$ grams in Turkish women $(p<0.05), 14.8$ grams in Moroccan women $(p<0.001)$ and 29.9 grams in Hindu women $(\mathrm{p}<0.01)$. Dutch women had mean fructosamine levels of $222.4 \mu \mathrm{mol} / \mathrm{L}$ (SD: $17.5 \mu \mathrm{mol} / \mathrm{L}$ ). Turkish, Moroccan and Hindu women had significant lower fructosamine levels $(\mathrm{p}<0.01)$. $\mathrm{pBMI}$ was in general associated with lower fructosamine levels, with small differences between the ethnic groups ( $p$ for interaction $<$ $0.01)$. Every unit increase in pBMI was associated with a decrease in fructosamine levels in Dutch women of $1.3 \mu \mathrm{mol} /$ $\mathrm{L}(\mathrm{p}<0.001)$, in Turkish women of $1.1 \mu \mathrm{mol} / \mathrm{L}(\mathrm{p}<0.01)$, in Moroccan women of $0.8 \mu \mathrm{mol} / \mathrm{L}(\mathrm{p}<0.01)$ and in Black Caribbean women of $0.9 \mu \mathrm{mol} / \mathrm{L}(\mathrm{p}<0.05)$. Higher fructosamine levels were only associated with lower birth weight in Moroccan women $(\beta=-4.6$ grams, $\mathrm{p}<0.01)$.

Conclusion: Increased pBMI is associated with increased birth weight and decreased fructosamine levels in early gestation, with ethnic specific associations. Despite ethnic differences in fructosamine levels, fructosamine is not associated with birth weight, except for Moroccan women. These results suggest that glycemic control in early pregnancy is not associated with birth weight. Therefore fructosamine in early pregnancy cannot explain the ethnic specific association between pBMI and birth weight.

Disclosure of Interest: None Declared. 


\section{DOHaD13-1415}

Ethnicity, education and marital status are associated with weight gain in pregnancy

Amal Khanolkar ${ }^{1 *}$, Gillian E. Hanley ${ }^{2}$, Ilona Koupil ${ }^{1}$, Patricia A. Janssen ${ }^{2}$

${ }^{1}$ Centre for Health Equity Studies, Karolinska Institutet, Stockholm, Sweden; ${ }^{2}$ Public Health and the Child and Family Research Institute, University of British Columbia,

Vancouver, Canada

Background: Maternal overweight/obesity and adverse weight gain in pregnancy are associated with detrimental pregnancy outcomes in both mother and infant. The Institute of Medicine (IOM) recommended revised guidelines for weight gain in pregnancy in 2009. Our aim was to investigate ethnic differences in weight gain in pregnancy according to the guidelines and if differences could be explained by maternal education and marital status.

Method: We used the Washington Birth Events Record Database (BERD) to conduct an observational cohort study on all singleton pregnancies (2006 to 2008, $n=209587$ ) in Washington State. Exposures included self-reported ethnicity (Black, Native American, Asian, Hispanic, and Indian), maternal education $\left(12^{\text {th }}\right.$ grade or less, High school graduate, some college, Associate degree, Bachelor's degree and Master's/PhD degree) and marital status. Using the 2009 IOM guidelines for weight gain in pregnancy, mothers were categorized into low, appropriate and high weight gain groups. Associations between 1)IOM weight gain groups and pregnancy outcomes and 2)ethnicity, education and marital status and weight gain groups were analysed using multivariate- and multinomial-logistic regression respectively. Analyses were adjusted for maternal age, parity, smoking, gestational hypertension, diabetes, BMI and number of prenatal visits.

Results: Gaining too much weight in pregnancy was associated with increased odds for gestational hypertension (OR 1.93, 95\% CI 1.83-2.03), preeclampsia or eclampsia $(1.90,1.77-2.03)$, caesarean-delivery $(1.36,1.33-1.40)$, and extended hospital stay $(1.15,1.12-1.18)$. White mothers gained more weight in pregnancy than any other ethnic group. Black and Indian mothers had the highest relative risk ratios (RRR) for gaining less weight than recommended compared to white mothers (adjusted RRR 1.35, 1.30-1.43 and 1.37, 1.27-1.50 respectively). Hispanic, Indian and Asian mothers had the lowest risk for gaining too much weight in pregnancy (adjusted RRR 0.67, 0.64-0.70, 0.73, $0.67-0.80$ and $0.74,0.71-0.77$ respectively). Adjustment for education/marital status marginally reduced the ethnicityweight gain RRR in Black, Native-American and Hispanic groups. Maternal educational level was inversely associated with gaining too much or too little weight in pregnancy with higher educated mothers most likely to gain within recommendations. Mothers with Bachelor and post-graduate degrees had a significantly lower risk of gaining too little compared to least educated mothers (adjusted RRR 0.70, $0.67-0.74$ and $0.66,0.63-0.70$ respectively). In stratified analysis, a linear association with education was observed within all ethnic groups, with highest educated mothers having lowest risk for gaining too little weight.

Conclusion: Both ethnicity and education independently predict weight gain in pregnancy. Lower educated mothers, regardless of ethnicity are at a higher risk for inappropriate weight gain. Public health interventions could specifically target all lower educated mothers to decrease both short-term and long-term consequences of inappropriate weight gain in pregnancy.

Disclosure of Interest: None Declared.

\section{DOHaD13-1591}

Obesity and overweight in children from a region of Italian immigration in Southern Brazil

Lucia C. Pellanda $^{1,2, *}$, Renata Geremia ${ }^{1}$, William B. de Souza $^{3}$, Hosana Cimadon ${ }^{4}$

${ }^{1}$ Post graduation program, Fundação Universitária de Cardiologia do Rio Grande do Sul (FUC RS); ${ }^{2}$ Public Health; ${ }^{3}$ Curso de Medicina, Universidade Federal de Ciências da Saúde de Porto Alegre (UFCSPA), Porto Alegre; ${ }^{4}$ Curso de Nutriçáo, FEEVALE, Novo Hamburgo, Brazil

Background: The increasing prevalence of obesity among children is a significant public health problem both in developed as in developing countries. The main modifiable risk factors for obesity are related to lifestyle, such as dietary habits and sedentary behavior, are significantly influenced by the family and environment. Therefore, local culture and traditions can be an important factor in this scenario. Migration patterns offer the opportunity to study interactions between the original and local cultures. Thus, the objective of the present study was to estimate the prevalence of overweight/obesity and associated lifestyle factors in children from Bento Gonçalves, a southern Brazil city with strong Italian immigration influence.

Method: Cross-sectional study of a population-based cluster sample, totaling 590 students aged 9-18 years. Questionnaires were applied to assess lifestyle based on time spent in sedentary behavior (TV/video game/computer), food frequency questionnaire and weekly hours of physical activity (supervised or unsupervised physical activity), and to assess family history. Also, school children underwent physical, anthropometric examination and measurement of blood pressure. Overweight and obesity were evaluated according to WHO percentile curves.

Results: A total of 590 students were evaluated. Mean age was $12.45 \pm 1.49$ years. The prevalence of overweight and obesity was $16.3 \%$ and $8.3 \%$ respectively $(16.2 \%$ and $5.5 \%$, 
among girls and $16.3 \%$ and $12.2 \%$, for boys). High blood pressure was observed in $11.1 \%$ of students, sedentary behavior (physical activity $<3$ times per week) in $52.3 \%$. Mean screen time was $5.38 \pm 2.88$ hours/day (including television, sedentary videogames and computer activities). Low consumption (less than 4 times per week) of vegetables and fruits was observed in $49.5 \%$ and $36.8 \%$, respectively. Overweight and obesity were more frequent among fathers $(62.8 \%)$ than in mothers $(46.3 \%)$. The prevalence of systemic high blood pressure was higher in obese $(30.6 \%)$ and overweight $(21.2 \%)$ children, comparing to eutrophic children $(6.8 \% ; p<0.001)$. Excess weight in mothers, but not fathers, was positively associated with excess weight in children. ( $p$ 0.048).

Conclusion: The city showed high prevalences of overweight and obesity. Since this is a city with a strong Italian cultural influence and with an agriculture-based economy, it was expected that health habits would be more adequate when compared to others regions of Brazil. However, we observed characteristics of an urban center sedentary lifestyle, low frequency of consumption of vegetables and high levels of screen time. Italian traditional foods were locally adapted since the immigrants' arrival in the XIX century, to include more fat and less vegetables, and physical activity levels are in decline among this population. These findings reinforce the importance of implementing prevention strategies aimed at children and their families, considering that health habits are shared and transmitted along generations

Disclosure of Interest: None Declared.

\section{DOHaD13-1718}

\section{Predictors of birth weight (BW) in a multiethnic British population}

H. Venkataraman ${ }^{1, *}$, N. Sukumar ${ }^{1}$, J. Wilson ${ }^{1}$, K. Khunti ${ }^{2}$, P. Saravanan ${ }^{1}$

\section{${ }^{1}$ Warwick Medical School, University of Warwick, Coventry; ${ }^{2}$ University Of Leicester, Leicester, United Kingdom}

Background: BW is a health indicator and is associated with metabolic outcome in adulthood. Several factors have been shown to affect birth weight across different populations. Customized growth charts correct for these 'physiological variables' by using fixed coefficients, to identify pathological BW outcomes. We studied a large population in the UK to analyse the predictors of BW in White Caucasians (WC) and South Asians (SA).

Method: Retrospective data was collected for 88,606 singleton pregnancies in Leicester, UK, between 1996-2006. 55,128 term pregnancies (37-42 weeks) were studied after excluding missing data, stillbirths \& preterm babies. We studied 13 variables: maternal age, height, weight, systolic and diastolic blood pressure (BP), ethnicity, deprivation (IMD-index of multiple deprivation), rural/urban status, gravidity, smoking status, presence of diabetes, gestational age and sex. IMD was analysed as quintiles. Multivariable linear and logistic regression analysis with interaction terms was used. Backward selection was used to build the model

Results: 44,661 (84.05\%) of the population were WC and $8,471(15.95 \%)$ were SA. SA mothers were lighter (BMI $23.5 \pm 4.6$ vs $\left.24.9 \pm 5 \mathrm{~kg} / \mathrm{cm}^{2}\right)$, shorter $(158.2 \pm 6.3$ vs $164.3 \pm 6.7 \mathrm{~cm})$, younger $(28.1 \pm 5$ vs $28.3 \pm 5.8 \mathrm{yrs})$, more deprived (IMD 27.8 vs 20.4) and had a lower systolic BP $(108.6 \pm 12.8$ vs $112.4 \pm 12.4)$ ( $p<0.001$ for all). Median IMD score was 15.58 (Caucasian: 13.54, Asian: 28.21; $\mathrm{p}<0.001)$. BW was higher in WC than SA $(3444.5 \pm 487.6$ vs $3102.4 \pm 453.4 \mathrm{~kg}, \mathrm{p}<0.001)$.

All the above variables were associated independently with BW $(\mathrm{p}<0.001)$, except urban index and diastolic BP. Together, these variables account for $30.6 \%$ of variation in BW. Gestational age is the major contributor, contributing to $15.4 \%$ whilst ethnicity and sex accounted for $6.3 \%$ and $1.7 \%$ respectively.

Smoking, deprivation and female sex had a negative impact on BW. In SAs, being female and more deprived, had a smaller negative impact on the BW $(\mathrm{p}<0.001$ and $\mathrm{p}=0.007)$ whereas gravidity had a greater positive impact on the BW ( $p=0.009)$, compared to WC.

The overall prevalence of low birth weight (LBW) $(\mathrm{BW}<$ $2.5 \mathrm{Kg})$ was $3.3 \%(\mathrm{n}=1753$; SA vs WC: 8.1 vs $2.3 \%$; $\mathrm{p}<0.001)$. All variables were independently associated with the risk of LBW $(\mathrm{p}<0.001)$ except diastolic BP and Urban status. SA had higher odds of having a LBW baby (OR: 2.2 SA vs WC, $\mathrm{p}<0.001)$ after adjusting for other variables. The effect of sex $(p=0.02)$ and gravidity $(p<0.001)$ on LBW was lower in Asians compared to WC.

The prevalence of macrosomia (BW $>4 \mathrm{Kg}$ ) was $11 \%$ overall, $2.9 \%$ in SA \& $12.6 \%$ in WC, $(p<0.001)$. SA had significantly lower odds of macrosomia (OR 0.35 SA vs WC, $\mathrm{p}<0.001)$. All variables other than BP and Urban status were significantly and independently associated with macrosomia. The effect of maternal weight on macrosomia was higher in SA compared to WC ( $\mathrm{p}=0.001)$.

Conclusion: These factors explain only a third of BW variation implying that the large proportion of factors affecting BW are still unknown.

Ethnicity not only independently affects BW, but also modifies the effect of other variables on BW. Being SA seems to protect against LBW and favors macrosomia compared to WC of comparable characteristics. Deprivation seems to disadvantage WC more than SA.

This supports the need for further research and use of separate customized charts for each ethnic group rather than single charts with common correction factors.

Disclosure of Interest: None Declared. 


\section{DOHaD13-1441}

The association of foetal growth rate with systolic blood pressure in young adulthood is modified by ethnicity

Amal Khanolkar ${ }^{1,2, *}$, Sara WEDRÉN ${ }^{1}$, George Ploubidis ${ }^{3}$, Pär Sparen ${ }^{4}$, Ilona Koupil ${ }^{2}$

${ }^{1}$ Institute of Environmental Medicine, Karolinska Institutet;

${ }^{2}$ Centre for Health Equity Studies, Stockholm University/

Karolinska Institutet, Stockholm, Sweden; ${ }^{3}$ Faculty of

Epidemiology \& Population health, London School of Hygiene

and Tropical Medicine, London, United Kingdom; ${ }^{4}$ Medical

Epidemiology and Biostatistics, Karolinska Institutet,

Stockholm, Sweden

Background: Previous studies on the association of adverse foetal growth and higher systolic blood pressure (SBP) in adulthood and its modification by ethnicity have been inconclusive. We investigated: 1) whether systolic blood pressure (SBP) differs by ethnicity in young Swedish men and 2) if the shape or strength of association between foetal growth rate and adulthood SBP differs between ethnic groups.

Method: We linked data from the Swedish Medical Birth Register to data on 450362 military conscripts (all men, mean age 18.2 years, born 1973-1990) with concurrent SBP and body mass index measurements available from conscript examinations. Ethnicity was based on parental country of birth as recorded in the Immigration register. Birth weight standardized to gestational age $-\mathrm{Z}$ scores was used as an indicator for foetal growth rate (FGR). Using linear regression, we first analyzed associations between 1 . Ethnicity and SBP. 2. FGR and SBP and 3.FGR and SBP stratified by ethnic group (to assess if the shape or strength of association between FGR and SBP differed by ethnicity). We used structural equation modeling (SEM) to analyze direct and indirect effects of FGR on SBP across ethnic groups. For linear regression analysis subjects were grouped into 13 ethnic groups (Western Europe and North America, Eastern Europe, countries of former Yugoslavia, Arab league, South Asia, East Asia, Latin America, Finland, Poland, Turkey, Iran, and Chile) with the ethnic Swedish group as reference. These were further collapsed into Swedish, Caucasian and nonCaucasian groups for the SEM analysis. All analyses were adjusted for age, and where appropriate, concurrent BMI, height and maternal education.

Results: Compared to ethnic Swedish men, men of all other ethnic groups had lower mean SBP with greatest differences in South Asian $(-4.48 \mathrm{mmHg}, 95 \% \mathrm{CI}-6.05$ to -2.91$)$, Turkish $(-4.63,-5.29$ to -3.97$)$, Arabic $(-3.60,-4.48$ to $-2.72)$ and Iranian $(-3.76,-5.45$ to -2.08$)$ ethnic groups. FGR was inversely associated with SBP $(-0.11 \mathrm{mmHg},-0.15$ to -0.08$)$ and the association was strengthened on adjustment for concurrent BMI $(-0.6,-0.64$ to -0.57$)$. In linear regression analysis stratified by ethnicity, FGR showed statistically significant inverse association with SBP only in Swedish $(-0.63 \mathrm{mmHg},-0.66$ to -0.60$)$, Finnish
$(-0.33 \mathrm{mmHg},-0.64$ to -0.02$)$, Yugoslavian $(-0.64 \mathrm{mmHg}$, -1.08 to -0.21$)$ and Iranian $(-1.99 \mathrm{mmHg},-3.98$ to -0.01 ) groups. In SEM, the total effect of FGR on SBP (the sum of the direct effect and indirect - via - concurrent BMI effect) were of similar magnitude in Swedish and Caucasians groups $(-0.042 \mathrm{mmHG}$ and $-0.022 \mathrm{mmHg}$ per SD increase in BW, $\mathrm{p}<0.05)$. On the other hand, we did not observe a similar effect in non-Caucasians $(-0.002 \mathrm{mmHg}$, n.s.).

Conclusion: The association of FGR with adulthood SBP was inverse but weak in Caucasians and negligible or nonexisting in non-Caucasians. This suggests that ethnicity modifies the effect of FGR on later adulthood SBP although larger data sets are needed to confirm our results.

Disclosure of Interest: None Declared.

\section{DOHaD13-1325}

A premature increase in circulating cortisol in the very preterm sheep fetus does not stimulate expression of genes regulating lung liquid reabsorption

Erin McGillick ${ }^{1}$, Sandra Orgeig ${ }^{1}$, I. Caroline McMillen ${ }^{1}$, Janna L. Morrison ${ }^{1, *}$

${ }^{1}$ School of Pharmacy \& Medical Sciences, Sansom Institute for Health Research, University of South Australia, Adelaide, Australia

Background: In late gestation, there is a natural increase in circulating concentrations of cortisol, the endogenous glucocorticoid (GC), in many mammalian species. This prepartum cortisol surge is vital for maturation of a range of organ systems, particularly the fetal lung. GC availability is controlled by two isoforms of the $11 \beta$-hydroxysteroid dehydrogenase (11ßHSD) enzyme and the effects of GC are largely mediated by the intracellular glucocorticoid receptor (GR). The minimum age at which the fetal lung is able to biochemically respond to GC has not been evaluated. The lung develops as a fluid filled sac and thus, the successful transition to air breathing at birth requires the reabsorption of fetal lung liquid. The process of lung liquid reabsorption is driven by transepithelial sodium and water movement. Here, we evaluate the ability of the lung of the very preterm sheep fetus to respond to a premature increase in plasma cortisol concentrations during the late canalicular phase (equivalent to $\sim 23-24$ wks gestation in humans; term, 40wks). Our aim was to influence expression of genes regulating lung liquid reabsorption and we have compared this to the level of expression in late gestation following exposure to the prepartum cortisol surge.

Method: Vascular catheters were implanted in the ewe and fetus. At 109-116d gestation (term $150 \pm 3 d$ ), either cortisol (Solucortef, $2-3 \mathrm{mg}$ in $4.4 \mathrm{ml}$ saline/24h; $\mathrm{n}=9$ ) or saline $(n=8)$ was infused intravenously into the fetus. Late gestation fetuses received saline infusion from 130-140d gestation $(n=12)$. Expression of GC regulatory genes 


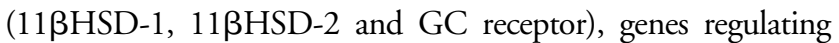
sodium (amiloride-sensitive epithelial sodium channel (ENAC) $-\alpha,-\beta$ and $-\gamma$ subunits; sodium potassium active transport pump ( $\mathrm{Na} \mathrm{K}$ ATPase) subunits, $-\alpha 1$ and $-\beta 1$ ) and water (aquaporin $(\mathrm{AQP})-1,-3,-4$ and -5$)$ ) reabsorption in the fetal lung were measured by qRT-PCR. Data were analysed using a one-way ANOVA with Duncan Post Hoc test. $P<0.05$ was considered statistically significant.

Results: Cortisol infusion had no impact on mRNA expression of the GC receptor or $11 \beta \mathrm{HSD}-2$ in the lung; however, there was an increase in expression of $11 \beta \mathrm{HSD}-1$. Lung mRNA expression of ENAC $-\alpha,-\beta,-\gamma$, AQP $-1,-3$ and -5 increased with age but not cortisol infusion. Whilst there was no change in mRNA expression of Na-K-ATPase- $\beta 1$ subunit or AQP-4, expression of the catalytic Na-K-ATPase- $\alpha 1$ subunit increased with both age and cortisol infusion.

Conclusion: Despite a potential increase in availability of cortisol in the lung, a premature elevation of GC concentrations in the very preterm sheep fetus did not induce expression of genes regulating lung liquid reabsorption. These data suggest that there may be a point before which the lung is unable to respond to GCs. These findings may have implications for very preterm infants born at the limits of viability in terms of fetal lung liquid reabsorption which may complicate the transition to air breathing at birth.

Disclosure of Interest: None Declared.

\section{DOHaD13-1469}

\section{Developmental expression of leptin and its receptor in fetal ovine heart and lungs}

Miles J. De Blasio ${ }^{1, *}$, Sarah L. Kempster ${ }^{2}$, Gordon C. Smith $^{3}$, Katie L. Davies ${ }^{1}$, Brett S. Bernstein ${ }^{1}$, FB Peter Wooding $^{1}$, Dominique Blache ${ }^{4}$, Abigail L. Fowden ${ }^{1}$, Alison J. Forhead ${ }^{1}$

${ }^{1}$ Department of Physiology, Development and Neuroscience;

${ }^{2}$ Department of Medicine; ${ }^{3}$ Department of Obstetrics and Gynaecology, University of Cambridge, Cambridge, United Kingdom; ${ }^{4}$ School of Animal Biology, University of Western Australia, Perth, Australia

Background: Leptin, a hormone typically associated with the postnatal control of energy balance, is also present in the fetal circulation. Plasma leptin in fetal sheep rises with the endogenous prepartum increase in cortisol and after exogenous glucocorticoid treatment ${ }^{1}$. Furthermore, leptin receptors are present in the lungs of the baboon fetus ${ }^{2}$ and the heart of the pig fetus ${ }^{3}$, therefore leptin may play a role in maturation of these tissues near term. This study examined the protein localisation, and mRNA abundance, of leptin and its receptor in the heart and lungs of sheep fetuses towards term.

Method: After maternal and fetal euthanasia $(200 \mathrm{mg} / \mathrm{kg}$ sodium pentobarbitone iv), heart and lung samples were collected from 20 ovine fetuses at 100, 115, 130 and 144 days (d) of gestation ( $n=5$ per group, term $\sim 145 \mathrm{~d}$ ) and were fixed or frozen. Umbilical arterial blood samples were collected for plasma cortisol, T4, T3 and leptin using ovine validated ELISA or RIA. Gene expression was measured by Taqman qRT-PCR using probes for leptin, all forms of the leptin receptor (R-A) and the functional long form of the leptin receptor (R-L). Immunohistochemistry determined the localisation of leptin R-L. Data were assessed by one-way ANOVA with Tukey's post-hoc and linear regression.

Results: No leptin mRNA was detected in fetal ovine lungs, and low levels were present in the fetal heart. Pulmonary leptin R-A and leptin R-L mRNA were increased at d144 compared to d100 $(\mathrm{P}<0.05)$. Fetal plasma cortisol and T3 were increased at $\mathrm{d} 144$ compared to $\mathrm{d} 100(\mathrm{P}<0.05)$. Plasma leptin showed an increasing trend at $\mathrm{d} 144$ compared to $\mathrm{d} 100$ $(\mathrm{P}=0.088)$ while plasma T4 remained unchanged. Positive correlations were observed between pulmonary leptin R-L mRNA and plasma cortisol $(\mathrm{r}=0.79, \mathrm{n}=20, \mathrm{p}<0.01)$ and $\mathrm{T} 3(\mathrm{r}=0.59, \mathrm{n}=20, \mathrm{P}<0.01)$. In contrast, fetal heart leptin R-A and leptin R-L mRNA levels were lower at d144 compared to $\mathrm{d} 100(\mathrm{P}<0.05)$. At each age, protein expression of leptin R-L was localised to Type II pneumocytes, bronchiolar and vascular endothelium and chondrocytes in cartilage around the bronchi of lungs, and cardiomyocytes and blood vessel endothelium in the heart.

Conclusion: Localisation of leptin R-L protein to Type II pneumocytes, and the increase in lung leptin R-L mRNA towards term, suggests that leptin may promote surfactant production. Expression of leptin R-L protein in fetal cardiomyocytes and vascular endothelium may indicate a role for leptin in cardiac growth, angiogenesis and vasodilatation. Furthermore, the ontogenic decrease in cardiac leptin R-L mRNA suggests that leptin action in the heart may be suppressed towards term. The absence of leptin mRNA in fetal lungs and low levels in the fetal heart also suggest that the actions of leptin are endocrine, rather than paracrine, in these tissues before birth.

This project was funded by the BBSRC.

\section{References}

1. Forhead AJ et al 2002 Endocrinology 143(4):1166-73.

2. Henson MC et al 2004 Reproduction 127(1):87-94.

3. Lin J et al 2000 Dom Anim Endocrinol 19(1):53-61.

Disclosure of Interest: None Declared.

\section{DOHaD13-1467}

Effect of glucocorticoid exposure on leptin and leptin receptor gene expression in fetal ovine heart and lungs

Miles J. De Blasio ${ }^{1, *}$, Sarah L. Kempster ${ }^{2}$, Gordon C. Smith $^{3}$, Brett S. Bernstein ${ }^{1}$, Katie L. Davies ${ }^{1}$, FB Peter Wooding $^{1}$, Dominique Blache ${ }^{4}$, Dino A. Giussani ${ }^{1}$, Abigail L. Fowden ${ }^{1}$, Alison J. Forhead ${ }^{1}$ 
${ }^{1}$ Department of Physiology, Development and Neuroscience;

${ }^{2}$ Department of Medicine; ${ }^{3}$ Department of Obstetrics and Gynaecology, University of Cambridge, Cambridge, United Kingdom; ${ }^{4}$ School of Animal Biology, University of Western Australia, Perth, Australia

Background: Cortisol is essential for pulmonary maturation in utero and respiratory function at birth. Synthetic glucocorticoids, such as dexamethasone, are administered to women at risk of preterm delivery to promote fetal lung maturation and prevent respiratory distress. However, glucocorticoids adversely affect cardiac growth and cardiomyocyte replication ${ }^{1}$. In fetal sheep, plasma leptin rises with the prepartum increase in endogenous cortisol and after maternal dexamethasone treatment ${ }^{2}$. Fetal ovine heart and lungs possess leptin receptors suggesting that leptin may play a role in fetal development of these organs. This study investigated (a) fetal cortisol infusion and (b) maternal dexamethasone treatment on gene expression of leptin, and its receptor in fetal ovine heart and lungs.

Method: After catheterisation at $\sim 116$ days (d) of gestation, 10 fetuses were infused iv with either cortisol (2-3 mg.kg ${ }^{-1} \mathrm{~d}^{-1} ; \mathrm{n}=5$; EF-Cortelan, GlaxoSmithKline, UK) or saline $(n=5)$ for $5 d$ from $125 d$ of gestation. In a separate cohort, 10 pregnant ewes were injected im with $12 \mathrm{mg}$ dexamethasone sodium phosphate $(\mathrm{n}=5$; Merck Sharpe and Dohme UK) or saline $(n=5)$, twice at $24 \mathrm{~h}$ intervals from $125 \mathrm{~d}$ of gestation. Tissues were collected $10 \mathrm{~h}$ after the second injection.

After maternal and fetal euthanasia, heart and lung samples were collected. Plasma cortisol, T3, T4 and leptin were measured in umbilical arterial samples using ELISA or RIA. Gene expression was measured by Taqman qRT-PCR using probes for leptin, all forms of the leptin receptor (R-A) and the functional long form of the leptin receptor (R-L). Data were analysed by unpaired $\mathrm{t}$-test.

Results: Glucocorticoid treatment did not alter fetal weight nor heart and lung weights. Fetal cortisol infusion and maternal dexamethasone increased plasma T3 and leptin concentrations; plasma cortisol increased with cortisol infusion and decreased in response to maternal dexamethasone $(\mathrm{P}<0.05$ for all). Leptin mRNA was not expressed in fetal ovine lungs, but was in the heart at low levels. Fetal cortisol infusion increased pulmonary leptin R-L (2.2 fold, $\mathrm{P}<0.05)$ but not leptin R-A mRNA expression, and maternal dexamethasone increased pulmonary leptin R-A (2.3 fold, $\mathrm{P}<0.05)$ and R-L $(3.5$ fold, $\mathrm{P}<0.01)$ mRNA expression. In fetal hearts, leptin R-A and R-L mRNA expression were unchanged by exposure to either glucocorticoid.

Conclusion: Both fetal cortisol infusion and maternal dexamethasone treatment increase pulmonary ovine mRNA expression of the long form of the leptin receptor. The effects of cortisol may be responsible for the developmental rise in pulmonary leptin R-L mRNA seen in fetal ovine lungs near term. Furthermore, the maturational effects of endogenous and synthetic glucocorticoids may be mediated by coincident increases in plasma leptin and pulmonary leptin receptor expression.

This project was funded by the BBSRC.

\section{References}

1. Rudolph AM et al. 1999 Pediatr Res 46:141-6.

2. Forhead AJ et al. 2002 Endocrinology 143(4):1166-73.

Disclosure of Interest: None Declared.

\section{DOHaD13-1268}

\section{Fetal leptin administration improves aspects of lung function and maturation in fetal sheep during late gestation}

Miles De Blasio ${ }^{1, *}$, Sarah L. Kempster ${ }^{2}$, Gordon C. Smith ${ }^{3}$, FB Peter Wooding ${ }^{1}$, Dominique Blache ${ }^{4}$, Abigail L. Fowden $^{1}$, Alison J. Forhead ${ }^{1}$

${ }^{1}$ Department of Physiology, Development and Neuroscience; ${ }^{2}$ Department of Medicine; ${ }^{3}$ Department of Obstetrics and Gynaecology, University of Cambridge, Cambridge, United Kingdom; ${ }^{4}$ School of Animal Biology, University of Western Australia, Perth, Australia

Background: Preterm delivery is associated with impaired lung function and inadequate gas exchange, leading to increased perinatal morbidity and mortality. Synthetic glucocorticoids are administered to promote lung maturation and prevent respiratory distress in fetuses at risk of preterm delivery. In fetal sheep, endogenous and synthetic glucocorticoid exposure increases adipose leptin and leptin receptor mRNA abundance and plasma leptin concentration ${ }^{1}$, although the extent to which leptin may mediate the maturational effects of glucocorticoids in the lung is unknown. This study investigated the effects of fetal leptin administration on indices of pulmonary development such as lung maturation, compliance and gene expression.

Method: Singleton sheep fetuses were catheterised at $\sim 118$ days (d) of gestation and infused iv for $5 \mathrm{~d}$ with either recombinant ovine leptin $\left(0.5 \mathrm{mg} \mathrm{kg}^{-1} \mathrm{~d}^{-1}, \mathrm{n}=10\right.$; Protein Laboratories Rehovot, Israel) or saline $(\mathrm{n}=15)$ from $125-130 \mathrm{~d}$ of gestation (term $\sim 145 \mathrm{~d}$ ). After maternal and fetal euthanasia $(200 \mathrm{mg} / \mathrm{kg}$ sodium pentobarbitone iv; $130 \mathrm{~d}$ ), lung deflation curves were performed using a water manometer, and lung samples were fixed and frozen. Lung structure was assessed by computer assisted stereology, and gene expression was measured by Taqman qRT-PCR using probes against surfactant proteins SP-A, SP-B and SP-C, vascular endothelial growth factor-A (VEGF-A) and its receptor VEGF-R2, elastin, all forms of the leptin receptor (R-A) and the functional long form of the leptin receptor (R-L). Data were assessed by unpaired t-test.

Results: Fetal leptin infusion did not alter fetal body weight, or absolute or relative lung weights. The closing pressure of 
the deflation limb of the pressure-volume curve was increased after leptin administration $(\mathrm{P}<0.05)$, with no effects of leptin on maximum volume at $40 \mathrm{cmH}_{2} \mathrm{O}$ or slope of deflation curve. Gene expression of leptin R-A and R-L, SP-A, B and C were increased (2-2.5 fold, $\mathrm{P}<0.05$ for all), whilst VEGF-A, VEGF-R2 and elastin were unaltered after leptin administration. There was no change in the percentage volume of lung tissue and alveolar airspace. Total elastin content in the alveolar walls was increased by $25 \%$ with leptin administration $(\mathrm{P}<0.05)$. Alveolar wall thickness (diffusion distance) was reduced by $20 \%$ with leptin infusion $(\mathrm{P}<0.01)$. Conclusion: Leptin administration in fetal sheep during late gestation improves aspects of lung maturation and function, and increases the pulmonary expression of genes involved in surfactant production and leptin signalling. Whether enhanced expression of these genes leads to an increase in lung surfactant content remains to be determined.

This project was funded by the BBSRC.

\section{References}

1. Forhead AJ et al. 2008 J Physiol 586:2393-2403.

Disclosure of Interest: None Declared.

\section{DOHaD13-1733}

Fetal stress physiology across gestation is influenced by maternal exposure to trauma in her own childhood

Nora Moog ${ }^{1}$, Claudia Buss ${ }^{2, *}$, Sonja Entringer ${ }^{3}$, Babak Shahbaba ${ }^{4}$, Daniel Gillen ${ }^{4}$, Curt Sandman ${ }^{1}$, Calvin Hobel $^{5}$, Pathik Wadhwa ${ }^{1}$

${ }^{1}$ Psychiatry; ${ }^{2}$ Pediatrics, University of California, Irvine, Irvine; ${ }^{3}$ Pediatrics, University of California, Irvine, Orange; ${ }^{4}$ Statistics, University of California, Irvine, Irvine; ${ }^{5}$ Obstetrics and Gynecology, University of California, Los Angeles, Los Angeles, United States

Background: The severity and persistence of the detrimental effects of exposure to trauma in childhood on an individual's mental and physical health outcomes over the life course is well established. Emerging evidence supports the intriguing possibility of trans-generational transmission of the effects of childhood trauma from an exposed mother to her child. The time period(s) and mechanism(s) underlying this transgenerational effect have yet to be clarified. In the context of human pregnancy and fetal development the placental corticotrophin-releasing hormone system may be particularly relevant because of its tripartite actions as a sensor, transducer and effector of gestational stress. We addressed the hypothesis that the trans-generational effect of history of maternal exposure to childhood trauma may start as early as the intrauterine period of a child's life and be associated with fetal stress physiology, as reflected by the trajectory of placental $\mathrm{CRH}$ production over the course of gestation.
Method: The study was conducted in a representative, population-based cohort 333 socio-demographically and ethnically diverse pregnant women with singleton intrauterine pregnancies attending prenatal care at two university-based medical centers in southern California. History of maternal exposure to childhood abuse or neglect was assessed using a validated questionnaire (CTQ). The trajectory of placental $\mathrm{CRH}$ production was assessed serially up to five time points over the course of gestation from 15 (T1) through 36 weeks (T5).

Results: The prevalence of moderate to severe childhood trauma in the study population ranged between none $(60.9 \%)$, at least one (41.1\%), and multiple events (22.5\%). After accounting for the effects of established determinants of placental CRH production the sum of exposure to different types of traumatic events in childhood was significantly and positively associated in a linear, dose-dependent manner with increased placental CRH production over the course of gestation $(p<.05)$ and with a steeper rate of increase over the latter half of gestation.

Conclusion: To the best of our knowledge this finding represents the first report linking exposure to traumatic events in childhood among pregnant women with fetal stress physiology, thus identifying a possible novel pathway of intergenerational transmission in this particular context.

Disclosure of Interest: None Declared.

\section{DOHaD13-1343}

Local effects of pregnancy on connexin 37 and 43 protein expression in ovine uterine artery endothelium mediate $\mathrm{Ca} 2+$-associated endothelial nitric oxide synthase activation

Ronald R. Magness ${ }^{1,2,3}$,*, Timothy Morschauser ${ }^{1}$, Jayanth Ramadoss ${ }^{4}$, FuXian Yi ${ }^{1}$, Gladys E. Lopez ${ }^{1}$, Ian M. Bird ${ }^{1}$

${ }^{1}$ Ob/Gyn; ${ }^{2}$ Pediatrics; ${ }^{3}$ Animal Sciences, UNIVERSITY OF WISCONSIN, Madison; ${ }^{4}$ Ob/Gyn, Univ of Texas Med Branch, Galveston, United States

Background: Coordinated and sufficient uterine endothelial adaptations during gestation facilitate the dramatic increases in uterine blood flow (UBF) needed for fetal growth. These adaptations include elevations in uterine artery (UA) endothelial eNOS expression and Nitric Oxide (NO) production that may be modulated by coordinated intercellular interactions via gap junction proteins that regulate eNOS activation. We hypothesized that the local expression and distribution of connexin proteins in ovine uterine vasculature play an important role in mediating in vivo gestational eNOS activation and NO production.

Method: We established an ovine model to restrict pregnancy to a single uterine horn $(\mathrm{n}=8)$ and compared this to nonpregnant (luteal \& follicular; $\mathrm{n}=5$ /group) and control late pregnant $[(120-130 \mathrm{~d}$, term $=147 \mathrm{~d} ; \quad(\mathrm{n}=8)]$ sheep, 
measuring bilateral UBF, uterine artery shear stress, connexins $[\mathrm{Cx} 37 / \mathrm{Cx} 43]$ and $\mathrm{P} 635$ eNOS levels in uterine artery (UA) and systemic artery [Omental (OA) and Renal (RA)] endothelium (endo) and connexins in vascular smooth muscle (VSM) via Western analysis.

Results: Compared to nonpregnant luteal and follicular sheep, UBF and UA shear stress were locally (unilaterally) and substantially elevated during late gestation. The patterns of Cx43 expression mimicked UA endo eNOS expression, as well as the dramatic physiologic rises in UBF and UA shear stress. Specifically, UAendo gap junction proteins Cx37/ $\mathrm{Cx} 43$ were locally regulated in the pregnant horn and elevated 10.3- and 25.6-fold; UAendo P635 eNOS and total eNOS were elevated 3.3- and 2.9-fold; whereas UAvsm connexins $37 / 43$ were locally elevated 12.5 - and 5.9-fold, respectively. Less pronounced changes in protein expression were seen in systemic vasculature except for significant pregnancy increases in OAvsm Cx43 and OAendo P635 eNOS and total eNOS, demonstrating primarily UA specific changes. Blockade of gap junction channels using $\mathrm{Cx} 43$ specific GAP peptides abrogated $\mathrm{Ca} 2+$-mediated $\mathrm{NO}$ production.

Conclusion: Thus there is a distinct difference in uterine vs systemic vascular programming of gap junction proteins $\mathrm{C} \times 43$ and $\mathrm{Cx} 37$ during pregnancy. In UAendo, Cx43, but not $\mathrm{Cx} 37$, is required for the $\mathrm{Ca} 2+$-mediated eNOS activation/NO production required for the vasodilatation needed to accommodate dramatic increases in UBF and shear stress during healthy pregnancies. These data suggest that the regulatory mechanisms pertaining to gap junction proteins may delineate a greater understanding of local rather then the systemic vascular adaptations during pregnancy. NIH HL49210, HD38843, HL87144, HL079020.

Disclosure of Interest: None Declared.

\section{DOHaD13-1231}

\section{Pharmacokinetics of theophylline in the fetal sheep}

Barent N. Dubois ${ }^{1,1, *}$, Samantha Louey ${ }^{2}$, George Giraud ${ }^{2,3}$, Sonnet Jonker ${ }^{2}$, Ganesh Cherala ${ }^{1,4,5}$

${ }^{1}$ Pharmacy Practice, Oregon State University; ${ }^{2}$ Knight Cardiovascular Institute, Oregon Health and Science University; ${ }^{3}$ Portland VA Medical Center; ${ }^{4}$ Heart Research Center; ${ }^{5}$ School of Medicine, Oregon Health and Science University, Portland, United States

Background: A thorough understanding of fetal pharmacokinetics is essential to characterize in utero exposure of xenobiotics. Fetal exposure to methylxanthines is relevant given their near ubiquitous presence in the Western diet and use in treating apnea in pre-term neonates. Fetal pharmacokinetics has been estimated from maternal exposure and in pre-term neonates. In the current study, we utilized a model of chronically instrumented fetal sheep which permitted us to directly administer and determine theophylline pharmacokinetics in utero.

Method: Six time-bred ewes (ovis aries) carrying singleton fetuses were studied. $60 \mathrm{mg}$ theophylline was given i.v. into the fetal vein, and blood samples were drawn from fetus and ewe over three days. Serum concentrations were measured by reverse phase HPLC with ultraviolet detection at $275 \mathrm{~nm}$ in an isocratic solvent system (15 mM sodium phosphate, $15 \mathrm{mM}$ potassium phosphate with $20 \%$ methanol, $\mathrm{pH} 4.5$ ). Maternal and fetal pharmacokinetic parameters were estimated with WinNonLin. Cytochrome P450 1A (CYP1A) activity was measured in vitro in maternal (liver, caruncles [placenta]) and fetal (liver, cotyledon [placenta], kidney) to estimate the relative contribution of each organ to drug elimination.

Results: Theophylline pharmacokinetic data fits to a twocompartment model. The mean half-life $(\mathrm{hr})$ was $6.70 \pm 1.33 ; \mathrm{Vd}$ (L) was $3.72 \pm 1.00 ;$ AUC (hr*ug/mL) was $13.85 \pm 2.60$; and CL (L/hr) was $4.45 \pm 0.74$. Rapid distribution of the drug across the placenta was observed, and the half-life of the drug in the maternal circulation was estimated to be $6.54 \pm 2.43 \mathrm{hr}$. In vitro CYP1A activity was robust in maternal liver, negligible in placenta (fetal or maternal), and undetectable in fetal liver and kidney.

Conclusion: The clearance of theophylline from the fetal circulation is likely attributable to its rapid distribution into the maternal circulation across the placenta. Given the minimal activity of CYP1A in fetal kidney and liver, the elimination of the drug would appear to be facilitated by the maternal liver. This is corroborated by the strong association between fetal and maternal half-life. These results suggest that the placenta offers no barrier to theophylline, and the fetus has little capacity to metabolize this compound. Elimination from the maternal-fetal circulation appears to be predominately via the maternal liver.

Disclosure of Interest: None Declared.

\section{DOHaD13-1488}

Renal function in preterm infants: a study in indigenous and non-indigenous Australians

Mary J. Black ${ }^{1, *}$, Belinda Davidson ${ }^{2}$, Daryl Hartigan ${ }^{2}$, Sian Graham ${ }^{2}$, Danica Ryan ${ }^{1}$, Shanti Diwakarla ${ }^{1}$, Wendy Hoy ${ }^{3}$, Gurmeet Singh ${ }^{2}$

${ }^{1}$ Anatomy and Developmental Biology, Monash University, Clayton; ${ }^{2}$ School of Health Research, Menzies, Darwin; ${ }^{3}$ Faculty of Health Sciences, University of Queensland, Brisbane, Australia

Background: Preterm birth affects $\sim 12 \%$ of all births worldwide. In Australia, the incidence of preterm birth is $\sim 8 \%$ with an increased incidence in the Indigenous population $(\sim 14 \%)$. Preterm birth is associated with an increased risk of morbidity and mortality in the neonatal period and this is primarily attributed to the underdevelopment 
of vital organs. Preterm infants are born at a time when nephrogenesis, the formation of nephrons in the kidney, is still ongoing. Renal function in preterm neonates may be adversely affected by renal immaturity and/or postnatal injury. However, to date, little is known about the effect of preterm birth and associated pre- and post-natal factors on renal function. The aim of this study, conducted at The Royal Darwin Hospital in Australia, was to evaluate renal function in the preterm neonate during the first month of life, as well as compare renal function of preterm Indigenous and non-Indigenous infants.

Method: Preterm Indigenous and non-Indigenous infants were grouped according to gestational age at birth: $\leq 28$ weeks (extremely preterm; 10 Indigenous infants and 6 nonIndigenous infants), 29-31 weeks (very preterm; 11 Indigenous infants and 5 non-Indigenous infants) and 32-36 weeks (moderately preterm; 10 Indigenous infants and 10 nonIndigenous infants). Twenty-four hour pooled urine samples were obtained at days 3 to 7, 14, 21 and 28 of life, via a cotton pad fitted to the nappies. From these samples, creatinine clearance $(\mathrm{CrCl}$; an estimate of glomerular filtration rate) and fractional excretion of sodium $\left(\mathrm{Fe}_{\mathrm{Na}}\right.$; an estimate of tubular function) were calculated.

Results: During the first week of life, $\mathrm{CrCl}$ increased with gestational age at birth (Indigenous: $\mathrm{P}=0.0006$; nonIndigenous: $\mathrm{P}=0.018)$, while $\mathrm{Fe}_{\mathrm{Na}}$ decreased with increasing gestational age at birth (Indigenous: $\mathrm{P}<0.0001$; nonIndigenous: $\mathrm{P}=0.054$ ) and with postnatal age (Indigenous: $\mathrm{P}<0.0001$; non-Indigenous: $\mathrm{P}<0.0001)$. Interestingly, unlike $\mathrm{CrCl}, \mathrm{Fe}_{\mathrm{Na}}$ during the first month of life was greater in extremely preterm Indigenous infants than non-Indigenous infants, indicating a difference in tubular function.

Conclusion: This study in Australian infants, demonstrates that renal function in preterm neonates is significantly dependent on gestational and postnatal age in both Indigenous and non-Indigenous infants, and the findings suggest that the kidneys of extremely preterm Indigenous infants may be more vulnerable.

Disclosure of Interest: None Declared.

\section{DOHaD13-1512}

\section{A pre-conceptional micronutrient-rich food supplement prevents gestational diabetes: Mumbai Maternal Nutrition Project}

Sirazul A. Sahariah ${ }^{1, *}$, Ella Marley-Zagar ${ }^{2}$, Patsy Coakley ${ }^{2}$, Meera Gandhi ${ }^{1}$, Harshad Sane ${ }^{1}$, Monika Dayama ${ }^{1}$, Harsha Chopra $^{1}$, Sarah Kehoe ${ }^{2}$, Nick Brown ${ }^{3}$, Ramesh Potdar ${ }^{1}$, Barrie Margetts ${ }^{4}$, Caroline H. Fall ${ }^{2}$

${ }^{1}$ Centre for the Study of Social Change, Mumbai, India; ${ }^{2} M R C$ Lifecourse Epidemiology Unit, University of Southampton, Southampton; ${ }^{3}$ Salisbury District Hospital, Salisbury; ${ }^{4}$ Public Health Nutrition, University of Southampton, Southampton, United Kingdom
Background: The Mumbai Maternal Nutrition Project was a food based randomised controlled trial which aimed to assess whether pre-conceptional micronutrient supplementation is associated with improved fetal growth. The intervention was a daily snack made from green leafy vegetables, fruit and milk, provided for at least three months prior to conception and throughout pregnancy. Control supplements were made from vegetables of low micronutrient content. This analysis examines the effect of the intervention on maternal outcomes. Method: The trial was conducted between 2006 and 2012 in slum areas of Mumbai. Married non-pregnant women aged $<40$ years, who had not completed their family, were recruited following written informed consent. They were randomised to receive intervention or control supplements daily (6 days/week) under supervision until delivery. Maternal plasma micronutrient concentrations (vitamin B12, folate and retinol) were measured (median gestation 10 weeks); haemoglobin concentration was measured (median 10 and 29 weeks) and an oral glucose tolerance test was carried out (median 29 weeks). Gestational diabetes (GDM) was defined as a fasting glucose $\geq 7 \mathrm{mmol} / \mathrm{l}$ or 120 -minute glucose $\geq 7.8 \mathrm{mmol} / \mathrm{l}$. Anaemia was defined as a haemoglobin concentration $<11 \mathrm{~g} / \mathrm{dL}$. B12, folate and retinol deficiencies were defined as concentrations $<150 \mathrm{pmol} / \mathrm{l},<7 \mathrm{nmol} / \mathrm{l}$ and $<0.7 \mathrm{nmol} / \mathrm{l}$ respectively.

Results: Of 6513 women recruited (mean age and pre-pregnant BMI 24 years and $20 \mathrm{~kg} / \mathrm{m}^{2}$ ), 1826 became pregnant and consumed the supplement $\geq 3$ months before conception. Baseline demographic and anthropometric measurements were similar in the intervention and control groups, except that control women had larger waist circumference $(+0.6 \mathrm{~cm}, \mathrm{p}=0.05)$. The prevalence of GDM was reduced in the intervention group (Intervention: $7.5 \%$ vs Control: $13.3 \%$; $=0.009$ ). There were no differences in BMI between intervention groups during pregnancy. The reduction in GDM was predominantly among thinner women $\left(\mathrm{BMI}<22 \mathrm{~kg} / \mathrm{m}^{2}\right)$. Median haemoglobin concentration and prevalence of anaemia were similar in both groups in the $1^{\text {st }}$ trimester (Intervention: $11.3 \mathrm{~g} / \mathrm{dL}, 39 \%$ vs Control: $11.3 \mathrm{~g} / \mathrm{dL}, 34 \% ; \mathrm{p}=0.6, \mathrm{p}=0.2)$ and $3^{\text {rd }}$ trimester $(10.8 \mathrm{~g} / \mathrm{dL}$. $55 \%$ vs $10.6 \mathrm{~g} / \mathrm{dL}, 61 \% ; \mathrm{p}=0.1, \mathrm{p}=0.1)$. After adjusting for gestational age at measurement, median haemoglobin was higher in the intervention group in the $3^{\text {rd }}$ trimester $(p=0.03)$. Median vitamin $\mathrm{B} 12$, folate and retinol concentrations, and rates of deficiency, were similar in both intervention groups.

Conclusion: A daily micronutrient-rich supplement made from GLV, fruit and milk taken by women from at least 3 months before, and throughout, pregnancy almost halved the incidence of gestational diabetes and produced a small increase in third trimester haemoglobin. It had no measurable effect on circulating vitamin B12, folate or retinol concentrations.

Acknowledgements: The project was funded by the Wellcome Trust and Medical Research Council, UK, ICICI Social Initiatives Group, Mumbai and Parthenon Trust, Switzerland.

Disclosure of Interest: None Declared. 


\section{DOHaD13-1632}

Association of intrauterine growth restriction, low Apgar score and high ponderal index with perinatal morbidity infants born from women with pre-gestational diabetes

Shireen Begum ${ }^{1, *}$, Laila A. Banu ${ }^{2}$, S M Keramat $\mathrm{Ali}^{3}$

${ }^{1}$ Previous Affiliation, NIPSOM; ${ }^{2}$ Gynae \& Obstetrics, Bangladesh Institute of Research and Rehabilitation in Diabetes, Endocrine and Metabolic Disorder (BIRDEM); ${ }^{3}$ Clinical Nutrition, Dhaka University, Institute of Nutrition and Food Science, Dhaka University., Dhaka, Bangladesh

Background: Diabetes in pregnancy is yet a challenge in the paediatric medicine and obstetrics due to adverse pregnancy outcomes. Infants of diabetic mother (IDMs) are at higher risk for congenital malformations, pre-term, large for gestational age, macrosomy, and perinatal morbidity than babies born from women with no diabetes. Intrauterine Growth Restriction (IUGR) has both short term and long term effect on child health. IUGR is a strong predictor of perinatal morbidity and mortality, and neonatal morbidity.

Objective: Assess the association of IUGR, low Apgar score, and Ponderal Index (PI) with perinatal morbidity in infants born from women with pre-gestational diabetes.

Method: A prospective matched cohort study was conducted on 200 pregnant women with pre-gestational diabetes (DM) and 200 women with no diabetes (NDM). Pregnant women with clinically diagnosed pre-gestational diabetes were enrolled at the antenatal care unit of the Bangladesh Institute of Research and Rehabilitation in Diabetes, Endocrine and Metabolic Disorder (BIRDEM), Dhaka. This paper is a part of a PhD study - diabetes in pregnancy outcome study. Pregnant women were followed-up from their first trimester $(<12 \mathrm{wk})$ until the perinatal period. Parity, income, and gestational age were matched between the groups. Ultrasound in early pregnancy (USG) confirmed the gestational age. IUGR cases were confirmed by the sonologist in the second-trimester and third trimester based on biometric parameters (USG). Standard techniques were used to measure birth weight and neonatal anthropometry. Basic and appropriate inferential statistical analyses were done to examine the relation of IUGR, low Apgar score, and Ponderal Index (PI) with perinatal morbidity.

Results: Preterm IUGR rates were more (about seven times) in infants of diabetic mothers (IDMs) than the control group. Eighty six percent of the pre term IUGR babies suffered from perinatal morbidity. The risk of developing perinatal morbidity was 18.2 times more $(\mathrm{P}<0.0001)$ in pre term IUGR than normal birth weight babies. Risk of developing perinatal morbidity in IDMs with ponderal index (PI) $>2.1$ was 1.6 times higher $(\mathrm{P}<0.01)$ than normal PI. Significantly more babies were severely asphyxiated (3.2\%) and mild asphyxiated $(23.7 \%)$ from DM groups compared to only $8.0 \%$ from NDM groups at 1 minute of birth. Risks of developing perinatal morbidity in infants with Apgar score below eight were (at 1 minute) 12 times higher $(\mathrm{P}<0.0001)$ than the normal group.

Conclusion: Pre-term IUGR is more in babies born from mothers with pre-gestational diabetes than babies of women with no diabetes. Pre-term IUGR, high Ponderal Index, and low Apgar score were significantly associated with perinatal morbidity in IDMs.

\section{References}

1. Barker DJ. The developmental origins of adult disease. J Am Coll Nutr 2004; 23(6 Suppl):588S-595S.

Acknowledgements: Professor Dr. Azad Khan, Professor Dr. Hazera Mahtab, Prof. Dr. Kishore Azad, Prof. Dr. TA Choudhury, and Prof. Dr. Liaquat Ali of BIRDEM and Professor Dr. Harun-Ar Rashid, Director BMRC.

Funding: Bangladesh Medical Research Council (BMRC).

Disclosure of Interest: None Declared.

\section{DOHaD13-1492}

Effects of maternal dietary protein, folate and vitamin B12 status on glucose intolerance during pregnancy

Mary F.-F. Chong ${ }^{1, *}$, Majorelee Colega ${ }^{2}$, Ling Wei Chen ${ }^{2}$, Rob M. van Dam ${ }^{2}$, Chuen Seng Tan ${ }^{2}$, Ai Lin Lim ${ }^{1}$, Wei Wei $\mathrm{Pang}^{2}$, Shirong $\mathrm{Cai}^{2}$, Yung Seng $\mathrm{Lee}^{2}$, Seang Mei Saw ${ }^{2}$, Kenneth Kwek ${ }^{3}$, Keith M. Godfrey ${ }^{4}$, Yap Seng Chong ${ }^{2}$, Peter Gluckman ${ }^{5}$ and GUSTO

${ }^{1}$ Singapore Institute for Clinical Sciences, $A^{*}$ STAR; ${ }^{2}$ National University of Singapore; ${ }^{3} K K$ Women's and Children's Hospital, Singapore, Singapore; ${ }^{4}$ University of Southampton, Southampton, United Kingdom; ${ }^{5}$ Liggins Institute, Auckland, New Zealand

Background: Current evidence supports the link between diet and the development of glucose intolerance, but limited studies have examined diets during pregnancy as risk factors in the development of gestational diabetes mellitus (GDM). We examined the association of dietary protein intakes, plasma folate and vitamin B12 with glucose intolerance during pregnancy in an Asian population.

Method: In the Singapore birth cohort study (GUSTO), women from three ethnic groups underwent oral glucose tolerance testing (OGTT) and provided blood samples for plasma folate and vitamin B12 measurements during 26th to 28th weeks of gestation. Dietary intakes were also ascertained using 24-hr food recalls. Relationships of nutrients with GDM status, fasting and 2-hr postprandial glucose concentrations were assessed using linear and logistic regression.

Results: Of 925 women, 21.4\% were diagnosed with GDM, based on WHO criteria. In regression models adjusted for maternal age, prepregnancy BMI and ethnicity, higher 
percentage dietary protein (\% of energy), higher plasma folate concentrations and lower plasma vitamin B12 were associated with higher 2-hr postprandial glucose concentrations $(ß=0.18 \mathrm{mmol} / \mathrm{L}, 95 \% \mathrm{CI}=0.07-0.29 ; \beta=0.49 \mathrm{mmol} / \mathrm{L}$, 95\% CI $=0.267-0.711 ; \quad \beta=-0.12 \mathrm{mmol} / \mathrm{L}, 95 \% \quad \mathrm{CI}=$ -0.22 , -0.021 for standardized nutrient values respectively). An ethnicity*folate interaction was observed $(p=0.011)$, suggesting largest effect of folate in Indian women. In adjusted logistic models, higher dietary protein, higher plasma folate and lower plasma vitamin B12 also predicted greater risk of GDM $(\mathrm{OR}=1.35$ per $\mathrm{SD}$ of percent protein, 95\% CI = 1.09-1.67; OR = 1.96 per SD of logfolate, $95 \% \mathrm{CI}=1.17-3.31 ; \mathrm{OR}=0.80$ per $\mathrm{SD}$ of vitamin $\mathrm{B} 12$, $95 \% \mathrm{CI}=0.64-0.98)$. Only lower plasma vitamin B12 was associated with higher fasting glucose concentrations $(ß=-0.037 \mathrm{mmol} / \mathrm{L}, 95 \% \mathrm{CI}=-0.07$ to -0.003$)$.

Conclusion: High protein and folate intakes as well as low vitamin B12 intakes maybe potential risk factors for the development of glucose intolerance during pregnancy. This would have important public health implications and should be further investigated.

Disclosure of Interest: None Declared.

\section{DOHaD13-1462}

Effects of pre-pregnancy BMI and gestational weight gain on LGA incidence among GDM mothers

\section{Dittakarn Boriboonhirunsarn ${ }^{1}$,* \\ ${ }^{1}$ Obstetrics and Gynecology, Faculty of Medicine Siriraj Hospital, Bangkok, Thailand}

Background: The objective of this study was to evaluate the effects of pre-pregnancy body mass index (BMI) and gestational weight gain (GWG) on the incidence of largefor-gestational age (LGA) infants among pregnant women with gestational diabetes (GDM).

Method: A total of 343 term, singleton pregnant women diagnosed with GDM at Siriraj Hospital, Thailand, were included. All received GDM screening and diagnostic procedures as well as management according to institutional guidelines. Data on antenatal care and delivery, including pre-pregnancy BMI, GWG, and pregnancy outcomes, were retrieved. Pre-pregnancy BMI and adequacy of GWG were classified according to Institute of Medicine (IOM) recommendation. LGA was defined as birth weight of greater than the 90th percentile at specific gestational age. Various characteristics were compared between women with LGA infants and those with appropriate-for-gestational age (AGA), and small-for gestational age (SGA) infants. Logistic regression analysis was used to determine independent associated risk factors for LGA infants.

Results: Mean age of pregnant women was $33 \pm 4.8$ years and $44 \%$ were nulliparous. The women first visit to antenatal clinic at $12.7 \pm 5.5$ weeks of gestation and GDM were diagnosed at $22.6 \pm 8.5$ weeks of gestation. GDM class A2 was found in 56 women (16.3\%). Mean pre-pregnancy BMI was $24.4 \pm 4.7 \mathrm{~kg} / \mathrm{m}^{2}$ and mean GWG was $12.4 \pm 4.8 \mathrm{~kg}$. Women were classified into overweight and obese in $24.2 \%$ and $13.4 \%$ respectively. GWG in the recommended range was found in $37.9 \%$, excessive GWG was found in $34.1 \%$, and $28 \%$ had inadequate weight gain. Mean gestational age at delivery was $38.4 \pm 1.1$ weeks of gestation and $39.1 \%$ were delivered by cesarean section. LGA was found in 96 cases (28\%) while AGA was found in 237 cases $(69.1 \%)$ and SGA in 10 cases (2.9\%). Multiparous women were significantly more likely to deliver LGA infants than nulliparous women $(34.4 \%$ vs $19.2 \%, \mathrm{p}=0.008)$. Pre-pregnancy BMI and GWG were significantly greater among women with LGA than those with AGA and SGA infants $(p<0.001)$. Overweight and obese women were significantly more likely to have LGA infants than normal and underweight women $(p=0.047)$. Excessive GWG significantly increased the rate of LGA infants than those with adequate and inadequate GWG $(p<0.001)$. Logistic regression analysis demonstrated that multiparity and excessive GWG were independently increased the risk of LGA infants, adjusted for pre-pregnancy BMI, age, parity, GDM classification, and gestational age. Adjusted odds ratio for multiparity was 2.7 (95\% CI 1.5-4.8, p = 0.001) and for excessive GWG was $2.4(95 \%$ CI 1.3-4.3, $\mathrm{p}=0.004)$.

Conclusion: Multiparity and GWG above the recommendation, but not pre-pregnancy BMI, were significant determinants of delivering LGA infants among GDM women. Since GWG is modifiable, caregivers should pay more attention to this specific issue among this group of pregnant women, especially in terms of nutritional surveillance, counseling, support, and monitoring, as well as behavioral modifications during antenatal care in order to minimize the risk of inappropriate fetal growth and related complications.

Disclosure of Interest: None Declared.

\section{DOHaD13-1354}

\section{Exosomes protect human umbilical arteries endothelial cells against hyperglycemia}

Carlos Salomon ${ }^{1}$, Katherin Scholz ${ }^{1}$, Miharu Kobayashi ${ }^{1}$, Murray Mitchell ${ }^{1, *}$, Gregory Rice ${ }^{1}$

${ }^{1}$ Centre for Clinical Diagnostics, The University of Queensland Centre for Clinical Research, Brisbane, Australia

Background: Exosomes are small (40-100 nm) membrane vesicles that contain an extensive repertoire of biologicallyactive proteins and oligionucleotides that are released by many cell type, including the human placenta, and exert effects at both maternal and fetal circulation. Hypoxia, hyperglycaemia and hyperinsulinemia are risk factors for complications of pregnancy and may adversely affect 
placentation and development of the materno-fetal vascular exchange. In particular, during early pregnancy such conditions may impact the remodeling of maternal spiral arterioles. We hypothesize that: (i) that the release of exosomes from chorionic villi is regulated by $\mathrm{D}$-glucose and insulin concentrations; and (ii) chorionic villi exosomes ameliorate the effects of hyperglicaemia on arterial endothelial cells.

Method: Chorionic villi (CV) were isolated from first trimester placental tissue (8-12 week of gestation, $\mathrm{n}=3$ per week each of gestation). CV were incubated (DMEM, 10\% FCS-exosomes free) with $5 \mathrm{mM}$ (control) and $25 \mathrm{mM} \mathrm{D}$-glucose (high) in absence or presence of insulin $(1 \mathrm{nM})$ at $48 \mathrm{~h}$ under $3 \% \mathrm{O}_{2}$ (BioSpherix). Parallel cultures were exposed to $5 \mathrm{mM}$ D-glucose plus $20 \mathrm{mM}$ L-glucose (osmotic control). CV-conditioned media was collected and exosomes (exo-CV) were isolated by differential and buoyant density centrifugation. Exosomes release was expressed as protein content of the isolated fraction and were characterised by electron microscopy, Western blot and ELISA using exosomes marker (CD63) and placenta-specific marker (PLAP). The effect of $100-\mu \mathrm{g} / \mathrm{ml}$ exo-CV on human umbilical arteries endothelial cells (HUAEC) migration in presence of $25 \mathrm{mM}$ D-glucose was established using an Incucyte ${ }^{\mathrm{TM}}$ live-cell imaging system.

Results: Exo-CV were identified (electron microscopy) as spherical vesicles, with a typical cup-shape and diameters ranging from 50 to $100 \mathrm{~nm}$ and were positive for the exosome markers: CD63 and PLAP. High D-glucose concentration $(25 \mathrm{mM})$ increased exosome released by $\sim 2.0$-fold (ANOVA, $\mathrm{p}<0.01)$ compared to control. Insulin $(1 \mathrm{nM})$ inhibited D-glucose-induced exosome release to control values. Exo-CV (from 5 or $25 \mathrm{mM} \mathrm{D}$-glucose in absence or presence of insulin) increased HUAEC migration by $\sim 1.5$-fold (ANOVA, $\mathrm{p}<0.05)$ to compared to control. High D-glucose reduced ( $60 \%)$ HUAEC migration, however, cells pre-treated with exosomes isolated from $\mathrm{CV}$ exposed to high $\mathrm{D}$-glucose blocked the effect of high D-glucose on HUAEC migration.

Conclusion: The data obtained in the study are consistent with the hypothesis that the release of exosomes from chorionic villi is responsive to hyperglicaemia and hyperinsulinemia, Furthermore, CV-derived exosomes promote endothelial cell migration and modify the responsive of endothelial to hyperglicaemia.

Disclosure of Interest: None Declared.

\section{DOHaD13-1073}

Fasting plasma glucose at the first prenatal visit is associated with fetal birth weight in gestational diabetes mellitus

Bin Liu ${ }^{1} *$, Yun $\mathrm{Xu}^{2}$, Chongyou $\mathrm{An}^{1}$, Ying Zhang ${ }^{1}$, Zilian Wang ${ }^{1}$

${ }^{1}$ The First Affiliated Hospital of Sun Yat-sen University;

${ }^{2}$ The Sixth Affiliated Hospital of Sun Yat-sen University,

Guangzhou, China
Background: Recent study showed high fasting plasma glucose (FPG) level at the first prenatal visit is related to the development of gestational diabetes mellitus. However, the relationship of high FPG and adverse pregnant outcome has not been clarified. Thus, the primary purpose of the present study is to investigate the relationship of FPG level at the first prenatal visit and major pregnant outcome in gestational diabetes mellitus.

Method: Pregnant women who took FPG test at the first prenatal visit during 10-24 gestational weeks, received regular prenatal care, and delivered in our center between March 2011 and March 2012 were recruited in the study population. Pregravid body mass index, FPG and maternal age were recorded at the first prenatal visit, while gestational diabetes mellitus, fetal birth weight and birth methods (vaginal vs. cesarean) were taken as major pregnant outcome. Partial correlations analysis were preformed to study the relationship of FPG (at the first prenatal visit) to GDM, fetal birth weight, and birth methods, adjusted by pregravid BMI and maternal age.

Results: A total of 1423 pregnant women were recruited in the research population, 279 of which developed GDM and the other 1144 were NGT. In the whole cohort, FPG at the first prenatal visit were correlated with GDM (adjusted $\mathrm{R}=0.187, \mathrm{p}<0.001$ ), fetal birth weight (adjusted $\mathrm{R}=0.054$, $\mathrm{p}=0.041$ ), and macrosomia (adjusted $\mathrm{R}=0.064, \mathrm{p}=0.017$ ), but has no relationship with birth methods. In GDM group, FPG at the first prenatal visit showed stronger correlation with fetal birth weight (adjusted $\mathrm{R}=0.222, \mathrm{p}<0.001$ ) and macrosomia (adjusted $\mathrm{R}=0.172, \mathrm{p}=0.004$ ).

Conclusion: Basal characteristics at the first prenatal visit provides effective information for pregnant outcome prediction. Independent of pre-gravid BMI and maternal age, FPG level is strongly associated with fetal birth weight, especially in those developed GDM in the future. More risk factors should be recruited in first prenatal care for early prediction of GDM and prevention of adverse pregnant outcome.

Disclosure of Interest: None Declared.

\section{DOHaD13-1559}

GDM among women who are overweight and obese: the effect of BMI category

Kate E. Martin ${ }^{1, *}$, Rosalie Grivell ${ }^{1,2}$, Jodie Dodd ${ }^{1,2}$, Lisa Yelland ${ }^{2}$

${ }^{1}$ Obstetrics and Gynaecology, Women's and Children's Hospital;

${ }^{2}$ Robinson Institute, University of Adelaide, Adelaide, Australia

Background: The aim of this study was to evaluate the role of maternal body mass index (BMI) on the development of gestational diabetes (GDM) and the risk of adverse pregnancy outcomes in women who are overweight or obese.

Method: A prospective cohort study nested within the LIMIT randomized control trial. Women were recruited 
between $10^{+0}$ and $20^{+0}$ weeks' gestation, with a BMI $\geq 25 \mathrm{~kg} / \mathrm{m}^{2}$, and were categorized as either overweight (BMI $25.0-29.9 \mathrm{~kg} / \mathrm{m}^{2}$ ) or obese subclass 1 (BMI $30.0-34.9 \mathrm{~kg} /$ $\mathrm{m}^{2}$ ), obese subclass 2 (BMI $35.0-39.9 \mathrm{~kg} / \mathrm{m}^{2}$ ), and obese subclass $3 \quad\left(\mathrm{BMI} \geq 40.0 \mathrm{~kg} / \mathrm{m}^{2}\right)$, utilizing World Health Organization criteria. Women underwent a fasting oral glucose tolerance test at 26-28 weeks' gestation, and a diagnosis of GDM was made if the results of fasting blood glucose were $\geq 5.5 \mathrm{mmol} / \mathrm{L}$ or blood glucose $\geq 7.8 \mathrm{mmol} / \mathrm{L}$ after two hours. Treatment for GDM was initiated routinely, reflecting current clinical practice. Maternal antenatal and postpartum complications, and neonatal outcomes were evaluated.

Results: A total of 1,010 women formed the cohort for this analysis, with 434 (42.97\%) categorized as overweight, 295 (29.21\%) obese subclass 1, 167 (16.53\%) obese subclass 2, and $114(11.29 \%)$ obese subclass 3 . The incidence of GDM increased with increasing maternal BMI (6.91\% overweight vs $12.54 \%$ obese subclass 1 vs $10.78 \%$ obese subclass 2 vs $19.30 \%$ obese subclass 3 ). Women who were diagnosed with gestational diabetes were significantly less likely to give birth to an infant with birth weight above $4 \mathrm{~kg}$ (RR 0.55; 95\% CI 0.32 to $0.95 ; \mathrm{p}=0.0333)$. Both the need for caesarean delivery, RR 1.27 (95\% CI 1.07 to $1.5 ; \mathrm{p}=0.0072)$, and infant admission to intensive care, RR 2.47 (95\% CI 1.00 to $6.10 ; \mathrm{p}=0.05)$, were significantly increased in women who were obese when compared with women who were overweight, independent of the presence of GDM. The effects of maternal BMI and gestational diabetes on risk of clinical outcomes were independent.

Conclusion: Increasing maternal BMI is a significant independent risk factor for the development of gestational diabetes. Our findings demonstrate a considerably higher prevalence of GDM than has been previously described in women who are overweight and obese. This has significant public health implications given that GDM is a major contributor to adverse pregnancy outcome, and has long term consequences for both women and their infants.

Disclosure of Interest: None Declared.

\section{DOHaD13-1342}

Impact of leptin and adiponectin and that of maternal obesity on the weights at birth and at one year old of infants born to women with gestational diabetes mellitus

Haitian Chen ${ }^{1, *}$, Linan $\mathrm{Xu}^{1}$, Wenjing $\mathrm{Zhu}^{1}$, Yanxin $\mathrm{Wu}^{1}$, Man $\mathrm{Xu}^{1}$, Zilian Wang ${ }^{1}$

${ }^{1}$ Department of Obstetrics, the First Affiliated Hospital of SUN Yat-sen University, Guangzhou, China

Background: With the increasing incidence of gestational diabetes mellitus (GDM) in recent years, the impact of GDM on neonates and their long-term health has become a heated topic among obstetricians. This study aimed to evaluate the association of leptin and adiponectin levels and maternal constitutional factors with the weights at birth and at one year old of infants born to women with gestational diabetes mellitus (GDM).

Method: Fifty-one women with GDM diagnosed by the new ADA criteria with medically manageable blood glucose were recruited in our hospital from June 2011 to September 2011. Leptin and adiponcetin level in maternal blood and in cord blood were measured and their relation with infant's birth weight and 1-year-old offspring's weight was evaluated using Pearson correlation analysis. In addition, maternal constitutional factors were recorded.

Results: The neonatal birth weight had positive correlation with levels of leptin $(\mathrm{r}=0.316, \mathrm{P}=0.024)$ and adiponectin $(r=0.855, P=0.026)$ in cord blood, while 1-year-old infant's weight was only correlated with levels of adiponectin $(\mathrm{r}=0.753, \mathrm{P}=0.036)$ in cord blood. The infant's birth weight had positive correlation with maternal constitutional factors such as pre-pregnancy weight $(\mathrm{r}=0.340, \mathrm{P}=0.015)$, pre-pregnancy BMI $(\mathrm{r}=0.289, \mathrm{P}=0.040)$, and weight gain $(\mathrm{r}=0.280, \mathrm{P}=0.047)$.

Conclusion: The infant's birth weight is closely associated with levels of leptin and adiponectin in cord blood and with the pregravid and prepartum maternal obesity. However, 1year-old offspring's weight is only associated with levels of adiponectin in cord blood in the previously diabetic mother.

\section{References}

1. Uvena-Celebrezze J, Fung C, Thomas AJ. Relationship of neonatal body composition to maternal glucose control in women with gestational diabetes mellitus. Journal of Maternal-Fetal \& Neonatal Medicine 2002; 12:396-401.

2. Gonzalez-Quintero VH, Ist wan NB, Rhea DJ et al. The impact of glycemic control on neonatal outcome in singleton pregnancies complicated by gestational diabetes. Diabetes Care 2007; 30:467-70.

3. Aman J, Hansson U, Ostlund I,et al. Increased fat mass and cardiac septal hypertrophy in newborn infants of mothers with well-controlled diabetes during pregnancy. Neonatology 2011; 100:147-54.

4. Sivan E, Lin WM, Homko CJ, et al. Leptin is present in human cord blood. Diabetes 1997; 46:917-9.

Disclosure of Interest: None Declared.

\section{DOHaD13-1211}

Intra-uterine exposure to maternal diabetes and stress-responses in Indian children

GV Krishnaveni ${ }^{1 *}$, SR Veena ${ }^{1}$, MP Malathi ${ }^{1}$, A Jones ${ }^{2}$, $\mathrm{CHD} \mathrm{Fall}{ }^{3}$

${ }^{1}$ Epidemiology Research Unit, CSI Holdsworth Memorial Hospital, Mysore, India; ${ }^{2}$ Centre for Cardiovascular Imaging, University College London Institute of Child Health, London; 


\section{${ }^{3}$ MRC Lifecourse Epidemiology Unit, University of South- ampton, Southampton, United Kingdom}

Background: Individuals exposed to intrauterine undernutrition as well as over-nutrition associated with maternal gestational diabetes (GDM) are at an increased risk of adiposity and later cardiometabolic disease. Recent studies suggest that exaggerated cortisol and autonomic responses to stress may be one mechanism leading to increased disease risk in low birth weight individuals. However, it is not known if altered stress-responses underlie the disease risk in the offspring of GDM mothers (ODM). We aimed to test the hypothesis that ODM have higher cortisol and cardiosympathetic responses during the Trier Social Stress Test for children (TSST-C) in a birth cohort in India.

Method: Adolescent children ( $\mathrm{N}=273,28$ ODM; mean age: 13.6 years) whose mothers completed an oral glucose tolerance test during pregnancy performed 5-minutes each of a public speaking and a mental arithmetic task in front of two unfamiliar 'evaluators' (TSST-C). Salivary cortisol concentrations were measured at baseline and at regular intervals after the stressor. Continuous measurements of heart rate, finger blood pressure, stroke volume, cardiac output and systemic vascular resistance were carried out before, during and for 10 minutes after the TSST-C using a finger cuff. Detailed anthropometry was carried out and fasting plasma glucose and insulin concentrations were measured on the following day. Insulin resistance was calculated using the Homeostasis Model Assessment equation (HOMA-IR). Pubertal status was assessed using the Tanner's method.

Results: Offspring of diabetic mothers were larger in all body measurements including skinfold thickness and percentage body fat than controls (offspring of non-GDM mothers; $\mathrm{P}<0.001)$. They also had higher fasting insulin concentrations and HOMA-IR $(\mathrm{P}<0.001)$.

In all children, the mean salivary cortisol concentrations, and heart rate, systolic and diastolic blood pressure, cardiac output, stroke volume and systemic vascular resistance $(\mathrm{P}<0.001$ for all $)$ increased from baseline values after inducing stress. The increment was significantly higher in ODM compared to controls for systolic blood pressure $(29 \mathrm{v}$ $24 \mathrm{mmHg}, \quad \mathrm{P}=0.03 ; \quad \mathrm{P}=0.050$ after adjusting for socio-economic status, current body mass index and pubertal stage), cardiac output $(1.1 \vee 0.6 \mathrm{~L} / \mathrm{min}, \mathrm{P}=0.001$ and 0.01$)$, and stroke volume $(7.7 \times 3.7 \mathrm{ml}, \mathrm{P}=0.001$ and 0.01 ). There were no statistically significant differences between ODM and controls in salivary cortisol concentrations after stress.

Conclusion: Maternal diabetes during pregnancy is associated with higher cardio-sympathetic responses to stress in the offspring. This may be a contributing factor for the higher risk of cardiometabolic disease in the ODM. Our findings set a stage for further studies to examine the neuro-endocrinal programming in ODM as a predictor of their later cardiometabolic risk.
Funding source: This study was supported by an intermediate fellowship from the Wellcome Trust, UK and by the Parthenon Trust, Switzerland and the Medical Research Council, UK.

Disclosure of Interest: None Declared.

\section{DOHaD13-1461}

\section{Maternal glucose intolerance alters offspring renal development}

Stacey Hokke ${ }^{1, *}$, James Armitage ${ }^{1,2}$, John F. Bertram ${ }^{1}$, Luise Cullen-McEwen ${ }^{1}$

${ }^{I}$ Department of Anatomy and Developmental Biology, Monash University, Clayton; ${ }^{2}$ School of Medicine (Optometry), Deakin University, Waurn Ponds, Australia

Background: Animal studies indicate that offspring exposed to a diabetic intrauterine environment demonstrate a nephron deficit and reduced renal function. Current literature is, however, limited to models of persistent severe hyperglycemia and fetal growth restriction, which do not reflect the typical metabolic abnormalities seen in the majority of human maternal diabetes. The aim of this study was to assess the effect of impaired glucose tolerance in pregnancy on offspring nephron endowment.

Method: Impaired maternal glucose tolerance was induced in C57Bl6 mice by high fat feeding $(21 \%$ fat w/w) for six weeks prior to pregnancy and throughout gestation. Control mice were fed a matched normal fat diet $(6 \%$ fat w/w). Offspring of dams with impaired glucose tolerance (IGT) or normal glucose tolerance (NGT) were collected just prior to birth at embryonic day (E) 18.5 and at postnatal day (PN) 21 for determination of nephron number using unbiased stereology. Results: At E18.5, offspring of IGT dams were $5 \%$ heavier (NGT $1.08 \pm 0.01 \mathrm{~g} \mathrm{n}=7$ litters vs IGT $1.13 \pm 0.01 \mathrm{~g} \mathrm{n}=8$ litters, $p=0.019$; values are mean \pm SEM) and had $25 \%$ more nephrons (NGT $1,376 \pm 96 \mathrm{n}=6$ litters vs IGT $1,720 \pm 78 \mathrm{n}=8$ litters; $p=0.009)$ than offspring of NGT dams. When adjusted for body weight, E18.5 offspring of IGT dams had significantly more nephrons than offspring of NGT dams (NGT 1,249 \pm 73 nephrons/g $n=6$ litters vs IGT $1492 \pm 59$ nephrons/g $\mathrm{n}=8$ litters, $p=0.014$ ). PN21 offspring of IGT dams had $27 \%$ more nephrons than offspring of NGT dams (NGT 14,981 $\pm 412 \mathrm{n}=6$ litters vs IGT $18,962 \pm 364 \mathrm{n}=8$ litters; $p<0.0001)$ and did not differ in body weight $(p=0.107)$. Nephron endowment at PN21 was correlated with maternal glucose tolerance prior to pregnancy (rho $=0.674, p=0.0002$ ).

Conclusion: This is the first study to assess renal development in offspring exposed to impaired maternal glucose tolerance. We show that offspring of IGT dams have an elevated nephron endowment which correlates with maternal glucose intolerance and is established before birth, relatively 
early in mouse nephrogenesis. This may occur as a result of increased energy and nutritional substrate transfer to the fetal compartment in glucose intolerant high fat fed dams, thereby promoting accelerated nephrogenesis in the offspring. This accelerated nephrogenesis may be associated with perturbed nephron morphology and renal function in later life. Additional studies are required to assess whether adult offspring of IGT dams develop renal disease including proteinuria, glomerular hyperfiltration, glomerular hypertrophy and glomerulosclerosis.

Disclosure of Interest: None Declared.

\section{DOHaD13-1546}

Metformin therapy reduces maternal weight gain in gestational diabetes: a potential for positive programming? Evidence from a systematic review and meta-analysis.

May Oo Khin ${ }^{1, *}$, Manu Vatish ${ }^{1}$, Simon Gates ${ }^{1}$, Ponnusamy Saravanan ${ }^{1}$

${ }^{1}$ Warwick Medical School, University of Warwick, Coventry, United Kingdom

Background: Gestational diabetes (GDM) is common and contributes to significant maternal and neonatal morbidity. Its prevalence may triple to $16-18 \%$, if universal screening and the new IADPSG (International Association of Diabetes and Pregnancy Study Groups) cut-off for diagnosis are adopted. Management of GDM is time and resource intensive. Treatment involves diet $\&$ lifestyle, followed by insulin therapy. Metformin is considered safe in pregnancy but not widely used, probably because of lack of studies examining its superiority over insulin. We carried out systematic review and meta-analysis to evaluate the efficacy of metformin over insulin in GDM. Our secondary aim was to identify a subgroup who would benefit with metformin monotherapy.

Method: 5 databases were searched by two independent reviewers without any restriction along with hand searching of relevant references in the primary publications. The search was updated on $1^{\text {st }}$ June, 2013. All primary studies comparing metformin to insulin and reporting maternal and neonatal outcomes of GDM were included. 12 studies compared with insulin (6 RCTs-n $=1307 \& 6$ non-RCTs/ NRCTs- $n=1883$ ) were identified. Quality assessment of RCTs and NRCTs used separate risk of bias tools, in line with PRISMA and MOOSE guidelines. Appropriate sensitivity analysis was undertaken.

Results: In RCT meta-analysis, metformin appeared superior to insulin in minimizing maternal weight gain (weighted mean difference (WMD): $-1.63 \mathrm{~kg} ; 95 \%$ confidence interval $(\mathrm{CI}):-2.14,-1.11)$ and preventing neonatal hypoglycaemia (odds ratio (OR): 0.65; CI: 0.47, 0.89) and macrosomia (OR: 0.53; CI: 0.3,0.91). Similar effects were observed in NRCT meta-analysis (neonatal hypoglycaemia:OR: 0.42; CI:
0.27, 0.64; macrosomia: OR: 0.63; CI: 0.42, 0.93; maternal weight gain: WMD: $-1.78 \mathrm{~kg}$; CI: $-1.91,-1.64)$ along with reduction in large for gestational age (OR: 0.60;CI: $0.46,0.77$ ), small for gestational age (OR: 0.66; CI: $0.45,0.95)$ and neonatal intensive care admissions rate (OR:0.57;CI:0.40,0.82). Metformin monotherapy failure rate varied from 0 to $46.5 \%$. Three studies (2 RCTs and 1 NRCT) compared baseline characters of metformin monotherapy and metformin plus insulin. Lower pre-treatment glucose values and GDM diagnosed later in the pregnancy supported the use of metformin monotherapy. In contrast, higher pre-treatment BMI suggested the need for additional insulin.

Conclusion: This meta-analysis show that metformin in GDM appears to have significant benefit on less maternal weight gain, lower neonatal hypoglycaemia and macrosomia. Our evidence calls for the routine first line use of metformin when lifestyle measures fail. This will also reduce the resource burden of treating the increasing number of GDM mothers. While our study provides some insight into GDM subgroup who can be successfully managed on metformin monotherapy, studies specifically designed to look this as an outcome measure are required. Both GDM and excess maternal weight gain have been shown to be independently associated with adverse metabolic traits in the offspring. As metformin appears to improve both glycaemic control and the maternal weight gain, it is conceivable that it may offer beneficial metabolic effects for the offspring in the long term.

Disclosure of Interest: None Declared.

\section{DOHaD13-1732}

Postnatal oral glucose tolerance test abnormalities (GTT) abnormalities in gestational diabetes (GDM) - can fasting plasma glucose (FPG) replace GTT?

H. Venkataraman ${ }^{1, *}$, N. Sukumar ${ }^{1}$, Z. D’Souza ${ }^{2}$, P. Saravanan ${ }^{1}$

${ }^{1}$ Warwick Medical School, University of Warwick, Coventry;

${ }^{2}$ Warwick Hospital, Warwick, United Kingdom

Background: Women with GDM are at an increased risk of developing type 2 Diabetes. This risk in the first year is between $2.5-16.7 \%$. Postnatal screening is hence vital. Current NICE guidance recommends only FPG for postnatal screening. ADA guidelines recommend GTT and Scottish (SIGN) guidelines recommend GTT in addition to FPG if clinically indicated. It is possible that mothers with antenatal post-prandial hyperglycemia (PPG) could have normal FPG but abnormal PPG postnatally. The aim of this study is to characterize postnatal GTT results, to assess whether postnatal FPG alone is sufficient for screening and examine the role of ethnicity.

Method: Retrospective data was collected for all pregnant women who had a $75 \mathrm{~g}$ GTT (selective screening) between 2005 \& 2021, across 3 hospitals in the West Midlands, UK. Postnatal GTT results and ethnicity were obtained for the 
women with GDM. A descriptive analysis was used to characterize the GTT results and the role of ethnicity. Simple percentages and Chi-square test were used.

Results: A total of 14477 OGTTs were performed over the study period. The prevalence of GDM by modified WHO criteria ( $\geq 6.1 \& \geq 7.8 \mathrm{mmol} / \mathrm{l})$ was $1332(9.2 \%) .82 .5 \%$ are diagnosed purely by postprandial readings. The prevalence by IADPSG criteria ( $\geq 5.1 \& \geq 8.5 \mathrm{mmol} / \mathrm{l})$ would be $12.2 \%$ ( $\mathrm{n}=1763 ; 40 \%$ diagnosed by postprandial values). IADPSG diagnoses additional 845 women but missed 414 (31\%) of the original WHO cohort.

$51.7 \%(\mathrm{n}=689)$ of mothers attended postnatal GTT between 5 \& 20 weeks after delivery. 27.3\% were South Asians (SA) and $64.2 \%$ were White Caucasians (WC). Mean FPG was $4.9 \pm 0.86 \mathrm{mmol} / \mathrm{l}$ and mean PPG reading was $5.6 \pm 1.8 \mathrm{mmol} / \mathrm{l}$. There was a moderate linear correlation between the fasting and post prandial readings $(\mathrm{r}=0.409 ; \mathrm{p}<0.001)$.

Of the 689 women who had postnatal GTT, $14.9 \%$ $(\mathrm{n}=103)$ were abnormal. The prevalence of overt diabetes was $1.9 \%(\mathrm{n}=13)$. Of these $0.6 \%$ were diagnosed on fasting values alone, $0.9 \%$ on postprandial alone and $0.4 \%$ on both readings. The prevalence of impaired fasting glycaemia was $4.4 \%($ IFG; $\mathrm{n}=30)$, IGT $-7.4 \%(\mathrm{n}=51)$ and both IFG \& IGT $-1.3 \%(n=9)$. IGT accounted for $49.5 \%$ of all abnormalities on OGTT. The proportion of abnormal GTTs within the ethnic groups was marginally higher in SA compared to WC ( $18 \%$ vs $12.2 \%, \mathrm{p}=0.052)$.

$53.4 \%(\mathrm{n}=55$, T2D -4 , IGT- 51$)$ of total abnormalities will be missed if a FPG alone $(<6.0 \mathrm{mmol} / \mathrm{L})$ is used for postnatal screening. Out of these $34.5 \%$ were SA and $49.1 \%$ were WC. The odds of a SA woman being missed by the FPG alone was 1.7 times than that for WC $(\mathrm{p}=0.06)$.

Conclusion: As a third of women diagnosed by the WHO criteria will be missed by IADPSG, intervention studies are needed before adopting the IADPSG criteria.

In this large cohort of GDM women, nearly 15\% had abnormal GTT postnatally and post-prandial hyperglycemia was more common than fasting hyperglycemia. Using FPG alone for postnatal screening will miss half of the women with persistent hyperglycaemia. SAs are at a marginally higher risk compared to WC of being labeled "normal" by FPG. Identifying these women with IGT, will provide a crucial window of opportunity to reduce the risk of future T2D and prepare for subsequent pregnancies. Postnatal GTT should be the investigation of choice for postnatal screening in GDM.

Disclosure of Interest: None Declared.

\section{DOHaD13-1504}

Prevelance of high risk pregnancy in tertiary care hospital Rozina Khalid ${ }^{1, *}$

${ }^{1}$ Maternal noenatal and child health, Health Services Academy, Islamabad, Pakistan
Background: Throughout human history, care for expectant mothers has been based on one paramount objective that each pregnancy should end with a healthy mother and baby. Over recent decades, as maternal mortality has decreased, the emphasis of antenatal and intrapartum care has broadened and is now increasingly focused on the well-being of the fetus and newborn. The purpose of the study was

1. To determine the prevalence of high-risk pregnancies presenting during two months period at PNS Shifa hospital, Karachi.

2. To identify various risk factors in women presenting with high risk pregnancies.

3. To point out those areas where interventions can be made to reduce complications of high risk pregnancies

Method: The study was carried out in obstetric ward by checking medical record of high risk pregnant patients who were admitted or were referred from different armed forces hospitals from all over Sindh. All high risk cases were identified subsequently and relevant case notes were checked for study end points like socioeconomic status, blood pressure, hemoglobin, parity, gravidity, history of abortions, previous caesarean sections, complications during present pregnancy including Pregancy induced hypertension, Gestational Diabetes, Anemia, post-date, multiple pregnanc Analysis was done on SPSS version 14.

Results: Prevalence of high risk pregnancy was found to be $37.8 \%$

There are various medical complications which may occur during pregnancy making it high risk. Among these anemia cases had the highest percentage, $70 \%$ while pregnancy induced hypertension was found to be at $35 \%$ and $21 \%$ gestational diabetes.

Conclusion: The study indicates that prevalence of high risk pregnancy is more in low socio-economic group as compared to high socio-economic group. In connection with risk factors anaemia was found to be significant among low socio-economic group. Advanced maternal age and gravidity are associated with an increased risk of various complications like Pregancy induced hypertension, Gestational Diabetes and anaemia.

\section{References}

1. Tinker AG. Improving women's health in Pakistan. Human Development Network Series. The World Bank, Washington, DC, 1998.

2. WHO.UNFPA/UNICEF/World bank. Reduction of maternal mortality. Geneva 1999

3. UNFPA, fast fact sheet on maternal mortality

4. National Health Survey of Pakistan. Pakistan medical Research Council, Islamabad, Pakistan 1995.

5. Mansoor M, Ahmed Z, Saeed M. Development of precoded pregnancy monitoring card for use in teaching hospitals.Pakistan J. Med. 2004; 43(4)

Disclosure of Interest: None Declared. 


\section{DOHaD13-1676}

\section{The offspring among pregnant diagnosed gestational diabetes in their first pregnancy}

Hanne B. Wielandt ${ }^{1, *}$, Charlotte H. Blunk ${ }^{1}$, Helena Schønemann-Rigel ${ }^{1}$, Mia K. Hansen ${ }^{1}$, Miriam N. Markman ${ }^{1}$

${ }^{1}$ Institute of Regional Health Services Research, University of Southern Denmark, Kolding, Denmark

Background: Gestational diabetes mellitus (GDM) is defined as glucose intolerance with onset or first recognition during pregnancy. Recently, the differences between GDM and the underlying patho-physiology of Type1 Diabetes Mellitus have been discussed, emphasising that factors are common for GDM, Type2 Diabetes Mellitus and overweight. Further, the implication of the intrauterine milieu on the development of the fetus and thus on the future individual has been emphasised (1). Both aspects point to the importance of studies considering the offspring among pregnant diagnosed GDM in order to discuss the development of health and disease.

Method: The present study considers a prospective follow-up study among GDM patients, diagnosed by a two-step procedure in accordance with the Danish national programme for antenatal care. The pregnant women with glucosuria; prior GDM; pre-pregnancy $B M I \geq 27.0 \mathrm{~kg} / \mathrm{m}^{2}$; a family history of diabetes and/or a previous offspring birth weight above 4,500 grams were invited to have a $75 \mathrm{~g}$-2hours OGTT. Women with previous gestational diabetes and women with two of the risk factors mentioned above were invited to OGTT at gestational age (GA) 14-20. Women with only one risk factor or a non-diabetic OGTT in early pregnancy were invited again at GA 27-30. The blood samples were analysed at the hospital laboratory in accordance with standard procedures. The pregnant women were diagnosed with GDM, if the blood glucose was $9.0 \mathrm{mmol} / \mathrm{l}$ or higher after two hours. Only pregnant women diagnosed with GDM before GA 34 are considered in the present context. Women with pregestational diabetes mellitus are not comprised of the screening program (OGTT) and they are therefore not considered in the present study. Further, if the GDM-patient presented blood glucose and HbAlc values exceeding the treatment goals and therefore needed treatment with insulin, she was excluded from the study-population. In conclusion, the study considers a homogen population of pregnant women with glucoseintolerance predominantly due to insulin resistance.

Results: During the four-year period 0101 2009-31 12 2012 in total 12.920 pregnant women were admitted for delivery at the Department of Gynaecology and Obstetrics, Lillebaelt Hospital, Kolding, Denmark and among them a studypopulation of 401 GDM-patients $(3,1 \%)$ was defined. In total 166 were pregnant for the first time. The presentation considers the offspring, in total 172 infants, as there was six pairs of twins

Conclusion: The relation between maternal BMI and weight of the offspring at birth and at five months old is analysed.
The birthweight correlates to the weight at five months of age. Data in relation to breastfeeding is presented.

\section{References}

1. Catalano PM, Hauguel-De MS. Is it time to revisit the Pedersen hypothesis in the face of the obesity epidemic? Am J Obstet Gynecol 2011 Jun; 204(6):479-87.

Disclosure of Interest: None Declared.

\section{DOHaD13-1561}

\section{Impact of mothers' hygiene practices on nutritional status of young children}

Sarika Verma ${ }^{1, *}$, Neha Lohia ${ }^{1}$, Rajlakshmi Nair ${ }^{2}$, Padmini S. Ghugre ${ }^{1}$, Shobha Udipi ${ }^{1}$

${ }^{1}$ FOOD SCIENCE AND NUTRITION, S.N.D.T WOMEN'S UNIVERSITY; ${ }^{2}$ NUTRITION SPECIALIST, UNICEF, $M U M B A I$, India

Background: In India, almost half the children under 3 years are undernourished. The role of care as an important input for child health and nutrition is well recognized. Care encompasses all behaviours and practices at the household level of caregivers translating available food and health care resources into a child's growth and development. This also includes appropriate hygiene practices since poor sanitation and hygiene increased risk of morbidity and under nutrition. Therefore the present study was assessed to study the impact of mother's education and hygiene practices on nutritional status of children.

Hypothesis: Hypothesis tested was: Maternal hygiene care practices have an influence on child nutritional status.

Method: Mothers of 4441 children (below 3 years) were interviewed to assess Knowledge, Attitude and Practices in relation to hygiene in urban slums of Mumbai city. Nutritional status (WAZ, WHZ and HAZ, based on WHO criteria) was assessed using anthropometric measurements. ANOVA and $\chi^{2}$ was used to analyze whether hygiene practices influenced nutritional status.

Results: A considerable percentage of children were stunted (26.1\%) but a smaller percentage (8.4\%) were severely wasted; $20.4 \%$ were moderately stunted and 8.1 percent were moderately wasted.

Various feeding hygiene practices of mothers regarding were examined for their association with child nutritional status. The hygiene practices which were significantly associated with better nutritional status (WHZ, HAZ and WAZ) were boiling drinking water, heating the food before feeding the child, habit of giving the child food that has fallen on the floor, whether mother washes breast before feeding the child. Practices like letting children eat street foods mother not washing hand before lifting the child were associated with HAZ and WAZ. Disinfecting the bottle after feeding her child, heating the milk left in bottle before feeding it for the 
next time, keeping the bottle open without any lid, washing hand after going to toilet and cleaning child faeces, paying more attention on cleaning nails while washing hands were associated with stunting (HAZ).

Conclusion: The findings indicated that good care giving hygiene practices are associated with improved child nutritional status. The results revealed that caregivers who exhibited better quality of care practice had better-nourished children. Such caregivers were more likely to practice good household and personal hygiene than those of poorly nourished children.

Disclosure of Interest: None Declared.

\section{DOHaD13-1281}

On Japanese female university students' perception of developmental origins of health and disease: a questionnaire survey

Aya Endo ${ }^{1, *}$, Masahito Oyamada ${ }^{2}$

${ }^{1}$ Institute of QOL, Fuji Women's University; ${ }^{2}$ Department of Food Science and Human Nutrition, Fuji Women's University, Ishikarishi, Japan

Background: In Japan, the percentage of low-birth-weight $(<2,500 \mathrm{~g})$ newborns increased from $5.1 \%$ in 1980 to $9.6 \%$ in 2010 , and $29.0 \%$ of women in their twenties were underweight (BMI, <18.5) in 2010. These data have raised concern that chronic adult diseases related to $\mathrm{DOHaD}$ may further increase in the future. Thus, dissemination of the $\mathrm{DOHaD}$ concept among Japanese young females is an important issue for the improvement of their nutrition. However, scant data are available in Japan on public awareness of the influence of early nutrition on lifelong health.

The purpose of this study was to determine the level of awareness among Japanese young women about the influence of early nutrition on lifelong health. For this purpose, we conducted a survey of Japanese female university students using the questionnaire developed by Gage et al [Am J Clin Nutr 2011; 94(suppl): 2018S].

Method: Female university students (first, second, third, and fourth year, aged from 18 to 22) from the three respective departments of nutrition, childhood care, and human life studies were invited to complete a questionnaire. The total number of invited students was approximately 900 . We translated the questionnaire originally developed by Gage et al. into Japanese and used it for this survey.

Results: A total of 865 students from the departments of nutrition (329), childhood care (297), and human life studies (239) completed the questionnaire. Overall, diet during infancy was rated as an extremely/very important influence on adult health by $57 \%$ of the students who responded, a percentage comparable to $59 \%$ for genetics/inheritance but lower than for other potential factors, such as diet and physical activity in childhood/adolescence $(82 \%, 75 \%)$ and adulthood $(66 \%, 74 \%)$, air pollution $(70 \%)$, and exposure to cigarette smoke (94\%). The present results of Japanese young females showing relatively low levels of awareness of the effect of early nutrition on lifelong health are consistent with the results of first-time mothers in five European countries reported by Gage et al. No statistically significant differences were found among the three departments with respect to the students' levels of awareness of the effect of early nutrition on lifelong health: the percentage of students who rated diet during infancy as an extremely/very important influence on adult health came to $60 \%$ for the nutrition group, $53 \%$ for the childhood care group, and $57 \%$ for the human life studies group. However, the nutrition group, who received $\mathrm{DOHaD}$ education from the second year and beyond, were distinct from the childhood care and human life studies groups in that their levels of awareness of the effect of early nutrition on lifelong health increased as the academic year advanced. These results suggest that nutrition education could help to raise public awareness of the effect of early nutrition on lifelong health.

Conclusion: In our questionnaire survey, Japanese young females showed relatively low levels of awareness of the effect of early nutrition on lifelong health.

Disclosure of Interest: None Declared.

\section{DOHaD13-1622}

Scores on the dietary guideline index for children and adolescents (DGI-CA) are associated with insulin sensitivity in adolescents

Wendy Chan She Ping-Delfos ${ }^{1, *}$, Lawrence Beilin ${ }^{1}$, Wendy Oddy ${ }^{2}$, Sally Burrows ${ }^{1}$, Trevor Mori ${ }^{1}$

${ }^{1}$ Medicine and Pharmacology, University of Western Australia;

${ }^{2}$ Population Science, Telethon Institute for Child Health Research, Perth, Australia

Background: The Dietary Guideline Index (DGI) is the only Australian tool available to assess adherence to the Australian Dietary Guidelines and has also been developed and validated on children and adolescents (DGI-CA) ${ }^{1}$. To-date, the DGICA has only been used to assess adherence to the Guidelines and associations with adiposity. Its relationship with insulin sensitivity has not been examined. The aim of this study is to firstly determine the level of adherence to the Guidelines using the DGI-CA in adolescents at ages 14 and 17 years and to consequently examine the relationship between the assessed diet quality and markers of insulin sensitivity.

Method: Data collected during the 14- and 17-year followups of the Western Australian Pregnancy (Raine) cohort were analysed. The DGI-CA was used to determine the participants' diet quality from the food frequency questionnaires collected. The tool consists of 11 components that reflect the Australian dietary guidelines. The index criteria are age 
specific and a score closer to the maximum score of 100 reflects better adherence to the Guidelines. Physical and fasting biochemical measures were taken during a clinic visit using standard procedures. Background information, including socio-economic measures, was collected via questionnaires. HOMA-IR was derived from insulin and glucose levels. The Index of Relative Socio-economic Advantage and Disadvantage (IRSEAD) from the Australian Bureau of Statistics was used as a measure of socio-economic status. Longitudinal Linear Mixed models were used to investigate associations between markers of insulin sensitivity and DGI-CA, adjusting for IRSEAD, physical activity, BMI and gender. Interactions were investigated between DGI-CA and aforementioned covariates to determine if the relationship between DGICA and outcome was modified by any of the covariates.

Results: 1015 participants from the 14-year and 773 participants from the 17-year follow-ups completed all components required to compute the DGI-CA scores. The mean DGI-CA scores ( \pm SD) were similar at both 14 and 17-year (47.7 \pm 9.9 and $47.9 \pm 11.0$ respectively) and not significantly different between gender. Associations were found between DGI-CA and IRSEAD (Spearman rho $=0.19, \mathrm{p}=0.000$ ) and physical activity (Spearman rho $=0.23, \mathrm{p}=0.000)$. An increase in DGI-CA was found to be associated with a decrease in insulin levels $(p=0.008)$ and similarly for HOMA $(\mathrm{p}=0.004)$. No significant interactions were detected. There were no associations between glucose levels and DGI-CA ( $\mathrm{p}=0.291)$.

Conclusion: The DGI-CA is the only Australian tool available to assess adherence to the Australian Dietary Guidelines in Children and Adolescents. The level of adherence amongst the Raine adolescents is similar to the National cohort as published in Golley et al. ${ }^{1}$ (DGI-CA score of $48.6 \pm 0.5$ for ages 12 - to 16 - year olds). The DGI-CA was able to detect that small improvements in diet quality is associated with better insulin sensitivity.

\section{References}

1. Golley RK, Hendrie GA, McNaughton SA. Scores on the Dietary Guideline Index for Children and Adolescents Are Associated with Nutrient Intake and Socio-Economic Position but Not Adiposity. The Journal of Nutrition. 2011 July 1, 2011;141(7):1340-7.

Disclosure of Interest: None Declared.

\section{DOHaD13-1552}

\section{"Welcoming the baby": the use of a social network as health-enhancing tool}

Ana Maria D. U. Escobar ${ }^{1, *}$, Joana A. Ribeiro ${ }^{1}$, Roberta Rivellino $^{1}$, Alexandra V. M. Brentani ${ }^{1}$, Filumena S. Gomes ${ }^{1}$, Maria Helena Valente ${ }^{1}$, Murilo Wadt ${ }^{2}$, Andre Covic ${ }^{2}$, Sandra Grisi $^{1}$, Danilo Almeida ${ }^{2}$, Patricia Carvalho ${ }^{1}$

${ }^{1}$ Pediatrics; ${ }^{2}$ Faculdade de Medicina da USP, Sao Paulo, Brazil
Background: Brazil is a continent-sized country. It has socially underserved areas and its public health system is complex and unable to meet all demands in an effective and consistent manner. $60 \%$ of pregnant women in Brazil attend less than 7 prenatal doctors' visits. About $8 \%$ of Brazilian children are low birth weight infants. Over 65 million Brazilians access social network, about one-third of Brazil's population. $84 \%$ of these users are in Facebook. The objective of the study is to discuss the methodology that proposes, for the first time, follow a group 2000 pregnant women through a social network.

Method: All pregnant women who are in the first trimester of pregnancy will be invited to participate, on a voluntary basis and must accept the terms and conditions and fill out an online form with questions on their current lifestyle: eating habits, exercising, and sociodemographic data. These women will receive daily posts with pregnancy-related information, also providing guidance on the importance of undergoing prenatal care. Questions will be shared and clarified. At the end of the survey, mothers will fill out a new form to check whether there were any changes in their habits and lifestyle. In addition, a comparison will be made between the rate of low birth weight and post-partum depression in the population covered by the survey and the Brazilian population, adjusted for the same sociodemographic parameters. Each sampling element will be compared to itself at the beginning and at the end of the survey. The categorical variables will be described according to their frequencies (95\% confidence intervals). The association with the outcome will be established with the chi-square test. Continuous variable will be described according to their averages and standard deviations. The association with the outcome will be established with the Student's t-test. Nonparametric variables will be described by using the median (interquartile range), and compared by means of the Kruskal Wallis test; the likelihood of rejecting the null hypothesis will be established at 5\%.

Results: Early in 2012, "Dra. Ana Escobar" fanpage appeared on Facebook. In about 1 year and a half, the fanpage has accumulated over 500,000 active followers of which $97 \%$ are women, being $62 \%$ between 18 and 45 years old. The high levels of interactivity and engagement from followers measured by number of shares, comments and likes - enabled an average reach of 209,735.89 users (unique impressions) per post in April, 2013. A relevant result, considering the content complexity and normal reach from Facebook pages.

Conclusion: The research project will help put together a health promotion tool for pregnant women and babies. It is expected that the low weight rates and post partum depression rates within the targeted population will be decreased. If the results actually point that way and are, therefore, satisfactory, this tool may be expanded so as to cover a larger group of pregnant women, enabling a new group to be opened every month. Public policy may fall into step with this new instrument that may even be employed to implement healthcare actions.

Disclosure of Interest: None Declared. 


\section{DOHaD13-1103}

Consumption of diets with graded fat and carbohydrate content in mice over three generations induces changes in indices of growth and metabolism that vary between generations

Samuel P. Hoile ${ }^{1}$, Leonie R. Grenfell ${ }^{1}$, Sheila Barton ${ }^{2}$, Mark A. Hanson ${ }^{1}$, Karen A. Lillycrop ${ }^{1}$, Graham Burdge ${ }^{1, *}$

${ }^{1}$ University of Southampton, Southampton, United Kingdom; ${ }^{2}$ MRC Lifecourse Epidemiology Unit, University of Southampton, Southampton, United Kingdom

Background: The passage of induced phenotypes between generations has been reported in several phyla (1). In mammals, environmental challenge in an F0 generation alone induces phenotypic variation in subsequent generations $(2,3)$. Less is known about the effects on phenotype of a persistent environmental challenge over several generations. Increased energy intake over three generations of rats induced adjustments in fat and carbohydrate metabolism that implied a compensatory response involving specific epigenetic changes and altered expression of heat-shock protein-90 (4). Here, we have tested the hypothesis that indices of growth and metabolism in mice adjust to a graded dietary challenge sustained over three generations.

Method: Virgin female F0 mice were mated and fed diets containing carbohydrate: fat $(\mathrm{w} / \mathrm{w})$ ratios of either 3:1 $(21 \%$ fat $(w / w)), 4: 1,9: 1,15: 1$ or $23: 1(2.7 \%$ fat $(w / w)$; standard chow) throughout pregnancy and lactation. Offspring were fed the same diet as their dams. On postnatal day 90, females were either mated with males fed the 23:1 diet or killed. Males were killed on day 90. This procedure was repeated until the $\mathrm{F} 3$ generation to generate five female dietary lines. Body weight and food intake over 24 hours were measured weekly and summarised as area-under-the-curve. Organ weights on day 90 are expressed relative to body weight. Fasting plasma glucose and $\beta$-hydroybutyrate $(\mathrm{bHB})$ were measured by colorimetric assays. Because of the expected bimodal distribution, males and females were analysed separately by general linear models for each outcome with diet and generation as covariates, and generation*diet interaction $(\mathrm{P}<0.05$ was considered significant).

Results: There was no significant $\operatorname{diet}^{*}$ generation interaction effect on body weight. The model explained $12 \%$ of the variation in energy intake in females (diet*generation $\beta=-0.25)$. The model explained $10 \%$ of the variation in relative heart weight, $6 \%$ of the variation in lung weight and $8 \%$ of variation in testis weight (diet*aeneration $\beta=-0.009 ;-0.008 ;-0.006$, respectively) in males, but not females. The model explained $20 \%$ of the variation in fasting glucose (generation*diet $\beta=4.5$ ) in males and $14 \%$ of the variation in fasting glucose (generation*diet $\beta=2.0$ ) in females. There was no significant effect on fasting bHB concentration.
Conclusion: These findings show that changing the balance of carbohydrate and fat induced sex-related changes in the growth of specific organs in a manner that implied amelioration of the effect of diet over generations. However, differences in nutrient balance on glucose metabolism were exacerbated between generations Together these findings are consistent with the suggestion that specific aspects of growth and metabolism adjusted to the persistent dietary challenge over several generations.

This work was support by European $7^{\text {th }}$ Framework award; IDEAL.

\section{References}

1. Jablonka E Q Rev Biol 2009; 84:131

2. Kaati $G$ et al. EJHG 2002; 10:682

3. Pinheiro AR et al. Clin Sci 2008; 114:381

4. Burdge GC et al. PLoS ONE 2011; 6:e28282

Disclosure of Interest: None Declared.

\section{DOHaD13-1449}

Elevated S-adenosylhomocysteine perturbs adipocyte function but not differentiation via epigenetic mechanisms

Sherry Ngo ${ }^{1, *}$, Xiaoling $\mathrm{Li}^{1}$, Renelle O’Neill ${ }^{1}$, Chandrakanth Bhoothpur $^{1}$, Peter Gluckman ${ }^{1}$, Allan Sheppard ${ }^{1}$ and Developmental Epigenetics Group

${ }^{1}$ Liggins Institute, University of Auckland, Auckland, New Zealand

Background: Maternal deficiencies in key micronutrients affecting one-carbon cycle metabolism before and during pregnancy can result in fetal pathologies, and can influence the metabolic status and degree of metabolic syndrome of the progeny in adulthood through the hallmarks of metabolic syndrome, insulin resistance and obesity. The observation that plasma S-adenosylhomocysteine (SAH) levels are elevated with maternal vitamin deficiencies, and also in the progeny, led us to investigate whether this key one-carbon cycle intermediate could directly affect adipocyte differentiation and function.

Method: 3T3-L1 preadipocytes were proliferated and differentiated $-/+10 \mathrm{mM}$ and $100 \mathrm{mM}$ SAH. Mature adipocytes were assessed for basal and insulin-stimulated (100 nM; $20 \mathrm{~min}$ ) glucose uptake (GU), basal and isoproterenol (ISO)-stimulated (10 mM) lipolysis and lipid accumulation. Total glucose transporters (GLUT) -1 and -4 that mainly mediate basal and insulin-stimulated GU respectively, were assessed by Western blot. Total RNA was extracted to assess adipogenic genes expression using semi-quantitative RTPCR. CpG methylation at promoter region of target genes were measured using Sequenom Massarray. Histones H3 K27 and $\mathrm{K} 4$ trimethylation (H3K27me3, H3K4me3) occupancy on the promoter of target genes were assessed using chromatin immunoprecipitation. 
Results: Basal GU was impaired by $45 \%(\mathrm{p}<0.05)$ at $10 \mathrm{mM}$ and $30 \%(p=0.05)$ at $100 \mathrm{mM}$ SAH. Insulin-stimulated GU was reduced by $50 \%$ at both $10 \mathrm{mM}(\mathrm{p}<0.05)$ and $100 \mathrm{mM}$ $(\mathrm{p}<0.01)$ SAH. However, SAH did not alter total GLUT1 and -4 levels. Exposure to $10 \mathrm{mM}$ SAH did not alter total lipid accumulation but impaired basal and ISO-stimulated lipolysis by $70 \%(\mathrm{p}<0.05)$ and $50 \%(\mathrm{p}<0.05)$ respectively. Whilst gene expression of the key adipogenic markers preadipocyte factor 1 and peroxisome proliferator activated receptor- $\gamma 2$ were unchanged by SAH, CAAT enhancer binding protein $($ Cebp $) \alpha$ and retinoid $\mathrm{x}$ receptor $(R x r) \alpha$ were dramatically reduced by $90 \%(\mathrm{p}<0.05)$ and $50 \%(\mathrm{p}<0.01)$ respectively at $100 \mathrm{mM}$ SAH. Interestingly, exogenous $10 \mathrm{mM}$ and $100 \mathrm{mM} \mathrm{SAH}$ treatment yielded dramatic increases in intracellular $\mathrm{SAH}$ without altering the level of methyl donor S-adenosylmethionine suggesting that these effects of SAH were independent of global methyl donor availability but perhaps due to SAH's inhibition of various methyltransferase activities. Compared to untreated adipocytes, $100 \mathrm{mM}$ SAH-treated adipocytes yielded unchanged methylation on Cebp $\alpha$ promoter but methylation of specific CpG's on $R x r \alpha$ first intronic region distal to the promoter, was increased by $10 \%(\mathrm{p}<0.01)$ which is consistent with the reduction in gene expression. We also found that the histone marks H3K27me3 and H3K4me3 were selectively increased in adipocytes exposed to SAH compared to untreated adipocytes. The $(100 \mathrm{mM})$ SAH-mediated reduction in Cebp $\alpha$ and $R x r \alpha$ expression was associated with increased promoter occupancy of H3K27me3 by 4-fold ( $\mathrm{p}<0.05$ ) for Cebp $\alpha$; 2-fold ( $\mathrm{p}<0.01$ ) for $R x r \alpha$ compared to untreated adipocyte control.

Conclusion: While exposure to SAH did not affect the process of adipogenesis per se the functionality of mature adipocytes was altered, such that they exhibited features of insulin resistance. We have identified some of the principal molecular changes underpinning this altered phenotype and provide evidence that epigenetic mechanisms regulate adipocyte physiological function.

Disclosure of Interest: None Declared.

\section{DOHaD13-1174}

\section{Impact of pre-pregnancy parental overweight and obesity on offspring obesity: systematic analysis and meta analysis}

\author{
Nurzalinda Zalbahar ${ }^{1, *}$, Mamun Abdullah ${ }^{1}$ \\ ${ }^{1}$ School of Population Health, The University of Queensland, \\ Brisbane, Australia
}

Background: Parental obesity is one of the strongest determinants of offspring obesity. Several studies showed that pre-pregnancy parental overweight and obesity contribute to the offspring overweight and obesity. Some studies reported maternal-offspring association is stronger than paternaloffspring association. However, the link of pre-pregnancy parental overweight and obesity with offspring obesity in short and long term is relatively less understood. Currently, there is no systematic review and meta-analysis on this issue. Therefore, the objectives of this study are to determine the prospective association of pre-pregnancy parental overweight and obesity with offspring obesity in short and long-term and also to examine whether maternal-offspring link of overweight and obesity is stronger than the paternal-offspring link.

Method: A comprehensive literature search on online databases (PubMed, EMBASE, CINAHL, Web of Science, BioSis and Google Scholar) was conducted. All longitudinal studies reporting on pre-pregnancy parental, paternal and/or maternal BMI and its categories with offspring obesity (infant, child, adolescent and adult) were systematically reviewed. Adult overweight and obesity were defined by using body mass index (weight $(\mathrm{kg}) /$ height $(\mathrm{m})^{2}$ cut-offpoints of 25 and 30, respectively; childhood overweight and obesity were defined based on the international standard cutoff of body mass index. Adjusted odds ratios (ORs) from selected studies were extracted, calculated, and a quality adjusted meta-analyses was performed.

Results: A total of 19 articles $(n=70,662)$ were systematically reviewed from a database of 3366 independent articles. Majority of the articles reported significantly positive association and six articles found no significant association between pre-pregnancy parental BMI and offspring obesity. A total of 7 publications were performed subgroup metaanalysis. Overall, mother and father who were overweight have a similar risk of having an overweight children (motheroffspring OR 2.42, \%CI: 1.69, 3.44 and father-offspring OR 2.39, 95\%CI: 1.88, 3.05). The odds of having obese children are higher for pre-pregnancy overweight mothers (OR 3.94, 95\%CI: 3.15, 4.94) compared to pre-pregnancy overweight fathers (OR 2.56, 95\%CI: 2.06, 3.18). The odds of being obese among older offspring are nearly twice than younger offspring.

Conclusion: Our meta-analysis showed that pre-pregnancy parental BMI associated with offspring obesity in short and long term. Findings of this study support the notion of implementing obesity intervention for offspring before their birth

\section{References}

1. Chivers, P., Parker, H., Bulsara, M., Beilin, L., \& Hands, B. (2012). Parental and early childhood influences on adolescent obesity: a longitudinal study. Early Child Development and Care, 182(8), 1071-1087. doi: 10.1080/ 03004430.2012 .678590

2. Davey Smith, G., Steer, C., Leary, S., \& Ness, A. (2007). Is there an intrauterine influence on obesity? Evidence from parent child associations in the avon longitudinal study of parents and children (ALSPAC). Arch Dis Child, 92(10), 876-880.

Disclosure of Interest: None Declared. 


\section{DOHaD13-1237}

\section{MAGA's: possible candidates for environmental factors mediating DOHaD effects}

William Johnson $^{1, *}$, Steven Buyske ${ }^{2}$, Edward S. Stenroos ${ }^{1}$

${ }^{1}$ Neurology, UMDNJ-RWJMS; ${ }^{2}$ Statistics \& Genetics,

Rutgers University, Piscataway, United States

Background: The developmental origin of health and disease $(\mathrm{DOHaD})$ hypothesis involves factors that affect fetal growth during gestation and are believed to be environmental in nature. Genetic factors acting prenatally include: 1. fetal genes of maternal or paternal origin acting in fetal tissues, some with parent-of-origin effects and 2. maternal genes acting in maternal tissues, i.e. maternally acting gene alleles, MAGAs. MAGAs act independently of any inheritance by the fetus. From the mother's perspective, MAGAs are genetic factors, producing gene products, e.g., proteins or perhaps microRNAs or circular RNAs that influence intrauterine environment. However, from the fetus' perspective, MAGAs are environmental factors since they act independently of any inheritance from mother to fetus and since their effects result from gene action in the mother not in the fetus. MAGAs are one component of the gene-teratogen model ${ }^{1-2}$ that requires additional interacting factors including fetal alleles. Other names for MAGAs are teratogenic alleles, maternally acting alleles and non-inherited maternal alleles. Statistical methods to document MAGAs include the log-linear method, maternal TDT or maternal-fetal genotype incompatibility testing if an interacting fetal allele is known. Since 1939 there are at least 72 reports of 33 alleles of 28 genes for MAGAs $^{3-7}$, some implicated in PTD, SGA, IUGR or low birth weight. MAGAs are attractive candidates for factors mediating $\mathrm{DOHaD}$ since they are environmental factors from the perspective of the fetus and since they follow the observed inheritance pattern in half-sibs for human birth weight ${ }^{8}$. We hypothesized that there would be many independent mechanisms for MAGAs since their only unifying feature so far is occurrence in many neurodevelopmental disorders and since in fetuses that develop certain neurodevelopmental disorders, e.g., autism, more than 1000 genes may contribute. Method: To test this hypothesis, we identified reports of prenatal maternal effects and excluded those resulting from: maternal environmental effects acting on the fetus or interacting with a fetal genotype; mitochondrial genes; microchimerism; or known genomic imprinting. We included MAGAs that affect phenotype of the embryo or fetus. We determined possible mechanisms of action, understanding that genes may have more than one mechanism and considering the possibility of ascertainment bias.

Results: Among 72 reports of MAGAs we found that 57\% involved folate-related genes, $18 \%$ detoxification genes, $13 \%$ immune-related genes; the rest did not fit these categories. Conclusion: We hypothesized that MAGAs would result from many independent mechanisms. However we found that nearly all of the known MAGAs could be grouped into three categories. This has implications for their actions in contributing to $\mathrm{DOHaD}$ and efforts to prevent the resulting disorders.

\section{References}

1. Johnson WG. Am J Med Genet. (Neuropsychiatr. Genet.) 1999; 88: 311-323.

2. Johnson WG. Bio Essays 2003; 25:464-477.

3. Johnson WG, et al. Am J Med Genet. 2004; 124A: 339-45.

4. Johnson WG, et al. Am J Clin Nutr, 2005; 81: 664-668. 5. Williams TA et al. Arch Pediatr Adolesc Med 2007; 161:356-361.

6. Johnson WG, et al. Arch Ped Adol Med 2009; 163: 542-546.

7. Johnson WG, et al. Eur Psychiatr Rev, Winter 2011, vol 4, issue 2, pp117-127.

8. Morton NE, Ann Hum Genet. 1955; 20: 125-34.

Disclosure of Interest: None Declared.

\section{DOHaD13-1310}

\section{Maternal PCOS diagnosis and its implication for congenital birth defects and hospital admissions in the offspring}

Dorota A. Doherty ${ }^{1,2, *}$, John P. Newnham ${ }^{1,2}$, Carol Bower ${ }^{3}$, Roger Hart ${ }^{2}$

${ }^{1}$ Women and Infants Research Foundation, Subiaco; ${ }^{2}$ School of Women's and Infants' Health, The University of Western Australia, Crawley; ${ }^{3}$ WA Register of Developmental Anomalies, King Edward Memorial Hospital, Subiaco, Australia

Background: Polycystic ovary syndrome (PCOS) is the commonest endocrinopathy in women of reproductive years. PCOS is associated with increased risk of infertility, pregnancy complications and adverse pregnancy outcomes for the mother. Whilst there has been extensive research on the perinatal consequences of PCOS, studies assessing the longer term consequences for the offspring are still required. The objective of this study was to examine the effects of PCOS on the offspring beyond the perinatal period.

Method: Data on the long-term outcomes were available from a case-control study initially designed to evaluate the health effects in women hospitalised with a PCOS diagnosis (cases) between 1997 and 2010. Every case was age matched to 10 women without a record of a PCOS diagnosis at any time (controls). Pregnancy outcomes and general health of women's offspring were examined using the Western Australian statewide data systems recording all hospitalisations (Hospital Morbidity System), pregnancies beyond 20 weeks gestation (Midwives' Notification System), and congenital anomalies (Register of Developmental Anomalies). 
Offspring hospitalisations and the age of their occurrence were categorised according to primary and secondary ICD-10 diagnoses, and ages of first hospitalisations were examined. Logistic regression and Cox proportional hazards regression analyses were used to assess the effects of PCOS on the likelihood of adverse neonatal outcomes, congenital defects and hospitalisations. The PCOS effects were summarised using adjusted odds and hazard ratios (OR, HR) and their 95\% confidence intervals (CI), after controlling for relevant maternal and perinatal characteristics.

Results: Among the 2,566 PCOS cases and 25,660 nonPCOS controls, $69.7 \%(\mathrm{n}=1,789)$ of cases and $62.9 \%$ $(\mathrm{n}=16,139)$ of controls had at least one birth. Offspring hospitalisations up to the maximum age of 31 years were examined for 38,663 individuals. Offspring of women with PCOS were at higher risk of stillbirth $(1.8 \%$ vs. $0.6 \%$, $\mathrm{p}<0.001)$, preterm birth $(14.2 \%$ vs. $5.2 \%, \mathrm{p}<0.001)$, low birthweight $(10.1 \%$ vs. $5.8 \%, \mathrm{p}<0.001)$, and ICD-10 neonatal diagnoses $(24.9 \%$ vs. $15.1 \%, \mathrm{p}<0.001)$. Significant increases in any congenital anomalies $(5.8 \%$ vs. $4.7 \%$, $\mathrm{OR}=1.26$, CI $1.09-1.47$ ), any major anomalies ( $4.6 \%$ vs. $3.8 \%, \mathrm{OR}=1.23 \mathrm{CI} 1.04-1.45)$, cardiovascular defects alone $(1.4 \%$ vs. $1.0 \%, \mathrm{OR}=1.44 \mathrm{CI} 1.07-1.94)$ and uro-genital defects alone ( $1.9 \%$ vs. $1.4 \%, \mathrm{OR}=1.42 \mathrm{CI} 1.10-1.84)$ were found in offspring of PCOS cases. PCOS was associated with increased hospitalisations for ICD-10 diagnoses of metabolic disorder $(7.3 \%$ vs. $5.3 \%, \mathrm{HR}=1.32 \mathrm{CI} 1.16-1.50)$, any disease of nervous system $(7.8 \%$ vs. $5.8 \%, \mathrm{HR}=1.23 \mathrm{CI}$ $1.09-1.40)$, any eye disease (3.8\% vs. $2.8 \%, \mathrm{HR}=1.27$, CI $1.06-1.53)$, any ear disease ( $12.4 \%$ vs. $9.6 \%, \mathrm{HR}=1.36 \mathrm{CI}$ $1.23-1.50)$, asthma $(5.3 \%$ vs. $4.1 \%, \quad \mathrm{HR}=1.31 \quad \mathrm{CI}$ $1.13-1.54)$, upper and lower respiratory diseases $(19.0 \%$ vs. $15.2 \%, \mathrm{HR}=1.33 \mathrm{CI} 1.23-1.45)$ and $(7.5 \%$ vs. $5.7 \%$, $\mathrm{HR}=1.23$ CI 1.08-1.40), respectively.

Conclusion: A PCOS diagnosis in the mother is associated with increased risks of important health outcomes in the offspring, which will have major implications for their lifelong health and wellbeing. The increased risk of maternal PCOS for the offspring is not explained by the perinatal risks associated with this diagnosis.

Disclosure of Interest: None Declared.

\section{DOHaD13-1234}

\section{Multigenerational programming of adult HPA function} and behavior by single course antenatal glucocorticoid

Vasilis G. Moisiadis ${ }^{1}$, , Paul Blakeley ${ }^{1}$, Alisa Kostaki ${ }^{1}$, Stephen G. Matthews ${ }^{1,2,3}$

${ }^{1}$ Physiology; ${ }^{2} O B G Y N ;{ }^{3}$ Medicine, University of Toronto, Toronto, Canada

Background: Preterm birth occurs in approximately $10 \%$ of pregnancies, with the majority of women receiving treatment with a single course of synthetic glucocorticoids (sGC) to mature the fetal lungs. Mounting evidence from both human and animal studies suggests that even a single course of $s \mathrm{GC}$ can result in perturbed HPA function and growth in young first and second generation offspring $\left(\mathrm{F}_{1}, \mathrm{~F}_{2}\right)$. Little is known regarding the impact of single course sGC treatment on HPA activity and behavior in adults, across generations. We hypothesized that single course antenatal sGC treatment would reduce measures of growth, HPA function and attention, as well as increase locomotor activity in $F_{1}$ and $\mathrm{F}_{2}$ adult offspring.

Method: Pregnant guinea pigs were treated with betamethasone (Beta; $1 \mathrm{mg} / \mathrm{kg} ; \mathrm{n}=12)$ or saline $(\mathrm{C} ; \mathrm{n}=11)$ on gestational days $50 \& 51$ (term $\sim 69$ days). Adult $\mathrm{F}_{1}$ females (Beta; $\mathrm{n}=10 \& \mathrm{C} ; \mathrm{n}=8$ ) were mated with control males to produce $\mathrm{F}_{2}$ offspring; there was no manipulation during pregnancy. For $F_{1}$ and $F_{2}$ offspring, morphometry and weight were measured at birth and on postnatal day (PND) 20. In adulthood, offspring HPA function was assessed under basal and stress-activated conditions. Animals were tested in an open-field (OF; $30 \mathrm{~min}$ ) to assess locomotor activity/anxiety. Attention was assessed by prepulse inhibition (PPI) testing. Female offspring were tested during the estrous and midluteal phases of the reproductive cycle.

Results: $\mathbf{F}_{1}$ : Beta reduced birth weight and body size in $F_{1}$ females $(P<0.05)$. Adult Beta females exhibited a greater daily production of cortisol than $\mathrm{C}$ (estrous), and elicited a greater HPA response to challenge (luteal; $\mathrm{P}<0.05$ ). Beta did not alter basal cortisol in males, but significantly attenuated HPA response to challenge. Locomotor activity was reduced in Beta females (estrous) and males $(\mathrm{P}<0.05)$. PPI was also reduced in Beta males $\left(\mathrm{P}<0.05\right.$; decreased attention). $\mathbf{F}_{2}$ : Beta reduced birth weight and abdominal circumference in $\mathrm{F}_{2}$ male offspring $(\mathrm{P}<0.01 ; \mathrm{P}<0.05)$. Beta males displayed a rise in basal cortisol production at midday and mounted a more robust response to stress $(\mathrm{P}<0.05)$. Beta did not affect basal cortisol levels in females, but resulted in a reduced HPA response to stress. PPI was increased in $\mathrm{F}_{2}$ Beta males $(\mathrm{P}<0.05$; increased attention).

Conclusion: Antenatal exposure to a single course of $s \mathrm{GC}$ affected measures of growth, HPA function, attention and locomotor activity in two generations of adult offspring. These effects were sexually dimorphic, with male and female offspring responding differently depending on age and hormonal status. Interestingly, the sGC effects in the $F_{1}$ offspring were quite different from those in the $\mathrm{F}_{2}$ offspring; this was especially evident with the switch in attention between $\mathrm{F}_{1}$ and $\mathrm{F}_{2}$ males. The complex, and long-term, nature of these results indicates the necessity for longitudinal studies to identify the effects of $s G C$ treatment in human populations. We are currently undertaking molecular analyses to identify the mechanisms that underlie sGC-induced endocrine and behavioral changes.

Disclosure of Interest: None Declared. 


\section{DOHaD13-1356}

Transgenerational maternal effects on dairy production in New Zealand

Boyd W. Gudex ${ }^{1, *}$, Dave L Johnson ${ }^{1}$, Kuljeet Singh ${ }^{2, *}$

${ }^{1}$ LIC; ${ }^{2}$ Ruakura Research Centre, AgResearch Ltd, Hamilton 3240, New Zealand

Background: The impact of a cow's nutritional status, in this study indicated by her milk prroduction during the first half of pregnancy, upon the subsequent milking ability (whole lactation) of the resultant daughter and grand-daughters was investigated in the New Zealand dairy cattle industry.

Method: To differentiate between trans generational maternal affects and 'traditional' additive genetic inheritance, the geneticists 'animal' model was used to estimate both the additive (h2) and maternal heritabilities (m2).

Results: The variation in the daughters and grand daughters milk ability due to transgenerational maternal effects (m2) were similar, with between 0.9 and $1.5 \%$ (depending on the $\mathrm{dam} /$ daughter/grand daughter trait combinations) of the daughters milking ability and between 1.1 and $1.5 \%$ of the grand daughters milking ability associated with the cows milk production during pregnancy.

Conclusion: Although these results are highly similar in both the daughter and grand daughter generations, the magnitude of these results suggest that either transgenerational maternal effects on milk production are very small or that a cows milking ability during pregnancy is not a good measure of the maternal environment.

Disclosure of Interest: None Declared. Keywords: None.

\section{DOHaD13-1537}

A community based randomised controlled trial of pre-conceptional micronutrient supplementation to influence programming of diabesity - the Pune Intervention Study

Kalyanaraman Kumaran ${ }^{1,2, *}$, Pallavi Yajnik ${ }^{1}$, Himangi Lubree ${ }^{1}$, Charudatta Joglekar ${ }^{1}$, Dattatray Bhat ${ }^{1}$, Prachi Katre ${ }^{3}$, Urmila Deshmukh ${ }^{1}$, Caroline Fall ${ }^{2}$, Chittaranjan Yajnik ${ }^{1}$

${ }^{1}$ Diabetes Unit, KEM Hospital Research Centre, Pune, India; ${ }^{2}$ MRC Lifecourse Epidemiology Unit, University of Southampton, Southampton, United Kingdom; ${ }^{3}$ Life Sciences Domain, Persistent Systems, Pune, India

Background: The Pune Maternal Nutrition Study (PMNS) was established in 1993 to prospectively study the influence of maternal nutrition on foetal growth, and later cardiometabolic risk of the offspring. The study showed that newborns had a 'thin-fat' body type, with markedly low birthweight but relatively preserved body fat. High homocysteine and low vitamin B12 levels in pregnancy predicted lower birthweight and higher insulin resistance at 6 years in the offspring. B12 deficiency was widespread in this population and is due to low intake. A pilot study showed it can be effectively treated using physiological B12 doses $(2 \mu \mathrm{g})$ given daily for 12 months. We therefore commenced a community based B12 intervention study as the logical next step. The main hypothesis is that vitamin B12 supplementation of adolescent girls improves their offspring birth weight and B12 status, newborn and childhood body composition (reduced adiposity and increased lean mass), and reduce future diabesity.

Method: After ethical approval and initial community sensitisation, the adolescent boys and girls in the PMNS $(\sim 17$ years $)$ were consented, screened, counselled and recruited into the trial. They were individually randomised into 3 groups, to receive daily for at least 3 years or until their first delivery: 1) vitamin B12 $2 \mu \mathrm{g}$; or 2) vitamin B12 $2 \mu \mathrm{g}$ plus multiple micronutrients (MMN) plus $5 \mathrm{~g}$ of milk protein or 3) placebo. The MMN was added as a pragmatic measure to cover other micronutrient deficiencies. Iron and folic acid is given to participants in all three groups according to Indian guidelines. Compliance is assessed by monthly supplement counts. Morbidity is monitored on a monthly basis via a standard questionnaire every month, and information on adverse events is collected.

Results: Of the 690 who underwent screening, 557 were eligible for our study and were randomised into three groups. Those who were anaemic $(n=2)$, severely B12 deficient $(<$ $100 \mathrm{pmol} / \mathrm{L} ; \mathrm{n}=117)$ or had chronic medical illness $(\mathrm{n}=14)$ were excluded and managed appropriately. There were no significant differences in the baseline characteristics of the three groups. Over $50 \%$ were B12 deficient according to international standards $(<150 \mathrm{pmol} / \mathrm{L})$ while folate deficiency was rare $(\sim 2 \%)$. These adolescents had higher levels of B12 deficiency compared to their mothers (34\%). The distribution of the micronutrient supplements commenced in September 2012. Compliance after 6 months of supplementation is over $80 \%$ in $\sim 80 \%$ of the participants in all three groups; there were no significant differences in compliance between the three groups.

Conclusion: A community based individually randomised controlled trial is complex to organise and involved detailed planning of logistics. About $20 \%$ of the cohort has migrated to other areas necessitating considerable travel. The later than predicted age of marriage may extend the study time. Our long standing rapport with the local community has been invaluable in securing high participation rates. Collaboration with the local government and using existing systems will help participation rates when the trial is scaled up to influence policy as the results will have significant implications for public health in India.

Disclosure of Interest: None Declared. 


\section{DOHaD13-1263}

A daily snack containing green leafy vegetables, fruit and milk for 12 weeks increases women's erythrocyte docosahexanoic acid - a randomised controlled trial in slums of Mumbai, India

Harsha V. Chopra ${ }^{1,2, *}$, Sarah Kehoe ${ }^{3}$, Dnyaneshwar Tarwade $^{4}$, Harshad N. Sane ${ }^{1}$, S A. Sahariah ${ }^{1}$, Vanessa Cox $^{3}$, Ramesh Potdar ${ }^{1}$, Caroline Fall ${ }^{3}$, Sadhana Joshi ${ }^{2}$

${ }^{1}$ Centre for the Study of Social Change, Mumbai; ${ }^{2}$ Department of Nutritional Medicine, Interactive Research School for Health Affairs, Bharati Vidyapeeth Deemed University, Pune, India; ${ }^{3}$ MRC Lifecourse Epidemiology Unit, University of Southampton, Southampton, United Kingdom; ${ }^{4}$ Apnalaya, Govandi, Mumbai, India

Background: Alpha linolenic acid (ALA) is an essential and parent $n-3$ fatty acid found mainly in green leafy vegetables (GLV) and is further converted to eicosapentanoic acid (EPA) and docosahexanoic acid (DHA). Recent studies indicate that DHA supplementation during pregnancy influences the gestation period and infant size. Arachidonic acid (AA) and DHA are highly enriched in the brain and play critical role in brain development and cognition. Snack containing GLV, fruit and milk, was recently developed and used in Mumbai Maternal Nutrition Project (MMNP), a large randomized controlled trial (RCT) which showed increased birth weight and reduced small-for-gestational age births.

Objective: To investigate whether supplementing with a snack made from GLV, fruit and milk for 12 weeks (wks) would increase $n$-3 LCPUFAs especially DHA in women living in Mumbai slums.

Method: In our RCT, non-pregnant women aged 14-35 yrs $(\mathrm{n}=222)$ were randomized to receive an experimental snack (containing $25 \mathrm{~g} \mathrm{GLV}, 4 \mathrm{~g}$ dried fruits and $12 \mathrm{~g}$ milk powder) or a control snack containing 'low micronutrient' foods such as potato. Both experimental and control supplements were fried in sunflower oil. Anthropometric and demographic information was collected. Women were asked to eat the snack 6 days/week for 12 wks. Venous blood was collected at 0 and 12 wks. Food frequency data were also collected at both time points. A total of 15 different fatty acids which included saturated, monounsaturated and polyunsaturated fatty acids (PUFA) were estimated by Gas Chromatography. Fatty acids were expressed as $\mathrm{g} / 100 \mathrm{~g}$ fatty acid, i.e. percentage of total fatty acids as $100 \%$. The $n-3$ fatty acids included ALA, EPA, and DHA while $n-6$ fatty acids included linoleic acid, gamma linolenic acid, dihomo gamma linolenic acid, docosapentaenoic acid, and AA.

Results: The median (IQR) erythrocyte DHA in the experimental group increased from $1.50(1.11,2.03) \mathrm{g} / 100 \mathrm{~g}$ at 0 wks to $1.86(1.50,2.43) \mathrm{g} / 100 \mathrm{~g}$ at $12 \mathrm{wks}$, while those in the control group reduced from $1.78(1.37,2.32) \mathrm{g} / 100 \mathrm{~g}$ at 0 wks and $1.60(1.32,2.04) \mathrm{g} / 100 \mathrm{~g}$ at $12 \mathrm{wks}$. The median difference in erythrocyte DHA between 0 and 12 wks was
$-0.21(-0.42,-0.11)$ in the experimental group and 0.02 $(-0.05,0.30) \mathrm{g} / 100 \mathrm{~g}$ in the control group. Multiple regression analysis showed that erythrocyte DHA increased significantly $(p<0.001)$ in the experimental group compared to control and these findings remained consistent when adjusted for age, body mass index (BMI), standard of living index, compliance status, and the women's usual dietary intakes of GLV, meat and fish. Group allocation did not affect erythrocyte saturated, monounsaturated and n- 6 PUFAS.

Conclusion: Daily supplementation of these snacks for at least 12 wks increases stores of erythrocyte DHA in the women. The increase in the birth weight produced by the supplement in the MMNP trial may be mediated by the changes in fatty acids. Further, it may also influence the brain and nervous system development of their offspring.

Disclosure of Interest: None Declared.

\section{DOHaD13-1392}

A novel antibiotic for the treatment of intrauterine ureaplasma and mycoplasma infection: pharmacokinetic studies in the pregnant sheep model

Jeffrey A. Keelan ${ }^{1, *}$, Matthew W. Kemp ${ }^{1}$, Matthew Payne ${ }^{1}$, David Johnson $^{2}$, Sarah Stock ${ }^{3}$, Masatoshi Saito ${ }^{4}$, Prabhavathi Fernandes $^{5}$, John P. Newnham ${ }^{1}$

${ }^{1}$ School of Women's and Infants' Health, University of Western Australia, Perth, Australia; ${ }^{2}$ Microconstants Inc., San Diego,

United States; ${ }^{3}$ Department of Obstetrics \& Gynaecology, University of Edinburgh, Edinburgh, United Kingdom; ${ }^{4}$ Division of Perinatal Medicine, Tohoku University Hospital, Sendai, Japan; ${ }^{5}$ Cempra, Inc., Chapel Hill, United States

Background: Intrauterine infection and inflammation play a key role in the aetiology of spontaneous preterm birth (PTB), particularly in deliveries less than 32 weeks' gestation. Macrolides (e.g. erythromycin) have been prescribed during pregnancy for the prevention of PTB as they are effective in treating important genital tract microorganisms such as Ureaplasma and Mycoplasma spp. However, there is evidence that maternal erythromycin administration is largely ineffective in eradicating intrauterine ureaplasma infection due to poor transplacental passage $(<4 \%)$. A more effective antibiotic with better maternal-amniotic-fetal transfer properties is required to eradicate both fetal and amniotic infection. Solithromycin is a novel broad-spectrum fluoroketolide antibiotic that is exceptionally potent against Ureaplasma and Mycoplasma spp. Here we explored the pharmacokinetics and maternal-amniotic-fetal transfer of solithromycin in our pregnant sheep model to assess its potential for treating intrauterine and antenatal infection.

Method: Chronically catheterized pregnant ewes $(n=6-7)$ at $\sim 115$ days gestation received either a single maternal IV infusion of solithromycin (Cempra, Inc.) $(10 \mathrm{mg} / \mathrm{kg}$ over $1 \mathrm{~h})$, 
a single intra-amniotic (IA) injection $(1.4 \mathrm{mg} / \mathrm{kg}$ estimated fetal weight), or a combined IV and IA dose. Maternal plasma (MP), fetal plasma (FP) and amniotic fluid (AF) samples were taken via catheter at $0.5,1,2,4,8,12,24,48$ and $72 \mathrm{~h}$ post administration. Samples were analysed by LC-MS/MS to determine concentrations of solithromycin and its bioactive $\mathrm{N}$-acetylated metabolite (NAc-S). Pharmacokinetic parameters were calculated using PKSolver.

Results: Following maternal IV infusion, peak solithromycin concentrations in MP, FP and AF were 1073, 353 and $214 \mathrm{ng} / \mathrm{ml}$, respectively. This represents a maternal-to-fetal transfer efficiency of $34 \%$. The $t \frac{1}{2}$ in plasma was $\sim 5 \mathrm{~h}$, whereas in $\mathrm{AF}$ it was much longer at $\sim 20 \mathrm{~h}$. A single maternal dose resulted in effective concentrations $(>30 \mathrm{ng} / \mathrm{ml})$ in MP, $\mathrm{FP}$ and $\mathrm{AF}$ sustained for $>12 \mathrm{~h}$. NAc-S was detected in considerable concentrations in FP and AF; it exhibited delayed accumulation and clearance, resulting in an extended antimicrobial effect $(>48 \mathrm{~h})$, particularly in AF. IA injection resulted in high $(\sim 50 \mu \mathrm{g} / \mathrm{ml})$ and sustained solithromycin concentrations in AF and significant solithromycin levels in FP, although the efficiency of amniotic-to-fetal transfer was low $(\sim 1.5 \%)$. Antimicrobial efficacy was augmented by accumulation of considerable concentrations of NAc-S in AF and FP. Combined IV and IA administration resulted in primarily additive concentrations in all three compartments. Conclusion: Our findings suggest that solithromycin is the first antibiotic of its class to exhibit significant maternal-tofetal and maternal-to-amniotic transfer. Solithromycin may provide, for the first time, an effective antimicrobial approach for the prevention and treatment of intrauterine infection and provide a new opportunity for early prevention of preterm birth. Studies in humans are underway to confirm these data.

Disclosure of Interest: J. Keelan: None Declared., M. Kemp: None Declared., M. Payne: None Declared., D. Johnson Consultant for: Cempra Inc. to provide analytical services, S. Stock: None Declared., M. Saito: None Declared., P. Fernandes Shareholder of: Cempra Inc., J. Newnham: None Declared.

\section{DOHaD13-1344}

\section{Chips-child: follow up of a RCT of differential blood pressure control during pregnancy on offspring health}

Tessa Rosebom ${ }^{1, *}$ and CHIPS CHILD working group

\section{${ }^{1}$ Academic Medical Centre Amsterdam, Amsterdam, Netherlands}

Background: The early environment shapes later health and wellbeing. Maternal blood pressure (BP) has been shown to affect offspring growth and development in observational studies. In a randomised controlled trial (RCT) of differential maternal blood pressure (BP) control in pregnancy, we investigate the programming effects of 'less tight' $\mathrm{BP}$ control [target diastolic BP (dBP) $100 \mathrm{mmHg}$ ] vs. 'tight' control (target dBP $85 \mathrm{mmHg}$ ) on offspring health. Our hypothesis is that 'tight' (vs. 'less tight') maternal BP control in pregnancy will be associated with fetal undernutrition, and as consequence, accelerated postnatal growth, hypothalamic-pituitary-adrenal (HPA) axis activation, and a modified DNA methylation profile. Method: The CHIPS RCT is designed to answer how maternal $\mathrm{BP}$ in pregnancy should be managed in order to optimise clinical outcomes for babies and keep mothers safe. From 95 international centres, CHIPS recruited 1,031 women with: pre-existing or gestational hypertension; office dBP $90-105 \mathrm{mmHg}$ (or dBP $85-105 \mathrm{mmHg}$ if on antihypertensive medication); live fetus; and 14-33 weeks; women were excluded if they: had severe systolic hypertension $(\geqslant 160 \mathrm{mmHg}$ ), proteinuria, or a contraindication to either arm of trial or to prolongation of pregnancy; had used ACE inhibitors at $\geqslant 14$ weeks; had a multiple gestation or lethal/ major fetal anomaly; planned to terminate pregnancy; or had already participated in CHIPS. Women were randomised to 'less tight' or 'tight' control of maternal BP, stratified by centre and type of hypertension (pre-existing or gestational).

In the ongoing CHIPS-Child study, we are extending the child outcome measures to age 12 months (and beyond) and focussing on in utero metabolic cues for developmental programming.

Results: We are measuring the size of babies at birth (birthweight), as well as their postnatal growth at 12 months of age (i.e., length, weight, head and waist circumference). We will assess function of the HPA axis through cortisol measurements in hair. We will collect buccal swabs for epigenetic analyses. In the future, neurodevelopmental followup as well as cardiometabolic outcomes will be considered.

Conclusion: CHIPS-Child is designed to improve our understanding of $\mathrm{DOHaD}$ in the setting of maternal $\mathrm{BP}$ control in pregnancy. It will undertake a 'naturalistic' test linked to a definitive RCT to determine if differential maternal BP control in pregnancy has a developmental programming effect. CHIPSChild will capitalise on the robust CHIPS RCT design, which provides the opportunity to tease out the independence of the in utero environment from genetic variability and prenatal nutrition; these should, along with other 'unknown' factors, be balanced between the groups in this large RCT. CHIPS-Child will also create future opportunities for epigenetic investigation by creating a DNA bank from paediatric follow-up samples.

Disclosure of Interest: None Declared.

\section{DOHaD13-1376}

Impact of an unsupervised walking intervention during pregnancy on post-partum weight retention and infant outcomes

Kai Ling Kong ${ }^{1, *}$, Christina G. Campbell ${ }^{2}$, Kelly A. Wagner ${ }^{2}$, Anna D. Peterson ${ }^{3}$, Lorraine M. Lanningham-Foster ${ }^{2}$

${ }^{1}$ Pediatrics, State University of New York at Buffalo, Buffalo;

${ }^{2}$ Food Science and Human Nutrition; ${ }^{3}$ Statistic, Iowa State

University, Ames, United States 
Background: Interventions have been designed to help pregnant women increase their physical activity (PA) participation to improve maternal and fetal health outcomes. Yet, few studies have investigated the impact of these interventions on post-partum weight retention, and/or infant growth. The purposes of this study were 1) to compare post-partum weight retention of participants enrolled in a walking intervention during pregnancy versus a nonintervention control group, as well as their offspring outcomes (weight-for-length z-score (WLZ score), fat mass and fat-free mass) at one and six months of age; 2) to examine the relationship between pre-pregnancy body mass index (BMI) and trimester-specific gestation weight gain rates with post-partum weight retention and child outcomes at one and six months of age.

Method: Thirty seven previously non-exercising, overweight or obese (BMI $\geq 25.0 \mathrm{~kg} / \mathrm{m}^{2}$ ) pregnant women were randomly assigned to an unsupervised, free-living walking intervention or non-intervention control group. The women were provided with a treadmill for home use. The length of the walking intervention was at least 20 weeks. For the follow-up study, weight of the mother and weight, length and body composition of the infant were collected at one month post-partum $(n=37)$ and six months post-partum $(n=33)$. ANOVA was used to determine the differences in maternal post-partum weight retention and child outcomes. A Pearson correlation coefficient analysis was conducted to examine the association between pre-pregnancy BMI and trimesterspecific GWG rates with post-partum weight retention and child outcomes.

Results: The walking program was successful in changing the walking behavior of the pregnant women in the intervention group. These women were able to significantly increase their moderately intense walking cadence, and were, most importantly, able to sustain these habits until late pregnancy (data published elsewhere). The results of this follow-up study showed that at six months post-partum, weight retention of obese women in the intervention group (Int-OB) was $-0.10 \pm 8.11 \mathrm{~kg}(0.8 \%$ of weight retention from prepregnancy weight); while, obese women in the control group (Con-OB) was $6.35 \pm 7.47 \mathrm{~kg}$ ( $7 \%$ of weight retention from pre-pregnancy weight). Lower WLZ scores were observed among infants who were born to obese women in the intervention group at one month (Int-OB $=-0.037$, Con$\mathrm{OB}=0.311 ; p=0.7904$ ) and six months (Int- $\mathrm{OB}=0.167$, Con-OB $=1.04 ; p=0.430$ ), however it was not statistically significant. Post-partum weight retention at one and six months post-partum were significantly correlated with all trimester-specific GWG rates, but not pre-pregnancy BMI. Infant WLZ score at six months was significantly correlated with third trimester GWG rate only.

Conclusion: The reduced post-partum weight retention observed among the obese women in the intervention group may be explained in part by the lifestyle modification during pregnancy. Targeting PA interventions for obese women early during pregnancy could be a promising starting point for obesity prevention.

Disclosure of Interest: None Declared.

\section{DOHaD13-1688}

Increasing women's intake of green leafy vegetables, fruit and milk pre-conceptionally and through pregnancy increases birthweight: a randomised controlled trial in Mumbai, India (Mumbai Maternal Nutrition Project)

Ramesh Potdar ${ }^{1,1, *}$, Sirazul Sahariah ${ }^{1}$, Meera Gandhi ${ }^{1}$, Monika Dayama ${ }^{1}$, Nick Brown ${ }^{2,3}$, Harshad Sane ${ }^{1}$, Patsy Coakley ${ }^{4}$, Ella Marley-Zagar ${ }^{4}$, Harsha Chopra ${ }^{1}$, Sarah Kehoe $^{4}$, Devi Sane ${ }^{1}$, Vanessa Cox ${ }^{4}$, Vijaya Taskar ${ }^{5}$, Barrie Margetts ${ }^{6}$, Caroline Fall ${ }^{4}$

${ }^{1}$ Centre for the Study of Social Change, Mumbai, India; ${ }^{2}$ Salisbury District Hospital, Salisbury, United Kingdom; ${ }^{3}$ Aga Khan University, Karachi, Pakistan; ${ }^{4}$ Lifecourse Epidemiology Unit, MRC, University of Southampton, Southampton, United Kingdom; ${ }^{5}$ Streehitakarini, Mumbai, India; ${ }^{6}$ Public Health Nutrition, University of Southampton, Southampton, United Kingdom

Background: Low birth weight (LBW) is a major public health problem in low- and middle-income countries, and is associated with increased infant mortality, childhood stunting, impaired cognitive function and an increased risk of adult chronic disease. The World Health Organization has set a global target to reduce LBW by $30 \%$ by 2025 . An earlier observational study among under-nourished rural Indian women showed that those who reported higher intakes during pregnancy of green leafy vegetables (GLVs), fruit and milk had larger newborns. The objective of this study was to determine the effect of a daily snack made from these foods, taken for at least three months before conception and until delivery, on newborn anthropometry.

Method: A non-blinded, individually randomised controlled trial was carried out among women living in slums in Mumbai, India, between 2006 and 2012. Married non-pregnant women aged $<40$ years were recruited and randomised to receive a daily snack made from green leafy vegetables, fruit and milk or a control snack made from vegetables of low micronutrient content, under supervision, until delivery. Trained staff measured newborns within 72 hrs of delivery.

Results: Of 6,513 non-pregnant women enrolled, 2,310 became pregnant and 1,826 were supplemented for $\geq 3$ months prior to conception. Of these, 1,562 delivered live singleton newborns, and 1,094 newborns were measured. The intervention increased birth weight by $48 \mathrm{~g}$ overall (control: 2583g, treatment: 2631g; $\mathrm{p}=0.05)$. The effect increased with maternal BMI $(+113 \mathrm{~g}, \mathrm{p}=0.008 ;+79 \mathrm{~g}, \mathrm{p}=0.07$ and $-8 \mathrm{~g}, \mathrm{p}=0.8$ in the highest, middle and lowest thirds of maternal BMI; $\mathrm{p}$ for interaction $=0.001$ ). Similar effects 
were observed for newborn chest, abdomen and mid-upperarm circumferences and skinfolds $(\mathrm{p}<0.05)$, but not length or head circumference. Low birthweight and small-forgestational-age births were reduced by approximately $20 \%$ (OR: $0.76,95 \%$ CI: $(0.59,0.98), \mathrm{p}=0.03$; and $0.78,95 \%$ CI: $(0.60,1.03), p=0.07$ respectively). There were no significant effects on gestation, or risk of pre-term birth or intra-uterine death/stillbirth.

Conclusion: A daily snack made from local micronutrientrich foods, for at least three months pre-conceptionally and throughout pregnancy, resulted in larger newborn size in a slum population with high rates of intra-uterine growth restriction. This effect was comparable with, and up to twice as effective as, pharmaceutical multiple micronutrients taken in pregnancy alone. There was no effect in mothers of low BMI $\left(<18.5 \mathrm{~kg} / \mathrm{m}^{2}\right) ;$ maternal diets need to have adequate macronutrients in addition to high micronutrient quality for optimal reproductive success.

Acknowledgements: The project was funded by the Wellcome Trust and Medical Research Council, UK, ICICI Social Initiatives Group, Mumbai and Parthenon Trust, Switzerland.

Disclosure of Interest: None Declared.

\section{DOHaD13-1599}

\section{Postprandial metabolism and inflammatory markers in overweight adolescents}

Lucia C. Pellanda ${ }^{1,2, *}$, Bianca C. Schauren ${ }^{1}$, Vera Lucia Portal ${ }^{1}$

${ }^{1}$ Post graduation Program, Fundação Universitária de Cardiologia do Rio Grande do Sul (FUCRS); ${ }^{2}$ Public Health, Universidade Federal de Ciências da Saúde (UFCSPA), Porto Alegre, Brazil

Background: Throughout the past decades, diet composition went from rich in fibers to rich in saturated fat, trans fatty acids and simple carbohydrates. These lifestyle changes have an impact in the lipids metabolism. Since modern occidental man lives most of the day in a postprandial state and because diet sources of lipids usually exceed its real requirements, the organism is constantly facing an overload of circulating lipids, which may lead to endothelial dysfunction, oxidative stress and exaggerated inflammatory response. Such changes may be further aggravated in the presence of overweight. Thus, the aim of this study aims is to describe the postprandial metabolism of lipids, carbohydrates and inflammatory response in overweight (OW) and normal weight (NW) adolescents.

Method: Sixty-two adolescents aged 11 to 18 years, with normal fasting blood glucose and triglycerides (TG), were dividided in two groups: overweight $(\mathrm{OW} ; \mathrm{n}=39)$ and normal weight (NW; $n=23$ ). Total cholesterol (TC), HDL$\mathrm{C}$, triglycerides (TG), glucose, insulin, hsCRP, fibrinogen (F) and leukocytes $(\mathrm{L})$ were collected in fasting and 4 and 6 hours after a meal with high fat content $(1.000 \mathrm{Kcal}, 27.4 \%$ de carbohydrates, $14.7 \%$ protein and $57,8 \%$ lipids $(30.4 \%$ saturated, $32.7 \%$ monounsaturated and $26.5 \%$ polyunsaturated fatty acids; and $288 \mathrm{mg}$ total cholesterol). ANOVA for repeated measures was performed.

Results: OW adolescents showed significantly higher fasting values of TC $(p=0.036)$, LDL-C $(p=0.010)$, TC/HDL $(p=0.013)$, fibrinogen $(p=0.036)$ and hsCRP $(p=0.004)$. TC $(p=0.037)$, LDL-C $(p=0.009)$, TC/HDL $(p=0.013)$ and fibrinogen $(p=0.029)$ increased and remained higher in the OW group in response to OFTT. Insulin, glucose and TG values did not differ significantly.

Conclusion: Adolescents with OW showed higher LDL-C and inflammatory markers levels, and a more exacerbated response after OFTT in relation to TC, LDL-C, TC/HDL, fibrinogen and hsCRP levels. These findings are clinically important, since the atherosclerotic process may begin early in the lifecourse and post prandial lipid and inflammatory metabolism may be early markers of cardiovascular risk. To identify early markers of risk in this population is of paramount importance if we are to prevent an epidemic rise in atherosclerotic manifestations in the future.

Disclosure of Interest: None Declared.

\section{DOHaD13-1698}

The effect of antenatal lifestyle advice for women who are overweight or obese on newborn anthropometry: the limit randomised trial

Jodie Dodd ${ }^{1,2,3, *}$, Sheryl Rifas ${ }^{4}$, Lisa Yelland ${ }^{1,2}$, Andrea Deussen $^{1,2}$, Matthew Gillman ${ }^{4}$, Rosalie Grivell ${ }^{1,2,3}$, Deborah Turnbull $^{5}$, Andrew McPhee ${ }^{6}$, Gary Wittert ${ }^{7}$, Julie Owens ${ }^{1,2}$, Jeffrey Robinson ${ }^{1,2}$

${ }^{1}$ Discipline of Obstetrics \& Gynaecology, The University of Adelaide, Adelaide; ${ }^{2}$ Robinson Institute, The University of Adelaide; ${ }^{3}$ Department of Perinatal Medicine, Women's and Children's Hospital, North Adelaide, Australia; ${ }^{4}$ Obesity Prevention Program, Harvard Pilgrim Health Care Institute, Boston, United States; ${ }^{5}$ School of Psychology, The University of Adelaide, Adelaide; ${ }^{6}$ Department of Neonatal Medicine, Women's and Children's Hospital, North Adelaide; ${ }^{7}$ School of Medicine, The University of Adelaide, Adelaide, Australia

Background: Overweight and obesity is a significant health concern during pregnancy and childbirth. Infants born to women who are overweight or obese are more likely to be large for gestational age compared with infants born to women with a normal BMI, which may have implications for longer-term health. Body weight at birth is a crude indicator of body composition and measuring skinfold thickness provides additional information about the proportion and distribution of fat free mass and body fat. Our aims were to report the effect of providing antenatal dietary and lifestyle advice to women who are overweight or obese on newborn anthropometry. 
Method: We conducted a randomised controlled trial, where pregnant women with a body mass index $\geq 25 \mathrm{~kg} / \mathrm{m}^{2}$ at their first antenatal appointment, with a singleton gestation between $10^{+0}$ and $20^{+0}$ weeks were recruited from public maternity units across metropolitan Adelaide, South Australia. Women were randomised to receive Lifestyle Advice or continued Standard Care. Anthropometric measurements were obtained within the first days after birth according to a protocol specifically developed for use in infants. Anthropometric measurements included biceps, triceps, abdominal, suprailiac, subscapular and thigh skinfold thicknesses and head, chest abdominal and right upper arm circumferences. Fat free mass and body fat was calculated based on infant sex, weight and triceps, subscapular and thigh skinfold thicknesses, in addition to the use of bioimpedance analysis (BIA). Results: A total of 970 infants with available anthropometric measures were included in the analyses, of which 394 had additional bioimpedance assessment. The mean birth weight was $3.544 \mathrm{~g}( \pm 502 \mathrm{~g})$, length $50.06 \mathrm{~cm}( \pm 2.15 \mathrm{~cm})$ and ponderal index $28.14 \mathrm{~kg} / \mathrm{m}^{3}\left( \pm 2.72 \mathrm{~kg} / \mathrm{m}^{3}\right)$. The mean calculated percentage body fat from skinfold thickness measurements was $14.39 \%( \pm 3.42 \%)$, and from bioimpedance analysis (R50 kHz) 10.86\% ( $\pm 3.18 \%)$. There were no statistically significant differences identified between infants born to women in the Lifestyle Advice group or Standard Care group, for any of the measures obtained.

Conclusion: For women who are overweight or obese, provision of an antenatal dietary and lifestyle intervention was not associated with differences in measures of newborn anthropometry and estimated fat mass.

References TRIAL REGISTRATION: Australian and New Zealand Clinical Trials Registry (ACTRN12607000161426).

Disclosure of Interest: None Declared.

\section{DOHaD13-1412}

Trials and tribulations of a randomised controlled trial the Mumbai Maternal Nutrition Project, Mumbai, India

Ramesh Potdar ${ }^{1, *}$, Sirazul Sahariah ${ }^{1}$, Meera Gandhi ${ }^{1}$, Harsha Chopra $^{1}$, Harshad Sane ${ }^{1}$, Sarah Kehoe ${ }^{2}$, Devi Sane ${ }^{1}$, G Subbulakshmi ${ }^{1}$, Monika Dayama ${ }^{1}$, Nick Brown ${ }^{3,4}$, Barrie Margetts $^{5}$, Caroline Fall ${ }^{2}$

${ }^{1}$ Centre for the Study of Social Change, Mumbai, India; ${ }^{2}$ Lifecourse Epidemiology Unit, MRC, University of Southampton, Southampton; ${ }^{3}$ Salisbury District Hospital, Salisbury, United Kingdom; ${ }^{4}$ Aga Khan University, Karachi, Pakistan;

${ }^{5}$ Public Health Nutrition, University of Southampton, Southampton, United Kingdom

Background: The Mumbai Maternal Nutrition Project was a randomised controlled trial of a food based micronutrientrich supplement for women for 3 months before and during pregnancy, in the slums of Mumbai. The primary outcome was birth weight. The 5-year trial is complete, and the results are emerging. This paper describes some of the challenges faced in carrying out the project.

Method: Women $(\mathrm{N}=6513)$ were recruited after community meetings explaining the pros and cons of the project. Randomisation was stratified by age and BMI. Supplements were developed by establishing a special kitchen under qualified nutritionists and 110 recipes were used, in four groups ( 2 experimental and 2 control) in order to obscure group allocation, because double blinding of a food-based intervention was not possible. Supplements were transported to and distributed via 56 centres daily. Consumption was supervised and recorded. Pregnancy surveillance was carried out using a computerised system, with the expected date of delivery calculated from last menstrual period dates and serial ultrasound scans. Babies were measured within 72 hours of birth in hospital or at home.

Results: Difficulties faced were as follows: Recruitment: Some infertile women joined the trial hoping for a better chance of conception after eating the supplement, a rumour created in the community by unknown sources. Rumour-mongers also suggested that the supplement may cause infertility. One-toone counselling solved this problem. Blinding and choice of supplement: Since participants were married women, it was impossible to hide the ingredients of the supplements and they asked why there was so little day-to-day variation. The ingenuity of our staff was stretched to the limits in satisfying their culinary inquisitiveness. Distribution, timing and compliance: Care had to be taken that the supplement did not become a substitute for the women's normal diet by giving it to them at tea time without tea. Eating it under observation and recording, without sharing it with a friend or their own child, was traumatic to many. Fasting practices during festivals, both Hindu and Muslim, required expert management strategies. Pregnancy surveillance and follow-up: Constant surveillance of the participants, delivering at numerous different institutions in Mumbai or wider Maharashtra was a challenge, as was shifting of the families when slums were demolished for redevelopment. Avoiding (pseudo) adverse effects: A common fear was that the supplements would make babies too large, requiring caesarean section.

Conclusion: Despite these challenges MMNP (SARAS) was a very satisfying experience for the whole team, who developed new expertise in management of big intervention trials in slum communities. Our trials and tribulations may be entertaining and informative to $\mathrm{DOHaD}$ scientists who want to undertake large trials.

Acknowledgements: The project was funded by the Wellcome Trust and Medical Research Council, UK, ICICI Social Initiatives Group, Mumbai and Parthenon Trust, Switzerland.

Disclosure of Interest: None Declared. 


\section{DOHaD13-1117}

\section{A health economic model to assess the impact of high birth weight on public health}

Irene Lenoir-Wijnkoop ${ }^{1}$, Mark Nuijten ${ }^{2}$, Eline M. Van Der Beek $^{3, *}$, Ricardo Uauy ${ }^{4}$

${ }^{1}$ Pharmaceutical Sciences, Utrecht University, Utrecht;

${ }^{2}$ Ars Assessus Medica, Rotterdam, Netherlands; ${ }^{3}$ Centre

for Specialised Nutrition, Danone Research, Singapore,

Singapore; ${ }^{4}$ Institute of Nutrition, INTA University of Chile,

Santiago, Chile

Background: Despite the general interest in the impact of overweight and obesity on public health, little is known about the social and economic impact of being born large for gestational age (LGA, $>90$ th centile) or macrosomic (birth weight $>4,000 \mathrm{~g})$. Both conditions are related to maternal obesity and/or gestational diabetes (GDM) and associated with increased short term morbidity for mother and child. Macrosomia is associated with an increased risk of developing obesity and type 2 diabetes mellitus (T2DM) later in life. The objective of this study is to assess the health-economic impact of macrosomia.

Method: A decision analytical model was designed to estimate the health-economic impact of macrosomia, including both short- and long-term consequences. Options for performing different base-case analyses and various scenario analyses are incorporated, allowing for country or population specific calculations.

Results: Poorly controlled diabetes during pregnancy, prepregnancy maternal obesity and/or excessive maternal weight gain during pregnancy are associated with intermittent periods of fetal exposure to hyperglycemia and subsequent hyperinsulinemia. Insulin acts as a growth hormone during fetal life leading to high birth weight, increased body adiposity and glycogen storage in the liver. Macrosomia and associated complications for mother and child are included in our model, considering both short- and long-term consequences. The first analysis mapping the related healtheconomic burden indicates that the annual budgetary impact of being born large could be substantial. An example of cost per case assessment and related approximation of budget impact analysis will be presented.

Conclusion: Although overweight and obesity are of concern in the general population worldwide, there exists a clear predisposition in Asian countries to develop diabetes. This puts women of child-bearing age in this part of the world at a particular risk of delivering macrosomic infants bearing short and long term consequences for mother and child health. The current prevalence and increasing incidence of GDM is worrying; effective preventive management strategies are needed.

The presented core-model offers a template for further health technology assessments in different parts of the world and based on country specific data to determine the cost-effectiveness of preventive educational interventions on life style, diet and physical activity to reduce the short- and long-term public health consequences of macrosomia.

References This study was supported by the Danone Institute International.

Disclosure of Interest: I. Lenoir-Wijnkoop Employee of: Danone, M. Nuijten: None Declared., E. Van Der Beek Employee of: Danone Baby Nutrition, R. Uauy: None Declared.

\section{DOHaD13-1702}

Intergenerational transmission of inequality: maternal endowments, investments, and birth outcomes

Gabriella Conti $^{1 *}$, James Heckman ${ }^{2}$, Pia Pinger ${ }^{3}$, Arianna Zanolini ${ }^{4}$

${ }^{1}$ Harris School of Public Policy; ${ }^{2}$ Economics, University of Chicago, Chicago, United States; ${ }^{3}$ Economics, University of Mannheim, Mannheim, Germany; ${ }^{4}$ CIDZR (Centre for Infectious Disease Research), Lusaka, Zambia

Background: Newborn health outcomes are an important ring in the chain of intergenerational transmission of disadvantage. This paper contributes to the literature on the determinants of health at birth in two ways.

Method: First, we analyze the role of maternal endowments and investments (education and smoking in pregnancy) in the probability of having a baby who is small for gestational age (SGA). We both estimate the total impact of maternal endowments on birth outcomes, and we also decompose it into a direct, "biological" effect and a "choice" effect, mediated by maternal behaviors. Secondly, we estimate the causal effects of maternal education and smoking in pregnancy, and we investigate whether women endowed with different traits have different returns.

Results: We find that cognition affects birth outcomes primarily through education, that personality traits mainly operate by changing smoking behavior, and that the physical fitness of the mother has a direct, "biological" effect on SGA. We also find significant heterogeneity in the effects of education and smoking along the distribution of maternal physical traits, suggesting that women with a less healthy physical constitution should be the primary target of prenatal interventions.

Conclusion: Our results suggest that prenatal interventions, such as home-visiting programs, aimed at (among other things) reducing the prevalence of risky behaviors in pregnant women, and targeting low-income mothers, seem to be an effective way to compensate for maternal endowments differentials, and to guarantee a healthy start of life for the next generation.

Disclosure of Interest: None Declared. 


\section{DOHaD13-1152}

Longitudinal determinants of the impact of the economic recession in Republic of Ireland: findings from the lifeways cross-generation cohort study

Cecily C Kelleher ${ }^{1}{ }^{*}$, Celine Murrin ${ }^{1}$, Karien Viljoen ${ }^{1}$, John O'Brien ${ }^{1}$, Ricardo Seguardo ${ }^{1}$ and Lifeways Cross-Generation Cohort Study Steering Group

${ }^{1}$ School of Public Health, Physiotherapy and Population Science, University College Dublin, Dublin, Ireland

Background: The population health impacts of the global economic recession may depend on the responsive austerity measures in individual countries or on ameliorating social protection systems. Studies to date are primarily ecological or cross-sectional with few prospective studies reported. The Lifeways study, which was established a priori to study economic impact on health, recruited index infants, mothers, fathers and at least one grandparent in 2001/3, followed up in 2011/12.

Method: This cohort linkage study, recruited through two maternity hospitals in East and West of Ireland, included at baseline a number of socio-demographic (age, sex, family, group and individual identifiers, marital status, housing tenure, means tested general health services eligibility), lifestyle (fruit \& vegetable compliance and physical activity) and health status (self-reported general health (SRH) and mental wellbeing score) variables and at 10-year follow-up, 3 self reported questions on experience of the economic environment, combined on a 15-point composite score. Mixed model uni-variate and multivariate models are reported, with family ID as a random effect and the composite economic score as the outcome variable.

Results: Responses were received from 590 of 1082 original families $(\mathrm{RR}=54.5 \%)$ of whom 1336 individuals in 433 families had complete data for this analysis. At uni-variate level only sex, region and fruit $\&$ vegetable consumption were insignificant predictors of economic adversity. In the final multivariate model, baseline higher education (B coefficient $0.190,95 \%$ CI $0.062,0.318$ ) and higher mental distress score (B coefficient $0.050,95 \%$ CI $0.030,0.071$ ), were each positively associated with adverse economic score, whereas those with poorer baseline SRH (B coefficient -0.145 , 95\% CI and owner or rental property dwellers (B coefficient -0.221 , 95\% CI $-0.360,-0.081)$ were less likely to report economic adversity. Conclusion: To date social welfare and means-tested comprehensive health care have been preserved in Ireland, so those in poor health originally and those financially disadvantaged appear relatively supported in the recession. This novel prospective analysis indicates it is those ineligible for such support, with financial mortgage commitments and existing mental ill-health who report most current economic adversity. These findings have general policy implications for economic planning and support, including the proposal to introduce universal health care in the Republic of Ireland.

Disclosure of Interest: None Declared.

\section{DOHaD13-1528}

A comparison of dual energy X-ray absorptiometry and clinical anthropometry in relation to cardio-metabolic risk factors in the Raine Study

Denise Demmer ${ }^{1, *}$, Lawrence J. Beilin ${ }^{1}$, Sally Burrows ${ }^{1}$, Beth Hands ${ }^{2}$, Craig Pennell ${ }^{3}$, Stephen Lye ${ }^{4}$, Jenny Mountain ${ }^{5}$, Trevor A. Mori ${ }^{1}$

${ }^{1}$ School of Medicine and Pharmacology, University of Western Australia; ${ }^{2}$ Institute for Health Research, University of Notre Dame; ${ }^{3}$ School of Women's and Infants Health, University of Western Australia, Perth, Australia; ${ }^{4}$ Samuel Lunenfeld Research Institute, University of Toronto, Toronto, Canada;

${ }^{5}$ Telethon Institute for Child Health Research, Centre for Child Health Research, University of Western Australia, Perth, Australia

Background: Direct measures of adiposity using dual energy X-ray absorptiometry (DXA) are considered superior to anthropometric indices to distinguish fat and fat free components of an individual. However, there is uncertainty regarding the value of these two approaches for predicting cardio-metabolic (CM) risk factors. The aim of this study was to compare adiposity determined by DXA, with anthropometry to determine which measure best estimates CM risk factors in young adults.

Method: This cross-sectional study included 1138 young adults aged 20 years from the Western Australian Pregnancy Cohort (Raine) Study. Adiposity was measured using DXA (total body fat percentage, Fat Distribution Index and midriff fat mass) or anthropometry (abdominal skinfolds, waist circumference (WC), waist to height ratio (WHtR) and body mass index (BMI)) and CM risk factors included fasting lipids, high sensitivity C-reactive protein (hsCRP), glucose, insulin, homeostatic model of insulin resistance (HOMA-IR) and clinic blood pressure (BP). Linear regression models were utilised to examine relationships between adiposity measures and CM risk factors. For each risk factor the adjusted Rsquared (pseudo R-squared from the Tobit regression) and Aikeke Information Criterion (AIC) values were obtained. The lowest AIC was obtained by comparing the AICs within an outcome to identify the best adiposity measure. From this, the highest R-squared and lowest AIC value for an adiposity measure was identified as the best model for a particular CM risk factor. In all models interactions between gender and adiposity measures were investigated.

Results: There were no differences between genders and further analyses combined females and males. Models using midriff fat mass produced the smallest AIC value for cholesterol, triglycerides, LDL-C, glucose, insulin, HOMAIR and diastolic BP. The anthropometric variable with the next smallest AIC value varied between outcomes with abdominal skinfolds for serum cholesterol, WHtR for triglycerides and LDL-C, WC for glucose and BMI for insulin and HOMA-IR. Anthropometric measures generated 
the smallest AIC for HDL-C (WHtR) and systolic BP (BMI). For hsCRP, total body fat percentage produced the smallest AIC followed by BMI. Based on the difference of $<2$ between AIC models, neither DXA nor anthropometric measure showed superior performance for cholesterol, LDL-C and glucose. For other outcomes, the difference between the lowest AIC for the DXA and anthropometry measure ranged from 3 to 10 , which suggests DXA is superior. However, the R-squared values between the best DXA and anthropometry generally differed by $<0.01$, with the exception of two measures that were approximately 0.04 . This suggests the majority of the performance differences between DXA and anthropometry is negligible.

Conclusion: Although central adiposity measured using DXA was the best predictor of CM factors in young adults, overall it was not superior to simple anthropometric measures. Anthropometric indicies such as WC and WHtR are less invasive and more practical for studying the complex relationships between adiposity and CM risk at a population level.

Disclosure of Interest: None Declared.

\section{DOHaD13-1654}

\section{Adverse effect of in-utero alcohol exposure on childhood academic ability: a parental comparison analysis supporting a causal influence}

Rosa Alati ${ }^{1}, *$, George Davey $S_{m i t h}{ }^{2}$, Sarah lewis ${ }^{2}$, kapil Sayal $^{3}$, E Draper ${ }^{4}$, Jean Golding ${ }^{2}$, Robert Fraser ${ }^{5}$, Ron Gray ${ }^{6}$

${ }^{1}$ University of Queensland, Brisbane, Australia; ${ }^{2}$ University of Bristol, Bristol; ${ }^{3}$ University of Nottingham, Nottingham;

${ }^{4}$ University of Leicester, Leicester; ${ }^{5}$ university of Sheffiled, Sheffeld; ${ }^{6}$ University of Oxford, Oxford, United Kingdom

Background: This study will test the hypothesis that low-tomoderate maternal alcohol use in pregnancy is associated with lower school test scores in offspring aged 11 via intrauterine mechanisms.

Method: We used data from the Avon Longitudinal Study of Parents and Children (ALSPAC), an observational birth cohort study based in the South West of England. Analyses were conducted on 7062 participants who had complete data on the following measures: - maternal and paternal patterns of alcohol use in the first trimester and at 18 weeks' gestation; child's academic ability measured at age 11 , gender, maternal age, parity, marital status, ethnicity, household crowding, home ownership status and parental education. We compared maternal - offspring and paternal - offspring associations of alcohol consumption with child's National Curriculum Key Stage 2 (KS2) test scores. We used multivariable linear regression to estimate mean differences and $95 \%$ confidence intervals $[\mathrm{CI}]$ in $\mathrm{KS} 2$ scores across the exposure categories and computed $f$ statistics to compare maternal and paternal associations.
Results: Our approach compares the association of offspring outcomes with maternal and paternal alcohol consumption, on the assumption that only maternal alcohol use will influence intrauterine processes. Drinking up to 1 unit a day during pregnancy was not associated with lower test scores. However, frequent prenatal consumption of 4 units (equivalent to 32 grams of alcohol) on each single drinking occasion was associated with reduced educational attainment in a parental comparison analysis.

Conclusion: This pattern of alcohol use in pregnancy may adversely affect childhood academic ability via intrauterine mechanisms. The project has achieved its objectives in that we aimed to demonstrate that low levels of alcohol consumption in pregnancy were detrimental to cognitive development in childhood. This study presents some of the most compelling evidence to date that this pattern of alcohol use consumed by mothers in early pregnancy may influence academic abilities in their offspring at age 11, via intra-uterine effects.

Disclosure of Interest: None Declared.

\section{DOHaD13-1304}

Adult height and change in height as predictors of cardiovascular risk factors in young adults from a transitioning population

Kalyanaraman Kumaran ${ }^{1,2, *}$, Suyog Joshi ${ }^{1}$, Himangi Lubree $^{1}$, Clive Osmond ${ }^{2}$, Anand Pandit ${ }^{3}$, Chittaranjan Yajnik $^{1}$, Caroline Fall ${ }^{2}$

${ }^{1}$ Diabetes Unit, KEM Hospital Research Centre, Pune, India; ${ }^{2}$ MRC Lifecourse Epidemiology Unit, University of Southampton, Southampton, United Kingdom; ${ }^{3}$ Department of Paediatrics, KEM Hospital Research Centre, Pune, India

Background: Short adult height has been associated with an increased risk of cardiovascular disease (CVD) and its risk factors although the reasons for this association remain unclear. Height is an indicator of nutrition and growth in early life, and impaired fetal and childhood growth have also been associated with CVD and its risk factors. It is therefore possible that the association between height and CVD may be related to the programming of metabolism in early life. The Pune Children's Study (PCS) was established to prospectively examine early life antecedents of adult disease. We previously demonstrated that children who had lower birthweight but grew tall at 8 years had higher levels of CVD risk factors. Short parental height was also associated with higher insulin resistance in offspring at 8 years. We followed them up at 21 years and examined the relationship of final adult height and the difference from predicted adult height to CVD risk factors.

Method: Of the 360 children studied at 21 years of age, 357 were also studied at 8 years. We measured plasma glucose, insulin, lipids, blood pressure and anthropometry. The relationship 
between height and CVD risk factors was examined using correlation and regression techniques. In order to examine associations between outcomes at 21 years and early growth (changes in height), we generated conditional growth variables for height at 8 years and at 21 years. We also examined for any interaction between parents' heights, change in height between generations and the height of their offspring on CVD risk factors.

Results: At 21 years, the mean height was $172 \mathrm{~cm}$ in men and $157 \mathrm{~cm}$ in women. There was an intergenerational increase in height between parents and their offspring $(\sim 6 \mathrm{~cm}$ for boys and $4 \mathrm{~cm}$ for girls). Higher socio-economic class was associated with greater height. Adult height was inversely associated with $120 \mathrm{~min}$ glucose $(\mathrm{r}=0.17, \mathrm{p}<0.01)$; leg length was inversely associated with body mass index (BMI), diastolic BP, fasting and 120 minute glucose, and body fat percent while sitting height was directly associated with BMI, systolic BP and body fat percent $(r=0.10$ to $0.18, p<0.05$ for all). A smaller gain in conditional height between 8 and 21 years was associated with higher 120 minute glucose (beta $[95 \% \mathrm{CI}]-0.139 \mathrm{SD}[-0.243,-0.304])$ and insulin (beta $[95 \% \mathrm{CI}]-0.107 \mathrm{SD}[-0.210,-0.004])$. A smaller increase in conditional leg length and greater increase in conditional sitting height were also associated with adverse CVD risk. There were no relationships between parental heights and CVD risk factors in the offspring at 21 years.

Conclusion: Our findings show that short adult height, short leg length and higher sitting height were associated with adverse CVD risk factors and are comparable with results from elsewhere. A lower gain in conditional total height and leg length, but greater gain in conditional sitting height, between childhood and young adulthood is also associated with adverse CVD risk. The exact mechanisms need further study but point to a role for intrauterine and childhood growth; leg length may be constrained by adverse intrauterine and childhood growth while greater sitting height may reflect accelerated sexual maturation and pubertal growth.

Disclosure of Interest: None Declared.

\section{DOHaD13-1523}

Antenatal, but not postnatal, factors are associated with an accelerating growth trajectory in early childhood

Lynne Giles ${ }^{1}$, Melissa Whitrow ${ }^{2}$, Alice Rumbold ${ }^{2,3}$, Chris Davies $^{1}$, Michael Davies ${ }^{2,3}$, , Vivienne Moore ${ }^{1}$

${ }^{1}$ Public Health; ${ }^{2}$ Obstetrics and Gynaecology; ${ }^{3}$ Robinson Institute, University of Adelaide, Adelaide, Australia

Background: Childhood obesity frequently persists into adulthood, so that obese children carry greatly increased risks of cardiovascular disease and other conditions into their adult lives. Identifying and better understanding differences between patterns of growth in children in early life may give important insights into the roots of childhood obesity.

Method: As part of an ongoing longitudinal study of 557 families, children's heights and weights were measured at

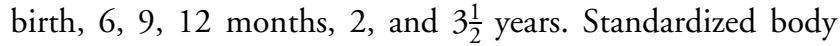
mass indices (BMI) were derived at each time, and latent class growth models used to identify groups of children with different growth trajectories. We investigated the relationship between distinct growth trajectory groups and a range of antenatal and postnatal exposures.

Results: We identified four growth trajectory groups across early childhood, corresponding to low, medium, high, and accelerating growth. Compared to children in the medium growth trajectory group, those in the accelerating group were more likely to have a mother with BMI $>30$ in early pregnancy (Odds Ratio $=4.5 ; 95 \%$ confidence interval 1.4-14.4) than a mother of normal weight. Parity was also significantly associated with accelerating growth trajectory. Neither breastfeeding nor timing of introduction to solids were significantly associated with growth trajectories.

Conclusion: Of the antenatal and postnatal exposures that we considered, the most important factor that differentiated between the growth trajectory groups was maternal BMI in early pregnancy. Initiatives to address intergenerational transmission of obesity and its consequences are vital for the health of Australian families.

Disclosure of Interest: None Declared.

\section{DOHaD13-1500}

\section{Association of maternal oxidative stress with infant anthropometry in a longitudinal study}

Sadhana Joshi ${ }^{1}$ on behalf of Nutritional Medicine, Suchitra Roy ${ }^{1, *}$, Kamini Dangat ${ }^{1}$, Anitha Kilari ${ }^{1}$, Asmita Joshi ${ }^{1}$, Sanjay Lalwani ${ }^{2}$, Savita Mehendale ${ }^{3}$

${ }^{1}$ Nutritional Medicine, Interactive Research School for Health Affairs; ${ }^{2}$ Pediatrics; ${ }^{3}$ Obstetrics and Gynecology, Bharati Medical College and Hospital, Pune, India

Background: An optimum amount of oxidative stress is essential for the progression of pregnancy. However, excessive oxidative stress adversely affects placental development and influences pregnancy outcome. There is limited data available on the association of maternal oxidative stress and its effect on the anthropometry of the baby during early infancy. The present study examined whether oxidative stress in the mother at the end of pregnancy is associated with the infant's weight, length, head and chest circumferences at various time points until 6 months of age.

Method: 100 pregnant women delivering at term (total gestation $\geqslant 37$ weeks and baby weight $\geqslant 2.5 \mathrm{~kg}$ ) were enrolled at the Department of Obstetrics and Gynecology, Bharati Hospital, Pune, India. Maternal blood was collected at delivery. Oxidative 
stress markers (Malondialdehyde (MDA)) and antioxidant enzymes superoxide dismutase (SOD) and glutathione peroxidase (GPx) were estimated from maternal plasma. Anthropometric measurements of the baby were recorded at birth, 1.5, 2.5, 3.5 and 6 months of age. Associations between maternal plasma MDA, antioxidant enzymes and baby anthropometric measures were examined. The data was analysed using the SPSS/PC+ package (Version 20, SPSS Inc., Chicago, IL, USA).

Results: MDA levels in mothers delivering male babies was $1.31 \pm 0.010 \mu \mathrm{mol} / \mathrm{ml}$ and in mothers delivering female babies it was $1.27 \pm 0.13 \mu \mathrm{mol} / \mathrm{ml}$. Maternal SOD and GPx levels were comparable in mothers delivering male and female babies. Maternal MDA levels were negatively associated with baby weight and chest circumference at all time points in female babies and in male babies only with weight at birth. Maternal plasma SOD levels were negatively associated with baby weight at 2.5 months of age only in female babies. Maternal plasma GPx levels showed a negative association with head circumference at 2.5 and 6 months of age only in male babies.

Conclusion: Our data suggests for the first time that increased oxidative stress in mothers has a differential effect on birth outcome in male and female babies and adversely affect growth of the female babies even after birth. Altered antioxidant enzymes during pregnancy may influence neurodevelopmental risk especially in male children.

Disclosure of Interest: None Declared.

\section{DOHaD13-1311}

Blood pressure in young adults born at very low birth weight - seven of the cohorts in APIC adults born preterm international collaboration

Petteri Hovi ${ }^{1,2, *}$, Sylvia van der $\mathrm{Pal}^{3}$, Betty Vohr ${ }^{4}$, Maureen $\mathrm{Hack}^{5}$, Lex Doyle ${ }^{6}$, Kari Anne Indredavik Evensen $^{7}$, Ann-Mari Brubakk ${ }^{7}$, Marit Indredavik ${ }^{7,8}$, Ruth E. Grunau ${ }^{9}$, Sture Andersson ${ }^{10}$, Saroj Saigal ${ }^{11}$, Eero Kajantie ${ }^{1,10}$

${ }^{1}$ National Institute for Health and Welfare; ${ }^{2}$ Children's Hospital, Helsinki University Central Hospital and University of Helsinki, Helsinki, Finland; ${ }^{3}$ TNO, Leiden, Netherlands; ${ }^{4}$ Women's and Infants Hospital, Providence; ${ }^{5}$ Case Western Reserve University CWRU, Cleveland, OH, United States; ${ }^{6}$ Royal Women's Hospital RWH, Melbourne, Australia; ${ }^{7}$ Norges teknisk-naturvitenskapelige universitet NTNU; ${ }^{8}$ Department of Child and Adolescent Psychiatry, St. Olav's University Hospital, Trondheim, Norway; ${ }^{9}$ University of British Columbia UBC, Vancouver, Canada; ${ }^{10}$ Children's Hospital, University Central Hospital and University of Helsinki, Helsinki, Finland;

${ }^{11}$ McMaster University, Hamilton, Canada

Background: Adults born at very low birth weight (VLBW; $<1.5 \mathrm{~kg}$ ) have higher blood pressure than their term born peers. Previous studies suggest that this difference is larger among women but had insufficient power to perform more detailed analysis and identify the most vulnerable VLBW subjects.

Our objectives were

1. To study whether the difference in systolic or diastolic blood pressure (SBP, DBP) between VLBW and control adults is larger among women than among men.

2. To study BP in VLBW adults born appropriate or small for gestational age (AGA/SGA).

Method: We pooled our datasets for an individual-subject meta-analysis of 1390 VLBW and 611 control subjects. We obtained datasets including current height and BMI and SBP and DBP data, at a mean measurement age 19.2 (15.4-27.1) yrs, from the Helsinki Study of VLBW Adults (164 VLBW/171 controls), from Cleveland (205/213), Melbourne (155/37), Trondheim (41/63), the Dutch POPS Study (475, VLBW only), the Vancouver Study (52/24), and the Indomethacin Study (296/95). For consistency, we used Finnish birth weight SDS scores for all subjects; SDS $<-2.0$ defined SGA. Differences were calculated with linear regression with adjustments for cohort (categorical), age at assessment, parental education (at least some university level), height and BMI.

Results: SBP differences (and 95\% confidence intervals) from the controls were 2.8 (0.8 to 4.7) $\mathrm{mmHg}$ in VLBW men and 4.7 (2.8 to 6.5$) \mathrm{mmHg}$ in VLBW women $\left(\mathrm{P}_{\text {sex }^{*} \mathrm{VLBW}} 0.04\right)$. Within the total of 1388 VLBW subjects, $574(41.4 \%)$ of whom were SGA, the SGA-AGA group-mean-difference in SBP was -1.0 (-3.1 to 1.0$) \mathrm{mmHg}$ among the $648 \mathrm{men}$ and -1.5 ( -3.4 to 0.4$) \mathrm{mmHg}$ among the 740 women.

DBP differences from the controls were 1.7 (0.4 to 2.9$) \mathrm{mmHg}$ in VLBW men and 2.4 (1.1 to 3.8) in VLBW women ( $\mathrm{P}_{\text {sex }}{ }^{*} \mathrm{VLBW}$ 0.37). Within the1388 VLBW subjects, the SGA-AGA groupmean-difference in DBP was $-0.7(-2.1$ to 0.7$) \mathrm{mmHg}$ among men and -0.8 ( -2.1 to 0.6$) \mathrm{mmHg}$ among women.

None of the differences mentioned were affected by adjustment for parental education. Adjusting the models further for current height and BMI resulted in 1 to $2 \mathrm{mmHg}$ increase in the difference estimates from the controls.

Conclusion: These preliminary results from the majority of the APIC collaboration sites reinforce previous suggestions that differences in SBP between VLBW and controls may be larger among women. There were no substantial differences within the VLBW cohort related to SGA status. These results may have implications for the future cardiovascular health of VLBW adults, especially women.

Disclosure of Interest: None Declared.

\section{DOHaD13-1158}

Childhood growth and weight gain and adult waist circumference in five low- and middle-income countries

Rebecca Kanter $^{1, *}$, Aryeh D. Stein ${ }^{2}$, Manuel Ramirez-Zea ${ }^{1}$ on behalf of the COHORTS investigators 
${ }^{1}$ INCAP Comprehensive Center for the Prevention of Chronic Diseases (CIIPEC), Guatemala, Guatemala; ${ }^{2}$ Emory University, Atlanta, United States

Background: In high-income countries, childhood weight gain predicts adult waist circumference (WC). It is unclear if childhood weight gain $(<5 y)$, independent of linear growth, predicts adult WC in low and middle-income countries (LMIC) and if there is also an association between childhood linear growth and adult WC. The study purpose was to investigate the differential associations of childhood linear growth and weight gain on adult WC in LMIC.

Method: We analyzed data from 7,241 subjects in prospective cohort studies from Brazil, Guatemala, India, Philippines, and South Africa that make up the Consortium for Health Oriented Research in Transitional Societies (COHORTS). All cohorts have $>1000$ subjects and follow up through to adulthood. Weight and length measurements were obtained at birth, $12 \mathrm{mo}, 24 \mathrm{mo}$, and mid-childhood (4 y for Brazil, Guatemala, and India; 5 y for South Africa; 8 y for Philippines). The mean age at which adult WC was collected varied from $18 \mathrm{y}$ in South Africa to $31 \mathrm{y}$ in Guatemala. In separate models by site and sex, we regressed childhood length-for-age $Z$ scores on all previous length-forage measurements. The standardized residuals from these models represent linear growth in specific age periods $(0-12$ mo, 12-24 mo, 24 mo-mid-childhood) that is not correlated with growth in the prior age periods. We computed conditional weights in a similar manner. We also regressed childhood weight-for age on present length-for-age and on all previous weight-for age and length-for-age measurements to represent conditional relative weight that is independent of height. We used generalized linear models to examine associations of conditional height, conditional weight, and conditional relative weight with adult WC. All models were adjusted for adult age, birth order, and childhood SES. To test for heterogeneity across sexes and sites F-tests were performed. Pooled models were also adjusted for site and sex. Results: Mean adult WC ranged from $67.8 \mathrm{~cm}$ (Filipino females) to $92.5 \mathrm{~cm}$ (Guatemalan females). The significant heterogeneity by site and sex reflected the variation in the magnitude of the coefficients and the sample size, rather than direction of the estimates. Thus, we present results from the pooled estimates. All conditional heights, conditional weights, and conditional relative weights were associated at $\mathrm{p}<0.001$ with greater adult WC. Across all ages and child growth measures, conditional weight in mid-childhood was the strongest predictor of WC in adulthood $(2.73 \mathrm{~cm}$ per SD increase in conditional weight in mid-childhood; $\mathrm{p}<0.001$ ). Conditional height at mid-childhood was also associated with adult WC $(1.65 \mathrm{~cm}$ per SD increase in conditional height; $\mathrm{p}<0.001)$. The association between conditional relative weight in mid-childhood was similar to that of conditional weight and WC $(2.41 \mathrm{~cm}$ per SD increase in conditional relative weight; $\mathrm{p}<0.001)$.
Conclusion: This study elucidates how childhood linear growth and relative weight gain predict greater adult WC, a known risk factor for cardiometabolic disease, in five LMIC. Yet, postnatal weight gain, especially in mid-childhood, has a stronger association with adult WC than linear growth. The efforts to reduce stunting by focusing on linear growth in the first 2 y do not have to conflict with preventing rapid weight gain in those 3-5y, when rapid weight gain is strongly associated with adult WC.

Funding: NHLBI.

Disclosure of Interest: None Declared.

\section{DOHaD13-1494}

\section{Circulating angiogenic factors in women with preeclampsia}

Sadhana Joshi ${ }^{1}$ on behalf of Nutritional Medicine, Deepali Sundrani ${ }^{1, *}$, Akriti Sahay ${ }^{1}$, Vidya Patil ${ }^{1}$, Asmita Joshi ${ }^{1}$, Girija Wagh ${ }^{2}$, Sanjay Gupte ${ }^{3}$

${ }^{1}$ Nutritional Medicine, Interactive Research School for Health Affairs; ${ }^{2}$ Obstetrics and Gynecology, Bharati Medical College and Hospital; ${ }^{3}$ Obstetrics and Gynecology, Gupte Hospital and Research Centre, Pune, India

Background: Our earlier studies in women with preeclampsia have demonstrated altered levels of key components of the one carbon cycle, reduced placental omega 3 fatty acids, and lower levels of angiogenic factors at delivery. We have also demonstrated differential placental gene specific methylation patterns of angiogenic factors in preeclampsia. This study examined gestation dependant changes in plasma angiogenic and anti-angiogenic factors in women with preeclampsia, and their association with cord angiogenic factors, maternal blood pressure and fetal birth weight.

Method: A total of 81 pregnant women (46 normotensive and 35 with preeclampsia) were followed at three different time points during pregnancy: 16-20 weeks (T1), 26-30 weeks (T2) and at delivery (T3). Plasma levels of angiogenic factors including vascular endothelial growth factor (VEGF), placental growth factor (PlGF), soluble fms-like tyrosine kinase-1 (sFlt-1) and soluble kinase insert domain receptor (sKDR) were determined in the maternal and cord plasma by commercial enzyme-linked immunosorbent assay kits.

Results: Maternal plasma VEGF and PlGF levels, were lower $(27.0 \pm 3.2$ versus $41.1 \pm 5.0, \mathrm{p}<0.05$ and $178.0 \pm 20.1$ versus $256.2 \pm 27.3, \mathrm{p}<0.05$ respectively) while sFlt-1 levels and sFlt-1/PlGF ratio $(\mathrm{p}<0.01)$ were higher $(1147.3 \pm$ 153.8 versus $754.6 \pm 84.7, \mathrm{p}<0.05$ and $11.6 \pm 2.4$ versus $3.7 \pm 0.8, \mathrm{p}<0.05$ respectively) in early pregnancy in preeclampsia than in the normotensive group. Cord plasma VEGF and sKDR levels were lower (156.7 \pm 45.1 versus $607.8 \pm 91.6, \mathrm{p}<0.01$ and $4638.1 \pm 395.4$ versus $5460.3 \pm$ $259.3, \mathrm{p}<0.05$ respectively) in women with preeclampsia. 
Maternal plasma VEGF levels in early pregnancy were positively associated with systolic blood pressure $(\mathrm{r}=0.423$, $\mathrm{p}=0.035, \mathrm{n}=25)$ while $\mathrm{sFlt}-1 / \mathrm{PlGF}$ ratio at T2 was negatively associated with baby weight $(\mathrm{r}=-0.501, \mathrm{p}=0.029$, $\mathrm{n}=21$ ) in preeclampsia.

Conclusion: Low levels of pro angiogenic factors (VEGF and PlGF) and high levels of anti-angiogenic factors (sFlt-1 and sFlt-1/PlGF ratio) exist in the maternal circulation from the second trimester of pregnancy in preeclampsia, and before the onset of clinical signs of preeclampsia. Future studies will examine changes in angiogenic factors in early gestation in relation to maternal micronutrition to seek evidence for modifiable factors to prevent preeclampsia.

Disclosure of Interest: None Declared.

\section{DOHaD13-1216}

Conditional fetal growth in the last trimester: the effect of BMI, gestational weight gain, fasting glucose and placental weight

Marie Cecilie P. Roland ${ }^{1}$, Camilla M. Friis ${ }^{1}$, Jens Bollerslev $^{2,3}$, Guttorm Haugen ${ }^{3,4}$, Tore Henriksen ${ }^{1,3, *}$

${ }^{1}$ Dept of Obstetrics; ${ }^{2}$ Dept of Endocrinology, Oslo University Hospital; ${ }^{3}$ University of Oslo; ${ }^{4}$ Dept of Obstetrics, Section for fetal medicine, Oslo University Hospital, Oslo, Norway

Background: Birthweight is considered as a crude measurement of fetal growth. Assessing fetal growth essentially requires two or more estimates of fetal size and the use of fetal growth charts. Conditional growth charts take into account the fetal size measured at one point and estimate the expected growth for a fetus of that particular size for a specified period of time. Conditioning on a previous measurement gives information on fetal growth - fast or slow - compared to expected growth for fetuses of the same size and same gestational age. Our aim was to study the influence of placental weight and maternal variables (parity, BMI, gestational weight gain (GWG), fasting glucose) on conditional fetal growth in the third trimester.

Method: The STORK project is a prospective cohort study with a longitudinal design. Biometric ultrasound measurements were performed in gestational weeks $30-32$ and 36-38 on 1031 healthy pregnant women, of which 842 had complete data on fetal growth. Fetal growth was estimated using conditional growth percentiles, based on reference curves from a Norwegian cohort. Fetal growth percentiles were conditioned on the estimated fetal weight (EFW) measured at weeks 30-32, giving the rate of growth between weeks 30-32 and weeks 36-38. The conditional growth percentile is given as a percentil between 0-100, with the 50 percentile representing the mean expected growth. The conditional fetal growth variable derived from these curves included information on whether a fetus grew fast or slow compared to other fetuses of the same size at weeks 30-32. The associations between maternal characteristics and fetal growth were analysed by univariate and multiple linear regression models.

Results: Mean maternal age was 31.3 years (SD3.9). Mean $( \pm S D)$ value for gestational age at birth was 39.5 weeks ( \pm 1.5 ), for birthweight $3588 \mathrm{~g}( \pm 574)$, and for placental weight $711 \mathrm{~g}( \pm 156)$. Only $2.7 \%$ smoked during pregnancy. Parity, BMI, GWG and placental weight were significant determinants of conditional fetal growth in the third trimester. Multipara had higher conditional growth percentiles than primipara (B 5.6, 95\% CI 2.1-9.2, $\mathrm{p}=0.002$ ). In the multiple model one unit higher BMI increased the conditional growth percentile by 1.04 (B1.04, 95\% CI $0.6-1.5, \mathrm{p}<0.001)$. One kg higher GWG and $100 \mathrm{~g}$ increase in placental weight increased the conditional fetal growth percentile by 1.16 (B 1.16, 95\% CI $0.5-1.8, \mathrm{p}=0.001$ ), and 5.13 (B 5.13, 95\% CI 3.9-6.3, p <0.001), respectively. Fasting glucose was not an independant determinant of conditional fetal growth in third trimester.

Conclusion: Fetal growth can be evaluated in various methods. We have presented a conditional growth model. Placental weight was an independent determinant of conditional fetal growth besides the maternal variables parity, BMI and GWG.

Disclosure of Interest: None Declared.

\section{DOHaD13-1627}

Developmental screening tools: evaluation of the agreement between 2 different screening tools (ASQ and PEDS-DM) at 9 months in the GUSTO cohort

Pratibha K. Agarwal ${ }^{1, *}$, Kok Hian Tan ${ }^{1}$, Helen Chen ${ }^{1}$, Sok Bee Lim ${ }^{1}$, LM Daniel ${ }^{1}$, Anne Rifkin-Graboi ${ }^{2}$, Iliana Magiati $^{3}$, Leher Singh ${ }^{3}$, Fabian K. P. Yap ${ }^{1}$, Kenneth Kwek ${ }^{1}$, Marielle Fortier ${ }^{1}$, Shirong Cai ${ }^{4}$, Shu E Soh ${ }^{5}$, Yin Bun Cheung $^{6}$, Sang Chee Chong ${ }^{7}$, Seang Mei Saw ${ }^{5}$, Yap Seng Chong $^{7}$, Michael J. Meaney ${ }^{2}$, Victor S. Rajadurai ${ }^{1}$

${ }^{1} K K$ Women's and Children's Hospital; ${ }^{2}$ Singapore Institute for Clinical Sciences; ${ }^{3}$ Psychology; ${ }^{4}$ O\& G; ${ }^{5}$ Saw Swee Hock School of Public Health, National University of Singapore; ${ }^{6}$ DukeNUS Graduate Medical School; ${ }^{7}$ National University Hospital, Singapore, Singapore

Background: Developmental screening tools has been recommended to be used by primary health care providers at 9, 18, 24-30 months in Singapore. Parent-report based screening tools are gaining recognition with their high validity, specificity and sensitivity on different populations. In Singapore, there are few reports of these tools, of which suggests that the American based scoring criteria provided are not suitable for the Singaporean population. Thus, there is a need to study these tools in Singapore. This study seeks to evaluate the agreement and the differences between two 
parent-report based developmental screening tools in a low risk healthy cohort at 9 months.

Method: This study is part of the GUSTO birth cohort study in Singapore, where 549 parents of children 9 months of age were recruited to complete the Parents' Evaluation of Developmental Status: Developmental Milestones (PEDS$\mathrm{DM}$ ) and Ages and Stages Questionnaire (ASQ-3) in the same visit. The PEDS:DM detects possible developmental delays by asking parents to evaluate the child's response to listed tasks and noting which developmental milestones the child may have achieved. ASQ-3 also requires parents to rate their child's performance to listed tasks, but results are separated by age. The two screening tools were compared to see areas of agreement and disagreement.

Results: There were 519 parents that completed both screening tests; 329 (63.4\%) were Chinese, 118 (22.7\%) were Malay and $72(13.9 \%)$ were Indian. The mean mother age was 32.3 years $(\mathrm{SD}=4.9), 135(26.0 \%)$ of the mothers had less than tertiary education and $258(49.7 \%)$ of the children were male. In total, 96 (18.5\%) passed both the PEDS:DM and ASQ, 181 (34.87\%) failed both, 242 children failed either one of the tests (38 ASQ, 204 PEDS:DM), in total, 134 (25.8\%) passed the PEDS:DM, and 300 (57.8\%) passed the ASQ. Agreement tests showed poor agreement $(\kappa=0.133, \mathrm{P}=0.00)$ between both tests and proportions were statistically different (McNemar, $P=0.00)$. When the domain results in ASQ were compared with equivalent concerns in PEDS:DM, both tests showed that the children failed fine motor development (ASQ - 17.9\%; PEDS:DM - 46.2\%), but there was poor agreement $(\kappa=0.162$, $\mathrm{P}=0.00$; McNemar, $\mathrm{P}=0.00)$.

Conclusion: There is poor agreement between the PEDS:DM and ASQ screening tools on which children in the GUSTO cohort that have developmental delays. There is a need to include a gold standard to determine the sensitivity and specificity of both tests in the GUSTO cohort or in a Singaporean population.

Disclosure of Interest: None Declared.

\section{DOHaD13-1749}

Developmental screening tools: evaluation of agreement between ASQ \& PEDS at 18 months in the Gusto cohort

Pratibha Agarwal ${ }^{1, *}$, Kok Hian Tan ${ }^{1}$, Helen Chen ${ }^{1}$, Sok Bee Lim $^{1}$, LM Daniel ${ }^{1}$, Anne Rifkin-Graboi ${ }^{2}$, Iliana Magiati ${ }^{3}$, Leher Singh ${ }^{3}$, Fabian K. P. Yap ${ }^{1}$, Kenneth Kwek ${ }^{1}$, Marielle Fortier $^{1}$, Shirong Cai ${ }^{4}$, Shu E Soh ${ }^{5}$, Yin Bun Cheung ${ }^{6}$, Sang Chee Chong $^{7}$, Seang Mei Saw ${ }^{5}$, Yap Seng Chong ${ }^{7}$, Michael J. Meaney $^{2}$, Victor S. Rajadurai ${ }^{1}$

${ }^{1} K K$ Women's and Children's Hospital; ${ }^{2}$ Singapore Institute for Clinical Sciences; ${ }^{3}$ Psychology; ${ }^{4} O \& G ;{ }^{5}$ Saw Swee Hock School of Public Health, National University of Singapore;

${ }^{6}$ Duke-NUS Graduate Medical School; ${ }^{7}$ National University Hospital, Singapore, Singapore
Background: It is reported that up to a $65 \%$ of children with developmental delays are undetected. Though it has been recommended in the Enabling Masterplan 2012 that children should take part in developmental screening at 9, 18, 24-30 months in Singapore, there are currently no recommended screening tools for the Singaporean population. Parent-report based developmental screening tools are a good method for detecting developmental delays early in young children; however, few of these tools have been studied in Singapore. This study seeks to evaluate the agreement and the differences between two developmental screening tools in a low risk healthy cohort and compare the concordance of the two tools at 18 months.

Method: The study is a smaller study within a large birth cohort study, GUSTO, in Singapore. 441 parents of children 18 months of age from the GUSTO cohort were recruited to complete the Parents' Evaluation of Developmental Status (PEDS) and Ages and Stages Questionnaire (ASQ-3) at the same visit. PEDS is a screening test that uses parental concerns reported to determine the developmental status of the child, while ASQ-3 is a test that uses the parents' rating of the child's response to listed tasks to determine is the child has any form of developmental delay. The two tools were analyzed for concordance and for differences.

Results: 347 parents took part in both screening tests, of which 194 (55.9\%) were Chinese, 106 (30.5\%) were Malay and 47 $(13.5 \%)$ were Indian. The mean mother age was 32.9 years $(\mathrm{SD}=5.19), 27.9 \%$ of the mothers had less than tertiary education and $42.4 \%$ of the children were male. In total, 236 (68\%) passed the ASQ -3, 204 (58.8\%) passed PEDS. 151 (43.5\%) children passed both the PEDS and ASQ-3, 58 failed both and 138 children failed either one of the tests (85 PEDS, 53 ASQ). Agreement tests showed that there was poor agreement $(\kappa=0.15, P=0.004)$ between both tests. When the domains from ASQ were compared with their equivalent concerns in PEDS, the two tools did not agree with each other, with more children failing in the fine motor and problem solving domains in ASQ, while more children failed in expressive language and behavior concerns in PEDS. The proportions between two tests were found to be statistically different (McNemar, $\mathrm{P}=0.008)$.

Conclusion: There is poor agreement between the PEDS and ASQ screening tools on which children have developmental delayed at 18 months. Further evaluation and expansion of the study to include a gold standard is needed to determine which tool is suitable for Singaporean children at 18 months.

Disclosure of Interest: None Declared.

\section{DOHaD13-1748}

Developmental screening tools: evaluation of agreement between two developmental screening tools (ASQ and PEDS) at 9 months in the Gusto cohort

Pratibha K. Agarwal ${ }^{1, *}$, Kok Hian Tan ${ }^{1}$, Helen Chen ${ }^{1}$, Sok Bee Lim ${ }^{1}$, LM Daniel ${ }^{1}$, Anne Rifkin-Graboi ${ }^{2}$, Iliana Magiati ${ }^{3}$, Leher Singh ${ }^{3}$, Fabian K. P. Yap ${ }^{1}$, 
Kenneth Kwek ${ }^{1}$, Marielle Fortier ${ }^{1}$, Shirong Cai ${ }^{4}$, Shu E Soh ${ }^{5}$, Yin Bun Cheung ${ }^{6}$, Sang Chee Chong ${ }^{7}$, Seang Mei Saw ${ }^{5}$, Yap Seng Chong ${ }^{7}$, Michael J. Meaney ${ }^{2}$, Victor S. Rajadurai ${ }^{1}$

${ }^{1} K K$ Women's and Children's Hospital; ${ }^{2}$ Singapore Institute for Clinical Sciences; ${ }^{3}$ Psychology; ${ }^{4}$ O\& $G ;{ }^{5}$ Saw Swee Hock School of Public Health, National University of Singapore; ${ }^{6}$ Duke-NUS Graduate Medical School; ${ }^{7}$ National University Hospital, Singapore, Singapore

Background: Early screening of developmental delays is important, as there is great benefit in early intervention. Parent-report based developmental screening tools are coming into common use, as it is proven as a good method for detecting delays. Though it has been encouraged that developmental screening should take place at 9, 18, 24-30 months in Singapore, there are few studies on parent-report developmental screening tool use and no standardized screening tool in Singapore; it is found that scoring criteria provided in tests are not suitable for Singapore. The study seeks to evaluate and compare the agreement and the differences between ASQ and PEDS in a low risk healthy cohort at 9 months.

Method: 549 parents of children 9 months of age within the GUSTO cohort, a large birth cohort study in Singapore, were recruited to complete the Parents' Evaluation of Developmental Status (PEDS) and Ages and Stages Questionnaire (ASQ-3) at the same visit. The PEDS detects developmental delays using parental concerns about the child and picks out predictive concerns that can indicate delays. ASQ-3 requires parents to rate their child's performance to a list of tasks. The agreement and disagreement of the two screening tools were determined.

Results: 516 parents took part in both screening tests, of which 327 (63.4\%) were Chinese, 119 (23.1\%) were Malay and 70 $(13.6 \%)$ were Indian. The mean mother age was 32.3 years $(\mathrm{SD}=4.9), 135(26.2 \%)$ of the mothers had less than tertiary education and $252(48.8 \%)$ of the children were male. In total, 208 (40.3\%) passed both the PEDS and ASQ, 74 (14.3\%) failed both, 355 (68.8\%) passed the PEDS, 295 (57.2\%) passed the ASQ and 234 children failed either one (147 ASQ, 87 PEDS). Analysis showed that there was poor agreement $(\kappa=0.04$, $\mathrm{P}=0.33$ ) between both tests and proportions were statistically different $(\mathrm{McNemar}, \mathrm{P}=0.00$ ). When ASQ domains were compared with the equivalent PEDS concerns, both tests showed high failures in ASQ Fine Motor (18.2\%) and Personal-Social (18.8\%) domains, and PEDS Behavior (30.30\%) and SocialEmotional $(25 \%)$ concerns. There was poor agreement between personal-social and behavior $(\kappa=0.048, \mathrm{P}=0.26$; McNemar: $\mathrm{P}=0.00$ ) or between personal-social and social-emotional $(\kappa=-0.014, \mathrm{P}=0.75$; McNemar: $\mathrm{P}=0.021)$.

Conclusion: There is poor agreement between the PEDS and ASQ on which children in the GUSTO cohort that have developmental delays. Further evaluation and expansion of the study is needed to determine which is suitable for Singaporean children.

Disclosure of Interest: None Declared.

\section{DOHaD13-1146}

\section{Determinants of age at menarche in rural Indian girls}

Charudatta Joglekar ${ }^{1}{ }^{1, *}$, Urmila Deshmukh ${ }^{1}$, Dattatray Bhat ${ }^{1}$, Kalyanraman Kumaran ${ }^{2}$, Caroline Fall ${ }^{2}$, Chittaranjan Yajnik ${ }^{1}$

${ }^{1}$ Diabetes Unit, King Edward Memorial Hospital Research Centre, Pune, India; ${ }^{2}$ MRC Lifecourse Epidemiology Unit, University of Southampton, Southampton, United Kingdom

Background: Menarche is a critical milestone in the reproductive development of female adolescents. World over the age at menarche is falling, and early menarche has been associated with increased risk of breast cancer and coronary heart disease. A study of determinants of menarche is therefore of interest.

Method: The Pune Maternal Nutrition Study (PMNS) was set up to study the association between maternal nutrition and fetal growth and adult NCDs in a rural Indian population. It collected information on mothers from before and during pregnancy, and fetal growth and neonatal measurements were obtained. Two thirds of women had low vitamin B12 status and only $2 \%$ had low folate status. They were supplied with 100 tablets of iron $(60 \mathrm{mg})$ and folic acid $(500 \mathrm{mg})$ at 18 weeks gestation. The offspring are regularly followed up for measurements of growth and metabolic-nutritional risk factors for NCDs. The offspring are now $17 \mathrm{y}$ old. This lifecourse approach provides an opportunity to investigate determinants of any outcome in a life-course model. The onset of menarche in girls was recorded as an exact date obtained during biannual follow up and provided an opportunity to analyse its determinants and associations with NCD risk factors.

Results: The follow up rate is more than $90 \%$. Out of 338 girls, 4 had not attained menarche by $17 \mathrm{y}$ of age. The average age at menarche was 13.5 years (Range 9-17y). Earlier age at menarche was associated with larger birth weight, earlier adiposity rebound (BMI) and more rapid growth of skinfolds during childhood. Higher cholesterol and leptin concentrations at $6 y$ predicted earlier menarche. Earlier menarche was also predicted by higher maternal pre-pregnant weight and fat mass, and lower physical activity during pregnancy, but not maternal height, weight gain during pregnancy, or macronutrient intake during pregnancy. Higher maternal erythrocyte folate during pregnancy predicted earlier menarche in the daughters. Vitamin B12, C and D concentrations during pregnancy were not related. Larger paternal size (weight, height, BMI) predicted earlier menarche.

On multiple regression analysis higher maternal erythrocyte folate and lower maternal physical activity during pregnancy and higher adiposity of the girl at 6y were independently associated with earlier menarche.

Conclusion: Age at menarche in rural Indian girls is associated with higher adiposity in the girl and her parents. In addition higher maternal folate concentrations and lower physical activity during pregnancy are also predictive. Falling 
age at menarche could be related to increasing adiposity epidemic. Unregulated folic acid supplementation may have a contributory role.

Disclosure of Interest: None Declared.

\section{DOHaD13-1285}

Does early growth predict blood pressure increase during late adult life? an analysis of longitudinal data from the Helsinki Birth Cohort Study (HBCS)

Samuel Sandboge $e^{1,2, *}$, Clive Osmond ${ }^{3}$, Eero Kajantie ${ }^{2,4}$, Johan G. Eriksson ${ }^{1,2,5,6,7}$

${ }^{1}$ Folkhälsan Research Centre; ${ }^{2}$ Department of Chronic Disease Prevention, National Institute for Health and Welfare, Helsinki, Finland; ${ }^{3}$ MRC Epidemiology Resource Centre, University of Southampton, Southampton, United Kingdom; ${ }^{4}$ Hospital for Children and Adolescents, Institute of Clinical Medicine, University of Helsinki; ${ }^{5}$ University of Helsinki, Department of General Practice and Primary Health Care; ${ }^{6}$ Helsinki University Central Hospital, Unit of General Practice, Helsinki; ${ }^{7}$ Vaasa Central Hospital, Vaasa, Finland

Background: The developmental origins of hypertension have been studied extensively. Previous studies show that a small body size at birth is associated with an increased risk of adult hypertension. According to findings from the Helsinki Birth Cohort Study (HBCS) and other cohort studies, slow growth in infancy, followed by a more than adequate catchup growth during childhood, increases this risk. Systolic blood pressure increases throughout adult life and some studies indicate that the association between birth weight and adult blood pressure is amplified with age. The aim of this study was to explore whether early growth predicts blood pressure increase during late adult life.

Method: We studied 586 individuals from the HBCS born 1934-44 who attended a clinical study in 2001-2004 as well as a follow-up study in 2006-2008. Only individuals without antihypertensive medication at both visits were included. Mean age at first visit was 61.5 years and mean time between visits was 4.9 years (range 2.2-7.2). Data on birth, childhood, and adult anthropometrics were available for all individuals, as well as adult clinical measurements. Growth trajectories were studied by conditional growth analysis measuring how much body size at a certain age differed (in SDs) from what would be predicted from size at a previous age. The associations between early growth and adult blood pressure at the second clinic visit were studied separately for men and women in a series of linear regression models controlling for gestational age, childhood and adult socio-economic status, smoking, salt intake, exercise frequency, blood pressure at first clinic visit as well as body mass index, and age at both visits.

Results: Among men, several childhood anthropometric measurements were inversely associated with both systolic and diastolic blood pressure at the second clinic visit. Systolic blood pressure was $2.1 \mathrm{mmHg}(95 \% \mathrm{CI}-3.8$; -0.3$)$ lower for every $\mathrm{kg}$ of weight at 2 years of age, $2.6 \mathrm{mmHg}(95 \% \mathrm{CI}-4.6 ;-0.7)$ lower for every SD of conditional weight gain and $2.7 \mathrm{mmHg}$ (95\% CI - 4.5; - 0.9) lower for every SD of conditional height gain between birth and 2 years of age. For women, systolic blood pressure was $1.8 \mathrm{mmHg}(95 \% \mathrm{CI}-2.5$; -0.4$)$ lower for every SD of conditional weight gain between 2 and 7 years and $1.9 \mathrm{mmHg}(95 \% \mathrm{CI}-3.4 ;-0.4)$ lower for every SD of conditional BMI gain between 7 and 11 years.

Conclusion: Among men, a slow weight and height gain between birth and 2 years of age, and a small body size at age 2 was associated with a more rapid increase in systolic and diastolic blood pressure in late adulthood, independent of traditional risk factors for hypertension. Among women, a more rapid weight and BMI growth in later childhood provides a slight protection against adult hypertension.

Disclosure of Interest: None Declared.

\section{DOHaD13-1475}

Early exposure to violence: trajectories of depression and anxiety symptoms among children and adolescents affected by war

Elizabeth A. Newnham ${ }^{1,2, *}$, Ryan K. McBain ${ }^{2}$, Robert Brennan $^{2}$, Theresa S. Betancourt ${ }^{2}$

${ }^{1}$ School of Psychology, The University of Western Australia, Perth, Australia; ${ }^{2}$ Harvard School of Public Health, Boston, United States

Background: One billion children live in countries affected by armed conflict. Early developmental experience of direct and indirect exposure to violence has significant implications for mental health outcomes later in life. High rates of depression, anxiety and post-traumatic stress disorder (PTSD) have been routinely documented among war-affected children and adolescents. However, there remains significant debate regarding the longitudinal course and persistence of mental health problems in youth affected by early trauma.

Method: The current study examined the course of internalizing emotional and behavioral problems (depression, anxiety and social withdrawal) among 529 war-affected youth in Sierra Leone over three waves of measurement (2002, 2004 and 2008). Participants (25\% female, ages $10-17$ at baseline) comprised former child soldiers $(73 \%)$ as well as youth not actively recruited into armed forces or rebel groups. Local research assistants administered all surveys in Krio. Latent class growth analyses were conducted to determine trajectories of psychological response to violent trauma. Multinomial logistic regression analyses determined specific risk and protective factors associated with the longitudinal progression of symptoms.

Results: Latent class growth analyses revealed four trajectories of internalizing symptoms. A large majority of youth 
maintained lower levels of internalizing problems (Low Symptoms; $41.4 \%$ ) or significantly improved over time (Improvers; $47.6 \%$ ) despite severely limited access to care. Smaller proportions continued to report severe difficulties six years post-war (High Symptoms; 4.5\%) or their symptoms worsened over time (Deteriorators; 6.4\%). Relative to the low symptoms group, those who maintained high symptoms over time were older $(p<.05)$, experienced family abuse and neglect $(p<.05)$, stigma $(p<.001)$, and social disorder $(p<.05)$ following the war. Those who reported deterioration in symptoms were more likely to have lost a parent during the war $(p<.01)$, and reported family abuse and neglect $(p<.05)$, stigma $(p<.01)$ and daily hardship $(p<.05)$. In contrast, improvement in symptoms was associated with older age $(p<.01)$, female gender $(p<.01)$ and community acceptance $(p<.05)$.

Conclusion: Despite exposure to extreme violence and adversity early in life, many war-affected youth demonstrated comparative resilience. Interventions that address family- and community-level stressors and incorporate a life-course perspective in addressing mental health among children exposed to war trauma are needed.

Disclosure of Interest: None Declared.

\section{DOHaD13-1381}

\section{Early life body mass trajectories as determinants of mortality: 70-year follow-up in the Helsinki Birth Cohort Study}

Mikaela von Bonsdorff ${ }^{1}$, Timo Törmäkangas ${ }^{1}$, Taina Rantanen ${ }^{1}$, Minna K. Salonen ${ }^{2,3}$, Clive Osmond ${ }^{4}$, David J. Barker ${ }^{4,5}$, Eero Kajantie ${ }^{3,6, *}$, Johan G. Eriksson ${ }^{2,3,7,8,9}$

${ }^{1}$ Gerontology Research Center and Department of Health Sciences, University of Jyväskylä, Jyväskylä; ${ }^{2}$ Folkhälsan Research Centre; ${ }^{3}$ Department of Chronic Disease Prevention, National Institute for Health and Welfare, Helsinki, Finland; ${ }^{4} M R C$ Lifecourse Epidemiology Unit, University of Southampton, Southampton, United Kingdom; ${ }^{5}$ Chair of Fetal Programming, College of Science, King Saud University, Riad, Saudi Arabia; ${ }^{6}$ Children's Hospital, Helsinki University Central Hospital and University of Helsinki; ${ }^{7}$ Department of General Practice and Primary Health Care, University of Helsinki; ${ }^{8}$ Unit of General Practice, Helsinki University Central Hospital, Helsinki; ${ }^{9}$ Vasa Central Hospital, Vasa, Finland

Background: Overweight and obesity in childhood have been linked with increased mortality in later life, but the evidence is scarce and inconsistent findings have been reported on the mediating effect of adult body size. Our aim was to identify different developmental trajectories of early life body mass index (BMI), examine whether the BMI trajectories were associated with risk of mortality in later life and if adult body size explained this potential association.
Method: The study population consisted of 4943 individuals from the Helsinki Birth Cohort Study, born between 1934 and 1944. They had serial measures of BMI extracted from birth hospital, child welfare clinic and school healthcare records from birth to age 11 years, self-reported weight and height in late adulthood and register-based mortality data with 10-year follow-up. Latent class growth mixture models were fitted to the data to capture heterogeneity in the body size development in an optimal number of classes.

Results: Three distinct trajectories of early BMI were identified and named 'accelerating' $(6.4 \%$ of men and $25.4 \%$ of women), 'normal' $(66.7 \%$ of men and $59.0 \%$ of women) and 'fluctuating' (26.9\% of men and $15.6 \%$ of women). Men with an accelerating BMI trajectory had lower BMI during the first months of life after which it was consistently higher throughout childhood than in those with a normal trajectory. Men with a fluctuating BMI trajectory had higher BMI in early childhood but at age 4 years, it dropped below the normal trajectory. Women with an accelerating BMI trajectory had a normal BMI until 6 months, after which it was higher throughout childhood. Women with a fluctuating BMI trajectory had lower BMI in early life but after the age of 7 years, it was consistently higher than the normal trajectory.

Women with an accelerating or fluctuating early BMI trajectory had increased mortality compared to those with a normal BMI trajectory, hazard ratio (HR) being 1.59 (95\% CI 1.10-2.89) and 1.57 (1.04-2.36), respectively. Late adulthood BMI did not explain the association. Among men, early BMI trajectories were not associated with mortality, but having high or low BMI compared to normal BMI in late adulthood increased mortality, HR being 1.37 , (95\% CI $1.05-1.80)$ and 1.62 (1.22-2.16), respectively. Adjustment for socioeconomic position in childhood and adulthood, and lifestyle factors such as smoking, alcohol consumption and physical activity did not attenuate these associations.

Conclusion: Distinct trajectories of early BMI were identified in the study population showing that among women accelerating and fluctuating early BMI development were associated with an increased mortality risk in later life. These findings underline the importance of early BMI development on later health decline and suggest that maintaining normal body size throughout childhood is beneficial for health outcomes in older age.

Disclosure of Interest: None Declared.

\section{DOHaD13-1728}

Early life nutritional programming of obesity: optimising mother-child cohort studies

Eline van der Beek ${ }^{1, *}$ on behalf of ILSI Europe Expert Group on Aligning Cohort Studies and The ILSI Europe Metabolic Imprinting Task Force, Expert Group on Aligning Cohort Studies 
${ }^{1}$ Centre for Specialised Nutrition, Danone Research, Helios \#09-01/02, Singapore

Background: As the incidence of overweight and obesity remains high across populations, so does the number of overweight or obese women before and during pregnancy. Data indicate that offspring of these women tend towards higher birth weight and increased risk of later life obesity. While gestational diabetes may be one contributing factor, other contributors to adverse outcomes in pregnancy through childhood may be important. If properly designed, motherchild cohort studies may serve as a key research tool for understanding these relationships.

Method: The Metabolic Imprinting Task Force of the International Life Sciences Institute, European Branch brought together leading global experts in a 2011 workshop to review and discuss how current and future cohort studies can increase our knowledge and understanding of the mechanisms that may be associated with increased risk of adverse pregnancy outcomes in obese pregnant women. The scope of the exercise was limited to the outcomes of 1) risk of obesity and 2) related metabolic disorders in childhood, as influenced by nutrition. The key aim of the workshop was to explore the value of combining cohort data in relation to how maternal obesity and diet influence the early nutritional environment of the foetus and interact with the postnatal environment.

Results: The Expert Group first reviewed the maternal factors that influence body composition of the child, finding that results of individual studies are quite inconsistent and meta-analyses suggest interventions are of limited value. At the same time, some animal data suggest that dietary changes just before pregnancy can have a significant effect. New findings suggest that higher activity of brown adipose tissue and specific depots of brown fat may impact energy balance. Gender differences in adipose tissue growth and differences in maternal and infant diet (e.g. formula vs. breastfeeding) may be important influencers of outcomes. Considering the data review, the Expert Group proposed optimal requirements and outputs when undertaking mother-child cohort studies to assess the role of nutrition on offspring obesity. The guidelines cover, for the mother, infant and child, best approaches to: dietary intake assessment, nutrient status assessment, body composition, other key measurements, stage of development for evaluation and potential confounders. Conclusion: The workshop provided insights into measurements critical for the characterisation of sustainable nutritional intervention strategies in the mother, infant and child for prevention of later life obesity.

\section{Reference}

1. M. Symonds, et al. Early Life Nutritional Programming of Obesity: Mother-Child Cohort Studies. Annals of Nutrition and Metabolism 2013; 62:137-145.

Disclosure of Interest: None Declared.

\section{DOHaD13-1745}

Growth of Chinese children in the first two years of life: a comparison of the GUSTO cohort with the WHO child growth standards'

Tuck Seng Cheng ${ }^{1, *}$, Ngee Lek ${ }^{1,2}$, Yap Seng Chong ${ }^{3}$, Kenneth Kwek ${ }^{1}$, Seang Mei Saw ${ }^{3}$, Keith M. Godfrey ${ }^{4,5}$, Peter Gluckman ${ }^{6,7}$, Yin Bun Cheung ${ }^{2}$, Yung Seng Lee ${ }^{3,7}$, Fabian Yap ${ }^{1,2}$

${ }^{1}$ KK Women's and Children's Hospital; ${ }^{2}$ Duke-NUS Graduate Medical School (GMS); ${ }^{3}$ NUS, National University Health

System (NUHS), Singapore, Singapore; ${ }^{4}$ University of Southampton; ${ }^{5}$ University Hospital Southampton NHS Foundation

Trust, Southampton, United Kingdom; ${ }^{6}$ University of Auckland, Auckland, New Zealand; ' Singapore Institute for Clinical Sciences (SICS), A*STAR, Singapore, Singapore

Background: The 2006 WHO Child Growth Standards (WHO-CGS) were based on the growth of breastfed, physically well children in 6 countries across all continents. As no Chinese data were included, the validity of WHO-CGS in monitoring growth in children of Chinese ethnic origin could be questioned. We assessed the utility of the WHOCGS in Chinese children and used the WHO-CGS to compare the growth patterns of breastfed and formula fed Chinese infants.

Method: We analysed growth data collected in GUSTO (Growing Up in Singapore Towards Healthy Outcome), an on-going birth cohort study in which eligible mothers were recruited at two large hospitals in Singapore during the first trimester of pregnancy and their children subsequently followed up at regular time intervals after birth. We included all Chinese children whose mothers did not smoke during pregnancy and after delivery, and who were born as singleton babies at term gestation, and either were exclusively or predominantly breastfed in the first 4 months and at least partially breastfed in the next 8 months $(\mathrm{BF})$, or were formula fed in the first 12 months of life (FF). Length and weight of the children at birth, 6, 12 and 24 months were measured and converted into WHO-CGS standard deviation scores (SDS). Deviation within \pm 0.4 SDS from the WHO-CGS was considered as normal. Length and weight velocities were calculated as changes in SDS during 3 time intervals: from birth to 6 months; 6 to 12 months; and 12 to 24 months. Linear mixed modeling was used to estimate the means.

Results: One hundred and forty-four children were included in the analyses, of whom 69 were BF (38 male) and 75 were FF (42 male). At 6, 12 and 24 months, respectively, the weight (mean SDS at the 3 time points: $0.13 ;-0.16 ;-0.15$ ) and length $(0.05 ; 0.02 ;-0.01)$ of $\mathrm{BF}$ children were consistent with the WHO-CGS in both boys and girls. BF boys and girls showed similar weight velocity SDS (0.56 vs $0.38 ;-0.36$ vs $-0.21 ; 0.04$ vs -0.03$)$ and similar length velocity SDS (0.41 vs $0.21 ; 0.12$ vs $-0.20 ; 0.01$ vs -0.06$)$ in the 3 time intervals. However, FF boys and girls differed in 
weight velocity SDS $(0.15$ vs $0.56 ;-0.06$ vs $-0.04 ;-0.01$ vs 0.11$)$ and length velocity SDS (0.66 vs $1.01 ; 0.03$ vs 0.23 ; -0.18 vs -0.32 ), especially in the first 6 months. Over the first 2 years of life, BF boys gained more weight (0.24 SDS) than FF boys (0.08 SDS) but no difference was observed in length velocity SDS (0.54 vs 0.51 ). Compared to BF girls, FF girls had higher weight velocity SDS (0.63 vs 0.14$)$ and higher length velocity SDS (0.92 vs -0.05$)$.

Conclusion: Our analyses suggest that the WHO-CGS can be used to monitor the growth of Chinese children in the GUSTO cohort. Compared with the WHO-CGS, formula fed Chinese girls had substantially faster growth in the first two years of life.

Disclosure of Interest: None Declared.

\section{DOHaD13-1448}

\section{Late bedtimes are associated with less total sleep in 6-month-old children}

Sara S. Tan ${ }^{1, *}$, Diaz A. Utama ${ }^{2}$, Daniel Goh ${ }^{3}$, Oon Hoe $\mathrm{Teoh}^{4}$, Michael Meaney ${ }^{5}$, Peter Gluckman ${ }^{5}$, Seang Mei Saw $^{6}$, Keith Godfrey ${ }^{5}$, Yap Seng Chong ${ }^{7}$, Kenneth Kwek ${ }^{8}$, Anne Rifkin-Graboi ${ }^{5}$, Joshua J. Gooley ${ }^{1}$

${ }^{1}$ Program in Neuroscience and Behavioral Disorders, Duke-NUS Graduate Medical School Singapore; ${ }^{2}$ National University of Singapore; ${ }^{3}$ Department of Paediatrics, Division of Paediatric Pulmonary \& Sleep, National University Hospital, Singapore; ${ }^{4}$ Department of Paediatrics, Respiratory Medicine Service, KK Women's and Children's Hospital, Singapore; ${ }^{5}$ Growth, Development and Metabolism Programme, Singapore Institute of Clinical Research; ${ }^{6}$ National University Hospital Singapore; ${ }^{7}$ Department of Obstetrics \& Gynaecology, National University Hospital Singapore; ${ }^{8}$ Department of Obstetrics \& Gynaecology, KK Women's and Children's Hospital, Singapore, Singapore

Background: Sleep is thought to play an important role in early development. Growing evidence suggests that, on average, school-aged children in Singapore sleep less than the amount recommended by most sleep researchers and clinicians. It is not known, however, whether such sleep habits are related to schoolwork, or if they arise at a much earlier age. We therefore examined sleep behavior in Singaporean children prior to formal education.

Method: We studied 127 children aged 6 months who were enrolled in the Growing Up in Singapore Towards healthy Outcomes (GUSTO) birth cohort study (47.7\% male; 60.6\% Chinese, 26.8\% Malay, 12.6\% Indian). Sleep was assessed objectively using an actigraphy device that was worn around the child's ankle for 5-7 days. Bedtimes and wake times were recorded in a sleep diary by the primary caretaker, and the duration of night sleep and day sleep (i.e., naps) was estimated based on scoring of actigraphy counts.
Results: Average bedtime was 9:40 pm $\pm 1 \mathrm{hr} 10 \mathrm{~min}$, and most children (83.5\%) slept fewer than 10 hours at night. As expected, later bedtimes associated with later wake times (Pearson's $r=0.65 ; P<0.001)$. Based on actigraphy-estimated sleep, however, bedtimes correlated negatively with night sleep duration $(r=-0.49 ; P<0.001)$. Children with later bedtimes did not show a compensatory increase in daytime napping, and therefore exhibited less total daily sleep, as compared to children with earlier bedtimes.

Conclusion: Our preliminary findings suggest that Singaporean infants have later bedtimes and sleep substantially less than the amount reported in predominantly Caucasian countries. Sleep duration was, to a large degree, determined by bedtime. Specifically, 6-month-old children with earlier bedtimes had more total sleep than those who went to bed late. Hence, our findings for sleep behavior in infants mirror those reported in school-aged children. Given that sleep is important for learning and memory consolidation, our findings have potential implications for cognitive development in Singaporean children.

Disclosure of Interest: None Declared.

\section{DOHaD13-1642}

Life-course obesity trajectories are associated with development of non-alcoholic fatty liver disease at age 17 years

Rae-Chi Huang ${ }^{1,2, *}$, Oyekoya T. Ayonrinde ${ }^{1,3,4}$, John K. Olynk ${ }^{1,3,4,5}$, Trevor A. Mori ${ }^{1}$, Lawrence J. Beilin ${ }^{1}$, Leon A. Adams ${ }^{1,6}$

${ }^{1}$ School of Medicine and Pharmacology; ${ }^{2}$ Telethon Institute for Child Health Research, University of Western Australia; ${ }^{3}$ Department of Gastroenterology, Fremantle Hospital; ${ }^{4}$ Curtin Health Innovation Research Institute, Curtin University; ${ }^{5}$ Institute for Immunology and Infectious Diseases, Murdoch University; ${ }^{6}$ Department of Gastroenterology, Sir Charles Gairdner Hospital, Perth, Australia

Background: Non-alcoholic fatty liver disease (NAFLD) is associated with insulin resistance and the metabolic syndrome, both of which are well-recognized outcomes that occur as a result of early life programming. Identification of latent patterns of lifecourse trajectories have been shown to be associated with insulin resistance and the metabolic syndrome. Our aim was to assess whether childhood adiposity trajectories were associated with NAFLD.

Method: The Western Australian Pregnancy Cohort (Raine) Study assessed NAFLD using ultrasound in 811 adolescents at 17 years of age. These participants also had anthropometry measured at birth, 1, 3, 5, 8, 10 and 14 years of age. We have previously used semi-parametric mixture modeling to identify 7 distinct adiposity trajectories. Multinomial regression was used to investigate the effect of the trajectories upon risk of NAFLD. 
Results: At 17 years, 14.2\% were identified with NAFLD. Compared with a stable adiposity trajectory the three rising trajectories (1, 2 and 4), from birth weights (mean \pm SD) of $3.5 \pm 0.6,3.3 \pm 0.5$ and $3.1 \pm 0.5 \mathrm{~kg}$ were associated with an increased likelihood of NAFLD development at 17 years (adjusted $\mathrm{OR}=9.2,95 \%$ CI 4.1 to 20.6; $\mathrm{OR}=10.1$, 95\%CI 5.1 to $20.2, \mathrm{OR}=3.1,95 \% \mathrm{CI} 1.5$ to 6.2 ).

Conclusion: NAFLD is associated with rapid preschool adiposity gains regardless of starting birth weight. This evidence of life-course effects upon development of NAFLD may reflect the underlying common etiologies of insulin resistance and NAFLD. It suggests that reducing rapid adiposity gains in childhood is likely to reduce NAFLD in young adults.

(Figure adapted from Huang et al. Diabetes Care 2011, 34(4), 1019-1025).

Disclosure of Interest: None Declared.

\section{DOHaD13-1399}

Lifetime socio-economic achievements and intergenerational social-mobility of individuals born late-preterm

Kati Heinonen $^{1, *}$, Johan G. Eriksson ${ }^{2,3,4,5,6}$, Eero Kajantie ${ }^{2,7}$, Anu-Katriina Pesonen ${ }^{1}$, David J. Barker ${ }^{8,9}$, Clive Osmond ${ }^{9}$, Katri Raikkonen ${ }^{1}$

${ }^{1}$ Institute of Behavioral Sciences; ${ }^{2}$ National Institute for Health and Welfare; ${ }^{3}$ Department of General Practice and Primary Health Care, Helsinki; ${ }^{4}$ Vasa Central Hospital, Vaasa;

${ }^{5}$ Helsinki University Central Hospital; ${ }^{6}$ Folkhälsan Research Centre; ${ }^{7}$ Children's Hospital, Helsinki University Central Hospital and University of Helsinki, Helsinki, Finland; ${ }^{8}$ Oregon Health and Science University, Oregon, United States; ${ }^{9} M R C$ Lifecourse Epidemiology Unit, Southampton, United Kingdom

Background: Approximately every $10^{\text {th }}$ birth worldwide is premature ( $<37$ weeks of gestation). Studies suggest that those born preterm have on average poorer neurocognitive abilities and they attain lower socio-economic position than those born at term. However, most of these studies have assessed only preterms born at the lower end of the gestational age or birth weight distribution as compared with controls. Yet, over $70 \%$ of all preterm births are late-preterm, defined as birth at 34 weeks 0 days through 36 weeks 6 days gestation. The extent to which individuals born late-preterm are at a risk of socio-economic disadvantage in subsequent life is less well understood. We examined if those born late-preterm differed from those born at term in their attained lifetime socioeconomic position (SEP) across the adult years, and in intergenerational social mobility from childhood parental SEP to own attained SEP.

Method: Participants were 8,993 Finnish men and women of the Helsinki Birth Cohort Study born between 1934-44. Gestational age was calculated based on last menstrual period, extracted from hospital birth records and socio-economic attainments from Finnish National Census. Logistic regression analyses were used to test if late-preterms and terms differed in their odds (Adjusted Odds Ratio [AOR], 95\% Confidence Interval [95\%CI]) for belonging to the lowest or the highest SEP category, and in their odds for downward or upward social mobility. All models included sex and year of birth. Analyses were further adjusted for birth order, maternal body-mass-index, maternal age, father's occupational status and birth weight adjusted for gestational age.

Results: Compared to those born at term those born latepreterm were more likely to be manual workers $(\mathrm{AOR}=1.61$, 95\%CI 1.26-2.05), have a basic or upper secondary level of education $(\mathrm{AOR}=1.31,95 \% \mathrm{CI} 1.07-1.61)$, and belong to the lowest third based on their incomes $(\mathrm{AOR}=1.34$, 95\%CI 1.11-1.62), and less likely to belong to the highest third based on their incomes (AOR $=0.75$, 95\%CI 0.62-0.93). Latepreterms were also less likely to be upwardly mobile and more likely to be downwardly mobile: they were less likely to have higher occupations (AOR $=0.72,95 \% \mathrm{CI} 0.58-0.91)$ and more likely to have lower occupations than their fathers $(\mathrm{AOR}=1.53$, 95\%CI 1.02-2.28). They were also less likely to be upwardly mobile if incomes were used as the outcome of own attained SEP $(A O R=0.70$, 95\%CI 0.58-0.86), and men were more likely to be downwardly mobile if education was used as the outcome of own attained SEP $(\mathrm{AOR}=1.48$, 95\%CI 1.02-2.14). Adjustments did not change the results.

Conclusion: This study demonstrates that there are considerable long-term socio-economic disadvantages associated with late-preterm birth, which are not explained by the parent-of-origin SEP.

Disclosure of Interest: None Declared.

\section{DOHaD13-1498}

Longitudinal assessment of maternal plasma fatty acids and their associations with cord and placental fatty acids in normotensive pregnancy

Sadhana Joshi ${ }^{1}$ on behalf of Nutritional Medicine, Nisha Wadhwani ${ }^{1, *}$, Vidya Patil ${ }^{1}$, Karuna Randhir ${ }^{1}$, Girija Wagh ${ }^{2}$, Sanjay Gupte ${ }^{3}$

${ }^{1}$ Nutritional Medicine, Interactive Research School for Health Affairs; ${ }^{2}$ Obstetrics and Gynecology, Bharati Medical College and Hospital; ${ }^{3}$ Obstetrics and Gynecology, Gupte Hospital and Research Centre, Pune, India

Background: During the last trimester of pregnancy there is an increased need for docosahexaenoic (DHA) and arachidonic (AA) acids for rapid fetal brain growth. Since the human fetus has a limited ability to synthesize these LCPUFA, it is dependent on maternal supplies transferred through the placenta. Our earlier cross sectional studies in women with preterm delivery and preeclampsia have demonstrated lower levels of maternal, cord and placental fatty acids at delivery which are associated 
with poor birth outcome. This study was undertaken to examine gestation dependant changes in the levels of maternal plasma fatty acids and to examine their association with cord and placental fatty acids.

Method: A longitudinal study of 49 normal pregnant women delivering at term (total gestation $\geqslant 37$ weeks and baby weight $\geqslant 2.5 \mathrm{~kg}$ ) was carried out at Gupte Hospital, Pune, India and maternal blood was collected at 3 time points $\left(\mathrm{T} 1=16-20^{\text {th }}\right.$ week, T2 $=26-30^{\text {th }}$ week and T3 $=$ at delivery). Cord blood and placenta were collected at delivery. Birth weight, length, head and chest circumference were recorded. Fatty acids were estimated using gas chromatography and expressed as g per $100 \mathrm{~g}$ fatty acid. The data were analysed using the SPSS/PC+ package (Version 20, SPSS Inc., Chicago, IL, USA).

Results: Maternal plasma DHA and omega 3 fatty acid concentrations were lower at T2 $(0.96 \pm 0.35, \mathrm{p}<0.05$; $1.54 \pm 0.54, \mathrm{p}<0.01$ respectively) and T3 $(0.85 \pm 0.33$, $1.7 \pm 0.76, \mathrm{p}<0.01$ for both) than at $\mathrm{T} 1$ (1.18 \pm 0.49 , $2.25 \pm 1.03$ respectively). AA and omega 6 fatty acids were lower at T3 $(5.49 \pm 1.51, \mathrm{p}<0.05 ; 36.38 \pm 5.2, \mathrm{p}<0.01$ respectively) than at $\mathrm{T} 1(6.32 \pm 1.24,40.05 \pm 6.37$ respectively). Cord plasma DHA and AA concentrations were higher than maternal values at all time points $(1.57 \pm 0.79$, $13.65 \pm 3.39 ; \mathrm{p}<0.01$ for both). Total cord plasma omega 3 fatty acids were higher $(2.33 \pm 0.93)$, whereas omega 6 fatty acids were lower $(28.63 \pm 3.48)$, than maternal values. There were positive associations between maternal plasma DHA concentrations at every time point of gestation and cord plasma and placental DHA concentrations $(r=0.786$, $\mathrm{p}<0.01, \mathrm{n}=41, \mathrm{r}=0.691, \mathrm{p}<0.01, \mathrm{n}=45, \mathrm{r}=0.629$, $\mathrm{p}<0.01, \mathrm{n}=45 ; \mathrm{r}=0.699, \mathrm{p}<0.01, \mathrm{n}=30, \mathrm{r}=0.773$, $\mathrm{p}<0.01, \mathrm{n}=32, \mathrm{r}=0.761, \mathrm{p}<0.01, \mathrm{n}=32$ respectively), and between placental DHA and cord DHA concentrations $(\mathrm{r}=0.820, \mathrm{p}<0.01, \mathrm{n}=31)$. There was a positive association between baby head circumference and maternal plasma omega 6 fatty acids at T1 $(\mathrm{r}=0.483, \mathrm{p}<0.05, \mathrm{n}=23)$.

Conclusion: Maternal fatty acids concentrations, from the second trimester of pregnancy, are positively correlated with cord blood and placental fatty acids. The alterations in the levels of these fatty acids throughout pregnancy could indicate gestation dependant changes in requirements for fetal growth. Maternal fatty acid levels during pregnancy may be useful in predicting birth outcome and in designing intervention programs for better pregnancy outcome.

Disclosure of Interest: None Declared.

\section{DOHaD13-1077}

\section{Longitudinal changes in adiponectin multimer levels in preterm infants}

Yuya Nakano ${ }^{1, *}$, Kazuo Itabashi ${ }^{1}$, Motoichiro Sakurai ${ }^{1}$, Madoka Aizawa ${ }^{1}$, Kazushige Dobashi ${ }^{1}$, Katsumi Mizuno ${ }^{1}$

${ }^{1}$ Pediatrics, Showa University School of Medicine, Tokyo, Japan
Background: Adiponectin is an adipocyte-secreted hormone that exerts positive health effects. High molecular weight adiponectin (HMW-Ad) level is a better indicator of glucose intolerance than total adiponectin (T-Ad) level. Moreover, HMW-Ad level and the ratio of HMW-Ad to T-Ad (HMW\%) can be used to predict insulin resistance. In term infants, we previously showed that HMW-Ad levels at 12 months of age were significantly lower than those at birth and 6 months, and that the cord serum HMW-Ad levels could significantly determine the levels at 12 months. Preterm infants have altered adiponectin levels and have a higher risk of future insulin resistance than term infants. However, there is no information on the longitudinal changes in adiponectin multimer levels in preterm infants. Therefore, we aimed to investigate the longitudinal changes in adiponectin multimer levels in preterm infants and compare the levels between term and preterm infants.

Method: The study included 31 preterm infants $(16$ male and 15 female) born at $\leqslant 34$ weeks' gestation. Serum levels of T-Ad, HMW-Ad, and low molecular weight adiponectin (LMW-Ad) were measured at birth and term-, 6 months-, and 12 months-equivalent age. We reviewed the longitudinal changes in adiponectin multimer levels in 46 term infants (19 male and 27 female) investigated previously. The differences between series of data from term and preterm infants were determined by Wilcoxon's rank-sum test. The MannWhitney test was used to compare variables at term-, 6 months-, and 12 months-equivalent age between term and preterm infants. Differences were considered statistically significant at $p<0.05$.

Results: The mean birth weight, birth weight SD score, and gestational age of the preterm infants were $1571 \mathrm{~g},-0.8 \mathrm{SD}$, and 32.1 weeks, respectively. In preterm infants, serum levels of T-Ad, HMW-Ad, and HMW\% at 6 months-equivalent age were similar to those at term-equivalent age; however the levels were significantly lower at 12 months-equivalent age than those at term-equivalent age and at 6 months-equivalent age; this finding was also obtained in term infants (all values $\mathrm{p}<0.001)$. The LMW-Ad levels did not differ $(\mathrm{p}=0.081)$. HMW\% in preterm infants at term- and 6 monthsequivalent age was significantly lower than that in term infants at corresponding time points $(\mathrm{p}<0.001)$; however, this significance was absent at 12 months-equivalent age. LMWAd levels and the ratio of LMW-Ad to T-Ad (LMW\%) in preterm infants at term- $(\mathrm{p}<0.001$ and $\mathrm{p}=0.001$, respectively), 6 months- ( $p=0.001$ and $p<0.001$, respectively), and 12 months-equivalent age $(p=0.021$ and $p=0.006$, respectively) were significantly higher than those in term infants at the corresponding time points.

Conclusion: The longitudinal changes in adiponectin multimer levels differ in preterm and term infants. In preterm infants, the T-Ad levels sustained from term- to 6 monthsequivalent age and decline up to 12 months-equivalent age, as well as term infants. The decrease in T-Ad levels during this period resulted from the decrease in HMW-Ad levels. LMW- 
Ad and LMW\% remained higher in preterm infants than term infants at least up to 12 months-equivalent age. The differences in adiponectin multimer levels may reflect fat tissue maldevelopment in preterm infants. Further studies are needed to identify the factors that alter adiponectin multimer levels in preterm infants.

Disclosure of Interest: Y. Nakano: None Declared., K. Itabashi Grant/Research support from: JSPS KAKENHI (N0. 22591208), M. Sakurai: None Declared., M. Aizawa: None Declared., K. Dobashi: None Declared., K. Mizuno: None Declared.

\section{DOHaD13-1387}

Longitudinal trends in vitamin B12 concentrations in a rural Indian cohort: the Pune Maternal Nutrition Study

C. S. Yajnik ${ }^{1, *}$, D. S. Bhat ${ }^{1}$, C. V. Joglekar ${ }^{1}$, H. G. Lubree ${ }^{1}$, K. Kumaran ${ }^{1}$, S. S. Naik ${ }^{1}$, C. H. D. Fall ${ }^{2}$

${ }^{1}$ Diabetes Unit, KEM Hospital Research Centre, PUNE, India; ${ }^{2} M R C$ Lifecourse Epidimiology Unit, Southampton General Hospital, Southampoton, United Kingdom

Background: Vitamin B12 deficiency is common in India, and may have an important role in fetal programming of diabesity. In the western world vitamin B12 concentrations fall with age, low concentrations in the elderly being attributed to reduced absorption of vitamin B12 because of gastritis-related 'food B12 malabsorption'. Vitamin B12 absorption is normal in the majority of Indians, and the deficiency is ascribed to dietary deficiency. There are few longitudinal studies of vitamin B12 in India. In the Pune Maternal Nutrition Study (PMNS) $\sim 65 \%$ of mothers had low B12 concentrations in pregnancy. Serial measurements of vitamin B12 in the children born in the PMNS offered an opportunity to document life-course evolution of vitamin B12 deficiency.

Method: We made serial measurements of vitamin B12 in 671 children (351 boys, 320 girls) at 6,11 and 17 years of age, along with anthropometric and biochemical measurements including hemoglobin, white cell count, folate, and homocysteine. Vitamin B12 and folate were measured using the same microbiological assays throughout the study. Dietary intake (food frequency questionnaire) was available at 11 years, and socio-economic status (SES) at 6 and 11 years.

Results: These children were born small (mean birth weight $2.7 \mathrm{~kg}$ ) and at 17 years had an average BMI of $18.4 \mathrm{~kg} / \mathrm{m}^{2}$. Mean vitamin B12 concentrations were 252, 207 and $159 \mathrm{pmol} / \mathrm{l}$, and mean plasma folate concentrations 19, 24 and $20 \mathrm{nmol} / \mathrm{l}$ respectively at 6,11 and 17 years of age. Mean total homocysteine concentration was $13.0 \mu \mathrm{mol} / \mathrm{l}$ at 12 years, and $32 \mu \mathrm{mol} / \mathrm{l}$ at 17 years $(\mathrm{n}=136$ at 17 years). Vitamin B12 deficiency $(<150 \mathrm{pmol} / \mathrm{l})$ rose from $16 \%$ to $25 \%$ and $58 \%$ while folate deficiency was stable and low (upto 3\%). During this follow up, there was no significant change in frequency of consumption of milk and nonvegetarian foods. However, SES parameters progressively improved, including safe water supply and hygiene. Mean hemoglobin concentrations progressively increased from 115 to 128 and $133 \mathrm{gm} / \mathrm{l}$, as did BMI from 13.4 to 14.9 and $18.4 \mathrm{~kg} / \mathrm{m}^{2}$. After adjusting for age, gender and SES, vitamin B12 was inversely associated with hemoglobin and BMI. Of dietary items, only milk consumption was positively related to vitamin B12. Maternal vitamin B12 concentration in pregnancy was a significant predictor of the child's vitamin $\mathrm{B} 12$ at the three time points $\left(\mathrm{r}^{2} \sim 3 \%\right.$, all $)$.

Conclusion: Progressive increase in vitamin B12 deficiency in the first two decades of life in the PMNS population is striking. This could be related to poor body stores at birth due to low vitamin B12 status in pregnant mothers, and improved SES, hygiene and body size. Vitamin B12 is synthesized only by microbes in the nature and it is intriguing to speculate the role of microbe in this association. These findings raise interesting questions about public health management of this problem in India.

Disclosure of Interest: None Declared.

\section{DOHaD13-1689}

Maternal diet during pregnancy according variation in perinatal environmental and effects of infant's health IVAPSA birth cohort

Juliana R. Bernardi ${ }^{1}$, Marina Nunes ${ }^{1}$, Ednara N. Gonçalves ${ }^{1}$, Mariana Brito ${ }^{1}$, Márcio B. Alves ${ }^{1}$, Priscyla Rocha ${ }^{1}$,

Rafaela D. S. Côrrea ${ }^{1}$, Tanara Vogel ${ }^{1}$, Vera L. Bosa ${ }^{1}$, Clécio H. da Silva ${ }^{1}$, Isabel C. Werlang ${ }^{1}$, Marcelo Z. Goldani $^{1, *}$ and IVAPSA Birth Cohort

${ }^{1}$ Universidade Federal do Rio Grande do Sul, Porto Alegre, Brazil

Background: It has been shown that different insults during the pregnancy and neonatal period bring long-term consequences to the offspring. Studies have shown that the diet during pregnancy may have effects on the health of the child that become apparent later in life. The aim was investigate the relation of maternal diet (energy and macronutrient intake) during pregnancy with variations in perinatal environmental and this effects of infant's health.

Method: This is a thematic, prospective, birth cohort to assess the interactions between the maternal phenotype during gestation (maternal smoking, hypertension, diabetesDM, intrauterine growth restriction-IGR and controls) and their associations with outcomes related with mothers and children. Participants were postpartum women assisted by Grupo Hospitalar Conceição and Hospital de Clínicas de Porto Alegre (HCPA) residing in Rio Grande do Sul, Brazil. Six interviews were performed at home (7, 15 days and 3 month) or at the clinical research center (CRC) in HCPA 
(postpartum, 1 and 6 month). Retrospectively food intake estimate was collected in 7 days interview and assessed by a 97-itens Food Frequency Questionnaire (FFQ), validated for the pregnant women in Brazil.

Results: Interviews were conducted with 159 mother-child pairs (lost to follow up: 16.8\%) with 25 DM; 19 hypertension; 50 smoking; 17 IGR; 48 control groups. The mean of maternal energy intake was $5.023 \mathrm{Kcal}$ ( $\pm 2938 \mathrm{Kcal}$; minimum: $1857 \mathrm{Kcal}$; maximum: $18.059 \mathrm{Kcal})$ and the median was $(\mathrm{P} 25$ : $3214 \mathrm{Kcal}$; P75: $5849 \mathrm{Kcal}$ ) with $60.8 \%$ carbohydrate, $14.5 \%$ protein and $26.6 \%$ total fat. There was no have differences of maternal energy and macronutrient intake between the mothers from different clinical backgrounds $(\mathrm{p}>0.05)$.

Conclusion: The quantitative maternal diet was similar between groups. More qualitative analyses should be done in our research for understand the mothers consumption from different clinical backgrounds.

References Bernardi et al. Impact of perinatal different intrauterine environments on child growth and development in the first six months of life - IVAPSA birth cohort: rationale, design, and methods. BMC Pregnancy Childbirth. 2012; 12: 25.

Bernardi et al. Impact of perinatal different intrauterine environments on child growth and development in the first sixmonths of life-IVAPSA birth cohort: rationale, design, and methods. BMC Pregnancy Childbirth. 2012; 12: 25.

Disclosure of Interest: None Declared.

\section{DOHaD13-1501}

\section{Maternal nerve growth factor levels in women with preeclampsia from two socioeconomic groups: a longitudinal study}

Vandita D'Souza $^{1, *}$, Savita Mehendale ${ }^{2}$, Girija Wagh ${ }^{2}$, Sanjay Gupte ${ }^{3}$, Vidya Patil ${ }^{1}$, Hemlata Pisal ${ }^{1}$, Madhavi Dhobale $^{1}$, Sadhana Joshi ${ }^{1}$

${ }^{1}$ Nutritional Medicine, Interactive Research School for Health Affairs; ${ }^{2}$ Obstetrics and Gynaecology, Bharati Medical College and Hospital; ${ }^{3}$ Obstetrics and Gynaecology, Gupte Hospital and Research Center, Pune, India

Background: Preeclampsia is a pregnancy complication with a placental pathology characterized by systolic blood pressure $\geqslant 140 \mathrm{~mm} \mathrm{Hg}$ or diastolic blood pressure $\geqslant 90 \mathrm{~mm} \mathrm{Hg}$ occurring after 20 weeks' gestation in a woman whose blood pressure has previously been normal accompanied by proteinuria of $\geqslant 300 \mathrm{mg}$ per $24 \mathrm{~h}$. Our earlier cross sectional study has shown altered nerve growth factor (NGF) levels at delivery in women with preeclampsia. However this may be a secondary effect where the pathology has progressed. Studies have shown that NGF is expressed and localized in the placenta making it a potent neuroendocrine organ through- out gestation Thus there is a need to examine the role of NGF in vascular development during different stages of a normal pregnancy and a pregnancy complicated with preeclampsia.

Method: Pregnant women $(n=163)$ were enrolled for this longitudinal study from 2 major hospitals from Pune, Bharati hospital mainly catering to women from the lower socioeconomic (LSE $\mathrm{n}=74$ ) group while Gupte hospital catering to the higher socioeconomic (HSE $\mathrm{n}=89$ ) group, classified on Kuppuswamy's socioeconomic status scale (Kumar et al., 2012), during the year 2010-2012. They were followed at three different time points [16-20 weeks (T1), 26-30 weeks (T2) and at delivery (T3)] during pregnancy and maternal blood at every time point and cord blood at delivery was collected and processed. 24 women from the LSE group and 35 women from the HSE group developed PE during pregnancy. NGF levels were measured from maternal and cord plasma using the Emax Immuno Assay System (Promega). The data was analyzed using the SPSS/PC+ package (Version 20.0, Chicago, IL, USA).

Results: Maternal NGF levels at T2 $(\mathrm{p}<0.05)$ and T3 $(\mathrm{p}<0.01)$ were lower in preeclampsia as compared to control in the whole cohort. Maternal NGF levels at T2 and T3 were lower $(\mathrm{p}<0.05)$ in preeclampsia as compared to control in the HSE group. In contrast, there was no change in maternal NGF levels in the LSE group in preeclampsia as compared to control. There was no change in cord NGF levels in preeclampsia as compared to control.

Conclusion: Our data suggests that NGF may play an important role in the development of the maternal-fetalplacental unit during pregnancy.

Disclosure of Interest: None Declared.

\section{DOHaD13-1553}

Mother's marital status and offspring's socio-economic position in adulthood. The Helsinki birth cohort study 1934-1944

Maiju Mikkonen ${ }^{1}$, Antti Häkkinen ${ }^{2}$, Minna K. Salonen ${ }^{1,3}$, Clive Osmond $^{4}$, Maarit Olkkola ${ }^{2}$, Johan G. Eriksson ${ }^{1,3,5}$, Eero Kajantie ${ }^{1,6, *}$

${ }^{1}$ The National Institute for Health and Welfare; ${ }^{2}$ Department of Political and Economic Studies, University of Helsinki;

${ }^{3}$ Folkhälsan Research Center, Helsinki, Finland; ${ }^{4}$ MRC Epidemiology Unit, University of Southampton, Southampton, United Kingdom; ${ }^{5}$ Department of General Practice and Primary Health Care, University of Helsinki; ${ }^{6}$ Hospital for Children and Adolescents, Helsinki, Finland

Background: It is well established that poor socio-economic circumstances early in life are associated with poor health throughout life. Few studies, however, have assessed how childhood social and economic hardship affects socioeconomic position throughout life. Our aim was to examine whether being born to an unmarried mother in a society 
where marriage is a norm is associated with socio-economic position in later life.

Method: The Helsinki Birth Cohort Study comprises 13345 men and women who were born between 1934 and 1944 in one of two public hospitals and who visited infant welfare clinics in the city and lived in Finland in 1971. Early life data was abstracted from birth records and child welfare clinic records. Using a unique personal identification number, we followed the subjects by linking their birth data to information on educational attainment, occupational status and taxable income, obtained from Statistics Finland in 5-year intervals between 1970 and 2000.

Results: Compared to children of married mothers the children of unmarried mothers were more likely to have attained lower education. When children with upper tertiary education served as a reference group, odds ratios adjusted for year and birth and sex were 1.67 (95\% CI 1.07 to 2.62) for lower tertiary, 2.87 (1.87 to 4.40) for upper secondary and 2.95 (1.95 to 4.47) for basic or less or unknown education. After adjustment for year of birth, sex, birth weight, birth order, mother's age, length of gestation and mother's and father's occupation, corresponding odds ratios were 1.53 (0.98 to 2.43 ), 2.28 (1.46 to 3.56 ) and 2.48 (1.61 to 3.83 ). Children of unmarried mothers had also lower occupational status and were less likely to reach the highest income third in adulthood.

Conclusion: This life-course study shows that being born to an unmarried mother is associated with socio-economic disadvantage throughout life.

Disclosure of Interest: None Declared.

\section{DOHaD13-1458}

\section{Parental growth and offspring birth weight: pooled analyses from four birth cohorts in low and middle income countries}

Yaw Addo ${ }^{1, *}$, Reynaldo Martorell ${ }^{1}$ and Consortium on Health Orientated Research in Transitional Societies

${ }^{1}$ Global Health, Rollins School of Public Health of Emory University, Atlanta, United States

Background: Growth failure has profound implications for individuals throughout the life cycle and for their offspring. Associations between parental and offspring size over the lifecourse are well established, but when parental growth matters most is a question that remains unanswered. Such information is needed to break the cycle of growth failure in low and middle-income countries (LMICs). Our objective was to relate parental birthweight and postnatal linear growth and weight gain to offspring birthweight.

Method: We analyzed data from 2588 parents and their 4042 newborns who had participated in one of four prospective birth cohorts from Brazil, Guatemala, India and the Philippines.

We applied conditional modeling techniques to correlated heights and weights measured at birth, $2 y$, mid-childhood and adulthood to generate orthogonal growth measures. Conditional height is current height accounting for all previous heights and weights (but not current weight). Conditional relative weight is current weight accounting for current height and all previous weights and heights. Conditional growth variables were expressed in standard deviation units to allow for comparisons of coefficients across measures, periods and parental sex.

We estimated associations of parental birthweight, conditional heights and relative weights with offspring birthweight, separately for mothers and fathers, using hierarchical linear models adjusted for parental level covariates, study site, and multiple births as random effects.

Results: There was no significant heterogeneity by study site or offspring sex. A 1 SD increase in maternal birthweight and in postnatal conditional linear growth from $0-2 y$ were associated with an $85.2 \mathrm{~g}$ (95\%CI: 56.0 -113.9) and 38.5 (95\%C: 9.3-67.7) g increase in offspring birthweight, respectively. Paternal birthweight and conditional linear growth $0-2 \mathrm{y}$ were associated with $52.1 \mathrm{~g} \quad(95 \% \mathrm{CI}$ : 22.0-82.5), and $45.1 \mathrm{~g}$ (95\%CI: 12.7-77.5) increases in offpring birthweight, respectively. Maternal (but not paternal) birthweight and linear growth from $0-2 y$ were independently associated with reduced risk of offspring low birthweight, with prevalence ratio $(\mathrm{PR})=0.69(95 \% \mathrm{CI}$ : $0.60-0.78)$ and $\mathrm{PR}=0.84$ (95\% CI: $0.73-0.96)$ respectively. There was no significant association between parental conditional relative weight and offspring birthweight except for the maternal mid-childhood to adulthood period $(\beta=50.0 \mathrm{~g}, 95 \% \mathrm{CI}: 22.0-78.2)$.

Conclusion: Both maternal and paternal birthweight and conditional linear growth from birth to 2 years, but not later, are associated with offspring birthweight. Maternal conditional relative weight from mid-childhood to adulthood was associated with offspring birthweight. These findings underscore the importance of adequate nutrition during the first 1000 days for both parents, and perhaps the preconception period for mothers, not only for the individual, but for the next generation as well. These associations could reflect nonmutually exclusive genetic, epigenetic and environmental mechanisms that influence fetal growth. Funding: Wellcome Trust; Bill and Melinda Gates Foundation.

Disclosure of Interest: None Declared.

\section{DOHaD13-1189}

\section{Parenteral nutrition accelerates growth even in healthy VLBWs}

Naho Morisaki ${ }^{1,2, *}$, Mandy B. Belfort ${ }^{3}$, Rintaro Mori ${ }^{4}$, Marie C. McCormick ${ }^{5}$, Hisashi Noma ${ }^{6}$, Satoshi Kusuda ${ }^{7}$, Masanori Fujimura ${ }^{8}$ and Neonatal Research Network of Japan

${ }^{1}$ Department of Health Policy, National Center for Child
Health and Development; ${ }^{2}$ Department of Pediatrics, 
University of Tokyo, Tokyo, Japan; ${ }^{3}$ Division of Newborn Medicine, Children's Hospital Boston, Boston, United States; ${ }^{4}$ Department of Health Policy, National Center fro Child Health and Development, Tokyo, Japan; ${ }^{5}$ Society, Health, and Human Development, Harvard School of Public Health, Boston, United States; ${ }^{6}$ Department of Data Science, The Institute of Statistical Mathematics; ${ }^{7}$ Department of Neonatology, Maternal and Perinatal Center, Tokyo Women's Medical University, Tokyo; ${ }^{8}$ Department of Neonatology, Osaka Medical Center and Research Institute for Maternal and Child Health, Osaka, Japan

Background: Increasing preference for enteral feeding for preterm infants, may shift parenteral nutrition to its temporary substitutes. Whether infants receiving parenteral nutrition only briefly would also benefit from its additional nutrition remains a question. In Japan, enteral feeding successfully advances at a fairly rapid rate, mainly thought to be due to the low background necrolizing-enterocolitis in neonates.

Method: We studied the effect of parenteral nutrition practices on in situ growth, in 4005 very low birth weight (VLBW) infants who reached full enteral feeding $(100 \mathrm{ml} / \mathrm{kg} /$ day) by day 14, from 75 institutions in the Neonatal Research Network Japan (2003-2007).

Results: Infants receiving parenteral nutrition were born earlier, showed lower SD score for weight and head circumference at birth and received longer stay. After adjusting for maternal, infant, and institutional characteristics, parenteral nutrition was associated with increased growth and less extra-uterine growth restriction (EUGR), i.e. below the $10 \%$ tile of the birth weight chart at corrected gestational age, at discharge. This increase was 0.09 standard deviation (SD) $(95 \% \mathrm{CI} 0.02,0.16)$ in weight, $0.16 \mathrm{SD}$ $(0.05,0.28)$ in head circumference, odds of being EUGR by weight was $0.85(0.66,1.08)$ and by head circumference $0.66(0.49,0.88)$. Parenteral nutrition also shortened length of stay by $1.2(0.3,2.4)$ days, but did not significantly change the odds of having broncho-pulmonary dysplasia $(0.85 ; 95 \% \mathrm{CI} 0.66$, $1.08)$ or periventricular hemorrhage $(1.19$; $95 \% \mathrm{CI} 0.76-1.87)$.

Conclusion: Even in VLBW infants who reached enteral feeding of $100 \mathrm{ml} /$ day within 2 weeks, parenteral nutrition accelerated weight gain, head growth and shortened stay without increasing complications.

Disclosure of Interest: None Declared.

\section{DOHaD13-1113}

\section{Pattern of variation of leptin in a cohort of Brazilian pregnant women}

Ana Beatriz Franco-Sena ${ }^{1, *}$, Livia C. Oliveira ${ }^{1}$, Thatiana J. P. Pinto $^{1}$, Dayana R. Farias ${ }^{1}$, Juliana S. Vaz ${ }^{1}$, Gilberto Kac ${ }^{1}$

${ }^{1}$ Nutritional Epidemiology Observatory, Federal University of Rio de Janeiro, Rio de Janeiro, Brazil

Background: Leptin concentrations have been associated with adverse gestational outcomes such as gestational weight gain and birth weight inadequacies ${ }^{1,2}$ and these conditions are related to long-term metabolic consequences ${ }^{3,4}$. Alterations in leptin levels during pregnancy are physiological and may be modified by maternal pre-pregnancy body mass index $(\mathrm{BMI})^{5}$. However, it is not known which other maternal characteristics may influence the longitudinal behavior of leptin in pregnancy. The objective of the current study was to assess if longitudinal trends of leptin in pregnancy are influenced by biochemical, anthropometric and life-style factors.

Method: Prospective cohort of 222 pregnant women followed at a public health care center in Rio de Janeiro, Brazil. Women met the following eligibility criteria when recruited: $20-40$ years of age; singleton pregnancy; $\leqslant 13$ weeks of pregnancy; and free from infectious or chronic diseases (except obesity). Blood samples were collected at $\leqslant$ $13^{\text {th }}, 20^{\text {th }}-26^{\text {th }}$ and $30^{\text {th }}-36^{\text {th }}$ gestational weeks, for the quantification of leptin, the dependent variable. Plasmatic concentrations were assessed by enzyme-linked immune sorbent assay kits (ELISA). Leptin concentrations were tested for normality using Shapiro-Wilk test and were logtransformed. The independent variables were collected at $\leqslant 13^{\text {th }}$ and were stratified after descriptive analysis, as follows: pre-pregnancy BMI $\left(<25 \geqslant 25 \mathrm{~kg} / \mathrm{m}^{2}\right)$; alcohol drinking (yes/no); total caloric intake and biochemical variables (categorized in tertiles of the sample distribution). For these variables, $\mathrm{p}$ for trend was considered. The effect of biochemical (adiponectin, insulin, glycaemia, triglycerides, total cholesterol and its fractions), anthropometric (prepregnancy BMI), socio-demographic (age, schooling and percapita family income) and life-style (alcohol drinking, smoking and practice of leisure time physical activity) variables on longitudinal behavior of plasmatic leptin concentrations were assessed using longitudinal linear mixed-effects model regressions with $\beta$ coefficients and standard error estimates.

Results: Pregnant women had a mean of $26.5(\mathrm{SD}=5.2)$ years of age and $8.6(S D=2.9)$ years of schooling. In the sample, $45 \%$ of the women had a BMI $\geqslant 25 \mathrm{~kg} / \mathrm{m}^{2}$ and $41 \%$ were nulliparous. Median leptin levels and interquartile ranges were $17.0(9.9-25.8), 26.4(16.8-43.9)$ and 24.9 $(16.0-38.9)$ for the $1^{\text {st }}, 2^{\text {nd }}$ and $3^{\text {rd }}$ trimesters, respectively. The multiple longitudinal regression model revealed association of pre-pregnancy BMI $(\beta=0.56, \mathrm{SE}=0.06)$, alcohol drinking $(\beta=0.25, S E=0.07)$, insulin plasmatic levels $\left(\beta^{2 \text { nd tertile }}=0.19, S E=0.07 ; \beta^{\text {3rd tertile }}=0.32, S E=0.08\right)$, total cholesterol serum levels $\left(\beta^{2 \text { nd tertile }}=0.22, \mathrm{SE}=0.10\right.$; $\left.\beta^{3 \text { rd tertile }}=0.35, S E=0.12\right)$ and total caloric intake $\left(\beta^{2 \text { nd }}\right.$ tertile $\left.=0.03, \mathrm{SE}=0.08 ; \beta^{3 \text { rd tertile }}=0.18, \mathrm{SE}=0.07\right)$ with prospective changes of log-leptin concentrations.

Conclusion: Women with excessive weight, without alcohol drinking, with higher caloric intake, and higher total cholesterol and insulin levels in the first trimester of pregnancy presented greater changes in leptin plasmatic levels along gestational trimesters. 


\section{References}

1. Stein TP et al. Am J Clin Nutr 1998; 68(6):1236-40.

2. Catov JM et al. Am J Obstet Gynecol 2007; 196: 558e1-558e8.

3. Levy-Marchal C et al. Pediatric Diabetes 2004; 5: 147-53.

4. Wrotniak BH et al. Am J Clin Nutr 2008; 87: 1818-24.

5. Misra VK et al. Obesity 2011; 19: 416-21.

Disclosure of Interest: None Declared.

\section{DOHaD13-1119}

Prenatal growth in a bi-ethnic cohort from Bradford, UK: the Born in Bradford birth Cohort

\author{
Tom Norris ${ }^{1, *}$, Derek Tuffnell ${ }^{2}$, John Wright ${ }^{3}$, Noel \\ Cameron $^{1}$ \\ ${ }^{1}$ Centre for Global Health and Human Development, Lough- \\ borough University, Leicester; ${ }^{2}$ Bradford Teaching Hospitals \\ NHS Foundation Trust; ${ }^{3}$ Bradford Institute for Health \\ Research, Bradford, United Kingdom
}

Background: Cardiovascular diseases (CVD), Type II diabetes and the Metabolic Syndrome (MetS), are of pandemic status. South Asians display increased risk for development of these NCDs. Within the developmental origins paradigm the acquisition of risk during fetal development is a core element of both the fetal origins hypothesis and the theory of Predictive Adaptive Responses. However, little research focusing on the prenatal growth experienced by this group has been conducted. This work aims to fill this gap, with an analysis of whether growth at the whole body (fetal weight) or using single dimensions (abdominal (AC) and head circumference (HC)) differs between Pakistani and White British fetuses and whether maternal size and demographic variables mediate any differences.

Method: Secondary analysis of fetal biometry (Estimated fetal weight (EFW), AC, HC) in 13776 fetuses enrolled in the Born in Bradford (BiB) birth cohort. Fetal biometry was collected from around 20 weeks gestation to birth. Analysis was limited to live-born full term singleton Pakistani and White British fetuses. Fractional polynomials of 1, 2 and 3 degree were used to model the growth patterns and the best model was identified using model fit and deviance statistics as well as residual diagnostics. Once the best fitting model was identified, the model was run using multilevel modelling. To see if there were ethnic differences in size and growth, Pakistani dummy and interaction (with age) variables were added to the model. At each stage, the $p$ values of the coefficients, along with the model fit, were assessed in order to determine whether inclusion of a particular variable improved the model. Variables were introduced into the model that adjusted for maternal pregnancy health status and potential maternal mediating variables and the effect on the Pakistani coefficient was identified.

Results: The best fitting model for $\ln (\mathrm{EFW})$ and $\ln (\mathrm{AC})$ was a two degree FP with the age terms $1 \& 2$, whereas for $\ln (\mathrm{HC})$, the best fitting model was a 2 degree FP with age terms $-0.5 \& 2$. Pakistani fetuses were $4.38 \%(\mathrm{p}<0.001)$ lighter and with smaller AC and $\mathrm{HC}(2.63 \%$ and $1.00 \%$ respectively, $\mathrm{p}<0.001)$ compared to White British throughout pregnancy. Although not reaching statistical significance in the weight or $\mathrm{HC}$ models, the Pakistani age interactions were statistically significant in the AC model, with the Pakistani mean curve displaying noticeable 'falling away' from the White British curve after 30 weeks.

Adjusting for maternal pre-/pregnancy health increased the deficits seen in all dimensions, whilst adjusting for maternal size and demographic variables reduced the deficits observed, with magnitude of the reduction depending on the outcome. Adjusting for SEP at the household level (maternal education) increased the deficits,whereas the neighbourhood level indicator of SEP (IMD_decile) reduced the deficits observed 6.29\% for weight, $4.35 \%$ for AC and 7.2\% for HC.

Conclusion: Pakistani fetuses may be characterized as having smaller dimensions throughout gestation, with a divergent growth profile that is limited only to AC. This reduced AC growth may be a reflection of reduced growth of the visceral organs and if so, offers a potential link between fetal growth and the increased risk for the previously mentioned NCDs observed in this group.

Disclosure of Interest: None Declared.

\section{DOHaD13-1218}

Pre-conceptional influences on infant and childhood body composition: findings from the Southampton Women's survey

Hazel Inskip ${ }^{1, *}$, Sarah Crozier ${ }^{1}$, Mark Hanson ${ }^{2}$, Nicholas Harvey $^{1}$, Siân Robinson ${ }^{1}$, Cyrus Cooper ${ }^{1}$, Keith Godfrey ${ }^{1,3}$ and Southampton Women's Survey Study Group

${ }^{1}$ MRC Lifecourse Epidemiology Unit; ${ }^{2}$ Faculty of Medicine, University of Southampton; ${ }^{3}$ NIHR Southampton Biomedical Research Centre, University of Southampton and University Hospital Southampton NHS Foundation Trust, Southampton, United Kingdom

Background: Many studies have researched parental influences on pregnancy and childhood outcomes, but, in most, prepregnancy data have been collected retrospectively. There has been increasing interest in parental influences on childhood body composition, notably in relation to obesity. However, few studies have collected detailed information before pregnancy.

Method: The Southampton Women's Survey interviewed 12,583 non-pregnant women aged 20-34y from the general population. 3,159 women were followed through a subsequent pregnancy and the children are being followed-up. We considered the following pre-conceptional influences: (a) socio-economic variables, (b) both parents: age, body composition, general health, (c) mother only: physical 
activity, alcohol consumption, diet, smoking, education, parity, menstrual cycle factors, haemoglobin, red cell folate concentration. Paternal factors were reported by the mother during pregnancy. Outcome variables were: birth length, height at 4 and 6y, fat and fat-free mass at birth, 4 and 6y obtained by dual X-ray absorptiometry, and body mass index (BMI) above the $90^{\text {th }}$ centile at 4 and $6 y$. Linear regression was used for continuous outcomes expressed as standard deviation (SD) scores, and Poisson regression with robust variance to calculate relative risks for binary outcomes. A manual forward stepwise procedure following a strict protocol was used. Adjustment for age, gestational age, length/height was made as appropriate. Due to the exploratory nature of the analysis, factors were only retained in the model when significant at the $1 \%$ level.

Results: Parental body composition measures were the dominant influences on child's body composition. Parous women had larger babies at birth but parity was unrelated to outcomes at 4 or 6 years, indicating a transient effect. Few women had been diagnosed with diabetes before pregnancy, but their children were at increased risk of high BMI at age 4 and 6y, the relative risk at 6y being 5.9 (95\%CI 2.4-14.6). Of the behavioural factors, only maternal pre-conceptional smoking featured in any final model, being associated with increased fatmass at $4 y$ and increased risk of high BMI at 4 and 6y.

Women with longer menstrual cycles had children who were taller, had greater fat mass at $4 y$, greater fat-free mass at $6 y$, and increased risk of high BMI at 4y. For each extra day in menstrual cycle length, the continuous outcomes increased by around $0.03 \mathrm{SD}$ (95\%CI 0.01-0.05). Women with irregular menstrual cycles (varying by $>5 \mathrm{~d}$ over the previous $6 \mathrm{~m}$ ), had children who were $0.3 \mathrm{SD}(95 \% \mathrm{CI} 0.1-0.4)$ shorter at age $4 \mathrm{y}$ than those with regular cycles. Child's height decreased by an average of $0.08 \mathrm{SD}(95 \% \mathrm{CI} 0.03-0.12)$ for each additional year of mother's age at menarche.

Conclusion: This work has identified various pre-conceptional factors associated with body composition up to 6 years of age, many of which were unsurprising. The only behavioural factor associated with the childhood body composition was pre-pregnancy smoking. The findings in relation to women's menstrual cycle may in part reflect maternal endocrine dysfunction. We encourage other researchers who have similar data to test these associations.

Disclosure of Interest: None Declared.

\section{DOHaD13-1369}

Sensitive developmental periods for atherosclerosis: insight from relationships between BMI and height across the growing years with carotid intima-media thickness in adulthood

William Johnson $^{1, *}$, Marietta Charakida ${ }^{2}$, Diana $\mathrm{Kuh}^{1}$, John E. Deanfield ${ }^{2}$, Rebecca Hardy ${ }^{1}$ and on behalf of the NSHD scientific and data collection teams
${ }^{1}$ MRC Unit for Lifelong Health and Ageing; ${ }^{2}$ National Centre for Cardiovascular Prevention and Outcomes, University College London, London, United Kingdom

Background: Carotid intima-media thickness (cIMT) is a marker of atherosclerosis that provides a surrogate end point for coronary artery disease. Although there is little evidence of a clinically significant birth weight - adulthood cIMT relationship, childhood/adolescent body mass index (BMI) does influence early adulthood cIMT. This suggests the presence of a sensitive period during postnatal development where faster BMI gain and/or slower height growth increases atherosclerosis risk, yet the timing of this period has not been characterised. The present study investigates the influences of BMI and height across the growing years on mid-late adulthood cIMT, with the aim to document sensitive periods for atherosclerosis development.

Method: The sample comprised 538 men and 609 women from the MRC National Survey of Health and Development (NSHD), a birth cohort born in 1946. Sex-stratified odds ratios (OR) of BMI/ height $Z$-scores at 2, 4, 6, 7, 11, 15, and 20 years of age, and conditional $Z$-score changes between $2-4,4-7,7-15$, and $15-20$ years of age, for cIMT at age 60-64 years in the upper quartile were estimated using logistic regression. In subsequent models, mediating by body size tracking and confounding by early childhood socioeconomic position (father's occupation and parental education), household environments (house quality/care and household crowding), nutrition (energy intake), physical illness (hospital admission), and stress (parental divorce and death) were investigated separately for each exposure mediator/confounder dyad.

Results: In males, a one SD increase in BMI at age four years incurred a $1.267(95 \%$ CI 1.021, 1.572) times increased odds of high cIMT, while a one SD increase in height at age four years incurred a $0.780(95 \%$ CI $0.624,0.975)$ times decreased odds. Limited evidence of association was seen for height at subsequent ages in childhood/ adolescence, but the positive association of BMI with cIMT re-emerged at 15 years of age and became stronger as age increased (OR of BMI at age 20 years 1.271; 95\% CI 1.002, 1.611). Accordingly, a one SD increase in BMI gain between seven to 15 years of age resulted in a $1.307(95 \%$ CI $0.956,1.787)$ increased odds of high cIMT, while a one SD increase in height growth between two to four years of age resulted in a 0.734 (95\% CI $0.554,0.973)$ decreased odds. The early childhood BMI and height effects on cIMT were robust to adjustment for mediators and confounders, but BMI during adolescence operated largely through BMI tracking. Results in females were null.

Conclusion: Early childhood BMI and height in males may have opposite effects on adulthood cIMT, which suggest that they represent two distinct mechanisms. The fact that height only in early childhood appeared to be an important determinant of cIMT suggests that this may be a sensitive 
period for the programming of atherosclerosis risk. Both early childhood and adolescence were identified as sensitive periods for the programming of atherosclerosis risk by excess relative weight; any risk acquired in early childhood may therefore be modifiable by weight gain in adolescence. The maintenance of healthy weight in males from adolescence onward may be a useful strategy to avoid the atherosclerotic complications of adiposity tracking.

Disclosure of Interest: None Declared.

\section{DOHaD13-1611}

\section{Slower postnatal growth is associated with increased sympathetic drive at age 5-6: the ABCD-study}

Maaike G. Gademan ${ }^{1}$, Arend W. van Deutekom ${ }^{2}$, Bert-Jan H. van den Born ${ }^{3}$, Marcel T. Twickler ${ }^{4}$, Tanja G. Vrijkotte ${ }^{1, *}$

${ }^{1}$ Public Health, Amsterdam Medical Center; ${ }^{2}$ Pediatrics, VU Medical Center; ${ }^{3}$ Vascular Medicine, Amsterdam Medical Center, Amsterdam, Netherlands; ${ }^{4}$ Endocrinology, Diabetology en Metabolic Diseases, Antwerp University Hospital, Antwerp, Belgium

Background: Accelerated postnatal growth is assumed to increase the a-priori risk to the occurrence of premature cardiovascular disease later in life. The origin of this relationship could be in the accumulation of visceral fat that disturbs the autonomic balance. Against this background, we analysed whether the cardiac autonomic balance found at age 5-6 years was associated with the postnatal growth profile, and whether the waist-to height ratio (at age 5-6 years) could explain this possible relationship.

Method: This analysis was part of the ABCD study (Amsterdam Born Children and their Development) ${ }^{1}$. Children with known postnatal growth (Youth Health Care) and known cardiac autonomic balance (measured at age 5-6) were included. Children who were premature $(n=118)$, had congenital anomalies $(n=52)$ and children of mothers with pre-existent or gestational diabetes mellitus $(n=42)$ were excluded from the current analysis. Electrocardiography and impedance cardiography were used to assess sympathetic drive (pre-ejection period, PEP), parasympathetic drive (respiratory sinus arrhythmia, RSA) and heart rate (HR) during supine rest. Two indicators of growth (weight and weight-for-height) were defined in 3 periods by calculating delta standard deviation scores (sds) in the periods $1-3,3-6$ and 6-12 months after birth. Linear regression models assuming normally distribution errors were used to investigate the association between postnatal weight gain and HR, RSA and PEP. In the final models adjustments were made for offspring sex, age and time of the day at outcome measurement, standardised birth weight, earlier growth (when applicable), waist-to-height ratio and the following maternal characteristics: pre-pregnancy BMI, height, educational level, ethnicity, alcohol use and smoking during pregnancy, hypertension, gestational age and parity. As, when applicable, adjustments for earlier growth were made, our results represent conditional growth. Results: After use of adjusted models, slower conditional postnatal growth (delta sds weight) between 1-3 months was associated with decreased PEP ( $\beta 0.97 \mathrm{msec}$, 95\% CI;0.04, $1.79)$ and increased heart rate $(\beta$ : $-0.99 \mathrm{msec}, 95 \% \mathrm{CI}$; $-1.94,-0.05)$. In addition, slower conditional postnatal growth (delta sds weight and delta sds height gain) between 3-6 months was associated with lower PEP ( $\beta$ : $1.09 \mathrm{msec}$, 95\%CI; 0.26, 1.92 and ( $\beta$ : $0.87 \mathrm{msec}, 95 \% \mathrm{CI} ; 0.10,1.63$, respectively). Moreover, slower postnatal conditional growth between 6-12 months was linearly associated with increased HR ( $\beta$ delta sds weight for height: -1.48 beats $/ \mathrm{min}$, 95\%CI;-2.55, -0.41 and $\beta$ delta sds weight: -1.08 beats/ $\min , 95 \% \mathrm{CI} ;-2.02,-0.14)$. These associations were not mediated by waist-to-height ratio at age 5-6 years. Postnatal growth, in general, was not associated with changes in RSA. Conclusion: Surprisingly, slower growth (and not faster growth) was associated with increased sympathetic drive and increased heart rate. We found no proof for a mediating role of visceral fat within this association.

\section{Reference}

1. Eijsden van M, Vrijkotte TGM, RJBJ Gemke and Wal, van der MF. Cohort Profile: The Amsterdam Born Children and their Development (ABCD) Study. Int J Epidemiol 2011, 40 (5): 1176-1186.

Disclosure of Interest: None Declared.

\section{DOHaD13-1641}

Study of asthma genes and the environment: cohort retention over time

Rishma Chooniedass ${ }^{1, *}$, Brenda Gerwing ${ }^{1}$, Saiful Huq ${ }^{1}$, Anita Kozyrskyj ${ }^{2}$, Clare Ramsey ${ }^{3}$, Allan Becker ${ }^{1}$

${ }^{1}$ Pediatrics and Child Health, University of Manitoba, Winnipeg; ${ }^{2}$ Epidemiology, University of Alberta, Edmonton; ${ }^{3}$ Respirology, University of Manitoba, Winnipeg, Canada

Background: The Study of Asthma Genes and the Environment (SAGE) investigated early life origins of asthma, using provincial healthcare administrative records. We sent surveys to 12556 families of children born in Manitoba and created a nested cohort for intensive research. We hypothesized that maternal education, smoking history, household income and child asthma would be predictors of attrition.

Method: From returned surveys ( $\mathrm{n}=3580 / 12556), 640$ children were recruited as a purpose built nested cohort. Children and parents were seen, homes visited, questionnaires, skin testing, pulmonary function and blood for genetic and immunologic testing were obtained at 3 timepoints. 
Results: Children were seen at $9.1 \pm 0.5 \quad(\mathrm{n}=640)$, $10.6 \pm 0.4 \quad(\mathrm{n}=512,80 \%)$, and $12.6 \pm 0.5 \quad(\mathrm{n}=451$, $70 \%)$ years. Initially $227 / 640(35.5 \%)$ had asthma, 374/ 640 (58\%) were urban, 361 (56\%) were male, $492(77 \%)$ mothers had post-secondary education, 246 (39.4\%) mothers smoked (ever), with $17 \%$ current smokers, $179(28 \%)$ were high income $(\geqslant \$ 80,000)$, and $113(17.7 \%)$ low income $(<\$ 39,999)$.

Of children seen on visit 3; $451(70 \%)$ returned (males:71.5\% vs. females:69.2\%, urban:73\% vs. rural:67\%). From these, more children at study entry had asthma (OR: 1.45, 95\%CI: 1.0-2.1), more mothers were not current smokers (OR: 1.97, 95\%CI: 1.1-3.4) and had higher education (OR: 1.6, 95\%CI: 1.1-2.3) and more were from urban settings (OR: 1.32, 95\%CI: 0.9-1.9). There is a trend towards higher income to return (73\% vs. $70.6 \%$ vs. $68.1 \%, p=0.11$ ).

Conclusion: Retention over years for SAGE was, in part, dependent on a child's asthma diagnosis (not unexpectedly), maternal education and smoking status. There was a trend towards greater retention in families with higher income. Recruitment and retention of participants are critical for a successful population-based study. For future cohort studies, specific retention strategies should be considered for those at greater risk of attrition.

Disclosure of Interest: None Declared.

\section{DOHaD13-1711}

Tea consumption during early pregnancy does not increase the risk of small for gestational age and preterm delivery in Southern China: a birth cohort study

Jianrong $\mathrm{He}^{1}$, Songying Shen ${ }^{1}$, Yu Liu ${ }^{1}$, Jinhua $\mathrm{Lu}^{1}$, Mingyang Yuan ${ }^{1}$, Jun Chen ${ }^{1}$, Lijuan Xiu ${ }^{1}$, Xiaoyan Xia ${ }^{1}$, Yong Guo ${ }^{1}$, Xiu Qiu ${ }^{1, *}$

${ }^{I}$ Guangzhou Women and Children's Medical Center, Guangzhou, China

Background: Few western studies have suggested tea consumption increased the risk of adverse pregnancy outcome. We aimed to evaluate the association of tea consumption during early pregnancy with pregnancy outcome in a Southern Chinese population.

Method: We prospectively recruited 2691 pregnant women before gestational age of 20 weeks at two branch hospitals of Guangzhou Women and Children's Medical Center, China between October 2011 and October 2012. By May 31st, 2013, 35 (1.3\%) participants terminated their pregnancies or ended in stillbirth, $45(1.7 \%)$ had multiple gestations, 186 (6.9\%) dropped out, and $95(3.5 \%)$ lost to follow-up. Finally, 2330 singleton live births were included in the present study. Information on tea consumption and potential confounders were collected by face to face interview. Small for gestational age (SGA) and large for gestational age (LGA) cases were defined according to the Guangzhou birthweight reference. Preterm delivery (PTD) was defined as delivery before 37 weeks of gestation.

Results: We found $16.3 \%$ of pregnant women reported tea consumption at least once per week during early pregnancy. Neither frequency (never vs $\geqslant 1$ times/week) nor type (never vs green tea and non-green tea only) of tea consumption was significantly associated with the risk of SGA, LGA and PTD, after adjusted for maternal age, educated years, income, prepregnancy body mass index, parity, passive smoking and offspring's gender. The results did not change substantially when the analysis was restricted to term deliveries or stratified by offspring's gender.

Conclusion: Our results do not support an association of tea consumption in early pregnancy with pregnancy outcome in Chinese population. Further studies are needed to evaluate whether tea consumption affects the long-term outcomes in offspring.

Disclosure of Interest: None Declared.

\section{DOHaD13-1525}

The impact of full breastfeeding, partial breastfeeding and formula feeding on infant growth during the first year of life

Izzuddin B. M. Aris ${ }^{1, *}$, Doris Loh ${ }^{2}$, Wei Wei Pang ${ }^{2}$, Seang Mei Saw ${ }^{3}$, Kenneth Kwek ${ }^{4}$, Peter D. Gluckman ${ }^{5}$, Fabian Yap ${ }^{4}$, Yung Seng Lee ${ }^{1}$, Yap Seng Chong ${ }^{2}$ and GUSTO Study Group

${ }^{1}$ Paediatrics; ${ }^{2}$ Obstetrics and Gynaecology, National University of Singapore; ${ }^{3}$ Saw Swee Hock School of Public Health; ${ }^{4} K K$ Women's and Children Hospital; ${ }^{5}$ Singapore Institute for Clinical Sciences, Singapore, Singapore

Background: We examined the impact of full breastfeeding (FBF), partial breastfeeding (PBF) and formula feeding (FF) on the infants' growth outcome during the first year of life in Growing Up in Singapore Towards healthy Outcomes (GUSTO) birth cohort

Method: 250 postnatal growth data were available from birth to 12 months of age: FBF $4-6$ months $(n=107)$, PBF 4-6 months $(n=69)$ and FF 4-6 months $(n=74)$. Crosssectional and mixed longitudinal analyses were utilized to examine the association between the feeding types and infant's body weight, body mass index (BMI), ponderal index (PI) and abdominal circumference (AC).

Results: Boys who were fully breast-fed for 4-6 months after birth had significantly higher BMI, PI and AC change in the first 6 months of life $(p<0.05)$, but had significantly lower changes in weight, BMI, PI and AC from 6 to 12 months of age $(p<0.05)$, when compared to boy who were fed with formula milk. Boys who were partially breast-fed for 4-6 months also had slower weight gain and BMI change from 6-12 months 
of age. In comparison to formula-fed girls, only those who were fully breast-fed had significantly slower weight change between 6-12 months of life $(p<0.05)$.

Conclusion: Full breastfeeding and partial breastfeeding for 4 to 6 months resulted in slower rates of weight gain and BMI changes in GUSTO infants from 6 to 12 months of age. Differences in weight gain in the early postpartum period between the breast-fed (full and partial) and formula-fed infants can perhaps be utilized to guide feeding and infant care recommendations given to mothers and care givers as a means of preventing the risk of obesity in children.

Disclosure of Interest: I. Aris: None Declared., D. Loh: None Declared., W. W. Pang: None Declared., S. M. Saw: None Declared., K. Kwek: None Declared., P. Gluckman Paid Instructor for: Have received reimbursement for speaking at conferences sponsored by companies selling nutritional products; part of an academic consortium that has received research funding from Abbot Nutrition, Nestec and Danone., F. Yap: None Declared., Y. S. Lee: None Declared., Y. S. Chong Paid Instructor for: Have received reimbursement for speaking at conferences sponsored by companies selling nutritional products; part of an academic consortium that has received research funding from Abbot Nutrition, Nestec and Danone.

\section{DOHaD13-1172}

The long-term effects on growth and blood pressure of antenatal multiple micronutrient supplementation in Nepal Delan Devakumar $^{1, *}$, Jonathan Wells ${ }^{2}$, Shiva S. Chaube ${ }^{3}$, Naomi M. Saville ${ }^{1}$, Dharma S. Manandhar ${ }^{3}$, Anthony Costello $^{1}$, Jonathan G. Ayres ${ }^{4}$, David Osrin ${ }^{1}$

${ }^{1}$ Institute for Global Health; ${ }^{2}$ Childhood Nutrition Research Centre, University College London, London, United Kingdom; ${ }^{3}$ Mother and Infant Research Activities, Kathmandu, Nepal;

${ }^{4}$ Institute of Occupational and Environmental Medicine, University of Birmingham, Birmingham, United Kingdom

Background: We followed up children from a double blind randomised controlled trial of antenatal multiple micronutrient (MMN) supplementation in Nepal (1). 600 pregnant women in the intervention group received the UNIMAPP supplement and 600 in the control group iron and folate. The trial and first follow-up found that infants born in the MMN group were $77 \mathrm{~g}$ heavier at birth (1) and $204 \mathrm{~g}$ heavier at 2.5 years, with a $2.5 \mathrm{mmHg}$ lower mean systolic blood pressure (2). The second follow-up investigated longer-term differences in growth and blood pressure.

Method: The study was conducted in Dhanusha district, Nepal, between September 2011 and December 2012. Children from the original trial were followed up at eight years of age. We measured anthropometry, body composition using bioelectrical impedance (with a population specific isotope calibration study), blood pressure, and kidney dimensions by ultrasound. Questionnaire data were collected on demography, illness history, socioeconomic status and food security. We used $\mathrm{t}$ tests and univariable linear regression to compare the intervention and control groups. We then constructed a causal diagram and controlled for potential confounding factors based on this using multiple linear regression.

Results: We followed up 841 children, $80 \%$ of the total potential number. Children lost to follow-up were no different, other than their mothers being more likely to have had some education and be urban residents $(\mathrm{p}<0.0001)$. The mean age was 8.5 years (range 7.2 to 9.9 ). No significant difference was found between intervention and control groups for any outcome. The difference in weight (intervention group minus control) was $0.101 \mathrm{~kg}(95 \% \mathrm{CI}-0.350$, $0.553 \mathrm{~kg})$ and in systolic blood pressure $0.017 \mathrm{mmHg}(95 \%$ CI $-1.016,1.051 \mathrm{mmHg})$. After excluding children with chronic diseases, multivariable regression adjusted for air pollution, asset score, diet, gestation, maternal education, maternal height, and parity showed no difference in weight for age $(0.058,95 \%$ CI $-0.067,0.183)$, height for age $(0.040,95 \% \mathrm{CI}-0.073,0.152)$ or BMI for age $(0.040,95 \%$ CI $-0.086,0.166)$.

Conclusion: At 8.5 years of age there was no difference in anthropometry or blood pressure between children born to antenatal MMN supplemented mothers and children whose mothers received iron and folate. While not ruling out physiological differences and longer-term effects, our findings do not suggest a sustained effect of antenatal micronutrient supplementation on growth.

\section{Reference}

1. Osrin D, Vaidya A, Shrestha Y, Baniya RB, Manandhar DS, Adhikari RK, et al. Effects of antenatal multiple micronutrient supplementation on birthweight and gestational duration in Nepal: double-blind, randomised controlled trial. Lancet. 2005 Mar 12-18; 365(9463): 955-62. 2. Vaidya A, Saville N, Shrestha BP, Costello AM, Manandhar DS, Osrin D. Effects of antenatal multiple micronutrient supplementation on children's weight and size at 2 years of age in Nepal: follow-up of a double-blind randomised controlled trial. Lancet. 2008 Feb 9; 371(9611): 492-9.

Disclosure of Interest: None Declared.

\section{DOHaD13-1490}

The relationship between active smoking and high-sensitive $\mathrm{C}$-reactive protein in late adolescence

Chi Le-Ha ${ }^{1}$, Lawrence J. Beilin ${ }^{1}$, Sally Burrows ${ }^{1}$, Rae-Chi Huang $^{1}$, Wendy H. Oddy ${ }^{2}$, Beth Hands ${ }^{3}$, Trevor A. Mori ${ }^{1, *}$ ${ }^{1}$ School of Medicine and Pharmacology; ${ }^{2}$ Telethon Institute for Child Health Research, University of Western Australia; ${ }^{3}$ Notre Dame University, Perth, Australia 
Background: Cigarette smoking contributes to the chronic inflammatory process which has a role in atherosclerosis. C-reactive protein (CRP), smoking, and the use of oral contraceptives (OC) are factors importantly related to cardiovascular risk in adults. Evidence of an association between active smoking and high-sensitive C-reactive protein (hs-CRP) in adolescents has been inconsistent. This study aimed to examine the association between active smoking and hs-CRP in adolescent boys and girls.

Method: One thousand and fifty seven adolescents (mean age, $17 \pm 0.25$ years) of the Western Australian Pregnancy Cohort (Raine) Study had lifestyle and metabolic measures recorded. The association between log-transformed hs-CRP concentrations and smoking was analysed using multivariable tobit linear regression, with adjustment for adiposity and lifestyle confounders. A 3-level variable (girls not using oral contraceptives (OC), girls using OC, and boys) was employed to assess the interactive effects of sex, OC use and smoking. Results: The cohort consisted of 546 boys and 511 girls; $17 \%$ of the adolescents smoked, 51\% consumed alcohol, and 31\% of girls took OC. Log hs-CRP concentrations were positively associated with BMI in boys and girls $(b=0.133 ; \mathrm{p}<0.001)$. Log hs-CRP was also positively associated with OC use in girls that did not smoke $(b=1.189 ; \mathrm{p}<0.001)$, and with smoking in girls that did not take OC $(b=0.571$; $\mathrm{p}=0.001)$. However, there was no association between $\log$ hs-CRP concentrations and smoking in boys, or in girls who took OC and smoked.

Conclusion: Our data in a large population-based birth cohort show that smoking associated with higher CRP levels in girls not using OC, and that OC use in non-smoking girls strongly associated with higher hs-CRP. Smoking has a more clear-cut effect on CRP in girls not using OC than in boys. As CRP levels track from childhood to adulthood, public health programmes aiming to reduce adolescent smoking will alleviate the cardiovascular disease burden in adults.

Disclosure of Interest: None Declared.

\section{DOHaD13-1166}

Tracking of cardiovascular risk factors from childhood to young adulthood - the Pune Children's Study

Suyog Joshi ${ }^{1}{ }^{1 *}$, Prachi Katre ${ }^{2}$, Kalyanaraman Kumaran ${ }^{1}$, Charudatta Joglekar ${ }^{1}$, Clive Osmond ${ }^{3}$, Dattatray Bhat ${ }^{1}$, Himangi Lubree ${ }^{1}$, Anand Pandit ${ }^{4}$, Chittaranjan Yajnik ${ }^{1}$, Caroline Fall ${ }^{3}$

${ }^{1}$ Diabetes Unit, KEM Hospital Research Centre; ${ }^{2}$ Persistent Systems, Pune, India; ${ }^{3}$ Lifecourse epidemiology unit, University of Southampton, Southampton, United Kingdom; ${ }^{4}$ Pediatric Unit, KEM Hospital Research Centre, Pune, India

Background: Longitudinal studies from developed countries have shown correlations between risk factor levels measured in childhood and adult ('tracking'). The implication is that efforts to control CVD risk factors should start in childhood. Since developmental origins of health and disease $(\mathrm{DOHaD})$ research is moving towards preconceptional interventions with the objective of improving fetal development and reducing later cardio-metabolic risk in the offspring, it is important to know what childhood risk profiles mean in terms of predicting adult risk factors. There are no studies with adequate follow-up time to study child-adult tracking in low and middle income countries. The Pune Children's Study is a cohort of children born in Pune, India, who had a range of CVD risk factors measured at the age of 8 years. We recently measured the same set of risk factors, using similar methods, at the age of 21 years, providing the first opportunity to assess the degree of child-adult tracking in an Indian population.

Method: We studied 357 men and women from the Pune Children's Study. Body mass index (BMI), skinfolds, waist circumference, blood pressure, plasma lipids (total cholesterol, triglycerides, HDL-cholesterol), glucose, insulin, insulin resistance and leptin were measured at 8 and 21 years of age. The degree of tracking was assessed using correlations, the risk of remaining in upper or lower fourths of the distribution, Cohen's kappa statistics, and predictive values.

Results: All 8-year risk factors were significantly positively correlated with their counterparts at 21 years, except 120minute glucose. Correlation coefficients (age- and sexadjusted) ranged from $\mathrm{r}=0.53$ (BMI and total cholesterol) to $\mathrm{r}=0.14$ (fasting insulin) $(\mathrm{p}<0.05$ for all). The relative risks for remaining in the upper fourth of the distribution at 21 years if also there at 8 years ranged from 4.19 for total cholesterol $(95 \%$ CI $2.94,5.97)$ to 1.47 for fasting glucose (95\% CI 1.00, 2.15). The relative 'benefit' for remaining in the lower fourth of the distribution at 21 years if also there at 8 years ranged from 2.88 for total cholesterol (95\% CI 2.05, 4.06) to 1.46 for fasting insulin $(95 \%$ CI $1.00,2.15)$. Positive predictive values for developing 'disease' outcomes (hypertension, dyslipidaemia, hyperglycaemia, central obesity or overweight/ obesity) if in the upper fourth of the distribution of the relevant measure at 8 years ranged from $73 \%$ (low HDL-cholesterol) to $8 \%$ (hypertension). Sensitivity values were less than $50 \%$, except for cholesterol and hypercholesterolaemia (75\%). Specificity values were in the range of $70-80 \%$. The strength of tracking as measured by Cohen's kappa statistics was strongest for cholesterol (23\%) and weakest for systolic BP (2\%).

Conclusion: BMI and cholesterol concentrations track strongly, and blood pressure, other lipids, glucose and insulin concentrations track moderately from childhood to young adulthood. Measurements in children as part of birth cohort studies to investigate associations between maternal and offspring exposures with CVD risk factors are informative about adult risk. Public health measures to reduce CVD risk should start in childhood or earlier.

Disclosure of Interest: None Declared. 


\section{DOHaD13-1296}

\section{What is rotten in the state of Denmark: why is Danish health behind the other Western European Nations?}

Stephen Bezruchka ${ }^{1, *}$

${ }^{1}$ Global Health, University of Washington, Seattle, United States

Background: Denmark is the only rich country with current health outcomes ranking with the United States of America and behind the other Western European nations. Forty years ago Denmark had considerably better mortality outcomes compared to the USA.

Method: The relative health decline in Denmark is explored in terms of stress, political, economic and early life issues.

Results: There is considerable evidence that state-level factors affected women in Denmark in the late 1960s when they entered the work force in temporary low-wage jobs. They were laid off in the 1970s and unemployment soared compared to other Scandinavian countries. Denmark lacked comparable welfare supports.

Health improvements for men and women in Denmark stagnated during the 1970s and 80 s at a time when they continued improving in the United States and Western European nations. There is likely a cohort effect affecting the subsequent generation, reflecting lack of attention to early life, that continues their continued relatively poor health status.

Consumption of cigarettes increased so that today the highest rates of female deaths from smoking related conditions in Western Europe are found in Denmark. Male smoking rates there have not changed significantly. Alcohol consumption in Denmark is among the highest levels in Western Europe with increasing trends in contrast to neighboring countries.

In contrast to the earlier period when relative health status declined, Denmark has a system called flexicurity embodying agreements among employers, workers and the state. Very generous welfare benefits result for the unemployed combined with flexibility in the labor market.

Studies have demonstrated a relationship between both income and wealth inequality with life expectancy among rich nations. While income inequality is low in Denmark, wealth inequality is on a par with the United States of America and may in part be related to status and stress issues that play out in adverse health-related behaviors.

Unlike the government of the USA which appears to ignore the poor health status of its people in comparison to other rich nations, the Danish government recognizes the problem and envisions the country regaining its relative health status compared to other nations.

Conclusion: Denmark represents a country that has experienced a relative health decline compared to other Western European nations where the government recognizes the problem and has a stated vision to improve.

\section{Reference}

1. Bjerregaard, P. and N. Hermann (1994). Lifetime in Denmark: Second report from the Life Expectancy Committee of the Ministry of Health, Denmark. Copenhagen, Ministry of Health, The Life Expectancy Committee.

Disclosure of Interest: None Declared.

\section{DOHaD13-1214}

All about ' $U$ ': associations between intra-uterine exposure to prenatal depression and offspring responses to stress

Michelle Fernandes ${ }^{1, *}$, Alan Stein ${ }^{2}$, Krishnamachari

Srinivasan $^{3}$, Gladys Menezes ${ }^{4}$, Paul Ramchandani ${ }^{5}$

${ }^{1}$ Nuffield Department of Obstetrics and Gynaecology; ${ }^{2}$ Department of Psychiatry, University of Oxford, Oxford, United

Kingdom; ${ }^{3}$ St. John's Research Institute, St. John's National Academy of Health Sciences, Bangalore; ${ }^{4}$ Snehalaya Hospital, Solur, India; ${ }^{5}$ Faculty of Medicine, Imperial College, London, United Kingdom

Background: The importance of the intra-uterine environment in influencing developmental trajectories has gained in scientific importance ${ }^{1,2}$. Intra-uterine exposure to maternal depression has been associated with increased risks of disturbances in stress responses, poorer selfregulation and behavioural problems during childhood and adolescence ${ }^{2}$. The influence of prenatal depression on foetal responses to a potential stressor are however unclear. Furthermore, there is very little research exploring this issue in socio-economically disadvantaged populations from the developing world. The objective of this study is twofold:first, to examine the association between prenatal maternal depression and (i) foetal and (ii) infant stress responses in a rural cohort from South India; and second, to explore whether continuity between foetal and infant stress responses exist.

Method: 67 depressed and 66 non-depressed mothers with singleton pregnancies in their third trimester were recruited from a specialist obstetric hospital in the village of Solur in South India. The Edinburgh Postnatal Depression Scale and the Kessler 10 Scale were used to assess mothers for prenatal depression. In the prenatal assessment, foetal responses to a potentially stressful vibroacoustic stimulus were examined by measuring changes in foetal heart rate during and after exposure. At 3 months after birth, infant responses to a potential stressor, i.e. immunization, were examined by measuring salivary cortisol before and after immunization. Infant temperament was assessed via maternal reports on the Infant Behaviour Questionnaire.

Results: The association between prenatal depression and foetal response to the vibroacoustic stimulus was $U$ shaped. Foetuses exposed to moderate levels of prenatal depression showed lower total stress responses than those exposed to very 
high or low levels of depression $\left(R^{2}=0.98, p=0.01\right)$. Prenatal depression predicted infant cortisol responsivity independent of postnatal depression $(B=13.08, \mathrm{p}=0.02)$. Further exploration revealed this relationship to be $\mathrm{U}$ shaped. Infants of mothers with moderate levels of prenatal depression showed lower cortisol responses to immunization than the infants of mothers with extreme levels of prenatal depression. Prenatal depression did not influence any of the dimensions of infant temperament based on maternal reports. Foetal responsivity correlated with infant cortisol responsivity $(\mathrm{r}=0.37, \mathrm{p}=0.02)$. Prenatal depression however did not effect this association $(\beta=0.81, \mathrm{p}=0.74)$.

Conclusion: Our findings suggest that exposure to some amount of intra-uterine stress may favour offspring development in certain environmental contexts ${ }^{1,2}$. The study represents the first attempt to investigate the programming of foetal responses in the context of maternal stress in the developing world and highlights the need for further research in this field in these regions.

\section{References}

1. Gluckman PD et al (2010). A conceptual framework for the developmental origins of health and disease. Journal of Developmental Origins of Health and Disease.

2. Glover V. (2011) Annual Research Review: Prenatal stress and the origins of psychopathology: an evolutionary perspective. Journal of Child Psychology and Psychiatry.

Disclosure of Interest: None Declared.

Keywords: None.

\section{DOHaD13-1495}

\section{Association of maternal dietary carbohydrate intake and plasma folate levels with antenatal low mood and depression}

Mary F.-F. Chong ${ }^{1, *}$, Jocelyn X. Wong ${ }^{2}$, Majorelee Colega ${ }^{2}$, Ling Wei Chen ${ }^{2}$, Rob M. van Dam ${ }^{2}$, Chuen Seng Tan ${ }^{2}$, Ai Lin Lim ${ }^{1}$, Wei Wei Pang ${ }^{2}$, Shirong Cai ${ }^{2}$, Birit Broekman ${ }^{3}$, Yung Seng Lee ${ }^{2}$, Seang Mei Saw ${ }^{2}$, Kenneth Kwek ${ }^{4}$, Keith M. Godfrey $^{5}$, Yap Seng Chong ${ }^{2}$, Peter Gluckman ${ }^{6}$, Michael J. Meaney ${ }^{7}$, Helen Chen ${ }^{4}$ and GUSTO

${ }^{1}$ Singapore Institute for Clinical Sciences, $A^{*} S T A R ;{ }^{2}$ National University of Singapore; ${ }^{3}$ National University Hospital; ${ }^{4} \mathrm{KK}$ Women's and Children's Hospital, Singapore, Singapore; ${ }^{5}$ University of Southampton, Southampton, United Kingdom; ${ }^{6}$ Liggins Institute, Auckland, New Zealand, ${ }^{7}$ McGill University, Montreal, Canada

Background: General population studies have proposed links between nutrition and depression, but less is known about the perinatal period. Depletion of nutrient reserves throughout pregnancy and delayed postpartum repletion could increase the risk of maternal depression. Relationships with maternal macronutrient intake and plasma folate concentrations were examined.

Method: At $26^{\text {th }} 28^{\text {th }}$ weeks of gestation, women from the birth cohort study (GUSTO) provided blood samples for plasma folate measurements, and their dietary intakes were ascertained using 24-hr food recalls. Symptoms of low mood and depression were measured with the Edinburgh Postpartum Depression Scale (EPDS) during the same period, as well as at 3-month postpartum. Validation studies have shown that EPDS score of $\geqslant 15$ during pregnancy or $\geqslant 13$ at postpartum are indicative of low mood and probable depression.

Results: Of the pregnant women, $6.1 \%(51 / 838)$ had an EPDS score $\geqslant 15$, and $9.7 \%(60 / 616)$ a post-partum score $\geqslant 13$. Univariate analyses showed a high antenatal EPDS score $(\geqslant 15)$ was associated with younger mothers, lower educational levels, lower household income, being of Indian ethic origin, smoking before pregnancy and being exposed to smoking during pregnancy ( $p<0.05$ for all). After adjusting for the above covariates and others known to be associated with perinatal depression, logistic regression showed that lower dietary carbohydrate intakes (OR: $0.95,95 \% \mathrm{CI}$ : $0.91-0.99, p=0.020)$ and lower plasma folate concentrations (OR: $0.98,95 \%$ CI: $0.97-0.99, p=0.046$ ) were associated with a higher risk of an antenatal EPDS score $\geqslant 15$. These associations did not persist for a high postpartum EPDS score, after adjusting for EPDS scores during pregnancy.

Conclusion: Maternal macronutrient intakes and folate status during pregnancy were not related to postpartum low mood and depression. Lower dietary carbohydrate intake and lower plasma folate concentrations during pregnancy were, however, associated with a higher risk of antenatal low mood and depression.

Disclosure of Interest: None Declared.

\section{DOHaD13-1111}

Between- and within-individual variability in first morning urinary cortisol in pre-pubertal children: potential explanatory variables

Cindy K. Barha ${ }^{1,2, *}$, Katrina G. Salvante ${ }^{1,2}$, Jianfeng Zhang ${ }^{3}$, Leilei Zeng ${ }^{3}$, Pablo A. Nepomnaschy ${ }^{1,2}$

${ }^{1}$ Health Sciences; ${ }^{2}$ Human Evolutionary Studies Program, Simon Fraser University, Burnaby; ${ }^{3}$ Department of Statistics and Actuarial Science, University of Waterloo, Waterloo, Canada

Background: The stress, or hypothalamic-pituitary-adrenal axis (HPAA), acts as a vital mediator between individuals and their environment, allowing them to respond and temporarily adapt to internal and external challenges. Basal HPAA dysregulation is associated with numerous adverse health 
outcomes across the lifespan, broadly ranging from metabolic syndrome to neuropsychiatric disorders. Importantly, a large degree of variability in HPAA activity has been observed both between and within individuals. We have recently observed this variability in first morning urinary cortisol (FMUC) levels, a biomarker of HPAA activity. Variability in HPAA functioning influences how individuals differentially respond to life challenges and may have important consequences for susceptibility to disease across the lifespan. Therefore, understanding the origins of variation in HPAA function is of critical importance to understanding health and wellbeing at both the individual and the population levels. Evidence is accumulating suggesting a crucial role for environmental exposures during early ontogenetic stages, including exposure to maternal cortisol, in differential programming of HPAA function. Here we explore between- and within-individual variability in basal HPAA functioning in pre-pubertal children in response to the commencement of a new school term and whether this variability is associated with maternal peri-conceptional cortisol levels.

Method: Eighteen Kakchiquel Mayan women at risk of conceiving were recruited 13 years ago in Guatemala and followed for one year in which they conceived and subsequently delivered a live baby. FMUC levels were assessed in samples collected thrice weekly during the periconceptional period from mothers. In their now pre-pubertal children, FMUC levels were assessed in specimens collected daily for three weeks. The relationship between maternal periconceptional HPAA activity and their children's basal HPAA function in response to the commencement of a new school term was explored using hierarchical regression analysis.

Results: We observed a substantial amount of variation in FMUC levels in the children across the three weeks evaluated (range of individual cortisol means: $38.75-85.24 \mathrm{ng} / \mathrm{mL}$; standard deviations: $9.83-65.01 \mathrm{ng} / \mathrm{mL}$ ). A large degree of variability was also observed in children's basal HPAA responses to starting school; some children showed sustained increases in cortisol for several days after school started (max increase $48.54 \mathrm{ng} / \mathrm{mL}$ ), whereas other children showed sustained decreases (max decrease $45.53 \mathrm{ng} / \mathrm{mL}$ ). Preliminary analyses indicated that children's FMUC levels in response to the commencement of school are negatively associated with early peri-conceptional cortisol levels in mothers $\left(R^{2}=0.23\right.$; $\mathrm{p}<0.05$ ), while children's FMUC levels prior to the first day of school were not $(\mathrm{p}>0.05)$.

Conclusion: Our preliminary results provide novel information critical to understanding how maternal HPAA during early gestation may be linked to basal pre-pubertal HPAA function in children. This knowledge will aid researchers in their evaluation of the ontogeny of the HPAA and the role of variation in HPAA functioning in the etiology of a variety of negative physical and mental health outcomes seen across the lifespan.

Disclosure of Interest: None Declared.

\section{DOHaD13-1729}

Disaster-related prenatal maternal stress affects cognitive and language abilities in toddlers: the Iowa Flood Study Suzanne King ${ }^{1, *}$, Michael W. O'Hara ${ }^{2}$, David P. Laplante ${ }^{3}$

${ }^{1}$ Psychiatry, MCGILL UNIVERSITY, Montreal, Canada;

${ }^{2}$ Psychology, University of Iowa, Iowa City, United States; ${ }^{3}$ Psychosocial Research, Dougals Hospital Research Centre, Verdun, Canada

Background: Research indicates that in utero exposure to maternal stress and/or anxiety results in deficits in cognitive and language functioning $(1,2)$. We have demonstrated that in utero exposure to disaster-related prenatal maternal stress also affects cognitive and language development (3,4). Higher levels of objective hardship (i.e., disaster related events), but not subjective distress (i.e., women's psychological reaction to these events) was associated with lower cognitive and language abilities in 2 year-old toddlers. Moreover, the effect was more pronounced in toddlers exposed to the effects of the disaster early in gestation.

The goal of the present study was to replicate our previous findings by assessing the cognitive and language abilities of 30 month-old toddlers whose mothers experienced varying degrees of objective hardship and subjective distress as a result of the 2008 Iowa Floods.

Method: Maternal objective hardship and subjective distress were assessed immediately after the flood and 16 months post-flood. The toddlers' cognitive and language abilities were assessed at 30 months using the Bayley Scales of Development - $3^{\text {rd }}$ Edition (5) and the short form of the MacArthur Communicative Development Inventory (MCDI) (6), respectively. Socioeconomic status was assessed using the Hollingshead scale (7). Data was available for 104 or 122 toddlers who were exposed in utero to varying degrees of maternal objective hardship and/or subjective distress during the $1^{\text {st }}(25$ or 30$), 2^{\text {nd }}(42$ or 50$)$, or $3^{\text {rd }}(37$ or 42$)$ trimester of pregnancy, respectively for the Bayley and MCDI.

Results: Higher subjective distress (especially early in the pregnancy) and lower economic status were associated with lower Bayley scores, accounting for $16,2 \%$ of the variance of the toddlers' Bayley scores. Higher subjective distress and an objective hardship x child's sex interaction accounted for $12.9 \%$ of the variance of the toddlers' receptive language abilities. Higher subjective distress, objective hardship $\mathrm{x}$ child's sex and subjective distress $\mathrm{x}$ timing of exposure interactions, and increased current maternal general depression accounted for $20.9 \%$ of the variance of the toddlers' productive language abilities.

Conclusion: The present findings differ from those observed in Project Ice Storm, in that, maternal subjective distress, and not objective hardship, was associated with the toddlers' functioning. We believe that the nature of the disasters might explain this difference. While the ice storm affected everybody to some degree, some women in the present study may either been affected to varying levels by the flooding while 
other women may not have been affected at all. Thus, the women's subjective reactions to the flooding may have played a larger role in the present sample. In conclusion, these results support our position that increased levels of disaster-related maternal stress is associated poorer cognitive and language abilities in toddlers.

Disclosure of Interest: None Declared.

\section{DOHaD13-1737}

DNA methylation signatures of prenatal maternal exposure to a natural disaster: Project Ice Storm

Suzanne King ${ }^{1, *}$, Lei $\mathrm{Cao}^{1}$, Moshe Szyf ${ }^{2}$, David P. Laplante ${ }^{3}$

${ }^{1}$ Psychiatry; ${ }^{2}$ Pharmacology, McGill University, Montreal;

${ }^{3}$ Psychosocial Research Division, Douglas Hospital Research

Centre, Verdun, Canada

Background: Prenatal maternal stress (PNMS) predicts a wide variety of outcomes in animal and human studies. In Project Ice Storm, a study concerning the PNMS derived from a natural disaster, findings show that different sets of developmental outcomes are predicted by objective maternal exposure and/or subjective maternal distress from the storm. However, the underlying molecular mechanism involved is poorly understood. Methylation, one of the most studied epigenetic mechanisms, may be involved in the effect of PNMS on outcomes in human offspring.

Our goal was to determine the extent to which the severity of objective and/or subjective PNMS from a natural disaster explains variance in genome-wide methylation patterns in young adolescent children exposed in utero to the January 1998 Ice Storm.

Method: In June 1998, women who were pregnant during the ice storm completed questionnaires about their experiences. Objective storm-related stress was assessed using a 32-point questionnaire of hardship (Storm32) which included items about duration of power loss, house changes, financial loss, etc. Subjective stress was assessed by the Impact of Events Scale-Revised, which measures PTSD-type symptoms. The children from these families have been followed since birth. In October 2011, 36 of the children agreed to provide a blood sample from which $\mathrm{T}$ cells were isolated and DNA was extracted. Methylation profiles were measured using the Illumina $450 \mathrm{~K}$ BeadChip and pyrosequenicng.

Results: We found that the methylation profiles of 1678 CpG sites were significantly associated with objective stormrelated stress. The correlations between objective stress levels and methylation of the top 100 significant $\mathrm{CpG}$ sites were illustrated in Heatmap. Pyrosequencing results for 6 selected candidate genes were comparable with that from $450 \mathrm{~K}$ array data. The set of genes contained numerous biological processes markers. No correlation was found between genome wide methylation and maternal subjective distress.
Conclusion: This is the first investigation of genome-wide methylation profile in relation to prenatal maternal stress derived from a natural disaster. Our study provides initial support for the notion that epigenetic mechanisms play an important role in mediating the effect of in utero exposure to maternal stress on adverse health outcomes in later life.

Disclosure of Interest: None Declared.

\section{DOHaD13-1370}

Maternal prenatal depression at critical windows during pregnancy is associated with increased placental serotonin and glucocorticoid sensitivity and transfer

Rebecca M. Reynolds ${ }^{1, *}$, Anu-Katriina Pesonen ${ }^{2}$, James R. O'Reiilly $^{1}$, Soile Tuovinen ${ }^{3}$, Marius Lahti ${ }^{3}$, Eero Kajantie ${ }^{3}$, Pia Villa ${ }^{3}$, Hannele Laivuori ${ }^{3}$, Esa Hämäläinen ${ }^{3}$, Jonathan R. Seckl ${ }^{1}$, Katri Räikkönen ${ }^{3}$

${ }^{1}$ University of Edinburgh, Edinburgh, United Kingdom;

${ }^{2}$ Institute of Behavioral Sciences; ${ }^{3}$ University of Helsinki, Helsinki, Finland

Background: Maternal prenatal depression is a strong predictor of postpartum depression and increases risk of prematurity and low birthweight. Mood disorders are commonly attributed to disordered signalling by monoamines, notably serotonin, and/or glucocorticoid 'stress' hormones. We hypothesised that maternal prenatal depression would be associated with increased fetal serotonin and glucocorticoid exposure by action in placenta on key genes involved in regulating placental serotonin and glucocorticoid transfer, metabolism and/or action.

Method: Placental biopsies were obtained from 54 healthy mothers aged $32.2 \pm 5.3$ years with singleton, term pregnancies (37-42 gestational weeks) a maximum of 90 mins after vaginal or caesarean delivery, and stored in RNA-later at -20C. Placental mRNAs encoding the serotonin transporter (SERT), glucocorticoid receptor (GR), mineralocorticoid receptor (MR), and 11beta-hydroxysteroid dehydrogenase type 1 (HSD1) and 2 (HSD2), which regenerate and inactivate glucocorticoids respectively, were determined by real-time PCR. Maternal depressive symptoms were assessed at two week intervals throughout pregnancy from 12 to 38 weeks gestation using the Center of Epidemiological Studies Depression Scale. Associations between depressive symptoms throughout pregnancy and placental mRNA levels were tested using mixed random-effects models. Analyses were adjusted for maternal education, age, parity, BMI, smoking, alcohol intake, mode of delivery, time between delivery and biopsy, birthweight, gender and gestation at delivery. Ethical approval and written, informed consent were obtained.

Results: Placental GR and MR mRNAs were higher in women who were more depressed across the whole pregnancy (0.23 increase in standard deviation $[S D]$ units per each SD 
increase in mRNA level; 95\% Confidence Interval, 0.02-0.45, $\mathrm{P}=0.036$ and 0.26 SDs, $0.05-0.48, \mathrm{p}=0.016$, respectively). HSD2 and SERT did not show linear associations with depression but there was a 'threshold' effect such that in those who had at or above median HSD2 mRNA levels, depression scores tended to increase towards the end of pregnancy (0.54 SDs, 0.03-1.049, $\mathrm{P}=0.039$; interaction with time $\mathrm{P}=0.02$ ) and in those with at or above median SERT, depressive symptoms scores were higher across the whole pregnancy, though more pronounced towards the end of pregnancy (0.53 SDs, $0.04-1.03, \mathrm{P}=0.036$ ). Increased maternal depressive symptoms in first trimester only were also associated with increased placental HSD1 mRNA (1.05 SDs, 0.23-1.86, $\mathrm{p}=0.01$ ).

Conclusion: Maternal depression throughout pregnancy increases placental glucocorticoid sensitivity at term (increased receptors). Depressive symptoms in early pregnancy associate with increased placental glucocorticoid regeneration, presumably ampifying the effect of increased receptors, whilst depression in late pregnancy associates with increased placental glucocorticoid inactivation, perhaps ameliorating receptor induction, and also with increased serotonin transportation. These findings offer potential gestational-age-specific mechanisms linking maternal prenatal depression via placental biology with adverse offspring outcomes.

Disclosure of Interest: None Declared.

\section{DOHaD13-1541}

\section{Maternal perinatal stress and depression predict parent-reported food allergy in offspring in the first three years of life}

Monique Robinson ${ }^{1, *}$, Renee D. Goodwin ${ }^{2}$, Peter D. Sly ${ }^{3}$, Craig E. Pennell ${ }^{4}$, Peter Jacoby ${ }^{1}$, Stephen R. Zubrick ${ }^{1}$, John P. Newnham ${ }^{4}$, Patrick G. Holt ${ }^{1}$

${ }^{1}$ Telethon Institute for Child Health Research, The University of Western Australia, Perth, Australia; ${ }^{2}$ Mailman School of Public Health, Columbia University, New York, United States;

${ }^{3}$ Queensland Children's Medical Research Institute, University of Queensland, Brisbane; 'School of Women's and Infants'

Health, The University of Western Australia, Perth, Australia

Background: In most Western countries the last decade has seen an unprecedented increase in children being diagnosed with food allergy, particularly for common foods such as nuts, milk and egg. To date, most of the epidemiological research in this area has taken a relatively standard toxicological approach, focusing on the identification of environmental agents to which mothers are exposed via food and ambient air. An important area that has not yet been investigated in this context is the possible role of maternal stress and mood disturbance. The mother's experience of stress may lead to a greater fetal exposure to the stress hormone cortisol in utero. However, altered nutrition due to stress or depression may influence the placental enzyme system (11betaHSD2) that protects the fetus from the mother's cortisol. Amongst its many functions, cortisol is known to be a potent suppressor of many immune functions including those related to resistance to allergic sensitization, and exposure may be an important contributor towards the development of allergic disease in postnatal life. We aimed to investigate the impact of perinatal maternal stress and depressive symptoms on the incidence of parent-reported food allergy in offspring in the first three years of life.

Method: In the Western Australian Pregnancy Cohort (Raine) Study, 2,498 women provided data on exposure to stressful life events at 18 and 34 weeks' gestation, and depressive symptoms were assessed at three days postpartum. Live born children were followed up at ages one, two and three years and mothers reported whether the child suffered from allergies to any foods. Serum $\operatorname{IgE}$ data were collected at age six. Logistic regression models were used to assess relationships between prenatal stress and postnatal depression and parent-report food allergy at ages one, two or three with adjustment for maternal self-report food allergy and selected sociodemographic factors.

Results: By the age of three, 220 children were reported to have an allergy to one or more foods. Mothers who reported exposure to three or more stressful life events during pregnancy were more likely to report that their child had a food allergy $(\mathrm{OR}=1.72,95 \% \mathrm{CI}=1.08,2.73)$. In further analyses, chronic rather than acute stress was significantly associated with increased report of food allergy $(\mathrm{OR}=1.16$, $95 \% \mathrm{CI}=1.03,1.32)$, as was stress in the first four months of pregnancy $(\mathrm{OR}=1.20,95 \% \mathrm{CI}=1.05,1.38)$ as opposed to later in pregnancy. Postpartum depressive symptoms also significantly predicted parent-reported food allergy by three years $(\mathrm{OR}=1.49,95 \% \mathrm{CI}=1.01,2.20)$. Results are compared with serum IgE data confirming food allergy at age six. Conclusion: Maternal stress and depression in the perinatal period were significantly associated with a higher risk for parent-report food allergy in the first three years of life. While this supports existing theory as to the biological programming of stress and mood on atopic disease in childhood, parent-report can often over-estimate the incidence of food allergy in children therefore further research is needed to validate these findings.

Disclosure of Interest: None Declared.

\section{DOHaD13-1233}

Maternal stress, anxiety and depression increase the risk of small for gestational age

Ali S. Khashan ${ }^{1, *}$, Claire Everard ${ }^{1}$, Lesley M. McCowan ${ }^{2}$, Gustaaf Dekker ${ }^{3}$, Rona Moss-Morris ${ }^{4}$, Philip N. Baker ${ }^{5}$, Lucilla Poston $^{6}$, James J. Walker ${ }^{7}$, Louise C. Kenny ${ }^{1}$ and SCOPE Consortium

${ }^{1}$ The Irish Centre for Fetal and Neonatal Transalational Research (INFANT), University College Cork, Cork, Ireland; ${ }^{2}$ Department of Obstetrics and Gynaecology, University of 
Auckland, Auckland, New Zealand; ${ }^{3}$ Department of Obstetrics and Gynaecology, University of Adelaide, Adelaide, Australia; ${ }^{4} 4$ Health Psychology Section, Psychology Department, Institute of Psychiatry, King's College, London, United Kingdom; ${ }^{5}$ National Centre for Growth \& Development, University of Auckland, Auckland, New Zealand; ' ${ }^{6}$ Division of Women's Health, Women's Health Academic Centre, King's College, London; ${ }^{7}$ Department of Obstetrics and Gynaecology, St James University Hospital, Leeds, United Kingdom

Background: Several studies have investigated the impact of prenatal stress on the risk of small for gestational age (SGA) and preterm birth but the results are conflicting ${ }^{1}$. We aimed to examine the effect of prenatal stress, anxiety and depression on the risk of SGA and preterm birth and whether any observed associations are dependent on infant sex.

Method: The study population consisted of 5,606 healthy nulliparous pregnant women who participated in the international prospective Screening for Pregnancy Endpoints Study (SCOPE). Women completed the Perceived Stress Scale (PSS), the Short-form Trait-Anxiety Inventory (STAI) and the Edinburgh Postnatal Depression Scale (EPDS) at $15 \pm 1$ and $20 \pm 1$ weeks' gestation. Fetal weight was estimated using data from the ultrasound examination at $20 \pm 1$ weeks' gestation. SGA was defined as birthweight below the $10^{\text {th }}$ customised percentile. Preterm birth was defined as spontaneous delivery before 37 week's gestation. Logistic regression was used for data analysis adjusting for several potential confounders including maternal age, body mass index, physical exercise, socio-economic status, education and ethnicity.

Results: The risk of SGA was increased in relation to mild $(\mathrm{aOR}=1.35[1.07-1.71])$, moderate $(\mathrm{aOR}=1.26[1.06-1.49])$, high $(\mathrm{aOR}=1.45[1.08-1.95])$ and very high stress scores $(\mathrm{aOR}=1.56[1.03-2.37])$; very high anxiety score $(\mathrm{aOR}=$ $1.45[1.13-1.86])$; and very high depression score $(\mathrm{aOR}=$ $1.14[1.05-1.24])$ at $20 \pm 1$ weeks' gestation. Sensitivity analyses showed that very high anxiety and very high depression increases the risk of SGA in males but not females while stress increases the risk of SGA in males and females. Excluding infants who were below the $10^{\text {th }}$ percentile of the ultrasound estimated fetal weight or born preterm did not change the SGA results. Furthermore, excluding offspring of women who had pre-eclampsia or were treated with steroids for threatened preterm labour did not change the SGA results. There was very little evidence for an association between prenatal stress, depression or anxiety at 15 or 20 weeks' gestation and the risk of spontaneous preterm birth.

Conclusion: The present findings suggest that prenatal stress, anxiety and depression measured at 20 weeks' gestation increase the risk of SGA but not spontaneous preterm birth. The observed associations were strongest in male infants and independent of the estimated fetal weight in mid pregnancy. These results are consistent with findings from populationbased studies on bereavenment and SGA. The specific finding is consistent with a recent study on bereavement and risk of infant mortality ${ }^{2}$. Animal studies suggested that maternal stress may increase the risk of SGA in the offspring and that the association may be sex-specific. However, the mechanistic line between the experience of stress and risk of SGA is still unclear although glucocorticoids are potential mediators ${ }^{3}$.

\section{References}

1) Khashan ei al., Psychosomatic Medicine 2008; 70: 688-694.

2) Class et al., Psychological Science 2013; Advanced online publication. doi: 10.1177/0956797612468010

3) Kapoor et al., J Physiology 2006; 572: 31-44

Disclosure of Interest: None Declared.

\section{DOHaD13-1616}

Maternal stress during pregnancy, in both first generation females born of normal Birth Weight and those born small, programs second generation male offspring hypertension

Mary E. Wlodek ${ }^{1, *}$, Andrew J. Jefferies ${ }^{1}$, Kristina Avevska ${ }^{1}$, Camille Vickneswaran ${ }^{1}$, Jean N. Cheong ${ }^{1}$, Alanna Hanvey ${ }^{1}$, Erica Gerace ${ }^{1}$, Karen M. Moritz ${ }^{2}$

${ }^{1}$ Physiology, The University of Melbourne, Parkville; ${ }^{2}$ Biomedical Sciences, University of Queensland, St. Lucia, Australia

Background: Being born small for gestational age increases the risk of adult cardiovascular disease later in life. Males exhibit a more severe cardiovascular phenotype than females. Increasing evidence suggests that these disease risks are not limited to the first, directly exposed generation (F1) but can be transmitted to the next generation (F2). Stress during pregnancy adversely impacts on fetal growth and development. We characterised the cardiovascular phenotype of F2 male offspring born to normally grown and growth restricted (F1) mothers and assessed the impact of maternal stress during late pregnancy.

Method: Late gestation uteroplacental insufficiency was induced on day 18 of pregnancy by bilateral uterine vessel ligation (Restricted) or sham (Control) surgery in F0 females. F1 female offspring (Control and Restricted) were mated with a normal male Wistar Kyoto rat and randomly allocated to Unstressed or Stressed groups. Physiological stressors (24h metabolic cage, tail cuff blood pressure, glucose tolerance test) were introduced during late pregnancy of $\mathrm{F} 1$ females in the Stressed group while their Unstressed counterparts were unhandled. Body weights were measured in F2 males from birth to 12 months, and blood pressure by tail cuff measured at 6, 9 and 12 months. Body, heart and kidney weights were measured at 12 months. All data were analysed by 2-way ANOVA.

Results: F2 males born to mothers exposed to maternal stress had reduced birth weight $(-4-6 \%, p<0.05)$. F2 Stressed Control males demonstrated early postnatal accelerated growth $(p<0.05)$ while Stressed Restricted males experienced late postnatal accelerated growth $(p<0.05)$. Body 
weight was not different across all groups from 5 weeks to 12 months of age. There were no differences in absolute nor relative heart, left ventricle or kidney weights at 12 months. Restricted Unstressed males had elevated blood pressure at all ages (6, 9 and 12 months) compared to Control Unstressed $(+8 \mathrm{mmHg} ; p<0.05)$. Control male offspring exposed to maternal stress during pregnancy had elevated blood pressure at 6 and 9 months compared to Control Unstressed $(+13 \mathrm{mmHg}$; $p<0.05)$. This maternal stress pregnancy response on Control males resulted in an elevated blood pressure that was not different to the high blood pressure of male offspring born to Unstressed mothers who were born small. Both Control and Restricted offspring exposed to maternal stress during pregnancy experienced high blood pressure (compared to Unstressed) at 12 months $(+7 \mathrm{mmHg} ; p<0.05)$.

Conclusion: Mothers, born of normal birth weight, but exposed to stress during late pregnancy produced male offspring who developed adult hypertension in early adulthood. This degree of hypertension was similar to male offspring of mothers who were born small but not exposed to maternal stress during pregnancy. Offspring of mothers born small developed high blood pressure later in life (at 12 months) when exposed to maternal stress during pregnancy. The different postnatal growth profiles for F2 Control and F2 Restricted offspring from Stressed pregnancies may predispose adults to differential cardiovascular responses to later life second-hits.

Disclosure of Interest: None Declared.

\section{DOHaD13-1524}

\section{Maternal stress during pregnancy programs glucose intolerance in second generation male offspring and exacerbates pancreatic deficits in offspring of females born small}

Jean N. Cheong ${ }^{1, *}$, Melanie Tran ${ }^{1}$, Andrew J. Jefferies ${ }^{1}$, Karen M. Moritz ${ }^{2}$, Mary E. Wlodek ${ }^{1}$

${ }^{1}$ Physiology, The University of Melbourne, Parkville; ${ }^{2}$ Biomedical Sciences, University of Queensland, St. Lucia, Australia

Background: Intrauterine growth restriction increases the risk of adult metabolic diseases, with males exhibiting a more severe phenotype than females. Evidence suggests that these disease risks are not limited to the first, directly exposed generation (F1) but can be transmitted to the next generation (F2). Stress is increasingly prevalent in today's society and mothers exposed to stress during pregnancy adversely impacts upon fetal growth and development. We aimed to characterise the metabolic phenotype of F2 male offspring born to growth restricted F1 mothers and determine if maternal stress during late pregnancy will exacerbate the phenotype.

Method: Late gestation uteroplacental insufficiency was induced on day 18 of pregnancy by bilateral uterine vessel ligation (Restricted) or sham (Control) surgery in F0 females.
F1 female offspring (Control and Restricted) were mated with a normal male Wistar Kyoto rat and randomly allocated to Unstressed or Stressed groups. Physiological stressors (24h metabolic cage, tail cuff blood pressure, glucose tolerance test) were introduced during late pregnancy of F1 females in the Stressed group while their Unstressed counterparts were unhandled. Body weights were measured in F2 males from birth to 6 months, and metabolic function characterised by conducting a fasting glucose tolerance test and insulin challenge at 6 months. Pancreatic $\beta$-cell and islet mass were quantified following post mortem. All data were analysed by two-way ANOVA ( $\mathrm{n}=6-10$ per group).

Results: F2 males born to mothers exposed to maternal stress had reduced birth weight $(-4-6 \%, p<0.05)$. F2 Stressed Control males demonstrated early postnatal accelerated growth $(p<0.05)$ while Stressed Restricted males experienced late postnatal accelerated growth $(p<0.05)$. Body weights were not different across all groups from 5 weeks to 6 months of age. At 6 months, both Control and Restricted F2 males born to Stressed mothers developed impaired glucose tolerance $(p<0.05)$ when compared with offspring of Unstressed mothers. Insulin sensitivity and secretion were not altered. Compared to Control counterparts, Unstressed Restricted males had decreased $\beta$-cell and islet mass $(-17-21 \%, p<0.05)$ at 6 months, and these deficits were exacerbated in Stressed Restricted males ( $-40-42 \%$, $p<0.05)$. There were no differences in pancreas weights.

Conclusion: Regardless of maternal birth weight, mothers exposed to modest stress during late pregnancy reduced birth weight and programmed metabolic dysfunction in F2 male offspring. Early catch up growth in Stressed Control males prevented pancreatic deficits but not glucose intolerance. The different postnatal growth trajectories and pancreatic phenotypes for F2 Control and Restricted males from Stressed pregnancies may predispose Restricted offspring to differential responses to later life second-hits such as aging and an unhealthy diet. These second-hits have the potential to unmask metabolic dysfunction in Unstressed Control males presenting with less severe pancreatic deficits. Limiting exposure to stress during pregnancy may reduce the risk of offspring developing metabolic dysfunction later in life.

Disclosure of Interest: None Declared.

\section{DOHaD13-1483}

Pre- and post-partum depression in women living in slums in Mumbai, India

Meera Gandhi ${ }^{1, *}$, Sirazul Sahariah ${ }^{1}$, Ella Marley-Zagar ${ }^{2}$, Harshad Sane ${ }^{1}$, Sunita Borhade ${ }^{1}$, Patsy Coakley ${ }^{2}$,

Vanessa Cox ${ }^{2}$, Ramesh Potdar ${ }^{1}$, Caroline Fall ${ }^{2}$

${ }^{1}$ Centre for the Study of Social Change, Mumbai, India;

${ }^{2}$ Lifecourse Epidemiology Unit, MRC, University of

Southampton, Southampton, United Kingdom 
Background: During pregnancy women are susceptible to emotional turbulence. Prospective studies in India have detected depression in $16-23 \%$ of pregnant women, persisting 6 months after childbirth in $11-14 \%$. We studied the prevalence of pre- and post-partum depression among women participating in a randomised controlled trial in Mumbai slums. 6,513 women were registered in the Mumbai Maternal Nutrition Project, in which micronutrient-rich food supplements were given for at least 3 months prior to conception and throughout pregnancy. Of these, 2,310 became pregnant and 1,976 delivered live singleton newborns.

Objectives: To assess:

-The prevalence of depression pre- and post-delivery -The role of socio-economic factors in depression.

Method: 743 women were studied at the $3^{\text {rd }}$ trimester ultrasound visit, and 480 were studied 30-60 days postdelivery at home. Interviews were conducted in private by a senior social worker and counselor, or by project assistants under her supervision. Depression was assessed using the Edinburgh Post Natal Depression Scale (EPDS) and scores were classified as Normal: $<8$ points; 'Baby Blues': $8-12$ points; Possible Depression: 13-14 points; or Depression: $\geq 15$ points. We examined the following factors as predictors of possible depression and depression using multiple linear regression: the child's sex and the woman's trial allocation, age, parity, education, religion, occupation, obstetric complications, anaemia, sleep problems and socio-economic factors including financial worries, family support, personal or family history of depression, partner-related problems (eg. domestic violence) and unexpected adverse events during pregnancy and post-delivery. Results: Of 743 women interviewed in the $3^{\text {rd }}$ trimester 99 (13\%) had possible depression and 141 (19\%) had depression. Of 480 women interviewed post-delivery 45 (9\%) had possible depression and 71 (15\%) had depression. Of 366 women interviewed at both times, the equivalent figures were: $3^{\text {rd }}$ trimester: $48(13 \%)$ possible depression and $66(18 \%)$ depression, and post-natally: $38(10 \%)$ possible depression and $55(15 \%)$ depression. 44 women who had depression in the $3^{\text {rd }}$ trimester were no longer depressed post-natally, while 33 women who were not depressed pre-natally had post-natal depression. Factors related to risk of possible depression or depression were (pre-natally) partner-related problems and (both pre- and post-natally) lack of family support, financial worries and perceived inadequate sleep (all $\mathrm{p}<0.01$ ). The other factors examined, including trial allocation, were unrelated to depression risk.

Conclusion: The prevalence of depressive symptoms is high among pregnant and post-partum women in this population, and higher pre-natally than post delivery. Lack of family support and financial worries related to poverty were key social factors predicting a higher risk. Supplementation with micronutrient-rich foods from the pre-conception period did not reduce the risk. Further work will assess associations of maternal depression with birth outcomes and child growth.
Acknowledgements: The project was funded by the Wellcome Trust and Medical Research Council, UK, ICICI Social Initiatives Group, Mumbai and Parthenon Trust, Switzerland.

Disclosure of Interest: None Declared.

\section{DOHaD13-1597}

Preconception maternal exposure to bereavement stress increases the risk of infant and childhood death: a Danish population-based study

Quetzal Q. Class ${ }^{1}$, Preben B. Mortensen ${ }^{2}$, Marianne G. Pedersen $^{2}$, Tine B. Henriksen ${ }^{3}$, Christina Dalman ${ }^{4}$, Brian M. D'Onofrio $^{1}$, Ali S. Khashan ${ }^{5, *}$

${ }^{1}$ Department of Psychological and Brain Sciences, Indiana University, Bloomington, United States; ${ }^{2}$ National Center for Register-based Research; ${ }^{3}$ Perinatal Epidemiology Research Unit, Department of Paediatrics, Aarhus University, Aarhus, Denmark; ${ }^{4}$ Department of Public Health Sciences, Karolinska Institute, Stockholm, Sweden; ${ }^{5}$ The Irish Centre for Fetal and Neonatal Transalational Research (INFANT), University College Cork, Cork, Ireland

Background: Neonatal, infant, and early childhood death remain major global concerns. The Developmental Origins of Health and Disease Hypothesis suggests that early maternal stress exposure may be associated with increased risk for early offspring mortality. We aimed to examine the effect of preconception and prenatal exposure to bereavement on the risk of neonatal, infant, and child mortality using populationbased data from Denmark.

Method: We used a Danish population-based sample of offspring $(\mathrm{N}=1,865,454)$ born between 1979 and 2009 to predict odds of neonatal (0-28 days), post-neonatal infant (29-365 days), and early childhood (29 days to 5 years) mortality following maternal bereavement stress in the preconception (6-0 months before conception) and prenatal (between conception and birth) periods. Bereavement stress was defined as death of a parent or sibling of the mother. We used logistic regression for the analyses of neonatal and infant mortality and log-linear Poisson regression with aggregated person-years for the analysis of child mortality. All models controlled for infant sex, year of birth, birth order, maternal and paternal age and country of origin, as well as maternal diabetes, hypertension, cardiovascular disease, and renal disease. Sensitivity analyses tested the robustness of associations by examining moderation by adverse birth outcomes and timing specificity.

Results: We identified 12,201 offspring that died between birth and age 5 years (6,541 between 0-28 days; 3,528 between 29-265 days of life; and 2132 between $1-5$ years of age). The association between preconception bereavement stress and neonatal mortality (OR $=1.14,95 \%$ CI: $0.86-1.53)$ was greatly attenuated when controlling for gestational age and 
being small for gestational age $(\mathrm{OR}=1.05,95 \% \mathrm{CI}$ : $0.74-1.48)$. Odds of infant mortality were independently increased following preconception stress exposure (OR $=1.31,95 \% \mathrm{CI}$ : 0.91-1.90). This association was primarily found in male offspring $(\mathrm{OR}=$ 1.60, 95\% CI: 1.02-2.49), though sample size may have limited our ability to detect an effect in female offspring. Similarly, there was a significant positive association, independent of gestational age and birth weight, between preconception bereavement stress and risk of child mortality in males $(\mathrm{RR}=1.58$; $95 \% \mathrm{CI}$ : 1.11-2.25). Associations were timing-specific (6 months prior to conception only) and consistent throughout sensitivity analyses. Prenatal stress, however, did not increase the risk of neonatal, infant, or child mortality.

Conclusion: Results suggest that the preconception period may be a sensitive developmental period incurring risk for infant and childhood mortality. The association is particular to the 6 month period immediately prior to conception and not due to increased risk for adverse birth outcomes or familial risk for death. These findings are consistent with and extend previous findings in the Swedish population (Class et al., 2013). Preconception nutritional insufficiency or insult during early organogenesis may be mechanisms responsible for the associations identified, though more research is needed.

\section{Reference}

1. Class et al., Psychological Science 2013. Epub. doi: 10.1177/ 0956797612468010

Disclosure of Interest: None Declared.

\section{DOHaD13-1614}

\section{Prenatal maternal exposure to a natural disaster predicts more masculine 2D:4D finger length ratio in girls: Project Ice Storm}

Suzanne King ${ }^{1, *}$, Peter L. Hurd ${ }^{2}$, Aihua Liu $^{3}$, David P. Laplante ${ }^{3}$

${ }^{1}$ Psychiatry, McGill University, Montreal; ${ }^{2}$ Psychology,

University of Alberta, Edmonton; ${ }^{3}$ Psychosocial Research,

Douglas Institute, Montreal, Canada

Background: Prenatal maternal stress appears to have masculinizing effects on the fetus in some systems and feminizing effects on others. Most experimental research is conducted in animals since one cannot ethically stress pregnant women. We capitalized on a natural disaster in January 1998 in Quebec, Canada to study the effects of stress exposure in pregnant women on their unborn children. The ratio of the lengths of the 2 nd and $4^{\text {th }}$ fingers (2D:4D ratio), a sexually dimorphic trait, reportedly reflects in utero testosterone exposure and may be sensitive to prenatal stress.

The purpose of the current study was to determine the extent to which the pregnant women's objective degree of exposure to the 1998 Quebec Ice Storm crisis, and their level of subjective distress about the storm, influenced their children's 2D:4D ratio.
Method: Subjects were 115 French-Canadian women who were pregnant during the ice storm, or conceived within 3 months of the storm; their children ( 58 girls, 57 boys) at age $5 \frac{1}{2}$ years; a matched comparison group of 47 girls and 50 boys; and 111 adult French Canadians (60 women, 51 men). Women were recruited 4-5 months after the ice storm and completed a questionnaire about objective stress exposure (Storm32) and their subjective distress about the storm (Impact of Event Scale - Revised; IES-R). Finger lengths of children and comparison adults were measured with digital calipers.

Results: Prenatal ice storm exposed girls had more masculine 2D:4D than their male peers $(\mathrm{p}<.05)$ while comparison children and adults had ratios in the expected direction. Ice storm girls had significantly more masculine 2D:4D than comparison girls; ice storm boys had slightly more feminine ratios than comparison boys. Timing in gestation of the stress exposure had effects for both boys and girls.

Conclusion: Prenatal maternal stress exposure, randomly distributed by a natural disaster, influences a sexually dimorphic trait in children, resulting in an overall masculinization of girls. Effects of timing and of objective or subjective stress differed by sex.

Disclosure of Interest: None Declared.

\section{DOHaD13-1731}

Prenatal maternal stress due to a natural disaster predicts birth weight and length: the Iowa Flood Study

Suzanne King ${ }^{1 *}$, David P. Laplante ${ }^{2}$, Kelsey N. Dancause ${ }^{2}$, Kimberly J. Nylen ${ }^{3}$, Michael W. O'Hara ${ }^{4}$

${ }^{1}$ Psychiatry, McGill University, Montreal; ${ }^{2}$ Psychosocial Research, Dougals Hospital Research Centre, Verdun, Canada; ${ }^{3}$ Psychology, Idaho State University, Pocatello, Idaho;

${ }^{4}$ Psychology, University of Iowa, Iowa City, Iowa, United States

Background: Prenatal maternal stress (PNMS) is increasingly accepted as a risk factor for adverse birth outcomes. These effects, which are largely thought to reflect the impact of maternal stress hormones on the developing fetus, vary by timing of exposure and newborn sex. However, our understanding remains limited because of the challenges of designing human studies of PNMS.

We have investigated PNMS by designing studies of women exposed to natural disasters during pregnancy. Natural disasters provide excellent models of PNMS because the exact timing of the stressor can be identified, and exposure tends to be randomly distributed with regard to psychological, medical, and other characteristics. Our goal was to determine whether and how the timing and severity of exposure to prenatal maternal stress (PNMS) due to a natural disaster influenced gestational age and growth measures at birth.

Method: We assessed objective hardship and subjective distress among 144 women exposed to severe flooding in 
the state of Iowa in June 2008 during or shortly before pregnancy, and assessed gestational age and growth measures (birth weight, birth length, head circumference, and growth ratios) for their infants. We analyzed associations between objective and subjective PNMS levels and birth outcomes, controlling for potential confounding variables.

Results: Later timing of exposure to the floods during gestation predicted predicted heavier birth weights. We observed interactions between PNMS and infant sex: more severe PNMS predicted shorter birth lengths among girls, but longer birth lengths among boys. Neither timing nor severity of PNMS exposure was associated with gestational age, ponderal index, head circumference, or head circumference to birth length ratio. Conclusion: Timing of PNMS from a natural disaster during pregnancy affected birth weight, and severity of PNMS affected birth length with variations by infant sex. These effects were independent of other maternal characteristics, suggesting that PNMS is an independent predictor of birth outcomes. Differences between boys and girls might reflect sex-specific effects of hormones such as testosterone on fetal growth. More research is necessary to clarify the effects of exposure to moderately stressful events on birth outcomes, and the underlying mechanisms.

Disclosure of Interest: None Declared.

\section{DOHaD13-1555}

Prenatal and postnatal maternal anxiety impact early and late cognitive processes detected by event-related potentials

Suet Chian Sam ${ }^{1, *}$, Diaz Adi Utama ${ }^{2}$, Hui Jun Chong ${ }^{1}$, Birit Broekman ${ }^{1,2}$, Helen Chen ${ }^{3}$, Jenny Richmond ${ }^{4}$, Seang Mei Saw ${ }^{5}$, Kenneth Kwek ${ }^{6}$, Peter Gluckman ${ }^{1,7}$, Yap Seng Chong ${ }^{8}$, Keith Godfrey ${ }^{9}$, Michael J. Meaney ${ }^{1,10}$, Anqi Qiu ${ }^{11}$, Anne Rifkin-Graboi ${ }^{1}$

${ }^{1}$ Singapore Institute for Clinical Sciences (SICS), Agency for Science, Technology and Research (A*STAR), ${ }^{2}$ Department of Paediatrics,

Yong Loo Lin School of Medicine, National University of Singapore, ${ }^{3}$ Department of Psychological Medicine (Mental Wellness Service), KK Women's and Children's Hospital (KKH), Singapore, Singapore, ${ }^{4}$ School of Psychology, University of New South Wales, New South Wales, Australia, ${ }^{5}$ Saw Swee Hock School of Public Health, National University of Singapore, ${ }^{6}$ Department of Maternal Fetal Medicine, KK Women's and Children's Hospital (KKH), Singapore, Singapore, ${ }^{7}$ Liggins Institute, University of Auckland, Auckland, New Zealand, ${ }^{8}$ Department of Obstetrics \& Gynaecology, Yong Loo Lin School of Medicine, National University of Singapore, National University Health System, Singapore, Singapore, ${ }^{9}$ MRC Lifecourse Epidemiology Unit \& NIHR Southampton Biomedical Research Centre, University of Southampton \& University Hospital Southampton NHS Foundation Trust, Southampton, United Kingdom,

${ }^{10}$ Department of Psychiatry and Neurology \& Neurosurgery, Douglas Mental Health University Institute, McGill University, Montreal, Canada, ${ }^{11}$ Department of Bioengineering, National University of Singapore, Singapore, Singapore
Background: Although maternal anxiety is a known risk for the development of anxiety in children, the nature of the association remains unclear. For example, the influence of maternal anxiety may operate through physical changes in the uterine environment, as well as alterations in parenting behavior. Moreover, the majority of studies examining endophenotypes assess children, rather than young infants. Unfortunately, this dilutes the ability to observe potential early life risks.

Method: Six month-old infants free of pregnancy or birth complications provided usable data while taking part in a passive auditory oddball paradigm. Maternal anxiety scores were rated using the Spielberger State Trait Anxiety (STAI) scale for both prenatal (at 26 weeks gestation) and postnatal (3 months following the birth). 87 and 71 data were collected for prenatal and postnatal anxiety measures respectively. During this paradigm event-related potentials were recorded via a 128 channel system. Four electrophysiological components were identified. In particular, we examined the influence of maternal anxiety upon components occurring earlier $(0-210 \mathrm{msec}$, Early Negativity- EN; $120-450 \mathrm{msec}$, Early Positivity-EP), and later (250-550 msec, Late Negativity- LN; $375-700 \mathrm{msec}$, Late Positivity- LP) Components occurring shortly after stimulus onset are thought to reflect earlier stages of information processing like pre-attention, with later processes indicating more complex processes such as memory. Past research suggests anxiety may be linked to hemispheric and regional asymmetries. Therefore, in the current analyses we assess the amplitude and latency of the electrophysiological response stemming from the region (i.e., right frontal) that is likely most sensitive to the potential effects of maternal mood.

Results: While both pre- and post-natal anxiety impact infant electrophysiology, the timing of the insult (i.e., preversus post-natal) influences the stage of affected processing (e.g., attention versus memory updating). Preliminary analysis suggests that prenatal anxiety predicted an increase in the firing speed of earlier component, EP $(\beta=-0.209$, $\left.\Delta \mathrm{R}^{2}=0.044, \mathrm{p}=0.052\right)$. In contrast, postnatal anxiety predicted a shorter latency of later component, LP $\left(\beta=-0.919, \Delta R^{2}=0.065, p=0.032\right)$. Consistent with past research, in both cases post-hoc analyses suggest that the increased firing speed occurs specifically when infants hear the infrequent oddball sound. This may suggest that the timing of the insult impacts differing cognitive resources associated with the processing of superfluous information.

Conclusion: These findings complement work with older children suggesting that maternal anxiety influences cognitive processes important to learning, memory, and the development of anxiety within the offspring, and underscore the importance of screening for maternal mental health during pregnancy and the early postnatal period.

Disclosure of Interest: S. C. Sam: None Declared, D. Adi Utama: None Declared, H. J. Chong: None Declared, B. Broekman: None Declared, H. Chen: None Declared, J. Richmond: None Declared, S. M. Saw: None Declared, 
K. Kwek: None Declared, P. Gluckman: None Declared, Y. S. Chong: None Declared, K. Godfrey: None Declared, M. Meaney Grant/Research support from: Abbott Nutrition and Translational Clinical Research (TCR) grant. A. Qiu: None Declared, A. Rifkin-Graboi: None Declared.

\section{DOHaD13-1365}

The effect of lifestyle counseling as prevention of gestational diabetes mellitus: a cluster-randomized controlled trial

Shuang Wang ${ }^{1, *}$, Jingmei Ma ${ }^{1}$, Huixia Yang ${ }^{1}$

${ }^{1} O B G Y N$, Peking University 1st Hospital, Beijing, China

Background: Hyperglycemia during pregnancy, as one of common pregnancy complications, has great influences on series of long-term issues, including women developing type 2 diabetes after pregnancy, and high risk of non-communicable diseases (NCDs) including metabolism syndrome in their offspring' adult period, which has been proved by a lot of evidence from epidemic and animal models. We try to examine whether gestational diabetes mellitus(GDM) can be prevented by lifestyle counseling in pregnant women at high risk of GDM.

Method: We conducted a cluster-randomized trial in the Department of Obstetrics and Gynecology of First Hospital of Peking University since September 2012, where 272 women with at least one GDM risk factor were included before 8 wk gestation and had finished their OGTT at 24-28 wk gestation by April, 19th, 2013. High risk-interference group (HR-1, $\mathrm{n}=134$ ) were given not only routine obstetrical examination counseling but also additional lifestyle, while High risk-noninterference group $(\mathrm{HR}-0, \mathrm{n}=138)$ were given routine obstetrical examination, and another group with no risk factors (NHR, $\mathrm{n}=155$ ) were treated as the same as HR-0 group.

Results: (1) No differences between HR-1 group and HR-0 group at age (31.01 \pm 3.80 vs. $30.27 \pm 3.64, P=0.087)$, family history of DM $[49(36.30 \%)$ vs. $51(36.96 \%)$, $P=0.947]$, body weight before pregnancy $(60.26 \pm 9.73 \mathrm{~kg}$ vs.61.36 $\pm 10.40 \mathrm{~kg}, P=0.371)$, BMI before pregnancy $\left(22.95 \pm 3.65 \mathrm{~kg} / \mathrm{m}^{2} \quad\right.$ vs. $\left.23.06 \pm 3.63 \mathrm{~kg} / \mathrm{m}^{2}, \quad P=0.798\right)$, FBG during early pregnancy $(4.95 \pm 0.40 \mathrm{mmol} / \mathrm{L}$ vs. $4.93 \pm 0.39 \mathrm{mmol} / \mathrm{L}, P=0.683)$, blood lipid during early pregnancy [triglyceride $(1.03 \pm 0.55 \mathrm{mmol} / \mathrm{L}$ vs. $0.95 \pm 0.44$ $\mathrm{mmol} / \mathrm{L}, P=0.163)$, cholesterol $(4.21 \pm 0.79 \mathrm{mmol} / \mathrm{L}$ vs. $4.16 \pm 0.83 \mathrm{mmol} / \mathrm{L}, P=0.621)$, HDL $(1.36 \pm 0.31 \mathrm{mmol} / \mathrm{L}$, $1.38 \pm 0.34 \mathrm{mmol} / \mathrm{L}, P=0.667) \mathrm{LDL}(2.31 \pm 0.67 \mathrm{mmol} / \mathrm{L}$ vs. $2.29 \pm 0.77 \mathrm{mmol} / \mathrm{L}, P=0.779)]$, FINS during early pregnancy $(7.74 \pm 4.75 \mathrm{ng} / \mathrm{ml}$ vs. $7.25 \pm 4.45 \mathrm{ng} / \mathrm{ml}, \quad P=0.384)$ and HOMA-IR $(1.74 \pm 1.19$ vs. $1.63 \pm 1.15, P=0.451)$. (2) Positive rate of GDM for HR-1 group 17.16\% (23/134), HR-0 group $23.91 \%$ (33/138), NHR group 11.61\% (18/155); HR-1 was lower than HR-0, and higher than NHR at the sametime, but there were no significant differences $(P=0.168, P=0.177)$, while HR-0 was significantly higher than $\operatorname{NHR}(P=0.006)$.
(3) Positive rate of GDM for those FBG $\geqslant 5.1 \mathrm{mmol} / \mathrm{L}$ during early pregnancy: HR-1 group $(11 / 36,30.55 \%)$ was lower than HR-0 group $(17 / 37,45.95 \%)$, but there was no significant difference $(P=0.076)$. (4) Compared with normal pregnant women, those who were diagnosed as GDM had significantly higher BMI before pregnancy $\left(23.75 \pm 3.22 \mathrm{~kg} / \mathrm{m}^{2}\right.$ vs. $\left.22.00 \pm 3.10 \mathrm{~kg} / \mathrm{m}^{2}, P<0.001\right)$, FBG during early pregnancy $(5.13 \pm 0.46 \mathrm{mmol} / \mathrm{L}$ vs. $4.89 \pm 0.33 \mathrm{mmol} / \mathrm{L}, P<0.001)$, triglyceride during early pregnancy $(1.20 \pm 0.62 \mathrm{mmol} / \mathrm{L}$ vs. $0.88 \pm 0.43 \mathrm{mmol} / \mathrm{L}, P<0.001)$, FINS during early pregnancy $(9.59 \pm 4.64 \mathrm{ng} / \mathrm{ml}$ vs.6.48 $\pm 3.87 \mathrm{ng} / \mathrm{ml}, \quad P<0.001)$, HOMA-IR $(2.24 \pm 1.28$ vs.1.43 $\pm 0.93, P<0.001)$, weight gain during early pregnancy $(2.01 \pm 1.97 \mathrm{~kg}$ vs. $1.27 \pm 2.21 \mathrm{~kg}$, $P=0.007)$ and weight gain during mid-term pregnancy $(6.18 \pm 2.32 \mathrm{~kg}$ vs. $5.59 \pm 2.01 \mathrm{~kg}, P=0.026)$.

Conclusion: The lifestyle counseling may decrease the positive rate of GDM in HR-1 group comparing with HR-0 group.

Disclosure of Interest: None Declared.

\section{DOHaD13-1106}

The foetal flaw in perinatal care: how best to engage women in intergenerational obesity prevention?

Lydia Hearn $^{1,2, *}$ and Chilld Health Promotion Research Centre

${ }^{1}$ School of Exercise and Health Science, Edith Cowan University;

${ }^{2}$ School of Dentistry, University of Western Australia, Perth, Australia

Background: Maternal overweight and obesity are costly challenges for obstetric care. International evidence highlights the close link between excessive weight gain during pregnancy and postpartum years with detrimental impacts on mother and child health. Yet, despite the limited capacity of perinatal health care workers to provide healthy lifestyle counselling, current research indicates they could play an active role in guiding today's internet savvy childbearing women to the use of appropriate 'quality-assured' online resources. Although there are few rigorous evaluations of online healthy lifestyle support interventions with perinatal women, literature on the effects of internet-based interventions on physical activity and dietary behaviour in wider populations of women is growing, with systematic reviews suggesting promising effects when self-assessment, goal-setting and tailored advice are used, and online mobile methods of communication, such as Smartphone applications and SMS, are employed. Using the example of Western Australia, this presentation will outline the process involved in the selection, implementation and reach of a statewide intervention aimed at offering a convenient option for primary health care providers to address service and geographical barriers to healthy lifestyle promotion.

Method: Consultations were conducted with senior health policy makers, as were focus groups and interviews with 116 perinatal mothers and 76 perinatal care providers, to 
determine what information was needed, in what form, and how best it should be presented.

Results: The outcome was the launch of a clinically-endorsed website and app that primary health care providers could officially recommend to women. Preliminary 6 month findings indicate average sign up to the app represents $10.5 \%$ of all and $26 \%$ of first pregnancies and reflect regional distribution of births. App self-assessments were completed at a rate of 259 per week, with an average of 4.2 assessments per person and one quarter using the weight tracker. Website views per week for lifestyle information averaged 570, with most users focusing on nutrition (35-39\%) and weight (28-36\%).

Conclusion: Engagement of service policy makers and practitioners in the promotion of personalised, clinicallyapproved online resources represents a low-cost, sustainable approach for the promotion of consistent antenatal and postnatal messages delivered equitably across geographical and socio-economic groups to improve nutrition, fitness and weight using practical activities tailored to the developmental stage of their children.

Disclosure of Interest: None Declared.

\section{DOHaD13-1727}

\section{Water and ice: using natural disasters to study prenatal} maternal stress

Suzanne King ${ }^{1, *}$, David P. Laplante ${ }^{2}$

${ }^{1}$ Psychiatry, McGill University, Montreal; ${ }^{2}$ Psychosocial Research, Douglas Hospital Research Centre, Verdun, Canada

Background: Well-controlled animal experiments suggest that maternal stress during pregnancy programs the fetus for a variety of developmental delays and illnesses. Little is known about the effects of prenatal maternal stress in humans, however. Because it would be unethical to randomly assign pregnant women to stress conditions, studying prenatal maternal stress in humans presents challenges to the researcher. These can be overcome by taking advantage of naturally-occurring stressors that distribute hardship in quasi-random fashion.

Our research program addresses these research questions: (1) to what extent do objective hardship and subjective distress during pregnancy influence the cognitive, behavioural, physical and motor development of the unborn child? (2) Are these effects moderated by the timing in pregnancy and the child's sex? (3) What are the mechanisms of these effects? And (4) will more intensive prenatal care buffer these effects?

Method: We are running three studies of pregnant women exposed to natural disasters. In each, we identified pregnant women within weeks of the disaster onset and assessed objective exposure and subjective distress (PTSD symptoms). We then evaluated pregnancy outcomes, and the cognitive, behavioural, motor and physical development of the children.
Results: We began Project Ice Storm in Quebec in 1998; The Iowa Flood Study in 2008, which added pre-disaster data from an on-going study of pregnant women; and The QF2011 Queensland Flood Study in Australia last year which included pre-trauma data on pregnant women, a pre-existing randomized controlled trial of two prenatal care programs, plus birth biological specimens that will reveal the mechanisms of action of prenatal stress in humans. We will give the background rationale for the research program, the methods of the three studies, and a sampling of results.

Conclusion: Our studies demonstrate strong and long-lasting effects of prenatal stress on outcomes such as IQ, language, memory, anxiety, coordination, and risk for obesity in children.

Disclosure of Interest: None Declared.

\section{DOHaD13-1414}

Effects of a vegetarian food-based intervention on markers of micronutrient status in low-income Indian women of reproductive age. A randomised controlled trial

Sarah Kehoe ${ }^{1, *}$, Harsha Chopra $^{2}$, Sirazul Sahariah ${ }^{2}$, Meera Gandhi $^{2}$, Dnyaneshwar Tarwande ${ }^{3}$, Nick Brown ${ }^{1}$, Harshad Sane $^{2}$, Vanessa $\operatorname{Cox}^{1}$, Clive Osmond ${ }^{1}$, Barrie Margetts ${ }^{1}$, Ramesh Potdar ${ }^{2}$, Caroline Fall ${ }^{1}$

${ }^{1}$ University of Southampton, Southampton, United Kingdom;

${ }^{2}$ Centre for Study of Social Change; ${ }^{3}$ Apnalaya, Mumbai, India

Background: Evidence indicates that the nutritional status of women before they become pregnant affects the growth and development of their children. There are high prevalences of micronutrient deficiencies among women of reproductive age in India and intakes of micronutrient-rich foods such as fruit, milk and green leafy vegetables are low compared with national and international recommendations. Our objective was to determine whether daily consumption of a micronutrient-rich snack containing these foods would lead to an increase in markers of blood micronutrient concentrations when compared with a control snack, among women living in a Mumbai slum.

Method: Non-pregnant women aged $14-35$ years $(n=222)$ were randomised, stratified by age and body mass index (BMI), to receive a treatment snack (containing 25g green leafy vegetables, $10 \mathrm{~g}$ fruit and $12 \mathrm{~g}$ milk powder) or a control snack containing 'low micronutrient' foods such as potato or tapioca. All snacks were fried in sunflower oil and prepared daily. Women attended a community centre in the area they lived and ate the snacks under observation 6 days per week for 12 weeks. Adherence to the study protocol was recorded and compliance was defined as consumption of $\geqslant 3$ snacks per week. Venous blood was collected at baseline and after 12 weeks supplementation and analysed for beta-carotene, retinol, ascorbate, folate and ferritin concentrations. Food frequency data were collected at both time points by a 
nutritionist along with information on synthetic micronutrient supplement use. We assessed the effect of group allocation on change in blood micronutrient concentrations using univariate regression models. We also ran multivariate models adjusted for age, BMI, intake of fruit and vegetables and synthetic micronutrient supplements, percentage of snacks consumed and nutritional status at baseline in a multivariate model.

Results: Compliance with the intervention was $85 \%$ in the treatment and $90 \%$ in the control group. Complete blood micronutrient data at both time points were available for 170 women ( $77 \%$ of those randomised). Median (IQR) betacarotene concentrations in the treatment group increased from $390(305,470) \mathrm{nmol} / \mathrm{L}$ at 0 weeks to $470(380,610)$ $\mathrm{nmol} / \mathrm{L}$ at 12 weeks, while those in the control group were $390(305,470) \mathrm{nmol} / \mathrm{L}$ at 0 weeks and $440(340,540) \mathrm{nmol} / \mathrm{L}$ at 12 weeks. The median difference in concentrations between 0 and 12 weeks was $+80 \mathrm{nmol} / \mathrm{L}(25,155)$ in the treatment group and $+40 \mathrm{nmol} / \mathrm{L}(-5,115)$ in the control group. Group allocation was a significant independent predictor of the change in beta-carotene concentrations $(p=0.025)$. There were no statistically significant effects of group allocation on change in concentrations of retinol, ascorbate, folate or ferritin.

Conclusion: Locally sourced foods can be made into acceptable snacks that enhance the beta-carotene status of women of reproductive age living in Indian slums. Our study may help to inform the design of interventions aimed at improving the nutritional status of young women before they become pregnant thus improving the long term health of the women themselves and the next generation.

Disclosure of Interest: None Declared.

\section{DOHaD13-1136}

Fish intake during pregnancy, fetal growth, and gestational length: a meta-analysis of 19 European Birth Cohort Studies

Vasiliki Leventakou ${ }^{1}$, Theano Roumeliotaki ${ }^{1}$, David Martinez ${ }^{2}$, Henrique Barros ${ }^{3}$, Anne-Lise Brantsaeter ${ }^{4}$, Maribel Casas $^{2}$, Marie-Aline Charles ${ }^{5}$, Sylvaine Cordier ${ }^{6}$, Merete Eggesbø $\varnothing^{7}$, Manon van Eijsden ${ }^{8}$, Francesco Forastiere 9 , Ulrike Gehring $^{10}$, Eva Govarts ${ }^{11}$, Thorhallur I. Halldórsson ${ }^{12}$, Wojciech Hanke ${ }^{13}$, Margaretha Haugen ${ }^{4}$, Denise H. Heppe $^{14}$, Barbara Heude ${ }^{15}$, Hazel M. Inskip ${ }^{16}$, Vincent W. Jaddoe $^{14}$, Maria Jansen ${ }^{17}$, Cecily Kelleher ${ }^{18}$, Helle Margrete Meltzer $^{4}$, Franco Merletti ${ }^{19}$, Carolina Moltó-Puigmartí ${ }^{20}$, Monique Mommers ${ }^{20}$, Mario Murcia ${ }^{21}$, Andreia Oliveira ${ }^{3}$, Sjúrður F. Olsen ${ }^{12}$, Fabienne Pele ${ }^{6}$, Kinga Polanska ${ }^{13}$, Daniela Porta ${ }^{9}$, Lorenzo Richiardi ${ }^{19}$, Siân M. Robinson ${ }^{16}$, Hein Stigum $^{22}$, Marin Strøm ${ }^{12}$, Jordi Sunyer ${ }^{2}$, Carel Thijs ${ }^{20}$, Karien Viljoen $^{18}$, Tanja G. Vrijkotte ${ }^{23}$, Alet H. Wijga ${ }^{24}$, Manolis Kogevinas ${ }^{2}$, Martine Vrijheid ${ }^{2}$, Leda Chatzi ${ }^{1, *}$
${ }^{1}$ Department of Social Medicine, Faculty of Medicine, University of Crete, Greece, Heraklion, Greece; ${ }^{2}$ Centre for Research in Environmental Epidemiology (CREAL), Barcelona, Spain; ${ }^{3}$ Department of Clinical Epidemiology, Predictive Medicine and Public Health and Cardiovascular Research \& Development Unit, University of Porto Medical School, Porto, Portugal; ${ }^{4}$ Norwegian Institute of Public Health, Division of Environmental Medicine, Oslo, Norway; ${ }^{5}$ Inserm, Centre for research in Epidemiology and Population Health (CESP), Villejuif; ${ }^{6}$ Inserm (Institut National de la Santé et de la Recherche Médicale) UMR 1085, IRSET (Institut de Recherche Santé Environnement \& Travail), Université de Rennes 1, Rennes, France; ${ }^{7}$ Department for Genes and Environment, Division of Epidemiology, Norwegian Institute of Public Health, Oslo, Norway; ${ }^{8}$ Department of Epidemiology, Public Health Service Amsterdam, Documentation and Health Promotion, Amsterdam, Netherlands; ${ }^{9}$ Department of Epidemiology, Lazio Regional health System, Rome, Italy; ${ }^{10}$ Institute for Risk Assessment, Utrecht University Sciences, Utrecht, Netherlands; ${ }^{11}$ Environmental Risk and Health, Flemish Institute for Technological Research (VITO), Mol, Belgium; ${ }^{12}$ Maternal Nutrition Group, Centre for Fetal Programming, Statens Serum Institut, Copenhagen, Denmark; ${ }^{13}$ Department of Environmental Epidemiology, Nofer Institute of Occupational Medicine, Lodz, Poland; ${ }^{14}$ The Generation R Study Group of the Erasmus Medical Center, the Generation R Study Group (Room Ae-012) Erasmus Medical Center, Rotterdam, Netherlands; ${ }^{15}$ Inserm, Centre for research in Epidemiology and Population Health (CESP), U1018, Lifelong epidemiology of obesity, diabetes and renal disease Team, Villejuif, France; ${ }^{16}$ MRC Lifecourse Epidemiology Unit, University of Southampton, Southampton General Hospital, Southampton, United Kingdom; ${ }^{17}$ Department of Health Services Research, Faculty of Health, Medicine and Life Sciences, Caphri, Maastricht University, Maastricht, Netherlands; ${ }^{18}$ School of Public Health, Physiotherapy and Population Science, University College Dublin, Dublin, Ireland; ${ }^{19}$ Department of Medical Sciences, Cancer Epidemiology Unit, University of Turin, Torino, Italy; ${ }^{20}$ Department of Epidemiology, Maastricht University, School for Public Health and Primary Care, Maastricht, Netherlands; ${ }^{21}$ CIBER Epidemiología y Salud Pública (CIBERESP), Instituto de Salud Carlos III, Madrid, Spain; ${ }^{22}$ Department of Chronic diseases, Division of Epidemiology, Norwegian Institute of Public Health, Oslo, Norway; ${ }^{23}$ Department of Public Health, Academic Medical Centre - University of Amsterdam, Amsterdam; ${ }^{24}$ Centre for Nutrition, Prevention and Health Services, National Institute for Public Health and the Environment, Bilthoven, Netherlands

Background: Fish is a rich source of nutrients such as polyunsaturated n-3 fatty acids, protein, selenium, iodine and vitamin $\mathrm{D}$, considered to be beneficial for fetal growth and development, but on the other hand, is also a well-known route of exposure to pollutants such as dioxins, polychlorinated biphenyls (PCB), methylmercury, and other heavy 
metals which may adversely affect fetal growth and gestational length. Findings from prospective birth cohort studies on the relationship between fish intake during pregnancy and birth weight are discrepant, with reports of either positive/null or negative effects. We assessed whether fish intake during pregnancy affects fetal growth and length of gestation in a meta-analysis of European birth cohort studies.

Method: Data from 19 birth cohorts conducted in 12 European countries comprising 151,880 singleton births were pooled and harmonized. Questionnaires were used to estimate fish intake during pregnancy. Birth outcomes were collected from maternity records and parental questionnaires. Adjusted cohort-specific effect estimates were combined using random and fixed-effects meta-analysis.

Results: Compared with women who rarely ate fish (once a week or less), those with a higher intake of fish during pregnancy had $13 \%$ decreased risk of preterm birth (RR 0.87; 95\% CI: $0 \cdot 82,0.92)$ for fish intake of more than once but less than three times a week, and $11 \%$ decreased risk (RR: 0.89; 95\% CI: $0 \cdot 84,0.96$ ) for three or more times a week. Women with a higher intake of fish during pregnancy gave birth to neonates with increased birth weight by $8.9 \mathrm{~g}$ $(95 \%$ CI: $3 \cdot 3,14 \cdot 6)$ for $1 \mathrm{CI}: 8 \cdot 9,21 \cdot 5)$ for $\geqslant 3$ times a week, independent of gestational age. The effect was greater in pregnant women who smoked during pregnancy and were overweight or obese before pregnancy. Findings were adjusted for several confounders, and were consistent across countries. Conclusion: This large international study indicates a moderate beneficial effect of fish intake during pregnancy on the risk of preterm birth, and a small but significant increase in birth weight.

Disclosure of Interest: None Declared.

\section{DOHaD13-1091}

Flaxseed oil during lactation changes milk composition and programming metabolic parameters in male and female rats

Mariana S. Figueiredo ${ }^{1, *}$, Deysla S. Guarda ${ }^{1}$, Ligia D. A. Maia ${ }^{1}$, Elaine de Oliveira ${ }^{1}$, Patricia C. Lisboa ${ }^{1}$, Egberto G. de Moura ${ }^{1}$

${ }^{1}$ Department of Physiological Sciences, State University of Rio de Janeiro, Rio De Janeiro, Brazil

Background: Flaxseed oil is a rich source of n-3 PUFA and its consumption has been reported to exert beneficial effects on cardiovascular diseases. However, there is no data about its safety during lactation and the possible programming effect on the progeny, since we had showed previously a programming effect of total flaxseed [1,2]. We aimed to evaluate the effects of flaxseed oil during lactation in the milk composition and metabolic parameters in male and female rats during development.
Method: Thirty lactating rats were divided in groups of 10 litters: control (C), with a 7\% lipid (soy oil); Hyper soy oil (HS), with a diet containing 19\% lipid (soy oil exclusively); and Hyper flaxseed oil (HF), with a diet containing 19\% lipid ( $2 \%$ from soy oil and $17 \%$ from flaxseed oil). After weaning, all pups received a standard diet until postnatal day (PN) 180 . Male and female offspring (2 from each litter) were killed at PN 21 (weaning) and PN 180 (adulthood). All significant data were $\mathrm{p}<0.05$. The body composition was determined by carcass analyses and DXA. Milk and serum analyses were determined by biochemical assays or RIA.

Results: Dams, male and female pups from HS and HF group presented lower body mass during lactation. HF mothers presented lower body fat mass $(-32 \%)$ and lower visceral fat mass $(-44 \%)$ during lactation. HS mothers presented lower body protein mass $(-15 \%)$. At 14 days of lactation we observed lower triglycerides and cholesterol in the milk from HS (-26\% and $-60 \%$, respectively) and HF mothers $(-54 \%$ and $-63 \%$, respectively). Similarly, at weaning, the milk of HS $(-59 \%$ and $-70 \%)$ and HF $(-62 \%$ and $-64 \%)$ mothers presented lower content of triglycerides and cholesterol. We observed lower total cholesterol and HDL-c in serum of HS mothers. Male HF offspring had a lower body fat mass at PN21 $(-20 \%)$ and higher at PN180 $(+26 \%)$ and lower lean mass $(-14 \%)$ at PN180. By the contrary, we observed no changes in body fat mass in female HF offspring at PN21 and PN180. At PN 21, the male and female HF offspring presented lower serum VLDL-c $(-41 \%$ and $-50 \%$, respectively) and triglycerides $(-40 \%$ and $-50 \%$, respectively). At adulthood, we observed higher serum VLDL-c and triglycerides in male HF offspring ( $+56 \%$ and $+57 \%$, respectively); no changes were observed in lipid profile of female HF offspring. Both female and male from HF group presented hypoinsulinemia $(-29 \%$ and $-27 \%$, respectively) at PN21, but at PN180 we observed hyperglycemia $(+12 \%)$ and hyperinsulinemia $(+68 \%)$, with hypoadiponectinemia $(-42 \%)$ only in female HF offspring. We observed hyperleptinemia in the adult male HF offspring $(+78 \%)$. Despite no change in body fat mass, adult female HF offspring presented hyperleptinemia (+47\%).

Conclusion: Our findings suggest a gender specific effect of maternal intake of flaxseed oil during lactation leading to changes in insulin sensitivity especially in female offspring as well as in adiposity and lipid profile on the male offspring. These effects differ from the obtained with total flaxseed both in male and female, showing specific programming effects of the oil. Thus, women should limit their intake of flaxseed oil during lactation to prevent altered adipogenesis and glucose homeostasis in their progeny.

\section{Reference}

[1]. Figueiredo et al. (LifeSci 2009;85:365-71). [2] Troina et al. (Food Chem Toxicol 2010;48:697-703).

Disclosure of Interest: None Declared. 


\section{DOHaD13-1459}

\section{Human yolk sac adaptation between mother and fetus}

Henriette K. Hellebust ${ }^{1,2, *}$, Synnøve L. Johnsen ${ }^{2}$, Svein Rasmussen $^{1,2}$, Mario Merialdi ${ }^{3}$, Torvid Kiserud ${ }^{1,2}$

${ }^{1}$ Department of Clinical Science, University of Bergen;

${ }^{2}$ Department of Obstetrics and Gynecology, Haukeland University Hospital, Bergen, Norway; ${ }^{3}$ Department of Reproductive Health and Research, WHO, Geneva, Switzerland

Background: The yolk sac has a wide functional variation in biology. The human yolk sac rarely exceeds $8 \mathrm{~mm}$ and is visible before 13 weeks of gestation, a time when diffusion is of relatively high importance for nutrient and gas transportation. Protein synthesis (e.g. albumin, prealbumin, apolipoproteins, transferrin, IGF, a1-antitrypsin and AFP) and hematopoiesis (including pluripotent stem cells) are important functions in addition to its close link with the developing gastrointestinal tract (1).

Based on the assumption that mothers' resource status would influence yolk sac formation we hypothesized that more resources such as body mass would condition larger yolk sac ensuring more resources transmitted to the growing embryo.

Method: The present study, approved by the ethics review board, was part of the ongoing "WHO Multicentre Study for the Development of Growth Standards from Fetal Life to Childhood: The Fetal Component". Of the 140 low-risk pregnancies included in this longitudinal study, 120 had an ultrasound scan at a stage that permitted the measurement of the yolk sac diameter 3 times to form a mean. Additionally, crown-rump length (CRL) and abdominal circumference (AC) were measured. Fetal biometry was reiterated during pregnancy. Pre-pregnancy maternal height, weight and BMI had been recorded, and maternal AC and body composition assessed before 13 weeks of gestation. Standard deviation scores (SDS) were used for the statistical analysis.

Results: Pre pregnancy maternal height had a negative influence on the yolk sac diameter $(95 \% \mathrm{CI}-7.8$; -1.0 for SDS) and so had maternal weight $(-0.05 ;-0.01)$ while BMI had no influence. Maternal AC and body composition did not influence yolk sac diameter. On the other hand, a small yolk sac/CRL ratio was associated with a high fetal AC at 24 weeks of gestation $(p=0.018)$. Yolk sac diameter did not influence other fetal morphometric measurements.

Conclusion: The human yolk sac shows adaptational capacity being larger when the mother is smaller, the opposite of our hypothesis. The result is suggesting an auto-regulation of the yolk sac, possibly a compensatory expansion of its diffusion surface and production capacity when resources are less available. Such a mechanism is also found later in pregnancy: the fetal liver increases its umbilical venous perfusion when the mother is slim and when she prefers junk food $(2,3)$. The concept is further supported by our present result that fetuses with such a relatively large yolk sac develop a smaller AC reflecting a small liver size at mid gestation.

\section{References}

1. Nogales FF (Ed.). The human yolk sac and yolk sac tumors. 1993;368 pp. Springer, New York. ISBN 0-387-56031-9

2. Haugen G, Hanson M, Kiserud T, Crozier S, Inskip H, Godfrey KM. Fetal liver-sparing cardiovascular adaptations linked to mother's slimness and diet. Circ Res 2005; 96: 12-14.

3. Godfrey KM, Haugen G, Kiserud T, Inskip HM, Cooper C, Harvey NCW, Crozier ZR, Robinson SM, Davies Lucy, the Southampton Women's Survey Study Group, Hanson MA. Fetal liver blood flow distribution: role in human developmental strategy to prioritize fat deposition versus brain development. PLoS ONE. 2012; 10.1371/journal. pone.0041759

Disclosure of Interest: None Declared.

\section{DOHaD13-1743}

Intrauterine growth, maternal predisposition and family background: evidence from São Paulo Brazil.

Alexandra Brentani ${ }^{1, *}$, Helena Brentani ${ }^{2}$, Filumena M. D. S. Gomes ${ }^{1}$, Maria H. Valente ${ }^{1}$, Ana P. Scoleze ${ }^{1}$, Ana Maria D. U. Escobar ${ }^{1}$, Sandra J. Grisi ${ }^{1}$, Günther Fink ${ }^{3}$

${ }^{1}$ Pediatrics; ${ }^{2}$ Psychiatry, Faculdade de Medicina Universidade de São Paulo, São Paulo, Brazil; ${ }^{3}$ Global Health and

Population, Harvard School of Public Health, Cambridge, United States

Background: A large literature has highlighted the importance of maternal characteristics such as stature and maternal education on low birth weight (LBW) and intra-uterine growth restrictions (IUGR). Relatively few studies have investigated the associations between IGUR and mother's larger family context.

Objectives: To determine the associations between maternal family characteristics and intrauterine growth restrictions.

Method: 535 mothers giving births at the University Hospital in São Paulo between April and October 2012 were enrolled and invited for a follow-up assessment with a pediatrician six months after births. Detailed records were collected on maternal and paternal characteristics such as education, income, stature and health behavior, as well as medical histories and socioeconomic characteristics of maternal grandparents. Hospital records were retrieved for children's birth weight, adjusted birth weight, type of delivery, gestational length and Apgar scores. Multivariate logistic models were used to assess the associations between intrauterine growth restriction and individual as well as family characteristics.

Results: Strong associations were observed both between IUGR and both maternal and family background characteristics. Short maternal stature, substance abuse and previous stillbirths were the biggest risk factor for IUGR at the 
maternal level, with estimated odds ratios of 3.84 (95\% CI [1.25, 11.82], p-value 0.019), 13.52 (95\% CI [2.50, 73.12], $\mathrm{p}$-value $=0.03)$ and $5.53(95 \%$ CI 1.34, 23.04), $\mathrm{p}$-value $=$ $0.018)$, respectively. While no statistically significant associations were found for maternal or paternal education and social class, low socioeconomic status of the maternal grandparents was found to increase the odds of IUGR by a factor of 2.29 (95\% CI [1.01, 5.26], p-value 0.05), while highly educated maternal grandmothers reduced the odds of IUGR by $81 \%$ (OR $0.17,95 \%$ CI $[0.03,1.13]$, $\mathrm{p}$-value $=0.068$ ).

Conclusion: The results suggest that fetal development in general, and intrauterine growth restrictions in particular are strongly influenced by mother's socioeconomic background and environment. The large effects found on grandparental education and social status underline the importance of family and network support during pregnancy, and suggest that a large fraction of fetal growth restrictions can be prevented if sufficient support is provided for mothers during pregnancy.

Disclosure of Interest: None Declared.

\section{DOHaD13-1213}

Maternal homocysteine concentrations during pregnancy are associated with higher cortisol response to stress in Indian adolescents

GV Krishnaveni ${ }^{1, *}$, SR Veena ${ }^{1}$, R Somashekara ${ }^{1}$, A Jones ${ }^{2}$, CS Yajnik ${ }^{3}$, CHD Fall ${ }^{4}$

${ }^{1}$ Epidemiology Research Unit, CSI Holdsworth Memorial Hospital, Mysore, India; ${ }^{2}$ Centre for Cardiovascular Imaging, University College London Institute of Child Health, London, United Kingdom; ${ }^{3}$ Diabetes Unit, KEM Hospital, Pune, India; ${ }^{4} M R C$ Lifecourse Epidemiology Unit, University of Southampton, Southampton, United Kingdom

Background: Maternal micronutrient deficiencies may impair the one-carbon pathway resulting in compromised fetal growth and elevated cardiometabolic risk later in life. Exaggerated hypothalamic-pituitary-adrenal (HPA) axis activity may link fetal undernutrition with adult cardiovascular disease. We aimed to examine (1) the association between maternal vitamin B12, folate and homocysteine concentrations and cortisol responses to the Trier Social Stress Test for children (TSST-C) and (2) the association between stressinduced cortisol increment and cardiovascular disease risk markers in a group of adolescent children.

Method: Plasma vitamin B12, folate and homocysteine concentrations were measured at 28-32 weeks gestation in women who delivered live, normal babies at one hospital in India. At 13.5 years of age, the offspring $(\mathrm{N}=273$, mean age: 13.6 years) performed 5 -minutes each of a public speaking and a mental arithmetic task in front of two unfamiliar 'evaluators' (TSST-C). Salivary cortisol concentrations were measured at baseline and at regular intervals after inducing stress. Detailed anthropometry was carried out and fasting plasma glucose, insulin and lipid concentrations, and systolic and diastolic blood pressure were measured on the following day. Insulin resistance was calculated using the Homeostasis Model Assessment equation (HOMA-IR).

Results: There was a consistent rise in the salivary cortisol concentrations from baseline after inducing stress in the children. Higher maternal homocysteine concentrations were associated with higher cortisol responses to stress adjusted for age, sex, pubertal status, current BMI, childhood vitamin B12 and folate concentrations and maternal BMI and socioeconomic status (peak increase: $0.2 \mathrm{SD}$ per SD rise in maternal homocysteine concentrations, $95 \%$ CI: 0.02 to 0.3 SD; $\mathrm{P}=0.01)$. Maternal vitamin $\mathrm{B} 12$ and folate concentrations were not associated with stress-induced salivary cortisol concentrations. There was a positive association between cortisol increment after TSST-C and systolic blood pressure (0.02 SD increase per SD rise in cortisol concentrations; $95 \%$ CI: 0.04 to $0.3 \mathrm{SD}, \mathrm{P}=0.002$ ). There were no associations between cortisol reactivity and other risk factors.

Conclusion: Associations of maternal homocysteine concentrations with stress-induced cortisol concentrations and of cortisol response to higher blood pressure in our study suggest that intrauterine exposure to nutritional imbalances in the one-carbon pathway may alter HPA axis reactivity to stress and increase cardiovascular disease risk in later life. Further studies are needed to elucidate the causality of these associations in different populations.

Funding source: This study was supported by an intermediate fellowship from the Wellcome Trust, UK and by the Parthenon Trust, Switzerland and the Medical Research Council, UK.

Disclosure of Interest: None Declared.

\section{DOHaD13-1739}

Maternal iron status related to infant iron status: a prospective study in Indonesia

Rima Irwinda ${ }^{1, *}$

${ }^{1}$ obstetrics and gynecology, university of indonesia, jakarta, Indonesia

Background: To identify relationship of maternal iron status and infant iron status in pregnant women in Indonesia.

Method: We conducted a prospective study in 44 pregnant Women in Budi Kemuliaan, Jakarta. Every subject took the same multimicronutrients supplementation from milk during study. Maternal hemoglobin, ferritin, and reticulocyte in first, second, and third trimester were measured. Cord blood hemoglobin, ferritin, and reticulocyte were also measured. Women with low hemoglobin or ferritin level got iron therapy until delivery. 
Results: There were significant changes of maternal $\mathrm{Hb}$, ferritin, iron, and reticulocyte levels during TM1,2,3 $(p<0.05)$. Thirty three percent, $9.1 \%$ and $11.4 \%$ of cord blood had low level of $\mathrm{Hb}$, reticulocyte and iron, on the other hand, none of cord blood ferritin level was low. Significant correlation between maternal $\mathrm{Hb}$ in TM3 and cord blood $\mathrm{Hb}$ ( $p$ 0.01) was found. In contrast, no correlation between maternal ferritin in TM1,2 or 3 and cord blood ferritin $(\mathrm{p}>0.05)$.

Conclusion: Maternal hemoglobin in third trimester has correlation with cord blood hemoglobin. No correlation was found between maternal ferritin in trimester 1,2 or 3 and cord blood ferritin.

Disclosure of Interest: None Declared.

\section{DOHaD13-1286}

Maternal malnutrition impacts structure, function and inflammation of key barriers along the nutritional pipeline

K. L. Connor ${ }^{1, *}$, E. Bloise ${ }^{2}$, S. G. Matthews ${ }^{2}$, S. J. Lye ${ }^{1}$

${ }^{1}$ Samuel Lunenfeld Research Institute, Mt Sinai Hospital;

${ }^{2}$ Physiology, U Toronto, Toronto, Canada

Background: The pathway to optimal development is complex and integrated along a pipeline that includes the maternal gut, placenta and fetal gut. Altered maternal nutrition impacts fetoplacental development and health long-term, yet it is unclear how malnutrition may influence structure/function throughout the pipeline. The $\mathrm{ABC}$ transporters P-glycoprotein (P-gp/Abcbla/b) and breast cancer resistance protein (BCRP/Abcg2) play a central role in the efflux translocation of nutrients and xenobiotics across cell membranes, limiting absorption of these compounds into maternal and fetal blood. Thus they confer protection at key barrier sites including the gut and placenta. We hypothesise that maternal malnutrition is associated with altered barrier function at the small intestine (SI) and placenta, in part through changes in inflammation and in P-gp and BCRP expression, stressing the importance of these integrated barriers in optimal development and health of the mother and fetus.

Method: Female mice $(n=7 /$ grp $)$ were fed a control diet throughout pregnancy $(\mathrm{CON})$, or calorically restricted by $30 \%$ from embryonic day 5.5-18.5 (CR) or fed a 60\% high fat diet from 8 weeks before mating and throughout pregnancy $(\mathrm{HF})$. At E18.5 (term = 19 d) tissues were collected. Maternal SI mucosal and placental architecture were measured following H\&E staining. Cytokines were measured in maternal SI intraepithelial lymphocytes (IEL) and placental tissue using 23-Plex assay. mRNA expression of $A b c b 1 a / b$ and $A b c g 2$ was determined in maternal and fetal SI and placenta. Immunohistochemistry for P-gp and BCRP was performed on maternal SI and placenta. Significance $\mathrm{p}<0.05$.
Results: Maternal SI crypt depth was longer in HF $(\mathrm{p}<0.001)$ and trended shorter in CR vs CON. Villus height was not altered by diet. HF diet increased levels of proinflammatory cytokines in SI IEL including IL-1 $\beta$, IL12p70, eotaxin $(p<0.05)$. Fetoplacental weights were reduced in CR vs CON and HF $(\mathrm{p}<0.0001)$ and were associated with increased placental TNF $\alpha$ levels $(\mathrm{p}<0.05)$. Junctional and labyrinth areas of CR and HF placentae were $>10 \%$ smaller vs CON. CR increased Abcbla mRNA expression 3.7-fold in maternal SI vs CON $(\mathrm{p}<0.01)$ but did not change in placenta or fetal gut. HF diet decreased Abcb1a mRNA expression in maternal SI $(\mathrm{p}<0.05)$, placenta $(\mathrm{p}=0.01)$ and fetal gut $(\mathrm{p}=0.001)$ vs CON. CR decreased Abcbg 2 mRNA expression in maternal SI $(\mathrm{p}<0.01)$ and placenta $(\mathrm{p}<0.05)$ but not fetal gut. Preliminary data show PgP staining was less intense in HF SI and BCRP was less intense in CR SI.

Conclusion: Maternal malnutrition alters structure and function of barriers along the nutritional pipeline and is associated with local inflammation, fetoplacental growth restriction and modified expression of drug transporters. If these changes hold true in humans, it may be of concern in low resource settings where the effects of poor nutrition during pregnancy are compounded by disease. Further, malnourished mothers and their fetuses may have deranged drug biodisposition due to altered transport expression along the pipeline. Urbanisation of populations and exposure to calorie dense but nutrient poor diets may also alter host defence and barrier permeability. These changes may not only impact maternal immunity and nutritional status, but also alter exposure of the fetus/placenta to both beneficial and harmful compounds during development.

Disclosure of Interest: None Declared.

\section{DOHaD13-1518}

Patterns of maternal weight and body composition in pregnancy and post-partum and fetal and infant growth in women with morbid obesity

Rebecca M. Reynolds ${ }^{1, *}$, Fiona C. Denison ${ }^{1}$, Shareen

Forbes ${ }^{1}$, Amanda J. Drake ${ }^{1}$, Jane E. Norman ${ }^{1}$

${ }^{1}$ University of Edinburgh, Edinburgh, United Kingdom

Background: Pregnant women with morbid (Class III) obesity $\left(\mathrm{BMI} \geqslant 40 \mathrm{~kg} / \mathrm{m}^{2}\right)$ are at greater risk of complications for mother and child yet little is known about changes in gestational weight gain (GWG), body composition, postpartum weight retention or fetal/infant growth patterns.

Method: We measured weight and body composition (by bio-electrical impedance and skin-fold thickness) in 255 morbidly obese women (BMI mean (sd) $44.3(4.0) \mathrm{kg} / \mathrm{m}^{2}$ ) and 111 lean controls (BMI $\left.22.8(2.6) \mathrm{kg} / \mathrm{m}^{2}\right)$ at 16,28 and 36 weeks gestation, and at 3 and 6 months postpartum in a 
subgroup (obese $n=117$, lean $n=96$ ). Ultrasonographic fetal biometry was assessed at 12, 20, 28 and 36 weeks gestation. Infant weight and length were recorded at birth $(\mathrm{n}=304), 3(\mathrm{n}=272)$ and 6 months $(\mathrm{n}=164)$ in those born at term ( $>37$ weeks gestation).

Results: Obese women had significantly less GWG than lean $(5.3 \mathrm{~kg}( \pm 6.0)$ vs $10.2 \mathrm{~kg}( \pm 3.7), \mathrm{p}<0.001)$ and no increase in overall percentage body fat $(-0.5 \%( \pm 4.1)$ vs $5.0 \%$ ( \pm 3.2$), \mathrm{p}<0.001)$ but were more likely to exceed Institute of Medicine guidelines for GWG (22\% vs $7 \%$, $\mathrm{p}<0.0001$ ). At 6 months postpartum weight did not differ from early pregnancy in either group, but percentage body fat had increased in obese $(+1.4 \%(4.3) \mathrm{p}=0.02)$ and decreased in lean $(-1.77 \%(4.9), \mathrm{p}=0.03)$. Fetal abdominal circumference was significantly greater in obese compared to lean at 28 and 36 weeks gestation (both $p<0.01$ ). Offspring of obese had higher birthweight than lean after adjustment for maternal age, parity, smoking, ethnicity, socio-economic status, gestation at delivery and gender (3584 (565) vs 3519 (533) g p $=0.03$ ). Prevalence of macrosomia (birthweight $>4000 \mathrm{~g})$ was significantly higher in obese $(25.0 \%$ vs $16.8 \%$, $\mathrm{p}_{\text {adj }}=0.036$ ). Infants of obese were also shorter with greater ponderal index at birth and 3 months (all $\mathrm{p}<0.05$ ). By 6 months, infants of obese remained heavier than lean $(p=0.023)$ though other measurements did not differ. Infants of obese mothers were more likely to be formula fed and were introduced to solids earlier (all $\mathrm{p}<0.001$ ).

Conclusion: Although morbidly obese women gain little weight during pregnancy compared to lean, at 6 months postpartum they have a greater percentage body fat compared with early pregnancy which may have implications for future metabolic health. Predisposition to obesity in offspring of obese starts in utero but is also influenced by postnatal feeding behaviours.

Disclosure of Interest: None Declared.

\section{DOHaD13-1397}

The D-tect study, a societal intervention to examine the influence of exposure to Vitamin D in fetal life for development of fractures, obesity, and type 1 diabetes later in life

Berit L. Heitmann ${ }^{1,2, *}$, Ramune Jacobsen ${ }^{2}$, Bo Abrahamsen ${ }^{3}$, Marta Bauerek ${ }^{2}$, Claus Holst ${ }^{2}$, Camilla B. Jensen ${ }^{2}$, Joachim Knop $^{2}$, Kyle Raymond ${ }^{2}$, Lone B. Rasmussen ${ }^{4}$, Thorkild I. Sørensen ${ }^{2,5}$, Allan Vaag ${ }^{6}$

${ }^{1}$ National Institute of Public Health, University of Southern Denmark, Odense, University of Southern Denmark, Odense; ${ }^{2}$ Institute of Preventive Medicine, Frederiksberg and Bispebjerg Hospitals - a part of Copenhagen University Hospital, The Capital Region, Copenhagen; ${ }^{3}$ Institute of Clinical Research, University of Southern Denmark, Odense; ${ }^{4}$ National Food Institute, DTU, Copenhagen; ${ }^{5}$ Novo Nordisk Foundation
Center for Basic Metabolic Research, University of Copenhagen; ${ }^{6}$ Institute of Clinical Medicine, Orthopedics and Internal Medicine, Rigshospitalet, Copenhagen, Denmark

Background: Vitamin D deficiency is common among otherwise healthy pregnant women and may have consequences for them as well as the early development and longterm health of their children. However, the importance of maternal vitamin $\mathrm{D}$ status on offspring health later in life has not been widely studied. The present program examines the influence of exposure to vitamin D early in life for development of fractures of the wrist, arm and clavicle; obesity, and type 1 diabetes during child- and adulthood, using a unique societal experiment that took place in Denmark during 1961 through 1985.

Method: The study is based on the fact that in 1961 fortifying margarine with vitamin D became mandatory in Denmark. Apart from determining the influences of exposure prior to conception and during prenatal life, we will examine the importance of vitamin D exposure during specific seasons and trimesters, by comparing disease incidence among individuals born before and after fortification. The Danish National databases assure that there are a sufficient number of individuals to verify any vitamin $\mathrm{D}$ effects during different gestation phases. Additionally, a validated method will be used to determine neonatal vitamin D status using stored dried blood spots from individuals who developed the aforementioned disease entities as adults and their time and gender-matched controls.

Results: The results of the study will contribute to our current understanding of the significance of supplementation with vitamin D. More specifically, they will enable new research in related fields, including interventional research designed to assess supplementation needs for different subgroups of pregnant women. Also, the results of the study will justify the debate of Danish health authorities whether to resume vitamin $\mathrm{D}$ supplementation policies.

Disclosure of Interest: None Declared.

\section{DOHaD13-1584}

The effect of folic acid addition to juvenile rat offspring from protein restricted dams on bone structure and function

Stephanie Meakins ${ }^{1, *}$, Cyrus Cooper $^{2}$, Stuart Lanham ${ }^{1}$, Richard Oreffo ${ }^{1}$

${ }^{1}$ Faculty of Medicine, University of Southampton; ${ }^{2} M R C$ Lifecourse Epidemiology Unit, University of Southampton, Southampton, United Kingdom

Background: Previous animal and epidemiological studies suggest that skeletal growth is programmed during intrauterine and early postnatal life with a key role for epigenetics and epigenetic modulation. Epidemiological studies have also 
shown that poor growth during fetal life, infancy and childhood is associated with an increased risk of fracture and decreased bone mass in adulthood. Folic acid addition may have an effect on the negative effects of the protein restriction due to its methyl donor properties. We hypothesised that folic acid addition, a methyl donor, to the young rats from a protein restricted mothers would alter and enhance bone structure and microarchitecture potentially through an epigenetic mechanism.

Method: Rat dams were fed either a protein restricted (PR) or control (C) diet during pregnancy and their offspring were fed either a control folic acid (CFA) or high folic acid (HFA) diet between day 29-56 postnatal. All offspring were fed a high fat diet between day 57-84 days. Females $(n=4-6)$ in the four offspring diet groups PR/CFA, C/CFA, PR/HFA and C/HFA were analysed at 12 weeks. Femurs from each group were analysed for bone matrix composition using Alcian Blue Sirius Red staining, bone microarchitecture and composition using $\mu \mathrm{CT}$ for trabecular and cortical analysis (Brucker CTAn custom analysis) and mechanical composition using three point bend testing. Vertebras were also analysed using $\mu \mathrm{CT}$.

Results: Differences in bone structure were observed in all groups using $\mu \mathrm{CT}$ : i) $\mathrm{C} / \mathrm{CFA}$ vs $\mathrm{C} / \mathrm{HFA}$ Within the vertebrae (cortical bone) bone volume was significantly increased $18 \%$ compared to the C/CFA group ( $\mathrm{p}<0.05$ ), cortical thickness was enhanced by $8 \%(\mathrm{P}<005)$, trabecular pattern factor was reduced by $110 \%(\mathrm{P}<0.05)$ as was the structural model index by $126 \%(\mathrm{P}<0.05)$ indicating stronger cortical bone. Vertebra trabecular no differences, femur cortical no differences, femur trabecular connectivity density; ii) C/ CFA vs PR/CFA vertebra cortical results bone volume and trabecular pattern factor, vertebra trabecular no differences, femur cortical no differences, femur trabecular no differences; iii) C/CFA vs PR/HFA vertebra cortical results bone volume, vertebra trabecular bone volume, femur cortical no differences, femur trabecular connectivity density.

Analysis of bone composition indicated no significant differences found between groups for Alcian Blue Sirius Red staining, bone mineral density analysis and in mechanical competency as confirmed by three point bend testing.

Conclusion: Addition of folic acid to juvenile offspring of mothers fed a low protein diet resulted in altered cortical vertebral bone structure; with changes in bone thickness, volume and structure in female offspring at 12 weeks. The current study demonstrates a site specific effect of dietary manipulation and enhancement using folic acid on vertebral bone development, not observed in control offspring.

Further analysis at later time points will elucidate if folic acid can ameliorate the deleterious effect of a low protein diet on bone development in these offspring. These studies indicate the importance of the skeletal developmental window on modulation of bone structure and the need to further evaluate the epigenetic mechanisms involved in this process.

Disclosure of Interest: None Declared.

\section{DOHaD13-1129}

The mother study: treatment of hyperemesis gravidarum with early enteral tube feeding. A randomized controlled trial study protocol

Iris J. Grooten ${ }^{1, *}$, Tessa J. Roseboom ${ }^{2,3}$, Joke M. J. Bais ${ }^{1}$, Carrie Ris-Stalpers ${ }^{3}$, Ben Willem J. $\mathrm{Mol}^{3}$, Joris A. M. van der Post $^{3}$, Rebecca C. Painter ${ }^{1,3}$

${ }^{1}$ Dept. of Obstetrics \& Gynaecology, Medical Centre Alkmaar, Alkmaar; ${ }^{2}$ Dept. of Clinical Epidemiology and Biostatistics; ${ }^{3}$ Dept. of Obstetrics \& Gynaecology, Academic Medical Centre, University of Amsterdam, Amsterdam, Netherlands

Background: Hyperemesis gravidarum (HG), or intractable vomiting during pregnancy, is the largest cause of hospital admission in early pregnancy. HG has a major impact on maternal quality of life and has repeatedly been associated with poor pregnancy outcome such as low birth weight. Previous studies have demonstrated that poor maternal nutrition in early pregnancy can have permanent detrimental effects on offspring health, primarily in the cardiometabolic disease spectrum. Currently, women with HG may be admitted to hospital for intravenous fluid replacement, without receiving specific nutritional attention. Nasogastric tube feeding is sometimes used as treatment of last resort. At present however, there is no evidence showing the effectiveness of any of the treatments for HG. We hypothesize that in women with HG, an early refeeding strategy can improve maternal quality of life, reduce hospital stay and improve markers of cardiometabolic risk in neonatal cord blood.

Method: Multicenter randomized controlled trial (RCT) in women hospitalized for HG under 20 weeks of gestation.

Inclusion criteria: gestational age between $5+0$ and $19+6$ weeks, singleton or multiple pregnancy, hospital admission because of HG, first admission or readmission for HG.

Exclusion criteria: maternal age $<18$ years, molar pregnancy, missed abortion, acute infection causing vomiting, contraindication for enteral tube feeding, HIV infection.

After informed consent a baseline measurement for quality of life will be performed. Subsequently participants will be randomized for either 'care as usual' or tube feeding in addition to care as usual. Continuation of tube feeding on discharge is encouraged until oral intake exceeds 1000 calories per day.

Results: The primary outcomes will be maternal Pregnancy Unique Quantification of Emesis score (PUQE) one week after randomization and neonatal birth weight. Secondary outcomes will be maternal quality of life, duration of hospital stay and admission rates, maternal weight, ketonuria, neonatal morbidity, small for gestational age (SGA), prematurity, costs and molecular outcomes in umbilical cord blood and placental tissue that relate to HG. Long-term follow-up of children will be performed.

All data will be analyzed according to the intention-to-treat principle. Based on an improvement of $20 \%$ of the PUQE 
score after tube feeding and a possible $10 \%$ loss to follow up we need a total of 120 participants (alpha error 5\%, beta error $20 \%)$. This sample size is also large enough to detect a difference in birth weight of 200 grams. Quality of life as well as symptoms scores will be analysed using repeated measures analysis of variance.

Conclusion: The MOTHER study is the first randomized controlled trial comparing an early tube feeding strategy with a rehydration and anti emetics only strategy for women with HG. It will be the first RCT among HG patients to assess neonatal outcomes and long-term offspring outcome. Alongside, we will investigate placental tissue and umbilical cord blood on molecular level for HG etiology and consequences. Currently, clinicians lack any treatment options with proven efficacy. This study will assess whether early tube feeding can benefit both maternal and neonatal outcomes.

Disclosure of Interest: None Declared.

\section{DOHaD13-1730}

\section{The relation of maternal plasma ferritin to neonatal abdominal adipose tissue composition}

Wei Wei Pang ${ }^{1, *}$, Mya Thway Tint ${ }^{1}$, Marjorelee T. Colega ${ }^{1}$, Yiong Huak Chan ${ }^{2}$, Rob M. van Dam ${ }^{3}$, Fabian K. Yap ${ }^{4}$, Marielle V. Fortier ${ }^{5}$, Seang-Mei Saw ${ }^{3}$, Peter Gluckman ${ }^{6}$, Kenneth Kwek ${ }^{7}$, Yap-Seng Chong ${ }^{1}$, Keith M. Godfrey ${ }^{8}$, Yung Seng Lee', Mary F. Chong ${ }^{6}$ and GUSTO Study Group ${ }^{1}$ Department of Obstetrics \& Gynaecology; ${ }^{2}$ Biostatistics Unit; ${ }^{3}$ Saw Swee Hock School of Public Health, National University of Singapore; ${ }^{4}$ Department of Paediatric Endocrinology; ${ }^{5}$ Department of Diagnostic and Interventional Imaging, KK Women's and Children's Hospital; 'Singapore Institute for Clinical Sciences (SICS), Agency for Science, Technology and Research ( $\left.A^{*} S T A R\right) ;{ }^{7}$ Department of Maternal Fetal Medicine, KK Women's and Children's Hospital (KKH), Singapore, Singapore; ${ }^{8}$ MRC Lifecourse Epidemiology Unit \& NIHR Southampton Biomedical Research Centre, University of Southampton \& University Hospital Southampton NHS Foundation Trust, Southampton, United Kingdom; ${ }^{9}$ Department of Paediatrics, National University of Singapore, Singapore, Singapore

Background: The micronutrient status of the mother has been linked with childhood body composition in the offspring. While influenced by inflammatory and other processes, serum ferritin in pregnancy is a measure of maternal iron status and we examined the relation of maternal ferritin concentration to neonatal adiposity in the birth cohort "Growing Up in Singapore Towards healthy Outcomes" (GUSTO).

Method: Maternal plasma ferritin concentrations were measured from blood samples collected at $26-28^{\text {th }}$ weeks' gestation. Neonatal abdominal adipose tissue volumes were determined by magnetic resonance imaging (MRI), performed
3-21 days after delivery. Abdominal adipose tissue was categorized into superficial (ASSC) and deep subcutaneous (ADSC) and internal (AI) adipose tissue compartments and quantified using in-house quantitative analysis software. Linear regression analyses were performed to determine the influence of maternal plasma ferritin on neonatal abdominal adipose tissue volumes. Log-transformed ferritin and abdominal adipose tissue volumes were used in all analyses.

Results: 260 mother-infant dyads had complete ferritin and neonatal MRI data. Adjusting only for infants' age at MRI measurement, significant inverse associations were observed between maternal plasma ferritin and neonatal volumes of ADSC and ASSC, but there was no association with AI volume; a $10 \%$ increase in plasma ferritin predicted a $2.1 \%$ decrease in ADSC volume $(\beta=-0.213$ [95\% CI: -0.36 , $-0.07], p=0.004)$ and a $0.9 \%$ decrease in ASSC volume $(\beta=-0.086 \quad[95 \% \quad$ CI:-0.17, -0.003$], p=0.042)$. In multivariable models further adjusting for infant gender, gestational age, birth length, ethnicity, maternal age, prepregnancy BMI and other covariates, only the association of maternal plasma ferritin with ADSC remained significant $(\beta=-0.166$ [95\% CI:-0.31, -0.02$], p=0.022)$. The use of plasma ferritin as quintiles in the multivariable model suggested that the inverse association with ADSC was graded across the distribution of ferritin (compared to Q1, Q2: $\beta=-0.02$ [95\% CI: $-0.17,0.12]$, Q3: $\beta=-0.10$ [95\% CI:-0.24, 0.05], Q4: $\beta=-0.15$ [95\% CI:-0.29, -0.006 ], Q5: $\beta=-0.15$ [95\% CI:-0.30, -0.002]).

Conclusion: Our findings suggest a graded inverse relationship between maternal plasma ferritin and neonatal abdominal deep subcutaneous adipose tissue volume. Further analyses are required to determine if this reflects an effect of maternal iron status or alternative underlying mechanisms.

Disclosure of Interest: None Declared.

\section{DOHaD13-1345}

Weight loss and nausea and vomiting in pregnancy: effects on cardiometabolic profile of the offspring at age 5

Iris J. Grooten ${ }^{1, *}$, Rebecca C. Painter ${ }^{1,2}$, Martina Pontesilli ${ }^{1}$, Joris A. M. van der Post ${ }^{2}$, Ben Willem J. Mol ${ }^{2}$, Manon van Eijsden $^{3}$, Tanja G. Vrijkotte ${ }^{4}$, Tessa J. Roseboom ${ }^{2,5}$

${ }^{1}$ Dept. of Obstetrics \& Gynaecology, Medical Centre Alkmaar, Alkmaar; ${ }^{2}$ Dept. of Obstetrics \& Gynaecology, Academic Medical Centre, University of Amsterdam; ${ }^{3}$ Dept. of Epidemiology, Documentation and Health Promotion, Public Health Service of Amsterdam; ${ }^{4}$ Dept. of Social Medicine; ${ }^{5}$ Dept. of Clinical Epidemiology and Biostatistics, Academic Medical Centre, University of Amsterdam, Amsterdam, Netherlands

Background: Poor maternal nutrition in early pregnancy is known to have permanent detrimental effects on offspring health, primarily in the cardiometabolic disease spectrum. In 
the Western world, weight loss in early pregnancy is a feature of hyperemesis gravidarum (HG), a severe form of nausea and vomiting in pregnancy. HG is thought to be associated with premature delivery and low birth weight but data on short and long term consequences are limited. Therefore we aimed to investigate the consequences of $\mathrm{HG}$ on pregnancy outcomes and cardiovascular and metabolic profiles of 5-year-old children.

Method: We used prospectively collected data from the Amsterdam Born Children and their Development study (ABCD-study). This is a large prospective multi-ethnic cohort study that was set up in Amsterdam between 2003 and 2004. Detailed information on life style, nutrition and socio-demographic background was recorded at antenatal booking, with pregnancy details and outcomes available for 8266 women. At booking participants answered questions about nausea, vomiting and weight loss. Nausea and vomiting of pregnancy (NVP) was defined as nausea and/or vomiting for at least two weeks, severe weight loss was defined as weight loss $\geqslant 5 \mathrm{~kg}$. Participants were considered to have HG when suffering from both NVP and severe weight loss. 3263 children were examined at the age of 5-6 years. Of 2167 children capillary blood samples were taken to assess glucose and lipid profile. Blood pressure, height and weight were measured.

Results: The prevalence of HG was 3,7\%. Women were younger ( $28 \pm 6$ vs $31 \pm 5$ years), more often of non-Western origin $(58,4 \%$ vs $22,8 \%)$ and more obese than women without HG (BMI $>29,5 \mathrm{~kg} / \mathrm{m}^{2}$ adjusted OR 3,0 (95\% CI $2,1-4,4)) .11,6 \%$ of hyperemetic women delivered prematurely, compared to $7,7 \%$ of women without HG. At 5 years of age, children born to mothers with HG had higher body mass indexes (BMI $16,3 \pm 2,2 \mathrm{~kg} / \mathrm{m}^{2}$ ) compared to children born to mothers that did not suffer from HG (BMI $15,5 \pm 1,4 \mathrm{~kg} / \mathrm{m}^{2}$ adjusted difference $0,3 \quad(95 \% \quad$ CI $0,1-0,6))$. Blood pressure was higher among children whose mother had had HG compared to those of mothers who had not experienced HG. But after adjustment for confounders this difference was not statistically significant.

Conclusion: This is the first report of long-term offspring health after pregnancies complicated by HG. At the age of 5, the children born after hyperemetic pregnancies had higher body mass indexes compared to their peers, even after adjusting for confounders including maternal obesity and prematurity. Blood pressure, glucose and lipid profiles were not significantly altered. This study shows that early pregnancy weight loss, usually occurring as a manifestation of $\mathrm{HG}$ in our urban western setting, has long-term consequences on offspring health. Interestingly, initial treatment of $\mathrm{HG}$ is often based on rehydration instead of nutritional support. Thus, an intervention among women with HG aimed at nutritional support in order to prevent weight loss may offer a method for improving offspring health, and requires investigation.

Disclosure of Interest: None Declared.

\section{DOHaD13-1134}

Anxiety of mothers during pregnancy and birth outcomes

Birit F. Broekman ${ }^{1, *}$, Yiong Huak Chan ${ }^{2}$, Yap-Seng Chong ${ }^{3}$, Kenneth Kwek ${ }^{4}$, Sharon Sung ${ }^{5}$, Charlotte L. Haley, Helen Chen ${ }^{7}$, Cornelia Chee ${ }^{8}$, Anne Rifkin-Graboi ${ }^{9}$, Peter D. Gluckman ${ }^{10}$, Michael Meaney ${ }^{11}$, Seang Mei Saw ${ }^{12}$ and Gusto Singapore

${ }^{1}$ Neurocognitive Development Centre (SICS), Psychological Medicine (NUH), Singapore Institute for Clinical Sciences, National University Hospital, National University of Singapore; ${ }^{2}$ Head biostatics unit, National University of Singapore; ${ }^{3}$ Department of Obstetrics and Gynaecology, National University Hospital; ${ }^{4}$ Department of Maternal Fetal Medicine, KK Hospital; ${ }^{5}$ Department of Child and Adolescent Psychiatry, Institute of Mental Health, Singapore; ${ }^{6}$ Office of Clinical Sciences, Duke-NUS Graduate Medical School Singapore, Singapore; ${ }^{7}$ Department of Psychological Medicine, KK Hospital; ${ }^{8}$ Psychological Medicine, National University Hospital; ${ }^{9}$ Neurocognitive Development Centre;

${ }^{10}$ Metabolism and Growth; ${ }^{11}$ Neurocognition, Singapore Institute for Clinical Sciences; ${ }^{12}$ Saw Swee Hock School of Public Health, National University of Singapore, Singapore, Singapore

Background: Mental health problems during pregnancy can have an influence on fetal growth. This study examines the associations between symptoms of maternal depression and anxiety during pregnancy on multiple birth outcomes in an Asian population.

Method: A cohort (Growing Up in Singapore Towards healthy Outcomes; GUSTO) of 915 Asian pregnant women with a mean age of 30.5 years old were recruited between 2009 to 2010 at two birthing hospitals in Singapore. Depressive symptoms were measured with the Edinburgh Postnatal Depression Scale (EPDS) and the Beck Depression Inventory (BDI-II), anxiety was measured with the Spielberger State-Trait Anxiety Inventory (STAI) at 26 weeks gestation. Health personnel recorded birth weight, birth length, gestational age and head circumference at birth.

Results: The mean birth weight across the sample was $3.086 \mathrm{~kg}$ (SD 450.96) and the mean birth length was $48.5 \mathrm{~cm}$ (SD 2.3). After controlling for multiple confounders the association between STAI and birth length was significant $(\beta=-0.15$, SE $0.07, P=0.026)$. No associations were found between scores on the EPDS, BDI-II and STAI with birth weight, head circumference or gestational age.

Conclusion: Depressive symptoms during the second trimester are not associated with body size and gestational age at birth. In contrast, anxiety is associated with a shorter birth length, which may be considered as a more stable measure of anabolic investment in growth.

Disclosure of Interest: None Declared. 


\section{DOHaD13-1272}

Does late childbearing impact upon offspring behaviours? A Longitudinal Cohort Study

Jessica Tearne ${ }^{1, *}$, Monique Robinson ${ }^{1}$, Jianghong $\mathrm{Li}^{1}$, Peter Jacoby ${ }^{1}$, Neil McLean ${ }^{2}$

${ }^{1}$ Telethon Institute for Child Health Research, West Perth; ${ }^{2}$ School of Psychology, The University of Western Australia, Crawley, Australia

Background: This study examined the relationship between parental age and longitudinal mental health outcomes in offspring, using data from the Western Australian Pregnancy Cohort (Raine) Study. We examined what links exist between maternal and paternal age and child behaviour outcomes, and whether these relationships persist when accounting for known perinatal risk factors.

Method: The Raine Study provided comprehensive data from 2900 pregnancies, resulting in 2868 live born children. Offspring were followed up at ages two, five, eight, 10, 14, and 17 years using the Child Behaviour Checklist (CBCL). The CBCL provided clinical cut-offs for behavioural morbidity for overall, internalizing (withdrawn, anxious/depressed, somatic complaints) and externalizing (delinquent, aggressive) behaviour $(T \geqslant 60)$. We used longitudinal logistic regression models with stepwise adjustment for known prenatal risk factors (gestational age, maternal smoking in pregnancy, maternal education at pregnancy, parity, total family income and gender).

Results: In the unadjusted models, as both maternal and paternal age increased, there was a significantly decreased risk for the later development of total, internalizing, and externalizing problems in children throughout childhood. In the adjusted models, maternal age remained a significant predictor of total $(\mathrm{OR}=.89,95 \% \mathrm{CI}=.81, .97)$, internalising $(\mathrm{OR}=.91,95 \%$ $\mathrm{CI}=.83, .99)$ and externalising $(\mathrm{OR}=.89,95 \% \mathrm{CI}=.82, .97)$ problems, with increased age related to decreased behaviour problems in children. Paternal age was no longer significantly associated with risk for total, internalising and externalising child behaviour problems.

Conclusion: Preliminary findings indicate both advancing maternal and paternal age at conception contribute to the child's later behavioural development.

Disclosure of Interest: None Declared.

\section{DOHaD13-1275}

Food allergy behavioral-cognitive abnormalities and neuroinflammation are prevented by a dietary intervention with bifidobacterium breve $\mathrm{M}-16 \mathrm{~V}$ in combination with short chain galacto-oligosaccharides and long-chain fructo-oligosaccharides

Yuliya E. Borre ${ }^{1,2}$, Sander De Kivit ${ }^{1}$, Mary E. Morgan ${ }^{1}$, Johan Garssen $^{1,3, *}$, Berend Olivier ${ }^{1,2}$, Aletta D. Kraneveld ${ }^{1}$
${ }^{1}$ Pharmaceutical Sciences, Division of Pharmacology, Faculty of Science, Utrecht University; ${ }^{2}$ Rudolf Magnus Institute of Neuroscience, Univerisity Medical Center Utrecht, Utrecht; ${ }^{3}$ Danone Research, Center for Specialized Nutrition, Wageningen, Netherlands

Background: The gut-immune-brain axis has been implicated in various neurodevelopmental disorders. Though the immune pathophysiology of food allergic reactions has been well characterized, little is known about its consequences on brain function and cognition. The aims of this study were to investigate whether inflammation associated with food allergy affects brain function and to examine the effect of dietary supplementation with Bifidobacterium breve $M-16 \mathrm{~V}$ in combination with short chain galacto-oligosaccharides and long-chain fructo-oligosaccharides ( $\mathrm{B} / \mathrm{GF})$ alleviates the food allergy-induced neuropathologies.

Method: Balb/c mice were fed a control or $B b / \mathrm{GF}$ diet for 2 weeks prior to and during a 5 week sensitization protocol to ovalbumin (OVA) by gavage. Around the oral challenge with OVA nesting behavior, open field and $T$ maze tests were performed to assess behavior and cognition. Fecal and serum samples, ileal lamina propria cells as well as hippocampal cells were collected for further analysis. Water content in stool, OVA-specific immunoglobulins, activated $\mathrm{T}$ cell subtypes and mRNA expression brain derived neutrophic factor (BDNF), FoxP3 (transcription factor specific for regulatory $\mathrm{T}$ cells) and p-glycoprotein (blood brain barrier marker) were assessed.

Results: The validity of the food allergy model was confirmed by increased OVA-specific IgE levels in serum, diarrhea and enhanced number OVA-specific Th2 cells and reduced numbers of Th1 and regulatory $\mathrm{T}$ cells in the ileum. OVAallergic mice demonstrated impaired nesting behavior, increased anxiety levels and spatial memory deficits. These aberrations were in parallel with decreased expression of mRNA of BDNF and p-glycoprotein in the hippocampi. FACS analysis of the hippocampal cells revealed elevation in the number of $\mathrm{CD} 11 \mathrm{c}^{+} \mathrm{F} 4 / 80^{+} \mathrm{CD} 68^{-}$macrophages and CD69+CD4+ T cells. Bb/GF diet normalized OVAinduced aberrant behavior and cognition and cellular and molecular changes in the brain.

Conclusion: The present data demonstrate that food allergic peripheral inflammation modifies the brain inflammatory status (activated macrophages and Thelper cells) and dampens the cognitive abilities suggesting that food allergy may play a role in the development and/or progression of neurodevelopmental disorders. In addition, targeting the gutimmune-brain axis with $B b / \mathrm{GF}$ diet may have implications for treatment of patients suffering from neurodevelopmental disorders.

Disclosure of Interest: Y. Borre: None Declared., S. De Kivit: None Declared., M. Morgan: None Declared., J. Garssen Employee of: Danone Research, Wageningen, B. Olivier: None Declared., A. Kraneveld: None Declared. 


\section{DOHaD13-1318}

Life stress events from pregnancy to adolescence and risk of young adult depression $\&$ anxiety

Carly E. Herbison ${ }^{1,2, *}$, Karina L. Allen ${ }^{2}$, Monique

Robinson ${ }^{2}$, Julie A. Marsh ${ }^{3}$, John P. Newnham ${ }^{1}$, Craig E. Pennell ${ }^{1}$

${ }^{1}$ School of Women's and Infants' Health; ${ }^{2}$ The Telethon Institute for Child Health Research, Centre for Child Health Research; ${ }^{3}$ School of Mathematics and Statistics, The University of Western Australia, Perth, Australia

Background: Prenatal stress, early life adversity and subsequent negative life events have been independently linked with adolescent and adult depression. The timing of these stressors may impact the development of brain regulatory regions such as the hippocampus, prefrontal cortex and amygdala that are crucial to determining an individual's response to stress and risk of mental health morbidity. The relative importance of stress timing with regard to the onset of depressive illness is debated. Therefore, the aim of this study was to determine whether prenatal, early childhood (ages 1-3), middle childhood (ages 5-10), and adolescent (ages 14-17) stressors, play different roles in predicting depression and anxiety in early adulthood. We hypothesised that the relationship between prenatal stress and depression and anxiety at age 20 would be partially mediated by life stress events in early childhood, middle childhood and adolescence. Method: Participants were from the prospective Western Australian Pregnancy Cohort (Raine) Study, a study of 2868 live births with longitudinal review at 18 and 34 weeks gestation and ages 1, 2, 3, 5, 8, 10,14, 17, 18 and 20. Comprehensive data have been collected allowing for adjustment of potential confounders. Experience of any of 11 life stress events in the past 4 months (in pregnancy) or the past year was reported by the mother or primary caregiver. Symptoms of depression and anxiety were measured using the Depression, Anxiety and Stress Scale (DASS-21) at age 20. At the same time, participants reported whether they had ever received a clinical diagnosis of depression or anxiety.

Analyses were conducted using linear and logistic regression. Univariate and multivariate analyses were performed adjusting for potential confounders. Mediation analyses for continuous outcomes (symptom scores) were performed using three regression equations as described by Baron and Kenny (1986) using Sobels test.

Results: Experience of three or more stress events at 18 weeks gestation, in early childhood, middle childhood and in adolescence was each associated with increased risk of a depression/anxiety diagnosis by age 20 after adjustment for potential confounders (OR 1.70-2.80 p <0.05). This effect was only observed in females.

Higher numbers of stress events during these same developmental periods were also associated with greater depression/anxiety symptoms in females at age 20 after similar adjustment (B 1.28-2.39 $\mathrm{p}<0.05$ ).
The association of prenatal stress at 18 weeks gestation with the development of symptoms of depression and anxiety at age 20 was not mediated by stress events at $2,3,5,8$ or 10 . However, it was partially or completely mediated by life stress events at ages 1,14 and 17 .

Conclusion: Stressful events over the lifespan from pregnancy to adulthood were associated with risk for depression and anxiety in females but not in males. The data suggest that key windows for exposure to stress affecting female mental health are around pregnancy, the first year of life and the time of adolescence. Gender differences require further evaluation and exploration of the factors that promote resilience or sensitivity to stressful life events may offer unique opportunities for prevention.

Disclosure of Interest: None Declared.

\section{DOHaD13-1699}

Maternal emotional reactivity during pregnancy and offspring externalizing symptoms 16 years later

Michelle Horner $^{1, *}$, Kevin Kim ${ }^{1}$, Ralph Tarter ${ }^{1}$

${ }^{1}$ University of Pittsburgh, PIttsurgh, United States

Background: Several studies in humans demonstrate that maternal stress and depression during the antenatal period predict lasting change in offspring's emotion and behavior. It is unclear what aspects of maternal stress and depression are related to this relationship. Chronic negative emotionality activates the maternal HPA-axis and may reflect a mechanism for programming of offspring's dysregulated emotion and behavior. We hypothesized that chronic antenatal emotional reactivity during pregnancy predicts externalizing behavior in offspring with stable, lasting effects.

Method: As part of the Teen Cohort Study, 413 consecutive pregnant teens (67.8\% African American and 32.2\% Caucasian) were recruited from the local regional community obstetric clinic. Mother-child dyads were prospectively tracked from around 17 weeks gestational age to offspring age 16 . Linear regression was used to determine the relation between third-trimester maternal "emotional reactivity" measured using Achenbach's Youth SelfReport and Profile and parent-rated Child-Behavior Checklist of externalizing symptoms of offspring age 10, 14 and 16 .

Results: Maternal emotional reactivity during the third trimester predicted offspring externalizing symptoms at age $10(\beta=.139$, $\mathrm{t}(327)=2.543, \mathrm{p}=.011)$, at age $14(\mathrm{~B}=.21, \mathrm{t}(314)=3.33$, $\mathrm{p}=.001)$, and age $16(\mathrm{~B}=.23, \mathrm{t}(322)=2.26, \mathrm{p}=.025)$.

Conclusion: Preliminary analysis demonstrates a relation between antenatal maternal emotional reactivity on longer-term offspring outcomes known to predict risk for psychiatric disorders such as substance use disorder. Additional studies are warranted to determine the mechanisms driving this relationship.

Disclosure of Interest: None Declared. 


\section{DOHaD13-1245}

\section{Placental weight and sleep onset among infants: Hamamatsu Birth Cohort (HBC) Study}

Kenji J. Tsuchiya ${ }^{1, *}$, Ryosuke Asano ${ }^{1}$, Taeko Harada ${ }^{1}$, Yumeno Kugizaki ${ }^{1}$, Ryuji Nakahara ${ }^{1}$, Chieko Nakayasu ${ }^{1}$, Akemi Okumura ${ }^{1}$, Yukiko Suzuki ${ }^{1}$, Shu Takagai ${ }^{1}$, Hiroaki Itoh $^{2}$, Nori Takei ${ }^{1}$ and HBC Study Team

${ }^{1}$ Research Centre for Child Mental Development, Hamamatsu University School of Medicine; ${ }^{2}$ Perinatal Center, Hamamatsu University School of Medicine, Uniiversity Hospital,

Hamamatsu, Japan

Background: Placental neuroactive steroids have been suggested to facilitate development of GABAergic neurons of the foetal brain [1]. Thus, induction of sleep onset among neonates and infants may be disrupted if secretion of neuroactive steroids is insufficient, since GABAergic system plays a key role in sleep induction [2]. To test this, we investigated whether placental dysfunction is associated with late sleep onset among 10-month-old infants using a birth cohort.

Method: The study participants are term-born, singleton 10-month-old infants, who took part in Hamamatsu Birth Cohort (HBC) Study [3] and had been longitudinally followed-up since their foetal period. Sleep patterns were assessed using Brief Infant Sleep Questionnaire [4], providing sleep-onset time at night, as well as nocturnal sleep duration and daytime sleep duration. Placental function was assessed by placental weight, a good proxy measure for overall placental function [5]. For statistical analyses, placental weight was regressed onto either sleep-onset time at night, adjusting for gender of the infant, parity, maternal socioeconomic class, nocturnal sleep duration, and daytime sleep duration.

Results: In a sample of 749 children, we found that placental weight was negatively associated with sleep-onset time, ie, the heavier the placental weight, the earlier the infants' onset of sleep. The association was significant (Rsquare $=2 \%, \mathrm{p}=.001$ ) even after controlling for potential confounders including sleep pattern properties. For appropriate-for-age (AGA) infants, those with placental weight $<350 \mathrm{~g}$ had an average sleep-onset time of $21.9 \mathrm{~h}$ (95\%CI: 21.5-22.2h) whereas those with placental weight $\geqslant 650 \mathrm{~g}$ had an average sleep-onset time of $21.4 \mathrm{~h}$ (21.2-21.6h). Such a contrast was more clearly replicated among small-for-gestational-age (SGA) infants; those with placental weight $<350 \mathrm{~g}$ had an average sleep-onset time of 22.5h (95\%CI: 21.9-22.9h) whereas those with placental weight $\geqslant 650 \mathrm{~g}$ had an average sleep-onset time of $21.5 \mathrm{~h}$ (21.0-22.0h).

Conclusion: Sleep-onset time, ie, time the infants were induced to fall asleep at night, was significantly associated with placental weight among term-born, singleton infant. The hypotheses we proposed were upheld.

\section{References}

1. Hirst JJ, et al. (2013) Neuroactive steroids in pregnancy: Key regulatory and protective roles in the foetal brain. J Steroid Biochem Mol Biol. In press.

2. Murillo-Rodriguez E, et al. (2012) Basic sleep mechanisms: an integrative review. Cent Nerv Syst Agents Med Chem 12: 38-54.

3. Tsuchiya KJ, et al. (2010) Searching for very early precursors of autism spectrum disorders: the Hamamatsu Birth Cohort for Mothers and Children (HBC). J Dev Origins Health Dis 1: 158-173.

4. Sadeh A (2004) A brief screening questionnaire for infant sleep problems: validation and findings for an Internet sample. Pediatrics 113: e570-577.

5. Salafia CM, et al. (2008) Placental characteristics and birthweight. Paediatr Perinat Epidemiol 22: 229-239.

Disclosure of Interest: None Declared.

\section{DOHaD13-1135}

Pre- and perinatal risk factors for severe mental disorders in adulthood

Marius Lahti ${ }^{1 * *}$, Johan G. Eriksson ${ }^{2}$, Kati Heinonen ${ }^{1}$, Eero Kajantie $^{3}$, Jari Lahti ${ }^{1}$, Kristian Wahlbeck ${ }^{4}$, Soile Tuovinen ${ }^{1}$, Anu-Katriina Pesonen ${ }^{1}$, Tom Forsën ${ }^{3}$, Maiju Mikkonen ${ }^{5}$, David J. P. Barker ${ }^{6}$, Clive Osmond ${ }^{7}$, Katri Räikkönen ${ }^{1}$

${ }^{1}$ Institute of Behavioural Sciences; ${ }^{2}$ Institute of Clinical Medicine, University of Helsinki; ${ }^{3}$ National Institute for Health and Welfare; ${ }^{4}$ The Finnish Association for Mental Health; ${ }^{5}$ University of Helsinki, Helsinki, Finland; ${ }^{6} \mathrm{DOHaD}$ Centre; ${ }^{7}$ MRC Lifecourse Epidemiology Unit, University of Southampton, Southampton, United Kingdom

Background: Increasing evidence suggests that pre-and perinatal adversity may predict an increased risk of mental disorders in childhood and young adulthood. However, it remains unclear whether these effects extend across adult ages. Hence, we examined whether pre-/post-term birth, small or large body size for gestational age, grand multiparity and/or young maternal age at childbirth predict the risk of severe mental disorders in adulthood.

Method: The current study sample included 12711 Helsinki Birth Cohort Study 1934-1944 participants (6624 men and 6087 women). Data on maternal age at childbirth, gestational age, body size at birth, and on parity was derived from hospital birth records. Our diagnostic follow-up extended for over four decades of adult life, from 1969 to 2010. The diagnoses of mental disorders were extracted from national hospital discharge- and causes of death- registers. There were 1676 participants (13.2\%) with severe mental disorders. Our main analytic method was Cox Proportional Hazards Model. All the analyses were stratified for sex and year of birth, and adjusted for socioeconomic position 
in participants' childhood and mothers' marital status at childbirth.

Results: Post-term birth predicted an increased risk of any severe mental disorder (Hazard Ratio $(\mathrm{HR})=1.18 p=.04$ ) and particularly of substance use $(\mathrm{HR}=1.31, p=.01)$ and anxiety $(\mathrm{HR}=1.43, p=.03)$ disorders, and among men, of suicide attempts $(\mathrm{HR}=2.37, p=.04)$. Men born preterm $(\mathrm{HR}=$ $1.98, p=.05)$ were at an increased risk of suicides. Being born small for gestational age predicted an increased risk of any severe mental disorder $(\mathrm{HR}=1.41, p=.02)$ and substance use $(\mathrm{HR}=1.66, p=.01)$ disorders, while women born large for gestational age were at an increased risk of psychotic disorders $(\mathrm{HR}=2.41, p=.02)$. On the other hand, women born to teenage mothers $(\mathrm{HR}=5.38, p=.01)$ were at increased risks of suicides. Grand multiparity predicted an increased risk of suicide attempts $(\mathrm{HR}=2.80, p=.03)$ and among women, it also predicted an increased risk of any severe mental disorder $(\mathrm{HR}=1.83, p=.003)$, and of substance use $(\mathrm{HR}=2.05$, $p=.05), \operatorname{mood}(\mathrm{HR}=2.12, p=.005)$, and psychotic $(\mathrm{HR}=$ $2.57, p=.01)$ disorders. The effects of grand multiparity were more evident among individuals with higher socioeconomic position in childhood, while post-term birth exerted effects on severe mental disorders particularly among individuals with lower childhood socioeconomic position.

Conclusion: Our findings suggest that a number of pre- and perinatal risk factors may predict an increased risk of severe mental disorders across the lifespan.

Disclosure of Interest: None Declared.

\section{DOHaD13-1266}

The effect of parental prenatal anxiety and depression on the risk of depression in the offspring at 18: evidence to support fetal programming?

Lauren E. Capron $^{1, *}$, Susannah Murphy ${ }^{2}$, Rebecca Pearson ${ }^{3}$, Glyn Lewis ${ }^{3}$, Alan Stein ${ }^{2}$, Vivette Glover ${ }^{4}$, Paul Ramchandani ${ }^{1}$

${ }^{1}$ Academic Unit of Child and Adolescent Psychiatry, Imperial College, London; ${ }^{2}$ Dept of Psychiatry, University of Oxford, Oxford; ${ }^{3}$ Academic Unit of Psychiatry, University of Bristol, Bristol; ${ }^{4} I R D B$, Imperial College, London, United Kingdom

Background: There is accumulating evidence that maternal prenatal anxiety and depression are associated with an increased risk of behavioural and emotional problems in the child ${ }^{1}$. Only a few studies have followed the children into adolescence ${ }^{2}$. Evidence from animal studies suggests direct effects on the fetus during pregnancy, which then leads to longer lasting developmental consequences (fetal programming). However, in human studies, it is difficult to disentangle direct prenatal effects from potential effects of enduring anxiety and depression in the postnatal period, and from other confounding effects.

Method: The aim of this study is to assess possible intrauterine effects of maternal depression and anxiety on the offspring by comparing the associations between maternal prenatal depression and anxiety and offspring depression at 18 years of age, with associations between paternal depression and offspring depression. If fetal programming effects are occurring we would expect exposure to maternal depression and anxiety in utero to result in an increased risk of offspring depression, whereas exposure to paternal depression would not. We used data from the ALSPAC study; a longitudinal birth cohort study based in Bristol, UK. Measures of parental depression and anxiety were measured, using the Edinburgh Postnatal Depression Scale (EPDS) and the Crown-Crisp Index at 18 weeks gestation (CCEI). At 18 years of age the children underwent the Clinical Interview Schedule (CIS-R), a structured interview assessment, which diagnoses Depressive Disorder. Standardised cut-offs were used for each measure, and logistic regression analyses undertaken, computing Odds Ratios as a measure of risk. All results were adjusted for confounding variables including postnatal anxiety or depression exposure.

Results: Women with depression (EPDS $\geqslant 13$ ) at 18 weeks gestation were more likely to have a child with a diagnosis of depression at 18 years of age (11.5\% vs.7.3\%; OR 1.45(1.01, 2.08); $\mathrm{p}=0.04)$. Similarly, women who suffered from maternal anxiety (CCEI-anxiety subscale $\geqslant 8$ ) were more likely to have a child who is diagnosed with depression at 18 years of age (11.4\% vs. 6.9\%; OR 1.66(1.22, 2.25); $\mathrm{p}=0.03)$. However, exposure to paternal depression (EPDS $\geqslant 13$ ) was not associated with an increased risk of depression in offspring at 18 years of age $(7.9 \%$ vs. $7.6 \%$; OR $0.45(0.13,1.48) ; \mathrm{p}=0.19)$. Those exposed to paternal anxiety (CCEI-anxiety subscale $\geqslant 5$ ) were not more likely to be diagnosed with depression at 18 years of age (9.5\% vs. $7.0 \%$; OR $1.11(0.78,1.59) ; \mathrm{p}=0.57)$.

Conclusion: There is evidence of an association between maternal prenatal depression and anxiety and the diagnosis of depression in the children at 18 years of age after allowing for postnatal maternal mood. The evidence for an association with paternal depression and anxiety was much weaker. This data shows a difference in the impact of paternal and maternal mood during pregnancy on child outcome and suggest that fetal programming effects may account, at least in part, for this association.

\section{References}

1. Talge, N. et al. JCPP 48, 245-261(2007). 2. Pearson, R. et al. The Am J Psych 170, 1-8(2013).

Disclosure of Interest: None Declared.

\section{DOHaD13-1549}

\section{Could intestinal microbiota be programmed before weaning?}

Fanny Morel ${ }^{1,2,3}$, Hugues Piloquet ${ }^{2}$, Raish Oozeer ${ }^{3}$, Anthony Pagniez ${ }^{2}$, Jan $\mathrm{Knol}^{3, *}$, Annemarie Oosting ${ }^{3}$, Dominique Darmaun $^{2}$, Catherine michel ${ }^{1,2}$ 
${ }^{1}$ INRA $;{ }^{2}$ Université de Nantes - UMR 1280 Physiologie des adaptations nutritionnelles, Nantes, France; ${ }^{3}$ Danone Research, Wageningen, Netherlands

Background: Increasing evidence indicates that nutrition in early life has sustained effects on adult health. Identifying mechanisms underlying this nutritional imprinting may enable new disease prevention strategies. Intestinal microbiota could be a key player in this since i) it affects metabolic homeostasis of its host, ii) its postnatal implement can be modulated by nutrition, iii) its initial set-up is thought to have a sustained impact on microbiota composition throughout life. Our objective was to determine whether preweaning modification of intestinal microbiota could have long-lasting effects in rats.

Method: Suckling rat pups were supplemented with short chain fructo-oligosaccharides (scFOS), galacto-oligosaccharides/ long chain fructo-oligosaccharides mix (scGOS/lcFOS, 9/1), acidic oligosaccharides (AOS), amoxicillin or control solution from postnatal day 5 to 15 then were weaned to standard chow until day 130. We characterized caecocolonic microbiota at day 15 and 130 using qPCR, pyrosequencing and metabolites analyses.

Results: At day 14-15, all the treatments did affect the microbiota. Amoxycillin had a higher impact without being very specific. All oligosaccharides decreased Firmicutes counts whereas bifidobacteria were specifically increased in scFOS and scGOS/lcFOS rats. At day 130 , solely the scGOS/lcFOS preweaning treatment exerted a sustained effect as reflected by an increased OTU richness and decreased counts of Roseburia intestinalis cluster and Erysipelotrichaceae.

Conclusion: scGOS/lcFOS provided before weaning exerted a mild imprinting impact on intestinal microbiota. Such a finding suggest that pioneer bacterial colonizers may be involved in the control of the adult microbiota structure and sustain the idea that intestinal microbiota may act as a long lasting relay of neonatal nutrition and thus contribute to nutritional programming.

Disclosure of Interest: F. Morel: None Declared., H. Piloquet: None Declared., R. Oozeer Employee of: Danone Research, A. Pagniez: None Declared., J. Knol Employee of: Danone Research, A. Oosting Employee of: Danone Research, D. Darmaun: None Declared., C. michel: None Declared.

\section{DOHaD13-1578}

\section{Elective caesarean delivery increases the risk of type 1 diabetes mellitus: a sibling design study}

Ali S. Khashan ${ }^{1, *}$, Louise C. Kenny ${ }^{1}$, Cecilia Lundholm ${ }^{2}$, Patricia M. Kearney ${ }^{3}$, Tong Gong ${ }^{2}$, Catarina Almqvist ${ }^{4}$

${ }^{1}$ The Irish Centre for Fetal and Neonatal Transalational Research (INFANT), University College Cork, Cork, Ireland; ${ }^{2}$ Department of Medical Epidemiology and Biostatistics,
Karolinska Institute, Stockholm, Sweden; ${ }^{3}$ Department of Public Health and Epidemiology, University College Cork, Cork, Ireland; ${ }^{4}$ Department of Public Health and Epidemiology \& Department of Women's and Children's Health at Astrid Lindgren Children's Hospital, Karolinska Institute, Stockholm, Sweden

Background: Caesarean section (CS) has been reported to increase the risk of offspring type 1 diabetes mellitus $(\mathrm{T} 1 \mathrm{DM})^{1}$. The gut microbiota, which are established during delivery and in the first few months of life, have been proposed to play a significant role in stimulating the immune system and increasing the risk of T1DM. We aimed to investigate if there is an association between elective (and hence 'sterile') CS and T1DM, and if the association remains after accounting for familial confounding using sibling-matching design.

Method: We conducted a population-based cohort study of all singleton live births in Sweden between 1973-2009 followed by a sibling-matched analysis. T1DM diagnoses were identified from the Swedish Hospital register using ICD-8 (250), ICD-9 (250) and ICD-10 (E10). Mode of delivery and confounders were retrieved from the Medical Birth Register. Mode of delivery was categorized into spontaneous vaginal birth (reference group), instrumental vaginal birth, emergency CS and elective CS. The statistical analysis was conducted in two steps. First, log-linear Poisson regression was used for the analysis of the cohort study adjusting for offspring age and calendar year as time dependent variables, offspring sex, maternal age, education, diabetes, country of birth and body mass index, small for gestational age, large for gestational age, preterm birth, preeclampsia and birth order. Second, to account for unmeasured familial environmental confounding, such as home environment and life style, and partly for genetic factors, sibling-matched analyses ${ }^{2}$ were conducted using conditional logistic regression. In this analysis siblings who were discordant for both mode of delivery and T1DM were included. The sibling-matched analyses were adjusted for the same potential confounders as the cohort analyses.

Results: 1$)$ In the cohort analyses $(\mathrm{N}=3,511,620)$, there was an increased risk of T1DM among children born by elective CS (adjusted RR $=1.27$, [95\% CI: 1.15-1.39]) compared to children born by spontaneous vaginal birth. However, there was little evidence of an association between emergency CS (adjusted RR $=1.03$, [95\% CI: $0.98-1.08]$ ) or instrumental vaginal delivery (adjusted $\mathrm{RR}=1.07$, [95\% CI: 1.01-1.13]) and T1DM. In sibling-matched analyses $(\mathrm{N}=2433$ pairs of siblings), the association between elective CS and T1DM remained the same but did not reach statistical significance (adjusted OR $=1.27$, [95\% CI: 0.89-1.81). The emergency CS (adjusted OR $=1.10, \quad[95 \%$ CI: $0.91-1.32]$ ) and instrumental vaginal birth (adjusted OR $=1.11$, [95\% CI: 0.94-1.32]) estimates increased slightly. Sensitivity analyses excluding major pregnancy complications did not change the results materially. 
Conclusion: The present findings suggest that mode of delivery may play an important role in increasing the risk of T1DM. We found that children born by elective CS, via a sterile route, had an increased risk of T1DM. These results indicate that the association is due to an independent effect of factors associated with elective CS, rather than familial factors shared by siblings. Given rising CS rates, this finding is of potential public health concern and warrants further investigation.

\section{References}

1) Cardwell et al., Diabetologia 2008;51:726-735.

2) Donovan and Susser, Int J Epidemiology 2011; 40: 345-349.

Disclosure of Interest: None Declared.

\section{DOHaD13-1413}

\section{Maternal and postweaning high fat diet alters caecal microbiota in the adult mouse offspring}

Felino R. Cagampang ${ }^{1, *}$, Yue Liu ${ }^{2}$, Gemma E. Walton ${ }^{2}$, Kimberley D. Bruce ${ }^{1}$, Glenn R. Gibson ${ }^{2}$, Christopher D. Byrne ${ }^{1}$

${ }^{1}$ Faculty of Medicine, Institute of Developmental Sciences, University of Southampton, Southampton; ${ }^{2}$ Department of Food and Nutritional Sciences, University of Reading, Reading,

\section{United Kingdom}

Background: The prevalence of obesity and metabolic diseases are rapidly increasing worldwide, and there are studies to indicate that nutrient excess during early development can increase susceptibility to obesity and features of the metabolic syndrome in adulthood. In mice, we have shown that in-utero and postnatal exposure to high fat diets increase the offspring's susceptibility to metabolic pathologies in adulthood [1]. It has been suggested that alterations in the intestinal microbiome (gut microbiota) are associated with the metabolic syndrome [2]. Diets with different fat contents have been shown to alter the caecal microbiota of mice [3]. Little however is known about the impact of a high fat diet fed to the mother during pregnancy and lactation on microbiota development in the offspring. The aim of the current study was to investigate in mice the effects of maternal and offspring high fat diets on caecal microbiota in the adult offspring.

Method: Female C57/BL6J mice were fed either a high-fat (HF; 45\% kcal fat, SDS, UK) or standard chow (C; 7\% kcal fat, RM1 diet, SDS, UK) diet 4 to 6 weeks prior to conception, through pregnancy and lactation. Resulting offspring were fed either the $\mathrm{C}$ or HF diet after weaning, creating 4 offspring groups; HF/HF ( $n=10$ males, $n=4$ females), HF/C ( $n=7$ males, $n=13$ females), C/HF $(n=5$ males, $\mathrm{n}=3$ females), $\mathrm{C} / \mathrm{C}(\mathrm{n}=11$ males, $\mathrm{n}=7$ females $)$, representing maternal vs. the post-weaning offspring diet, respectively. Offspring were killed at 15 weeks of age and caecal samples collected. Fluorescent in situ hybridisation (FISH) was used to determine bacterial populations. Results were analysed by one-way ANOVA ( $p<0.05$ was considered significant).

Results: The Bacteroides subgroup was found to be significantly reduced by $0.71 \mathrm{log}$ and $1.11 \mathrm{log}$ in the caecum of $\mathrm{HF} / \mathrm{C}$ and $\mathrm{HF} / \mathrm{HF}$ female offspring, respectively (both at $\mathrm{p}<0.01$, ANOVA), compared to $\mathrm{C} / \mathrm{C}$ females. In $\mathrm{HF} / \mathrm{HF}$ male offspring, this was reduced by $0.92 \log (\mathrm{p}<0.01)$ compared to $\mathrm{C} / \mathrm{C}$ males. Clostridium coccoides-Eubacterium rectale was also significantly reduced in the caecum of $\mathrm{HF} / \mathrm{C}$ (by $0.43 \mathrm{log}$ ) and HF/HF (by $0.71 \mathrm{log}$ ) male offspring, and in $\mathrm{HF} / \mathrm{HF}$ females (by $0.48 \mathrm{log}$ ) compared to the corresponding $\mathrm{C} / \mathrm{C}$ mice (all at $\mathrm{p}<0.01$ ). We also found sex-specific changes in microbiota composition; including a reduction by $0.55 \log (\mathrm{p}<0.01)$ in Lactobacillus-Enterococcus in the $\mathrm{HF} / \mathrm{HF}$ females, and an increase by $1.20 \log (\mathrm{p}<0.01)$ in Bifidobacterium in $\mathrm{HF} / \mathrm{HF}$ males compared to corresponding $\mathrm{C} / \mathrm{C}$ animals.

Conclusion: In conclusion, this study has shown that a maternal high fat diet during pregnancy and lactation, with or without further high fat feeding in postnatal life alters caecal microbiota composition in adult offspring. It suggests that the maternal diet plays an important role in the development of gut microbiota of the offspring.

This work is supported by the BBSRC

\section{References}

1. K. D. Bruce et al., Hepatology 50, 1696 (2009).

2. J. M. Kinross et al., Genome Med 3, 14 (2011).

3. N. de Wit et al., Am J Physiol Gastrointest Liver Physiol 303: G589 (2012).

Disclosure of Interest: None Declared.

\section{DOHaD13-1401}

Maternal gut microbiome is altered by maternal diet: the forgotten barrier in developmental programming

K L. Connor ${ }^{1, *}$, C Chehoud ${ }^{2}$, L Chan ${ }^{2}$, S J. Lye ${ }^{1}$

${ }^{1}$ Samuel Lunenfeld Research Institute, Mt Sinai Hospital,

Toronto, Canada; ${ }^{2}$ Second Genome, San Bruno,

United States

Background: The pathway to optimal development of the fetus/infant involves a complex integrated sequence of physiologic processes. Many studies have examined steps along this pipeline, but critical elements, relatively underexplored, are the efficiency of nutrient utilisation/transfer across the maternal gut and the gut microbiome. We speculate that the maternal gut microbiome is a key element in this pipeline during pregnancy and also contributes to the development of the fetal gut thereby supporting growth and 
lifelong health. We hypothesise that maternal malnutrition impacts fetal/infant development, in part through modifications in the maternal gut microbiome.

Method: Female mice ( $n=5 /$ grp) were fed a control diet throughout pregnancy $(\mathrm{CON})$, or calorically restricted by $30 \%$ from embryonic day (E) 5.5-18.5 of pregnancy (CR) or fed a $60 \%$ high fat diet from 8 weeks before mating and throughout pregnancy $(\mathrm{HF})$. At E18.5 (term $=19 \mathrm{~d}$ ) maternal and fetal tissues were collected. DNA was isolated from maternal caecal contents (MoBio DNA kit). 16S rRNA genes were amplified using degenerate primers, hybridised to the G3 PhyloChip ${ }^{\text {TM }}$ and scanned using GeneArray ${ }^{\circledR}$ (Affymetrix). Microbial sequences were filtered and exhaustively analysed using PhyCA-Stats ${ }^{\mathrm{TM}}$ analysis software to determine bacterial abundance and incidence scores. The Adonis test was used to determine significant $(\mathrm{p}<0.05)$ associations between the microbiome and continuous variables such as body weight and cytokine levels.

Results: Microbial communities of mothers sharing specific characteristics during pregnancy were more similar to each other than of mothers not sharing the characteristics. Mothers with similar weight at conception, at E18.5 and weight gain during pregnancy had similar microbiomes. Fetal but not placental weight was associated with the microbiome. Mothers with similar plasma levels of early response proinflammatory cytokines (IL-6, TNF $\alpha$, IFN $\gamma$ ), proinflammatory cytokines (IL-1 $\alpha$, IL-12p70), anti-inflammatory cytokines (IL-10), chemokines (MIP-1 $\alpha$, MIP-1 $\beta$ ) and leptin had similar microbiomes. In maternal small intestinal intraepithelial leukocytes similar levels of early response proinflammatory cytokines (IL-1 $\beta$, IFN $\gamma$ ), proinflammatory cytokines (IL-1 $\alpha$, IL-12p70), anti-inflammatory cytokines (IL-10, IL-2) and chemokines (eotaxin, MCP-1, MIP-1 $\alpha$ ) were associated with similar microbiomes. Maternal diet structured the gut microbiome such that HF mothers had less bacterial richness than CON and CR. HF mothers had significantly distinct microbiomes from $\mathrm{CON}$ and $\mathrm{CR}$ for abundance and incidence of bacterial taxa. Many significantly distinctive taxa were found between dietary groups; selective members of RikenellaceaeII and Lachnospiraceae were more abundant in HF mothers.

Conclusion: The maternal gut microbiome is shaped by a HF diet in the periconceptional period and is influenced by maternal weight as well as local and peripheral inflammatory status during pregnancy. These data suggest that as maternal nutrition and/or body composition are altered before or during pregnancy, shifts in the microbiome may occur. Although the functional significance of these shifts is currently unknown, they may not only impact maternal nutrient utilisation and immune barrier function, but also alter nutrient transfer to the fetus/infant and offspring immunity, influencing developmental trajectories and lifelong health.

Disclosure of Interest: None Declared.

\section{DOHaD13-1634}

Maternal high fat diet alters the pregnancy microbiome in a mouse model

Wajiha Gohir ${ }^{1}$, Fiona Whelan ${ }^{2}$, Michael Surette ${ }^{2}$, Caroline Moore ${ }^{1}$, Jonathan Schertzer ${ }^{1}$, Jay Patel ${ }^{1}$, Deborah M. Sloboda ${ }^{1, *}$

${ }^{1}$ Biochemistry and Biomedical Sciences; ${ }^{2}$ Medicine, McMaster University, Hamilton, Canada

Background: The bacterial composition of the gut microbiome has been shown to contribute to weight gain and altered metabolism. Recently, shifts in the bacterial composition of the maternal gut microbiome have been implicated in maternal metabolic adaptations to pregnancy; microbial shifts that are modified by maternal BMI and pregnancy weight gain. We investigated in the present study, bacterial population shifts in a mouse model of maternal HF feeding and asked how a disruption of the maternal gut microbiome may influence adaptation to pregnancy.

Method: In this pilot study, female C57BL/6 mice were fed either a control or a high fat diet for $8-10$ weeks prior to mating. In order to investigate the effects of gut dysbiosis on pregnancy adaptation, females were further divided into two groups; those that received antibiotics (ATB; bacitracin at $3.514 \mathrm{mM}$, pimaricin $0.13 \% \mathrm{v} / \mathrm{v}$, neomycin at $8.135 \mathrm{mM}$ ) and those that did not, resulting in four groups of mice Con, Con + ATB, HF and HF + ATB ( $n=5$ all groups). All females were introduced to a male and mating was confirmed with the appearance of a vaginal plug. In all cases throughout pregnancy, maternal weight gain, and food intake were recorded and maternal fecal pellets collected. Maternal gut microbiome was determined from fecal DNA by amplification and sequencing the $\mathrm{V} 3$ region of the 16s rRNA gene on an Illumina MiSeq. Relative abundances, taxonomic assignments, and $\mathrm{a} / \mathrm{b}$ diversity were determined using qiime.

Results: Mice fed a HF diet were modestly heavier than Con females at the time of mating and did not gain significantly more weight during pregnancy compared to Con. Although mating was confirmed in all Con + ATB females, none demonstrated gestational weight gain and pregnancy did not occur. In contrast, where mating was confirmed in all Con, HF and HF+ATB mice, gestational weight gain and pregnancy was established. Pregnant HF+ATB females showed significantly lower caloric intake and weight gain throughout pregnancy compared to Con and HF mice. HF feeding was associated with changes in the microbiome. A HF diet prior to pregnancy resulted in a decrease in the Bacteroidetes: Firmicutes ratio compared to Con and this shift was maintained in pregnant mice fed a high fat diet. The most striking result was that the microbiome of HF fed pregnant mice that had gut dybiosis due to ATB intake, was dominated by a single genus; Lactococcus. Conclusion: It appears that disruption of the female gut microbiome (with ATB) compromised the establishment of pregnancy in Con fed mice but not in HF fed mice. Whether 
the observed shifts in the maternal gut microbiota modulate the maternal-placental-fetal relationship is unknown. Ongoing studies are investigating the association between shifts in maternal gut bacterial populations and fetal and placental inflammatory pathways.

Disclosure of Interest: None Declared.

\section{DOHaD13-1346}

\section{Maternal perinatal microbiota composition and transfer to infants}

Olga Sakwinska ${ }^{1, *}$, Catherine Ngom-Bru ${ }^{1}$, Shaillay Dogra ${ }^{2}$, Wolfram Brueck ${ }^{1}$, Bernard Berger ${ }^{1}$, Melissa Lepage ${ }^{1}$, Ivan Montoliu Roura ${ }^{1}$, Harald Bruessow ${ }^{1}$, Lee Yung Seng ${ }^{2}$, Kenneth Kwek ${ }^{3}$, Chong Yap-Seng ${ }^{4}$, Peter D. Gluckman ${ }^{2}$, Joanna D. Holbrook ${ }^{2}$

${ }^{1}$ Nestle Reasearch Center, Lausanne, Switzerland; ${ }^{2}$ Singapore Institute of Clinical Sciences (SICS); 'Women's and children's hospital; ${ }^{4}$ National University of Singapore, Singapore, Singapore

Background: Mother to infant microbiota transfer has been suggested to influence long-term health outcomes of infants. Compared to vaginal delivery, altered microbiota acquisition has previously been observed in infants delivered by cesarean section. This led to the hypothesis that early dysbiosis may be the cause of the known negative impacts of cesarean section on the infant, such as increased risk of allergies and childhood overweight.

Method: The composition of perinatal microbiota and the transfer routes from mother to infant remain poorly known. We have collected the microbiota samples from 42 motherinfants pairs who are part of the Growing Up in Singapore Towards healthy Outcomes (GUSTO) birth cohort. Maternal body sites were sampled at the delivery, and included breast skin $(\mathrm{n}=12)$, rectum $(\mathrm{n}=33)$, vulva $(\mathrm{n}=27)$, and vagina $(\mathrm{n}=33)$. Vaginal samples were also collected at 34-36 weeks of pregnancy. Infant fecal samples were collected 3 days $(\mathrm{n}=29)$ and 3 weeks $(\mathrm{n}=27)$ after birth. The microbiota composition was assessed by 454 pyrosequencing of PCR-amplified fragments of the bacterial 16S rRNA gene. Taxonomic assignation was performed with RDP classifier. The sequencing reads were clustered in operational taxonomic units (OTUs) with $97 \%$ identity.

Results: While 61\% (20/33) of the women harboured Lactobacilla-dominated normal vaginal microbiota, 27\% (9/33) had mixed microbiota with large proportion of Gardnerella, a genus strongly associated with bacterial vaginosis. Surprisingly, the vaginal microbiota of 3 women was composed predominantly of Bifidobacteria. As expected, different maternal body sites harboured highly differentiated microbial communities. The analysis based on unifrac distances indicated that infant gut microbiota was highly dissimilar to maternal microbiota. However, it resembled the most vaginal microbiota at 34-36 weeks gestation, followed by vaginal microbiota at birth and vulval and rectal microbiota.
None of the 8 infants born by cesarean section harboured Bifidobacteria in the gut on day 3; in contrast, microbiota of $43 \%$ (9/21) infants born vaginally was composed mostly (>80\%) of Bifidobacteria. By week 3, 52\% (11/21) vaginally born infants, and 33\% (2/6) infants delivered by cesarean section had Bifidobacteria-dominant microbiota. Even thought the difference in overall microbiota composition between vaginally and cesarean born infants decreased from day 3 to week 3 , it remained statistically significant (unifrac distances, $\mathrm{p}<0.001)$.

Conclusion: Maternal vaginal and rectal microbiota appeared to seed the infant gut microbiota. Genetic proximity between Gardnerella and Bifidobacterium, and mismatches to primers commonly used in microbiota profiling studies, has very likely led to misclassification and non-detection of these two taxa in previous studies. Specific qPCR for both groups will be used to confirm the identity of these taxa in vaginal samples.

Infants born via cesarean section showed delayed establishment of normal infant gut microbiota dominated by Bifidobacteria. In contrast, infants born vaginally already harboured more developed, Bifidobacteria-dominated microbiota 3 days after birth.

Disclosure of Interest: O. Sakwinska Employee of: Nestec Ltd, C. Ngom-Bru Employee of: Nestec Ltd, S. Dogra: None Declared., W. Brueck Employee of: Nestec Ltd, B. Berger Employee of: Nestec Ltd, M. Lepage Employee of: Nestec Ltd, I. Montoliu Roura Employee of: Nestec Ltd, H. Bruessow Employee of: Nestec Ltd, L. Yung Seng: None Declared., K. Kwek: None Declared., C. Yap-Seng: None Declared., P. Gluckman: None Declared., J. Holbrook: None Declared.

\section{DOHaD13-1643}

\section{The effect of low birth weight on indoxyl sulfate and OAT1/3 expression}

Jacob Pearson ${ }^{1}$, Barent DuBois ${ }^{1, *}$, Bonnie Hastings ${ }^{1}$, Alison Edelman $^{2}$, Ganesh Cherala ${ }^{1}$

${ }^{1}$ OSU/OHSU; ${ }^{2}$ Department of Obstetrics \& Gynecology, OHSU, Portland, United States

Background: Indoxyl Sulfate (IndS) is a product of dietary tryptophan metabolism; tryptophan is first metabolized by the gut microbiota before undergoing hepatic conjugation into IndS. Elevated serum IndS is nephrotoxic, cardiotoxic, decreases free-radical scavenging, and increases oxidative stress. Renal elimination of IndS is reported to prevent these adverse effects; IndS increase may contribute to cardiovascular risk with low GFR. Low birth weight (LBW) prognosticates adult onset of CVD and CKD. The risk of disease is thought to emanate from differential programming in utero which resonates into the adult phenotype, permanently altering organ morphology and physiology. Since LBW and elevated serum IndS share similar pathophysiologies, we hypothesize that LBW subjects are 
susceptible to elevated IndS. To test this, we investigated the status of serum IS in LBW subjects in multiple species.

Method: Human birth weights are self-reported. LBW was induced in rats through maternal protein restriction during gestation and lactation; the resultant offspring were then studied as adults. Only woman and female animals were investigated. High Pressure Liquid Chromatography was used to quantify IndS in serum, liver, kidney, brain and creatinine clearance in rats. Western Blotting was performed for drug transporters OAT1 and OAT3.

Results: All data expressed as mean \pm standard deviation. In rats, serum IndS was significantly increased $(0.89 \pm 0.18 \mu \mathrm{M}$ control to $2.45 \pm 1.35$ LBW; $\mathrm{p}<0.05, \mathrm{n}=5)$ while a trending increase was found in human $(<5.5 \mathrm{lbs}$ or $>8.0 \mathrm{lbs}$ at birth; $\mathrm{p}=0.06$ ). LBW reduced absolute creatinine clearance in rats $(4.22 \pm 0.63 \mathrm{~mL} / \mathrm{min}$ control to $3.41 \pm 0.25 \mathrm{LBW}$; $\mathrm{p}=0.017, \mathrm{n}=5-6$ ). However, there was no difference in creatinine clearance when normalized to body weight, implying that increased IndS in LBW is not entirely explained by altered renal function. We also measured, in the rat, the distribution of IndS in multiple organs, as well as the expression of transporters that mediate the flux of IndS across these compartments. LBW had no effect on hepatic or renal IndS concentration or transporter expression; however, decreased brain IndS $(22.18 \pm 11.94 \mu \mathrm{M}$ control to $14.03 \pm 2.50 \mathrm{LBW} ; \mathrm{p}=0.052$, $\mathrm{n}=5-6)$ and increased Oat3 expression (31.7\%, $\mathrm{p}<0.05$, $\mathrm{n}=4-5$ ) was observed. There was no difference in food consumption in rats, suggesting increased serum IndS cannot be explained by increased tryptophan consumption.

Conclusion: Our findings indicate that LBW subjects tend to have elevated IndS, a finding that is not explained by increased food consumption; however, decreased renal function is possible as suggested by creatinine clearance. Gut bacteria have not been investigated and could also help explain this finding. Additionally, LBW rats exhibit organ-specific interaction with IndS, possibly due to changes in transporter expression.

Disclosure of Interest: None Declared.

\section{DOHaD13-1086}

Medication influencing the gastro intestinal milieu detrimentally affects sperm motility in men visiting the preconception outpatient clinic

Nicole A. Huijgen ${ }^{1}, *$, Hedwig Goijen ${ }^{1}$, John Twigt ${ }^{1}$, Annemarie Mulders ${ }^{1}$, Jan Lindemans ${ }^{2}$, Gert Dohle ${ }^{3}$, Eric Steegers ${ }^{1}$, Joop Laven ${ }^{1}$, Régine Steegers-Theunissen ${ }^{1}$

${ }^{1}$ Obstetrics and Gynaecology; ${ }^{2}$ Clinical Chemistry; ${ }^{3}$ Department of Urology, Erasmus MC, University Medical Centre,

Rotterdam, Netherlands

Background: In the Netherlands, subfertility occurs in around $10 \%$ of the couples, in which $45 \%$ is due to a male factor. Medication influencing the gastro intestinal (GI) tract is frequently used in the reproductive population. The uptake of (micro) nutrients, such as vitamin B12 and folate, is dependent on the composition of the GI milieu. It is hypothesized that medication affecting the GI tract induces a shortage of these micronutrients and subsequently deranges the one carbon pathway important for spermatogenesis.

Method: In a periconception cohort study, performed between October 2007 and March 2011, 1251 men visited the preconception outpatient clinic 'Achieving a Healthy Pregnancy' of the department of Obstetrics and Gynaecology at the Erasmus MC, Rotterdam in the Netherlands. General questionnaires were completed and anthropometric measurements, blood samples and semen analysis were obtained. From this cohort a group of men without use of vitamin supplements and with a semen analysis performed between 70 days prior up to 21 days after the first visit were included. Medication influencing the GI milieu comprises antacids, proton pump inhibitors (PPI's), histamine-2 receptor antagonists, metformin, carbasalate calcium and antibiotics. Multivariable linear and logistic regression models adjusted for potential confounders were performed and effect estimates were calculated.

Results: In total, 503 men met the inclusion criteria, with a median age of 34 years, a BMI of $26.4 \mathrm{~kg} / \mathrm{m}^{2}$ and $62.8 \%$ was of Dutch ethnicity, $22.7 \%$ used medication, $32.2 \%$ smoked and $68.6 \%$ used alcohol. Men using medication influencing the GI milieu, showed a nearly 3-fold higher risk for having a low motile semen concentration $(\mathrm{VCM}=$ semen volume $\mathrm{x}$ semen concentration $\mathrm{x}$ percentage motile semen $=<3 \times 10^{6}$ ) (OR 2.71; 95\%CI 1.17-6.30), after adjustment for smoking. Men using antacids, histamine-2 receptor antagonists and/or PPI's showed a more than 3-fold increased risk for having a low VCM, 3.47 OR (95\%CI 1.13-10.68), adjusted for smoking.

Univariable linear regression showed an inverse association between BMI and semen volume $(\beta-0.013 \mathrm{p}<0.01)$ and between smoking and VCM $(\beta-0.195 \mathrm{p}<0.05)$, semen count $(\beta-1.317 \mathrm{p}<0.05)$ and semen volume $(\beta-0.124$ $\mathrm{p}<0.01)$. Alcohol use and the Dutch ethnicity were associated with a higher semen volume (respectively $\beta$ $0.104 \mathrm{p}>0.05$ and $\beta 0.157 \mathrm{p}<0.01$ ).

Multivariable linear regression showed an inverse association between GI medication and semen concentration $(\beta-0.399 \mathrm{p}<0.05)$ adjusted for smoking and alcohol use, age, ethnicity and BMI. Red blood cell (RBC) folate is positively associated with the percentage motile semen $(\beta 7.246 \mathrm{p}<0.05)$ and with VCM $(\beta 0.441 \mathrm{p}<0.05)$. Serum folate is positively associated with semen volume $(\beta$ 0.008; $\mathrm{p}<0.05)$ and semen count $(\beta 0.108 \mathrm{p}<0.05)$.

Conclusion: A significant detrimental effect of very commonly used medication influencing the GI milieu on motile semen concentration is shown. Furthermore, smoking men, just as men with low RBC and/or serum folate, or men with a high BMI are more prone to have a poor semen quality. Hence, these findings support the importance to offer preconception counselling to men as well.

Disclosure of Interest: None Declared. 


\section{DOHaD13-1647}

\section{The contribution of paternal obesity to newborn weight and body composition}

Lodewyk DuPlessis $^{1, *}$, Andrea Deussen ${ }^{1,2}$, Rosalie Grivell $^{1,2,3}$, Lisa Yelland ${ }^{1,4}$, Andrew McPhee ${ }^{5}$, Jeffrey Robinson ${ }^{1,4}$, Julie Owens ${ }^{1,4}$, Jodie Dodd ${ }^{1,3,4}$

${ }^{1}$ Discipline of Obstetrics \& Gynaecology; ${ }^{2}$ The Robinson Institute, The University of Adelaide; ${ }^{3}$ Department of Perinatal Medicine, Women's and Children's Hospital; ${ }^{4}$ Robinson Institute, The University of Adelaide; ${ }^{5}$ Department of Neonatal Medicine, Women's and Children's Hospital, North Adelaide, Australia

Background: While the association between maternal and childhood obesity is relatively well recognised, paternal contributions to fetal growth, infant birth weight and infant body composition are less clearly described. While paternal height has been correlated with birth weight and measures of skeletal growth, the precise effects of paternal weight, and BMI on early infant body composition are less clearly defined.

Method: We conducted a nested prospective cohort study within the LIMIT randomised trial. Detailed anthropometric measurements from 257 maternal, paternal and infant triads were taken, using a standardised protocol. Infant anthropometric outcomes were analysed using linear regression models to test for the effect of four paternal body mass index (BMI) categories $\left(<25.0 \mathrm{~kg} / \mathrm{m}^{2}, \quad 25.0-29.9 \mathrm{~kg} / \mathrm{m}^{2}, \quad 30.0-34.9 \mathrm{~kg} / \mathrm{m}^{2}\right.$ and $\geq 35.0 \mathrm{~kg} / \mathrm{m}^{2}$ ), whilst controlling for maternal and infant factors. Results: Increasing paternal BMI was not associated with a statistically significant increase in mean infant birth weight, birth length, or body circumferences. However, increasing paternal BMI was associated with a significant increase in both infant suprailiac and thigh skinfold thickness measurements, particularly where paternal BMI was above $35 \mathrm{~kg} / \mathrm{m}^{2}$. While increasing paternal BMI was not associated with statistically significant differences in infant fat mass or fat free mass, increasing BMI was associated with an increase in percentage body fat in infants.

Conclusion: Our findings of an association between paternal BMI and increased skin-fold measures and percentage body fat in infants, particularly for men with BMI above $35 \mathrm{~kg} / \mathrm{m}^{2}$, highlights the need for ongoing research targeting interventions among overweight and obese men.

Disclosure of Interest: None Declared.

\section{DOHaD13-1139}

\section{A diet high in sugar and fat alters the lipid metabolic profile of the mouse placenta}

Barbara Musial $^{1, *}$, Owen Vaughan ${ }^{1}$, Abigail Fowden ${ }^{1}$, Amanda Sferruzzi-Perri ${ }^{1}$

${ }^{1}$ Physiology, Development and Neuroscience, Cambridge University, Cambridge, United Kingdom
Background: In developed societies, diets high in sugar and fat (HSHF) are now the norm and contribute to the increasing rates of maternal obesity during pregnancy. Maternal obesity and/or enhanced fat accumulation during pregnancy are associated with abnormal birth weight and an increased risk of the infant developing metabolic disease in later life. Similarly, feeding pregnant mice HSHF diets alters the metabolic phenotype of their adult offspring. However, little is known about the mechanisms involved prenatally. Our preliminary work has shown that HSHF feeding of pregnant mice increases adiposity and influences expression of proteins involved in lipid metabolism in several maternal tissues. These changes were also associated with altered fetoplacental growth. This study examined the effect of HSHF feeding on placental fat content and abundance of proteins involved in lipid metabolism and transport at day (D) 16 (maximum placental growth) and D19 (maximum fetal growth) of mouse pregnancy (term D20.5).

Method: C57BL6 mice were fed a control diet (energy from fat $11 \%$, sugar $7 \%$ ) or a HSHF diet (energy; fat 23\%, sugar $20 \%$ ) from D1 of pregnancy. On D16 and D19, dams were killed and placentas and fetuses were dissected, weighted and snap frozen. Total maternal fat content was measured by dual emission X-ray absorbance on the hysterectomised dams. Western blotting was used to determine the abundance of peroxisome proliferator-activated receptor (PPAR) $\gamma$ and $\alpha$, sterol regulatory element of binding protein (SREBP), lipoprotein lipase (LPL), fatty acid transport protein 1 (FATP1) and fatty acid synthase (FAS) in one placenta from 5-6 litters per diet. Fat content was measured by a modified Folch method in another placenta from 7-9 litters per diet. Statistical significance was assessed using t-test and accepted when $\mathrm{P}<0.05$. All experiments were carried out under the UK Animals (Scientific Procedures) Act 1986.

Results: Total maternal fat content increased 30-40\% with HSHF feeding at both ages $(\mathrm{P}<0.05)$. HSHF feeding reduced placental and fetal weight by $6-10 \%$ compared to controls at D16 and D19 $(\mathrm{P}<0.001)$. At D16, HSHF feeding reduced placental abundance of PPAR $\alpha(-43 \%$, $\mathrm{P}=0.01)$ and increased abundance of SREBP $(+41 \%$, $\mathrm{P}=0.04)$. In contrast, at $\mathrm{D} 19$, placental expression of PPAR $\alpha$ and SREBP had normalised but placental FATP1 was now twice as abundant in HSHF than control dams $(+106 \%, P=0.01)$. HSHF feeding had no effect on placental expression of LPL, PPAR $\gamma$ and FAS or on placental fat content at either age.

Conclusion: HSHF feeding increased maternal adiposity at the expense of feto-placental growth. This was associated with altered placental expression of proteins involved in fatty acid catabolism and utilisation at day 16 and those involved in lipid uptake and transport at day 19. Since placental fat content was not increased, more fat may have been utilised by the placenta or transferred to the fetus, which could help maintain fetal growth when placental growth was compromised by the obesogenic diet. However, these placental 
adaptations and the fetal growth restriction may have important roles in programming metabolic dysfunction and overt metabolic disease in later life.

Disclosure of Interest: None Declared.

\section{DOHaD13-1680}

\section{A maternal high fat diet results in elevation in amniotic fluid lipid concentrations}

Sarah Henry ${ }^{1, *}$, Jacqui Weir ${ }^{2}$, Peter Meikle ${ }^{2}$, Ryan WoodBradley $^{1}$, Luise Cullen-McEwen ${ }^{1}$, John Bertram ${ }^{1}$, James Armitage ${ }^{3}$

${ }^{1}$ Anatomy \& Developmental Biology, Monash University;

${ }^{2}$ Baker IDI Heart and Diabetes Institute, Melbourne;

${ }^{3}$ Optometry, Deakin University, Waurn, Australia

Background: An understanding of how maternal fat intake alters fetal development and lipid metabolism is limited. Until keratinisation of the fetal skin occurs, there is no epithelial barrier so amniotic fluid lipid content reflects fetal plasma lipid status, allowing us to probe fetal lipid metabolism under conditions of altered maternal dietary intake. We hypothesise that exposure to excess saturated fats during development will alter fetal metabolism of lipids, resulting in increased accretion of fatty acids and altered fetal growth. In addition, we investigated the mechanism potentially involved in altered fatty acid accumulation in the amniotic fluid, including changes in placental transport genes or lipid synthesis in the fetal liver

Method: Female Sprague-Dawley rats $(\mathrm{n}=4-7$ per group) were fed either a control (7\% canola oil) or lard rich (HF) (3\% canola oil and $20 \%$ lard) diet for 3 weeks prior to mating and throughout pregnancy until embryonic day 14.25 (E14.25) and 20 (E20). Dams were euthanised, a blood sample taken and the uterus removed. Amniotic fluid was collected and frozen. Embryos and placentas were dissected and weighed. Lipid profiles were determined in amniotic fluid and maternal plasma by performing lipid extraction followed by liquid chromatography mass spectrometry (LCMS) at E14.25 and E20. Internal standards were used to quantify diacylglyceride (DG), triacylglyceride (TG) and phosphatidylcholine (PC) species and expressed as $\mathrm{pmol} / \mathrm{ml}$. Data were compared by 1-way ANOVA with maternal diet as the factor. Data were analysed as total (measured) DG, TG and PC, then individual species were compared. Placental and fetal liver gene expression was determined using real time PCR. Data are presented as mean \pm SEM.

Results: At E14.25, there was an effect of in utero HF exposure on feto-placental ratio, with a significant decrease in embryo:placenta ratio in HF exposed animals $(\mathrm{p}<0.05)$. Although summed lipid totals in amniotic fluid were unaffected, HF feeding had a significant effect on lipid concentrations of a number of individual lipids at E14.25.
At E14.25, placental gene expression of GLUT4 was significantly decreased in placentas from HF exposed dams but there was no difference in mRNA expression of enzymes involved in lipid transport. By E20, maternal HF feeding was associated with hypertriglyceridemia of the amniotic fluid. There were significant increases in total and saturated triglycerides, increased in the amniotic fluid of HF exposed embryos by $96 \%$ and $64 \%$ respectively $(\mathrm{P}<0.05)$, however there was no effect of maternal diet on embryo or placental weight, nor was there any change in the expression of placental fatty acid transporters. Analysis of fetal livers showed no effect of maternal diet on the mRNA expression of proteins involved in fatty acid synthesis.

Conclusion: Maternal consumption of a HF diet in pregnancy is associated with profound increases in amniotic fluid fatty acid profiles but this accumulation is not related to increased mRNA expression of key transporters and enzymes in the placenta or fetal liver. Further investigation of lipid fluxes across the placenta; using functional assay is warranted, in order to determine how this fetal dyslipidaemia is brought about.

Disclosure of Interest: None Declared.

\section{DOHaD13-1496}

Altered levels and expression of maternal, placental and cord blood antioxidant enzymes in preterm deliveries

Sadhana Joshi ${ }^{1}$ on behalf of Nutritional Medicine, Madhavi Dhobale $^{1, *}$, Hemlata Pisal ${ }^{1}$, Savita Mehendale ${ }^{2}$

${ }^{1}$ Nutritional Medicine, Interactive Research School for Health Affairs; ${ }^{2}$ Obstetrics and Gynaecology, Bharati Medical College and Hospital, Pune, India

Background: During pregnancy, the intrauterine environment generates oxidative stress which influences both the physiology and pathology of the feto-placental unit, thereby affecting pregnancy and fetal outcome. It is therefore important to understand the mechanistic role of antioxidant enzymes in preterm deliveries. This study for the first time examines the mRNA levels of placental superoxide dismutase (SOD), catalase (CAT) and glutathione peroxidase (GPx) and their levels from maternal and cord erythrocyte samples in term and preterm deliveries.

Method: 97 women delivering preterm $(<37$ weeks gestation) and 84 women delivering at term $(=>37$ weeks gestation) were recruited for the study. All samples were taken at the time of delivery. Placental mRNA levels of SOD, GPx and CAT were analysed by real time quantitative polymerase chain reaction. SOD, GPx and CAT levels were estimated using kits. Mean values of the estimates of various parameters for the preterm group was compared with those of term group at conventional levels of significance $(\mathrm{p}<0.05$, $\mathrm{p}<0.01$ ) using student ' $\mathrm{t}$ ' test. 
Results: Placental mRNA levels of SOD, CAT and GPx were higher ( $\mathrm{p}<0.05$ for all) in the preterm group as compared to the term group. Maternal erythrocyte GPx $(\mathrm{n}=39$; $146.6 \pm 43.5 \mathrm{mU} / \mathrm{ml})(\mathrm{p}<0.05)$ levels were higher but lower in the SOD $(\mathrm{n}=76 ; 2791.1 \pm 739.9 \mathrm{U} / \mathrm{ml})(\mathrm{p}<0.01)$ and CAT $(\mathrm{n}=97 ; \quad 10682.5 \pm 5082.2 \mathrm{U} / \mathrm{ml}) \quad(\mathrm{p}<0.05) \quad$ in the preterm group. Further, the cord $\operatorname{SOD}(\mathrm{n}=61$; $2829.4 \pm 989.6 \mathrm{U} / \mathrm{ml}), G P x(\mathrm{n}=44 ; 94.8 \pm 23.6 \mathrm{mU} / \mathrm{ml})$ and CAT $(\mathrm{n}=93 ; 9496.5 \pm 4511.2 \mathrm{U} / \mathrm{ml})$ levels were lower in the preterm group as compared to the term group.

Conclusion: Our findings suggest that the higher mRNA expression of placental antioxidant enzymes may be a response to increased oxidative stress during pregnancy. The altered response of placental antioxidant enzymes may have important implications in fetal development and preterm delivery. This altered maternal antioxidant enzyme defense mechanism may increase the risk for adult diseases through fetal programming via epigenetic mechanisms in preterm deliveries.

Disclosure of Interest: None Declared.

\section{DOHaD13-1150}

\section{Cognitive performance in Indian children: relationship to placental morphology}

Sargoor R. Veena ${ }^{1, *}$, Ghattu Krishnaveni ${ }^{2}$, Krishnamachari Srinivasan ${ }^{3}$, Caroline Fall ${ }^{4}$

${ }^{1}$ Epidemiology Research Unit, CSI Holdsworth Memorial Hospital, Mysore; ${ }^{2}$ Epidemiology Research Unit, CSI Holdsworth Memorial Hospital, Myosre; ${ }^{3}$ St John's Research Institute, St. John's National Academy of Health Sciences, Bangalore, India; ${ }^{4} M R C$ Lifecourse Epidemiology Unit, University of Southampton, Southampton, United Kingdom

Background: The size, morphology and nutrient transfer capacity of the placenta that determines fetal growth may also influence health in later life. The role of placental morphology in predicting cognitive performance either during childhood or adult life is largely unknown. We aimed to determine whether the size and shape of the placenta predict cognitive ability in school aged Indian children.

Method: We studied healthy boys $(\mathrm{n}=255)$ and girls $(n=278)$ from a birth cohort, for whom placental weight, length and breadth were measured. The children's cognitive function (learning, long-term retrieval/storage, short-term memory, reasoning, visuo-spatial and verbal abilities, attention and concentration) was assessed at a mean age of 9.7 years using 3 core tests from the Kaufman Assessment Battery for children-II edition, Wechsler Intelligence Scale for Children-III edition and additional tests. Data on a variety of potential confounders (birthweight, maternal age and BMI in pregnancy, parental education, socio-economic status, occupation and income) were also recorded. The association between placental morphology and childhood cognitive function was examined using multiple linear regression analysis.

Results: Placental weight, length, and breadth were similar in boys and girls. Girls scored better than boys in tests of reasoning, short-term memory, verbal ability-names and attention and concentration ( $\mathrm{p}<0.05$ for all). Adjusted for gestational age and current age, among girls, placental weight was positively associated with learning/long-term retrieval $(\beta=0.18 \mathrm{SD} / 100 \mathrm{~g}$ increase in placental weight), reasoning and verbal ability ( $\beta=0.14$ for both) $(\mathrm{p}<0.05$ for all). In boys there was an inverse association between placental weight and these outcomes $(\beta=-0.08,-0.10$ and -0.17 respectively), significant only for verbal ability $(p=0.03$ ). After adjusting for potential confounders, the associations in girls were largely attenuated $(\beta=0.15,0.10$ and 0.04 respectively) and lost their significance. In boys, while the negative effect on learning, long-term retrieval and reasoning became stronger and significant $(\beta=-0.22$, and -0.25 respectively; $\mathrm{p}<0.05$ for both), the effect size on verbal ability attenuated $(\beta=-0.10)$ and lost significance. There was a positive association between placental length and visuospatial ability in boys $(\beta=0.09 \mathrm{SD} / \mathrm{cm}$ increase in length; $\mathrm{p}=0.004)$ but the association diminished $(\beta=0.05)$ and lost significant after adjusting for confounders. In girls this association was negative and not significant $(\beta=-0.008$; $\mathrm{p}=0.8)$ but became stronger and significant after controlling for confounders $(\beta=-0.07 ; p=0.047)$. There was no association of placental breadth with any of the cognitive ability. Conclusion: In this Indian population we found different associations of placental morphology with cognitive ability in boys and girls. Changes in these associations after adjustment for potential confounders are likely to reflect confounding effect by socio-environmental factors.

Disclosure of Interest: None Declared.

\section{DOHaD13-1273}

\section{Essential fatty acid and prostaglandin pathways are altered in the placentae of fat fed rats}

Marloes Dekker Nitert ${ }^{1}$, Kanchan Vaswani ${ }^{1}$, Melissa Hum ${ }^{1}$, Hsiu-Wen Chan ${ }^{1}$, Sarah L. Henry ${ }^{2}$, Ryan J. Wood-Bradley ${ }^{2}$, Murray D. Mitchell ${ }^{1}$, Gregory E. Rice ${ }^{1}$, James A. Armitage ${ }^{2,3,4, *}$

${ }^{1}$ Centre for Clinical Research, University of Queensland, Brisbane; ${ }^{2}$ Anatomy and Developmental Biology, Monash University, Clayton; ${ }^{3}$ School of Medicine (Optometry), Deakin University, Waurn Ponds; ${ }^{4}$ Baker IDI Heart and Diabetes Institute, Melbourne, Australia

Background: Offspring of obese dams can go on to demonstrate programmed disease, however there is little understanding as to whether maternal high fat intake (in the absence of obesity) programmes offspring disease or dysfunction. Moreover, the role of the placenta in programming offspring 
requires greater investigation. We employed a microarray approach, combined with pathway analysis algorithms to determine genome wide expression in the placentae of rats fed a high fat diet during pregnancy.

Method: Sprague Dawley rats were fed either a high fat $(23 \%$ $\mathrm{w} / \mathrm{w}$ lard, $\mathrm{n}=10)$ or control $(6 \% \mathrm{w} / \mathrm{w}$ soy oil, $\mathrm{n}=10)$ diet ad libitum for 3 weeks prior to mating (which occurred in a 3 hour window to carefully control embryonic age) and throughout pregnancy. In late gestation (embryonic day 17.5) animals were humanely killed and the placentae snap frozen. Tissues were homogenized and aliquots of homogenate processed to isolate total RNA or protein. Placental RNA expression was determined by Illumina beadChip microarray and data analysed (Lumi package). Pathway analysis was undertaken. Individual gene and protein expression were confirmed by qPCR and Western blot.

Results: Pathway analysis indicated upregulation of placental MAPK signaling $(\mathrm{P}<0.003)$ arachidonic acid metabolism $(\mathrm{P}<0.039)$, chemokine signaling $(\mathrm{P}<0.039)$ and glycosphyingolipid biosynthesis $(\mathrm{P}<0.043)$ in offspring of high fat fed rats. Downregulated gene sets included regulators of angiogenesis $(\mathrm{P}<0.044)$, apoptosis $(\mathrm{P}<0.044)$ and developmental growth $(\mathrm{P}<0.044)$. Offspring of fat fed dams demonstrated elevated placental COX2 protein (1.9 fold; $\mathrm{P}<0.05$ ), and LIMK1 (4.1 fold, $\mathrm{P}<0.05$ ) expression.

Conclusion: Maternal high fat intake in the absence of maternal obesity appears to be associated with elevated fetal inflammatory pathways and a reduction in apoptotic pathway expression. These data indicate that placental-derived inflammatory processes can programme altered fetal growth; presumably rendering individuals prone to programmed disease in adult life.

Disclosure of Interest: None Declared.

\section{DOHaD13-1244}

\section{Identification of candidate genes in human placenta involved in the development of childhood allergy}

Astrud Tuck ${ }^{1}$, Annette Osei-Kumah ${ }^{1}$, Zarqa Saif ${ }^{1}$, Vicki Clifton ${ }^{1, *}$

\section{${ }^{1}$ Robinson Insitute, University of Adelaide, Adelaide, Australia}

Background: The prevalence of childhood allergic disease has increased dramatically in developed countries. The increasing prevalence of allergy with modern urbanisation and the early onset of disease in childhood indicates that allergy originates during prenatal life. Modern environmental changes are hypothesised to be causing deviations in fetal programming, in which the placenta plays a central role, increasing the susceptibility and prevalence of disease throughout life. We hypothesised that susceptibility to childhood allergy is determined by changes in placental function that programs immune function in the fetus in a sex-specific manner. In this study we aimed to identify candidate genes and pathways in human placental tissue that may contribute to the development of childhood allergy.

Method: Human placental tissue was obtained after delivery from women giving informed consent at the Lyell McEwin Hospital, Adelaide, and global gene expression was examined via microarray analysis. Placentae from pregnancies that gave rise to children with allergy by 4 years of age $(n=45)$ were compared to placentae from children with no allergy $(\mathrm{n}=17)$, and sex-specific differences were also examined. Differentially expressed genes and pathways were identified using Ingenuity Pathway Analysis software.

Results: Microarray analysis identified increased expression of cytokine growth factor kit ligand (KITL), its receptor kit, and matrix metalloproteinase 2 and 9 (MMP2, MMP9) in placenta associated with allergy compared to control. $\mathrm{qPCR}$ validation showed increased expression of these genes in female placentae compared to male placentae in control and allergy groups.

Conclusion: This study has identified genes known to be involved in inflammation that appear to be differentially regulated in male and female placentae, suggesting sexspecific roles in fetal development. The roles of each gene in placental function and fetal development are unknown, and may have sex-specific roles in the programming of fetal immune function and contribution of allergy susceptibility.

Disclosure of Interest: None Declared.

\section{DOHaD13-1085}

In vivo studies on fetoplacental glucose consumption in the human

Ane M. Holme ${ }^{1, *}$, Marie Cecilie P. Roland ${ }^{1}$, Bjørg Lorentzen $^{1}$, Trond M. Michelsen ${ }^{1}$, Tore Henriksen ${ }^{1,2}$

${ }^{1}$ Obstetrics, Oslo University Hospital; ${ }^{2}$ University of Oslo, Oslo, Norway

Background: Placenta has major impact on fetal environment. In vivo studies of placental function in the human are few. We have established a method to obtain arterial and venous blood samples from both sides of placenta in vivo. Glucose is the main source of energy for the intrauterine fetus. The fetus has no significant gluconeogenesis and is dependent on glucose transfer over the placenta. Several studies have established that there is a correlation between mother's glucose levels and birth weight, yet it is not fully understood what regulates the amount of glucose offered to the fetus or the fetal glucose consumption.

\section{Objectives:}

1. To determine if maternal fasting glucose, maternal BMI and maternal arterio-venous glucose-gradient are associated with the concentration of glucose in the umbilical vein.

2. To determine if maternal fasting glucose and maternal BMI are associated with fetal glucose consumption. 
3. To determine if the concentration of glucose in the umbilical vein and the fetal glucose consumption is associated with birth weight.

Method: The study includes women $(\mathrm{n}=39)$ with uncomplicated pregnancies undergoing planned caesarean section. Fasting blood samples were obtained from the radial artery and the uterine vein just before uterine incision, and the umbilical artery and vein immediately after cord clamping. The maternal arterio-venous glucose gradient $(\Delta \mathrm{Gm})$ was calculated as the difference in glucose concentration between the radial artery (substitute for uterine artery) and the uterine vein. The umbilical arterio-venous difference in glucose concentration $(\Delta \mathrm{Gf})$ was used as a proxy for fetal glucose consumption.

Comparisons between concentrations of glucose in maternal and fetal vessels, respectively, were done by paired t-tests, and associations were explored by univariate and multiple linear regression models.

\section{Results:}

1. Maternal arterial fasting glucose concentration was positively associated with the concentration of glucose in the umbilical vein (in univariate linear regression $\mathrm{B} 0.75$, 95\% CI 0.60-0.90, p <0.001). Neither maternal BMI nor $\Delta \mathrm{Gm}$ were associated with umbilical vein glucose.

2. In univariate analyses maternal BMI was positively associated with $\Delta$ Gf (B 0.03, 95\% CI $0.01-0.06, \mathrm{p}=0.013$ ) and the association between maternal fasting glucose concentration and $\Delta$ Gf was borderline (B 0.20, 95\% CI -0.02-0.41, $\mathrm{p}=0.07$ ). In a multiple model only BMI remained statistically significant (B 0.03, 95\% CI 0.01-0.06, $\mathrm{p}=0.01$ ).

3. In univariate analyses $\Delta \mathrm{Gf}$ was associated with birthweight (B 535.4, 95\% CI 2.7-1068.2, $\mathrm{p}=0.049$ ) whereas the glucose concentration in the umbilical vein was not.

Conclusion: This human in vivo study suggests that in a fasting state maternal BMI is a stronger determinant of fetal glucose consumption than maternal glucose. Fetal glucose consumption was associated with birthweight, whereas the umbilical vein glucose concentration was not.

Disclosure of Interest: A. Holme Grant/Research support from: South-Eastern Norway Regional Health Authority, M. C. Roland Grant/Research support from: The Norwegian ExtraFoundation for Health and Rehabilitation, Norwegian Health Association, B. Lorentzen: None Declared., T. Michelsen Grant/Research support from: Norwegian Resource Centre for Women's Health, T. Henriksen: None Declared.

\section{DOHaD13-1723}

Markers of early endothelial dysfunction in IUGR-derived human umbilical vein endothelial cells revealed by 2D-Dige and mass spectrometry analyses

Andrés Caniuguir ${ }^{1}$, Bernardo Krause ${ }^{1}$, Paola Casanello ${ }^{1, *}$

${ }^{1}$ Obstetrics and Gynecology, Pontificia Universidad Católica de Chile, Santiago, Chile
Background: Intrauterine growth restriction (IUGR) is a clinical condition in which the fetus does not reach its expected growth potential, and clinically diagnosed by fetal biometry (ultrasound) and fetal wellbeing followed up by umbilical artery Doppler. The etiology is mainly due to placental dysfunction, leading to fetal chronic hypoxia. The main alteration in IUGR placenta and umbilical vessels is a higher vascular tone in umbilical artery and vein, as well as in chorionic arteries (Casanello et al., 2009; Krause et al., 2013). We hypothesize that IUGR-derived Human Umbilical Vein Endothelial Cells (HUVEC) show important changes at the proteome level that could serve a placental dysfuncion biomarkers. For this purpose 2D-Difference in Gel Electrophoresis (DIGE) and mass spectrometry analysis of HUVEC proteome from fetuses diagnosed with IUGR and control placentae were performed.

Method: Umbilical cords from IUGR $(n=8)$ or control $(n=8)$ fetuses were obtained immediately after birth previous informed consent from their mothers. Primary cultured HUVEC were grown to confluence in normoxia $\left(\begin{array}{ll}5 \% & \mathrm{O}_{2}\end{array}\right)$. Cells were lysed, proteins were cleaned (2-D CleanUp kit) and labeled (50 $\mu \mathrm{g})$ using $100 \mathrm{pmol}$ of CyDye DIGE minimal Fluor dyes (GE, Healthcare). The samples (IUGR, Control and a pool of proteins used as an internal control) were transferred to IPG Immobiline DryStrip (3-7 and 6-11 pH strips), rehydrated overnight and submitted to IEF (12 h, 60000 total Vh). The focused proteins were later separated by $12 \%$ SDS-PAGE (16 h, $15 \mathrm{~mA} / \mathrm{gel})$. Gels were scanned (Ettan DIGE Imager), and analyzed using Delta 2D software (Decodon). The spots with significant changes (IUGR/control ratio) were analyzed using a MALDI-TOF Microflex (Bruker Daltonics Inc., USA) and the peptide mass fingerprints identified using MASCOT search program (MatrixScience Inc., USA). The protein patterns were assessed by univariate and multivariate analysis to distinguish IUGR and control samples, hierarchical clustering performed. Results: A total of 81 protein spots were found differentially expressed between IUGR and control samples. After 2-D univariate analysis the Principal Component Analysis (PCA) calculated eigenvalues from 37 spots matched from differentially expressed proteins in IUGR vs control samples showing about an $87 \%$ of cumulative variance, allowing a clear separation of two components. Spots that showed significant changes were hierarchically clusted using a complete linkage algorithm and Euclidean distance as a distance metric. Finally, the proteins were classified in specific groups according to their biological function. Some of the proteins that show major changes included cytoskeleton protein isoforms (vimentin, actin, moesin), chaperones (ORP-150), proteasome proteins (PA28alpha, PSMD10), and others with different biological functions.

Conclusion: The identified protein groups that showed significant changes between IUGR and control-derived HUVEC were associated with different cellular functions, including protein sub-cellular location, and stability. Further analyses are 
required to determine the role of these proteins in the basis of the pathophysiology of vascular dysfunction and further validate these proteins as potential biomarkers.

\section{References}

1. Casanello P et al. Placenta 2009;30:625-633.

2. Krause BJ et al. Placenta. 2013;34:20-8.

Disclosure of Interest: None Declared.

\section{DOHaD13-1522}

Maternal vitamin $\mathrm{D}$ and vitamin $\mathrm{D}$-binding protein: relationship with mRNA expression and epigenetic regulation of placental facilitated amino acid transporters

Jane K. Cleal ${ }^{1, *}$, Sheila J. Barton ${ }^{2}$, Claire L. Simner ${ }^{1}$, Karen A. Lillycrop ${ }^{3}$, Hazel M. Inskip ${ }^{2}$, Cyrus Cooper ${ }^{2}$, Mark A. Hanson ${ }^{1}$, Keith M. Godfrey ${ }^{1,2,4}$, Nicholas C.

Harvey $^{2}$, Rohan M. Lewis ${ }^{1}$ and The Southampton Woman's Survey Study Group

${ }^{1}$ Human Development and Health Academic Unit; ${ }^{2} M R C$ Lifecourse Epidemiology Unit; ${ }^{3}$ Centre for Biological Sciences, University of Southampton; ${ }^{4}$ NIHR Southampton Biomedical Research Centre, University of Southampton and University Hospital Southampton NHS Foundation Trust, Southampton, United Kingdom

Background: The facilitated transporters TAT1 (SLC16A10), LAT3 (SLC43A1) and LAT4 (SLC43A2) mediate efflux of amino acids across the basal membrane of the placental syncytiotrophoblast into the fetal circulation. In placentas from Southampton Women's Survey (SWS) pregnancies the mRNA levels of TAT1 and LAT3 positively related to several measures of fetal growth and TAT1 negatively related to the micro RNA miR27a. All three transporters contain putative vitamin D receptor response elements within their promoters, suggesting that these transporters may be modulated by vitamin $\mathrm{D}$ status which is known to be related to adiposity and bone health of the SWS offspring. This study aimed to establish whether maternal vitamin D and vitamin D-binding protein (DBP) levels relate to the expression and epigenetic regulation of facilitated amino acid transporters in human placenta.

Method: We used data and samples from the SWS, a cohort of study of 3,159 pregnancies with information collected from the mothers before conception. With informed consent and ethical approval maternal serum 25-hydroxyvitamin D $[25(\mathrm{OH}) \mathrm{D}]$ and DBP levels were measured at 34 weeks gestation by radioimmunoassay and placental tissue samples collected within $30 \mathrm{~min}$ of delivery. Tissue samples used for this analysis were selected from 300 collected in total, based on availability of neonatal DXA data and maternal serum measures. Quantitative real-time PCR was used to measure TAT1, LAT3 and LAT4 mRNA expression and miR27a micro-RNA expression; all normalized to appropriate housekeeping genes. Pearson's correlation $\left(r_{p}\right)$ was used to explore the relationship between maternal $25(\mathrm{OH}) \mathrm{D}$ $(\mathrm{n}=91)$ and $\operatorname{DBP}(\mathrm{n}=85)$ concentrations and placental amino acid transporter mRNA and miR27a levels.

Results: Maternal 25(OH)D levels correlated positively with expression of LAT3 mRNA $\left(r_{p}=0.31, p=0.003\right)$, and DBP levels with expression of LAT3 $\left(r_{p}=0.22, p=0.04\right)$, LAT4 $\left(r_{p}=0.28, p=0.01\right)$ and TAT1 $\left(r_{p}=0.20, p=0.06\right)$ mRNA. Maternal DBP levels also correlated positively with miR27a expression $\left(r_{p}=0.26, p=0.02\right)$.

Conclusion: These data suggests that maternal $25(\mathrm{OH}) \mathrm{D}$ and DBP may regulate the expression of the facilitated amino acid transporters and influence the transfer of amino acids to the fetus. The correlation between DBP and the putative TAT1 repressor miR27a suggests that vitamin D may exert its effect through both transcriptional and post transcriptional mechanisms. This finding may indicate a mechanism to underpin the development of interventions to improve placental function.

Disclosure of Interest: None Declared.

\section{DOHaD13-1629}

Omega-3 fatty acid treatment stimulates proliferation of the placental trophoblast cell line HTR8/SVneo

Jing Zhou ${ }^{1, *}$, Amanda Highet ${ }^{2}$, Tina Bianco-Miotto ${ }^{2,3}$, Sam Buckberry $^{2}$, Claire T. Roberts ${ }^{2}$, Stefan Hiendleder ${ }^{2,4}$, Beverly Muhlhausler $^{1}$

${ }^{1}$ FOODplus Research Centre, School of Agriculture, Food \& Wine, University of Adelaide; ${ }^{2}$ The Robinson Institute, School of Pediatrics and Reproductive Health, University of Adelaide; ${ }^{3}$ School of Agriculture, Food \& Wine; ${ }^{4}$ School of Animal \&. Veterinary Science, University of Adelaide, Adelaide, Australia

Background: Previous clinical studies have demonstrated that maternal omega-3 long chain polyunsaturated fatty acid (n-3 LCPUFA), mainly Docosahexaenoic acid (DHA), supplementation is associated with a significant increase in the length of gestation and reduced incidence of preterm delivery. The underlying mechanisms are not well understood, but we hypothesise that this is due to effects of DHA on the differentiation and/or function of the developing placenta. Proliferation of invasive extravillous cytotrophoblasts from the tips of the placental anchoring villi is an importanant step in early placental development and contributes to sucessful pregnancy outcome. The aim of this study was to investigate the effects of DHA on placental development by studying the effect of DHA treatment on proliferation and gene expression in the human first trimester extravillous cytotrophoblast cell line HTR8/SVneo.

Method: HTR8/SVneo cells were treated with $50 \mu \mathrm{l}$ or $100 \mu \mathrm{l}$ DHA emulsion (NuMega Ingredients Pty Ltd) and compared to cells with no treatment. After 24 hours, proliferation was assessed by Calcein-AM assay. One-way ANOVA was used for comparison of proliferation rate 
between different treatment groups. To measure the effect on gene expression, HTR8/SVneo cells were seeded in 6-well plates $(200,000$ cells/well) and cultured for 24 hours with either no treatment or $50 \mu \mathrm{M}$ DHA emulsion. After 24 hours, RNA was extracted from the cells and gene expression was assessed using Illumina Human HT-12 version 4 microarrays. Differentially expressed genes were functionally annotated and analysed using bioinformatics tools.

Results: DHA treatment increased the rate of proliferation by $45 \%$ compared to control $(\mathrm{P}<0.05)$. 175 genes were differentially expressed between control and DHA treatment $(\mathrm{P}<0.01)$. The majority of these differentially expressed genes were involved in cell signaling, cell death and survival, lipid metabolism, cardiovascular disease and post-translational modification. The gene with the greatest increase in expression in DHA treated cells was GAR1, which is essential for ribosome biogenesis and telomere maintenance. Conversely, the gene with the greatest down regulation was ANGPTL4, which is involved in lipid metabolism and also acts as an apoptosis survival factor for vascular endothelial cells. We also identified many other novel genes which may be involved in mediating DHA action on trophoblast cells which require further investigation.

Conclusion: This study has provided evidence that DHA treatment increases the proliferation rate of a placental trophoblast cell line in vitro. DHA altered the expression of genes involved in cell signaling, death and survival as well as genes involved in lipid metabolism. This work provides novel insights into the mechanisms by which maternal DHA supplementation increases gestational length.

\section{References}

1. Makrides M, Gibson RA, McPhee AJ, et al. Effect of DHA Supplementation During Pregnancy on Maternal Depression and Neurodevelopment of Young Children A Randomized Controlled Trial. Jama-Journal of the American Medical Association 2010;304:1675-83.

Disclosure of Interest: None Declared.

\section{DOHaD13-1393}

Placental myostatin expression is induced by hypoxia and hyperglycemia and represses trophoblast migration

Hassendrini Peiris ${ }^{1}$, Carlos Salomon ${ }^{1}$, Katherin Scholz ${ }^{1}$, Maximilian Clissold ${ }^{2}$, Alexander Grainger ${ }^{2}$, Gregory Rice ${ }^{1}$, Murray Mitchell ${ }^{1, *}$

${ }^{1}$ Centre for Clinical Research, University of Queensland;

${ }^{2}$ Brisbane Boys' College, Brisbane, Australia

Background: Inadequate placental development is associated with pregnancy disorders and can adversely affect the postnatal life of offspring. Myostatin is a highly conserved secretory protein that inhibits muscle development affecting both cellular proliferation and differentiation. It also regulates fat deposition and blood glucose concentrations, glucose metabolism and homeostasis. Moreover, myostatin is up-regulated in muscle tissue of humans with chronic hypoxemia. Recently, we demonstrated that myostatin: expression decreases in the human placenta with gestational age; facilitates glucose uptake in placental explants and is localized to cytotrophoblast, syncytiotrophoblast and extravillous trophoblast cells of human placentae. The aim of this study was to determine the effect of low oxygen tension $(1 \%$ $\mathrm{O}_{2}$ ) and hyperglycaemia on the expression of the active myostatin protein and the effect of myostatin treatment on extravillous trophoblast migration.

Method: $1^{\text {st }}$ trimester placentae were collected after clinicallyindicated termination of pregnancy (Human Research Ethics Committees of the Royal Brisbane and Women's Hospital, and the University of Queensland). Placental explants: Chorionic villi were isolated and cultured for $48 \mathrm{~h}$ in DMEM/10\% FBS with normal $(5 \mathrm{mM})$ or high $(25 \mathrm{mM})$ D-glucose concentration at hypoxic $\left(\begin{array}{lll}1 \% & \mathrm{O}_{2}\end{array}\right)$ and physiologically relevant $\left(\begin{array}{ll}3 \% & \mathrm{O}_{2}\end{array}\right)$ oxygen tensions. Protein detection: Following protein extraction and quantitation, the expression of dimeric myostatin $(\sim 28 \mathrm{kDa})$ was assessed by Western blot analysis. Densitometry was performed utilising Quantity One software. Migration assay: Collagen 1-coated wells were seeded with $5 \times 10^{5}$ HTR-8/ SVneo cells* and grown to confluence. Scratch wounds were generated with 96-pin WoundMaker ${ }^{\mathrm{TM}}$. Cell migration into the wound was assessed in the absence (control) and presence of recombinant myostatin $(0.05 \mu \mathrm{g} / \mathrm{ml}$; treatment using an Incucyte $^{\mathrm{TM}}$ live-cell imaging system.

Results: The expression of the myostatin dimer was $\sim 1.5$ fold $(p<0.05)$ greater in first trimester chorionic villi explants exposed to $1 \% \mathrm{O}_{2}$ compared to $3 \% \mathrm{O}_{2}$. At an atmosphere of $3 \% \mathrm{O}_{2}$, myostatin expression was $\sim 1.6$-fold $(\mathrm{p}<0.05)$ greater in the presence of $25 \mathrm{mM} \mathrm{D}$-glucose compared to $5 \mathrm{mM}$ D-glucose. This effect was not observed under $1 \% \mathrm{O}_{2}$. Myostatin $(0.05 \mu \mathrm{g} / \mathrm{ml})$ significantly reduced HTR-8/SVneo migration $(20 \pm 2 \%, \mathrm{p}<0.03)$

Conclusion: The data obtained in this study establish that placental myostatin expression increases in response to physiological stress, i.e. hypoxia and hyperglycaemia, and that increased myostatin concentrations reduced the motility of trophoblast cells. We propose that myostatin expression is increased in association with abnormal placentation and thereby alters placental cell function.

Disclosure of Interest: None Declared.

\section{DOHaD13-1120}

Placental vitamin D homeostasis and reduced ovine fetal vitamin D status following maternal gestational undernutrition

Jane K. Cleal ${ }^{1}$, Melissa R. Hargreaves ${ }^{1}$, William D. Fraser ${ }^{2}$, Kirsten R. Poore ${ }^{1}$, Mark A. Hanson ${ }^{1}$, Lucy R. Green ${ }^{1, *}$ 
${ }^{1}$ Institute of Developmental Sciences, University of Southampton, Southampton; ${ }^{2}$ Norwich Medical School, University of East Anglia, Norwich, United Kingdom

Background: Substantial portions of the human population, including pregnant women, have levels of the secosteroid Vitamin D (VD) at the lower end of the measurable range ${ }^{1}$. The fetus is dependent on placental transport of the inactive 25-hydroxyvitamin $\mathrm{D}_{3}(25(\mathrm{OH}) \mathrm{D})$ from the mother, and $\mathrm{VD}$ is implicated in skeletal, cardiovascular and muscle development. In sheep, maternal undernutrition during critical windows of development impacts on the distribution of cardiovascular resources to the fetal hindlimb and fetal skeletal muscle development ${ }^{2-4}$. Here we determined in sheep the effect of such maternal undernutrition in early and late gestation on materno-fetal $25(\mathrm{OH}) \mathrm{D}$ status, and on the expression of genes involved in VD homeostasis and nutrient transport in the placenta.

Method: Pregnant ewes received either $100 \%$ of total nutrient requirements throughout gestation $(\mathrm{C}, n=7)$, $40 \%$ requirements from 1-31 days of gestation (dGA) (PI40 $n=8$ ), or $50 \%$ from $104 \mathrm{dGA}$ until post mortem at 127 dGA (L50 $n=6$ ), with 100\% requirements at other times. At post mortem, materno-fetal plasma $25(\mathrm{OH})-\mathrm{D}_{2}$ and $-\mathrm{D}_{3}$ levels were measured by HPLC tandem mass spectroscopy. Placental mRNA levels of genes involved in VD homeostasis (the gene encoding 1- $\alpha$-hydroxylase, which converts $25(\mathrm{OH}) \mathrm{D}$ to the active $1,25(\mathrm{OH})_{2} \mathrm{D}$ [CYP27B1]; $\mathrm{VD}$ receptor [VDR]) and nutrient transport (insulin receptor [IR], the amino acid transporter TAT-1; lipoprotein lipase [LPL]) were measured by quantitative real-time RT-PCR and normalized using the geometric mean of the housekeeping genes $\beta A c t i n$ and GAPDH. CYP27B1 and VDR probe/ primer sequences were designed by alignment of bovine and human mRNA sequences and validated. Data were analysed by ANOVA and Bonferroni post hoc tests.

Results: $25(\mathrm{OH}) \mathrm{D}$ levels were greater in maternal than in fetal plasma $(\mathrm{P}<0.0001)$. Fetal, but not maternal, plasma levels of $25(\mathrm{OH}) \mathrm{D}$ were lower in PI40 $(\mathrm{P}<0.01)$ and $\mathrm{L}$ $(\mathrm{P}<0.01)$ compared to control groups. CYP27B1 mRNA levels tended to be increased in PI40 and L compared to control groups $(\mathrm{P}=0.0598)$. There was no significant difference in the placental VDR, IR, TAT-1 or LPL mRNA levels between dietary groups.

Conclusion: We have confirmed the expected materno-fetal gradient in plasma levels of the $25(\mathrm{OH}) \mathrm{D}$. Our finding that maternal undernutrition during critical early and late gestation periods affected fetal, but not maternal, plasma $25(\mathrm{OH}) \mathrm{D}$ suggests effects on either placental transport/ homeostasis of VD, or on fetal homeostasis of VD. The trend for increased placental CYP27B1 mRNA levels in the dietary restricted groups warrants investigation of 1- $\alpha$-hydroxylase activity, which if elevated might increase placental metabolism of $25(\mathrm{OH}) \mathrm{D}$ to the active $1,25(\mathrm{OH})_{2} \mathrm{D}$ and contribute to the observed reduction in fetal $25(\mathrm{OH}) \mathrm{D}$ status. Reduced fetal VD status may contribute to fetal cardiovascular and musculo-skeletal adaptive responses to maternal undernutrition.

\section{References}

1. Harvey, N.C. et al. (2012) BMJ 345, e4695.

2. Burrage, D.M. et al. (2009) J. Physiol 587, 611-624.

3. Costello, P.M. et al. (2008) J. Physiol 586, 2371-2380.

4. Hawkins, P. et al. (2000) Reprod. Fertil. Dev. 12, 443-456.

Disclosure of Interest: None Declared.

\section{DOHaD13-1554}

\section{Role of $\mathrm{xCT}$ in the delivery of antioxidant precursors in the human placenta}

Emma Lofthouse ${ }^{1}$, Jane Cleal ${ }^{2}$, Mark Hanson ${ }^{1}$, Kirsten Poore $^{1}$, Rohan M. Lewis ${ }^{1, *}$

${ }^{1}$ Faculty of Medicine; ${ }^{2}$ University of Southampton, Southampton, United Kingdom

Background: We have shown previously that the glutamate/ cystine exchanger $\mathrm{xCT}$ (SLC7A11) is active in human placenta and transports the antioxidant $\mathrm{N}$-acetylcysteine (NAC). xCT also meditates uptake of cystine which is converted to cysteine for glutathione synthesis. We have also shown that the volume regulated anion channel (VRAC), which is known to be activated by oxidative stress, is present in the placenta. NAC is an antioxidant in its own right and is converted to cysteine within the cytoplasm providing a substrate for glutathione synthesis. In this study we address whether uptake of NAC affects subsequent activity of the oxidant sensitive VRAC channel.

Method: Isolated perfused placental cotyledons from normal, term pregnancies were perfused on the maternal side with physiological buffer containing $0.1 \mathrm{Ci} / \mathrm{L}{ }^{14} \mathrm{C}$-glutamate and 1 $\mathrm{Ci} / \mathrm{L}{ }^{3} \mathrm{H}$-proline at $14 \mathrm{ml} / \mathrm{min}$ and allowed to reach steady state. A $1.5 \mathrm{ml}$ bolus of $16 \mu \mathrm{mol} / \mathrm{L} \mathrm{NAC}$ was given to the maternal side of the placenta to stimulate release of glutamate via $\mathrm{xCT}$. This was followed by a maternal side infusion of $100 \mathrm{ml}$ of $100 \mathrm{mmol} / \mathrm{L}$ urea to create an osmotic shock to activate the VRAC, stimulating glutamate release. Following each bolus of urea, release of ${ }^{14} \mathrm{C}$-glutamate and ${ }^{3} \mathrm{H}$-proline into the maternal venous outflow was determined for ten minutes and the area under the curve compared to baseline release using a Wilcoxon signed rank test.

Results: NAC boli $(\mathrm{n}=7)$ stimulated efflux of ${ }^{14} \mathrm{C}$-glutamate $(\mathrm{p}=0.03)$ but not ${ }^{3} \mathrm{H}$-proline $(\mathrm{p}=0.16)$. Urea infusion stimulated the release of ${ }^{14} \mathrm{C}$-glutamate $(\mathrm{p}=0.047, \mathrm{n}=7)$ but not ${ }^{3} \mathrm{H}$-proline $(\mathrm{p}=0.38)$. However, in placentas previously perfused with NAC, no efflux of ${ }^{14} \mathrm{C}$-glutamate $(\mathrm{n}=7, \mathrm{p}=0.82)$ or ${ }^{3} \mathrm{H}$-proline $(\mathrm{n}=7, \mathrm{p}=0.66)$ was observed.

Conclusion: This study demonstrates that prior exposure of the placenta to a single bolus of NAC inhibits VRAC 
channel activity. We propose that placental NAC uptake is mediated by $\mathrm{xCT}$ and has its effect on VRAC activity by decreasing oxidative stress within the placenta. If the level of $\mathrm{xCT}$ are reduced in pathological pregnancies such as preeclampsia, this may lead to increased oxidative stress and placental dysfunction.

Disclosure of Interest: None Declared.

\section{DOHaD13-1373}

\section{Sex-specific effects of a single course of antenatal betamethasone treatment on maternal and fetal ACTH and cortisol levels in humans}

Franziska Braun $^{1,2, *}$, Anne-Katrin Gramzow ${ }^{1,2}$, Loreen Ehrlich $^{2}$, Annett Husar ${ }^{1}$, Deborah M. Sloboda ${ }^{3,4,5}$, John R. G. Challis ${ }^{6,7,8}$, Wolfgang Henrich ${ }^{1}$, Andreas Plagemann ${ }^{1,2}$, Thorsten Braun ${ }^{1,2}$

${ }^{1}$ Department of Obstetrics; ${ }^{2}$ Division of Experimental Obstetrics, Study Group Perinatal Programming, Charite' University, Berlin, Germany; ${ }^{3}$ Department of Biochemistry and Biomedical Sciences; ${ }^{4}$ Department of Obstetrics and Gynecology; ${ }^{5}$ Department of Pediatrics, McMaster University, Hamilton; ${ }^{6}$ Department of Physiology; ${ }^{7}$ Department of Obstetrics and Gynecology, University of Toronto, Toronto; ${ }^{8}$ Faculty of Health Sciences, Simon Fraser University, Vancouver, Canada

Background: We have previously shown that repeated antenatal glucocorticoid treatment in sheep resulted in decreased birth weight and short and long-term alterations in hypothalamic-pituitary-adrenal axis (HPA) function. Even a single course of betamethasone (BET) treatment in women facing high risk of preterm delivery for induction of lung maturation, is associated with decreased fetal growth and development. Less is known about the effect of a single course of antenatal BET treatment on the HPA-axis and the role of the placenta. We therefore analysed the sex-specific effects of a single course of antenatal BET treatment on maternal and fetal cortisol and ACTH plasma levels.

Method: BET (single course, $2 \times 12 \mathrm{mg}$ ) exposed women $(\mathrm{n}=86$ ), who delivered between $24+2$ and $41+6$ weeks of gestation (wks), were compared to aged-matched controls $(n=92)$. Maternal and umbilical cord blood samples were obtained at delivery. ACTH and cortisol plasma levels were analysed in maternal venous and fetal umbilical cord blood samples. Time of day and delivery mode were accounted for in the analyses. P-values were adjusted for major confounders e.g. maternal weight parameters, smoking, days of gestation at birth etc.

Results: In both females and males, a single course of BET treatment significantly decreased birth weight (up to 7.6\%) and head circumference (up to $2.3 \%$ ) as compared to controls. In mothers with male fetuses, delivered during daytime and by spontaneous delivery or secondary caesarean section, BET treatment significantly lowered maternal ACTH and cortisol plasma levels compared to controls. In the BET group, maternal cortisol levels were significantly correlated with birth weight, fetal head circumference and body length $(\mathrm{p}<0.05)$. Umbilical cord ACTH and cortisol levels in BET males were not different from controls.

In contrast, maternal ACTH and cortisol plasma levels in mothers with female fetuses were not different after BET treatment compared to controls. In the BET group, maternal cortisol levels were significantly correlated with body length and fetal head circumference $(\mathrm{p}<0.05)$. Umbilical cord ACTH plasma levels in females were significantly lower after BET treatment compared to controls. Umbilical cord cortisol plasma levels tended to be higher in BET treated female fetuses born $<37$ weeks.

Conclusion: Maternal ACTH and cortisol blood levels were lower in BET treated women pregnant with male fetuses only, indicating a sex-specific suppression of the maternal HPA-axis. This effect is particularly distinct in spontaneous daytime delivery. In female fetuses, umbilical cord ACTH plasma levels were significantly lower without associated changes in umbilical cord cortisol levels. We speculate that this could be due to either a dysregulation of the fetal HPA-axis or due to a higher placental transfer of maternally derived cortisol to the fetus, thus suppressing fetal adrenal cortisol production.

Disclosure of Interest: None Declared.

\section{DOHaD13-1309}

The crosstalk between cAMP and MAPK pathways in the regulation of placental 11 beta hydroxysteroid dehydrogenase 2

Gang Sun ${ }^{1, *}$, Jianneng $\mathrm{Li}^{2}$

${ }^{1}$ Center of Reproductive Medicine, Renji Hospital Shanghai Jiaotong University; ${ }^{2}$ School of Life Sciences, Fudan University, Shanghai, China

Background: Appropriate amount of glucocorticoids is vital for the normal development of the fetus. However excessive exposure to glucocorticoids not only causes intrauterine growth retardation but also programs the development of chronic diseases in later life. The passage of maternal glucocorticoids to the fetus is controlled by the placental $11 \beta$-hydroxysteroid dehydrogenase 2 (11ß-HSD2). Thus understanding of the regulation of $11 \beta$-HSD2 expression in human placenta will help us understand how the normal development of the fetus in utero is ensured.

Method: In this study, we prepared human placental cytotrophoblasts from normal and term placentae without labor and studied the role of MAPK in the upregulation of $11 \beta$ HSD2 by activation of hCG/cAMP pathway upon maximal syncytialization of the trophoblasts. The syncytiotrophoblasts were treated with hCG and dibutyl cAMP (dbcAMP) in the 
presence or absence of $\mathrm{p} 38$ or ERK1/2 inhibitors. The phosphorylation of p38 and ERK1/2, 11ß-HSD2 protein levels were measured with Western blotting, and $11 \beta-\mathrm{HSD} 2 \mathrm{mRNA}$ level were determined with real time PCR (qRT-PCR).

Results: Treatment of the syncytiotrophoblasts with hCG $(10 \mathrm{UI} / \mathrm{ml})$ or dbcAMP $(100 \mu \mathrm{M})$ increased the levels of both phosphorylated p38 and ERK1/2 at 15,30 or $60 \mathrm{~min}$. Treatment with hCG and dbcAMP for $24 \mathrm{~h}$ increased $11 \beta$ HSD2 mRNA and protein significantly, which was blocked by p38 inhibitor SB203580 $(10 \mu \mathrm{M})$ but not by ERK1/2 inhibitor PD98059 $(50 \mu \mathrm{M})$. SB203580 also reduced the basal $11 \beta$-HSD2 level while PD98059 increased basal $11 \beta$ HSD2 level. There was no additive effect of hCG/cAMP and PD98059 on $11 \beta-H S D 2$ expression.

Conclusion: In conclusion, activation of the p38 but not ERK1/2 pathway by hCG/cAMP pathway may be involved in the maintenance of $11 \beta-H S D 2$ expression in human placental syncytiotrophoblasts. How PD98059 inhibits $11 \beta$-HSD2 expression remains to be studied.

Disclosure of Interest: G. Sun Grant/Research support from: Natural Science Foundation of China, J. Li: None Declared.

\section{DOHaD13-1337}

The selection of placental morphological and histopathological variables with the LASSO

Yanjun Zhao ${ }^{1, *}$, Jun Zhang $^{1}$

${ }^{1}$ XinHua Hospital affiliated to Shanghai Jiao Tong University School of Medicine, Shanghai, China

Background: The placenta pathology plays a pivotal role in ascertaining the cause of the adverse perinatal outcome. Placental morphological and histopathological measures can be numerous and it is very time consuming to collect all the information. The aim of this study was to evaluate the importance of each measure in predicting child diseases and systematically select measures that have significant clinical implications.

Method: We used placental pathology information from Collaborative Perinatal Project (CPP), a large prospective birth cohort study that was conducted in the U.S. from 1959 to 1976. A total of 59,500 births were included in CPP, of whom 45,785 had comprehensive information on placental morphology and histology (103 items per placenta). The placentas were evaluated by reference pathologists using a standardized protocol. Children were followed up to 7 years of age. We chose 20 major child diseases as outcomes, including physical growth and development, cardiovascular, respiratory, neurological, digestive, and blood diseases. The statistical method of LASSO (least absolute shrinkage and selection operator) was used to select important placental measures that have better predictability for outcomes.

Results: From 103 placental measures, LASSO selected 78 measures as candidates. After consulting placental pathologists, we narrowed down to 37 most important measures to form our shortened evaluation system that could be used in clinical practice and research. They include placental weight, length of cord, calcification in cut surface and so on.

Conclusion: The shortened version of placental information collection form needs validation before introducing it into clinical practice.

Disclosure of Interest: None Declared.

\section{DOHaD13-1673}

In vitro culture and embryo transfer alters cardiac metabolism in early postnatal life

Monalisa Padhee $^{1, *}$, I. Caroline McMillen ${ }^{1}$, Song Zhang ${ }^{1}$, Severence M. MacLaughlin ${ }^{1}$, David O. Kleemann ${ }^{2}$, Simon K. Walker $^{2}$, Jen Kelley ${ }^{2}$, Skye Rudiger ${ }^{2}$, Janna L. Morrison ${ }^{1}$

${ }^{1}$ Sansom Institute for Health Research, School of Pharmacy and Medical Sciences, University of South Australia; ${ }^{2}$ Turretfield Research Centre, South Australian Research and Development Institute, Adelaide, Australia

Background: Nutrition during the periconceptional period is critical in determining cardiometabolic health in postnatal life. Previous studies provide evidence that assisted reproductive technologies are associated with cardiometabolic dysfunction in postnatal life. In this study, we aimed to determine if in vitro culture and transfer of the embryo, which are part of assisted reproductive technologies as well as manipulations to the nutritional environment during the periconceptional period, alter cardiac metabolism in postnatal life.

Method: Embryos were either transferred to an intermediate ewe (ET) or cultured in vitro in the absence (IVC) or presence of human serum (IVCHS) and a methyl donor (IVCHS + M) for 6d. Naturally mated (NM) ewes acted as controls. At 24wks, hearts were collected and mRNA expression of receptors and signalling molecules involved in cardiac metabolism were measured using qRT-PCR. Plasma non-esterified fatty acids (NEFA) and glucose concentrations were also measured at 24 wks. Results: There was a decrease in mRNA expression of Peroxisome proliferator-activated receptor- $\alpha$ (PPAR $\alpha$ ), a master transcriptional regulator of genes involved in fatty acid metabolism, in females of all treatment groups compared to controls. There were increased plasma NEFA concentrations in IVCHS $+\mathrm{M}$ females only. However, there was no change in other signaling molecules involved in fatty acid metabolism such as CD36, acetyl CoA carboxylase, fatty acid transporter protein 1 and carnitine palmitoyltransferase-Ib. There was no change in plasma glucose concentrations in any of the treatment groups but an increase in the gene expression of pyruvate dehydrogenase kinase-4 (PDK4) in IVCHS+M females only but no difference in other signaling molecules involved in cardiac glucose metabolism such as glucose transporter- 4 and Pyruvate carboxylase. 
Conclusion: This study demonstrates that culture and transfer of the embryo results in a decrease in cardiac PPAR $\alpha$ gene expression in females, suggesting a decrease in fatty acid metabolism. The increase in plasma NEFA concentrations in females of IVCHS $+\mathrm{M}$ with a decrease in PPAR $\alpha$ gene expression suggest an abnormal balance between fatty acid availability and fatty acid oxidation, which may result in a vulnerability to lipid accumulation by increasing the flux of NEFA in the heart in later life. The increase in PDK 4 in the females of the IVCHS+M group suggests a potential decrease in glucose oxidation with no change in glycolysis and this may lead to acidosis and impaired contractility. These results suggest that early nutritional manipulation of the embryo can alter cardiac metabolism in females, but not males, in early postnatal life.

Disclosure of Interest: None Declared.

\section{DOHaD13-1625}

\section{Mothers' peri-conceptional cortisol levels and their children's postnatal stress reactivity}

Pablo A. Nepomnaschy ${ }^{1,2, *}$, Cindy K. Barha ${ }^{1,2}$, Katrina G. Salvante ${ }^{1,2}$, Huiting $\mathrm{Ma}^{3}$, Leilei Zeng ${ }^{3}$

${ }^{1}$ Health Sciences; ${ }^{2}$ Human Evolutionary Studies Program, SIMON FRASER UNIVERSITY, Burnaby; ${ }^{3}$ Statistics and Actuarial Science, University of Waterloo, Waterloo, Canada

Background: Adverse prenatal conditions can have important consequences for fetal development and increase disease susceptibility across the lifespan. High levels of maternal stress during pregnancy have been shown to affect children's behavioural, emotional, cognitive, immune, and metabolic functioning. Existing evidence suggests that these outcomes may be linked to the way the hypothalamic-pituitary-adrenal axis (HPAA) is programmed in utero. This evidence is mostly derived from research focused on the gestational period following the clinical detection of pregnancy ( $>$ week 6 ). Until now logistical challenges have made it very difficult to evaluate the effects that maternal physiologic stress may exert at earlier stages of fetal development ( $<$ week 6 or peri-conceptional period). We propose, however, that the peri-conceptional period should represent a "critical window of vulnerability". Crucial epigenetic processes take place during this period, and the embryo is highly exposed to the maternal hormonal milieu at this point as it is without the protection of a fully developed, functional placenta. Here we indirectly test this hypothesis by evaluating if maternal cortisol levels (a marker of HPAA function and activation) during the first 6 weeks of gestation are associated with children's postnatal HPAA responsivity to an experimental stress challenge.

Method: As part of a prospective study, 107 women at risk of conceiving a pregnancy provided first morning urinary specimens (FMUS) every other day for up to a year between the years 2000 and 2001. We captured the first 6 weeks following conception for 18 of those women and quantified cortisol levels in the FMUS during that period. Then in 2013 we quantified changes in salivary cortisol levels in their 18 children (now 11- and 12-years old) in response to the Trier Social Stress Test-Child (TSST-C). The link between maternal cortisol levels during the first 6 weeks of gestation and their children's HPAA reactivity was evaluated using stepwise multiple regression.

Results: Mother's peri-conceptional cortisol levels positively correlate with change in cortisol levels during recovery from exposure to an experimental stressor in children $\left(R^{2}=0.35\right.$, $\mathrm{p}<0.05)$. There is a trend towards this relationship being stronger in boys than girls (interaction: $\mathrm{p}=0.09$ ).

Conclusion: This is the first study to explore and find an association between peri-conceptional stress levels and postnatal stress physiology in children. Dysregulation of the HPAA greatly impacts many aspects of development, including physical and mental health outcomes across the lifespan. Thus, these results are critically important for understanding HPAA programming, child development in general, and the development of public health interventions.

Disclosure of Interest: None Declared.

\section{DOHaD13-1491}

Preconceptional omega 3 fatty acid supplementation to a micronutrient deficient diet influences the expression of hepatic transcription factors in Wistar rats

Akshaya Meher ${ }^{1, *}$, Asmita Joshi ${ }^{1}$, Sadhana Joshi ${ }^{1}$

${ }^{1}$ Nutitional Medicine, Interactive Research School for Health Affairs, Pune, India

Background: Nutritional status of the mother influences various metabolic adaptations for optimal fetal development and is mediated by transcription factors like peroxisome proliferator activated receptors (PPARs) and hepatocyte nuclear factor 4 alpha (HNF4- $\alpha$ ). Docosahexaenoic acid (DHA) acts as natural ligand and activates these transcription factors. A series of our earlier studies both in animals and humans have demonstrated that maternal folic acid, vitamin B12 and omega 3 fatty acids are interlinked in one carbon cycle. We therefore hypothesize maternal micronutrient deficient diet will influence the fatty acid levels and thereby the expression of transcription factors in the liver.

Method: Female rats were divided into five groups $(n=816 /$ group) as follows; control, folic acid deficient (FD), vitamin B12 deficient (BD) and omega 3 supplemented groups (FDO and $\mathrm{BDO}$ ). Half the dams were dissected on the gestational day 20 while the remaining half of the dams were allowed to deliver normally. Pups were dissected on the postnatal day 22. Results: Maternal micronutrient deficiency resulted in lower $(\mathrm{p}<0.05)$ levels of DHA and arachidonic acid (AA) as compared to the control group in pup liver while there was no change in the dam plasma DHA levels. Pup liver PPAR $\alpha$ and 
PPAR $y$ expression was lower $(\mathrm{p}<0.05)$ in the $\mathrm{BD}$ group although there was no difference in the expression of SREBP1c, LXRa and RXRa expression. Pup liver global methylation levels were higher $(\mathrm{p}<0.05)$ in both the micronutrient deficient groups. In the presence of omega 3 fatty acids most of the above effects were ameliorated.

Conclusion: Our data for the first time suggests a role for omega 3 fatty acids in the one-carbon cycle in influencing the hepatic expression of transcription factors in the dam and the offspring possibly due to altered gene specific methylation. This may have implications in the fetal lipid metabolism and the subsequent risk for the metabolic syndromes in the adults.

Disclosure of Interest: None Declared.

\section{DOHaD13-1198}

Twin conception results in increased fat mass and altered metabolism in adult sheep

Anne L. Jaquiery ${ }^{1, *}$, Danielle Smeitink ${ }^{1,2,3}$, Mark Oliver ${ }^{1,3}$, Serina Hancock ${ }^{1}$, Clare McLean ${ }^{1}$, Frank Bloomfield ${ }^{2,3,4}$

${ }^{1}$ Liggins Institute, University of Auckland, Rotorua,

${ }^{2}$ Paediatrics: Child and Youth Health, University of Auckland,

${ }^{3}$ Gravida, National Centre for Growth and Development,

${ }^{4}$ Liggins Institute, University of Auckland, Auckland,

New Zealand

Background: Previous studies in sheep have shown that being conceived as a twin alters birth weight and postnatal growth trajectories and results in increased \% fat mass in young adulthood, even if one fetus was removed in early gestation. This suggests that, in twins, early growth and body composition outcomes are largely determined in the periconceptional period. We hypothesised that the effects of twin conception on growth and development would be amplified with age, resulting in altered body composition and deranged metabolism in mature adulthood.

Method: Twin pregnancies in sheep were randomised to reduction of one twin on day 42 of a $148 \mathrm{~d}$ pregnancy by intra-thoracic $\mathrm{KCl}$ (Reductions, $\mathrm{n}=30$ ) or a sham procedure (Twins, $n=19$ ). Singleton-bearing ewes also underwent a sham procedure $(n=23)$. Ewes lambed spontaneously. A cohort of the offspring (Twins $n=11$, Reductions $n=10$, Singletons $n=9$ ) was retained and studied at 3 years of age. Body composition was assessed by dual X-ray absorptiometry (DXA). Glucose tolerance was assessed by intravenous glucose tolerance test and insulin sensitivity by an hyperinsulinaemic euglycameic clamp technique. Groups were compared using ANOVA.

Results: Body weight was greater in males than females in all groups, but not different between groups in either sex. In males, Twins and Reductions had greater \% fat mass and less \% lean mass than Singletons (\% Fat mass: Twins $15 \pm 2 \%$, Reductions $19 \pm 2 \%$, Singletons $8 \pm 2 \%$, group effect $\mathrm{p}<0.0001$; Lean mass: Twins $63 \pm 2 \%$, Reductions $61 \pm 2 \%$, Singletons $68 \pm 2 \%$; group effect $p=0.001$ ). Body composition was not different between groups in females. Glucose tolerance was not different between sexes, or between groups in either sex. Males were more insulin sensitive than females in all groups (sex effect $p=0.001$ ). Twin males tended to be less insulin sensitive than Singletons, with Reductions intermediate (Insulin Sensitivity: Twins $3.7 \pm 0.3$, Reductions $4.6 \pm 0.3$, Singletons $5.1 \pm$ $0.5 \mathrm{mg} . \mathrm{ml} . \mu \mathrm{U}^{-1} \cdot \mathrm{kg}^{-1} \cdot \mathrm{min}^{-1}$; group effect $\mathrm{p}=0.06$ ). Insulin sensitivity followed a similar pattern in females, but results were not statistically significant (Twins $2.9 \pm 0.4$, Reductions $2.7 \pm 0.4, \quad$ Singletons $\left.3.9 \pm 0.6 \mathrm{mg} \cdot \mathrm{ml} \cdot \mu \mathrm{U}^{-1} \cdot \mathrm{kg}^{-1} \cdot \mathrm{min}^{-1}\right)$. There was no independent effect of birth weight or early growth velocity on adult glucose tolerance or insulin sensitivity. Conclusion: In sheep, the periconceptional event of being conceived a twin has sex specific effects on body composition and metabolism, independent of birth weight or postnatal growth velocity, that persist into mid adult life. Events occurring in the crucial periconceptional period have more profound effects on growth and metabolism in males than females.

Disclosure of Interest: None Declared.

\section{DOHaD13-1267}

A large scale prospective metabolomic cohort study of first trimester maternal serum reveals system-wide associations between metabolite concentrations and later pre-eclampsia

Louise C. Kenny ${ }^{1, *}$, David I. Broadhurst ${ }^{2}$, Rupasri Mandal ${ }^{3}$, Beamsoo $\mathrm{Han}^{3}$, David Wishart ${ }^{3}$, Philip N. Baker ${ }^{4}$

${ }^{1}$ The Irish Centre for Fetal and Neonatal Translational Research, University College Cork, Cork, Ireland; ${ }^{2}$ Department of Medicine; ${ }^{3}$ Metabolomics Innovation Centre, University of Alberta, Alberta, Canada; ${ }^{4}$ The Liggins Institute, University of Auckland, Auckland, New Zealand

Background: Pre-eclampsia is a pregnancy specific disorder associated with increased fetal, neonatal and maternal morbidity and mortality. Although the precise aetiology is not completely understood, the condition is associated with deficient placentation and fetal growth restriction and the maternal syndrome shares some features with metabolic conditions such as cardiovascular disease and type II diabetes. Both mothers and infants have an increased risk of a range of adverse health outcomes. We have previously performed small scale metabolomic profiling of early pregnancy plasma samples to investigate the pathophysiological mechanisms underpinning the disease and to search for clinically useful biomarkers. Here we report the results of a large scale clinical metabolomics study of a prospective cohort using a highly stable semi-targeted assay.

Method: In the present study, serum samples taken at 15 weeks' gestation from 1,747 low risk, first time pregnant women enrolled in the Irish Screening for Pregnancy Endpoints (SCOPE) study (www.scopestudy.net) were subject to a targeted quantitative metabolomics approach using a combination of DI-MS (Absolute IDQTM Kit) with a reverse-phase LC-MS/MS Kit (BIOCRATES Life Sciences AG, Austria) on ABI 4000 Q-Trap (Applied Biosystems/MDS 
Sciex, CA) MS. Metabolites with $\mathrm{QC}_{\mathrm{RSD}}>15 \%$ were removed. A multivariate model was constructed using Logistic Regression and stepwise variable selection from a base data set derived from women who subsequently developed pre-eclampsia (PET) and women with uncomplicated pregnancies including the 139 metabolites with $\mathrm{QC}_{\mathrm{RSD}}<15 \%$, together with age, $\mathrm{BMI}$, and Mean Arterial Pressure (MAP) at 15 weeks' gestation. ROC curve analysis was then performed on this fixed model (with bootstrap 95\% confidence interval). The performance of the model was then tested in the total cohort and score distributions were calculated for PET (versus non-PET) and for other pregnancies complications including small for gestational age and gestational hypertension.

Results: There were $67(3.84 \%)$ cases of PET in the total cohort of 1747 pregnancies. 804 women had uncomplicated pregnancies and the remainder had one or more other pregnancy complications including small for gestational age. The final model selected 11 variables (10 metabolites and MAP). The ten metabolites were selected in pairs and acted within the model as metabolite ratios. For example the univariate $\mathrm{p}$-value of phenylalanine is 0.06 whereas the $\mathrm{p}$-value of the ratio phenylalanine/serine is 0.008 . The AUC (PET $\mathrm{v}$ uncomplicated) of the final model $=0.81 ; 95 \% \mathrm{CI}$ (0.75-0.86). This compares favorably with the AUC of a MAP and BMI alone of $0.71 ; 95 \%$ CI (0.64-0.72). Using the same Critical Decision Boundary, the AUC for PET versus the whole cohort (non-PET) $=0.76 ; 95 \%$ CI $(0.69-0.81)$.

Conclusion: Large-scale prospective clinical metabolomics studies are very rare and to our knowledge this is the largest performed to date. The prospective cohort design of the SCOPE study protects against the introduction of bias. Metabolomic profiling of early pregnancy serum samples can provide insights into the pathogenesis of pre-eclampsia as well as the potential of an early pregnancy screening test.

Disclosure of Interest: L. Kenny Shareholder of: LCK is a minority shareholder in Metabolomic Diagnostics, a company with an interest in the development of metabolomic biomarkers for pregnancy outcome, D. Broadhurst: None Declared., R. Mandal: None Declared., B. Han: None Declared., D. Wishart: None Declared., P. Baker Shareholder of: PNB is a minority shareholder in Metabolomic Diagnostics, a company with an interest in the development of metabolomic biomarkers for pregnancy outcome.

\section{DOHaD13-1515}

Specific LC-PUFA's can promote beneficial adiponectin secretion in primary human adipocytes

Marieke Schoemaker ${ }^{1, *}$, Tania Romacho ${ }^{2}$, Philipp Glosse ${ }^{2}$, Eric A. van $\mathrm{Tol}^{1}$, Juergen Eckel $^{2}$

${ }^{1}$ Mead Johnson Nutrition, Nijmegen, Netherlands;

${ }^{2}$ Paul-Langerhans-Group, Integrative Physiology, German

Diabetes Center, Duesseldorf, Germany
Background: Obesity is a rising epidemic which requires novel approaches. Early postnatal nutrition factors may benefit healthy weight development in adult life. Early dietary supplementation with n-3 series long-chain polyunsaturated fatty acids (LCPUFAs) like docohexaenoic acid (DHA) and eicosapentaenoic acid (EPA) in adult mice have been shown to reduce adipogenesis and adipose tissue inflammation. However, the direct effects of these factors on human adipocyte function and metabolism remain largely unknown. Therefore, the aim of the present study was to evaluate the impact of DHA, EPA, and arachidonic acid (ARA) on adipokine secretion, and to determine their effect on TNF $\alpha$-induced activation of the proinflammatory nuclear factor (NF)- $\mathrm{B}$ in human adipocytes.

Method: Primary human adipocytes were isolated from subcutaneous adipose tissue. Differentiated adipocytes were characterized by immunofluorescence. Adipogenesis was assessed by Oil Red staining. Adiponectin and leptin secretion were assessed by ELISA. Adiponectin intracellular protein levels and TNF $\alpha$ induced NF- $\mathrm{KB}$ activation were measured by Western blot.

Results: DHA and EPA $(50 \mu \mathrm{M}$ and $100 \mu \mathrm{M})$ significantly upregulated adiponectin secretion but did not affect leptin secretion. A combination of DHA/ARA (1:2) in a similar ratio as reported in human breast and formula milk significantly upregulated adiponectin secretion in the adipocytes. Nevertheless, the effect of the DHA/ARA combination on adiponectin secretion was not due to de novo synthesis as determined by Western blot analyses. Importantly, DHA $(100 \mu \mathrm{M})$ prevented TNF $\alpha$-induced $(5 \mathrm{ng} / \mathrm{ml}) \mathrm{NF}-\kappa \mathrm{B}$ activation $(43,0 \pm 15,7 \%$ vs. TNF $\alpha$-induced NF- $\mathrm{B}$ activation levels). Although EPA showed a trend towards inhibition, DHA was found to be the most potent inhibitor of $\mathrm{TNF} \alpha$-induced $N F-\kappa B$ activation, whereas ARA did not impact NF- $\mathrm{kB}$ activation.

Conclusion: DHA, EPA, as well as the DHA/ARA combination arise as promising nutritional factors that are capable of upregulating adiponectin secretion, thus promoting a more beneficial cardiometabolic profile. Moreover, DHA can significantly reduce $\mathrm{TNF} \alpha$ - induced $\mathrm{NF}-\kappa \mathrm{B}$ activation in human adipocytes suggesting a direct protective role of this $n-3$ LC-PUFA in inflammatory conditions where TNF- $\alpha$ levels are upregulated, such as is the case of obesity and Type 2 Diabetes.

Disclosure of Interest: M. Schoemaker Employee of: Mead Johnson Nutrition, T. Romacho Grant/Research support from: Caja Madrid Foundation, P. Glosse: None Declared., E. van Tol Employee of: Mead Johnson Nutrition, J. Eckel: None Declared.

\section{DOHaD13-1144}

The effect of pubertal exposure to dietary soy on markers of estrogen activity in the breast of cynomolgus macaques

Fitriya N. Dewi ${ }^{1, *}$, Charles E. Wood ${ }^{1}$, Thomas C. Register ${ }^{1}$, Cynthia J. Lees ${ }^{1}$, Timothy D. Howard ${ }^{2}$, Zhiqing Huang ${ }^{3}$, Susan K. Murphy ${ }^{3}$, Janet A. Tooze ${ }^{4}$, J. Mark Cline ${ }^{1}$ 
${ }^{1}$ Pathology/Comparative Medicine; ${ }^{2}$ Center for Genomics and Personalized Medicine Research, Wake Forest School of Medicine, Winston-Salem, NC; ${ }^{3}$ Gynecologic Oncology, Duke University Medical Center, Durham, NC; ${ }^{4}$ Biostatistical

Sciences, Wake Forest School of Medicine, Winston-Salem, NC, United States

Background: Puberty is highly influenced by estrogen, and is a critical period for mammary gland development. Epidemiologic studies show that soy intake during childhood/ adolescence is associated with reduced breast cancer risk later in life. This effect of soy may be driven in part by isoflavones (IFs), a biological compound of the soybean that has structural similarity to estradiol and can bind to estrogen receptor (ER) to elicit estrogen-agonist/antagonist action. Utilizing a nonhuman primate model, we assessed the effect of dietary soy on the expression of ERs and ER-regulated markers in the breast across pubertal development.

Method: Pubertal female cynomolgus macaques were fed a diet with either soy $(n=17)$ or casein $(n=12)$ as the protein source; the soy dose approximated a human equivalent dose of $120 \mathrm{mg}$ IF/day. Using breast biopsies collected serially over 4 years, we measured mRNA of ERs and markers for ER activity (TFF1, PGR, GREB1) by qRT-PCR; promoter methylation was assessed by pyrosequencing. Using immunohistochemistry, we measured the immunolocalization of ERs and GATA-3 within epithelial structures of the mammary gland. We used a mixed model ANOVA with a random animal effect to model main and interactive effects of diet and time across pubertal transition (i.e. 2 years premenarche to 2 years post-menarche). Relationships between markers were analyzed using Spearman's rank correlation.

Results: We found lower mRNA expression of $\mathrm{ER} \alpha$ and GREB1 in the breast of soy-fed animals after menarche $(P<0.01$ for both markers). Soy treatment showed lower ER $\beta$ only in the ductal structures $(P<0.05)$. Differential expression was not accompanied with a change in the methylation of promoter region $\mathrm{B}$ of the $\mathrm{ER} \alpha$ gene, $\mathrm{CpG}$ island within $300 \mathrm{bp}$ upstream of $\mathrm{ER} \beta$, or $\mathrm{CpG}$ sites flanking estrogen responsive element (ERE) $1.6 \mathrm{~kb}$ upstream of GREB1. Soy treatment appeared to alter GATA-3, a transcription factor critical for mammary gland differentiation that co-regulates ER $\alpha$ activity. GATA-3 was higher before menarche in the transitional ducts of soy-fed animals $(P<0.05)$, and showed a diet $\mathrm{x}$ time interaction in the mature lobules post menarche $(P<0.05)$. PGR and TFF1 mRNA did not differ with treatment. These markers along with $\mathrm{ER} \alpha$ and GREB1, showed a decrease in expression with maturity independent of diet. TFF1 showed an increase of methylation in $\mathrm{CpG}$ sites near the ERE within the promoter (time effect $P<0.0001$ ); this was the only marker that showed an inverse correlation between mRNA expression and promoter methylation (Spearman's $\rho=-0.31, P<0.01$ ).

Conclusion: The effect of soy on ER and ER activity markers was not mediated by methylation of the specific promoter sites examined. The downregulation of $\mathrm{ER} \alpha$ with pubertal soy exposure may be associated with an altered GATA-3 and/ or breast differentiation. Our results suggest that exposure to soy diet before menarche may transiently dampen estrogen responsiveness of the breast in young adulthood. Further studies are warranted to investigate whether the phenotype will be carried-over to later life and alter susceptibility to cancer.

Disclosure of Interest: None Declared.

\section{DOHaD13-1205}

\section{Chronic disease morbidity and treatment behavior of older adults in India}

Mini Gomathyamma Krishnakurup ${ }^{1, *}$

${ }^{1}$ Achutha Menon Centre for Health Science Studies, SREE CHITRA TIRUNAL INSITUTE FOR MEDICAL SCIENCE, Trivandrum, India

Background: India has the second largest population (1210 million) and number of elderly (77 million) in the world. Non Communicable Diseases (NCDs) are the major health issue for men and women in most countries including India among adults in all age group. However epidemiological studies of chronic disease in India have mostly been carried out in adult populations. The result of these studies are not generalisable to the whole population group especially that of older adults in India. There are limited nationally representative data on chronic disease morbidity and treatment behavior among older adults in India. The present study examined the inter-state variations on self reported chronic disease morbidity of diabetes, hypertension, heart disease and cancer and its treatment pattern in India.

Method: This study used raw data collected by the NSSO (2004) on Morbidity, Health Care and Conditions of the Aged. We analysed some most common chronic diseases such as diabetes, hypertension, heart disease and cancer from selfreports based on a nationally representative sample of 34,831 elderly persons aged 60 years and above (males; 51\%). The treatment pattern of the selected chronic diseases was also studied.

Results: Morbidity rate (prevalence of ailment during 15 days leading up to the survey date per thousand population) among older adults was 310 in India as a whole, ranging from 584 in Kerala to 126 in Delhi. Proportion of older adults with diabetes was 32, hypertension 49, heart disease 22, and cancer 3 per thousand population. Proportion of older adults with at least one of these conditions 92 per thousand population. Diabetes was higher among men (Odds Ratio (OR): 1.07), urban, (OR: 3.96), unemployed (OR: 2.22) and literates (OR: 4.60) compared to their counterparts. Hypertension was higher among women (OR: 1.34), urban (OR: 3.20), unemployed (OR: 2.49) and literates (OR: 2.72) compared to their counterparts. Heart Disease was higher 
among men (OR: 1.56), urban (OR: 3.67), unemployed (OR: 1.80) and literates (OR: 4.27) compared to their counterparts. Majority of the older adults having the chronic conditions were under treatment (diabetes: 95\%; hypertension: 94\% and heart disease: 94\%).

Conclusion: Self reported prevalence of chronic diseases will be an underestimate of the actual burden. Older people with chronic disease were more likely to seek treatment. Considering the prolonged nature of chronic diseases there is an urgent need to integrate care of Indian elderly with chronic disease in to the existing health system. Conditions associated with the chronic diseases should also be streamlined with affordable health services to the older adults.

Disclosure of Interest: None Declared.

\section{DOHaD13-1109}

Combination of early life stress and self-reported traumas across the lifespan associates with shorter leukocyte telomere length in later adulthood

Katri Savolainen $^{1, *}$, Johan G. Eriksson ${ }^{2}$, Laura Kananen ${ }^{3}$, Eero Kajantie $^{2}$, Anu-Katriina Pesonen ${ }^{1}$, Kati Heinonen ${ }^{1}$, Katri Räikkönen ${ }^{1}$

${ }^{1}$ Institute of Behavioural Sciences, University of Helsinki;

${ }^{2}$ Department of Chronic Disease Prevention, National Institution for Health and Welfare; ${ }^{3}$ Research Programs Unit,

Molecular Neurology, University of Helsinki, Helsinki, Finland

Background: Early life stress (ELS) poses a risk for mental disorders and aging-related disorders. Accelerated biological aging, reflected in shorter leukocyte telomere length (LTL), may underlie these risks. Yet, studies examining associations between ELS and LTL in adulthood have been scarce and retrospective. We examined if objectively-recorded separation from both parents and retrospectively self-reported traumatic experiences across the lifespan are associated with LTL in later adulthood.

Method: Data on separation, traumatic experiences and LTL were available for 1486 participants of the Helsinki Birth Cohort Study. Of them 215 had been exposed to objectively-recorded temporarily separation from both parents during World War II when Finnish children were evacuated unaccompanied by their parents to temporary foster care abroad (median age at separation $=4.1$ years, median length of separation $=1.4$ years). Participants selfreported traumatic experiences at age 63.2 years $(\mathrm{SD}=2.8)$ using questions derived from the Traumatic Experiences Checklist. LTL was measured at age 61.5 years $(\mathrm{SD}=2.9)$ using real-time quantitative PCR method.

Results: Separated individuals did not differ in LTL from the non-separated and self-reported traumatic experiences were not associated with LTL. In comparison to non-separated participants who did not report traumatic experiences, participants who were separated and in addition reported emotionally or physically traumatic experiences, had shorter LTL ( $\mathrm{p}$-values $<0.008$ for separation $\mathrm{x}$ traumatic experiences interactions).

Conclusion: ELS may not per se be associated with LTL measured decades later. However, when combined with subjectively traumatic experiences across lifespan, individuals with ELS experiences have shorter LTL.

Disclosure of Interest: None Declared.

\section{DOHaD13-1613}

Maternal folate concentration in early pregnancy predicts newborn telomere length

Sonja Entringer ${ }^{1}$, Elissa S. Epel ${ }^{2}$, Jue Lin ${ }^{3}$, Elizabeth Blackburn $^{3}$, Claudia Buss ${ }^{1}$, Babak Shahbaba ${ }^{4}$, Daniel L. Gillen $^{4}$, Raman Venkataramanan ${ }^{5}$, Hyagriv N. Simhan ${ }^{6}$, Pathik D. Wadhwa ${ }^{7, *}$

${ }^{1}$ Pediatrics, University of California, Irvine, Orange; ${ }^{2}$ Psychiatry; ${ }^{3}$ Biochemistry \& Biophysics, University of California, San Francisco, San Francisco; ${ }^{4}$ Statistics, University of California, Irvine, Irvine; ${ }^{5}$ Pharmaceutical Sciences; ${ }^{6}$ Obstetrics \& Gynecology, University of Pittsburgh, Pittsburgh; ${ }^{7}$ Psychiatry, University of California, Irvine, Irvine, United States

Background: Telomere biology exerts a pervasive and potentially causal role in key aspects of genomic integrity, physiology, aging, disease risk and mortality. The newborn setting of telomere length (TL) likely plays a major role in telomere dynamics throughout human lifespan; however, its determinants are poorly understood. Because folate is essential for DNA integrity and methylation and because the maternal compartment is the only source of folate for the developing fetus, we tested the hypothesis that variation in maternal folate concentrations in early gestation would influence the developing fetal telomere system.

Method: In a prospective study of a population-based cohort of 119 mother-newborn dyads, maternal serum folate levels were assessed in early gestation (week 10). Newborn TL was assessed in peripheral blood mononuclear cells (PBMCs) isolated from cord blood.

Results: After accounting for the effects of other potential determinants of newborn TL (maternal pre-pregnancy BMI, birth weight, gestational age at birth, infant sex, and obstetric complications), maternal total folate concentration in early gestation was significantly associated with newborn TL. Specifically, a $10 \mathrm{ng} / \mathrm{ml}$ increase in total folate was associated with a $6.2 \%$ increase in median TL (95\% CI: 1.0\%-9.4\%; $\mathrm{p}=0.018)$. The magnitude of this effect translates to an approximately $10 \%$ relative difference of TL in infants of mothers in the top vs. bottom quartile of folate concentration. Secondary analyses of folate species revealed this effect was primarily driven by 5 -methyltetrahydrofolate ( $5 \mathrm{MeTHF})$. 
Conclusion: Our findings support the concept of developmental plasticity in the fetal telomere biology system and provide the first evidence in humans that during pregnancy maternal folate may exert a "programming" effect on this system that is already apparent at birth. This finding identifies a potentially modifiable factor and supports the eventual development and testing of early interventions to optimize health and reduce disease susceptibility over the lifespan.

Disclosure of Interest: S. Entringer: None Declared., E. Epel Other: EE is a co-founders of Telomere Health, Inc, a company focused. on telomere measurement. Assays and all other activity for the current report are, however, unrelated to this company, J. Lin Other: JL is a co-founders of Telomere Health, Inc, a company focused. on telomere measurement. Assays and all other activity for the current report are, however, unrelated to this company, E. Blackburn Other: EB is a co-founders of Telomere Health, Inc, a company focused. on telomere measurement. Assays and all other activity for the current report are, however, unrelated to this company, C. Buss: None Declared., B. Shahbaba: None Declared., D. Gillen: None Declared., R. Venkataramanan: None Declared., H. Simhan: None Declared., P. Wadhwa: None Declared.

\section{DOHaD13-1298}

Periodization of postnatal ontogeny, according to agerelated morbidity and mortality: the impact on programming-of-aging hypothesis in the frame of $\mathrm{DOHaD}$ concept

Viktor I. Goudochnikov ${ }^{1, *}$

${ }^{1}$ Council, International Society for DOHaD, Santa Maria, Brazil

Background: Earlier we have evaluated age-related patterns and gender differences of morbidity and mortality caused by a number of disorders: cardiometabolic, neuropsychiatric, respiratory, gastro-intestinal ones, etc., as well as bone fractures and various cancer types, using epidemiologic data in three brazilian states of Southern region. On the basis of such evaluation, we have made a conclusion on the absence of evidence in favor of unique general scheme of aging. In the present work we have re-evaluated these data, focusing on their utility for periodization of postnatal ontogeny, in order to advance the programming-of-aging hypothesis.

Method: Epidemiologic data were retrieved from brazilian national DataSus database during the periods 1998-2000, 2001-2004 and 2005-2007. Raw data for both sexes together were recalculated in each age category as a percentage of total morbidity or mortality. Besides, feminine fraction was calculated in percent of epidemiologic indices in each age category. Thereafter, descriptive statistical treatment was performed, calculating arithmetic mean and standard error of the mean in each time period, with curve plotting by means of Excel software.
Results: The age categories evaluated were 8 decades, from $0-9 \mathrm{yr}$ to $70-79 \mathrm{yr}$ and lastly, more than $80 \mathrm{yr}$. These categories were grouped in 3, creating three stages of postnatal ontogeny: early $(0-29 \mathrm{yr})$, middle $(30-59 \mathrm{yr})$ and late (from 60 to more than $80 \mathrm{yr}$ ). As expected, the majority of disorders have shown the maxima of morbidity and mortality in the late stage of ontogeny. However, the morbidity maxima for affective disorders, schizophrenia, gastro-duodenal ulcers, cholecystitis and non-infectious skin diseases, as well as mortality maxima for epilepsy (and related disorders) were observed in the middle stage, whereas the morbidity maxima for epilepsy, pneumonia, asthma, femoral and other bone fractures and skin infections were registered in early stage of ontogeny. Besides, as expected, the majority of cancer types have shown the maxima of morbidity and mortality in the late stage of ontogeny. However, the morbidity maxima for the cancer of breast, uterine body and cervix and for benign and malignant skin cancers were observed in the middle stage, whereas the morbidity maxima for the cancer of eye (and adnexa) and bone/cartilage were registered in the early stage of ontogeny. Some disorders have demonstrated morbidity or mortality maxima on the borderline between middle and late stage. On the other hand, some diseases (pneumonia and femoral bone fractures) have shown two maxima of morbidity in different stages of ontogeny (early and late).

Conclusion: Considering that aging can be defined via increase in the risk of morbidity and mortality, the results obtained allow to conclude that programming of aging may be as complex, as the process of aging itself and should be studied by means of systems biology and medicine.

\section{References}

1. Goudochnikov VI. Comparison of age-related dynamics and gender differences in morbidity and mortality caused by several groups of diseases: no evidence for unique general scheme of aging potentially modifiable by perinatal programming. J. Dev. Orig. Health Dis. 2009, 1 (Suppl.1): S123.

Disclosure of Interest: None Declared.

\section{DOHaD13-1247}

Region-centric analysis on the Infinium $450 \mathrm{~K}$ array: an aging example

Mei-Lyn Ong ${ }^{1 * *}$, Joanna D. Holbrook ${ }^{1}$

${ }^{1}$ Growth, Development and Metabolism, SICS, Singapore, Singapore

Background: Infinium $450 \mathrm{~K}$ arrays are a cost-effective tool for interrogating the methylome on a genome-wide scale. Substantial bioinformatics challenges are inherent in their analysis, including multiple testing and the scarcity and spatial non-uniformity of the data.

Method: We used publicly available Infinium 450K datasets from three age-related studies GSE30870, GSE36064 and 
GSE 40279 [1, 2, 3] with 39, 78 and 656 samples respectively. All analyses were performed directly on the pre-processed data available online. A candidate DMR/VMR is defined as at least two spatially contiguous probes within $1 \mathrm{~kb}$ distance of each other and with differential statistic greater than the $95^{\text {th }}$ percentile. To evaluate the significance of candidate DMRs/VMRs, the area of the region (defined by genomic distance and the differential statistic) is compared to Monte Carlo simulations of null distributions, and corrected for multiple testing using FDR.

Results: We describe a methodology for detecting differentially methylated regions (DMRs) and variable methylated regions (VMRs), in data from Infinium $450 \mathrm{~K}$ arrays. We apply our method to three recently published datasets investigating DNA methylation change with age across the life course. Our method is more specific than the single $\mathrm{CpG}$ analysis since it returns many fewer DMRs and reduces the multiple testing problem. When aging associated DMRs were compared to results from other studies, the overlap was $\sim 2-4$ times greater than at the single $\mathrm{CpG}$ level, also evidencing the specificity of our method. These DMRs are significantly enriched in GenAge, which is a human curated database of genes whose function is implicated in the ageing process [4], suggesting differential methylation may reflect or cause differential transcription during aging. The consensus ageing DMRs are significantly enriched for muscle biogenesis pathways. We find a massive increase in VMRs with age and in regions of the genome associated with open chromatin and neurotransmission.

Conclusion: We propose a new method for detecting differentially and variably methylated regions on Infinium $450 \mathrm{~K}$ arrays. We have used multiple publicly available Infinium $450 \mathrm{~K}$ datasets to generate a consensus list of regions which are differentially methylated with age, supporting the hypothesis that ageing is associated with specific epigenetic modifications. We are able to show a massive increase in inter-individual variability of methylation levels by age and in targeted regions of the genome, suggesting the effect of environment on the divergence in the methylome profiles over the life course.

\section{References}

1. Heyn H, Li N, Ferreira HJ, Moran S, Pisano DG, Gomez A, Diez J, Sanchez-Mut JV, Setien F, Carmona FJ et al: Distinct DNA methylomes of newborns and centenarians. Proc Natl Acad Sci U S A 2012, 109(26):10522-10527.

2. Alisch RS, Barwick BG, Chopra P, Myrick LK, Satten GA, Conneely KN, Warren ST: Age-associated DNA methylation in pediatric populations. Genome Res 2012, 22(4):623-632.

3. Hannum G, Guinney J, Zhao L, Zhang L, Hughes G, Sadda S, Klotzle B, Bibikova M, Fan JB, Gao Y et al: Genome-wide Methylation Profiles Reveal Quantitative Views of Human Aging Rates. Mol Cell 2013, 49(2):359-367.

4. Tacutu R, Craig T, Budovsky A, Wuttke D, Lehmann G, Taranukha D, Costa J, Fraifeld VE, de Magalhaes JP: Human Ageing Genomic Resources: integrated databases and tools for the biology and genetics of ageing. Nucleic Acids Res 2013, 41(Database issue): D1027-1033.

Disclosure of Interest: None Declared.

\section{DOHaD13-1208}

The developmental origins of osteoarthritis: findings from the Hertfordshire Cohort Study

Elaine M. Dennison ${ }^{1, *}$, Camille Parsons ${ }^{1}$, Mark Edwards ${ }^{1}$, Karen Jameson ${ }^{1}$, Nicholas Harvey ${ }^{1}$, Avan Aihie Sayer ${ }^{1}$, Cyrus Cooper ${ }^{1,2}$

${ }^{1} M R C$ Lifecourse Epidemiology Unit, SOUTHAMPTON UNIVERSITY, Southampton; ${ }^{2}$ NIHR Musculoskeletal Biomedical Research Unit, Oxford University, Oxford, United Kingdom

Background: Many previous studies have linked growth in early life to musculoskeletal health in late adulthood. Specifically, investigators have suggested a link between birthweight and both hand and lumbar spine osteoarthritis (OA). In this study we sought to extend these observations by investigating relationships between growth in early life and self-reported, clinical and radiological diagnoses of OA at the hand, knee and hip among participants from the Hertfordshire Cohort Study (HCS).

Method: Complete data were available for 207 men and 202 women, born in Hertfordshire UK during 1931 -9 who still lived there in adult life, for whom birthweight and weight at one year were available from birth records, and who participated in this study of musculoskeletal health. Participants completed a questionnaire detailing self-reported OA, demographics and lifestyle. Clinical OA was defined based on American College of Rheumatology criteria. Radiographs were taken of the knees and hips and graded accorded for the presence of osteophytes, joint space narrowing and overall Kellgren and Lawrence score.

Results: The median age in this group was 75 years (interquartile range 73-77); the mean body mass index (BMI) was $28.2 \mathrm{~kg} / \mathrm{m}^{2}$ (SD 4.4). Higher birthweight and weight at one year were associated with lower rates of selfreported hip osteoarthritis, with relationships remaining robust to adjustment for age, sex, BMI, alcohol intake and smoking status (birthweight: OR 0.97, 95\% CI 0.96, 0.99 $\mathrm{p}=0.001$; weight at one year: OR 0.99, 95\% CI 0.98, 1.00, $\mathrm{p}=0.02)$. Similar relationships were also seen between weight at one year and clinical hand OA (OR 0.99, 95\% CI $0.98,1.00 \mathrm{p}=0.02$ ) but relationships were weakened by adjustment for confounders. Individuals of higher birthweight were less likely to have osteophytic growth scored as 2 or more at the hip (OR 0.98, 95\% CI 0.96, $0.99 \mathrm{p}=0.005$ ) after adjustment for age, sex, BMI, smoking and alcohol consumption but no relationships were observed with weight at one year. In general, relationships at the knee were weaker. 
Conclusion: We have found further evidence of a relationship between early life factors and adult OA. These results accord with findings from previous studies.

Disclosure of Interest: None Declared.

\section{DOHaD13-1236}

\section{A maternal 'junk food' diet alters the response of the mesolimbic reward system to naloxone in offspring postweaning}

Jessica Gugusheff ${ }^{1}$, Zhi Yi Ong ${ }^{1}$, Beverly Muhlhausler ${ }^{1, *}$

${ }^{1}$ School of Agriculture, Food and wine, University of Adelaide, Adelaide, Australia

Background: The consumption of highly palatable but nutrient poor 'junk foods' in western society is escalating and thus determining what mediates an increased preference for these foods has become of growing importance. We have previously shown that offspring of mothers who consume a junk food diet during pregnancy and lactation not only consume more junk food as adults ${ }^{1}$ but also have a desensitized opioid pathway ${ }^{2}$ (part of the mesolimbic reward system), which may impair their response to reward stimuli. The aim of the present study was to determine whether the gene expression response to the opioid antagonist naloxone in rat offspring at the 3 weeks could be influenced by exposure to a maternal junk food diet in the perinatal period.

Method: 17 Albino Wistar female rats were randomly divided into groups to receive either a junk food (JF, $\mathrm{n}=9$ ), or control chow diet $(C, \mathrm{n}=8)$, during pregnancy and lactation. At weaning, pups received daily intraperitoneal injections of either naloxone $(5 \mathrm{mg} / \mathrm{kg})$ or an equivalent volume of saline for 10 days. Real time PCR was used to determine the expression of mu-opioid receptor (MOR) and the endogenous opioid enkephalin in the nucleus accumbens (NAc) and ventral tegmental area (VTA) in the brains of offspring.

Results: A significant interaction $(P<0.05)$ was present between maternal and pup treatment for MOR expression in the VTA of male offspring indicating opposing effects of naloxone treatment depending on maternal diet. Increased MOR in males (C $0.003 \pm 0.0007$, JF $0.006 \pm 0.0008$, $P<0.05)$ and increased enkephalin (C $1.29 \pm 0.04$, JF $1.43 \pm 0.04, P<0.05)$ expression in females was observed in the NAc of junk food offspring independent of naloxone treatment.

Conclusion: These results indicate that exposure to a junk food diet during the perinatal period has functional consequences on gene expression in the opioid pathway of the offspring and provides evidence to suggest that dysregulation of the this pathway could be responsible for the increased preference for junk food observed in offspring exposed to a junk food diet in early life.

\section{References}

1. Ong, Z. Y., \& Muhlhausler, B. S. (2011). Maternal “junkfood" feeding of rat dams alters food choices and development of the mesolimbic reward pathway in the offspring. The FASEB Journal, 25(7), 2167-2179.

2. Gugusheff, J. R., Ong, Z. Y., \& Muhlhausler, B. S. (2013). A maternal "junk-food" diet reduces sensitivity to the opioid antagonist naloxone in offspring postweaning. The FASEB Journal, 27(3), 1275-1284

Disclosure of Interest: None Declared.

\section{DOHaD13-1352}

Alteration of hypothalamic gene expression by maternal obesity and exercise

Hasnah Bahari ${ }^{1}$, Mukesh Raipuria ${ }^{1}$, Margaret J. Morris ${ }^{1}$ **

${ }^{1}$ Pharmacology, University of New South Wales, Sydney, Australia

Background: Maternal obesity during pregnancy is directly associated with the growth of the fetus and can lead to an increased risk of obesity in adulthood. As beneficial effects of exercise have been shown in individuals, we investigated whether maternal exercise has positive effects on offspring. This study examined the effects of maternal obesity on offspring hypothalamic mRNA expression of appetite regulators and inflammatory markers and whether these can be influenced by maternal exercise.

Method: Female Sprague Dawley rats were fed standard chow or high fat diet (HFD), yielding lean (C) and obese (F) dams. After 6 weeks of diet, half were exercised (running wheels) (CE or FE) while half remained sedentary (CS or FS). Mating began after 8-10 days of exercise and pre-pregnancy diet was maintained throughout gestation/lactation. Dams were exercised until the end of pregnancy. Blood and whole hypothalamus were collected from both sexes at postnatal day (PND) 19 in the fed state.

Results: The exercise level was modest and did not differ between lean and obese dams $(8.1 \pm 2.4$ vs $5.1 \pm 1.5 \mathrm{~km}$ total distance run) with no significant effect on final dam's body weight. At PND1, male pups from obese dams were significantly smaller (FS vs CS; $P<0.01$ ) with no significant difference in female pups. In lean dams, maternal exercise was associated with smaller pups regardless of sex (CE vs CS, $P<0.01)$ and no difference was observed in pups of obese dams. At PND19, body weights of pups were significantly higher in pups from obese dams (FS vs CS, FE vs CE; $P<0.01)$ regardless of pup sex and maternal activity. In lean dams, exercise significantly reduced pup body weight (CE vs CS; $P<0.05)$. The level of circulating leptin was significantly increased by maternal HFD regardless of maternal activity in both male and female pups (FS vs CS, FE vs CE; $P<0.01$ ). Maternal exercise only significantly decreased leptin level in male pups of obese dams (FE vs FS; $P<0.05$ ). 
Hypothalamic fat mass and obesity associated gene (FTO) mRNA expression was increased in pups of obese dams regardless of maternal exercise (FS vs CS, FE vs CE; $P<0.01)$. Exercise downregulated FTO mRNA expression in pups of lean dams (CE vs CS; $P<0.01$ ) with no significant difference in obese dams. Maternal obesity did not significantly affect the hypothalamic leptin receptor $(\mathrm{ObRb})$ and suppressor of cytokine signalling 3 (SOCS3) mRNA expression in either male or female pups. Interestingly, exercise had different effects on ObRb between male and female pups. In male pups, maternal exercise significantly increased ObRb mRNA expression in both lean and obese dams (CE vs CS, FE vs FS; $P<0.01$ ). However in female pups, maternal exercise significantly decreased $\mathrm{ObRb}$ mRNA expression (FE vs FS; $P<0.01$ ). SOCS3 mRNA expression was increased by exercise in male pups regardless of maternal $\operatorname{diet}(\mathrm{CE}$ vs $\mathrm{CS}, \mathrm{FE}$ vs FS; $P<0.01$ ) with no significant difference observed in female offspring. Maternal HFD (FS vs $\mathrm{CS}$ ) and maternal exercise (CE vs CS) significantly increased hypothalamic interleukin 1 beta (IL-1 $\beta$ ) mRNA expression with no difference between sexes $(P<0.01)$.

Conclusion: As expected, maternal obesity impacted pup body weight and leptin concentration in both male and female offspring. Maternal exercise effects on pups were observed in hypothalamic FTO, ObRb, SOCS3 and IL-1 $\beta$ mRNA gene expression, with larger impacts on male pups. Surprisingly maternal exercise may increase hypothalamic inflammation in pups.

Disclosure of Interest: None Declared.

\section{DOHaD13-1157}

\section{Developmental contributions to dietary intake in adult survivors of kwashiorkor and marasmus}

Claudia P. Campbell ${ }^{1, *}$, Asha Badaloo ${ }^{1}$, David Raubenheimer $^{2}$, Peter Gluckman ${ }^{2}$, Mark Hanson ${ }^{3}$, Claudia Martinez ${ }^{2}$, Alison Gosby ${ }^{4}$, Stephen Simpson ${ }^{2}$, Clive Osmond ${ }^{5}$, Michael Boyne $^{1}$, Terrence Forrester ${ }^{1}$

${ }^{1}$ Tropical Medicine Research Institute, University of the West Indies, Mona, Kingston, Jamaica; ${ }^{2}$ Liggins Institute and National Research Centre for Growth and Development, University of Auckland, Auckland, New Zealand; ${ }^{3}$ Institute of Developmental Sciences, University of Southampton, Southampton, United Kingdom; ${ }^{4}$ Institute and National Research Centre for Growth and Development, University of Auckland, Auckland, New Zealand; ${ }^{5}$ MRC Lifecourse Epidemiology Unit, University of Southampton, Southampton, United Kingdom

Background: Evidence indicates that in utero conditions may influence the clinical presentation of childhood severe acute malnutrition (SAM) phenotypes i.e. kwashiorkor and marasmus. These phenotypes may have different risks to develop obesity in later life including variation in energy intake in relation to having set target for dietary protein. We studied adult survivors of SAM to determine if birthweight and SAM phenotype were associated with energy intake and body weight change when subjects consumed foods with different fixed percentage of energy as protein (PEP).

Method: We recruited 63 adults, aged $17-46$ years, who had been treated for SAM in infancy. For 3 consecutive days, they consumed a combination of foods containing 10, 15 and 25 PEP, ad libitum (phase 1). Protein target was defined as protein intake during this phase. On the next 5 days (phase 2) the subjects were randomized to choose from foods that contained one fixed PEP $(10 \% ; \mathrm{n}=22,15 \% ; \mathrm{n}=20$ or $25 \% ; n=21)$. This tested whether they would increase their energy intake (EI) on lower PEP diet and decrease their EI on higher PEP diet (i.e. protein leveraging) in order to maintain their protein target. Regression analyses were used to explore the association of these outcomes with age, sex, birth weight, SAM phenotype and dietary PEP.

Results: In phase 1 , protein intake (mean \pm SD) contributed $402 \pm 114 \mathrm{kcal} / \mathrm{d}$ and $6.4 \pm 1.9 \mathrm{kcal} / \mathrm{d}$ per kg bodyweight. It was similar in the SAM phenotypes but higher in males compared to females. Also, PEP was similar in survivors of kwashiorkor (14.7) and marasmus (14.9), and was not associated with subject's current age or anthropometry. However, proportion of total energy intake from protein fell by $0.36 \%$ per kg birth weight (range: 0.04 to $0.69 ; P=0.03$ ). This fall was similar in SAM phenotypes. In phase 2, participants allocated the $10 \%$ protein diet maintained their total EI and hence protein intake was reduced. Subjects allocated to the $15 \%$ protein diet maintained similar protein and total energy intakes to phase 1 experiment. Participants allocated the $25 \%$ protein diet decreased total EI but protein intake was increased. The change in EI from phase 1 to 2 was not related to age, sex, SAM phenotype or birth weight, but was inversely associated with PEP in the allocated diet ( $P$ gradient across groups $=0.03)$. Also, weight change was inversely correlated to EI and allocated diet ( $P$ gradient across groups $=0.002$ ), but not with age, sex, other measures of anthropometry, SAM phenotype or birth weight.

Conclusion: There is a significant birthweight effect on protein intake in adulthood, suggesting that antenatal factors influence later appetite control, energy balance and thus, obesity risk. The inverse relationship between EI and dietary PEP in phase 2 supports protein leveraging, but the estimated protein target was not maintained.

Disclosure of Interest: None Declared.

\section{DOHaD13-1048}

Early programming of aerobic and neuromuscular fitness at primary school age. The $\mathrm{ABCD}$-study

Arend W. Van Deutekom ${ }^{1, *}$, Mai J. Chinapaw ${ }^{2}$, Manon van Eijsden $^{3}$, Tanja G. Vrijkotte ${ }^{4}$, Reinoud J. Gemke ${ }^{1}$ 
${ }^{1}$ Dept of Pediatrics; ${ }^{2}$ Dept of Public Health, VU medical center; ${ }^{3}$ Dept of Epidemiology and Health Promotion, Public Health Service; ${ }^{4}$ Dept of Social Medicine, Academic Medical Centre, Amsterdam, Netherlands

Background: Obesity plays a critical role in the association of low birth weight and early growth with adult disease, often preceding insulin resistance, dyslipidemia and hypertension. As obesity is fundamentally a disorder of energy regulation, research of the pathogenesis of obesity also includes developmental origins of energy intake and expenditure. A series of animal studies demonstrated that an adverse perinatal environment reduces later physical activity levels, as a main component of energy expenditure, partly arising from observed disruptions in aerobic and neuromuscular fitness. These findings have been replicated in adults and adolescents, but not in prepubescent children. We hypothesize that this developmental programming of physical fitness is also evident at 8-9 years of age, and thereby represents a possible cause for the association of low birth weight and accelerated growth with childhood obesity.

Method: We assessed aerobic and neuromuscular fitness in 194 children (104 boys) of Dutch ethnicity at 8.6 years (range: 8.1-9.3y) from the ABCD birth cohort. This is a prospective birth cohort, including extensive questionnaires, detailed anthropometrics and blood samples. We measured aerobic fitness using a 20 meter multistage shuttle run test (20m-MSRT), and neuromuscular fitness using the standing broad jump (SBJ) and handgrip strength test. Low birth weight was defined as below the $10^{\text {th }}$ percentile for gestational age and accelerated infant growth as a gain in SD score $>0.67$ for weight between birth and 12 months. Multivariable linear regression analyses were used to evaluate the relationships.

Results: Subjects with low birth weight and accelerated infant growth $(\mathrm{n}=16)$ attained a mean $( \pm S D) 20 \mathrm{~m}-M S R T$ level of 3.9 ( \pm 0.4$)$, which was significantly lower than subjects with normal birth weight and normal infant growth $(n=106$; difference: $1.1 ; 95 \% \mathrm{CI}, 0.3-1.9 ; \mathrm{p}=0.009)$; subjects with low birth weight and normal infant growth $(\mathrm{n}=28$; difference: $1.5 ; 95 \% \mathrm{CI}, 0.6-2.5 ; \mathrm{p}=0.002$ ); and subjects with normal birth weight and accelerated infant growth $(\mathrm{n}=44$; difference: $1.1 ; 95 \% \mathrm{CI}, 0.2-1.9 ; \mathrm{p}=0.02)$. Normal birth weight subjects had a mean grip strength of $13.6 \mathrm{~kg}$ $( \pm 3.0)$, independent of infant growth. This was significantly higher than low birth weight children (difference: -1.3 ; $95 \% \mathrm{CI},-2.4-0.3 ; \mathrm{p}=0.01$ ), with no effect of infant growth on this relationship. There was no association of infant growth with grip strength or SBJ, or of birth weight with SBJ. All analyses were adjusted for age, gender, height, gestational age, maternal age, and socio-economic status.

Conclusion: Low birth weight with subsequent accelerated infant growth was associated with diminished aerobic fitness at age 8-9. Aerobic fitness of children with only low birth weight or accelerated growth was not significantly different from the reference group. Birth weight was linearly associated with hand grip strength, indicative of increased neuromuscular fitness with higher birth weight. These early developmentallyinduced deficiencies in aerobic and neuromuscular fitness may lead to increased susceptibility to obesity and related disease in low birth weight and early growth accelerated children.

Disclosure of Interest: None Declared.

\section{DOHaD13-1049}

Early programming of physical activity and sedentary behavior at primary school age. The ABCD-study

Arend Van Deutekom ${ }^{1, *}$, Mai J. Chinapaw ${ }^{2}$, Manon van Eijsden $^{3}$, Tanja G. Vrijkotte ${ }^{4}$, Reinoud J. Gemke ${ }^{1}$

${ }^{1}$ Dept of Pediatrics; ${ }^{2}$ Dept of Public Health, VU medical center; ${ }^{3}$ Dept of Epidemiology and Health Promotion, Public Health Service; ${ }^{4}$ Dept of Social Medicine, Academic Medical Centre, Amsterdam, Netherlands

Background: There is growing evidence that developmental programming of the energy balance is a possible link between early life experiences and adult-onset disease. As energy balance is to a great extent regulated through variations in energy expenditure, programming of physical activity in the offspring is plausible. Indeed, animal studies have illustrated plasticity of control regarding physical activity, with offspring being hypoactive after perinatal malnutrition. In human research the impact of early life influences on physical activity levels has received far less attention. In addition, few studies assessed the perinatal effects on later sedentary behavior. We hypothesize that low birth weight and growth acceleration in infancy leads to lower physical activity levels and more sedentary behavior at 8-9 years of age, rendering these subjects more susceptible to obesity.

Method: In the ABCD study, a prospective birth cohort encompassing 8266 pregnant women and their offspring, physical activity and sedentary time were measured in a group of 183 children (100 boys) of Dutch ethnicity at 8.7 years (range: 8.1-9.3y) using an Actigraph accelerometer for at least 10 hours on at least three school days and one weekend day. Outcome measures were minutes per day above moderate intensity activity (>3000 counts per minute (c.p.m.)) and minutes per day spent sedentary $(<100$ c.p.m.). We expressed low birth weight as weight below the $10^{\text {th }}$ percentile for gestational age. Average standardized growth velocity, defined as the difference in weight SD score between birth and 12 months, was linearly associated with the outcome, and therefore assessed as a continuous determinant. Multivariable linear regression analyses were used to evaluate the relationships.

Results: On average $( \pm S D)$, children participated in 37 $( \pm 14)$ minutes of physical activity and $412( \pm 45)$ minutes of sedentary behavior per day. Low birth weight $(n=41)$ was not significantly associated with either physical activity $(\mathrm{p}=0.2)$ or sedentary time $(\mathrm{p}=0.4)$ in our cohort. The average 
standardized growth velocity, however, was significantly positively associated with sedentary time, with an average increase of 7.8 minutes in daily sedentary time (95\%CI, 0.7-15.0; $\mathrm{p}=0.03$ ) for every increase in SD score for weight gain in infancy. This association was adjusted for birth weight, gender, age, height, BMI, gestational age, Actigraph wearing dates, wearing time, maternal age, and socio-economic status, and was evident across the whole range of growth. Growth velocity was not significantly associated with physical activity $(\beta=-0.81$; 95\%CI, $-2.7-1.1 ; \mathrm{p}=0.4)$.

Conclusion: Our study indicates that infant growth may program sedentary behavior, but not physical activity levels at age 8-9. Birth weight was not related to either physical activity or sedentary behavior. This suggests that the developmental effects of growth on childhood energy expenditure is manifested by variations in sedentary behavior, rather than physical activity levels. Future research should provide a better understanding of the independent mediating roles of sedentary behavior and physical activity in the developmental processes of health and disease.

Disclosure of Interest: None Declared.

\section{DOHaD13-1508}

Facial responses do basic taste in newborns from mothers with and without gestational diabetes mellitus

Joao G. Alves ${ }^{1, *}$, Andrea Zacche ${ }^{1}$

${ }^{1}$ Instituto de Medicina Integral Prof. Fernando Figueira (IMIP), Recife, Brazil

Background: Taste preferences play a key role in formation of food habits and can be influenced by genetic and environmental factors. Flavor, experienced by taste, smell, and hemosensory irritation, has been demonstrated in human fetal life. Food flavors transmitted from the mother's diet through amniotic fluid can influence food acceptance. However it is not known if blood stimulus in fetal life as higher glucose level can influence preference to basic taste. Our aim is to compare facial responses in newborns from mothers with and without gestational diabetes mellitus

Method: A cross-sectional study was developed at the Instituto de Medicina Integral Prof. Fernando Figueira (IMIP), Recife, Brazil, between August 2012 to April 2013. This study was previously approved by IMIP Ethical Committee in Research, and written informed consent was obtained. One hundred and eighty newborns, all born full term, were studied. Each newborn was tested within the first 24 hours of life. The stimulus was given in the following sequence with an interval of 1 minute: glucose $(0.3 \mathrm{M})$, sodium chloride $(0.3 \mathrm{M})$, sodium citrate $(0.001 \mathrm{M})$, and urea $(0.18 \mathrm{M})$. A $1-\mathrm{mL}$ disposable syringe was used to apply $0.2 \mathrm{~mL}$ of solution on the dorsal surface of the tongue. All infants were held upright facing forward during the test and had their face videotaped during a 1-minute interval. The facial responses were coded into nine action units according to the Baby Facial Action Coding System: Alrepresents no distinct mouth action or sucking on the face; $\mathrm{A} 2$ is A1with a negative expression on themid-face; A3 is A1with a negative expression on the mid-face and brows; B1 represents the facial response of a pursingmouth; B2 is B1with a negative expression on the midface; $\mathrm{B} 3$ is $\mathrm{B} 1$ with a negative expression on the mid-face and brows; $\mathrm{C} 1$ represents a mouth-gaping action; $\mathrm{C} 2$ is $\mathrm{C} 1$ with a negative expression on the mid-face; and C3 is C1 with a negative expression on the mid-face and brows. Two trained professionals who were unaware of the study aims watched the videotape recordings to classify facial responses. Reliability for scoring was $88 \%(\mathrm{p}<0.001)$.

Results: A total of 169 newborns were studied; 83 from mothers with gestational diabetes and 86 without. Facial action units to sweet taste were less pronounced in newborns from mothers with gestational diabetes as compared to newborns from mothers without gestational diabetes, respectively $71 \%$ vs $86 \%$; $<0.004$. Facial action units to salty, sour and bitter showed no differences among newborns from mothers with and without gestational diabetes, salty $(56 \%$ vs $66 \% ; \mathrm{p}=0.09)$, sour $(30.0 \%$ vs $33 \% ; \mathrm{p}=0 ; 07)$, and bitter $(28.3 \%$ vs $24 ; 6 \% . \mathrm{P}=0.59)$ tastes respectively.

Conclusion: Our fundings suggest that a higher exposition to glucose during fetal life may change newborn taste preference to sweet. Further studies should explore these implications throughout childhood.

Disclosure of Interest: None Declared.

\section{DOHaD13-1433}

\section{Facial responses to basic taste in preterm newborns}

Joao G. Alves ${ }^{1, *}$, Andrea Z. Sa ${ }^{1}$, José Roberto Junior ${ }^{1}$

${ }^{1}$ Instituto de Medicina Integral Prof. Fernando Figueira (IMIP), Recife, Brazil

Background: The ability to detect sensory stimuli, such as taste seems to develop during fetal life and term newborns can identify basic tastes. However it is not know if preterm newborns can recognize basic tastes. Our aim is to verify taste preferences to sweet, salty, bitter and sour among preterm newborns with different gestational age.

Method: 60 preterm newborns, 30 with $34 / 36$ and 30 with $31 / 33$ gestational weeks were tested to facial responses to four solutions (glucose, sodium chloride, sodium citrate, and urea). Two drops of this solution were applied on the tongue and facial responses of the infants were recorded. Facial responses, A1 to $\mathrm{C} 3$, were coded using the Baby FACS. Chi-squared test was used to detect differences in facial responses. This project was approved by IMIP Ethical Committee on Research and all participants signed an informed consent before admission to this research. 
Results: Facial responses to taste solutions showed differences among preterm newborns with more positive and negative responses within $34 / 36$ gestational age group as compared to $31 / 33$ gestational age group, respectively: sweet $-88.3 \%$ vs $51.2 \%$ ( $\mathrm{p}<0.001)$, salty $-51.7 \%$ vs $31 \%(\mathrm{p}<0.001)$, sour $-30.0 \%$ vs $18 \%(\mathrm{p}<0.04)$, and bitter $-28.3 \%$ vs $18.1 \%$ $(\mathrm{p}<0.047)$.

Conclusion: Facial responses to basic taste solutions seems to be not completely developed in preterm newborns at $31 / 33$ gestational weeks. Our findings suggest that facial responses develops during the last trimester of pregnancy.

\section{References}

1. Alves JG, Russo PC, Alves GV. Facial Responses to Basic Tastes in Breastfeeding and Formula-Feeding Infants. Breast Feeding Medicine 2013; 8(2):235-6.

Disclosure of Interest: None Declared.

\section{DOHaD13-1400}

In-utero and postnatal exposure to high fat diet alters expression of genes that regulate circadian clock function and feeding behaviour in the adult mouse offspring brain

Felino R. Cagampang ${ }^{1, *}$, Jasmin L. Shearer ${ }^{1}$, Ravi Mani ${ }^{1}$, Jane K. Cleal ${ }^{1}$, Kerry L. Hyde ${ }^{1}$, Rohan M. Lewis ${ }^{1}$, Mark A. Hanson $^{1}$, Christopher D. Byrne ${ }^{1}$, Kimberley D. Bruce ${ }^{1}$

${ }^{1}$ Faculty of Medicine, Institute of Developmental Sciences, University of Southampton, Southampton, United Kingdom

Background: The prevalence of obesity and metabolic diseases are rapidly increasing. We have previously shown in mice that suboptimal in-utero and postnatal nutritional environments can increase the offspring's susceptibility to metabolic pathologies in adulthood [1, 2]. Moreover, these mouse offspring have altered $24 \mathrm{~h}$ (circadian) locomotor activity and feeding behaviour, which may contribute to the development of metabolic disturbance. In mammals, circadian pattern of physiological processes, including activity cycle and feeding, are regulated by an endogenous timing system in the suprachiasmatic nuclei (SCN) of the brain. Therefore, in this study, we examined whether in-utero and postnatal exposure to a high fat diet alters the expression pattern of genes that regulate clock function in the SCN and those that regulate food intake in the hypothalamic arcuate nucleus (ARC).

Method: Female C57/BL6J mice were fed either a high-fat (HF; $45 \%$ kcal fat, SDS, UK) or control chow (C; 7\% kcal fat, RM1 diet, SDS, UK) diet 4 to 6 weeks prior to conception, through pregnancy and lactation. Resulting male offspring were fed the same $\mathrm{C}$ or $\mathrm{HF}$ diet after weaning, creating two offspring groups; $\mathrm{C} / \mathrm{C}$ and $\mathrm{HF} / \mathrm{HF}$, representing maternal vs. the post-weaning offspring diet, respectively. At 15 weeks of age, offspring were killed over 6 time points
( $\mathrm{n}=5$ per time point) across the $24 \mathrm{~h}$ light-dark cycle $(12 \mathrm{~h}$ light: $12 \mathrm{~h}$ dark) and brains were collected. The SCN and ARC regions were micropunched from coronal brain sections and processed for RT-PCR quantification of the clock genes CLOCK, BMAL1, PER2 and CRY2, and the appetite regulating genes $N P Y$ and $P O M C$. The geNorm program was used in selecting $\beta$-actin and $G A P D H$ as the appropriate reference genes to normalize expression levels. Cosine wave analysis (using the circadian software ACRO) was used to calculate the acrophase (time of peak) and mesor (mean levels) of the $24 \mathrm{~h}$ rhythm in gene expression. Difference in mean mRNA levels for each gene between the two offspring groups were statistically analysed using $t$-test $(\mathrm{p}<0.05$ was considered significant).

Results: Peak mRNA expression for CLOCK, BMAL1 and $C R Y 2$ in the SCN shifted by $4 \mathrm{~h}, 8 \mathrm{~h}$ and $4 \mathrm{~h}$, respectively. In addition, there was a 1.8 and 1.3 -fold reduction in mean $24 \mathrm{~h}$ mRNA levels for PER2 and CRY2, respectively (both at $\mathrm{p}<0.05$ ), in the HF/HF compared to C/C animals. In the ARC, peak mRNA expression for $N P Y$ shifted by $4 \mathrm{~h}$ and mean $24 \mathrm{~h}$ mRNA levels for POMC was reduced by 1.7 -fold $(\mathrm{p}<0.05)$ in the HF/HF versus $\mathrm{C} / \mathrm{C}$ group.

Conclusion: Prenatal and postnatal exposure to HF diet alters circadian rhythms in expression of molecular clock components in the offspring $\mathrm{SCN}$, and the appetite regulating genes in the ARC. These novel findings could provide some mechanistic explanation to changes in locomotor and feeding rhythms observed in the HF/HF offspring, and provides further evidence for the involvement of an altered circadian clock network in the development of metabolic disturbance.

This work is supported by the BBSRC \& Diabetes UK

\section{References}

1. K. D. Bruce et al., Hepatology 50, 1696 (2009).

2. M. M. Elahi et al., Br. J. Nutr. 102, 514 (2009).

Disclosure of Interest: None Declared.

\section{DOHaD13-1249}

Maternal sildenafil treatment increases neuropeptide $y$ innervation of the paraventricular nucleus in the brains of offspring

Joanna L. Stanley ${ }^{1, *}$, Christine Jasoni ${ }^{2}$, Irene J. Andersson ${ }^{3}$, Sandra T. Davidge ${ }^{4}$, Philip N. Baker ${ }^{1}$

${ }^{1}$ Liggins Institute, University of Auckland, Auckland; ${ }^{2}$ Anatomy, University of Otago, Dunedin, New Zealand; ${ }^{3}$ Medicine;

${ }^{4}$ Obstetrics/Gynecology \& Physiology, University of Alberta, Edmonton, Canada

Background: Fetal growth restriction (FGR) is associated with long-term health consequences in adults, including the development of obesity. Studies have observed that the 'fetal programming' of adult obesity may be linked with, among 
other changes, perturbations in neuronal development, release of hormones and expression of receptors involved in appetite/ body weight regulation. We hypothesized that offspring from a mouse model of FGR, the catechol-O-methyl transferase knockout mouse $\left(\mathrm{COMT}^{-1-}\right)$, would display abnormalities of hypothalamic neuropeptide Y (NPY)-containing neurons, which stimulate food intake and decrease energy expenditure. Further, we hypothesized that maternal treatment with Sildenafil citrate during pregnancy, which normalizes fetal growth, would also normalize NPY-containing neuron development.

Method: Female $\mathrm{COMT}^{-1-}$ and control C57BL/6J mice were mated with males of a corresponding genotype. At gestational day 12.5 they were randomized to receive either Sildenafil citrate-treated $(0.2 \mathrm{mg} / \mathrm{ml})$ or untreated drinking water. Pups were removed at gestational day 18.5, weighed, measured then placed in $10 \%$ formalin. Brains were removed and $20 \mu \mathrm{m}$ frozen sections were taken in the coronal plane. NPY fibers were imaged using immunohistochemistry (rabbit anti-NPY antibodies, 1:2000, followed by goat anti-rabbitAlexa-488, 1:1000), followed by confocal microscopy (Zeiss LSM-510). The paraventricular nucleus (PVN) was identified, and average pixel intensity was analysed using ImageJ. The brains of 6 pups per experimental group were analysed, from 3 mothers per group. Data was analysed by 2-way ANOVA followed by Bonferroni post-hoc test.

Results: There was no effect of genotype on NPY fiber density in the PVN $(p=0.26)$. There was, however, a significant effect of Sildenafil treatment on NPY fiber density $(p<0.001)$ in the PVN across treatment groups. Fiber density was significantly increased in both C57BL/6J $(\mathrm{p}<0.01)$ and $\mathrm{COMT}^{-1-}$ $(p<0.05)$ pups from mothers treated with Sildenafil citrate compared with their untreated controls of matched genotype.

Conclusion: There is considerable evidence that NPY neurons, which project to the PVN, are extremely important in regulating energy homeostasis. Increased NPY release in the PVN results in increased food intake, as well as increased energy storage in the form of fat. Given that Sildenafil treatment increased pup birth weight, NPY-mediated increases in fat storage may be one mechanism by which this was achieved. There may, however, also be long-term consequences for offspring of mothers treated with Sildenafil, such as an increased risk of obesity, which should be studied further.

Disclosure of Interest: None Declared.

\section{DOHaD13-1575}

\section{Sensitivity of housekeeping genes in the suprachiasmatic region of the mouse brain to diet and the daily light-dark cycle}

Jane K. Cleal ${ }^{1, *}$, James N. Shepherd ${ }^{1}$, Jasmin L. Shearer ${ }^{1}$, Kimberly D. Bruce ${ }^{2}$, Mark A. Hanson ${ }^{1}$, Christopher D. Byrne $^{1}$, Felino R. Cagampang ${ }^{1}$
${ }^{1}$ Institute of Developmental Sciences, University of Southampton, Southampton, United Kingdom; ${ }^{2}$ The Scripps Research Institute, Florida, United States

Background: In gene expression studies housekeeping genes are used as internal controls. The expression of housekeeping genes in mice can vary according to tissue type and experimental treatment such as nutrition [1,2]. Many genes involved in nutrient homeostasis are entrained to $24 \mathrm{~h}$ (circadian) rhythms regulated by an endogenous timing system in the suprachiasmatic nuclei $(\mathrm{SCN})$ of the brain. Housekeeping genes within the SCN may exhibit changes in expression levels throughout the $24 \mathrm{~h}$ light-dark cycle and as such the time of sample collection may influence the stability of the gene selected as internal controls.

Aim: to establish whether the expression of housekeeping genes in the SCN is influenced by a high fat diet and the time of sample collection.

Method: Female C57/BL6J mice were fed either a high fat (HF, $45 \% \mathrm{kcal}$ fat) or control chow (C, 7\% kcal fat) diet 4 weeks prior to conception and throughout pregnancy and lactation. Weaned male offspring were fed $\mathrm{C}$ or $\mathrm{HF}$ diet generating 2 groups $\mathrm{C} / \mathrm{C}$ and $\mathrm{HF} / \mathrm{HF} .15$ week old offspring were killed over 6 time points ( $\mathrm{n}=4-6$ per time point) across the $24 \mathrm{~h}$ light-dark cycle and brains were collected. The SCN region was micropunched from coronal brain sections and processed for quantitative RT-PCR measurements of the housekeeping genes: adenosine triphosphate synthase subunit (Atp5b), cytoplasmic beta actin $(B-A c t)$, beta-2-microglobulin $(B 2 M)$, cytochrome c-1 (Cycl), calnexin (Canx), eukaryotic translation initiation factor $4 \mathrm{~A}$ isoform 2 (Eif4a2), glyceraldehyde 3-phostphate dehydrogenase (Gapdh), succinate dehydrogenase complex, subunit A $(S d h a)$, ubiquitin $\mathrm{C}(U b c)$, and tyrosine 3-monooxygenase/tryptophan 5-monooxygenase activation protein $(Y w h a z)$. CT values were transformed into relative quantification data using the deltaCT method. Data were compared by two-way ANOVA (factors: diet and day/night). Significance was accepted with $\mathrm{p}<0.05$ (trend $\mathrm{p}<0.1$ ). Expression stability and the optimum number of control genes required were calculated using geNorm software.

Results: SCN samples collected during the dark compared to the light period had significantly decreased Atp $5 b$ (4.5-fold, $\mathrm{p}=0.01), \quad B$-Act $\quad$ (3.6-fold, $\quad \mathrm{p}=0.02), \quad$ Cycl $\quad$ (3.9-fold, $\mathrm{p}=0.03), \quad$ Eif4a2 (5.4-fold, $\mathrm{p}=0.001), \quad U b c$ (3.4-fold, $\mathrm{p}=0.01)$, Gapdh (4.7-fold, $\mathrm{p}=0.04)$, Ywhaz (2.8-fold, $\mathrm{p}=0.04)$ expression and a trend towards reduction in $S d h a$ (2.8-fold, $\mathrm{p}=0.08)$ and $B 2 M(1.5$-fold, $\mathrm{p}=0.06)$. The pairwise variation with the sequential addition of each reference gene indicated that three genes are sufficient as internal controls. Gapdh, B-Act and Atp5b showed highest stability with all samples analyzed together and when samples in the dark period were analyzed separately. For samples in the light period, Sdha replaced $A t p 5 b$ as the third most stable gene.

Conclusion: We suggest Gapdh, B-Act and Atp5b as housekeeping genes recommended as internal controls. These appear 
to be the most stably expressed genes in the SCN regardless of diet or time within the $24 \mathrm{~h}$ light-dark cycle. However SCN samples collected during the light and dark periods did show differences in expression and as such the timing of collection should be considered when carrying out qPCR studies.

\section{This work is supported by the BBSRC \& Diabetes UK}

\section{References}

1. Bruce et al., J. Clinic. Experiment. Cardiol 2011.

2. Bruce et al., Liver Int 2012.

Disclosure of Interest: None Declared.

\section{DOHaD13-1404}

Behavioural and neurodevelopmental effects of prenatal stress in neonatal guinea pigs

Greer A. Bennett ${ }^{1}$, Hannah K. Palliser ${ }^{1}$, David W. Walker ${ }^{2}$, Jonathan J. Hirst ${ }^{1, *}$

${ }^{1}$ Mothers and Babies Research Centre and School of Biomedical Sciences and Pharmacy, University of Newcastle, Callaghan;

${ }^{2}$ Obstetrics and Gynaecology, Ritche Centre For Baby Health

Research, Monash University, Melbourne, Australia

Background: Maternal psychosocial stress has been linked with many adverse neonatal outcomes, particularly effects on behaviour and neurodevelopment. Epidemiological studies have shown a striking relationship between maternal stress exposure and altered cognitive functioning of offspring at school age. In animal studies, prenatal stress is not only associated with behavioural alterations in offspring but is also linked with altered size and structure of various brain regions, including the hippocampus. The aim of the current study was to characterize the effects of prenatal stress on neurodevelopment in term fetuses and assess the behavioural outcomes in these guinea pig neonates at 5 postnatal days (neonatal) and 21 postnatal days (juvenile).

Method: Stress was induced in pregnant guinea pigs by exposure to strobe light for 2hours/day at 50, 55, 60 and 65 gestational days (term 70days). Maternal salivary cortisol was measured before and after each stress episode. Fetal brains were collected at term and stained for myelin basic protein expression to assess myelination in three brain regions: the CA1 region of the hippocampus, the white matter tracts of the cerebral cortex and the cerebellum. Those pregnancies allowed to spontaneously deliver at term underwent behavioural testing for ambulatory activity and anxiety measures, using open field testing on post-natal days 5 and 21 .

Results: Maternal salivary cortisol was significantly increased $(\mathrm{p}<0.01)$ after exposure at each stress induction event, indicating large elevations in circulating glucocorticoid levels at this time. Term fetuses (males $\mathrm{n}=5$, females $\mathrm{n}=4$ ) exposed to prenatal stress showed reduced $(\mathrm{p}<0.01)$ expression of myelin basic protein in the hippocampus, cerebral cortex and cerebellum compared to controls (males $n=7$, females $n=5$ ). At 21 days of age, prenatally stressed offspring (males $\mathrm{n}=4$, females $\mathrm{n}=4$ ) showed significantly $(\mathrm{p}<0.05)$ reduced ambulatory activity compared to controls (males $\mathrm{n}=5$, females $\mathrm{n}=4$ ). These prenatally stressed offspring also spent less time in the inner zone of the field $(\mathrm{p}<0.01)$ at 21 days postnatal age, indicating a more anxious phenotype. There were no significant effects of prenatal stress on behaviour at day 5 .

Conclusion: These findings indicate that prenatal stress induces disruptions in fetal brain development. These disruptions may have ongoing functional effects shown by changes in behavioural outcomes of juvenile guinea pigs exposed to prenatal stress. These observations suggest that elevated maternal stress levels and thus, excess glucocorticoid exposure, may adversely affect fetal brain development and neonatal brain functioning after birth. Previous studies indicate elevated glucocorticoid exposure and prenatal stress suppress neurosteroid producing enzyme expression in the placenta and disrupt neurosteroid levels in the fetus, respectively, which may be processes mediating these adverse effects. These changes may contribute to the increased incidences of behavioural disorders in childhood observed in human studies following exposure to prenatal stress.

Disclosure of Interest: None Declared.

\section{DOHaD13-1540}

Cerebral echo3D and neurodevelopment in newborn. Preliminary study

Benito Cappuccini ${ }^{1 * *}$, Graziano Clerici ${ }^{2}$, Elena Laurenti ${ }^{2}$, Giancarlo Barboni ${ }^{1}$, Gian Carlo Di Renzo ${ }^{2}$

${ }^{1}$ Neonatology, Perugia Hospital, Italy; ${ }^{2}$ Perinatal Medicine, University of Perugia, Perugia, Italy

Background: The "Early Development" concept represented the beginning of a new era in neonatal and perinatal medicine. Prematurity, IUGR, epigenetic damages, pain and stress are the most remarkable insults of the brain in fetal life and in neonatal period. These damages arouse several deleterious effects on behavioural development, later in life. Animals and humans show evidence of correlation among impaired neurocomportamental development and the volume reduction of brain structures such as Cortex, Thalamus, Hippocampus; also volume reduction is associated with dementia and cognitive performance. Data from literature are scarce. Recently, the echo 3D has been proposed to estimate the volume of the above in newborns. Aim of the study: feasibility of brain's structures measures. Evaluate possible differences among Preterms, IUGR, Healthy newborns.

Method: We estimated the volumes of Cortex, Thalamus and Hippocampus (mean of 4 measures, in ml.) with the cerebral echo 3D in 45 neonates, 30-40 days old (at corrected age in 
preterms and IUGR). 16 were preterms (gestational age 24-31 w.), Group A; 13 were IUGR (gestational age 36-38) Group B; 16 were healthys at term (Group C).

Results: Median Values of volume $(\mathrm{ml})$ Frontal Cortex: $\mathrm{A}=28.4, \mathrm{~B}=31.8, \mathrm{C}=45.8$ (A vs $\mathrm{B}$ and $\mathrm{C} \mathrm{p}<0.001, \mathrm{~B}$ vs $\mathrm{C} p<0.001$ ). Thalamus: $\mathrm{A}=4.8, \mathrm{~B}=5.4, \mathrm{C}=7.2$ (A vs $\mathrm{B}$ $\mathrm{p}<0.01$, A vs $\mathrm{C} \mathrm{p}<0.001, \mathrm{~B}$ vs $\mathrm{C} \mathrm{p}<0.001)$.

Hippocampus: $\mathrm{A}=0.52, \mathrm{~B}=0.72, \mathrm{C}=1.2$ (A vs $\mathrm{B}$ and $\mathrm{C}$ $\mathrm{p}<0.001$, B vs $\mathrm{C} \mathrm{p}<0.001)$. Frontal Cortex + Thalamus: $\mathrm{A}=33.5, \mathrm{~B}=37.8, \mathrm{C}=52.8$, (A vs $\mathrm{B}$ and $\mathrm{C} \mathrm{p}<0.001, \mathrm{~B}$ vs C p $<0.001)$.

Conclusion: The Variance was $<10 \%$ for each measure, proving that it is feasible to evaluate the volume of frontal cortex, thalamus, hippocampus, with the cerebral echo 3D. All volumes were smaller in group $\mathrm{A}$ vs $\mathrm{B}-\mathrm{C}$ and in group $\mathrm{B}$ vs $\mathrm{C}$. Interestingly, also the volume of frontal cortex + thalamus were smaller in the same way. The differences among volumes were statistically significant $(\mathrm{p}<0.001)$. Conclusion: The estimate of brain's structures volume in newborns with echo3D is feasibile. The preterms and IUGR showed a significant reduction of these volumes, probably acquired in fetal life, so these differences may be a major cause of impaired development. Ultrasounds might be useful to conduct studies and screenings. Also, they can be instrumental to predict the cognitive and neurocomportamental risk later in life. Further and larger studies are necessary, as well as fetal 3D ultrasounds and epigenetic-molecular investigations; however the system medicine approach based on cross disciplinary collaborations represents the future.

\section{References}

1. "BRAIN" in Encyclopedia on Early Childhood Development, 2010. Riccabona M. "Potential role of 3DUS in infants and children" Pediatr. Radiol., 2011. Pohlack ST. "Bigger is better! Hippocampal volume and declarative memory performance in healthy young men" Brain Struct. Funct., 2012. Brain Development Cooperative Group “Total and regional brain volumes in a population based normative sample from 4 to 18 years" Cereb. Cortex, 2012. Ball G. "The effect of preterm birth on thalamic and cortical development" Cereb. Cortex, 2012.

Disclosure of Interest: None Declared.

\section{DOHaD13-1391}

Early life influences on cortical excitability and cognitive outcomes in adolescents

Luke Schneider $^{1, *}$, Nicholas R. Burns ${ }^{2}$, Ted Nettelbeck ${ }^{2}$, Michael C. Ridding ${ }^{1}$, Julia B. Pitcher ${ }^{1}$

${ }^{1}$ Robinson Institute; ${ }^{2}$ School of Psychology, UNIVERSITY OF ADELAIDE, Adelaide, Australia

Background: Increasing evidence shows that even late preterm birth can impair neurocognitive development in children without clinical brain lesions. The pathophysiology underlying this is unknown, although cerebral white matter maturation and integrity is sensitive to damage following preterm birth, and is one possible mediator. Threshold responses to non-invasive transcranial magnetic brain stimulation (TMS), a measure of cortical excitability, reflect white matter integrity, so may provide a cheaper alternative to other imaging modalities, in the clinical assessment of these children. However, cognitive development in these children is commonly further influenced by other prenatal factors such as suboptimal fetal growth, maternal pregnancy health, as well as early postnatal factors such as social disadvantage.

The main aim was to determine the associations between threshold responses to TMS and the development of cognitive abilities in 12 year old children born $24-41$ weeks of completed gestation (GA). A second aim was to investigate the relative importance of other prenatal and postnatal factors on these outcomes.

Method: 145 children who were members of the PREMOCODE study were administered the Woodcock-Johnson Tests of Cognitive Abilities. Resting motor thresholds (rMT) were obtained from left and right hand muscles using single pulse TMS and surface electromyography. Socioeconomic information at birth and at 12 years was obtained from labour ward records and national census data. Maternal and perinatal health data were obtained with consent from clinical records. Relative importance regression modelling was used to determine the influence of various factors on specific and broad cognitive abilities at 12 years.

Results: Both rMT and GA were correlated with overall general intellectual ability (GIA). However, the best model of poorer GIA included a higher degree of social disadvantage at birth (but not at 12 years), shorter stature at 12 years and reduced cortical excitability $\left(R^{2}=0.20, \mathrm{~F}_{[3,125]}=9.4, P \leq\right.$ 0.0001). Models of more specific cognitive outcomes (e.g. verbal and thinking abilities, working memory) also included degree of social disadvantage at birth and measures of preand postnatal growth (particularly birthweight centile and height at 12 years), but not GA or rMT.

Conclusion: Preterm birth increases the risk of reduced cognitive abilities in non-cerebral palsy children at 12 years, but this appears to be mediated by the commonly associated conditions of poor fetal and postnatal growth, rather than by reduced GA per se. The observation that these outcomes are influenced by social disadvantage at birth, but not later in childhood, suggest fetal and early postnatal growth are more potent programmers of cognitive development. However, the findings also raise questions about the intergenerational transmission of disadvantage and its contribution to the risk of preterm birth, low birthweight, child growth and cognitive development. TMS may offer a useful tool in clinical assessment of cortical development and overall GIA in these children, but appears to have limited application to more specific cognitive abilities.

Disclosure of Interest: None Declared. 


\section{DOHaD13-1502}

\section{Effect of gestational diabetes on infants' brain electrophysiological profile}

Shirong Cai ${ }^{1, *}$, Anqi Qiu ${ }^{2}$, Suet Chian $\mathrm{Sam}^{3}$, Michaela Bruntraeger $^{3}$, Hui Jun Chong ${ }^{3}$, Jenny Richmond ${ }^{4}$, Keith Godfrey ${ }^{5,6}$, Peter Gluckman ${ }^{3,7}$, Seang Mei Saw ${ }^{8}$, Kenneth Kwek' ${ }^{9}$, Yap Seng Chong ${ }^{1}$, Michael Meaney ${ }^{3,10}$, Anne Rifkin-Graboi ${ }^{3}$

${ }^{1}$ Obstetrics and Gynaecology, National University of Singapore, National University Health System; ${ }^{2}$ Bioengineering, National University of Singapore; ${ }^{3}$ Singapore Institute for Clinical Sciences (SICS), Agency for Science, Technology and Research, Singapore, Singapore; ${ }^{4}$ Department of Psychology, University of New South Wales, New South Wales, Australia; ${ }^{5}$ NIHR Southampton Biomedical Research Centre, University of Southampton \& University Hospital Southampton NHS Foundation Trust; ${ }^{6} M R C$ Lifecourse Epidemiology Unit, Southampton, United Kingdom; ' Liggins Institute, University of Auckland, Auckland, New Zealand; ${ }^{8}$ Saw Swee Hock School of Public Health, National University of Singapore; 'KK Women's and Children's Hospital, Singapore, Singapore; ${ }^{10}$ Department of Psychiatry and Neurology \& Neurosurgery, Douglas Mental Health University Institute, McGill University, Montreal, Canada

Background: Infants born to diabetic mothers have been shown to have impaired recognition memory. Experimental and some human data have implicated hippocampal damage attributed to fetal iron deficiency. Gestational diabetes mellitus (GDM) has a high prevalence in Asian populations, but its impact on offspring brain development has not been studied in Asians. Here we employ an electrophysiological approach (Event Related Potential -ERP) to evaluate the effect of GDM on infant brain development in an Asian population.

Method: At $26^{\text {th }}$ to $28^{\text {th }}$ weeks of gestation, women from the Singapore birth cohort study (GUSTO) were assessed for GDM with the oral glucose tolerance test (OGTT). Both the fasting and $2 \mathrm{~h}$ post OGTT blood glucose levels were recorded. When the infants reached 6 and 18 months of age, a subset of them participated in the ERP task with auditory oddball stimuli. For our analysis, low risk infants (last recorded APGAR score $\geq 9$, birth weight $\geq 2500 \mathrm{~g}$, full term, no fetal and maternal complications other than GDM, singletons) with good quality ERP data were included. The electroencephalograms of the control infants and infants born to mothers with GDM (IGDM) were compared [ 6 months $\mathrm{n}=140,116$ controls and 24 IGDM; 18 months $\mathrm{n}=39,32$ controls and 7 IGDM], with special reference to a late occurring component likely indicative of memory processes.

Results: IGDM infants had a lower late positive (LP) amplitude compared to control infants, both at 6 months (IGDM: $1.20 \pm 0.23 \mu \mathrm{V}$, control: $1.61 \pm 0.13 \mu \mathrm{V}, \mathrm{p}=0.104$ ) and 18 months (IGDM: $0.22 \pm 0.33 \mu \mathrm{V}$, control: $1.29 \pm$ $0.17 \mu \mathrm{V}, \mathrm{p}=0.006$ ), after adjusting for procedural and participant covariates including ethnicity. Higher maternal fasting and $2 \mathrm{~h}$ post OGTT blood glucose concentrations were also associated with a lower LP amplitude at 6 months $(\beta=-0.15$, $\mathrm{p}=0.053)$ and 18 months $(\beta=-0.34, \mathrm{p}=0.067)$ respectively even across the normal range of blood glucose concentrations.

Conclusion: Our data showed that there are indeed subtle differences in the electrophysiology of infants born to mothers with GDM, particularly the memory component (LP). The differences are found to persist up to 18 months of age, long after any conditions associated with maternal diabetes are resolved. We also find that increase in maternal blood glucose, even across the normal range, can potentially have an impact on infants' memory.

Disclosure of Interest: S. Cai: None Declared., A. Qiu: None Declared., S. C. Sam: None Declared., M. Bruntraeger: None Declared., H. J. Chong: None Declared., J. Richmond: None Declared., K. Godfrey: None Declared., P. Gluckman: None Declared., S. M. Saw: None Declared., K. Kwek: None Declared., Y. S. Chong: None Declared., M. Meaney Grant/ Research support from: Abbott Nutrition, A. Rifkin-Graboi: None Declared.

\section{DOHaD13-1752}

\section{Endocrine disruptor-mediated altered neurogenesis: bisphenol a enhances neural progenitor cell proliferation and differentiation}

Mina Desai Ph.D ${ }^{1, *}$, Guang Han, M.D ${ }^{1}$, Marie H. Beall, $\mathrm{MD}^{2}$, Michael G. Ross ${ }^{1}$

${ }^{1}$ Department of Obstetrics and Gynecology, Los Angeles Biomedical Research Institute at Harbor-UCLA Medical Center, Torrance, ${ }^{2}$ Los Angeles Perinatal Association, Los Angeles, United States

Background: Increases in a diversity of childhood and adult diseases (eg, autism, behavioral/learning abnormalities, obesity) have been attributed, in part, to programming effects resulting from developmental exposures. Bisphenol A (BPA) is a ubiquitous chemical widely used in plastics (eg, water bottles, food can liners) and paper industries. BPA is an endocrine disruptor (EDC) chemical which has estrogen receptor effects, and significant levels are consistently observed in pregnant women and fetal plasma and amniotic fluid. We sought to determine if fetal/newborn BPA exposure modifies neurogenesis, potentially resulting in altered cerebral structure or function.

Method: Newborn rats born from normal dams were sacrificed and brains dissected. Hypothalamic neuroprogenitor cells (NPCs) from control newborns were cultured in both complete and differentiation medium and treated with BPA (1, 10, $20 \mathrm{uM}$ ) or DMSO (control) for 5 days. NPC proliferation (MTT assay) was assessed, and protein expression 
(Western Blot) of NPC markers (Nestin), NPC proliferative and neurogenic factors (Hes1, Mash1), and markers neuronal and astrocyte cell types (Tuj1, GFAP, respectively) were analyzed. ${ }^{*} \mathrm{P}<0.05$ vs. untreated cells.

Results: In response to BPA exposure, NPC proliferation markedly increased, as measured by MTT assay as well as Nestin and Hes1. In differentiation media, BPA (10 uM) increased NPC expression of Mash1, Tuj1 and GFAP (Fig A, B), with a greater increase in Tuj1 as compared to GFAP.

Conclusion: Exposure to BPA causes increased NPC proliferation, in part via upregulated Hes1. Increased NPC differentiation to neurons as compared to astrocytes may alter cerebral structure and/or function and thus contribute to neurobehavioral abnormalities.

Disclosure of Interest: None Declared.

\section{DOHaD13-1474}

Environmental enrichment reduces anxiety-like behavior in rats programmed by neonatal hyperleptinemia

Egberto G. Moura ${ }^{1, *}$, Mabel C. Fraga ${ }^{1}$, Dayse G. CorreaAlmeida $^{1}$, Alex C. Manhaes ${ }^{1}$, Elaine Oliveira ${ }^{1}$, Patricia C. Lisboa $^{2}$

${ }^{1}$ Physiology; ${ }^{2}$ STATE UNIVERSITY OF RIO DE JANEIRO, Rio de Janeiro, Brazil

Background: Neonatal leptin treatment during the first ten days of lactation is associated with high anxiety and leptin resistance of the adult progeny ${ }^{1}$. Enriched environment (EE) is used as a form of reduction of anxiety ${ }^{2,3}$ and increases leptin sensitivity ${ }^{3}$. Here, we hypothesize that EE can prevent programming of anxiety in the neonatal leptin induced programming model.

Method: All experiments were carried out under institutional Ethical Commitee approval (CEA/002/2013). Sixteen litters of Wistar rats were used. At postnatal day PN1, all litters were culled to six male pups and they were divided into 2 experimental groups: leptin (L: $8 \mu \mathrm{g} / 100 \mathrm{~g} / \mathrm{Kg} /$ once a day s.c.) during the first 10 days of lactation (from PN1 to PN10) or saline (C: $50 \mu \mathrm{l})$. From PN 30 until PN 45, groups were randomly assigned to the nonenriched housing (NE: cage size: $39 \mathrm{~cm}$ long $\times 32 \mathrm{~cm}$ widex $14 \mathrm{~cm}$ high) or enriched housing (EE). The EE consisted of cage $(80 \mathrm{~cm}$ long x $75 \mathrm{~cm}$ wide $x 50 \mathrm{~cm}$ high), which contained stimulus designed to induce exploratory behavior, such as toys, toys that makes sound, and stimulus designed to provide the animals with free access to exercise, such as tunnels, running wheels, stairs and ramps. The objects were changed two times a week. After this period, the rats returned to standard cage. We analyzed the anxiety levels before and after the EE (PN 21, 30, 45 and 150). The animals were submitted to elevated plus maze test (EPM) over a period of 10 minutes and the time spent and \% of time spent in open arm (Time OA and \% Time OA) and the number and \% of the number of open arm entries (Entries OA and \%Entries OA) were recorded and used as anxiety measurements. The increased of these measurements are indicative of a reduced anxiety. Twelve rats were used for each group and each ages.

Results: At PN 21, was observed a significantly increased in the Time $\mathrm{OA}$ in the $\mathrm{L}$ group $(+11.7$ fold; L: $78191 \pm 13301 s$ vs. $6700 \pm 2841 s \mathrm{P}<0.001)$ and increased in the Entries OA $(+380 \%$; $\quad$ : $6.75 \pm 1.37$ and $\mathrm{C}: 1.40 \pm 0.67, \mathrm{P}<0.001)$. At $\mathrm{PN} 30, \mathrm{~L}$ rats showed an increased in the \%Time OA $(+438 \% ; 28 \pm 8.5 \%$ vs. C: $5.2 \pm 2.3 \%, \mathrm{P}<0.05)$. Curiously, at PN 45 the EE was not able to reduce anxiety in the $\mathrm{C}$ animals but had a modest anxiolytic effect in the programmed $\mathrm{L}$ group $(+141 \%$ in Time OA; ANOVA: $\mathrm{P}<0.05)$ and also in \%Time OA (+119\%; ANOVA: $\mathrm{P}<0.05)$. This effect is accentuated at PN 150, where EE had a higher anxiolytic effect in $\mathrm{L}$ group observed by an increased in Time OA (+656\%; ANOVA: $\mathrm{P}<0.001)$ and \%Entries OA (+154\%; ANOVA: $\mathrm{P}<0.001)$.

Conclusion: $\mathrm{EE}$ in fact reduces anxiety in both $\mathrm{C}$ and $\mathrm{L}$ group, but much more in $\mathrm{L}$ group, while $\mathrm{L}$ animals in standard housekeeping conditions were more anxious.

\section{References}

1. Fraga-Marques MC et al. (2009). Hormones and Behavior. 55: 272-279.

2. Fox C et al. (2006). Behav Brain Res. 175(1):1-8. 3. Morley-Fletcher $S$ et al. (2003). Eur J Neurosci. 18(12):3367-74.

4. Mainardi M et al. (2010). Proc Natl Acad Sci U S A. 107(38):16673-8.

Disclosure of Interest: None Declared.

\section{DOHaD13-1589}

Gradual introduction of vegetables in milk and rice at the beginning of weaning: the effects of early, varied and repeated exposure on intake of vegetables.

Marion Hetherington ${ }^{1}$, Camille Schwartz ${ }^{2}$, Jerome Madrelle $^{1}$, Fiona Croden ${ }^{1}$, Carel Vereijken ${ }^{3}$, Martine Alles $^{3, *}$, Hugo Weenen ${ }^{3}$

${ }^{1}$ Biopsychology group, Institute of Psychological Science, Leeds, United Kingdom, ${ }^{2}$ Centre de Recherche, Institute Paul Bocuse, Ecully, France, ${ }^{3}$ Danone Research, Danone Baby Nutrition, Utrecht, Netherlands

Background: Food learning starts very early, especially the first two years of life seem important for the development of healthy eating habits (Cashdan, 1994) as this is a period in which new foods are relatively easily accepted. Once food habits are established they tend to be stable. Various studies (see Nicklaus and Remy 2013 for review) have shown that food preferences which are developed at an early age have a 
long-lasting influence. Thus, the first 2 years can be seen as the basis for development of later healthy eating habits. Although the health benefits of fruits and vegetables are generally accepted (Boeing et al. 2012), the consumption of fruits and especially vegetables is much lower than recommended both in adults and in children. Ways to promote intake of vegetables and fruits throughout life are therefore needed. Breastfed infants are more willing to try new foods (Maier et al. 2008; Hausner et al. 2010), breastfeeding duration is associated with eating a more varied diet at two years (Scott et al. 2012) and exclusive breastfeeding for at least 3 months is associated with a higher consumption of vegetables at 4 years (Burnier et al. 2011). This is thought to be due to flavour exposure via breast milk (Menella et al. 2001, Hausner et al. 2010). A similar but stronger means to provide flavour exposure to infants is to add vegetable flavour to milk. The present study investigated the effect of a step-by-step introduction of pure vegetables added first to milk and then to rice at the start of weaning on liking and intake of vegetables.

Method: Just before the start of weaning, enrolled mothers were randomised to either the intervention group (IG, $\mathrm{n}=18$ of which 6 were breastfed) or to the control group (CG, $n=18$ of which 6 were breastfed). IG infants received 12 daily exposures to vegetable puree added to milk, then 12 daily exposures to vegetable puree, added to baby rice at home. Plain milk and cereal were given to the control group. Then both groups received 11 daily exposures of vegetable puree; intake and liking were recorded and eating behaviour filmed on days 25-26 and 33-35 after the start of weaning at the laboratory. Vegetables were provided in rotation (carrots, green beans, spinach and broccoli) and a new vegetable (parsnip) given on the final day. Results: IG infants liked and ate the exposed vegetable purees more than CG infants. Carrots were liked and consumed more than green beans. New vegetable intake was marginally greater in IG infants. Furthermore, mothers reported that the approach was acceptable and they appreciated the structure and guidance which the step-by-step approach provided.

Conclusion: Early exposure to vegetables in a step-by-step process enhanced liking and intake of vegetables during weaning. This approach could provide infants with a good start to healthy eating habits by improving vegetable acceptance and it may have a positive impact on healthy food preferences later in life.

Disclosure of Interest: M. Hetherington: None Declared., C. Schwartz: None Declared., J. Madrelle: None Declared., F. Croden: None Declared., C. Vereijken: None Declared., M. Alles Employee of: Danone Research, H. Weenen: None Declared.

\section{DOHaD13-1687}

\section{Identification of perinatal epigenetic marks associated with later neuropsychological function}

Karen Lillycrop ${ }^{1, *}$, Paula Costello ${ }^{2}$, AiLing Teh ${ }^{3}$, Robert Murray ${ }^{2}$, Rebecca Clarke-Harris ${ }^{2}$, Shelia Barton ${ }^{4}$,
Emma Garratt ${ }^{2}$, Sherry $\mathrm{Ngo}^{5}$, Allan Sheppard ${ }^{5}$, Johnny Wong $^{3}$, Shaillay Dogra ${ }^{3}$, Graham Burdge ${ }^{2}$, Cyrus Cooper ${ }^{4}$, Hazel Inskip ${ }^{4}$, Catherine Gale ${ }^{4}$, Peter Gluckman ${ }^{5}$, Nicholas C. Harvey ${ }^{4}$, Mark Hanson ${ }^{2}$, Joanna Holbrook ${ }^{3}$, Keith Godfrey ${ }^{4}$ and The Epigen consortium

${ }^{1}$ Centre for Biological Sciences; ${ }^{2}$ Human Development and Health, University of Southampton, Southampton, United Kingdom; ${ }^{3}$ Singapore Institute of Clinical Sciences, $A^{*}$ Star, Singapore, Singapore; ${ }^{4}$ MRC Lifecourse Epidemiology Unit, University of Southampton, Southampton, United Kingdom; ${ }^{5}$ Liggins Institute, University of Auckland, Auckland, New Zealand

Background: Early life environment has been linked to longterm changes in neuropsychological development. The mechanism by which environmental cues in early life are transmitted to the fetus and the process by which different, stable phenotypes are induced may involve the altered epigenetic regulation of genes. However to date there is no direct evidence in humans that early life environment may affect later neuropsychological function through an epigenetic mechanism. To investigate the contribution of epigenetic processes to later neuropsychological function, we used a genome wide methylation assay (MBD-array) to identify methylation differences at birth associated with neuropsychological function at age 4 years.

Method: Genomic DNA from umbilical cord samples was obtained from 24 children from the Southampton Women's Survey (SWS) and analysed using a MBD array. Their Wechsler Preschool and Primary Scale of Intelligence (WPPSI) scores at age 4 years were grouped into 4 categories with group 1 having the lowest WPPSI scores and group 4 having the highest. Absolute methylation levels were estimated using the Bayesian algorithm BATMAN. Pathway analysis was performed to search for known interactions between the genes containing differentially methylated regions of interest (DMROIs). Sodium bisulfite pyrosequencing was used to validate the DMROIs on a further 176 samples from the SWS cohort.

Results: The bioinformatics analysis identified 110 DMRs and 38 DMROIs. Pathway enrichment analyses found that the top pathway enriched amongst the DMROIs in the GO process category were diencephalon development (4/59 genes, $\mathrm{p}=0.000033)$. Differential methylated CpGs which were contained in the diencephalon development pathway were selected for further validation. The sodium bisulfite pyrosequencing validation confirmed that umbilical cord methylation of specific $\mathrm{CpGs}$ at birth was associated with later cognitive function. Functional validation of the $\mathrm{CpG}$ loci associated with later cognitive performance is ongoing.

Conclusion: This study shows that methylation of specific genes at birth are associated with later cognitive outcomes providing novel evidence regarding the importance of the developmental contribution to later cognitive function. Moreover such epigenetic marks at birth may have prognostic value and could be used for monitoring programmes to 
optimize maternal health and nutrition for long-term benefits to the offspring.

This work was supported by funding from the Medical Research Council, British Heart Foundation, NIHR Southampton Biomedical Research Centre, University of Southampton and University Hospital Southampton NHS Foundation Trust, UK Foods Standards Agency and Abbott Nutrition.

Disclosure of Interest: None Declared.

\section{DOHaD13-1576}

Intake of artificially sweetened soft drinks during pregnancy and offspring risk of mood and conduct problems at age 7: A study from the Danish National Birth Cohort

Marin Strøm ${ }^{1, *}$, Ekaterina Maslova ${ }^{1}$, Sjúrdur F. Olsen ${ }^{1}$, Thorhallur I. Halldorsson ${ }^{1}$

${ }^{1}$ Dept. Epidemiology Research, Centre for Fetal Programming, Statens Serum Institut, Copenhagen S, Denmark

Background: Evidence has shown early life nutrition to play an important role in aspects of physiology and behavior, incl neurodevelopment and predisposition to mental disorders.

Artificial sweeteners are suspected to exert adverse health effects, incl potential carcinogenic effects of aspartame, which is broken down into phenylalanine, aspartic acid, and methanol during metabolism in the body.

We have recently shown that artificial sweeteners in pregnancy may increase risk of preterm birth, and that it may play a role in programming of immunological effects.

Although speculative, there are indications that artificial sweeteners, especially aspartame, may affect the central nervous system through effects on glial cells (astrocytes) and neurons with excess phenylalanine blocking transport of important amino acids to the brain resulting in reduced levels of dopamine and serotonin as one of the proposed mechanisms. Few studies are available in humans investigating potential effects of low level exposures through the diet in normal free living individuals.

Method: Among 38,039 subjects from the Danish National Birth Cohort (DNBC) we investigated the association between maternal intake of artificially sweetened soft drinks, as reported in gestation week 25 by a validated Food Frequency Questionnaire, and offspring emotional and conduct problems by means of parental report of the validated Strengths and Difficulties Questionnaire (SDQ (www.sdqinfor.org)) at age 7. Based on the SDQ we assessed internalizing and externalizing problems, and classified children who were probable to suffer from emotional disorder (ED) or conduct/oppositional disorder (COD); we present here ORs and $95 \%$ CIs comparing daily $\geq 4$ servings/day vs no intake adjusted for a wide range of potential confounders, incl. maternal education and parental attention/academic problems.
Results: Based on parental report at age 7 among the 38,039 children, 6,053 (15.9\%) were categorized as experiencing internalizing problems, 1,847 (4.9\%) externalizing problems, whereas $469(1.2 \%)$ and $267(0.7 \%)$ were classified as probable to suffer from ED or COD, respectively. We saw that children whose mother had higher intake of artificially sweetened carbonated soft drinks were more likely to have ED $2.98(1.43 ; 6.22)$ but not COD $0.86(1.19 ; 4.21)$. For sugar sweetened soft drinks ORs were $1.37(0.70 ; 2.68)$ and 2.30 $(1.12 ; 4.72)$ for ED and COD, respectively. Maternal intake of artificially sweetened soft drinks was not associated with internalizing and externalizing problems, whereas intake of sugar sweetened soft drinks showed consistent associations with these outcomes. Results for non-carbonated soft drinks were very similar to those for the carbonated soft drinks.

Conclusion: We found similar associations for artificially and sugar sweetened soft drinks during pregnancy with offspring mood and conduct problems, and we can therefore not substantiate a role of the artificial sweeteners in fetal programming of behavior and mood. We propose that the observed associations may be due to residual confounding by lifestyle factors, the high content of sugar in soft drinks, which women may substitute with artificially sweetened drinks during the course of pregnancy, or they may be caused by other factors contained in this group of beverages, such as food additives incl. colourings.

Disclosure of Interest: None Declared.

\section{DOHaD13-1530}

Maternal consumption of cafeteria diet ameliorates anxiety-like behaviour in male rat offspring of mothers who exercised before and during pregnancy

Mukesh Raipuria $^{1, *}$, Hasnah Bahari ${ }^{2}$, Jayanthi Maniam ${ }^{1}$, Margaret J. Morris ${ }^{1}$

${ }^{1}$ Pharmacology; ${ }^{2}$ UNIVERSITY OF NEW SOUTH WALES, SYDNEY, AUSTRALIA, Sydney, Australia

Background: Many studies in rodents have shown that high fat or western diets during pregnancy are associated with the development of metabolic disorders in offspring. An anxiolytic effect of early postnatal overfeeding (Spencer and Tilbrook 2009) and maternal cafeteria diet during the lactation period (Wright et al., 2011) was reported in rat offspring. Effects of maternal obesity on the behaviour of offspring are less explored. Physical exercise is known to influence cognitive and emotional processes, so the present study was designed to address the question of whether voluntary exercise prior to mating and during pregnancy differentially affects anxiety-related behaviours of offspring.

Hypothesis: Voluntary exercise prior to mating and during pregnancy ameliorates anxiety-like behaviour differentially in offspring from lean and obese mothers. 
Method: Female Sprague-Dawley rats were fed chow (C) or western high fat $(\mathrm{F})$ diet and half of each underwent voluntary exercise (E), (CE or FE) with a running wheel prior to mating and during gestation while the others remained sedentary (S), (CS or FS). Two male pups from each mother across all groups were tested at the age of 5-6 weeks on the Elevated Plus Maze (EPM) for 10 min using video camera recording (Ethovision XT, USA). Frequency of entry and time spent in the open, exposed arm was assessed over 10 minutes as an indicator of anxiety-like behaviour. Data were analysed by 2-way ANOVA, followed by post hoc LSD test.

Results: Maternal obesity in sedentary dams had no effect on anxiety-like behaviour in the male offspring, as there were no significant differences in frequency to enter and time spent in the open arm (CS $17.8 \pm 1.3$ vs FS $16.0 \pm 1.5$ entries and CS $116.3 \pm 10.3$ vs FS $112.7 \pm 15.0 \mathrm{sec}$ ) of the EPM. Voluntary exercise in lean mothers (CE) during pregnancy increased anxiety-like behaviour in the male offspring. Thus pups from $\mathrm{CE}$ had fewer entries and spent significantly less time in the open arm of the EPM (CE $12.9 \pm 1.9$ vs CS $17.8 \pm 1.3$ entries and CE $45.5 \pm 7.5$ vs CS $116.3 \pm 10.3 \mathrm{sec})$. However the combination of $\mathrm{E}$ and $\mathrm{F}$ restored anxiety-like behaviour to control levels (FE 93.5 $\pm 17.0 \mathrm{sec}$ ). There was no difference in the number of entries to the closed arm across groups, but pups from lean exercised mothers spent $33 \%$ more time in the closed arm and showed less overall locomotor activity.

Conclusion: Maternal obesity had no effect on anxiety-like behaviour in male offspring of sedentary dams at 5-6 weeks of age. Exercise during pregnancy increased anxiety likebehaviour but only in offspring of dams consuming chow. The molecular mechanisms underlying the anxiogenic effects of maternal exercise require further investigation. Interestingly maternal consumption of high fat diet appeared to attenuate the anxiety-like behaviour induced by maternal exercise.

\section{References}

1. Spencer, SJ and A Tilbrook (2009) Neonatal overfeeding alters adult anxiety and stress responsiveness. Psychoneuroendocrinology 34: 1133-1143.

2. Wright, TS et al. (2011). The impact of maternal cafeteria diet on anxiety-related behaviour and exploration in the offspring. Physiology \& Behavior 103: 164-172.

Disclosure of Interest: None Declared.

\section{DOHaD13-1505}

\section{Maternal licorice consumption during pregnancy: offspring's cognitive, psychiatric, and neuroendocrine outcomes in early adolescence}

Silja Martikainen ${ }^{1, *}$, Anu-Katriina Pesonen ${ }^{1}$, Kati Heinonen ${ }^{1}$, Riikka Pyhälä ${ }^{1}$, Jari Lahti ${ }^{1}$, Kimmo Feldt ${ }^{1}$, Timo Strandberg ${ }^{2}$, Eero Kajantie ${ }^{3}$, Johan Eriksson ${ }^{2,3}$, Katri Räikkönen ${ }^{1}$
${ }^{1}$ Institute of Behavioural Sciences; ${ }^{2}$ Institute of Clinical Medicine, UNIVERSITY OF HELSINKI, ${ }^{3}$ Department of Chronic Disease Prevention, National Institute for Health and Welfare, Helsinki, Finland

Background: Overexposure to glucocorticoids may link prenatal adversity with detrimental outcomes in later life. Glycyrrhizin, a natural constituent of licorice, inhibits placental 11-beta-hydroxysteroid dehydrogenase type 2, the feto-placental "barrier" to higher maternal levels of cortisol. We have previously found that 8-year-old children with fetal exposure to high levels of glycyrrhizin demonstrated increased salivary cortisol levels diurnally and after psychosocial stress when compared to a low exposure group. Furthermore, these children had decrements in cognitive abilities and higher odds for psychiatric problems. It is not known, however, whether these associations persist into adolescence, which is marked with changes in hypothalamic - pituitaryadrenocortical axis (HPAA) functioning as well as in psychological well-being.

Method: We studied whether prenatal exposure to glycyrrhizin in licorice is associated with cognitive abilities (subtests of the Wechsler Intelligence Scale for Children III as well as the Children's Developmental Neuropsychological Assessment), psychiatric problems (Child Behavior Checklist), and HPAA functioning (diurnal salivary cortisol profile and responses to the dexamethasone suppression test) in 398 (51.5\% girls) Finnish adolescents 12.3 years of age born in 1998 as healthy singletons (the Glaku project).

Differences between glycyrrhizin exposure groups (zero-low exposure, $0-249 \mathrm{mg} /$ week, $\mathrm{n}=289$; moderate exposure, 250-499 mg/week, $\mathrm{n}=64$; high exposure, $\geq 500 \mathrm{mg} / \mathrm{week}$, $\mathrm{n}=45$ ) were tested by multiple linear regression analyses adjusted for time at awakening (in salivary cortisol analyses), sex, age, pubertal development, body mass index, and parental education (highest of either parent) at testing, as well as length of gestation, birth weight, birth head circumference, mother's age at delivery, maternal smoking and use of alcohol during pregnancy.

Results: In comparison to the group with zero-low glycyrrhizin exposure, those with high exposure had decrements in verbal abilities, narrative memory, and memory for faces $(P$-values $<.045)$ and increased odds for somatic complaints, aggressive behavior, and attention/deficit hyperactivity problems (range of odds ratios 2.9 to 4.4 ; $P$-values <.014). In comparison to the group with zero-low glycyrrhizin exposure, adolescents with moderate exposure had elevated salivary cortisol concentrations at peak after awakening, awakening area under the curve, and nadir $(P$-values $<.032)$. However, the group with high exposure did not differ from the low-exposure group ( $P$-values $>.70)$. No differences in HPAA responses to the dexamethasone suppression test were found ( $P$-values $>.45)$.

Conclusion: Our findings at the mean age of 12.3 years are similar to the previous results on the same participants at 
8 years of age. Regarding diurnal salivary cortisol concentrations, however, instead of the high-exposure group, the group with moderate glycyrrhizin exposure showed increased diurnal salivary cortisol levels. Data are compatible with adverse fetal "programming" by overexposure to glucocorticoids and caution against excessive intake of licoricecontaining foodstuffs during pregnancy.

Disclosure of Interest: None Declared.

\section{DOHaD13-1131} Maternal obesity and offspring's neurodevelopment: a
prospective cohort study

Lisu Huang ${ }^{1, *}$, Jun Zhang $^{1}$

${ }^{1}$ MOE-Shanghai Key Laboratory of Children's Environmental Health, Xinhua Hospital, Shanghai Jiao Tong University School of Medicine, Shanghai, China

Background: Both underweight and overweight women have an increased risk of adverse perinatal outcomes in offspring. Our aim was to examine the association of maternal pre-pregnancy body mass index (BMI) and/or weight gain and offspring's neurodevelopment at age 7 years.

Method: We used data from the Collaborative Perinatal Project, a large prospective cohort study with 46,021 of women. Prepregnancy BMI $\left(\mathrm{kg} / \mathrm{m}^{2}\right)$ was classified as underweight $(<18.5)$, normal weight (18.5-24.9), overweight (25.0-29.9), and obese ( $\geq 30.0)$. Intelligence quotient (IQ) were obtained using Wechsler Intelligence Scale for Children (WISC I) at $7.1 \pm 0.8$ years old.

Results: Two piecewise linear regression suggested that among underweight women, the lower the maternal BMI was, the lower the children IQ score was. Among overweight and obese women, however, the higher the maternal BMI the lower the children IQ score. Women with a pre-pregnancy BMI at around $20 \mathrm{Kg} / \mathrm{m}^{2}$ appeared to have had the highest offspring's IQ score. In the generalized estimating equation (GEE) model, suboptimal weight gain, both too little and too much, was associated with an increased risk of low IQ score of the offspring at age 7 years. Compared with normal weight women with $10-12 \mathrm{Kg}$ weight gain during pregnancy, children born to obese women who had excessive gain $(>20 \mathrm{Kg})$ had 4.7 $(95 \% \mathrm{CI}=-7.18,-2.24)$ points deficit in IQ after adjustment for potential confounders.

Conclusion: In this large prospective cohort study, extreme maternal prepregnancy BMI and weight gain were associated with an increased risk of neurodevelopmental delay, independence of pregnancy complications. With the pandemic of obesity in childbearing women around the world, great maternal prepregnancy BMI and weight gain may have a large population attributable risk for the decrease of IQ score.

Disclosure of Interest: None Declared.

\section{DOHaD13-1624}

\section{Maternal prepregnancy-BMI and infant attention at 9 months of age}

Bea R. Van Den Bergh ${ }^{1,2,3, *}$, Marion Van Den Heuvel ${ }^{1}$, Renée A. Otte ${ }^{1}$, Marijke A. Braeken ${ }^{1,4}$, Franc C. L. Donkers ${ }^{1,5}$, Jens Henrichs ${ }^{1}$, István Winkler ${ }^{6,7}$

${ }^{1}$ Psychology, Tilburg University, Tilburg, Netherlands; ${ }^{2}$ Psychology, Catholic University Leuven, Leuven; ${ }^{3}$ Department of Welfare, Public Health and Family, Flemish Government, Brussels; ${ }^{4}$ Biomedical Research Institute, Hasselt University, Hasselt, Belgium; ${ }^{5}$ Psychiatry, University of North Carolina at Chapel Hill, Chapel Hill, NC, United States; 'Institute of Cognitive Neuroscience and Psychology, Research Centre for Natural Sciences, MTA, Budapest; ' Institute of Psychology, University of Szeged, Szeged, Hungary

Background: Nutrition and stress are the major environmental influences during prenatal development which can program health and diseases in later life. Much more uncertain is the link between maternal obesity and neurocognitive development. We examined the association between prepregnancy-body mass index (BMI) and infant attention.

Method: Participants included 72 pregnant women and their 9 month old offspring participating in a prospective longitudinal study. Passive auditory attention was measured during a visit to the lab. To this end, event-related brain potentials (ERPs) were recorded in an auditory oddball paradigm with 4 types of stimuli: (1) standard tones (70\%) presented at a regular $300 \mathrm{~ms}$ inter-stimulus interval (ISI); (2) the same tone preceded by a $100 \mathrm{~ms}$ ISI (10\%); (3) white noise sounds (10\%); and (4) novel environmental sounds (10\%). In order to examine the effect of prepregnancy-BMI on latency and amplitude of the ERP components, separate ANOVAs were performed for each of the four types of stimuli with prepregnancy-BMI as a continuous predictor.

Results: Preliminary analysis of the ERP responses revealed that for the white noise sounds, higher maternal prepregnancy-BMI score was associated with shorter latency of the P300 $(p<.01)$ and N400 $(p<.05)$. No effects were found for any of the other types of stimuli.

Conclusion: Our results may indicate that infants of obese mothers are more easily distracted by the intrusive white noise sounds. This disturbance in passive auditory attention processes in infancy may enhance the risk for neurocognitive problems in childhood. As the prevalence of obesity has dramatically risen among women of reproductive age in the Western World, an increasing number of future children may 
be at risk of developing neurocognitive problems later in life due to maternal obesity.

Disclosure of Interest: None Declared.

\section{DOHaD13-1452}

\section{Negative maternal perceptions of infant behaviour are associated with increased placental glucocorticoid and serotonin sensitivity}

Katri Räikkönen ${ }^{1, *}$, Anu-Katriina Pesonen ${ }^{1}$, James R. O’Reilly $^{2}$, Soile Tuovinen ${ }^{1}$, Marius Lahti ${ }^{1}$, Eero Kajantie ${ }^{1}$, Pia Villa ${ }^{1}$, Hannele Laivuori ${ }^{1}$, Esa Hämäläinen ${ }^{3}$, Jonathan R. Seckl ${ }^{2}$, Rebecca M. Reynolds ${ }^{2}$

${ }^{1}$ University of Helsinki, Helsinki, Finland; ${ }^{2}$ University of Edinburgh, Edinburgh, United Kingdom; ${ }^{3}$ Helsinki University Central Hospital HUSLAB, Helsinki, Finland

Background: Animal models have shown that fetal glucocorticoid overexposure is one key biological mechanism explaining how environmental effects during prenatal life may mediate adverse offspring outcomes. Fetal overexposure may result from an increase in placental glucocorticoid regeneration, transfer and sensitivity. Whether placental glucocorticoid mechanisms play a role in programming adverse outcomes in human offspring have not been studied in detail. The serotonin biosynthetic pathway in the placenta has also not been studied in humans in detail, though recent work has suggested that this pathway may underlie the pronounced impact of serotonin on long-lasting mental health outcomes. We therefore tested the hypothesis that increased placental expression of genes involved in glucocorticoid sensitivity and transfer, and in serotonin sensitivity between the mother and fetus is associated with more negative maternal perceptions of infant behaviours.

Method: Biopsies of placental tissue were obtained from 54 healthy mothers aged $32.2 \pm 5.3$ years with singleton, term pregnancies (37-42 gestational weeks) a maximum of $90 \mathrm{~min}$ after vaginal or caesarean delivery, and stored in RNA-later at $-20 \mathrm{C}$. Placental mRNAs encoding mineralocorticoid receptor (MR), glucocorticoid receptor (GR), serotonin transporter (SERT), and 11-beta hydroxysteroid dehydrogenase type 1 (HSD1) and type 2 (HSD2), which regenerate and inactivate glucocorticoids respectively, were determined by real-time PCR. Perceptions of the infant at mean age of $15(S D=4.2)$ days were rated by their mothers using the Neonatal Perception Inventory capturing more problems in own infant's crying, feeding, spitting, elimination, sleeping and predictability behaviors in relation to an average infant.

Results: More negative maternal perceptions of the infant behaviours were related to higher placental GR ( 0.44 increase in standard deviation $[\mathrm{SD}]$ units per each SD unit increase in mRNA level; 95\% Confidence Interval, 0.19-0.68, $\mathrm{P}=0.001$ ), HSD1 (0.30 SDs, 0.03-0.57, P=0.03), HSD2 (0.41 SDs,
0.13-0.69, $\mathrm{P}=0.005)$ and SERT (0.26 SDs, 0.00-0.53, $\mathrm{P}=0.05)$ mRNA levels. The associations of GR, HSD1 and HSD2 remained significant when we made adjustments for concurrent maternal depressive symptoms measured using the Center of Epidemiological Studies Depression Scale (P-values $<0.05)$, while the association of SERT was rendered nonsignificant $(P=0.13)$; after controlling for a number of other important covariates and confounders, the associations of GR, HSD2 and SERT remained significant (P-values <0.04), while the association of HSD1 was rendered non-significant $(\mathrm{P}=0.20)$.

Conclusion: Mothers who perceive their own infants behaviours to be more negative at age 15 days have higher placental GR, HSD1, HSD2 and SERT gene expression. This combination will both regenerate glucocorticoids in placenta, increase placental sensitivity to glucocorticoids and to serotonin, but also inactivate glucocorticoids, perhaps ameliorating receptor induction, potentially leading to greater fetal glucocorticoid and serotonin exposure and altered infant behaviour.

Disclosure of Interest: None Declared.

\section{DOHaD13-1121}

The opposite effects of prenatal maternal mindfulness and anxiety on auditory event-related potentials of 9-month old infants

Marion Van Den Heuvel ${ }^{1, *}$, Renée Otte ${ }^{1}$, Franc Donkers ${ }^{1,2}$, István Winkler, ${ }^{3,4}$, Bea Van den Bergh ${ }^{1,5,6}$

${ }^{1}$ Tilburg University, Tilburg, Netherlands; ${ }^{2}$ University of North Carolina at Chapel Hill, Chapel Hill, United States;

${ }^{3}$ Hungarian Academy of Sciences, Budapest; ${ }^{4}$ University of Szeged, Szeged, Hungary; ${ }^{5}$ Catholic University of Leuven, Leuven; ${ }^{6}$ Flemish Government, Brussels, Belgium

Background: Recently, mindfulness-based interventions are developed and evaluated for decreasing stress during pregnancy. Prenatal stress, such as maternal anxiety, is known to have negative effects on the fetus. However, not much is known about the effects of maternal mindfulness on the child. By recording event-related brain potentials (ERPs) during a passive auditory oddball-paradigm, the current study examines the effects of the mother's prenatal mindfulness and anxiety on the processing of frequently occurring standard (regular) and infrequently occurring deviant (irregular) sounds. Our hypothesis was that mindfulness and anxiety exert opposite effects on the ERP amplitudes.

Method: In total, 78 mother-infant dyads (1 twin; 42 girls) were included in the study. Maternal mindfulness was measured using the Freiburg Mindfulness Inventory (FMI) and maternal anxiety was measured using the anxiety subscale of the Symptom Checklist (SCL-90). Both were assessed at the beginning of the second trimester. Infant ERPs were 
measured at 9-months of age in response to an auditory oddball paradigm with 4 types of stimuli: a complex tone of $500 \mathrm{~Hz}$ base frequency (standard, $\mathrm{p}=.7$ ) delivered with $300 \mathrm{~ms}$ inter-stimulus interval (ISI), the same tone preceded by a shorter $(100 \mathrm{~ms})$ inter-stimulus interval (ISI-deviant, $\mathrm{p}=.1)$, white noise $(\mathrm{p}=.1)$, and novel sounds $(\mathrm{p}=.1)$. Average peak amplitudes from $60 \mathrm{~ms}$ long windows centred on the peaks observed at electrodes F3, Fz, F4, C3, Cz, C4, P3, Pz, and P4 were measured for analysis. Two series of repeated-measures ANOVAs were conducted: one with "Mindfulness" and one with "Anxiety" as a continuous predictor; two electrode position within-subject factors "Frontal-CentralParietal" x "Left-Medial-Right" were also included. Separate ANOVAs were performed per stimulus type (standard, ISIdeviant, white noise, and novel sounds), per peak.

Results: Preliminarily analyses revealed that for the standard tones, higher maternal mindfulness was associated with lower $\mathrm{N} 250$ amplitude $\left[\mathrm{F}(1,77)=8.275, p<.01, \eta^{2}=.097\right]$, whereas higher maternal anxiety was associated with higher N250 amplitude $\left[\mathrm{F}(1,73)=4.757 ; p<.05 ; \eta^{2}=.061\right]$. Moreover, higher maternal mindfulness was also found to be associated with higher P150 amplitude $[\mathrm{F}(1,77)=11.496$, $\left.p<.01, \eta^{2}=.130\right]$. No effects were found for any of the rare sound events.

Conclusion: We tentatively interpret these findings as infants prenatally exposed to higher levels of maternal mindfulness devote less processing to regular occurring irrelevant stimuli or they habituate faster to these stimuli (i.e. lower N250). In contrast, infants prenatally exposed to higher levels of maternal anxiety process such uninformative sounds more extensively or they habituate slower to these stimuli (i.e. higher N250). This difference might stem from infants prenatally exposed to higher maternal mindfulness having better pre-attentive access to perceptual representations as indexed by the higher P150. If replicated, our results may indicate that being more mindful during pregnancy is beneficial for both mother and child. Furthermore, the findings could contribute to a better understanding of the underlying mechanisms of prenatal mindfulness-based interventions.

Disclosure of Interest: None Declared.

\section{DOHaD13-1484}

The relationship between nutrition in early childhood and cognitive performance during adolescence

Anett Nyaradi ${ }^{1,2, *}$, Wendy Oddy ${ }^{1}$, Siobhan Hickling ${ }^{1,2}$, Jianghong $\mathrm{Li}^{1,3,4}$, Jonathan Foster ${ }^{1,5,6}$

${ }^{1}$ Telehon Institute for Child Health Research, Centre for Child Health Reseach; ${ }^{2}$ School of Population Health, The University of Western Australia; ${ }^{3}$ Curtin Health Innovation Research Institute, Centre for Population Health Research, Curtin University, Perth, Australia; ${ }^{4}$ WZB Berlin Social Research Center, Berlin,
Germany; ${ }^{5}$ School of Paediatrics \& Child Health, The University of Western Australia; ${ }^{6}$ School of Psychology or Speech Pathology, Curtin University, Perth, Australia

Background: It is well known that the first two years of life represent a sensitive period for neurodevelopment $(1,2)$, that is genetically programmed, but influenced by environmental factors including nutrition (1, 3, 4). Optimal nutrition during this critical developmental period may have long lasting positive effects on the cognitive abilities of the individuals. The aim of the study was to investigate the long term association between the duration of breastfeeding and diet quality at one year of age and cognitive performance during adolescence.

Method: Participants $(\mathrm{n}=717)$ were drawn from the Raine Study cohort, which is a prospective longitudinal study of 2868 children and their families located in Perth, Western Australia. Breastfeeding duration and an Eating Assessment in Toddlers (EAT) diet score at one year of age were used as the main predictor variable. Four components, including Detection Task (DET; psychomotor speed), Identification Task (IDN; psychomotor speed and visual attention), One Card Learning Task (OCL - visual learning and, memory) and Continuous Paired Association Learning Task (CPALvisual learning), of a computerized cognitive battery (CogState) assessed adolescents' cognitive performance at 17 years. Associations between breastfeeding duration, EAT score and cognitive performance were assessed in multivariate regression models.

Results: For males, there was a significant main effect of breastfeeding ( $\geq 4$ months) $(\beta=-0.026$, 95\% CI: -0.046 ; $-0.006, p=0.010)$ on the reaction time of the DET task. There was no other significant main effect of breastfeeding on cognitive performance. The main effect of diet score in the whole sample at age one year was significantly associated with the reaction time of DET task $(\beta=-0.005,95 \% \mathrm{CI}$ : $-0.009 ;-0.001, \mathrm{p}=0.026)$. There were no other significant associations identified.

Conclusion: Nutrition in early childhood (including breastfeeding and diet quality at age one year) has a long term influence on simpler cognitive tasks that possibly start developing during the first year of life.

\section{References}

1. Bryan J, Osendarp S, Hughes D, Calvaresi E, Baghurst K, van Klinken J-W. Nutrients for Cognitive Development in School-aged Children. Nutr Rev. 2004;62(8):295-306.

2. Lenroot RK, Giedd JN. Brain development in children and adolescents: Insights from anatomical magnetic resonance imaging. Neurosci Biobehav Rev. 2006;30(6):718-29.

3. Toga AW, Thompson PM, Sowell ER. Mapping brain maturation. Trends Neurosci. 2006;29(3):148-59.

4. Giedd J, Stockman M, Weddle C, Liverpool M, Alexander-Bloch A, Wallace G, et al. Anatomic Magnetic Resonance Imaging of the Developing Child and Adolescent 
Brain and Effects of Genetic Variation. Neuropsychol Rev. 2010;20(4):349-61.

Disclosure of Interest: None Declared.

\section{DOHaD13-1265}

Size at birth and physical growth predict neurocognitive function in late adulthood: the Helsinki Birth Cohort Study

Jari Lahti ${ }^{1,2, *}$, Kati Heinonen ${ }^{1}$, Anu-Katriina Pesonen ${ }^{1}$, Marius Lahti ${ }^{1}$, Soile Tuovinen ${ }^{1}$, Eero Kajantie ${ }^{3}$, Clive Osmond $^{4}$, David J. Barker ${ }^{5}$, Johan G. Eriksson ${ }^{6}$, Katri Räikkönen ${ }^{1}$

${ }^{1}$ Institute of Behavioural Sciences, University of Helsinki;

${ }^{2}$ Folkhailsan Rersearch Centre; ${ }^{3}$ National Public Health Institute, Helsinki, Finland; ${ }^{4}$ Medical Research Council Lifecourse Epidemiology Unit; ${ }^{5}$ Developmental Origins of Health and Disease Division, University of Southampton, Southampton, United Kingdom; ${ }^{6}$ Department of General Practice and Primary Health Care, University of Helsinki, Helsinki, Finland

Background: Small body size at birth and slow postnatal growth, markers for suboptimal environment, have been associated with lower neurocognitive test scores in childhood and in adulthood, but data on late adulthood remain scarce. We tested in the Helsinki Birth Cohort Study (HBCS) whether anthropometric measures from birth to late adulthood or growth after birth predicted cognitive function or Mild Cognitive Impairment (MCI) in late adulthood.

Method: The sample comprised 1030 men and women without a history of stroke $(54.3 \%$ female) from the HBCS sample who were born 1934-1944 in Helsinki, Finland and who participated in the neurocognitive assessment with the Consortium to Establish a Registry for Alzheimer's Disease (CERAD) test battery at the mean age of 68.1 years. There were 33 cases with MCI when using pre-defined cut-off in the composite CERAD score. Data on anthropometric measures were extracted from medical records. All regression models were adjusted for sex, age at testing, education, and gestational age (only models pertaining to birth size).

Results: After adjusting for confounders, lower ponderal index at birth was associated with lower composite CERAD score $(\mathrm{p}=.049)$, and lower birth weight $(\mathrm{p}=.048)$ and head circumference $(\mathrm{p}=.01)$ with MCI status. Higher gain in weight $(\mathrm{p}=.03)$ and in BMI $(\mathrm{p}=.01)$ particularly between 7 and 11 years predicted lower composite CERAD score. A smaller difference in heights between 11 and 68 years predicted lower composite CERAD score $(\mathrm{p}=.046)$ and MCI status $(\mathrm{p}=.05)$.

Conclusion: Our results indicate that slower prenatal growth and smaller difference in heights between 11 and 68 years may predict poorer neurocognitive functioning and higher risk for MCI in late adulthood. Moreover, higher weight gain between 7 and 11 years may associate with poorer neurocognitive functioning in late adulthood.

Disclosure of Interest: None Declared.

\section{DOHaD13-1312}

A maternal low GI diet improves glucose tolerance and reduces visceral fat mass in female offspring

Mohammed Al-Nussairawi ${ }^{1}$, Amanda Kheng ${ }^{1}$, Jennie BrandMiller $^{2}$, Sheridan Gentili ${ }^{3}$, Bev Muhlhausler ${ }^{1, *}$

${ }^{1}$ FOODplus Research Centre, School of Agriculture, Food and Wine, The University of Adelaide, Adelaide; ${ }^{2}$ Boden Institute of Obesity, Nutrition, Exercise \& Eating Disorders, School of Molecular Bioscience, The University of Sydney, Sydney;

${ }^{3}$ Sansom Institute for Health Research, School of Pharmacy and Medical Sciences, University of South Australia, Adelaide, Australia

Background: Low glycaemic index (GI) diets have been associated with improved insulin sensitivity and lower risk of type 2 diabetes. Other data suggest that a low GI diet during pregnancy can improve maternal glucose tolerance and reduce the incidence of large for gestational age deliveries; however the effect on the longer term metabolic heath of the offspring has not been investigated. This study aimed to test the hypothesis that consuming a low GI diet during pregnancy and lactation would improve metabolic health of the offspring at weaning.

Method: 25 female Albino Wistar rats were fed either a low GI $(n=13)$ or high GI $(n=12)$ diet (Specialty Feeds, Glen Forrest, Australia) from 4 weeks prior to mating until the end of lactation. Glucose tolerance was assessed in the dam during lactation and in one male and one female pup from each litter prior to weaning at 3 weeks of age. Tissues were collected from male and female offspring at 3 weeks of age for the determination of fat mass and hepatic lipid content. Hepatic expression of lipogenic genes (G3PDH, ACC $\beta$, SREBP-1 $\alpha$, PPAR $\alpha$ ) was determined by qRT-PCR. The outcomes in the low GI and high GI groups were compared using 2-way ANOVA and repeated measures ANOVA as appropriate.

Results: Glucose tolerance was significantly improved in the low GI dams compared to high GI dams (AUC: $1322 \pm 55$ vs $1522 \pm 39 \mathrm{mmol} / \mathrm{min} / \mathrm{L}, P<0.04)$. There was no difference in birth weight, gestational age or litter size between the low GI and high GI groups. At 3 weeks of age, female offspring of low GI dams had improved glucose tolerance (AUC: $1242 \pm 28$ vs $1350 \pm 39 \mathrm{mmol} / \mathrm{min} / \mathrm{L} /, \quad P<0.02$ ) and a lower visceral fat mass relative to body weight $(0.45 \pm 0.003 \mathrm{~g} / \mathrm{g}$ vs $0.53 \pm 0.003 \mathrm{~g} / \mathrm{g}, P<0.05)$ compared with offspring of high GI dams. These effects were not seen in males. There were no differences in relative liver weight or hepatic liver content between the low GI and high GI groups in either males or females. In males, but not in females, mRNA expression for the lipogenic gene, G3PDH, was lower 
in the low GI compared to the high GI group $(0.28 \pm 0.026$ vs $0.68 \pm 0.15, P<0.03)$. There were no differences between the low GI and high GI groups in hepatic expression of other key lipogenic genes at 3 weeks of age, ACC $\beta$, SREBP- $1 \alpha$ or $\operatorname{PPAR} \alpha$ in either male or female offspring.

Conclusion: We have shown that consuming a low GI diet during pregnancy and lactation improves maternal glucose tolerance and is associated with an improved metabolic profile in female offspring at weaning in the absence of significant reductions in hepatic liver content or alterations to hepatic gene expression. This study highlights the potential for low GI diets in pregnancy to improve the metabolic health of future generations.

Disclosure of Interest: None Declared.

\section{DOHaD13-1701}

\section{Abdominal adipose tissue growth during the first year of life}

Joao G. Alves ${ }^{1, *}$, vIVIANNE Barros ${ }^{2}$, Adriana Melo ${ }^{2}$, Jousilene Tavares ${ }^{2}$, Melania Amorim ${ }^{2}$

${ }^{1}$ Instituto de Medicina Integral Prof. Fernando Figueira (IMIP), Recife; ${ }^{2} I P E S Q$, Campina Grande, Brazil

Background: Fetal growth restriction may lead to permanent anatomo-physiological deficit and is associated with chronic disease in latter life. Few studies have showed that fetal growth restriction may lead to a redistribution of fetal fat and accumulation of visceral fat as a mechanism to preserve energy to ensure the concept survival. Thus the knowledge of the early patterns of visceral adipose tissue growth it is an important issue to understand the genesis of visceral obesity and its comorbidities. Our aim is to verify the growth of visceral adipose tissue during the first year of life.

Method: This cohort study was performed from June 2010 to September 2011. 116 health newborns were followed during the first 12 months of life. All infants were recruited from the ISEA, Campina Grande, Brazil. This project was previously approved by the ISEA review board and all mothers agreed to participate and signed an informed consent. All the infants were evaluated at 1, 3, 6 and 12 months of life through an interview with the mother, anthropometric measurements and ultrasonographic exams. Visceral fat thickness was measured by an ultrasound. Statistical significance was set at a $\mathrm{P}$-value $<0.05$, and analyses were performed using SAS Version 9.3.

[LA1]ACRESCENTAR

Results: A total of 830 study observations were performed. Abdominal fat growth rate was higher during the first trimester especially subcutaneous tissue; $2.87(+0.89)$ to 5.14 $(+0.75), \mathrm{p}<0.001$. Visceral fat showed high growth rate during the first semester; $8.14(+1.73)$ to $12.33(+1.94)$, $\mathrm{p}<0.001$. The growth rate was minimum during the second semester of life; $0.001 \mathrm{~mm} / \mathrm{month}$ and $0.19 \mathrm{~mm} / \mathrm{month}$, respectively for subcutaneous and visceral fat thickness. This growth remained consistent after control to gestational age, gender, birth weight, height and exclusively breast feeding.

Conclusion: A rapid visceral and subcutaneous abdominal fat thickness increase occurs during the first semester of life, especially during the first trimester.

Disclosure of Interest: None Declared.

\section{DOHaD13-1217}

Association between birth weight and body adiposity in childhood: a Brazilian cohort study

Patricia H. Rondó ${ }^{1, *}$, Joilane Pereira-Freire ${ }^{2}$, Jesuana

Lemos $^{1}$, Artemizia Souza ${ }^{2}$, Camila Menezes ${ }^{2}$

${ }^{1}$ Nutrition, School of Public Health, University of Sao Paulo, Sao Paulo; ${ }^{2}$ Nutrition, Federal University of Piaui, Picos, Brazil

Background: A worldwide increase in the prevalence of childhood overweight and obesity has been seen over the past few decades, including in developing countries such as Brazil. In addition to psychosocial consequences, children with excess weight are at an increased risk of developing metabolic and endocrine diseases in adult life. The objective of this study was to determine the association between birth weight and body adiposity in childhood.

Method: Prospective cohort study carried out in Jundiai city, Brazil, between November 2004 and December 2006. Four hundred eighty (480) children aged 5 to 8 years were involved in the study. Birthweight was assessed from a previous cohort ${ }^{1}$ that involved the mothers of those children. Anthropometric measures (weight, height, triceps and subscapular skinfold thickness and waist circumference) were determined using a standard method. Body fat percentage, fat mass and fat-free mass were assessed by electrical bioimpedance analysis utilising the 310 Body Composition Analyzer from Biodynamics ${ }^{\circledR}$ (USA). Five multiple linear regression models were developed considering waist circumference, triceps skinfold thickness, fat mass percentage, fat-free mass and fat mass as markers of body adiposity, and outcomes.

Results: Significant positive associations were observed between birth weight and waist circumference $(p=0.002)$, triceps skinfold thickness $(p=0.03)$, and fat-free mass $(\mathrm{p}<0.001)$. Almost $16 \%$ of the children presented excess body fat and approximately $28 \%$ of them had central adiposity (waist circumference $\geq 95^{\text {th }}$ percentile) ${ }^{2}$, characterizing obesity.

Conclusion: The results of this study demonstrate that childhood is a critical period for the development of overweight and obesity in later life, as confirmed by the associations of birthweight with markers of body adiposity in healthy children aged 5 to 8 years. 


\section{References}

1. Rondó PHC, Ferreira RF, Nogueira F et al. (2003). Maternal psychological stress and distress as predictors of low birth weight, prematurity and intra-uterine growth retardation. Eur J Clin Nutr 57, 266-272.

2. McCarthy HD, Jarrett KV \& Crawley HF (2001). The development of waist circum-ference percentiles in British children aged 5.0-16.9 y. Eur J Clin Nutr 55, 902-907.

Disclosure of Interest: None Declared.

\section{DOHaD13-1087}

Association between change in maternal adiposity during pregnancy and birth weight and subcutaneous fat of the newborn

Christine Sommer ${ }^{1, *}$, Line Sletner ${ }^{2}$, Anne K. Jenum ${ }^{3}$, Kjersti Mørkrid $^{1}$, Annhild Mosdøl ${ }^{4}$, Kåre I. Birkeland ${ }^{1}$

${ }^{1}$ Department of Endocrinology, Morbid Obesity and Preventive Medicine; ${ }^{2}$ Norwegian Resource Centre for Women's Health, Oslo University Hospital; ${ }^{3}$ Institute of Health and Society, Department of General Practice, University of Oslo; ${ }^{4}$ Department of Health, Nutrition and Management, Oslo and Akershus University College of Applied Sciences, Oslo, Norway

Background: Maternal adiposity is thought to affect the body composition of neonates. The aim was to explore the separate effect of change in total body weight, total fat and truncal fat during pregnancy on neonatal skinfold thickness at birth.

Method: STORK Groruddalen is a population-based prospective cohort study of 823 (74\% of the invited, $59 \%$ of non-Western origin) pregnant women and their offspring attending primary antenatal clinics in Eastern Oslo, Norway. The study population comprised the 475 pregnant women (57.7\% of total sample) who 1 had two valid measures from a bioelectrical impedance analysis (Tanita-BC $418 \mathrm{MA}$ ) at gestational week $14 \pm 3$ (mean \pm SD) and $28 \pm 1$, and 2 who had four valid skinfold measurements (triceps, thigh, suprailiac and subscapular sites) performed (by midwives) on their neonate at birth. We compared sample characteristics across quartiles of neonatal sum of skinfolds (sum of the four performed). To ease comparison, maternal gain of body weight, total fat and truncal fat were standardized, using z-score. Linear regression analyses were performed separately for the z-score variables (gain of total weight, total fat and truncal fat from gestational week $14 \pm 3$ to $28 \pm 1$ ) with neonatal sum of skinfolds $(\mathrm{mm})$ as the dependent variable. Model 0 was univariate analyses. In model 1 we adjusted for maternal age, pre-pregnant BMI, parity, gestational diabetes (GDM) defined by the World Health Organization criteria, offspring crown-heel-length, gestational age at delivery and sex of the baby. In model 2 we additionally adjusted for ethnic origin. Statistical analyses were performed using IBM SPSS Statistics 19.

Results: Mean maternal weight gain was $0.51 \mathrm{~kg} /$ week $(S D=0.22)$, mean fat gain $0.38 \mathrm{~kg} /$ week $(S D=0.20)$, and mean truncal fat gain was $0.22 \mathrm{~kg} /$ week $(S D=0.14)$. The univariate analyses (model 0) revealed significant effects of weight and fat gain (SD) on sum of skinfolds $(\mathrm{mm})$ in the newborn, whereas the effect of truncal fat gain became significant in model 1 . Weight $(\beta=0.59, p=0.004)$, fat gain $(\beta=0.62, p=0.001)$ and truncal fat gain $(\beta=0.42$, $\mathrm{p}=0.021$ ) remained significant predictors in the final model, meaning that for each SD increase in maternal fat gain, a $0.62 \mathrm{~mm}$ increase in neonatal sum skinfolds was observed. In concordance with other studies, maternal age, pre-pregnant BMI, neonatal crown-heel-length and female sex and GDM during pregnancy, were all positively associated with neonatal sum of skinfolds. A South Asian origin was associated with a lower neonatal sum skinfold, but only in the analysis using weight gain per week as the explanatory variable. We found similar effects of $z$-scores of maternal weight gain $(\beta=56.0$, $\mathrm{p}<0.001)$, total fat $(\beta=44.9, \mathrm{p}<0.001)$ and truncal fat gain $(\beta=35.3, p=0.005)$ on offspring birth weight.

Conclusion: Excessive maternal gain of weight and fat during pregnancy were associated with neonatal birth weight and adiposity, which may increase the risk of later obesity in the offspring and potentially other chronic disease in adulthood (1).

\section{References}

1. Poston L. Gestational weight gain: influences on the longterm health of the child. Curr Opin Clin Nutr Metab Care. 2012 May;15(3):252-7.

Disclosure of Interest: None Declared.

\section{DOHaD13-1636}

Association between gestational weight gain in overweight/obese women and infant body composition

Andrea R. Deussen ${ }^{1, *}$, Roslaie M. Grivell ${ }^{1}$, Lisa N. Yelland ${ }^{1}$, Matthew W. Gillman ${ }^{2}$, Andrew J. McPhee,

Jodie M. Dodd

${ }^{1}$ Discipline of Obstetrics and Gynaecology, School of Paediatrics and Reproductive Health, The University of Adelaide, North Adelaide, Australia; ${ }^{2}$ Department of Nutrtition, Harvard Pilgrim Healthcare Institute, Boston, United States; ${ }^{3}$ Neonatology, Womens and Childrens Health Network; ${ }^{4}$ The Robinson Institute; ${ }^{5}$ Discipline of Obstetrics and Gynaecology, School of Paediatrics and Reporductive Health, The University of Adelaide, North Adelaide, Australia

Background: Infants born to overweight or obese women are more likely to be large for gestational age compared with infants born to women with a normal $\mathrm{BMI}^{1}$. This may have 
implications for the infant's long-term health ${ }^{2,3 ;}$. Body weight at birth is a crude indicator of body composition and measuring skinfold thickness provides additional information about the proportion and distribution of fat free mass and body fat. Skinfold thickness measurements are an inexpensive and portable method to assess infant body composition. We assessed skin fold thickness measurements on newborn infants of women who were overweight or obese and described newborn infant body composition in relation to early pregnancy BMI and gestational weight gain (GWG).

Method: We studied 482 infants born to women who were overweight (BMI $\left.25.0-29.9 \mathrm{~kg} / \mathrm{m}^{2}\right)$ or obese $\left(>30 \mathrm{~kg} / \mathrm{m}^{2}\right)$. Infant weight and length were measured at birth and anthropometric measurements obtained within the first days after birth. Anthropometric measurements included biceps, triceps, abdominal, suprailiac, subscapular and thigh skinfold thicknesses and head, chest abdominal and right upper arm circumferences. Fat free mass and body fat was calculated based on sex, weight and triceps, subscapular and thigh skinfold thicknesses. The effect of BMI and GWG on infant body composition was assessed using linear regression.

Results: Infant body composition did not vary with maternal BMI category. Mean birth weight of infants born to women who were overweight was $3539.6 \mathrm{~g}$ and $3557.6 \mathrm{~g}$ for infants born to women who were obese $(\mathrm{p}=0.1695)$. Fat mass $520.4 \mathrm{~g}$ and $525.6 \mathrm{~g}$ respectively $(\mathrm{p}=0.4095)$ Mean birth weight was $190 \mathrm{~g}$ higher $(95 \%$ CI 85 to $295, \mathrm{p}=0.0004)$, fat free mass was $118 \mathrm{~g}$ higher (95\% CI 45 to $190, \mathrm{p}=0.0015$ ) and percent body fat was $1.2 \%$ higher $(95 \%$ CI 0.4 to 2.0 , $\mathrm{p}=0.003)$ in infants born to mothers whose GWG was above compared with below Institute of Medicine recommended guidelines for weight gain in pregnancy.

Conclusion: Among women who were overweight or obese, higher GWG was associated with higher infant birth weight and percent body fat. Our findings indicate the importance of GWG as a potentially modifiable antenatal factor influencing infant birth weight and body composition. The identified association of higher birth weight with excessive maternal GWG appears to reflect both fat free mass and adiposity. Evaluation of the effect of antenatal interventions designed to limit GWG on measures of infant body composition, at birth and in later childhood, are therefore of high importance.

\section{References}

1. Callaway LK, Prins JB, Chang AM et al. (2006) The prevalence and impact of overweight and obesity in an Australian obstetric population. MJA 184, 56-59.2.

2. Gale CR, Martyn CN, Kellingray SD et al. (2001) Intrauterine programming of adult body composition. J Clin Endocrinol Metab 86, 267-272.3.

3. Singhal A, Wells JE, Cole TJ et al. (2003) Programming of lean body mass: a link between birth weight, obesity, and cardiovascular disease? Am J Clin Nutr 77, 726-730.4.

Disclosure of Interest: None Declared.

\section{DOHaD13-1219}

\section{Birthweight, catch-up growth in infancy and markers of overweight and obesity in childhood}

Patricia H. Rondó ${ }^{1, *}$, Martha R. Sacco ${ }^{1}$, Natalia P. Castro ${ }^{1}$, Veronica L. Euclydes ${ }^{1}$, José M. Souza ${ }^{1}$

${ }^{1}$ Nutrition, School of Public Health, University of Sao Paulo, Sao Paulo, Brazil

Background: Data published by the World Health Organization ${ }^{1}$ have shown a worrisome increase of overweight and obesity during the first years of life. In infancy, hyperplasia and/or hypertrophy of adipose cells results in an exacerbated expansion of adipose tissue, a characteristic of obesity, which tends to persist into adulthood. This mechanism can be explained by the developmental origins of health and disease hypothesis, which suggests that an insult or stimulus during critical periods of development, such as growth in the intrauterine period and infancy, has long-term effects on the physiology, structure and functions of the organism. The objective of this study was to evaluate the relationship between birthweight and catch-up growth in infancy and markers of overweight/obesity in childhood, using different cut-off values for catch-up growth.

Method: Cross-sectional study involving 98 five-year old preschool Brazilian children. Catch-up growth was considered as weight gain in standard deviation (SD) of z-scores above $+0.67,+1$ and +2 in relation to birthweight, at any time during the first 2 years of life. The nutritional status of the children was determined by anthropometry and electrical bioimpedance. Multiple linear regression analysis was used considering fat mass percentage, body mass index (BMI), waist and neck circumferences as outcomes.

Results: Birthweight, catch-up growth (assessed by different cut-off values) and maternal obesity were positively associated with increased fat mass percentage, BMI, waist and neck circumferences. Different cut-off values of catch-up growth did not change the positive associations between catch-up growth and fat mass percentage $(>+0.67 \mathrm{SD}, \mathrm{p}=0.007$; $>+1$ SD, $p=0.007 ;>+2$ SD, $p=0.01)$; BMI $(>+0.67$ SD, $\mathrm{p}=0.002 ;>+1$ SD, $\mathrm{p}=0.007 ;>+2 \mathrm{SD}, \mathrm{p}<0.001$ ), waist circumference $(>+0.67 \mathrm{SD}, \mathrm{p}=0.002$; $>+1 \mathrm{SD}, \mathrm{p}=0.002$; $>+2 \mathrm{SD}, \mathrm{p}<0.001)$ and neck circumference $(>+0.67 \mathrm{SD}$, $\mathrm{p}=0.01 ;>+1 \mathrm{SD}, \mathrm{p}=0.03 ;>+2 \mathrm{SD}, \mathrm{p}<0.001)$.

Conclusion: The use of different cut-off values for the definition of catch-up growth did not interfere in the associations between birthweight and catch-up growth with fat mass percentage, BMI, waist and neck circumferences. Children with higher birthweight, those who undergo catchup growth in infancy and whose mothers are obese, seem to be more at risk for overweight/obesity.

\section{References}

1. World Health Organization. WHO child growth standards: length/ height-for-age, weight-for-age, weight-for-length, weight-for-height and 
body mass index-for-age: methods and development, World Health Organization: Geneva, 2006.

Disclosure of Interest: None Declared.

\section{DOHaD13-1064}

\section{Bodycomposition in young adults born preterm - the Ester Study}

Marika Sipola-Leppänen ${ }^{1,2,3, *}$, Marjaana Tikanmäki ${ }^{1}$, Hanna-Maria Matinolli ${ }^{1}$, Satu Miettola ${ }^{1}$, Petteri Hovi ${ }^{1,4}$, Anneli Pouta ${ }^{1,5}$, Johan G. Eriksson ${ }^{1,6}$, Marjo-Riitta $J_{a ̈ r v e l i n}{ }^{2,7,8}$, Marja Vääräsmäki ${ }^{1,5}$, Eero Kajantie ${ }^{1,4}$

${ }^{1}$ National Institute for Health and Welfare, Oulu and Helsinki, ${ }^{2}$ Institute for Health Science, University of Oulu, ${ }^{3}$ Department of Pediatrics and Adolescence, Oulu University Hospital, Oulu, ${ }^{4}$ Children's Hospital, Helsinki University Central Hospital and University of Helsinki, Helsinki, ${ }^{5}$ Department of Obstetrics and Gynaecology, Oulu University Hospital, Oulu, ${ }^{6}$ Department of General Practice and Primary Health Care, University of

Helsinki, Helsinki, ${ }^{7}$ Biocenter Oulu, Oulu, Finland, ${ }^{8}$ Imperial College, London, United Kingdom

Background: Studies have shown that adults born preterm have lower lean body mass (LBM) than their peers born at term (1) and there is some evidence of more central fat distribution $(2,3)$. Most of the studies have assessed subjects born with very low birth weight. We focused on all degrees of preterm birth and its association with adiposity and body composition.

Method: This study is a part of the ESTER study, in which we assessed the effects of preterm birth on health in adulthood. In this study we included subjects with data on body composition: 1) 134 born preterm <34 wk of GA, 2) 242 born between 34 and $<37$ wk of GA ("late preterm"), and 3) 339 born $\geq 37 \mathrm{wk}$ of GA (controls). At mean age of 23.3 years (SD 1.2) the subjects participated in a clinical examination, which included measurements of height, weight, waist and hip circumference and body composition (bioelectric impedance, InBody 3.0, Biospace). Data were analyzed by linear regression, adjusted for sex, age, height, birth weight SD score, maternal smoking during pregnancy, parental educational attainment, physical activity and daily smoking ("full model").

Results: Adults born preterm $<34 \mathrm{wk} / 34$ to $<37$ wk of GA (mean differences and 95\% CI's) were 3.5\% (95\% CI -0.3 , 7.3)/4.5\% (1.4, 7.7) heavier, had 3.5\% (-0.2, 7.4)/4.4\% (1.3, 7.6) higher body mass index (BMI), 4.2\% (1.6, 6.9)/ $4.0 \%(1.8,6.2)$ higher waist circumference, $1.4 \%(0.3,2.6) /$ $1.2 \%(0.3,2.2)$ higher waist-hip ratio, $0.6 \%(-1.4,2.8) /$ $1.8 \%(0.03,3.6)$ higher $L B M, 11.6 \%(1.3,23.0) / 14.6 \%(5.8$, 24.1) higher fat mass, and $7.7 \%(0.9,15.0) / 9.5 \%(3.7,15.5)$ higher body fat percentage than controls, when adjusted for full model. The difference in weight, BMI and LBM were statistically significant only for groups born late preterm. The differences with controls were slightly smaller when adjusted only for sex and age, although most remained statistically significant. There was no interaction between preterm birth and sex on the body composition (all $P>0.7$ ).

Conclusion: Adults born preterm, including those born late preterm, have higher body fat percentage and abdominal fat than their peers born at term. They may be at a greater risk developing obesity and its complications such as cardiovascular disease.

\section{References}

1) Hovi et al, NEJM, 2007; 2) Edstedt Bonamy et al, J Inter Med, 2005; 3) Inderavik Evensen et al, Early Hum Dev, 2009.

Disclosure of Interest: None Declared.

\section{DOHaD13-1566}

\section{Bodycomposition in young adults born preterm - the Ester Study}

Marika Sipola-Leppänen ${ }^{1,2,3, *}$, Marjaana Tikanmäki ${ }^{3}$, Hanna-Maria Matinolli ${ }^{3}$, Satu Miettola ${ }^{3}$, Petteri Hovi ${ }^{3,4}$, Anneli Pouta ${ }^{3,5}$, Johan G. Eriksson ${ }^{3,6}$, Marjo-Riitta Järvelin $^{2,7,8}$, Marja Vääräsmäki ${ }^{3,5}$, Eero Kajantie ${ }^{3,4}$ ${ }^{1}$ Department of Pediatrics and Adolescence, Oulu University Hospital, ${ }^{2}$ Institute for Health Science, University of Oulu, Oulu, ${ }^{3}$ National Institute for Health and Welfare, Oulu and Helsinki, ${ }^{4}$ Children's Hospital, Helsinki University Central Hospital and University of Helsinki, Helsinki, ${ }^{5}$ Department of Obstetrics and Gynaecology, Oulu University Hospital, Oulu, ${ }^{6}$ Department of General Practice and Primary Health Care, University of Helsinki, Helsinki, ${ }^{7}$ Biocenter Oulu, Oulu, Finland, ${ }^{8}$ Imperial College, London, United Kingdom

Background: Studies have shown that adults born preterm have lower lean body mass (LBM) than their peers born at term (1) and there is some evidence of more central fat distribution (2,3). Most of the studies have assessed subjects born with very low birth weight. We focused on all degrees of preterm birth and its association with adiposity and body composition.

Method: This study is a part of the ESTER study, in which we assessed the effects of preterm birth on health in adulthood. In this study we included subjects with data on body composition: 1) 134 born preterm <34 wk of GA, 2) 242 born between 34 and <37 wk of GA ("late preterm"), and 3) 339 born $\geq 37 \mathrm{wk}$ of GA (controls). At mean age of 23.3 years (SD 1.2) the subjects participated in a clinical examination, which included measurements of height, weight, waist and hip circumference and body composition (bioelectric impedance, InBody 3.0, Biospace). Data were analyzed by linear regression, adjusted for sex, age, height, 
birth weight SD score, maternal smoking during pregnancy, parental educational attainment, physical activity and daily smoking ("full model").

Results: Adults born preterm $<34 \mathrm{wk} / 34$ to $<37$ wk of GA (mean differences and 95\% CI'S) were 3.5\% (95\% CI -0.3 , 7.3)/4.5\% (1.4, 7.7) heavier, had 3.5\% (-0.2, 7.4)/4.4\% (1.3, 7.6) higher body mass index (BMI), 4.2\% (1.6, 6.9)/ $4.0 \%(1.8,6.2)$ higher waist circumference, $1.4 \%(0.3,2.6) /$ $1.2 \%(0.3,2.2)$ higher waist-hip ratio, $0.6 \%(-1.4,2.8) /$ $1.8 \%(0.03,3.6)$ higher $L B M, 11.6 \%(1.3,23.0) / 14.6 \%(5.8$, 24.1) higher fat mass, and 7.7\% (0.9, 15.0)/9.5\% (3.7, 15.5) higher body fat percentage than controls, when adjusted for full model. The difference in weight, BMI and LBM were statistically significant only for groups born late preterm. The differences with controls were slightly smaller when adjusted only for sex and age, although most remained statistically significant. There was no interaction between preterm birth and sex on the body composition (all $P>0.7$ ).

Conclusion: Adults born preterm, including those born late preterm, have higher body fat percentage and abdominal fat than their peers born at term. They may be at a greater risk developing obesity and its complications such as cardiovascular disease.

References 1) Hovi et al, NEJM, 2007; 2) Edstedt Bonamy et al, J Inter Med, 2005; 3) Inderavik Evensen et al, Early Hum Dev, 2009.

Disclosure of Interest: None Declared.

\section{DOHaD13-1651}

\section{Can neonatal exendin- 4 prevent obesity after IUGR?}

Hong Liu ${ }^{1,2, *}$, Christopher G. Schultz ${ }^{3}$, Miles De Blasio ${ }^{4}$, Damien Hunter ${ }^{1,2,5}$, Rebecca Simmons ${ }^{6}$, Karen L. Kind ${ }^{2,5}$, Julie A. Owens ${ }^{1,2}$, Kathryn L. Gatford ${ }^{1,2}$

${ }^{1}$ School of Paediatrics and Reproductive Health, and the Robinson Institute, ${ }^{2}$ The Robinson Institute, University of Adelaide, ${ }^{3}$ Nuclear Medicine \& Bone Densitometry, Royal Adelaide Hospital, Adelaide, Australia, ${ }^{4}$ Physiology, Development \& Neuroscience, University of Cambridge, Cambridge, United Kingdom, ${ }^{5}$ School of Animal \& Veterinary Sciences, University of Adelaide, Adelaide, Australia, ${ }^{6}$ Medical School, University of Pennsylvania, Philadelphia, United States

Background: In humans, low birth weight and accelerated neonatal growth predict later obesity and metabolic disorders. Whilst central adiposity (a predictor of later metabolic disease) develops by 4 years of age in intrauterine growth restricted (IUGR) children ${ }^{1}$, there are limited and conflicting findings regarding obesity and fat distribution in the IUGR adult human ${ }^{2,3}$. Similarly, IUGR sheep exhibit catch-up growth and increased visceral fat mass as juveniles ${ }^{4,5}$. In the sheep, treatment of IUGR offspring with the GLP-1 analogue, exendin-4, prevented catch-up growth and fat deposition at the end of treatment at $\mathrm{d} 16$ of age ${ }^{6}$. The effects of placentally restricted (PR) and neonatal exendin- 4 on adult fat mass and distribution are unknown. We are therefore investigating the distribution of fat mass and long-term effects of neonatal exendin- 4 on growth and fat distribution of PR sheep.

Method: PR was induced by surgical removal of most uterine implantation sites of ewes before mating. Weight and size were measured at birth and throughout life in control (CON; $\mathrm{n}=26 \mathrm{~F}, 20 \mathrm{M}$ ), and PR offspring. A subset of PR offspring were treated with exendin-4 (PR+EX-4; $1 \mathrm{nmol} / \mathrm{kg}$ s.c., daily from day 1 to day 16 of age; $n=13 F, 9 M)$, and the remainder were untreated $(\mathrm{PR} ; \mathrm{n}=18 \mathrm{~F}, 13 \mathrm{M})$. Fat and lean tissue masses were assessed at $\sim 43$ weeks age by dual X-ray absorptiometry for total body, total abdominal region, and upper abdominal regions (primarily omental fat depots). ANOVA was used to test for differences between groups, and 1 -sided Pearson's correlation analyses were used to test for associations with birth weight.

Results: PR reduced birth weight (13\% overall: CON: $5.24 \pm 0.15 \mathrm{~kg}, \quad \mathrm{PR}: 4.57 \pm 0.20 \mathrm{~kg}, \mathrm{p}=0.002 ; 27 \%$ in singletons: $\mathrm{CON}: \quad 6.21 \pm 0.35 \mathrm{~kg}, \quad \mathrm{PR}: \quad 4.52 \pm 0.20 \mathrm{~kg}$, $\mathrm{p}<0.001)$ but not adult weight $(\mathrm{p}=0.969$, overall: $41.2 \pm 0.8 \mathrm{~kg})$. Total body fat ( $\%$ body weight $)$ was not correlated with birth weight $(\mathrm{CON}: \mathrm{r}=0.001, \mathrm{p}>0.4$; PR: $\mathrm{r}=-0.063, \quad \mathrm{p}>0.3 ; \quad \mathrm{PR}+\mathrm{EX}-4: \quad \mathrm{r}=0.355, \quad \mathrm{p}=0.052)$. Whilst total abdominal fat (\% total body fat) was not correlated with birth weight $(\mathrm{CON}$ and $\mathrm{PR}: \mathrm{r}=-0.09$, $\mathrm{p}=0.219 ; \mathrm{PR}+\mathrm{EX}-4 \mathrm{r}=0.268, \mathrm{p}=0.114)$, fat mass in the upper abdominal region (\%total body fat) was negatively correlated with birth weight in animals not treated with EX-4 (CON and PR: $r=-0.219, \mathrm{p}=0.028$ ) but not in animals treated with EX-4 (PR+EX-4: $r=0.261, p=0.120)$.

Conclusion: IUGR is associated with unchanged overall adiposity in adult sheep, similar to that reported in humans, but with redistribution of fat centrally, possibly contributing to IUGR-associated adverse cardiovascular and metabolic health outcomes. Neonatal exendin- 4 treatment of IUGR offspring normalises pattern of fat distribution.

\section{References}

1. Ibanez, L. et al. (2008) J Clin Endocrinol Metab 93 (3): 925-8.

2. Yajnik, C. S. (2004) Proc Nutr Soc 63 (3): 387-96.

3. Rogers, I. (2003) Int J Obes Relat Metab Disord 27 (7): 755-77.

4. De Blasio, M. J. et al. (2007) Am J Physiol Regul Integr Comp Physiol 292 (2): R875-86.

5. Muhlhausler, B. S. et al. (2008) Domest Anim Endocrinol 35 (1): 46-57.

6. Gatford, K. L. et al. (2013) PLoS One 8 (2): e56553.

Disclosure of Interest: None Declared. 


\section{DOHaD13-1359}

\section{Choosing the best anthropometric measures at birth to identify intrauterine growth restriction}

Leixiaoping Lei $^{1,2, *}$, Jun Zhang $^{1}$

${ }^{1} M O E$ and Shanghai Key Laboratory of Children's Environmental Health, Xinhua Hospital, Shanghai Jiaotong University School of Medicine, Shanghai, China, shanghai;

${ }^{2}$ Department of Neonatology, Affiliated Hospital of Luzhou

Medical College, Luzhou, China

Background: Intrauterine growth restriction (IUGR) is associated with various perinatal and long term morbidity and mortality. However, there is no "gold standard" for the diagnosis of IUGR. The purpose of the study is to find the most sensitive anthropometric measure to identify IUGR at birth.

Method: Data from the Collaborative Perinatal Project, a multicenter prospective cohort study in the U.S. from 1959 to 1976 were used. A total of 46,021 women were enrolled in the study during pregnancy and 56990 children were followed up to 7 years of age. Only non-Hispanic White and Black infants of 37-42 weeks of gestation were used $(\mathrm{N}=28,326)$. An array of anthropometric measures at birth were tested, including birthweight (BW), birth length (L), head circumference (HC), chest circumference (CC), BW to $\mathrm{L}$ ratio, body mass index (BMI), Ponderal index (PI), BW to $\mathrm{HC}$ ratio (WHR), $\mathrm{CC}$ to $\mathrm{HC}$ ratio (CHR), placental weight (PW) and BW to PW ratio (WPR). Widely-accepted, IUGR-related prenatal risk factors and postnatal outcomes, such as maternal current smoking, severe hypertension during pregnancy, neonatal infection disease, IQ at age 4 years, mental retardation at age 4 years and body mass index at age 7 years, were chosen as dependent variables in this analysis. Odds ratios (for categorical outcomes in logistic model) or regression coefficients (for continuous outcomes in linear regression model) were computed for each measure, separated by gender (male and female) and race (black and white). According to the size and consistency of the statistical value in different subgroups, we selected anthropometric measures for the diagnosis of IUGR that are more sensitive to adverse outcomes.

Results: Among all measures tested, WHR and WLR were most closely associated with perinatal risk conditions and long term outcomes in all 4 subgroups. Less associated were BW, CC, L, BMI and HC. CHR, PW, PI, CLR and WPR are least associated with perinatal and long-term outcomes. It is interesting to note that the magnitude of association of the three weight/length index (WLR, BMI, PI) decreased with increasing the power of the numerator.

Conclusion: Using perinatal and long-term adverse outcomes as the "gold standard", WHR and WLR, as markers for asymmetric growth restriction, are more sensitive to identify IUGR than BW alone or other measures.

Disclosure of Interest: None Declared.

\section{DOHaD13-1534}

Consumption of sucrose, not high fructose corn syrup, leads to an increase in visceral and subcutaneous adiposity in the rat

Carla R. Toop ${ }^{1}$, Bev Muhlhausler ${ }^{2}$, Kerin O’Dea ${ }^{3}$, Sheridan Gentili ${ }^{1, *}$

${ }^{1}$ School of Pharmacy and Medical Sciences, Sansom Institute for Health Research, University of South Australia, ${ }^{2}$ FOODplus Research Centre, School of Agriculture, Food and Wine, The University of Adelaide, ${ }^{3}$ School of Population Health, Sansom Institute for Health Research, University of South Australia, Adelaide, Australia

Background: Consumption of sugar-sweetened beverages is steadily increasing worldwide, including in pregnant and lactating women. Excess intake of such beverages has been associated with an increased risk of obesity and type 2 diabetes in non-pregnant adults, however the effects of excess consumption during pregnancy and lactation remains unclear. Sucrose, also known as table or cane sugar (glucosefructose disaccharide), is the most commonly used natural sweetener in food and beverages worldwide. This is in contrast to the USA, where high fructose corn syrup (HFCS; $55 \%$ fructose: $42 \%$ glucose: $3 \%$ polysaccharides) is used in place of sucrose. Excess consumption of both sucrose and fructose have been associated with increased body fat mass, dyslipidemia, and the development of type 2 diabetes, although unlike glucose the long term metabolic consequences of fructose are likened to that of ethanol. While there is evidence that excess maternal intake of either fructose or glucose can have detrimental effects on the offspring, the impact of commonly used sweeteners is unclear. The aim of this study was to test the hypothesis that maternal HFCS consumption would result in greater impairments in glucose tolerance, and increase in fat deposition, when compared to sucrose consumption.

Method: 57 female Albino Wistar rats were randomly assigned to either a control $(C, n=22)$, sucrose $(S ; n=18)$ or HFCS (HFCS, $\mathrm{n}=17$ ) group. All rats were provided ad libitum access to standard laboratory rat chow and water. In addition, rats in the $S$ and HFCS groups were provided with a $10 \% \mathrm{w} / \mathrm{v}$ sucrose or HFCS beverage respectively for $4 \mathrm{wk}$ prior to mating, during pregnancy and lactation. Glucose tolerance was assessed in the dam prior to commencing their respective diets, after $4 \mathrm{wk}$ on the diet and immediately prior to postmortem at $3 \mathrm{wk}$ postpartum. At postmortem all tissues were collected and weighed. The effect of $S$ or HFCS consumption were compared using a one-way ANOVA.

Results: There was no effect of treatment on body weight (C, $309.8 \pm 5.5 ; \quad$ S, $\quad 317.0 \pm 4.8 ; \quad$ HFCS,$\quad 309.8 \pm 3.4 \mathrm{~g})$. Consumption of sucrose, but not HFCS, during pregnancy and lactation was associated with a significant increase in relative parametrial $(\mathrm{P}<0.001)$, retroperitoneal $(\mathrm{P}<0.001)$, omental $(\mathrm{P}<0.001)$ and subcutaneous $(\mathrm{P}<0.001)$ fat mass 
at 3wk postpartum. Dams consuming the HFCS tended to have an increase in relative liver weight $(C, 4.37 \pm 0.09$; $S$, $4.61 \pm 0.08$; HFCS, $4.67 \pm 0.05 \%$ body weight, $P=0.052$ ) compared to controls. There was no significant effect of $S$ or HFCS consumption on glucose tolerance at $3 \mathrm{wk}$ postpartum (AUC: C, 1001.6 $\pm 26.2 ; \mathrm{S}, 1109.7 \pm 35.9$; HFCS, $1011.4 \pm 42.3 \mathrm{mmol} / \mathrm{L} / \mathrm{min}, P=0.08)$.

Conclusion: We report for the first time a sugar specific effect of sucrose versus HFCS on relative liver weight, and on both absolute and relative fat mass in the adult rat. Interestingly neither sucrose nor HFCS consumption had a significant effect on body weight, despite an increase in relative liver, visceral, and subcutaneous fat masses. Furthermore, these data suggest that the impact of sucrose on fat deposition is greater than that of HFCS. We speculate that the reported changes in fat deposition in this cohort are associated with an increase in hepatic lipid accumulation and lipogenic gene expression.

Disclosure of Interest: None Declared.

\section{DOHaD13-1313}

\section{Delivery mode and feeding specifies gut microbiota of} infants which influences body composition

Shaillay Dogra ${ }^{1, *}$, Olga Sakwinska ${ }^{2}$, Catherine Ngom-Bru ${ }^{2}$, Wolfram Bruek ${ }^{2}$, Bernard Berger ${ }^{2}$, Harald Bruessow ${ }^{2}$, Yung Seng Lee ${ }^{1,3}$, Fabian Yap ${ }^{4}$, Yap-Seng Chong ${ }^{3}$, Peter Gluckman ${ }^{1}$, Joanna Holbrook ${ }^{1}$

${ }^{1}$ Singapore Institute of Clinical Sciences (SICS), A*STAR, Singapore, Singapore; ${ }^{2}$ Nestle Research Centre, Lausanne, Switzerland; ${ }^{3}$ Yong Loo Lin School of Medicine, National University of Singapore; 'Woman's and children's hospital, Singapore, Singapore

Background: The microbes residing in human gut have a profound influence of our physiology. Bacterial colonisation of the gut starts at birth and is influenced by mode of delivery and subsequent nutritional and environmental exposures. In this study we surveyed the gut microbiomes of 75 infants who are part of the Growing Up in Singapore Towards healthy Outcomes (GUSTO) birth cohort.

Method: Infant fecal samples were collected at 3 days, 3 weeks, 3 months and 6 months of age. The microbiota composition was assessed by 454 pyrosequencing of the amplified V456 region of $16 \mathrm{~S}$ ribosomal RNA. The data set consisted of 292 samples. Taxonomic assignation was performed with RDP classifier. The sequencing reads were clustered in operational taxonomic units (OTUs) with 97\% identity. Data was floored at one read density and filtered for presence in at least two samples. Differential analyses were run segregated by timepoint.

Results: Infant microbiomes changed profoundly over the timecourse. Early samples contained fewer bacteria and were very different across individuals. At later timepoints the samples contained more diverse bacteria but they converged to become more similar across individuals. There was a strong effect of mode of delivery, with infants born by caesarean section having less diverse microbiomes at day 3 than those born vaginally. There was also a marked effect of mode of feeding with some taxa unique to formula fed babies and some predominant in breast fed babies. As mode of feeding has been shown to influence adiposity of infants, we modelled the relationship between these variables and showed that it can be mediated by microbial profile.

Conclusion: We hypothesise that one molecular mechanism whereby early environment can influence development is microbial. We show the profound effect of early environment on seeding infant gut microbiomes and the association with early indicators of growth trajectory.

Disclosure of Interest: None Declared.

\section{DOHaD13-1705}

Disproportionate growth of abdominal adipose tissue in first 6 months of life in Asian infants

Mya-Thway Tint $^{1, *}$, Marielle V Fortier ${ }^{2}$, Keith M Godfrey ${ }^{3}$, Borys Shuter ${ }^{1}$, Jeevesh Kapur ${ }^{4}$, Victor S Rajadurai ${ }^{2}$, Pratibha Agarwal $^{2}$, Amutha Chinnadurai ${ }^{4}$, Krishnamoorty Niduvaje ${ }^{4}$, Yiong Huak Chan ${ }^{1}$, Izzuddin Bin Mohd Aris ${ }^{1}$, Shu E Soh ${ }^{1,5}$, Fabian K.P. Yap ${ }^{2}$, Seang Mei Saw ${ }^{5}$, Yap-Seng Chong ${ }^{1}$, Peter D Gluckman ${ }^{6,7}$, Yung Seng Lee ${ }^{1,7}$

${ }^{1}$ Yong Loo Lin School of Medicine, NUS, NUHS; ${ }^{2}$ KK Women's and Children's Hospital, Singapore, Singapore; ${ }^{3}$ University of Southampton, Southhampton, United Kingdom; ${ }^{4} N U H$, NUHS; ${ }^{5}$ Saw Swee Hock School of Public Health, NUS, NUHS, Singapore, Singapore; ${ }^{6}$ University of Auckland, Auckland, New Zealand; ${ }^{7}$ SICS, A*STAR, Singapore, Singapore

Background: Early growth and weight gain in early infancy has been linked with increased propensity for later obesity. Abdominal deep subcutaneous adipose tissue was recently reported to be strongly related to insulin resistance and type 2 diabetes in a manner nearly identical to that of visceral adipose tissue. The longitudinal growth of abdominal adipose tissue of infants may thus yield valuable information that could help devise strategies to prevent later metabolic disease. The aim of this study is to examine the abdominal adipose tissue growth in first 6 months of life of infants of birth cohort study, Growing Up in Singapore Towards healthy Outcomes (GUSTO).

Method: 334 subjects born $\geq 34$ weeks of gestation and birth weight $\geq 2000 \mathrm{~g}$ from the GUSTO birth cohort study had complete data for abdominal adipose tissue volumes assessed using magnetic resonance imaging (MRI) at first week (3-21 days) of life. A subset of infants $(\mathrm{N}=38)$ returned for repeat MRIs at ages 6 weeks (36-64 days) and/or 6 months 
(171-213 days). Abdominal adipose tissue was categorized into superficial (ASSC) and deep subcutaneous (ADSC) and internal (AI) adipose tissue compartments and quantified accordingly using in-house quantitative analysis software. Statistical Analysis: Age and gender adjusted standard deviation scores (SDS) for abdominal adipose tissue volumes and infant weight were derived at ages 1 week, 6 weeks and 6 months. The growth of each adipose tissue compartment or weight between time points was calculated taking account of the SDS at earlier time point(s). Regression analysis was performed taking account of ethnicity, birth weight, gestational age at delivery and birth order. Regression coefficients obtained from this residual regression analysis indicate the growth of the adipose tissue in SDS in relation to a 1SDS increase in weight between time points.

Results: Between ages 1 and 6 weeks, abdominal adipose tissue growth was disproportionately high relative to infant weight gain; conditional increases in standard deviation scores relative to weight were $1.15(0.34-2.00, \mathrm{p}=0.009)$ for ASSC, $1.32(0.59-2.01$, $\mathrm{p}=0.002)$ for ADSC and $1.25(0.80-1.69, \mathrm{p}<0.001)$ for AI. Conditional adipose tissue growth between ages 6 weeks and 6 months was, however, proportional to infant weight gain.

Conclusion: Disproportionately rapid growth of abdominal adipose tissue in the first six weeks of life supports the notion that nutritional intervention during the neonatal and early infancy period may influence the risk of subsequent metabolic diseases later in life.

Disclosure of Interest: None Declared.

\section{DOHaD13-1690}

Distribution and determinants of abdominal adipose tissue depots in Asian neonates: quantification by MRI

Mya-Thway Tint ${ }^{1, *}$, Marielle V Fortier ${ }^{2}$, Keith M Godfrey ${ }^{3}$, Borys Shuter ${ }^{1}$, Jeevesh Kapur ${ }^{4}$, Victor S. Rajadurai ${ }^{2}$, Pratibha Agarwal $^{2}$, Yiong-Huak Chan ${ }^{1}$, Izzuddin Bin Mohd Aris ${ }^{1}$, Shu-E Soh ${ }^{1}$, Kenneth Kwek ${ }^{2}$, Fabian K. P. Yap ${ }^{2,5}$,

Seang Mei Saw ${ }^{1}$, Yap-Seng Chong ${ }^{1}$, Peter D Gluckman ${ }^{6,7}$, Yung Seng Lee ${ }^{1,6}$

${ }^{1}$ NUS, NUHS; ${ }^{2} K K$ Women's and Children's Hospital,

Singapore, Singapore; ${ }^{3}$ University of Southampton,

Southhampton, United Kingdom; ${ }^{4} N U H$, NUHS;

${ }^{5}$ Duke-NUS Graduate Medical School; ${ }^{6}$ SICS, A*STAR,

Singapore, Singapore; ${ }^{7}$ Liggins Institute University of Auckland, Auckland, New Zealand

Background: In-utero developmental exposures significantly influence later susceptibility to metabolic diseases. These prenatal exposures can affect the development of adiposity at birth and may determine subsequent metabolic phenotype and contribute to ethnic variations in metabolic risk. We examined the quantity, distribution, and determinants of abdominal adipose tissue depots in Asian neonates.
Method: Magnetic Resonance Imaging (MRI) at 1 week (3-21days) post-partum was performed in 334 neonates from the GUSTO birth cohort study, born at $\geq 34$ weeks gestation and birthweight $\geq 2000 \mathrm{~g}$. Using in-house quantitative analysis software, we quantified abdominal superficial subcutaneous (ASSC), deep subcutaneous (ADSC) and internal (AI) adipose tissue depot volumes. Statistical Analysis: Neonatal abdominal adipose tissue depot volumes were log transformed and multiple linear regression analysis performed with ethnicity, gender and parity as independent variables. Ethnicity (for analysis of gender), gender (for analysis of ethnicity), birthweight, gestation duration, birth order, age on the day of MRI and breastfeeding status were adjusted as confounders.

Results: MRI scans at age 1 week were completed for 334 neonates; 183 male (54.8\%), 151 female (45.2\%); 145 Chinese (43.4\%), 128 Malay (38.3\%) and 61 Indian (18.3\%). Mean birthweights were 3145, 3127 and $3061 \mathrm{~g}$ in Chinese, Malay and Indian neonates, respectively. The neonates had substantial ASSC but small ADSC and AI depots. Female neonates had greater neonatal adiposity than male neonates, particularly at the ASSC 13\% (95\%CI: 9, 17\%, p < 0.001) and ADSC $24 \%$ (95\%CI: 15, 33\%, p < 0.001) depots; AI tended to be greater in female neonates but the difference was not significant, 3\% (95\%CI: $-3,8 \%, p=0.369)$. Compared with Chinese neonates, ASSC and ADSC were $8 \%(95 \% \mathrm{CI} 3,13 \%$, $\mathrm{p}=0.001)$ and $22 \%(95 \% \mathrm{CI}: 11,35 \%, \mathrm{p}<0.001)$ greater in Indian neonates despite having a lower mean birth weight. Malay neonates had 14\% (95\% CI: 6, 24\%, $\mathrm{p}=0.001)$ greater ADSC compared to Chinese neonates. ASSC, ADSC and AI were generally lower in the neonates of primiparous mothers compared to neonates of multiparous mothers ( 89.0 vs. 91.6, 13.4 vs. 14.0 and 28.3 vs. $29.0 \mathrm{ml}$, respectively), however, none of these differences were statistically significant.

Conclusion: The significant differences in adipose tissue distribution at birth provide a greater understanding of characteristics of the Asian metabolic phenotype and ethnic based differences in health and disease. An understanding of these differences will facilitate the development of specifically targeted and more efficacious interventions. The longitudinal tracking of adipose tissue growth may help us understand better the impact of the different abdominal adipose tissue depots on subsequent metabolic diseases.

Disclosure of Interest: None Declared.

\section{DOHaD13-1203}

Effects of age on the developmental timeline for the manifestation of the programmed bone deficits associated with fetal growth restriction

Tania Romano ${ }^{1, *}$, John D. Wark ${ }^{2}$, Mary E. Wlodek ${ }^{3}$

${ }^{1}$ Human Biosciences, La Trobe University; ${ }^{2}$ Bone and Mineral Medicine; ${ }^{3}$ Physiology, The University of Melbourne, Melbourne, Australia 
Background: Recent evidence links low birth weight and poor adult bone health. Uteroplacental insufficiency (UPI) complicates $10 \%$ of human pregnancies causing intrauterine growth restriction and programming of bone deficits. We characterized bone size, mineral content, density and stress stain index of strength (SSI) in rats from weaning to 12 months.

Method: Bilateral uterine vessel ligation (Restricted) or sham surgery (Control) was performed on gestational day 18 (term $=22$ days) in rats inducing UPI and growth restriction. Post mortem of Restricted and Control male and female offspring was performed at postnatal day 35 (weaning), 2, 4, 6 and 12 months. Right femur mineral content, density and strength were measured (pQCT).

Results: Male and female Restricted pups were born 10-15\% lighter, remaining smaller and having shorter femurs than Controls to 12 months $(p<0.05)$. Male and female Restricted rats had lower trabecular content compared to Controls across all ages $(\mathrm{p}<0.05)$, without trabecular density changes. Cortical content at day 35, and 6 and 12 months was reduced in Restricted males, this deficit increasing between $6(7 \%)$ and 12 months (11\%). Cortical content was reduced across all ages in Restricted females $(p<0.05)$. Cortical density was lower at day 35 in Restricted males only $(\mathrm{p}<0.05)$. Cortical thickness was reduced in Restricted males on day 35 and 12 months $(\mathrm{p}<0.05)$. SSI was lower at day 35, and $6(13 \%)$ and 12 months (15\%) in Restricted males, and across all ages for Restricted females $(\mathrm{p}<0.05)$.

Conclusion: Skeletal deficits were observed in Restricted males as early as 35 days, and at 6 and 12 months. Restricted females demonstrated deficits across all ages, highlighting gender differences regarding programming of adult bone. The findings that deficits observed at 6 months in males are increased at 12 months indicate that aging may be important in the manifestation of programmed adult bone phenotypes.

Disclosure of Interest: None Declared.

\section{DOHaD13-1724}

\section{Effect of maternal glycemia on neonatal adiposity in a multi-ethnic Asian Birth Cohort}

Izzuddin B. M. Aris ${ }^{1, *}$, Shu E Soh ${ }^{1}$, Mya Thway Tint ${ }^{1}$, Shen Liang ${ }^{1}$, Seang Mei Saw ${ }^{2}$, Kenneth Kwek ${ }^{3}$, Amutha Chinnadurai ${ }^{4}$, Peter D. Gluckman ${ }^{5}$, Keith Godfrey ${ }^{6}$, Victor S. Rajadurai ${ }^{3}$, Yap Seng Chong ${ }^{1}$, Fabian Yap $^{3}$, Yung Seng Lee ${ }^{1}$

${ }^{1}$ Yong Loo Lin School of Medicine, NUS, NUHS; ${ }^{2}$ Saw Swee Hock School of Public Health; ${ }^{3} K K$ Women's and Children Hospital; ${ }^{4}$ National University Hospital; ${ }^{5}$ Singapore Institute for Clinical Sciences, Singapore, Singapore; ${ }^{6}$ University of Southampton, Southampton, United Kingdom

Background: Maternal hyperglycemia is known to increase the risk of obesity and diabetes in offspring later in life.
We examined the relationship between maternal glycemia and neonatal adiposity in a multi-ethnic cohort of Singaporean neonates.

Method: A prospective observational birth cohort study recruited pregnant mothers $(57.2 \%$ Chinese, $25.5 \%$ Malay and $17.3 \%$ Indian) and performed $75 \mathrm{~g} 2$-hour oral glucose tolerance tests at 26-28 weeks gestation. Among 1247 eligible study participants, results for glucose level were available for 1081 mothers. Neonatal anthropometrics (birth weight, length, triceps and subscapular skinfolds) were measured. Neonatal percentage body fat $(\% \mathrm{BF})$ was predicted using our published equation. Associations of maternal glucose with excessive neonatal adiposity (defined as large-for-gestational age (LGA), \%BF and sum of skinfolds ( $\mathrm{SFT})>90^{\text {th }}$ percentile) were assessed using multiple logistic regression analyses, with adjustment for potential confounders.

Results: For measures of excessive neonatal adiposity, we observed strong, positive and continuous association across the range of maternal glucose levels for both glucose measures, after adjustment for potential confounders. In fully adjusted continuous variable models, odds ratios ranged from 1.07-1.64 for LGA, 1.32-2.73 for \%BF and 1.24-2.50 for $\Sigma$ SFT $>90^{\text {th }}$ percentile for fasting and 2-hour glucose higher by $1 \mathrm{mmol} / \mathrm{L}$. The influence of high maternal fasting glucose on neonatal $\sum \mathrm{SFT}$ is significantly more pronounced in Indians compared to Chinese (interaction $p=0.005$ ).

Conclusion: These findings confirm a continuous relationship between maternal glycemia and excessive neonatal adiposity, which extends across the range of maternal glycemia. Indian babies may be more susceptible to increased adiposity from high maternal glucose levels compared to Chinese babies.

Disclosure of Interest: I. Aris: None Declared., S. E. Soh: None Declared., M. Thway Tint: None Declared., S. Liang: None Declared., S. M. Saw: None Declared., K. Kwek: None Declared., A. Chinnadurai: None Declared., P. Gluckman Paid Instructor for: Have received reimbursement for speaking at conferences sponsored by companies selling nutritional products; part of an academic consortium that has received research funding from Abbot Nutrition, Nestec and Danone., K. Godfrey: None Declared., V. Rajadurai: None Declared., Y. S. Chong Paid Instructor for: Have received reimbursement for speaking at conferences sponsored by companies selling nutritional products; part of an academic consortium that has received research funding from Abbot Nutrition, Nestec and Danone., F. Yap: None Declared., Y. S. Lee: None Declared.

\section{DOHaD13-1735}

Excessive gestational weight gain is associated with greater perinatal deep and superficial abdominal subcutaneous adipose tissue deposition assessed by neonatal MRI

Mya-Thway Tint ${ }^{1, *}$, Shu E Soh ${ }^{1}$, Marielle V Fortier ${ }^{2}$, Borys Shuter ${ }^{1}$, Fabian K.P Yap ${ }^{2,3}$, Seang Mei Saw ${ }^{1}$, 
Peter D Gluckman ${ }^{4,5}$, Yung Seng Lee ${ }^{1,4}$, Keith M Godfrey ${ }^{6}$, Yap-Seng Chong ${ }^{1}$

${ }^{1}$ NUS, NUHS; ${ }^{2} K K$ Women's and Children's Hospital;

${ }^{3}$ Duke-NUS Graduate Medical School; ${ }^{4}$ SICS, A*STAR,

Singapore, Singapore; ${ }^{5}$ Liggins Institute, University of Auckland,

Auckland, New Zealand; 'University of Southampton \&

University Hospital Southampton NHS Foundation Trust,

Southampton, United Kingdom

Background: Body composition at birth reflects intrauterine developmental influences and high neonatal adiposity has been associated with obesity in later childhood. Maternal gestational weight gain has been linked with greater offspring adiposity persisting from birth to age 6 years, but little is known about the influence of gestational weight gain on regional abdominal adipose tissue deposition in the offspring. Using neonatal magnetic resonance imaging (MRI) to quantify abdominal adipose tissue volumes, we have examined the influence of pregnancy weight gain in the birth cohort study, Growing Up in Singapore Towards healthy Outcomes (GUSTO).

Method: We analyzed data on 210 GUSTO infants born $\geq 37$ weeks of gestation who had MRI performed during the neonatal period and whose mothers had complete information on gestational weight gain (GWG) and did not have gestational diabetes. GWG was derived from mother's selfreported pre-pregnancy weight and her last weight recorded at antenatal clinic visit close to delivery (37-41 weeks gestation). Pregnancy weight gain was categorized using 2009 recommendations of the Institute of Medicine. Weight gains below the recommended ranges were categorized as inadequate, those within the range as adequate and those above the upper range as excessive. Neonatal abdominal adipose tissue volumes were separated into superficial and deep subcutaneous and internal adipose tissue and quantified accordingly using in-house quantitative analysis software.

Results: $42.5 \%$ of mothers in this MRI subgroup had excessive GWG and $25.1 \%$ had inadequate GWG. Neonates of mothers with excessive, adequate and inadequate GWG had mean(SD) birth weights of 3294(423), 3091(416) and 2996(344) g, respectively. The birth weight of neonates of mothers with excessive GWG was significantly greater compared to those with adequate GWG (difference 217 (95\%CI: 101, 332) g, p < 0.001). However, the difference in birth weight in neonates between mothers with inadequate and adequate GWG was not significant. Compared to neonates born to mothers with adequate GWG, taking account of neonatal and maternal confounders, those born to mothers with excessive GWG had greater superficial subcutaneous (difference 0.371 (95\%CI: $0.093,0.649$ ) SDS, $\mathrm{p}=0.009$ ) and greater deep subcutaneous (difference 0.352 (95\%CI:0.043, 0.661) SDS, $\mathrm{p}=0.026)$ tissue. Internal adipose tissue was similar (difference $0.220 \quad(95 \% \mathrm{CI}$ : $-0.072,0.511)$ SDS, $p=0.14$ ) in both GWG groups. Neonates of mothers with inadequate GWG tend to have lower adipose tissue in all 3 compartments compared to adequate GWG group, but the differences were not statistically significant.

Conclusion: Greater neonatal abdominal adipose tissue mass in neonates of mothers with excessive GWG suggests that excessive maternal weight gain may make a significant contribution to the increased risk of metabolic disease in the offspring later in life.

Disclosure of Interest: None Declared.

\section{DOHaD13-1646}

\section{H19/IGF2 methylation is associated with body composition changes in young adults}

Rae-Chi Huang ${ }^{1,2, *}$, Trevor A. Mori ${ }^{1}$, Lawrence J. Beilin ${ }^{1}$, Craig E. Pennell ${ }^{3}$, Stephen Lye ${ }^{4}$, Jenny Mountain ${ }^{2}$, Jeffrey M. Craig ${ }^{5}$

${ }^{1}$ School of Medicine and Pharmacology; ${ }^{2}$ Telethon Institute for Child Health Research, University of Western Australia;

${ }^{3}$ Department of Obstetrics and Gynaecology, King Edward Memorial Hospital, Perth, Australia; ${ }^{4}$ The Samuel Lunenfeld Institute, Mount Sinai Hospital, Toronto, Canada; ${ }^{5}$ Murdoch Children's Research Institute, Melbourne, Australia

Background: Periconceptional dietary changes have been associated with altered methylation at the H19/Insulin like growth factor (IGF)2 locus. Further H19/IGF2 DMR (differentially methylated region) methylation has been associated with obesity. We aimed to investigate the associations between H19/IGF2 DMR methylation and body fat distribution at age 17 years old.

Method: Adolescents (age $=16.5 \pm 0.5$ years) $(n=207)$ of the Western Australian Pregnancy Cohort (Raine study) had H19/IGF2 DMR methylation measured at 17 years and body composition measured by Dual Energy X-ray Absorptiometry (DEXA) at 20 years old. DNA methylation was measured at twelve cytosine-phosphate-guanine sites (CpGs), analysed as Sequenom MassARRAY EpiTYPER units within the IGF2/H19 $I C R$. DNA methylation was investigated for its association with body composition using linear regression analysis.

Results: There were 115 males and 91 females participants. The principal component of IGF2/H19 ICR DNA methylation (representing mean methylation across all $\mathrm{CpG}$ units) positively correlated with soft tissue and fat mass (total, chest, head, midriff and pelvic) (all $\mathrm{p}<0.05$ ). It was not associated with lean mass in any region or with lean or fat mass in limbs. Conclusion: H19/IGF2 DMR methylation is specifically associated with central body fat and soft tissue. This provides further evidence that methylation of specific loci is associated with adiposity patterns associated with increased cardiovascular risk. It suggests that $H 19 / I G F 2 D M R$ methylation may play a role in mediating the effects of early life environments on subsequent obesity-related disease.

Disclosure of Interest: None Declared. 


\section{DOHaD13-1507}

\section{Longitudinal variance of visceral fat thickness in pregnant adolescents}

Joao G. Alves ${ }^{1, *}$, Luciana Dutra ${ }^{2}$, Rosangela Cisneiros ${ }^{2}$, Alex Souza $^{1}$, Carolina P. Diniz ${ }^{2}$, Lais Alves ${ }^{2}$, José N. Figueiroa ${ }^{1}$

${ }^{1}$ Instituto de Medicina Integral Prof. Fernando Figueira (IMIP), Recife, ${ }^{2}$ UNIVASF, Petrolina, Brazil

Background: Visceral adipose tissue excess is associated with metabolic risk. Nevertheless few studies have assessed fat distribution during pregnancy, Our aim is to verify the longitudinal variance of visceral fat thickness (VFT) among pregnant adolescents

Method: A prospective cohort study with pregnant adolescents was carried out from February 2011 to May 2012 in Petrolina, Brazil. Pregnant adolescents were followed from the 12th-20th gestational week until delivery. The study was previously approved by the Ethics Committee in Research, Universidade Federal do Vale do São Francisco (UNIVASF) and all patients signed a consent form. VFT was evaluated between 12 th to 20 th and 33rd to 36th gestational weeks by abdominal ultrasound according to Armellini et al method.

Results: 75 adolescents were admitted to this study and 67 completed the second ultrasound exam. They aged 13-19 years $(17.7 \pm 1.9)$ and had a BMI of $23.0 \mathrm{~kg} / \mathrm{m}^{2}\left(\mathrm{SD}=3.9 \mathrm{~kg} / \mathrm{m}^{2}\right)$ during early pregnancy $(<20$ th gestational week). VFT determined at $35.1 \mathrm{st}(\mathrm{SD}=1.1)$ was higher than VFT at 16.9th $(\mathrm{SD}=2.2)$ gestational, respectively 1.6 to $7.8 \mathrm{~cm}$ $(4.5 \pm 1,2)$ and 1.5 to $6.6 \mathrm{~cm}(3.2 \pm 1.0) ; \mathrm{p}<0.001$. Longitudinal variance of VFT between early and late pregnancy was $1.1 \mathrm{~cm}$ ( \pm 1.0$)$. No correlation was observed between VFT increase and maternal anthropometric variables. A positive correlation was found between VFT and newborn weight $(\mathrm{r}=0.39 ; \mathrm{p}<0.001)$.

Conclusion: Abdominal visceral fat increases in thickness from early to late pregnancy in adolescents. Fat thickness increase is positively correlated with birth weight.

Disclosure of Interest: None Declared.

\section{DOHaD13-1568}

Maternal consumption of high fructose corn syrup, but not sucrose, is associated with increased fat deposition in the rat offspring post weaning

Carla R. Toop ${ }^{1, *}$, Bev Muhlhausler ${ }^{2}$, Kerin O'Dea ${ }^{3}$, Sheridan Gentili ${ }^{1}$

${ }^{1}$ School of Pharmacy and Medical Sciences, Sansom Institute for Health Research, University of South Australia; ${ }^{2}$ FOODplus Research Centre, School of Agriculture, Food and Wine, The University of Adelaide; ${ }^{3}$ School of Population Health, Sansom Institute for Health Research, University of South Australia, Adelaide, Australia
Background: Consumption of sugar-sweetened beverages has been associated with an increased risk of developing obesity and type 2 diabetes. Few studies, however, have investigated the immediate and long terms effects of maternal sugarsweetened beverage consumption during pregnancy and lactation on the offspring. Sucrose, also known as table or cane sugar (glucose-fructose disaccharide), is the most commonly used natural sweetener in food and beverages worldwide. This is in contrast to the USA, where high fructose corn syrup (HFCS; 55\% fructose: 42\% glucose: $3 \%$ polysaccharides) is used in place of sucrose. The aim of this study was to determine the effects of maternal sucrose and HFCS consumption during pregnancy and lactation on the offspring post weaning. We hypothesise that maternal consumption of HFCS during pregnancy and lactation would be associated with an increase in body weight, liver weight and fat mass of the offspring at $21 \mathrm{~d}$ postnatal when compared to maternal sucrose consumption.

Method: 27 female Albino Wistar rats were randomly assigned to either a control $(C, n=8)$, sucrose $(S, n=11)$ or HFCS (HFCS, $\mathrm{n}=8$ ) group. Dams were given ad libitum access to a standard rat chow and, in addition to water, either a $10 \% \mathrm{w} / \mathrm{v}$ sucrose or HFCS drink for $4 \mathrm{wk}$ prior to mating and throughout pregnancy and lactation. Within $24 \mathrm{~h}$ of birth, litters were culled to 8 pups. At P20, an intraperitoneal glucose tolerance test was performed on 1 male and 1 female from each litter. A postmortem was performed on the same pups at P21 with tissues weighed and collected. The effect of sex and maternal diet during pregnancy and lactation on the offspring were determined using a two-way ANOVA with litter as a covariate, maternal diet and sex as factors with Bonferroni post hoc analysis.

Results: We report a significant effect of treatment on offspring weight at $\mathrm{P} 21$, such that $\mathrm{S}$ offspring were significantly smaller than $\mathrm{C}$ offspring (C, 44.9 $\pm 1.2 ; \mathrm{S}$, $41.6 \pm 0.7 ; \mathrm{H}, 44.1 \pm 1.0 \mathrm{~g}, P<0.05)$, independent of sex. Absolute liver weight tended to be lower in the $S$ offspring (C, $1.67 \pm 0.06 ;$ S, $1.51 \pm 0.04 ;$ HFCS, $1.62 \pm 0.05 \mathrm{~g}$, $P=0.054$ ) compared to $\mathrm{C}$ offspring independent of sex, however there was no difference in relative liver weight between the groups. We report a significant increase in the relative gonadal fat mass $(\mathrm{C}, 0.17 \pm 0.01 ; S, 0.17 \pm 0.01$; HFCS, $0.22 \pm 0.02 \%$ body mass, $P<0.05)$ in the HFCS offspring compared to $\mathrm{S}$ and $\mathrm{C}$ offspring, and interscapular fat mass (C, $0.43 \pm 0.02 ; \mathrm{S}, 0.46 \pm 0.02$; HFCS, $0.51 \pm 0.01 \%$ of body mass, $P<0.01$ ) in the HFCS offspring compared to $\mathrm{C}$ offspring, independent of sex.

Conclusion: We have shown that maternal consumption of either sucrose or HFCS during pregnancy and lactation significantly impacts on the developing offspring in a sugar specific manner. Maternal consumption of sucrose during pregnancy and lactation resulted in significantly smaller offspring post weaning. In contrast, maternal consumption of HFCS during pregnancy and lactation, despite having no effect on body weight, resulted in an increase in fat deposition 
post weaning. This is the first study to show a differential effect of maternal consumption of sucrose and HFCS on the development of the rat offspring, and highlights the importance of the different metabolic outcomes of these two sweeteners.

Disclosure of Interest: None Declared.

\section{DOHaD13-1334}

\section{Maternal obesity and excessive weight gain during pregnancy and childhood cardiovascular risk factors.}

Romy Gaillard ${ }^{1,2,3, *}$, Albert Hofman ${ }^{2}$, Eric Steegers ${ }^{4}$, Vincent Jaddoe ${ }^{1,2,3}$

${ }^{1}$ Generation R; ${ }^{2}$ Epidemiology; ${ }^{3}$ Pediatrics; ${ }^{4}$ Obstetrics and Gynaecology, Erasmus Medical Center, Rotterdam, Netherlands

Background: Maternal prepregnancy obesity is associated with a higher risk of childhood obesity. Less is known about the associations of maternal prepregnancy obesity with the development of other cardiovascular risk factors in the offspring. Excessive gestational weight gain might also influence long-term cardiovascular health of the offspring. Not much is known about critical periods of gestational weight gain in relation to childhood cardiovascular health outcomes. We examined the associations of maternal prepregnancy obesity, excessive gestational weight gain and trimester specific weight gain with cardiovascular outcomes in childhood.

Method: The study was embedded in a population-based prospective cohort study among 4871 mothers and their children. Information about maternal weight before pregnancy and in each trimester of pregnancy, and maximum weight during pregnancy was obtained by physical examinations and questionnaires. At the age of 6 years, childhood anthropometrics, body fat distribution, left ventricular mass, blood pressure, blood lipids and insulin levels were measured. Clustering of cardiovascular risk factors was defined as having 3 or more of the following components: android fat mass \% $=>75$ th percentile; systolic or diastolic blood pressure $=>75$ th percentile; HDL-cholesterol $<=25$ th percentile or triglycerides $=>75$ th percentile; and insulin level $=>75$ th percentile.

Results: As compared to children from mothers with a normal prepregnancy weight, children from mothers with prepregnancy obesity had higher levels of childhood body mass index, total fat mass, android/gynoid fat mass ratio, systolic blood pressure, triglycerides and insulin ( $p$-values $<0.05$ ). However, these associations of maternal prepregnancy obesity with childhood systolic blood pressure, triglycerides and insulin attenuated towards non-significant after adjustment for current childhood body mass index. As compared to normal prepregnancy weight, maternal prepregnancy obesity was associated with an increased odds of childhood overweight and obesity and clustering of cardiovascular risk factors (Odds Ratio(OR): 3.59 (95\%CI: 2.80, 4.61) and OR 3.19 (95\%CI: 2.18, 4.66), respectively). As compared to non-excessive gestational weight gain, excessive gestational weight gain was associated with higher childhood body mass index, total fat mass and android/gynoid fat mass ratio, but not with other cardiovascular risk factors. Conditional analyses showed that when maternal prepregnancy body mass index and weight gain in all trimesters was taken into account, the strongest associations were present for maternal prepregnancy body mass index and gestational weight gain in the first half of pregnancy with childhood adiposity outcomes.

Conclusion: Our study shows that maternal prepregnancy obesity and weight gain during early pregnancy are associated with increased levels of childhood adiposity. Associations of maternal prepregnancy obesity with other childhood cardiovascular risk factors are largely explained by offspring body mass index. Future preventive strategies to reduce overweight and obesity in pregnant women may lead to better cardiovascular health in their children.

Disclosure of Interest: None Declared.

\section{DOHaD13-1169}

Maternal obesity and lipid levels in early pregnancy and the risk of childhood obesity: the mother child "RHEA" cohort in Crete, Greece

Vasiliki Daraki ${ }^{1,2}$, Vaggelis Georgiou ${ }^{1}$, Georgia Chalkiadaki ${ }^{1}$, Stella Koinaki ${ }^{1}$, Marianna Karahaliou ${ }^{1}$, Katerina Sarri ${ }^{1}$, Maria Vassilaki ${ }^{1}$, Stathis Papavasiliou ${ }^{2}$, Manolis Kogevinas ${ }^{3}$, Leda Chatzi ${ }^{1, *}$

${ }^{1}$ Department of Social Medicine, Faculty of Medicine, University of Crete, Greece, ${ }^{2}$ Clinic of Endocrinology Diabetes and Metabolic Diseases, University Hospital of Crete, Heraklion, Greece, ${ }^{3}$ Centre for Research in Environmental Epidemiology, CREAL, Barcelona, Spain

Background: Childhood obesity has increased dramatically over the last decades and is recognized as a serious public health concern. Maternal pre-gestational body size as well as metabolic factors in early pregnancy may be independent determinants of fetal and child growth. Many studies support an association between increased pre-pregnancy body mass index (BMI) and a higher risk of obesity in infancy and childhood, but there is limited evidence on the association of serum lipid levels in early pregnancy with child growth. The aim of this study was to assess the associations between maternal obesity and lipid levels in early pregnancy with the risk of obesity in offspring at 4 years of age.

Method: The mother-child "Rhea" study in Crete, Greece is a prospective cohort examining pregnant women that became pregnant during one year starting in February 2007. Eight hundred twenty four pregnant women and their children were available for the present analyses after excluding twin 
pregnancies, pregnancies with confirmed pre-gestational diabetes, and those with missing data. Height, measured at the first prenatal visit, and self-reported pre-pregnancy weight, were used to calculate the pre-pregnancy BMI $\left(\mathrm{Kg} / \mathrm{m}^{2}\right)$. Maternal fasting serum samples collected at the first prenatal visit (Mean: 12 weeks, SD: 1,5) were used to determine levels of triglycerides, total cholesterol, high density lipoprotein (HDL) and low density lipoprotein (LDL) cholesterol in early pregnancy. Weight, height, abdominal circumference, and skinfold thickness of triceps, thigh, subscapular, and suprailiac were measured in 824 children at 4 years of age. Multivariable linear and poisson regression models were used to estimate the effect of maternal obesity and lipid levels in early pregnancy on the risk of obesity in preschool children after adjusting for potential confounders.

Results: Children of overweight/obese mothers pre-pregnancy $\left(B M I \geq 25 \mathrm{Kg} / \mathrm{m}^{2}\right)$ had increased risk of being overweight/obese at 4 years of age (RR: 1.75, 95\% CI: 1.15 -2.68) after adjusting for maternal age, education, parity, smoking during pregnancy, gestational diabetes, weight gain during pregnancy, birth weight, and breastfeeding. Overweight prior to gestation was associated with increased fat mass as measured by the sum of skinfolds measurements (b coef: 2.04 , 95\% CI: 0.18-3.9), while maternal obesity was associated with increased risk of central adiposity at 4 years of age (waist circumference $\geq 90$ th percentile; RR: 1.53 , 95\% CI: 1.07-2.20). An elevation of $50 \mathrm{mg} / \mathrm{dl}$ in fasting triglyceride levels in early pregnancy increased significantly the risk of overweight/obesity in preschool children (RR: 1.29, 95\% CI: 1-1.67). No associations were observed between total cholesterol, HDL and LDL levels in early pregnancy and offspring weight status at 4 years of age.

Conclusion: Maternal overweight/obesity and elevated triglyceride levels in early pregnancy may contribute to increased risk for the development of obesity and central adiposity in preschool children. Further follow up of this cohort will allow to determine whether these results sustain in later life increasing the risk for cardiovascular disease.

Disclosure of Interest: None Declared.

\section{DOHaD13-1485}

\section{Metabolic relations of adipose tissue during and after cigarette smoke exposition associated to a high-fat diet intake, in young male mice}

Celly Cristina Nascimento-Saba ${ }^{1, *}$, Rejane P. Gaspar-Reis ${ }^{1}$, Cynthia C. Silva ${ }^{1}$, Aline S. Santos ${ }^{1}$, Yael A. Villaça ${ }^{1}$, Erica Patricia Garcia-Souza ${ }^{1}$

${ }^{1}$ Physiological Sciences, STATE UNIVERSITY OF RIO DE JANEIRO, Rio de Janeiro, Brazil

Background: Excess body weight is the sixth most important risk factor contributing to the overall burden of diseases.
Overweight and obesity are predisposing factors for many metabolic diseases as type 2 diabetes mellitus, steatohepatitis, hypertension and cardiovascular disease. It has been suggested that poor diet and physical inactivity could soon replace smoking as the leading cause of avoidable death. However, human studies have shown that smoking itself is linked to the development of abdominal or central obesity and both, obesity and cigarette smoking, are risk factors for insulin resistance, cardiovascular disease, and cancer. Smoking reduces appetite, which makes many people reluctant to quit but few studies have documented the metabolic impact of combined smoke exposure and high-fat diet $(1,2,3,4,5)$. We hypothesized that in animals feeding high-fat diet (HFD) the cigarette smoke exposure (SE) would reduce caloric intake and body weight, accompanied by reduced fat accumulation, thereby reducing metabolic risk. So, the aim of this study was to explore and to evaluate the influence of smoking in puberty and the consequences of withdrawal in adulthood on the adiposity of males mice feeding a palatable HFD.

Method: At 35 days old, mice received control diet containing $7 \%(\mathrm{C}, \mathrm{n}=12)$ or a high-fat diet containing $19 \%(\mathrm{HF}, \mathrm{n}=12)$ of soybean oil and were exposed to $3 \mathrm{R} 4 \mathrm{~F}$ cigarette (Tobacco and Health Research Institute) smoke, 8 h/day for 15 days. At 50 days old, half of animals were then sacrificed and intra-abdominal (IA) and subcutaneous (SC) adipose tissues were collected and evaluated. The other half of mice was evaluated thirty days after stop exposition. An unexposed group accompanied the events (NS, $n=24$ ). During all the period, body mass, length and food intake were controlled.

Results: The body mass $(\mathrm{p}<0.001)$, body length $(\mathrm{p}<0.001)$ and food intake $(\mathrm{p}<0.05)$ were higher in HF exposed group throughout all the experimental period. During exposure the HFD intake did not alter IA adipose tissue mass however, in this group, SC adipose tissue mass was lower than in $\mathrm{C}$ and NS groups $(\mathrm{p}<0.001)$. Thirty days after exposure, the mass of IA and SC adipose tissues were higher $(\mathrm{p}<0.03$ and $\mathrm{p}<0.009$, respectively) in HF group. At the $14^{\text {th }}$ day of smoke exposure, the ipGTT showed high glucose concentration in HF group. However, after 29 days of withdrawal the ipGTT did not differ between the groups.

Conclusion: The set of results indicates different response of high-fat diet intake associated with cigarette smoke that seems to start in young males and lead to malefic metabolic alterations in adulthood.

\section{References}

1. Meyers AW et al. Are weight concerns predictive of smoking cessation? A prospective analysis. J. Consult. Clin. Psychol. 1997;448-452.

2. Yeh HC et al. Smoking, smoking cessation, and risk for type 2 DM: a cohort study. Ann. Intern. Med. 2010;10-17. 3. Fulkerson JA \& French SA. Cigarette smoking for weight loss or control among adolescents: gender and racial/ethnic differences. J. Adolesc. Health. 2003;306-313. 
4. Chen $\mathrm{H}$ et al. Detrimental metabolic effects of combining long-term cigarette smoke exposure and high-fat diet in mice. Am J Physiol Endocrinol Metab. 2007;E1564-E1571.

5. Bray GA. Medical consequences of obesity. J Clin Endocrinol Metab 2004;2583-2589.

Disclosure of Interest: None Declared.

\section{DOHaD13-1375}

\section{Modifying effect of birth weight on the association between healthy diet score and body composition in adulthood}

Mia-Maria Perälä ${ }^{1, *}$, Satu Männistö ${ }^{1}$, Eero Kajantie ${ }^{1}$, Johan G. Eriksson ${ }^{2}$

${ }^{1}$ Department of Chronic Disease Prevention, National Institute for Health and Welfare; ${ }^{2}$ Department of General Practice and Primary Health Care, University of Helsinki, Helsinki, Finland

Background: Body size at birth is associated with body mass index (BMI), body composition and indices of obesity in adult life. Diet also has a strong impact on BMI and body composition. We aimed to investigate whether birth weight modifies the associations between a healthy diet score (HDS) that illustrates Nordic nutrition recommendation and body composition in adulthood.

Method: We studied 2003 participants from the Helsinki Birth Cohort Study, born 1934-44, whose birth weight was abstracted from birth records. At the study clinic, weight, height, waist circumference (WC), lean body mass (LBM) and body fat \% (BF\%) were measured at adulthood. Diet was assessed with a validated food-frequency questionnaire. Participants were divided into birth weight tertiles $(<3200 \mathrm{~g}, 3200-3600 \mathrm{~g},>3600 \mathrm{~g})$. The HDS was calculated according to the quartiles of consumption of fruits, vegetables, rye, a ratio of white meat to red and processed meat and a ratio of polyunsaturated to saturated and transfatty acids. The lowest intake was given 0 points, the second 1 , the third 2 and the highest 3 points. In addition, the highest quartile of intake of salt, sucrose and alcohol were given 0 points, the second 1 , the third 2 and the lowest 3 points. The HDS ranged from 0 to 24 . The associations between HDS and body compositions were tested by multivariate regression analysis which was adjusted for sex, age, height and BMI.

Results: In the total study population, a positive relationship between birth weight and BMI and LBM as well as an inverse relationship between birth weight and $\mathrm{WC}$ and $\mathrm{BF} \%$ was observed. In the total study population, the HDS was positively associated with BMI $\left(0.08\right.$ points higher HDS per $1 \mathrm{~kg} / \mathrm{m}^{2}$ increase in BMI; $\mathrm{P}=0.02)$ and $\mathrm{LBM}(0.06$ points per $1 \mathrm{~kg}$ increase in LBM, $\mathrm{P}=0.002$ ) and inversely associated with WC $(-0.12$ points per $1 \mathrm{~cm}$ increase in WC; $\mathrm{P}<0.001)$ and $\mathrm{BF} \%$ $(-0.06$ points per $1 \mathrm{BF} \% ; \mathrm{P}=0.009)$. When participants were divided into birth weight groups, the HDS was inversely related to WC $(-0.18$ points per $1 \mathrm{~cm}$ increase in WC; $\mathrm{P}=0.003)$ and positively related to LBM $(0.07$ points per $1 \mathrm{~kg}$ increase in LBM; $\mathrm{P}=0.048$ ) among participants born with low birth weight. No such associations were observed within the other birth weight groups.

Conclusion: A healthy diet that follows nutrition recommendations has positive effects on adult life body composition. Low birth weight subjects seem to have especially beneficial impact of this diet on their body composition.

Disclosure of Interest: None Declared.

\section{DOHaD13-1283}

\section{Neonatal body composition by peapod in relation to maternal variables}

Laura Breij ${ }^{1 * *}$, Anita Hokken-Koelega ${ }^{1}$, Marieke Abrahamse ${ }^{2}$, Dennis Acton ${ }^{2}$

${ }^{1}$ Department of Pediatrics, Erasmus University Medical Centerl Sophia Children's Hospital, Rotterdam; ${ }^{2}$ Danone Research Centre for Specialised Nutrition, Wageningen, Netherlands

Background: There is increasing evidence that body composition early in life has both immediate and far-reaching influence on health. Until recently it was impossible to assess infant body composition properly, but air-displacement plethysmography creates the opportunity to study the effect of prenatal and early postnatal factors on body composition in newborns. Prenatal maternal factors that could influence neonatal body composition are prepregnancy obesity and gestational weight gain. To improve child health outcome, the Institute of Medicine (IOM) published revised guidelines recommending lower gestational weight gain for obese women. The aim of this study was to generate reference values of percentage body fat $(\% \mathrm{BF})$ and fat mass in Dutch newborn infants and to assess the relationship with neonatal weight, prepregnancy obesity and gestational weight gain.

Method: Study population comprised 180 healthy neonates, with a gestational age between 35.2 and 42 weeks, born in Erasmus MC - Sophia Children's Hospital. Neonatal data and adiposity measurements included birth weight, birth length and birth head circumference, extracted from birth records. Within 3 days after birth, weight, crown-to-heel length, head circumference and whole-body composition was assessed using air-displacement plethysmography (PEA POD, Infant Body Composition System, COSMED). Maternal data, i.e. weight before and at end of pregnancy, height and parity were obtained from medical records or were selfreported.

Results: Dutch newborn infants showed a large range in \%BF (1.4-19.9). Weight was related to fat mass $(r=0.56$ $\mathrm{p}<0.001)$, but there was a large variation in $\% \mathrm{BF}$ in children with the same weight. Mean \%BF in girls and boys 
(10.46\% vs $9.94 \%)$ were similar. Mean fat mass increased with gestational age $(r=0.24, p=0.001)$, but $\% B F$ did not. The BMI of the mother before pregnancy was associated with fat mass and $\% B F$ of the infant at birth (both $r=0,2$, $\mathrm{p}=0.01)$. Average weight gain during pregnancy was $14.34 \mathrm{~kg}$ and mothers who were overweight or obese had similar weight gain as normal weight mothers. Gestational weight gain of the mother did not correlate with fat mass, lean body mass and \% BF of the neonate.

Conclusion: This study has generated accurate $\% \mathrm{BF}$ and fat mass reference data of a large population of healthy newborns in the Netherlands. Newborn infants showed a large variation in body fat. Neonates born after a longer pregnancy have more body fat in grams but a similar $\% \mathrm{BF}$ as compared to those born more prematurely. We observed an association of maternal prepregnancy BMI with the fat mass and \%BF of the newborns. We did not find a correlation between gestational weight gain and the body composition of the neonates. Contrary to the IOM guidelines, the obese or overweight pregnant women in this study population had a similar gestational weight gain as the lean women.

Disclosure of Interest: None Declared.

\section{DOHaD13-1409}

Neonatal body composition in a multi-ethnic population; the impact of maternal socioeconomic conditions over the life course

Line Sletner ${ }^{1,2,3, *}$, Britt Nakstad ${ }^{2,3}$, Kjersti Mørkrid ${ }^{2,4}$, Siri Vangen ${ }^{1}$, Kåre I. Birkeland ${ }^{2,4}$, Anne K. Jenum ${ }^{5}$

${ }^{1}$ Norwegian Resource Center of Womens health, Oslo University Hospital; ${ }^{2}$ Institute of Clinical Medicine, University of Oslo, Oslo; ${ }^{3}$ Department of Child and Adolescents Medicine, Akershus University Hospital, Lørenskog; ${ }^{4}$ Dep. of Endocrinology,

Oslo University Hospital; ${ }^{5}$ Institute of Health and Society, University of Oslo, Oslo, Norway

Background: Birth weight and neonatal body composition differ between ethnic groups, also within European welfare states. In ethnic Europeans, a socioeconomic gradient for several adverse health outcomes, including low offspring birth weight, is observed. The inverse associations with present socioeconomic position (SEP) are not as evident in ethnic minority groups. It has been hypothesized that the differential effect of SEP may partly be caused by differences in early life living conditions.

Our aim was to examine the impact of ethnic origin, maternal early life and present SEP on neonatal body composition in a multi-ethnic population.

Method: Data are drawn from a population-based cohort study of healthy pregnant women living in Oslo, Norway (59\% ethnic minorities). One individual early life SEP score and one present SEP score were extracted through two separate principal components analyses. The early-life SEPscore was further dichotomized ( \pm median score). General linear models were used to assess the association between maternal present SEP and three neonatal anthropometric measures (gender- and gestational-specific z-scores for length, abdominal circumference $(A C)$ and sum of skin folds). Analyses were stratified by ethnic country of origin classified according to the UN Human Development Index (HDI) and merged into two categories (Very High/High = High-HDI, $\mathrm{n}=287$ ), Medium and Low = Low-HDI, $\mathrm{n}=250)$.

Results: Women with Low HDI origin (90\% born in a Low HDI country) had lower early life- and present SEP scores. A relative "thin-fat-phenotype" was seen in Low HDI neonates; irrespective of maternal early life SEP. No significant differences were observed for length or skin folds, compared with high $\mathrm{HDI}$ neonates, but $\mathrm{AC}$ was significantly smaller (mean z-score difference: $0.40(-0.60,-0.20)$.

However, a strong interaction $(\mathrm{p}<0.001)$ was observed between maternal early life and present SEP for the dependent variables length and sum of skin folds, both in neonates with High and Low HDI origin. In mothers with a high early life SEP, a strong positive association was found between maternal present SEP and these two anthropometric measures, but not between present SEP and AC. In other words; a relative decline in maternal SEP from early life to the actual pregnancy, was associated with shorter length and less subcutaneous fat in the offspring, compared with neonates of women with persistent, high SEP, while AC was not significantly affected by a decline in SEP. In contrast, in mothers with low early life SEP, present SEP had no significant impact on any of the three offspring anthropometric measures.

Conclusion: These novel findings suggest that there is an ethnic universal interactional effect between maternal early life and present SEP on offspring size. This interaction seems to be relatively stronger for neonatal length and skin folds compared with AC (reflecting lean mass). This is in accordance with findings from animal studies suggesting that phenotypic changes are driven by interactions between the maternal phenotype (influenced by her early life environment) and her present environment, leading to changes in the developmental context of the offspring in each generation.

Disclosure of Interest: None Declared.

\section{DOHaD13-1284}

Postnatal medium chain fatty acids prevent excessive body fat accumulation and improve metabolic profile in adult mice

Annemarie Oosting ${ }^{1}$, Diane Kegler ${ }^{1}$, Inga C. Teller ${ }^{1}$, Bert J. van de Heijning ${ }^{1}$, Eline M. van der Beek ${ }^{2, *}$

${ }^{1}$ Danone Research - Centre for Specialised Nutrition, Wageningen, Netherlands; ${ }^{2}$ Danone Research, Singapore, Singapore 
Background: Breastfeeding is associated with reduced risk on later life obesity. It is unknown which factors contribute to this protective effect, but our previous experimental studies suggest that lipid quality, e.g. fatty acid composition ${ }^{1}$ and physical properties of lipid droplets ${ }^{2}$, plays a role. We have demonstrated that dietary $\mathrm{n} 6$ and $\mathrm{n} 3$ PUFA are translated into maternal milk and their composition in early postnatal life programs adult body composition and metabolic phenotype in mice ${ }^{1}$. Besides PUFA, medium chain fatty acids (MCFA; C8-C10) are key fatty acids in human milk. After absorption MCFA are preferentially oxidized offering fast energy supply and protect PUFA from beta-oxidation. We hypothesized that postnatal dietary MCFA modulate growth and metabolic development and thereby beneficially program adult body composition and metabolic health.

Method: Litters of C57Bl/6j mice were fed either a control diet (CTRL; 3\% C8-C10 of total fatty acids) or a MCFA enriched diet (MCFA; 20\% C8-C10 of total fatty acids from postnatal day (PN) 2 to 21 , and the male offspring continued this diet up to PN42. Subsequently, mice of both experimental groups were challenged with a moderate Western style diet (WSD) until dissection on PN98. Body composition was measured by dual X-ray absorptiometry at PN42, 70 and 98. After dissection, plasma lipid profile, glucose, insulin, adipokines and epididymal adipocyte size distribution were measured.

Milk samples were drawn from dams on CTRL or MCFA diet at PN7, 10 and 13 in a separate study investigating whether the diet intervention affected maternal milk MCFA content.

Results: In contrast to previous PUFA interventions, analyses of the milk samples showed its FA composition was not affected by dietary MCFA fed to the dams. In the offspring, MCFA did not affect body composition at PN42. However, body fat accumulation during the WSD challenge was reduced by $28 \%$ compared to the CTRL group $(\mathrm{p}<0.001)$. This was accompanied by a shift in cell size distribution towards reduce numbers of large epididymal adipocytes and reduced plasma leptin and triglyceride concentrations.

Conclusion: Postnatal dietary MCFA protect against excessive body fat accumulation in a mild obesogenic adult environment. These data emphasize the importance of dietary lipid quality in early postnatal life and indicate that besides $\mathrm{n} 6$ and n3 PUFAs, MCT may be relevant. Additionally, the data indicate that the critical window for programming by dietary lipids extends beyond lactation into the weaning period, since MCFA enrichment did not affect maternal milk FA composition.

\section{References}

1 Oosting A, Kegler D, Boehm G, et al. (2010) Pediatr Res 68, 494-499.

2 Oosting A, Kegler D, Wopereis HJ, et al. (2012) Pediatr Res 72, 362-369.

Disclosure of Interest: None Declared.

\section{DOHaD13-1079}

\section{Predicitive biomarkers for obesity in a humanized mouse model}

Peter Y. Wielinga ${ }^{1}$, Marieke Schoemaker ${ }^{2, *}$, Robert Kleemann ${ }^{1}$, Eric A. van Tol ${ }^{2}$, Teake Kooistra ${ }^{1}$

${ }^{1}$ Metabolic Health Research, TNO, Leiden; ${ }^{2}$ Global Research \& Discovery, Mead Johnson Nutrition, Nijmegen, Netherlands

Background: Obesity is a major health problem that is associated with high morbidity and mortality rates. The prevalence of obesity in children and young adolescents is increasing worldwide. It is important to identify subjects at risk to become obese at an early time point. Up to now, however, there are no good markers available that can predict whether a subject will become obese. The aim of this study is to identify early biomarkers reflecting the susceptibility of an individual to become obese later in life and thus could potentially identify high-risk groups. This was studied in an established humanized mouse model for hyperlipidemia with mild obesity.

Method: Male ApoE*3Leiden mice were fed a standard chow diet until 12 weeks of age which is comparable to the age of young adolescent humans. Subsequently, mice were fed a lard based high fat diet (HFD) for another eight weeks to induce obesity. Blood collected prior to and during HFD feeding was extensively analyzed by lipidomics and proteomics. Blood parameters were correlated to endpoints of obesity including body weight gain, body composition, adipose tissue quantity and quality.

Results: All mice responded to HFD but showed considerable variation in body weight gain (between 2.6 and 11.8 gram). Furthermore other obesity parameters such as body adiposity and body composition showed considerable variation. When sampled prior to HFD feeding, the circulating levels of four specific cholesteryl esters correlated significantly with body weight gain, body composition, adipose tissue quantity and quality. When sampled during and after the HFD feeding, the correlations were even stronger. Furthermore, other lipids were identified to correlate to obesity endpoints as well.

Conclusion: The identified markers are early predictive markers for the susceptibility to obesity prior to HFD feeding in a humanized mouse model. Such markers may open up new strategies for dietary counseling or nutritional intervention while monitoring the development of obesity in early life. Follow-up studies are aimed at evaluating a broader array of potential biomarkers, confirm their validity and translational value in terms of application to human subjects.

Disclosure of Interest: P. Wielinga: None Declared., M. Schoemaker Employee of: Mead Johnson Nutrition, R. Kleemann: None Declared., E. van Tol Employee of: Mead Johnson Nutrition, T. Kooistra: None Declared. 


\section{DOHaD13-1061}

\section{Prenatal DNA damage level is associated with infant adiposity during the first year of life}

See Ling Loy ${ }^{1, *}$, Sirajudeen $\mathrm{KNS}^{2}$, Hamid Jan B. Jan Mohamed ${ }^{1}$

${ }^{1}$ Nutrition Programme; ${ }^{2}$ Department of Chemical Pathology,

Universiti Sains Malaysia, Kubang Kerian, Malaysia

Background: Although several studies have been carried out to investigate childhood obesity, the pathway implications of intrauterine oxidative stress on short and long term adiposity development remain to be elucidated. The Universiti Sains Malaysia Birth Cohort Study was thus established in year 2009 to investigate the effects of prenatal oxidative stress levels on the development of maternal and infant adiposity.

Method: From April 2010 to December 2012, pregnant women aged 19-40 years were recruited from the Obstetrics and Gynaecology Clinic of Hospital Universiti Sains Malaysia and Kubang Kerian Health Clinic in the second trimester of pregnancy $(+18$ weeks of gestation) and followed-up in the third trimester $(+34$ weeks of gestation) until postnatal stage. Baseline data which comprised of 153 pairs of healthy motherchild were collected. Maternal blood samples were collected in the second and third trimesters to analyze for oxidative stress markers. Infant anthropometric measurements were taken at birth, 2 months, 6 months and 12 months of age. Infant weight, body mass index-for-age $\mathrm{Z}$ score (BAZ) and abdominal circumference were used as the adiposity indicator. Statistical analysis was performed by using multiple linear regression.

Results: After adjusting for maternal age, prepregnancy body mass index, gestational weight gain, dietary pattern, nicotine exposure, gestational age, infant sex and breastfeeding practice, greater maternal deoxyribonucleic acid (DNA) damage in the second trimester resulted in lower infant weight at birth $(\beta=-0.12, p<0.001), 2$ months $(\beta=-0.20, p<0.001)$, 6 months $(\beta=-0.28, p<0.001)$ and 12 months of age $(\beta=-0.31, p=0.001)$. Similar findings were observed for infant BAZ and abdominal circumference. Of the antioxidant markers, maternal glutathione peroxidase activity in the second trimester was positively associated with infant BAZ $(\beta=0.01$, $p=0.012)$ and abdominal circumference at birth $(\beta=0.03$, $p=0.001)$. Prenatal lipid peroxidation, protein oxidation, total antioxidant capacity, superoxide dismutase and catalase activities did not seem to affect postnatal growth and adiposity. Conclusion: The present study shows a consistent inverse association between maternal DNA damage in the second trimester and infant adiposity during the first year of life. These infants with reduced growth and adiposity in early postnatal life may have a high tendency to experience catchup growth during childhood, which is strongly associated with later obesity. Monitoring oxidative stress levels during pregnancy could be helpful to strategize in combating obesity epidemic among future generations.

Disclosure of Interest: None Declared.

\section{DOHaD13-1675}

\section{The influences of and implications of neonatal body fat percentage}

Fergus Mccarthy ${ }^{1, *}$, Ali Khashan ${ }^{1}$, Mairead Kiely ${ }^{2}$, Jonathan Hourihan $^{3}$, Deirdre Murray ${ }^{3}$, Louise Kenny ${ }^{1}$

${ }^{1}$ The Irish Centre for Fetal and Neonatal Translational Research (INFANT) and Department of Obstetrics and Gynaecology, University College Cork, Ireland., ${ }^{2}$ School of Food and Nutritional Sciences, University College Cork, ${ }^{3}$ Department of Paediatrics and Child Health, University College Cork, Cork, Ireland

Background: Many factors influence fetal size which has significant implications for both mother and child. The optimal method for assessing neonatal size is unclear. Air displacement plethysmography as measured by the 'Peapod' is a new technique for assessing body composition in neonates. The aims of this study were:

1. Determine the effect of maternal biometrics and lifestyle choices on neonatal body fat percentage

2. Investigate the correlation between body fat percentage (BF\%) and Birthweight (BW), Ponderal Index (PI) and Individualised Birthweight Ratio (IBR).

3. To investigate the influence of $\mathrm{BF} \%$ on mode of delivery.

Method: Study participants $(\mathrm{n}=1240)$ were drawn from the BASELINE study (www.baselinestudy.net). BF\% was measured by 'Peapod' air displacement plethysmography performed within 48 hours of birth. Multivariate linear regression in SPSS was used to analyse the effect of maternal age group, gravidity, BMI, waist-hip ratio, waist-height ratio, smoking, educational attainment and alcohol consumption on neonatal percentage body fat. BF\% was compared with BW, IBR and PI using a combination of Pearson's and Spearman's correlation coefficient. Finally, a binary variable was created to indicate whether neonatal \%BF was above the 90th percentile. Logistic regression was used to examine the association between neonatal $\% \mathrm{BF}$ above the 90th percentile and mode of delivery and LGA and mode of delivery. All analyses were adjusted for infant gender; maternal BMI, smoking and alcohol; and maternal and paternal \%BF determined 4 site skin fold.

Results: Mean $(+/-\mathrm{SD})$ neonatal body fat was calculated at $11.1 \%(+/-4.1 \%)$. Maternal obesity $(30-35 \mathrm{~kg} / \mathrm{m})$ significantly increased neonatal body fat \% (Mean difference $1.56 \%$, (95\% CI 0.67, 2.44)). Increasing paternal birth weight $(\mathrm{PBW})$ was associated with increased neonatal body fat $\%$ (Mean difference $0.5 \% / \mathrm{KgPBW},(95 \%$ CI $0.05,0.95)$. Maternal birthweight, maternal smoking, paternal smoking and 4 -site skin fold determined body fat $\%$ had no effect on neonatal body fat percentage.

Pearson's Correlation coefficients showed a significant correlation between \%BF and Bwt (0.48), IBR (0.45), BMI (0.44), PI (0.33) and head circumference (0.25). 
Mean BF\% was significantly increased in assisted (11.2 \pm 4.1$)$ or C-Section births (elective $11.5 \pm 4.5$ and emergency $11.9 \pm 4.4)$ compared to normal vaginal deliveries (10.4 \pm 3.8$)$. Conclusion: Our study links maternal obesity with an increase in neonatal fat percentage. BF\% correlates significantly with BW, IBR and PI. Mean BF\% was significantly increased in assisted or C-Section births compared to normal vaginal deliveries.

Disclosure of Interest: None Declared.

\section{DOHaD13-1478}

Transgenerational transmission of bone deficits in growth restricted offspring

Tania Romano ${ }^{1, *}$, Kristina Anevska ${ }^{1}$, Linda Gallo ${ }^{2}$, Melanie Tran ${ }^{2}$, John D. Wark ${ }^{3}$, Mary E. Wlodek ${ }^{2}$

${ }^{1}$ Human Biosciences, La Trobe University; ${ }^{2}$ Physiology;

${ }^{3}$ Bone and Mineral Medicine, The University of Melbourne, Melbourne, Australia

Background: Uteroplacental insufficiency (UPI) reduces uterine blood flow and nutrient supply leading to low birth weight offspring. We reported that rat mothers with UPI do not gain and lose bone during pregnancy for fetal skeletal mineralisation as normal rat mothers do. These mothers deliver low birth weight offspring (F1) with adult bone deficits. When pregnant, these F1 females have impaired adaptations which, together with germ line inheritance, may program bone deficits in the F2 generation. We aimed to determine the effects of UPI on F1 maternal bone during pregnancy and whether bone deficits are passed on to the F2 generation via inheritance or impaired pregnancy adaptations using embryo-transfer (ET) techniques.

Method: Late gestation UPI was induced by bilateral uterine vessel ligation (Restricted, R) or sham surgery (Control, C) in WKY rats. F1 R and C female offspring were mated with normal males. One cohort of pregnant F1C and F1R females was sacrificed on gestational day 20. ET was performed on gestational day 1 in another cohort of $\mathrm{F} 1$ females, with $\mathrm{F} 2$ embryos gestated in either Control (C-in-C, R-in-C) or Restricted (C-in-R, R-in-R) mothers. Non-transfer F2R and F2C offspring were also generated. Growth and pQCT bone analysis was performed on femurs of F1 mothers and F2 offspring to determine cortical and trabecular content, density and strength.

Results: Restricted F1 females were born 10-15\% lighter than Controls and had shorter femurs, lower trabecular and cortical content, density and strength compared to nonpregnant Controls $(\mathrm{p}<0.05)$. These differences were absent between Control and Restricted F1 pregnant rats. F2 non-ET offspring were born of normal weight and underwent periods of slowed and accelerated growth $(\mathrm{p}<0.05)$ with no transmission of major F2 bone deficits at 35 days or 6 months.
Day 35 male Restricted embryos (R-in-C, R-in-R) were heavier and had longer femurs, increased cortical content, thickness and strength compared to $\mathrm{C}$-in- $\mathrm{C}$ and $\mathrm{C}$-in- $\mathrm{R}$ groups $(\mathrm{p}<0.05)$. Male Restricted embryo groups had accelerated followed by slowed growth $(\mathrm{p}<0.05)$ so that by 6 months no differences in body weight existed between male ET groups. Male Restricted embryos maintained increased cortical content of $4 \%$ at 6 months compared to all other groups $(\mathrm{p}<0.05)$, although this may not be physiologically relevant. Restricted ET females did not develop bone deficits at 35 days or 6 months of age.

Conclusion: Deficits observed in non-pregnant F1 Restricted females were absent on gestational day 20 in pregnant Restricted females, indicating restoration of normal skeletal adaptations in F1 pregnancy. This potentially resulted in adequate calcium supply to the F2 fetus who was born of normal birth weight thus explaining why bone deficits were absent in F2 non-transfer as well as female ET offspring. The increased body weight in male Restricted ET groups at day 35 relates to these offspring having larger and stronger bones at this age. The small increase in cortical content only in male Restricted ET groups may not be a physiologically relevant change in bone phenotype. Importantly, these results highlight that growth restriction and bone deficits are not passed on from a growth restricted mother to her offspring aged to 6 months.

Disclosure of Interest: None Declared.

\section{DOHaD13-1593}

Validation of anthropometry and bioelectrical impedance against a two-compartment model to assess total body water in Indian rural preschool children

Urmila Deshmukh $^{1, *}$, Suyog Joshi ${ }^{1}$, Charudatta Joglekar ${ }^{1}$, Elaine Rush ${ }^{2}$, Anura Kurpad ${ }^{3}$, Chittaranjan Yajnik ${ }^{1}$

${ }^{1}$ Diabetes Unit, KEM Hospital Research Centre, Pune, India;

${ }^{2}$ Faculty of Health and Environmental Sciences, Auckland University of Technology, Auckland, New Zealand;

${ }^{3}$ Departrment of Physiology, Division of Nutrition, St John's Medical College, Bangalore, India

Background: In community child nutrition surveys body weight, height/ length and mid-upper arm circumference (MUAC) are the commonest anthropometric measurements used. Composition of weight gain and consequently tissue deposition pattern could be assessed in children if age, gender and population specific body composition equations based on anthropometry are available.

Our objectives were 1) To develop age and gender specific predictive equations of total body water (TBW) estimation using different field methods, using a reference value derived by a two-compartment model (2C; deuterium dilution technique) in rural, pre-school Indian children, and, 2) To cross validate TBW estimated by new equations with those developed in other populations 
Method: A standard measurement protocol of anthropometry, bio-impedance analysis (BIA; single frequency, $50 \mathrm{kHz}$ ) and deuterium dilution technique were carried out in 25 rural, preschool children at baseline and after 6 and 18 months (total 59 valid data points). A $2 \mathrm{C}$ model was used as the reference method. TBW prediction equations were developed using multiple linear regressions. The new equations were tested for agreement with published equations of TBW developed in Jamaican and Peruvian children, using methods described by Altman and Bland.

Results: The study children were (median, IQR) 34.2 (22.3, 43.3) months old. Using 2006 WHO Growth Standards, mean (SD) weight for age (WAZ), weight for height (WHZ), height for age (HAZ) and MUAC z scores were - 2.9 (1.18), -1.7 (1.23), $-3.2(1.23)$ and $-1.6(1.16)$ respectively.

The best predictive models using only anthropometry included body weight $(\mathrm{Wt})$, height $(\mathrm{Ht})$, MUAC and gender $(\mathrm{G}$; Boy $=1$, Girl $=2$ ) as independent variables, and explained $88 \%$ of the TBW variance. Combining impedance and anthropometry variables, body weight, height ${ }^{2} /$ impedance $\left(\mathrm{Ht}^{2} / \mathrm{I}\right)$ and gender explained $89 \%$ of the TBW variance.

The new predictive equations developed are as follows,

1) $\mathrm{TBW}(\mathrm{kg})=-2.803+(0.080 \mathrm{Ht})+(0.308 \mathrm{Wt}) \quad$ (0.356G) Eq 1

2) TBW $(\mathrm{kg})=0.794+\left(0.376 \mathrm{Ht}^{2} / \mathrm{I}\right)+(0.317 \mathrm{Wt}) \quad$ $(0.341 \mathrm{G})$ Eq 2

Cross validation of Eq 1 with published anthropometry based equation (Mellits-Cheek and Morgenstern) showed good agreement $\left[\mathrm{R}^{2}\right.$ (difference) $=0.04 ; \mathrm{p}>0.05$ for both]. Cross validation of $\mathrm{Eq} 2$ with published BIA based equations (Bocage and Fjeld) showed good agreement with Bocage equation $\left[R^{2}\right.$ (difference) $\left.=0.01 ; \mathrm{p}>0.05\right]$. The Fjeld equation under-predicted TBW [mean difference 0.38, LoA $-0.14,+0.90 ; \mathrm{R}^{2}$ (difference) $\left.=0.47, \mathrm{p}<0.01\right]$. The mean bias for the Fjeld equation was $6 \%$.

Conclusion: Anthropometry based new equations could be utilised to study body composition and pattern of fat mass and fat free mass deposition in longitudinal epidemiological studies or community based surveys in Indian undernourished, preschool children. Bio-impedance model is equally predictive but the technique is cumbersome and time consuming in non-co-operative small children. Cross validation results need careful interpretation after comparing the nutritional status of the study population.

Disclosure of Interest: None Declared.

\section{DOHaD13-1423}

Visceral adiposity in the first half of pregnancy: correlation with lipid profile, glucose, insulin and HOMA in the second half of pregnancy

Joao G. Alves ${ }^{1, *}$, Andrea Cardoso ${ }^{1}$, José N. Figueroa ${ }^{1}$

${ }^{1}$ Instituto de Medicina Integral Prof. Fernando Figueira (IMIP), Recife, Brazil
Background: Pregnancy leads to visceral fat tissue (VAT) excess, glucids and lipids metabolic changes aiming to offer nutrient supply to the fetus growth. These maternal metabolic alterations resemble the metabolic syndrome. Our hypothesis is that VAT excess in early pregnancy is associated with insulin resistance and dyslipidemia during late pregnancy. Our aim is to correlate visceral fat thickness, measured by ultrasound between 15th and 20th weeks of gestation, with the lipoprotein profile, glucose tolerance test, insulin and HOMA-IR between 36th and 38th gestational weeks.

Method: A cohort study was performed at the Instituto de Medicina Integral Professor Fernando Figueira (IMIP), Recife, Brazil. All participants were admitted to the study after they signed an informed consent. This project received previous approval from IMIP Ethics Committee in Research. Visceral fat thickness was measured by an ultrasound using Armellini et al technique*. Blood samples were collected after 12 hours of fasting to the determination of glucose, triglycerides, total cholesterol, HDL-cholesterol, LDL-cholesterol and VLDLcholesterol by colorimetric methods and chemiluminescence. Data analysis was performed using the correlation coefficient of Pearson and linear regression. A significance level of 0.05 was adopted. Pearson correlation test was used to measure a linear association between variables. The role of visceral fat thickness as associated variable with lipoprotein profile, glucose, insulin and HOMA-IR was tested by linear regression with the use of univariate and multivariate models. All $\mathrm{p}$ values presented are two-tailed, and values less than 0.05 were considered to indicate statistical significance.

Results: VAT showed a positive correlation with 2-hour GTT, insulin, HOMA-IR and triglycerides in univariate analysis $(\mathrm{r}=0.303, \mathrm{p}=0.004 ; \mathrm{r}=0.336, \mathrm{p}<0.001 ; \mathrm{r}=0.388$, $\mathrm{p}<0.001 ; \mathrm{r}=0.279, \mathrm{p}<0.001$, respectively). However, multiple linear regression analysis showed that HOMA-IR was the most significant dependent correlate with VAT excess $(\mathrm{R} 2=0.206, \mathrm{p}=0.042)$.

Conclusion: The present study suggests that visceral adipose tissue excess in early pregnancy may be associated with insulin resistance in later pregnancy.

\section{Reference}

1. Armellini F, Zamboni M, Rigo L, et al. The contribution of sonography to the measurement of intra-abdominal fat. J Clin Ultrasound. 1990;18(7):563-567.

Disclosure of Interest: None Declared.

\section{DOHaD13-1481}

Association between maternal plasma polyunsaturated fatty acid (PUFA) status and childhood atopy at 18 months of age

Yamei $\mathrm{Yu}^{1, *}$, Yiong Huak Chan ${ }^{2}$, Shu E Soh ${ }^{1,3}, \mathrm{Ai}$ Lin $\mathrm{Lim}^{4}$, Oon Hoe Teoh ${ }^{5}$, Anne Goh ${ }^{5}$, Philip C. Calder ${ }^{6}$, 
Seang Mei Saw ${ }^{7}$, Kenneth Kwek ${ }^{5}$, Peter Gluckman ${ }^{4,8}$, Keith M. Godfrey ${ }^{9}$, Yap Seng Chong ${ }^{10}$, Lynette P.-C. Shek ${ }^{1}$, Mary F.-F. Chong ${ }^{11}$, Hugo P. S. Van Bever ${ }^{1}$ and GUSTO

${ }^{1}$ Department of Paediatrics, Yong Loo Lin School of Medicine, National University of Singapore; ${ }^{2}$ Biostatistics Unit; ${ }^{3}$ Saw Swee Hock School of Public Health, National University of Singapore; ${ }^{4}$ Growth, Development and Metabolism Programme, Singapore Institute for Clinical Sciences (SICS), Agency for Science, Technology and Research ( $\left.A^{*} S T A R\right) ;{ }^{5} K K$ Women's and Children's Hospital (KKH), Singapore, Singapore; ${ }^{6}$ Faculty of Medicine, University of Southampton, Southampton, United Kingdom; ${ }^{7}$ Saw Swee Hock School of Public Health, Yong Loo Lin School of Medicine, National University of Singapore, Singapore, Singapore; ${ }^{8}$ Liggins Institute, University of Auckland, Auckland, New Zealand; ${ }^{9}$ MRC Lifecourse Epidemiology Unit \& NIHR Southampton Biomedical Research Centre, University of Southampton \& University Hospital Southampton NHS Foundation Trust, Southampton, United Kingdom; ${ }^{10}$ Department of Obstetrics \& Gynaecology, Yong Loo Lin School of Medicine, National University of Singapore; ${ }^{11}$ Clinical Nutrition Research Centre, Singapore Institute for Clinical Sciences (SICS), Agency for Science, Technology and Research ( $\left.A^{*} S T A R\right)$, Singapore, Singapore

Background: Prevalence of childhood allergic disorders is on the rise. Increased intake of pro-inflammatory n-6 PUFAs and reduced intake of anti-inflammatory n-3 PUFAs have been suggested to influence childhood atopy although findings are inconclusive. We prospectively examined the association of maternal PUFA status with childhood atopy.

Method: In the GUSTO Birth Cohort Study, maternal blood samples were collected antenatally at 26-28 weeks and assayed for relative abundance of PUFAs (\% of total fatty acids). Offspring were followed up to 18 months of age, and allergic sensitization was assessed by standardized skin prick test (SPT) to common inhalant and food allergens. A subject with at least one positive SPT was considered atopic, independent of clinical manifestations. Using multivariate logistic regression, the relationship between maternal PUFA status, divided by quintiles, and the presence or absence of childhood allergy was assessed.

Results: Among 755 children, 105 of them (13.9\%) had sensitization on skin prick test. In multivariate models adjusted for maternal ethnicity, maternal education level, maternal age, maternal energy intake, parental history of allergic disease, infant gender, birth weight, gravidity, gestational age, length of breastfeeding and environmental tobacco exposure, very low (lowest quintile, adjusted OR 2.93, 95\% CI 1.51, 5.66, $p=0.001$ ) and high (highest two quintiles, adjusted OR 2.14, 95\% CI 1.15, 3.99, p = 0.016) maternal total n-6 PUFA abundance were associated with increased risk of atopy, compared to intermediate total n- 6 PUFA abundance $\left(2^{\text {nd }}\right.$ and $3^{\text {rd }}$ quintiles). Maternal plasma $n-3$ PUFA and the ratio of $n-6$ to $n-3$ PUFA were not associated with childhood atopy.
Conclusion: Findings from this study provide evidence for the hypothesis that the risk of childhood atopy is modified by variation in exposure to $\mathrm{n}-6$ PUFAs during pregnancy. Our data also suggest a dose-response effect in the relationship between total n-6 PUFAs and childhood atopy.

Disclosure of Interest: None Declared.

\section{DOHaD13-1580}

\section{Characterizing the spinal and supraspinal changes associated with exposure to an immune challenge during the neonatal life}

I. Zouikr ${ }^{1}$, M. A. Tadros ${ }^{2}$, M. James ${ }^{2}$, A. F. Ahmed ${ }^{2}$, K. W. Beagley ${ }^{3}$, V. L. Clifton ${ }^{4}$, R. F. Thorne ${ }^{2}$, C. V. Dayas ${ }^{2}$, R. J. Callister ${ }^{2}$, D. M. Hodgson ${ }^{1, *}$

${ }^{1}$ Laboratory of neuroimmunology; ${ }^{2}$ School of Biomedical Sciences \& Pharmacy, The University of Newcastle, Callaghan; ${ }^{3}$ Institute of Health Biomedical Innovation, Queensland University of Technology, Brisbane; ${ }^{4}$ Robinson Institute, University of Adelaide, Adelaide, Australia

Background: Sensory input from the environment is essential in wiring the spinal cord neurocircuitry and the brain. During the neonatal period these systems are highly plastic, and adverse events occurring during this period can alter spinal and supraspinal processing of pain. Evidence suggests that exposure to repetitive pain during the neonatal period is associated with alterations in pain sensitivity later in life. However less is known about the impact of neonatal exposure to a bacterial mimetic, Lipopolysaccharide (LPS) on pain in later life, which was the aim of the current study. This is of clinical relevance since infections in neonates are a common occurrence which not only impacts short term survival, but may also have lifelong effects on health outcomes.

Method: Wistar rats were subjected to either LPS (salmonella enteriditis, $0.05 \mathrm{mg} / \mathrm{kg}$, ip) or saline (equivolume) on postnatal days (PND) 3 and 5. At PND22, and 80-97, rats were injected with $1.1 \%$, and $2.25 \%$ formalin (respectively) into the hindpaw. 1h later, blood and hippocampus were collected to assess plasma IL-1 $\beta$ (ELISA) as well as hippocampal IL-1 $\beta$ mRNA (qRT-PCR) and protein level (Western Blotting). A separate group of animals were subjected to the formalin test at PND22 and transverse spinal cord slices (300 $\mu \mathrm{m}$ thick) were prepared for whole-cell patch-clamp recording (KCH3SO4-based internal) from superficial dorsal horn (SDH) neurons. At PND22, another subset of male rats was used for cFos immunohistohemistry labelling. Data analyses were carried out using ANCOVA.

Results: Neonatal LPS challenge increased formalin-induced nociception in preadolescent and adult rats. These changes were associated with increased plasma IL-1 $\beta$ levels in preadolescent but not adult rats $(\mathrm{p}<.01)$. Neonatal exposure to LPS altered the intrinsic properties of SDH neurons such 
that neonatally-treated rats displayed decreased input resistance and action potential amplitude at PND $22(\mathrm{p}<.05)$. This change was observed ipsilateral to the formalin injection $(\mathrm{n}=10$ neurons saline; $\mathrm{n}=17$ neurons LPS) and suggests neonatal LPS exposure results in subtle developmental changes rendering adult SDH neurons more excitable to sensory input. No changes were observed in hippocampal IL-1 $\beta$ mRNA and protein levels at this age. However, in adulthood, an increase in IL-1 $\beta$ protein level in the hippocampus was observed $(\mathrm{p}<.05)$. Moreover, we demonstrated that cFos positive cells were significantly increased in the dorsal and ventrolateral periaqueductal gray (DPAG; $\mathrm{p}<.01)$ and VLPAG; $\mathrm{p}<.05$ respectively) in saline controls, implying that the inhibitory descending control pathways were enhanced in this group which might explain the decreased nociception compared to LPS group.

Conclusion: We have demonstrated that sensory information processing at the spinal and supraspinal levels is altered in a developmentally regulated fashion by neonatal exposure to LPS. Understanding the mechanisms that modulate pain in response to early life events, especially the role of the immune and endocrine system, is of crucial importance in generating an understanding of how the early life microbial environment can program pain sensitivity later in development.

Disclosure of Interest: None Declared.

\section{DOHaD13-1360}

Early life overfeeding leads to exacerbated responses to lipopolysaccharide via changes to the hypothalamicpituitary-adrenal axis

Sarah Spencer ${ }^{1, *}$, Guohui $\mathrm{Cai}^{1}$, Ilvana Ziko ${ }^{1}$, Rachel Kenny ${ }^{1}$

${ }^{1}$ School of Health Sciences and Health Innovations Research Institute (HIRi), RMIT University, Melbourne, Australia

Background: Dietary influences in early life can have crucial programming effects on adult physiology. We have previously seen overfeeding in the first three weeks of life in the rat leads to an overweight phenotype long-term and exacerbated fevers in response to the bacterial mimetic, lipopolysaccharide (LPS), later in life. This exacerbated immune response is partially due to neonatally overfed rats having increased expression of toll-like receptors (TLR) 2 and 4, the receptors for LPS. These exacerbated fevers are also associated with changes in the hypothalamic-pituitary-adrenal (HPA) "stress" axis. Thus, neonatally overfed rats have greater activation of the paraventricular nucleus of the hypothalamus (PVN) in response to LPS, and a less efficient glucocorticoid (GC) response resulting in compromised GC negative feedback and less effective GC-mediated inhibition of the immune response. In this study we aimed to determine which aspects of the HPA axis are altered in neonatally overfed rats to contribute to exacerbated febrile responses to LPS in adulthood.
Method: To derive neonatally overfed and control-fed rats we manipulated the litter sizes in which the rats were suckled. Thus, on the day of birth we allocated Wistar rat pups to small litters of 4 (SL; neonatally overfed) or control litters of 12 (CL). Pups were suckled in their new litters until being weaned into same-sex littermate pairs at postnatal day (P) 21 where they were given ad libitum access to normal rat chow. This manipulation results in an overweight phenotype in SL rats that persists throughout life. In adulthood (approximately P70) the rats were given a i.p. LPS $(100 \mu \mathrm{g} / \mathrm{kg})$ and tissue collected $2 \mathrm{hr}$ later for rt-PCR.

Results: As we have reported previously, the neonatal litter size manipulation resulted in male and female SL rats that were significantly heavier at P70 than CL rats of the same sex. There were no differences between the CL and SL groups in adrenal size when corrected for body weight. LPS caused a significant increase in melanocortin 2 receptor (MC2R) mRNA expression in the adrenal glands of male but not female rats, but there were no differences between the CL and SL groups. In males, but not females, there was a significant effect of litter size on pro-opiomelanocortin (POMC) mRNA expression in the pituitary, with SL rats having reduced POMC expression compared with CL.

Conclusion: These findings, that neonatal overfeeding leads to reduced pituitary POMC expression in male rats, suggest there would be less adrenocorticotropic hormone (ACTH) released in response to LPS in these animals leading to reduced or slower GC release from the adrenal glands and a less-efficient GC negative feedback to shut down the immune response. These results remain to be confirmed and we will also further explore the mechanism for the exacerbated immune response to LPS in neonatally overfed females.

Disclosure of Interest: None Declared.

\section{DOHaD13-1429}

\section{Formula milk ages enhances kidney programming in IUGR piglets}

Ghada Elmhiri ${ }^{1, *}$, Thibaut Larcher ${ }^{2}$, Céline NiquetLéridon $^{3}$, Philippe Jacolot ${ }^{4}$, Narges Bahi-Jaber ${ }^{5}$, Samir Boukthir ${ }^{6}$, Abalo Chango ${ }^{6}$, Frederic J. Tessier ${ }^{6}$, Latifa Abdennebi-Najar ${ }^{6}$

${ }^{1}$ UPSP EGEAL, Institut polytechnique LaSalle, Beauvais; ${ }^{2}$ INRA, UMR 703, Ecole Nationale Vétérinaire Agroalimentaire et de l'Alimentation Nantes-Atlantique (Oniris), Nantes 44307; ${ }^{3}$ UPSP EGEAL, Institut polytechnique LaSalle Beauvais; ${ }^{4} U P S P$ EGEAL, Institut polytechnique LaSlle Beauvais; ${ }^{5}$ UPSP EGEAL, polytechnique LaSalle Beauvais; ${ }^{6} U P S P$ EGEAL, Institut polytechnique LaSalle Beauvais, Beauvais, France

Background: Infants suffering from intrauterine growth restriction (IUGR) are largely fed with infant formula which shows high concentrations of Advanced Glycation End 
Products (AGEs). As IUGR is associated with a low nephron number and a high risk of hypertension in adulthood, accumulation of AGEs could induce the initiation and progression of kidney dysfunction that may also contribute to increased disease in later life. We aimed to test whether AGEs from formula milk affect kidney development and function in the IUGR.

Method: Newborn IUGR piglets were randomly divided into 2 groups and they were fed until weaning either with unheated (Control; $\mathrm{n}=8$ ) or heated formula milk at $120^{\circ} \mathrm{C}$ for $20 \mathrm{~min}$ (Treated; $\mathrm{n}=8$ ). Then, all the animals received an ad libitum solid diet until euthanasia on day 54. During development, $N^{\prime}$-carboxymethyl-lysine (CML), a major indicator of known AGEs, was quantified in milk, plasma, feces and kidneys by HPLC-MS/MS. Blood samples were collected before and after weaning and used for sodium, potassium, urea, and albuminemia and creatinine analysis. At day 54, histomorphometric and CML immunofluorescence studies were performed on kidney tissues. The angiotensin receptor (AT2), SIRT1, FOXOa3, RAGE and the transcription factor $\mathrm{NFkB}$ genes expressions were quantified by qPCR. Results: A 15000 fold significant increase of CML, was observed in the heated milk when compared to control $(\mathrm{p}<0.05)$. During artificial suckling, the level of total CML in the plasma and feces was significantly higher in the IUGR treated animals as compared to controls, $(\mathrm{p}<0.05, \mathrm{p}<0.05$ respectively). Free CML level in the plasma was significantly increased in treated group $(\mathrm{p}<0.05)$, while no difference in the level of CML (free and total) was observed when animals received the solid diet. During artificial suckling, u rea level in serum was significantly higher in the treated group as compared to control $(113.5 \pm 9.04 \mu \mathrm{mol} / \mathrm{L}$ vs $108.75 \pm 11.87 \mu \mathrm{mol} / \mathrm{L}, \mathrm{p}<0.05)$, nevertheless no difference was observed on day 54 for this parameter. Interestingly, immunofluorescence study revealed the presence of CML in the kidney's nuclear cells in both groups. A structural alteration in kidneys of treated animals was evidenced by histomorphometric studies with a significant increase of methylene blue positive cells surrounding the tubular lumen, and a significant decrease of glomeruli diameter $(p<0.05)$. Furthermore, treatment with heated milk resulted in a significant up regulation of several studied genes. A significant increase of AT2 $(1.8$ fold, $p<0.05)$ and NFkB (1.3 fold, $\mathrm{p}<0.05)$ gene expression was observed in the treated group with a tendency of an up regulation of SIRT1 (1.3 fold). These results suggest an inflammatory and /or an apoptotic state induced by milk AGEs in renal cells of IUGR treated animals.

Conclusion: During perinatal development, high levels of AGEs in milk formula might induce a delay in IUGR renal maturity and/or an inflammatory process. All these data strongly show an enhancement of renal programming by milk AGEs.

Disclosure of Interest: None Declared.

\section{DOHaD13-1519}

Influence of early life exposures on gut immunity in Canadian infants at 4 months - pilot results from synergy in microbiota research (SyMBIOTA) project

SL Bridgman ${ }^{1, *}$, MB Azad $^{1}$, AS Hill ${ }^{1}, \mathrm{~T}$ Konya ${ }^{2}$, H Maughan $^{2}$, D Guttman ${ }^{2}$, MR Sears ${ }^{3}, \mathrm{AB}$ Becker ${ }^{4}$, $S$ Turvey ${ }^{5}$, JA Scott ${ }^{2}$, CJ Field ${ }^{1}$, AL Kozyrskyj ${ }^{1}$ and CHILD Research Team

${ }^{1}$ University of Alberta, Edmonton; ${ }^{2}$ University of Toronto,
Toronto; ${ }^{3}$ McMaster University, Hamilton; ${ }^{4}$ University of
Manitoba, Winnipeg; ${ }^{5}$ University of British Columbia,
Vancouver, Canada

Background: Immunoglobulin A (IgA) in breast milk provides passive immunity to infants. Compared to breast milk from mothers with existing children, breast milk of primgaravida mothers is reported to contain higher levels of immune factors. The pre and postnatal environment may influence IgA levels in infancy, with implications for later health.

As part of the Synergy in Microbiota Research (SyMBIOTA) project, in collaboration with the Canadian Healthy Infant Longitudinal Development (CHILD) birth cohort, the present sub-study investigates the influence of breastfeeding, microbiota composition and other early life exposures on infant fecal IgA levels at 4 months.

Method: Forty-six infants enrolled in the CHILD study were included. Demographic information was collected by parent questionnaire. At age 4 months, fecal samples were collected and mothers reported current breastfeeding status. Fecal IgA was quantified in duplicate by enzyme-linked immunosorbent assay. Fecal microbiota was characterized in a subset of 24 infants by sequencing the bacterial $16 \mathrm{~S}$ rDNA gene (hypervariable regions V5-V7). The association of fecal IgA and early life exposures, including birth order, breastfeeding status and gut microbiota composition was evaluated with appropriate non-parametric statistical tests.

Results: First born infants (no older maternal siblings) and breastfed infants had higher fecal IgA levels than their infant counterparts $(p=0.03$ and $p=0.04$ respectively). The degree of breastfeeding (none, partial, exclusive) was associated with increasingly higher concentrations of fecal IgA ( $\mathrm{p}$ for trend $=0.01$ ). Stratification by breastfeeding (exclusive/partial vs none) and birth order (older maternal siblings, yes/no) revealed that fecal IgA levels were highest in breastfed, first-born infants, and lowest in non-breastfed infants with siblings $(\mathrm{p}<0.05)$. Among other demographic and environmental exposures, infant fecal $\operatorname{IgA}$ was not associated with gender, mode of delivery, birth weight, hospitalization after birth, household pets, and maternal history of allergy.

Regarding infant gut microbiota composition, we observed a positive correlation between the relative abundance of Veillonella and the level of fecal IgA (Spearman $r=0.47$; $\mathrm{p}=0.04)$. There was also a trend towards negative correlation 
with the absolute abundance of Clostridium difficile $(\mathrm{r}=-0.27 ; \mathrm{p}=0.07)$. Fecal IgA was not correlated with microbiota diversity or any other individual taxa, including Lactobacillus and Bifidobacterium.

Conclusion: As expected, infant fecal IgA levels were determined by the extent of breastfeeding. Our novel finding was that $\operatorname{IgA}$ levels in first born infants were higher than infants with siblings, suggesting that breast milk IgA may be influenced by maternal gravidity. We also found an association between intestinal colonization by specific bacterial taxa and IgA. The effect of these exposures on early IgA concentrations may prove significant in the later risk of childhood disease. Further analysis on a larger sample of the CHILD cohort is required to confirm significance of these results, and explore underlying biological mechanisms.

Disclosure of Interest: None Declared.

\section{DOHaD13-1473}

\section{Lipids and inflammatory markers in newborn umbilical vein blood and associated maternal diabetes, body mass index and weight gain during pregnancy}

Lucia C. Pellanda $^{1,2, *}$, Tatiana Brittos ${ }^{1}$, Fernando Anschau ${ }^{3}$

${ }^{1}$ Post graduation Program, Fundação Universitária de Cardiologia (FUCRS); ${ }^{2}$ Public Health, Universidade Federal de Ciências da Saúde de Porto Alegre (UFCSCPA); ${ }^{3}$ Fundação Universitária de Cardiologia (FUCRS), Porto Alegre, Brazil

Background: Maternal hypercholesterolemia, obesity and diabetes during pregnancy influence fetal development and may predispose offspring to cardiovascular disease. Thus, the aim of this study is to evaluate lipid profile, high-sensitivity C-reactive protein (hs-CRP) and leukocyte counts in the newborns and their relationship to maternal pre-pregnancy BMI, diabetes and excessive weight gain during pregnancy.

Method: Cross-sectional study of 245 mothers and their newborns, consecutively born in a general hospital in southern Brazil. Blood was collected from the umbilical vein of newborns and assayed for total cholesterol and fractions, triglycerides, hs-CRP and leukocyte count. Statistical analysis included Pearson and Spearman correlations and partial correlation coeficients.

Results: Newborns average weight was 3241 grams, with $53.9 \mathrm{mg} / \mathrm{dL}$ of total cholesterol, $21.9 \mathrm{mg} / \mathrm{dL}$ HDL cholesterol, $26.2 \mathrm{mg} / \mathrm{dL}$ LDL cholesterol, $29.5 \mathrm{mg} / \mathrm{dL}$ triglyceride and 13777 leukocytes $/ \mathrm{mm}^{3}$. There was a direct correlation of pre-pregnancy BMI of overweight and obese mothers with total cholesterol $(\mathrm{r}=0.220, \mathrm{p}=0.037)$ and LDL-C $(\mathrm{r}=0.268, \mathrm{p}=0.011)$ of newborns. Total cholesterol, LDL and HDL-C were higher in preterm newborns (66.3 \pm 19.7 , $35.9 \pm 14.6$ and $25.2 \pm 7.7 \mathrm{mg} / \mathrm{dL}$, respectively) that in fullterm infants $(52.4 \pm 13.1,25.0 \pm 8.7$ and $21.5 \pm 6.0 \mathrm{mg} /$ $\mathrm{dL}$ ), with $\mathrm{p}=0.001, \mathrm{p}=0.001$ and $\mathrm{p}=0.003$ respectively.
Leukocyte counts were higher in full-term newborns (14268 \pm $3982 / \mathrm{mm}^{3}$ ) compared with pre-term infants (9792 $\pm 2836 /$ $\left.\mathrm{mm}^{3}, \mathrm{p}<0.0001\right)$. There was an inverse correlation of birth weight with total cholesterol $(\mathrm{r}=-0.160, \mathrm{p}=0.012)$ and with LDL-C $(\mathrm{r}=-0.175, \mathrm{p}=0.006)$, and a direct correlation between birth weight and leukocyte counts of newborns $(\mathrm{r}=0.282, \mathrm{p}<0.0001)$.

Conclusion: These results suggest the possible interaction of maternal weight and fetal growth with lipid metabolism and leukocyte count in the newborn, which may be linked to programming of the immune system.

Disclosure of Interest: None Declared.

\section{DOHaD13-1424}

Maternal dietary supplementation with specific nondigestible oligosaccharides leads to reduced allergic asthma symptoms in their offspring

Astrid Hogenkamp ${ }^{1, *}$, Suzan Thijssen ${ }^{1}$, Naomi van Vlies ${ }^{1}$, Leon Knippels ${ }^{2}$, Elena Sandalova ${ }^{2}$, Johan Garssen ${ }^{1,2}$

${ }^{1}$ Immunopharmacology, Utrecht University, Utrecht;

${ }^{2}$ Immunology, Danone Research Centre for Specialised Nutrition, Wageningen, Netherlands

Background: Previous studies from our laboratory indicate that maternal supplementation with non-digestible carbohydrates may beneficially affect maternal and fetal immune status in mice ${ }^{1}$. We therefore chose to investigate whether maternal supplementation during pregnancy could have long-term programming effects on the immune response in the offspring of both sensitized and non-sensitized dams. To this end, we studied immune responses in the offspring in an ovalbumin (OVA)-induced model for experimental allergic asthma.

Method: Female mice were sensitized twice via i.p. injection to OVA or PBS as a control, and transferred to either a control diet or a diet supplemented with short-chain galactoand long-chain fructo-oligosaccharides (scGOS/lcFOS; ratio 9:1). After 2 weeks, mice were mated. All dams were transferred to control diet after birth of the offspring. Male offspring were sensitized to OVA at 6 weeks, and the acute allergic skin response was measured at 8 weeks. The offspring were challenged 3 times with nebulized OVA, after which lung function was measured and animals were sacrificed.

Results: In offspring of non-sensitized dams supplemented with scGOS/lcFOS a reduction in the acute allergic skin response could be observed as compared to the offspring of non-sensitized dams fed the control diet. This effect was not observed in offspring of sensitized dams. Correspondingly, a dramatic decrease in lungresistance as a response to metacholine challenge was seen in offspring of non-sensitized dams supplemented with scGOS/lcFOS. Furthermore, whereas IgE levels were elevated in the offspring of non-sensitized dams fed the control diet, no such increase was observed for the 
offspring of non-sensitized dams supplemented with scGOS/ lcFOS. IgG2a levels were significantly higher in the latter group compared to the other experimental groups, and a significant increase in the percentage of regulatory $\mathrm{T}$ cells in the spleen was observed.

Conclusion: Maternal supplementation with a specific mixture of oligosaccharides during pregnancy leads to a significant decrease in allergic symptoms in the offspring of non-sensitized mice, suggesting a beneficial programming effect. Results from this study indicate a role for regulatory $\mathrm{T}$ cells.

\section{Reference}

1. Effects of short-chain galacto- and long-chain fructooligosaccharides on systemic and local immune status during pregnancy Journal of Reproductive Immunology 94 (2012) $161-168$.

Disclosure of Interest: None Declared.

\section{DOHaD13-1482}

\section{Maternal weight, gestational weight gain and offspring health at 2 years of age}

Shu E. Soh ${ }^{1,2, *}$, Antony Hardjojo ${ }^{2}$, Keith M. Godfrey ${ }^{3,4}$, Peter D. Gluckman ${ }^{5,6}$, Seang Mei Saw ${ }^{1}$, Bee Wah Lee ${ }^{2}$, Oon Hoe Teoh ${ }^{7}$, Anne Goh ${ }^{7}$, Hugo P. S. Van Bever ${ }^{2}$, Lynette Pei-Chi Shek ${ }^{2}$, Yap Seng Chong ${ }^{8}$

${ }^{1}$ Saw Swee Hock School of Public Health, NUS; ${ }^{2}$ Paediatrics, National University Health System, Singapore, Singapore; ${ }^{3}$ Medical Research Council Lifecourse Epidemiology Unit; ${ }^{4}$ NIHR Southampton Biomedical Research Centre, University of Southampton and University Hospital Southampton NHS Foundation Trust, Southampton, United Kingdom; ${ }^{5}$ Liggins Institute, Auckland, New Zealand; ' ${ }^{6}$ Singapore Institute for Clinical Sciences, Agency for Science and Technology Research (A*STAR); ${ }^{*}$ Paediatrics, KK Women's and Children's Hospital; ${ }^{8}$ Obstetrics \& Gynaecology, National University Health System, Singapore, Singapore

Background: Maternal pre-pregnancy obesity has been associated with children's health including respiratory morbidity and neuropsychological function. Excess weight gain during pregnancy is associated with offspring adiposity and increased risk of wheezing and asthma. We sought to determine whether maternal pre-pregnancy weight and gestational weight gain (GWG) have an effect on offspring health at 2 years of age.

Method: The Growing Up in Singapore Towards healthy Outcomes (GUSTO) cohort study in Singapore includes women from the major Asian ethnic groups (Chinese, Malays and Indians) studied throughout pregnancy at two hospitals, KK Women's and Children's Hospital and National University Hospital, from 2009-2011. Out of the whole cohort of 1247 mother-child pairs, 787 women aged 18-46 years, without gestational diabetes mellitus or preeclampsia, and with live term singleton healthy infants were included in this analysis. The children health outcomes were monitored to 2 years of age and plans for future visits are ongoing.

Results: Using the Asian body mass index (BMI) cut-offs of $<18.5 \mathrm{~kg} / \mathrm{m}^{2}$ (underweight), $18.5-22.9 \mathrm{~kg} / \mathrm{m}^{2}$ (normal range), $23.0-27.4 \mathrm{~kg} / \mathrm{m}^{2}$ (overweight), $\geq 27.5 \mathrm{~kg} / \mathrm{m}^{2}$ (obese), $8.5 \%$ of the women were underweight, $47.9 \%$ normal, $26.5 \%$ overweight and $17.1 \%$ obese before pregnancy. Offspring of overweight and obese mothers had an increased risk of doctor-diagnosed rhinitis (adjusted OR: 1.416, 95\% CI: $1.015,1.976)$ at 2 years of age, independent of family history of atopy, birth order, gender and mothers' age. Mean gestational weight gain was $11.7 \mathrm{~kg}$ (SD 4.4). For each prepregnancy BMI category, the top quartile GWG is taken as excessive weight gain and offspring of mothers in these top quartiles had an increased risk of doctor-diagnosed eczema (adjusted OR: 1.833, 95\% CI: 1.189, 2.825). No relationship was found between age of diagnosis of eczema and GWG. Maternal pre-pregnancy BMI and GWG were not found to be associated with wheezing, use of nebulizer, lower respiratory tract infections (LRTI) such as pneumonia, bronchiolitis, bronchitis and atopy (positive skin prick test) by 2 years old.

Conclusion: Pre-pregnancy BMI and GWG were found to be associated with rhinitis and eczema respectively. Familial atopic predisposition did not modify the effects. As the children in our cohort are still young, we planned to follow them up to further assess respiratory symptoms and functions. The underlying mechanisms of these associations will be explored as adipose tissue is an important source of inflammatory cytokines. Compared with Institute of Medicine 2009 GWG guidelines, Asian women with healthy pregnancies showed differences in mean pregnancy weight gain and we will also re-evaluate the definition and impact of excess GWG in this cohort.

Disclosure of Interest: None Declared.

\section{DOHaD13-1388}

mTOR plays an important role in cow's milk allergy-induced behavioral and immunological deficits

Jiangbo $\mathrm{Wu}^{1, *}$, Caroline de Theije ${ }^{1}$, Sofia Lopes da Silva ${ }^{1,2}$, Martien $\mathrm{Kas}^{3}$, Kim van $\mathrm{Elst}^{3}$, Johan Garssen ${ }^{1,2}$,

Aletta Kraneveld ${ }^{1}$

${ }^{1}$ Division of Pharmacology, Utrecht Institute for Pharmaceutical Sciences, Faculty of Science, Utrecht University, Utrecht;

${ }^{2}$ Danone Research, Center for Specialized Nutrition, Wageningen; ${ }^{3}$ Rudolf Magnus Institute of Neuroscience, University Medical Center Utrecht, Utrecht, Netherlands

Background: Autism spectrum disorder (ASD) is multifactorial, with both genetics and environmental factors working together to develop the autistic phenotype. Immunological disturbances 
in autistic individuals have been reported and a role of cow milk allergy (CMA) has been suggested in ASD. Previously, we have demonstrated that cow's milk allergic mice display behavioural and neuroimmunoligical deficits. In ASD, several mutations in genes are found that are strongly linked to an enhanced mammalian target of rapamycin (mTOR) signaling pathway and enhanced mTOR also plays a central role in directing immune responses towards allergy. Therefore, mTOR pathways may be the link between the immune disturbances and behavioral deficits observed in ASD. In this study we have investigated the role of the mTOR pathway in CMA-induced behavioral and immunological deficits.

Method: To induce CMA, male $\mathrm{C} 3 \mathrm{H}$ mice were orally sensitized with whey protein and cholera toxin (CT) or CT alone for 5 weeks and 1 week later subsequently orally challenged with whey protein once. Rapamycin treatment $(0.5$ or $2 \mathrm{mg} / \mathrm{kg})$ was performed i.p. 5 days per week for 6 weeks. One day after challenge, social interaction and grooming tests were performed. After sacrificing, blood was collected for analysis of whey-specific immunoglobulins and mouse mast cell protease 1 (mMCP-1) measurements. In addition, ileum and brain samples were collected for mTOR activity via Western Blotting.

Results: The validity of the CMA model was confirmed by increased whey-specific $\operatorname{IgE}, \mathrm{IgG}_{1}$ and $\mathrm{IgG}_{2}$ levels in serum and mast cell activation assessed by increased serum levels of mMCP-1. In the social interaction test, cow's milk allergic mice showed less social interaction, while their motor activity was barely affected. Furthermore, repetitive behavior was evident in cow's milk allergic mice as they demonstrated enhanced grooming duration and number. Analysis of brain and ileal tissue samples demonstrated that the cow's milk allergy-induced immune response and behavioral deficits were associated with enhanced mTOR activity. Inhibition of mTOR with rapamycin showed a dose dependent reduction of the immune and behavioral disturbances in cow's milk allergic mice.

Conclusion: CMA animals showed less social interaction and increased repetitive behavior and had enhanced mTOR activity in brain and gut. Inhibition of mTOR with rapamycin ameliorated both social and repetitive behaviors and attenuated the allergic response. In future studies, the mTOR pathway in the development of disturbed behavior will be studied as possible target for interventions.

Disclosure of Interest: J. Wu Grant/Research support from: This study is part of the Utrecht University Focus en Massa program and is financially supported by Danone Centre for Specialised Nutrition, C. de Theije: None Declared., S. Lopes da Silva Employee of: Dr. Sofia Lopes da Silva is the employee at Danone Research Centre for Specialised Nutrition, M. Kas: None Declared., K. van Elst: None Declared., J. Garssen Employee of: Prof. Dr. Johan Garssen is the employee at Danone Research Centre for Specialised Nutrition, A. Kraneveld: None Declared.

\section{DOHaD13-1383}

Neonatal hyperoxia leads to persistent oxidative stress and inflammation in the lungs of mice

Sheena Bouch ${ }^{1 * *}$, Richard Harding ${ }^{1}$, Foula Sozo ${ }^{1}$

${ }^{1}$ Department of Anatomy and Developmental Biology, Monash University, Melbourne, Australia

Background: Very preterm infants (born $<32$ weeks of gestation) usually require supplemental oxygen $\left(\mathrm{O}_{2}\right)$ due to lung immaturity, and the hyperoxia may contribute to their greater risk of respiratory infections and asthma later in life. It is likely that this increased risk may be a result of persistent pulmonary oxidative stress and inflammation, and structural alterations to the bronchioles and alveolar region of the lung. Therefore, our aim was to examine the effects of neonatal exposure to clinically relevant levels of $\mathrm{O}_{2}$ on lung pathology, immune status and oxidative stress.

Method: Neonatal mice (C57BL/6J) were raised in either $40 \%$ or $65 \% \mathrm{O}_{2}$ (hyperoxia groups) from birth until postnatal day $7(\mathrm{P} 7 \mathrm{~d})$. At birth, the lungs of mice are at a similar stage of development as very preterm babies. Controls $(\mathrm{CON})$ breathed room air. Mice were either euthanized immediately following exposure at P7d (CON n $=8,40 \%$ $\mathrm{O}_{2} \mathrm{n}=20,65 \% \mathrm{O}_{2} \mathrm{n}=8$ ), or kept in room air after the 7 days of hyperoxia until adulthood at P56d (CON n $=8,40 \%$ $\mathrm{O}_{2} \mathrm{n}=19,65 \% \mathrm{O}_{2} \mathrm{n}=9$ ). At P7d and P56d, we analysed the wall composition of the bronchioles, the architecture of the alveolar region of the lung, and the area of nitrotyrosine staining in the alveolar region, as a marker of oxidative stress. At P7d, the proportion of macrophages in the alveolar region was determined using an immunofluorescent marker for galectin-3. At P56d, broncho-alveolar lavage fluid (BALF) was collected and the number of immune cells counted. Data were analysed using a one-way ANOVA.

Results: At P7d, the 65\% $\mathrm{O}_{2}$ group, but not the $40 \% \mathrm{O}_{2}$ group, had less alveolar tissue, larger alveoli and fewer bronchiolar-alveolar attachments than controls. In the bronchioles of both hyperoxia groups, the epithelial area was not altered. At P7d, there was significantly less nitrotyrosine staining in the $40 \% \mathrm{O}_{2}$ group compared to the $\mathrm{CON}$ and $65 \% \mathrm{O}_{2}$ groups; however the proportion of macrophages was not significantly different between groups. At P56d, exposure to hyperoxia $\left(40 \%\right.$ and $\left.65 \% \mathrm{O}_{2}\right)$ did not result in structural alterations. However, there was a tendency for the percentage of nitrotyrosine staining to be increased in the hyperoxia groups, and there was a significant increase in number of immune cells in the BALF from both the $40 \% \mathrm{O}_{2}$ group (by $117 \%$ ) and the $65 \% \mathrm{O}_{2}$ group (by 150\%) compared to controls.

Conclusion: Although neonatal exposure to $65 \% \mathrm{O}_{2}$ alters lung architecture at P7d, exposure to $40 \% \mathrm{O}_{2}$ has no apparent effect. Exposure to $40 \%$ and $65 \% \mathrm{O}_{2}$ causes a persistent increase in pulmonary immune cells and a 
tendency for the level of oxidative stress to be increased, suggesting ongoing inflammation and oxidative stress. Our findings may help explain why preterm infants are more susceptible to respiratory infections and asthma later in life.

Disclosure of Interest: None Declared.

\section{DOHaD13-1321}

Neonatal modulation of serum cytokine profiles by a specific mixture of neutral and acidic oligosaccharides in preterm infants

Jolice Van Den Berg ${ }^{1, *}$, Ninke van Zwieteren ${ }^{1}$, Elisabeth Westerbeek ${ }^{1}$, Johan Garsen ${ }^{2}$, Ruurd van Elburg ${ }^{2}$

${ }^{1}$ pediatrics, VUmc, Amsterdam; ${ }^{2}$ Danone Research, Centre for Specialized nutrition, Wageningen, Netherlands

Background: Early nutrition may contribute to the development and programming of the immune system for later life. The rising numbers of preterm infants are vulnerable for infections during early life which cause differences in cytokine levels. Supplementation of a combination of neutral and acidic oligosaccharides (scGOS/lcFOS/pAOS) showed a trend towards a decreases in the incidence of infections in preterm infants. Aim of this study was to measure cytokine levels in preterm infants during the first year of life and to determine the effect of supplementation of neutral and acidic oligosaccharides (scGOS/lcFOS/pAOS) to either own mother's milk or preterm formula. Furthermore, other perinatal factors (breastfeeding, infections, GA and BW) in relation to these cytokine levels were analysed.

Method: In a randomized controlled trial, preterm infants (GA $<32$ weeks and/or birth weight $<1500 \mathrm{~g}$ ) received a scGOS/lcFOS/pAOS mixture or a placebo (maltodextrin) between days 3 and 30 of life. Cytokine levels (IL-1 $\beta$, IL-2, IL-4, IL-6, IL-8, IL-10, IL-17, IFN- $\gamma$, TNF- $\alpha$ ) were analysed at 5 time points during the study: before start of the study, at day 7 , at day 14 and at 5 and 12 months after the start of the intervention.

Results: In total, 55 preterm infants in the scGOS/lcFOS/ pAOS group and 58 in the placebo group were included. During the neonatal period all measured cytokine levels increased at day 7 ( $p<0.05$ except for IL-8) and returned to the level at birth at day $14(\mathrm{p}>0.05$, except for Il- $1 \beta$ and IL10 , who remained at higher level). After the neonatal period, IL-6, IL-8, IL-10, IL-17 and TNF- $\alpha$ decreased at 5 months and remained at low level at 12 months (all $\mathrm{p}<0.05$ ). IL-1 $\beta$ decreased at 12 months $(\mathrm{p}<0.05)$ (fig 1$)$.

Preterm infants in the scGOS/lcFOS/pAOS group showed lower cytokine levels of IL-1 $\beta$, IFN $\gamma$ and TNF $\alpha(\mathrm{p}<0.05)$ and a trend towards lower levels of IL-4, IL-6, IL-8 and IL-17 (all $\mathrm{p}<0.10$ ) at day 7 compared to preterm infants in the placebo group. This effect disappeared at day 14. In the neonatal period, only serious infection before sampling increased all cytokine levels $(\mathrm{p}<0.05)$. There was no effect of BW, GA or breastfeeding on the cytokine levels (fig 1).

Figure:
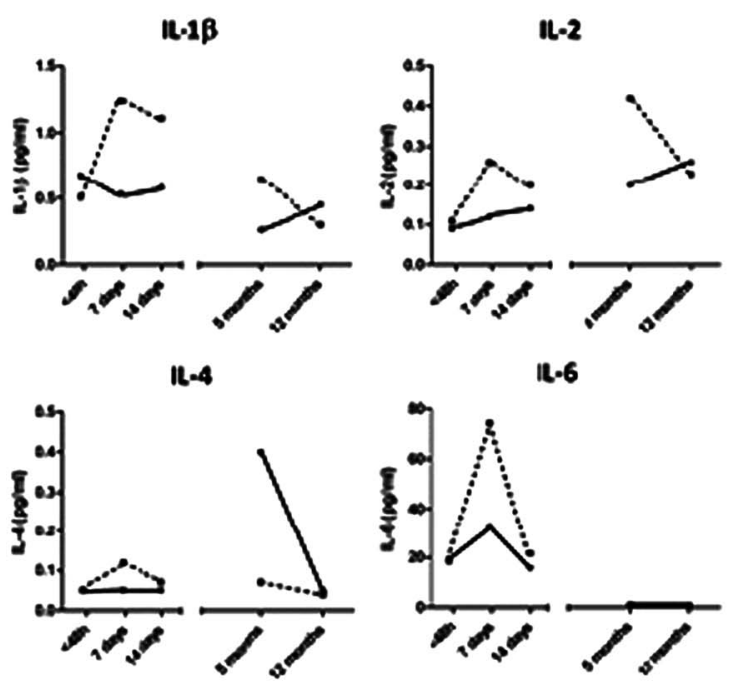

ILA
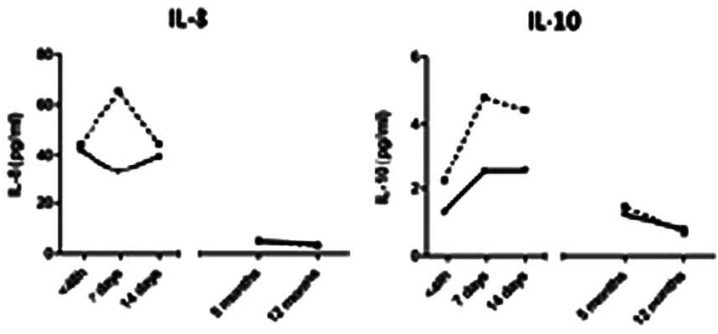

IL-17
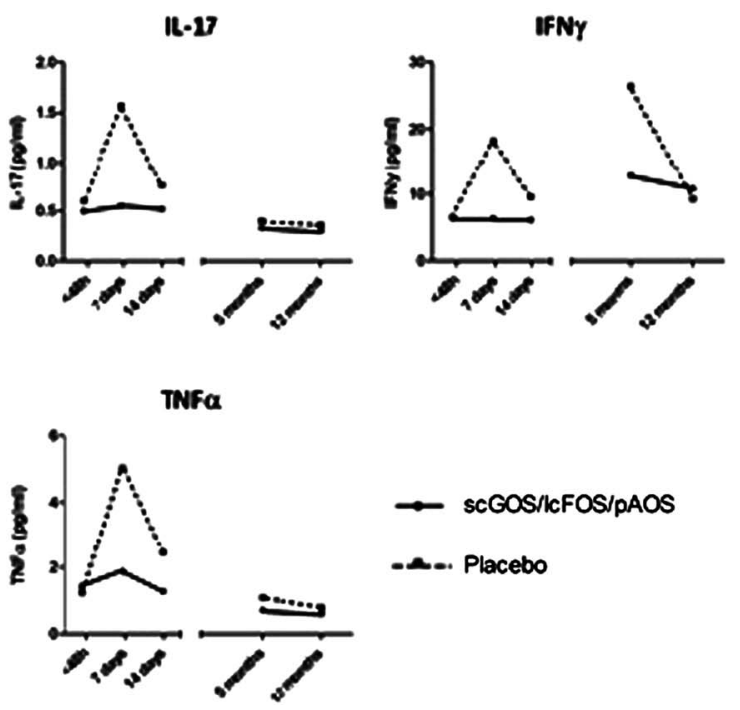

Conclusion: Cytokine levels increase during the neonatal period, followed by a decrease at 5 and 12 months for most cytokines. Enteral supplementation of neutral and acidic oligosaccharides (scGOS/lcFOS/pAOS) decreased proinflammatory cytokine levels in preterm infants at day 7 of life. 
Thereafter, this effect disappeared suggesting a temporary anti-inflammatory effect of scGOS/lcFOS/pAOS supplementation which may play a role in the development and programming of the immature immune system.

Disclosure of Interest: J. Van Den Berg: None Declared., N. van Zwieteren: None Declared., E. Westerbeek: None Declared., J. Garsen Employee of: Danone Research, R. van Elburg Employee of: Danone Research.

\section{DOHaD13-1426}

\section{Offspring of ovalbumin-sensitized mice supplemented with specific non-digestible oligosaccharides during pregnancy or lactation display attenuated allergic responses in a model for hen's egg food allergy}

Astrid Hogenkamp ${ }^{1, *}$, Betty van esch ${ }^{1}$, Elena Sandalova ${ }^{2}$, Leon Knippels ${ }^{2}$, Johan Garssen ${ }^{1}$

${ }^{1}$ Immunopharmacology, Utrecht University, Utrecht;

${ }^{2}$ Immunology, Danone Centre for Specialised Nutrition, Wageningen, Netherlands

Background: Non-digestible carbohydrates, resembling oligosaccharides present in human milk, have been shown to have beneficial effects in infants and young children. However, not much is known about their potential effects during pregnancy or lactation. Previous studies from our laboratory indicate that maternal supplementation with nondigestible carbohydrates may beneficially affect maternal and fetal immune status in mice. We therefore investigated whether supplementation during pregnancy or lactation could have long-term programming effects on the immune response in the offspring of both sensitized and non-sensitized dams.

Method: Female mice were sensitized orally once a week for 5 weeks with ovalbumin (OVA) using cholera toxin (CT) as adjuvant. Control groups received either PBS or PBS+CT. Two weeks after the last sensitization mice were mated and fed either a control diet or a specific non-digestible oligosaccharide mixture containing shortchain-Galacto-, longchain-Fructo- and Acidic-oligosaccharides (9:1:1) (scGOS/lcFOS/pAOS) during pregnancy or lactation. After weaning, female offspring were transferred to control diet and sensitized orally once a week for four weeks with OVA + CT. One week after the last sensitization, the offspring were challenged intradermally with OVA, and the acute allergic skin response, temperature and shock scores were measured after one hour. Mice were subsequently orally challenged with OVA, and euthanized the following day.

Results: Compared to offspring of OVA-sensitized mice fed the control diet throughout pregnancy and lactation, the acute allergic skin response in the offspring of scGOS/lcFOS/ pAOS supplemented OVA-sensitized dams was significantly reduced. This effect was observed for both the offspring of OVA-sensitized dams supplemented during pregnancy as well as lactation. In contrast to these results, supplementation did not significantly attenuate the acute allergic skin response in the offspring of dams who received PBS or PBS $+\mathrm{CT}$ prior to mating. Shock scores were significantly lower in offspring of dams supplemented during lactation compared to the offspring of non-supplemented dams or dams supplemented during pregnancy. Temperature drops were not observed in offspring of OVA-sensitized dams and offspring of nonsensitized dams supplemented during lactation. In offspring of OVA-sensitized dams supplemented during lactation, OVA-specific Ig-levels corresponded with these effects, but supplementation of OVA-sensitized dams during pregnancy does not appear to affect Ig-levels. In the offspring of non-sensitized dams similar effects can be observed, but the results are less clear.

Conclusion: A specific mixture of non-digestible carbohydrates significantly reduced the allergic skin response in the offspring of OVA-sensitized dams. Furthermore, shock scores and temperature changes were significantly different in offspring of dams supplemented during lactation. These results suggest that maternal supplementation with a specific mixture of oligosaccharides could have a beneficial programming effect on the immune response of offspring of sensitized mice.

Disclosure of Interest: None Declared.

\section{DOHaD13-1095}

\section{Role of maternal distress in the perinatal programming} of allergic disease

Meghan B. Azad ${ }^{1, *}$, Matthew E. Dahl ${ }^{2,3}$, Marni Brownell ${ }^{2,3}$, Mariette J. Chartier ${ }^{2,3}$, Nicole Letourneau ${ }^{4}$, Anita L. Kozyrskyj ${ }^{1}$

${ }^{1}$ Pediatrics, University of Alberta, Edmonton; ${ }^{2}$ Community Health Sciences, University of Manitoba; ${ }^{3}$ Manitoba Centre for Health Policy, Winnipeg; ${ }^{4}$ Nursing, University of Calgary, Calgary, Canada

Background: Psychosocial stress in the mother is increasingly being appreciated for its perinatal programming effects on childhood asthma and allergic disease. This evidence is derived from a limited number of studies in inner-city US families, burdened with high rates of asthma and allergic disease. Studies often evaluate prenatal or postnatal maternal distress in isolation, yet prenatal distress has a strong likelihood of continuation into the postpartum period. Both periods are critical for the formation of the respiratory and immune systems, which are established in utero and continue to develop during infancy. We undertook a study to determine collectively, the association between maternal distress in the pre and postnatal time periods, and the development of atopic (allergic) dermatitis and asthma in a general population of Canadian children.

Method: This was a database linkage study between the provincial postnatal public health nurse screening and health 
care utilization records of 12,587 children born in 2004. A measure of maternal distress (depression or anxiety) was implemented according to the time period of occurrence: pregnancy, recurrent from or after the postnatal period, or the postnatal period only. Expressed as an Odds Ratio[OR] and 95\% Confidence Level[CI], the association between maternal distress and childhood atopic dermatitis (AD) at age 5 and asthma at age 7 was determined from logistic regression, adjusting for known confounding factors, including fetal distress (documented fetal distress, or resuscitation, low Apgar score or respiratory symptoms at birth).

Results: Over $50 \%$ of mothers experienced distress at some time during their pregnancy or within 5 years of the birth of their child. Prenatal distress (14\%) was less common than postnatal distress (39\%), which was further classified as selflimiting (limited to the first year postpartum, 6\%), recurrent (occurring throughout the first 5 years, 12\%), or late-onset (originating after the first year postpartum and before the child's fifth birthday, 22\%). Independent of maternal parity, history of asthma or urban residence, preterm birth or low birth weight, and infant gender or antibiotic use, $\mathrm{AD}$ was 1.3 times more likely following prenatal maternal distress (95\%CI: 1.1-1.5). Recurrent or late-onset maternal distress increased the risk of $\mathrm{AD}$ or asthma by a similar magnitude. Maternal distress during pregnancy had an especially strong impact on asthma development $(\mathrm{OR}=1.6,95 \% \mathrm{CI}$ : 1.3-1.9). Asthma outcome models were additionally adjusted for fetal distress, which also increased the risk for asthma $(\mathrm{OR}=1.2,95 \% \mathrm{CI}: 1.01-1.35)$. Of note, self-limiting maternal distress during the postnatal period was not associated with $\mathrm{AD}$ or asthma in children.

Conclusion: Depression, anxiety or stress is common among new mothers in Canada. Originating during pregnancy or postnatally, maternal distress has the potential to affect fetal lung development and maturation of the infant immune system towards an allergic profile. Maternal prenatal distress appears to have a strong influence on asthma development that cannot be attributed to obvious signs of fetal distress.

Disclosure of Interest: None Declared.

\section{DOHaD13-1746}

\section{Role of respiratory viruses in early onset childhood rhinitis}

Antony Hardjojo ${ }^{1, *}$, Anne Goh' Oon Hoe Teoh ${ }^{2}$, Lynette P.-C. Shek ${ }^{1}$, Hugo P. S. Van Bever $^{1}$, Jian Yi Soh ${ }^{1}$, Thomas Biju ${ }^{2}$, Boon Huan Tan ${ }^{1}$, Yiong Huak Chan ${ }^{1}$, Mahesh Babu Ramamurthy ${ }^{1}$, Seang Mei Saw ${ }^{1}$, Kenneth Kwek ${ }^{2}$, Yap Seng Chong ${ }^{1}$, Keith M. Godfrey, ${ }^{3,4}$, Peter D. Gluckman ${ }^{5}$, Bee Wah Lee ${ }^{1}$
${ }^{1}$ National University of Singapore; ${ }^{2} K K$ Children's and Women's Hospital, Singapore, Singapore; ${ }^{3}$ Medical Research Council Lifecourse Epidemiology Unit; ${ }^{4}$ NIHR Southampton Biomedical Research Centre, University of Southampton and University Hospital Southampton NHS Foundation Trust, Southampton, United Kingdom; ${ }^{5}$ Singapore Institute for Clinical Sciences, Singapore, Singapore

Background: Rhinitis in infancy and early childhood is poorly understood as allergic rhinitis is considered to occur late in the atopic march. The role of respiratory viruses in development of rhinitis is also less studied compared to wheeze/asthma. We evaluated the role of respiratory viruses in the development and persistence of early childhood rhinitis. Method: Subjects ( $\mathrm{n}=1248$ at birth) in the Growing Up in Singapore Towards healthy Outcomes (GUSTO) birth cohort were screened by questionnaire for rhinitis (defined as runny, blocked nose or sneezing lasting 2 or more weeks) at 7 scheduled visits at 3 weeks, 3, 6, 9, 12, 15, and 18 months of age. Nasal swabs were taken during both scheduled and additional symptomatic visits for analysis by quantitative PCR for respiratory viruses. The viruses targeted were: Human Rhinovirus (HRV), Respiratory Syncytial Virus (RSV), Human Influenza A and B, Human Metapneumovirus (HMPV), Human Adenovirus, Human Bocavirus, Parainfluenza virus (PIV) 1-4, and Human coronavirus (OC43, HKU1, NL63, and 229E). From the cohort, 26 prolonged rhinitis (at least 4 weeks) cases and 26 healthy controls matched for month of birth and number of siblings had been analyzed.

Results: Evaluation of swabs obtained during scheduled visits till $12^{\text {th }}$ month of age showed that respiratory viruses were detected in $14.0 \%$ of the 222 nasal swabs analyzed. HRV was the most frequently detected (10.4\% of analyzed swabs). Other detected viruses were adenovirus, bocavirus, PIV3, and coronavirus. No significant difference in virus detection rate was observed between rhinitis $($ mean $=14.9 \%)$ and control groups $($ mean $=13.9 \%)(p=0.59)$. However, subjects who had upper respiratory infection symptoms during scheduled visit swabbing, irrespective of cases or controls, had significantly $(\mathrm{p}<0.05)$ higher detection rates for any virus, HRV, and coronavirus. Respiratory viruses were detected in $21.8 \%$ of 119 additional swabs taken during clinic visits by rhinitis subjects, HRV being the most frequently detected ( $12.6 \%$ of samples). PIV 1 and 2 were not detected in any of the samples.

Conclusion: In conclusion, young infants with rhinitis have a common pattern of respiratory viruses isolated from nasal swabs, HRV being the most common. Prolonged rhinitis symptoms did not appear to contribute towards higher rates of persistence of virus carriage in the subjects. Analysis of larger sample size will be conducted to substantiate this observation.

Disclosure of Interest: None Declared. 


\section{DOHaD13-1358}

Stimulated cord blood responses from preterm infants are skewed towards a pro-inflammatory profile following pathogen exposure

Nicolette Hodyl ${ }^{1, *}$, Natalie Aboustate ${ }^{1}$, Vicki Clifton ${ }^{1}$, Michael Stark ${ }^{1}$

${ }^{1}$ Obstetrics and Gynaecology, School of Paediatrics and Reproductive Health, UNIVERSITY OF ADELAIDE, North Adelaide, Australia

Background: Preterm neonates are at a significantly higher risk of adverse short and long term health outcomes compared to their term born counterparts. This is evident in the high rates of neonatal morbidities observed in preterm neonates, with $75 \%$ of these conditions characterized by inflammation. Preterm males are over-represented in these statistics. Contributory factors predisposing preterm neonates to these morbidities are poorly defined, but may relate to an immaturity of the innate pathogen recognition system. The Toll-like receptors (TLRs) are a critical component of the innate immune system that recognize viral and bacterial products. Following TLR binding, an intracellular signaling cascade is activated, resulting in the release of cytokines and induction of an inflammatory state. This system may be additionally compromised in preterm neonates by the administration of antenatal betamethasone therapy, which is routinely administered to women in preterm labor to accelerate fetal lung maturation. Betamethasone, however is a potent immunosuppressive and therefore may result in an inhibited pro-inflammatory response. This study aimed to determine cytokine responses of term and preterm cord blood, following activation of three different Toll-Like Receptors (TLRs): TLR2 by peptidoglycan (PGN), TLR3 by Poly I:C, and TLR4 by lipopolysaccharide (LPS) exposure. The potential impact of prenatal betamethasone exposure and infant sex on cord blood responses was assessed.

Method: Cord blood was collected immediately following delivery from preterm $(n=18)$ and term $(n=23)$ neonates. Blood was cultured, either alone or with PGN $(1 \mu \mathrm{g})$, Poly I:C $(5 \mu \mathrm{g})$ or LPS $(10 \mu \mathrm{g})$ for 2, 6 and 24 hours, and cytokine levels were measured by multiplex ELISA. Clinical data was collected at time of birth from maternal and neonatal health records. Data were analysed according to gestational age, sex, mode of delivery and betamethasone exposure.

Results: Despite no differences in baseline cytokine production, preterm neonates produced a significantly greater proinflammatory response compared to term neonates following exposure to TLR2, TLR3 and TLR4 agonists $(p<0.05$ in each case). Importantly, no compensatory anti-inflammatory cytokine response was observed, with levels of IL10 and IL-1 ra equivalent in term and preterm cord blood responses to each TLR agonist. Prenatal betamethasone exposure was associated with an increase in interleukin-6 (IL-6) following TLR2, TLR3 and TLR4 exposure $(\mathrm{p}<0.05)$ compared to unexposed infants. Preterm males demonstrated increased IL8 production compared to preterm females $(p<0.05)$. Cytokine responses were not altered by mode of delivery.

Conclusion: Cord blood from preterm neonates, particularly males, produce an exaggerated pro-inflammatory cytokine response to pathogen exposure compared to term-born neonates. An inability to balance this response through the production of anti-inflammatory cytokines may leave the preterm neonate more prone to inflammatory conditions in the neonatal period than their term-born counterparts. The long term consequences of this early pro-inflammatory environment are yet to be determined, however given that both innate and adaptive immune responses are shaped by early life exposures, long term alterations to immune function may occur following preterm birth.

Disclosure of Interest: None Declared.

\section{DOHaD13-1328}

The effect of enteral supplementation of neutral and acidic oligosaccharides on the response to pneumococcal, DTaP and Hib vaccinations in preterm infants.

Jolice Van Den Berg ${ }^{1, *}$, Elisabeth Westerbeek ${ }^{1}$, Guy Berbers ${ }^{2}$, Fiona van der Klis ${ }^{2}$, Ruurd van Elburg ${ }^{3}$ ${ }^{1}$ pediatrics, VUmc, Amsterdam; ${ }^{2}$ Laboratory for Infectious Diseases and Screening, National Institute for Public Health and the Environment, Bilthoven; ${ }^{3}$ Danone Research, Centre for Specialised Nutrition, Wageningen, Netherlands

Background: The rising number of preterm births is a concern, as it has implications for long term health. Early nutrition may contribute to programming e.g. of the immune system later in life. We found a trend towards decreased incidence of serious infectious morbidity during the neonatal period after enteral supplementation of neutral and acidic oligosaccharides (scGOS/ lcFOS/pAOS) to preterm infants. Preterm infants follow the Dutch Immunization Programme, not corrected for gestational age. Aim of this study was to determine the long term effect on the immune system of scGOS/lcFOS/pAOS in the response to $\mathrm{DTaP}-\mathrm{Hib}$ vaccine and heptavalent pneumococcal conjugate vaccine (PCV7) in preterm infants.

Method: In a RCT, infants with a gestational age $<32$ weeks and/or birth weight $<1500 \mathrm{~g}$ received enteral supplementation of scGOS/lcFOS/pAOS or placebo (maltodextrin) between days 3-30 of life. Serum samples were taken at 5 and 12 months of age, after the $3^{\text {th }}$ and $4^{\text {th }}$ DTaP-Hib and PCV7 vaccination. Samples were analyzed by multiplex immune assay (MIA, Luminex). The serum samples at 5 months were compared with 41 infants from Pienter II, a Dutch population-based seroprevalence study, conducted in the same year as the study in preterm infants.

Results: In total, 88 preterm infants at 5 months and 85 preterm infants at 12 months were included. At 5 months, the 
response to Pneumococcal vaccinations was lower in the scGOS/ lcFOS/pAOS group. This effect was not related to GA, BW or cord blood antibody concentration. At 12 months, there were no significant differences between both groups for any of the vaccinations (table 1). At 5 months, protective antibody concentrations were shown in $64 \%$ of preterm infants for Hib, $85 \%$ for Pertussis toxin and $88 \%$ for all pneumococcal serotypes. At 12 months, protective antibody concentrations were shown in $73 \%$ for Hib, 95\% for Ptx and 93\% for all pneumococcal serotypes. All preterm infants showed protective antibody concentrations for Dtx and Ttx at 5 and 12 months.

Antibody concentrations of preterm infants at 5 months and 12 months of age

\begin{tabular}{llcccccc}
\hline & DTaP-Hib & Ptx & FHA & Prn & Dtx & Ttx & Hib \\
& & & & & & & \\
\hline \multirow{3}{*}{5 months } & scGOS/lcFOS/pAOS & 37 & 78 & $78+$ & 0.40 & 0.74 & 0.35 \\
& Placebo & 44 & 96 & $80^{*}$ & 0.57 & 0.99 & 0.63 \\
& Dutch population & 46 & 70 & $41^{*}+$ & 0.45 & 0.69 & 0.57 \\
& 12 months & 55 & 122 & 116 & 0.88 & 1.64 & 2.91 \\
& scGOS/lcFOS/pAOS & 66 & 119 & 106 & 1.11 & 1.79 & 2.55 \\
& Placebo & & & & & & \\
\hline
\end{tabular}

\begin{tabular}{|c|c|c|c|c|c|c|c|c|}
\hline & Pneumococcal serotype & 4 & $6 \mathrm{~B}$ & 14 & $9 \mathrm{~V}$ & $18 \mathrm{C}$ & $23 \mathrm{~F}$ & $19 \mathrm{~F}$ \\
\hline \multirow[t]{3}{*}{5 months } & scGOS/lcFOS/pAOS & $1.5^{*}$ & $0.3^{*}$ & $2.4^{*}$ & $1.2^{*}$ & $1.9^{*}$ & $0.8^{*}$ & $7.4^{*}$ \\
\hline & Placebo & $3.3^{*}+$ & $0.8^{*} \dagger$ & $4.5^{*}$ & $2.6^{*}$ & $3.2^{*}+$ & $1.9^{*}+$ & $14.6^{*} \dagger$ \\
\hline & Dutch population & $1.0+$ & $0.3+$ & 3.2 & 1.7 & $2.0 \dagger$ & $0.6+$ & $5.1 \dagger$ \\
\hline \multirow[t]{2}{*}{12 months } & scGOS/lcFOS/pAOS & 4.3 & 3.3 & 5.8 & 4.0 & 4.7 & 4.6 & 10.7 \\
\hline & Placebo & 4.6 & 5.4 & 8.1 & 5.3 & 5.4 & 7.4 & 11.5 \\
\hline
\end{tabular}

Ptx, FHA, Prn: IU/ml, Dtx, Ttx: EU/ml, Hib, pneumococci: $\mu \mathrm{g} / \mathrm{ml},{ }^{*} \mathrm{p}<0,01,+\mathrm{p}<0.05$

Conclusion: At 5 months, preterm infants in the scGOS/lcFOS/ pAOS group showed a lower antibody response to pneumococcal vaccinations, which was more comparable to the Dutch general population than the placebo group. There were no differences for the DTaP-Hib vaccination, except for higher Prn levels in both preterm groups compared to the Dutch control group.

After the booster vaccination at 12 months, there were no differences between the scGOS/lcFOS/pAOS and placebo group, but $27 \%$ of preterm infants did not reach protective concentrations for Hib. These results show a potential beneficial effect of early nutrition on the immune system in preterm infants.

Disclosure of Interest: None Declared.

\section{DOHaD13-1443}

\section{Acute dual effects of methyglyoxal on the secretion of insulin in isolated rat pancreas cells: implication for programming of the metabolism}

Ghada Elmhiri $^{1, *}$, Luiz F. Barella ${ }^{2}$, Paulo C. D. F. Mathias ${ }^{3}$, Sylvaine Camous ${ }^{4}$, Dler F. D. Mahmood ${ }^{5}$, Latifa Abdennebi-Najar ${ }^{1}$
${ }^{1}$ UPSP-EGEAL Institut Polytechnique LaSalle Beauvais, Beauvais, France; ${ }^{2}$ Laboratory of Secretion Cell Biology, Maringa; ${ }^{3}$ Laboratory of Secretion Cell Biology, Department of Cell Biology and Genetics, State University of Maringa', Maringá, Brazil; " INRA, UMR1198, Biologie du Développement et reproduction, Jouy en Josas; ${ }^{5}$ Unité de Recherche, UR-04. Vieillissement, Stress et Inflammation. Université Pierre et Marie Curie, Paris, France

Background: Chronic diseases, such as type 2 diabetes, are the leading cause of death worldwide. Evidences from observational and experimental studies links adverse exposures during gestation, particularly related to nutrition, to chronic disease susceptibility in adulthood. Advanced glycation end products (AGEs) are formed and accumulated by endogenous and exogenous mechanisms. They are thought to play a role in diabetes complications. Methylglyoxal (MG), a highly reactive metabolite of glucose, is a strong precursor for a number of known advanced glycation end products (AGE) involved in the resistance to insulin and diabetes.

Method: To study the acute effects of MG on insulin production and secretion, islets of Langerhans were isolated from adult rats and incubated in vitro with $\mathrm{MG}$, in the presence of different metabolic factors.

Results: The results showed different patterns of MG effects on insulin secretion at the concentration of $10 \mu \mathrm{M}$. In basal glucose secretion $(5.6 \mathrm{mM}), \mathrm{MG}$ induced significant increase $(\mathrm{p}<0.001)$ in insulin secretion as compared to untreated cells. By contrast, in high glucose concentrations $(8.3 \mathrm{mM}$ and $16.7 \mathrm{mM}$ ), MG inhibited insulin secretion significantly $(\mathrm{p}<0.01)$. Furthermore, in the presence of potassium, forskolin, and epinephrine, MG enhanced insulin secretion $(\mathrm{p}<0.05)$, while when it was incubated with acetylcholine and leucine, MG resulted in a decreased insulin secretion $(\mathrm{p}<0.01)$.

Conclusion: Based on these results, our preliminary data showed that MG seems to modulate the secretory activity of $\beta$-cells depending on its level of stimulation by other metabolic factors. These results provide insights into a dual acute effect of MG on pancreatic cell.

Disclosure of Interest: None Declared.

\section{DOHaD13-1190}

BAT to WAT - early programming obesity induces brown adipose tissue hypoactivity in rats.

Douglas Lopes Almeida $^{1, *}$, Kesia P. Rigo ${ }^{1}$, Gabriel S. Fabrício $^{1}$, Laize P. Tofolo ${ }^{1}$, Amanda B. Trombini ${ }^{1}$, Audrei Pavanello $^{1}$, Paulo Cezar D. F. Mathias ${ }^{1}$

${ }^{1}$ Laboratory of Secretion Cell Biology, Department of Cell Biology and Genetics, State University of Maringa' - Maringa'l PR, Brazil, Maringa, Brazil 
Background: Overfeeding in the early postnatal period leads human and animal to obesity. This profile is maintained in adult life, being a predictor to different deleterious health conditions such as diabetes and cardiovascular diseases. While white adipose tissue (WAT) acts storing energy contributing to obesity, brown adipose tissue (BAT) has an antiobesity potential due to its ability to expend energy through thermogenisis. BAT mitochondria uncoupling protein-1 (UCP1) dissipates energy from respiratory chain as heat. Rats bred in small litters (SL) present body weight gain and lower UCP1 expression in adult life. Although, the role of UCP1 in weight gain under thermoneutrality seems controversial in literature. Interscapular BAT (IBAT) is the main site of thermogenisis in rats. Furthermore changes in local temperature reflect its activity. We investigated whether the obesity programmed by early overfeeding is able to modify IBAT thermogenisis in adulthood.

Method: Pregnant Wistar rats were placed in individual cages until pups birth (day 0). At day 2 litters were adjusted to 9 pups in normal litters (NL) and 3 pups in SL groups. Just male rats were used. At day 21 pups were weaned and then housed in 3 rats per cage. At day 81 animals had a temperature transponder implanted underneath IBAT pad $(\mathrm{n}=20 \mathrm{NL}, 18 \mathrm{SL})$. IBAT temperature was measured at light and dark periods between day 87 and 90 . At day 91 animals were weighed and euthanized; IBAT, epididymal and peritoneal fat was collected and weighed; blood samples were collected for analysis of insulin, glucose and corticosterone concentration. Histological analysis of IBAT was made. Vagus and retroperitoneal sympathetic nerves activity was recorded in 90 days old rats. Animals were kept in acclimated room with 12-h dark/12-h light cycle, controlled temperatures $\left(22 \pm 2^{\circ} \mathrm{C}\right)$ and free access to water and chow. Data were submitted to Student's t-test and ANOVA two-way (factors: litter and period) to compare groups. $\mathrm{P}<0.05$ was considered statistically significant.

Results: Lower IBAT temperature was observed in SL rats compared to NL group during light $(\mathrm{p}<0.001)$ and dark $(\mathrm{p}<0.001)$ periods, both groups showed higher IBAT temperature in dark compared to light turn $(\mathrm{p}<0.001)$. IBAT of SL rats was heavier than NL group $(\mathrm{p}<0.001)$. Histological analysis showed that SL rats have lower lipids droplets, except in the periphery of tissue where larger lipids droplets were observed. SL Vagus nerve presented 2 fold higher activity than NL; no difference was observed in sympathetic nerve activity. SL rats was heavier in adulthood than NL $(\mathrm{p}<0.001)$, also presenting higher glycemia $(\mathrm{p}<0.05)$, insulinemia $(\mathrm{p}<0.05)$ and corticosteronemia $(\mathrm{p}<0.01)$.

Conclusion: The obesity programmed by overfeeding in early life changes IBAT composition on adulthood, developing characteristics similar to WAT and being less active in obese animals. Hypercorticosteronemia finding in SL rats could induce lower Dio2 protein in BAT, which impair local thyroid hormones action contributing to thermogenisis reduction. IBAT changes leads to local thermogenisis hypoactivity, which reduce energy expenditure and may contribute to obesity maintained in adult life.

Disclosure of Interest: None Declared.

\section{DOHaD13-1742}

Birth weight and child growth as risk factors for subclinical atherosclerosis in young adults: evidence from the butantá cohort, Brazil

Maria H. Valente ${ }^{1}$, Filumena M. D. S. Gomes ${ }^{1}$,

Ana Maria de Ulhôa Escobar ${ }^{1, *}$, Alexandra Brentani ${ }^{1}$, Isabela Bensenor ${ }^{2}$, Sandra J. Grisi ${ }^{1}$

${ }^{1}$ Pediatrics; ${ }^{2}$ Internal Medicine, Faculdade de Medicina Universidade de Sáo Paulo, São Paulo, Brazil

Background: Environmental adverse conditions during pregnancy and early life are associated with higher risk of cardiovascular disease and metabolic programming. This study aims identifying the relationship between birth weight, early life growth and subclinical atherosclerosis in young adults.

Method: In 2009, 88 young adults aged 20-31 years, followed since birth at the CSE primary care Unit, were enrolled. From medical records, birth weight gestational length, and growth during the first year of life were extracted. The adults were then interviewed and medically assessed. Information related to sociodemographic characteristics, anthropometric data, blood pressure measurements, metabolic profile, and evaluation of subclinical atherosclerosis by ultrasound and graphical methods were obtained and analyzed according to two birth weight cutoff points $(<2,500$ grams and $>3500$ grams $)$ and weight gain during the first year of life.

Results: Low birth weight, less than 2.500 grams (g), was negatively correlated with higher waist-hip ratio (WHR), (regression coefficient $(\mathrm{RC})=$ to $-0.323,95 \% \mathrm{CI}[-0.571$, $-0.075], \mathrm{p}<0.05)$, diastolic blood pressure $(\mathrm{RC}=-4.744$, $95 \%$ CI $[-9.017,-0.470], \mathrm{p}<0.05)$, reduced HDL cholesterol $(\mathrm{RC}=-0.272,95 \%$ CI $[-0.516,-0.029]$, $p<0.05$ ), and frequency of the intima-media thickness (IMT) of the left carotid (LC) above the $75^{\text {th }}$ percentile $(\mathrm{RC}=-0.242,95 \% \mathrm{CI}[-0.476,-0.008], \mathrm{p}<0.05)$. Birth weight $>=3.500 \mathrm{~g}$ was positively associated with BMI $(\mathrm{RC}=2.832,95 \% \mathrm{CI}[0.433,5.232], \mathrm{p}<0.05)$, bigger waist circumference $(\mathrm{RC}=0.284, \quad 95 \%$ CI $[0.054,0.513]$, $\mathrm{p}<0.05)$, higher WHR $(\mathrm{RC}=0.280,95 \%$ CI $[0.054$, $0.505, \mathrm{p}<0.05)$, fasting glucose $(\mathrm{RC}=3.808,95 \% \mathrm{CI}$ [0.558, 7.058], $\mathrm{p}<0.05)$, minimum subcutaneous adipose tissue (SAT) $\quad(\mathrm{RC}=4.354, \quad 95 \%$ CI $[0.821,7.888]$, $\mathrm{p}<0.05)$, maximum SAT (CR $=7.095,95 \%$ CI $[0.608$, 13.583], $\mathrm{p}<0.05)$, right lobe liver size $(\mathrm{RC}=6.896,95 \%$ CI $[1.946,11.847], \mathrm{p}<0.01)$. Weight gain during the first year of life was negatively correlated with mean frequency of 
IMT media of left carotid above the $75^{\text {th }}$ percentile $(\mathrm{RC}=-0.253,95 \% \mathrm{CI}[-0.487,-0.018, \mathrm{p}<0.05$, and the frequency of the IMT (left carotid + right carotid) $/ 2$ above the $75^{\text {th }}$ percentile $(\mathrm{RC}=-0.241,95 \%$ CI $[-0.442$, $-0.041], \mathrm{p}<0.05)$.

Conclusion: Adults with birth weight less than $2.500 \mathrm{~g}$. and heavier than $3.500 \mathrm{~g}$. and insufficient weight gain during the first year of life had different metabolic phenotypes, suggesting different adaptation pathways.

Disclosure of Interest: None Declared.

\section{DOHaD13-1072}

\section{Bone metabolism in obese rats programmed by early weaning}

Egberto G. Moura ${ }^{1, *}$, Ligia D. A. Maia ${ }^{1}$, Carmen C. PazosMoura $^{2}$, Elaine Oliveira ${ }^{1}$, Patricia C. Lisboa ${ }^{1}$

${ }^{1}$ Physiology, State University of Rio de Janeiro; ${ }^{2}$ Biophysic Institute, Federal University of Rio de Janeiro, Rio de Janeiro, Brazil

Background: It is supposed that obesity and osteoporosis have a common pathogenesis, especially because the development of bone and adipose tissue has common origins. Since early weaning (EW) decreases adipogenesis in the neonate, further programming for obesity and hyperleptinemia, we hypothesized that these changes in adipogenesis could affect bone metabolism. We reported before that depending on the type of EW (mechanical EW (MEW), in which dams were involved with a bandage interrupting suckling in the last 3 days of lactation or pharmachological EW (PEW), in which the mothers were bromocriptinetreated during the same period), pups leptin serum levels at weaning were different, lower in $\mathrm{MEW}^{1}$ and higher in $\mathrm{PEW}^{2}$ group, but the two groups presents hyperleptinemia and leptin resistance at adulthood ${ }^{3,4}$.

Method: We evaluated bone metabolism in 2 models of EW in adult rat offspring. Lactating rats were separated into 3 groups: control $(\mathrm{C}, \mathrm{n}=8)-$ dams whose pups ate milk throughout lactation; MEW $(\mathrm{n}=8)$ and PEW $(\mathrm{n}=8)-$ dams were bromocryptine-treated $(0.5 \mathrm{mg} /$ twice a day i.p.) 3 days before weaning. At different periods, the animals were subjected to dual-energy X-ray absorptiometry and bone tissue was also evaluated by computed tomography, microcomputed tomography and biomechanical tests. Data were analyzed by one-way ANOVA, followed by Newman-keuls post-test. The $25(\mathrm{OH}) \mathrm{D}$ and CTX-I data were analyzed by Kruskal-Wallis test, followed by Dunn's Multiple Comparison Test. All results showed here presents significance level of 0.05 or less.

Results: MEW and PEW groups had higher visceral fat mass. MEW and PEW presented higher total bone mineral density (BMD), total bone mineral content, spine BMD and bone area in PN150. In PN180, both groups also presented increase of these parameters and higher femur BMD and fourth lumbar vertebra (LV4) BMD, femoral head radiodensity and LV4 vertebral body radiodensity, trabecular number, stiffness and break load; and lower trabecular separation, maximal deformation and break deformation. Only MEW offspring presented higher bone volume fraction (13\%), trabecular thickness (6\%) and lower trabecular pattern factor (159\%). MEW and PEW groups had higher 25-hydroxivitamin D (97\% and 136\%, respectively), whereas parathyroid hormone was higher only in MEW group (33\%). Serum C-terminal cross-linked telopeptide of type I collagen was lower for both MEW and PEW groups (67\% in both groups).

Conclusion: Since both models program for obesity and increased bone mass, and leptin increases plasma vitamin D levels, probably leptin is the link between obesity and higher bone mass. The differences in bone parameters that seem to be more marked in MEW group may be related to different leptin imprinting of the two groups during lactation.

\section{References}

1. Lima et al., Horm Metab Res. 45(1):22-30, 2013.

2. Bonomo et al., Horm Metab Res. 37(4):220-5, 2005.

3. Bonomo et al., J Endocrinol. 192(2):339-44, 2007.

4. Lima et al., Br. J. Nutr 105(9):1405-13, 2011.

Disclosure of Interest: None Declared.

\section{DOHaD13-1665}

Comparative study of 1-C metabolites during methionine load test in young Indian and US women

Pallavi Hardikar ${ }^{1, *}$, Kejal Joshi Reddy ${ }^{1}$, Suyog Joshi ${ }^{1}$, Prachi Katre $^{2}$, Deepa Raut ${ }^{1}$, Himangi Lubree ${ }^{1}$, Anura Kurpad ${ }^{3}$, Satish Kalhan ${ }^{4}$, Chittaranjan Yajnik ${ }^{1}$

${ }^{1}$ Diabetes Unit, KEM Hospital Research Centre; ${ }^{2}$ Life Sciences, Persistent Systems Ltd, Pune; ${ }^{3}$ Department of Physiology,

Division of Nutrition, St John's Medical College, Bangalore, India; ${ }^{4}$ Department of Pathobiology, Lerner Research Institute (NC22) The Cleveland Clinic Foundation, Cleveland, Ohio, United States

Background: Indians are largely vegetarian (consume no or only small amount of animal origin foods) and manifest multiple deficiencies, including vitamin B12 and proteins. Folate status is relatively normal. We have demonstrated that these nutritional eficiencies are associated with disturbance in 1-C metabolism, like hyperhomocystenemia. We have also demonstrated significant associations between maternal nutrients affecting 1-C metabolism and fetal programming of adiposity, insulin resistance, and neurocognitive function. There is little information on other nutrients and metabolites in 1-C pathway in Indians. 
We have used methionine load test (MLT) to investigate and compare transmethylation and transulphuration pathways of homocysteine metabolism in well-nourished American and micronutrient deficient Indian women.

Method: Young women from Pune, India $(\mathrm{n}=10$, age $20.64 \pm 4.86)$ and Cleveland, US $(n=13$, age $27.30 \pm 3.27)$ were challenged with methionine load $(50 \mathrm{mg} / \mathrm{kg}$ body weight) in orange juice. Baseline and hourly samples were collected for 5 hours, to measure vitamin B12, folate, and vitamin B6 status in the baseline sample and homocysteine, cysteine and glutathione in all the samples.

Results: Indian women were shorter (median 152.0 vs $171.2 \mathrm{~cm}$ ) and lighter $(52.6$ vs $71.8 \mathrm{Kg}$ ) but had similar BMI $\left(22.0\right.$ vs $23.3 \mathrm{Kg} / \mathrm{m}^{2}$ ) compared to the US women. Indian women had lower vitamin B12 (130 vs $308 \mathrm{pmol} / \mathrm{l}$ ), folate (19.5 vs $30.0 \mathrm{nmol} / \mathrm{l})$ and vitamin B6 (6.9 vs $19.3 \mu \mathrm{gm} / \mathrm{l})$ concentrations compared to the US women. Homocysteine concentrations were 2.5 times higher in Indian women (20.4 vs $7.9 \mu \mathrm{mol}$ ) and showed a rise upto $41 \mu \mathrm{mol}$ after methionine challenge. Cysteine (173 vs $234 \mu \mathrm{mol})$ and glutathione (3.3 vs $6.5 \mu \mathrm{mol})$ concentrations were lower in Indian women $(\mathrm{p}<0.05$ for both). After methionine challenge, cysteine remained lower in Indian women throughout the test while glutathione showed a rise upto the levels in the American women.

Conclusion: Indian women are deficient in vitamin B12, and have low folate and vitamin B6 status as compared to that in American women. This reflects in disturbances in both the transmethylation (higher homocysteine) and transsulfuration (lower cysteine and glutathione) pathways. This is the first demonstration of a combined nutritional-metabolic disturbance in 1-Carbon pathway in Indians. We will investigate relative contribution of disturbances in the two cycles through isotopic challenge test and their reversibility by nutrient supplementation studies.

Disclosure of Interest: None Declared.

\section{DOHaD13-1635}

\section{Correlational study on expression of IGF-2 and PGC-1A in IUGR rats}

Meihui Zhang ${ }^{1}$, Lihong Liao ${ }^{1}$, Xuemei Xie ${ }^{1}$, Xiaoping Luo ${ }^{1, *}$

${ }^{1}$ Pediatrics, Tongji Hospital, Tongji Medical College, Huazhong University of Science and Technology, Wuban, China

Background: To explore expression changes of insulin-like Growth factor-2(IGF-2), phos-phoinositide 3-kinase(PI3K), serine/threonine-protein kinases(AKT2), peroxisome proliferator-activated receptor- $\gamma$ coactivator- $1 \alpha(\mathrm{PGC}-1 \alpha)$ in liver and placenta tissues of intrauterine growth retardation rats with catch-up growth (CG-IUGR) for the purpose of elucidating potential mechanism of insulin resistance in CG-IUGR offspring.
Method: We established a CG-IUGR rats models with maternal nutritional restriction during the whole pregnant period and reducing the litter size of IUGR offspring after birth. The mRNA levels of IGF-2 in placenta and liver tissues were detected via Real-time PCR respectively and protein expression changes of PI3K, AKT2 and PGC-1 $\alpha$ were also evaluated by western blot. Besides, plasma was also isolated from IUGR and untreated SD rats of 8 weeks to detect triglyceride(TG)levels, which have a strong positive correlation with metabolic syndrome, and fatty degeneration was observed in liver tissues via $\mathrm{HE}$ and Oil red $\mathrm{O}$ staining.

Results: IGF2 mRNA expression of placenta, fetal liver,newborn liver and liver of 4 weeks dramatically declined in IUGR group compared with untreated group.The mRNA and protein expression of PI3K, AKT2 and PGC-1a was obviously lower in placenta, fetal liver and newborn liver tissues of IUGR than untreated SD rats $(P<0.05)$. The results above also applied to CG-IUGR and control groups of 8 weeks, however, the mRNA expressions of IGF-2 were not detected in both groups.TG level in plasma of IUGR group was obviously higher than untreated groups, and mild fatty degeneration was only observed in hepatocytes of IUGR group via Oil red staining, however, there was no difference in HE staining.

Conclusion: Decreases in transcriptional levels of IGF-2 of CG-IUGR rats might cause metabolic syndrome by downregulatingPGC-1a, and PGC-1a might be a downstream molecule of PI3K/AKT2 pathway.

Disclosure of Interest: None Declared.

\section{DOHaD13-1685}

\section{Detailed phenotyping of infant dried blood spot samples by metabolomics and lipidomics reveals extensive differences by age and nutrition}

Philippa Prentice ${ }^{1, *}$, Albert Koulman ${ }^{2}$, Lee Matthews ${ }^{2}$, Neil Dalton ${ }^{3}$, Charles Turner ${ }^{3}$, Marieke Schoemaker ${ }^{4}$, Eric vanTol ${ }^{4}$, Carlo Acerini ${ }^{1}$, Ken Ong ${ }^{1}$, David Dunger ${ }^{1}$

${ }^{1}$ University of Cambridge; ${ }^{2}$ MRC Human Nutrition Research, Cambridge; ${ }^{3}$ WellChild Laboratory, London, United Kingdom; ${ }^{4}$ Mead Johnson Nutrition, Nijmegen, Netherlands

Background: Links between early life growth and long-term health outcomes may in part be due to nutritional programming in infancy. Methodology is now available for detailed metabolic phenotyping, which may inform early programming mechanisms. However, there are no previous large-scale infancy metabolomic/lipidomic studies. We therefore set out to characterise the metabolomic and lipidomic phenotype in infancy, focusing on influences of early nutrition and age.

Method: Capillary dried blood spot (DBS) samples at ages 3 months ( $\mathrm{N}=292$ for metabolomics \& lipidomics) were 
collected from infants with detailed data collection including feeding practices. A pilot subgroup of DBS samples at age 12 months were also studied, from infants' exclusively breastfeeding $\geq 3$ months $(\mathrm{N}=80$ for metabolomics, 36 for lipidomics).

Metabolites were extracted from a $3.2 \mathrm{~mm}$ DBS and analysed by liquid-chromatography electrospray ionisation tandem mass spectrometry, to fully quantify 34 metabolites. A second DBS was used for novel lipidomic profiling of 95 lipid species, using high resolution mass spectrometer (bench top orbitrap) using chip based nanospray. Supervised multivariate statistics (OPLS-DA) were performed, using SIMCA-P v.13. Univariate analysis was performed, using SPSS and Excel, with Bonferroni correction for multiple comparison.

Results: Nutrition: At age 3 months, DBS metabolite and lipid profiles both differed significantly between exclusively breast-fed and formula-fed infants (fed a wide range of standard formulas). OPLS-DA could robustly predict class membership.

Breast-fed infants showed higher levels of free cholesterol, but also higher levels sphingomyelins (such as $\mathrm{SM}(34: 1)$ ) and polyunsaturated fatty acid containing phosphatidylcholines (PC(40:6)). Formula-fed infants had higher branched chain amino acids (BCAA), creatine, short chain acylcarnitines, polyunsaturated fatty acid triglycerides and linoleic acid containing phosphatidylcholines ( $\mathrm{PC}(34: 2)$ ). (All $\mathrm{p}$ values $<0.0003$ for lipids $/<0.001$ for metabolites).

Age: OPLS-DA shows clear separation of 12 month samples, from all 3 month samples. Older age in breast-fed infants (12 vs. 3 months) was associated with higher levels of BCAA and uric acid, but lower 3-0-methyldopa (30MD) and sialic acid. In the preliminary lipid analyses, older infants had strikingly lower sphingomyelin $(\mathrm{SM}(34: 1))$ and differences in specific cholesterol ester and phosphocholine groups (for example the palmitate vs. stearate fraction dramatically decreased in the first year of life).

Conclusion: A novel methodology is described, allowing detailed metabolic profiling of infants, using DBS samples and suggesting nutrition and age-related biochemical variations. There are differences between exclusively breast-fed and formula-fed infants at 3 months, in both metabolite and lipid classes. In addition there are large changes seen with infancy age in breast-fed infants; higher metabolites at 3 months such as $30 \mathrm{MD}$ and sphingomyelins, possibly reflecting the faster rate of neurological development during earlier infancy. Knowledge of differential metabolic patterns may help to further elucidate the early programming of later disease risk.

Disclosure of Interest: P. Prentice: None Declared., A. Koulman: None Declared., L. Matthews: None Declared., N. Dalton: None Declared., C. Turner: None Declared., M. Schoemaker Employee of: Mead Johnson Nutrition, E. vanTol Employee of: Mead Johnson Nutrition, C. Acerini: None Declared., K. Ong: None Declared., D. Dunger: None Declared.

\section{DOHaD13-1465}

Dietary supplementation with DHA in preterm neonates alters adipokine levels over the first four weeks of life

Michael Stark ${ }^{1, *}$, Nicolette Hodyl ${ }^{1}$

${ }^{1}$ Obstetrics and Gynaecology, School of Paediatrics and Reproductive Health, University of Adelaide, Adelaide, Australia

Background: Nutrition and growth during early fetal life and postnatal life are linked to health and disease in later life. Preterm infants account for over $80 \%$ of infants born with low birth weight (LBW) and are at significantly greater risk of developing late-onset diseases such as obesity, type 2 diabetes and hypertension. The adipokines leptin and adiponectin play central roles in glucose and lipid metabolism, energy homeostasis, inflammation and immunity and have been proposed to play an important role in the developmental origins of obesity and insulin resistance. Dietary long-chain polyunsaturated fatty acids (LCPUFAs) may have significant effects on developmental processes that affect both short and long-term outcomes related to growth, infant development and long-term health. Emerging evidence supports significant modulatory effects of the LCPUFA docosahexanoic acid (DHA) on circulating adipokines in child and adulthood. This study aimed to determine the effect of DHA supplementation on plasma leptin and adiponectin in LBW preterm neonates from initiation of oral milk feeds to 1 month of age.

Method: Infants $<33$ weeks gestation $(n=40)$ were randomised to receive placebo or one of three oral DHA supplements (DHA at 40,80 or $120 \mathrm{mg} / \mathrm{kg} /$ day) within 3 days of commencing enteral feeds. No infants received formula milk. Serial plasma samples were taken on days 0,7 , 14, 21, and 28 and plasma leptin and adiponectin determined by ELISA. Data were analysed by Pearson correlation and repeated measures ANOVA.

Results: No significant differences in gestational age, birth weight centile or proportion of small for gestational age infants were observed between the groups. Leptin and adiponectin levels were positively correlated on day 0 $(p<0.05)$ and did not differ at baseline between the groups. Birth weight positively correlated with both day 0 leptin $(p=0.005)$ and adiponectin $(p=0.003)$. A significant time and group interaction was observed with leptin decreasing $(\mathrm{p}=0.01)$ and adiponectin levels increasing $(p=0.03)$ over time in the mid- and high-DHA dose groups, temporal changes not observed in the placebo and low-dose DHA groups.

Conclusion: This study demonstrates that DHA supplementation of low birth weight preterm infants, at risk of developing late-onset programmed metabolic disorders, results in dose dependent decreases in leptin and increases in adiponectin. These effects on circulating adipokines may beneficially influence growth, body composition and development in postnatal life through known effects on glucose and lipid metabolism, energy homeostasis, inflammation and 
immunity. With leptin and adiponectin playing important roles in insulin resistance and obesity, the current data would suggest that intervention studies in preterm infants should examine dose-response relationships and elucidate not only the potential immediate but long-term benefits of LCPUFA supplementation.

Disclosure of Interest: None Declared.

\section{DOHaD13-1107}

Early life cardiovascular status, metabolic programming and growth in very young children

Kim Wijnands ${ }^{1, *}$, Sylvia Obermann-Borst ${ }^{1}$, Bas Heijmans ${ }^{2}$, Régine Steegers-Theunissen ${ }^{1,3}$

${ }^{1}$ Obstetrics and Gynecology, Erasmus MC, University Medical Center, Rotterdam; ${ }^{2}$ Molecular Epidemiology, Leiden University Medical Center, Leiden; ${ }^{3}$ Clinical Genetics, Erasmus MC, University Medical Center, Rotterdam, Netherlands

Background: Lipid abnormalities are an increasing problem in children and can persist into adulthood. Derangements during early postnatal life may induce stable epigenetic changes and alter metabolic programming. Our aim was to study whether blood total cholesterol, triglyceride, high-density lipoprotein (HDL) cholesterol and low-density lipoprotein (LDL) cholesterol concentrations in both mothers and very young children are associated with DNA methylation of LEP (leptin) and $T N F \alpha$ (tumor necrosis factor-alpha) in the child.

Method: In 120 healthy children, overall DNA methylation of $L E P$ and $T N F \alpha$ loci was measured in DNA derived from peripheral white blood cells. All children were recruited from the public child health care centers in the Rotterdam area in The Netherlands. At the standardized study moment and hospital visit at 17 months of age, we retrospectively collected questionnaire data of the periconception, prenatal and postnatal period and obtained venous blood samples from all children and their mothers.

Results: The overall mean DNA methylation levels of LEP and $T N F \alpha$ were $23.8 \%$ and $16.6 \%$, respectively. Total cholesterol levels in the children were associated with decreased methylation of $T N F \alpha,-7.5 \%(\mathrm{p}=0.036)$ and HDL-cholesterol levels were associated with decreased methylation of both $L E P,-13.2 \%(\mathrm{p}=0.021)$ and $T N F \alpha$, $-27.9 \%(\mathrm{p}=0.013)$. Additional adjustment for gestational age at birth, birth weight and gender showed comparable results $(L E P(-12.1 \% ; \mathrm{p}=0.031)$ and $T N F \alpha(-27.2$; $\mathrm{p}=0.017))$. Triglycerides and LDL-cholesterol were not significantly associated with DNA methylation.

In the mothers the HDL-cholesterol levels were associated with decreased methylation of $T N F \alpha$ in the child, $-26.1 \%$ $(\mathrm{p}=0.001)$. Total cholesterol, triglycerides and LDL-cholesterol levels in the mother were not associated with DNA methylation in the child.
Conclusion: Our data support the $\mathrm{DOHaD}$ hypothesis by showing that total cholesterol and HDL-cholesterol levels in very young children are associated with epigenetic changes, which may affect their cardiometabolic risk in later life.

Disclosure of Interest: None Declared.

\section{DOHaD13-1367}

Effects of adiponectin on gluconeogenesis via AMPK pathway in adult offspring rats exposed to intrauterine mild hyperglycemia

Kai Zhang ${ }^{1 * *}$, Jingmei $\mathrm{Ma}^{1}$, Huixia Yang $^{1}$

${ }^{1}$ OBGYN, Peking University 1st Hospital, Beijing, China

Background: To study the role of adiponectin on hepatic gluconeogenesis in adult offspring through a rat model suffered from intrauterine mild hyperglycemia and/or high fat diet in early life after birth.

Method: Streptozotocin (STZ, $25 \mathrm{mg} / \mathrm{kg}$ ) was given to rats of intrauterine hyperglycemia group by a single intraperitoneal injection to induce intrauterine mild hyperglycemia model (blood glucose between 10-20 mmol/L). Offsprings were divided into 4 groups according to intrauterine blood glucose level and dietary patterns (high fat/DF\&CF or normal/DN\&CN) after weaning to mimic the western lifestyle and at 28 weeks, metabolism syndrome were induced among hyperglycemia and high fat group (DF). The serum adiponectin was measured by ELISA; Real-time fluorescence quantitative was used to measure the adiponectin mRNA expression in visceral adipose tissue and the hepatic mRNA expression of AdipoR1, PEPCK, G-6-Pase and PGC- $1 \alpha$.

Results: At the age of 28 weeks, the serum adiponectin level of group DF was remarkably decreased than that of group CF $(P<0.05)$; Compared to group $\mathrm{CN}$, the adiponectin mRNA expression of group $\mathrm{DN}$, group $\mathrm{CF}$ and group $\mathrm{DF}$ were significantly decreased in perirenal fat $(P<0.05)$, the adiponectin mRNA expression of epididymal fat in group DF was also decreased $(P<0.05)$; The adiponectin mRNA expression of perirenal fat and epididymal fat in group DF were remarkably decreased compared with group DN and group CF $(P<0.05)$; 6 . At the age of 28 weeks, the hepatic AdipoR1mRNA expression in group DF were remarkably decreased compared with group $\mathrm{DN}$ and group $\mathrm{CF}$ $(P<$ O.05); The hepatic PEPCK, G-6-Pase, PGC-1 $\alpha$ mRNA levels of group DF were significantly elevated compared with other three groups $(P<0.05)$; The adiponectin mRNA expression and the AdipoR1mRNA expression were highly positive correlation $(P<0.05)$; The adiponectin mRNA levels and the hepatic PEPCK, G-6-Pase, PGC-1 $\alpha$ mRNA levels were highly negative correlation $(P<0.05)$. The hepatic PEPCK, G-6-Pase expression and the PGC- $1 \alpha$ mRNA expression were highly positive correlation $(P<0.05)$; Hepatic P-AMPK was located in the cytoplasm, the level of 
group DF was significantly decreased compared to other three groups $(P<0.05)$; Hepatic TRCT2 was expressed in the nucleus, the level of group DF was significantly increased compared with other three groups $(P<0.05)$.

Conclusion: The possible mechanism of adiponectin inhibits gluconeogenesis: Adiponectin + AdipoR1 $\rightarrow$ P-AMPK $\leftarrow \rightarrow$ CRTC2 $\downarrow \rightarrow$ PGC-1 $\alpha$, PEPCK, G-6-Pase $\downarrow$.

Disclosure of Interest: None Declared.

\section{DOHaD13-1202}

Effects of diet and voluntary maternal exercise during pregnancy on markers of glucose metabolism in skeletal muscles in rat offspring.

Mukesh Raipuria $^{1, *}$, Hasnah Bahari ${ }^{1}$, Margaret J. Morris ${ }^{1}$

${ }^{1}$ Pharmacology, University of New South Wales, Sydney, Australia, Sydney, Australia

Background: Obesity is increasing worldwide across all age groups and is associated with a range of adverse outcomes. Animal studies suggest a critical role for maternal phenotype and nutritional status during pregnancy in the development of obesity and metabolic disorders in offspring. Recent interest has turned to ways of limiting gestational weight gain to reduce the impact of maternal obesity.

Hypothesis: Voluntary exercise during pregnancy would limit excessive weight gain in pregnancy and ameliorate metabolic disorders in offspring of obese mothers.

Method: Female Sprague-Dawley rats were fed chow (C) or high fat $(\mathrm{F})$ diet and half of each underwent voluntary exercise (CE or FE) with a running wheel while the others remained sedentary (CS or FS). Pup body weight was monitored and male and female pups were killed at postnatal day (PND) 19 for further metabolic assessment.

Results: Both lean and obese mothers had similar modest levels of exercise $(8.1 \pm 2.4$ vs $5.1 \pm 1.5 \mathrm{~km})$, with no significant effect on body weight of mothers. The effects of maternal exercise on birth weight were sex and diet specific; At PND 1, lean exercised mothers had lighter male (CS $7.4 \pm 0.1$ vs CE $6.6 \pm 0.1 \mathrm{~g}$ ) and female pups (CS $6.8 \pm 0.1$ vs $\mathrm{CE} 6.2 \pm 0.1 \mathrm{~g}$ ), with no effect in those from obese mothers. As expected, at PND 19, maternal obesity increased offspring body weight and adiposity more than 2-fold without any significant impact of exercise. At PND19, male pups from obese exercised mothers had low blood glucose (FS $8.7 \pm 0.2$ vs FE $7.9 \pm 0.4 \mathrm{mmol} / \mathrm{l})$. Plasma leptin concentrations were significantly increased in pups of obese mothers $(\mathrm{P}<0.01)$, with no significant effect of exercise at PND 19. Insulin concentration was reduced in male pups from both lean and obese exercised mothers (CS $1.74 \pm 0.21$ vs $\mathrm{CE}$ $1.0 \pm 0.13$, FS $1.76 \pm 0.21$ vs FE $0.99 \pm 0.13 \mathrm{ng} / \mathrm{ml}$ ) with no significant effect of maternal diet on plasma insulin concentration. In females insulin concentration was three times higher in pups of obese mothers. Glut4 is an insulinregulated glucose transporter found in muscles. In the males, gastrocnemius muscle Glut4 mRNA expression was downregulated in pups from obese mothers (CS vs FS, P<0.01), while it was returned to normal level in pups from obese exercised mothers (FS vs FE, P < 0.05). Expression of MyoD, an important regulator of myogenesis, was decreased in male pups from obese mothers $(\mathrm{P}<0.05)$, suggesting downregulation of myogenesis.

Conclusion: In line with our previous work and other animal studies, maternal obesity was associated with higher body weight, adiposity, hyperleptinemia, hyperinsulinemia in rat offspring. In male offspring only, voluntary maternal exercise by obese mothers during pregnancy decreased blood glucose, plasma insulin and ameliorated glucose metabolism markers in skeletal muscles in rat's offspring. Thus sex-specific effects of maternal exercise were observed.

Disclosure of Interest: None Declared.

\section{DOHaD13-1280}

Effects of high-fat feeding during pregnancy and lactation on the behavioral indexes and metabolic changes, in rat's offspring

Celly Saba ${ }^{1, *}$, Gabrielle L. Gonzalez ${ }^{1}$, Aline S. Santos ${ }^{1}$, Sanderson Alves ${ }^{1}$, Karine Albuquerque ${ }^{1}$, Penha Barradas ${ }^{2}$

${ }^{1}$ Physiological Sciences; ${ }^{2}$ Pharmacology, State University of Rio de Janeiro, Rio de Janeiro, Brazil

Background: Obesity is a global health problem, a epidemic, that contributes to diabetes mellitus, hypertension and dementia and can results from changes in lifestyle, as increased intake of fats and carbohydrates and reduced physical activity (1). An increase in the prevalence of obese women of childbearing age is also a public health concern. Maternal obesity has been documented to play a direct role in the transmission of obesogenic and diabetogenic traits to the next generation. The obesity and overnutrition have also been shown to influence the nervous system of offspring $(2,3)$. A high-fat diet disrupts cognition and contributes to neurodegenerative diseases as well as metabolic diseases impairing hippocampal synaptic plasticity and cognitive abilities such as learning and memory (4). The aim of these study was investigated the effects of maternal diet containing 19\% soybean oil on metabolic parameters at different days of age (P10, P60 and P90) and behavioral indexes of offspring at 90 days of age. Method: Adult female Wistar rats were fed either a normal diet (ND, 4\% fat, $\mathrm{n}=6$ ) or a high-fat diet (HFD, 19\% fat, $\mathrm{n}=7$ ) during pregnancy and lactation. Mother and offspring body mass and food intake were monitored. Lipid profile and glucose were evaluated. Intra-abdominal adipose tissue was collected and weighted. At P60 body composition was analyzed by dual energy X-ray absortiometry (DEXA). From PN84 to PN90, male rats were submitted to the behavioral 
analysis based on the tests for determine anxiety (elevated plus-maze/EPM) and for learning and memory (radial arm water maze/RAWM)

Results: In pregnancy body mass was $234.2 \pm 10.91 \mathrm{~g}$ and at the end of the lactation rats feeding HFD increased body mass $26 \%$, without change on food consumption. During the postnatal period (1 to 27 day) no difference in the evolution of body mass was observed between the HF and ND groups, except for a significant increase (17\%) in group HFD at P28. At P90 the HFD group showed an increase $(6 \%)$ in the gain in body mass in regard to ND group. However, there is no difference on offspring food consumption.

In regard to intra-abdominal adipose tissue mass, no difference was observed between the groups at P10. However, HFD groups showed a significant increase in the mass at P60 $(23 \%)$ and at P90 (43\%). Body composition was determined by DEXA only for P60 showing an increase of $31 \%$ in fat mass and no change in lean mass in HFD rats. Serum evaluations showed a significant increase (20\%) in triglycerides concentrations at P10, P60 and P90 HFD groups. The ipGTT determined elevated glucose concentrations only at P60 HFD group in fasting and in all postprandial intervals analyzed. Concerning behavioral tests, the EPM showed HFD a more anxious group, meanwhile at RAWN no differences in learning and memory process were verified between the groups.

Conclusion: Maternal exposure to HFD during pregnancy and lactation has a direct effect on offspring phenotype. It can permanently alter metabolic and behavior parameters by promoting anxiogenic effect.

\section{References}

1. Park HR et al. Neuroscience Letters.2009, 482:235-239.

2. Samuelsson AM et al. Hypertension. 201055 (1):76-82.

3. Samuelsson AM et al. Hypertension. 2008. 51:383-392.

4. Tozuca $Y$ et al. Neurochemistry International. 2010, 57:235-247.

Disclosure of Interest: None Declared.

\section{DOHaD13-1366}

Epigenetic influences on long-term phenotype of offspring exposed to intrauterine hyperglycemia

Jie Yan ${ }^{1, *}$, Jingmei $\mathrm{Ma}^{1}$, Huixia Yang ${ }^{1}$

${ }^{1}$ OBGYN, Peking University 1st Hospital, Beijing, China

Background: Gestational Diabetes Mellitus (GDM) is a common medical complication in pregnancy. GDM will bring health issues for both mothers and offspring, not only the early complications, but also the long-term effects. Overnutrition in early life or high birth weight is reported to be associated with an increased susceptibility to metabolic disease. The underlying pathophysiology of GDM is still under discussion but insulin resistance is believed to play an important role. Changes in DNA methylation may be an early event in programming the metabolic profile in humans. The aim of this thesis was to illustrate the role of DNA methylation in the development of insulin resistance in metabolic diseases induced by intrauterine hyperglycemia exposure induced by streptozotocin in a rat model.

Method: Using a DNA methylation chip, we detected more than 3000 gene promoters were differentially methylated. Similar DNA methylation change was found for specific promoter sequences through bisulfite sequencing strategies.

Results: Inflammation is associated with insulin resistance. We show that Tumor necrosis factor (Tnf) is up-regulated, in visceral fat from $\mathrm{F} 1$ offspring of diabetic mother. The promoter region of $\operatorname{Tnf}$, a gene involved in inflammation, was differentially methylated in F1 offspring. Methylation level of $\operatorname{Tnf}$ was negatively correlated with mRNA expression. Furthermore, similar findings were revealed in F2 offspring indicating the intergenerational inheritance of methylation marks.

Conclusion: we identify the existence of DNA methylation changes in offspring exposed to intrauterine hyperglycemia and report a functional role in regulating gene associated with inflammation, which is of relevance to the pathogenesis of insulin resistance. We also provide evidence that DNA methylation is intergenerational inherited. Intrauterine environmental factors are potential triggers for changes in DNA methylation.

Disclosure of Interest: None Declared.

\section{DOHaD13-1720}

Excessive arginine intake by lactating rats induces visceral fat accumulation and insulin resistance in later life of offspring

Lila Otani ${ }^{1, *}$, Tomomi Mori ${ }^{1}$, Ayaka Koyama ${ }^{1}$, Shin-Ichiro Takahashi $^{1}$, Hisanori Kato ${ }^{1}$

${ }^{1}$ The university of Tokyo, Tokyo, Japan

Background: Rapid weight gain in early life is linked to the risk of obesity rand adverse metabolic health problems after maturation. Arginine is a semi-essential amino acid and known to be necessary for the optimal growth in young mammals. It has been reported that arginine supplementation in suckling piglets enhances their growth. Therefore we hypothesized that excessive maternal arginine intake during the suckling period may lead to increased susceptibility to obesity and insulin resistance in rat offspring. In addition, we investigated the effect of arginine supplementation on the composition of breast milk. Method: Wistar rat dams and their pups were divided randomly into control group $(\mathrm{CN})$ that fed on a normal diet ( $15 \%$ protein) and arginine group (ARG) that fed on the normal diet supplemented with $2 \%$ arginine. Breast milk was collected from dams of CN and ARG groups 2 weeks after delivery. After the termination of supplementation at 3 weeks of age, offspring from $\mathrm{CN}$ and $\mathrm{ARG}$ groups were fed the $15 \%$ 
protein diet until 6 weeks of age. Thereafter, the offspring of $\mathrm{CN}$ and ARG group was each subdivided into a normal diet $(\mathrm{CN}-\mathrm{CN}$ and ARG-CN) or high fat diet group (CN-HF and ARG-HF) at 6 weeks of age. Oral glucose tolerance test (OGTT) and body fat composition analysis were performed at 11 weeks of age. Mesenteric fat was collected from 12 wks old rats. Total RNA was extracted from mesenteric fat and then subjected to DNA microarray.

Results: In response to high fat diet feeding, abdominal adipose volume was significantly increased in ARG-HF group as compared to $\mathrm{CN}-\mathrm{HF}$ group, although no differences were observed between $\mathrm{CN}-\mathrm{CN}$ and ARG-CN group under the condition of feeding normal diet. In addition, blood glucose and insulin levels after glucose loading was significantly higher in ARG-HF group than that of CN-HF group. We have classified the genes that were differently expressed by using Ingenuity Pathway Analysis (IPA) and identified "Inflammatory Response" as the most significantly enriched set of bio functions in response to ARG in both $\mathrm{CN}$ and HF groups. $C x c l 2$ and $I L-6$ were commonly up-regulated in both the ARG-CN and ARG$\mathrm{HF}$ group as compared to $\mathrm{CN}$ group. We validated that there was a significantly higher expression of $I L-6$ in adipocytes of ARG-HF group using RT-PCR. There were no differences in hormonal profiles (IGF-1, leptin, corticosterone, adiponectin, and insulin) between $\mathrm{CN}$ and ARG group in breast milk, as well as the plasma of dams and pups. However, altered profiles of free amino acids in the breast milk were observed where the concentrations of Thr and Gly were lower in dams of ARG group than those of dams in $\mathrm{CN}$ group.

Conclusion: We demonstrated that offspring of dams supplemented with arginine during lactation acquired higher susceptibility to obesity, insulin resistance, and elevated inflammatory response when a high fat diet was given in later life. Interaction among these factors could lead to alternation of susceptibility to diet-induced metabolic disorders. In addition, arginine supplementation to dams lowered the supply of Thr and Gly to pups, and this may be also one of the causes contributing to the programming of lifelong risk of obesity in offspring. This work was supported by research and development projects for application in promoting new policy of agriculture, forestry and fisheries.

Disclosure of Interest: None Declared.

\section{DOHaD13-1118}

Extensive genome-wide gene expression changes in adipose tissue after 5 days high-fat overfeeding in healthy young men

Linn Gillberg ${ }^{1, *}$, Alexander Perfilyev ${ }^{2}$, Charlotte Brøns ${ }^{1}$, Tina Rönn ${ }^{2}$, Allan Vaag ${ }^{1}$, Charlotte Ling ${ }^{2}$

${ }^{1}$ Department of Endocrinology, Rigshospitalet, Copenhagen, Denmark; ${ }^{2}$ Department of Clinical Sciences, Lund University, Malmö; Sweden
Background: We have previously shown that a 5-day high-fat overfeeding (HFO) diet induced DNA promoter methylation changes in $45 \%$ of all genes in skeletal muscle from healthy young men. Subjects born with low birth weight (LBW) who have an increased risk of type 2 diabetes (T2D) seem however to be epigenetically inflexible in muscle in response to HFO. Adipose tissue is the main depot of excess energy and moreover an active endocrine organ influencing whole-body metabolism, insulin resistance and T2D development. We hypothesized that increased risk of T2D in LBW subjects may be explained by a different epigenetic and transcriptional response in subcutaneous adipose tissue (SAT) in response to HFO. Therefore, we investigated genome-wide gene expression and DNA methylation in SAT from 16 healthy LBW and 24 matched normal birth weight (NBW) men during control (30E\% fat) and HFO (60E\% fat, $+50 \%$ calories) conditions.

Method: Genome-wide gene expression was measured with Affymetrix Human Gene 1.0 ST arrays covering $>99 \%$ RefSeq genes and global DNA methylation was assessed with Illumina Infinium 450K BeadChips covering $>99 \%$ RefSeq genes and $>96 \%$ CpG islands.

Results: In vivo hepatic insulin resistance $(p<0.001)$, increased insulin $(p<0.01)$ and decreased FFA $(p<0.001)$ levels indicated glucose intolerance but efficient lipid storage in response to HFO in all men. The genome-wide mRNA expression in SAT from LBW men was not significantly different from NBW men after correction for multiple testing, and all 40 individuals were therefore analyzed together. 3890 corresponding to $12 \%$ of all transcripts on the array were differentially expressed in SAT after HFO $(q<0.05$, Benjamini Hochberg correction). Oxidative phosphorylation $\left(q=10^{-26}\right)$, citrate cycle $\left(q=10^{-10}\right)$ and biosynthesis of unsaturated fatty acids $\left(q=10^{-6}\right)$ were the top-three among twelve significant pathways of transcripts upregulated by HFO. $5 \%$ of the 64,920 analyzed methylation sites in and around the differentially expressed genes changed DNA methylation in SAT after HFO $(p<0.05)$, but these changes were not significant after correction for multiple testing.

Conclusion: $12 \%$ of all genes were up- or downregulated after 5 days HFO diet in SAT from healthy young men. The genomewide transcriptional profile and response to HFO was not significantly different in LBW compared to NBW men.

Disclosure of Interest: None Declared.

\section{DOHaD13-1427}

\section{Growing children with bovine metabolism: follow-up results from Russia.}

Alevtina Durmashkina ${ }^{1, *}$, Elena Lukushkina ${ }^{1}$, Olga Netrebenko ${ }^{2}$

${ }^{1}$ Pediatrics, Nizhny Novgorod State Medical Academy, Nizhny Novgorod; ${ }^{2}$ Moscow State Medical University, Moscow, Russian Federation 
Background: Number of recent studies examining the effects of early formula feeding revealed a significant effect on later obesity. However, there are no studies investigating a longterm impact of feeding whole cow milk on metabolism. Initial nutritional survey of a random sample of 436 mothers conducted in Nizhny Novgorod, Russia in 2001 had revealed a low prevalence of exclusive breastfeeding and high incidence of whole cow's milk feeding in infants. The objective of our follow up study was to evaluate consequences of whole cow's milk feeding in infancy on Body-Mass Index (BMI), blood pressure (BP) and insulin metabolism. We hypothesize that such feeding practice has long-term negative effect on metabolic health.

Method: Case-control cohort analysis of 79 children, aged 6 years (74.95 \pm 17.8 months), recruited from a clinical population $(\mathrm{n}=436)$ who had participated during infancy in the 2001 feeding practice survey. Participants were divided into 2 groups according to type of feeding in infancy: those breastfed for a minimum of 9 months (BF; $\mathrm{n}=36$ ), and those who had a high daily volume of cow's milk during the first year $(\mathrm{CM} ; \mathrm{n}=43)$. We measured BMI, BP, fasting and 2-hour glucose and insulin levels following an oral glucose load $(1.75 \mathrm{~g} / \mathrm{kg} ; 75 \mathrm{~g}$ max).

Results: There was a clear trend separating BMI between the groups, beginning at 6 months and persisting through the most recent measurement (6 years) at which time the difference had become 1.3 times higher $(\beta=1.697$, $\mathrm{p}=.003) . \mathrm{CM}$ children showed higher systolic and diastolic BP (99.58 vs $93.39 \mathrm{~mm} \mathrm{Hg}, \mathrm{p}<0.001 ; 68.23$ vs $63.67 \mathrm{~mm}$ $\mathrm{Hg}, \mathrm{p}=0.003)$, and a marginally significant upward trend in 2-hour insulin (18.5 vs $9.52 \mathrm{mcIU}, \mathrm{p}=0.049$ ). There was no significant difference in glucose level between the groups either in fasting or 2-hour levels. There was a significant correlation between BMI, BP and insulin level ( $\mathrm{p}=0.001-0.006)$.

Conclusion: These findings suggest that dietary patterns in infancy have immediate effects into toddlerhood and through age 6 years. Cow's milk feeding in infancy may predispose children to increased body mass, BP and insulin resistance.

Disclosure of Interest: None Declared.

\section{DOHaD13-1047}

Impact of short-term high-fat overfeeding on whole-body energy metabolism in young, healthy men born with a low birth weight.

Charlotte Brøns ${ }^{1, *}$, Søren K. Lilleøre ${ }^{2}$, Arne Astrup ${ }^{3}$, Allan Vaag ${ }^{4}$

${ }^{1}$ Dept. of Endocrinology, Rigshospitalet, Copenhagen;

${ }^{2}$ Novo Nordisk A/S, Søborg; ${ }^{3}$ University of Copenhagen;

${ }^{4}$ Rigshospitalet, Copenhagen, Denmark

Background: Low birth weight (LBW), which is a surrogate marker of disturbed fetal growth, is associated with an increased risk of developing type 2 diabetes (T2D). We recently showed that LBW subjects during a standardized diet exhibit increased nocturnal fat oxidation possibly associated with insufficient postprandial meal fat retention in subcutaneous adipose tissue. We hypothesized, that short-term, high fat overfeeding could further unmask key defects of whole body energy metabolism in men born with a low birth weight.

Method: Forty-six healthy lean young men matched for age and body mass index (BMI) were included in the study. All subjects were born at term (week 39-41). Twenty men had a birth weight $\leqslant 10^{\text {th }}$ percentile for gestational age $(2691 \pm 277 \mathrm{~g})$ and 26 had a normal birth weight (NBW) between the $50-90^{\text {th }}$ percentile $(3847 \pm 315 \mathrm{~g})$. The subjects were provided with a high-fat overfeeding diet (HFO) for 5 days including 60\% fat and $50 \%$ more calories than their individual energy requirements. The 24-h energy expenditure (EE), respiratory quotient (RQ) and substrate oxidation rates were assessed on the fourth day of overfeeding using whole body respiratory chambers. Baseline blood samples were drawn and a hyperinsulinemic euglycemic clamp was performed after 5 days HFO.

Results: When exposed to high fat overfeeding the LBW subjects had an increased 24-h $(P=0.04)$ as well as nighttime (23:00-08:00) $(P=0.01)$ EE compared to NBW control subjects after adjusting for differences in body composition. After HFO, the adjusted nighttime glucose oxidation rate was decreased $(P=0.05)$ whereas nighttime fat oxidation rate was increased in LBW men $(P=0.01)$ compared to NBW controls. Furthermore, the LBW men had a tendency towards increased fat oxidation $(P=0.07)$ during the whole 24-h period. Interestingly, when looking at the relative contribution of fat oxidation to the total EE, it was increased in the LBW subjects compared to the NBW controls both during the entire 24-h period ( $49.3 \pm 1.0$ vs. $52.8 \pm 1.6 \%, P=0.06)$ as well as during the nighttime ( $49.2 \pm 1.3$ vs. $54.1 \pm 1.7 \%, P=0.03)$.

Conclusion: In conclusion, our results indicate that a disproportionately elevated $24-\mathrm{h}$ as well as nighttime fat oxidation rate may contribute to the elevated risk of T2D in LBW subjects when exposed to a diet with excess fat and calories.

Disclosure of Interest: None Declared.

\section{DOHaD13-1340}

Influence of diet on the methylome of the male germline Thomas Chambers ${ }^{1, *}$, Amanda Drake ${ }^{2}$, Catherine Rose ${ }^{2}$, Sander van den Driesche ${ }^{1}$, Richard Sharpe ${ }^{1}$

${ }^{1}$ MRC Centre for Reproductive Health; ${ }^{2}$ BHF Centre for Cardiovascular Sciences, University of Edinburgh, Edinburgh, United Kingdom

Background: Environmental factors, for example diet, can influence the transgenerational inheritance of metabolic and reproductive health. The details of how this occurs are unclear, but cytosine methylation is one potential mechanism by which epigenetic information can be inherited. Establishment 
of cytosine methylation in male germ cells occurs during gestation, and is catalysed by the DNA methyltransferases $3 a$ and $3 b$ (DNMT3a and 3b). This methylation of DNA could be a point at which the epigenetic code is established for transmission to the next generation and is potentially vulnerable to influences via diet, lifestyle or chemical exposures. In pregnancy, these effects could occur in the fetus as a consequence of diet/exposures in the mother, and is perhaps most likely to occur during the period when germ cell demethylation and remethylation occurs. Recent evidence also suggests that a high fat diet, when fed to adult males can alter the sperm epigenome and lead to reproductive dysfunction in subsequent generations. Whether fetal germ cells are more vulnerable to diet-induced epigenetic modification than adult germ cells is unknown. The aim of the present studies was to address this by comparing the effect of feeding a high fat diet to pregnant rats or to adult male rats on the methylome of germ cells in the testis.

Method: Transgenic Sprague-Dawley rats expressing GFP in the germ line were fed a high fat (HFD) or control (CD) diet for 14 weeks. Females were fed up to and during pregnancy and males into adulthood and for sufficient time to cover one complete cycle of spermatogenesis. Immunoexpression of DNMT3a, 3b and 5-methyl cytosine was characterised in fetal germ cells in relation to loss of expression of Oct4 (as a result of methylation). Metabolic health was assessed by measuring fasting lipids and by oral glucose tolerance testing. Adult male sperm quality was assessed by counting total and motile epididymal sperm and by assessing sperm DNA damage using the TUNEL assay. The methylome of the germ cells from rats fed HFD or CD will be compared by MeDIP-seq.

Results: In the fetal testis, DNMT3a and 3b expression was present in developing fetal germ cells, with perhaps slightly increased expression between e17.5 and e21.5 (for Dnmt3a). Cytosine methylation was not detectable in germ cells at e17.5 but was present at e21.5. These changes were not obviously associated with loss of Oct 4 expression. HFD fed adult males demonstrated perturbed metabolic and reproductive health. Work regarding the impact of diet on the germline methylome is on-going and will be presented at the meeting.

Conclusion: The expression of the de novo DNA methyltransferases DNMT3a and $3 \mathrm{~b}$ in germ cells following the loss of pluripotency indicates that methylation patterns can be modulated in mature spermatogonia. This could be a means by which paternal environmental factors could induce transgenerational changes to health. Whether this is a direct effect of diet or secondary to hormonal changes in response to obesity is yet to be elucidated.

\section{Reference}

1. Palmer, N. O., Bakos, H. W., Fullston, T. \& Lane, M. Impact of obesity on male fertility, sperm function and molecular composition. Spermatogenesis 2, 253-263 (2012).

Disclosure of Interest: None Declared.

\section{DOHaD13-1406}

\section{Influence of grand-paternal obesity on F2 offspring metabolism}

Virginie Lecomte $^{1, *}$, Christopher A. Maloney ${ }^{1}$, Neil A. Youngson ${ }^{1}$, Margaret J. Morris ${ }^{1}$

${ }^{1}$ School of Medical Sciences, University of New South Wales, Sydney, Australia

Background: The global prevalence of overweight and obesity is rising alarmingly, with an estimated 55 to $65 \%$ of adults over the age of 20 overweight or obese in western countries. A strong contributor to this epidemic may be the transgenerational inheritance of metabolic disease.

Parental obesity is a major predictor of child obesity. While the impact of maternal obesity on offspring metabolism is well documented, strong evidence for a paternal obesity influence is just emerging. Our lab recently showed that female rat offspring from high fat diet (HFD) fed fathers developed glucose intolerance and defective insulin secretion (Ng et al. 2010). This demonstrated that metabolic defects from the father can be passed on to the F1 generation. Multigenerational human studies have revealed alterations to offspring, but also grand-offspring metabolism to be associated with the nutrition of the paternal grand-father (Kaati et al. 2002; 2007). In an attempt to model and understand these human observations, we extended the previous study by searching for abnormalities in the F2 generation from obese grandfathers.

Method: A cohort of obese and control F0 males (HFD $\mathrm{n}=8 /$ Con F0 $\mathrm{n}=8$ ) was established by feeding male Sprague Dawley rats either a control or a HFD from 3 weeks of age for 13 weeks prior to mating. These F0 males were then mated with control females. The resulting F1 males were fed a control diet then mated at 14 weeks of age with control females to generate F2.

The F2 males and females were fed either a control or a HFD until 14 weeks of age. The F2 rats underwent a glucose tolerance test (GTT) at 12 weeks of age and an insulin tolerance test (ITT) a week later.

Results: Grand-paternal obesity did not influence the metabolic profile of F2 offspring fed a control diet. However, when challenged with a HFD, F2 from HFD fed F0 grandfathers developed sex-specific metabolic defects. At 12 weeks of age, the F2 females from HFD F0 grand-fathers were glucose intolerant while the F2 females from control F0 presented a normal glucose clearance during the GTT. Insulin secretion during the GTT revealed that the F2 females from HFD F0 secreted significantly less insulin in response to the glucose injection. The ITT showed normal insulin sensitivity for these animals.

On the other hand, GTT of the HFD fed F2 males at 12 weeks did not show any significant difference. But ITT revealed that 13 week old HFD fed F2 males from HFD F0 were more insulin resistant than the F2 males from Con F0. 
Insulin secretion during the GTT confirmed this; the F2 males from HFD F0 secreted more insulin to achieve the same glucose clearance as the F2 from Con F0.

Conclusion: Grand-offspring rats from HFD fed grandfathers develop sex specific metabolic defects when they consume a HFD. On one hand, F2 females from HFD F0 developed glucose intolerance possibly due to a defect of insulin secretion in response to glucose. On the other hand, F2 males from HFD F0 presented peripheral insulin resistance compensated by an over-secretion of insulin in response to glucose. For both sexes, having an obese grandfather predisposed them to develop a poor metabolic phenotype when fed a HFD.

\section{References}

1. Ng SF et al. 2010. Chronic high-fat diet in father programs beta-cell dysfunction in female rat offspring. Nature 467:963-6.

2. Kaati $G$ et al. 2002. Cardiovascular and diabetes mortality determined by nutrition during parents' and grandparents' slow growth period. Eur. J. Hum. Genet. 10:682-8.

Disclosure of Interest: None Declared.

\section{DOHaD13-1648}

Interleukin 18, soluble tumour necrosis factor receptor-1 and adipokines may be more specific prognostic indicators of early life metabolic risk than systemic C-reactive protein

Rae-Chi Huang ${ }^{1,2, *}$, I-Jung Tsai ${ }^{1}$, Lawrence J. Beilin ${ }^{1}$, Patricia Price ${ }^{3}$, Sally Burrows ${ }^{1}$, Trevor A. Mori ${ }^{1}$

${ }^{1}$ School of Medicine and Pharmacology; ${ }^{2}$ Telethon Institute for Child Health Research; ${ }^{3}$ School of Pathology and Laboratory

Medicine, University of Western Australia, Perth, Australia

Background: Immune mediators such as interleukin-18 (IL18), soluble tumour necrosis factor receptors (TNFR)-1 and2 have previously been associated with adult atherosclerosis. Other immune mediators have previously been associated with the metabolic syndrome in adults ((interferon inducible protein-10 (IP-10)) and in children (C-reactive protein (CRP), leptin and adiponectin). Progression of cardiovascular disease (CVD) is silent and endpoints are not seen until middle age and older. Therefore, identification of surrogate biomarkers in adolescence may assist in predicting future CVD. Our aim was to identify inflammatory biomarkers that associate with metabolic risk in youth preceding CVD.

Method: This study was undertaken on 17 year olds in the Western Australian Pregnancy Cohort (Raine) Study. Immune mediators IL-18, IL-18 binding protein, TNFR-1 and TNFR-2, IP-10, high sensitive CRP, leptin and adiponectin were investigated as independent variables to predict the risk of metabolic syndrome in adolescents. Serum leptin and adiponectin, and plasma IL-18 and IL-18 binding protein were measured by ELISA. Plasma IP-10, sTNFR1 and sTNFR2 were quantified using Cytometric Bead Array. Two step cluster analysis incorporating systolic blood pressure, body mass index, fasting plasma triglycerides and HOMA-IR was used to define a "high metabolic risk" cluster. Forward selection logistic regression identified the model with greatest area under receiver operator characteristic (ROC) C-statistic.

Results: Of the 1027 adolescents, 17.5\% were identified with increased metabolic risk. Compared to the low risk, the high metabolic risk males had greater levels of IL-18 $(\mathrm{pg} / \mathrm{ml})$ (mean, 95\%CI) (287, 276-298 vs 309, 284-337), IL-18BP $(\mathrm{ng} / \mathrm{ml})(13.8,13.5-14.1$ vs $14.7,13.7-15.8)$, sTNFR1 (pg/ml) (354, 341-366 vs 397, 361-436), sTNFR2 (pg/ml) (3181, 3094-3271 vs 3556, 3318-3811) and IP10 (pg/ml) (106, 98-114 vs 138, 115-166). Compared to the low risk, the high metabolic risk females had greater levels of IL-18 $(\mathrm{pg} / \mathrm{ml})(280,269-291$ vs 309, 284-337), IL-18BP (ng/ml) (12.3, 12.0-12.6 vs 12.7, 12.1-13.4), sTNFR1 (pg/ml) (290, 276-305 vs 351, 321-383), sTNFR2 (pg/ml) (3037, 2940-3138 vs 3351, 3127-3591) and IP10 (pg/ml) (99.2, 90.5-108.6 vs 96.9, 81.4-115.4).

The model with the inflammatory components of IL-18, TNFR1, leptin and adiponectin (but not hsCRP) had an area under ROC C-statistic of 0.91. TNFR1 (OR = 1.6, 95\%CI 1.0-3.3), IL-18 (OR =6.7, 95\%CI 2.2-20.0) and serum leptin $(O R=25,95 \%$ CI 10-59) were associated with increased metabolic risk. Serum adiponectin was associated with decreased risk $(\mathrm{OR}=0.4,95 \%$ CI 0.3-0.6).

Conclusion: This study shows for the first time that IL-18 and TNFR-1 are independent markers for metabolic risk in healthy adolescents before overt atherosclerosis. These results demonstrate that markers of inflammation, specifically IL-18, TNF-R1 as well as leptin and adiponectin, may be useful for the early diagnosis and management of subclinical cardiovascular disease in youth.

Disclosure of Interest: None Declared.

\section{DOHaD13-1264}

\section{Maternal cholestasis during pregnancy programs metabolic disease in offspring}

Georgia Papacleovoulou ${ }^{1,2, *}$, Oscar Briz ${ }^{3}$, Shadi AbuHayyeh $^{4}$, Vanya Nikolova ${ }^{5}$, Xiao Huang ${ }^{6}$, Caroline Ovadia ${ }^{5}$, Maria Vaarasmaki ${ }^{7}$, Marc Baumann ${ }^{6}$, Eugene Jansen ${ }^{8}$, Christiane Albrecht ${ }^{6}$, Marjo-Riita Jarvelin ${ }^{2}$, Jose Marin ${ }^{3}$, AS Knisely ${ }^{5}$, Catherine Williamson ${ }^{1,2}$

${ }^{1}$ Division of Women's Health, Women's Health Academic Centre, King's College London; 'Imperial College London, London, United Kingdom; ${ }^{3}$ University of Salamanca, Salamanca, Spain; ${ }^{4}$ Women's Health; ${ }^{5}$ King's College London, London, United Kingdom; ${ }^{6}$ University of Bern, Bern, 
Switzerland; ${ }^{7}$ University of Oulu, Oulu, Finland; ${ }^{8}$ Public Health and the Environment, Bilthoven, Netherlands

Background: The intrauterine environment is a major contributor to increased rates of metabolic disease in adults. Intrahepatic cholestasis of pregnancy (ICP) is a liver disease of pregnancy that affects $0.5-2 \%$ pregnant women and is characterized by increased bile acid (BA) levels in the maternal serum. We hypothesized that exposure of the offspring to increased BA during intrauterine life can program metabolic disease in the offspring.

Method: To address our hypothesis, we performed anthropometric and metabolic profile analysis in 16-year old children of mothers with ICP that were recorded in the Northern Finland birth cohort 1985-86 (NFBC 1986) database. To further investigate potential programming effects, we developed a mouse cholestatic model and we assessed the phenotype of the offspring. We also performed lipid transport studies in a cholestatic gestational environment and we screened clinical ICP placentas and fetal cord serum samples to address whether metabolic programming effects originate in fetal life. In parallel, we used the agouti viable yellow (Avy) mouse model to address whether bile acids in gestation can affect the epigenome of the offspring.

Results: From the NFBC 1986 database, we identified 45 ICP pregnancies (27 male and 18 female offspring). Males had increased body mass index and females exhibited increased waist and hip girth compared to the offspring of uncomplicated pregnancies. Moreover, the male teenagers had increased levels of insulin and the female offspring decreased levels of HDL-cholesterol. In the mouse offspring, 18 -week old females from cholestatic mothers developed a severe obese, diabetic phenotype with hepatosteatosis following Western diet, whereas matched mice not exposed to cholestasis in utero did not. Female littermates were susceptible to metabolic disease before dietary challenge. Human and mouse studies in cholestatic placentas showed an accumulation of lipids, accompanied by increased transplacental cholesterol transport in the fetoplacental unit. Increased lipid levels were also observed in the fetal cord serum of cholestatic term pregnancies. In addition, in the Avy mouse model, we showed that increased BA levels in pregnancy trigger alterations in the epigenome of the offspring.

Conclusion: This is the first report showing that cholestatic pregnancy in the absence of altered maternal BMI or diabetes can program metabolic disease in offspring. This programming effect can be partially triggered by epigenetic alterations and/or increased transplacental cholesterol transport as a consequence of increased BA in pregnancy.

\section{Reference}

1. This work was financially supported by Genesis Research Trust and Wellcome Trust

Disclosure of Interest: None Declared.

\section{DOHaD13-1666}

Maternal high-fat diet alters expression and phosphorylation of key mammalian target of rapamycin signalling proteins in offspring livers in a mouse model of developmental origins of non-alcoholic fatty liver disease

Hugh Thomas ${ }^{1, *}$, Dawid Szczepankiewicz ${ }^{1}$, Aditi Jalali ${ }^{1}$, Kimberley D. Bruce ${ }^{1}$, Felino R. Cagampang ${ }^{1}$, Christopher D. Byrne ${ }^{1}$

${ }^{1}$ Institute of Developmental Sciences, University of Southampton Faculty of Medicine, Southampton, SO16 6YD, United Kingdom

Background: Non-alcoholic fatty liver disease (NAFLD) is a feature of metabolic syndrome and a major cause of chronic liver disease. Recent research has suggested that maternal obesity caused by a high fat (HF) diet during pregnancy increases offspring risk of NAFLD, exacerbates progression to non-alcoholic steatohepatitis (NASH) and impairs insulin sensitivity [1]. Of potential interest in NAFLD development is the role of mammalian target of Rapamycin (mTOR) signalling that is known to regulate hepatic lipogenesis and insulin signalling, which are key factors involved in the pathogenesis of NAFLD [2]. We tested the hypothesis that HF maternal diet during pregnancy influences expression levels and activation of key liver proteins involved in regulating $\mathrm{mTOR}$ and insulin signalling pathways in adult offspring.

Method: Female C57/BL6J mice were fed a high-fat (HF; $45 \% \mathrm{kcal}$ fat) or control chow (C; $7 \% \mathrm{kcal}$ fat) diet 6 weeks prior to mating, through pregnancy and lactation. Male offspring were fed either $\mathrm{C}$ or $\mathrm{HF}$ diet after weaning, creating four offspring groups; $\mathrm{C} / \mathrm{C}, \mathrm{C} / \mathrm{HF}, \mathrm{HF} / \mathrm{C}$ and $\mathrm{HF} / \mathrm{HF}$. At 15 weeks of age, a subset from each group ( $n=5-7$ per group) underwent a 2-hour fasting intra-peritoneal glucose tolerance test (IPGTT). Another set of offspring ( $\mathrm{n}=4-6$ per group) were killed and livers collected. NAFLD severity in fixed tissues was determined by histological Kleiner scoring. Liver protein levels was analysed by Western blot using antibodies that recognised proteins involved in insulin signalling, i.e. Akt and phospho-Akt (p-Akt T308 and S473), and in mTOR signalling, i.e. tuberous sclerosis 2 (TSC2) and phospho-TSC2 (p-TSC T1462). Data were analysed by ANOVA (significance at $\mathrm{p}<0.05$ ).

Results: Histological analysis showed that HF/C livers had some lipid droplets, while C/HF livers exhibited mild steatosis (mild NAFLD). HF/HF livers had extensive fat accumulation indicating a NASH-like condition. The areas under the curve (AUC) following IPGTT were greater in $\mathrm{C} / \mathrm{HF}, \mathrm{HF} / \mathrm{C}$ and HF/HF animals by 1.43-, 1.25-, and 1.66fold, respectively, vs. C/C (all at $\mathrm{p}<0.05$ ). Liver Akt protein levels were similar in all groups, but p-Akt $S 473$ and p-Akt T308 levels were reduced by 3.2- and 2.6-fold in C/HF, by 3.5- and 2.86-fold in HF/C, and by 2.3 and 1.6-fold in HF/ $\mathrm{HF}$, respectively, vs C/C (all at $\mathrm{p}<0.05$ ). Liver TSC2 level was unchanged in $\mathrm{C} / \mathrm{HF}$ but decreased by 2.15 - and 2.2 -fold in $\mathrm{HF} / \mathrm{C}$ and $\mathrm{HF} / \mathrm{HF}$ animals, respectively, vs. $\mathrm{C} / \mathrm{C}$ (all at 
$\mathrm{p}<0.01)$. Liver $\mathrm{p}$-TSC2 T1462 levels were unchanged in $\mathrm{C} / \mathrm{HF}$ but decreased by 1.97 - and 1.54-fold in $\mathrm{HF} / \mathrm{C}$ and $\mathrm{HF} / \mathrm{HF}$, respectively, vs. $\mathrm{C} / \mathrm{C}$ (both at $\mathrm{p}<0.01$ ).

Conclusion: A maternal HF diet markedly impairs offspring glucose tolerance and may cause insulin resistance and altered hepatic mTOR activity via decreased Akt activation (decreased T308 and S473 phosphorylation) and decreased total TSC2 levels, independent of the post-natal HF diet. These data suggest the insulin signalling-mTOR linked pathway may be involved in the developmental origins of NAFLD.

This work is supported by the BBSRC.

\section{References}

1. K. D. Bruce et al., Hepatology 50, 1696 (2009).

2. T. R. Peterson et al., Cell 146, 408 (2011).

Disclosure of Interest: None Declared.

\section{DOHaD13-1493}

\section{Maternal Omega 3 fatty acid supplementation to vitamin $B_{12}$ deficient diets improves markers of metabolic syndrome in dams}

Sadhana Joshi ${ }^{1}$ on behalf of Nutritional Medicine, Amrita Khaire ${ }^{1}$, Richa Rathod ${ }^{1}$, Anvita Kale ${ }^{1, *}$

\section{${ }^{1}$ Nutritional Medicine, Interactive Research School for Health} Affairs, Pune, India

Background: Several epidemiological and animal studies have suggested that there exists a close link between maternal nutrition and chronic metabolic disease in later life. The rise in non communicable diseases is alarming especially in populations with vitamin $\mathrm{B}_{12}$ deficiency who may also be omega 3 fatty acid deficient. Studies in our department have highlighted the importance of micronutrients and omega 3 fatty acids in determining pregnancy outcome. The present study analyzed the effect of maternal vitamin $\mathrm{B}_{12}$ deficiency and omega 3 fatty acid supplementation on markers of metabolic syndrome using Wistar albino rats.

Method: Pregnant dams were randomly assigned to three dietary treatment groups: control, vitamin $\mathrm{B}_{12}$ deficient group (BD), vitamin $\mathrm{B}_{12}$ deficient group supplemented with omega 3 fatty acid (BDO).

Results: Our results showed that dams who were vitamin $\mathrm{B}_{12}$ deficient had higher plasma homocysteine $(\mathrm{p}<0.01)$, insulin $(p<0.05)$ and lower DHA levels $(p<0.01)$ and showed a trend for lower levels of glucose. Omega 3 fatty acid supplementation to these dams normalized plasma homocysteine $(\mathrm{p}<0.01)$, insulin $(\mathrm{p}<0.05)$ and showed higher DHA levels $(\mathrm{p}<0.01)$.

Conclusion: In conclusion maternal vitamin $\mathrm{B}_{12}$ deficiency increased a few markers of metabolic syndrome and reduced DHA levels while omega 3 fatty acid supplementation ameliorated these effects. Our study highlights the importance of omega 3 fatty acids in reducing the biochemical markers of metabolic syndrome.

Disclosure of Interest: None Declared.

\section{DOHaD13-1327}

Maternal pre-pregnancy BMI, offspring's postnatal early weight gain and offspring's metabolic profile at age 5-6 years: the ABCD-study

Adriëtte Oostvogels ${ }^{1, *}$, Karien Stronks ${ }^{1}$, Manon Van Eijsden $^{2}$, Tanja Vrijkotte ${ }^{1}$

${ }^{1}$ Public Health, AMC; ${ }^{2}$ Epidemiology, Documentation and Health Promotion, Public Health Service, Amsterdam, Netherlands

Background: High maternal pre-pregnancy body mass index (pBMI) and offspring's postnatal accelerated growth might program the child for adverse metabolic outcomes. We assess the association between pBMI and offspring's metabolic profile at age 5-6 years and determine the role of offspring's postnatal weight gain between 1-3 months in this association (mediation or moderation).

Method: Data from the prospective ABCD study was used. We included mother-child pairs with known maternal pBMI (self-reported), offspring's postnatal weight gain between 1-3 months ( $\Delta$ SDS score) and offspring's metabolic components at 5-6 years (waist-to-height-ratio (WHtR), systolic and diastolic blood pressure (SBP/DBP), fasting glucose, triglycerides and HDL cholesterol values. Individual components were analysed continuously (z-scores) and combined as the sum of z-scores (continuous adverse metabolic profile) and as the presence of 3 or more adverse components (dichotomous adverse metabolic profile). Models were adjusted for characteristics of the mother (age, education, ethnicity, parity, hypertension and smoking during pregnancy) and the child (birth weight, pregnancy duration, sex and age at health check, sedentary behaviour and energy intake at 5 year, in addition also for height of the child at health check for SBP/DBP and the sum of z-scores). We tested for interaction between maternal pBMI and offspring's postnatal weight gain in the full models.

Results: We included 1550 mother-child pairs. In adjusted models, we found an independent association of higher maternal pBMI on higher offspring's WHtR $(\beta=0.028$, $\mathrm{p}<0.001)$, SBP $(\beta=0.020, \mathrm{p}<0.01)$, and higher sum score of the metabolic components $(\beta=0.072, p<0.001)$. After adding postnatal weight gain to the models, these effect sizes did not change to a relevant degree, which indicated no mediation. For postnatal weight gain we found an independent effect on WHtR $(\beta=0.152, \mathrm{p}<0.01)$, higher fasting glucose values $(\beta=0.112, \mathrm{p}<0.05)$ and higher sum score of the metabolic components $(\beta=0.394, \mathrm{p}<0.01)$. Furthermore an increased risk of offspring's adverse metabolic profile 
was found for every unit increase in maternal pBMI $(\mathrm{n}=54$; $\mathrm{OR}=1.086, \mathrm{p}<0.05$ ). Increased postnatal weight gain did not increase the risk on an adverse metabolic profile in the offspring. We found an interaction between maternal pBMI and postnatal weight gain in the association between maternal pBMI and SBP $(\mathrm{p}<0.05)$, which indicated moderation. Offspring of women with higher pBMI who had increased postnatal weight gain have higher systolic blood pressure compared to all other offspring.

Conclusion: Maternal pBMI and offspring's postnatal weight gain are both independently associated with some components of the metabolic profile. Offspring's postnatal weight gain does not mediate the association between maternal pBMI and offspring's metabolic profile. The combination of high maternal pBMI and accelerated offspring's postnatal weight gain was most detrimental for offspring's SBP. Awareness should be raised in women of childbearing age that a good pregnancy preparation starts with obtaining a healthy BMI to improve the metabolic profile in their offspring. Furthermore postnatal weight gain in the offspring should be carefully monitored and possibly controlled to further improve the metabolic outcome.

Disclosure of Interest: None Declared.

\section{DOHaD13-1215}

Maternal western diet primes non-alcoholic fatty liver disease in adult offspring in mice

Torsten Plosch ${ }^{1, *}$, Maurien G.M. Pruis ${ }^{2}$, Agnes Lendvai ${ }^{2}$, Vincent W. Bloks ${ }^{2}$, Mathijs V. Zwier ${ }^{2}$, Albert K. Groen ${ }^{2}$

${ }^{1}$ Obstetrics and Gynaecology; ${ }^{2}$ Pediatrics, University Medical Center Groningen, Groningen, Netherlands

Background: Metabolic programming via components of the maternal diet during gestation may play a role in the development of different aspects of the metabolic syndrome. Using a mouse model we aimed to characterise the role of maternal western type diet in the development of nonalcoholic fatty liver disease (NAFLD) in the offspring.

Method: Female mice were fed either a western (W) or lowfat control (L) semi-synthetic diet before and during gestation and lactation. At weaning, male offspring were assigned either the $\mathrm{W}$ or the $\mathrm{L}$ diet, generating four experimental groups: WW, WL, LW and LL offspring. Biochemical, histological and epigenetic indicators were investigated at 29 weeks of age. Results: Male offspring exposed to prenatal and post-weaning western-style diet (WW) showed hepatomegaly combined with accumulation of hepatic cholesterol and triglycerides. This accumulation was associated with up-regulation of de novo lipid synthesis, inflammation and dysregulation of lipid storage. Elevated hepatic transaminases and increased expression of Tnfa, $C d 11, M c p 1$ and Tgfb underpin the severity of liver injury. Histopathological analysis revealed the presence of advanced steatohepatitis in WW offspring. In addition, alterations in DNA methylation in key metabolic genes (Ppara, Insig, and Fasn) were detected.

Conclusion: Maternal dietary fat intake during early development programs susceptibility to liver disease in male offspring, mediated by disturbances in lipid metabolism and inflammatory response. Long lasting epigenetic changes may underlie this dysregulation.

Disclosure of Interest: None Declared.

\section{DOHaD13-1324}

Mental stress during pregnancy affects to the lipid metabolism mediated by activating the $11 \beta$-hydroxysteroid dehydrogenase type1 (11 $\beta$-HSD1) in the liver of the offspring mouse

Hiroki Maeyama ${ }^{1, *}$, Chie Obata $^{1}$, Kazuki Motizuki ${ }^{2}$, Takae Hirasawa ${ }^{1}$, Takeo Kubota ${ }^{1}$

${ }^{1}$ Epigenetic medicine; ${ }^{2}$ The Faculty of Life and Environmental Sciences, University of Yamanashi, chuo, Japan

Background: Nutritional and environmental condition in fetal period is known to cause obesity in the adulthood. It has been reported that mental stress during pregnancy induces adiposity in human fetuses. To evaluate the pathogenic mechanism of the obesity induced by mental stress during fetal period, we investigated whether restraint stress to the pregnant mothers causes the origin of adult diseases. In this study, we examined the expression of $11 \beta-H S D 1$, an enzyme that converts cortisol to cortisone, in the offspring of stressed mothers in order to clarify the adipose accumulation in the liver is caused by glucocorticoid increase due to up-regulation of expression of $11 \beta$-HSD1.

Method: Restraint stress in a $50-\mathrm{ml}$ tube for 3 hours/day from E8 to P0 was given to the pregnant mother mice. We analyzed protein and mRNA expression of $11 \beta-H S D 1,11 \beta-$ HSD2, peroxisome proliferator-activated receptor (PPAR), and glucocorticoid receptor (GR) using with the western blotting and real time PCR method. Lipid accumulation was detected in the liver tissue by oil red $\mathrm{O}$ staining after fixation and preparation with cryosection.

Results: As a result, protein and mRNA expression of PPARa and PPARg in the liver were increased in the offspring of the stressed mother on P37, compared with those of the non-stress mother. Lipid was predominantly observed in the liver in the offspring of the stressed mother on P37 by oil red O staining. Although there were no significant difference between the control and restrain mice in body weight changes, serum CORT and GR expression level in the liver, the protein expression of $11 \beta$-HSD1 increased greatly in the offspring of the stressed mother on P37, in spite of no change of the mRNA 11ß-HSD1.

Next, we examined whether these changes by the restrain stress started from fetal state. In the embryos of the stressed 
mother on E18, the expression of PPAR $\alpha$, PPAR $\gamma$ and GR was not changed compared with control group, but the $11 \beta$-HSD 1 mRNA expression was increased. To examine the difference between E18 and P37 is related to their diet change by weaning, we checked the weaning period mice (P21). In P21 mice, there were no change of expression of PPAR $\alpha$, PPAR $\gamma$, GR and $11 \beta-H S D 1$.

Conclusion: In this study, we showed that mental stress in the pregnancy mice leads the enhancement of the lipid accumulation and the increase of expression $11 \beta$-HSD 1 in the liver of offspring. These changes did not appear in the embryos and non-weaning mice. Therefore, these results suggest that the lipid metabolic change observed adult period is induced by not only environmental stress (i.e. mental stress via the mother) during fetal period but may be associated with nutrient factor after birth. Furthermore, the observed long-term up-regulation of $11 \beta$-HSD 1 starting from embryo may be caused by the epigenetic change of this gene. It is reported that $11 \beta-H S D 1$ is activated in type 2 diabetes patients. Therefore, these results indicated that environmental stress to fetuses via mental stress to the mother might be associated with development of type 2 diabetes and further suggested that prevention of the type 2 diabetes may be designed based on the attenuation $11 \beta$-HSD1 expression in the fetal period.

Disclosure of Interest: None Declared.

\section{DOHaD13-1242}

Mesenchymal stem cells derived from small for gestational age newborns exert properties promoting rapid catch-up growth and insulin resistance via early response Factor-1 and A COX-2-PGE2 feedback loop

Rami Sukarieh $^{1, *}$, Roy Joseph ${ }^{1}$, Shi Chi Leow ${ }^{1}$, Yhee Cheng Chng $^{1}$, Mona Löffler ${ }^{1}$, Jun Hao Tan ${ }^{1}$, Ai Ling Teh ${ }^{1}$, Joanna D. Holbrook ${ }^{1}$, Kai Lyn $\mathrm{Ng}^{2}$, Yap Seng Chong ${ }^{2}$, Peter D. Gluckman $^{1}$, Walter Stünkel ${ }^{1}$

${ }^{1}$ Growth, Development \& Metabolism Programme, Singapore Institute for Clinical Sciences, Agency for Science Technology and Research ( $\left.A^{*} S T A R\right),{ }^{2}$ Obstetrics \& Gynaecology, NUHS,

Singapore, Singapore

Background: Individuals exposed to an inadequate intrauterine environment are at risk to develop adverse health outcomes later in life. Those born with IUGR are insulin hyper-sensitive in infancy and evolve over childhood to become insulin resistant. We developed a cellular model system using human umbilical cords derived from normal or small for gestational age (SGA) neonates and identified physiological pathways associated with immediate insulin hypersensitivity.

Method: We isolated Wharton's jelly Mesenchymal Stem Cells (MSCs) from the umbilical cords of normal and SGA babies born at the National University Hospital (NUH), Singapore. We characterized these primary MSC lines and measured physiological and biochemical properties such as glucose uptake and AKT-phosphorylation, as well as cell proliferation rates. In order to identify global gene-expression pattern associated with these properties, we conducted comparative microarray experiments prior and post acute insulin stimulation.

Results: We observed that the metabolic physiology typical of SGA neonates are imprinted in Wharton's jelly derived MSCs such as enhanced insulin sensitivity - as evidenced by increased uptake of glucose, as well as enhanced AKTphosphorylation response. In basal, cycling MSCs from SGA babies, we identified Early Response Factor-1 (EGR-1) as a significantly under-expressed candidate gene compared to the control group. In contrast, upon acute insulin stimulation, EGR-1 expression was found to be reactivated concomitantly with an increase of Cyclooxygenase 2 (COX2). The under-expression of EGR-1 may explain the interesting finding that SGA MSC lines exert significantly higher basal cell proliferation rates compared to their normal counterparts. Additionally, enhanced COX2 expression is coupled with an increase in PGE2 production in SGA MSCs, which in turn further stimulates EGR-1, as well as COX-2 expression. We further demonstrate that ectopic overexpression of an EGR-1 vector in SGA derived MSCs inhibits cell proliferation, whereas RNAi-mediated EGR-1 knockdown in control MSCs stimulates cell growth.

Conclusion: Our findings indicate that umbilical cord derived mesenchymal stem cells retain physiological properties specific to intrauterine growth conditions. Molecular pathways involving EGR-1, and the COX2-PGE2 regulatory axis may play an important role in the biology of growth restricted individuals following an impaired intrauterine environment. Both, EGR-1 and COX2 have been linked to insulin resistance and therefore it is tempting to speculate that these genes are early mediators of catch-up growth associated with the development of insulin resistance in individuals born with SGA.

Disclosure of Interest: None Declared.

\section{DOHaD13-1725}

Micronutrients in pregnancy as a risk factor for gestational diabetes and effects on mother and baby: pride study

$\mathrm{N}_{\text {Sukumar }}{ }^{1, *}, \mathrm{H}_{\text {Venkataraman }}{ }^{1}$, A Antonysunil ${ }^{1}$, CS Yajnik ${ }^{2}$, C Fall ${ }^{5}$, P Saravanan ${ }^{1,3,4}$ and PRiDE study team

${ }^{1}$ Warwick Medical School, University of Warwick, Coventry, United Kingdom; ${ }^{2}$ Diabetes Centre, KEM Hospital, Pune, India; ${ }^{3}$ WISDEM centre, University Hospital, Coventry; ${ }^{4}$ Diabetes and Endocrinology, George Eliot Hospital, Nuneaton; 


\section{${ }^{5}$ MRC Lifecourse Epidemology Unit, Southampton, United Kingdom}

Background: GDM is described as glucose intolerance first recognised in pregnancy, with a prevalence of up to $17 \%$. Traditional risk factors such as obesity do not completely explain this risk. Recent research suggests that early foetal exposure to adverse maternal environment increases diabetes and obesity risk in later life, providing evidence for adverse foetal programming. Vitamin $B_{12}$ and folate, which are crucial for DNA methylation, could play an important part in this. Our recent work has shown that maternal vitamin $B_{12}$ insufficiency and hyper-homocysteinaemia, in combination with normal/high maternal folate status, is associated with an increased risk of GDM in the mothers, growth restriction in the foetus, adiposity and insulin resistance in the children.

The main aim of the study is to determine whether early pregnancy $\mathrm{B}_{12}$, folate and homocysteine levels independently predict the onset of GDM.

Method: This prospective observational case-control study will recruit 4500 women with GDM risk factors, in early pregnancy ( $<16$ weeks) across West Midlands, UK. All participants undergo a $75 \mathrm{~g}$ oral Glucose tolerance test (GTT) at 24-28 weeks and continue to get routine medical care throughout the study. Diagnosis of GDM is based on the modified WHO criteria (widely used in the UK; Fasting glucose $\geq 6 \mathrm{mmol} / \mathrm{l}$ or 2 -hr glucose $\geq 7.8 \mathrm{mmol} / \mathrm{l}$ ). All participants are seen at recruitment, GTT (24-28 weeks) and at delivery.

Demographic data, anthropometry, blood and urine samples are obtained from the participants at the study visits. At delivery, anthropometry of the baby, cord blood, placenta and cord tissue samples are also collected. Participants also complete wellbeing, quality of life, socio-economic, physical activity and psychological questionnaires at each visit.

Results: We present baseline data for the first 204 participants recruited since Sept 2012. This included 18.1\% Asians (SA) and 80.4\% White Caucasians (WC). Mean Age was $29.8 \pm 5.5$ years. Mean BMI was $31.1 \pm 6.8 \mathrm{Kg} / \mathrm{cm}^{2}$, (SA: $25.8 \pm 4.3 \&$ WC: $32.3 \pm 6.8 \mathrm{Kg} / \mathrm{cm}^{2}$ ). Mean abdominal circumference was $98 \pm 18.3 \mathrm{~cm}$ (SA: $83.6 \pm 14.2 \mathrm{~cm} \&$ WC: $101.3 \pm 17.6)$ The overall prevalence of GDM so far in our population of $18.3 \%$.

Conclusion: The proportion of GDM is according to our expectation, which may go up further when centres with higher South Asian population start recruitment. South Asians were lighter with lower abdominal circumference. PRiDE study will help to indentify whether early pregnancy nutritional biomarkers related to $1-\mathrm{C}$ metabolism predicts future GDM. The study will also have a biobank of samples including tissues which will be a useful repository for future research in both the mothers and offspring. The study is expected to complete by end of 2015 and early results may be available for $\mathrm{DOHaD} 2015$.

Disclosure of Interest: None Declared.

\section{DOHaD13-1196}

Muscarinic receptor subtypes composition in pancreatic islets from obese-programmed rats

Clarice Gravena $^{1, *}$, Aryane R. Agostinho ${ }^{1}$, Isis H. Trevenzolli ${ }^{2}$, Júlio C. de Oliveira ${ }^{1}$, Luiz F. Barella ${ }^{1}$, Claudinéia C. S. Franco ${ }^{1}$, Amanda B. Trombini ${ }^{1}$, Ananda Malta ${ }^{1}$, Rosana Torrezan ${ }^{3}$, Paulo C. F. Mathias ${ }^{1}$, Rosiane A. Miranda ${ }^{1}$

${ }^{I}$ Department of Biotechnology, Genetics and Cell Biology, State University of Maringá, Maringá; ${ }^{2}$ Institute of Biophysics Carlos Chagas Filho, Federal University of Rio de Janeiro, Rio de

Janeiro; ${ }^{3}$ Department of Physiological Sciences, State University of Maringá, Maringá, Brazil

Background: Impaired pancreatic $\beta$-cells function and insulin secretion is a link between obesity and type 2 diabetes. Regarding the obese humans and animals, it has been showed increased parasympathetic activity and hyperinsulinemia associated with insulin oversecretion, which can suggest that the composition of muscarinic acetylcholine receptors $(\mathrm{mAChR})$ in pancreatic islets may be compromised. Thus, the use of an experimental model that presents these features is essential for the characterization of $\mathrm{mAChR}$ in pancreatic $\beta$-cells of obese individuals. In fact, an experimental model that reconciles these features is the obese-programmed rats by neonatal administration of monosodium L-glutamate (MSG). This aminoacid in excessive concentrations have a neurotoxic effect crossing the blood brain barrier damaging cells in hypothalamic arcuate nucleus (ARC). Neurons from ARC are crucial to regulate metabolic homeostasis, which include insulin secretion as well as insulin tissue action, turns this model interesting for the investigation on the $\mathrm{mAChR}$ composition changes. We aimed to characterize the M1-M4 mAChR subtypes in pancreatic islets from pre-diabetic MSG-obese rats. Method: During the first five days of life, male pups received subcutaneous injections of MSG at a dose of $4 \mathrm{~g} / \mathrm{kg}$ body weight/day group (MSG). Control animals received equimolar saline solution. At 90-day-old, rats of both groups were used to biometric and biochemical evaluation. Anti-muscarinic drugs were used to study the mAChR function, either in vivo or in vitro. Superior vagus nerve branch electrical activity was recorded in both animal groups. Isolated pancreatic islets were used to $\mathrm{mAChR}$ characterization by western blotting and immunofluorescence staining.

Results: High vagus activity was observed in MSG-obese rats. Insulin oversecretion stimulated by glucose was observed in programed-obese rats, while, acetylcholine (Ach) induced low insulin release in vivo and in isolated pancreatic islets. Atropine, a non-selective muscarinic antagonist reduced the insulin secretion stimulated by ACh in MSG and control group; whereas, M2 mAChR selective antagonist increased cholinergic response. Moreover, insulinostatic effect of M3 mAChR selective antagonist was significantly high in MSG group. The M1 mAChR and M3 mAChR expression were increased in MSG-obese group by 55\% and 73\%, 
respectively $(p<0.05)$. Inversely, the expression of $\mathrm{M} 2$ $\mathrm{mAChR}$ decreased by $25 \%$ in MSG group ( $\mathrm{p}<0.05$ ), while it was unchanged to M4 mAChR. Immunofluorescence staining confirmed the changes observed by western blotting. Conclusion: Altered composition of the mAChR (M1-M4) subtypes from $\beta$-cell might be associated to insulin oversecretion displayed by MSG-obese rats; which can be directly related to vagal hyperactivity. This study might bring important pharmacological contributions to treatment of the increasing burden of metabolic syndrome that are important hallmark to type 2 diabetic condition and other metabolic disorders.

Disclosure of Interest: None Declared.

\section{DOHaD13-1722}

\section{Neonatal insulin resistance and endoplasmic reticulum stress markers in offspring of pregnancies with maternal obesity, subclinical and cellular perspectives}

Pablo J. Saez ${ }^{1}$, Francisco Westermeier ${ }^{1}$, Roberto Villalobos ${ }^{1}$, Francisco Mardones $^{2}$, Juan P. Kusanovic ${ }^{1}$, Jose A. Poblete ${ }^{1}$, Luis Sobrevia $^{3}$, Paola Casanello ${ }^{4}$, Marcelo Farias ${ }^{1, *}$

${ }^{1}$ Division of Obstetrics and Gynecology, Faculty of Medicine;

${ }^{2}$ Division of Public Health, Faculty of Medicine; ${ }^{3}$ Cellular and Molecular Physiology Laboratory (CMPL), Center of Medical Research (CIM), Faculty of Medicine; ${ }^{4}$ Perinatal Research Laboratory (PRL), Faculty of Medicine, Pontificia Universidad Catolica de Chile, Santiago, Chile

Background: Maternal obesity (MO) has been associated to increased insulin resistance at birth and long-term development of diabetes mellitus in the offspring. Since multiple signaling branches of endoplasmic reticulum (ER) stress pathway have been correlated with obesity-related IR at cellular level. Thus, we evaluated the effect of MO on IR and ER stress markers in newborn.

Method: Maternal nutritional status and gestational weight gain (GWG) was evaluated in 2112 pregnancies from PUC Chile Hospital. Neonatal plasma levels of insulin, glucose and $\mathrm{C}$-peptide were measured in $\mathrm{MO}$ and normal pregnancies samples. Primary cultures of human umbilical vein endothelial cells (HUVEC) were used to evaluate insulin response and ER stress in normal (HUVEC-N) or MO (HUVEC-OB) cells. Phosphorylated and total protein levels of IRS-1, Akt, p42/44MAPK, ATF6, IRE-1, PERK and eIF2- $\alpha$, were evaluated by Western blot. Immunofluorescence analysis was used to evaluate changes in intracellular distribution of ATF6 and ATF4. Ethic Committee approval and informed consent were obtained.

Results: An increased GWG, evaluated as body mass index variation $(\Delta \mathrm{BMI})$, was found in pregnant with $\mathrm{MO}$ at the end of gestation, compared to normal pregnancies $(5.8 \pm 0.008$ vs $4.9 \pm 0.06$, respectively, mean $\pm \mathrm{SE})$. As expected, an inverse correlation was observed between gestational BMI gain and pre-gestational BMI in normal pregnancies $\left(r^{2}=0.75\right)$, which was reduced in pregnancies with $\mathrm{MO}\left(\mathrm{r}^{2}=0.27\right)$. Similar plasma levels of insulin and glucose were found in both groups. Interestingly, C-peptide level was significantly increased in newborns from pregnancies with $\mathrm{MO}$, in relation to normal pregnancies. In HUVEC-OB we found a reduced and delayed Akt and p42/44MAPK phosphorylation after insulin exposure, compared to HUVEC-N. In addition, we found an increased phosphorylation of IRS-1 on the inactivating residue serine 307 in HUVEC-OB, compared to HUVEC-N. We observed no changes in activation of IRE-1, or in downstream XBP-1 mRNA splicing in both cell types. In HUVEC-OB we observed a slight increase in total levels of ATF6, which regulates another signaling branch of ER stress. Interestingly, this transcription factor showed an increased translocation to the nucleus in HUVEC-OB, compared to HUVEC-N. Finally, in HUVEC-OB we observed an increased phosphorylation of PERK and eIF2- $\alpha$, which remain low in HUVECN. ATF4 is also downstream of PERK branch, and we observed increased co-localization of ATF4 with ATF6 at nucleus of HUVEC-OB, but not HUVEC-N.

Conclusion: In this study we have shown subclinical and cellular evidence that MO promotes neonatal IR, probably through impairment of insulin signaling, which is associated with increased levels of C-peptide in cord blood. In addition, MO was related to activation of ATF6 and PERK, but not IRE-1, branches of ER stress in HUVEC. That profile is compatible with ER stress induced by inflammation, which might occur during MO.FONDECYT:1121145,1110977, 1090594,CONICYT ACT-73PIA.

Disclosure of Interest: None Declared.

\section{DOHaD13-1271}

Offspring of non-obese rats fed a high fat diet in pregnancy and suckling develop insulin resistance, cytokine mediated inflammation and mitochondrial dysfunction

Celine Latouche ${ }^{1}$, Sarah Haywood ${ }^{1}$, Sarah L. Henry ${ }^{2}$, Mark Ziemann ${ }^{1}$, Ross Lazarus ${ }^{1}$, Assam El Osta ${ }^{1}$, Bronwyn Kingwell ${ }^{1}$, James A. Armitage ${ }^{1,2,3, *}$

${ }^{1}$ Baker IDI Heart and Diabetes Institute, Melbourne; ${ }^{2}$ Anatomy and Developmental Biology, Monash University, Clayton; ${ }^{3}$ School of Medicine (Optometry), Deakin University, Waurn Ponds, Australia

Background: Maternal obesity during pregnancy and sucking can programme a metabolic-like syndrome in offspring. This study aimed to determine whether maternal consumption of a high fat, high sucrose diet per se (in the absence of maternal obesity) can have deleterious effects on offspring health and to probe the mechanisms underlying this programming. 
Method: Female Sprague Dawley rats were fed a high saturated fat high sucrose diet (HFD, 23\% lard, 20\% sucrose $\mathrm{w} / \mathrm{w} \mathrm{n}=5$ ) or normal fat diet (NFD, 6\% canola oil, $10 \%$ sucrose $w / w, n=6)$ for 3 weeks prior to mating, throughout gestation and suckling. At 21 days of age all animals were weaned to the NFD and fed ad libitum. At 12 months of age, male offspring were killed humanely and blood samples drawn for measurement of glucose and insulin and skeletal muscle (soleus) snap frozen. Next Generation Sequencing was used to identify differentially expressed genes and Gene Set Enrichment Analysis (GSEA) to detect coordinated changes that are characteristic of a biological function. Quantitative real time PCR and Western blotting were used to examine aspects of mitochondrial function and insulin signaling.

Results: Offspring of high fat/high sucrose-fed dams were not heavier than controls but demonstrated elevated plasma insulin concentration compared with control rats $(+40 \%$, $\mathrm{P}<0.05)$. GSEA indicated 15 pathways, which were significantly upregulated; including cytokine signaling $(\mathrm{P}<0.005)$, starch and sucrose metabolism $(\mathrm{P}<0.017)$, inflammatory response $(\mathrm{P}<0.024)$, cytokine-cytokine receptor interaction $(\mathrm{P}<0.037)$. A further 8 pathways were significantly down regulated; all were associated with mitochondrial function including oxidative phosphorylation $(\mathrm{P}<0.004)$, mitochondrial matrix $(\mathrm{P}<0.006)$ and electron transport/ uncoupling $(\mathrm{P}<0.022)$. Western blotting analysis indicated $\sim 60 \%$ reduction in phosphorylated Akt $(\mathrm{P}<0.05)$ and $\sim 70 \%$ reductions in mitochondrial complex II $(\mathrm{P}<0.05)$ and complex V $(\mathrm{P}<0.05)$ expression.

Conclusion: Offspring of non-obese high saturated fat/ sucrose fed rats do not become obese in adulthood when maintained on a normal diet, however they develop an insulin resistant phenotype, which is associated with changes in multiple pathways including cytokine activation and mitochondrial dysfunction. These data support moderation of saturated fat and sucrose intake during pregnancy (even in the absence of obesity) to prevent insulin resistance in offspring.

Disclosure of Interest: None Declared.

\section{DOHaD13-1598}

\section{Outcomes from a dietary 2nd hit: adolescent over nutrition in IUGR baboon offspring}

Laura Cox ${ }^{1, *}$, Robert E. Shade ${ }^{2}$, Kenneth Lange ${ }^{1}$, Shifra Birnbaum $^{1}$, Robert Baker ${ }^{2}$, Peter W. Nathanielsz ${ }^{3}$, Mark J. Nijland ${ }^{3}$

${ }^{1}$ Genetics; ${ }^{2}$ Southwest National Primate Research Center, Texas Biomedical Research Institute; ${ }^{3}$ Center for Pregnancy and Newborn Research, University of Texas Health Science Center, San Antonio, United States

Background: In previous studies we demonstrated that moderate global maternal nutrient reduction (MNR) from
0.16 gestation $(\mathrm{G})$ to $0.9 \mathrm{G}$ (term 184 days) in the baboon results in intrauterine growth restriction (IUGR). In addition, we found impaired fetal kidney nutrient sensing via the mTOR signaling cascade, down regulation of genes directing kidney branching morphogenesis, and decreased tubule length ${ }^{(1,2)}$. In addition, fetal liver showed, increased glycogen storage, and increased PEPCK ${ }^{(3,4)}$. The key questions arising from these studies are whether IUGR primate offspring (OFF) differ metabolically from control (CON) OFF and whether IUGR OFF differ in their response to overnutrition compared with CON OFF. We hypothesized that IUGR OFF show metabolic dysregulation for serum, morphometric, cardio-renal function and molecular genetic responses to a diet high in fat, cholesterol, simple carbohydrates and salt.

Method: At 3.5 years of age, IUGR $(n=6)$ and CON $(n=6)$ matched OFF were fed a high-fat, high-salt, high-simple carbohydrate diet (protein, 18\%, cholesterol, $0.1 \%$; lard, 18\%; Crisco, 4\%; Coconut Oil, 4\%; NaCI, 2.30\%; plus sugar drink (2.83 Kcal/g, $76 \%$ sugar, $41.8 \%$ fructose, $34.2 \%$ dextrose)) for 7 weeks. Liver, kidney, fat and skeletal muscle biopsies, blood samples, heart rate, blood pressure and morphometrics were collected before and after the challenge. Lipoproteins and markers of kidney function were quantified in serum. RNA was extracted from tissue and blood and analyzed by gene arrays.

Results: IUGR OFF weighed less than CON before the challenge but were the same at the end of the challenge. Gene expression and networks differed between CON and IUGR for all tissues. The greatest differences were seen in skeletal muscle with more than 1200 genes upregulated in CON OFF in response to the challenge with the majority of genes downregulated in IUGR OFF. In addition, genes involved with mitochondrial function were upregulated in CON OFF and downregulated in MNR OFF.

Conclusion: We found that MNR effects persist postnatally with the greatest impact on skeletal muscle mitochondrial gene abundance. These results indicate that moderate MNR during primate development programs IUGR OFF metabolically. These findings suggest that IUGR OFF are pre-diabetic when fed an energy-rich diet with dysregulation of energy management in skeletal muscle. In addition, skeletal muscle dysregulation precedes dysregulation of lipoprotein metabolism and blood pressure.

\section{References}

1. Cox LA, Nijland MJ, Gilbert JS, Schlabritz-Loutsevitch NE, Hubbard GB, McDonald TJ, Shade RE and Nathanielsz PW. J Physiol 572: 67-85, 2006.

2. Nijland MJ, Schlabritz-Loutsevitch NE, Hubbard GB, Nathanielsz PW and Cox LA. J Physiol 579: 643-56, 2007. 3. Li C, Schlabritz-Loutsevitch NE, Hubbard GB, Han V, Nygard K, Cox LA, McDonald TJ and Nathanielsz PW. Endocrinology 150: 4634-42, 2009.

4. Nijland MJ, Mitsuya K, Li C, Ford S, McDonald TJ, Nathanielsz PW and Cox LA. J Physiol 588: 1349-59, 2010.

Disclosure of Interest: None Declared. 


\section{DOHaD13-1640}

Paternal obesity impacts growth and skeletal muscle metabolism of male offspring

Chris Maloney $^{1, *}$, Virginie Lecomte ${ }^{1}$, Neil A. Youngson ${ }^{1}$, Margaret J. Morris ${ }^{1}$

${ }^{1}$ School of Medical Sciences, The University of New South Wales, Sydney, Australia

Background: The number of obese and overweight adults has reached pandemic proportions. There is clear evidence that perturbation of the maternal environment can impact the offspring's development and predispose them to metabolic disease in adult life. This may explain the relatively sudden increase in adults at risk of metabolic disease. Until recently the paternal contribution to the programming of metabolic disease has been thought to be minimal. Our group was one of the first to demonstrate that paternal obesity also confers metabolic changes to the offspring ( $\mathrm{Ng}$ et al, 2010) at 6 and 12 weeks of age. As the study by $\mathrm{Ng}$ et al, 2010 focused on females, the current study investigated metabolic alterations in adult male offspring.

Method: Obese $(n=8)$ and control groups $(n=8)$ of F0 males (HFD/Con F0) were established by feeding male Sprague Dawley rats either a control or a high fat diet (HFD) from 3 weeks of age for 13 weeks prior to mating. These F0 males were then mated with females maintained on a control diet. The resulting F1 offspring were weaned onto, and maintained on, a control diet. A group of male offspring from each litter was sacrificed at 6 months of age. A range of organs and tissues was collected (e.g. 2 skeletal muscles, 3 fat pads, liver). The tibialis anterior (TA) skeletal muscle was carefully dissected and removed from its capsule prior to freezing for later analysis. TA skeletal muscle triglycerides were measured. Gene expression in TA skeletal muscle was analysed using SABiosciences PCR arrays to assess the expression of genes involved in glucose and lipid metabolism ( $n=4$ per group). Results: The F1 offspring from obese fathers had a smaller birth weight. The male offspring from HFD F0 were smaller than the control group offspring from 9 weeks until 6 months of age. There was no indication of perturbed glucose or insulin metabolism. At 6 months of age the offspring from HFD fathers had smaller epididymal fat pads (HFD: $5.7 \mathrm{~g}$, $\mathrm{CON}$ : $7.2 \mathrm{~g} \mathrm{p}<0.02$ ), the skeletal muscle tended to be smaller with increased triglyceride content (HFD: $3.6 \mu \mathrm{g} / \mathrm{mg}$, CON: $5.2 \mu \mathrm{g} / \mathrm{mg} \mathrm{p}<0.02)$. Results of an 84 gene PCR array indicated that the skeletal muscle had increased expression of genes involved in lipid metabolism (e.g. Acaca, Fasn, FABP4), inflammation (e.g. Il1b, Il1r1, Nfkbia), and adipocyte like cell markers (e.g. Adipoq, Retn).

Conclusion: The male phenotype appears to be clearly different from that described in the females by $\mathrm{Ng}$ et al. 2010. F1 Male offspring from HFD fed fathers in this new study displayed mild intra-uterine growth restriction; early catch up growth followed by an earlier plateau of growth in adolescence.
This resulted in smaller animals that had less muscle and adipose tissue at 6 months of age. Gene expression in the muscle suggests perturbed lipid metabolism, increased inflammation and presence of adipocyte-like cells. Changes in growth and metabolism had no impact on glucose tolerance of the offspring at 6 months. As observed in other models of programming, (e.g. maternal low protein), investigation of late adult stages may uncover more direct metabolic consequences indicative of disease.

\section{References}

1. Ng SF et al. 2010. Chronic high-fat diet in father programs beta-cell dysfunction in female rat offspring. Nature 467: 963-6.

2. Fernandez-Twinn DS and Ozanne SE. 2010. Early life nutrition and metabolic programming. Ann N Y Acad Sci 1212: 78-96.

Disclosure of Interest: None Declared.

\section{DOHaD13-1586}

Perinatal fructose intake alters maternal, fetal and neonatal hepatic regulation of fatty acid metabolism

Zoe Clayton $^{1}$, Mark H. Vickers ${ }^{1,2}$, Angelica Bernal ${ }^{1,2}$, Deborah M. Sloboda ${ }^{3, *}$

${ }^{1}$ Liggins Institute, University of Auckland; ${ }^{2}$ Gravida: National Research Centre for Growth and Development, Auckland, New Zealand; ${ }^{3}$ Biochemistry and Biomedical Sciences, McMaster University, Hamilton, Canada

Background: We have previously reported that maternal fructose intake resulted in maternal metabolic compromise associated with sex specific changes in placental growth and fetal and neonatal metabolic development. In the present study, we therefore aimed to determine which regulatory pathways govern these changes in hepatic glucose and fatty acid metabolism and to determine whether they are associated with hepatic inflammation.

Method: Pregnant Wistar rats were randomized to either control (CON) or high-fructose (FR) diets. Fructose was provided in solution and comprised $20 \%$ of total caloric intake. Blood and liver samples were collected at embryonic day 21 (E21); postnatal day 2 (P2) and P10. Plasma metabolites were measured using an autoanalyzer and mRNA levels of key genes regulating fructose (fructokinase, GLUT5), glucose (PEPCK) and lipid metabolism as well as inflammatory processes (NfkB, NLRP3, IL1beta) were measured using qPCR. Results: At E21, FR mothers demonstrated significant suppression in hepatic lipase, ALT and AST levels $(p<0.05)$; an effect that was reversed at P10. These changes were associated with an increase in the fructose transporter (GLUT5 mRNA), a suppression in fructose metabolism (fructokinase mRNA) and an increase hepatic glycogen 
content $(\mathrm{p}<0.05)$. Similar changes were observed at P10. FR mothers also showed increased mRNA levels of SREBP1c and PPARa and suppressed IL1beta and NLRP3 levels. In offspring, FR exposure did not alter birth weight or neonatal growth but induced clear sex-specific changes at E21 and at P10. Hepatic GLUT5 mRNA levels were higher in FR female fetuses and fructokinase was suppressed in FR males and FR females $(\mathrm{p}<0.05)$ compared to CON. In P10 neonates, sexspecific changes in pathways regulating fatty acid transport, metabolism and beta oxidation were observed where the majority of genes investigated were reduced in FR males (ACAT, CRAT, FABP, LPL and Scl27a, p < 0.05) compared to CON. P10 females showed little or no changes in these enzymes. Preliminary data also show that FR exposure increased hepatic mRNA levels of IL1beta, NfkB and decreased NLRP3 (inflammasome) predominantly in male but not female FR neonates compared to CON.

Conclusion: The current study demonstrates that maternal fructose intake results in age- and sex-specific alterations in maternal, fetal and neonatal free fatty acid metabolism with associated changes in hepatic genes regulating key metabolic pathways. How these changes are associated with hepatic inflammatory processes is still unclear, although suppression of the hepatic inflammasome, at least in mothers and male neonates may point to impaired immune sensing. Further studies are needed to determine whether the observed sex differences elicit different risk profiles for metabolic disease into the post-weaning period.

\section{Reference}

1. Vickers, M.H., Clayton, Z.E., Yap, C. \& Sloboda, D.M. Maternal Fructose Intake during Pregnancy and Lactation Alters Placental Growth and Leads to Sex-Specific Changes in Fetal and Neonatal Endocrine Function. Endocrinology, en.2010-1093 (2011).

Disclosure of Interest: None Declared.

\section{DOHaD13-1532}

Placental restriction altered insulin actions and increased microRNA expression in insulin sensitive tissues of adult offspring in the rat

Himawan Harryanto ${ }^{1,2, *}$, Ezani M. Jamil ${ }^{1,2}$, Brooke L. Summers-Pearce ${ }^{1,2}$, Patricia A. Grant ${ }^{1,2}$, Miles J. De Blasio $^{1,3}$, Simon Moretta ${ }^{1,2}$, Wee-Ching Kong ${ }^{1,2}$, Kathy Gatford ${ }^{1,2}$, Marie Dziadek ${ }^{4}$, Mary Wlodek ${ }^{5}$, Julie A. Owens ${ }^{1,2}$

${ }^{1}$ The Robinson Institute; ${ }^{2}$ School of Paediatrics and Reproductive Health, The University of Adelaide, Adelaide, Australia; ${ }^{3}$ Department of Physiology, Development and Neuroscience, The University of Cambridge, Cambridge, United Kingdom; ${ }^{4}$ Garvan Institute of Medical Research, Sydney; ${ }^{5}$ Department of Physiology, The University of Melbourne, Melbourne, Australia
Background: Intrauterine growth restriction (IUGR) increases the risk of developing type 2 diabetes, in part through insulin resistance, which emerges after enhanced insulin sensitivity early in life. Why this occurs is unknown and we hypothesized that IUGR alters expression and actions of microRNA. MicroRNA is a small non-coding RNA that can co-ordinately regulate many molecules and pathways, in insulin sensitive tissues of offspring.

Method: Placental restriction (PR) and IUGR was induced in rats by bilateral uterine vessel ligation, at day 18 of pregnancy to restrict fetal growth. Insulin secretion and sensitivity assessed in vivo in adult offspring (at 3 and 6 months of age). Expression of selected insulin signalling and related molecules in liver, skeletal muscle and omental fat in older offspring was analysed by qRT-PCR. MicroRNA expression was analysed by Exiqon miRCURY arrays v11 and qRT-PCR. Predicted targets were identified by miRecords database then subjected to Ingenuity Pathway Analysis.

Results: PR induces insulin deficiency in young adult male offspring, which persists, with later onset in females. PR increases insulin sensitivity in young adult female offspring which also persists, with no effect in males. In older offspring, PR reduces hepatic expression of insr, p110b and Slc2a2 and skeletal muscle expression of insr and $p 110 b$ in males; and reduces hepatic expression of $p 110 \beta$ but increases that of $p 85 a$ in females, with no changes in skeletal muscle.

PR increased microRNA expression in insulin sensitive tissues in older offspring only (liver: miR-126, miR-199b; skeletal muscle: rno-451) and in fat of males only (miR-16, 18a, 19b, 20b, 21, 106a, 142-3p). Their predicted targets include insulin signallers and other molecules regulating metabolism, and functions including lipid metabolism, molecular transport and small molecule biochemistry.

Conclusion: PR alters expression of microRNAs in insulin sensitive tissues in older offspring, which may contribute to changes in insulin signalling, impaired lipid and metabolic control.

Disclosure of Interest: None Declared.

\section{DOHaD13-1175}

Preterm birth and its complications in sheep alter adult pancreatic beta-cell mass, beta- cell apoptosis and proliferation rates

Amita Bansal ${ }^{1,2, *}$, Jane M. Alsweiler ${ }^{1,2,3}$, Kristin L. Connor ${ }^{1,4}$, Mike Dragunow ${ }^{2,5}$, Jane E. Harding ${ }^{1}$, Frank H. Bloomfield ${ }^{1,2,3}$

${ }^{1}$ The Liggins Institute, University of Auckland; ${ }^{2}$ Gravida: National Centre for Growth \& Development, Gravida;

${ }^{3}$ Department of Paediatrics: Child \& Youth Health, University of Auckland, Auckland, New Zealand; ${ }^{4}$ Samuel Lunenfeld Research Institute, Mount Sinai Hospital, Toronto, Canada; ${ }^{5}$ Centre of Brain Research, University of Auckland, Auckland, New Zealand 
Background: The preterm birth rate worldwide is $11.1 \%$ of all live births. Babies born preterm have been shown to have impaired glucose tolerance in adulthood. We have demonstrated that sheep born preterm have reduced beta-cell mass in adulthood. However, the mechanisms underlying this are unknown. We hypothesized that (i) Sheep born preterm have altered beta-cell apoptosis and proliferation rates resulting in reduced beta-cell mass, and (ii) Hyperglycaemia, a common complication of preterm birth, and its treatment with insulin, further alter beta-cell apoptosis and proliferation rate in adulthood.

Method: Pancreata were collected at 12 months of age from sheep born at term (TermC; $148 \mathrm{~d}$ ) or preterm (corticosteroid induced, 137 d) ( $n=12-13$ per group). Preterm sheep were randomised to control (PremC), hyperglycaemic (HYPER; 50\% dextrose infused intravenously for $12 \mathrm{~d}$ to maintain blood glucose concentration (BGC) at $10-12 \mathrm{mmol} / \mathrm{L}$ ) or insulin-treated (INS; hyperglycaemia treated with insulin to achieve euglycaemia $(\mathrm{BGC}=4-6 \mathrm{mmol} / \mathrm{L}))$ groups. Immunofluorescent staining was performed on six $5 \mu \mathrm{m}$ sections, $100 \mu \mathrm{m}$ apart, for each animal to identify cell nuclei and insulin, Ki67 or active-Caspase 3 proteins. 12 images were taken from each slide and analysed by Metamorph automated image analysing software to determine proliferating and apoptotic beta-cells. Averages from each field of view were analysed by two-way ANOVA random effect model with Tukey's post-hoc test. Data are least square mean [SE].

Results: PremC had a lower percentage of proliferating betacells than TermC ( $\log _{10}$ values: TermC 0.67 [0.05], PremC 0.41 [0.08], HYPER 0.58 [0.06], INS 0.57 [0.05], overall effect $\mathrm{p}<0.05)$. HYPER had a higher percentage of apoptotic betacells than other groups (TermC 9.49 [1.18], PremC 16.55 [1.19], HYPER 21.63 [1.13], INS 12.28 [1.13], overall effect $\mathrm{p}<0.0001$ ). INS had a similar percentage of proliferating and apoptotic beta-cells to TermC.

Conclusion: In adult sheep born preterm, reduced pancreatic betacell mass was associated with a reduction in beta-cell proliferation. Neonatal hyperglycaemia increased beta-cell apoptosis which may further reduce beta-cell mass with advancing age. Treatment of hyperglycaemia with insulin restored beta-cell proliferation and apoptosis to control levels. Further investigation is required to determine if these changes are secondary to prematurity or related to antenatal corticosteroid use.

Disclosure of Interest: None Declared.

\section{DOHaD13-1240}

Pre-weaning growth hormone treatment rescues maternal undernutrition-induced meta-inflammation in adult male offspring

Clare M. Reynolds ${ }^{1, *}$, Minglan $\mathrm{Li}^{1}$, Clint Gray ${ }^{1}$, Mark H. Vickers ${ }^{1}$

${ }^{1}$ Liggins Institute, University of Auckland, Auckland, New Zealand

Background: Maternal undernutrition (UN) is associated with the development of obesity and metabolic complications in adult offspring. While it is clear that obesity gives rise to a state of chronic low-grade meta-inflammation, there is little evidence regarding the role of inflammatory processes in the adipose tissue of UN offspring. This study examines the effects growth hormone $(\mathrm{GH})$ treatment, during a critical period of developmental plasticity, on systemic, bone marrow and adipose tissue inflammatory processes in adult male offspring.

Method: Female Sprague-Dawley rats were randomly assigned to either a standard diet (C) or undernourished $(50 \%$ of ad libitum; UN) throughout gestation. At postnatal day 3, male $\mathrm{C}$ and UN pups received either saline (CS, UNS) or GH (2.5 $\mu \mathrm{g} /$ $\mathrm{g} / \mathrm{d})(\mathrm{CGH}, \mathrm{UNGH})$ by daily subcutaneous injection until day 21. Post-weaning offspring were fed either the standard diet or high-fat diet ad-libitum until postnatal day 150. Adipose explant insulin sensitivity was monitored by glucose uptake assay and cytokine expression and secretion by ELISA and RT-PCR. Primary adipocytes and stromal vascular fraction (SVF) cells were isolated following collagenase digestion of adipose tissue. Bone marrow hematopoietic cells were differentiated to a macrophage phenotype in the presence of macrophage colony stimulating factor (M-CSF; 50ng/ml). Differentiated bone marrow macrophages (BMM) were stimulated with lipopolysaccharide (LPS; $100 \mathrm{ng} / \mathrm{ml}$ ) for $6 \mathrm{~h}$.

Results: UN offspring displayed significant adipocyte hypertrophy compared to CS offspring which was corrected by early life GH treatment. An ex-vivo glucose uptake assay demonstrated that adipose tissue from UN offspring had attenuated insulin-stimulated glucose uptake accompanied by reduced adipose tissue glucose transporter type 4 (GLUT4) and insulin receptor substrate (IRS) 1 expression compared to $\mathrm{CS}, \mathrm{CGH}$ and UNGH. In addition to these metabolic alterations, UN offspring displayed a more potent immunophenotype with significant infiltration of adipose with proinflammatory M1 macrophages, enhanced cytokine secretion and expression from whole adipose tissue explants, adipose SVF, primary adipocytes and LPS-stimulated BMM when compared to CS, CGH and UNGH groups.

Conclusion: Early life exposure to maternal UN primes both adipose tissue and hematopoietic immune cells to a more potent pro-inflammatory phenotype with heightened cytokine secretion and receptor expression. This is accompanied by infiltration of pro-inflammatory M1 macrophages which may exacerbate adipose dysfunction. Pre-weaning GH treatment negates these detrimental effects indicating potential for reversing metabolic dysfunction and associated meta-inflammation in UN adult offspring.

Disclosure of Interest: None Declared.

\section{DOHaD13-1257}

Rapid infantile growth as a risk of adult fatty liver - a mouse animal model with intrauterine undernourishment

Keiko Muramatsu Kato ${ }^{1, *}$, Hiroaki Itoh ${ }^{1}$, Yukiko K Kohmura ${ }^{1}$, Kaori Yamazaki ${ }^{1}$, Kotomi Nagahashi ${ }^{1}$, Toshiaki 
Shibata ${ }^{1}$, Naomi Furuta ${ }^{1}$, Naoaki Tamura ${ }^{1}$, Chizuko Yaguchi ${ }^{1}$, Toshiyuki Uchida ${ }^{1}$, Kazunao Suzuki ${ }^{1}$, Kazuhiro Sugihara ${ }^{1}$, Naohiro Kanayama ${ }^{1}$

${ }^{1}$ Department of Obstetrics and Gynecology, Hamamatsu University School lf Medicine, Hamamatsu, Japan

Background: Increasing evidences have shown that neonatal or infantile rapid growth subsequent to undernourishment in utero is a risk factor of adult metabolic syndrome (MS). Recently, in the liver, phenotype of MS was also noticed as Non-alcoholic Fatty Liver Disease (NAFLD). Although, some reports suggested that intrauterine growth restriction is a risk of NAFLD, the pathological involvement has been established, partly because NAFLD was usually diagnosed by histology of the liver.In this study, we hypothesized that rapid growth during infantile period subsequent to undernourishment in utero is causatively associated with the increase of fat deposit in the liver under western life style in later life. To prove the hypothesis, we developed a mouse animal model of adult NAFLD following undernourishment in utero.

Method: Maternal caloric restriction (40\% reduction) was apply to C57/BL6 pregnant mice. After that, we fed high fat $\operatorname{diet}(\mathrm{HFD})$ from 9 to $17 \mathrm{wks}$. At $17 \mathrm{wks}$, weight of the liver, hematoxylin and eosin stain and oil-red $\mathrm{O}$ stain of the hepatic tissue. The gene expressions of fatty acid synthesis enzyme e.g. SREBP-1c, LXR and SCD and marker of liver injury such as TNF- $\alpha$ and TGF- $\beta 1$ were measured by quantitative RT-PCR. The rate of infantile body weight gain of the offspring was calculated with the formula of $Z$ score; [(body weight) - (mean body weight)]/standard deviation (SD) of body weight.

Results: At $17 \mathrm{wks}$, mean liver weight in undernourished offspring $(2.40 \pm 0.56[\mathrm{SD}] \mathrm{g})$ was significantly heavier than that in normally nourished offspring $(2.09 \pm 0.60 \mathrm{~g})$ $(\mathrm{p}<0.05)$. Moreover, in undernourished offspring, hepatic pathology showed that hepatic cell swelling, i.e. hepatocellular ballooning, concomitant with numerous lipid droplet, being compatible with NAFLD. The gene expression of TNF- $\alpha$ was accelerated in undernourishment offspring. $Z$ score of the rate of body weight gain around initiation of feeding was positively correlated with $\mathrm{Z}$ score of liver/body weight ratio $(\mathrm{r}=0.51, \mathrm{p}<0.05)$ and NAFLD score $(\mathrm{r}=0.71, \mathrm{p}<0.05)$ at $17 \mathrm{wks}$. The infantile rapid growth following undernourishment in utero accelerates deterioration of fatty liver, especially NAFLD pattern.

Conclusion: It was suggested that severity of undernourishment in utero may affect differently the development of obesity and/or NAFLD in later life. Undernourishment in utero followed by rapid infantile growth, specially at around the introduction of oral feeding, may contribute to the development of NAFLD in later life.

Disclosure of Interest: None Declared.

\section{DOHaD13-1141}

Rapid infantile growth after undernourishment in utero induce transformation from a "metabolically healthy obese" into a "metabolically morbidly obese" by adipose tissue remodeling - a possible involvement of chronic inflammation in adipose tissue

Hiroaki Itoh ${ }^{1, *}$, Yukiko Kohmura-Kobayashi ${ }^{1}$, Keiko Kato-Muramatsu ${ }^{1}$, Kaori Yamazaki ${ }^{1}$, Kotomi Nagahashi ${ }^{1}$, Naoaki Tamura ${ }^{1}$, Toshiyuki Uchida ${ }^{1}$, Kazunao Suzuki ${ }^{1}$, Naohiro Kanayama ${ }^{1}$ and Hamamatsu Birth Cohort (HBC) Study Team

${ }^{1}$ Department of Obstetrics and Gynecology, Hamamatsu University School of Medicine, Hamamatsu, Japan

Background: The infantile rapid growth after undernourishment in utero was reported to be a risk factor of adult obesity. Recently, it was highlighted that the chronic inflammation-associated adipose tissue remodeling, with a relative increase of small size adipocytes among enlarged adipocytes, in the white adipose tissues (WAT) plays as a "metabolically morbidly obese". In this study, we hypothesized that rapid infantile growth after undernourishment in utero accelerates adipose tissue remodeling and constitutes a risk of metabolic syndrome. To prove the hypothesis, we developed a mouse animal model of undernourishment in utero and compared between the growth at weaning and parameters of adipose tissue remodeling at $17 \mathrm{wks}$.

Method: Maternal caloric restriction (30\% reduction) was apply to C57/BL6 pregnant mice. At 3 wks, body weight distribution of the offspring was assessed by Z-score [(body weight) - (mean body weight)]/standard deviation (SD) of body weight] at weaning. After a HFD (60\% fat) from 9 to 17 wks, the body weight and weight of subcutaneous WAT, serum levels of glucose and total cholesterol, rate of small adipocyte (less than $30 \mathrm{um}$ in diameter), immunohistochemical detection rate of macrophage specific F4/80 in WAT were assessed. The gene expression of inflammatory M1 macrophage-specific CD11c and anti-inflammatory M2 macrophage-specific CD163 in WAT were measured by quantitative RT-PCR.

Results: At 17 wks, all of the body weight $(r=0.85)$, weight of subcutaneous WAT $(r=0.07)$, serum glucose levels $(r=0.67)$, total cholesterol levels $(r=0.46)$, the rate of small adipocyte $(r=0.49)$, immunohistochemical detection rate of macrophage specific F4/80 $(r=0.54)$, the M1/M2 macrophage ratio of CD11c/CD163 gene expression were positively correlated with Z-score at weaning ( $3 \mathrm{wks})$ in undernourished offspring (all $\mathrm{P}<0.001$ ), but not in normally nourished offspring.

Conclusion: Undernourishment in utero and rapid infantile growth may play a role of "Two Hits" in the development of metabolic syndrome by transforming a "metabolically healthy obese" into a "metabolically morbidly obese" with adipose tissue remodeling in later live. 


\section{References}

1. Association Between Body Weight at Weaning and Remodeling in the Subcutaneous Adipose Tissue of Obese Adult Mice With Undernourishment In Utero.

2. Kohmura YK, Kanayama N, Muramatsu K, Tamura N, Yaguchi C, Uchida T, Suzuki K, Sugihara K, Aoe S, Sasaki T, Suganami T, Ogawa Y, Itoh H. Reprod Sci. in press 2013, PMID: 23296035.

Disclosure of Interest: None Declared.

\section{DOHaD13-1431}

\section{Sex dependent glucose intolerance in adult offspring from pregnant rats fed low protein diet}

Blesson C. Selvanesan ${ }^{1}$, Vijayakumar Chinnathambi ${ }^{1}$, K Sathishkumar ${ }^{1}$, Chandra Yallampalli ${ }^{1, *}$

${ }^{1}$ Obstetrics and Gynecology, University of Texas Medical Branch, Galveston, United States

Background: Low protein (LP) diet during pregnancy leads to the development of various metabolic diseases including type II diabetes in adulthood. The onset, severity and progression of the disease appear to be sex dependent. However, the role of sex steroid hormones is not clearly understood.

Aim: To determine if there are sex differences and assess the role of sex steroids in the development of glucose intolerance in the adult rat offspring of dams fed LP diet during gestation.

Method: Pregnant Wistar rats were fed with control $(20 \%$ protein) or isocaloric LP (8\%) diet from gestational day 4 to until delivery. After weaning, offspring were given normal diet. A subset of male and female offspring was gonadectomized at 5 weeks of age. At 3 months of age, plasma glucose and insulin responses were assessed following oral glucose tolerance test. Plasma glucose and insulin responses were calculated using trapezoidal rule. Plasma levels of testosterone and estradiol were measured. Western blot analysis for the detection of glucose transport protein subtype 4 (glut4) expression in the skeletal muscle (gastrocnemius) at basal and following ex-vivo insulin challenge were also performed.

Results: Plasma glucose and insulin responses were expressed as $\Delta$ glycemia area under the curve (AUC) $\left(\mathrm{mg} / \mathrm{dL}^{*} 120 \mathrm{~min}\right.$ $\mathrm{x} 10^{3}$ ) and $\Delta$ Insulin AUC (ng/dL*120 min). In LP males, plasma glucose $(6.9 \pm 0.6)$ and insulin $(117 \pm 16)$ were significantly elevated after oral glucose administration compared with control males (glycemia: $2.9 \pm 0.5$; insulin: $83 \pm 1.8)$. This glucose intolerance in the LP males was associated with decreased plasma testosterone $(1.5 \pm 0.3 \mathrm{ng} /$ $\mathrm{ml}$ vs. $5.7 \pm 1.4 \mathrm{ng} / \mathrm{ml}$ in controls) and unchanged estradiol levels. Deprivation of testosterone levels by orchiectomy reversed glucose intolerance in LP males (glycemia: $4.8 \pm 0.6$; insulin: $49 \pm 10$ ) and was without significant effect in control males (glycemia: $4.3 \pm 0.5$; insulin: $55 \pm 5$ ). The LP females with intact ovaries exhibited glucose intolerance with elevated glucose $(6.2 \pm 0.5)$ and insulin $(80 \pm 7)$ following oral glucose administration compared with control females (glycemia: $2.8 \pm 0.4$, insulin: $57 \pm 15$ ). The plasma estradiol or testosterone levels in LP females were comparable to control females. Ovariectomy exacerbated glucose intolerance in LP females (glycemia: $8.7 \pm 0.7$; insulin: $51 \pm 10$ ) but was without significant effect in control females (glycemia: $4.5 \pm 0.7$; insulin: $50 \pm 7)$. In addition we found that insulin-stimulated glut 4 translocation to membrane was lower in the skeletal muscles of LP offspring and correlated with their glucose intolerance.

Conclusion: Gestational low protein diet leads to the development of glucose intolerance in sex dependent manner. Sex steroids appear to regulate glucose intolerance, with orchiectomy negating the effect in LP males and ovariectomy exacerbating the effect in LP females. Further studies on sex steroid mediated regulation of glucose homeostatic signaling may help develop sex-specific therapies for diabetes mellitus.

Disclosure of Interest: None Declared.

\section{DOHaD13-1432}

\section{Sex-specific plasma glucose intolerance in the prenatal} testosterone exposed adult offspring

Blesson C. Selvanesan ${ }^{1}$, Vijayakumar Chinnathambi ${ }^{1}$, Chandra Yallampalli ${ }^{1}$, K Sathishkumar ${ }^{1, *}$

${ }^{1}$ Obstetrics and Gynecology, University of Texas Medical Branch, Galveston, United States

Background: Plasma testosterone (T) levels are elevated in preeclampsia, PCOS mothers and pregnant African-American women. We have shown that prenatal testosterone exposure leads to sex-specific cardiovascular dysfunction in adult offspring with more pronounced hypertensive effect in the males than females. However, the impact of elevated maternal androgens on metabolic disorders including glucose intolerance in adult life is not known.

Aim: To evaluate whether prenatal $\mathrm{T}$ exposure leads to the development of glucose intolerance in adult males and females and to assess the influence of gonadal hormones on glucose homeostasis in these animals.

Method: Pregnant Wistar rats were injected with vehicle or $\mathrm{T}$ propionate $(0.5 \mathrm{mg} / \mathrm{Kg} /$ day from gestation day $15-19)$ to increase plasma $\mathrm{T}$ levels twofold, similar to that observed in clinical conditions like preeclampsia. The offspring were weaned to normal rat chow and their growth parameters were monitored. A subset of both male and female offspring was gonadectomized at 5 weeks of age. At 3 months of age, oral glucose tolerance test was performed to measure plasma glucose and insulin levels. Plasma glucose and insulin responses were expressed as $\Delta$ glycemia area under the curve (AUC) $\left(\mathrm{mg} / \mathrm{dL}^{*} 120 \mathrm{~min} \times 10^{3}\right)$ and $\Delta$ Insulin AUC (ng/dL*120 min) calculated using trapezoidal rule.

Results: Plasma T levels significantly increased by 2 -fold in $\mathrm{T}$ injected rats $(2.1 \pm 0.17 \mathrm{ng} / \mathrm{ml})$ compared to controls 
$(1.0 \pm 0.11 \mathrm{ng} / \mathrm{ml})$. Placental weights (control: $0.54 \pm 0.08 \mathrm{~g}$; T-treated: $0.43 \pm 0.13 \mathrm{~g}$ ) and birth weight of pups (control: $6.30 \pm 0.07 \mathrm{~g}$; T-treated: $5.21 \pm 0.09 \mathrm{~g}$ ) were significantly decreased in $\mathrm{T}$ treated group compared to controls. Both the male and female offspring of T-treated dams exhibited catchup growth such that at 4 week of age there were no differences in body weights between the offspring of $\mathrm{T}$-treated and control groups. At 12 week of age, there were no differences in glucose tolerance between control males and prenatal $\mathrm{T}$ treated males. Intriguingly, castration induced glucose intolerance in prenatal $\mathrm{T}$ treated males but not in controls ( $\Delta$ Glycemia AUC: Control, $5.0 \pm 0.8$ vs. T-treated, $7.9 \pm 1.1 \mathrm{mg} / \mathrm{dL}^{*} 120 \mathrm{~min} \times 10^{3} ; \Delta$ Insulin AUC: Control, $57 \pm 9$ vs. T-treated, $\left.71 \pm 5 \mathrm{ng} / \mathrm{dL}^{*} 120 \mathrm{~min}\right)$. In contrast, the female prenatal $\mathrm{T}$ treated offspring with intact ovaries exhibited glucose intolerance with elevated plasma glucose and insulin levels ( $\Delta$ Glycemia AUC: Control, $3.6 \pm 0.4$ vs. T-treated, $5.2 \pm 1.0 \mathrm{mg} / \mathrm{dL}^{*} 120 \mathrm{~min} \times 10^{3} ;(\Delta$ Insulin AUC: Control, $30 \pm 6$ vs. T-treated, $58 \pm 12 \mathrm{ng} /$ $\left.\mathrm{dL}^{*} 120 \mathrm{~min}\right)$. Interestingly, ovariectomy reversed glucose intolerance in prenatal $\mathrm{T}$ exposed females but was without significant effect in control females.

Conclusion: We demonstrate for the first time that elevated maternal $\mathrm{T}$, at concentrations relevant to those observed in abnormal clinical conditions, caused dysregulation of glucose metabolism in the female but not male offspring. Presence of testis in males contributes for the protective effect while ovaries in females contribute for glucose dysregulation in prenatal $\mathrm{T}$ exposed offspring.

Disclosure of Interest: None Declared.

\section{DOHaD13-1192}

Short-term treatment with a cholinergic antagonist drug, buscopan, during lactation attenuates obesity onset in small litter-programed rats

Kesia Palma Rigo ${ }^{1, *}$, Ananda Malta ${ }^{1}$, Aline A. de Souza ${ }^{1}$, Júlio C. de Oliveira ${ }^{1}$, Luiz F. Barella ${ }^{1}$, Rosiane A. Miranda ${ }^{1}$, Rosana Torrezan ${ }^{2}$, Amanda B. Trombini ${ }^{1}$, Claudinéia C. D. S. Franco ${ }^{1}$, Clarice Gravena ${ }^{1}$, Kelly V. Prates ${ }^{1}$, Josiane M. Martin $^{1}$, Paulo C. D. F. Mathias ${ }^{1}$

${ }^{1}$ Department of Biotechnology, Genetics and Cell Biology, State University of Maringá; '2 Department of Physiological Sciences, State University of Maringa', Maringá, Brazil

Background: Growth factors and hormones that participate in neuronal connections are crucial to brain development and its implication on metabolic programming. It was observed that calorie restriction during pregnancy in rats provokes rise in insulin blood levels, while similar insult during lactation induces low insulin secretion and lean phenotype in adult life. Furthermore, insulin treatment in the last thirty of gestation in rats compromises the fetus and programs pups to metabolism dysfunctions later in life. One mechanism that contributes to metabolic malprogramming from protein restriction could be offspring low insulin secretion. Studies have shown that muscarinic inhibition induces reduction in insulin secretion. Additionally postnatal early overfeeding leads to obesity and increased insulinaemia. We hypothesised that obesity observed in metabolic malprogramming may involve muscarinic-dependent insulin secretion. To test this hypothesis we treated milksucking rats from normal and reduced litters with the muscarinic antagonist drug, buscopan, and observed its effect on phenotype when the pups turn to adult life.

Method: Just after birth offspring rats from litters with 9 (B-NL) and 3 (B-SL) pups were intraperitoneally injected with buscopan [0.5 mg/Kg body weight (BW)] during the 12 first days of life. Control pup rats (S-NL and S-SL) were injected with saline solution. Diet intake and BW were measured each two days. After 85 days rats were submitted to intravenous glucose tolerance test (ivGTT). Blood glucose and insulin were measured. Fat pad from retroperitoneal, periepididymal, inguinal and visceral tissues were weighted. Data were submitted to two-way ANOVA or Student t-test with GraphPad Prism software version 6.01.

Results: Perinatal treatment with buscopan attenuated the accretion over life on BW, food intake and fat tissue in both litters type; however, change was bigger in B-SL than B-NL. Glucose intolerance observed in obese SL rats did not change with buscopan treatment; however, NL rats showed glucose intolerance when treated with the muscarinic agonist drug. It was also observed a reduced fasting insulinemia in groups treated early in life with buscopan.

Conclusion: Perinatal short-term treatment with buscopan programs rat offspring to a lean phenotype with metabolism dysfunction associated to poor insulin secretion. Finally, obesity programmed by reducing litter is attenuated by early treatment with buscopan.

Disclosure of Interest: None Declared.

\section{DOHaD13-1305}

Single course of antenatal betamethasone administration decreased fetal growth and altered maternal-fetal glucose homeostasis

Anne-Katrin Gramzow ${ }^{1,2, *}$, Franziska Braun ${ }^{1,2}$, Loreen Ehrlich $^{2}$, Annett Husar ${ }^{1,2}$, Deborah M. Sloboda ${ }^{3,4,5}$, John R.G. Challis ${ }^{6,7,8}$, Wolfgang Henrich ${ }^{1}$, Andreas Plagemann ${ }^{1,2}$, Thorsten Braun ${ }^{1,2}$

${ }^{1}$ Department of Obstetrics; ${ }^{2}$ Division of Experimental Obstetrics, Study Group Perinatal Programming, Charite' University, Berlin, Germany; ${ }^{3}$ Department of Biochemistry and Biomedical Sciences; ${ }^{4}$ Department of Obstetrics and Gynecology; ${ }^{5}$ Department of Pediatrics, McMaster University, Hamilton; ${ }^{6}$ Department of Physiology; ${ }^{7}$ Department of Obstetrics and Gynecology, University of Toronto, Toronto; ${ }^{8}$ Faculty of Health Sciences, Simon Fraser University, Vancouver, Canada 
Background: Antenatal betamethasone (BET) administration during pregnancy may decrease fetal growth and development and is associated with potential long term disease. Considering the fetal dependency on maternal glucose supply and the role of insulin as one of the key regulators of human fetal growth, we examined the effects of a single course of BET on maternal and fetal glucose and insulin plasma levels and the role of placental glucose transporter 1 (Glut-1) as a cause for low birth weight. Method: BET (single course, $2 \times 12 \mathrm{mg}$ ) exposed women $(\mathrm{n}=86)$, delivered between $24+2$ and $41+6$ weeks of gestation (wks) were compared to age-matched controls $(\mathrm{n}=92)$. Maternal and umbilical cord blood samples as well as placental tissues were obtained at delivery. Maternal, fetal venous and arterial umbilical cord glucose (MG, VUG, AUG) and insulin (MI, VUI, AUI) plasma levels were measured. Placental Glut-1 protein levels were analyzed by Western Blot. Statistics were compiled by multivariate analyzes, significance was accepted for $\mathrm{p}<0.05$.

Results: A single course of BET significantly decreased birth weight $(-7.6 \%)$ and head circumference $(-2.3 \%)$ as compared to controls. MI and MG plasma levels were sig. increased after BET treatment in pregnant women who delivered $\geq 37$ wks as compared to controls. BET administration did not sig. change AUI and VUI plasma levels, but resulted in a decreased AUG and VUG in fetuses born $<37$ wks. AUG and VUG plasma levels in fetuses born $\geq 37 \mathrm{wks}$ were sig. increased after BET administration as compared to controls. The ratio of MG over VUG/AUG tended to be higher, while the ratio of VUG over AUG and vice versa was not sig. changed as compared to controls. BET did not significantly change placental Glut-1 protein levels. In contrast to controls, MG plasma levels did not correlate to MI plasma levels in BET treated women.

Conclusion: BET induced long term changes in the maternal compartment with increased insulin and glucose plasma levels, indicative for an augmented insulin resistance. Despite no differences in Glut-1 protein levels, changes in umbilical cord insulin and glucose plasma levels suggest an impairment of the steadily fetal glucose homeostasis. Further investigations are required in regard to the mechanisms of an altered transplacental glucose transport after a single course of BET and its potential role for fetal growth impairment.

Disclosure of Interest: None Declared.

\section{DOHaD13-1378}

The impact of breast milk on obesity: a possible epigenetic mechanism influencing obesity

Heide Temples ${ }^{1, *}$

${ }^{1}$ School of Nursing, Clemson University, Clemson, United States

Background: The purpose of the study is to assess the impact of breast milk on the risk of obesity and risk of being overweight in infants and children. It is hypothesized the body mass index (BMI), skin fold thickness and waist circumference will be less in the participants with longer duration of breastfeeding. Background research includes a meta-analysis by Harder (2005) which demonstrated the risk of being overweight was reduced by $4 \%$ for each month of breastfeeding and the effect lasted until adulthood. Von Kries (1999) found the prevalence of obesity in 13,345 children was $4.5 \%$ in children who had never been breastfed as compared to $2.8 \%$ in breastfed children. A clear dose-response effect was identified on the prevalence of obesity and theorized that breastfeeding might have a programming effect.

Method: This cross sectional research study consists of 250 pairs of Australian twins and their mothers enrolled in the Peri/postnatal Epigenetic Twins Study (PETS). Infant cheek swabs were collected at birth and 18 months of age for DNA methylation analysis along with nutritional data, the health of the twin and anthropometric measurements including weight, length, head circumference and skinfold thickness. Data analysis for this abstract includes the duration of breastfeeding and the anthropometric measurements at birth and 18 months for the 500 twins enrolled in the PETS.

Results: The preliminary results are from a small subset of PETS participants $(\mathrm{n}=45)$, that had never received breast milk $(\mathrm{n}=28)$, as compared to receiving breast milk exclusively for 6 months $(\mathrm{n}=17)$. At 18 months of age, the body mass index (BMI) of the exclusively breast fed group was significantly lower than the non-breast fed group $(\mathrm{p}=0.09)$ at a $10 \%$ level of significance. Further research results with anthropometric measurements including head circumference, skinfold thickness, arm and abdominal circumference will be available at the DOHaD conference in November, 2013.

Conclusion: Breast feeding may play an epigenetic role in the prevention of obesity through metabolic programming. Since obese children have a high risk of becoming obese adults, these preventative measures may result in a reduction of cardiovascular disease and other diseases related to obesity.

\section{References}

1. Harder et al. (2005). Duration of breastfeeding and risk of overweight: A meta-analysis. American Journal of Epidemiology. 162, (5), 397-403.

2. von Kries, R., Koletzko, B., Sauerwald, T., et al. (1999). Breast feeding and obesity: cross sectional study. BMJ 319: 147-150.

Disclosure of Interest: None Declared.

\section{DOHaD13-1531}

Twin conception results in adult adipose tissue with sexually dimorphic changes in glucose transporter and inflammatory cytokine expression

Elise Donovan ${ }^{1,2, *}$, Anne Jaquiery ${ }^{1,2,3}$, Ana-Mishel Spiroski ${ }^{1,2}$, Mark Oliver $^{1,2}$, Serina Hancock ${ }^{1}$, Frank Bloomfield ${ }^{1,2,3}$

${ }^{1}$ The Liggins Institute, University of Auckland; ${ }^{2}$ Gravida: National Centre for Growth and Development; ${ }^{3}$ Department of 


\section{Paediatrics: Child and Youth Health, University of Auckland, Auckland, New Zealand}

Background: Sheep conceived and carried as twins, and twins reduced to a singleton pregnancy (reductions) in early gestation, have altered growth trajectories, greater fat mass, and less lean mass in adulthood than those conceived as singletons. Adult males conceived as twins tend to be less insulin sensitive than singletons, with reductions intermediate (see abstract by AL Jaquiery et al). We therefore hypothesized that adipose tissue from twins and reductions would exhibit a phenotype characterized by increased inflammatory marker and glucose transporter expression compared with singletons. Method: Twin pregnancies in sheep were randomised to reduction of one twin on day 42 of a $148 \mathrm{~d}$ pregnancy by intrathoracic $\mathrm{KCl}$ or a sham procedure. Singleton-bearing ewes also underwent a sham procedure and all ewes lambed naturally. At $2 \mathrm{yr}$ of age omental adipose tissue was collected (Twins: 6 male (M), 9 female (F); Reductions: 7 M, 7 F; Singleton: 6M, 9 F). Glucose transporter 4 (SLC2A4), glucose transporter 1 (SLC2A1), interleukin 6 (ILG), and tumour necrosis factor $(T N F)$ mRNA expression were measured with quantitative PCR. Data were normalized to three housekeeping genes and presented as fold difference with $99 \%$ confidence intervals.

Results: In males, twins and reductions both had significantly greater expression of SLC2A4 (1.41 (1.23-1.62) and 1.74 $(1.74-1.74))$ and SLC2A1 (1.22 (1.18-1.26) and 2.38 (2.17-2.60)) compared to singletons, and expression levels of both genes in twins were significantly lower than in reductions (0.81 (0.76-0.87) and $0.51(0.41-0.64))$. TNF expression was significantly greater in twins than reductions (1.16 (1.09-1.24)) but neither were different from singletons; IL6 was significantly greater in reductions compared to singletons $(1.54(1.38-1.71))$ and less in twins compared to reductions $(0.62(0.57-0.68))$. In females, SLC2A4 expression was significantly greater in twins compared to singletons and reductions (1.15 (1.12-1.19) and 1.04 (1.03-1.04)), whereas SLC2A1 expression was significantly less in twins and reductions compared with singletons $(0.89(0.87-0.91)$ and $0.86(0.75-0.98))$, although the size of these effects was small. TNF expression was significantly greater in reductions than in singletons (1.30 (1.23-1.39)) and less in twins compared to reductions $(0.71(0.63-0.80))$. IL6, however, was significantly greater in twins compared to both singletons and reductions (1.48 (1.30-1.68) and $1.31(1.12-1.52))$.

Conclusion: In sheep, twin conception results in elevated glucose transporter expression and increased inflammatory cytokine expression in omental adipose tissue in male adult offspring, consistent with a phenotype of adiposity and insulin resistance. In contrast, there were few differences amongst female offspring. We conclude that being conceived as a twin, regardless of whether the twin pregnancy is carried to term, alters adult omental adipose inflammatory and glucose transporter phenotype.

Disclosure of Interest: None Declared.

\section{DOHaD13-1558}

Vitamin B12 deficiency causes adipocyte dysfunction by inducing cholesterol biosynthesis and unfolded protein response

Antonysunil Adaikalakoteswari ${ }^{1}$, Sarah Finer $^{2}$, Jonathan Moore $^{3}$, Melissa Smart-Halajko ${ }^{2}$, Ciara McCarthy ${ }^{1}$, Philip Voyais ${ }^{1}$, Graham A. Hitman ${ }^{2}$, Philip G. McTernan ${ }^{1}$, Ponnusamy Saravanan ${ }^{1,4, *}$, Gyanendra Tripathi ${ }^{1}$

${ }^{1}$ Division of Metabolic and Vascular Health, University of Warwick, Warwick; ${ }^{2}$ Centre for Diabetes, The Blizard Institue, Barts and The London School of Medicine and Dentistry, London; ${ }^{3}$ Systems Biology Centre, University of Warwick, Warwick; ${ }^{4}$ Academic department of Diabetes, George Eliot Hospital, Nuneaton, United Kingdom

Background: Vitamin B12 deficiency has been shown to play an important role in the prediction of metabolic risk, but its significance and mechanism in the development of adiposity and adipose tissue (AT) dysfunction is largely unknown. Adipose tissue plays a central role in integrating energy metabolism including glucose homeostasis. It is the major site of fatty acid storage as triglycerides and is body's largest cholesterol pool. In AT, cholesterol is mostly found in its free, non-esterified form. Cholesterol imbalance in AT is closely associated with adipocyte dysfunction. B12 and folate regulate the levels of S-adenosylmethionine (AdoMet) and homocysteine (Hcy). Low levels of methyl donor AdoMet has been shown to activate SREBP-1 (Sterol regulatory elementbinding transcription factor) and induce lipogenesis in metazoans. The SREBP family (SREBP-1a, $-1 \mathrm{c}$ and SREBP-2) responds to nutrient levels and regulates transcription of genes required for lipid metabolism. Hcy has been shown to induce unfolded protein response (UPR). Therefore we investigated the effect of B12 on cholesterol synthesis and UPR in adipocytes and its underlying mechanisms.

Method: Human pre-adipocytes were differentiated in various B12 concentrations: (1) Control: (B12-500nM); (2) Low B12 (0.15nM) (3) No B12: (B12-0nM). DNA, RNA and protein was extracted from different B12 treated adipocytes and used for respective analysis such as methylation, microarray, qRT-PCR and western blotting. Conditioned media was used for Hcy analysis by HPLC. Glucose uptake was measured using 2-deoxy-D- $\left[{ }^{3} \mathrm{H}\right]$ glucose by scintillation counting.

Results: Microarray analysis of human pre-adipocytes in varying concentrations of $\mathrm{B} 12$ led to the identification of two important pathways; cholesterol synthesis and UPR. Validation by qRT-PCR confirmed that the genes involved in cholesterol biosynthesis and UPR were up-regulated in B12 deficient conditions (all $\mathrm{p}<0.05$ ). Adipocytes cultured in low B12 or no B12 conditions had increased intracellular cholesterol and extracellular Hcy levels and reduced glucose uptake capacity (all $\mathrm{p}<0.05$ ) compared to adipocytes cultured in normal B12 conditions. Global DNA methylation 
profiling of these adipocytes showed that the promoter regions of SREBF1 and LDLR (low density lipoprotein receptor) were hypomethylated $(\mathrm{p}<0.001)$ in the low and no B12 conditions. The corresponding mRNA expressions (SREBF1 and LDLR) and SREB proteins (SREBP1 and 2) (all $\mathrm{p}<0.05$ ) were also increased in the same conditions.

Conclusion: These data suggest that B12 deficiency can lead to adipocyte dysfunction by inducing excess cholesterol biosynthesis, homocysteine production and UPR. Some of these effects may be due to B12's role in methylation of DNA and regulation of homocysteine levels.

Disclosure of Interest: None Declared.

\section{DOHaD13-1588}

\section{Altered balance of apoptosis and proliferation in the testes of male offspring born to nutritionally compromised mothers}

Graciela Pedrana $^{1, *}$, Mark H. Vickers ${ }^{2,3}$, Angelica Bernal ${ }^{2,3}$, M H. Viotti ${ }^{4}$, E Souza ${ }^{4}$, Caroline Moore ${ }^{5}$, Deborah M. Sloboda ${ }^{5}$

${ }^{1}$ Facultad de Veterinaria, Universidad de la Republica, Montevideo, Uruguay; ${ }^{2}$ Liggins Institute, University of Auckland; ${ }^{3}$ Gravida: National Research Centre for Growth and Development, Auckland, New Zealand; ${ }^{4}$ Facultad de Veterinaria, Universidad de la República, Montevideo, Uruguay; ${ }^{5}$ Biochemistry and Biomedical Sciences, McMaster University, Hamilton, Canada

Background: We have previously reported that female offspring of nutritional compromised mothers have low birth weight, early puberty and impaired postnatal reproductive development. Low birth weight in males is associated with a reduced sperm count, reduced testicular size and infertility. We thus investigated apoptosis and proliferation within the testes of low birth weight male offspring born to nutritionally compromised mothers to determine whether maternal nutritional history modifies male reproductive development. Method: Pregnant Wistar rats were randomized and fed the following diets: 1) maintained on a standard diet (cont) during pregnancy and lactation or fed $50 \%$ of control during lactation (UNL), 50\% of control during pregnancy (UNP), $50 \%$ of control during pregnancy+lactation (UNPL), or fed a high fat diet $(45 \% \mathrm{kcal}$ fat $)$ during pregnancy+lactation (PLHF). Post-weaning, male offspring were maintained on a control diet until day 160, when testes were dissected, and either frozen or fixed. Fixed testicular tissues were processed to investigate active-caspase 3, Bax, Bcl-2, PCNA and Ki-67 immunolocalization. The percentage of immunopositive staining in testicular parenchyma was analyzed by image analysis. Total testicular gene expression levels were analyzed using qPCR.

Results: Cont offspring showed caspase-3 immunolocalization in spermatids with little staining in sertoli cells. A greater amount of caspase-3 immunostaining was observed in UNP and UNPL offspring in both Sertoli cells and spermatogonia (15.2\%, $\mathrm{p}=0.001$ and $15.7 \%, \mathrm{p}=0.0005$ respectively). This was associated with increased testicular caspase- 3 mRNA levels in UNP offspring compared to cont. Bax was localized often in Sertoli cells, rarely in Leydig cells and spermatocytes and $\mathrm{Bcl}-2$ was localized in round spermatids and elongated spermatids. Bax immunostaining was greater in UNPL offspring (15.8\%, $\mathrm{p}=0.002)$. Bcl-2 immunostaining was higher all offspring of nutritionally compromised mothers: PLHF (29.8\%, p <0.0001); UNP (27.7\%, p < 0.0001); UNPL: $\quad(27.6 \% \quad \mathrm{p}<0.0001)$; and UNL: $(27.3 \%$, $\mathrm{p}<0.0001)$ compared to cont offspring. Testicular Bax mRNA levels were unchanged in nutritional compromised offspring although Bcl2 mRNA levels were lower in PLHF compared to cont. The number of Ki-67-positive cells was increased in nutritionally challenged offspring: UNPL (22.1\%, p $<0.0001)$; UNL (21.7\%, p $<0.0001)$, UNP (19.7\%, $\mathrm{p}=0.0004)$ and PLHF $(18.9 \%, \quad \mathrm{p}=0.0002)$ compared to controls. PCNA-positive cell number was higher in UNPL $(p=0.009)$ compared to control offspring.

Conclusion: We demonstrate that maternal nutritional compromise increases apoptosis in localized testicular regions associated with increased $\mathrm{Ki}-67$ and PCNA protein immunolocalization - which may be suggestive of increased local spermatogonial cell number. We speculate that in offspring of nutritional compromised mothers, an imbalance occurs between testicular cell loss (through apoptosis) and cell proliferation and may prove to be harmful in the process of spermatogenesis.

Disclosure of Interest: None Declared.

\section{DOHaD13-1550}

\section{Early life inflammatory determinants of reproductive development}

Luba Sominsky ${ }^{1}$, Erin A. Fuller ${ }^{1}$, Alexander P. Sobinoff ${ }^{2}$, Matthew S. Jobling ${ }^{2}$, Victoria Pye ${ }^{2}$, Eileen A. McLaughlin ${ }^{2}$, Deborah M. Hodgson ${ }^{1, *}$

${ }^{1}$ Laboratory of Neuroimmunology, School of Psychology, Faculty of Science and IT, ${ }^{2}$ PRC in Reproductive Science and Chemical Biology, School of Environmental \& Life Sciences, Faculty of Science and IT, The University of Newcastle, Callaghan, Australia

Background: The increased rate of young women presenting with reduced fecundity worldwide suggests an increase in exposure to environmental factors, such as bacterial infections, which impair fertility. The incidence of Chlamydia has tripled in the last decade, and is continuing to increase in individuals of reproductive age. Moreover, up to $15 \%$ of infants are infected with Chlamydia at birth. Given that the fundamentals of healthy reproductive functioning are 
established during the early developmental period, alteration in the neonatal/juvenile microbial environment may play a role in the epidemiology of subfertility. The aim of the current study was to examine the impact of a mild immune activation during early neonatal development on ovarian and circulatory inflammatory markers.

Method: Female Wistar rats were administered with $0.05 \mathrm{mg} / \mathrm{kg}$ of LPS (Salmonella Enteritidis) or treated as saline controls, on postnatal days 3 and 5 (Birth $=$ day 1 ). The efficacy of LPS treatment was assessed on plasma levels of interleukin-6 (IL-6) and C-Reactive protein (CRP) at $90 \mathrm{~min}$ post LPS injection on day 5 . Ovaries were collected from an additional subset of animals on postnatal day 7 and microarray analysis was conducted. The gene data was confirmed by qRT-PCR analysis. Western blotting and immunofluorescence were conducted on selected number of proteins. Data were analysed using analyses of variances (ANOVA) design. The significance level was set at $\mathrm{p} \leqslant 0.05$

Results: LPS-treated animals exhibited a significant increase in both IL-6 and CRP plasma levels $(\mathrm{p}<.05)$. Microarray analysis of the ovarian genome indicated a significant upregulation in expression of 710 genes $(\geq 2$-fold change, $\mathrm{p}<.05$ ). Ingenuity Pathway Analysis identified a number of canonical pathways involved in immune response were significantly upregulated in the ovaries of LPS-treated animals, including NF-kB activation signalling pathway, macrophage-stimulating protein (MSP)-RON signalling pathway and LPS-stimulated mitogen-activated protein kinase (MAPK) signalling pathway. GRT-PCR analysis was conducted on the significantly altered genes identified as components of the LPS-stimulated MAPK signalling pathway and confirmed the microarray results $(\mathrm{p}<.05)$. Western blotting and immunofluorescence analysis of several components of the MAPK signalling pathway revealed a significant upregulation in the expression of Toll-like receptor 4 (TLR4), the receptor for LPS, in the neonatal ovary of LPS-treated animals $(\mathrm{p}<.05)$.

Conclusion: The current study indicates that neonatal exposure to LPS not only induces general peripheral inflammation, but a pro-inflammatory response is also expressed locally in the ovaries. These findings suggest that neonatal LPS induced ovarian inflammation is mediated through an upregulation of TLR4 signalling, which may be sustained into later life. Bacterial infections, such as E.coli and Chlamydia, are associated with alterations in TLR4 expression and pathological outcomes such as impaired fertility. Additionally, common ovarian diseases such as polycystic ovarian syndrome and endometriosis are associated with chronic increases in inflammatory mediators. Together, the current findings provide valuable insight into the link between early life infection and reproductive fitness.

Disclosure of Interest: None Declared.

\section{DOHaD13-1223}

Early life influences on testicular function in adulthood using the western Australian pregnancy (Raine Cohort)

Roger Hart ${ }^{1,2, *}$, Dorota A. Doherty ${ }^{1,3}$, David J. Handels$\operatorname{man}^{4}$, Robert McLachlan ${ }^{5}$, Niels E. Skakkebaek ${ }^{6}$, Jeffrey A. Keelan ${ }^{1,3}$, John Newnham ${ }^{1,3}$, Jan E. Dickinson ${ }^{1}$, Robert J. Norman ${ }^{7}$

${ }^{1}$ School of Women's and Infants Health, University of Western Australia; ${ }^{2}$ Fertility Specialists of Western Australia; ${ }^{3}$ Women's and Infants Research Foundation, King Edward Memorial Hospital, Perth; ${ }^{4}$ ANZAC Research Institute, ANZAC Research Institute, Sydney; ${ }^{5}$ Prince Henry's Institute, Monash Medical Centre, Melbourne, Australia; ${ }^{6}$ Department of Growth and Reproduction, Rigshospitalet, Copenhagen, Denmark, ${ }^{7}$ Robinson Institute, University of Adelaide, Adelaide, Australia

Background: It is alleged that sperm counts are decreasing, with evidence for adverse effects of chronic smoking and in-utero exposure, although there is conflicting evidence for the effect of intrauterine growth restraint upon sperm production. Adult adiposity is associated with adverse effects on sperm counts, and hormonal markers of testicular function. Such studies are prone to selection bias as the cohorts have very low recruitment rates and compromised by individuals with biased fertility status. This study set out to prospectively study the early life influences, in-utero, childhood and adolescence, that may impact on markers of testicular function as assessed by: testicular volume (TV), sperm production (total motile sperm [TMS], and hormone production (serum FSH, LH, and inhibin B [InhB]), in men aged 20-22 years.

Method: Prospective cohort study, using the Western Australian Pregnancy (Raine) Cohort, of men who underwent regular assessment since birth in 1990-1991, including fetal measurements for half the cohort. Part of the 20 year followup involved testicular assessment. Of the available 911 men; 696 attended general assessment and 423 took part in the testicular study.

404 men underwent testicular ultrasound examination, 365 underwent $\geq 1$ semen assessments (recruitment was $40 \%$ overall, $46.1 \%$ of available cohort, $60.3 \%$ of attendees of the 20 year assessments). The men underwent a dual energy X-ray absorptiometry scanning for adiposity, and testicular ultrasound examination and semen assessments at separate locations.

Results: World Health Organisation criteria for a normal sperm assessment were not met at initial assessment: seminal volume $<1.5 \mathrm{mls}(14.8 \%)$, total sperm $<39$ million (18.9\%), concentration $<15$ million/ml $(17.5 \%)$, progressive motility $<32 \%(14.4 \%)$ and $<4 \%$ morphologically normal sperm (26.4\%).

Men with good intrauterine growth were less likely to have a semen assessment with the TMS in the lowest quartile $(\mathrm{p}=0.011)$. Maternal smoking exposure $(18.6 \%)$ was 
associated with lower TMS $(\mathrm{p}=0.046)$. No relation was detected between cord blood testosterone, prematurity or birthweight with TMS, or TV in adulthood. TV correlated with childhood growth $(\mathrm{p}=0.001)$, height $(\mathrm{p}<0.0001)$.

This cohort has detailed measurements throughout life, with a retention rate of $>70 \%$, making it one of the largest cohorts worldwide. However, there is potential for participation bias in assessments as only $60.3 \%$ of men that attended the general assessment volunteered for the testicular study.

Conclusion: The results from this study suggest that perturbations in fetal growth, exposure to maternal smoking, lower childhood growth trajectory and increased adiposity may lead to reduced semen parameters in adulthood. Hence, public health measures to address these influences may help to mitigate these risks.

\section{References Study funding/competing interest(s)}

1. This abstract will be presented at the European Society of Human Reproduction and Embryology, London 2013.

2. This research was funded by NHMRC Project Grant Number 634457 and an unrestricted Grant for research from Merck-Serono to fund the testicular ultrasound examinations.

Disclosure of Interest: R. Hart Shareholder of: Western IVF, Grant/Research support from: NHMRC \& Merck-Serono, Consultant for: Merck-Serono \& MSD, D. Doherty: None Declared., D. Handelsman: None Declared., R. McLachlan: None Declared., N. Skakkebaek: None Declared., J. Keelan: None Declared., J. Newnham: None Declared., J. Dickinson: None Declared., R. Norman: None Declared.

\section{DOHaD13-1486}

\section{Higher incidence of preterm births with pregnancy exposure to high temperatures}

Jagjit S. Teji ${ }^{1, *}$, Rajdeep Singh ${ }^{2}$, Namrata K. Sekhon ${ }^{2}$

${ }^{1}$ Pediatrics, University of Chicago, Chicago, United States;

${ }^{2}$ Medical College, School of Medicine at Ross University,

Dominica, Saint Kitts and Nevis

Background: There have been several publications on temperature impact on pregnancy. Term low birthweight associated with high temperature exposed pregnancy have been previously reported by us. There have been some scattered reports of preterm births occurring more commonly in higher temperature both in animals and humans. The purpose of this study is to analyze whether preterm births incidence is impacted by higher temperature exposure of pregnancies in the USA.

Method: Data from birth certificates from the years 1995 to 2002 were analyzed by logistical regression on preterm birth, PT outcome with independent variable as ambient temperature, T. Following variables were controlled for: maternal age, education, exposure to tobacco and alcohol, prenatal care, and marital status. Other variables adjusted for were maternal risks such as incompetent cervix, diabetes, hypertension, rupture of membranes, placenta previa, induction, delivery type, plurality, sex, and race.

Results: Usable data for over 25 million newborns out of possible 32 million delivered during the study period. Delivering preterm babies from pregnancies exposed to ambient temperature greater than 55 degrees $\mathrm{F}$, had OR 1.188, 95\% CI (1.185-1.191) adjusted for the above variables, irrespective race, socioeconomic status. Incidentally higher ambient temperatures occur more commonly in the southern USA.

Conclusion: 1 . This is the first largest population based study demonstrating increased preterm births occurring in pregnancies exposed to temperatures greater than 55 degrees F. 2 . Complications of both being low birthweight and preterm can impact acutely and on later adult onset diseases. 3 . Biological reason for this association needs to be determined. 4. Global warming and its association with burden of disease should be studied for every stage of human lifetime.

\section{References}

1. Fulford AJC, Rayco-Solon P, Prentice AM. Statistical modelling of the seasonality of preterm delivery and intrauterine growth restriction in rural Gambia. Paediatric and Perinatal Epidemiology 2006; 20: 251-259.

2. Rupa Basu, Brian Malig, and Bart Ostro. High Ambient Temperature and the Risk of Preterm Delivery. Am J Epidemiol 2010; 172: 1108-1117.

3. Wolf J, Armstrong B (2012) The Association of Season and Temperature with Adverse Pregnancy Outcome in Two German States, a Time-Series Analysis. PLoS ONE 7(7): e40228. doi:10.1371/journal.pone.0040228

Disclosure of Interest: None Declared.

\section{DOHaD13-1151}

Influence of anthropometry and body composition on attainment of menarche in urban school-going girls in India

Leena Raje ${ }^{1, *}$, Amrita Behel ${ }^{2}$, Devaki Gokhale ${ }^{1}$

${ }^{1}$ Nutrition, SPND College; ${ }^{2}$ Nutrition, SPND College, Mumbai, Mumbai, India

Background: The age at menarche, the first indicator of reproductive capacity in women is known to be regulated by genetic and environmental factors like anthropometry, body composition (BC) and nutrition. Globally menarcheal age is consistently lowering.Body Mass Index (BMI) influences onset of menarche. Studies show that girls reaching menarche at a younger age have higher BMI and increased body fatness and obesity. BC during puberty is a marker of metabolic changes holding key information regarding current and future 
health. During puberty, the main components of BC i.e. total body fat and lean body mass increase. Early onset of menarche seems to be closely linked to cardiovascular disease, diabetes, obesity, cancer etc. and psychological and emotional problems.

To record the currently prevailing age at menarche in Indian population and to observe influence of anthropometry and $\mathrm{BC}$ on this important reproductive parameter, a study was taken up on 750 girls of $10-14$ years from standard $5^{\text {th }}$ to $9^{\text {th }}$ from 5 schools in 2 urban cities in Maharashtra.

Method: Weight $(\mathrm{kg})$ was measured to the nearest $100 \mathrm{~g}$ using an electronic weighing scale. Height was measured to the nearest centimeter using a stadiometer. BMI $\left(\mathrm{kg} / \mathrm{m}^{2}\right)$ was calculated as weight in kg divided by the square of height in meters. Body Composition Analysis was conducted for these girls using BC analyzer (Tanita- MA 420) based on bioelectrical impedance. Statistical analysis was performed using SPSS (Version 18).

Results: Final data is presented for 156 girls who had recently attained menarche $[\mathrm{AM}](<3$ months) and 156 girls from peri-menarcheal $[\mathrm{PM}]$ category showing $>1$ clinical sign of puberty. $72 \%$ of girls attained menarche between 10 to 12 years of age and $8 \%$ attained menarche as early as 10 years of age. Girls from AM group had a greater height $(151.62 \pm 6.55 \mathrm{~cm})$, weight $(44.36 \pm 9.28 \mathrm{wt})$ and BMI $(19.18 \pm 3.18)$ than girls from PM group $146.90 \pm 7.95$, $38.00 \pm 9.53,17.47 \pm 3.42$ respectively. The t-test showed that there was a significant effect $(\mathrm{p}=0.00$, sig 2-tailed) of height, weight and BMI on age at menarche.BC Analysis showed that means \pm sd for all parameters except waist/hip ratio (WHR) were higher in the AM group than PM group [AM group: fat mass $11.60 \pm 5.70(\mathrm{~kg})$; muscle mass $31.35 \pm 4.55(\mathrm{~kg})$; Waist circumference (WC) $70.50 \pm 6.16($ $\mathrm{cm})$; Hip $83.37 \pm 6.99(\mathrm{~cm}) \quad$ PM group: fat mass $8.74 \pm 5.88(\mathrm{~kg}) \quad$ Muscle Mass $27.94 \pm 4.23(\mathrm{~kg}) \quad$ Waist $62.66 \pm 4.62(\mathrm{~cm})$ and hip $74.93 \pm 6.45(\mathrm{~cm})]$. The t-test results showed that there was a significant positive effect of all the parameters of $\mathrm{BC}$, except WHR, on age at menarche $(\mathrm{p}=0.00$, sig 2-tailed) Discriminant analysis showed that changes in BC leading to accrual of body fat are important in influencing the onset of menarche. WC (1.029), an indicator of gluteal fat deposition, followed by fat\% (.975) has been shown to exert maximum effect followed by BMI $(-.834)$, fat mass (-.735), and muscle mass (.392). Thus, not only fat deposition but its distribution may be very important in attainment of menarche. Chronological age (.259) has been found to be the least important differentiating factor influencing the onset of menarche.

Conclusion: To conclude, changes in anthropometric profile and body composition leading to accrual of body fat were found to be significant determinants of early onset of menarche which has an important bearing on future health of women.

Disclosure of Interest: None Declared.

\section{DOHaD13-1583}

Maternal caloric restriction during critical developmental windows induces offspring ovarian follicle loss through ovarian inflammation and ER stress

Angelica Bernal ${ }^{1,2, *}$, Mark H. Vickers ${ }^{1,2}$, Kaitlyn $\mathrm{Chan}^{3}$, Wajiha Gohir ${ }^{3}$, Deborah M. Sloboda ${ }^{3}$

${ }^{1}$ Liggins Institute, University of Auckland; ${ }^{2}$ Gravida: National Research Centre for Growth and Development, Auckland, New Zealand; ${ }^{3}$ Biochemistry and Biomedical Sciences, McMaster University, Hamilton, Canada

Background: We have shown that offspring born to undernourished (UN) mothers have significant ovarian follicle loss as adults that is associated with increased ovarian oxidative stress and reduced antioxidant capacity, but a reduction in levels of pro-apoptotic Bax and increased antiapoptotic factor Bcl2. Since apoptosis does not appear to be regulating this loss of follicles in offspring, we investigated in the present study, whether other signaling pathways are governing this phenotype. We hypothesize that changes in the PI3 kinase signaling pathway may contribute to alterations in autophagy, ER stress and/or inflammation and may explain a loss of follicles in adult offspring of UN mothers.

Method: Using a rat model, pregnant dams were randomized to either a standard diet throughout pregnancy and lactation (Cont), or a calorie-restricted (50\% of control) diet during pregnancy alone (UNP), during pregnancy and lactation (UNPL) or during lactation (UNL). All offspring were fed a control diet postweaning. At 160 days of age, offspring ovaries were collected, frozen and mRNA levels of key factors in the PI3K and inflammatory signaling pathways (PIK3r1, PIK3ca, RelA, IL1beta, IL6, VEGFa, VEGFR2) as well as markers of ER stress (Xbp1s, Xbp1t) and autophagy (Beclin 1, LC3) were determined using qPCR. Immunohistochemistry was used to localize autophagy, VEGF and VEGFR2 in fixed ovarian sections.

Results: Offspring of UNP and UNPL mothers had increased ovarian mRNA IL6 but suppressed IL1beta levels compared to Cont. These changes were associated with an up regulation of PI3K signaling where UNP and UNPL offspring showed increased PI3ca levels as well as downstream targets RelA, and Bcl2. Despite no change in mRNA levels, immunostaining of VEGFa and its receptor, VEGFR2 was increased in UNP offspring. VEGF was highly localized to granulosa and theca cells, as well as the oocyte of small and large follicles. VEGFR2 was highly localized in the nucleus of antral follicles. UNP and UNPL ovaries showed a reduction LC3 and Beclin 1 mRNA and protein levels but increased ER stress (increased Xbp1s:Xbp1t ratio) markers compared to Cont. In all analyses, UNL offspring were no different than Cont.

Conclusion: These data show that maternal UN, particularly during the pregnancy time point - ie. UNP and UNPL, resulted in ER stress in adult offspring ovaries. Our data suggest that compensation for this cellular stress may be 
occurring through the up regulation of the PI3K survival pathway and may explain a reduction in key pro-apoptotic factors well as increases in the pro-angiogenic factor VEGFa, particularly in secondary and antral follicles. The up regulation of this key signaling pathway in the ovary may explain a loss of primordial follicles through accelerated follicle recruitment, with a concomitant loss of antral follicles through ER stress and inflammation. Thus, this may be one mechanism underlying the link between early life undernutrition and accelerated postnatal ovarian follicle loss in UN offspring.

Disclosure of Interest: None Declared.

\section{DOHaD13-1489}

Metabolic programming, lipid and hormonal profile and weight of male reproductive organs of animals fed with a diet rich in soybean oil

Celly Cristina Nascimento-Saba ${ }^{1, *}$, Cynthia C. Silva ${ }^{1}$, Aline S. Santos ${ }^{1}$, Eliane G. Aguiar ${ }^{1}$, Rejane P. Gaspar-Reis ${ }^{1}$

${ }^{1}$ Physiological Sciences, STATE UNIVERSITY OF RIO DE JANEIRO, Rio de Janeiro, Brazil

Background: Amount of body fat stored is known to influence fertility, indicating a link between adipose tissue and the reproductive system, but the exact mechanism connecting these processes is still not fully known (1). The excess of lipids on diet influences metabolism and affects testis reproductive function. The concept of metabolic programming is a permanent change related to a particular function, as a result of some event which occurs during the perinatal period (2). Several experimental animals have shown that hormones as leptin and nutrition status; which affect development during sensitive periods early in life, permanently program the structure and function of body tissues and systems (3-5). Thus, the aim of the study was evaluate the effect of high fat diet, containing soybean oil, on weight organs and on body composition and male reproductive system of young rats.

Method: Male Wistar rats, at 21days, received diet containing 7\% (control diet) or 19\% (high-fat diet, HF) of soybean oil, after the weaning until 30 and 60 days of age. Food intake and body mass were monitored during all experimental period. At the end, body composition was evaluated by DEXA and blood, liver, adipose tissue, testis and epidydimis were collected. Triglycerides (TG), cholesterol, HDL, VLDL, insulin, leptin and testosterone were assessed. Qualitative characteristics of the testis were verified by morphometry.

Results: Food intake, body mass gain, total fat mass and bone mineral content did not differ between the groups at 30 and 60 days. At 30 days, glycemia (164.5 \pm 9.80 vs $177.3 \pm 14.19)$ cholesterol $(60.2 \pm 2.25$ vs $63.5 \pm 2.72)$, HDL (21.0 \pm 2.97 vs $40.0 \pm 3.0)$, VLDL $(10.45 \pm 1.06$ vs $12.0 \pm 1.58)$ and testosterone $(1.35 \pm 1.09$ vs $10.89 \pm 1.12)$ were increased in rats fed with $\mathrm{HF}$, while TG was low. At 60 days, glycemia $(183.5 \pm 1.93$ vs $284.8 \pm 16.28)$, TG (71.74 \pm 8.72 vs $73.83 \pm 4.85)$, the mass of mesenteric adipose tissue $(2.15 \pm 0.16$ vs $2.82 \pm 0.21)$, the mass of subcutaneous adipose tissue $(1.82 \pm 0.15$ vs $2.73 \pm 0.30)$, the mass of epidydimal adipose tissue $(2.90 \pm 0.07$ vs $3.14 \pm 0.10)$, liver $(8.40 \pm 0.14$ vs $10.45 \pm 0.55)$ and epidydimis $(0.30 \pm 0.18$ vs $0.41 \pm 0.02)$ were high after $\mathrm{HF}$ ingestion. At 30 and 60 days the leptin and the testis weight were higher after $\mathrm{HF}$ ingestion, $(0.85 \pm 0.13$ vs $1.45 \pm 0.16),(1.57 \pm 0.06$ vs $2.53 \pm 0.18)$ and $(0.37 \pm 0.03$ vs $0.46 \pm 0.04),(1.55 \pm 0.03$ vs $1.66 \pm 0.05)$ respectively. The testis area $(31.87 \pm 0.98$ vs $37.17 \pm 1.97)$ was increased at 60 day, while the seminiferous tubules $(102.7 \pm 5.29$ vs $79.75 \pm 4.21)$ decreased.

Conclusion: The consumption of HF promoted hyperglycemia and excess of lipid reflecting negatively on intraabdominal adipose tissue from 30 days. It maintained hyperglycemia, hyperleptinemia without insulin alteration. At the same time, it could predict some testis function change, once epidydimis and testis mass increased, as well as, the results of morfometry in these young animals.

\section{References}

1. Frisch RE. Ballières Clin Gynaecol. 1990;4(3):419-39.

2. Molina-Carballo A, Fernandez-Tardaguila E, UberosFernandez J. Horm Res. 2007;68:11-9.

3. Fischbeck KL, Rasmussen KM. J Nutr. 1987;117(11): 1967-75.

4. Dörner G, Plagemann A. Horm Metab Res. 1994;26(5): 213-21.

5. Cravo CO, Teixeira CV, Passos MC, Dutra SC, Moura EG, Ramos C. Horm Metab Res. 2002;34(7):400-5.

Disclosure of Interest: None Declared.

\section{DOHaD13-1379}

Sexual orientation in adults born preterm - the Ester Study Eero Kajantie $^{1,2,3, *}$, Marika Sipola-Leppänen ${ }^{1,4}$, Marjaana Tikanmäki ${ }^{1}$, Hanna-Maria Matinolli ${ }^{1}$, Anu-Katriina Pesonen $^{5}$, Satu Miettola ${ }^{1}$, Anneli Pouta ${ }^{1}$, Marjo-Riitta Järvelin ${ }^{4,6}$, Katri Räikkönen ${ }^{5}$, Marja Vääräsmäki ${ }^{1,3}$

${ }^{1}$ National Institute for Health and Welfare, Helsinki and Oulu; ${ }^{2}$ Children's Hospital, Helsinki University Central Hospital and University of Helsinki, Helsinki; ${ }^{3}$ Department of Obstetrics and Gynaecology, Oulu University Hospital; ${ }^{4}$ Department of Health Sciences, University of Oulu, Oulu; ${ }^{5}$ Department of Behavioural Sciences, University of Helsinki, Helsinki, Finland; ' Imperial College, London, United Kingdom

Background: Sexual orientation, an individual's sexual preference to opposite or own sex, may be in part programmed through exposure of the fetal and infant brain 
to sex hormones. However, human evidence is scarce. Preterm birth is associated with altered hormonal exposure both before and after birth, and women and men born preterm have as adults less children than those born at term. We hypothesised that women and men born preterm have higher rates of homosexual orientation.

Method: The subjects participate in the Ester study, a casecontrol birth cohort study of people born in 1985-1989 in the two northernmost provinces of Finland. The study includes three groups: those born before 34 weeks of gestation (" $<34$ weeks"), those born between 34 and $<37$ weeks ("late preterm") and at 37 weeks of more ("at term"; controls). At present we have data ready for 500 subjects (268 women): 106 (59) born <34 weeks, 162 (83) late preterm and 232 (126) at term. The subjects completed the Klein Sexual Orientation Scale, in which the subject is asked to rate on a 7 point scale to what extent she/he rates her/himself as homosexual or heterosexual. Our primary outcome was predominantly homosexual identity (rating 1 to 3 ). Data were analysed by logistic regression, adjusted for sex, age, birth weight SD score, parental education and maternal age at birth and parity.

Results: 7 women (2.6\%) and 3 men (1.3\%) rated themselves as predominantly homosexual. Of these women, 5 were born $<34$ weeks, 1 late preterm and 1 at term; corresponding numbers for men were 0,2 and 1 . As compared with those born at term, adjusted odds ratio (aOR) for a predominantly homosexual rating in both sexes combined was 5.7 (95\% CI 1.1 to 29.7$)$ for those born $<34$ weeks and 2.2 (0.4 to 13.4) for those born late preterm. For each week lower length of gestation, aOR was 1.18 (1.03 to 1.34). Among women, corresponding aORs were 12.2 (1.3 to 118.1$)$ for $<34$ weeks, 1.7 (0.1 to 29.6) late preterm and 1.26 (1.07 to 1.48$)$ for each week lower length of gestation. The small numbers in men did not allow a meaningful analysis.

Conclusion: Our preliminary report suggests that women born preterm are more likely to identify themselves as predominantly homosexual than women born at term. If sustained, the finding adds to the scarce human evidence on fetal or infant programming of human sexual orientation.

Disclosure of Interest: None Declared.

\section{DOHaD13-1590}

Transgenerational effects of maternal high fat diet on offspring reproductive cyclicity and ovarian function

\author{
Michael Tsoulis ${ }^{1}$, Mark H. Vickers ${ }^{2,3}$, Caroline Moore ${ }^{1}$, \\ Kristin L. Connor ${ }^{4}$, Deborah M. Sloboda ${ }^{1, *}$ \\ ${ }^{1}$ Biochemistry and Biomedical Sciences, McMaster University, \\ Hamilton, Canada; ${ }^{2}$ Liggins Institute, University of Auckland; \\ ${ }^{3}$ Gravida: National Research Centre for Growth and Development, \\ Auckland, New Zealand; ${ }^{4}$ Samuel Lunenfeld Research Institute, \\ Mt Sinai Hospital, Toronto, Canada
}

Background: We have recently shown in a rat model that female offspring born to mothers fed a high-fat (HF) diet throughout pregnancy and lactation enter puberty early, have increased prepubertal fat mass and display aberrant reproductive cyclicity. As oxidative stress and inflammation are known contributing factors to ovarian dysfunction, we investigated whether maternal HF diet predisposes offspring to ovarian oxidative stress and inflammation in a manner that disrupts reproductive cyclicity. Further, since gametes of the next generation (F2) are present during HF exposure (F0), we hypothesized that reproductive compromise and impaired ovarian function may persist into future generations.

Method: Three generations of rats are currently being investigated. F0 pregnant rats were fed a control (Con) diet or a HF diet during pregnancy and lactation. Offspring were fed a control diet at weaning and as adults were bred with control males for 3 generations; offspring were fed either a HF or a control diet for 2 of the three generations. Within each generation, maternal and offspring growth, metabolic indicators (plasma insulin, leptin) were analyzed, and offspring cytokines (TNFa, IL6) and ovarian oxidative stress markers (4HNE, SOD2) were investigated using Western blotting and ELISA.

Results: F0 HF-fed mothers were fatter, hyperinsulinemic and hyperleptinemic compared to Con. HF-F1 mothers were also fatter and hyperleptinemic at weaning, despite being fed a control diet. Offspring of HF-fed F0 mothers had prolonged estrous cycles and offspring of HF-fed F1 mothers tended to have irregular estrous cycles. Preliminary work shows that these changes are not associated with changes in circulating or ovarian levels of TNFa, and IL6. Ovarian protein levels of $4 \mathrm{HNE}$ and SOD2 were similar between groups in the $\mathrm{F} 1$ generation.

Conclusion: Maternal high-fat diet compromises offspring reproduction and adaptation to pregnancy producing F2 offspring with reproductive abnormalities although these effects were absent in the F3 generation. F1 generation reproductive dysfunction was not associated with elevations in ovarian levels of TNFa, IL-6, SOD2, or 4-HNE suggesting that these factors, at the level of the ovary, are not driving the observed reproductive cycle irregularities. Ongoing analyses are investigating other markers of oxidative stress, as well as evaluating ovarian follicle populations and sex steroid regulation.

Disclosure of Interest: None Declared.

\section{DOHaD13-1471}

Aliskiren in early postnatal life prevents hypertension and reduces asymmetric dimethylarginine in offspring exposed to maternal caloric restriction

You-Lin Tain ${ }^{1, *}$, Chien-Ning $\mathrm{Hsu}^{2}$, Chien-Te Lee ${ }^{3}$, Chih-Min Tsai ${ }^{1}$, Li-Tung Huang ${ }^{1}$ 
${ }^{1}$ Pediatrics; ${ }^{2}$ Pharmacy; ${ }^{3}$ Nephrology, Kaohsiung Chang Gung Memorial Hospital and Chang Gung University, College of Medicine, Taiwan, Kaohsiung, Taiwan

Background: Asymmetric dimethylarginine (ADMA), an endogenous inhibitor of nitric oxide synthase (NOS), and renin-angiotensin system (RAS) are involved in the development of hypertension. We recently found that 3-month-old offspring of dams exposed to $50 \%$ caloric restriction (CR) during late pregnancy and lactation had low nephron number, increased ADMA, and increased blood pressure (BP) (Tain et al., 2010). Also, we fount that early aliskiren treatment can reduce ADMA, enhance NO bioavailability, and ameliorate the development of hypertension in young spontaneously hypertensive rats (Tain et al., 2011). Given that blockade of RAS by ACE inhibitor or AT1 receptor blocker early in life can offset the effects of nutritional programming on BP (Sherman et al., 2000), we tested whether renin inhibitor aliskiren can reduce ADMA and regulate RAS components, to prevent the development of hypertension in offspring exposed to maternal CR. RAS is critical in nephrogenesis and RAS genes can be epigenetically controlled via histone deacetylases (HDACs). We also intended to elucidate whether type I HDAC isoforms are differentially regulated by $\mathrm{CR}$ and aliskiren in this study.

Method: Four groups of male offspring ( $\mathrm{n}=5-7 /$ group) were sacrificed at 12-weeks old of ages: control, caloric restriction (CR), CR + aliskiren (CR + A), and CR + losar$\tan (\mathrm{CR}+\mathrm{L})$ group. CR group recruited male offspring from $50 \%$ food-restricted maternal SD rats received $11 \mathrm{~g} / \mathrm{d}$ of a standard chow from day 11 of pregnancy until the day of delivery (day 23) and $20 \mathrm{~g} / \mathrm{d}$ of the same chow during the entire lactation period. $C R+A$ group was conducted by treating CR offspring with oral aliskiren $10 \mathrm{mg} / \mathrm{kg} /$ day via gastric gavage between 2 and 4 weeks of age. Third group of $\mathrm{CR}$ offspring $(\mathrm{CR}+\mathrm{L})$ was conducted by treating $\mathrm{CR}$ offspring with oral losartan $20 \mathrm{mg} / \mathrm{kg} /$ day via gastric gavage between 2 and 4 weeks of age. This group was used as antihypertensive control.

Results: Blood pressure increased in CR rats, which was prevented by aliskiren or losartan. CR reduced L-arginine levels, increased ADMA levels, and reduced L-arginine-toADMA ratio in the plasma. These changes were prevented by aliskiren therapy. Renal renin and prorenin receptor expression increased in CR rats treated with aliskiren, while both were reduced by losartan therapy. Both aliskiren and losartan treatment decreased renal mRNA expressions of angiotensinogen (AGT), angiotensin II type 2 receptor (AT2R), and Mas in CR rats. However, aliskiren increased protein levels of AT2R and Mas in the CR kidneys.

Conclusion: We conclude that early blockade of RAS by aliskiren or losartan both prevents maternal CR-induced programmed hypertension. The protective effects of aliskiren on CR-induced programmed hypertension include reduction of ADMA, decreases of AGT expression, and increases of renal AT2R and Mas protein. Next, CR upregulated renal HDAC-1 expression, which losartan prevented. However, aliskiren therapy increased renal expression of HDAC-2, -3 , and -8 in CR offspring.

Disclosure of Interest: None Declared.

\section{DOHaD13-1517}

Continued protein restriction during lactation protects adult, one year old offspring from the detrimental metabolic effects of gestational low protein diet exposure

Anil Dmello ${ }^{1, *}$, Rani Qasem ${ }^{1}$, Hee Man Tang ${ }^{1}$, Jing $\mathrm{Li}^{1}$, Laura Pontiggia $^{1}$

${ }^{1}$ UNIVERSITY OF THE SCIENCES IN PHILADELPHIA, Philadelphia, United States

Background: Gestational exposure to low protein diet produces metabolic abnormalities in the adult offspring. Recent studies show that exposure to low protein diet selectively during lactation results in offspring with lower body weight at weaning, and young adult offspring characterized by lower body weight and adiposity, increased insulin sensitivity, and resistance to high fat diet induced obesity. While selective protein restriction during lactation programs a healthy phenotype, it is not known if this nutritional intervention can alleviate the detrimental effects of gestational low protein diet exposure. The objective of the present study was to determine if protein restriction during lactation can protect older, one year old animals from the adverse metabolic effects and increased susceptibility to high fat diet induced obesity produced by gestational low protein diet exposure.

Method: Pregnant rats were exposed to control (C, 19\% protein) or low protein (LP, $8 \%$ protein) diets throughout gestation and lactation. Upon parturition pups in all groups were weighed and litters culled to 8 pups. On day 28 , male pups were weaned on to laboratory chow. At one year of age, basal food consumption, body weight, and body composition were measured. Serum levels of glucose, insulin, leptin, and lipid fuels were measured in the fed state and after a 24 hour fast. Next, offspring in both groups were subject to a leptin sensitivity test conducted by the sequential administration of i.p. injections of saline and leptin $(1 \mathrm{mg} / \mathrm{kg})$ and the measurement of food consumption in discrete intervals for 24 hours. Finally, offspring were fed a short term high fat diet and daily body weight gain and food consumption measured for 14 days. At the end of the high fat diet feeding period serum levels of glucose, insulin, leptin, and lipid fuels were measured in the fed and fasted states.

Results: Under basal laboratory chow feeding conditions, male LP offspring exhibited hyperphagia that was mediated by lower leptin sensitivity. Despite the hyperphagia, LP offspring had lower body weight and similar $\%$ fat mass and 
\% lean mass compared to $\mathrm{C}$ offspring. The lower areal bone density and bone mineral content of LP offspring are likely evidence of higher sympathetic nervous system (SNS) drive. Insulin sensitivity and serum levels of lipid fuels in the fed and fasted state were similar between C and LP offspring. Upon high fat diet feeding, there were no differences in food consumption and body weight gain between $\mathrm{C}$ and LP offspring. Chronic high fat diet feeding increased adiposity and produced the expected deterioration in insulin sensitivity and alterations in lipid fuel profile but there were no differences between $\mathrm{C}$ and LP offspring.

Conclusion: In one year old male offspring, protein restriction during lactation did not protect against hyperphagia produced by gestational exposure to low protein diet probably because neural circuits for appetite are established during gestation. However, continued protein restriction during lactation protected LP offspring against increased adiposity, deterioration of insulin sensitivity, and increased susceptibility to high fat diet induced obesity possibly by programming an increased SNS drive.

Disclosure of Interest: None Declared.

\section{DOHaD13-1069}

Dietary intake of alpha-linolenic (omega-3) fatty acid in childhood and improved cardiovascular risk profile in those born small for gestational age: the STRIP study

Michael Skilton ${ }^{1, *}$, Katja Pahkala ${ }^{2}$, Jorma Viikari ${ }^{2}$, Tapani

Rönnemaa ${ }^{2}$, Olli Simell ${ }^{2}$, Antti Jula ${ }^{3}$, Harri Niinikoski ${ }^{2}$, David Celermajer ${ }^{4}$, Olli Raitakari ${ }^{2}$ on behalf of STRIP Study Group

${ }^{1}$ Boden Institute, University of Sydney, Sydney, Australia;

${ }^{2}$ University of Turku; ${ }^{3}$ National Public Health Institute, Turku, Finland; ${ }^{4}$ University of Sydney, Sydney, Australia

Background: Low birth weight is an important risk factor for adult cardiovascular disease, and is associated with higher blood pressure and arterial intima-media thickness, a marker of subclinical atherosclerosis, from infancy through adulthood $(1,2)$. We have previously demonstrated that dietary omega-3 fatty acids, assessed at a single time point in childhood, are associated with lower blood pressure and reduced arterial wall thickness in children born small for gestational age $(3,4)$.

Whether long-term intake of omega-3 fatty acids has beneficial effects on blood pressure and subclinical atherosclerosis in those born small for gestational age, has not previously been determined.

We sought to determine whether dietary intake throughout childhood (from 6 months to 19 years of age) of alphalinolenic acid, a predominantly plant-derived omega-3 fatty acid, is associated with concurrent blood pressure, and whether the average intake of alpha-linolenic acid over this period is associated with reduced aortic intima-media thickness at age 19 years.

Method: STRIP study participants were recruited at age 6 months and followed up every 6-12 months until 19 years of age. Of the 1009 participants with complete birth weight and gestational age data, and at least one blood pressure measure, $115(11 \%)$ were small for gestational age (birth weight $\leq 10^{\text {th }}$ percentile for gestational age and gender). Blood pressure and a 4 day food record were assessed at each study visit. Aortic intima-media thickness was assessed by high resolution ultrasound at 19 years of age. Linear mixed models were used to determine the association of alpha-linolenic acid intake with blood pressure derived from all visits $(n=115)$. Multivariable linear regression was used to determine the association of the average childhood alpha-linolenic acid intake with aortic intima-media thickness at age 19 years $(n=47)$. All analyses were adjusted for sex, energy intake, maternal and paternal age, and intervention group; mixed models were further adjusted for age. Alpha-linolenic acid was $\log$ transformed.

Results: In children born small for gestational age, dietary intake of alpha-linolenic acid was inversely associated with blood pressure. For example, for each $100 \%$ increase in dietary alpha-linolenic acid, systolic blood pressure was $2.0 \mathrm{~mm} \mathrm{Hg}$ lower ([95\% CI 0.8, 3.3]; $P=0.001$ ), diastolic blood pressure was $1.2 \mathrm{~mm} \mathrm{Hg}$ lower ([95\% CI 0.3, 2.2]; $P=0.01)$, and pulse pressure was $1.4 \mathrm{~mm} \mathrm{Hg}$ lower $[95 \% \mathrm{CI}$ $0.3,2.4] ; P=0.01$ ). Furthermore, the average long-term intake of alpha-linolenic acid was inversely associated with aortic intima-media thickness at age 19 years $(-0.32 \mathrm{~mm}$ $[95 \%$ CI $-0.53,-0.10]$ per $100 \%$ higher dietary alphalinolenic acid; $P=0.005$ ).

Conclusion: Dietary alpha-linolenic acid intake during childhood and adolescence is associated with improved blood pressure and lower aortic wall thickness in those born small for gestational age, consistent with reduced risk of cardiovascular events.

\section{References}

1. Skilton MR, et al. Lancet 2005;365:1484-1486.

2. Skilton MR, et al. Arterioscler Thromb Vasc Biol 2011;31:2975-2981.

3. Skilton MR, et al. Hypertension 2013;61:972-976.

4. Skilton MR, et al. Pediatrics 2012;129:e698-e703.

Disclosure of Interest: None Declared.

\section{DOHaD13-1261}

Early life nutrition with a complex lipid matrix diet reverses adverse metabolic programming in IUGR rats

Heike K. Hoyer-Kuhn ${ }^{1}$, Inga Teller ${ }^{2}$, Hella Brönneke ${ }^{3}$, Pia Nosthoff-Horstmann ${ }^{1}$, Annemarie Oosting ${ }^{2}$,

Gregor Lippach ${ }^{1}$, Maria Wohlfarth ${ }^{1}$, Eline M. van der Beek ${ }^{4}$, Jörg Dötsch ${ }^{1}$, Kai D. Nüsken ${ }^{1, *}$ 
${ }^{1}$ Department of Pediatrics and Adolescent Medicine, University of Cologne, Cologne, Germany; ${ }^{2}$ Department of Developmental Physiology \& Nutrition, Danone Research, Centre for Specialised Nutrition, Wageningen, Netherlands; ${ }^{3}$ Cologne Excellence Cluster on Cellular Stress Responses in Aging Associated Diseases (CECAD), Cologne, Germany; ${ }^{4}$ Danone Research, Centre for Specialised Nutrition, Singapore, Singapore

Background: Early feeding with a diet containing a complex lipid matrix of large lipid droplets (CLM Nuturis) and thereby resembling more closely the fat architecture of raw milk, has previously shown to reduce body fat accumulation in mice that were challenged with a Western style diet in later life [1]. Placental insufficiency and concomitant intrauterine growth restriction (IUGR) is associated with a developmental programming of impaired glucose tolerance and visceral adiposity in rat offspring [2]. We hypothesized that in this compromised situation, a formula based diet with CLM would be beneficial for the metabolic phenotype, compared to a control diet (CTRL).

Method: We investigated male offspring of Wistar rat dams that either underwent bilateral uterine artery and vein LIG or sham operation (SOP) on day 19 of their first pregnancy. Offspring of dams without treatment served as controls (C). CLM or CTRL diet was provided for 4 weeks between postnatal days P15 and P42. Both diets were identical except the physical configuration of the lipids. Subsequently, rats were fed with a western style diet (WSD; 20\% fat) for 8 weeks until to P98. An additional control group received AIN-M diet instead of WSD. Thus, seven groups were built: LIGCLM, $\mathrm{n}=8$; SOP-CLM, $\mathrm{n}=10$; C-CLM, $\mathrm{n}=20$; LIGCTRL, $n=9$; SOP-CTRL: $n=10$; C-CTRL, $n=10$; and C-CTRL-AINM, $n=10$. Accumulation of body weight was recorded weekly. Crown-rump-length and body composition were detected via $\mu \mathrm{CT}$ scan on P42 and P98. Fat depots were weighed on P98. Circulating glucose, insulin (ELISA) and leptin (ELISA) were analyzed and compared on P42 and P98. Results: Neonatal body weight and length of LIG and SOP offspring were significantly reduced compared to C offspring. On P98, all groups had similar body weight and length, indicating catch-up growth. Masses of retroperitoneal, mesenterial and epididymal fat, as well as visceral fat mass measured by $\mu \mathrm{CT}$, were increased in LIG-CTRL compared to both C-CTRL and LIG-CLM ( $\mathrm{p}<0.001$ ). Fasted blood glucose and leptin also were increased in LIG-CTRL compared to both C-CTRL and LIG-CLM ( $\mathrm{p}=0.001)$. Fasting insulin concentrations were increased in C-CTRL compared to C-CTRL-AINM ( $p=0.003$ ), the other groups did not show significant differences.

Conclusion: LIG offspring nourished by standard diet in early life shows an altered body composition with increased visceral fat mass, increased fasting glucose, and leptin resistance in later life. Exposure to a CLM based formula diet in early life, counteracts the programming of the adverse metabolic phenotype associated with IUGR. The physical configuration of dietary lipids is crucial to achieve this effect. Our findings underline the potential protective properties of early life nutrition on metabolism throughout life.

\section{References}

1. Oosting A, et al. Size and phospholipid coating of lipid droplets in the diet of young mice modify body fat accumulation in adulthood. Pediatr Res. 2012;72:362-9.

2. Nüsken KD, et al. Uteroplacental insufficiency after bilateral uterine artery ligation in the rat: impact on postnatal glucose and lipid metabolism and evidence for metabolic programming of the offspring by sham operation. Endocrinology. 2008;149:1056-63.

Disclosure of Interest: H. Hoyer-Kuhn: None Declared., I. Teller Employee of: Danone, H. Brönneke: None Declared., P. Nosthoff-Horstmann: None Declared., A. Oosting Employee of: Danone, G. Lippach: None Declared., M. Wohlfarth: None Declared., E. van der Beek Employee of: Danone, J. Dötsch Grant/Research support from: Danone, K. Nüsken Grant/Research support from: Danone.

\section{DOHaD13-1204}

Intra-Amniotic IGF1 treatment of the growth-restricted fetus alters size at birth and perinatal body composition, early postnatal growth velocity and mRNA levels of key somatotrophic genes in liver and muscle

Ana-Mishel Spiroski ${ }^{1,2, *}$, Mark H. Oliver ${ }^{1,2}$, Travis D. Gunn ${ }^{1}$, Anne L. Jaquiery ${ }^{1,2,3}$, Jane E. Harding ${ }^{1}$, Frank H. Bloomfield ${ }^{1,2,3}$ ${ }^{1}$ The Liggins Institute, University of Auckland; ${ }^{2}$ Gravida: National Centre for Growth and Development; ${ }^{3}$ Department of Paediatrics: Child and Youth Health, University of Auckland, Auckland, New Zealand

Background: Fetal growth restriction (FGR) reduces size at birth and often accelerates postnatal growth, with associated altered body composition and increased risk of later disease. Intra-amniotic insulin-like growth factor 1 (IGF1) treatment of FGR in sheep increases in utero growth, but postnatal effects on growth, body composition and the somatotrophic axis are unknown.

Method: Ovine FGR was induced by uteroplacental embolisation. FGR fetuses received $360 \mu \mathrm{g}$ IGF1 (FGRIGF1; $n=27$ ) or saline (FGR-Saline; $n=23$ ) intra-amniotically once weekly for five weeks from $107 \mathrm{~d}$ (term, $148 \mathrm{~d}$ ). Controls $(\mathrm{n}=31)$ were un-operated and un-embolised. 1 week after birth lambs underwent dual X-ray absorptiometry and percutaneous liver and muscle biopsies. mRNA expression of somatotrophic axis genes was measured by quantitative PCR. Data were analysed by ANOVA with Tukey's post hoc test when appropriate and are mean \pm SEM. mRNA data are calculated as fold change relative to 3 housekeeping genes and are significant at $p<0.01$. 
Results: Control females had greater birthweights than FGRSaline lambs, with FGR-IGF1 lambs intermediate $(5.6 \pm 0.4$ vs $4.4 \pm 0.4$ vs $5.2 \pm 0.4 \mathrm{~kg} ; p<0.05)$ and higher plasma IGF1 on d1, with FGR-IGF1 lambs intermediate (127.7 \pm 9.5 vs $78.1 \pm 9.5$ vs $\left.97.0 \pm 8.7 \mathrm{ng} \cdot \mathrm{mL}^{-1} ; p<0.05\right)$. In males, FGR-IGF1 lambs were smallest $(6.5 \pm 0.2$ vs $5.7 \pm 0.2$ vs $5.3 \pm 0.2 \mathrm{~kg} ; \quad p<0.05)$ with the lowest plasma IGF1 $\left(141.6 \pm 10.9\right.$ vs $123.7 \pm 12.7$ vs $102.5 \pm 12.1 \mathrm{ng} \cdot \mathrm{mL}^{-1}$; $p<0.05)$.

FGR-Saline lambs grew fastest in the first week after birth $\left(53.4 \pm 2.2\right.$ vs $65.3 \pm 2.5$ vs $56.7 \pm 2.3 \mathrm{~g} \cdot \mathrm{kg}^{-1} \cdot \mathrm{d}^{-1}$; $p<0.05)$ and FGR-IGF1 lambs grew fastest in the second week $\left(39.3 \pm 1.9\right.$ vs $43.5 \pm 2.2$ vs $46.4 \pm 2.0 \mathrm{~g} \cdot \mathrm{kg}^{-1} \cdot \mathrm{d}^{-1}$; $p<0.05)$. There was no difference amongst groups in measures of body composition at 1 week in females, whereas in males, Controls had greater lean mass than FGR-IGF1 lambs $(7.0 \pm 0.2$ vs $6.3 \pm 0.3$ vs $6.2 \pm 0.2 \mathrm{~kg}$; $p<0.05$ ).

FGR decreased hepatic IGF1 mRNA (19\% in males, 37\% in females,) and decreased muscle IGF1 mRNA $16 \%$ in females. IGF1 treatment increased hepatic $I G F 1(81 \%$ and $26 \%$ ) and muscle IGF1 mRNA in males (21\%) compared with FGR-Saline lambs but decreased expression in females 27\%. FGR increased hepatic IGF1 receptor (IGF1R) mRNA $238 \%$ in females; IGF1 treatment reduced both liver and muscle $I G F 1 R$ mRNA of both males ( 20\%) and females $(\sim 47 \%)$ compared with FGR-Saline.

FGR decreased hepatic glucose transporter 2 (SLC2A2) mRNA in females $(50 \%)$ but not males; IGF1 treatment restored hepatic SLC2A2 mRNA to normal levels in females but decreased levels in males $\sim 33 \%$ compared with both FGR-Saline and Controls. There was no effect of FGR or IGF1 treatment on hepatic growth hormone receptor $(G H R)$ mRNA, but IGF1 treatment decreased muscle GHR mRNA $\sim 30 \%$ in males $40-55 \%$ in females.

Conclusion: Intra-amniotic IGF1 treatment of FGR has sexspecific effects on plasma IGF1, size, body composition and neonatal growth. These may be mediated via tissue-specific effects on the somatotrophic axis. Effects of intra-amniotic IGF1 treatment of FGR on size, body composition, metabolism and the somatotrophic axis in adulthood need to be determined before this could be considered as a potential therapy.

Disclosure of Interest: None Declared.

\section{DOHaD13-1703}

Maternal folic acid intake, offspring kidney development and adult blood pressure

Ryan J. Wood-Bradley ${ }^{1, *}$, Sarah L. Henry ${ }^{1}$, Roger G. Evans ${ }^{1}$, Luise A. Cullen-McEwen ${ }^{1}$, John F. Bertram ${ }^{1}$, James A. Armitage ${ }^{2}$

${ }^{1}$ Monash University, Melbourne; ${ }^{2}$ Deakin, Waurn Ponds, Australia
Background: Maternal dietary protein restriction may programme hypertension and kidney disease. Whilst there is some evidence that folic acid (FA) supplementation of a maternal low protein diet may ameliorate hypertension, effects on kidney development and adult renal function are unknown.

To determine kidney development, nephron endowment adult blood pressure and kidney function in offspring of FA supplemented rats

Method: Female Sprague-Dawley rats ( $\mathrm{n}=5-8$ per group) were fed a control (NP, $18 \%$ casein, $2 \mathrm{mg} / \mathrm{kg}$ FA), low protein (LP, $9 \%$ casein, $2 \mathrm{mg} / \mathrm{kg} \mathrm{FA}$ ) or low protein high folic acid (FA) (LP+FA, 9\% casein, $200 \mathrm{mg} / \mathrm{kg}$ FA) for 3 weeks prior to mating, during pregnancy and lactation. After weaning, offspring were chow fed ad libitum. Branching morphogenesis was assessed at E14.25, after 48h culture. The ureteric epithelium was visualized by cytokeratin immunohistochemistry and branching morphogenesis (branch points $[\mathrm{BP}]$ and tips) manually quantified. At PN21, nephron number was estimated using the dissector/ fractionator. At PN360 glomerular filtration (GFR) and effective renal plasma flow (ERPF) were estimated in anaesthetised rats by radiolabelled $\left[{ }^{3} \mathrm{H}\right]$-inulin and $\left[{ }^{14} \mathrm{C}\right]$-para-aminohippurate clearance. Mean arterial pressure (MAP) and heart rate (HR) were determined by radiotelemetry. Offspring body composition was determined at PN21, 180 and 360 using DEXA. Data were analysed using a mixed linear model, weighted for litter with maternal diet and offspring sex as main factors.

Results: Maternal LP and LP+FA diet did not alter BP or tip number E14.25 compared with NP (BP; $\mathrm{P}_{\text {diet }}=0.49$, tips; $\mathrm{P}_{\text {diet }}=0.49$ ). Maternal LP diet lead to a $26 \%$ and $17 \%$ nephron deficit in female and male offspring compared with $\mathrm{NP}\left(\mathrm{P}_{\text {diet }}<0.05, \mathrm{P}_{\text {sex }}=0.50, \mathrm{P}_{\text {diet }^{*} \text { sex }}=0.40\right) . \mathrm{LP}+\mathrm{FA}$ did not normalise nephron number compared to LP. At PN360 LP and LP+FA offspring did not show altered kidney function compared with NP offspring (GFR $P_{\text {diet }}=0.58$, $\mathrm{P}_{\text {sex }}<0.05, \mathrm{P}_{\text {diet }}{ }^{* e x}=0.15$, ERPF $\mathrm{P}_{\text {diet }}=0.52, \mathrm{P}_{\text {sex }}<0.001$, $\left.\mathrm{P}_{\text {diet }{ }^{*} \text { sex }}=0.43\right)$. Maternal LP and LP+FA diet did not change HR $\left(\mathrm{P}_{\text {diet }}=0.76, \mathrm{P}_{\mathrm{sex}}<0.05, \mathrm{P}_{\text {diet }} *_{\text {sex }}=0.25\right)$ or MAP $\left(P_{\text {diet }}=0.75, \quad P_{\text {sex }}=0.70, \quad P_{\text {diet }^{*} \text { sex }}=0.52\right)$ in LP offspring compared with NP. Maternal LP lead to lighter offspring compared to NP offspring $(\mathrm{P}<0.001)$. Lean muscle (\% mass) and adiposity (\%body weight) were similar between LP, LP+FA and NP offspring. In contrast bone mineral content was reduced by $25 \%$ at $\mathrm{PN} 21\left(\mathrm{P}_{\text {diet }}<0.05\right.$, $\left.\mathrm{P}_{\text {sex }}=0.08, \mathrm{P}_{\text {diet }^{*} \text { sex }}<0.01\right), 14 \%$ at PN180 $\left(\mathrm{P}_{\text {diet }}<0.05\right.$, $\left.\mathrm{P}_{\text {sex }}<0.01, \mathrm{P}_{\text {diet }^{*}{ }^{*} \text { sex }}=0.63\right)$ and $17 \%$ at PN360 $\left(\mathrm{P}_{\text {diet }}<0.05\right.$, $\mathrm{P}_{\text {sex }}<0.05, \mathrm{P}_{\text {diet }}{ }^{*}$ sex $\left.=0.66\right)$ in LP offspring compared with NP but $12 \%$ greater in offspring exposed to LP+FA at PN360 compared with LP $\left(\mathrm{P}_{\text {diet }}<0.05\right)$.

Conclusion: Maternal LP does not appear to impair branching morphogenesis but does delay or impair nephrogenesis. It is yet to be determined exactly when this reduced nephron number is programmed. High maternal supplementation 
of FA did not alter growth patterns, rescue nephron number reduction or change cardiovascular haemodynamics induced by maternal protein intake. The lack of catch up growth may have prevented the onset of high blood pressure and reduced glomerular filtration rate. Interestingly maternal FA intake lead to increased bone mineral content compared to LP offspring. The potential benefits of maternal FA intake need to be further studied with regards to offspring growth and health.

Disclosure of Interest: None Declared.

\section{DOHaD13-1472}

\section{Maternal melatonin treatment prevents programmed hypertension in offspring exposed to maternal caloric restriction}

You-Lin Tain ${ }^{1, *}$, Chien-Ning $\mathrm{Hsu}^{2}$, Li-Tung Huang ${ }^{1}$, Chien-Te Lee ${ }^{3}$

${ }^{1}$ Pediatrics; ${ }^{2}$ Pharmacy; ${ }^{3}$ Nephrology, Kaohsiung Chang Gung Memorial Hospital and Chang Gung University, College of Medicine, Taiwan, Kaohsiung, Taiwan

Background: Hypertension and kidney disease might originate during early life. Pre-, peri-, or post-natal insults can elicit epigenetic alterations in gene for organogenesis, morphological changes, and adaptive physiological responses, leading to chronic disease in later life. Asymmetric dimethylarginine (ADMA), an endogenous nitric oxide synthase (NOS) inhibitor, level is elevated in patients with CKD and hypertension. Using a 50\% maternal caloric restriction (CR) model, we recently found reduced nephron numbers, increased ADMA, and decreased L-arginine to ADMA ratio contribute to the programmed adult kidney disease and hypertension (Tain et al., 2010). Melatonin, an indolamine produced from the pineal gland, and its metabolites are potent antioxidants. We found that melatonin prevents ADMA increases and hypertension concurrently in young SHR (Tain et al., 2010). Therefore, we tested whether melatonin can restore the ADMA-NO balance to prevent the development of hypertension using a $50 \%$ maternal CR model. As development-related genes can be epigenetically controlled via histone deacetylases (HDACs), we also intended to study whether HDAC isoforms are differentially regulated by $\mathrm{CR}$ and melatonin.

Method: Food-restricted maternal SD rats received $11 \mathrm{~g} / \mathrm{d}$ of a standard chow from day 11 of pregnancy until the day of delivery (day 23) and $20 \mathrm{~g} / \mathrm{d}$ of the same chow during the entire lactation period. Another group of CR offspring $(\mathrm{CR}+\mathrm{M}, \mathrm{n}=6)$ was conducted by treating CR mother rats with $0.01 \%$ melatonin solution dissolved in drinking water during the whole period of pregnancy. Control group $(C, n=6)$ consisted of 6 male offspring of maternal rats with free access to standard rat chow. Another group of control rats $(C+M, n=6)$ were with free access to standard rat chow and treated with $0.01 \%$ melatonin in drinking water. All offspring were sacrificed at $12 \mathrm{wks}$ old. Plasma L-citrulline, L-arginine, and dimethylarginine levels were measured by HPLC. Renal mRNA expression of sodium transporter (e.g., NKCC2 and NCC), RAS components (e.g., renin and $\mathrm{ACE}$ ), and HDACs were analyzed by real-time PCR.

Results: We found CR caused elevated plasma ADMA, decreased L-arginine, and decreased L-arginine-to-ADMA ratio (AAR) following maternal $\mathrm{CR}$, which were prevented by melatonin treatment. Next, CR upregulates NHE3 and NCC expressions in the kidney. Melatonin therapy had no effects on sodium transporters. CR had no effects on RAS gene expression, while melatonin treatment upregulated renal expressions of renin, prorenin receptor, and angioteninconverting enzyme (ACE)-2 in melatonin-treated CR offspring. Furthermore, CR upregulated histone deacetylase (HDAC)-2, -3, and -8 expressions in the kidney. Melatonin therapy did not alter renal expression of class I HDACs in $\mathrm{CR}+\mathrm{M}$ vs. $\mathrm{CR}$ group.

Conclusion: We concluded that maternal CR-induced programming hypertension, related to dysregulation of ADMA/NO pathway and sodium transporters in the kidney. Melatonin restored ADMA/NO balance and regulated RAS to prevent CR-induced programmed hypertension. Given that class I HDAC isoforms were regulated by $\mathrm{CR}$, the possible epigenetic regulation of HDAC on RAS and sodium transporters awaits further evaluation.

Disclosure of Interest: None Declared.

\section{DOHaD13-1380}

Ouabain decreases the risk of hypertension and renal damage by rescueing rat nephrogenesis during IUGR

Juan $\mathrm{Li}^{1}{ }^{1 *}$, Liang Chen ${ }^{1}$, Jing Yue ${ }^{1}$, Yali $\mathrm{Hu}^{1}$

${ }^{1}$ Department of Obstetrics and Gynecology, Nanjing Drum Tower Hospital,Nanjing University Medical School, nanjing, China

Background: Intrauterine growth restriction (IUGR) contributing to renal growth retardation results from increased apoptosis and reduction in nephron endowment. Due to this, the offspring of IUGR has a high risk of hypertension and renal disease in later life. In our previous work in vitro study, using explanted rat embryonic kidneys, we found that low doses of ouabain, a specific ligand of Na,K-ATPase(NKA), without inhibiting NKA activity, rescued nephrogenesis in growth factor deprived embryonic rat kidney by triggering a calcium-NF-kappaB signal pathway. Here we report that ouabain rescues rat nephrogenesis during IUGR in vivo study and decreases the risk of offspring's hypertension and renal damage in later life. 
Method: Animals Pregnant Sprague-Dawley rats (SD) were divided into three groups: 1) Normal group(CON); 2) IUGR group(IUGR);3) IUGR+ ouabain group(IUGR + OUA). The off spring was followed up for 18 months. Glomeruli counting The off spring was killed at 15 or 18 months, the left kidney was processed for stereological examination of glomerular number. Blood Pressure (BP) SBP was measured each month by tail plethysmography using an instrument obtained from Japan (Softron). Renal function Blood urea nitrogen (BUN) and creatinine and urinary microalbumin were measured using the VetACE clinical chemistry system7600(Hitachi, Japan). Statistical analysis One-way ANOVA followed post-hoc was applied $(\mathrm{p}<0.05)$.

Results: 1$)$ Birth weight was significantly reduced in IUGR $(6.3 \pm 0.8 \mathrm{~g}, \mathrm{p}<0.05)$ and IUGR + OUA $(7.0 \pm 0.9 \mathrm{~g}, \mathrm{p}<0.05)$ compared with CON $(8.1 \pm 0.9 \mathrm{~g})$. 2) The glomerular number was significantly reduced in IUGR $(23,528 \pm 2015, \mathrm{p}<0.05)$ compared with $\operatorname{CON}(26,621 \pm 2755)$. There was no difference between CON and IUGR+OUA(25,050 \pm 2059$)$ or IUGR and IUGR+OUA.3) The urinary microalbumin showed a significant difference between genders and between CON and IUGR $(p<0.05)$, There was no difference between CON and IUGR + OUA or IUGR and IUGR + OUA. 4) The blood urea nitrogen (BUN) and creatinine showed a significant difference between CON and IUGR $(\mathrm{p}<0.05)$. There was no difference between CON and IUGR+OUA or IUGR and IUGR + OUA.5). From 5 months, the blood pressure was increased accompanied with age increased. There was a difference between genders and male rats' blood pressure increased higher than femals. At the age of 15 months and 18 months, for IUGR, several male rats' systolic blood pressure was higher than $150 \mathrm{mmHg}$ and diastolic blood pressure was higher than $110 \mathrm{mmHg}$. For female rats of IUGR, just several rats' diastolic blood pressure was higher than $110 \mathrm{mmHg}$. For the other two groups, the systolic blood pressure wasn't higher than $150 \mathrm{mmHg}$ and the diastolic blood pressure wasn't higher than $110 \mathrm{mmHg}$.

Conclusion: Our study shows that IUGR results in a lower glomerular number, an increased urinary microalbumin, blood urea nitrogen and creatinine and blood pressure of rat off spring. Ouabain rescues nephrogenesis and decrease the risk of hypertension and renal damage. Thus we have identified a novel medicine by which kidney development can be rescued under IUGR.

\section{References}

1. Li J, et.al. Low doses of ouabain protect from serum deprivation -triggered apoptosis and stimulate kidney cell proliferation via activation of NF-kappaB. J Am Soc Nephrol. 2006 Jul;17(7):1848-57.

2. Li J, et.al. Ouabain protects against adverse developmental programming of the kidney Nature Commun. 2010 Jul $27: 1: 42$.

Disclosure of Interest: None Declared.

\section{DOHaD13-1633}

Paternal lifestyle interventions in obese males restores early embryo development and fetal weights, improving the metabolic health and adiposity status in subsequent female offspring

Nicole McPherson ${ }^{1,2,3, *}$, Hassan Bakos ${ }^{4,5}$, Brian Setchell ${ }^{6}$, Julie A. Owens ${ }^{1,2}$, Michelle Lane ${ }^{1,2,7}$

${ }^{1}$ Obstetrics and Gynaecology; ${ }^{2}$ Robinson Institute; ${ }^{3}$ Freemasons Center for Mens Health, University of Adelaide, Adelaide;

${ }^{4}$ Andrology, Repromed, Dulwich; ${ }^{5}$ Medicine; ${ }^{6}$ Anatomy, University of Adelaide; ${ }^{7}$ Repromed, Adelaide, Australia

Background: Having an obese father impairs embryo development, reduces pregnancy rates and increases the likelihood of an obese child. Rodent models of male obesity have additionally showed increased birth weight and adult onset impaired glucose tolerance and hyperinsulinemia in female offspring. Whether lifestyle interventions in the father to reduce adiposity and improve health can prevent these consequences for pregnancy and the metabolic health of their daughters is unknown.

Method: C57BL6 male mice $(\mathrm{n}=32)$ were fed a control diet (CD 6\% fat) or a high fat diet (HFD; 21\% fat) for 10 weeks before HFD mice were allocated to diet (HC), exercise (HE) or combinded diet/exericse interventions (HCE) or continuation of a HFD (HH) CD for a further 8 wks. Mice fed a CD continued with a $\mathrm{CD}$ during the intervention period. Males were mated with superovulated $\mathrm{CD}$ females for in-vitro embryos and natural cycling CD females for offspring. Ontime embryo development and cell numbers were determine by embryo culture $(\mathrm{n}=300$ per group). At 8 and 17 wks of age female offspring ( $\mathrm{n}=12$ per group) underwent $2 \mathrm{~g} / \mathrm{Kg}$ glucose tolerance testing (GTT), $0.75 \mathrm{IU}$ insulin tolerance testing (ITT) and insulin secretion during a GTT testing (IGT). Gonadal fat $(\mathrm{n}=6$ per group) was collected post-mortem (18wks) and stained with $H \& E$ for determining adipocyte cell size.

Results: Any form of exercise intervention in fathers (HE, HCE) increased ontime blastocysts development $(+14 \%$, $\mathrm{p}<0.05)$ and cell numbers $(+12 \%, \mathrm{p}<0.05)$ compared with $\mathrm{HH}$ fathers, with all interventions having smaller female fetues $(-15 \%, \mathrm{p}<0.05)$ to that of $\mathrm{HH}$ males 5 days post partum. HE female offspring had improved glucose tolerance $(+15 \%, \mathrm{p}<0.05)$ and insulin sensitivity $(+43 \%, \mathrm{p}<0.05)$ at $8 \mathrm{wks}$ persisting to $17 \mathrm{wks}$, with normalisation of hyperinsulinaemia to that of $\mathrm{CD}$ offspring as seen by a reduction in IGT during an GTT $(-39 \%, \mathrm{p}<0.05)$ at 17 wks. Both HE and HC female offspring displayed a reduction in adipocyte cell size $(-42 \%, \mathrm{p}<0.05)$ compared with $\mathrm{HH}$ female offspring.

Conclusion: This is the first study to show that the impaired embryo development, as well as poor metabolic health of female offspring, sired by obese males can be improved by paternal diet and exercise interventions. This suggests that interventions to father's health peri-conceptionly could 
significantly improve pregnancy outcomes and the metabolic health of the next generation.

Disclosure of Interest: N. McPherson: None Declared., H. Bakos Employee of: Repromed, B. Setchell: None Declared., J. Owens Grant/Research support from: NHMRC, M. Lane Shareholder of: Repromed, Grant/Research support from: NHMRC, Employee of: Repromed.

\section{DOHaD13-1083}

Resveratrol prevents hyperleptinemia and central leptin resistance in adult male rats programmed by early weaning

Patricia C. Lisboa $^{1, *}$, Elaine Oliveira ${ }^{1}$, Juliana G. Franco ${ }^{1}$, Natália S. Lima ${ }^{1}$, Nayara Peixoto-Silva ${ }^{1}$, Magna C. Passos ${ }^{1}$, Egberto G. Moura ${ }^{1}$

${ }^{1}$ Physiology, STATE UNIVERSITY OF RIO DE JANEIRO, Rio de Janeiro, Brazil

Background: Obesity and its clustering of cardiovascular risk factors are increasing worldwide. Resveratrol has been shown to improve cardiovascular disease and much speculation about its use in the treatment of obesity has emerged in the last years. In this study, we evaluated the effects of resveratrol on visceral obesity, hyperleptinemia and, insulin and leptin resistance in a developmental plasticity model of obesity in adult early weaned rats.

Method: To induce early weaning, Wistar lactating dams were separated into two groups: early weaning (EW) - dams were wrapped with a bandage to interrupt lactation in the last 3 days of lactation and control (C) - dams whose pups had free access to milk during the entire lactation period (21 days). At 150 days-old, male offspring from EW group were subdivided into: $\mathrm{EW}+$ resveratrol $(30 \mathrm{mg} / \mathrm{kg} / \mathrm{BW})$ during 30 days and EW offspring treated with diluent solution both by gavage. Significant differences had $\mathrm{p}<0.05$ or less.

Results: Resveratrol prevented the higher body weight, hyperphagia, visceral obesity, hyperleptinemia, hyperglycemia, insulin resistance and hypoadiponectinemia at adulthood in animals that were early weaned. Leptin resistance associated with lower JAK2 $(-27 \%)$ and pSTAT3 (-40\%) in total hypothalamus of $\mathrm{EW}$ rats was normalized by resveratrol, and this seems to be independent of SOCS3 normalization. In addition, EW rats showed higher hypothalamic content of NPY $(+39 \%)$ and resveratrol normalized it.

Conclusion: The amount of our data showed that resveratrol prevented the hyperphagia and regulated leptin and adiponectin levels contributing to correction of insulin resistance and central leptin action in adult rats programmed by early weaning. These present data suggest the importance of the future use of resveratrol as therapeutic tool in treating obesity because this compound could modulate fat accumulation and appetite control.

Disclosure of Interest: None Declared.

\section{DOHaD13-1556}

Resveratrol treatment rescues hiperleptinemia and improves central leptin signaling in adult rats programmed by maternal high-fat diet consumption in gestation and lactation

Isis H. Trevenzoli ${ }^{1}$, Juliana G. Franco ${ }^{2}$, Camilla P. Dias-Rocha ${ }^{3}$, Tatiana P. Fernandes ${ }^{3}$, Patricia C. Lisboa ${ }^{4}$, Egberto G. Moura ${ }^{4}$, Carmen C. Pazos- Moura ${ }^{1,1,1, *}$

${ }^{1}$ Instituto de Biofisica Carlos Chagas Filho, FEDERAL UNIVERSITY OF RIO DE JANEIRO; ${ }^{2}$ State University of Rio de Janeiro; ${ }^{3}$ FEDERAL UNIVERSITY OF RIO DE JANEIRO; ${ }^{4}$ Physiology, State University of Rio de Janeiro, Rio de Janeiro, Brazil

Background: Maternal high fat diet consumption during the perinatal period programs for offspring obesity. Obesity is associated with hyperleptinemia but also with the development of hypothalamic leptin resistance and hyperphagia. Resveratrol, which is a polyphenol found in grapes, has been shown to improve several obesity related co-morbidities, including hypertension and insulin resistance. However, the effects of resveratrol on leptin levels and signaling are poorly understood. We hypothesized that obesity programmed by maternal high fat diet consumption during gestation and lactation in rats would be reversed by chronic resveratrol treatment in the adulthood. We further hypothesized that resveratrol would decrease leptinemia and improve leptin signaling in the hypothalamic Arcuate Nucleus of the male adult offspring

Method: Female Wistar rats were divided into two groups: the control group $(\mathrm{C})$, which received a standard diet for rodents ( $9 \%$ of the calories as fat), and a high-fat group (HF), which received a high-fat diet ( $28 \%$ of the calories as fat), during 8 weeks before mating and throughout gestation and lactation. Maternal body fat content was evaluated by DEXA. The $\mathrm{C}$ and HF offspring were fed in the standard diet throughout life. From day 150 until day 180 of life, offspring were orally treated with resveratrol $(30 \mathrm{mg} / \mathrm{Kg}$ body weight/ day) or vehicle (carboxymethylcellulose) by gavage followed by killing. We accessed body weight and body composition, food intake, leptinemia and leptin expression, and hypothalamic leptin signalling (JAK2-STAT3) of the male offspring after treatment. ${ }^{*} \mathrm{p}<0.05$

Results: HF offspring were overweight from weaning to 180 days old, compared to $\mathrm{C}$ group $\left(+17 \%^{*}\right)$. This phenotype was associated with increased subcutaneous and visceral fat mass $\left(+72 \%^{*}\right.$ and $\left.+60 \%^{*}\right)$ without changes in body protein content. Resveratrol treatment decreased subcutaneous $\left(-30 \%^{*}\right)$ and visceral $(-20 \%)$ fat mass. HF offspring were also hyperphagic at adulthood $(+20 \% *)$ but resveratrol treatment did not alter food intake. Interestingly, HF offspring presented severe hyperleptinemia $\left(4\right.$ fold $\left.^{*}\right)$ and increased leptin mRNA expression in the adipose tissue $(2$ fold ${ }^{*}$, that were completely rescued by resveratrol treatment. 
Also, HF offspring had higher content of SOCS3 in the Arcuate Nucleus of the hypothalamus $(+50 \% *)$, which is associated with impairment of leptin signaling. Resveratrol treatment did not change SOCS3 content, but increased STAT3 phosphorylation $(+45 \% *)$, suggesting improvement of the hypothalamic leptin sensitivity in the HF offspring.

Conclusion: Collectively, our data suggest that resveratrol treatment can reverse hyperleptinemia and possibly other obesity co-morbidities programmed by maternal high fat diet consumption during the perinatal period.

Disclosure of Interest: None Declared.

\section{DOHaD13-1078}

Running wheel training reverse programming effects in adult obese rats programmed by maternal prolactin inhibition

Carmen C. Pazos- Moura ${ }^{1, *}$, Gabriel Boaventura ${ }^{2}$, Gustavo Casimiro-Lopez ${ }^{2}$, Elaine Oliveira ${ }^{2}$, Patricia C. Lisboa ${ }^{2}$, Egberto G. Moura ${ }^{2}$

${ }^{1}$ Instituto de Biofisica Carlos Chagas Filho, FEDERAL UNIVERSITY OF RIO DE JANEIRO; ${ }^{2}$ Physiology,

State University of Rio de Janeiro, Rio de Janeiro, Brazil

Background: Maternal prolactin inhibition at the end of lactation programs for metabolic syndrome ${ }^{1}$ and hypothyroidism $^{2}$ in adult offspring, impairing physical performance ${ }^{3}$. We hypothesize that physical training could be a therapeutic strategy to prevent or reverse these conditions.

Method: We evaluated the effect of a running wheel training program of short duration and low intensity in the metabolic and hormonal alterations observed in this model. Lactating Wistar rats were treated with bromocriptine (BRO, $1 \mathrm{mg}$ twice a day) or saline on days 19, 20 and 21 of lactation and offspring training began at 35 days old. They were divided into: sedentary and trained controls (C-Sed, C-Tr); sedentary and trained BRO (BRO-Sed, BRO-Tr) and studied when they were 180 days old. Data were analyzed by two-way ANOVA with the level of significance set at $p<0.05$.

Results: Chronic exercise delayed in 49 days the appearance of weight gain in BRO-Tr offspring. At 180 days, visceral fat mass was higher $(+46 \%)$ in Bro-Sed offspring compared with $\mathrm{C}-\mathrm{Sed}$ and BRO-Tr groups. Running capacity was higher in trained animals as expected. BRO-Sed group showed higher levels of total cholesterol (+32\%), VLDL-c $(+63 \%)$, LDL-c $(+76 \%)$ and triglycerides $(+63 \%)$, while HDL-c levels were lower $(-16 \%)$ when compared with C-Sed. Lipid profile was not altered in $\mathrm{C}-\mathrm{Tr}$ animals, but BRO-Tr group showed lower levels of total cholesterol $(-11 \%)$, LDL-c $(-32 \%)$, VLDL-c $(-26 \%)$ and triglycerides $(-27 \%)$ when compared with Bro-Sed. Insulin resistance index was higher in BROSed group $(+25 \%)$ and normalized with exercise. Thyroid hormones showed a hypothyroid-like behavior in animals from BRO-Sed group showing lower levels for total T3 $(-20 \%)$ and T4 $(-19 \%)$, and training stimulated higher levels of total T3 $(+29 \%)$ when compared with BRO-Sed group. BRO-Sed group showed higher levels of serum corticosterone $(+52 \%)$ that was attenuated by physical training $(-37 \%)$. Chronic exercise did not influence muscle glycogen in $\mathrm{C}-\mathrm{Tr}$ group, however liver glycogen was higher $(+30 \%)$, although it was unchanged in BRO offspring even the trained ones. BRO-Tr animals showed higher plasma LDH (Lactate Dehydrogenase), indicating skeletal muscle cell damage, which suggests intolerance to the training program. Conclusion: Most parameters observed in BRO-Sed offspring consistent with hypothyroidism and metabolic syndrome was reversed in BRO-Tr group. These results show that low intensity and chronic duration training is able to normalize many clinical aspects in BRO animals. However, they could have a lower threshold for exercise adaptation than control rats, since they have impaired liver glycogen and LDH response to exercise.

\section{References}

1. Moura et al., Maternal prolactin inhibition during lactation programs for metabolic syndrome in adult progeny. J Physiol. 587:4919-29, 2009.

2. Bonomo et al., Prolactin inhibition at the end of lactation programs for a central hypothyroidism in adult rat. J Endocrinol. 198:331-7, 2008.

3. Casimiro-Lopes et al., Maternal prolactin inhibition during lactation affects physical performance evaluated by acute exhaustive swimming exercise in adult rat offspring. Horm Metab Res. 44:123-9, 2012.

Disclosure of Interest: None Declared.

\section{DOHaD13-1301}

Taurine supplementation in the setting of maternal obesity partially reverses hepatic pro-inflammatory profiles in neonatal offspring in a sex-specific manner

Minglan $\mathrm{Li}^{1,2, *}$, Clare M. Reynolds ${ }^{1,2}$, Clint Gray ${ }^{1,2}$, Mark H. Vickers ${ }^{1,2}$

${ }^{1}$ Liggins Institute University of Auckland; ${ }^{2}$ Gravida: National Centre for Growth and Development, Auckland, New Zealand

Background: Maternal obesity is strongly associated with detrimental metabolic effects in both mothers and offspring. There is therefore growing interest in identifying intervention strategies that can reverse these programmed effects in offspring and serve to alleviate adverse effects in the mother. Previous studies have shown that maternal taurine supplementation during pregnancy in undernutrition models can improve offspring outcomes. However, the role of taurine supplementation in the setting of maternal obesity models has yet to be determined. The present study examined the effects 
of maternal taurine supplementation on maternal and neonatal outcomes, specifically related to metabolic inflammation and lipid metabolism, using a maternal obesogenic rat model.

Method: Time-mated Wistar rats were randomised to four groups: 1) control group (CON), chow diet during pregnancy and lactation; 2) control taurine group (CT), chow diet with taurine $(1.5 \% \mathrm{w} / \mathrm{v}$ taurine in drinking water); 3) maternal obesogenic diet group (MO), high fat, high fructose diet during pregnancy and lactation; 4) maternal obesogenic diet taurine group (MOT), high fat, high fructose diet with taurine $(1.5 \% \mathrm{w} / \mathrm{v}$ in drinking water). Dam body weight and food intakes were recorded daily. Maternal and neonatal plasma insulin, leptin and pro-inflammatory cytokine concentrations were measured by ELISA. Maternal liver morphology was determined using haematoxylin and eosin staining. Maternal and neonatal hepatic markers related to lipid metabolism and inflammation were measured using RT-PCR.

Results: The MO diet induced maternal hyperinsulinemia, hyperleptinemia hyperglycaemia and increased plasma TNF$\alpha$ concentrations. Taurine supplementation normalised maternal plasma TNF- $\alpha$ concentrations in MOT dams. Both $\mathrm{MO}$ and MOT mothers displayed evidence of fatty liver accompanied by alterations in key markers of hepatic inflammation and lipid metabolism. A marked pro-inflammatory phenotype was also observed in the livers of MOT mothers compared to MO dams with increased TNF- $\alpha$, IL- $1 \beta$ and IL-1R1 expression. Conversely, neonatal offspring from $\mathrm{MO}$ dams displayed a pro-inflammatory hepatic profile which was partially rescued in a sex-specific manner in MOT animals.

Conclusion: These data suggest that maternal taurine supplementation may ameliorate the adverse developmental programming effects in offspring following a maternal obesogenic diet. Interestingly, the observed hepatic inflammatory phenotype induced by taurine supplementation in MOT dams suggests a possible maternal trade-off to protect the neonate.

Disclosure of Interest: None Declared.

\section{DOHaD13-1371}

\section{Association between prenatal PCB exposure and obesity development at age 5 and 7: a prospective cohort study of 656 children from the Faroe Islands}

Jeanett L. Tang-Péronard ${ }^{1,2, *}$, Berit L. Heitmann ${ }^{2,3}$, Helle R. Andersen $^{1}$, Ulrike Steuerwald ${ }^{4}$, Philippe Grandjean ${ }^{1}$, Pál Weihe ${ }^{5}$, Tina K. Jensen ${ }^{1}$

${ }^{1}$ Department of Environmental Medicine, University of Southern Denmark, Odense C, Denmark; ${ }^{2}$ Institute of Preventive Medicine, Copenhagen University Hospital, Frederiksberg, Denmark; ${ }^{3}$ National Institute of Public Health, University of Southern Denmark, Copenhagen, Denmark; ${ }^{4}$ Department of Occupational Medicine and Public Health; ${ }^{5}$ Department of Environmental Medicine, Faroese Hospital System, Tórshavn, Denmark
Background: Increasing evidence suggests that some chemicals with endocrine disrupting abilities (EDCs) can act by promoting obesity and interfere with the body's natural weight-control mechanisms, especially if exposure occur during prenatal life. The objective was to examine the association between prenatal exposure to polychlorinated biphenyls (PCBs) or $p$, $p$ '-dichlorodiphenyldichloroethylene (DDE) and subsequent obesity at 5 and 7 years of age.

Method: From 1997 to 2000656 pregnant Faroese women were recruited. PCB/DDE was measured in maternal serum and breast milk, and the children's weight, height and waist circumference (WC) were measured at clinical examinations at 5 and 7 years of age. Difference between BMI at 5 and 7 years of age (delta BMI) was calculated. Analyses were performed using multiple linear regression models for girls and boys separately, taking into account maternal pre-pregnancy BMI.

Results: For girls at age 7 who had overweight mothers, prenatal PCB exposure in the highest quartile was associated with increased BMI ( $\beta=2.07$; 95\%CI: $0.59,3.55)$, and PCB/DDE exposure in the highest quartile with increased WC (PCB: $\beta=2.48$; 95\% CI $=1.10$, 3.85. DDE: $\beta=2.21$; $95 \% \mathrm{CI}$ : $0.84,3.56)$ as well as an increased delta BMI (PCB: $\beta=1.23$; $\mathrm{CI}=0.42,2.05$. DDE: $\beta=1.11 ; \mathrm{CI}=0.30,1.92)$. An association between $\mathrm{PCB}$ and increased WC was also seen for girls with normal weight mothers $(\beta=1.25$; 95\%CI: 0.04 , 2.45). For boys associations were not significant.

Conclusion: The results suggest that prenatal exposure to PCB and DDE may play a role for subsequent obesity development. Girls whose mothers had a high pre-pregnancy BMI seem most affected

Disclosure of Interest: None Declared.

\section{DOHaD13-1255}

Effect of fetal exposure to bisphenol a on brain mediated by $\mathrm{X}$-chromosome inactivation

Takayuki Kumamoto $^{1, *}$, Shigeru Oshio ${ }^{1}$

${ }^{1}$ Pharmaceutical Sciences, Ohu university, Koriyama, Fukushima, Japan

Background: Recent studies have reported that bisphenol A (BPA) influences brain development in fetal exposure to mice. The X-chromosome codes many neurodevelopmentrelated genes leading to abnormal development, such as mental retardation and intellectual deficiency. For females, most of expressions of $\mathrm{X}$-linked genes are regulated by $\mathrm{X}$ chromosome inactivation (XCI), which occurs during fetal period, and this mechanism is regulated by Xist and its antisense, Tsix. We have already reported that expressions of these genes are changed in fetal exposure to diesel exhaust in a concentration dependent manner in cerebrums (Kumamoto et al., 2013). To clarify the mechanisms of neurodevelopmental disorders by BPA, we exposed BPA to pregnant mice 
and examined mRNA expression of X-chromosome inactivation factor, X-linked neurodevelopment related genes and epigenetical changes of these genes.

Method: Pregnant ICR mice were orally administered at doses of 0.02 or $50 \mathrm{mg} / \mathrm{kg}$ of BPA on gestational days 6 and 15. Pups were sacrificed and the cerebrums were removed on postnatal days 2, 4, 26, and 46. Total RNA was isolated and mRNA expression of XCI-regulating factors (Xist and Tsix), X-linked neurodevelopment related genes (Fmr1, Gdi1, Nlgn3, Pak3 and Ophn1), and sexual differentiation-related genes (ER $\alpha, E R \beta$ and AR) were examined using real-time PCR system. The silence and maintenance mechanism of X-linked genes are epigenetical changes caused by Xist RNA, so that methylated DNA levels of Xist, Tsix, Nlgn3, Gdil were examined by bisulfite and methylation-specific PCR method. Anogenital distance (AGD) and serum estradiol concentration were also examined.

Results: In the $50 \mathrm{mg} / \mathrm{kg}$ exposed-group, reduction in mRNA expressions of Xist, Fmr1, Gdi1, Nlgn3, and Pak3 and increment in that of Tsix were observed simultaneously. In the $0.02 \mathrm{mg} / \mathrm{kg}$ exposed group, moderately reduced mRNA expressions of Xist, Gdi1, Nlgn3 and Pak3 were observed. Methylated DNA levels were not changed. Changes in mRNA expressions of ER $\alpha$, ER $\beta$ and $\mathrm{AR}$, and reduction in both AGDs and estradiol concentrations were observed in the exposure groups.

Conclusion: The present study represents that the fetal exposure to BPA changed expression of XCI-regulating factors. However, methylation levels of XCI-regulating factors and X-linked neurodevelopment related genes were not changed. These results suggest that fetal BPA exposure may alter the expression levels of X-linked neurodevelopment related genes disrupting the XCI mechanism and function, and the effects may mediated by the changes of Xist and Tsix RNA expressions (these genes regulate XCI as noncoding RNA) or changes of histone modifications like H4, H3 Lys9, macro $\mathrm{H} 2 \mathrm{~A}$, and other variants. This X-mediated effect is considered as one of the mechanisms of various BPA-induced neurodevelopmental disorders. This study was partly supported by the Grant-in-Aid for Scientific Research, Challenging Exploratory Research to TK (23651054).

\section{References}

1. Kumamoto et al.: Fetal exposure to diesel exhaust affects $\mathrm{X}$-chromosome inactivation factor expression in mice. J Toxicol Sci. 2013;38(2):245-54.

Disclosure of Interest: None Declared.

\section{DOHaD13-1477}

\section{Effects of perfluoroalkylcarboxylic acids and phthalate metabolite on transcriptional activity Via PPARA And PPARA-V227A Variant}

Hiroyuki Kojima $^{1, *}$, Shinji Takeuchi ${ }^{1}$, Kenichi Takahashi ${ }^{1}$, Fumihiro Sata ${ }^{2}$

\section{${ }^{1}$ Hokkaido Institute of Public Health, Sapporo, ${ }^{2}$ National Institute of Public Health, Wako, Japan}

Background: The activation of peroxisome proliferatoractivated receptor (PPAR) $\alpha$ by a variety of compound groups of diverse structure is deeply involved in lipid homeostasis. PPAR $\alpha$ is highly expressed in liver, heart, muscle, and kidney, and regulates the expression of genes encoding enzymes and proteins involved in the transport and oxidation of fatty acids. The locus encoding PPAR $\alpha$ is polymorphic in humans, and over a dozen missense polymorphisms resulting in amino-acid changes have been described. In particular, the V227A polymorphism at the hinge region of PPAR $\alpha$ has been observed in Japanese populations with relatively high allelic frequencies ${ }^{1)}$. Perfluoroalkylcarboxylic acids (PFCAs) are both hydrophobic and oleophilic and are used in the preparation of protectants and surfactant for nonstick surfaces. The potential health concerns for PFCAs arise from its ubiquitous distribution and persistence in the environment, and its presence in humans and wildlife. The aims of this study were to elucidate the effects of environmental chemicals, such as PFCAs and phthalate metabolite mono(2-ethylhexyl) phthalate (MEHP), on hPPAR $\alpha$-mediated transcriptional activity and to examine the effects of the V227A variant on PPAR $\alpha$ function.

Method: COS-7 cells were transfected with either pcDNAhPPAR $\alpha$ or pcDNA-hPPAR $\alpha$ V227A, pGL4-10xAOXluc2 and $p C M V-\beta G a l$ using the FuGene6 transfection reagent. Then, PFCA, MEHP or ciprofibrate as a positive control was added into the cells. After an incubation period of $24 \mathrm{hr}$, we lysed the cells and measured the firefly luciferase activity of the cell lysate. Luciferase activity was normalized against the $\beta$-galactosidase activity for each treatment ${ }^{2}$. To estimate the receptor activity of compounds, the $20 \%$ relative effective concentration $\left(\mathrm{REC}_{20}\right)$ of the compound was obtained from the dose-response curve as the concentration causing $20 \%$ of the response to $1 \times 10^{-5} \mathrm{M}$ ciprofibrate.

Results: Eleven PFCAs with linear alkyl chains ranging in length from $\mathrm{C}_{4}$ to $\mathrm{C}_{14}$, and MEHP were tested on human PPAR $\alpha$ wild type (WT)- and PPAR $\alpha$ V227A-mediated transcriptional activities. In hPPAR $\alpha$ WT assay, perfluorooctanoic acid (PFOA; $\mathrm{C}_{8}$ ) showed most potent agonistic activity among eleven PFCAs. The activities decreased in a stepwise manner as the alkyl chain was shortened to $\mathrm{C}_{4}$ or lengthened to $\mathrm{C}_{14}$. In addition, the PPAR $\alpha$ agonistic activity of MEHP exhibited approximately 3.7 -fold higher activity than that of PFOA. The $\mathrm{REC}_{20}$ values of PFOA and MEHP were $2.4 \times 10^{-5} \mathrm{M}$ and $6.5 \times 10^{-6} \mathrm{M}$, respectively. This indicates that the PPAR $\alpha$ agonistic activity of PFOA and MEHP was about 20- and 5.4-fold smaller than that of ciprofibrate $\left(\mathrm{REC}_{20} ; 1.2 \times 10^{-6} \mathrm{M}\right)$, respectively. On the other hand, in hPPAR $\alpha$ V227A assay, the above compounds showed agonistic activity similar to that in hPPAR $\alpha$ WT assay.

Conclusion: In this study, we found that PFCAs and MEHP have agonistic activity against PPAR $\alpha$ V227A as well as 
PPAR $\alpha$ WT, although this variant had been reported to be associated with perturbations in plasma lipid levels ${ }^{3)}$ and modulated the association between dietary polyunsaturated fatty acid and high-density lipoprotein cholesterol ${ }^{4)}$.

\section{References}

1. Naito et al., J. Occup. Health, 49, 353-362 (2007).

2. Kojima et al., Toxicology, 280, 77-87 (2011).

3. Naito et al., Pharmacogenet. Genomics, 16, 569-577 (2006).

4. Chan et al., Atherosclerosis, 187, 309-315 (2006).

Disclosure of Interest: None Declared.

\section{DOHaD13-1751}

Endocrine disruptor (bisphenol A) increases proliferation and lipid storage of adipocyte progenitor cells

Mina Desai Ph.D ${ }^{1, *}$, Juanita Jellyman, Ph.D ${ }^{1}$, Guang Han, M.D ${ }^{1}$, Marie H. Beall, M.D ${ }^{2}$, Michael G. Ross, M.D., M.P.H. ${ }^{1}$

${ }^{1}$ Department of Obstetrics and Gynecology, Los Angeles Biomedical Research Institute at Harbor-UCLA Medical Center, Torrance; ${ }^{2}$ Los Angeles Perinatal Association, Los Angeles, United States

Background: The past 40 years has witnessed an epidemic of childhood and adult obesity, attributed in part to programming effects of the in utero environment. During this time period, the use of plastics (e.g., water bottles, food can liners) containing bisphenol A (BPA) has accelerated in parallel to obesity rates. BPA, an endocrine disruptor (EDC) chemical which has significant estrogen receptor agonist effects, is ubiquitous, with significant levels in pregnant women and fetal plasma and amniotic fluid. In vivo and in vitro studies have shown that BPA promotes obesity, and obese adults have higher levels of BPA exposure. We hypothesized that fetal/ newborn BPA promotes the development of programmed obesity via stimulation of adipocyte proliferation and differentiation. We treated primary cultures of newborn preadipocytes from subcutaneous adipose tissue with varying doses of BPA and quantified adipocyte proliferation and differentiation.

Method: At 1 day of age, subcutaneous adipose tissue was collected from control newborns. Preadipocytes were isolated and cultured in standard medium for 5 days, after which cells were treated with BPA $(1,10,20 \mu \mathrm{g} / \mathrm{ml})$ for $72 \mathrm{~h}$. Cell proliferation (MTT assay) was determined and terminal adipocyte differentiation assessed by lipid staining (Oil Red $\mathrm{O})$ and protein expression of transcription factor markers of adipogenesis (PPAR $\gamma, \mathrm{C} / \mathrm{EBP} \alpha$ ) and lipogenesis (SREBP1c). ${ }^{*} \mathrm{P}<0.05$ vs. untreated cells.

Results: Preadipocytes showed dose-dependent increased proliferation in response to BPA (Fig A). Despite no change in the medium lipid content, BPA-exposed adipocytes exhibited dramatic increase in lipid staining (Fig B).
Consistent with this, protein expression of PPAR $\gamma, \mathrm{C} / \mathrm{EBP} \alpha$ and SREBP1c was significantly increased.

Conclusion: Adipocyte progenitor cell exposure to BPA causes increased proliferation and a marked increase in intracellular lipid. Mechanisms for enhanced lipid storage may include upregulation of lipogenic enzymes or BPAmediated differentiation to terminal adipocytes. These results suggest that early developmental exposure to BPA may contribute to programmed adipogenesis and obesity.

Disclosure of Interest: None Declared.

\section{DOHaD13-1228}

Intrauterine growth restriction decreases diuretic response of furosemide in rats during adulthood

Barent N. DuBois ${ }^{1, *}$, Jacob Pearson ${ }^{1}$, Tahir Mahmood ${ }^{1}$, Duc Nguyen ${ }^{1}$, Kent Thornburg ${ }^{2}$, Ganesh Cherala ${ }^{1,2,3}$

${ }^{1}$ Pharmacy Practice, Oregon State University; ${ }^{2}$ Heart Research Center, ${ }^{3}$ School of Medicine, Oregon Health and Science University, Portland, United States

Background: The perinatal environment powerfully influences the risk for chronic disease later in life. The perinatal programming of disease is attributed to morphological and physiological changes in organ systems. Intrauterine growth restriction (IUGR) is highly correlated with decreased nephron number, altered renal function and subsequent hypertension. We hypothesize that such renal maladaptations result in altered pharmacologic patterns for life.

Method: In this study we investigated the diuretic response of furosemide in IUGR rats. Pregnant rats were fed a control (19\%protein) or a low-protein (8\%protein) diet during gestation and lactation to induce IUGR in offspring. At three-months offspring were dosed with furosemide $(2 \mathrm{mg} / \mathrm{kg}$; i.p), and urine collected over a four-hour period. A week later animals were sacrificed, kidneys and liver harvested, and snap frozen for later analysis.

Results: In adult IUGR rats diuresis, renal excretion of furosemide, sodium ion excretion were all significantly reduced relative to controls, indicative of decreased efficacy. The in-vitro renal as well as the excretion of the urinary invivo metabolite was unaltered; however, the in vitro hepatic metabolism was significantly decreased in IUGR males only. There was no detectable difference in the expression of drug transporters responsible for proximal tubule secretion of furosemide. In IUGR rats, creatinine and uric acid clearances were significantly decreased, suggestive of renal insufficiency. We observed in IUGR female rats a significant increase in the expression of sodium-potassium-chloride cotransporter protein. Conclusion: These data indicate an adverse perinatal environment alters furosemide diuretic response. The decrease in efficacy is likely due to impaired renal clearance of furosemide to its site of action at the thick ascending limb 
in the nephron. Given that we observed no increases in metabolism or changes in drug transporter expression, the decrease in creatinine and uric acid clearance suggest altered renal function in IUGR rats which could explain the significant decrease in urinary furosemide excretion. In summary, IUGR could permanently imprint pharmacokinetic processes thus altering drug responses.

Disclosure of Interest: None Declared.

\section{DOHaD13-1197}

\section{Long-term environmental exposure and fetal growth restriction}

Karolina Sulek ${ }^{1,2, *}$, Ting-Li Han ${ }^{1,2}$, Silas G. Villas-Boas ${ }^{2}$, Lesley McCowan ${ }^{3}$, David Wishart ${ }^{4}$, Louise Kenny ${ }^{5}$, Philip N. Baker ${ }^{1}$

${ }^{1}$ Liggins Institute; ${ }^{2}$ School of Biological Sciences; ${ }^{3}$ Department of Obstetrics and Gynaecology, Faculty of Medical and Health Science, University of Auckland, Auckland, New Zealand; ${ }^{4}$ Metabolomics Innovation Centre, University of Alberta, Edmonton, Canada; ${ }^{5}$ The Anu Research Centre, Department of Obstetrics and Gynaecology, Cork University Maternity Hospital, Cork, Ireland

Background: Fetal Growth Restriction (FGR), defined as a fetus which fails to reach its genetic growth potential, is a massive unsolved healthcare problem. FGR babies are more likely to be stillborn, or die after delivery. Survivors are at increased risk of chronic diseases, such as hypertension, diabetes and stroke throughout their lives. We hypothesise that maternal exposure to various environmental factors plays a major role in FGR, but conventional methods (questionnaires and surveys) have failed to identify individual factors. We therefore developed a cutting edge methodology, applying metabolomics to hair samples, to discover environmental factors that women have been exposed to, periconceptually and through their pregnancy (the pregnancy exposome).

Method: Hair samples are ideal for analysis of longer-term environmental exposure. Different sample preparations were used, always pre-treating samples ( $25 \mathrm{mg}$ or $5 \mathrm{mg}$ ) with water and methanol, to remove surface pollutions. Hair metabolites were extracted using either acid or base solutions. Metabolite profiling was based on a Gas Chromatography combined with Mass Spectrometry (GC-MS) platform. Before the GCMS analysis, metabolites were modified by Methyl ChloroFormate (MCF) or TriMethyl Silyl (TMS) derivatization, increasing the volatility of compounds, or analysed without prior chemical modification. Compound identification was based on in-house and commercially available (National Institute of Standards and Technology NIST) libraries.

Results: Base treatment (1M KOH), followed by $70 \%$ water/ methanol extraction and derivatization was shown to be the most promising method for metabolome analysis from hair samples. We were able to extract metabolites even from a very small amount of hair $(5 \mathrm{mg})$, successfully identifying 44 metabolites. This method will be applied to compare normal pregnancies to those complicated by FGR.

Conclusion: We have developed a new method for exposome analysis. This metabolomic-based approach will allow determination of many of the chemicals, pollutants and toxins that a pregnant woman has been exposed to.

Disclosure of Interest: None Declared.

\section{DOHaD13-1276}

\section{Maternal exposure to DEHP and sex hormone levels in cord blood}

Atsuko Araki ${ }^{1}{ }^{1 *}$, Takahiko Mitsui ${ }^{2}$, Chihiro Miyashita ${ }^{1}$, Masahiro Tagawa ${ }^{3}$, Tamie Nakajima ${ }^{3}$, Seiko Sasaki ${ }^{4}$, Tamiko Ikeno $^{1}$, Sachiko Itoh ${ }^{1}$, Emiko Okada ${ }^{4}$, Kazutoshi Cho ${ }^{5}$, Katsuya Nonomura ${ }^{6}$, Reiko Kishi ${ }^{1}$

${ }^{1}$ Center for Environmental and Health Sciences, Hokkaido University; ${ }^{2}$ Department of Urology, Hokkaido University Hospital, Sapporo; ${ }^{3}$ Graduate School of Medicine, Nagoya University, Nagoya; ${ }^{4}$ Hokkaido University Graduate School of Medicine; ${ }^{5}$ Deapartment of Obsterics, Hokkaido University Hospital; ${ }^{6}$ Department of Renal and Genitourinary Surgery, Hokkaido University Graduate School of Medicine,

Sapporo, Japan

Background: Phthalates are a group of industrial chemicals that have been used for a variety of purposes including as a plasticizer. Di(2-ethylhexyl)phthalate (DEHP) is one of the major compounds and has more than $50 \%$ share of the phthalate production in Japan. Animal experimental studies addressed that postnatal exposure to phthalates may induce abnormalities of the reproductive system and reduce less testosterone (T) and insulin-like factor 3 (INSL3). Previous epidemiological studies suggested that fetal exposure to phthalates has adverse impact on genital development. There is one study, which showed that phthalates in breast milk were positively correlated with serum luteinizing hormone (LH)/ T ratio in newborns. The aim of the study is to investigate the association between maternal exposure to DEHP and reproductive hormone levels in cord blood.

Method: The study is a part of a prospective cohort study of pregnant women in Sapporo, Japan (Hokkaido Study/ Sapporo Cohort $)^{1}$. Participants were Japanese women who enrolled at 23-35 weeks of gestation in 2002-2005. Of 1796 potentially eligible women, 514 agreed to participate. Maternal blood samples were collected in late pregnancy, and mono(2-ethylhexyl)phthalate (MEHP), metabolite of DEHP, was measured by GC/MS. The hormone levels in cord blood were measured as following; estradiol (E2), T, and progesterone (P4) by LC/MS/MS, LH, follicle stimulating hormone (FSH), sex-hormone binding globulin (SHBG), 
and prolactin (PRL) by IRMA, inhibin B by ELISA, and INSL3 by EIA. Characteristics of mothers and their infants were obtained from self-administered questionnaires and medical records.

Results: MEHP was detected from all 487 samples and its median (range) level was $0.036(0.007-0.365) \mathrm{nmol} / \mathrm{ml}$. There was no case diagnosed as hypospadias or cryptorchidism. Hormone levels were measured from 295 cord blood; 135 boys and 159 girls (except INSL3, which was measured from all boys and 25 girls). If the levels of hormones were below their detection limits (DL), a half of their DL values were assigned as their hormone levels. Significantly higher levels of T, LH, FSH, T/E2, T/SHBG, inhibin B, and INSL3 were observed in boys than girls. Multivariate regression model was used to examine the associations between $\log 10$ transformed MEHP and hormone levels and the model was adjusted for maternal age, smoking and alcohol consumption during pregnancy, family income, and blood sampling period. Among boys, MEHP was negatively correlated to P4 $(\beta=-0.257: 95 \%$ CI $-0.404,-0.111)$, inhibin $B$ $(\beta=-0.212:-0.325,-0.100)$, and INSL3 $(\beta=-0.083$ : -0.163 , -0.002). Among girls, MEHP was negatively correlated to PRL $((\beta=-0.228:-0.394,-0.062)$.

Conclusion: The results are in accordance with animal data and suggested that DEHP exposure in utero may potentially affect testicular function reducing the levels of inhibin B and INSL3 of fetus. Although our data have several limitations, prenatal DEHP exposure at environmental levels may adversely affect reproductive development.

\section{Reference}

1. Kishi et al. Int J Epidemiol 2011;40:611-618.

Disclosure of Interest: None Declared.

\section{DOHaD13-1626}

\section{Multigenerational effects of air pollution on reproductive capacity and fetal development: a experimental study}

Natália S. X. Costa ${ }^{1, *}$, Sabrina A. Santos ${ }^{1}$, Karina V. Marques $^{2}$, Luiz Fernando F. da Silva ${ }^{1}$, Paulo H. N. Saldiva ${ }^{1}$, Mariana M. Veras ${ }^{1}$

\section{${ }^{1}$ Pathology, University of São Paulo - School of Medicine,} Sáo Paulo, ${ }^{2}$ Federal University of Tocantins, Palmas, Brazil

Background: The intrauterine environment is determinant for fetal development and perturbations that occur during this critical period of life can predispose individuals to later life diseases. Maternal exposure to a variety of substances may impair the normal development of pregnancy. Air pollution (AP), a widespread environment contaminant, is considered a threat to normal fetal development. Data have shown that gestational exposure to urban AP is associated with adverse effects on pregnancy outcome and fetal development, e.g. low birth weight and intrauterine growth restriction. To further explore this, we conducted a multigenerational study in mice to evaluate if fetal development is impaired by gestational exposure to AP.

Method: Mice were exposed to "real world" ambient concentrations of AP, using open top chamber situated close to a high traffic density road. Couples and their litters were continuously (24h/day) exposed inside chambers to either filtered $(\mathrm{F})$ or non filtered air $(\mathrm{nF})$ for 3 generations $(\mathrm{G})$. Couple mediated effects and gestational outcomes were assessed in all G. From each G 5 pregnant females were randomly selected and on gestational day 18.5 euthanized for fetal evaluation. Fetal and placental weight (W) were recorded, major organs (Lung, brain, heart, kidney, liver) were dissected, weighted and processed for histopathological examination. Skeletal development was also evaluated.

Results: The daily mean PM2.5 concentration inside chambers were $6.4 \mu \mathrm{m}^{3}$ (F) and $32.8 \mu \mathrm{m}^{3}(\mathrm{nF}), \mathrm{p}=0.002$. In the $1^{\text {st }} \mathrm{G}$ no effects were observed in couple mediated and gestational outcomes. In the $2^{\text {nd }} \mathrm{G}$ we observed that pregnancy rate $(\mathrm{F}=0.95, \mathrm{nF}=0.55 ; \mathrm{p}=0.003)$ and fetal $\mathrm{W}(\mathrm{F}=0.86 \mathrm{~g}, \mathrm{nF}=0.68 \mathrm{~g} ; \mathrm{p}=0.001)$ were reduced and $\mathrm{a}$ slight reduction (not significant) in the number of pups/litter $(\mathrm{F}=6.8, \mathrm{nF}=5.9)$. Fetal organ $\mathrm{W}$ and the histopathological evaluation did not shown differences between the groups. In the $3^{\text {rd }} \mathrm{G}$ no difference in couple mediated endpoints were observed, however gestation and fertility rate were reduced, the number of pups/litter were decreased and fetal W was markedly reduced $(\mathrm{F}=0.9 \mathrm{~g}, \mathrm{nF}=0.64 \mathrm{~g} ; \mathrm{p}<0.01)$, a mean reduction of $28 \%$. Organ $\mathrm{W}$ were reduced and fetuses seem to present a symmetric decrease in all organs $\mathrm{W}$, on average $24 \%$ less compared to control. Heart and kidneys were the most affected organs (36\% reduction in weight). Evaluation of the fetal growth by measuring femur and craniocaudal length is also reduced on $\mathrm{nF}$ group. Histopathological evaluation demonstrated that kidneys presented reduced numbers of glomeruli; in the lungs, total surface area of saccular structures were decreased; analysis of brain compartments revealed that $\mathrm{nF}$ fetuses presented reduced cortex, hippocampus and cerebellum volumes; also brain ventricles (Lateral, III and IV) were less developed.

Conclusion: The gestational exposure to AP has a relevant impact through generations on the fetal development. Our data suggests that these effects occur in a systemic way, affecting multiple organs that could lead to several health issues on adult life. These data are important if we consider the Barker's hypothesis that postulates that birth weight is determinant on health status later in life; we also have to consider the public health consequences of having a widespread exposure of urban population.

\section{Reference}

1. Veras, MM, et al. Biol Reprod, 2008.

Disclosure of Interest: None Declared. 


\section{DOHaD13-1521}

Multigenerational effects of BPA alone or in combination with genistein and/or vinclozolin on maternal behavior in Wistar rats

Latifa Abdennebi-Najar ${ }^{1, *}$, Sofiane Boudalia ${ }^{2}$, Folia Mireille $^{3}$, Claire Chabannet ${ }^{2}$, Bruno Pasquis ${ }^{2}$, Laurence Decocq Laurence ${ }^{2}$, Raymond Bergès ${ }^{2}$, Marie-Chantal Canivenc-Lavier ${ }^{2}$

${ }^{1}$ UPSP-EGEAL Institut Polytechnique LaSalle, Beauvais;

${ }^{2}$ Centre des Sciences du Gout et de l'Alimentation. UMR6265 CNRS, UMR1324 INRA, Université de Bourgogne, Agrosup;

${ }^{3}$ Centre Hospitalier Universitaire de Dijon, Dijon, France

Background: Inducing changes in sex and social behavior has been identified as neuroendocrine disrupting effects of endocrine disruptors (Frye et al 2012 ${ }^{1}$ ). This study aimed at comparing the multi-generational effects occurred by chronic low dose exposure to bisphenol A (BPA) alone or combined with the estrogen-like genistein $(\mathrm{G} ; 1 \mathrm{mg} / \mathrm{kg} \mathrm{bw})$ and/or the anti-androgen fungicide vinclozolin $(\mathrm{V} ; 10 \mu \mathrm{g} / \mathrm{kg}$ bw) on maternal behavior in F0 and F1 Wistar rat dams.

Method: Endocrine disruptors were daily administered by gavage to F0 pregnant rats from the first day of gestation (G1) until the last day of lactation (LD21) and to F1 female offsring from weaning (PND21) up to the day before mating at adulthood (PND100). Maternal behavior was assessed daily from the parturition to lactational day (LD1-LD8) by observing within the first three hours of the light cycle the occurrence of the following behaviours: - Nursing [Arched, blanket and passive position] - Resting [(Inside/outside) the nest] - Anogenital licking - Self grooming - Pup licking - Pup carrying - Feeding Drinking - Exploration - Rearing - Nest building.

Results: As a main result, maternal behaviour was lowly affected in F0 but strongly decreased in F1 dams. In F0 dams, Nest building and Pup carrying only were decreased by exposure to BPA and the GV and GB mixtures, but not by the GVB mixture. In F1 dams, nursing, nest and pups caring as well as pups grooming were strongly decreased in all groups resting in similar extents than BPA alone.

Conclusion: The present findings indicate that adverse effects of low-dose endocrine disruptors on maternal behaviour were strengthened by an early exposure (F1) as compared to an adult exposure (F0) does. Because of similar responses, the eendocrine properties of each chemical could not predict the endocrine effect of the mixture. The significant effects of BPA alone or in combination on maternal behaviour suggest possible common selective neuroendocrine defects and or epigenetic events.

\section{References}

1. Frye CA, Bo E, Calamandrei G, Calzà L, Dessi-Fulgheri F, Fernández M, Fusani L, Kah O, Kajta M, Le Page Y, Patisaul HB, Venerosi A, Wojtowicz AK, Panzica GC. Endocrine disrupters: a review of some sources, effects, and mechanisms of actions on behaviour and neuroendocrine systems J Neuroendocrinol. 2012 Jan;24(1):144-59.

Disclosure of Interest: None Declared.

\section{DOHaD13-1149}

Prenatal exposure to persistent organic pollutants (POPs) and child neuropsychological development AT 4 years of age

Marina Vafeiadi ${ }^{1}$, Katerina Koutra ${ }^{1}$, Theano Roumeliotaki ${ }^{1}$, Eleni Fthenou ${ }^{1}$, Georgia Chalkiadaki ${ }^{1}$, Maria Kabouri ${ }^{1}$, Andriani Kyriklaki ${ }^{1}$, Panu Rantakokko ${ }^{2}$, Hannu Kiviranta ${ }^{2}$, Soterios A. Kyrtopoulos ${ }^{3}$, Manolis Kogevinas ${ }^{4}$, Leda Chatzi ${ }^{1, *}$

${ }^{1}$ Department of Social Medicine, Faculty of Medicine, University of Crete, Heraklion, Greece; ${ }^{2}$ Department of Environmental Health, National Institute for Health and Welfare, Kuopio, Finland; ${ }^{3}$ Institute of Biology, Medicinal Chemistry and Biotechnology, National Hellenic Research Foundation, Athens, Greece; ${ }^{4}$ Centre for Research in Environmental Epidemiology (CREAL), Barcelona, Spain

Background: The potential adverse effects of environmental chemicals on children's health and development are a matter of widespread public health concern. Persistent organic pollutants (POPs), including polychlorinated biphenyls (PCBs) and pesticides bioaccumulate through the food chain and cross the placenta with potential neurotoxic effects. Despite a growing body of studies investigating the health effects associated with these compounds, their specific effects on early neuropsychological development remain unclear. This study examined the effects of in utero exposure to different POPs on neuropsychological development at 4 years of age in 696 mother-child pairs of the "Rhea" mother-child cohort study in Crete, Greece.

Method: Concentrations of several PCBs and organochlorine pesticides (dichlorodiphenyl dichloroethene [DDE], dichlorodiphenyl trichloroethane [DDT], and hexachlorobenzene [HCB]) were determined by triple quadrupole mass spectrometry in maternal serum samples collected in the first trimester of pregnancy. The psychomotor and mental development of the children was assessed at 4 years of age using the McCarthy Scales of Children's Abilities (MCSA) by two trained psychologists. We defined total PCBs as the sum of the 6 congeners, non dioxin-like PCBs as the sum of PCB $153,138,170$ and 180, and dioxin-like PCBs as the sum of PCB 118 and 156. Moreover we constructed two overall exposure scores (Score 1: HCB, DDE and total PCBs, score 2: HCB, DDE, dioxin-like and non dioxin-like PCBs) based on the concentrations of each pollutant or groups of pollutants that took values from 0 , with all chemicals below the median, to 3 for score 1 and 4 for score 2 with all chemicals above. General additive models were used to 
evaluate the shape of the relationship between organochlorine compounds and neurodevelopmental scores. These models indicated linear relationships for all outcomes (P-gain defined as the difference in normalized deviance between the GAM model and the linear model for the same predictor $<0.05$ ). Multiple linear regression models were used to investigate the associations of in utero exposure to different POPs with MCSA scores.

Results: The highest concentrations were found for DDE $($ median $=1955.1 \mathrm{pg} / \mathrm{ml})$, followed by PCBs $($ median $=$ $321.2 \mathrm{pg} / \mathrm{ml}), \mathrm{HCB} \quad($ median $=82.5 \mathrm{pg} / \mathrm{ml})$, and DDT (median $=25.0 \mathrm{pg} / \mathrm{ml}$ ). After adjusting for confounders, we observed negative relationships between prenatal exposure to HCB and DDE and MCSA scores at 4 years of age, though statistical significant only for $\mathrm{HCB}$, contributing to a 6 points decrease in the scale of perceptual-performance by $10 \mathrm{pg} / \mathrm{ml}$ increase in $\mathrm{HCB}$ (adjusted $\beta=-6.09$; 95\% CI: -11.31 to $-0.87)$. These results were more pronounced in girls and if mothers smoked during pregnancy. We found no statistically significant effects of the sum of prenatal PCBs or individual PCB congeners, and overall exposure scores on neuropsychological development at 4 years of age.

Conclusion: These findings suggest that even low exposure levels to HCB prenatally may have neurotoxic effects on mental development in preschool children, whereas associations with other POPs remain inconsistent.

Disclosure of Interest: None Declared.

\section{DOHaD13-1262}

\section{Relationship between blood polychlorinated biphenyl level and distance towards the nearest fishing port: a birth cohort study}

Masamichi Hanazato $^{1, *}$, Masahiro Watanabe ${ }^{1}$, Emiko Todaka ${ }^{1}$, Yoshiharu Matsuno ${ }^{1}$, Hiroko Nakaoka ${ }^{1}$, Chisato Mori ${ }^{1,2}$

${ }^{1}$ Center for Preventive Medical Science; ${ }^{2}$ Graduate School of Medicine, Chiba University, Chiba, Japan

Background: In previous studies, we have indicated that the blood polychlorinated biphenyl (PCB) level is affected by certain factors including age and past delivery experience. Moreover, seafood is the source of $>80 \%$ of the PCBs in Japan. In the present study, we aimed to examine the relationship between maternal blood PCB level and the distance towards the nearest fishing port.

Method: We measured the serum total PCB levels of pregnant women in Chiba Prefecture who participated in the Japan Environment and Children's Study (JECS; a Japanese national birth cohort study) in 2012. In total, 264 subjects (age range, 18-43 years) from 13 municipalities across suburban and rural areas were included in the study. We measured the total PCB levels from serum by using a gas chromatograph equipped with a packed column and electron capture detector. In the JECS, the geographic information (residential address) of the participants was recorded. Using the geographic information system, we measured the distance towards the nearest fishing port from the residences of the subjects. Based on this distance, the subjects were divided into the short-distance group (residence located within $5 \mathrm{~km}$ from the nearest fishing port) and the long-distance group (residence located within $5-20 \mathrm{~km}$ from the nearest fishing port). The statistical difference was determined using Mann-Whitney's U test.

Results: PCB was detected in 95\% of nulliparous (average PCB level, $0.30 \pm 0.14 \mathrm{ng} / \mathrm{g}$-wet) and $90 \%$ of multiparous subjects (average PCB level, $0.25 \pm 0.12 \mathrm{ng} / \mathrm{g}$-wet). Moreover, we noted that the blood PCB level increased with age. In addition, the blood PCB level was found to be associated with the distance towards the nearest fishing port $(\mathrm{P}<0.05)$. The PCB level was significantly higher in the short-distance group than in the long-distance group $(\mathrm{P}<0.05)$.

Conclusion: The results of the present study indicate that the distance towards the nearest fishing port could indicate the possible high risk group with a high exposure to $\mathrm{PCB}$ and persistent organic pollutants. As the current data of the JECS is speculative and is limited to individuals in Chiba Prefecture, we suggest that geographical information should be used to detect possible high risk group at a high exposure to these substances, in a similar time- and cost-effective manner, in a large-scale cohort study.

Disclosure of Interest: None Declared. 